\title{
Merging Electrosynthesis with 3d Transition Metal-Catalyzed C-H Transformations
}

\author{
Dissertation \\ for the award of the degree \\ "Doctor rerum naturalium" \\ of the Georg-August-Universität Göttingen

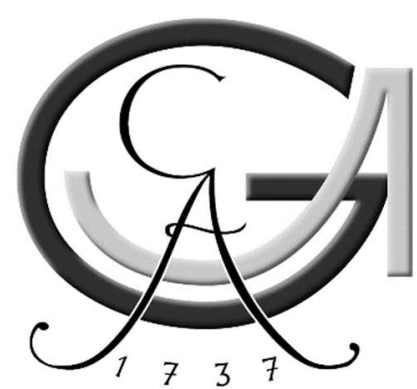

within the doctoral program of chemistry of the Georg-August-University School of Science (GAUSS)

submitted by

Tjark Hannes Meyer

from Leer

Göttingen, 2021 



\section{Thesis Committee}

Prof. Dr. Lutz Ackermann

Institut für Organische und Biomolekulare Chemie

Georg-August-Universität Göttingen

Prof. Dr. Shoubhik Das

Department of Chemistry

University of Antwerp

\section{Members of the Examination Board}

$1^{\text {st }}$ Reviewer: Prof. Dr. Lutz Ackermann

Institut für Organische und Biomolekulare Chemie

Georg-August-Universität Göttingen

$2^{\text {nd }}$ Reviewer: Prof. Dr. Shoubhik Das

Department of Chemistry

University of Antwerp

\section{Further Members of the Examination Board}

Prof. Dr. Konrad Koszinowski

Institut für Organische und Biomolekulare Chemie, Göttingen Jun.-Prof. Dr. Johannes C. L. Walker

Institut für Organische und Biomolekulare Chemie, Göttingen Dr. Holm Frauendorf

Institut für Organische und Biomolekulare Chemie, Göttingen Dr. Daniel Janßen-Müller

Institut für Organische und Biomolekulare Chemie, Göttingen

Date of the Oral Examination: June 28, 2021 



\section{TABLE OF CONTENTS}

1 Introduction 1

1.1 Transition Metal-Catalyzed C-H Activation ....................................................1

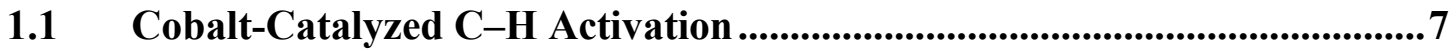

1.1.1 Low-Valent Cobalt-Catalyzed C-H Activations …................................... 7

1.1.2 High-Valent Cobalt-Catalyzed C-H Activations........................................ 11

1.1.3 Oxidative Cobalt-Catalyzed C-H Activation without Cp*-Ligands .............. 13

$1.2 \quad$ Undirected C-H Functionalization......................................................................34

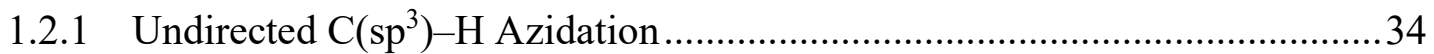

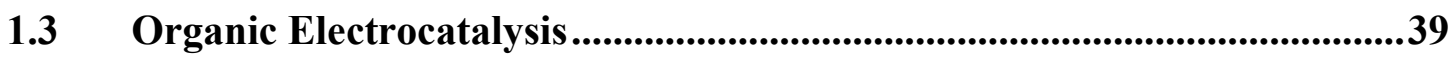

1.3.1 Electrocatalysis: Merging C-H Activation and Electrosynthesis ..................41

1.3.2 Cobaltaelectro-Catalyzed C-H Activation ..............................................4 44

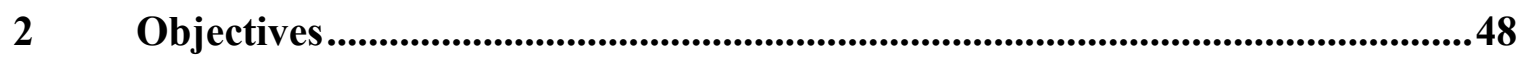

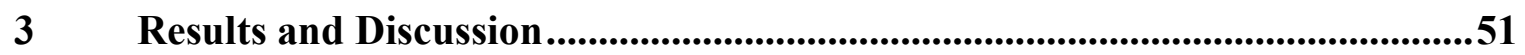

3.1 Cobaltaelectro-Catalyzed C-H Oxygenation ................................................51

3.1.1 Optimization Studies for the Cobaltaelectro-Catalyzed C-H Oxygenation .................................................................................... 51

3.1.2 Substrate Scope of the Cobaltaelectro-Catalyzed C-H Alkoxylation ............57

3.1.3 Mechanistic Studies for the Cobaltaelectro-Catalyzed C-H

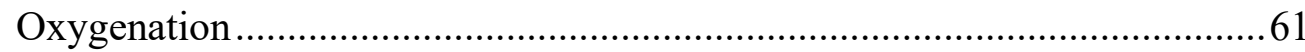

3.2 Cobaltaelectro-Catalyzed C-H Activation for Allene Annulations ...........66

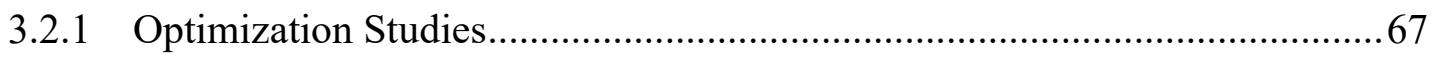

3.2.2 Versatility of the Cobaltaelectro-Catalyzed $\mathrm{C}-\mathrm{H} / \mathrm{N}-\mathrm{H}$ Allene

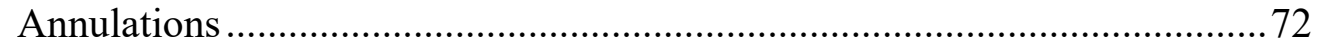

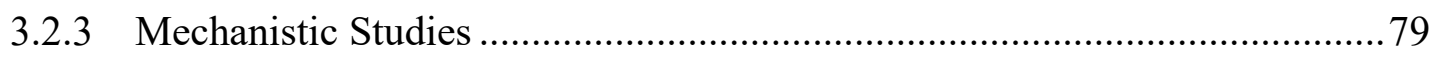

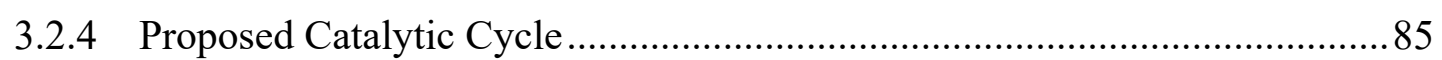

3.2.5 Cobaltaelectro-Catalyzed C-H Allene Annulation in Flow ..........................86

3.3 Cobaltaelectro-Catalyzed $\mathrm{C}-\mathrm{H}$ Activation in Biomass-Derived Glycerol Powered by Renewable Energy Sources .....................................88

3.3.1 Optimization Studies for Cobaltaelectro-Catalyzed C-H Activation in Biomass-Derived Solvents. 


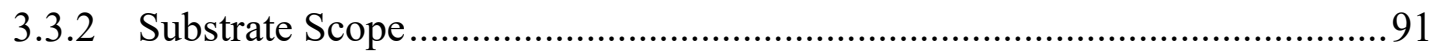

3.3.3 Cobaltaelectro-Catalyzed C-H Activation Powered by Renewable

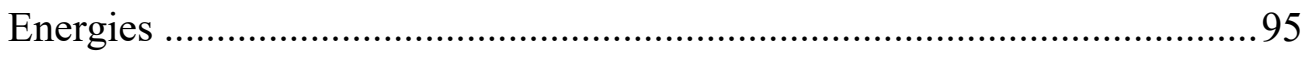

3.4 Mechanistic Insights into Cobalta(III/IV/II)-Electrocatalysis ...................98

3.4.1 Isolation and Characterization of Cobalta(III)cycle 190 ........................... 98

3.4.2 Reaction Monitoring by Mass Spectrometry .............................................. 106

3.4.3 Reactivity of Cyclometalated Cobalt(III) Complex 190 .............................109

3.4.4 Hammett-Zuman Correlation .................................................................. 112

3.4.5 Cobaltaelectro-Catalyzed C-H Arylation via Twofold C-H Scission ........ 115

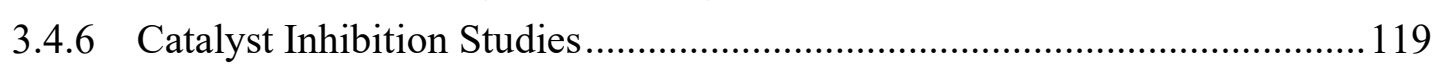

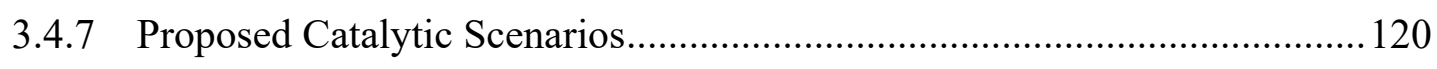

3.5 Mechanistic Studies of Metallaelectro-Catalyzed C-H Activations........123

3.5.1 Mechanistic Studies for Ruthenaelectro-Catalyzed C-H

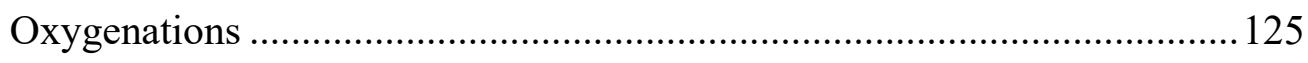

3.6 Manganaelectro-Catalyzed Undirected $\mathrm{C}\left(\mathrm{sp}^{3}\right)-\mathrm{H}$ Azidation....................133

3.6.1 Preliminary Studies toward Metal-Free Electrochemical C-H Azidation of Benzylic C-H Bonds ........................................................133

3.6.2 Optimization Studies for Manganaelectro-Catalyzed $\mathrm{C}\left(\mathrm{sp}^{3}\right)-\mathrm{H}$ Azidation................................................................................ 136

3.6.3 Substrate Scope of the Manganaelectro-Catalyzed C( $\left.\mathrm{sp}^{3}\right)-\mathrm{H}$ Azidation................................................................................ 141

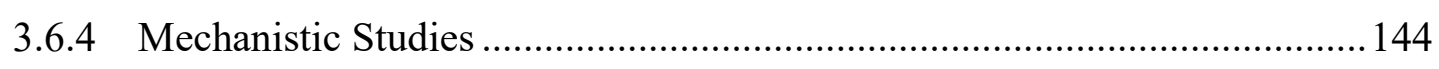

3.6.5 Proposed Mechanism for Manganaelectro-Catalyzed $\mathrm{C}\left(\mathrm{sp}^{3}\right)-\mathrm{H}$

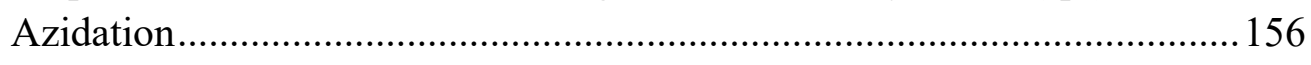

$4 \quad$ Summary and Outlook ..........................................................................................158

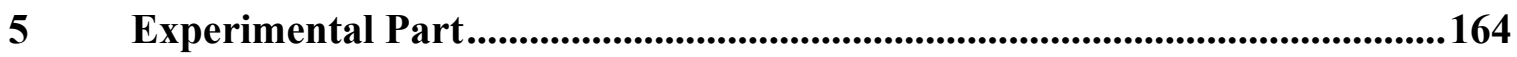

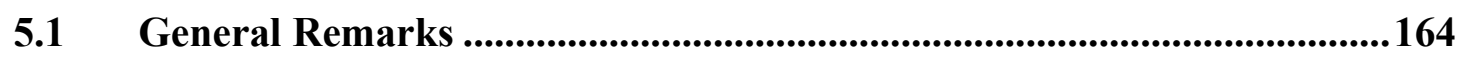

5.1.1 Caution: Experimentation with Organic Azides.......................................... 164

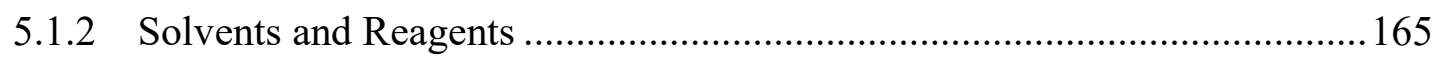

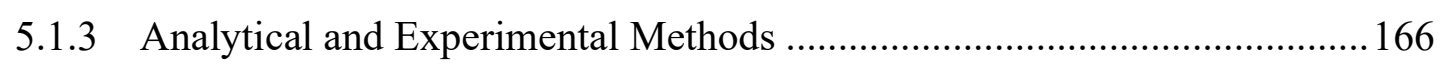

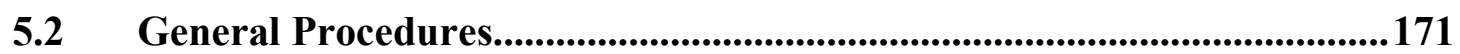

5.2.1 General Procedure A: Cobaltaelectro-Catalyzed C-H Oxygenation in a Divided Cell Setup ......................................................................... 171

5.2.2 General Procedure B: Cobaltaelectro-Catalyzed C-H Oxygenation in an Undivided Cell Setup 
5.2.3 General Procedure C: Cobaltaelectro-Catalyzed C-H Oxygenation in an Undivided Cell Setup

5.2.4 General Procedure D: Cobaltaelectro-Catalyzed C-H Activation for Allene Annulations

5.2.5 General Procedure E: Cobaltaelectro-Catalyzed C-H Activation in Aqueous Glycerol for Alkyne Annulations

5.2.6 General Procedure F: Cobaltaelectro-Catalyzed C-H Activation in Aqueous Glycerol for Allene Annulations

5.2.7 General Procedure G: Electrosynthesis of Cobalta(III)cycle 190................ 173

5.2.8 General Procedure H: Metal-Free Electro C $\left(\mathrm{sp}^{3}\right)-\mathrm{H}$ Azidation

5.2.9 General Procedure I: Manganaelectro-Catalyzed $\mathrm{C}\left(\mathrm{sp}^{3}\right)-\mathrm{H}$ Azidation

5.3 Cobaltaelectro-Catalyzed C-H Oxygenation 175

5.3.1 Characterization Data. 175

5.4 Cobaltaelectro-Catalyzed C-H Activation for Allene Annulations 188

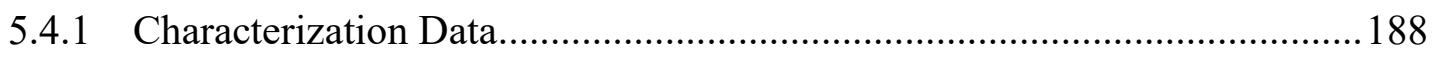

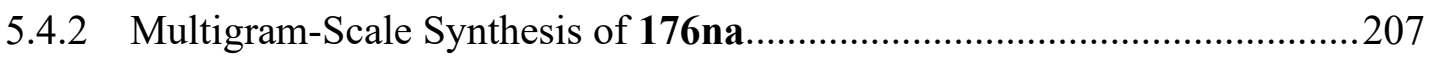

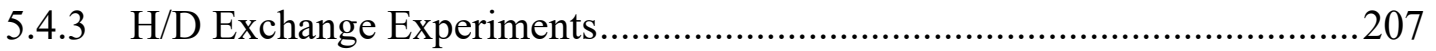

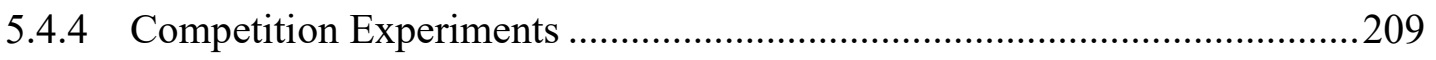

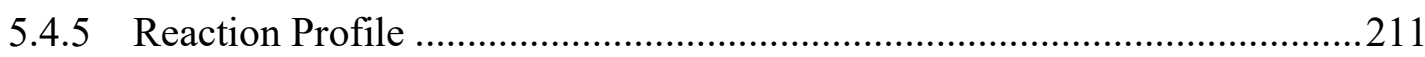

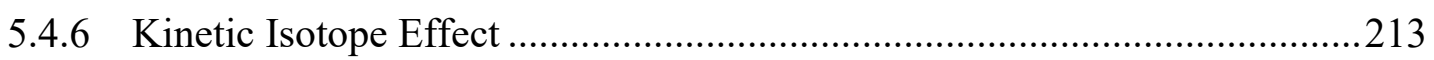

5.4.7 KIE Studies by In-Operando IR Analysis ...............................................2213

5.5 Cobaltaelectro-Catalyzed $\mathrm{C}-\mathrm{H}$ Activation in Biomass-Derived Glycerol Powered by Renewable Energy Sources .....................................215

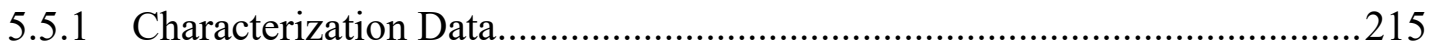

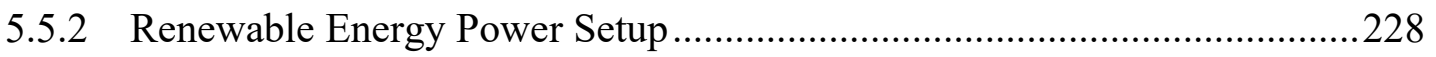

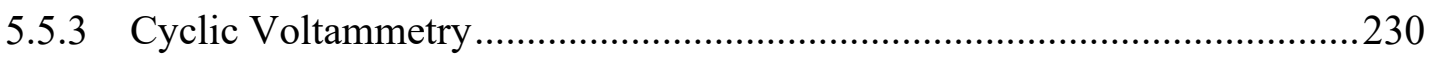

5.6 Mechanistic Insights into Cobalta(III/IV/II)-Electrocatalysis..................231

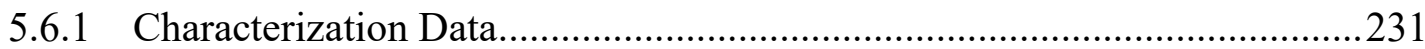

5.6.2 Reaction Monitoring by Mass Spectrometry ...........................................2. 234

5.6.3 Reactivity of Cyclometalated Cobalt(III) Complex 190............................238

5.6.4 Hammett-Zuman Plot ............................................................................. 242

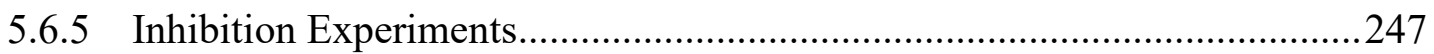

5.7 Mechanistic Studies for Ruthenaelectro-Catalyzed C-H Oxygenation ...................................................................................................2249

5.7.1 Reaction Profile for the Formation of Hypervalent Iodine Reagents ..........249 
5.7.2 Evaluation of the Electron Transfer Number by CV and RDE .................250

5.8 Manganaelectro-Catalyzed Undirected C( $\left.\mathbf{s p}^{3}\right)-\mathrm{H}$ Azidation...................253

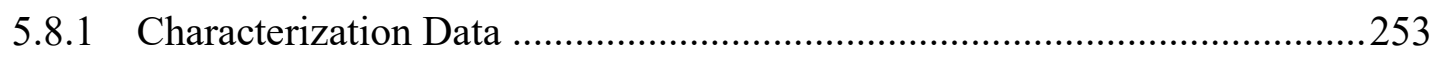

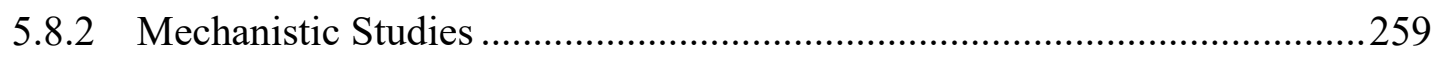

$6 \quad$ Crystallographic Data................................................................................................268

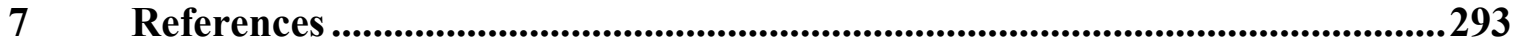




\section{List OF ABbREVIATions}

$\begin{array}{ll}\text { A } & \text { ampere } \\ A & \text { area } \\ \text { A } & \text { angstrom(s) } \\ \text { Ac } & \text { acetyl } \\ \text { acac } & \text { acetylacetonate } \\ \text { alk } & \text { alkyl } \\ \text { Am } & \text { amyl } \\ \text { AMLA } & \text { ambiphilic metal-ligand activation } \\ \text { API } & \text { active pharmaceutical ingredient } \\ \text { aq. } & \text { aqueous } \\ \text { AQ } & \text { 8-aminoquinoline } \\ \text { Ar } & \text { aryl } \\ \text { atm } & \text { atmospheric pressure } \\ \text { ATR } & \text { attenuated total reflectance } \\ \text { BDE } & \text { bond dissociation energy (at 298 K) } \\ \text { BHT } & 2,6 \text {-di-tert-butyl-4-methylphenol } \\ \text { BIES } & \text { base-assisted internal electrophilic substitution } \\ \text { Bn } & \text { benzyl } \\ \text { BQ } & 1,4 \text {-benzoquinone } \\ \text { br } & \text { broad (spectral) } \\ \text { Bu } & \text { butyl } \\ \text { Bz } & \text { benzoyl } \\ \text { C } & \text { Celsius } \\ c & \text { concentration } \\ \text { calcd. } & \text { calculated } \\ c a t . & \text { catalytic } \\ \text { CE } & \text { counter electrode; current efficiency } \\ c f . & \text { confer } \\ \text { CCE } & \text { constant current electrolysis } \\ \text { CMD } & \text { concerted metalation deprotonation } \\ \text { conc. } & \text { concentrated } \\ \text { Cp } & \text { cyclopentadienyl } \\ \text { Cp* } & \text { pentamethylcyclopentadienyl } \\ \text { CPE } & \text { constant potential electrolysis } \\ \text { CV } & \text { cyclic voltammetry } \\ \text { Cy } & \text { cyclohexyl } \\ \delta & \text { chemical shift } \\ \text { d } & \text { doublet (spectral) } \\ D & \text { diffusion coefficient } \\ \text { DCE } & 1,2 \text {-dichlorethane } \\ \text { DCM } & \text { dichloromethane } \\ \text { de } & \text { diastereomeric excess } \\ \text { DFT } & \text { density functional theory } \\ \text { DG } & \text { directing group } \\ \text { DMA } & \text { N,N-dimethylacetamide } \\ \text { DME } & 1,2 \text {-dimethoxyethane } \\ \text { DMF } & \text { N,dimethamide } \\ & \end{array}$




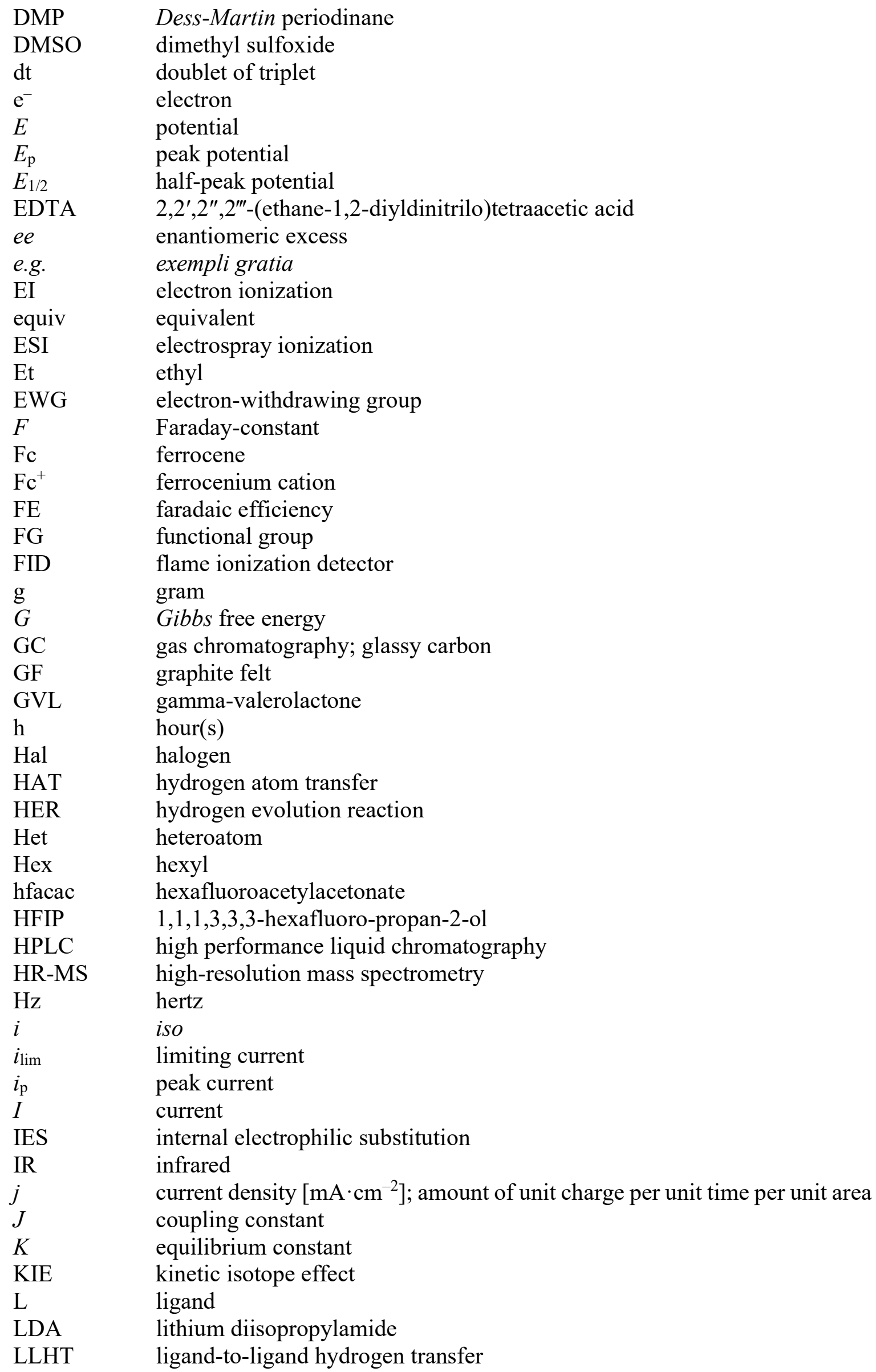




\begin{tabular}{|c|c|}
\hline$\mu$ & micro \\
\hline$\mu$ & dynamic viscosity \\
\hline $\mathrm{m}$ & multiplet (spectral); milli \\
\hline M & molar \\
\hline $\mathrm{M}^{+}$ & parent molecular ion \\
\hline$m$-CPBA & meta-chloroperoxybenzoic acid \\
\hline $\mathrm{mA}$ & milliampere \\
\hline $\mathrm{Me}$ & methyl \\
\hline Mes & mesityl \\
\hline $\mathrm{mg}$ & milligram \\
\hline $\mathrm{MHz}$ & megahertz \\
\hline $\min$ & minute \\
\hline $\mathrm{mL}$ & milliliter \\
\hline mmol & millimole \\
\hline M.p. & melting point \\
\hline MS & mass spectrometry \\
\hline MTBE & methyl tert-butyl ether \\
\hline$m / z$ & mass-to-charge ratio \\
\hline$v$ & scan rate \\
\hline$n$ & number of transferred electrons; amount of substance in mole \\
\hline n.d. & not detected \\
\hline NFSI & $N$-fluorobenzenesulfonimide \\
\hline NMP & $N$-methylpyrrolidinone \\
\hline NMR & nuclear magnetic resonance \\
\hline$o$ & ortho \\
\hline OD & outer diameter \\
\hline$\omega$ & rotation rate \\
\hline$p$ & para \\
\hline $\mathrm{p}$ & pentet \\
\hline PA & picolinamide \\
\hline PCET & proton-coupled electron transfer \\
\hline $\mathrm{Ph}$ & phenyl \\
\hline PIDA & (diacetoxyiodo)benzene \\
\hline PIP & 2-pyridylisopropylamine \\
\hline Piv & pivaloyl \\
\hline PMB & para-methoxybenzyl \\
\hline ppm & parts per million \\
\hline $\operatorname{Pr}$ & propyl \\
\hline Py & pyridyl \\
\hline pym & pyrimidyl \\
\hline $\mathrm{PyO}$ & pyridine- $N$-oxide \\
\hline $\mathrm{q}$ & quartet \\
\hline$Q$ & total electric charge \\
\hline$\rho$ & density; proportionality constant \\
\hline $\mathrm{R}$ & rest \\
\hline$R$ & gas constant \\
\hline rad & radian \\
\hline RDE & rotating disc electrode \\
\hline $\mathrm{RE}$ & reference electrode \\
\hline ref. & reference \\
\hline
\end{tabular}




$\begin{array}{ll}\text { RT } & \text { room/ambient temperature } \\ \text { RVC } & \text { reticulated vitreous carbon } \\ \text { s } & \text { singlet (spectral); second } \\ S & \text { slope } \\ \sigma_{\mathrm{p}} & \text { Hammett-value (para) } \\ \text { sat. } & \text { saturated } \\ \text { SCE } & \text { saturated calomel electrode } \\ \text { SET } & \text { single electron transfer } \\ \text { SPS } & \text { solvent purification system } \\ T & \text { temperature } \\ t & \text { tert } \\ \text { t } & \text { triplet } \\ \text { TBAI } & \text { tetra- } n \text {-butylammonium iodide } \\ \text { Tf } & \text { trifluoromethanesulfonyl } \\ \text { TFA } & \text { trifluoroacetic acid } \\ \text { TFAA } & \text { trifluoroacetic anhydride } \\ \text { TBAF } & \text { tetra- } n \text {-butylammonium fluoride } \\ \text { TBS } & \text { tert-butyldimethylsilyl ether } \\ \text { TFE } & \text { 2,2,2-trifluoroethanol } \\ \text { TEMPO } & \text { 2,2,6,6-tetramethylpiperidine 1-oxyl } \\ \text { THF } & \text { tetrahydrofuran } \\ \text { TLC } & \text { thin layer chromatography } \\ \text { TM } & \text { transition metal } \\ \text { TOF } & \text { time-of-flight } \\ \text { TS } & \text { transition state } \\ \text { USB } & \text { Universal Serial Bus } \\ \text { V } & \text { volt } \\ \text { WE } & \text { working electrode } \\ \text { X } & \text { (pseudo)halide }\end{array}$


Für Sarah und meine Familie. 

"Der Chemiker, welcher Alles gelernt hat, was man in chemischen Vorlesungen lernen kann, hat es in der Chemie eben so weit gebracht, wie ein Schwimmer, welcher sich über die Kunst des Schwimmens mündlich hat unterrichten lassen, und zugleich andere hat schwimmen sehen, er kann darum noch nicht schwimmen. Beides will geübt sein."[1]

- Hermann Kolbe 



\section{INTRODUCTION}

Almost two centuries after Wöhler's fundamental work in organic synthesis, ${ }^{[2]}$ the discipline has propelled to a central position in molecular assembly of various compounds with transformative applications to drug development, crop-protecting agents, energy storage or material sciences, among others. Despite significant advances, chemical synthesis continues to be inefficient and often large amounts of resources such as solvents, leaving groups and stoichiometric additives are consumed to achieve certain levels of molecular complexity. As a consequence, numerical concepts ${ }^{[3]}$ and theoretical principles ${ }^{[4]}$ such as the 12 Principles of Green Chemistry ${ }^{[4 \mathrm{~g}]}$ have been designed by chemists to provide practitioners in the field with guidelines to design overall more efficient and future oriented synthetic methods.

\subsection{Transition Metal-Catalyzed C-H Activation}

The past several decades have witnessed the development of more sustainable transformations with the aid of metal-catalyzed manifolds that have revolutionized the art of forming chemical bonds. ${ }^{[5]}$ The importance of these methodologies was appreciated by a number of Nobel Prizes for Chemistry within the past few years, such as 2010 in recognition of palladium-catalyzed cross-coupling reactions to Heck, Negishi and Suzuki. ${ }^{[6]}$ Furthermore, the use of catalytic reactions is often advantageous for economic reasons, since mild reaction conditions, increased throughput and overall reduced waste formation lead to cost savings. ${ }^{[7]}$

Despite the tremendous influence of cross-coupling reactions for modern carbon-carbon $(\mathrm{C}-\mathrm{C})$ or carbon-heteroatom $(\mathrm{C}-\mathrm{Het})$ bond formations, ${ }^{[8]}$ these approaches often fall short in addressing key green chemistry criteria, ${ }^{[4 \mathrm{~g}]}$ such as waste reduction, the minimization of pre-functionalization and the common requirement of precious metal catalyst (Scheme 1). ${ }^{[9]}$ In sharp contrast, the direct activation of omnipresent $\mathrm{C}-\mathrm{H}$ bonds has emerged as an increasingly powerful tool to minimize waste products and at the same time significantly improve the atom economy ${ }^{[10]}$ of coupling reactions. ${ }^{[11]}$ In addition, the direct formation of the desired bond avoids laborious pre-functionalization of the synthetic building blocks 
and thus enables excellent levels of step economy. ${ }^{[12]}$ Although isohypsic $\mathrm{C}-\mathrm{H}$ activation obviates the manipulation of one substrate, additional reaction steps are required to synthesize the second preactivated coupling partner, such as organic (pseudo)halides. ${ }^{[13]}$ In contrast, oxidative $\mathrm{C}-\mathrm{H} / \mathrm{C}-\mathrm{H}$ or $\mathrm{C}-\mathrm{H} / \mathrm{Het}-\mathrm{H}$ functionalizations are particularly desirable in terms of atom and step economy since

Atom economy: maximizing the number of atoms of the raw materials that are incorporated in the desired product. ${ }^{[10]}$

Step economy: reducing the number of reaction steps for efficient organic synthesis. ${ }^{[12]}$ they are devoid of additional pre-functionalization steps, and in theory, only hydrogen is formed as the byproduct. ${ }^{[9,14]}$

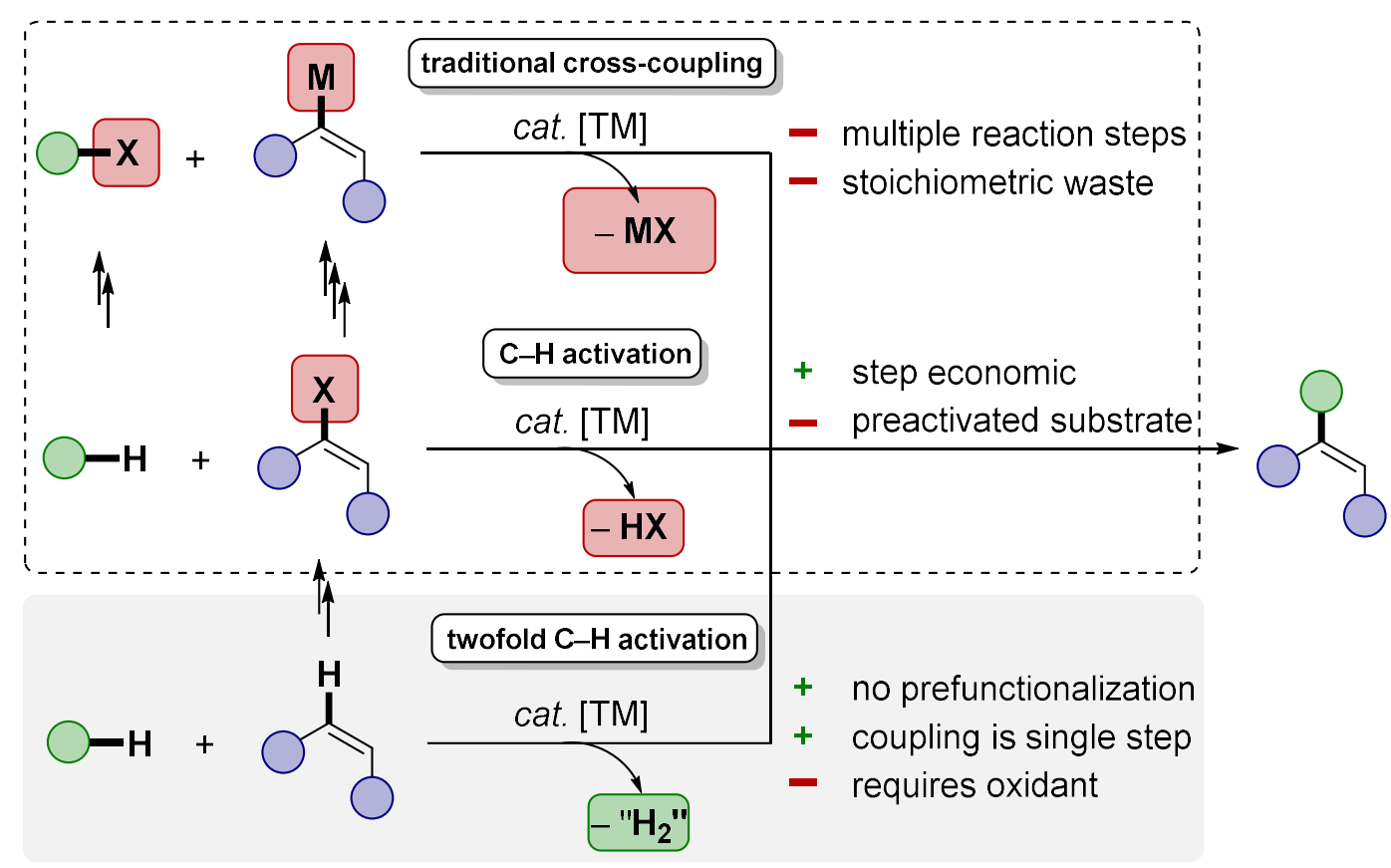

Scheme 1. Comparison of metal-catalyzed coupling reactions.

However, twofold $\mathrm{C}-\mathrm{H}$ activations or crossdehydrogenative couplings $(\mathrm{CDC})^{[9,}$ 14] inherently require stoichiometric amounts of often toxic and environmentally-harmful chemical oxidants and therefore result in poor levels of oxidant economy.

Oxidant economy: minimizing stoichiometric and nonrenewable oxidants to affect oxidative transformations. ${ }^{[9]}$ Moreover, dehydrogenative coupling reactions frequently suffer from harsh reaction conditions, strongly limiting their application to the synthesis of complex organic molecules. 
In comparison to classical cross-coupling reactions, the direct functionalization of omnipresent $\mathrm{C}-\mathrm{H}$ bonds with similar bond dissociation energies ${ }^{[15]}$ also features additional challenges in terms of site-selectivity control. ${ }^{[16]}$ To discriminate among different $\mathrm{C}-\mathrm{H}$ bonds, chemists employ different approaches (Scheme 2a). First, activated arenes or heterocycles, such as indoles, exhibit distinct $\mathrm{p} K_{\mathrm{a}}$-values ${ }^{[17]}$ of the $\mathrm{C}\left(\mathrm{sp}^{2}\right)-\mathrm{H}$ bonds and consequently $\mathrm{C}-\mathrm{H}$ metalation via proton-transfer proceeds at the kinetically most acidic $\mathrm{C}-\mathrm{H}$ bond. ${ }^{[18]}$ Likewise, sterically demanding groups lead to a steric bias of the adjacent $\mathrm{C}-\mathrm{H}$ bonds and hence $\mathrm{C}-\mathrm{H}$ activation will occur in the most accessible position. ${ }^{[19]}$ Since these two approaches are inherently substrate dependent, their range of application in organic synthesis is rather limited. In contrast, the most common way to achieve siteselective $\mathrm{C}-\mathrm{H}$ metalation is the use of auxiliary groups that contain Lewis-basic heteroatoms which coordinate the metal complex and bring the catalytically active center in close proximity to a specific $\mathrm{C}-\mathrm{H}$ bond (Scheme $2 \mathrm{~b}$ ). In recent years, the interest in chelation-assisted $\mathrm{C}-\mathrm{H}$ activation has increased dramatically and notable efforts have been made to expand the approach to weakly coordinating, ${ }^{[20]}$ removable $^{[21]}$ or transient ${ }^{[22]}$ directing groups.

(a) Differentiation of $\mathrm{C}-\mathrm{H}$ bonds

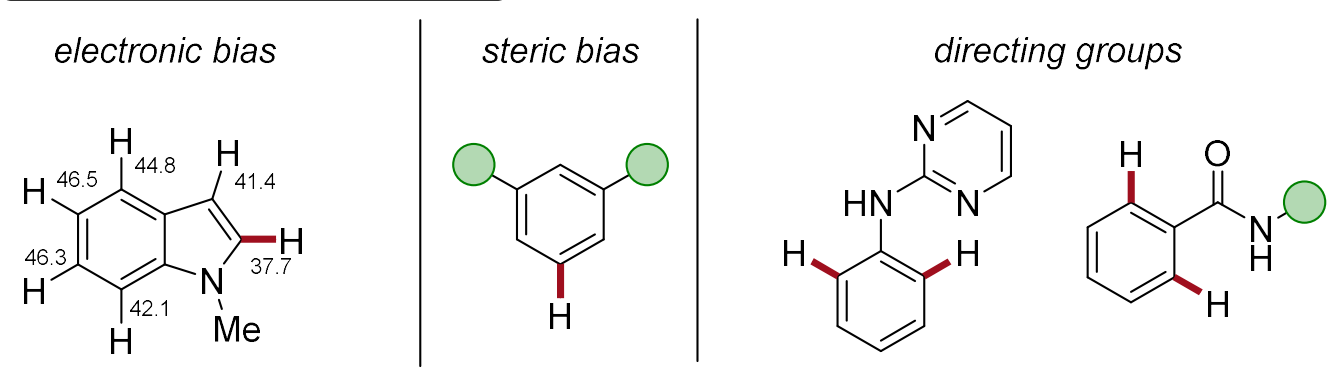

(b) Influence of the directing group

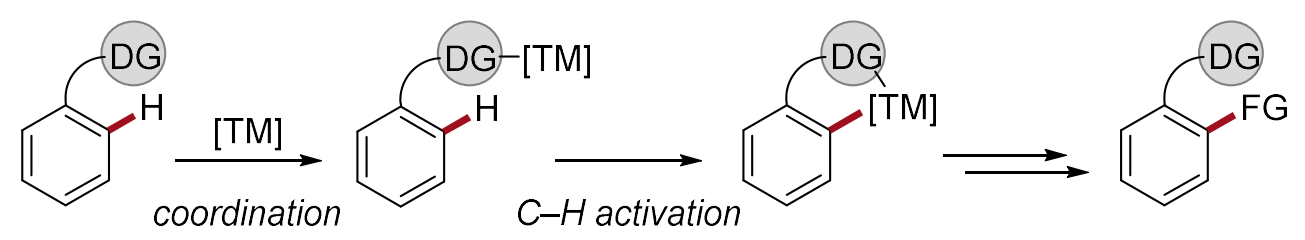

Scheme 2. Site-selectivity control in $\mathrm{C}-\mathrm{H}$ activation.

Although there are different definitions describing $\mathrm{C}-\mathrm{H}$ transformations, ${ }^{[23]} \mathrm{C}-\mathrm{H}$ activation in this thesis refers to an organometallic reaction step and the involvement of a resulting $\mathrm{C}-\mathrm{Met}$ bond, whereas $\mathrm{C}-\mathrm{H}$ functionalization is used in a broader context and can involve 
the abstraction of an electron or proton via outer-sphere/radical-type mechanism, thus creating radical intermediates before a new functional group is introduced. ${ }^{[24]}$

One of the key challenges in developing novel $\mathrm{C}-\mathrm{H}$ activations is to elucidate the underlying reaction mechanism. Over the past decades, different mechanistic pathways have been proposed for the key elementary step of the organometallic $\mathrm{C}-\mathrm{H}$ activation event (Scheme 3). ${ }^{[23 b, 25]}$ In this context, oxidative addition was mainly described to occur for electron-rich late transition metals in low oxidation states, such as iridium(I) or rhodium(I) complexes (Scheme 3a). ${ }^{[26]}$ In contrast, early transition metals of group 3 and 4 or actinides and lanthanides were prevalently reported to undergo isohypsic $\sigma$-bond metathesis (Scheme 3b). ${ }^{[27]}$ Often, the catalytically active complex features alkyl or hydride ligands. A closely related mechanistic scenario was mainly suggested for electrophilic late transition metals such as $\mathrm{Pd}^{2+}, \mathrm{Pt}^{2+}$ or $\mathrm{Pt}^{4+}$ (Scheme 3c). ${ }^{[28]}$ Here, the metal acts as a Lewis acid and undergoes electrophilic attack with the $\mathrm{C}-\mathrm{H}$ containing substrate. ${ }^{[29]}$

(a) Oxidative addition

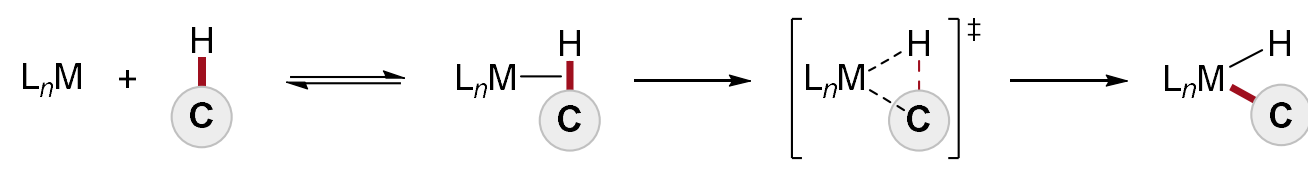

(b) $\sigma$-Bond metathesis

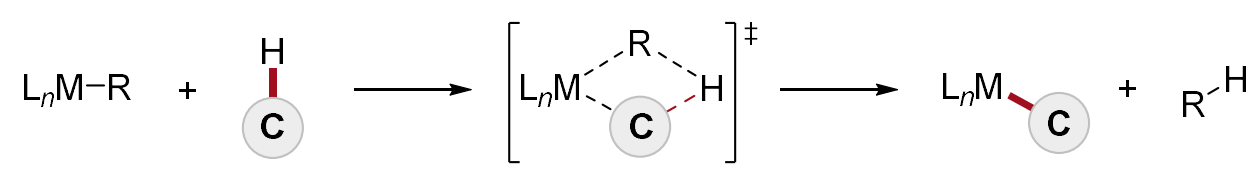

(c) Electrophilic substitution

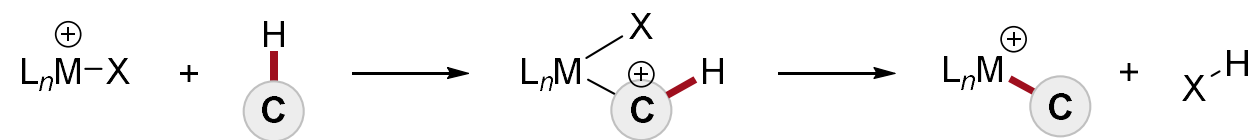

(d) 1,2-Addition

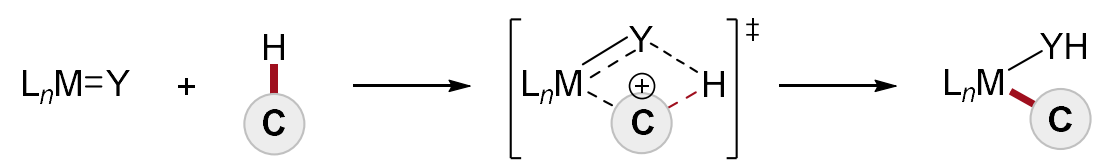

(e) Base-assisted metalation

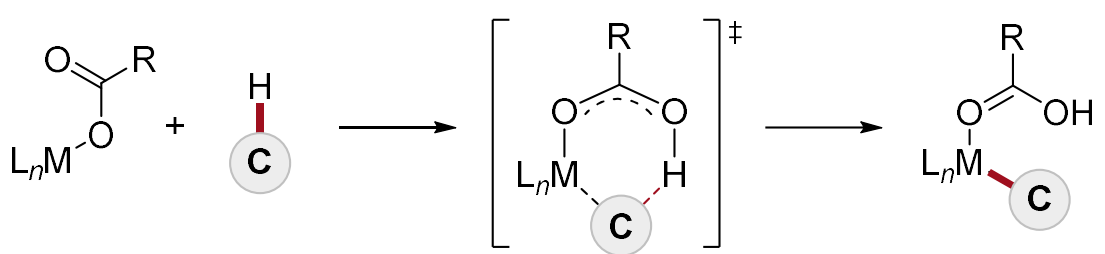

Scheme 3. Established mechanistic pathways for organometallic $\mathrm{C}-\mathrm{H}$ activation. 
The concept was later extended by Periana and Goddard for internal electrophilic substitutions (IES) in where the deprotonation is facilitated by oxy-ligands in a concerted fashion. ${ }^{[30]}$ Among these, a 1,2-addition was proposed for early transition metals or complexes which contain $\mathrm{M}=\mathrm{Y}$ double bonds, with $\mathrm{Y}$ as $\pi$-donating ligands such as oxo, imido or alkylidines (Scheme $3 \mathrm{~d}) .{ }^{[31]}$ Within the last two decades, base-assisted $\mathrm{C}-\mathrm{H}$ activation has received significant attention, which commonly proceeds via a five- or sixmembered transition state (Scheme 3e). Here, bifunctional basic ligands such as carbonates, ${ }^{[32]}$ secondary phosphine oxides $^{[33]}$ or carboxylate facilitate the hydrogen abstraction. ${ }^{[23 b, 34]}$ Indeed, detailed mechanistic studies have unravelled manifold mechanistic pathways for base-assisted $\mathrm{C}-\mathrm{H}$ metalation (Figure 1). ${ }^{[35]}$

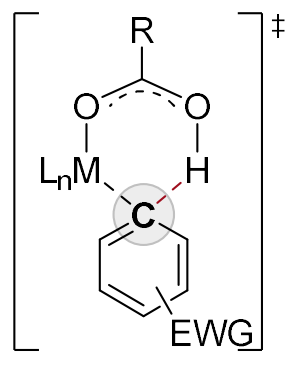

CMD/AMLA

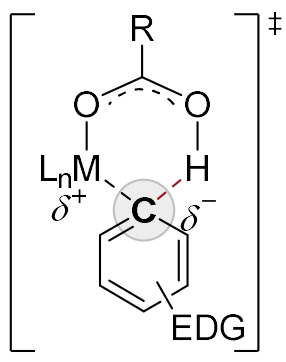

BIES

Figure 1. Proposed transition states for base-assisted $\mathrm{C}-\mathrm{H}$ metalation.

After pioneering theoretical studies by Sakaki on undirected benzene activation, ${ }^{[34 b]}$ detailed mechanistic work by Fagnou and Gorelsky have suggested that the $\mathrm{C}-\mathrm{H}$ metalation proceeds via a simultaneous metalation and intramolecular deprotonation within a sixmembered transition state. ${ }^{[36]}$ Hence, they have termed the pathway concerted metalation deprotonation (CMD). ${ }^{[37]}$ Important experimental observations included typically a preference of electron-deficient arenes for palladium-catalyzed $\mathrm{C}-\mathrm{H}$ arylations ${ }^{[38]}$ and the presence of large kinetic isotope effects (KIEs). ${ }^{[39]}$ Subsequently, MacGregor and Davies have proposed a related scenario but, based on theoretical calculations, explicitly postulated an agostic interaction between the metal center and the $\mathrm{C}-\mathrm{H}$ bond and summarized their findings as ambiphilic metal-ligand activation (AMLA). ${ }^{[40]}$

More recently, Ackermann has identified the pivotal role of bifunctional basic ligands within electrophilic substitution-type $\mathrm{C}-\mathrm{H}$ activations. In contrast to $\mathrm{CMD} / \mathrm{AMLA}$, intermolecular competition reactions revealed a strong preference for electron-rich substrates and theoretical studies were suggestive of a six-membered transition state. Based 
on their findings, the mechanistic pathway was termed base-assisted internal electrophilic substitution (BIES). ${ }^{[35,41]}$

In spite of major progress, $\mathrm{C}-\mathrm{H}$ activations and foremost cross-dehydrogenative couplings are largely dominated by cost-intensive and toxic precious metal catalysts. In addition, due to their low abundance, their extraction represents a serious environmental impact. ${ }^{[42]}$ Also, among poor levels of oxidant economy, oxidative couplings usually demand toxic, halogenated solvents, which contradicts the inherently green nature of $\mathrm{C}-\mathrm{H}$ activations.

Within recent years a remarkable progress has been made to address those limitations and to achieve ideal levels of resource economy in molecular syntheses. ${ }^{[9,43]}$ Notable efforts include the use of Earth-abundant catalyst, ${ }^{[23 a, 44]}$ the employment of biomass-derived solvents ${ }^{[45]}$ and alternative concepts for the catalyst reoxidation to avoid sacrificial oxidants (vide infra).

Resource economy: minimizing the in the overall footprint of chemical transformations as to the complete life cycle analysis, including, but not being limited to the use of naturally abundant or renewable feedstocks, solvents, metal catalysts, energy, and redox reagents. ${ }^{[9,43]}$

\section{Precious vs. Earth-abundant catalyst}

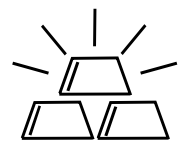

\section{$\mathrm{Pd}, \mathrm{Rh}$ Ir etc.} VS.
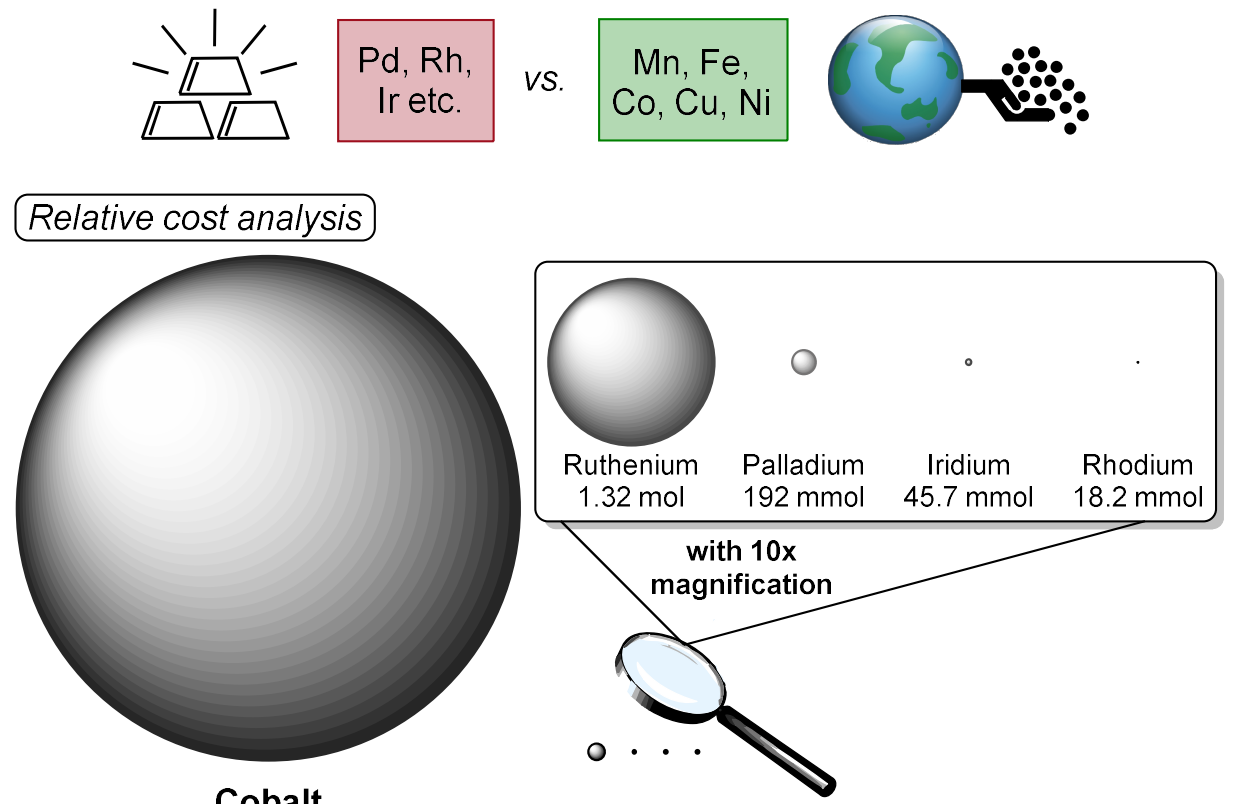

Cobalt $40.5 \mathrm{~mol}$

Figure 2. Precious Metal versus Earth-abundant metal catalyst. Molar amount of transition metal per $100 €{ }^{[46]}$ 


\subsection{Cobalt-Catalyzed C-H Activation}

In the context of $3 \mathrm{~d}$ base metal catalysts for molecular transformations, cobalt has received considerable attention, mainly due to its low cost compared to precious $4 \mathrm{~d}$ and $5 \mathrm{~d}$ elements (Figure 2) and its versatile reactivity in organometallic chemistry. Indeed, already in 1938 pioneering industrial applications for hydrocarbonylations of ethylene to yield propanal in the Fischer-Tropsch process were enabled with cobalt complexes $\left[\mathrm{CoH}(\mathrm{CO})_{4}\right]_{,}^{[47]}$ thus ensuring an important alternative for conventional oil-based fuels during World War II. ${ }^{[48]}$ Shortly thereafter, in 1941 Kharasch and Fields observed the catalytic effect of cobalt(II) salts in homocoupling reactions of phenylmagnesium bromide toward the synthesis of biphenyls. ${ }^{[49]}$ About seven years later, in 1948, Folkers and Smith successfully isolated vitamin $\mathrm{B}_{12}$ (cobalamin), which was found to be an important cofactor in biocatalytic processes, such as methylation or dehalogenation. ${ }^{[50]}$ Furthermore, cobalt complexes have been identified as versatile catalysts for various transformations of $\pi$-bond containing substrates, such as alkene, alkyne, allene or aryne, as was exemplified for cycloaddition reactions, ${ }^{[51]}$ the Nicholas reaction, ${ }^{[52]}$ or the Pauson-Khand reaction. ${ }^{[53]}$ In addition, cross-coupling reactions could be carried out with cobalt catalysts, providing a cost-efficient alternative to their more toxic noble metal counterparts. ${ }^{[54]}$ In spite of the rich nature of cobalt-catalyzed organic transformations, $\mathrm{C}-\mathrm{H}$ activation reactions under cobalt catalysis have been rarely reported in the last century, in particular compared to palladium-, rhodium- or ruthenium-catalyzed $\mathrm{C}-\mathrm{H}$ transformations. ${ }^{[11]}$ Nevertheless, since the report by Kharasch, some remarkable pioneering work has been performed in this context and extensive research, especially in the last decade, has made cobalt one of the most promising $3 \mathrm{~d}$ metals for $\mathrm{C}-\mathrm{H}$ activation reactions. In this regard, cobalt-catalyzed $\mathrm{C}-\mathrm{H}$ activations can be mainly divided into two concepts, namely, low-valent and high-valent cobalt catalysis, which will be discussed in the following sections. In addition, cobalt-catalyzed $\mathrm{C}-\mathrm{H}$ activations have recently been extensively covered in numerous excellent review articles by Ackermann, ${ }^{[23 a, 44 c, 55]}$ Chatani,${ }^{[56]}$ Cheng, ${ }^{[57]}$ Matsunaga,${ }^{[58]}$ Niu and Song, ${ }^{[59]}$ Ribas ${ }^{[54 b, 60]}$ Yoshikai, ${ }^{[61]}$ among others ${ }^{[62]}$ and therefore, only selected examples will be discussed in more detail.

\subsubsection{Low-Valent Cobalt-Catalyzed C-H Activations}

Low-valent cobalt catalysis is characterized by simple cobalt(II) salts and stoichiometric reductants, such as Grignard-reagents, or by the use of well-defined 
electron-rich cobalt(0) or cobalt(I) complexes. In this context, in 1955, Murahashi disclosed not only the first cobalt-catalyzed $\mathrm{C}-\mathrm{H}$ activation, but also the first directing group-assisted $\mathrm{C}-\mathrm{H}$ activations in general. In this pioneering work, isoindolinones 2 were synthesized from imines 1 under carbonylative cyclization with carbon monoxide as the coupling partner under relatively harsh reaction conditions (Scheme 4). ${ }^{[63]}$ Soon thereafter, the scope of the reaction was extended to the transformation of azobenzenes $3{ }^{[64]}$ The remarkable findings also served as an inspiration for other metal-catalyzed $\mathrm{C}-\mathrm{H}$ activation reactions, such as for pioneering work on nickel- ${ }^{[65]}$ or manganese-catalyzed $\mathrm{C}-\mathrm{H}$ activations. ${ }^{[66]}$

\section{(a) C-H Activation of imines 1}

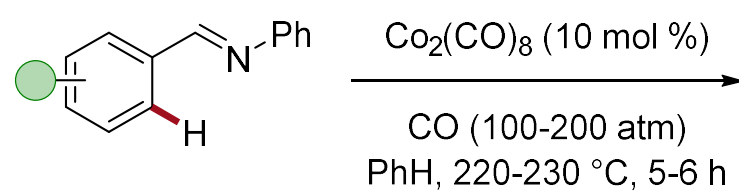

1

(b) C-H Activation of azobenzenes 3<smiles>c1ccc(N=Nc2ccccc2)cc1</smiles>

3<smiles>O=C1c2ccccc2CN1c1ccccc1</smiles>

2: $70-80 \%$<smiles>O=c1c2ccccc2[nH]n1-c1ccccc1</smiles>

4: $69-80 \%$

Scheme 4. Pioneering work for cobalt-catalyzed $\mathrm{C}-\mathrm{H}$ activation. Carbonylative cyclization of a) imines $\mathbf{1}$ and b) azobenzenes $\mathbf{3}$.

About 40 years later, Klein reported on the stoichiometric $\mathrm{C}-\mathrm{H}$ cleavage of similar aryl substrates 3, albeit with the use of low-valent cobalt(I) phosphine complex $\left[\mathrm{Co}\left(\mathrm{CH}_{3}\right)\left(\mathrm{PMe}_{3}\right)_{4}\right]$ (Scheme 5). ${ }^{[67]}$ The key $\mathrm{C}-\mathrm{H}$ cobaltation was reported to occur via oxidative addition with the $\mathrm{C}-\mathrm{H}$ bond and concomitant loss of methane, thus furnishing cyclocobaltated complex 5. The concept proved to be viable for a broad range of Lewisbasic directing groups, such as ketones, ${ }^{[68]}$ phosphines ${ }^{[69]}$ or pyridine. ${ }^{[70]}$ Furthermore, methane elimination was found to be an essential part of the catalysis since no cyclometalated complexes were observed when using $\left[\mathrm{Co}\left(\mathrm{C}_{2} \mathrm{H}_{4}\right)\left(\mathrm{Ph}_{3}\right)_{3}\right]$ as the precatalyst. 


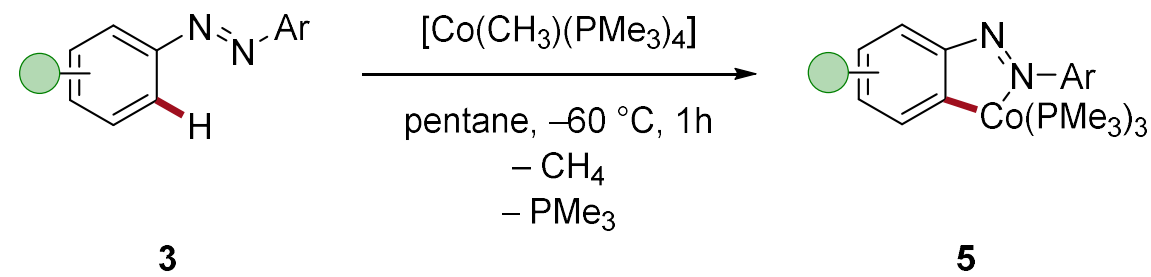

Scheme 5. Low-valent stoichiometric cyclometalation with $\left[\mathrm{Co}\left(\mathrm{CH}_{3}\right)\left(\mathrm{PMe}_{3}\right)_{4}\right]$.

Almost at the same time, in 1994, a seminal report on cobalt-catalyzed hydroarylations of azobenzenes 3 with diphenylacetylene (6a) was reported by Kisch (Scheme 6). ${ }^{[71]}$ Notably, no further additives were required to perform the catalysis under relatively mild conditions with overall good levels of efficiency. The key to obtain a catalytic turnover ${ }^{[72]}$ was found to be the employment of the well-defined cobalt(I) hydride complexes $\left[\mathrm{CoH}\left(\mathrm{N}_{2}\right)\left(\mathrm{PPh}_{3}\right)_{3}\right]$ or $\left[\mathrm{CoH}\left(\mathrm{N}_{2}\right)\left(\mathrm{PPh}_{3}\right)_{3}\right]$. The authors proposed that the active catalytic species was formed insitu after the loss off either $\mathrm{H}_{2}$ or $\mathrm{N}_{2}$, respectively, followed by coordination of azobenzene 3 and subsequent oxidative $\mathrm{C}-\mathrm{H}$ activation to yield a dihydrido cobalt(III) complex. Insertion of the alkyne $\mathbf{6 a}$ into one of the $\mathrm{Co}-\mathrm{H}$ bonds and final reductive elimination resulted the desired product 7 and the active cobalt(I) species. Notably, depending on the substitution pattern of the arene moieties also 2,3-dihydrocinnolines $(\mathbf{8})$ were isolated. The annulated products were most likely formed in a post-catalytic thermal cyclization reaction. ${ }^{[71]}$

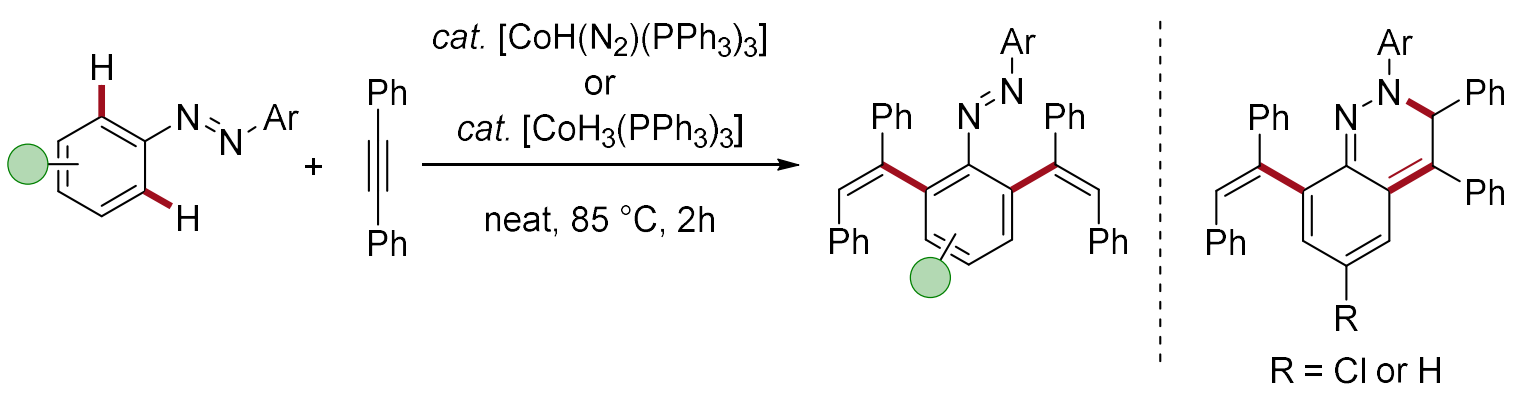
3 $6 a$

7: $70-80 \%$

8

Scheme 6. Pioneering cobalt-catalyzed hydroarylations of azobenzenes 3.

Another key finding was later reported by Brookhart for $\mathrm{Cp} * \mathrm{Co}(\mathrm{I})$-catalyzed hydroacylations of benzaldehydes 9 with trimethyl(vinyl)silane (10a) (Scheme 7). ${ }^{[73]}$ The catalytic transformation was characterized by a low catalyst loading of $1 \mathrm{~mol} \%$ and mild reaction conditions. Interestingly, mechanistic studies were suggestive of the formation of cobalt(III) hydride complex $\mathbf{1 3}$ after oxidative $\mathrm{C}-\mathrm{H}$ activation. Migratory insertion of the 
alkene 10a into the $\mathrm{Co}-\mathrm{H}$ bond and subsequent reductive elimination furnished the desired products 12. Additionally, detailed kinetic studies were supportive that the reductive elimination was the rate-limiting step of the catalysis. Indeed, the same $\mathrm{Cp}{ }^{*} \mathrm{Co}(\mathrm{I})$ complex 11 was recently employed by Pérez-Temprano for the synthesis of various cyclometalated $\mathrm{Cp}{ }^{*} \mathrm{Co}(\mathrm{III})$ complexes, albeit via oxidative addition of the corresponding aryl halides. ${ }^{[74]}$

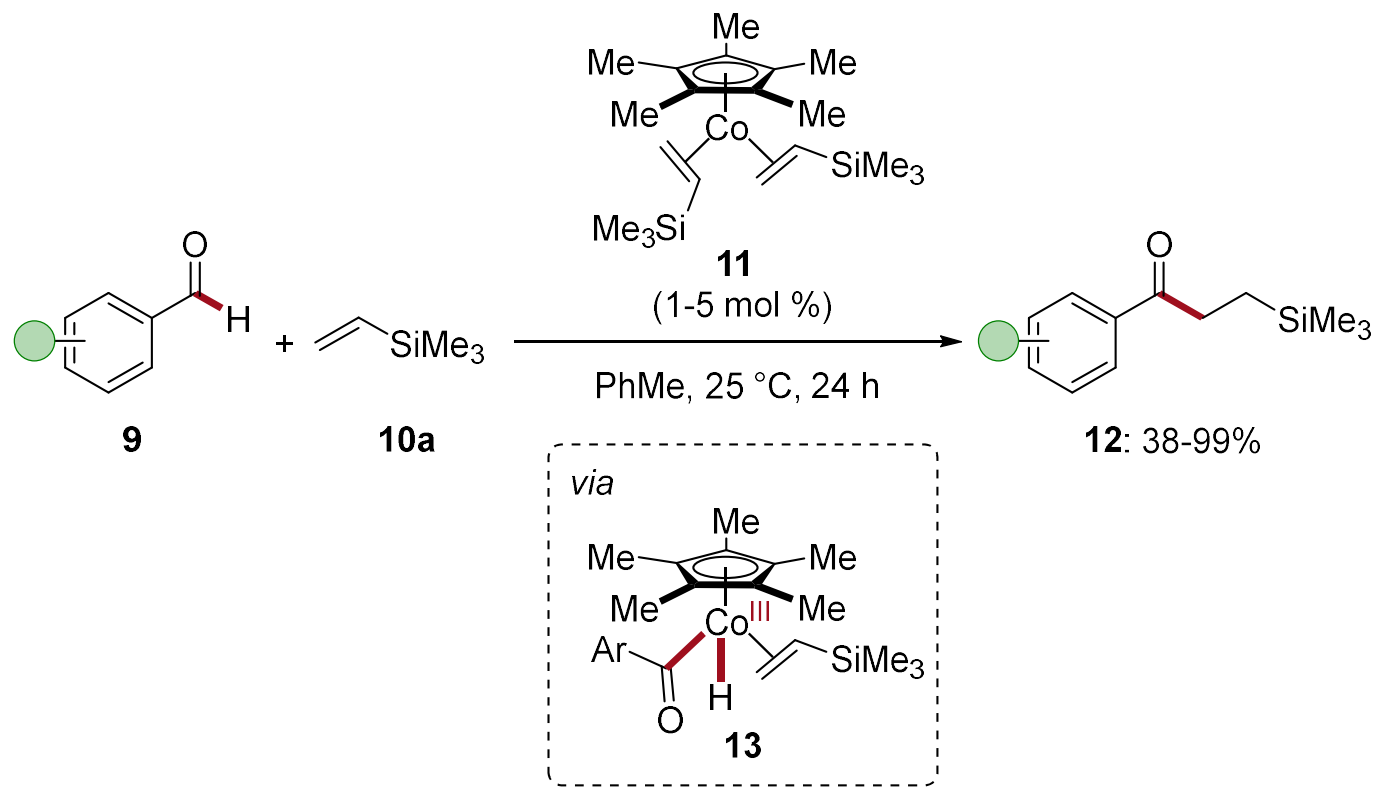

Scheme 7. Cobalt-catalyzed hydroacylation of benzaldehydes 9 with alkene 10a.

Likely inspired by the report of Kisch, in 2010, Yoshikai devised a cobalt-catalyzed hydroarylation of internal alkynes 6 with 2-arylpyridines 14 (Scheme 8). ${ }^{[75]}$

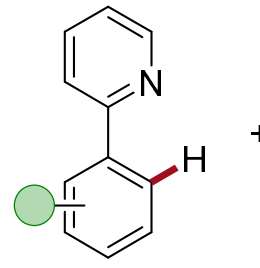

14

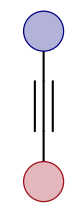

6

6

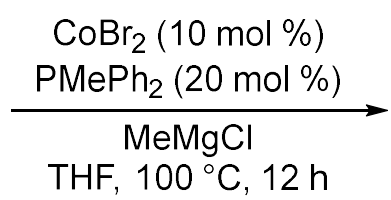

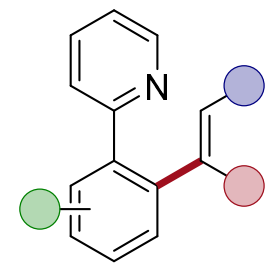

15: $13-78 \%$

Scheme 8. Cobalt-catalyzed hydroarylation of alkynes 6 with 2-arylpyridines 14.

Here, the key low-valent cobalt species was proposed to be formed by in-situ reduction from simple cobalt(II) salts with stoichiometric amounts of Grignard-reagents. Further addition of phosphine ligands stabilized the thus formed cobalt( 0$)$ or cobalt(I) complexes responsible for the $\mathrm{C}-\mathrm{H}$ activation. The scope of the reaction was later extended to the hydroarylation of other aromatic scaffolds, such as ketimines, ${ }^{[76]}$ aldimines ${ }^{[77]}$ and 
indoles. ${ }^{[78]}$ These important results initiated a plethora of notable developments for low-

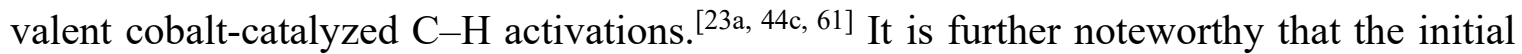
findings of Klein were later rediscovered by Petite for Grignard-free low-valent cobaltcatalyzed $\mathrm{C}-\mathrm{H}$ activations with the well-defined and air-sensitive cobalt(0) phosphine complex $\left[\mathrm{Co}\left(\mathrm{PMe}_{3}\right)_{4}\right] .{ }^{[79]}$

\subsubsection{High-Valent Cobalt-Catalyzed C-H Activations}

In spite of these remarkable contributions, low-valent cobalt-catalyzed $\mathrm{C}-\mathrm{H}$ activations were found to be experimentally tedious, often limited in functional group tolerance and the excess of Grignard-reagents diminished the overall atom-economy of the strategy. At the same time, after initial findings by Miura,${ }^{[80]}$ rhodium(III)-complexes were established as robust and bench-stable catalyst for a plethora of $\mathrm{C}-\mathrm{H}$ activation reactions, ${ }^{[11 \mathrm{v}, 81]}$ thus setting the stage for their more abundant and cost-efficient $3 \mathrm{~d}$ homologous. In fact, early reports on stoichiometric high-valent cobalt-catalyzed $\mathrm{C}-\mathrm{H}$ activations were already disclosed in 1986 by an elegant study of Broderick and Legg using a tetradentate macrocyclic ligand system to stabilize the high-valent cobalt(III) center (Scheme 9). ${ }^{[82]}$ Here, intramolecular electrophilic $\mathrm{C}\left(\mathrm{sp}^{3}\right)-\mathrm{H}$ activation via the agostic $\mathrm{C}-\mathrm{H}$ coordinating complex 17 took place after oxidation of the chelated cobalt(II) complex 16, to yield the cyclometalated complex 18. The authors noted that oxidation to the coordinatively unsaturated 16-electron cobalt(III) species $\mathbf{1 7}$ was essential for the successful $\mathrm{C}-\mathrm{H}$ activation as well as the presence of strong field ligands, such as $\mathrm{CN}^{-}, \mathrm{SO}_{3}{ }^{2-}$ or $\mathrm{NH}_{3}$. The isolation of an air-stable $\mathrm{Co}(\mathrm{III})-\mathrm{C}$ containing organometallic species $\mathbf{1 8}$ was a remarkable finding, since earlier reports for cobalt-catalyzed alkane functionalization ${ }^{[83]}$ with simple cobalt(III) salts mainly proposed an outer-sphere homolytic bond cleavage manifold. ${ }^{[84]}$

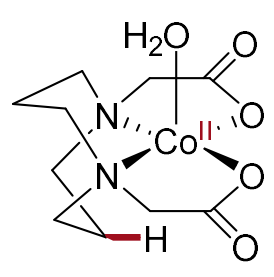

16

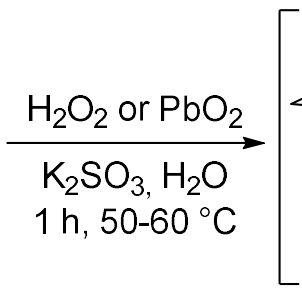

$\mathrm{K}_{2} \mathrm{SO}_{3}, \mathrm{H}_{2} \mathrm{O}$ .

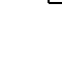

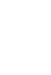

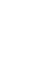

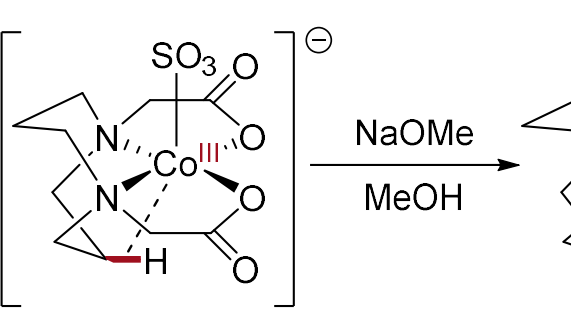

17

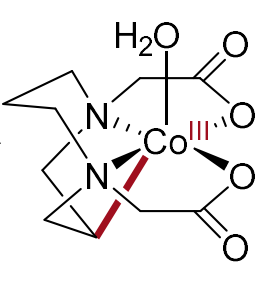

18

Scheme 9. Intramolecular high-valent cobalt(III)-mediated $\mathrm{C}-\mathrm{H}$ activation.

However, it took almost another 15 years before the first intermolecular high-valent cobaltmediated $\mathrm{C}-\mathrm{H}$ activation was reported. In a seminal approach, Avilés employed a mono- 
cyclopentadienyl cobalt(III) complex 19 for the ortho-metalation of azobenzene 3a (Scheme 10). ${ }^{[85]}$ An important feature of the study was the formation of a cationic cobalt(III) complex 20 via silver-mediated dehalogenation. In fact, Brookhart ${ }^{[86]}$ previously showed that the $\mathrm{Cp}^{*}$-ligated cobalt(I) complex 11 could even be oxidized to a rarely reported bishydrido cobalt(V) complex under appropriate reaction conditions. ${ }^{[87]}$

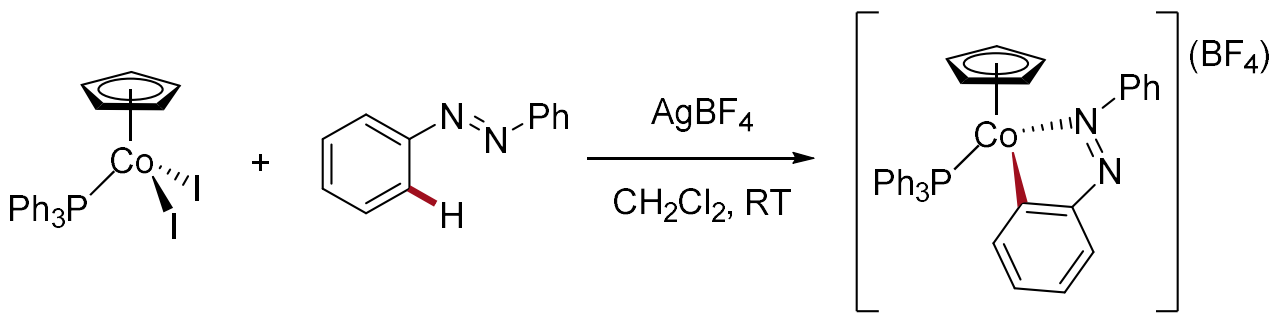

19

$3 a$

20: $88 \%$

Scheme 10. Intermolecular high-valent cobalt(III)-mediated $\mathrm{C}-\mathrm{H}$ activation.

In light of the successes for Cp*-rhodium(III)-catalyzed $\mathrm{C}-\mathrm{H}$ activations and the early work on high-valent cobalt complexes, ${ }^{[88]}$ a major breakthrough for synthetically useful applications of high-valent $\mathrm{Cp}^{*}$-cobalt(III)-catalyzed $\mathrm{C}-\mathrm{H}$ activation was made by Matsunaga and Kanai in 2013. ${ }^{[58 \mathrm{~d}]}$ Within their study, a cationic cobalt(III) complex $[\mathrm{Cp} * \mathrm{Co}(\mathrm{PhH})]\left(\mathrm{PF}_{6}\right) 21$ was employed for the additive-free hydroarylation of enones 22 or $N$-sulfonyl imines 24 with 2-arylpyridines 14 (Scheme 11).

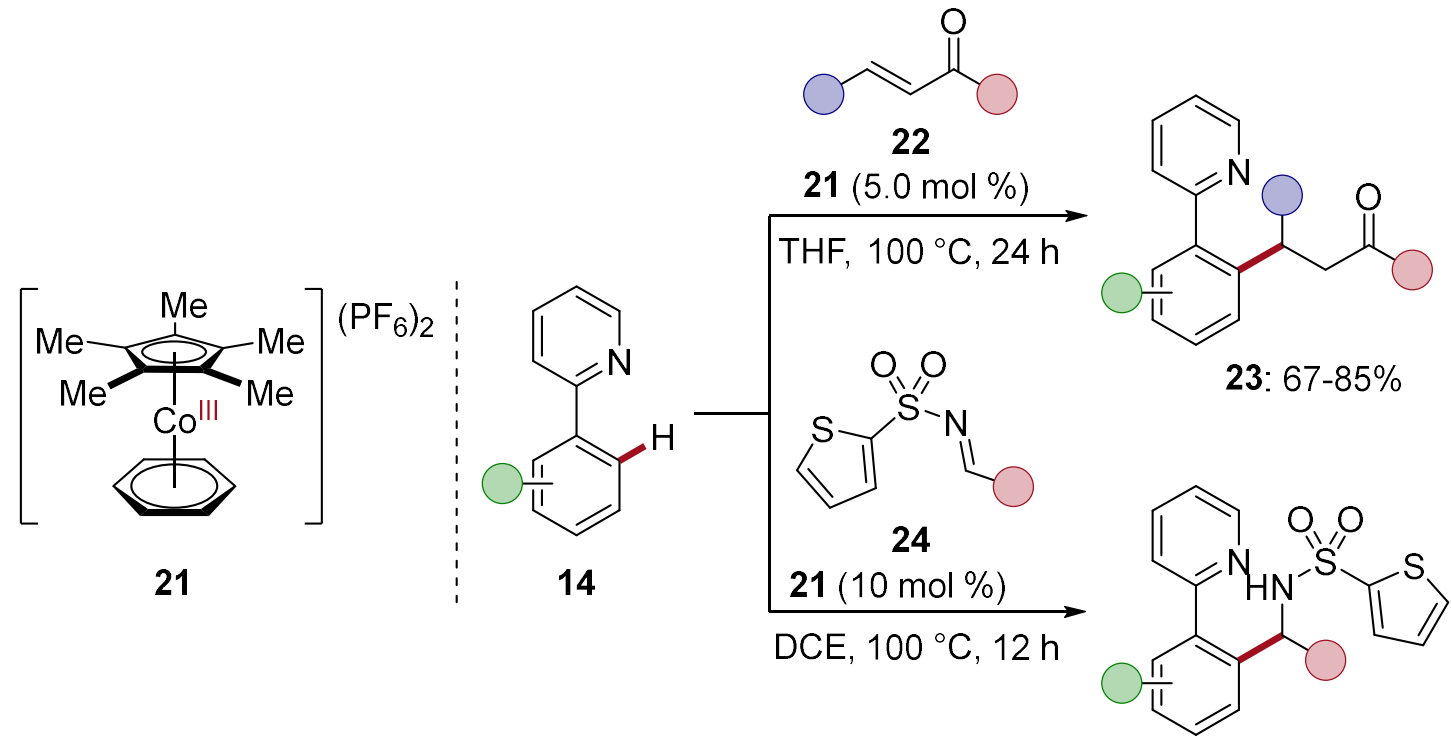

25: $57-83 \%$

Scheme 11. Intermolecular cobalt(III)-catalyzed C-H hydroarylations. 
The proposed redox-neutral mechanistic scenario was based on previously reported transformations with $\mathrm{Cp}^{*}$-rhodium(III) complexes. ${ }^{[89]}$ Indeed, all three of the aforementioned high-valent cobalt-catalyzed $\mathrm{C}-\mathrm{H}$ activation strategies mechanistically differ from the pathways reported for low-valent cobalt complexes. Low-valent cobalt complexes have been reported to cleave the $\mathrm{C}-\mathrm{H}$ bond within an oxidative addition-type mechanism and thus resulting in a cobalt hydride species ${ }^{[44 c, 61 b, 79 c]}$ or via a ligand-to-ligand hydrogen transfer (LLHT) mechanism. ${ }^{[90]}$ In contrast, the high-valent cobalt(III) complexes most likely facilitated $\mathrm{C}-\mathrm{H}$ scission via either electrophilic substitution or base-assisted metalation (cf. Scheme 3). ${ }^{[60]}$

Based on these notable findings, a wide range of $\mathrm{Cp}^{*}$-cobalt(III)-catalyzed $\mathrm{C}-\mathrm{H}$ activations were developed, including largely redox-neutral transformations ${ }^{[23 a, 58,60]}$ whereas oxidative $\mathrm{C}-\mathrm{H}$ transformations were only sporadically achieved. ${ }^{[55 \mathrm{a}]}$ Moreover, in contrast to cyclometalated rhoda(III)cycles, cost-efficient organometallic cobalt complexes and thus the $\mathrm{Co}-\mathrm{C}$ bond were often found to be particularly nucleophilic, leading to decreased $\mathrm{Co}-\mathrm{C}$ bond distances, ${ }^{[91]}$ which allowed new reactivities and unique chemo- or regioselectivities. $^{[44 c, 92]}$

\subsubsection{Oxidative Cobalt-Catalyzed C-H Activation without Cp*-Ligands}

Since the early reports by Lewis and $\operatorname{Smith}^{[93]}$ and following work by Murai ${ }^{[94]}$ on ruthenium-catalyzed hydroarylations by $\mathrm{C}-\mathrm{H}$ activation, notable studies have been conducted on precious metal-catalyzed $\mathrm{C}-\mathrm{H}$ alkyne annulations. In particular, oxidative transformations within rhodium(III)-catalyzed $\mathrm{C}-\mathrm{H}$ annulation reactions with the aid of stoichiometric amounts of chemical oxidants were flourishing. ${ }^{[80 \mathrm{a}, 81 \mathrm{i}]}$

In contrast, considerable recent efforts have been devoted to more sustainable, Earthabundant cobalt-catalyzed $\mathrm{C}-\mathrm{H}$ activations, yet oxidative transformations toward the stepeconomical formation of heterocyclic scaffolds have remained largely untapped until 2014. In a seminal report, Daugulis disclosed the oxidative $\mathrm{C}-\mathrm{H} / \mathrm{N}-\mathrm{H}$ activation of benzamides 26 and subsequent alkyne $\mathbf{6}$ annulation with simple cobalt(II) salts as the catalysts (Scheme 12)..$^{[95]}$ 
(a) $\mathrm{C}-\mathrm{H} / \mathrm{N}-\mathrm{H}$ Activation of benzamides 26

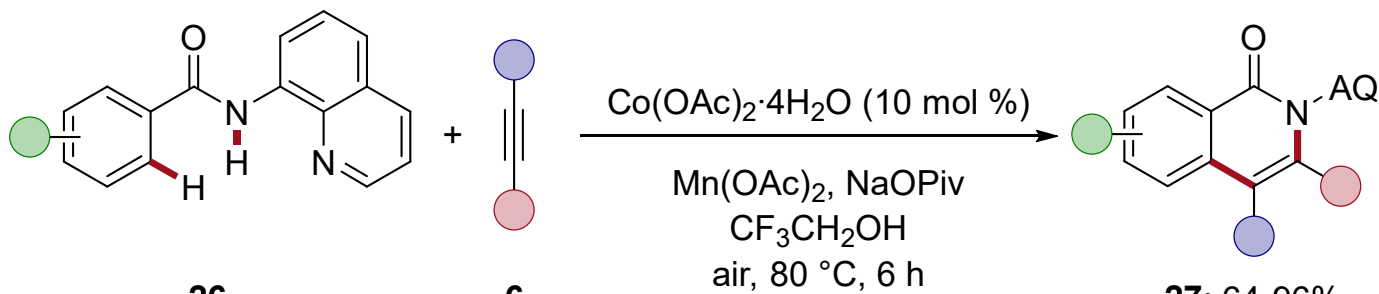

air, $80{ }^{\circ} \mathrm{C}, 6 \mathrm{~h}$

27: $64-96 \%$

(b) $\mathrm{C}-\mathrm{H}$ Activation of picolinamide $\mathbf{2 8 a}$

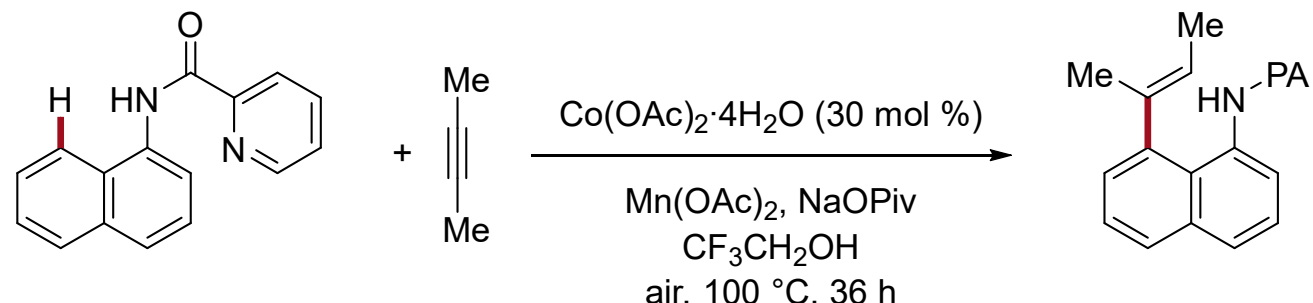

$28 \mathrm{a}$

$6 \mathrm{~b}$

29ab: $48 \%$

(c) Stoichiometric $\mathrm{C}-\mathrm{H}$ cobabs

26a

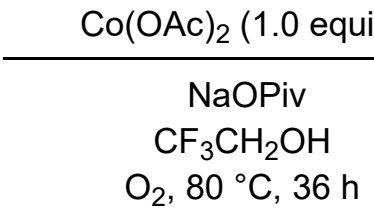

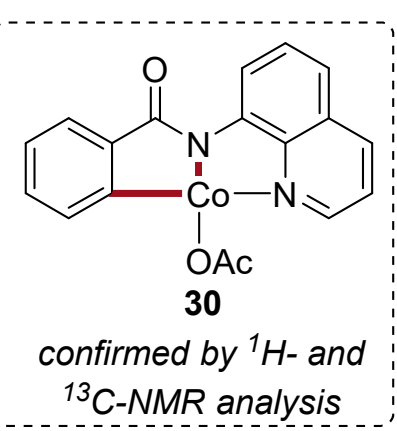

Scheme 12. Cobalt-catalyzed oxidative $\mathrm{C}-\mathrm{H} / \mathrm{N}-\mathrm{H}$ alkyne annulation with alkynes 6.

The key to success was the employment of an additional directing group, such as 8aminoquinoline (AQ) (Scheme 12a) or picolinamide (PA) (Scheme 12b) on the amide scaffold to stabilize high-valent cobalt(III) species. The use of these $N, N$ '-bidentate directing groups was previously introduced for high-valent palladium- ${ }^{\left[{ }^{[6]}\right.}$ and later also for other $3 \mathrm{~d}$, $4 \mathrm{~d}$ and $5 \mathrm{~d}$ transition metal-catalyzed $\mathrm{C}-\mathrm{H}$ activations, ${ }^{[11 \mathrm{~b}, 97]}$ albeit not for cobalt. The reaction optimization revealed that TFE was the solvent of choice and a combination of oxygen/air atmosphere as well as stoichiometric amounts of a metal-based co-oxidant were necessary to enable good catalyst turnover. In addition, carboxylate additives proved to be essential for the cobalt-catalyzed $\mathrm{C}-\mathrm{H}$ activation. Furthermore, the authors were able to synthesize an electrophilic cyclometalated cobalt(III) complex $\mathbf{3 0}$ under an atmosphere of oxygen (Scheme 12c), which was shown to be catalytically potent for the $\mathrm{C}-\mathrm{H} / \mathrm{N}-\mathrm{H}$ annulation reaction. ${ }^{[95]}$ Unfortunately, the characterization of the organometallic species was limited to NMR spectroscopic studies. Based on control experiments and the detection of intermediate 30, a simplified reaction mechanism was put forward (Scheme 13). Initially, 
base-assisted cobaltation generates the cyclometalated complex 31, followed by alkyne 6 insertion to generate the seven-membered intermediate 32. Subsequent reductive elimination releases the desired product 27 and a reduced cobalt(I) species 33. Hence, the overall mechanistic manifold most-likely proceeds within a cobalt(II/III/I) catalytic cycle. However, no further studies were conducted toward the generation of the active cobalt(III) species, the mechanism of the $\mathrm{C}-\mathrm{H}$ activation step or the nature of the oxidation.

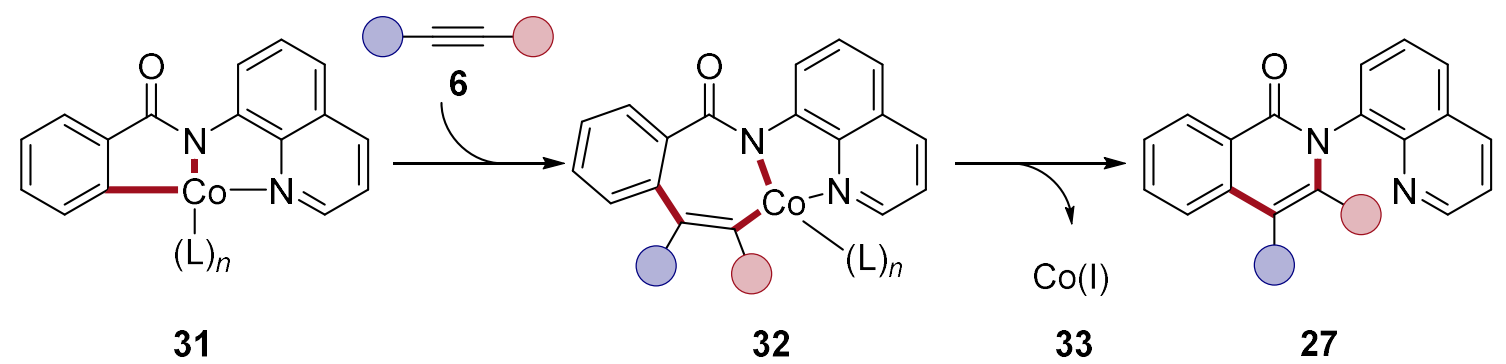

Scheme 13. Mechanistic proposal for the cobalt-catalyzed alkyne annulation.

Based on the groundbreaking work, a large number of oxidative cobalt-catalyzed $\mathrm{C}-\mathrm{H}$ transformations were disclosed under either mono- or bidentate directing group assistance (Scheme 14). Thus, the in-situ formed high-valent cobalt complexes enabled various oxidative $\mathrm{C}-\mathrm{C}$ or $\mathrm{C}-\mathrm{Het}$ bond formations. ${ }^{[55 \mathrm{a}, 60]}$ Therein, $N, N^{\prime}$-bidentate 8 -aminoquinoline (AQ), $N$-2-pyridylhydrazide or $N, O$-bidentate pyridine- $N$-oxide (PyO) proved to be among the most prominent DGs for the stabilization of high-valent cobalt species. ${ }^{[11 \mathrm{~b}, 97]}$ The DG is commonly attached to an amide group or an arene ring and consequently directs the cobalt center to the $\mathrm{C}-\mathrm{H}$ bond of interest (vide supra).

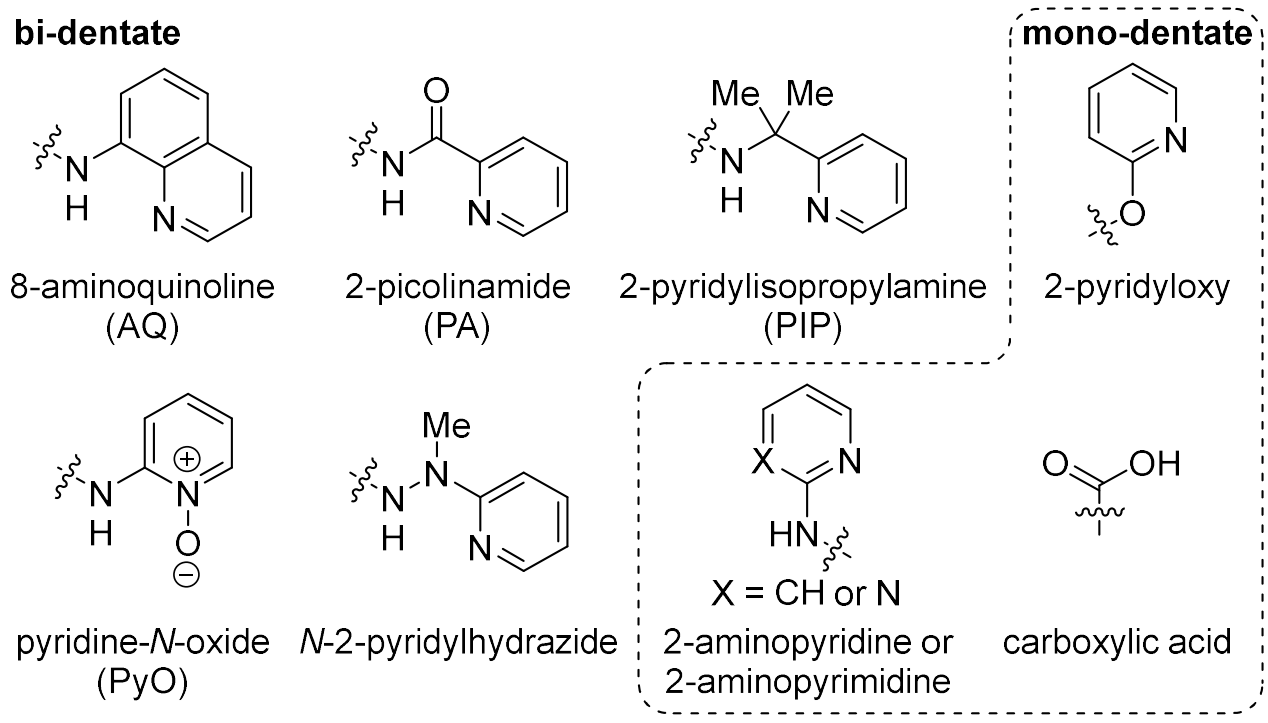

Scheme 14. Selected directing groups for oxidative cobalt-catalyzed $\mathrm{C}-\mathrm{H}$ activation with simple cobalt(II) salts. 


\subsubsection{Oxidative Cobalt-Catalyzed C-H Oxygenation}

During the last six years, significant progress has been made toward step economical oxidative cobalt-catalyzed $\mathrm{C}-\mathrm{Het}$ formations, including valuable $\mathrm{C}-\mathrm{N}, \mathrm{C}-\mathrm{X}(\mathrm{X}=\mathrm{Cl}, \mathrm{Br}, \mathrm{I}$, $\mathrm{CN}), \mathrm{C}-\mathrm{S}$ or $\mathrm{C}-\mathrm{O}$ bonds. ${ }^{[23 \mathrm{a}, 44 \mathrm{c}, 55 \mathrm{a}, 59-60]}$ Due to the wealth of reported transformations, the following section will be limited to intermolecular $\mathrm{C}-\mathrm{O}$ formations.

Early reports for SET-type cobalt-mediated $\mathrm{C}-\mathrm{H} / \mathrm{O}-\mathrm{H}$ transformations date back to $1973,{ }^{[98]}$ however it was not until recently that Niu and Song reported on cobalt-catalyzed two-fold $\mathrm{C}-\mathrm{H}$ activation of benzamide $\mathbf{3 4}$ or acryl amides $\mathbf{3 7}$ with simple alcohols $\mathbf{3 5}$ as the coupling partner (Scheme 15) ${ }^{[99]}$ The reaction proceeded under relatively mild reaction conditions of $40-60{ }^{\circ} \mathrm{C}$ with catalytic amounts of cost-efficient and bench-stable $\mathrm{Co}(\mathrm{OAc})_{2} \cdot 4 \mathrm{H}_{2} \mathrm{O}$ as the catalyst and stoichiometric amounts of silver(I) salts as the terminal oxidant. Among other directing groups, pyridine- $N$-oxide gave the best results. Overall, good functional group compatibility and ample substrate scope was accomplished under the reaction conditions. Mechanistic studies indicated the formation of radical intermediates and a KIE of 1.0 was supportive for a non-rate limiting $\mathrm{C}-\mathrm{H}$ activation step. However, the authors did provide only limited mechanistic insights.

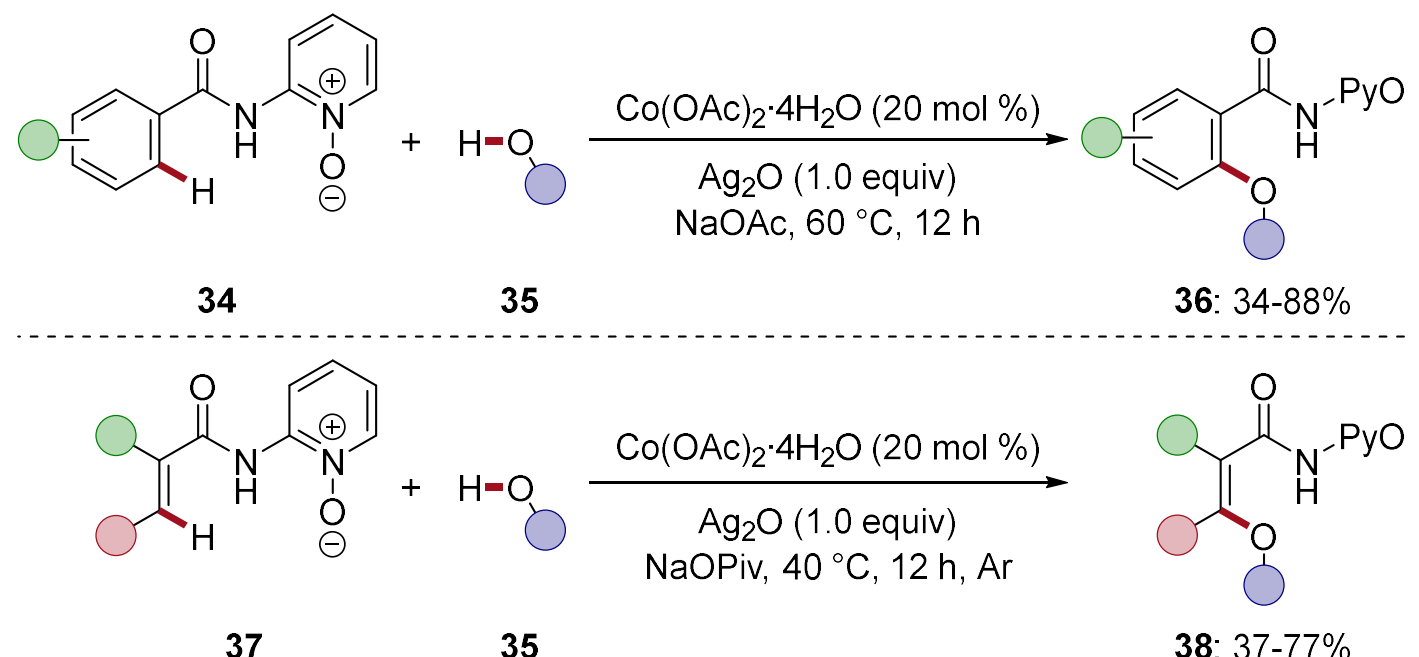

Scheme 15. Cobalt-catalyzed $\mathrm{C}-\mathrm{H}$ alkoxylations with $\mathrm{Ag}_{2} \mathrm{O}$ as the oxidant.

Thereupon, Niu and Wei disclosed more detailed mechanistical work with the aid of DFT calculations that further supported the preliminary working hypothesis for a cobalt(II/III) catalytic cycle (Scheme 16). ${ }^{[100]}$ Initially, the in-situ generated cobalt(III) species 39 undergoes SET with substrate $\mathbf{3 4}$ to form the arene radical cation $\mathbf{4 0 .}$ 


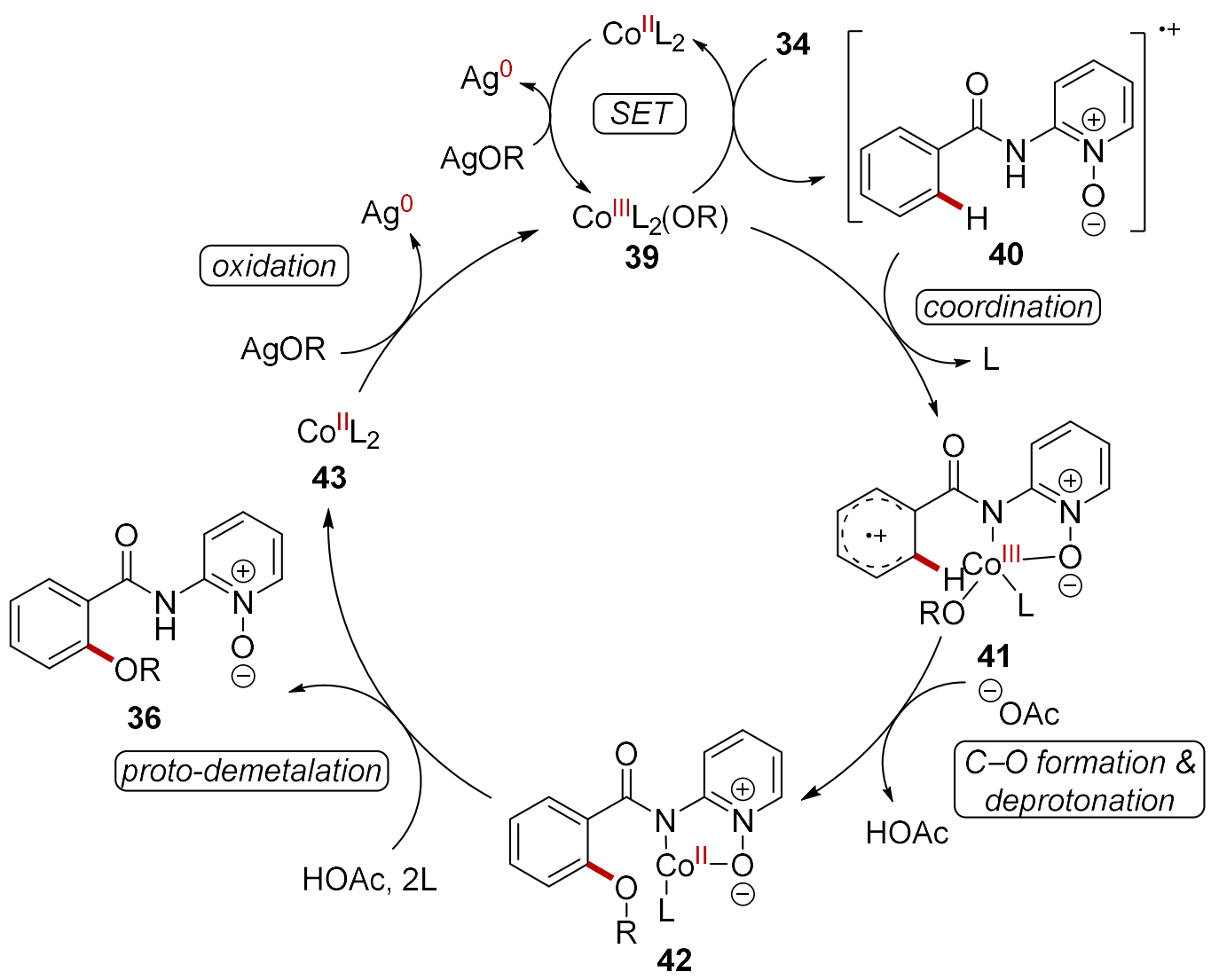

Scheme 16. Proposed catalytic cycle for the cobalt-catalyzed C-H alkoxylation.

At the same time, the cobalt(III) species 39 is regenerated after oxidation with the sacrificial silver salt. Subsequent coordination of amide $\mathbf{4 0}$ to $\mathbf{3 9}$, followed by a nucleophilic attack of the coordinating alkoxy species to the arene ring yields the Wheland intermediate 41. Thereafter, base-assisted deprotonation gives cobalt(II) species $\mathbf{4 2}$ and following protodemetalation releases the product 36. Finally, reoxidation of the cobalt(II) species $\mathbf{4 3}$ with the consumable silver oxidant reinitiates the catalysis. Moreover, the calculations indicated that the single electron transfer might be the rate-limiting step of the catalysis. The scope of the strategy was later extended by Huang for $\mathrm{C}-\mathrm{H}$ alkoxylation of quinolinamides $\mathbf{2 6}$ with $\mathrm{Co}(\mathrm{acac})_{2}$ as the catalyst. ${ }^{[101]}$ In the same context, Das disclosed regioselective cobaltcatalyzed C-H methoxylation of $\mathrm{N}$-phenylpyridin-2-amine (44) with $\mathrm{Ag}_{2} \mathrm{O}$ and oxygen as the oxidants (Scheme 17). ${ }^{[102]}$ Interestingly, DFT calculations were supportive of a PCETtype mechanistic manifold and the generation of an aminyl radical on the aniline derivative 44. 
<smiles>c1ccc(Nc2ccccn2)cc1</smiles>

44

$$
\begin{aligned}
& \underset{\mathrm{Co}(\mathrm{OAc})_{2} \cdot 4 \mathrm{H}_{2} \mathrm{O}(10 \mathrm{~mol} \%)}{\mathrm{Ag}_{2} \mathrm{O}(1.5 \text { equiv })} \\
& \mathrm{MeOH}, 80^{\circ} \mathrm{C}, 6 \mathrm{~h}, \mathrm{O}_{2}(1 \mathrm{~atm})
\end{aligned}
$$<smiles>COc1ccccc1Nc1ccccn1</smiles>

45: $82 \%$

Scheme 17. Cobalt-catalyzed C-H methoxylation of aniline derivative 44 .

In addition to cobalt-catalyzed $\mathrm{C}-\mathrm{H}$ alkoxylation, Zeng recently disclosed the cobaltcatalyzed $\mathrm{C}-\mathrm{H}$ acyloxylation of arenes $\mathbf{2 8}$ with picolinamide as the directing group (Scheme 18a). ${ }^{[103]}$ Here, readily available carboxylic acids 46 were employed as the coupling partner with $\mathrm{Ag}_{2} \mathrm{CO}_{3}$ as the chemical oxidant at $120{ }^{\circ} \mathrm{C}$ reaction temperature. Concurrently, Chatani ${ }^{[104]}$ and Zhang ${ }^{[105]}$ independently reported on cobalt-catalyzed C-H acyloxylations of benzamides $\mathbf{2 6}$ under slightly different reaction conditions (Scheme 18b). Both approaches required the use of silver salts as the oxidant. Notably, all three studies suggested a organometallic cyclometalated cobalt(III) complex as the key intermediate within a cobalt(II/III/I) catalytic manifold. Shortly thereafter, Deb and Cai independently reported on $\mathrm{C}-\mathrm{H}$ acetoxylations of benzamides and anilides under slightly modified reaction conditions. ${ }^{[106]}$ Very recently, Guo and Cao developed a C-H acetoxylation ${ }^{[107]}$ with PIDA as the oxidant/acetyl transfer reagent and $\mathrm{Co}(\mathrm{acac})_{2}$ as the catalyst (Scheme 18c). ${ }^{[108]}$ Notably, no reaction was detected under an atmosphere of nitrogen. The reaction exhibited a broad substrate scope, and the synthetic utility was demonstrated by late-stage acetoxylation of the herbicide diflufenican (49). 
(a) Picolinamide DG

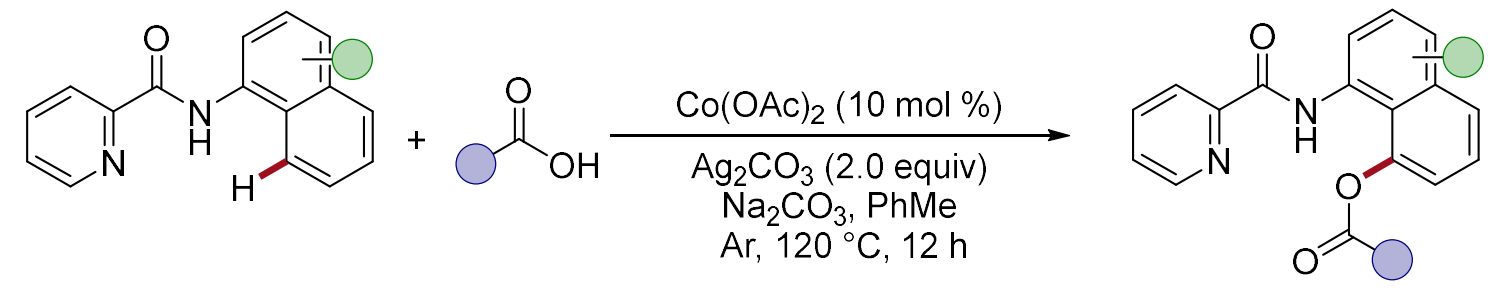

28

46

47: $42-92 \%$

(b) 8-Aminoquinoline $D G$

Zhang

$\mathrm{Co}(\mathrm{OAc})_{2}(20 \mathrm{~mol} \%)$

$\mathrm{Ag}_{2} \mathrm{SO}_{4}$ (2.2 equiv)

(6)

Chatani<smiles>O=C(O)NC(=O)c1ccccc1OC(=O)O</smiles>

(Zhang) 48: 41-99\% (Chatani) 48: 33-94\%

(c) Acetoxylation of diflufenican (49)<smiles>O=C(Nc1ccc(F)cc1F)c1cccnc1Oc1cccc(C(F)(F)F)c1</smiles>

49<smiles>CC(=O)Oc1ccc(C(F)(F)F)cc1Oc1ncccc1C(=O)Nc1ccc(F)cc1F</smiles>

50: $46 \%$

Scheme 18. Cobalt-catalyzed $\mathrm{C}-\mathrm{H}$ acyloxylation of arenes a) 28 and b) $\mathbf{2 6}$. C-H Acetoxylation of diflufenican (49).

\subsubsection{Oxidative Cobalt-Catalyzed $\mathrm{C}-\mathrm{H} / \mathrm{N}-\mathrm{H}$ Alkyne or Allene Annulations}

Since the pioneering study by Daugulis (cf. Scheme 12$),{ }^{[109]}$ a plethora of oxidative C$\mathrm{H} / \mathrm{N}-\mathrm{H}$ activations with inter alia alkynes, allenes, alkenes, carbon monoxide or isocyanides as the coupling partner have been reported in the following years. ${ }^{[55 \mathrm{a}, 60]}$ Only selected relevant examples are discussed in the following section with a focus on oxidative cobalt-catalyzed $\mathrm{C}-\mathrm{H} / \mathrm{N}-\mathrm{H}$ annulations with alkynes ${ }^{[110,57]}$ or allenes. ${ }^{[110]}$ 
Despite the significant impact of the study by Daugulis, the method relied on the use of stoichiometric amounts of manganese salts as the oxidant. In contrast, Ackermann elegantly developed an alternative cobalt-catalyzed $\mathrm{C}-\mathrm{H}$ alkyne annulation toward a step-economical synthesis of pharmaceutically-relevant isoquinolones ${ }^{[111]} 51$ with the aid of the bidentate directing group 2-pyridyl- $N$-oxide (PyO) and oxygen as the sole oxidant (Scheme 19). ${ }^{[12]}$ The cobalt oxidase catalysis ${ }^{[13]}$ was characterized by mild reaction conditions of $60{ }^{\circ} \mathrm{C}$, water as the sole byproduct and broad functional group tolerance. It is noteworthy that the use of fluorinated TFE as the solvent was essential to observe catalytic turnover. Besides other solvent effects, ${ }^{[14]}$ this observation can be rationalized by the highly $\mathrm{pH}$-dependent standard oxidation potential of oxygen in different solvents. ${ }^{[115]}$ The synthetic utility of the concept was further highlighted by the step-economical synthesis of a rosettacin derivative 52, which belongs to the class of aromathecin alkaloids that show activity as topoisomerase-I inhibitors. ${ }^{[116]}$

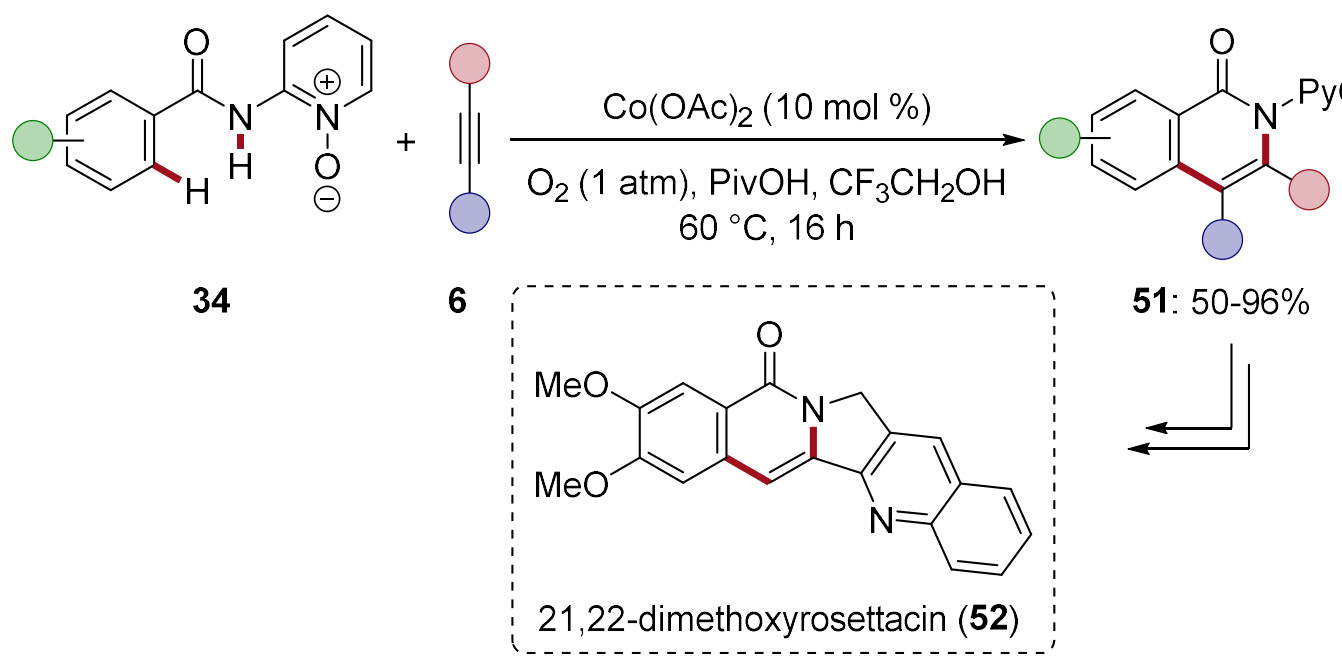

Scheme 19. Aerobic cobalt-catalyzed $\mathrm{C}-\mathrm{H} / \mathrm{N}-\mathrm{H}$ annulations with alkynes 6; stepeconomical synthesis of anticancer drug 52.

Furthermore, intermolecular competition studies indicated a clear preference for electronrich substrates and $\mathrm{H} / \mathrm{D}$-exchange gave support for an irreversible $\mathrm{C}-\mathrm{H}$ activation step. In addition, detailed DFT-calculations were indicative of a cobalt(II/III/I) catalytic cycle. An extension of the approach was later accomplished by Niu and Song for cobalt-catalyzed decarboxylative $\mathrm{C}-\mathrm{H} / \mathrm{N}-\mathrm{H}$ alkyne annulations with alkynyl carboxylic acids as the coupling partner. ${ }^{[117]}$ Remarkably, the cobalt catalysis delivered either isoquinolones $\mathbf{5 1}$ or isoindolinone derivatives 54. Likewise, the same authors previously reported on the 
formation of $\mathbf{5 4}$ for cobalt-catalyzed alkyne annulations with pyridine- $N$-oxide as the auxiliary group (Scheme 20). ${ }^{[118]}$

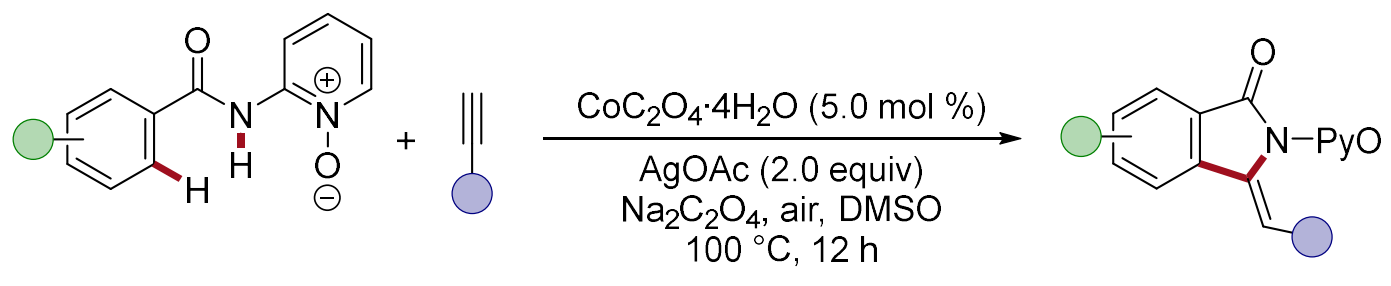

34

53

54: $55-96 \%$

Scheme 20. Cobalt-catalyzed isoindolinone 54 synthesis with terminal alkynes 53.

Here, and in contrast to the oxidase catalysis, ${ }^{[112]}$ silver(I) salts were employed as the terminal oxidant. The authors reasoned that the in-situ formation of a silver-acetylide complex 55 with terminal alkynes $\mathbf{5 3}$ was responsible for the drastic change in selectivity. Additionally, kinetic studies revealed a KIE of 2.5, hence being suggestive of a ratelimiting $\mathrm{C}-\mathrm{H}$ scission step. Thus, a reasonable working profile was drawn for both reaction motifs (Scheme 21). Coordination of the cobalt(II) salt and concomitant oxidation to the cobalt(III) species set the stage for a base-assisted $\mathrm{C}-\mathrm{H}$ activation of benzamides $\mathbf{3 4}$. The key cyclometalated cobalt(III) complex 56 can now undergo different follow-up reactions depending on the reaction conditions. In the presence of internal alkynes $\mathbf{6}$ or, in the case of terminal alkynes $\mathbf{5 3}$ and the absence of an excess of silver salts, a migratory insertion of the coordinating alkyne occurs and forms the seven-membered cobalt(III) species 57 (left cycle). Then, reductive elimination releases the isoquinolone $\mathbf{5 1}$ and a reduced cobalt(I) species 58. In contrast, the cobalta(III)cycle 56 undergoes transmetalation with the alkynyl silver(I) species 55 to furnish a cobalt(IV) species 60, which undergoes a facile reductive elimination to yield the ortho-alkynylated ${ }^{[119]}$ product $\mathbf{6 1}$ and a cobalt(II) species 59 (right cycle). Subsequently, 5-exo-dig cyclization delivers the desired isoindolinone 54. Finally, oxidation of the reduced cobalt(I) 58 or cobalt(II) species 59, respectively, reinitiates the catalysis. However, it should be noted that at this stage experimental support for the working hypothesis was scarce and robust information for the formation of a high-valent cobalt(IV) intermediate $\mathbf{6 0}$ was lacking. 


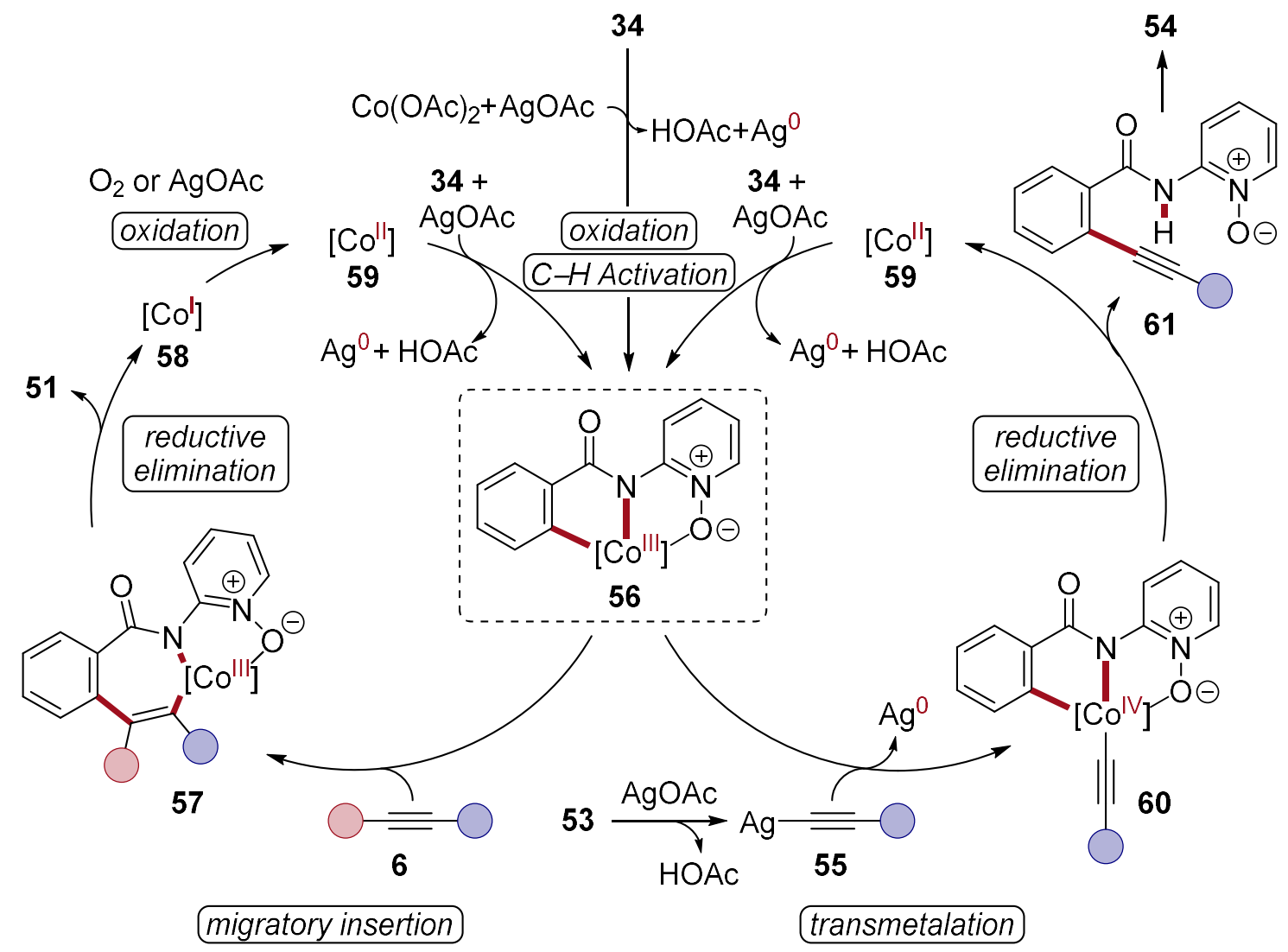

Scheme 21. General mechanistic proposal for cobalt-catalyzed C-H/N-H alkyne annulations toward isoquinolones 51 (left) or isoindolinones 54 (right).

Importantly, the reactivity of the cobalt(III)-catalyzed $\mathrm{C}-\mathrm{H} / \mathrm{N}-\mathrm{H}$ alkyne annulation was not limited to 8-aminoquinoline or pyridine- $N$-oxide as the directing group. Indeed, within the last five years, numerous strategies were disclosed for step-economical syntheses of different heterocycles (Scheme 22). Thus, Zhai developed an easily removable $N, N^{\prime}$ bidentate auxiliary, based on a N-2-pyridylhydrazide group, for the synthesis of isoquinolones 63 with alkynes 6 (Scheme 22a). ${ }^{[120]}$ Notably, the hydrazyl group was removed via reductive $\mathrm{N}-\mathrm{N}$ cleavage in a traceless fashion. The approach was later extended for a spirocyclization cascade of benzamides 63 with maleimides. ${ }^{[121]}$ Next, Cui reported on alkyne annulations toward the assembly of isoquinoline scaffolds $\mathbf{6 5}$ under the use of a traceless picolinamide auxiliary group (Scheme 22b). ${ }^{[122]}$ Likewise, Carretero disclosed oxidative cobalt-catalyzed $\mathrm{C}-\mathrm{H} / \mathrm{N}-\mathrm{H}$ alkyne annulations of the benzylamine derivatives 64 to access dihydroisoquinolines 66 (Scheme 22c). ${ }^{[123]}$ Notably, the authors used ESI-MS studies to detect several elusive cobalt(III) species, such as a five-membered cobaltacycle $\mathbf{6 7}$, which they assumed to be an on-cycle catalytically active intermediate. 
(a) Isoquinolone 63 synthesis

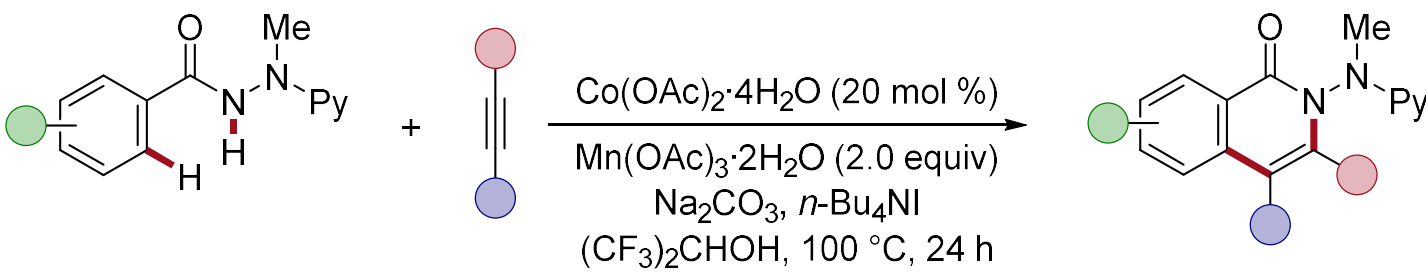

62
$\left(\mathrm{CF}_{3}\right)_{2} \mathrm{CHOH}, 100^{\circ} \mathrm{C}, 24 \mathrm{~h}$

6
63: $43-94 \%$

(b) Isoquinoline 65 synthesis

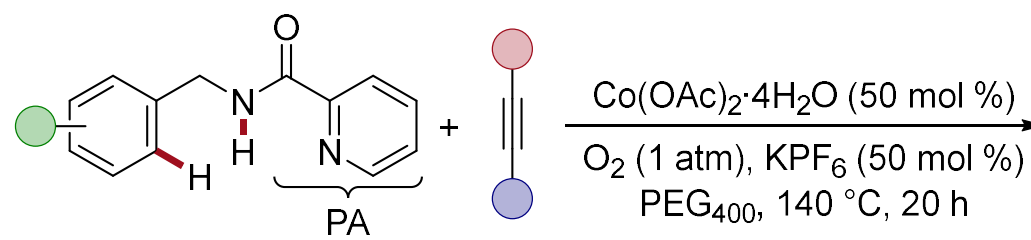

64

6<smiles>Oc1cc2oc3c(c2cn1)OCCO3</smiles>

65: $43-94 \%$

(c) Dihydroisoquinoline 66 synthesis

6<smiles>CCCN1Cc2cc(-c3ccccc3)ccc2C(O)=C1O</smiles>

64

(d) Isocoumarin 69 synthesis

66: $57-94 \%$

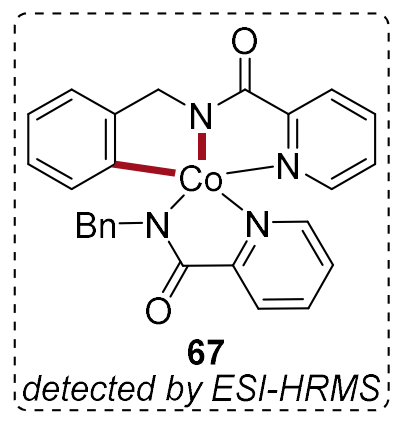<smiles></smiles>

68 6<smiles>O=c1oc(O)c(O)c2ccccc12</smiles>

69: $47-81 \%$

(e) Isoquinolone 71 synthesis with 1,3-diynes 70<smiles>O=C(N[N+](=O)[O-])c1ccccc1</smiles>

26<smiles>OC#COC1CCCCO1</smiles>

70

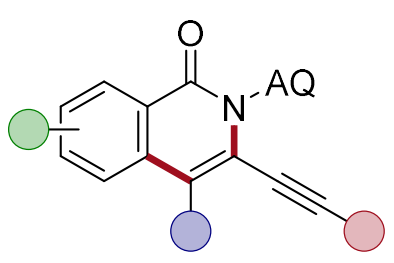

71: $50-93 \%$

Scheme 22. Cobalt-catalyzed C-H activations for the synthesis of various heterocycles.

The strategy of cobalt-catalyzed alkyne annulations with picolinamides as the directing group was later extended to the synthesis of dibenzo- $[b, d]$ azepines. ${ }^{[124]}$ In addition to carboxamides 26, 62 or 64, also sulfonamides ${ }^{[125]}$ and phosphonic amides ${ }^{[126]}$ proved viable for oxidative cobalt-catalyzed $\mathrm{C}-\mathrm{H} / \mathrm{N}-\mathrm{H}$ activations with alkynes $\mathbf{6}$ as the coupling partner. 
Very recently, Daugulis further accomplished a rare example of oxidative cobalt-catalyzed $\mathrm{C}-\mathrm{H} / \mathrm{O}-\mathrm{H}$ alkyne annulation of readily accessible aromatic carboxylic acids $68 .^{[127]}$ Here, TFE was used as the solvent, along with $\mathrm{O}_{2}$ and $\mathrm{CeSO}_{4}$ as chemical oxidants (Scheme 22d). Moreover, instead of simple alkynes 6, Nicholls recently demonstrated that 1,3-diynes 70 were broadly applicable in $\mathrm{C}-\mathrm{H} / \mathrm{N}-\mathrm{H}$ annulation reactions with benzamides $\mathbf{2 6}$ to deliver a variety of alkynylated isoquinolones 71 (Scheme 22e). ${ }^{[128]}$ In light of the pioneering work on cobaltaelectrocatalysis (vide infra), in 2019, Rueping and Sundararaju reported on cobalt-catalyzed $\mathrm{C}-\mathrm{H} / \mathrm{N}-\mathrm{H}$ alkyne annulations of benzamides $\mathbf{2 6}$ with the aid of photoredox catalysis and oxygen as the terminal oxidant. The merger of cobalt catalysis and photoredox catalysis enabled mild reaction conditions. ${ }^{[129]}$ The scope of the method was later extended to oxidative alkene annulations of benzamides 26. ${ }^{[130]}$

Indeed, the oxidative $\mathrm{C}-\mathrm{H} / \mathrm{N}-\mathrm{H}$ alkyne annulations with inexpensive cobalt(II) salts were not restricted to the activation of $\mathrm{C}\left(\mathrm{sp}^{2}\right)-\mathrm{H}$ bonds. Zhang recently exemplified cobaltcatalyzed $\mathrm{C}\left(\mathrm{sp}^{3}\right)-\mathrm{H}$ activations of amides $\mathbf{7 2}$ with alkynes $\mathbf{5 3}$ for the step-economical synthesis of $\gamma$-lactams 73 (Scheme 23). ${ }^{[131]}$ Under relatively harsh reaction conditions of $150{ }^{\circ} \mathrm{C}$ and an excess of chemical oxidants, several different aliphatic amides 72 were successfully transformed into the desired products 73. Interestingly, the authors found that TBAI was essential for the catalysis and a combination of two bases $\mathrm{Na}_{2} \mathrm{CO}_{3}$ and pyridine gave the best results. Mechanistic studies provided strong support for an organometallic $\mathrm{C}-\mathrm{H}$ cleavage event and the formation of a catalytically-relevant cobalta(III)cycle $\mathbf{7 4}$ was supported by MALDI-TOF mass spectrometric analysis.

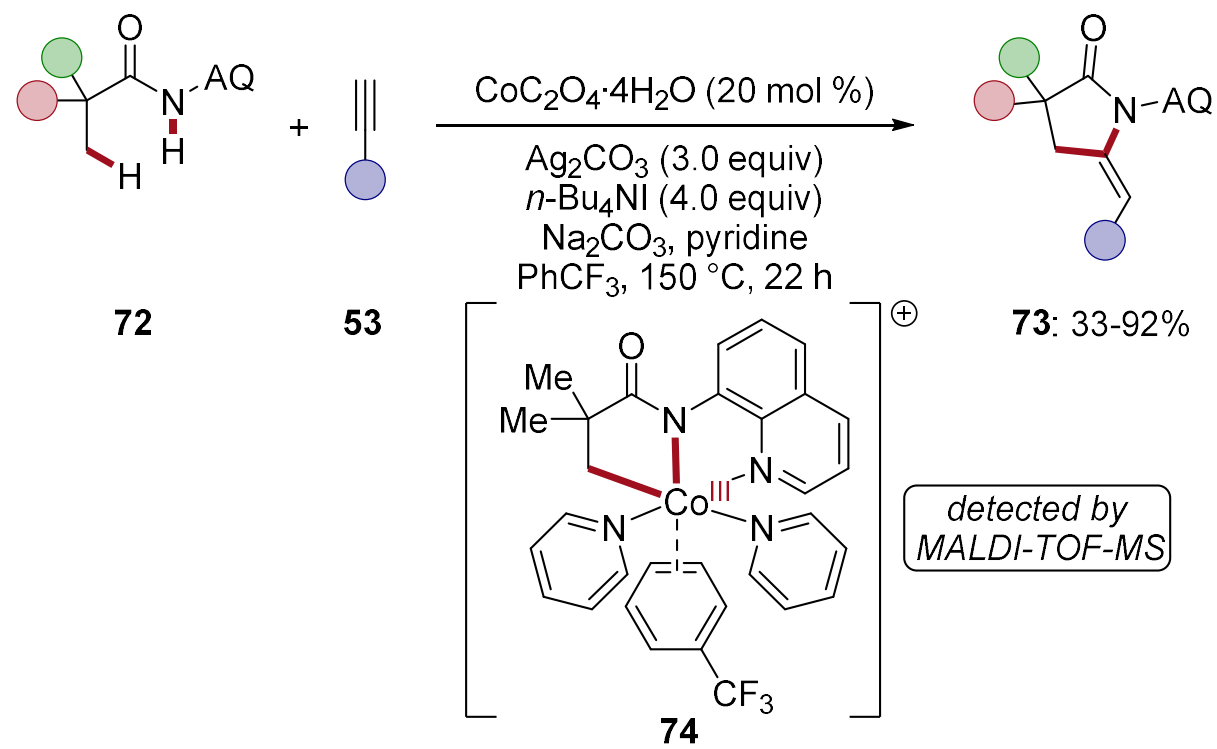

Scheme 23. Cobalt-catalyzed annulation of aliphatic amides $\mathbf{7 2}$ with alkynes $\mathbf{5 3}$. 
Similar to the vast number of reports on alkyne annulations, several groups have recently shown great interest in the use of more versatile allenes $\mathbf{7 5}^{[132]}$ as an unsaturated coupling partner for oxidative cobalt-catalyzed $\mathrm{C}-\mathrm{H} / \mathrm{N}-\mathrm{H}$ activations. ${ }^{[23 \mathrm{a}, 55 \mathrm{a}, 110]}$ The first report on cobalt-catalyzed heterocyclization reactions of aromatic or alkenyl amides $\mathbf{2 6}$ with allenes 75 was devised by Volla in 2016 (Scheme 24). ${ }^{[133]}$

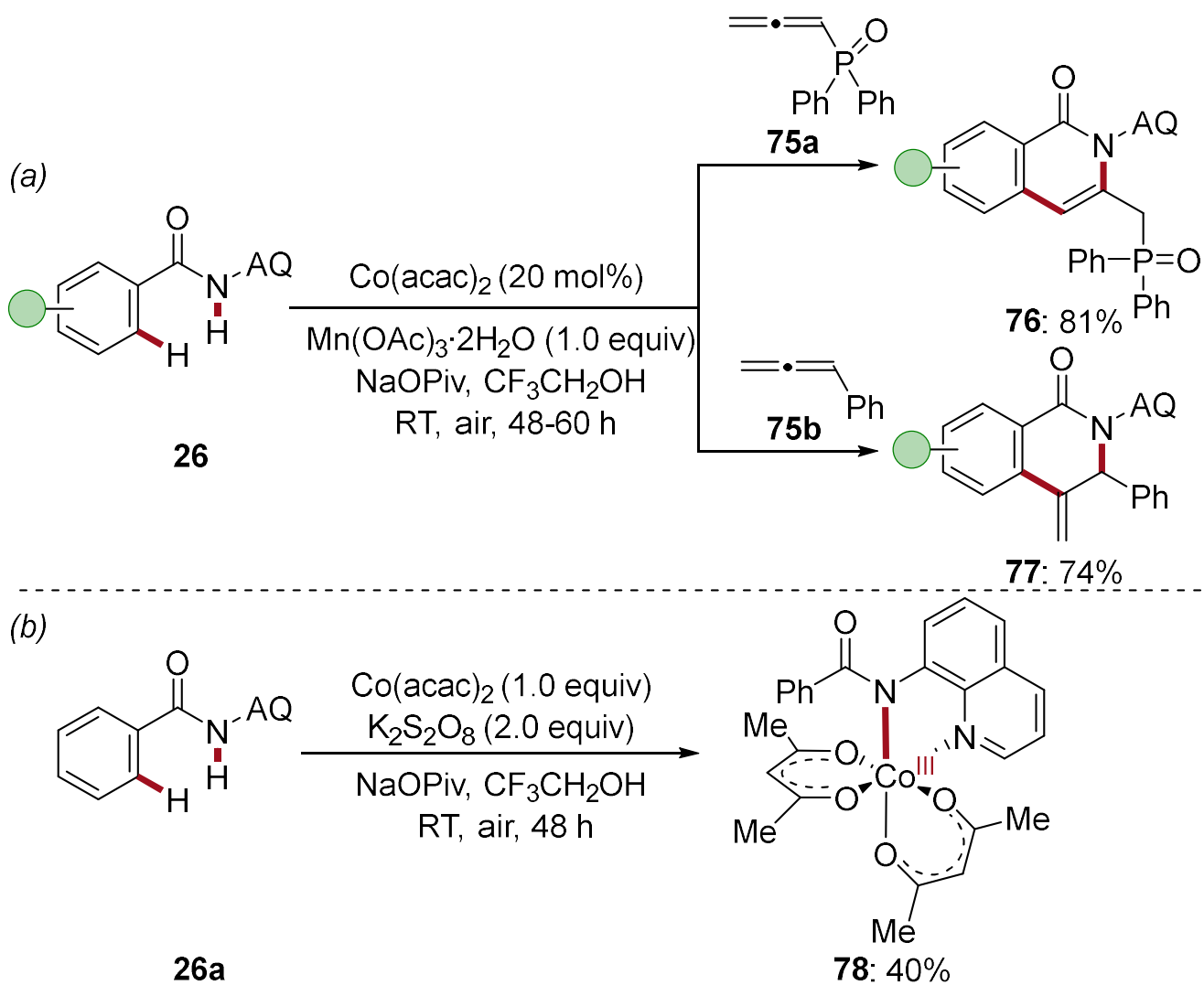

Scheme 24. Cobalt-catalyzed C-H/N-H allene annulations of benzamides 26.

The catalysis comprised mild reaction conditions at ambient temperature, $\mathrm{Mn}(\mathrm{OAc})_{3} \cdot 2 \mathrm{H}_{2} \mathrm{O}$ and $\mathrm{O}_{2}$ as the oxidants, TFE as the ideal solvent and bench-stable $\mathrm{Co}(\mathrm{acac})_{2}$ as the catalyst. In line with previous studies on precious metal-catalyzed $\mathrm{C}-\mathrm{H}$ allene annulations, ${ }^{[134]}$ regioand stereoselectivity control proved to be challenging and was particularly sensitive to the steric and electronic nature of the allene's substituents. Thus, electron-poor or sterically hindered allenes, for instance allene 75a, delivered the unsaturated heterocycle such as isoquinolone 76 (Scheme 24a). In contrast, when more electron-rich allene $\mathbf{7 5 b}$ was employed, the addition of the aryl group proceeded at the central carbon atom of the allene to furnish dihydroisoquinolin-1 $(2 H)$-ones 77 . Besides the synthetic contributions, a set of mechanistic experiments were performed to elucidate the reaction's working mode. First, $\mathrm{H} / \mathrm{D}$ exchange experiments revealed an irreversible $\mathrm{C}-\mathrm{H}$ activation step, whereas kinetic 
isotope effect studies were indicative of a facile $\mathrm{C}-\mathrm{H}$ cleavage with a $\mathrm{KIE}$ of 1.1. Second, intermolecular competition experiments excluded the presence of an electrophilic cobaltation mechanism since electron-rich benzamides 26 were favored over electrondeficient arenes 26. Finally, stoichiometric experiments of benzamide 26a with $\mathrm{Co}(\mathrm{acac})_{2}$ in the presence of $\mathrm{K}_{2} \mathrm{~S}_{2} \mathrm{O}_{8}$ as the oxidant, delivered an octahedral cobalt(III) complex 78, which was structurally confirmed by X-ray crystallography (Scheme 24b). Based on the mechanistic results and the previous findings, the authors proposed a general catalytic cycle (Scheme 25). Initially, oxidation of the cobalt(II) precursor results in the catalytically active cobalt(III) species, which along with coordination of substrate $\mathbf{2 6}$ generates the detected cobalt(III) complex 78. Thereafter, base-assisted $\mathrm{C}-\mathrm{H}$ activation takes place and furnishes the cyclometalated species 79. Then, allene coordination results in complex 80 and subsequent migratory insertion is mainly dictated by the electronic nature of the allene, thus affording two distinct seven-membered cobaltacycles 81 or 82, respectively. Finally, reductive elimination of intermediate $\mathbf{8 1}$, decorated with the electron-rich allene $\mathbf{7 5 b}$, results in the exo-methylene product 77. In contrast, when electron-deficient allene 75a was employed, reductive elimination takes place from a cobalt(III)-alkenyl complex 82 to release intermediate $\mathbf{8 3}$, which undergoes a 1,3-hydrogen shift to yield the desired isoquinolones 76 . 


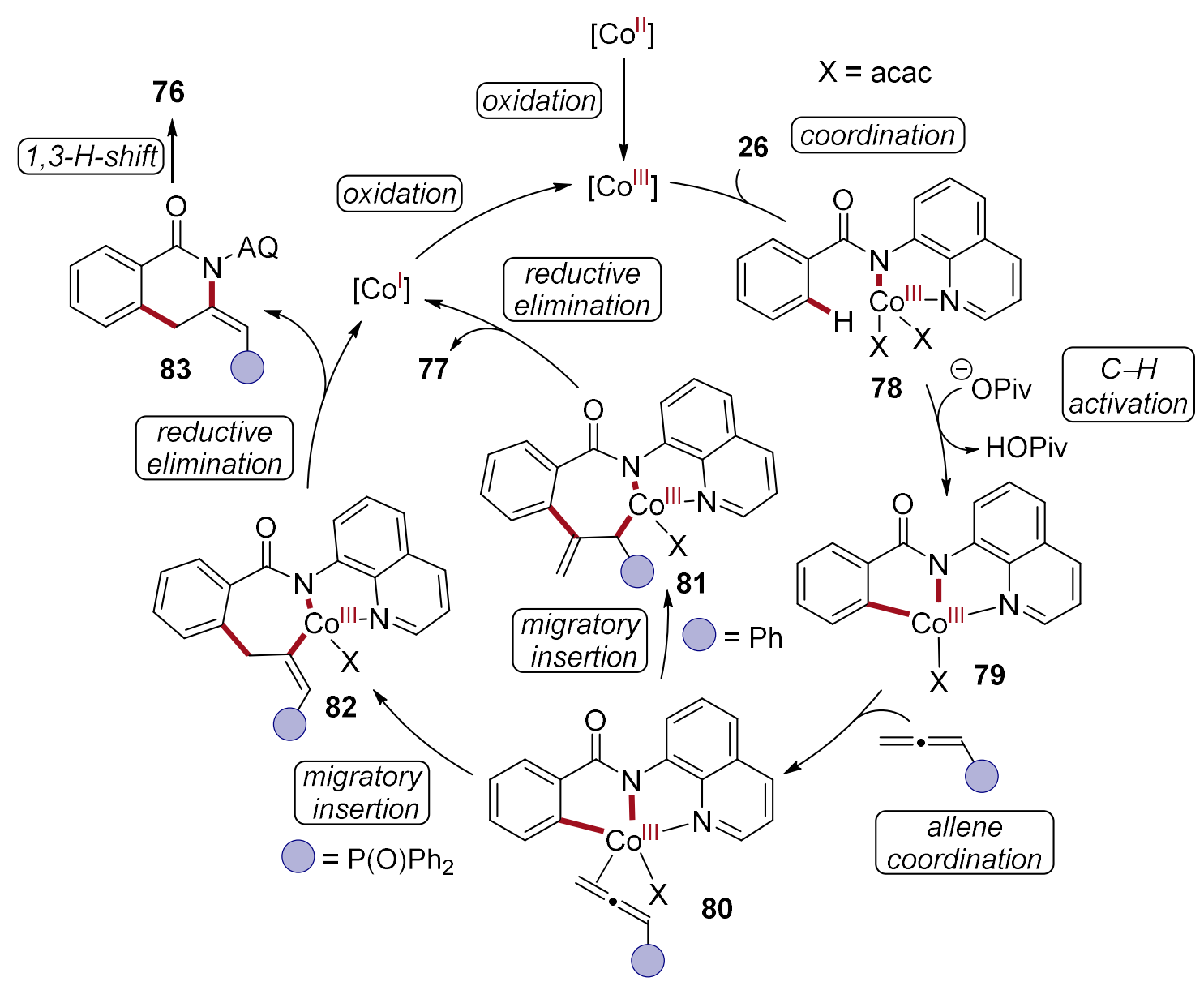

Scheme 25. Proposed mechanism for regioselective cobalt-catalyzed allene annulations.

Shortly thereafter, $R a o^{[135]}$ and Cheng ${ }^{[136]}$ independently disclosed related oxidative cobaltcatalyzed heterocyclizations of arylamides $\mathbf{2 6}$ with electron-rich or electron-deficient allenes to afford either the exo-cyclized 87 or endo-cyclized products $\mathbf{8 5} / \mathbf{8 8}$ (Scheme 26a and 26b). Interestingly, Rao observed that in addition to the electronic properties of the allenes 86, the use of different bases could alter the formation of the final products. Notably, the groups of Volla ${ }^{[137]}$ and $R a o^{[138]}$ concurrently expanded the scope of the cobaltcatalyzed $\mathrm{C}-\mathrm{H} / \mathrm{N}-\mathrm{H}$ allene annulation for the functionalization of sulfonamides 89 (Scheme 26c) and later for oxidative transformations of phosphonic amides 91 (Scheme 26d). ${ }^{[139]}$ Furthermore, allene annulations were not restricted to the use of 8 -aminoquinoline as the directing group. In this context, Zhai recently accomplished cobalt-catalyzed heterocyclizations of benzamides 62 and allenes 75 with the aid of $N, N$ '-bidentate $N$-2pyridylhydrazides as the directing auxiliary (Scheme 26e). ${ }^{[140]}$ Interestingly, in the presence of oxygen and DMP as the terminal oxidant, the authors observed oxidation of the benzylic position of the corresponding isoquinolones. 


\section{(a) Endocyclic allene annulation}

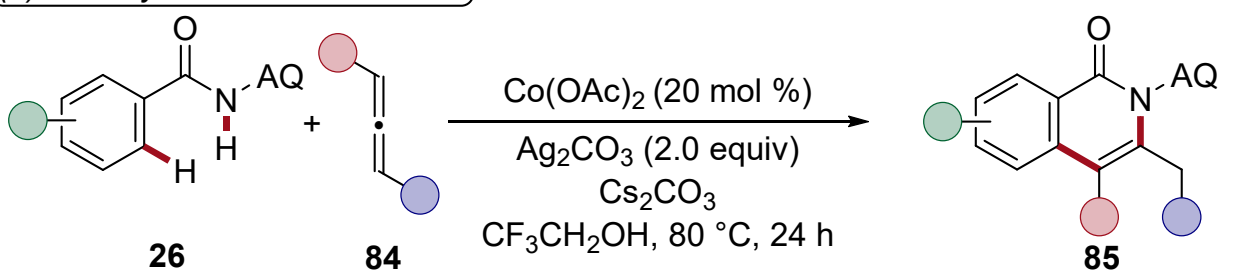

(b) Exo- or endocyclic allene annulation

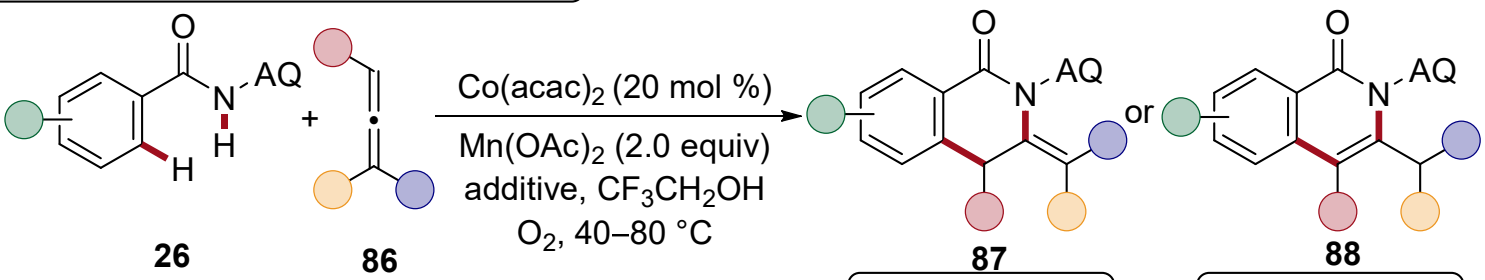

(c) Sulfonamides

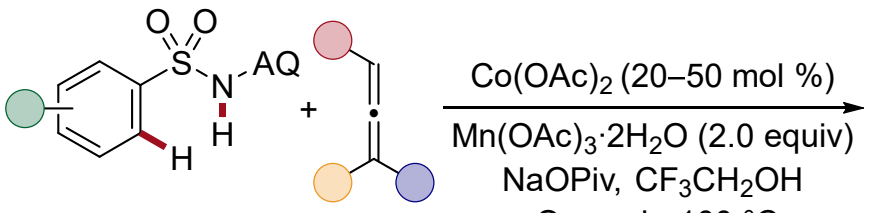

89
86

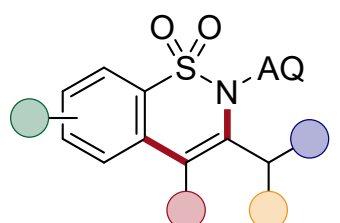

90

(d) Phosphonic amides

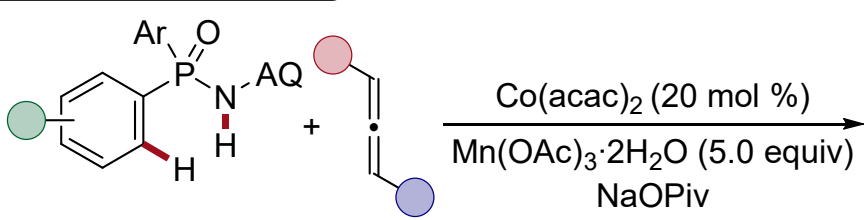

91

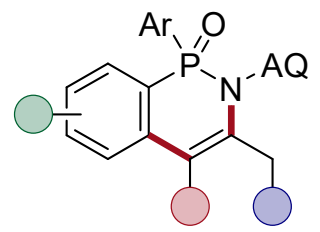

92

(e) Trifunctionalization of allenes

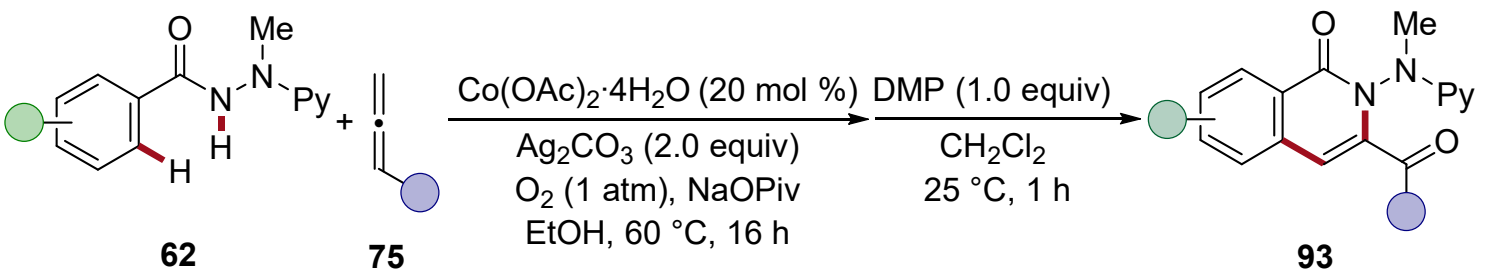

Scheme 26. Cobalt-catalyzed C-H/N-H annulations with allenes $\mathbf{7 5 , 8 4}$ or 86 .

\subsubsection{Oxidative Cobalt-Catalyzed Two-Fold C-H Arylation}

The development of cost-efficient and sustainable synthetic methods for the preparation of bi(hetero)aryls is of high demand for inter alia pharmaceutical and agrochemical research.$^{[141]}$ In this context, particularly $\mathrm{C}-\mathrm{H}$ activation, ${ }^{[5 \mathrm{~d}, 11 \mathrm{y}, 142]}$ ideally catalyzed with Earth-abundant metal catalysts, ${ }^{[23 a, 143]}$ has attracted considerable attention within the past decades. Despite the notable advances in canonical methodologies of viable isohypsic $\mathrm{C}-\mathrm{H}$ arylations, an ideal strategy in terms of step-economy and minimization of byproduct 
formation (vide supra) represents the direct coupling of $\mathrm{C}\left(\mathrm{sp}^{2}\right)-\mathrm{H} / \mathrm{C}\left(\mathrm{sp}^{2}\right)-\mathrm{H}$ bonds. ${ }^{[9,14]}$ For this purpose, oxidative cobalt-catalyzed dehydrogenative arylations via $\mathrm{C}-\mathrm{H}$ activation are highly desirable, but only rarely achieved. ${ }^{[54 \mathrm{~b}, 55 \mathrm{a}, 60]}$ However, it should be noted that highvalent cobalt-catalyzed $\mathrm{C}-\mathrm{H}$ arylations with pre-functionalized arenes as the coupling partner have previously been reported..$^{[144]}$

Likely inspired by the pioneering work of Kharash ${ }^{[49]}$ and earlier reports on Earth-abundant copper-catalyzed homocoupling reactions, ${ }^{[145]}$ in 2015, Daugulis devised a cobaltcatalyzed two-fold $\mathrm{C}-\mathrm{H}$ arylation of benzamides $\mathbf{2 6}$ with simple cobalt(II) salts as the precatalyst and a combination of oxygen and $\mathrm{Mn}(\mathrm{OAc})_{2}$ as the sacrificial oxidants (Scheme $27 a) .{ }^{[146]}$ Although the reaction was performed in ethanol, the authors did not report on competing alkoxylation reactions under the optimized reaction conditions ( $c f$. Chapter 1.1.3.1). ${ }^{[101]}$ Shortly thereafter, Liu, Zhu and $W u$ reported similar findings and reoptimized the reaction conditions for cobalt-catalyzed $\mathrm{C}-\mathrm{H} / \mathrm{C}-\mathrm{H}$ coupling of mono-dentate 2arylpyridines 14 with the aid of PIDA as the terminal oxidant (Scheme 27b).

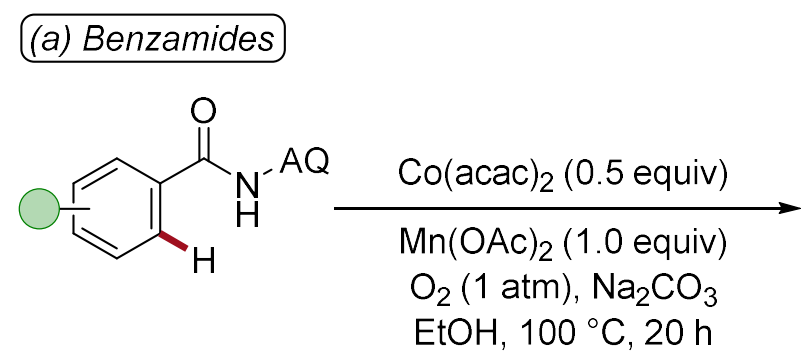

26<smiles>O=C(NC(=O)c1ccccc1-c1ccccc1)c1ccccc1</smiles>

94: $46-91 \%$

\section{(b) 2-Arylpyridines}

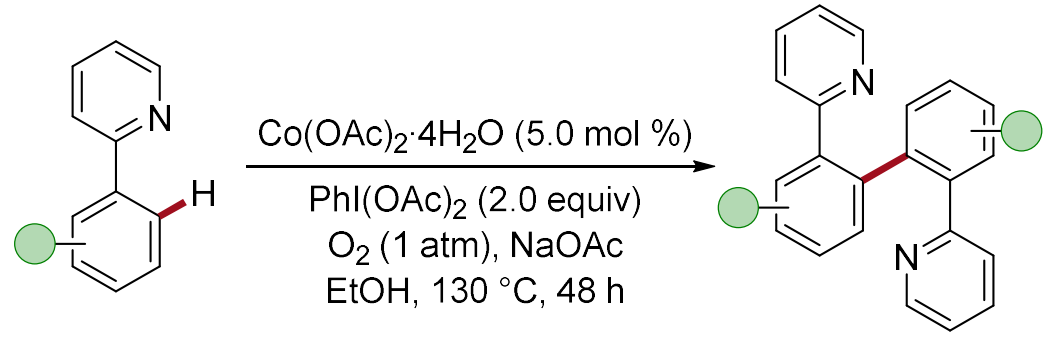

14

95: $43-77 \%$

Scheme 27. Cobalt-catalyzed oxidative biaryl-coupling of arenes 14 or 26.

In striking contrast, Niu and Song merged both strategies and disclosed an efficient crossdehydrogenative coupling of benzamides 26 with 2-arylpyridines 14 with inexpensive cobalt(III) salts as the catalyst (Scheme 28). ${ }^{[147]}$ The reaction was characterized by excellent levels of regio- and chemoselectivity and broad functional group tolerance. However, 
relatively harsh reaction conditions and super-stoichiometric amounts of chemical oxidants were required to enable high yields.<smiles>O=C(NC(=O)c1ccccc1)c1ccccc1</smiles><smiles>c1ccc(-c2ccccn2)cc1</smiles>
14

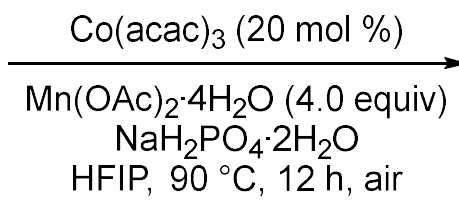

HFIP, $90^{\circ} \mathrm{C}, 12 \mathrm{~h}$, air<smiles>O=C(NC(=O)c1ccccc1-c1ccccn1)c1ccccc1</smiles>

96: $52-82 \%$

Scheme 28. Mixed two-fold $\mathrm{C}-\mathrm{H}$ arylation under oxidative cobalt catalysis.

Moreover, based on detailed mechanistic studies, the authors proposed a plausible catalytic cycle, which involves two different mechanistic pathways for the cobalt-catalyzed $\mathrm{C}-\mathrm{H}$ cleavage step (Scheme 29).

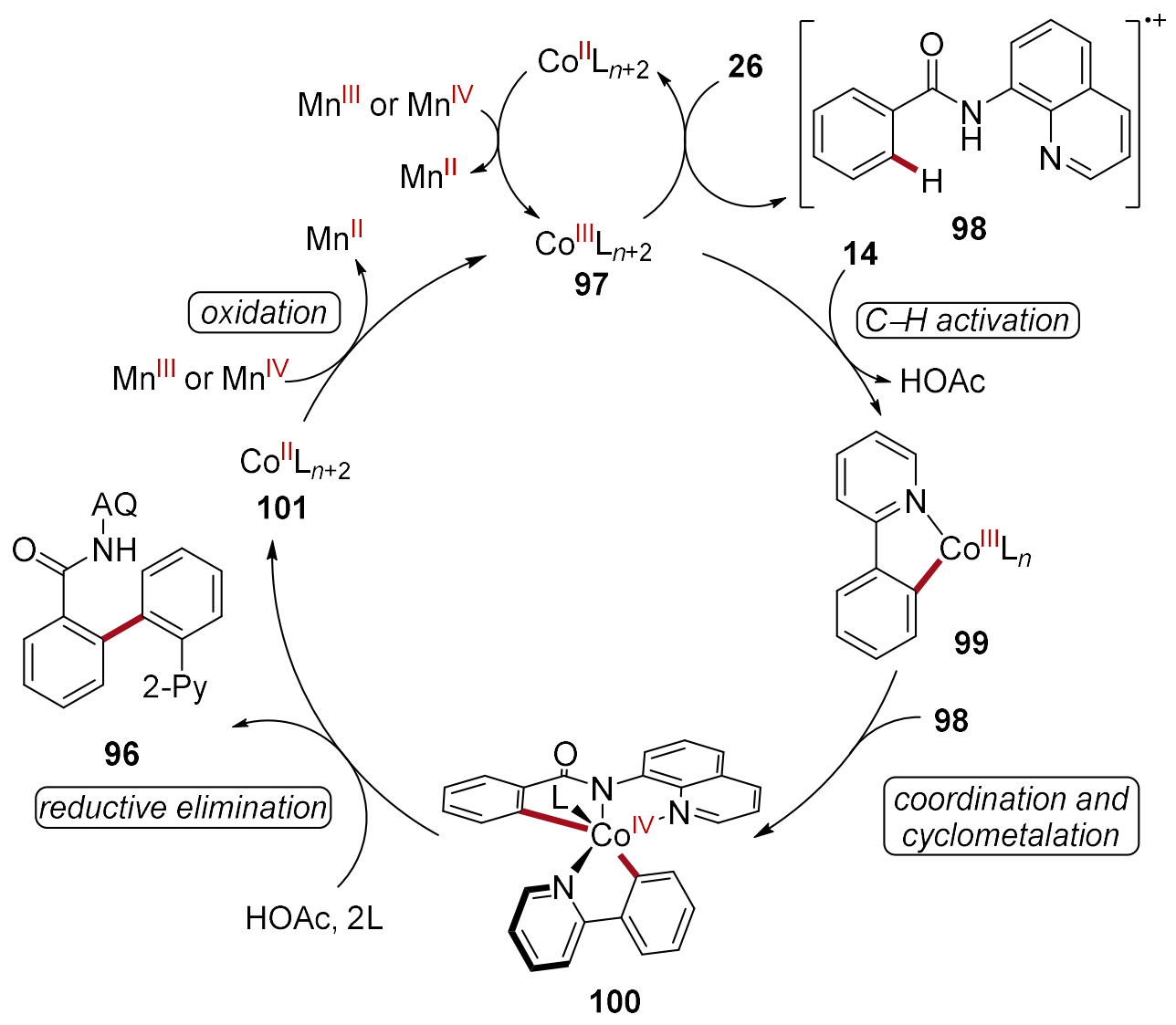

Scheme 29. Proposed catalytic cycle for cross-dehydrogenative coupling.

Thus, similar to their previous report on cobalt-catalyzed $\mathrm{C}-\mathrm{H}$ alkoxylation reactions, ${ }^{[99-}$ ${ }^{100]}$ the catalytic cycle is initiated by single-electron transfer (SET) of the catalytically active cobalt(III) species 97 to the benzamide $\mathbf{2 6}$ to generate the radical cation $\mathbf{9 8}$. Concurrently, 
the cobalt(III) species 97 is prone to undergo base-assisted $\mathrm{C}-\mathrm{H}$ activation with the 2phenylpyridine substrate 14 to generate the cobalt(III)cycle 99. Subsequently, coordination of the radical cation and a second SET-type cyclometalation forms the putative cobalt(IV) species 100. Finally, facile reductive elimination releases the biaryl product 96 and a reduced cobalt(II) species $\mathbf{1 0 1}$. The catalytic cycle is then reinitiated by oxidation of $\mathbf{1 0 1}$ to the corresponding cobalt(III) species 97. The mechanistic proposal goes in line with dehydrogenative couplings reported for $4 \mathrm{~d}$ and $5 \mathrm{~d}$ transition-metals, which are commonly suggested to proceed via high-valent metal complexes such as $\operatorname{Pd}(\mathrm{IV}),{ }^{[148]} \operatorname{Ir}(\mathrm{IV})^{[149]}$ or $\mathrm{Ru}(\mathrm{IV})$ intermediates. ${ }^{[33 \mathrm{~b}, 150]}$

Furthermore, the scope of the cobalt-catalyzed two-fold $\mathrm{C}-\mathrm{H}$ activation was recently extended by Zhang for the cross-dehydrogenative coupling of benzamides $\mathbf{2 6}$ with various other arenes, decorated with directing groups such as oximes, hydrazones or imidates. ${ }^{[151]}$ In contrast to the report by Nui and Song, the authors proposed two consecutive cobaltcatalyzed CMD-type $\mathrm{C}-\mathrm{H}$ activation events.

In addition to the presented strategies, You devised a cobalt-catalyzed crossdehydrogenative arylation of (hetero)aromatic carboxamides 26 with benzoxazoles and other $\mathrm{C}-\mathrm{H}$ acidic azoles $\mathbf{1 0 2}$ as the coupling partner (Scheme 30 ). ${ }^{[152]}$ Remarkably, with a reduced catalyst loading of $0.5 \mathrm{~mol} \%$ of $\mathrm{Co}(\mathrm{OAc})_{2} \cdot 4 \mathrm{H}_{2} \mathrm{O}$, selected products were efficiently generated in 74-98\% yield at slightly elevated reaction temperatures and longer reaction times. The method was later expanded by Wang for cobalt-catalyzed $\mathrm{C}-\mathrm{H}$ arylations with viable picolinamide as the directing group. ${ }^{[153]}$

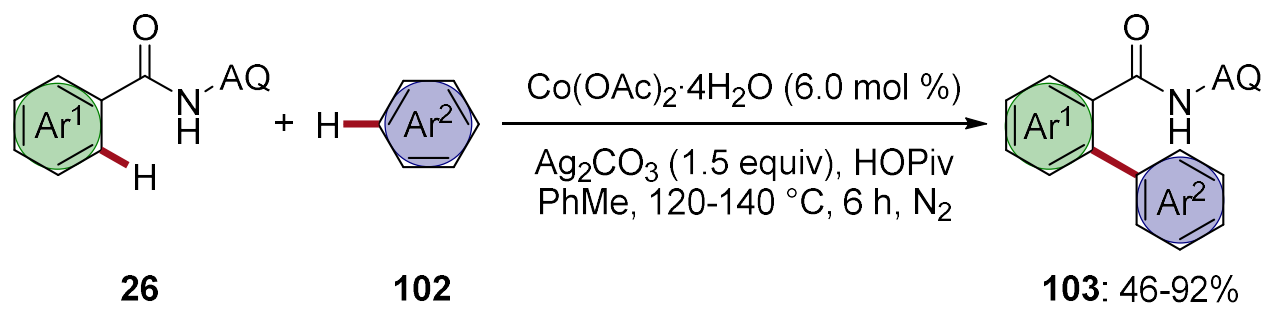

Scheme 30. Cobalt-catalyzed oxidative (het)aryl-coupling.

\subsubsection{Mechanistic Insights into High-Valent Cobalt-Catalyzed C-H Activations}

Despite the vast amount of reports on high-valent cobalt(III)-catalyzed C-H activations, detailed analytical data or snapshots of reaction intermediates are scarce and often restricted to mass spectrometry or limited NMR spectroscopic analysis. The initial findings of Daugulis on cyclometalated cobalt(III) species 30 (vide supra) have indicated the 
difficulties associated with the isolation and full characterization of mechanistically relevant organometallic reaction intermediates. However, there has also been a longstanding interest in the isolation and full characterization of highly oxidized species containing a Co-C bond, such as cobalt(IV) or cobalt(V) complexes. ${ }^{[24 \mathrm{j}, 154]}$ Thus, one of the first precedence for cobalt(IV)-carbon complexes was already reported in 1972 by reaction of alkyllithium reagents with the corresponding cobalt salt. ${ }^{[155]}$ Although, most of the reports presented to date remain as academic curiosities and have rarely been reported for synthetically useful applications. ${ }^{[87,156]}$ Nevertheless, within the last few years, few elusive cobalta(III)cycles were accessible via stoichiometric $\mathrm{C}-\mathrm{H}$ activation reactions. Cyclometalated $\mathrm{Cp}^{*}$-cobalt(III) complexes will not be discussed and can be found elsewhere. ${ }^{[74,157]}$

The first reported isolation of an organometallic $\mathrm{Co}-\mathrm{C}$ containing cobalt(III) complex 104 with a bidentate 8 -aminoquinoline ligand was accomplished by Maiti ${ }^{[158]}$ in 2016 (Scheme 31a), and later independently by Ribas with bipyridine as the co-ligand 105 (Scheme $31 \mathrm{~b}) \cdot{ }^{[159]}$ Both groups were able to fully characterize the elusive cobalt(III) cycles by means of X-ray diffraction crystallography. Moreover, the well-defined complex 104 was shown to be catalytically active for the $\mathrm{C}-\mathrm{H}$ allylation of benzamides $\mathbf{2 6}$ with unactivated alkenes. Based on these studies, similar cyclometalated cobalt(III) complexes have been reported by inter alia Song, ${ }^{[160]}$ Chatani, ${ }^{[161]}$ Zhang $^{[105]}$ and Grigorjeva. ${ }^{[162]}$

(a) Maiti<smiles>Cc1ccccc1C(=O)NC=O</smiles>

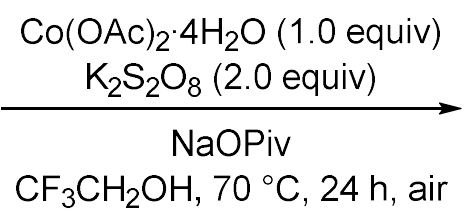

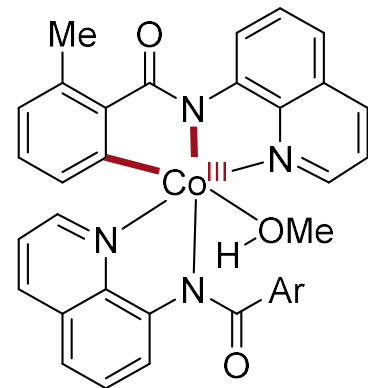

104: $15 \%$

$26 b$

(b) Ribas<smiles>O=C(Nc1ccc([N+](=O)[O-])c2cccnc12)c1ccccc1</smiles>

(1) $\mathrm{Co}(\mathrm{OAc})_{2}(1.0$ equiv) NaOPiv, $\mathrm{O}_{2}, \mathrm{CF}_{3} \mathrm{CH}_{2} \mathrm{OH}$ $80^{\circ} \mathrm{C}, 48 \mathrm{~h}$

(2) bipy (4.0 equiv)

$26 c$

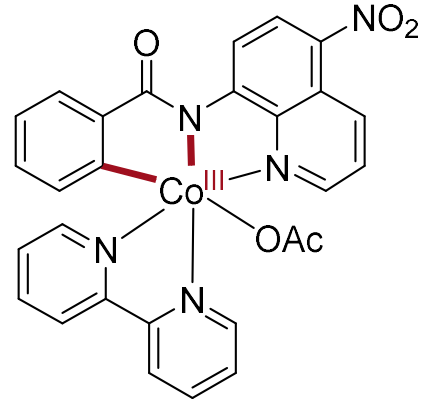

105: $42 \%$

Scheme 31. Reported cyclometalated cobalt(III) arylamide complexes 104 and 105. 
Further attempts toward the synthesis of cyclometalated cobalt(III) complexes via direct $\mathrm{C}-\mathrm{H}$ activation were made by Ribas with the aid of macrocyclic arene ${ }^{[163]}$ ligands (Scheme 32). ${ }^{[159]}$ The reactivity of the well-defined cobalt complex 106 was studied for selective alkyne 53 annulation reactions to afford either five- or six-membered ring products. Shortly thereafter, the same group disclosed a lactam 110 synthesis, when complex 106 was treated with diazo ester 107. ${ }^{[164]}$ Here, the formal $\mathrm{S}_{\mathrm{N}} 2$ reaction proceeded via an uncommon cobalt(III) enolate complex in which the aryl moiety acts as the nucleophile and the coordinating carboxylate as the leaving group. ${ }^{[165]}$

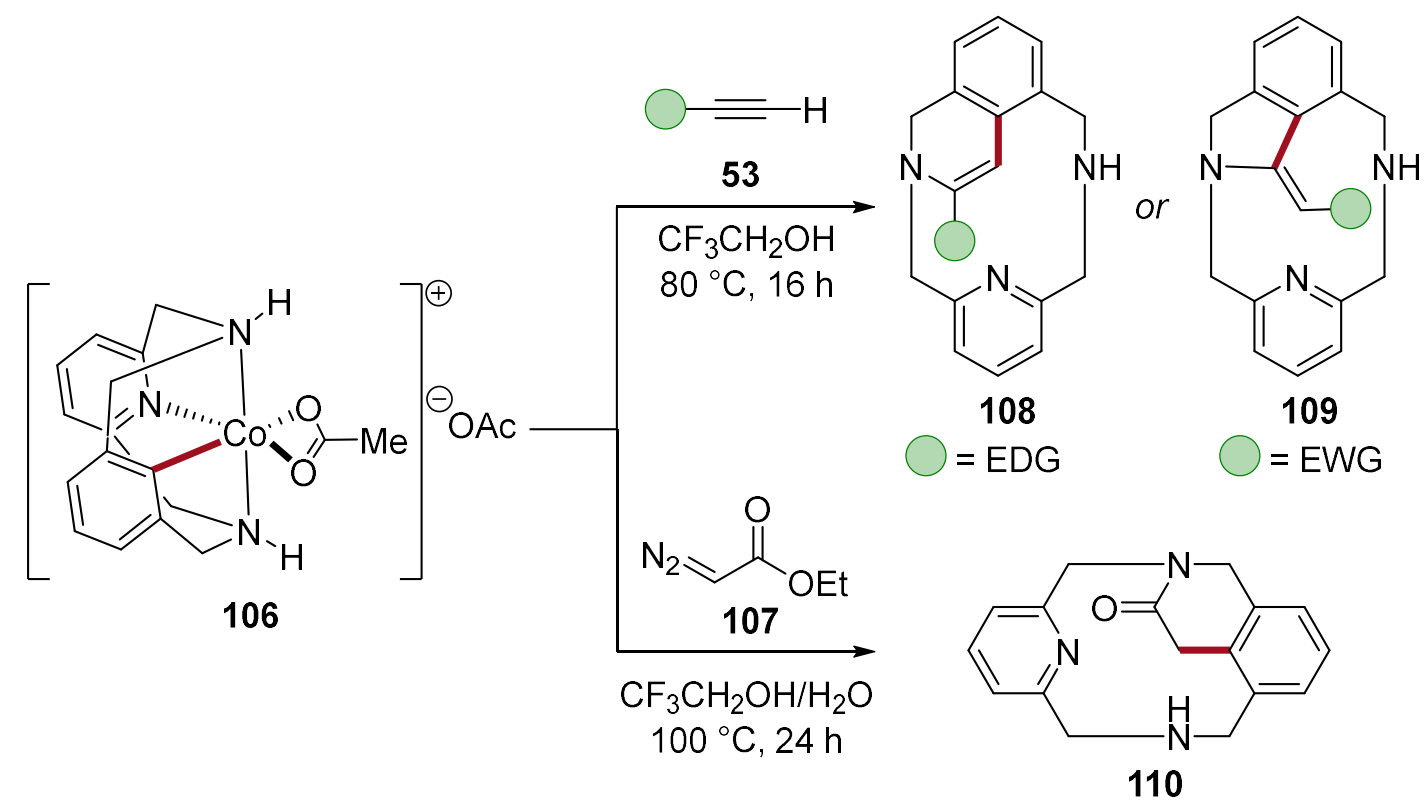

Scheme 32. Organometallic cobalt(III) complex 106 with a multidentate ligand system. 


\subsection{Undirected C-H Functionalization}

In the previous chapters, a key strategy to selectively activate the $\mathrm{C}-\mathrm{H}$ bond is presented by employing a Lewis-basic directing group, which brings the metal catalyst in close proximity to the target. While this concept has obvious benefits which justify its raison d'être, the auxiliary often needs to be present within the molecule or additional reaction steps are required to install or modify existing functional groups to meet this purpose. In addition, this prerequisite inherently limits the valuable approach for the activation of only selected $\mathrm{C}-\mathrm{H}$ bonds. In contrast, to functionalize $\mathrm{C}-\mathrm{H}$ bonds distant to the directing group or to transform unactivated aliphatic hydrocarbons without any Lewis-basic functional groups at all, chemists have developed innovative concepts that can be classified as undirected $\mathrm{C}-\mathrm{H}$ functionalization. ${ }^{[23 \mathrm{~g}, 25 \mathrm{c}, 166]}$ Among others, ${ }^{[167]}$ hydrogen atom abstraction via homolytic bond cleavage has proven to be particularly powerful within this context. ${ }^{[24,}$ 168] Generally, these free radical pathways include photochemical irradiation, ${ }^{[169]}$ electrochemistry ${ }^{[170]}$ or highly oxidizing chemical intermediates. ${ }^{[171]}$ However, controlling the site- and chemoselectivity in intermolecular alkane functionalizations remains a key challenge and is commonly dictated by the inherent electronic properties or steric environment of the $\mathrm{C}-\mathrm{H}$ bond. To overcome these limitations, transition-metal catalysis has become prevalent within the last decades to significantly improve the selectivity and efficiency of $\mathrm{C}-\mathrm{H}$ functionalizations. In this context, biological systems have evolved powerful enzymes that are capable of selective $\mathrm{C}-\mathrm{H}$ functionalizations for inter alia $\mathrm{C}-\mathrm{H}$ oxygenations ${ }^{[172]}$ or $\mathrm{C}-\mathrm{H}$ halogenations. ${ }^{[173]}$ Inspired by this toolbox, ${ }^{[174]}$ scientist have put large efforts in the development of biomimetic metal-catalyzed $\mathrm{C}-\mathrm{H}$ transformations to

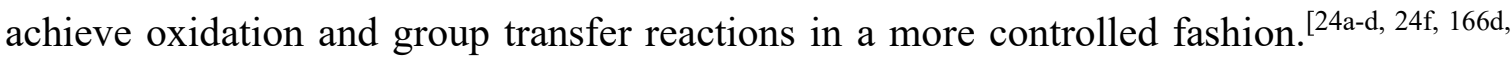
170d, 175]

\subsubsection{Undirected C( $\left.\mathrm{sp}^{3}\right)-H$ Azidation}

Organic azides ${ }^{[176]}$ have been recognized as key structural motifs in numerous molecules of interest to medicinal chemistry, ${ }^{[177]}$ material sciences, ${ }^{[178]}$ peptide chemistry or molecular biology. ${ }^{[179]}$ Due to the vast utility of azide-containing molecules, ${ }^{[180]}$ a plethora of functional group interconversion strategies have been developed, exploiting organic halides, alcohols or epoxides, among others. ${ }^{[176 e, 181]}$ However, more stepeconomical methods that directly install the azido-group into otherwise inert $\mathrm{C}\left(\mathrm{sp}^{3}\right)-\mathrm{H}$ bonds continue to be scarce. ${ }^{[182]}$ 
In 1992, Magnus disclosed a pioneering study on metal-free $\mathrm{C}\left(\mathrm{sp}^{3}\right)-\mathrm{H}$ azidation in the $\beta$ position of enol ethers to afford 112, with the aid of potentially explosive hypervalent iodine reagents and $\mathrm{TMSN}_{3}$ as the azide source (Scheme 33a). ${ }^{[183]}$ Based on this reagent combination, the substrate scope was later extended to the azidation of other functional groups such as carbamates and ureas, ${ }^{[184]}$ among others. ${ }^{[185]}$ Encouraged by these findings, notable contributions for metal-free $\mathrm{C}-\mathrm{H}$ azidations of aliphatic substrates $\mathbf{1 1 1}$ with the aid of stoichiometric amounts of hypervalent iodine reagents were independently reported by Kita ${ }^{[186]}$ Zhdankin $^{[187]}$ and Bols. ${ }^{[188]}$ More recently, Tang devised a metal-free C-H azidation of unactivated hydrocarbons 111 with $\mathrm{K}_{2} \mathrm{~S}_{2} \mathrm{O}_{8}$ as a strong oxidant and sulfonyl azide $\mathbf{1 1 5}$ as the electrophilic azide source, to deliver the desired azidation products $\mathbf{1 1 3}$ (Scheme 33b). ${ }^{[189]}$ In 2016, Chen disclosed photoredox-catalyzed $\mathrm{C}\left(\mathrm{sp}^{3}\right)-\mathrm{H}$ azidations of tertiary $\mathrm{C}-\mathrm{H}$ bonds under visible-light irradiation using the Zhdankin reagent ${ }^{[187 \mathrm{a}]} \mathbf{1 1 6}$ as the azide source (Scheme 33c). ${ }^{[190]}$ Likewise, photocatalytic azidations of benzylic $\mathrm{C}-\mathrm{H}$ bonds were subsequently reported by Greaney ${ }^{[191]}$ and Kamijo. ${ }^{[192]}$

(a) Magnus

(c) Chen

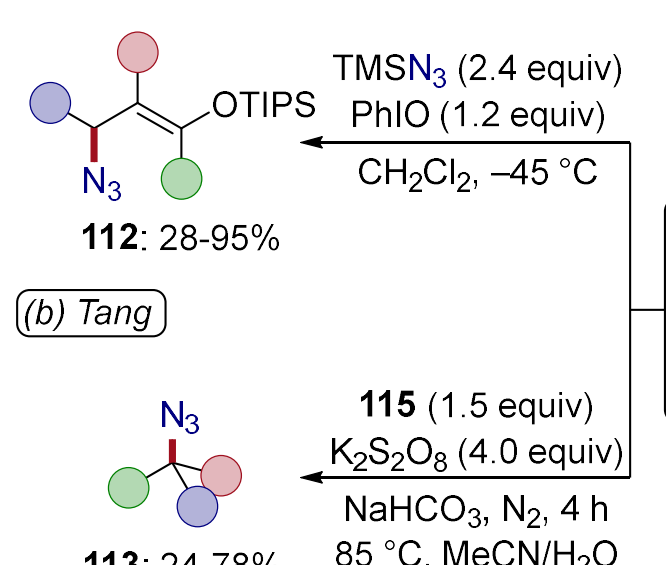

113: $24-78 \% \quad 85^{\circ} \mathrm{C}, \mathrm{MeCN} / \mathrm{H}_{2} \mathrm{O}$
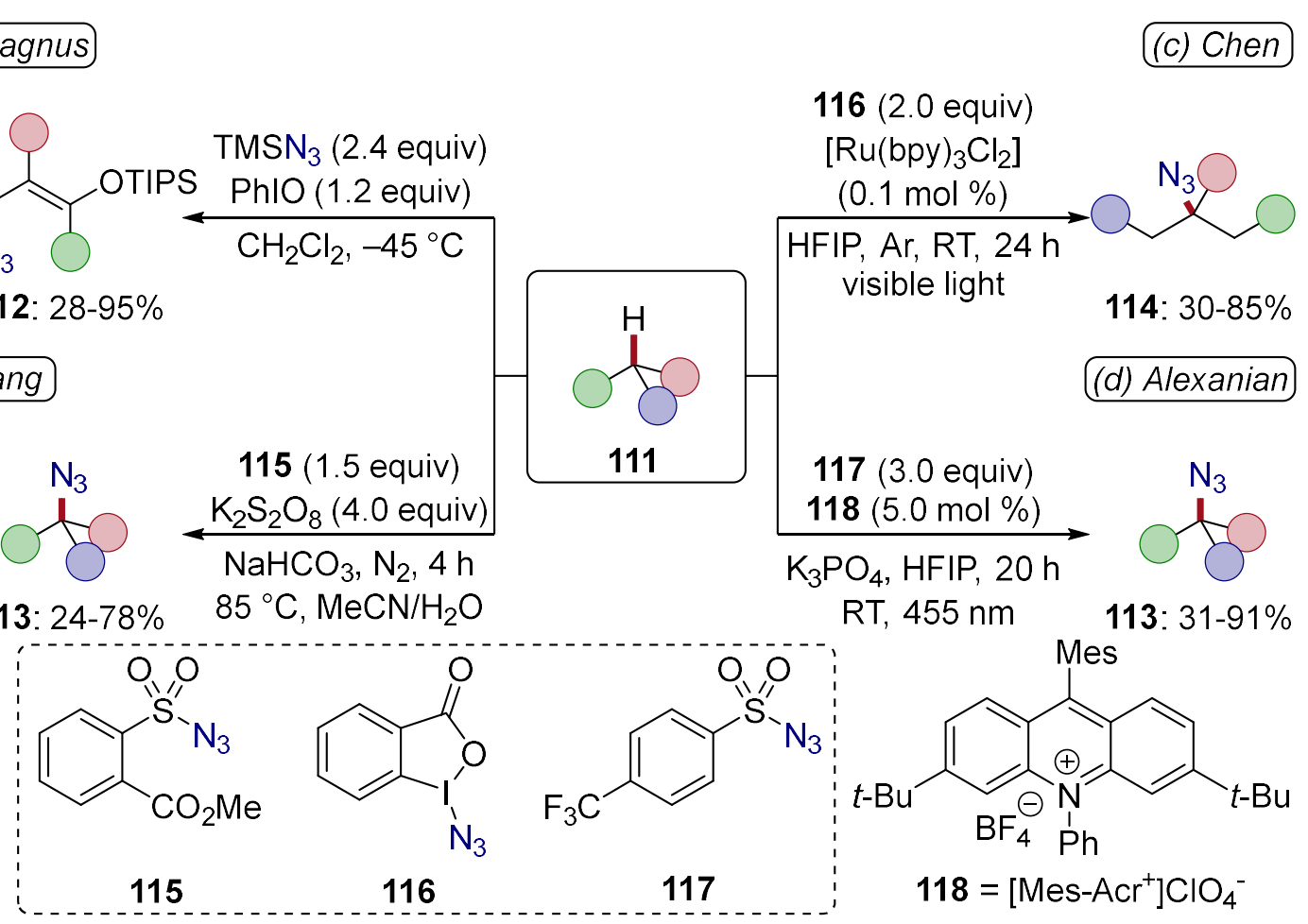

RT, $455 \mathrm{~nm}$

113: $31-91 \%$

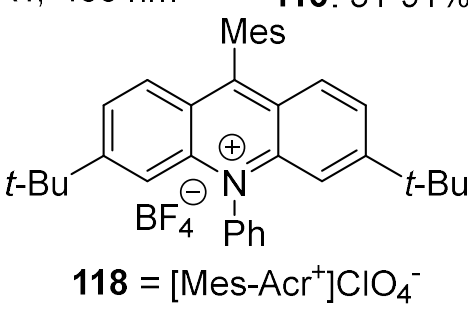

Scheme 33. Metal-free and photoredox-catalyzed $\mathrm{C}\left(\mathrm{sp}^{3}\right)-\mathrm{H}$ azidation.

A general approach for the photocatalytic azidation of unactivated $\mathrm{C}-\mathrm{H}$ bonds was later devised by Alexanian and Nicewicz (Scheme 33d). ${ }^{[169 b]}$ The method encompassed an essential phosphate salt, sulfonyl azide $\mathbf{1 1 7}$ and an acridinium photoredox catalyst 118, 
powered by blue LED irradiation. Moreover, the reaction conditions allowed for a broad substrate scope, including unactivated cyclic hydrocarbons. In the case of unsymmetrical, acyclic hydrocarbons, $\mathrm{C}-\mathrm{H}$ azidation occurred at the inherently more electron-rich $\mathrm{C}-\mathrm{H}$ bond with moderate regioselectivities. Detailed Stern-Volmer plot analysis was suggestive of a SET process from the phosphate salt to the photoexcited catalyst $118^{*}$, thus generating an oxygen-centered radical. Next, the highly oxidizing radical abstracts the most electronrich $\mathrm{C}-\mathrm{H}$ bond from substrate 111, producing a carbon-centered radical, which upon trapping by the sulfonyl azide transfer reagent 117 affords the desired organic azides $\mathbf{1 1 3}$. In spite of these notable contributions, the site-selectivity of metal-free $\mathrm{H}$ atom abstractors such as alkoxyl, iodany ${ }^{[193]}$ or sulfate radicals are inherently limited to the innate reactivity of the substrate. These radicals are typically highly electrophilic and therefore abstract the most electron-rich, less polarized, and weakest $\mathrm{C}-\mathrm{H}$ bonds in terms of bond dissociation energy. ${ }^{[15,194]}$ In contrast, a specifically designed metal catalyst can bypass these restrictions and thereby significantly enhance the reactivity profile for aliphatic $\mathrm{C}-\mathrm{H}$ functionalizations. ${ }^{[182 \mathrm{~d}]}$

In 2014, Bollinger employed a modified iron-dependent wild-type halogenase SyrB2 for the direct azidation of aliphatic $\mathrm{C}-\mathrm{H}$ bonds. ${ }^{[175 \mathrm{~h}]}$ In this proof-of-concept study, a highvalent iron(IV)-oxo active center abstracted the C4-hydrogen from L-threonine, followed by azidation via a radical-rebound mechanism. However, this approach was limited to the functionalization of substrates that were directly bonded to the carrier protein. Although early attempts to develop biomimetic iron- or manganese-catalyzed $\mathrm{C}-\mathrm{H}$ azidations of unactivated $\mathrm{C}\left(\mathrm{sp}^{3}\right)-\mathrm{H}$ bonds date back to 1983, these approaches suffered from low efficiency and large excesses of chemical oxidants. ${ }^{[195]}$ In contrast, from 2010 onwards, Groves developed a series of highly efficient manganese-catalyzed C-H halogenations ${ }^{[196]}$ that showed reactivity beyond typical hydroxylation of the previously used manganese porphyrin complexes. ${ }^{[197]}$ Inspired by these findings, the same group elegantly devised an efficient and selective manganese-catalyzed $\mathrm{C}-\mathrm{H}$ azidation of aliphatic hydrocarbons $\mathbf{1 1 1}$ with user-friendly $\mathrm{NaN}_{3}$ as the azide source, and a biphasic solvent mixture (Scheme 34). ${ }^{[198]}$ The manganese catalysis was characterized by low catalyst loadings of 1.5 $5 \mathrm{~mol} \%$, high functional group tolerance and efficient transformation of activated, as well as unactivated hydrocarbons $\mathbf{1 1 1}$ to the corresponding organic azides $\mathbf{1 1 3}$. The synthetic utility of the approach was highlighted by late-stage diversification ${ }^{[199]}$ of bioactive and pharmaceutically-relevant compounds. 
(a) Manganese-catalyzed C-H azidation

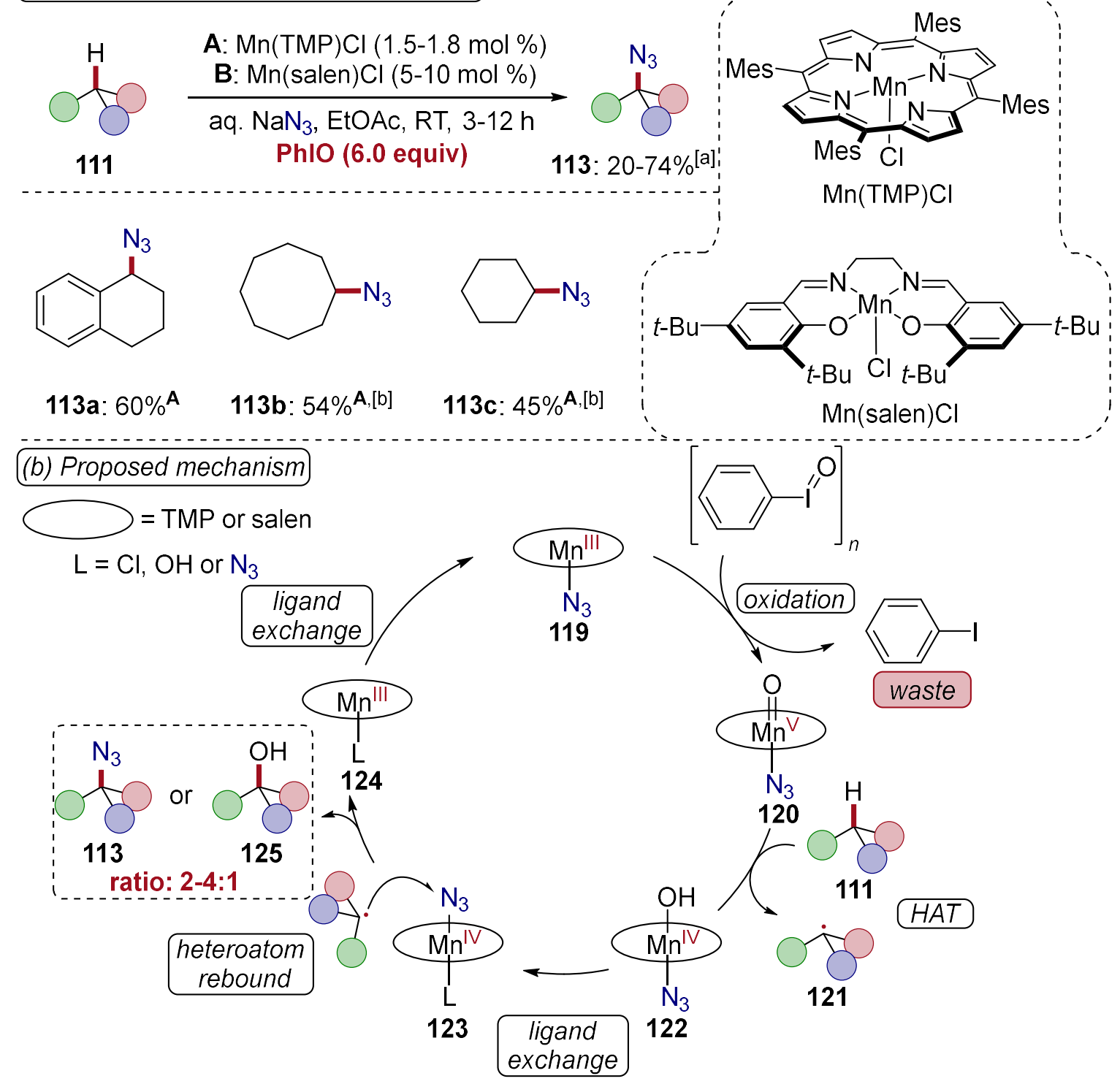

Scheme 34. Manganese-catalyzed aliphatic $\mathrm{C}-\mathrm{H}$ azidation. [a] Azide 113 to oxygenated 125 product ratios: $2-4: 1$. [b] Yield was determined relative to starting material by GC-MS.

Moreover, based on previous reports and mechanistic studies by experiment and DFTcalculations, the authors proposed a catalytic cycle of the manganese-catalyzed $\mathrm{C}-\mathrm{H}$ azidation (Scheme 34b). Initially, PhIO-mediated oxidation of the manganese(III) complex 119 generates the manganese(IV)-oxo complex 120. The highly oxidizing species 120 now undergoes hydrogen atom transfer (HAT) with substrate $\mathbf{1 1 1}$ to afford the carbon-centered radical 121 and a manganese(IV) species 122. Finally, azide transfer takes place between an azidomanganese(IV) complex 123 and radical 121 via a heteroatom rebound mechanism, thus forming the desired $\mathrm{C}-\mathrm{N}_{3}$ product 113. Despite the broad synthetic 
applicability of the method, a major drawback is the use of super-stoichiometric amounts of hypervalent iodine reagents ${ }^{[200]}$ and the relatively poor levels of chemoselectivity.

Concurrently, Hartwig reported iron-catalyzed C-H azidations of aliphatic substrates 111 with a chiral iron PyBOX catalyst and the Zhdankin azidation reagent 116 (Scheme 35). ${ }^{[201]}$ The mild reaction conditions enabled broad functional group tolerance and the strategy was later also applied to the late-stage diversification of drugs and other complex organic molecules. ${ }^{[202]}$ However, in contrast to Groves' report, iron-catalyzed C-H azidations were limited to the functionalization of tertiary and benzylic $\mathrm{C}-\mathrm{H}$ bonds.

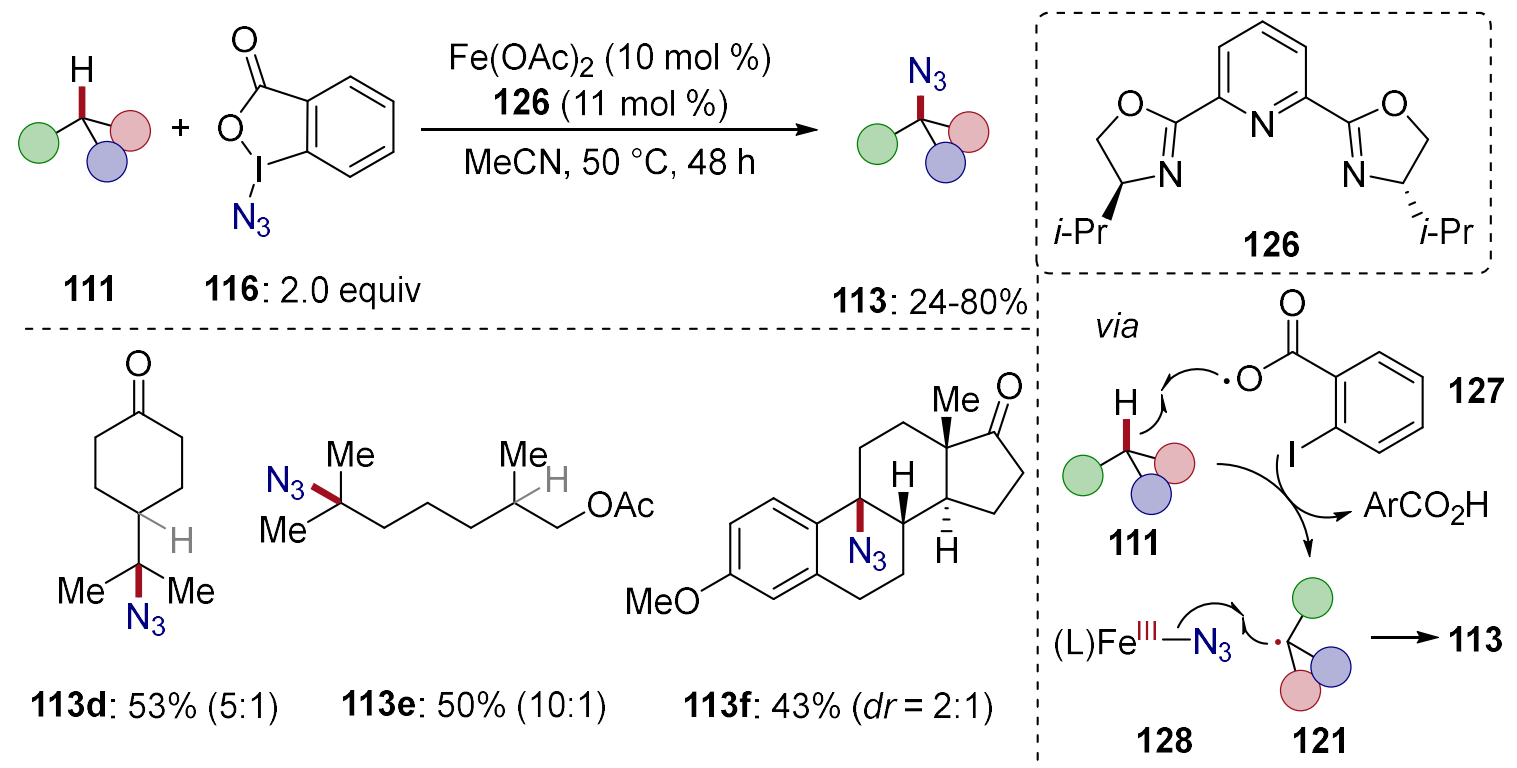

Scheme 35. Iron-catalyzed $\mathrm{C}-\mathrm{H}$ azidation of tertiary and benzylic $\mathrm{C}-\mathrm{H}$ bonds

Although detailed mechanistic studies were not conducted, the authors proposed that the reaction proceeds through a radical pathway, since radical traps such as BHT or TEMPO completely inhibited the catalysis. Moreover, kinetic studies with deuterated ethylbenzene$d_{10}$ and the non-deuterated compound revealed a large KIE of 5.0. Based on the obtained diastereomeric excess for some azidated products $\mathbf{1 1 3}$ and the relatively mild reaction conditions when comparing to Zhdankin's report, ${ }^{[187 \mathrm{a}]}$ Hartwig proposed a key catalystmediated azide-transfer step. With these observations in hand, the hydrogen abstraction likely proceeds by oxo-radical 127 , followed by azidoiron(III)-catalyzed azide transfer to afford the azidated products $\mathbf{1 1 3}$.

In addition to these notable contributions, other metal-catalyzed $\mathrm{C}\left(\mathrm{sp}^{3}\right)-\mathrm{H}$ azidations were reported, which however were limited to activated $\mathrm{C}-\mathrm{H}$ bonds such as benzylic, ${ }^{\text {[203] }}$ allylic $^{[204]}$ or acidic $\beta$-keto ester substrates. ${ }^{[205]}$ 


\subsection{Organic Electrocatalysis}

In organic synthesis, energy input is required in order to promote a desired chemical transformation. Indeed, since the very first chemical transformations were conducted, external heat was often applied to overcome intrinsic activation barriers. Likewise, scientist commonly use energy carriers to perform chemical oxidation or reduction reactions. ${ }^{[206]}$ However, apart from transferring or accepting the electrons, the carrier molecule is consumed after the transfer step and typically results in stoichiometric amounts of toxic and expensive waste products. Moreover, chemical oxidation reactions, although often used on a laboratory scale, constitute major safety concerns when performed on preparative scale and are therefore generally avoided for industrial synthetic processes. ${ }^{[207]}$ Also, most chemical energy carriers need to be chemically produced, which commonly occurs through electricity. Consequently, chemists have sought for more environmentally-benign and safe alternatives to sacrificial reagents and instead directly utilize adjustable electrical energy for synthetic operations - ideally generated by renewable energy sources such as hydro, wind or solar power (Scheme 36a). ${ }^{[13 a, 208]}$ In electrooxidation, a positive electrical potential decreases the Fermi-level of the working electrode and thus allows for oxidation of the substrate in solution with the lowest oxidation potential. To close the electrochemical circuit, electrons are transferred from the cathode to the substrate with the lowest reduction potential, which often results in the formation of hydrogen as the sole byproduct of the electrosynthetic reaction. ${ }^{[170 \mathrm{~g}, 209]}$
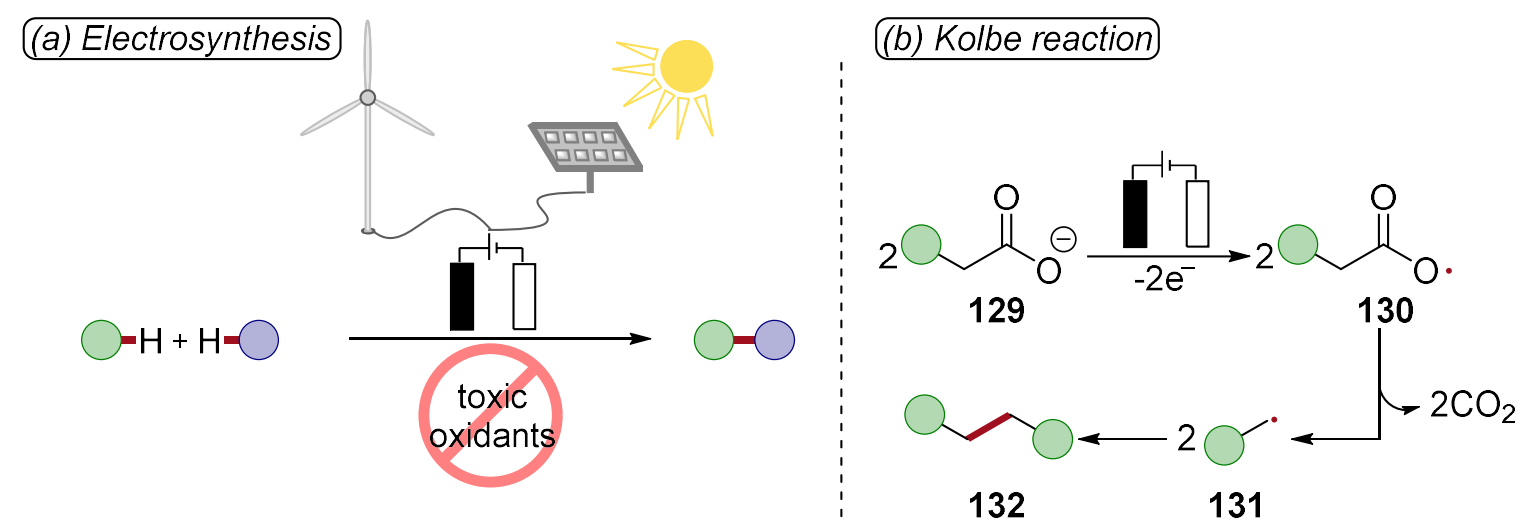

Scheme 36. (a) Sustainable energy sources for electrolysis. (b) Kolbe reaction.

In 1848, inspired by the recent reports of Faraday on the laws of electrolysis and preliminary experiments, ${ }^{[210]}$ Kolbe from Göttingen understood, interpreted and performed the first electroorganic transformation. ${ }^{[211]}$ In the later termed Kolbe reaction, organic aliphatic carboxylates 129 undergo anodic oxidation to the corresponding carboxyl radicals 
130, followed by decarboxylation to provide $\mathrm{CO}_{2}$ and the alkyl radical 131, which finally leads to the formation of alkane 132 (Scheme 36b). ${ }^{[212]}$

Since this pioneering work, electrosynthesis has evolved enormously and a variety of elegant reactions powered by electricity have been realized during the past century, ${ }^{[213]}$ ranging from applied industrial processes such as the hydrodimerization of acrylonitrile for the production of polyamide fibers - the Monsanto process, ${ }^{[214]}$ to fundamental transformations including Shono oxidation, ${ }^{[215]}$ Simons fluorination process ${ }^{[216]}$ or notable contributions to total synthesis. ${ }^{[217]}$ However, despite the powerful synthetic potential of electrochemistry, ${ }^{[218]}$ organic electrosynthesis has largely been underestimated by the synthetic community and the direct use of electric current for modern metal-catalyzed transformations was largely neglected. ${ }^{[219]}$

Stimulated by the global increase in awareness of the importance of circular ${ }^{[4 \mathrm{~b}, 4 \mathrm{e}]}$ or green chemistry $^{[4 \mathrm{a}, 4 \mathrm{~d}, 4 \mathrm{~g}]}$ and the determined pursuit of unprecedented synthetic methodology by organic chemists, electrosynthesis has experienced a resurgence in recent years. ${ }^{[170 b, 170 \mathrm{i}, 220]}$ Apart from the inherently green and cost-effective nature of electrosynthesis, a major advantage is the full control of oxidation/reduction potential applied to a chemical reaction. This provides practitioners in both academia and industrial settings with a convenient and knowledge-based tool for controlling reagent-free redox operations. ${ }^{[208]}$ However, the charge transfer selectivity is inherently dictated by the substrate's HOMO level and metalfree synthetic operations suffer from low levels of step economy for $\mathrm{C}-\mathrm{C}$ or $\mathrm{C}-\mathrm{Het}$ formations, since direct homolytic transformations would occur at the $\mathrm{C}-\mathrm{H}$ bond with the lowest bond dissociation energy (Scheme 37). ${ }^{[15]}$

\begin{tabular}{|c|c|}
\hline Electrosynthesis & Oxidative $\mathrm{C}-\mathrm{H}$ activation \\
\hline 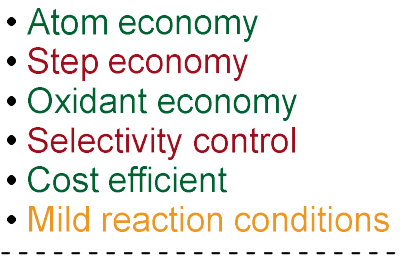 & $\begin{array}{l}\text { - Atom economy } \\
\text { - Step economy } \\
\text { - Oxidant economy } \\
\text { - Selectivity control } \\
\text { - Cost efficient } \\
\text { - Mild reaction conditions }\end{array}$ \\
\hline $\begin{array}{l}\text { Electrocatalysis } \\
\text { - Atom economy } \\
\text { - Step economy } \\
\text { - Oxidant economy } \\
\text { - Selectivity control } \\
\text { - Cost efficient } \\
\text { - Mild reaction conditions }\end{array}$ & $\begin{array}{l}\text { innate } \\
\text { reactivity electrocatalysis }\end{array}$ \\
\hline
\end{tabular}

Scheme 37. Electrosynthesis vs. oxidative $\mathrm{C}-\mathrm{H}$ activation and the merger of both: electrocatalysis. $\mathrm{BDE}_{\mathrm{C}-\mathrm{H}}\left(\mathrm{red} \approx 90 \mathrm{kcal} \cdot \mathrm{mol}^{-1}\right.$; green $\left.\approx 112 \mathrm{kcal} \cdot \mathrm{mol}^{-1}\right) \cdot{ }^{[15]}$ 
In this context, directed oxidative metal-catalyzed $\mathrm{C}-\mathrm{H}$ activation has emerged as a transformative tool to overcome the substrate's innate reactivity and constitutes one of the most promising strategies for breaking otherwise inert bonds and consequently allows for more atom- and step-economic molecular assembly with full selectivity control (Scheme 37). However, oxidative $\mathrm{C}-\mathrm{H}$ activation features an additional level of complexity in terms of oxidant economy for the key catalyst reoxidation step ( $c f$. Scheme 1). Thus, based on the previously introduced economies of organic synthesis (vide supra), a detailed assessment of the respective synthetic strategies: electrosynthesis and metal-catalyzed $\mathrm{C}-\mathrm{H}$ activation, revealed that both concepts have specific advantages and disadvantages. Hence, the direct merger of the two methodologies would eliminate the respective weaknesses and hence led to unmatched levels of resource economy and reactivity control (Scheme 37).

\subsubsection{Electrocatalysis: Merging C-H Activation and Electrosynthesis}

Despite an early work by Bercaw for anodic oxidation of benzylic $\mathrm{C}-\mathrm{H}$ bonds by means of Shilov-type ${ }^{[167 \mathrm{e}]}$ chemistry, ${ }^{[221]}$ one of the first reports on directed oxidative $\mathrm{C}-\mathrm{H}$ activation was disclosed by Jutand and Amatore for palladium-catalyzed alkenylation of acetanilide 133 with alkenes 10 (Scheme 38). ${ }^{[22]}$ The electrified Fujiwara-Moritani alkenylation $^{[223]}$ employed catalytic amounts of benzoquinone $\mathbf{1 3 6}$ as a redox mediator to reoxidize the palladium $(0)$ species $\mathbf{1 3 7}$ after reductive elimination to the catalytically active palladium(II) complex 138. However, the reaction had to be performed by strong Ncoordination in a divided cell setup to avoid electrodeposition of the palladium catalyst and the requirement of a redox mediator diminished the overall atom economy. Nevertheless, this example demonstrated the compatibility of metal-catalyzed $\mathrm{C}-\mathrm{H}$ activation with electrosynthesis and indicated the significant potential of electrocatalysis ${ }^{[224]}$ for organic synthesis. ${ }^{[9,208,213 a, 225]}$ 


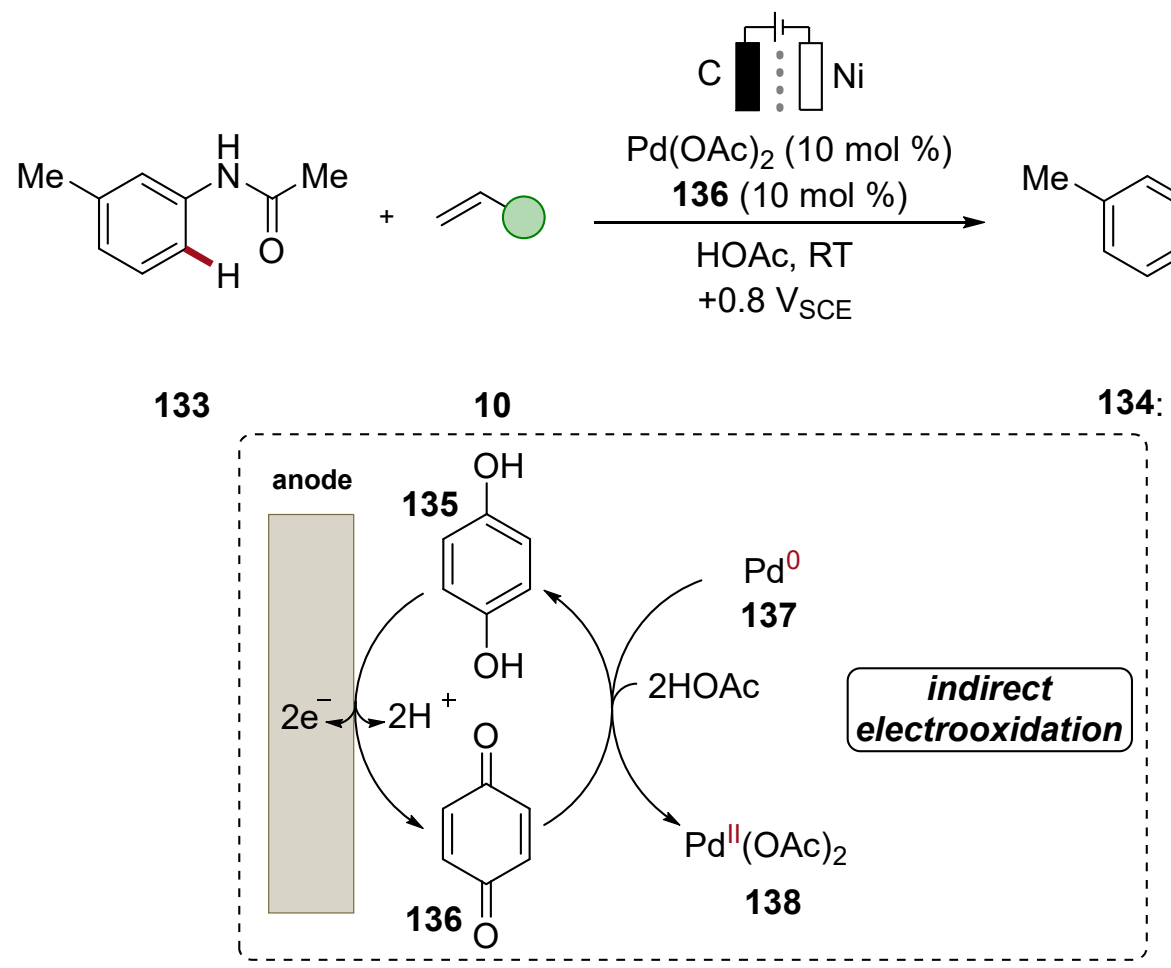

Scheme 38. Pioneering work on electrocatalytic palladium-catalyzed $\mathrm{C}-\mathrm{H}$ alkenylation.

In 2009, Kakiuchi disclosed a palladaelectro-catalyzed C-H halogenation of 2-aryl pyridines 14 with simple aqueous hydrogen halides as the halogenation reagents (Scheme 39). ${ }^{[226]}$<smiles>c1ccc(-c2ccccn2)cc1</smiles>

14
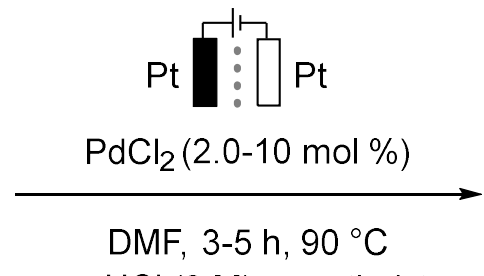

aq. $\mathrm{HCl}(2 \mathrm{M})$ as catholyte

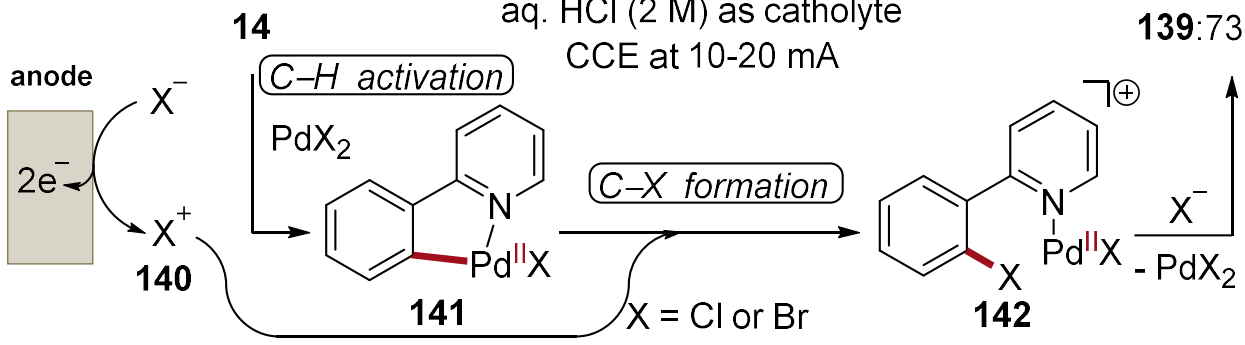

Scheme 39. Palladaelectro-catalyzed C-H halogenation of 2-arylpyridines 14 .

The proposed working mode followed previously established palladium(II)-catalyzed C-H halogenations with $N$-halosuccinimides as the electrophilic halogen surrogate. ${ }^{[227]}$ Thus, site-selective $\mathrm{C}-\mathrm{H}$ activation generates palladacycle 141 and subsequent electrophilic attack of the anodically generated halonium ion $\mathbf{1 4 0}$ furnishes the halogenated products $\mathbf{1 3 9}$ 
in an isohypsic fashion. The scope of the approach was later extended to $\mathrm{C}-\mathrm{H}$ iodination, ${ }^{[228]}$ including palladium-catalyzed domino-reactions ${ }^{[229]}$ and the $\mathrm{C}-\mathrm{H}$ halogenation of valuable quinolinamides. ${ }^{[230]}$ Notably, Mei recently discovered that less corrosive ammonium bromides were likewise viable for electrochemical palladiumcatalyzed $\mathrm{C}-\mathrm{H}$ brominations. ${ }^{[231]}$ However, in the presented examples, the utilization of electricity was limited to ensure a sufficient concentration of halonium ions, while the redox chemistry of the metal catalyst was not directly involved in the heterogeneous electron transfer step. To exploit the potential of electro-catalyzed $\mathrm{C}-\mathrm{H}$ activations, the direct oxidation of the metal catalyst on the electrode surface would be highly desirable.

In this context, Mei devised a palladaelectro-catalyzed $\mathrm{C}\left(\mathrm{sp}^{3}\right)-\mathrm{H}$ oxygenation of aliphatic oximes 143 featuring strong N-coordination with carboxylic acids 46 as the solvent and the corresponding sodium salts as the base (Scheme 40). ${ }^{[232]}$
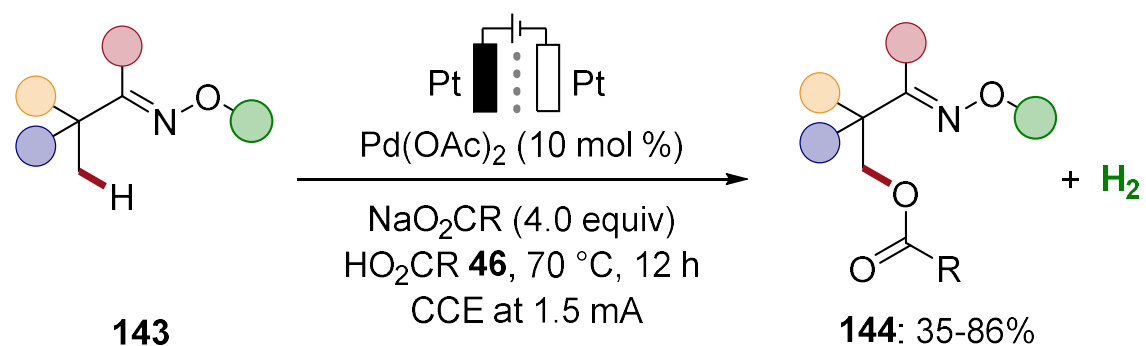

144: $35-86 \%$

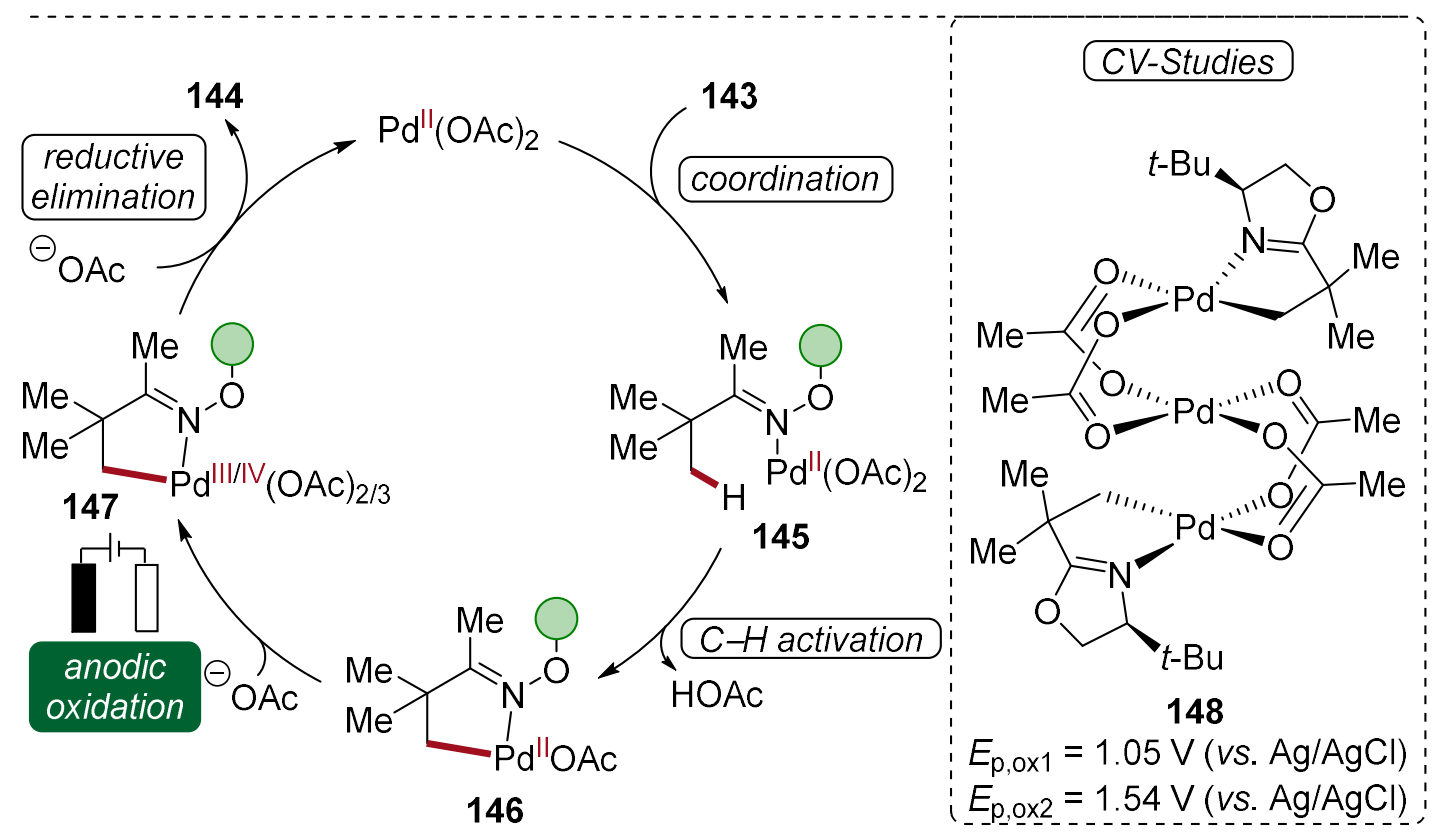

Scheme 40. Palladaelectro-catalyzed $\mathrm{C}\left(\mathrm{sp}^{3}\right)-\mathrm{H}$ oxygenation and a proposed catalytic cycle. $\mathrm{CV}$ studies were performed in $\mathrm{Ac}_{2} \mathrm{O}\left(n-\mathrm{Bu}_{4} \mathrm{NOAc}, 0.1 \mathrm{M}\right)$. 
Notably, the electro-catalyzed $\mathrm{C}-\mathrm{H}$ oxygenation enabled high levels of chemo- and regioselectivity and was compatible with valuable functional groups, such as ester, nitrile, and aliphatic halides. Based on previous reports and preliminary mechanistic studies, the authors proposed that catalysis is initiated by coordination of substrate 143 to a palladium(II) species to form species 145. Next, base-assisted $\mathrm{C}-\mathrm{H}$ palladation occurs at the neighbouring primary $\mathrm{C}\left(\mathrm{sp}^{3}\right)-\mathrm{H}$ bond to form palladacycle 146. Direct anodic oxidation of 146 furnishes a high-valent palladium(III) or palladium(IV) complex 147, which degrades under facile reductive elimination to release the acetoxylated product 144 and a reduced palladium(II) species. In order to substantiate the mechanistic proposal, the authors successfully isolated a cyclometalated palladium(II) complex 148. Subsequent CV studies revealed two distinct oxidation events at $E_{\mathrm{p}, \mathrm{ox}}=1.05 \mathrm{~V}$ and $1.54 \mathrm{~V} v s . \mathrm{Ag} / \mathrm{AgCl}$, respectively. Although a detailed interpretation of the electroanalytical results was not performed, the authors considered a direct anodic oxidation of the organometallic species to be sufficiently supported.

The scope of palladaelectro-catalyzed $\mathrm{C}-\mathrm{H}$ oxygenations was shortly thereafter extended to aromatic oximes by $M e i^{[233]}$ and subsequently by Sanford. ${ }^{[234]}$ Likewise, Chu and Sun developed palladaelectro-catalyzed $\mathrm{C}-\mathrm{H}$ oxygenations with Oxone as the hydroxylation reagent. ${ }^{[235]}$ Indeed, pallada-electrocatalysis was not limited to $\mathrm{C}-\mathrm{H}$ oxygenations. ${ }^{[236]}$ Accordingly, $\mathrm{Mei}$ later expanded the concept of palladaelectro-catalyzed $\mathrm{C}-\mathrm{H}$ transformations with $\mathrm{N}$-containing directing groups for $\mathrm{C}-\mathrm{H}$ alkylations ${ }^{[237]}$ or $\mathrm{C}-\mathrm{H}$ benzoylation. ${ }^{[238]}$ Moreover, in 2020, Ackermann devised the first asymmetric palladaelectro-catalyzed $\mathrm{C}-\mathrm{H}$ olefination with a transient directing group, thus exemplifying full selectivity control under exceedingly mild reaction conditions. ${ }^{[239]}$

Despite these early advances, electrocatalytic $\mathrm{C}-\mathrm{H}$ activation was at the outset of this thesis limited to the use of costly noble palladium catalysts. In contrast, the pioneering work presented in Chapter 3.1 represents the first electrocatalytic $\mathrm{C}-\mathrm{H}$ activation with Earthabundant metal catalysts. ${ }^{[23 a, 44]}$ Since these early findings, metallaelectro-catalyzed C-H activation has become an increasingly vibrant research arena with key contributions by Ackermann, ${ }^{[13 \mathrm{a}, 43,55 \mathrm{a}, 240]} \mathrm{Mei},{ }^{\left[236 \mathrm{~d},{ }^{241]}\right.} \mathrm{Lei}{ }^{[168,242]}$ and $X u,{ }^{[243]}$ among others. ${ }^{[244]}$

\subsubsection{Cobaltaelectro-Catalyzed C-H Activation}

While electrochemical cobalt-catalyzed cross-couplings, ${ }^{[245]}$ reductive couplings with $\mathrm{CO}_{2}{ }^{[246]}$ or outer-sphere transformations ${ }^{[247]}$ had been reported earlier, ${ }^{[248]}$ cobaltaelectro- 
catalyzed $\mathrm{C}-\mathrm{H}$ activations were unprecedented until the beginning of this thesis. Based on the pioneering work on $\mathrm{C}-\mathrm{O}$ formations ( $c f$. Chapter 3.1), ${ }^{[249]}$ further cobaltaelectrocatalyzed $\mathrm{C}-\mathrm{H}$ transformations have been investigated and shall be discussed in the following chapter.

At the beginning of 2018, Ackermann developed oxidative $\mathrm{C}-\mathrm{H} / \mathrm{N}-\mathrm{H}$ annulations of benzamides 34 with terminal alkynes $\mathbf{5 3}$ as versatile coupling partners in a cobaltaelectrocatalyzed manifold (Scheme 41a). ${ }^{[250]}$ The reaction proceeded at exceedingly mild reaction conditions and tolerated a broad range of functional groups, including halides, ethers and oxidatively labile heterocycles such as thiophene. Notably, the same group later expanded the electrooxidative alkyne annulation for the functionalization of benzamides $\mathbf{6 2}$ with internal alkynes 6 by means of an electroreductively removable benzhydrazide auxiliary (Scheme 41b). ${ }^{[251]}$

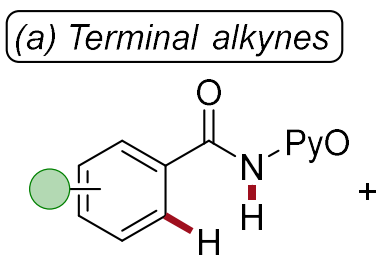

34

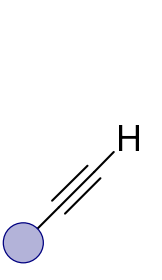

53

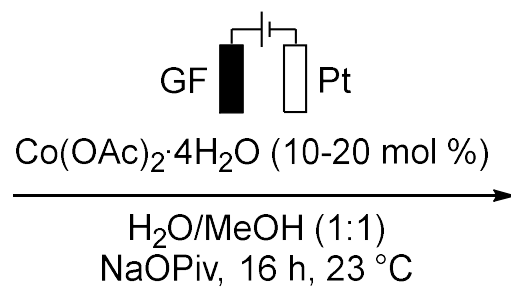

CCE at $4 \mathrm{~mA}$<smiles>O=c1c2cc3ccc2cc3n1[18O]</smiles>

51: $51-89 \%$

(b) Internal or terminal alkynes<smiles>CN([P+])NC(=O)c1ccccc1</smiles>

62

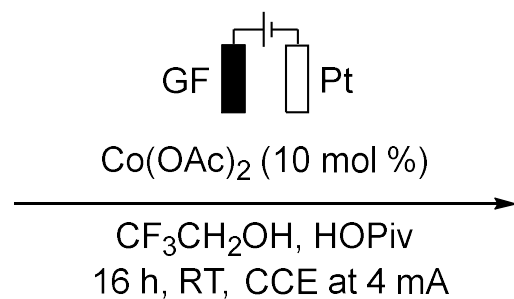

$16 \mathrm{~h}, \mathrm{RT}, \mathrm{CCE}$ at $4 \mathrm{~mA}$<smiles>CN([PH3+])n1c(O)c(O)c2ccccc2c1=O</smiles>

63: $47-95 \%$

(c) Gaseous acetylene<smiles>CC(=O)c1ccccc1</smiles>

26
6

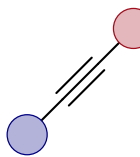

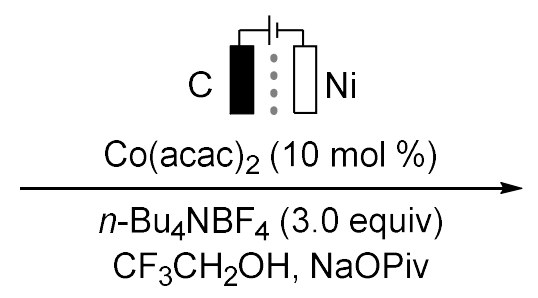

$3 \mathrm{~h}, 70^{\circ} \mathrm{C}, \mathrm{CCE}$ at $5 \mathrm{~mA}$<smiles>O=C([OH2+])n1ccc2occc2c1=O</smiles>

27: $49-85 \%$

Scheme 41. Cobaltaelectro-catalyzed isoquinolone synthesis via $\mathrm{C}-\mathrm{H} / \mathrm{N}-\mathrm{H}$ activation.

Thereafter, Lei reported a similar $\mathrm{C}-\mathrm{H} / \mathrm{N}-\mathrm{H}$ activation of benzamides 26 with 8aminoquinoline as the directing group (Scheme 41c). Key findings of their report included the use of gaseous acetylene (6c) or ethylene as the coupling partner. Also, kinetic studies were indicative of an overall rate-limiting anodic oxidation event. However, in direct 
comparison with the previous reports of Ackermann, the activation of benzamides $\mathbf{2 6}$ required inherently higher reaction temperatures, additional conducting salts and a divided cell setup.

Later in 2018, Ackermann elegantly devised valuable cobaltaelectro-catalyzed C-H aminations of benzamides 34 under mild reaction conditions and the absence of chemical oxidants (Scheme 42). ${ }^{[252]}$ Moreover, biomass-derived GVL was used as the reaction medium, thus enabling full resource economy. However, to overcome the low conductivity of the solvent, $n$-Bu $\mathrm{NPF}_{6}$ was required as the conducting salt. Under the optimized reaction conditions, various aromatic or heteroaromatic amides 34 were aminated, using a broad range of secondary amines $\mathbf{1 4 9}$ as the coupling partner.

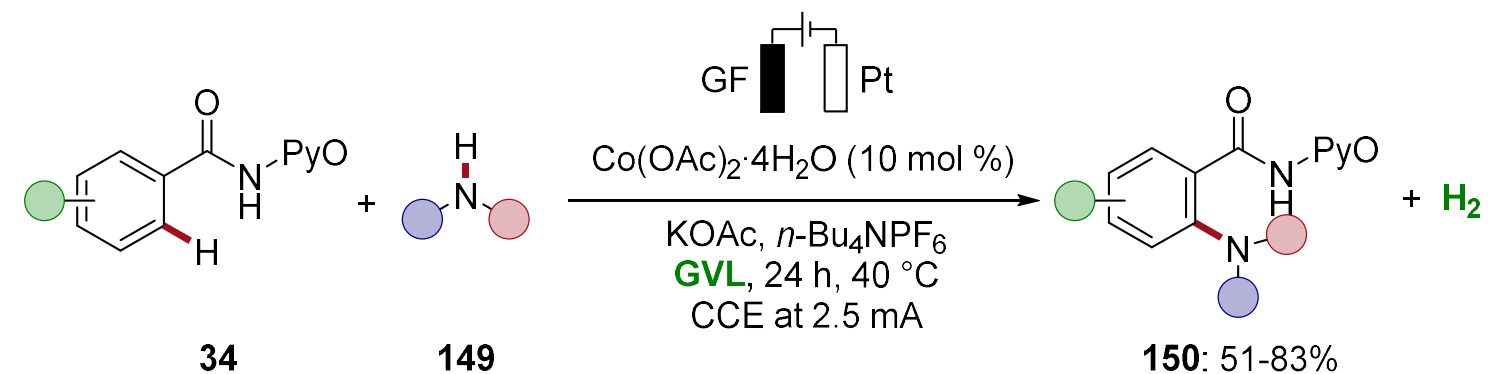

Scheme 42. Cobaltaelectro-catalyzed $\mathrm{C}-\mathrm{H}$ amination with $\mathrm{H}_{2}$ as the sole byproduct.

In addition, in-depth mechanistic studies were performed. Here, headspace gas chromatography unambiguously confirmed the formation of hydrogen as the sole byproduct. Furthermore, kinetic reaction profiles were recorded by in-operando electroIR-spectroscopic analysis and revealed a non-rate-limiting $\mathrm{C}-\mathrm{H}$ activation step. The proposed working mode followed previously established cobaltaelectro-catalyzed $\mathrm{C}-\mathrm{O}$ formations (vide infra). Concurrently, Lei reported a related cobaltaelectro-catalyzed $\mathrm{C}-\mathrm{H}$ amination of benzamides 26. To enable high yields, higher reaction temperatures of $65^{\circ} \mathrm{C}$ and the use of a divided cell setup were required. ${ }^{[253]}$

Based on these seminal reports, a plethora of dehydrogenative cobaltaelectro-catalyzed C$\mathrm{C}$ or $\mathrm{C}-\mathrm{Het}$ formations with hydrogen as a valuable byproduct were subsequently disclosed (Scheme 43). Thus, Ackermann developed C-H acyloxylation of benzamides 34 with abundant carboxylic acids 46 (Scheme 43a). ${ }^{[254]}$ Notably, biomass-derived GVL proved to be the optimal solvent for the electrocatalysis. Almost at the same time, Ackermann demonstrated the effective conversion of isocyanides $\mathbf{1 5 2}$ or gaseous carbon monoxide (153) for the synthesis of valuable heterocycles 154 (Scheme 43b) and 155 (Scheme 43c), 
respectively. ${ }^{[255]}$ Here, $N$-2-pyridylhydrazide proved to be the optimal directing group and allowed for ample substrate scope with high levels of functional group tolerance. Concurrently, Lei reported a similar cobaltaelectro-catalyzed $\mathrm{C}-\mathrm{H} / \mathrm{N}-\mathrm{H}$ carbonylation of quinolineamides 26 with a divided cell setup (Scheme 43d). ${ }^{[256]}$ On the basis of the previously reported electrooxidative alkyne annulations, Lei recently devised the synthesis of pharmaceutically-relevant sultams 157 under anodic cobalt catalysis (Scheme 43e). ${ }^{\text {257] }}$ The scalability of the approach was highlighted in a gram-scale electrocatalytic reaction with no loss of efficiency. The cobalta-electrocatalysis gained further momentum for the conversion of $\pi$-containing substrates. In this context, in 2020, Ackermann devised a C-H allylation of benzamides 26 with unactivated alkenes 158, delivering the corresponding allylated arenes 159 with high levels of chemo- and regiocontrol (Scheme 43f). ${ }^{[258]}$ The robust cobalta-electrocatalysis proved likewise effective for regio- and chemoselective $\mathrm{C}-\mathrm{H} / \mathrm{N}-\mathrm{H}$ annulation of hydrazides 62 with 1,3-diynes 70 (Scheme 43g). ${ }^{[259]}$ Remarkably, unsymmetrical 1,3-diynes $\mathbf{7 0}$ were inserted with excellent levels of regiocontrol.

(a)<smiles>O=C(N[O+])Oc1ccccc1C(=O)N[O+]</smiles>

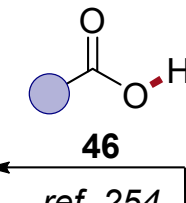

151: $32-66 \%$

(b)<smiles>CN(P)N1C(=O)c2ccccc2/C1=N/O</smiles>

154: $41-85 \%$

(c)

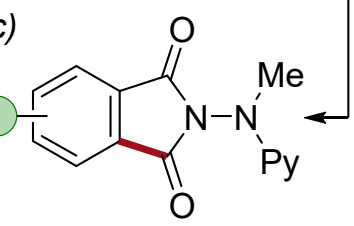

ref. 254

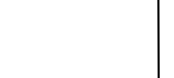
$\mathrm{H}$

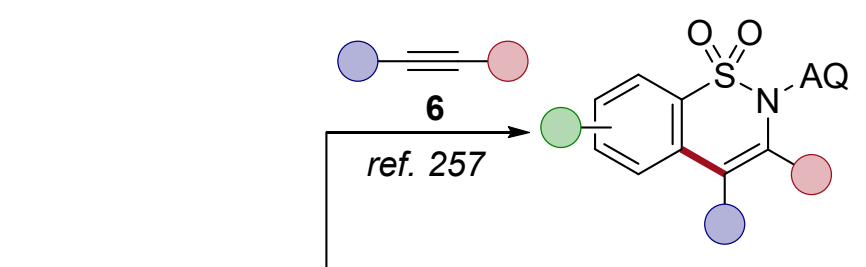

157: $51-88 \%$

(e)

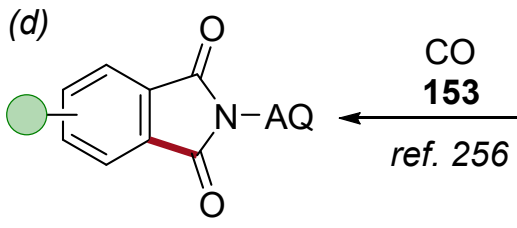

156: $52-85 \%$

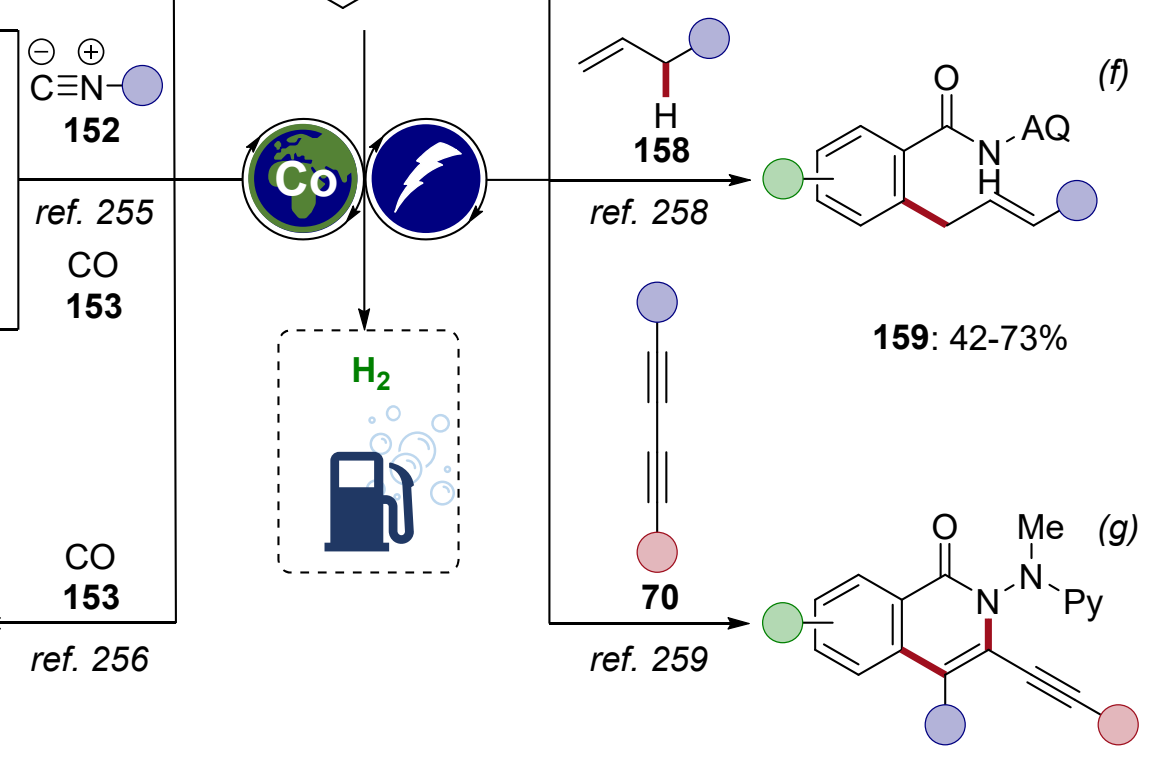

160: $44-92 \%$

Scheme 43. Recent advances in cobaltaelectro-catalyzed C-H activation. 


\section{OBJECTIVES}

The development of novel concepts for selective metal-catalyzed $\mathrm{C}-\mathrm{C}$ or $\mathrm{C}-\mathrm{Het}$ bond forming processes is of key-importance to expand the toolbox of modern organic synthesis. The several past decades have been witnessed major advances toward these goals by implementing step- and atom economical $\mathrm{C}-\mathrm{H}$ activation manifolds. But in spite of the indisputable advances, most of the developed approaches fall short in fulfilling sustainable or green synthetic criteria and rely on harsh reaction conditions, generation of undesired waste, and the use of precious metal catalysts. Thus, the focus of this thesis is directed toward the exploration of novel resource economical $\mathrm{C}-\mathrm{H}$ functionalizations, with a major center of attention on the previously underdeveloped merger of metal-catalyzed $\mathrm{C}-\mathrm{H}$ activation and electrosynthesis. ${ }^{[9,13 a, 213 a, ~ 225, ~ 240 f] ~}$

In recent years, Earth-abundant and cost-effective cobalt complexes have emerged as viable catalyst for oxidative $\mathrm{C}-\mathrm{H}$ activations. ${ }^{[23 \mathrm{a}, 44 \mathrm{c}, 55,61]}$ Despite major progress, ${ }^{[55 \mathrm{a}]}$ these transformations largely suffer from the use of stoichiometric amounts of toxic metal-based oxidants, which contradicts the inherently green nature of the $\mathrm{C}-\mathrm{H}$ activation strategy. Within this thesis the first cobalt-catalyzed oxidative $\mathrm{C}-\mathrm{H} / \mathrm{C}-\mathrm{H}$ or $\mathrm{C}-\mathrm{H} / \mathrm{Het}-\mathrm{H}$ coupling should be explored, employing anodic oxidation to reactivate the catalyst and cathodic proton reduction to generate molecular $\mathrm{H}_{2}$, which would obviate the use of sacrificial oxidants (Scheme 44). The envisioned concept would be highly desirable for the environmentally-benign formation of $\mathrm{C}-\mathrm{O}$ bonds or the synthesis of heterocycles via straightforward $\mathrm{C}-\mathrm{C} / \mathrm{C}-\mathrm{N}$ formation.

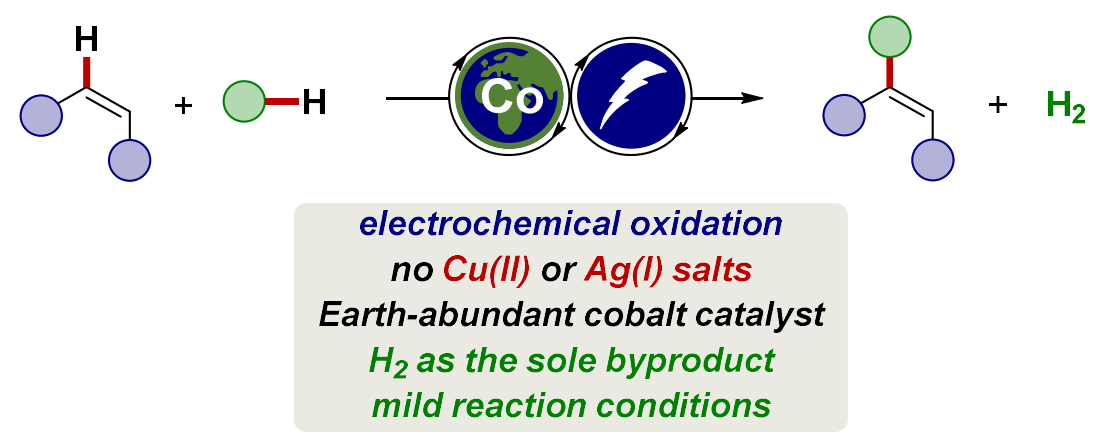

Scheme 44. The merger of cobalt-catalyzed C-H activation and electrochemistry.

In previous studies on cobalt-catalyzed $\mathrm{C}-\mathrm{H}$ activations, the catalysis was largely reported to proceed via a cobalt(II/III/I) catalytic manifold. ${ }^{[60]}$ Likewise, cobaltaelectro-catalyzed 
$\mathrm{C}-\mathrm{H}$ activations were postulated to follow similar pathways, albeit detailed experimental or computational studies were lacking. ${ }^{[240 \mathrm{c}, 240 \mathrm{e}, 240 \mathrm{f}]}$ For this purpose, comprehensive mechanistic insights would be highly desirable to not only elucidate the working-mode of the existing methodologies, ${ }^{[249-250,260]}$ but also to unravel novel reactivities (Scheme 45).
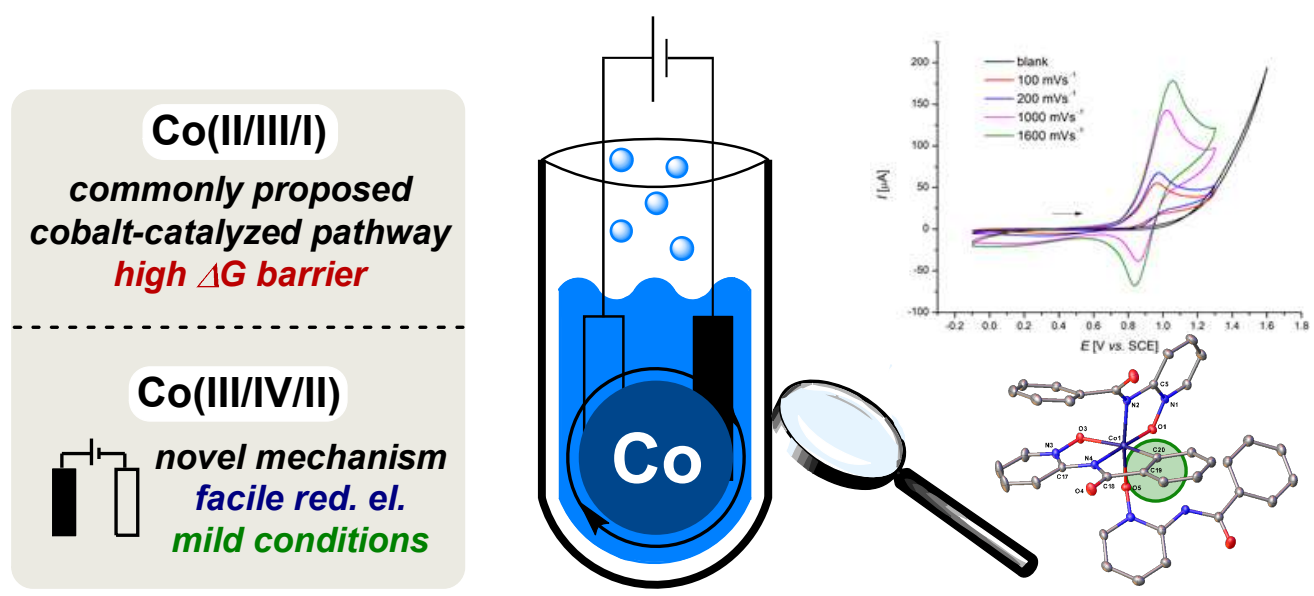

Scheme 45. Mechanistic insights into cobaltaelectro-catalyzed C-H activation.

Electrochemical synthesis offers the possibility to directly utilize electrical power and to transform the harvested energy in value-added chemical products. ${ }^{[261]}$ This concept represents an ideal scenario for a sustainable energy economy since unprofitable energy conversions from electricity to chemical charge carriers can be circumvented. ${ }^{[13 a]}$ Furthermore, a major environmental drawback of metal-catalyzed $\mathrm{C}-\mathrm{H}$ activation is reflected in the reaction media of the catalysis as commonly toxic halogenated organic solvents are used. ${ }^{[3 a, 4 e, 4 i, 45,262]}$ Thus, in a proof-of-concept study, a cobaltaelectrocatalyzed $\mathrm{C}-\mathrm{H}$ activation was intended to be powered by renewable energy sources and performed within a biomass-derived reaction medium to enable full resource economy (Scheme 46).

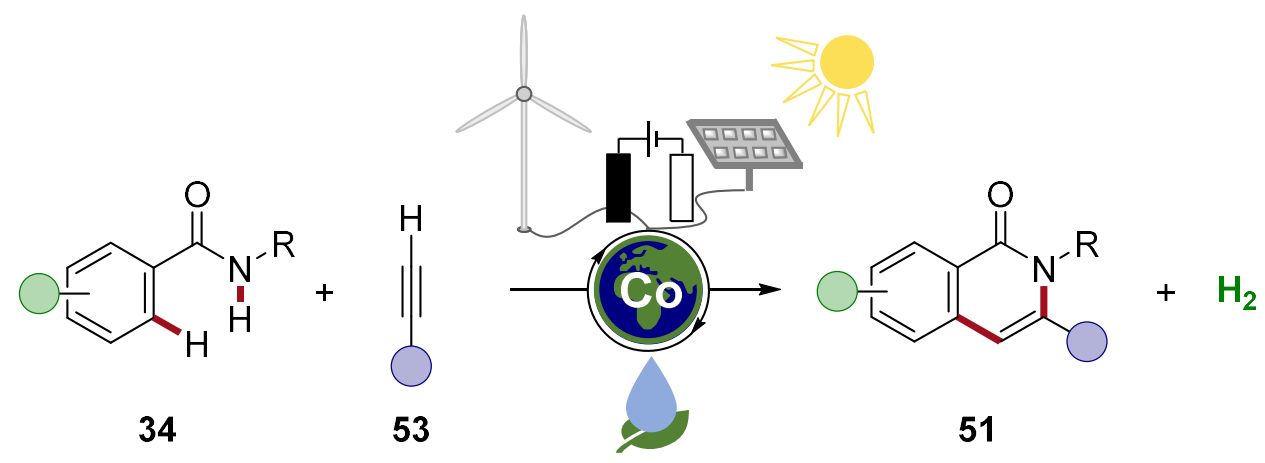

Scheme 46. Cobalta-electrocatalysis powered by renewable energy sources and performed in biomass-derived solvents. 
Electroanalytical methods represent a powerful tool for the elucidation of short-lived redoxactive reaction intermediates. ${ }^{[263]}$ During the course of this thesis, a selection of these tools such as cyclic voltammetry or rotating disc electrode experiments should be utilized to examine the reaction mechanism of various organic electrocatalytic transformations (Scheme 47).

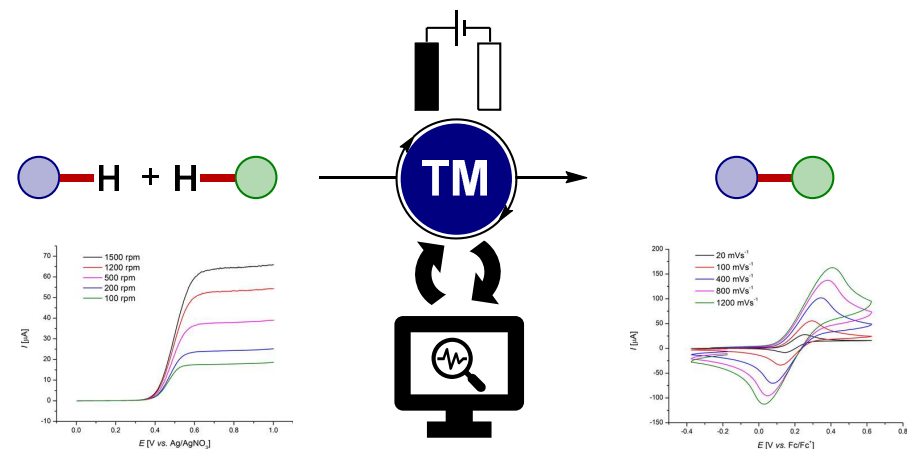

Scheme 47. Modern electroanalytical tools for reaction mechanism elucidation.

Efficient synthetic methods toward direct $\mathrm{C}-\mathrm{N}$ formations are in strong demand. ${ }^{[11 \mathrm{~h}]}$ In this context, bioinspired manganese(V)oxo complexes have proven to be particularly powerful for various undirected $\mathrm{C}\left(\mathrm{sp}^{3}\right)-\mathrm{H}$ functionalizations, such as $\mathrm{C}-\mathrm{H}$ azidation reactions. ${ }^{[182 \mathrm{~d} \text {, }}$ 196a, ${ }^{198]}$ However, these methodologies unfortunately require the employment of sacrificial oxidants, such as iodosobenzene, to generate the high-valent oxo species, thus resulting in undesired waste-products and low chemoselectivity due to competing $\mathrm{C}-\mathrm{H}$ oxygenation reactions. ${ }^{[24 \mathrm{c}, 264]}$ Here, the exploration of an electrochemical method for direct manganesecatalyzed $\mathrm{C}-\mathrm{H}$ functionalization of otherwise unactivitated $\mathrm{C}\left(\mathrm{sp}^{3}\right)-\mathrm{H}$ bonds would be highly desirable and of prime importance for inter alia late-stage drug diversification (Scheme 48).

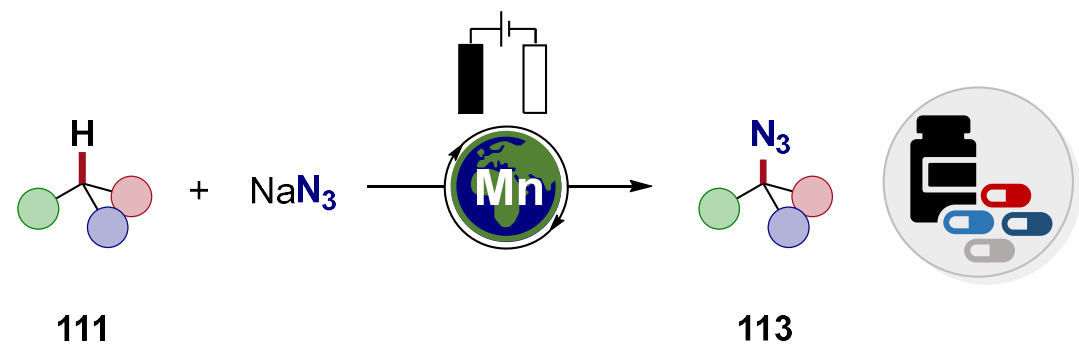

Scheme 48. Manganaelectro-catalyzed $\mathrm{C}-\mathrm{H}$ azidation of unactivated $\mathrm{C}\left(\mathrm{sp}^{3}\right)-\mathrm{H}$ bonds. 


\section{RESULTS AND DISCUSSION}

\subsection{Cobaltaelectro-Catalyzed $\mathrm{C}-\mathrm{H}$ Oxygenation}

There is a high demand for novel synthetic strategies to facilitate the construction of $\mathrm{C}\left(\mathrm{sp}^{2}\right)-\mathrm{O}$ bonds owing to their utmost importance as structural motif in a variety of pharmaceuticals and functional materials. ${ }^{[265]}$ Traditional cross-couplings by palladium ${ }^{[266]}$ or copper ${ }^{[267]}$ catalysis have been established as versatile tools for the synthesis of aryl ethers, yet rely on pre-functionalized substrates, which inevitably result in undesired amounts of byproducts and solvent waste. In contrast, the direct oxidative coupling of ubiquitous $\mathrm{C}\left(\mathrm{sp}^{2}\right)-\mathrm{H}$ and $\mathrm{O}-\mathrm{H}$ bonds would be highly desirable since pre-functionalization of the substrates could be entirely avoided. Despite major progress, previously developed oxidative $\mathrm{C}-\mathrm{H} / \mathrm{O}-\mathrm{H}$ couplings commonly exploit stoichiometric amounts of toxic and costintensive chemical oxidants, such as copper(II) or silver(I) salts, to reinitiate the catalysis ${ }^{[99]}$ and often rely on precious metal catalysts. ${ }^{[14 f, 145 i, 268]}$ Based on studies on palladiumcatalyzed $\mathrm{C}-\mathrm{H}$ transformations under electrochemical conditions, ${ }^{[222,226,232]}$ we envisioned to establish the first electrooxidative $\mathrm{C}-\mathrm{H}$ activation with an Earth-abundant metal catalyst ${ }^{[23 a]}$ such as cobalt, ${ }^{[44 c, 55 b]}$ with molecular hydrogen as the sole side product.

Even though I have initiated the project, it is particularly noteworthy that the development and execution of the following electrocatalytic experiments were performed together with Dr. N. Sauermann and both authors contributed equally to the work. ${ }^{[249]}$ Hence, larger parts of the presented project ${ }^{[249]}$ have been previously published in his doctoral thesis. ${ }^{[269]}$

\subsubsection{Optimization Studies for the Cobaltaelectro-Catalyzed C-H Oxygenation}

With the general working hypothesis in mind, various oxidative cobalt-catalyzed $\mathrm{C}-\mathrm{H}$ activations were evaluated. ${ }^{[55 a]}$ Here, particularly arenes with electron-deficient bidentate amide directing groups ${ }^{[11 \mathrm{~b}]}$ were chosen as the model substrate to stabilize the cobalt intermediates ${ }^{[99]}$ and to avoid undesired direct anodic amide oxidation. ${ }^{[270]}$ Furthermore, alcoholic solvents were envisioned to serve as the ideal reaction medium for the metallaelectro-catalyzed $\mathrm{C}-\mathrm{H}$ transformation, due to its protic nature and high conductivity. ${ }^{[271]}$ Inert electrode materials, such as platinum or graphite, were chosen as the anode and cathode material, ${ }^{[272]}$ respectively, to avoid electrode fouling under the 
electrolysis conditions and in case of platinum, to facilitate the hydrogen evolution reaction (HER). ${ }^{[273]}$

After initial successes for the cobaltaelectro-catalyzed $\mathrm{C}-\mathrm{H}$ oxygenations with benzamide 34a and readily available ethanol (35a), it was found that the electrochemical conditions, inter alia electrolysis cell dimensions, ${ }^{[274]}$ electrode materials as well as the mode of electrolysis had a strong impact on the reaction outcome (Table 1). ${ }^{[43]}$ Thus, by the use of an undivided electrolysis cell ( $c f$. Figure 77 ), constant potential electrolysis at $2.0 \mathrm{~V} v s$. $\mathrm{Ag} / \mathrm{Ag}^{+}$and two platinum electrodes, product 36aa was isolated in $18 \%$ yield and significant metal-deposition was observed on the cathode (entry 1). To avoid electrodeposition of the cobalt catalyst, a H-type divided cell was developed, based on previously reported designs. ${ }^{[275]}$ With the custom-made divided setup in hand (cf. Figure 78), the $\mathrm{C}-\mathrm{H}$ alkoxylation of benzamide $34 \mathrm{a}$ was achieved in $51 \%$ yield (entry 2 ).

Table 1. Preliminary results for the cobaltaelectro-catalyzed C-H oxygenation. ${ }^{[\mathrm{a}]}$

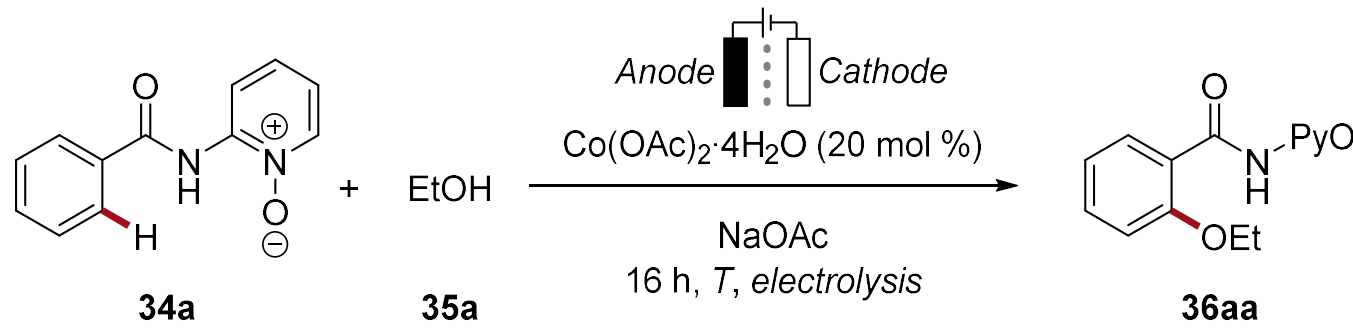

\begin{tabular}{cccccc}
\hline Entry & Mode of Electrolysis & Anode & Cathode & $T\left[{ }^{\circ} \mathrm{C}\right]$ & Yield [\%] \\
\hline 1 & $\mathrm{CPE}\left(2.0 \mathrm{~V} v s . \mathrm{Ag} / \mathrm{Ag}^{+}\right)$ & $\mathrm{Pt}$ & $\mathrm{Pt}$ & 60 & $18^{[\mathrm{b}]}$ \\
2 & $\mathrm{CPE}\left(2.0 \mathrm{~V} v s . \mathrm{Ag} / \mathrm{Ag}^{+}\right)$ & $\mathrm{Pt}$ & $\mathrm{Pt}$ & 60 & 51 \\
3 & $\mathrm{CPE}\left(2.0 \mathrm{~V} v s . \mathrm{Ag} / \mathrm{Ag}^{+}\right)$ & $\mathrm{Pt}$ & $\mathrm{Pt}$ & 23 & 48 \\
4 & $\mathrm{CPE}\left(1.4 \mathrm{~V} v s . \mathrm{Ag} / \mathrm{Ag}^{+}\right)$ & $\mathrm{Pt}$ & $\mathrm{Pt}$ & 23 & 40 \\
5 & $\mathrm{CPE}\left(2.0 \mathrm{~V} v s . \mathrm{Ag} / \mathrm{Ag}^{+}\right)$ & $\mathrm{Pt}$ & $\mathrm{Pt}$ & 23 & $24^{[\mathrm{c}]}$ \\
6 & $\mathrm{CPE}\left(2.0 \mathrm{~V} v s . \mathrm{Ag} / \mathrm{Ag}^{+}\right)$ & $\mathrm{GF}$ & $\mathrm{Pt}$ & 23 & $65^{[\mathrm{d}]}$ \\
7 & $\mathrm{CCE}$ at $6 \mathrm{~mA}$ & $\mathrm{GF}$ & $\mathrm{Pt}$ & 23 & 65 \\
8 & $\mathrm{CCE}$ at $8 \mathrm{~mA}$ & $\mathrm{GF}$ & $\mathrm{Pt}$ & 23 & $58^{[\mathrm{c}]}$ \\
\hline
\end{tabular}

[a] Reaction conditions: 34a $(0.50 \mathrm{mmol}), \mathrm{Co}(\mathrm{OAc})_{2} \cdot 4 \mathrm{H}_{2} \mathrm{O}(20 \mathrm{~mol} \%)$, NaOPiv (2.00 equiv in each halfcell), EtOH (35a) $\left(7.0 \mathrm{~mL}\right.$ in each half-cell), $T\left[{ }^{\circ} \mathrm{C}\right]$, electrolysis, $16 \mathrm{~h}$, graphite felt anode, Pt-plate cathode, divided cell. Isolated yields are given. [b] Undivided cell, EtOH (35a) $(14 \mathrm{~mL})$. [c] Electrolysis for $6 \mathrm{~h}$. [d] Reaction was performed by Dr. N. Sauermann.

Notably, decreasing the reaction temperature from $60{ }^{\circ} \mathrm{C}$ to $23{ }^{\circ} \mathrm{C}$ resulted in negligible differences in the catalytic efficacy (entry 3), while lowering the applied potential or the reaction time proved less efficient (entries 4 and 5). In contrast, by changing the anode 
material from platinum to graphite felt allowed for significantly higher catalytic efficacy (entry 6). Likewise, the more user-friendly constant current electrolysis was found to generate aryl ether 36aa in comparable yields (entries 7 and 8). Additionally, several repetitions of entries 6 and 7 revealed that the constant current electrolysis was more robust in terms of reproducibility and was therefore favored for further optimization studies.

Encouraged by these preliminary findings, various reaction parameters were evaluated for the cobaltaelectro-catalyzed $\mathrm{C}-\mathrm{H}$ oxygenation in a divided cell setup (Table 2). For the screening of different carboxylate additives, $\mathrm{Dr}$. N. Sauermann observed that the countercation did not have a significant influence on the reaction outcome, ${ }^{[269]}$ whereas $\mathrm{NaOPiv}$ was found to outperform the sterically less demanding acetate salts (entry 2). In contrast, carbonate or trifluoroacetate bases led to a significant drop in reactivity (entries 3 and 4). The optimization of different cobalt(II) catalysts revealed that $\mathrm{Co}(\mathrm{OAc})_{2} \cdot 4 \mathrm{H}_{2} \mathrm{O}$ was indeed the best catalyst for the electrocatalytic manifold (entries 5-7), while no conversion was observed when copper(II) salts were employed as the catalyst (entry 8). [268b] Furthermore, increasing the reaction temperature under the optimized reaction conditions or lowering the catalyst loading did not improve the product formation (entries $9-11)$. The reversal of the polarity on the electrodes highlighted the essential role of the platinum cathode for the HER (entry 12). Additional control experiments verified that the cobaltaelectrocatalysis was not viable in the absence of either electrical current, base, or cobalt catalyst (entries 13-15). Although the preliminary studies in undivided electrolysis cells did not lead to a satisfactory reaction outcome ( $c f$. Table 1, entry 1), a more standardized undivided cell setup was later developed by a subsequent extensive cell design ( $c f$. Figures 79-82). ${ }^{[274]}$ Notable contributions for the design and construction have been made by $D r$. N. Sauermann, Dr. C. Tian, Dr. L. Finger, M. Stangier and the mechanical workshop of the chemistry department at the University of Göttingen. Here, important features of the revised electrolysis cell included a fixed and reduced inter-electrode distance as well as a decreased cell volume. These key characteristics allowed for a reduction in cell resistance and according to Ohm's law, a lower applied potential to ensure the desired constant current. When using the novel undivided cell for the cobaltaelectro-catalyzed $\mathrm{C}-\mathrm{H}$ oxygenation, product 36aa was obtained in $70 \%$ yield under slightly modified reaction conditions (entry 16). 
Table 2. Optimization studies for the cobaltaelectro-catalyzed $\mathrm{C}-\mathrm{H}$ oxygenation. ${ }^{[\mathrm{a}]}$

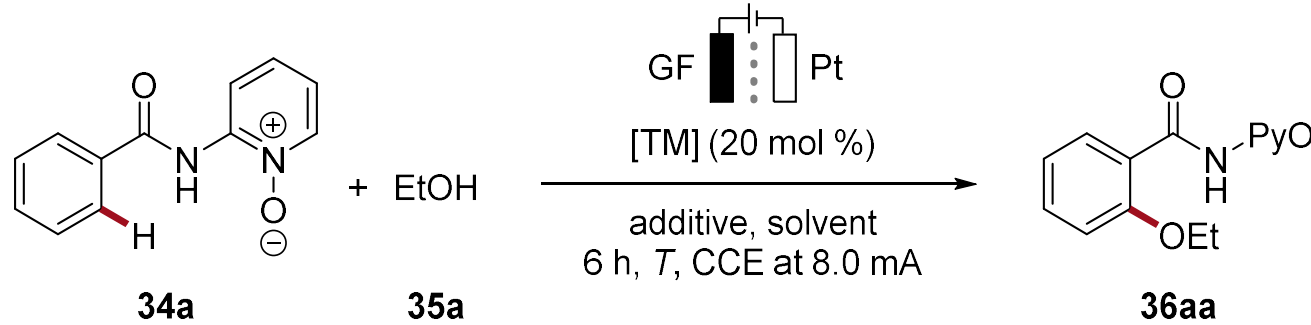

\begin{tabular}{ccccc}
\hline Entry & {$[\mathrm{TM}]$} & Additive & $T\left[^{\circ}\right]$ & Yield [\%] \\
\hline 1 & $\mathrm{Co}(\mathrm{OAc})_{2} \cdot 4 \mathrm{H}_{2} \mathrm{O}$ & $\mathrm{NaOAc}$ & 23 & 58 \\
$\mathbf{2}$ & $\mathrm{Co}(\mathrm{OAc})_{2} \cdot 4 \mathrm{H}_{2} \mathrm{O}$ & $\mathrm{NaOPiv}$ & $\mathbf{2 3}$ & $\mathbf{7 5}$ \\
3 & $\mathrm{Co}(\mathrm{OAc})_{2} \cdot 4 \mathrm{H}_{2} \mathrm{O}$ & $\mathrm{Na}_{2} \mathrm{CO}_{3}$ & 23 & 34 \\
4 & $\mathrm{Co}(\mathrm{OAc})_{2} \cdot 4 \mathrm{H}_{2} \mathrm{O}$ & $\mathrm{NaO}_{2} \mathrm{CCF}_{3}$ & 23 & traces \\
5 & $\mathrm{Co}(\mathrm{acac})_{2}$ & $\mathrm{NaOAc}$ & 23 & 44 \\
6 & $\mathrm{CoCl}{ }_{2}$ & $\mathrm{NaOPiv}$ & 23 & 64 \\
7 & $\mathrm{CoBr}{ }_{2}$ & $\mathrm{NaOPiv}$ & 23 & 55 \\
8 & $\mathrm{Cu}(\mathrm{OAc})_{2}$ & $\mathrm{NaOPiv}$ & 23 & --- \\
9 & $\mathrm{Co}(\mathrm{OAc})_{2} \cdot 4 \mathrm{H}_{2} \mathrm{O}$ & $\mathrm{NaOPiv}$ & 40 & $68^{[\mathrm{b}]}$ \\
10 & $\mathrm{Co}(\mathrm{OAc})_{2} \cdot 4 \mathrm{H}_{2} \mathrm{O}$ & $\mathrm{NaOPiv}$ & 60 & $58^{[\mathrm{c}]}$ \\
11 & $\mathrm{Co}(\mathrm{OAc})_{2} \cdot 4 \mathrm{H}_{2} \mathrm{O}$ & $\mathrm{NaOPiv}$ & 23 & $26^{[\mathrm{d}]}$ \\
12 & $\mathrm{Co}(\mathrm{OAc})_{2} \cdot 4 \mathrm{H}_{2} \mathrm{O}$ & $\mathrm{NaOPiv}$ & 23 & traces \\
13 & $\mathrm{Co}(\mathrm{OAc})_{2} \cdot 4 \mathrm{H}_{2} \mathrm{O}$ & NaOPiv & 23 & $---[\mathrm{f}]$ \\
14 & $\mathrm{Co}(\mathrm{OAc})_{2} \cdot 4 \mathrm{H}_{2} \mathrm{O}$ & --- & 23 & --- \\
15 & --- & NaOPiv & 23 & --- \\
16 & $\mathrm{Co}(\mathrm{OAc})_{2} \cdot 4 \mathrm{H}_{2} \mathrm{O}$ & NaOPiv & 23 & $70^{[\mathrm{g}]}$ \\
\hline
\end{tabular}

[a] Reaction conditions: 34a $(0.50 \mathrm{mmol})$, [TM] $(20 \mathrm{~mol} \%)$, additive (2.00 equiv in each half-cell), EtOH (35a) $(7.0 \mathrm{~mL}$ in each half-cell), $T, 8.0 \mathrm{~mA}, 6 \mathrm{~h}$, graphite felt anode, Pt-plate cathode, divided cell. Isolated yields are given. [b] $40^{\circ} \mathrm{C}$. [c] $60{ }^{\circ} \mathrm{C}$. [e] $\mathrm{Co}(\mathrm{OAc})_{2} \cdot 4 \mathrm{H}_{2} \mathrm{O}(10 \mathrm{~mol} \%)$. [f] Graphite felt cathode, Pt-plate anode. [g] Without current. [g] Undivided cell, 16 h, 4 mA.

The robustness of the protocol was further demonstrated conducting the electrolysis with the commercially available electrosynthesis kit ElectraSyn 2.0 from the IKA company (Table 3 and Figure 83). Here, a carbon electrode material other than graphite felt proved to be equally viable to promote the cobalta-electrocatalysis in a user-friendly undivided cell setup (entries 1 and 2). However, the use of more cost-efficient nickel cathodes resulted in a diminished reaction outcome (entry 3 ). 
Table 3. Cobaltaelectro-catalyzed C-H oxygenation with IKA ElectraSyn 2.0. ${ }^{\text {[a] }}$

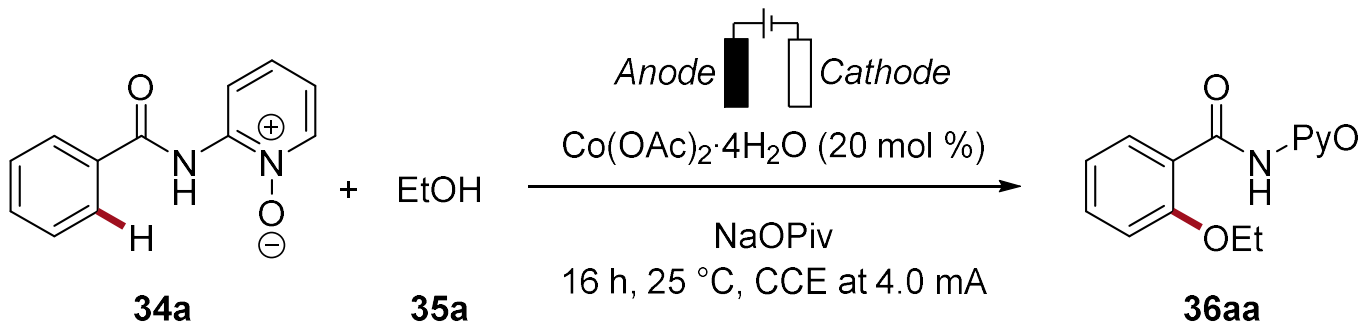

\begin{tabular}{cccc}
\hline Entry & Anode & Cathode & Yield [\%] \\
\hline 1 & $\mathrm{RVC}$ & $\mathrm{Pt}$ & 68 \\
2 & $\mathrm{GF}$ & $\mathrm{Pt}$ & 69 \\
3 & $\mathrm{RVC}$ & $\mathrm{Ni}$ & 38 \\
\hline
\end{tabular}

[a] Reaction conditions: 34a $(0.50 \mathrm{mmol}), \mathrm{Co}(\mathrm{OAc})_{2} \cdot 4 \mathrm{H}_{2} \mathrm{O}(20 \mathrm{~mol} \%)$, NaOPiv (2.00 equiv), EtOH (35a) $(5.0 \mathrm{~mL}), 25^{\circ} \mathrm{C}, 4.0 \mathrm{~mA}, 16 \mathrm{~h}$, undivided cell $(10 \mathrm{~mL})$. Isolated yields are given. Platinum-plated electrodes. $\mathrm{Ni}=$ nickel foam electrode.

In spite of the success with the undivided electrolysis cells, the subsequent co-solvent optimization studies were completed with the more efficient divided cell setup (Table 4).

Table 4. Evaluation of the co-solvent. ${ }^{[\mathrm{a}]}$

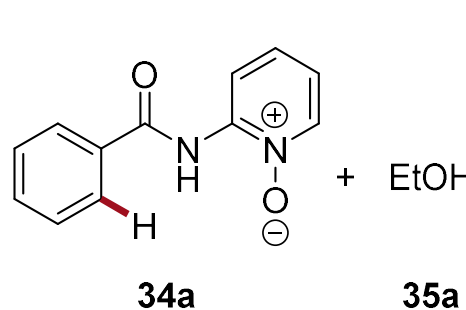

$35 a$

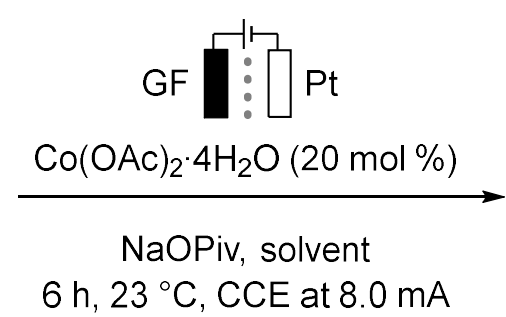

$6 \mathrm{~h}, 23^{\circ} \mathrm{C}, \mathrm{CCE}$ at $8.0 \mathrm{~mA}$<smiles>CCOc1ccccc1C(=O)NP=O</smiles>

36aa

\begin{tabular}{cccc}
\hline Entry & Solvent & Ratio (Solvent/EtOH) & Yield [\%] \\
\hline $\mathbf{1}$ & $\mathbf{E t O H}$ & --- & $\mathbf{7 5}$ \\
2 & $\mathrm{MeCN}$ & $16: 1$ & 12 \\
3 & $\mathrm{MeCN}$ & $1: 1$ & 19 \\
4 & $\mathrm{DMSO}$ & $16: 1$ & --- \\
5 & $\mathrm{DMSO}$ & $1: 1$ & --- \\
6 & $\mathrm{CH}_{2} \mathrm{Cl}_{2}$ & $1: 1$ & --- \\
\hline
\end{tabular}

[a] Reaction conditions: 34a $(0.50 \mathrm{mmol}), \mathrm{Co}(\mathrm{OAc})_{2} \cdot 4 \mathrm{H}_{2} \mathrm{O}(20 \mathrm{~mol} \%)$, $\mathrm{NaOPiv}(2.00$ equiv in each halfcell), solvent $\left(7.0 \mathrm{~mL}\right.$ in each half-cell), $23{ }^{\circ} \mathrm{C}, 8.0 \mathrm{~mA}, 6 \mathrm{~h}$, graphite felt anode, Pt-plate cathode, divided cell. Isolated yields are given.

The investigation of a suitable co-solvent to decrease the used equivalents of the alcohol coupling partner 35 proved to be challenging (entries 1-6). ${ }^{[269]}$ Thus, different mixtures of $\mathrm{MeCN}$ and 35a were found to be tolerated under the standard conditions, however, aryl 
ether 36aa was isolated in significantly reduced yields (entries 2 and 3). Unfortunately, other co-solvents failed to serve as a reaction medium for the electrocatalysis (entries 4-6).

Next, different $N$-substituted benzamides $26,34,64,162-167$ were tested as the directing group to chelate the cobalt catalyst, and thus to guide the $\mathrm{C}-\mathrm{H}$ activation event (Table 5).

Table 5. Studies on the directing group effect for the $\mathrm{C}-\mathrm{H}$ oxygenation. ${ }^{[\mathrm{a}]}$
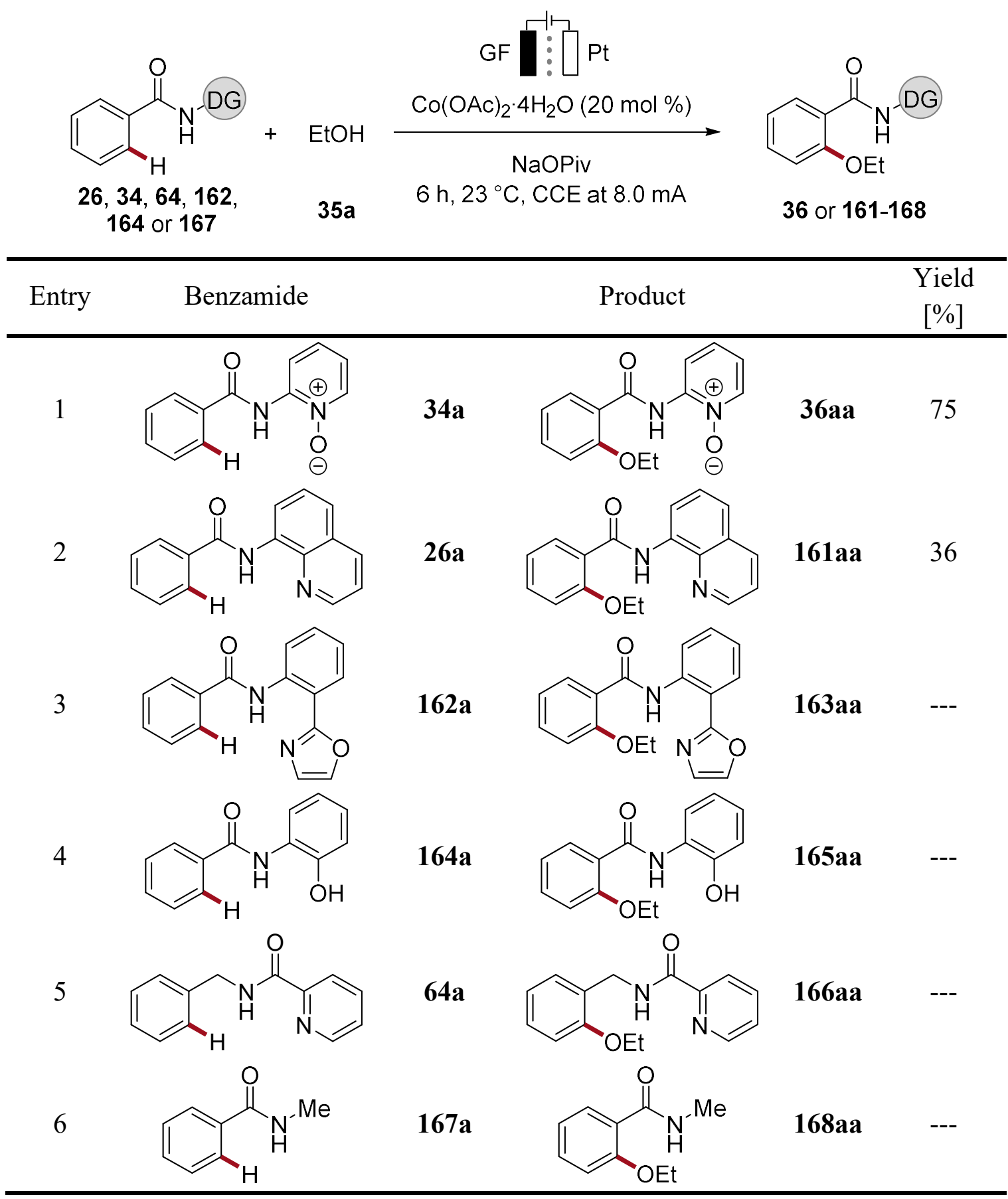

[a] Reaction conditions: Benzamide $(0.50 \mathrm{mmol}), \mathrm{Co}(\mathrm{OAc})_{2} \cdot 4 \mathrm{H}_{2} \mathrm{O}(20 \mathrm{~mol} \%), \mathrm{NaOPiv}$ (2.00 equiv in each half-cell), EtOH (35a) $\left(7.0 \mathrm{~mL}\right.$ in each half-cell), $23^{\circ} \mathrm{C}, 8.0 \mathrm{~mA}, 6 \mathrm{~h}$, graphite felt anode, Pt-plate cathode, divided cell. Isolated yields are given. 
Here, substrate 34a bearing a Lewis-basic pyridine- $N$-oxide group was found to promote the cobaltaelectro-catalyzed $\mathrm{C}-\mathrm{H}$ oxygenation in high yield (entry 1). In addition, the commonly employed 8-aminoquinoline directing group ${ }^{[96 a]}$ enabled electrochemical $\mathrm{C}-\mathrm{H}$ oxygenation in moderate yield (entry 2). Other structural motifs, including monodentate $\mathrm{N}$ methylbenzamide (167a) failed to provide any alkoxylated products (entries 3-6), thus showcasing the unique features of the bidentate scaffold of 34a (entry 6). [269]

\subsubsection{Substrate Scope of the Cobaltaelectro-Catalyzed C-H Alkoxylation}

With the optimized reaction conditions identified, the robustness of the first cobaltaelectro-catalyzed C-H activation of benzamides 34 was probed (Table 6).

Table 6. Scope of cobaltaelectro-catalyzed C-H oxygenation of benzamides 34. ${ }^{[\mathrm{a}]}$

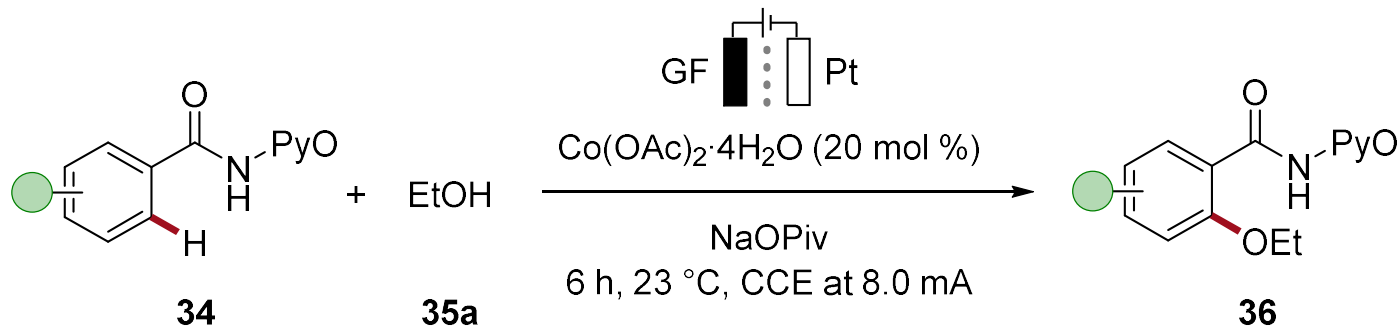

Entry


6<smiles>O=C(N[Pb])c1ccc(-c2ccccc2)cc1</smiles>

7<smiles>O=C(N[Pb])c1ccc(I)cc1</smiles>

8<smiles>O=C(N[Pb])c1cccc(Cl)c1</smiles>

9
$34 f$<smiles>CCOc1cc(-c2ccccc2)ccc1C(=O)NP(=O)(O)OC</smiles>

36fa

61

$34 \mathrm{~g}$

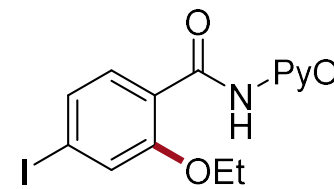

36ga

34h<smiles>CCOc1ccc(Cl)cc1C(=O)NP(=O)(O)O</smiles>

36ha

$34 \mathbf{i}$

36ia

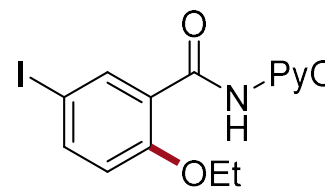

[a] Reaction conditions: Benzamide $34(0.50 \mathrm{mmol}), \mathrm{Co}(\mathrm{OAc})_{2} \cdot 4 \mathrm{H}_{2} \mathrm{O}(20 \mathrm{~mol} \%), \mathrm{NaOPiv}(2.00$ equiv in each half-cell), EtOH (35a) $\left(7.0 \mathrm{~mL}\right.$ in each half-cell), $23{ }^{\circ} \mathrm{C}, 8 \mathrm{~mA}, 6 \mathrm{~h}$, graphite felt anode, Pt-plate cathode, divided cell. Isolated yields are given.

Thus, various substituents in the C4-position of the benzamide $\mathbf{3 4}$ were well tolerated, including electron-donating methyl or methoxy groups, as well as the electron-withdrawing trifluoromethyl group (entries 1-4). Notably, even oxidatively sensitive thioether 34e, as well as phenyl and iodo substituents were compatible under the electrooxidative reaction conditions and delivered the desired oxygenated products in moderate to good yields with excellent chemoselectivities (entries 5-7). Moreover, sterically demanding substituents in the C3-position were likewise tolerated, furnishing halogenated aryl ethers 36ha and 36ia as the sole products (entries 8 and 9). Furthermore, Dr. N. Sauermann tested a wealth of other substituents in the meta and para-position of benzamide 34, including amines or enolizable ketones with overall moderate to good yields of the desired products 36 . However, ortho-substituted benzamides 34 were generally not tolerated and remained unaffected under the reaction conditions (vide infra). ${ }^{[269]}$

In addition to various benzamides $\mathbf{3 4}$, the versatility of the electrocatalytic manifold was further illustrated by the successful conversion of different alcohols 35 (Table 7). Here, methanol (35b) or propanol (35c) proved to be equally viable (entries $1-3)$. However, the functionalization of electron deficient TFE (35d) turned out to be more challenging under the standard conditions and furnished the corresponding product 36ad in a reduced yield (entry 3). Nonetheless, the reaction of fluorinated alcohol 35d was later reoptimized by $D r$. $N$. Sauermann to enable the synthesis of $36 \mathrm{ad}$ in $62 \%$ yield at a slightly elevated reaction temperature of $60{ }^{\circ} \mathrm{C}$. Furthermore, he was able to extend the scope of $\mathbf{3 5}$ to oxidation- 
sensitive benzylic alcohols or even more complex alcoholic scaffolds, such as citronellol. ${ }^{[269]}$

Table 7. Scope of cobaltaelectro-catalyzed C-H oxygenation of alcohols $35{ }^{\text {[a] }}$

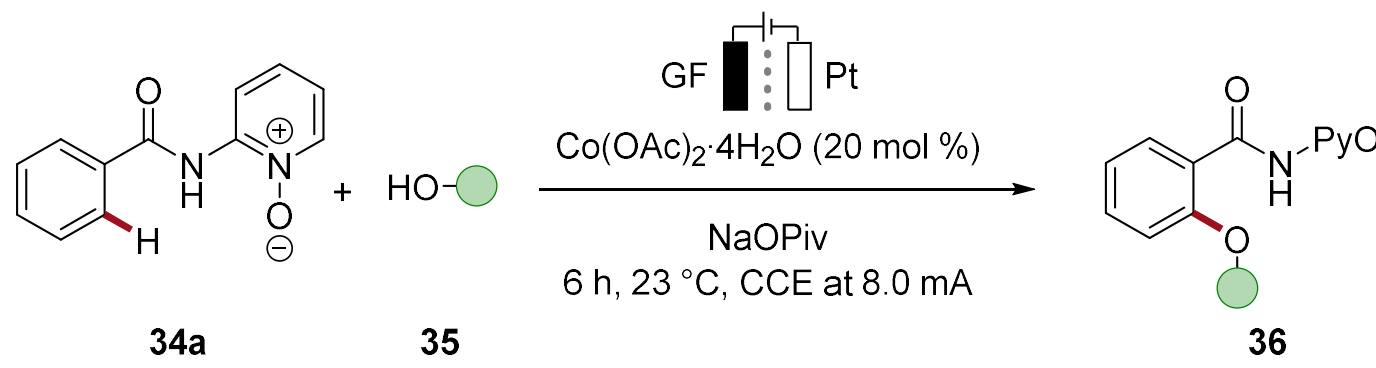

\begin{tabular}{|c|c|c|c|c|c|}
\hline Entry & Alcohol & 35 & Product & 36 & $\begin{array}{c}\text { Yield } \\
{[\%]}\end{array}$ \\
\hline 1 & $\mathrm{MeOH}$ & $35 b$ & & $36 a b$ & 71 \\
\hline 2 & $n-\mathrm{PrOH}$ & $35 \mathrm{c}$ & & $36 a c$ & $65^{[\mathrm{b}]}$ \\
\hline 3 & $\mathrm{CF}_{3} \mathrm{CH}_{2} \mathrm{OH}$ & 35d & & 36ad & 40 \\
\hline
\end{tabular}

[a] Reaction conditions: Benzamide 34a $(0.50 \mathrm{mmol}), \mathrm{Co}(\mathrm{OAc})_{2} \cdot 4 \mathrm{H}_{2} \mathrm{O}(20 \mathrm{~mol} \%), \mathrm{NaOPiv}(2.00$ equiv in each half-cell), alcohol 35 ( $7.0 \mathrm{~mL}$, in each half-cell), $23^{\circ} \mathrm{C}, 8 \mathrm{~mA}, 6 \mathrm{~h}$, graphite felt anode, Pt-plate cathode, undivided cell. Isolated yields are given. [b] Addition of $n$-Bu ${ }_{4} \mathrm{NOAc}(1.00 \mathrm{mmol}$ in each half-cell).

Additionally, along other limitations reported by Dr. N. Sauermann, ${ }^{[269]}$ allylic alcohol 35e or secondary alcohols $\mathbf{3 5 f}$ and $\mathbf{3 5 g}$ failed to furnish the desired oxidative $\mathrm{C}-\mathrm{H}$ alkoxylated products 36 (Scheme 49). Based on previous reports on electrochemical phenol coupling reactions, ${ }^{[276]}$ differently substituted aromatic alcohol derivatives were tested for the cobaltaelectro-catalyzed C-H oxygenation. Unfortunately, no desired biaryl ether 36 formation was observed. 


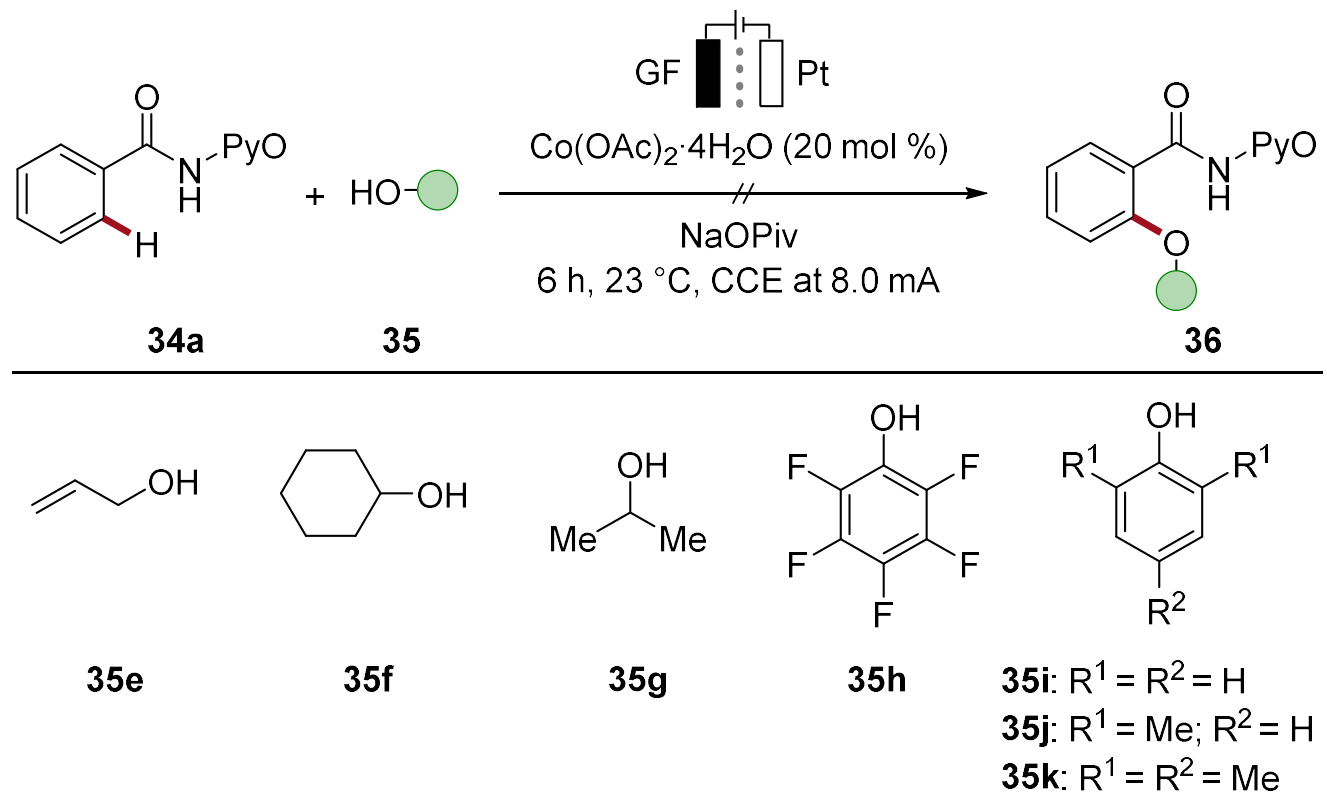

Scheme 49. Limitations of the cobaltaelectro-catalyzed C-H alkoxylation.

Besides the applicability of the cobalta-electrocatalysis toward the resource economical alkoxylation of arenes 34, also more challenging ${ }^{[99]}$ alkenes 37 were tested (Table 8). Interestingly, after extensive experimentation, it was found that an undivided cell setup and lower constant current conditions were beneficial to convert acrylic amides $\mathbf{3 7}$ in high yields and selectivities into the desired enol ethers $\mathbf{3 8}$.

Table 8. Scope of cobaltaelectro-catalyzed C-H oxygenation of alkenes 37. ${ }^{[\mathrm{a}]}$

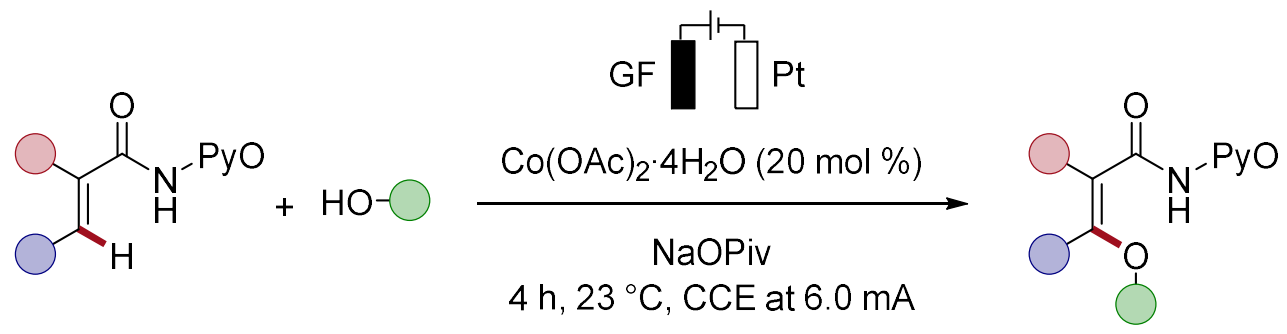

37

35

38

\begin{tabular}{|c|c|c|c|c|c|}
\hline Entry & Alkene & 37 & Product & 38 & $\begin{array}{c}\text { Yield } \\
{[\%]}\end{array}$ \\
\hline 1 & & $37 \mathbf{a}$ & & 38aa & 69 \\
\hline 2 & & $37 \mathbf{a}$ & & 38ab & 62 \\
\hline
\end{tabular}


3<smiles>O=C(N[Pb])C1=CCCCC1</smiles>

4<smiles>O=C(N[Pb])/C(=C/c1ccccc1)c1ccccc1</smiles>

5<smiles>C/C(=C\c1ccccc1)C(=O)NP</smiles>

6<smiles>C/C(=C\c1ccccc1)C(=O)N[Pb]</smiles>

7<smiles>O=C(/C=C/c1ccccc1)NP(=O)(O)c1ccccc1</smiles>

37b<smiles>CCOC1=C(C(=O)NP)CCCC1</smiles>

38ba 72

$37 c$<smiles>COC(=C(C(=O)N[OH2+])c1ccccc1)c1ccccc1</smiles>

$38 \mathrm{cb}$ 62

37d<smiles>CCO/C(=C(\C)C(=O)NP)c1ccccc1</smiles>

38da 53

37d<smiles>CO/C(=C(/C)C(=O)NP)c1ccccc1</smiles>

38db $\quad 71$<smiles>CO/C(=C/C(=O)NP)c1ccccc1</smiles>

38eb traces

[a] Reaction conditions: Alkene $37(0.25 \mathrm{mmol}), \mathrm{Co}(\mathrm{OAc})_{2} \cdot 4 \mathrm{H}_{2} \mathrm{O}(20 \mathrm{~mol} \%), \mathrm{NaOPiv}$ (2.00 equiv in each half-cell), alcohol $35(7.0 \mathrm{~mL}), 23{ }^{\circ} \mathrm{C}, 4 \mathrm{~mA}, 6 \mathrm{~h}$, graphite felt anode, Pt-plate cathode, undivided cell. Isolated yields are given.

Thus, both ethanol (35a) and methanol (35b) were found to be suitable reactants for the alkoxylation of alkene 37a (entries 1 and 2). Also, more rigid internal alkene 37b and diphenyl substituted alkene 37c were efficiently converted into the corresponding enol ethers 38ba and 38ca, respectively (entries 3 and 4). Asymmetrically substituted acryl amide 37d was likewise oxygenated to the corresponding ethyl- 38da or methyl ethers $\mathbf{3 8 d b}$ (entries 5 and 6). In this particular case, methanol (35b) was found to outperform ethanol (35a) as the coupling partner in terms of product yield. This is likely due to the increased conductivity of methanol $(\mathbf{3 5 b})$, which resulted in a decreased cell resistance and thus in lower working potentials. Interestingly, when using the trans-cinnamamide derivative 37e for the cobalta-electrocatalyzed $\mathrm{C}-\mathrm{H}$ methoxylation, only traces of product 38eb were detected (entry 7).

\subsubsection{Mechanistic Studies for the Cobaltaelectro-Catalyzed C-H Oxygenation}

The mechanistic studies were commenced by detailed electrochemical analysis of the redox properties of the reaction components. Cyclic voltammetry of substrate 34a in $\mathrm{MeOH}$ 
(35b) $\left(n-\mathrm{Bu}_{4} \mathrm{NPF}_{6}, 0.1 \mathrm{M}\right)$ as the electrolyte (Figure 3, red line), revealed an irreversible oxidation at $E_{\mathrm{p}, \mathrm{ox}}=1.51 \mathrm{~V} v s$. SCE. In contrast, oxidation of a mixture of $\mathrm{Co}(\mathrm{OAc})_{2} \cdot 4 \mathrm{H}_{2} \mathrm{O}$ and $\mathrm{NaOPiv}$ occurred at a significantly lower anodic oxidation event at $E_{\mathrm{p}, \mathrm{ox}}=1.19 \mathrm{~V} v \mathrm{~s}$. SCE (blue line). The irreversible redox event can be assigned to the oxidation of the cobalt(II) complex to the corresponding cobalt(III) carboxylate species. ${ }^{[277]}$ In the presence of substrate 34a, a considerable prewave formation at approximately $0.70 \mathrm{~V} v s$. SCE was detected, thus being indicative of a precatalyst formation (green line). ${ }^{[278]}$

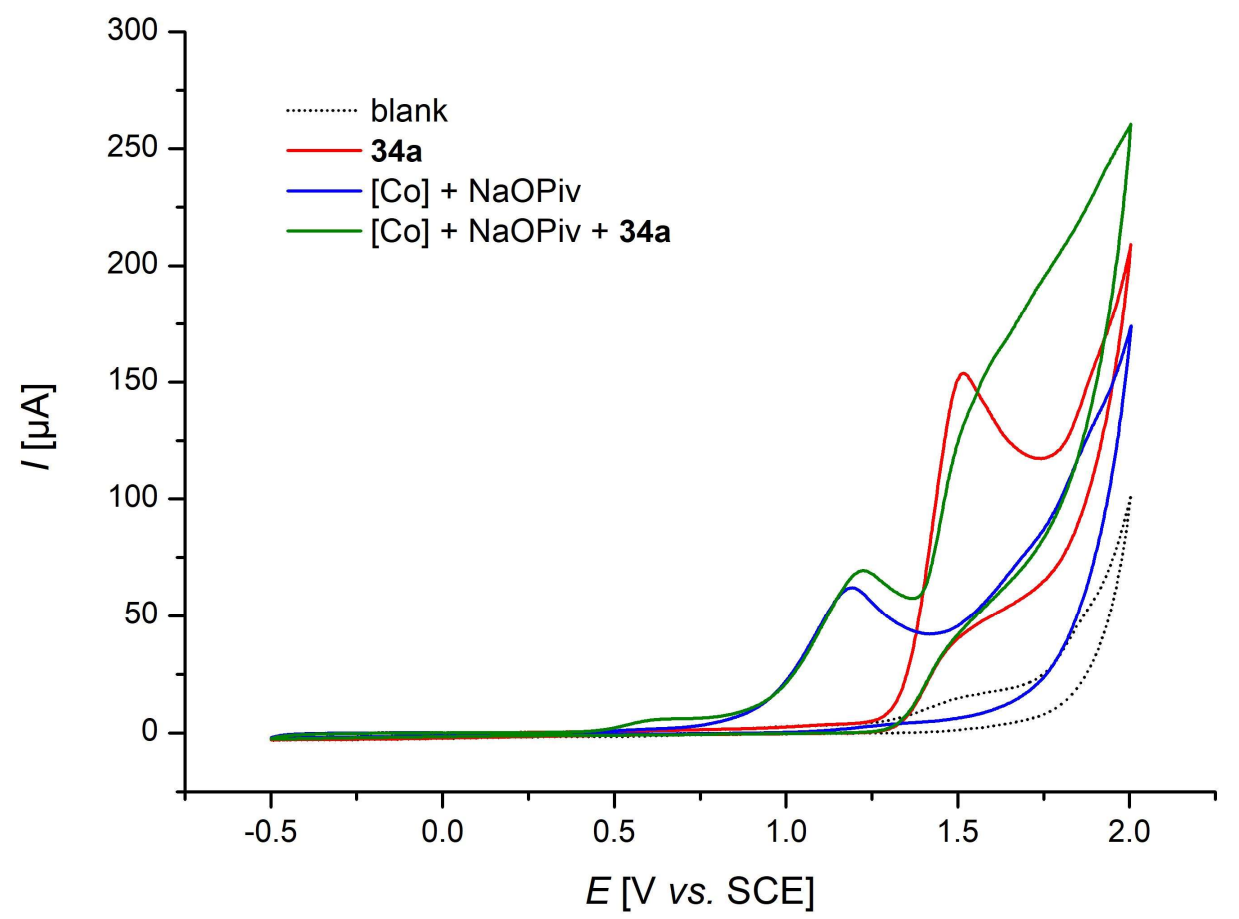

Figure 3. Cyclic voltammograms at $25{ }^{\circ} \mathrm{C}$ and $100 \mathrm{mV} \cdot \mathrm{s}^{-1}$ using $\mathrm{MeOH}(\mathbf{3 5 b})$ and $n-\mathrm{Bu}_{4} \mathrm{NPF}_{6}(0.1 \mathrm{M})$ as the electrolyte and a $\mathrm{GC}$ working electrode; concentration of substrates $341.0 \mathrm{mM}$ (NaOPiv $4.0 \mathrm{mM}$ ). (black) blank; (red) 34a; (blue) $\mathrm{Co}(\mathrm{OAc})_{2} \cdot 4 \mathrm{H}_{2} \mathrm{O}$ and NaOPiv; (green) $\mathrm{Co}(\mathrm{OAc})_{2} \cdot 4 \mathrm{H}_{2} \mathrm{O}$, $\mathrm{NaOPiv}$ and 34a.

When using MeCN ( $\left.n-\mathrm{Bu}_{4} \mathrm{NPF}_{6}, 0.1 \mathrm{M}\right)$ as the electrolyte instead of MeOH (35b) (Figure 4), similar redox properties were found for substrate 34a and for the cobalt(II) precatalyst (red and blue line). However, several redox events were detected for a mixture of 34a, $\mathrm{NaOPiv}$ and the cobalt(II) salt (green line). Notably, a significant irreversible current response at $E_{\mathrm{p}, \mathrm{ox}}=0.70 \mathrm{~V} v s$. SCE was indicative of the formation of a novel cobalt complex. Interestingly, upon addition of different amounts of the reactant EtOH (35a), the current response was diminished, and a new irreversible oxidation event emerged at a potential of approximately $1.22 \mathrm{~V} v s$. SCE (purple, orange and magenta lines), thus being 
suggestive of the generation and subsequent consumption of a catalytically relevant cobalt species. $^{[249,269]}$

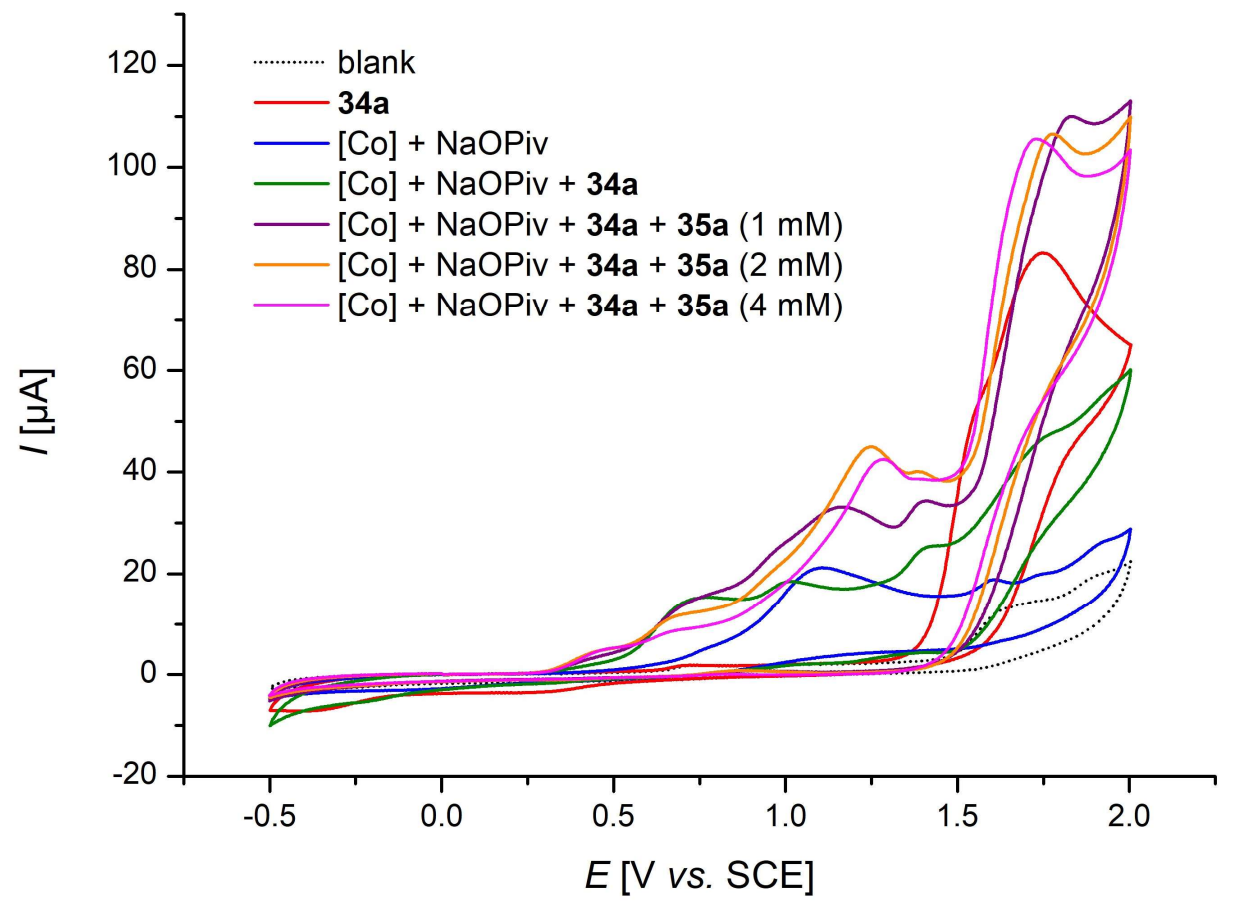

Figure 4. Cyclic voltammograms at $25^{\circ} \mathrm{C}$ and $100 \mathrm{mV} \cdot \mathrm{s}^{-1}$ using $\mathrm{MeCN}$ and $n-\mathrm{Bu}_{4} \mathrm{NPF}_{6}(0.1 \mathrm{M})$ as the electrolyte and a GC working electrode; concentration of substrates $341.0 \mathrm{~mm}$ (NaOPiv $4.0 \mathrm{~mm}$ ). (black) blank; (red) 34a; (blue) $\mathrm{Co}(\mathrm{OAc})_{2} \cdot 4 \mathrm{H}_{2} \mathrm{O}$ and NaOPiv; (green) $\mathrm{Co}(\mathrm{OAc})_{2} \cdot 4 \mathrm{H}_{2} \mathrm{O}$, $\mathrm{NaOPiv}$ and 34a; (purple) as green with 35a $(1.0 \mathrm{~mm})$; (orange) as green with 35a $(2.0 \mathrm{mM})$; (magenta) as green with 35a (3.0 mM).

Furthermore, ortho-substituted substrates, such as $\mathbf{3 4 s}$, were found to be unreactive under the electrocatalytic reaction conditions. Likewise, the cobaltaelectro-catalyzed $\mathrm{C}-\mathrm{H}$ oxygenation selectively provided the mono-functionalized benzamides $\mathbf{3 6}$ and no difunctionalization was observed, even though the obtained aryl ethers $\mathbf{3 6}$ are more electron-rich compared to the substrates $\mathbf{3 4}$ and overoxidation could become predominant. Hence, additional CV experiments were performed and revealed that substrate $34 \mathrm{~s}$ (1.71 V) or products $36 \mathbf{a b}(1.82 \mathrm{~V})$ and $36 \mathbf{d b}(1.76 \mathrm{~V})$ in fact possess very similar oxidation potentials when compared to model substrate 34a (1.51 V) (Figure 5). Consequently, these findings indicated that unfavorable steric interactions within organometallic cobalt intermediates 169 were likely responsible for the high levels of chemoselectivity (Scheme 50). 


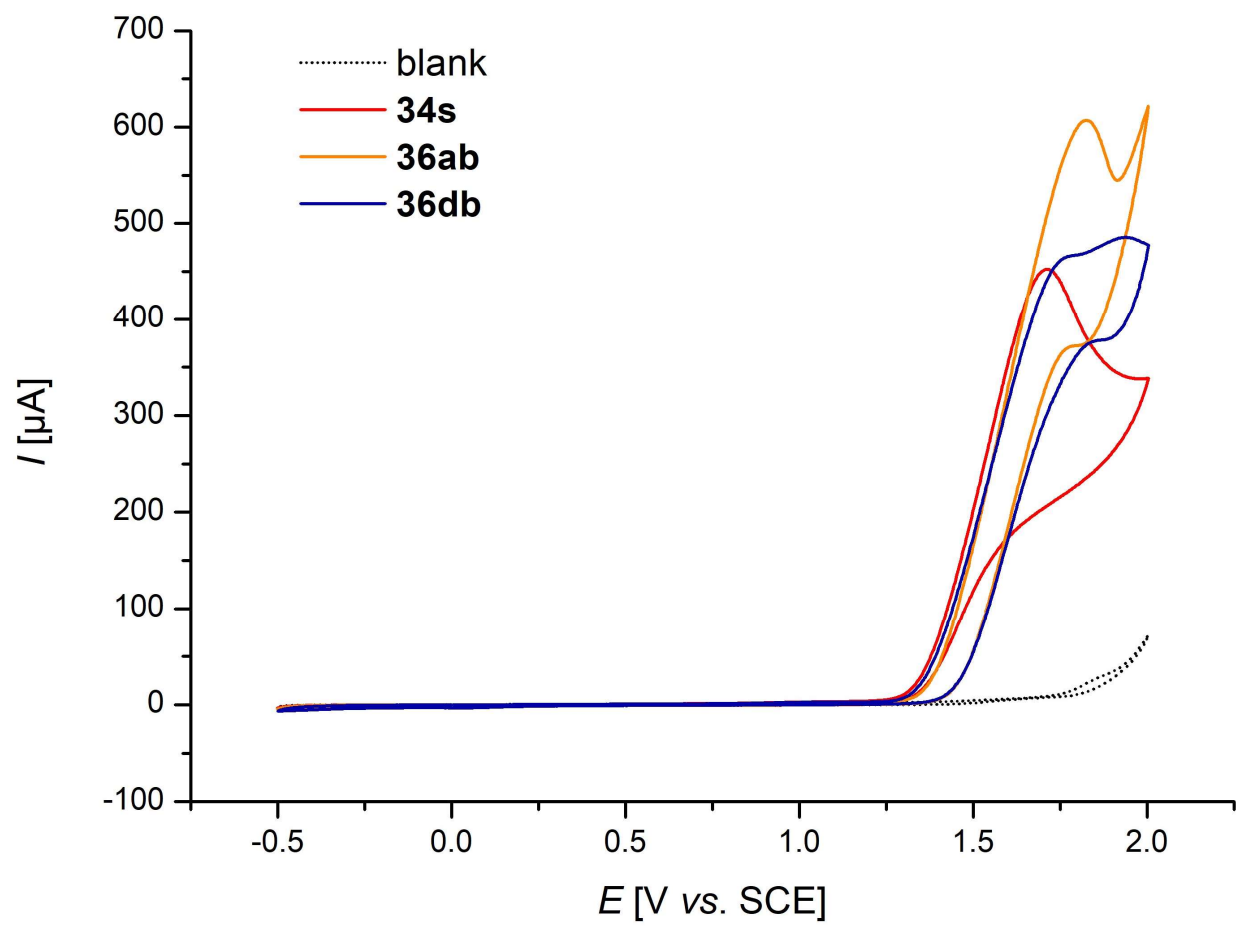

Figure 5. Cyclic voltammograms at $25^{\circ} \mathrm{C}$ and $100 \mathrm{mV} \cdot \mathrm{s}^{-1}$ using $\mathrm{MeOH}$ and $n-\mathrm{Bu}_{4} \mathrm{NPF}_{6}(0.1 \mathrm{M})$ as the electrolyte and a $\mathrm{GC}$ working electrode; concentration of substrates $4.0 \mathrm{mM}$. (black) blank; (red) 34s; (orange) 36ab; (dark blue) 36db.<smiles>Cc1cc(C)c(C(=O)N[Po])c(C)c1</smiles>

$34 s$<smiles>COc1ccccc1C(=O)NP=O</smiles>

$36 a b$<smiles>COc1ccc(OC)c(C(=O)NP=O)c1</smiles>

$36 \mathrm{db}$<smiles></smiles>

169

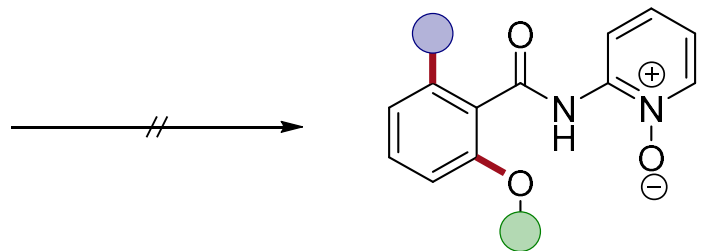

36

Scheme 50. Proposed model for the observed chemoselectivity.

Given the unique features of the first cobaltaelectro-catalyzed $\mathrm{C}-\mathrm{H}$ activation, additional mechanistic studies were performed by Dr. N. Sauermann to unravel its mode of action. ${ }^{[249 \text {, }}$ ${ }^{269]}$ To this end, isotopic labeling studies were suggestive of an irreversible $\mathrm{C}-\mathrm{H}$ metalation event since no significant H/D scrambling was observed. Nonetheless, kinetic isotope effect (KIE) studies provided strong support for a facile and non-rate-determining $\mathrm{C}-\mathrm{H}$ cleavage step. In agreement with the findings obtained during the substrate scope ( $c f$. Table 6 , entries 
2 and 3), intermolecular competition experiments showed that electron-rich benzamides, such as $\mathbf{3 4 b}$, were inherently more reactive than electron-poor substrates, such as $\mathbf{3 4 c}$. Similar findings were obtained for intermolecular competition reactions with $\mathrm{MeOH}(\mathbf{3 5 b})$ and TFE (35c).

On the basis of the mechanistic studies, a plausible catalytic cycle was proposed (Scheme 51). Initial anodic oxidation of the cobalt(II) salt forms the catalytically competent cobalt(III) species 170. Thereafter, coordination of substrate 34 occurs, followed by irreversible $\mathrm{C}-\mathrm{H}$ activation via a base-assisted internal substitution (BIES) manifold. ${ }^{[35,41]}$ Thereafter, the five-membered cobalta(III)cycle 171 undergoes reductive elimination to form a cobalt(I) complex 172 and the desired $\mathrm{C}-\mathrm{O}$ bond with excellent levels of positional control. Subsequent proto-demetalation releases the $\mathrm{C}-\mathrm{H}$ oxygenated product 36 . Finally, anodic oxidation of the reduced cobalt(I) species 173 regenerates the catalytically active cobalt(III) catalyst $\mathbf{1 7 0}$ and cathodic HER closes the electrochemical cycle.

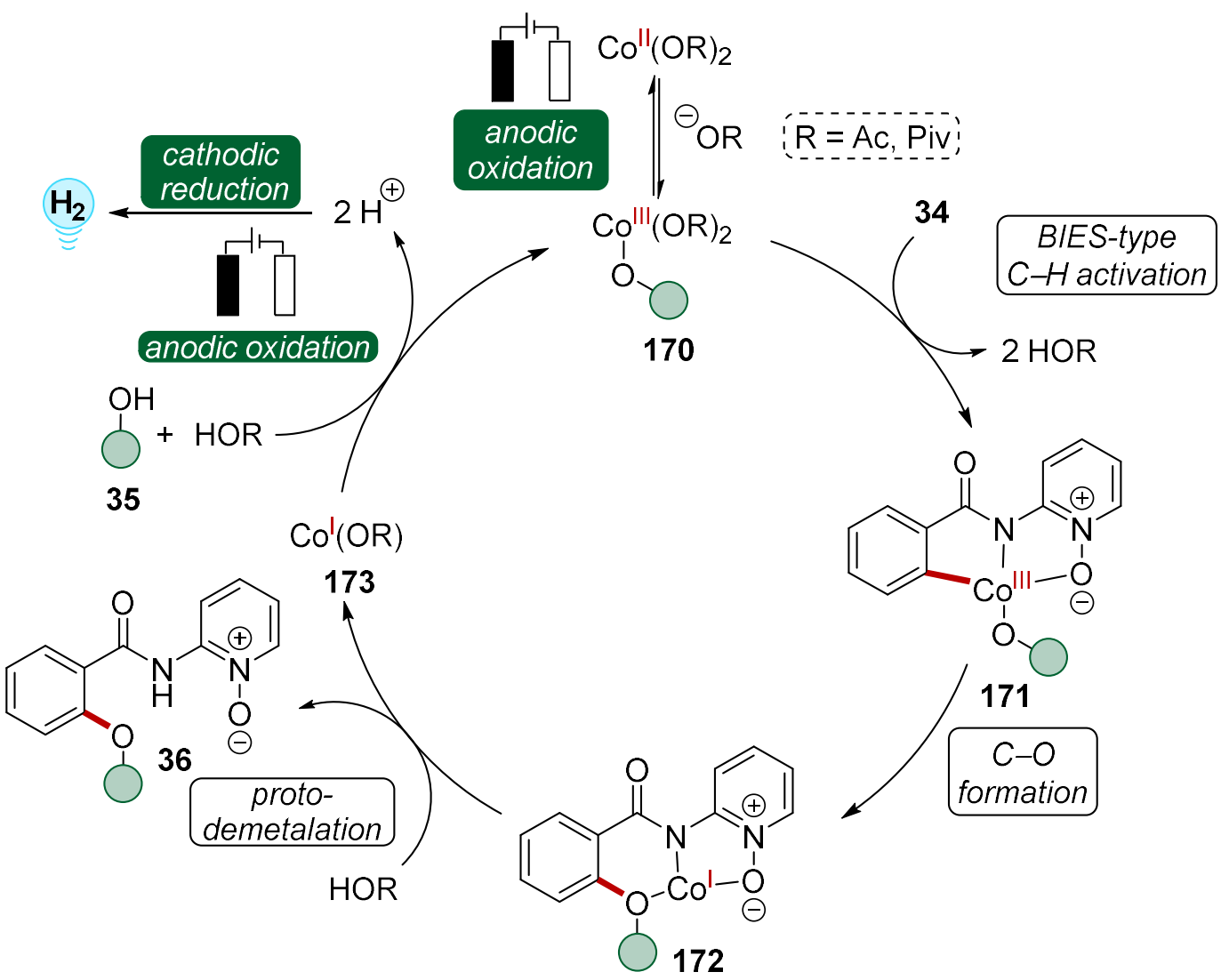

Scheme 51. Proposed catalytic cycle for the cobaltaelectro-catalyzed C-H oxygenation.

It is noteworthy that at this stage, single electron transfer between benzamide $\mathbf{3 4}$ and a cobalt(III) species as was previously proposed by Niu, Wei ${ }^{[100]}$ and Song, ${ }^{[99]}$ could not be fully ruled out (vide infra). ${ }^{[249,269]}$ 


\subsection{Cobaltaelectro-Catalyzed C-H Activation for Allene}

\section{Annulations}

Allenes, a subclass of cumulenes, have attracted considerable attention as versatile building blocks for organic syntheses during the last two decades. ${ }^{[132]}$ At the same time, $\mathrm{C}-\mathrm{H}$ activation has proven as a powerful strategy for step-economical molecular assembly toward molecular complexity within a single reaction step. ${ }^{[1 \mathrm{q}, 21,166 c, 279]}$ In this context, the unique reactivity of allenes, compared to their alkenyl or alkynyl complements, ${ }^{[132 \mathrm{~d}]}$ renders the allenylic structural motive a particularly versatile substrate class for intermolecular C-H annulation reactions. ${ }^{[57]}$ Despite major advances with Earth-abundant metals, ${ }^{[110,133 \text {, }}$ 135-140, 280] most of the reported $\mathrm{C}-\mathrm{H}$ transformations with allenes rely on precious metal catalyst. ${ }^{[81 \mathrm{~d}, 132 \mathrm{a}, 132 \mathrm{~b}, 134,281]}$ Furthermore, all developed oxidative $\mathrm{C}-\mathrm{H}$ activation reactions with allenes require stoichiometric amounts of chemical oxidants such as silver(I) or copper salts. However, until this study, ${ }^{[260]}$ allenes were only rarely presented in electrochemical transformations ${ }^{[282]}$ and are fully unprecedented within metalla-electrocatalysis. Based on the pioneering work for cobaltaelectro-catalyzed $\mathrm{C}-\mathrm{H}$ oxygenation ${ }^{[249]}$ and alkyne annulation reactions, ${ }^{[250]}$ an electrochemical $\mathrm{C}-\mathrm{H}$ annulation protocol was envisioned with allenes as the coupling partner. A major challenge here is the control of regioselectivity for the desired electrocatalytic $\mathrm{C}-\mathrm{H}$ transformation (Scheme 52). ${ }^{[283]}$

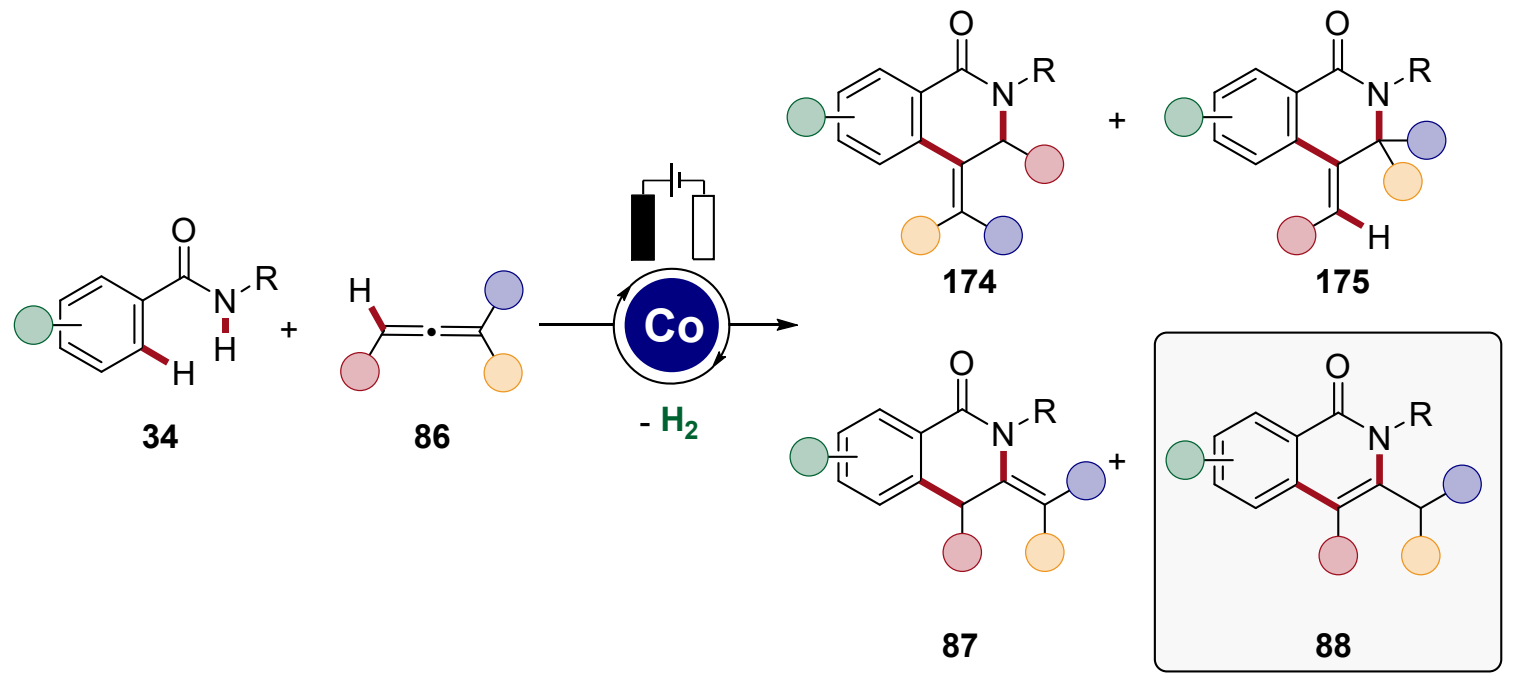

Scheme 52. The quest for selectivity; multiple insertion products are possible for the cobaltaelectro-catalyzed C-H activation with allenes $\mathbf{8 6}$. 


\subsubsection{Optimization Studies}

The study was commenced by exploring various reaction conditions for the desired cobaltaelectro-catalyzed $\mathrm{C}-\mathrm{H} / \mathrm{N}-\mathrm{H}$ functionalization of benzamide 34a and allene 75a in a user-friendly undivided cell setup with a platinum plate cathode and a graphite felt (GF) anode. Initially, different cobalt sources were tested as the catalyst (Table 9) with TFE as the solvent and NaOPiv as the additive. ${ }^{[112,133]}$ The reaction proceeded in good yield and high selectivity when $\mathrm{Co}(\mathrm{OAc})_{2} \cdot 4 \mathrm{H}_{2} \mathrm{O}$ was used as the precatalyst (entry 1). Other cobalt(II) or cobalt(III) sources proved less efficient (entries 2-4). Interestingly, a pentamethylcyclopentadienyl cobalt(III) complex furnished isoquinolone 176aa in 60\% yield (entry 5). Notably, this represents the first and thus far sole report of an electrocatalytic $\mathrm{C}-\mathrm{H}$ activation with a $\mathrm{Cp} *$ cobalt(III) catalyst. ${ }^{[55 \mathrm{a}]}$

Table 9. Catalyst optimization. ${ }^{[\mathrm{a}]}$

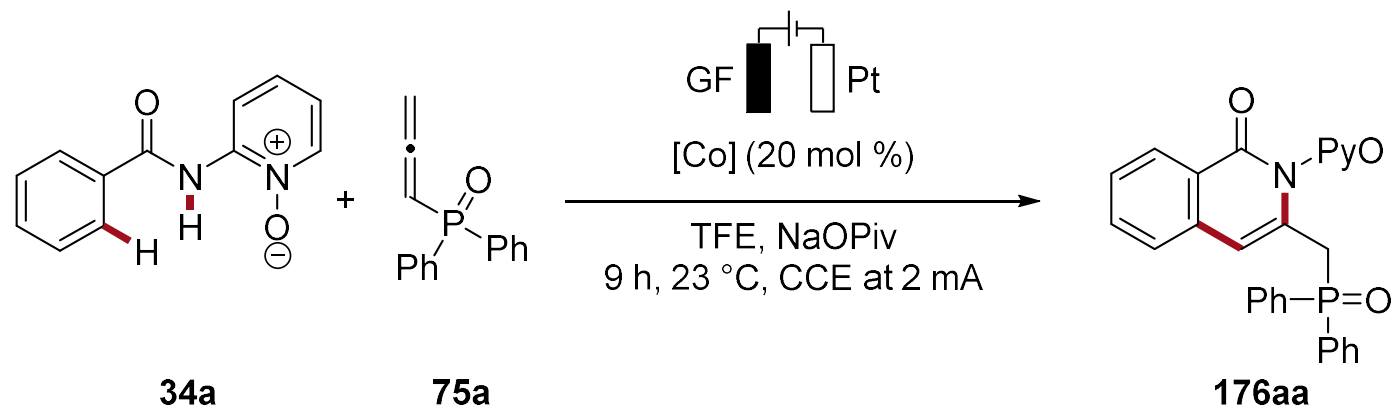

\begin{tabular}{ccc}
\hline Entry & {$[\mathrm{Co}]$} & Yield $[\%]$ \\
\hline 1 & $\mathrm{Co}(\mathrm{OAc})_{2} \cdot 4 \mathrm{H}_{2} \mathrm{O}$ & $82(72)$ \\
2 & $\mathrm{Co}(\mathrm{acac})_{2}$ & 50 \\
3 & $\mathrm{Co}(\mathrm{acac})_{3}$ & 40 \\
4 & $\mathrm{CoBr}_{2}$ & 70 \\
5 & {$\left[\mathrm{Cp} * \mathrm{Co}(\mathrm{CO}) \mathrm{I}_{2}\right]$} & $62(60)$ \\
\hline
\end{tabular}

[a] Reaction conditions: Undivided cell, 34a $(0.30 \mathrm{mmol}), \mathbf{7 5 a}(0.36 \mathrm{mmol}),[\mathrm{Co}$ ] $(20 \mathrm{~mol} \%)$, NaOPiv (2.00 equiv), TFE $(5.0 \mathrm{~mL}), 23^{\circ} \mathrm{C}, 2.0 \mathrm{~mA}, 9 \mathrm{~h}$, graphite felt anode, Pt-plate cathode. Conversion of $176 \mathbf{a a}$ determined by ${ }^{1} \mathrm{H}-\mathrm{NMR}$ analysis with 1,3,5-trimethoxybenzene as an internal standard is given. Isolated yields are shown in parentheses.

Encouraged by the preliminary optimization results, different reaction media were tested for the oxidative cobalt-catalyzed $\mathrm{C}-\mathrm{H}$ annulation reaction (Table 10). A variety of solvents were found to be tolerated, including protic solvents, such as benign $\mathrm{MeOH}$ or $\mathrm{EtOH}$, delivering the desired isoquinolone 176aa with similar levels of efficiency, compared to harmful TFE (entries 1-3). When aprotic solvents were used, an additional conducting salt 
was required to enable sufficient conductivity (entries 4-6). Acetic acid failed to furnish product 176aa in synthetically useful yields (entry 7).

Table 10. Optimization of the solvent. ${ }^{[\mathrm{a}]}$

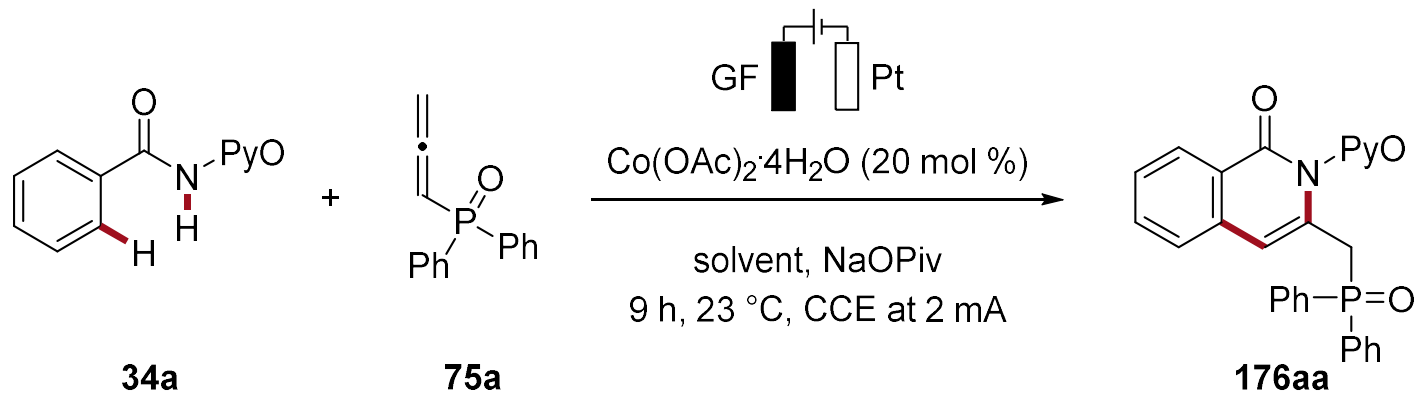

\begin{tabular}{ccc}
\hline Entry & Solvent & Yield [\%] \\
\hline 1 & $\mathrm{TFE}$ & $82(72)^{[\mathrm{b}]}$ \\
2 & $\mathrm{MeOH}$ & $78(75)^{[\mathrm{b}]}$ \\
3 & $\mathrm{EtOH}$ & $74^{[\mathrm{b}]}$ \\
4 & $\mathrm{MeCN}$ & $52^{[\mathrm{c}]}$ \\
5 & $\mathrm{CH}_{2} \mathrm{Cl}_{2}$ & $73^{[\mathrm{c}]}$ \\
6 & $\mathrm{THF}$ & $69^{[\mathrm{c}]}$ \\
7 & $\mathrm{HOAc}$ & 9 \\
\hline
\end{tabular}

[a] Reaction conditions: Undivided cell, 34a $(0.30 \mathrm{mmol}), \mathbf{7 5 a}(0.36 \mathrm{mmol}), \mathrm{Co}(\mathrm{OAc})_{2} \cdot 4 \mathrm{H}_{2} \mathrm{O}(20 \mathrm{~mol} \%)$, NaOPiv (2.00 equiv), solvent $(5.0 \mathrm{~mL}), 23^{\circ} \mathrm{C}, 2.0 \mathrm{~mA}, 9 \mathrm{~h}$, graphite felt anode, Pt-plate cathode. Conversion of 176aa determined by ${ }^{1} \mathrm{H}$-NMR analysis with 1,3,5-trimethoxybenzene as an internal standard is given. Isolated yields are shown in parentheses. [b] Traces of $\mathrm{C}-\mathrm{H}$ oxygenated product $\mathbf{3 6}$ was detected. [c] Addition of $n-\mathrm{Bu}_{4} \mathrm{NPF}_{6}$ (1.0 equiv) as supporting electrolyte.

Thereafter, different additives were evaluated for the cobaltaelectro-catalyzed $\mathrm{C}-\mathrm{H}$ activation (Table 11). Similar to previous reports on cobaltaelectro-catalyzed $\mathrm{C}-\mathrm{H}$ oxygenation ${ }^{[249]}$ or alkyne annulations, ${ }^{[250]} \mathrm{NaOPiv}$ proved to be the ideal additive, outcompeting other carboxylate salts (entries 1-3). ${ }^{[23 \mathrm{~b}, 34 \mathrm{a}, 284]}$ When triflate salts were employed, 176aa was formed with decreased efficacy (entry 4) and carbonate or phosphate salts failed to facilitate the desired $\mathrm{C}-\mathrm{H}$ transformation (entries 5 and 6). 
Table 11. Additive effects. ${ }^{[\mathrm{a}]}$<smiles>O=C(N[O+])c1ccccc1</smiles>

$34 a$<smiles>C=IC=[PH](=O)(c1ccccc1)c1ccccc1</smiles>

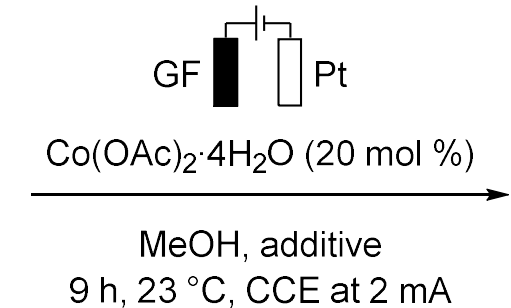

$9 \mathrm{~h}, 23^{\circ} \mathrm{C}, \mathrm{CCE}$ at $2 \mathrm{~mA}$<smiles>O=c1c2ccccc2cc(CP(=O)(c2ccccc2)c2ccccc2)n1[O-]</smiles>

176aa

\begin{tabular}{ccc}
\hline Entry & Additive & Yield [\%] \\
\hline 1 & NaOPiv & $78(75)$ \\
2 & $\mathrm{NaOAc}$ & $71(70)$ \\
3 & $\mathrm{KOAc}$ & $76(73)$ \\
4 & $\mathrm{KOTf}$ & 37 \\
5 & $\mathrm{Na}_{2} \mathrm{CO}_{3}$ & --- \\
6 & $\mathrm{~K}_{3} \mathrm{PO}_{4}$ & --- \\
\hline
\end{tabular}

[a] Reaction conditions: Undivided cell, 34a $(0.30 \mathrm{mmol}), \mathbf{7 5 a}(0.36 \mathrm{mmol}), \mathrm{Co}(\mathrm{OAc})_{2} \cdot 4 \mathrm{H}_{2} \mathrm{O}(20 \mathrm{~mol} \%)$, additive (2.00 equiv), $\mathrm{MeOH}(5.0 \mathrm{~mL}), 23^{\circ} \mathrm{C}, 2.0 \mathrm{~mA}, 9 \mathrm{~h}$, graphite felt anode, Pt-plate cathode. Conversion of 176aa determined by ${ }^{1} \mathrm{H}$-NMR analysis with 1,3,5-trimethoxybenzene as an internal standard is given. Isolated yields are shown in parentheses.

Finally, the electrolysis parameters, reaction temperature, reaction time and catalyst loading were optimized (Table 12). Changing the reaction time from $9 \mathrm{~h}\left(Q \cdot \mathrm{mol}^{-1}=2.24 \mathrm{~F}\right)$ to $15 \mathrm{~h}$ $\left(Q \cdot \mathrm{mol}^{-1}=3.73 \mathrm{~F}\right)$ led to decreased yields of $\mathbf{1 7 6 a a}$ (entries $\left.1-2\right)$, which can be rationalized by possible overoxidation of the product (vide infra). Likewise, an increased constant current of $5 \mathrm{~mA}\left(Q \cdot \mathrm{mol}^{-1}=5.60 \mathrm{~F}\right)$ resulted in diminished yields (entry 3$)$, as well as a constant current of $4 \mathrm{~mA}\left(Q \cdot \mathrm{mol}^{-1}=2.24 \mathrm{~F}\right)$ along with a reduced reaction time (entry 4). A platinum anode failed to promote the electrocatalysis (entry 5). Fortunately, a slight increase of the reaction temperature to $40{ }^{\circ} \mathrm{C}$ (entry 6) resulted in superior formation of isoquinolone 176aa and even allowed a significant reduction of catalyst loading without notable losses in the catalyst's efficacy (entry 7 and 8). Additionally, when the concentration of the benzamide 34a was raised to $0.1 \mathrm{M}$, while maintaining the overall applied charge $\left(Q \cdot \mathrm{mol}^{-1}=2.24 \mathrm{~F}\right)$, almost quantitative yield of the desired product 176aa was obtained (entry 9). Mild constant potential electrolysis at potentials as low as $1.3 \mathrm{~V} v \mathrm{~s}$. $\mathrm{Ag} / \mathrm{Ag}^{+}$proved equally viable (entry 10 ). Remarkably, in contrast to electrocatalysis with precious metal catalyst such as palladium, ${ }^{[226,232,236 \mathrm{a}, 236 \mathrm{c}, 236 \mathrm{~d}]}$ the cobaltaelectro-catalyzed $\mathrm{C}-\mathrm{H} / \mathrm{N}-\mathrm{H}$ annulation reaction did not require the use of a more laborious divided-cell setup 
(entry 11). Control experiments verified the essential role of the cobalt catalyst and electricity (entries 12 and 13). Finally, the cobalta-electrocatalysis was performed with a standardized electrochemistry kit, ElectraSyn 2.0 from IKA. The reaction proceeded equally well (entry 14), although reticulated vitreous carbon (RVC) was used as the anode material.

Table 12. Optimization of various reaction parameters. ${ }^{[\mathrm{a}]}$

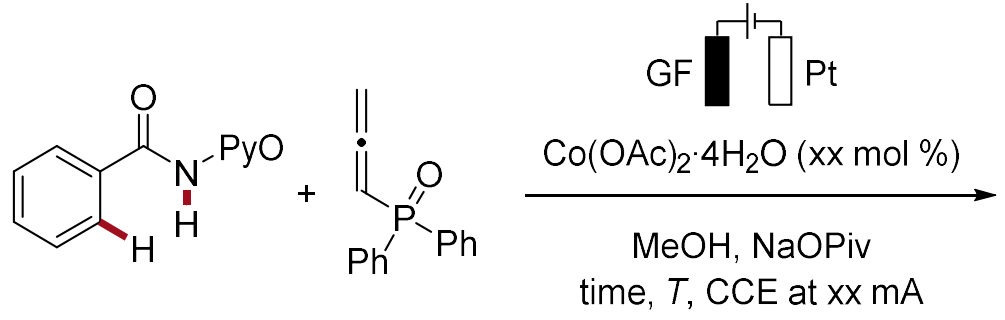

$34 a$<smiles>O=c1c2ccccc2cc(CP(=O)(c2ccccc2)c2ccccc2)n1[O-]</smiles>

176aa

\begin{tabular}{cccccc}
\hline Entry & {$[\mathrm{Co}][\mathrm{mol} \%]$} & Current $[\mathrm{mA}]$ & $T[\%]$ & Time $[\mathrm{h}]$ & Yield $[\%]$ \\
\hline 1 & 20 & 2 & 23 & 9 & $78(75)$ \\
2 & 20 & 2 & 23 & 15 & 65 \\
3 & 20 & 5 & 23 & 9 & 52 \\
4 & 20 & 4 & 23 & 4.5 & 49 \\
5 & 20 & 2 & 23 & 15 & $3^{[\mathrm{b}]}$ \\
6 & 20 & 2 & 40 & 9 & 86 \\
7 & 10 & 2 & 40 & 9 & $85(79)$ \\
8 & 10 & 2 & 23 & 9 & 48 \\
$\mathbf{9}$ & $\mathbf{1 0}$ & $\mathbf{2}$ & $\mathbf{4 0}$ & $\mathbf{1 5}$ & $\mathbf{9 2}(\mathbf{9 1})^{[\mathrm{c}]}$ \\
10 & 10 & $1.30 \mathrm{~V}$ & 40 & 12 & $91^{[\mathrm{c}, \mathrm{d}]}$ \\
11 & 10 & 2 & 40 & 9 & $55^{[\mathrm{e}]}$ \\
12 & --- & 2 & 40 & 9 & ---- \\
13 & 10 & ---- & 40 & 15 & $---{ }^{[\mathrm{f}]}$ \\
14 & 10 & 2 & 23 & 9 & $81(79)^{[\mathrm{g}]}$ \\
\hline
\end{tabular}

[a] Reaction conditions: Undivided cell, 34a $(0.30 \mathrm{mmol}), \mathbf{7 5 a}(0.36 \mathrm{mmol}),[\mathrm{Co}](\mathrm{xx} \mathrm{mol} \%), \mathrm{NaOPiv}$ (2.00 equiv), $\mathrm{MeOH}(5.0 \mathrm{~mL}), T\left[{ }^{\circ} \mathrm{C}\right]$, constant current $[\mathrm{mA}]$, time, graphite felt anode, Pt-plate cathode. Isolated yields are given. Conversion of 176aa determined by ${ }^{1} \mathrm{H}-\mathrm{NMR}$ analysis with 1,3,5trimethoxybenzene as an internal standard is given. Isolated yields are shown in parentheses. [b] Pt-plate anode and cathode. [c] 34a $(0.50 \mathrm{mmol})$. [d] Constant potential electrolysis at $1.30 \mathrm{~V} v s . \mathrm{Ag} / \mathrm{Ag}^{+}$. [e] Divided cell. [f] $\mathrm{N}_{2}$ Atmosphere. [g] Performed with IKA ElectraSyn 2.0, RVC anode, Pt cathode.

Utilizing the optimized conditions, different $N$-substitution patterns were probed for the desired cobaltaelectro-catalyzed $\mathrm{C}-\mathrm{H} / \mathrm{N}-\mathrm{H}$ allene annulation (Table 13). It was found that 
similar to the electrochemical cobalt-catalyzed $\mathrm{C}-\mathrm{H}$ oxygenation reactions (vide supra), pyridine- $N$-oxide served as the ideal directing group within the cobaltaelectro regime (entry 1). In contrast, commonly employed 8 -aminoquinoline derivative $\mathbf{2 6 a}^{[133,285]}$ did not furnish the desired annulated product 76aa under the optimized conditions. However, when the reaction was performed with TFE as the solvent, 76aa was isolated in moderate yields (entry 2). Notably, also the electroreductive removable ${ }^{[251]}$ hydrazide directing group $^{[120 \text {, }}$ ${ }^{140]}$ 62a proved to be viable under the exceedingly mild reaction conditions (entry 3) providing product 177aa in excellent yields. The scope of this reaction was later extended in an independent work by $\mathrm{Mei}$ and Ackermann. ${ }^{[286]}$ Here, the authors used slightly modified reaction conditions with TFE as the solvent, $\mathrm{NaOAc}$ as the additive and the electrocatalysis was performed under an atmosphere of argon.

Table 13. Studies on the $N$-substitution pattern for the $\mathrm{C}-\mathrm{H} / \mathrm{N}-\mathrm{H}$ activation. ${ }^{\text {[a] }}$

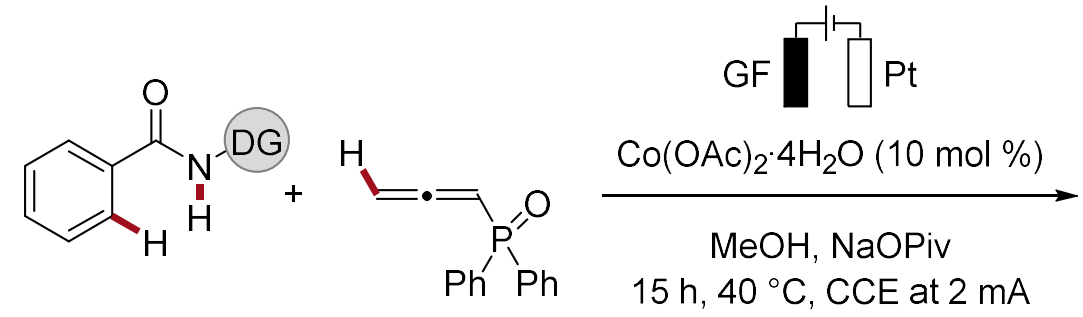

$26,34,62,162$, 164 or 180
$75 a$<smiles>Cn1c(CP(=O)(c2ccccc2)c2ccccc2)cc2ccccc2c1=O</smiles>

$76,176-181$<smiles>CN(C(=O)c1ccccc1)N(Nc1ccccn1)c1ccccc1</smiles> 
4<smiles>O=C(Nc1ccccc1-c1ncco1)c1ccccc1</smiles>

5<smiles>O=C(Nc1ccccc1O)c1ccccc1</smiles>

6<smiles>O=C(NC(F)(F)F)c1ccccc1</smiles>

$162 \mathrm{a}$<smiles></smiles>

178aa

179aa

$164 a$<smiles>O=c1c2ccccc2cc(CP(=O)(c2ccccc2)c2ccccc2)n1-c1ccccc1O</smiles><smiles></smiles>

$\mathrm{Ph}$

[a] Reaction conditions: Undivided cell, benzamide $(0.50 \mathrm{mmol})$, allene $75 \mathbf{a}(0.60 \mathrm{mmol}), \mathrm{Co}(\mathrm{OAc})_{2} \cdot 4 \mathrm{H}_{2} \mathrm{O}$ (10 mol \%), NaOPiv (2.00 equiv), $\mathrm{MeOH}(5.0 \mathrm{~mL}), 40^{\circ} \mathrm{C}, 2.0 \mathrm{~mA}, 15 \mathrm{~h}$, graphite felt anode, Pt-plate cathode. Isolated yields are given. [b] Reaction was performed in TFE $(5.0 \mathrm{~mL})$.

\subsubsection{Versatility of the Cobaltaelectro-Catalyzed $\mathrm{C}-\mathrm{H} / \mathrm{N}-\mathrm{H}$ Allene Annulations}

With the optimized conditions in hand, the robustness of the cobaltaelectro-catalyzed $\mathrm{C}-\mathrm{H} / \mathrm{N}-\mathrm{H}$ allene annulation was evaluated through an extensive substrate scope (Table 14).

Table 14. Substrate scope of benzamides 34 with allene 75a. ${ }^{\text {[a] }}$<smiles>O=C(Nc1cccc[n+]1[O-])c1ccccc1</smiles>

34

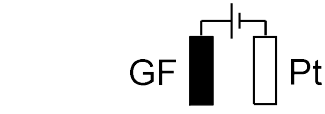

$\mathrm{Co}(\mathrm{OAc})_{2} \cdot 4 \mathrm{H}_{2} \mathrm{O}(10 \mathrm{~mol} \%)$

$\mathrm{MeOH}, \mathrm{NaOPiv}$

$15 \mathrm{~h}, 40^{\circ} \mathrm{C}, \mathrm{CCE}$ at $2 \mathrm{~mA}$<smiles>O=c1c2cc3ccc2cc3n1P(=O)(O)c1ccccc1</smiles>

176

\begin{tabular}{|c|c|c|c|c|c|}
\hline Entry & Benzamide & 34 & Product & 176 & $\begin{array}{c}\text { Yield } \\
{[\%]}\end{array}$ \\
\hline 1 & & $34 a$ & & 176aa & 91 \\
\hline
\end{tabular}


2<smiles>Cc1ccc(C(=O)N[Pb])cc1</smiles>

3<smiles>COc1ccc(C(=O)NP(=O)(O)O)cc1</smiles>

4<smiles>CSc1ccc(C(=O)NP(=O)(O)O)cc1</smiles>

5<smiles>O=C(N[Pb])c1ccc(-c2ccccc2)cc1</smiles>

6<smiles>O=C(N[Pb])c1ccc(I)cc1</smiles>

7<smiles>CC(C)(C)c1ccc(C(=O)NPO)cc1</smiles>

34j<smiles></smiles>

$34 k$<smiles>O=C(N[Pb])c1ccc(F)cc1</smiles>

$34 g$<smiles>O=c1c2ccc(I)cc2cc(CP(=O)(c2ccccc2)c2ccccc2)n1P(O)O</smiles>

176ga 56 34b<smiles>Cc1ccc2c(=O)n(C(=O)O)c(CP(=O)(c3ccccc3)c3ccccc3)cc2c1</smiles>

176ba 96<smiles>O=c1c2ccc(F)cc2cc(CP(=O)(c2ccccc2)c2ccccc2)n1P(O)O</smiles>

8

9<smiles>CC(=O)c1ccc(C(=O)N[Pb])cc1</smiles>

341<smiles>CC(=O)c1ccc2c(=O)n(P(=O)(O)O)c(CP(=O)(c3ccccc3)c3ccccc3)cc2c1</smiles> 
10<smiles>COC(=O)c1ccc(C(=O)NP=O)cc1</smiles>

11<smiles>Cc1ccc(C(=O)NP(=O)=O)cc1C</smiles>

12<smiles>O=C(NP)c1cccc2c1Cc1ccccc1-2</smiles>

13<smiles>COc1cccc(C(=O)NP(=O)(O)O)c1</smiles>

14<smiles>[2H]c1cc2c(cc1C(=O)N[Pb])OCO2</smiles>

15<smiles>O=C(N[Pb])c1cccc(F)c1</smiles>

16<smiles>Cc1ccc(C)c(C(=O)NP(=O)(O)c2ccccc2)c1</smiles>

$34 \mathrm{~m}$<smiles>CC(=O)c1ccc2c(=O)n(C(=O)O)c(CP(=O)(c3ccccc3)c3ccccc3)cc2c1</smiles>

176ma 51

34n<smiles>Cc1cc2cc(CP(=O)(c3ccccc3)c3ccccc3)n([Po])c(=O)c2cc1C</smiles>

176na 97

340<smiles>O=c1c2c3c(ccc2cc(CP(=O)(c2ccccc2)c2ccccc2)n1[Pb])-c1ccccc1C3</smiles>

176oa 89<smiles>COc1ccc2cc(CP(=O)(c3ccccc3)c3ccccc3)n(P(=O)(O)c3ccccc3)c(=O)c2c1</smiles>

176pa 96

$34 q$<smiles>O=c1c2ccc3c(c2cc(CP(=O)(c2ccccc2)c2ccccc2)n1P(=O)(O)c1ccccc1)OCO3</smiles>

176qa<smiles></smiles>

176ra

34s<smiles>Cc1cc(C)c2c(=O)n(C(=O)O)c(CP(=O)(c3ccccc3)c3ccccc3)cc2c1</smiles>

176sa 73 
17<smiles>O=C(N[OH2+])c1csc2ccccc12</smiles>

$34 t$

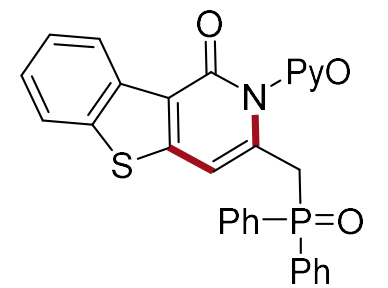

176ta 53

[a] Reaction conditions: Undivided cell, benzamide $34(0.50 \mathrm{mmol})$, allene 75a $(0.60 \mathrm{mmol}), \mathrm{Co}(\mathrm{OAc})_{2} \cdot 4 \mathrm{H}_{2} \mathrm{O}$ (10 mol \%), NaOPiv (2.00 equiv), $\mathrm{MeOH}(5.0 \mathrm{~mL}), 40{ }^{\circ} \mathrm{C}, 2.0 \mathrm{~mA}, 15 \mathrm{~h}$, graphite felt anode, Pt-plate cathode. Isolated yields are given.

First, a variety of para-substituted benzamides 34 were tested for the $\mathrm{C}-\mathrm{H} / \mathrm{N}-\mathrm{H}$ annulation reaction with allene 75a. In comparison to the unsubstituted substrate 34a (entry 1) the electrocatalytic manifold was fully tolerant of functional groups, including benzylic $\mathrm{C}-\mathrm{H}$ bonds, methoxy, thioether, phenyl, halogens and even iodo (entries 2-8). Second, also enolizable ketones or strongly deactivated ester groups were compatible with the cobaltaelectrocatalysis (entries 9 and 10). More sterically demanding substrates 34n and 340 furnished the desired isoquinolones 176na and 1760a in excellent yields and with full positional control (entries 11 and 12). It is noteworthy that the significantly weaker benzylic $\mathrm{C}-\mathrm{H}$ bond $\left(\mathrm{BDE}^{[15]}=82.2 \mathrm{kcal} \cdot \mathrm{mol}^{-1}\right)$ remained untouched, as the mass balance accounted for unreacted starting material $\mathbf{3 4 0}$.

Furthermore, the positional selectivity for the cobaltaelectro-catalyzed allene annulation was evaluated with differently meta-substituted benzamides 34 (entries 13-15). Hence, the electrocatalytic $\mathrm{C}-\mathrm{H}$ activation was mainly controlled by repulsive steric interactions, unless a secondary directing group effect was present, as was the case for substrate $\mathbf{3 4 q}$ and 34r (entries 14 and 15). In contrast to the previously reported cobaltaelectro-catalyzed C-H manifolds, ${ }^{[249-250]}$ the highly efficient allene annulation was also compatible with challenging ortho-methyl substituted substrate 34s, providing isoquinolone 176sa in $73 \%$ yield (entry 16). Finally, also heteroarenes such as a benzothiophene derivate $\mathbf{3 4 t}$ proved viable under the reaction conditions, furnishing the annulated product 176ta in moderate yield (entry 17). In terms of limitations for the benzamide 34, strongly electronwithdrawing groups such as nitro- or cyanide substituents resulted in low yields, as well as nicotinic acid derivates did not provide the desired annulated products in satisfactory yields. As a proof-of-concept, acrylic amide was likewise successfully transformed into the corresponding pyridine-2(1H)-one derivative 182aa (Scheme 53). 
<smiles>C/C=C(/C)C(=O)Nc1cccc[n+]1[O-]</smiles><smiles>COC(=O)OCCOC(=O)OCC(=O)OCc1ccccc1</smiles>

$37 a$<smiles>Cc1cc(CP(=O)(c2ccccc2)c2ccccc2)n(P([O-])[O-])c(=O)c1C</smiles>

182aa: $65 \%$

Scheme 53. Cobaltaelectro-catalyzed C-H annulation toward pyridines $\mathbf{1 8 2}$.

The versatility of the cobaltaelectro-catalyzed $\mathrm{C}-\mathrm{H}$ activation was further substantiated by the successful use of diversly substituted allenes $\mathbf{7 5}$ (Table 15).

Table 15. Cobaltaelectro-catalyzed $\mathrm{C}-\mathrm{H}$ annulations of allenes 75. ${ }^{[\mathrm{a}]}$

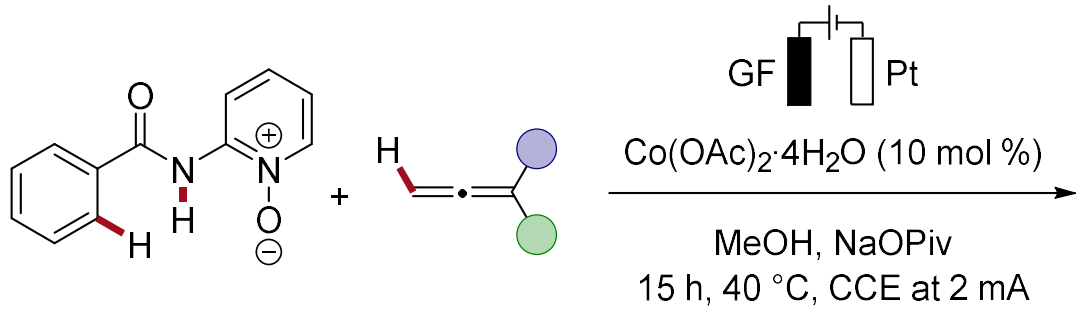

$34 a$

75<smiles>O=c1c2ccccc2cc(C(O)C(O)O)n1P(O)O</smiles>

176

Entry


4

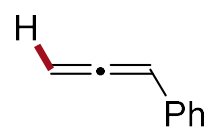

$75 b$<smiles>O=c1c2ccccc2cc(Cc2ccccc2)n1[Po]</smiles>

176ab $51^{[\mathrm{c}]}$

[a] Reaction conditions: Undivided cell, $34(0.50 \mathrm{mmol}), 75(0.60 \mathrm{mmol}), \mathrm{Co}(\mathrm{OAc})_{2} \cdot 4 \mathrm{H}_{2} \mathrm{O}(10 \mathrm{~mol} \%)$, NaOPiv (2.00 equiv), $\mathrm{MeOH}(5.0 \mathrm{~mL}), 40^{\circ} \mathrm{C}, 2.0 \mathrm{~mA}, 15 \mathrm{~h}$, graphite felt anode, Pt-plate cathode. Isolated yields are given. [b] $60{ }^{\circ} \mathrm{C}, \mathrm{Co}(\mathrm{OAc})_{2} \cdot 4 \mathrm{H}_{2} \mathrm{O}(20 \mathrm{~mol} \%)$, performed by $\mathrm{Dr}$. S. C. Sau. [c] Reaction was performed using constant potential electrolysis (CPE) at $1.25 \mathrm{~V} v \mathrm{~s} . \mathrm{Ag} / \mathrm{Ag}^{+}$.

Under the standard conditions, ester substituted allenes $\mathbf{7 5 c}$ and $\mathbf{7 5 d}$ smoothly provided the annulated products with excellent control of chemo- and regioselectivity (entries 1 and 2). Sterically demanding 3,3-disubstuted allene 75e required slightly modified reaction conditions to provide the annulated product 176ae in synthetically useful yields (entry 3 ). However, the annulation solely occurred in the allene's terminal position. In contrast, propa-1,2-dien-1-ylbenzene $\mathbf{( 7 5 b )}$ resulted in various reaction products under the less selective constant current electrolysis conditions. Thus, cyclic voltammetry revealed a relatively low oxidation potential for allene $\mathbf{7 5 b}$ with an onset potential of $1.35 \mathrm{~V} v s$. SCE (Figure 6, magenta line). In comparison, allene 75a proved to be significantly more redoxstable with an anodic onset potential at around $2.0 \mathrm{~V} v s$. SCE (Figure 6, blue line).

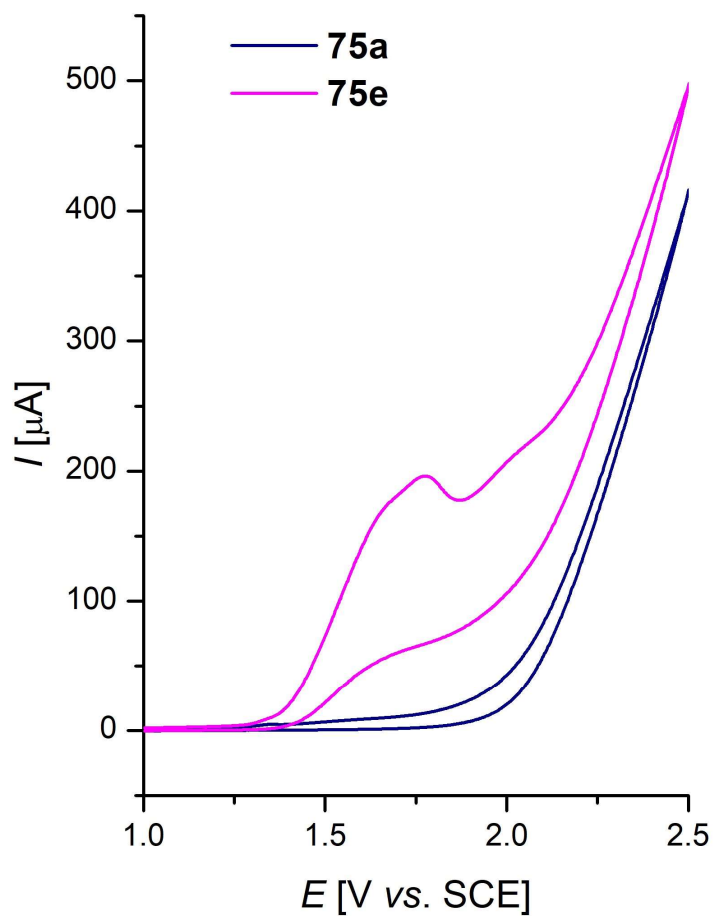

Figure 6. Cyclic voltammograms at $100 \mathrm{mV} \cdot \mathrm{s}^{-1}: n-\mathrm{Bu}_{4} \mathrm{NPF}_{6}(0.1 \mathrm{M}$ in $\mathrm{MeOH})$, concentration of allenes $3.0 \mathrm{mM}$. Allene 75a (blue); Allene 75b (magenta). 
Based on these information, mild constant potential electrocatalysis was performed at $1.25 \mathrm{~V}$ vs. $\mathrm{Ag} / \mathrm{Ag}^{+}$, thus furnishing the desired product 176ab with excellent regioselectivity control (entry 4). It is noteworthy that previous studies on cobalt-catalyzed $\mathrm{C}-\mathrm{H} / \mathrm{N}-\mathrm{H}$ annulation reactions using manganese(III) salts as the chemical oxidant, reported dihydroisoquinolin-1(2H)-ones 77 as the major product of different regioisomers, when allene 75b was used (cf. Scheme 24). ${ }^{[133]}$ However, when silver(I) salts were employed as the oxidant along with an elevated temperature of $80^{\circ} \mathrm{C}, 85$ was reported as the major product, albeit in $37 \%$ yield ( $c f$. Scheme $26 a) .{ }^{[136]}$

In addition to terminally substituted allenes, $N$. Ang successfully explored the scope for cobaltaelectro-catalyzed C-H/N-H annulations with internal allenes 84 (Scheme 54).

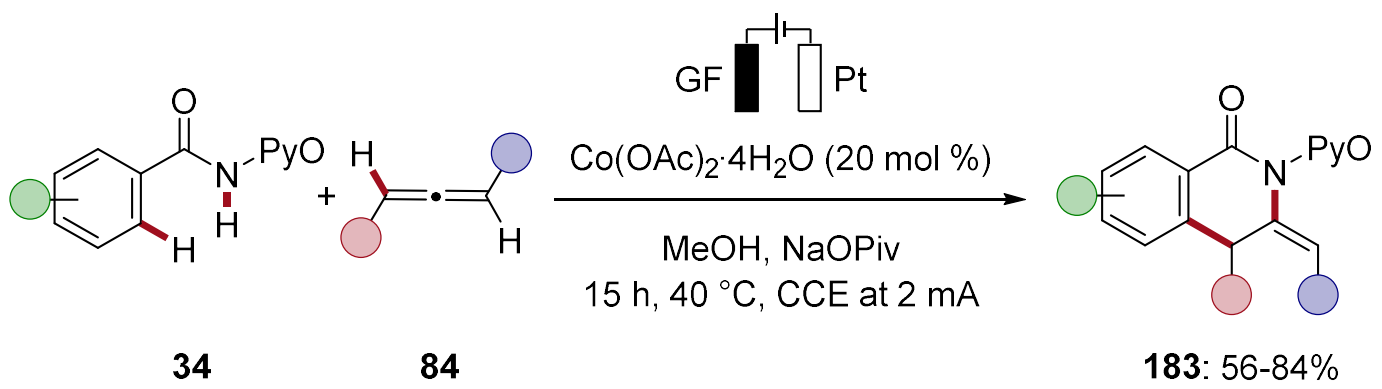

Scheme 54. $\mathrm{C}-\mathrm{H} / \mathrm{N}-\mathrm{H}$ annulation with internal allenes 84, performed by $N$. Ang.

Interestingly, when allenes $\mathbf{8 4}$ were used with a 1,3-disubstitution pattern, the corresponding exo-methylen isoquinolones 183 were obtained. Thus, the reaction outcome is supportive of a base-assisted post-catalytic allene isomerization manifold.

Apart from the broadly applicable substrate scope of the electrocatalytic $\mathrm{C}-\mathrm{H}$ activation, some limitations were disclosed (Scheme 55). Particularly, differently substituted allenes 75 were found to be challenging and resulted in complex product mixtures or did not provide synthetically useful product yields. Based on the findings for allene 75e, it cannot be excluded that extensive optimization studies or constant potential electrolysis could be beneficial for some of the presented substrates to enable the desired transformation. 


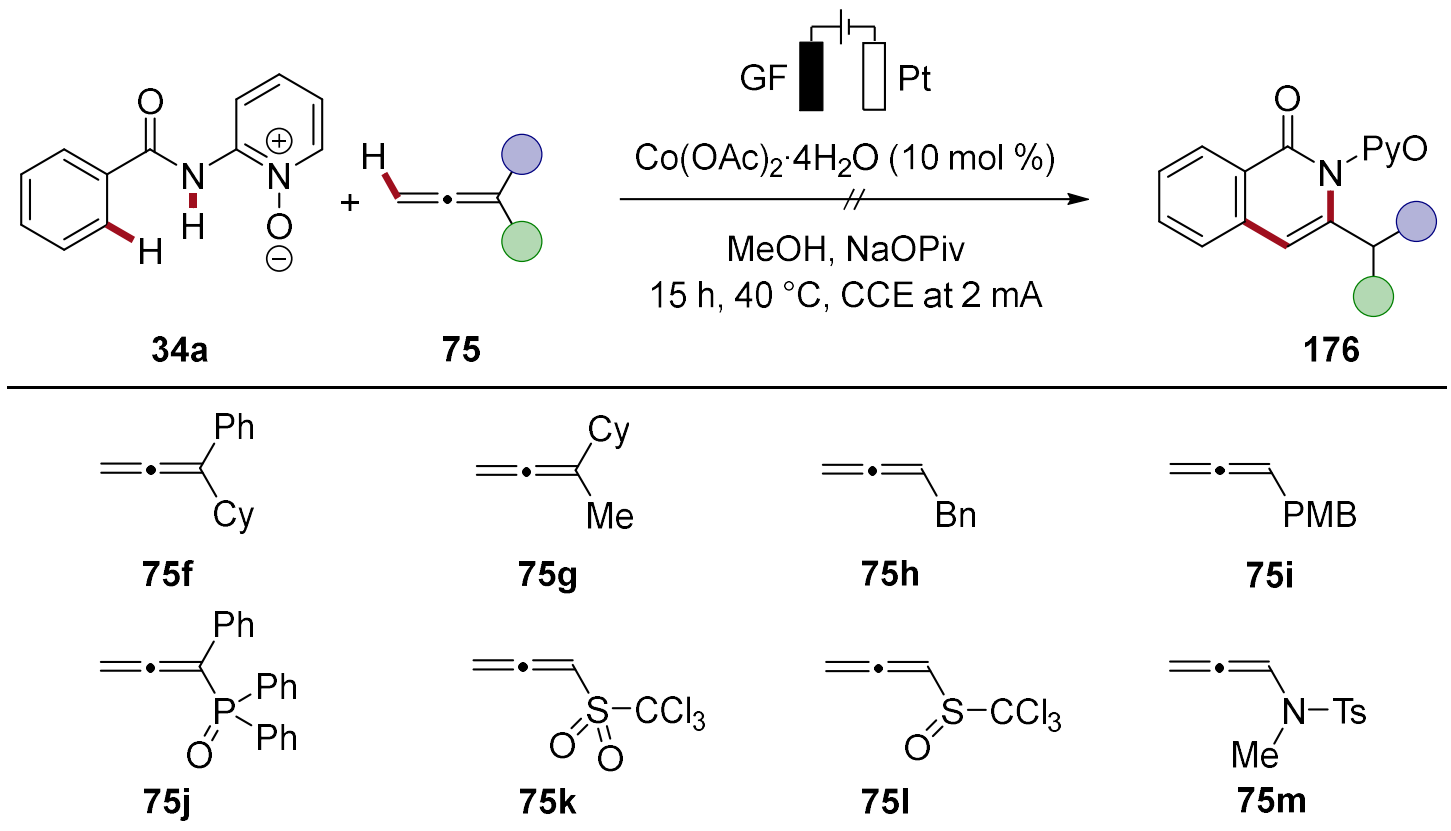

Scheme 55. Limitations for the allene $\mathbf{7 5}$ substrate scope.

Finally, the practical utility of the cobalta-electrocatalysis was reflected by a multigramscale electrocatalytic preparation of isoquinolone 176 na (Scheme 56) with similar levels of efficiency and regioselectivity ( $c f$. Table 14, entry 11). Notable features of the gramscale synthesis include an user-friendly undivided cell setup, cost-efficient graphite felt anode material, Earth-abundant cobalt catalyst, industrially compatible ${ }^{[287]} \mathrm{MeOH}$ as the solvent and a high current efficiency (CE) of $76 \%$.<smiles>Cc1ccc(C(=O)N(c2ccccc2)c2cccc[n+]2[O-])c(C)c1</smiles>

34n: $9.00 \mathrm{mmol}$

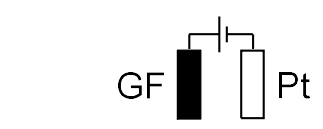

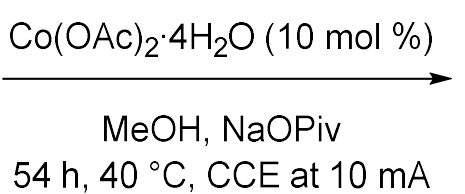

$54 \mathrm{~h}, 40^{\circ} \mathrm{C}, \mathrm{CCE}$ at $10 \mathrm{~mA}$<smiles>COc1cc2cc(CP(=O)(c3ccccc3)c3ccccc3)n([O-])c(=O)c2cc1C</smiles>

176 na: $85 \%, 3.66 \mathrm{~g}$

Scheme 56. Multigram-scale electrocatalytic isoquinolone 176na synthesis.

\subsubsection{Mechanistic Studies}

Given the unique features of the first cobaltaelectro-catalyzed $\mathrm{C}-\mathrm{H}$ activation with allenes, detailed mechanistic studies by experiment and computation ${ }^{[260]}$ were performed to delineate the catalyst's mode of action and to rationalize the high levels of regioselectivity. 


\subsubsection{H/D-Exchange Experiment}

In order to gain deeper knowledge on the $\mathrm{C}-\mathrm{H}$ activation mechanism, the electrocatalytic reaction was carried out under standard conditions with isotopically labelled $\mathrm{CD}_{3} \mathrm{OD}$ as the solvent (Scheme 57). The reisolated substrate 34a did not undergo $\mathrm{H} / \mathrm{D}$ exchange, thus being indicative of an irreversible $\mathrm{C}-\mathrm{H}$ scission event. It has to be noted that in-situ ${ }^{1} \mathrm{H}-\mathrm{NMR}$ studies were suggestive for full $\mathrm{H} / \mathrm{D}$ exchange of the acidic amide proton. Here, the detected $\mathrm{N}-\mathrm{H}$ protonation likely occurred during the subsequent column chromatographic purification. In case of the product 176aa, significant deuterium incorporation was observed in the vinylic position, which can be rationalized by facile baseassisted H/D exchange following the electrocatalysis.

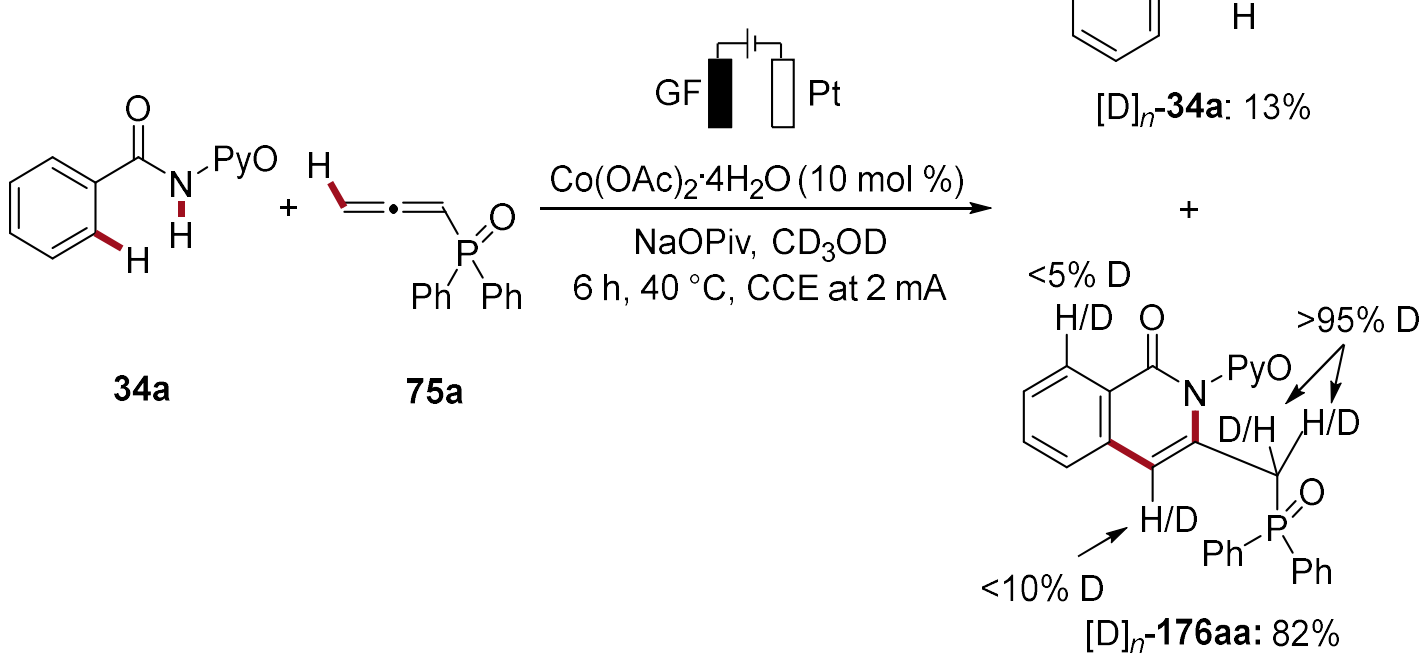

Scheme 57. H/D-Exchange study for the cobaltaelectro-catalyzed allene annulation.

\subsubsection{Intermolecular Competition Experiments}

To further examine the mechanistic rationale of the cobalt-catalyzed $\mathrm{C}-\mathrm{H}$ activation event, two intermolecular competition experiments were performed with electronically distinct substrates $\mathbf{3 4 d} / \mathbf{3 4 k}$ or $\mathbf{3 4 b} / \mathbf{3 4} \mathbf{c}$, respectively (Scheme 58 ). 


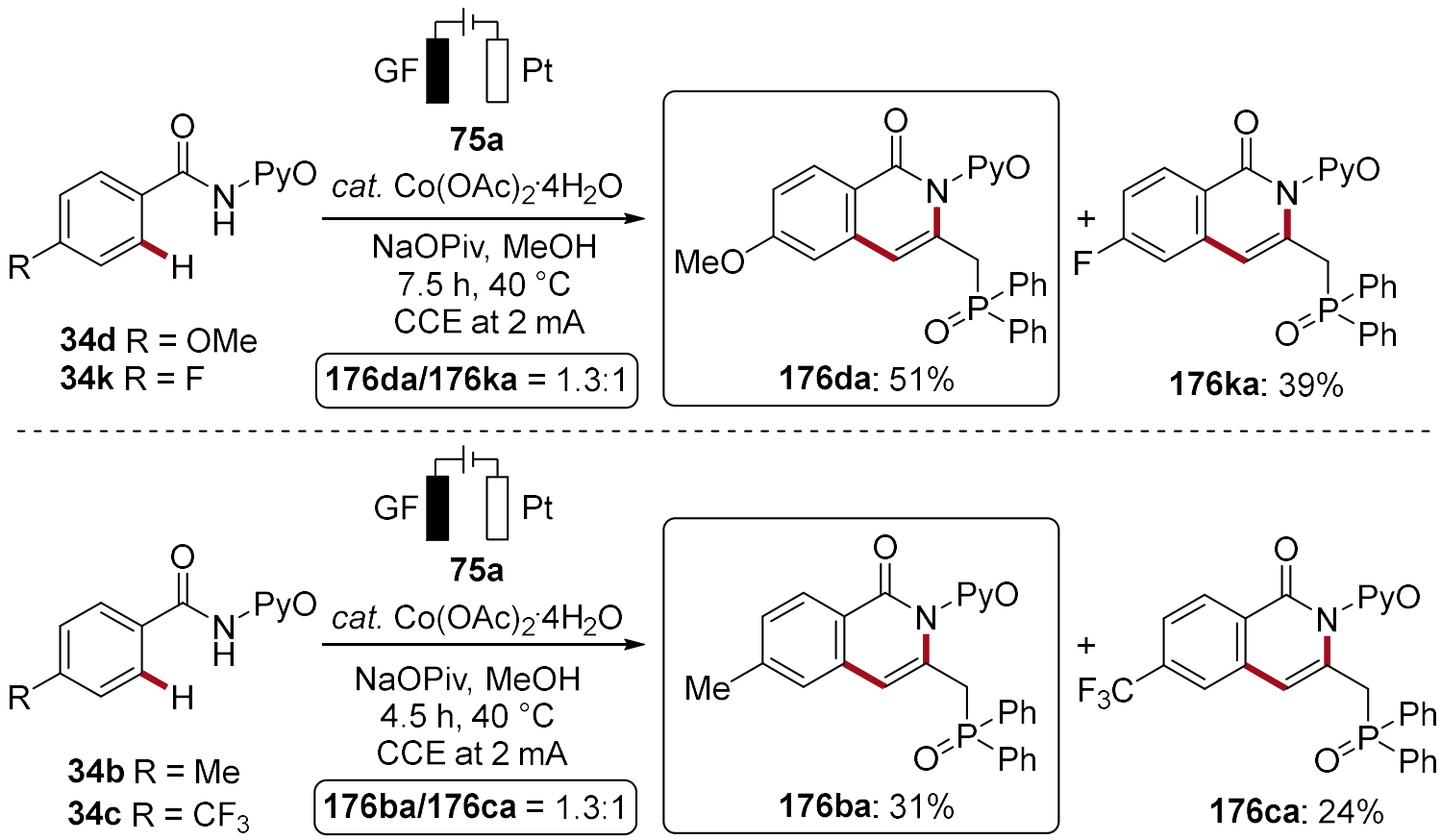

Scheme 58. Intermolecular competition studies.

Both experiments highlighted the preference for the electron-rich arenes $\mathbf{3 4 d}$ or $\mathbf{3 4 b}$, which reacted approximately 1.3 times faster than the electron-poor arenes $34 \mathbf{k}$ and $\mathbf{3 4 c}$. These findings are in good agreement with a BIES-type ${ }^{[35,41]} \mathrm{C}-\mathrm{H}$ activation to be operative for the cobaltaelectro-catalyzed $\mathrm{C}-\mathrm{H} / \mathrm{N}-\mathrm{H}$ allene annulation manifold. Furthermore, electronrich substrates would increase the nucleophilicity of the cobalt(III)-carbon bond and thus enhance a kinetically relevant migratory insertion of the allene. These findings were further substantiated by detailed computational mechanistic studies performed by $D r . J . C . A$. Oliveira. Indeed, the allene insertion showed a relatively high activation barrier of $16 \mathrm{kcal} \cdot \mathrm{mol}^{-1} \cdot{ }^{[260]}$

\subsubsection{Kinetic Reaction Profile}

Next, in-operando IR spectroscopy (ReactIR) was performed to study the kinetic profile of the cobaltaelectro-catalyzed $\mathrm{C}-\mathrm{H}$ activation (Scheme 59). Unfortunately, preliminary attempts under the standard conditions failed to deliver suitable data, due to overlapping IR-bands of $\mathrm{MeOH}$ with the signals of interest. However, when $\mathrm{CH}_{2} \mathrm{Cl}_{2}$ was used as the solvent, two characteristic IR-bands were identified for the product 176aa $\left(1675 \mathrm{~cm}^{-1}\right)$ and the starting material $34 \mathbf{a}\left(1510 \mathrm{~cm}^{-1}\right)$, respectively. 


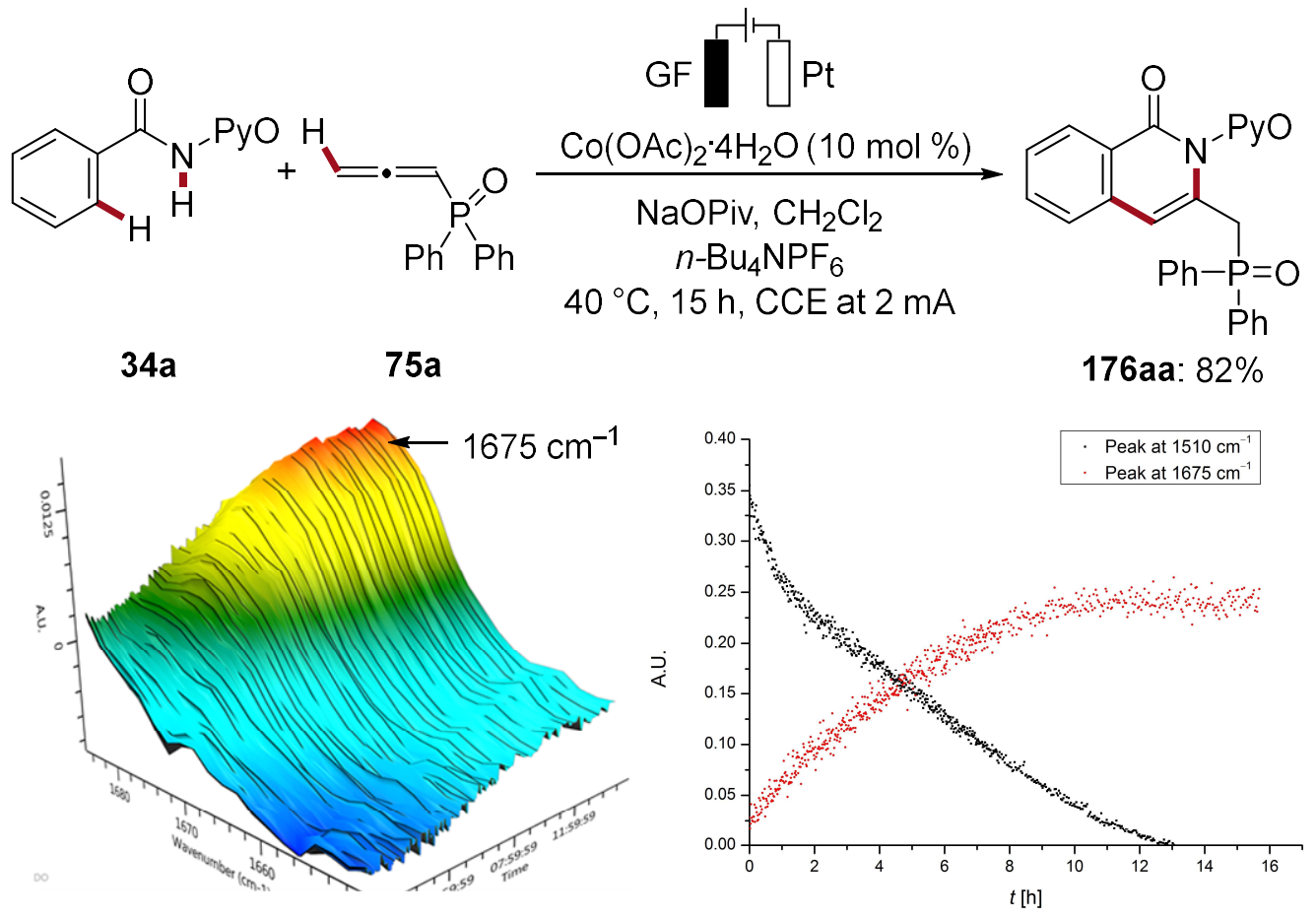

Scheme 59. In-operando IR studies. 3D surface plot and kinetic reaction profile of the electrocatalytic $\mathrm{C}-\mathrm{H}$ activation.

Additionally, product conversion was monitored by ${ }^{1} \mathrm{H}-\mathrm{NMR}$ spectroscopic analysis and allowed the quantification of the ReactIR data (Figure 7). Thus, the gathered data showed a linear product formation within the first two hours of the reaction without a significant induction period.

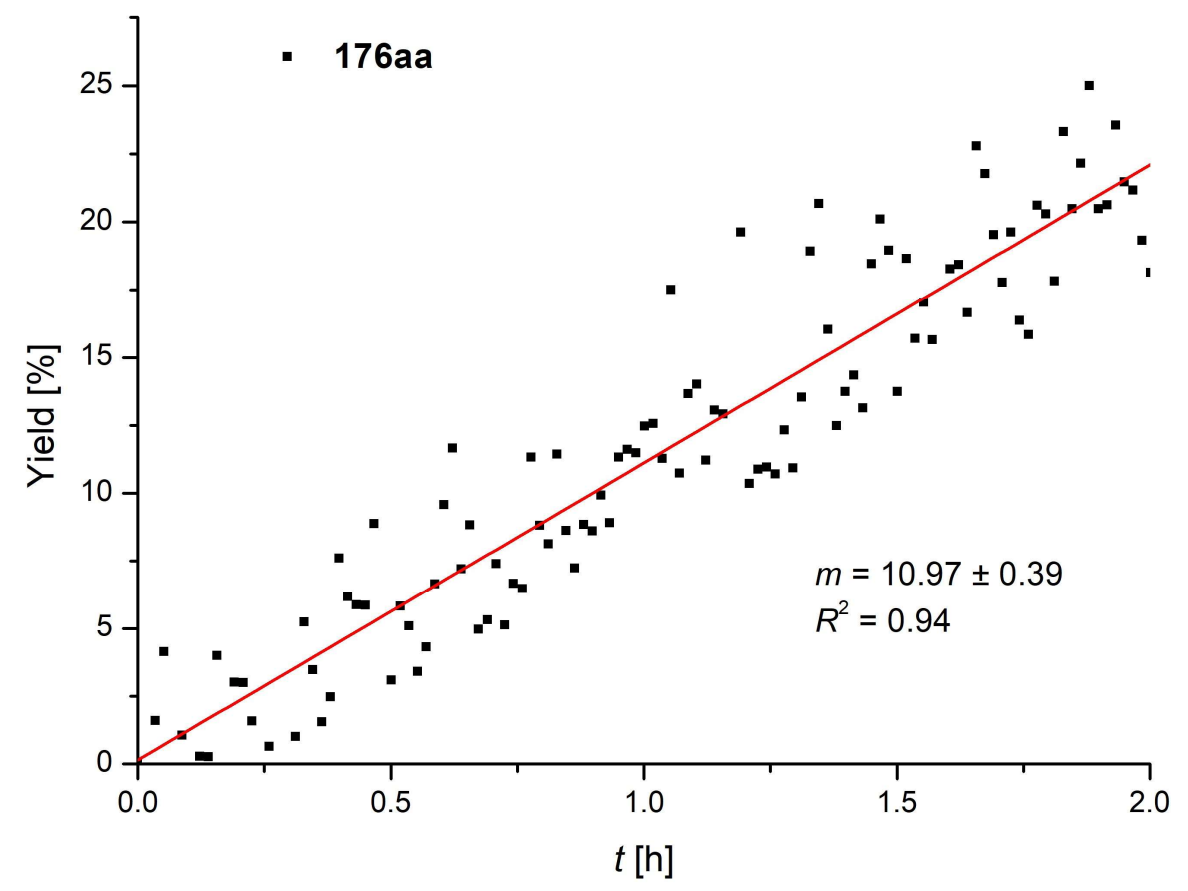

Figure 7. Initial rate plot for the electrocatalytic formation of 176aa. 


\subsubsection{KIE Studies}

The kinetic isotope effect (KIE) of the cobaltaelectro-catalyzed $\mathrm{C}-\mathrm{H} / \mathrm{N}-\mathrm{H}$ allene annulation was determined via the comparison of two independent reaction rates for substrate 34a and its deuterated analogue $[\mathrm{D}] 5-\mathbf{3 4 a}$ (Scheme 60). The experiments revealed a minor KIE of $k_{\mathrm{H}} / k_{\mathrm{D}}=1.2$ (Figure 8 ).

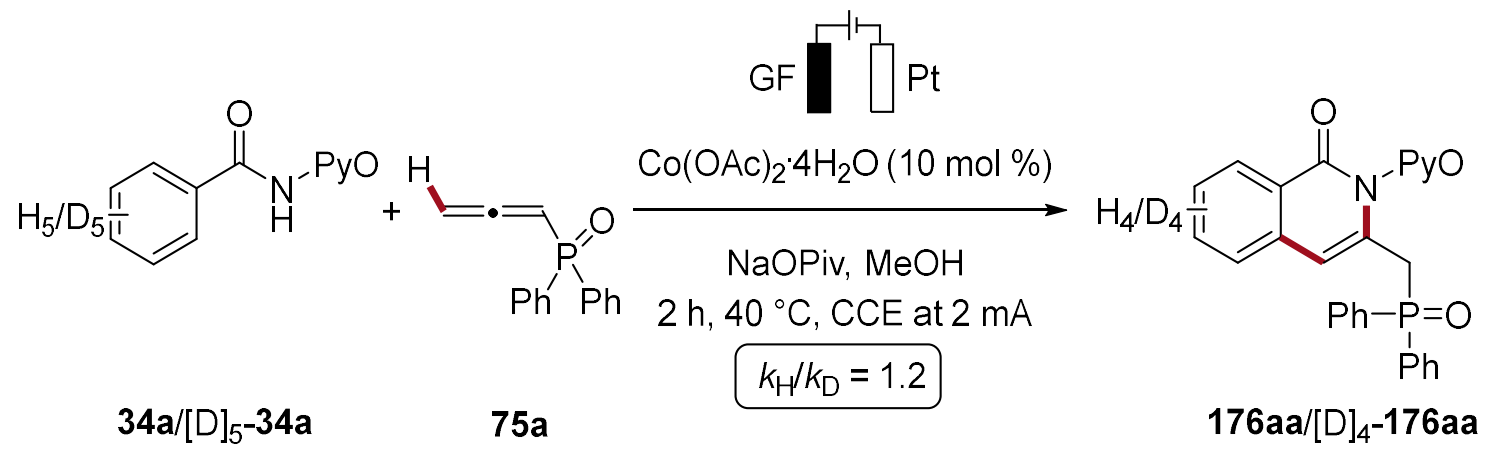

Scheme 60. KIE studies for the cobalta-electrocatalysis.

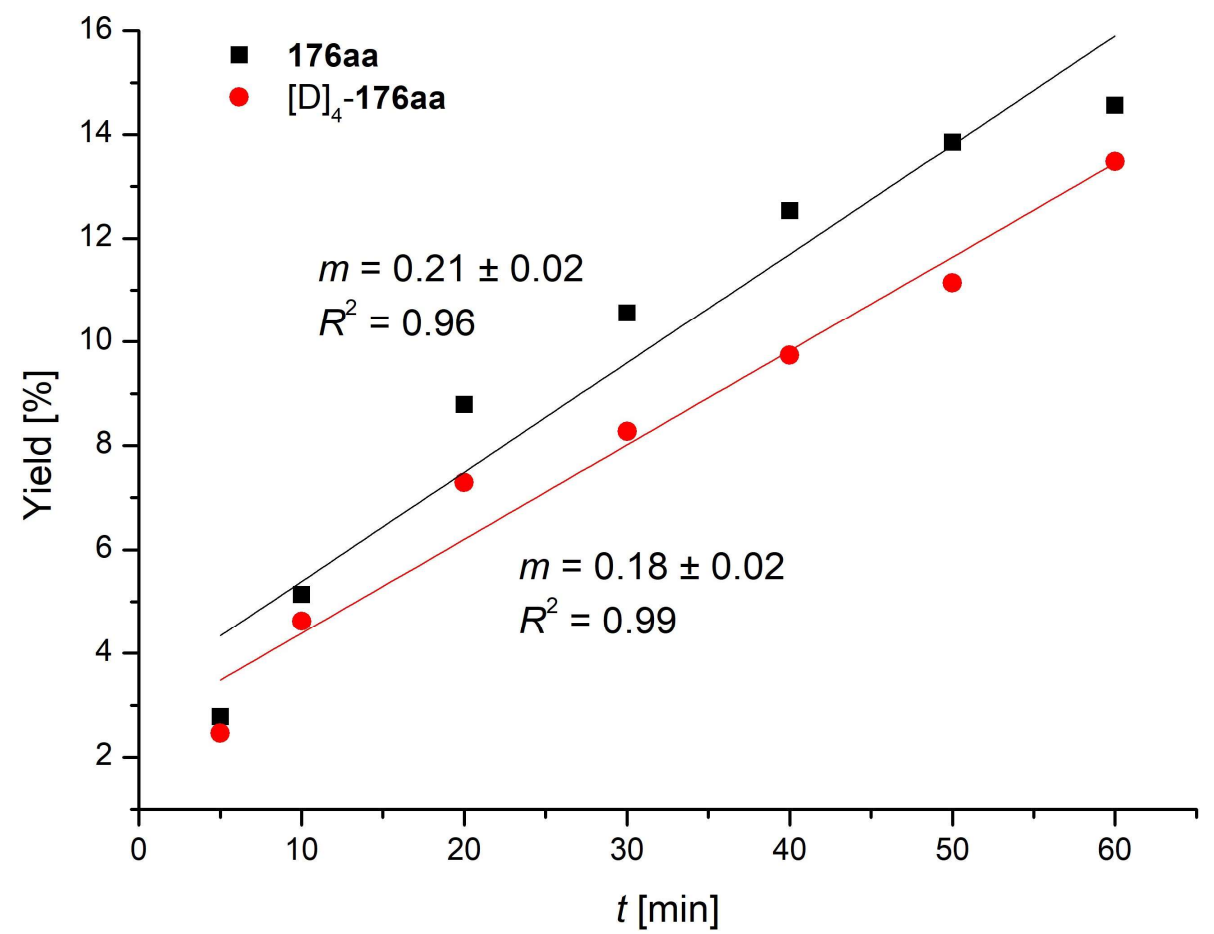

Figure 8. KIE studies for the cobalta-electrocatalysis.

Based on the previously developed method for the in-operando reaction monitoring of cobaltaelectro-catalyzed $\mathrm{C}-\mathrm{H}$ activation with allenes 75a (vide supra), additional $\mathrm{KIE}$ studies were performed and followed via ReactIR analysis (Scheme 61). Likewise, a minor KIE of $k_{\mathrm{H}} / k_{\mathrm{D}}=1.2$ was detected (Figure 9). These observations are in good agreement with 
previous mechanistic studies on cobaltaelectro-catalyzed $\mathrm{C}-\mathrm{H}$ activations ${ }^{[249-250]}$ and indicate that the $\mathrm{C}-\mathrm{H}$ cleavage is not the rate-determining step of the electrocatalysis.

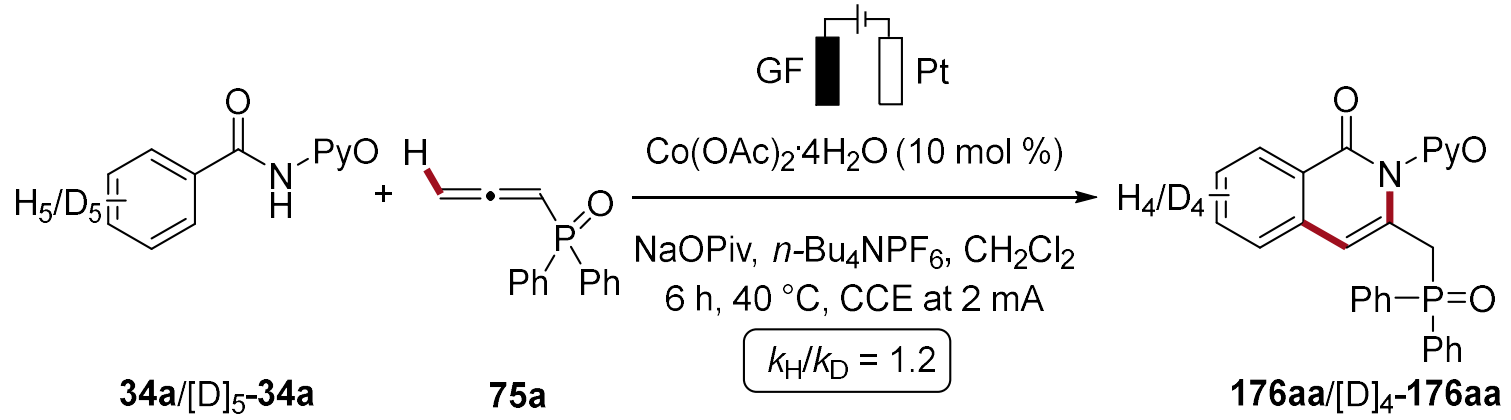

Scheme 61. KIE studies followed by in-situ IR analysis.
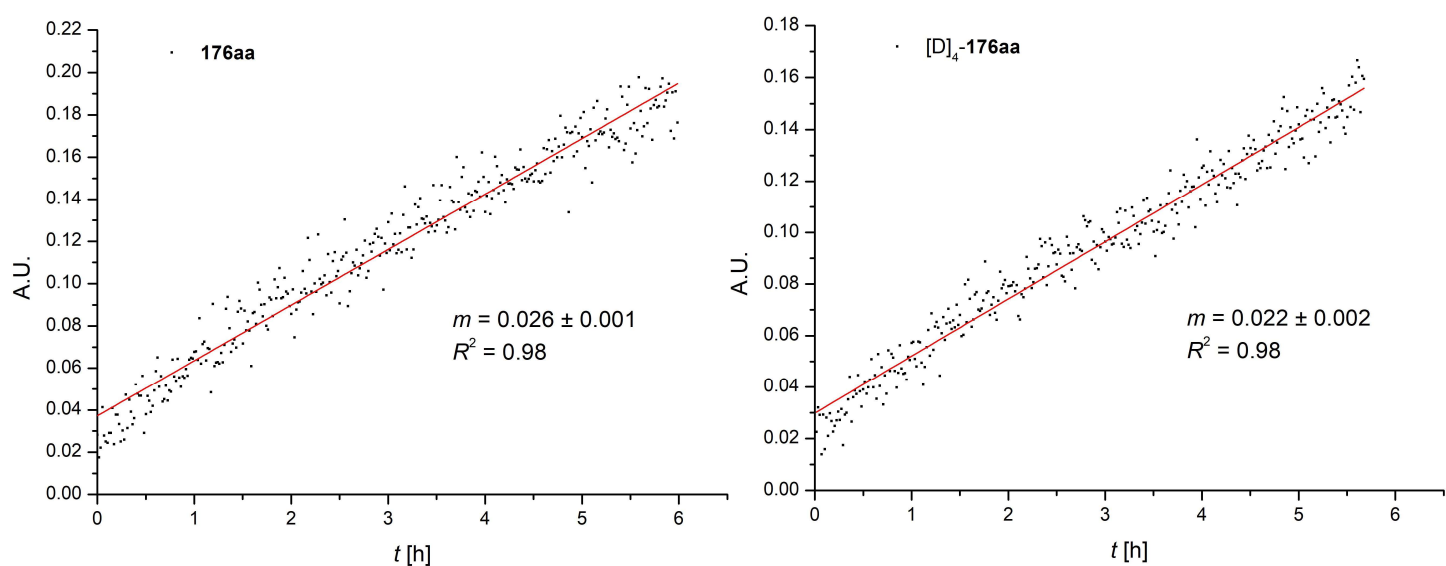

Figure 9. Initial rate analysis of 176aa and [D]4-176aa.

\subsubsection{CV Studies}

Finally, the oxidative cobaltaelectro-catalyzed $\mathrm{C}-\mathrm{H}$ activation was analyzed by means of cyclic voltammetry (Figure 10). The voltammograms were recorded with a scan rate of $100 \mathrm{mV} \cdot \mathrm{s}^{-1}$ in $\mathrm{MeOH}$ with $n-\mathrm{Bu}_{4} \mathrm{NPF}_{6}(0.1 \mathrm{M})$ as the conducting salt. In accordance to previous projects on cobalta-electrocatalysis (vide supra), ${ }^{[249-250]}$ substrate 34a was found to be relatively stable toward overoxidation with an irreversible oxidation at $E_{\mathrm{p}, \mathrm{ox}}=1.51 \mathrm{~V}$ vs. SCE. Likewise, allene 75a was found to be voltammetrically silent in the tested potential window ( $c f$. Figure 6). The cobalt(II) precatalyst revealed an irreversible oxidation peak at $1.19 \mathrm{~V} v s$. SCE, which can be assigned to the respective formation of the active cobalt(III) carboxylate species. ${ }^{[277]}$ Moreover, irreversible oxidation of product 176aa was observed at potentials beyond $1.44 \mathrm{~V} v s$. SCE, which is in accordance with the previous findings for overoxidation at higher applied constant currents or longer reaction times (cf. Table 12). 


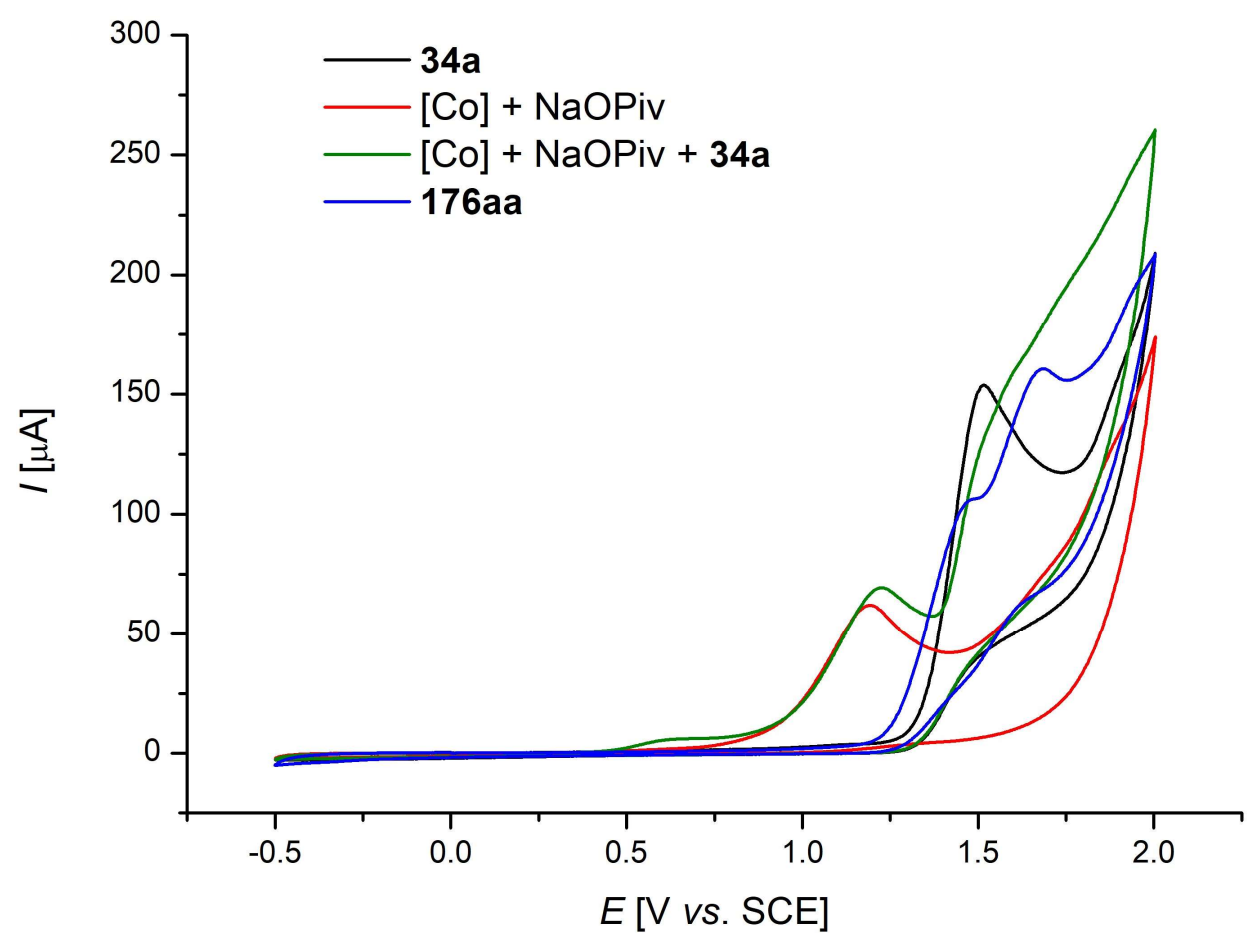

Figure 10. Cyclic voltammograms at $25^{\circ} \mathrm{C}$ and $100 \mathrm{mV} \cdot \mathrm{s}^{-1}$ using $\mathrm{MeOH}$ and $n-\mathrm{Bu}_{4} \mathrm{NPF}_{6}(0.1 \mathrm{M})$ as the electrolyte and a $\mathrm{GC}$ working electrode; concentration of substrates $3.0 \mathrm{mM}$. (black) 34a; (red) $\mathrm{Co}(\mathrm{OAc})_{2} \cdot 4 \mathrm{H}_{2} \mathrm{O}$ and $\mathrm{NaOPiv}$; (green) $\mathrm{Co}(\mathrm{OAc})_{2} \cdot 4 \mathrm{H}_{2} \mathrm{O}, \mathrm{NaOPiv}$ and 34a; (blue) $176 \mathbf{a a}$.

\subsubsection{Proposed Catalytic Cycle}

Based on the mechanistic findings, a catalytic cycle was proposed for the cobaltaelectro-catalyzed $\mathrm{C}-\mathrm{H} / \mathrm{N}-\mathrm{H}$ allene annulation (Scheme 62). The electrocatalysis commences by anodic oxidation of the cobalt(II) salt to the cobalt(III) carboxylate complex 184, followed by irreversible $\mathrm{C}-\mathrm{H}$ activation of substrate 34a in a BIES-type fashion to furnish 185. Next, kinetically relevant migratory insertion of allene $\mathbf{7 5}$ generates cobalt(III) complex 186 and the desired $\mathrm{C}-\mathrm{C}$ bond. Additionally, computational studies performed by Dr. J. C. A. Oliveira showed that the allene $\mathbf{7 5}$ insertion distal to the substituent is favoured by $2.2 \mathrm{kcal} \cdot \mathrm{mol}^{-1}$ over the insertion pathway proximal to the substituent via complex 188 and thus enables the high levels of regioselectivity for the cobalta-electrocatalysis. ${ }^{[260]}$ Thereafter, reductive elimination releases a reduced cobalt(I) species $\mathbf{1 8 8}$ and the exomethylene isoquinolone $\mathbf{1 8 3}$, which in the presence of base, undergoes irreversible isomerization to the desired product 176. Finally, anodic oxidation regenerates the active cobalt(III) complex 184 and cathodic proton reduction closes the electrochemical cycle and hence obviates the necessity for toxic metal-based terminal oxidants. 


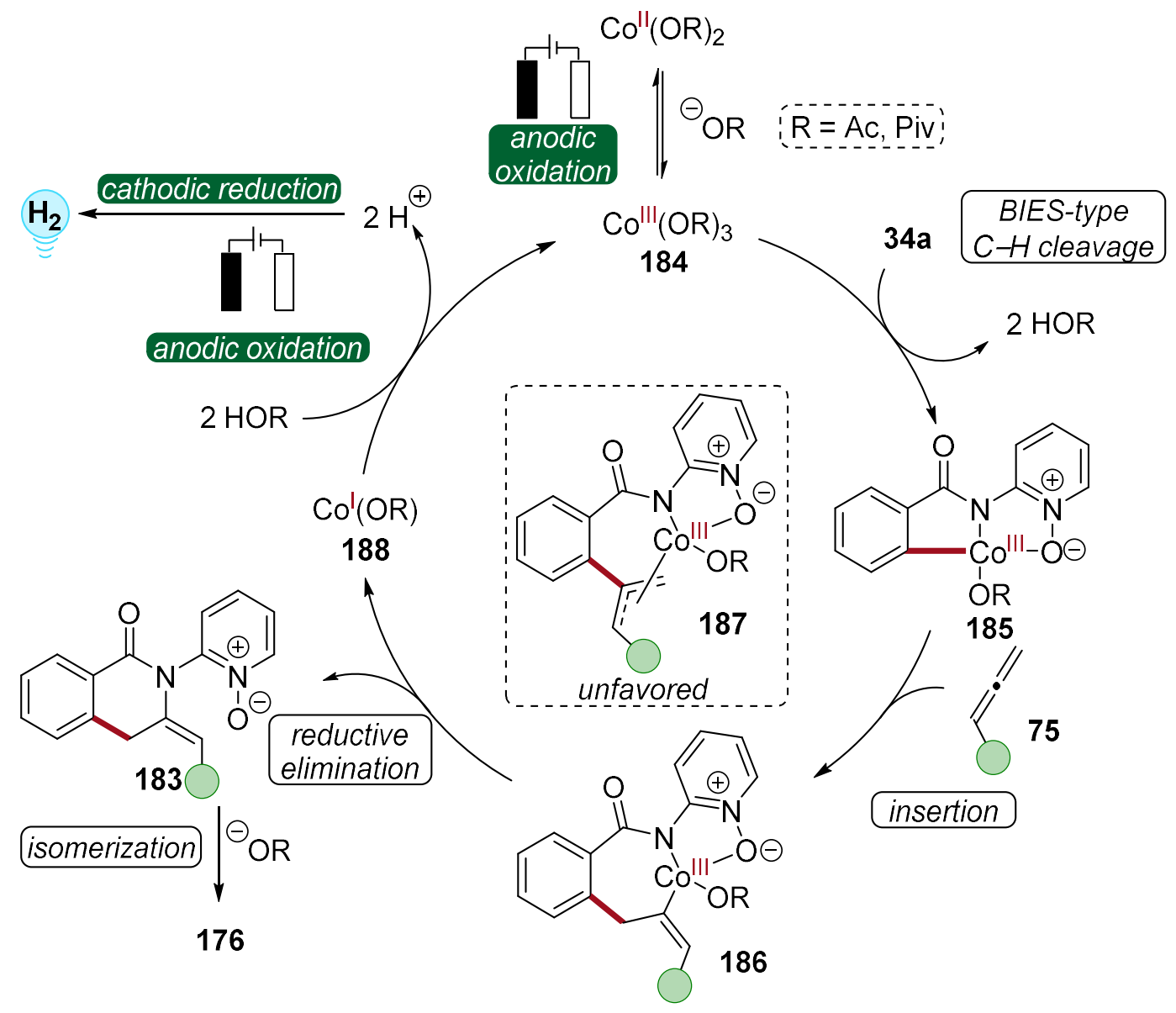

Scheme 62. Proposed catalytic cycle for the cobaltaelectro-catalyzed allene annulation.

\subsubsection{Cobaltaelectro-Catalyzed C-H Allene Annulation in Flow}

The combination of electrosynthesis and continuous-flow chemistry ${ }^{[288]}$ represents a particularly attractive concept in terms of efficient heat and mass transfer, high current efficiency and potential reaction scale-up. ${ }^{[289]}$ In spite of major advances in metal-free electroflow reactions, ${ }^{[290]}$ until the beginning of this study, flow-metallaelectro-catalyzed $\mathrm{C}-\mathrm{H}$ activations ${ }^{[291]}$ were unprecedented. Based on the achievements on cobaltaelectrocatalysis, preliminary studies for the oxidative $\mathrm{C}-\mathrm{H} / \mathrm{N}-\mathrm{H}$ annulation of allenes under flow conditions were conducted, using benzamide 34a and allene 75a as the model substrates (Table 16). The study was commenced with the commercially available flowelectrosynthesis kit from IKA with a GC plate as the anode and a nickel plate cathode. ${ }^{\text {[289i] }}$ Remarkably, product 176aa was isolated in 44\% yield after constant potential electrolysis for $4 \mathrm{~h}$ (entry 1). It should be noted that the electrolysis was performed in a two-electrode setup and non-referenced cell potentials are reported. Also, the efficiency of the flowelectrocatalysis drastically decreased upon lowering the flow rate to $8.2 \mu \mathrm{L} \cdot \mathrm{min}^{-1}$ (entry 2 ). 
When a custom-made graphite-felt anode compartment was employed, the desired isoquinolone 176aa was isolated in $48 \%$ yield (entry 3 ). These promising, yet not optimized preliminary results highlight the potential of cobaltaelectro-catalyzed $\mathrm{C}-\mathrm{H}$ activations in flow. It is noteworthy that the latter flow-setup was significantly advanced and modified by $D r$. L. Finger in the Ackermann group and was recently successfully applied for rhodaelectro-catalyzed $\mathrm{C}-\mathrm{H}$ annulation reactions with alkynes under flow conditions. ${ }^{\text {[292] }}$

Table 16. Attempts for cobaltaelectro-catalyzed $\mathrm{C}-\mathrm{H}$ allene annulation in flow. ${ }^{[\mathrm{a}]}$

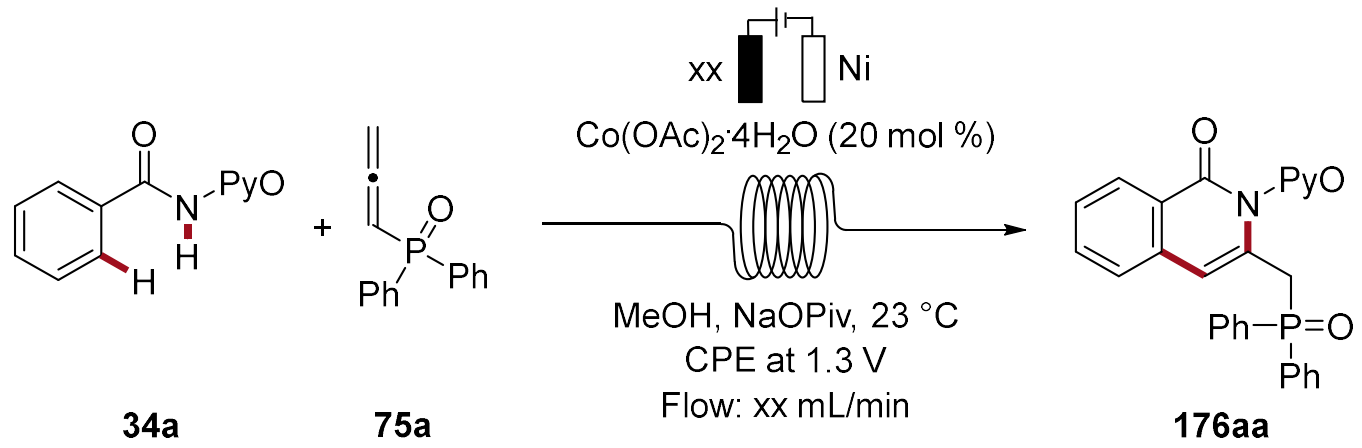

\begin{tabular}{ccccc}
\hline Entry & Anode & Flow-rate $\left[\mu \mathrm{L} \cdot \mathrm{min}^{-1}\right]$ & Time $[\mathrm{h}]$ & Yield $[\%]$ \\
\hline 1 & GC & 42.2 & 4 & 44 \\
2 & GC & 8.2 & 6 & 15 \\
3 & GF & 818 & 2 & 48 \\
\hline
\end{tabular}

[a] Reaction conditions: Undivided flow cell, 34a $(0.25 \mathrm{mmol}), \mathbf{7 5 a}(0.30 \mathrm{mmol}), \mathrm{Co}(\mathrm{OAc})_{2} \cdot 4 \mathrm{H}_{2} \mathrm{O}$ (20 mol \%), NaOPiv (2.00 equiv), $\mathrm{MeOH}(10.0 \mathrm{~mL}), 23{ }^{\circ} \mathrm{C}$, constant cell potential of $1.3 \mathrm{~V}$, time, $\mathrm{xx}$ anode, Ni-plate cathode. Isolated yields are given. 


\subsection{Cobaltaelectro-Catalyzed $\mathrm{C}-\mathrm{H}$ Activation in Biomass-Derived Glycerol Powered by Renewable Energy Sources}

Molecular electrosynthesis is often classified as a green methodology for organic transformations, since toxic and dangerous chemical energy carriers can be replaced by traceless electric charge. ${ }^{[218 c]}$ The sustainability of the approach can be further substantiated by the direct interconversion of renewable energies into value-added products. ${ }^{[293]}$ Here, electrosynthesis has particularly proven useful for the valorisation of biomass-derived solvents ${ }^{[45 \mathrm{~d}, 294]}$ or waste-products such as glycerol. ${ }^{[295]}$ At the same time, electrochemical cobalt-catalyzed $\mathrm{C}-\mathrm{H}$ activation has been established as a versatile and resource economical tool for molecular transformations (vide supra). ${ }^{[240 \mathrm{e}]}$ In this context, previous studies on cobaltaelectro-catalyzed C-H annulation reactions with alkynes $\mathbf{5 3}^{[250,259,285,296]}$ or allenes $\mathbf{7 5}^{[260]}$ as the coupling partner, largely employed short-chained alcohols as the solvent such as methanol, ethanol or halogenated 2,2,2-trifluoroethanol (TFE). However, these solvents, especially the latter, ${ }^{[112]}$ can be classified as noxious and competing undesired cobalt-catalyzed alkoxylation reactions were commonly observed in small amounts (vide infra). ${ }^{[249]}$ To obviate these drawbacks, we turned our attention toward the identification of less hazardous and environmentally-benign solvents ${ }^{[45 b, 45 c, 262,297]}$ for the resource economical ${ }^{[9,13 a, 43]}$ cobaltaelectro-catalyzed $\mathrm{C}-\mathrm{H}$ activation of benzamides 34 . Here, the use of glycerol as the solvent would be highly desirable, since it is non-flammable and is produced on large scale as the byproduct of bio-diesel production. ${ }^{\text {[295a-c, 295e] }}$ Furthermore the high dielectric constant of glycerol $\left(\varepsilon=42.5\right.$ at $\left.25^{\circ} \mathrm{C}\right),{ }^{[298]}$ would render it as an ideal electrolyte-free ${ }^{[299]}$ solvent for organic electrochemistry. However, until the beginning of this study its use as a reaction medium for sustainable $\mathrm{C}-\mathrm{H}$ activation reactions has been elusive. ${ }^{[300]}$

\subsubsection{Optimization Studies for Cobaltaelectro-Catalyzed C-H Activation in Biomass-Derived Solvents}

The optimization studies were commenced by the testing of different organic solvent mixtures for the desired cobaltaelectro-catalyzed $\mathrm{C}-\mathrm{H} / \mathrm{N}-\mathrm{H}$ annulation of benzamide 34a with alkyne 53a (Table 17). Initial results with biomass-derived solvents ${ }^{[45 \mathrm{~b}, 45 \mathrm{c}]}$ or aqueous mixtures ${ }^{[301]}$ thereof showed promising results for the green synthesis of isoquinolone $\mathbf{5 1 a a}$ (entries 1-9). Most notably, aqueous glycerol mixtures outperformed other, more commonly employed biomass-derived solvents, such as $\gamma$-valerolactone (GVL) (entries 4 
and 7). Due to the high viscosity of glycerol, the addition of water and slightly elevated temperatures of $40{ }^{\circ} \mathrm{C}$ were essential to obtain isoquinolone 51aa in high yields (entries 69).

Table 17. Optimization of the solvents and the reaction temperature. ${ }^{[\mathrm{a}]}$<smiles>O=C(Nc1cccc[n+]1[O-])c1ccccc1</smiles>

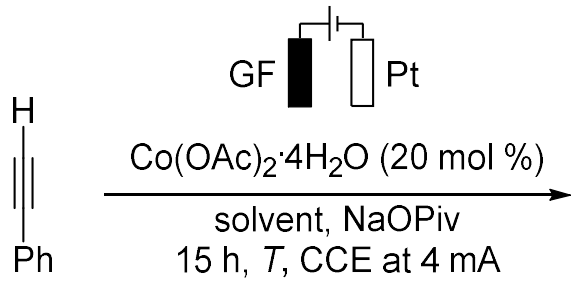<smiles>O=c1c2ccccc2cc(-c2ccccc2)n1P(O)O</smiles>

$34 a$

$53 a$

51 aa

\begin{tabular}{cccc}
\hline Entry & Solvent & $T\left[{ }^{\circ} \mathrm{C}\right]$ & Yield $[\%]$ \\
\hline 1 & $2-\mathrm{MeTHF}$ & 40 & $69^{[\mathrm{b}]}$ \\
2 & $2-\mathrm{MeTHF} / \mathrm{H}_{2} \mathrm{O}$ & 40 & 65 \\
3 & furfuryl alcohol/ $\mathrm{H}_{2} \mathrm{O}(1: 1)$ & 40 & 61 \\
4 & $\mathrm{GVL} / \mathrm{H}_{2} \mathrm{O}(1: 1)$ & 40 & 56 \\
5 & GVL/glycerol $(1: 1)$ & 40 & 9 \\
6 & glycerol & 40 & 24 \\
7 & glycerol/H2O $(1: 1)$ & 40 & $70(71)$ \\
8 & glycerol/ $\mathrm{H}_{2} \mathrm{O}(1: 1)$ & 23 & 47 \\
9 & glycerol/ $\mathrm{H}_{2} \mathrm{O}(1: 1)$ & 60 & $72(70)$ \\
10 & $\mathrm{MeOH}$ & 40 & $82(78)^{[\mathrm{c}]}$ \\
11 & $\mathrm{TFE}$ & 40 & $87^{[\mathrm{d}]}$ \\
12 & $\mathrm{THF}$ & 40 & $18^{[\mathrm{b}]}$ \\
13 & $\mathrm{DCE}$ & 40 & $43^{[\mathrm{b}]}$ \\
14 & $\mathrm{CH}{ }_{2} \mathrm{Cl} 2$ & 40 & $53^{[\mathrm{b}]}$ \\
15 & $\mathrm{DMF}$ & 40 & $21^{[\mathrm{b}]}$ \\
16 & $\mathrm{DMSO}$ & 40 & $29^{[\mathrm{b}]}$ \\
17 & $\mathrm{MeCN}$ & 40 & $51(51)^{[\mathrm{b}]}$ \\
18 & $\mathrm{HFIP}$ & 21 \\
\hline
\end{tabular}

[a] Reaction conditions: Undivided cell, 34a $(0.50 \mathrm{mmol}), \mathbf{5 3 a}(1.00 \mathrm{mmol}), \mathrm{Co}(\mathrm{OAc})_{2} \cdot 4 \mathrm{H}_{2} \mathrm{O}(20 \mathrm{~mol} \%)$, NaOPiv (2.00 equiv), solvent $(5.0 \mathrm{~mL}), \mathrm{CCE}=4 \mathrm{~mA}, 15 \mathrm{~h}$, graphite felt anode, Pt-plate cathode. Yield of 51 aa determined by ${ }^{1} \mathrm{H}-\mathrm{NMR}$ analysis with 1,3,5-trimethoxybenzene as an internal standard. Isolated yield in parentheses. [b] Addition of $\mathrm{LiClO}_{4}$ (1.00 equiv). [c] Oxygenated product 36ab (11 mg, $\left.45 \mu \mathrm{mol}, 9 \%\right)$ was formed as a byproduct. [d] Oxygenated product 36ad was detected in 5\%, based on ${ }^{1} \mathrm{H}-\mathrm{NMR}$ analysis. 
In contrast, conventional toxic organic solvents were less efficient in terms of product $\mathbf{5 1}$ yield (entries 10-18) and selectivity (entries 10 and 12). In comparison to other commonly employed biomass-derived solvents such as GVL, it is noteworthy that water-soluble glycerol was easily removed after the reaction. Furthermore, different cobalt sources and additives were tested for the cobalta-electrocatalysis in aqueous glycerol (Table 18).

Table 18. Catalyst optimization and additive effects. ${ }^{[\mathrm{a}]}$<smiles>O=C(Nc1cccc[n+]1[O-])c1ccccc1</smiles>

$34 a$

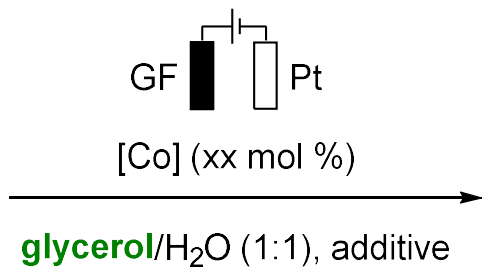

$15 \mathrm{~h}, 40^{\circ} \mathrm{C}, \mathrm{CCE}$ at $4 \mathrm{~mA}$<smiles>O=c1c2ccccc2cc(-c2ccccc2)n1[Po]</smiles>

51 aa

\begin{tabular}{ccccc}
\hline Entry & Additive (equiv) & {$[\mathrm{Co}]$} & $\mathrm{xx}[\mathrm{mol} \%]$ & Yield [\%] \\
\hline 1 & $\mathrm{NaOPiv}(2.00)$ & $\mathrm{Co}(\mathrm{OAc})_{2} \cdot 4 \mathrm{H}_{2} \mathrm{O}$ & 20 & $70(71)$ \\
2 & $\mathrm{Na}_{2} \mathrm{CO}_{3}(2.00)$ & $\mathrm{Co}(\mathrm{OAc})_{2} \cdot 4 \mathrm{H}_{2} \mathrm{O}$ & 10 & 29 \\
3 & $\mathrm{NaOAc}(2.00)$ & $\mathrm{Co}(\mathrm{OAc})_{2} \cdot 4 \mathrm{H}_{2} \mathrm{O}$ & 10 & 31 \\
4 & $\mathrm{KOAc}(2.00)$ & $\mathrm{Co}(\mathrm{OAc})_{2} \cdot 4 \mathrm{H}_{2} \mathrm{O}$ & 10 & 33 \\
5 & $\mathrm{NaOH}(2.00)$ & $\mathrm{Co}(\mathrm{OAc})_{2} \cdot 4 \mathrm{H}_{2} \mathrm{O}$ & 10 & --- \\
6 & $\mathrm{HOPiv}(2.00)$ & $\mathrm{Co}(\mathrm{OAc})_{2} \cdot 4 \mathrm{H}_{2} \mathrm{O}$ & 10 & 14 \\
7 & $\mathrm{NaOPiv}(2.00)$ & $\mathrm{Co}(\mathrm{OAc})_{2} \cdot 4 \mathrm{H}_{2} \mathrm{O}$ & $\mathbf{1 0}$ & $\mathbf{9 6}(\mathbf{9 2})$ \\
8 & $\mathrm{NaOPiv}(1.00)$ & $\mathrm{Co}(\mathrm{OAc})_{2} \cdot 4 \mathrm{H}_{2} \mathrm{O}$ & 10 & 93 \\
9 & $\mathrm{NaOPiv}(2.00)$ & $\mathrm{Co}(\mathrm{OAc})_{2} \cdot 4 \mathrm{H}_{2} \mathrm{O}$ & 5 & $90(85)$ \\
10 & $\mathrm{NaOPiv}(1.00)$ & $\mathrm{Co}(\mathrm{OAc})_{2} \cdot 4 \mathrm{H}_{2} \mathrm{O}$ & 5 & 73 \\
11 & $\mathrm{NaOPiv}(0.50)$ & $\mathrm{Co}(\mathrm{OAc})_{2} \cdot 4 \mathrm{H}_{2} \mathrm{O}$ & 5 & 67 \\
12 & $\mathrm{NaOPiv}(2.00)$ & $\mathrm{Co}(\mathrm{OAc})_{3}$ & 10 & $92(91)$ \\
13 & $\mathrm{NaOPiv}(2.00)$ & $\mathrm{Co}(\mathrm{OAc})_{3}$ & 5 & 82 \\
14 & $\mathrm{NaOPiv}(2.00)$ & $\mathrm{Co}(\mathrm{OAc})_{2} \cdot 4 \mathrm{H}_{2} \mathrm{O}$ & 10 & $(71)^{[\mathrm{c}]}$ \\
15 & $\mathrm{NaOPiv}(2.00)$ & $\mathrm{Co}(\mathrm{OAc})_{2} \cdot 4 \mathrm{H}_{2} \mathrm{O}$ & 10 & traces \\
16 & $\mathrm{NaOPiv}(2.00)$ & --- & --- & -- \\
17 & ---- & $\mathrm{Co}(\mathrm{OAc})_{2} \cdot 4 \mathrm{H}_{2} \mathrm{O}$ & 20 & 19 \\
\hline
\end{tabular}

[a] Reaction conditions: Undivided cell, 34a $(0.50 \mathrm{mmol})$, 53a $(1.00 \mathrm{mmol}),[\mathrm{Co}](\mathrm{xx} \mathrm{mol} \%)$, additive (xx equiv), glycerol/ $\mathrm{H}_{2} \mathrm{O}(1: 1,5.0 \mathrm{~mL}), 40^{\circ} \mathrm{C}, 4 \mathrm{~mA}, 15 \mathrm{~h}$, graphite felt anode, Pt-plate cathode. Yield of 51 aa determined by ${ }^{1} \mathrm{H}$-NMR spectroscopy with 1,3,5-trimethoxybenzene as an internal standard. Isolated yield in parentheses. [b] No current. [c] $8 \mathrm{~mA}, 6 \mathrm{~h}$. 
Among a series of different bases, sodium pivalate proved to be the additive of choice (entries 1-5), while pivalic acid delivered the desired product 51aa in only unsatisfactory amounts of yield (entry 6). Optimization of the catalyst and additive loading revealed increased reactivity at lower concentrations of the cobalt(II) catalyst (entries 7 and 8). Remarkably, in contrast to the previously reported cobaltaelectro-catalyzed $\mathrm{C}-\mathrm{H}$ alkyne annulation reactions, ${ }^{[250,259,285,296]}$ the electrocatalytic reaction proceeded with high turnover at catalyst loadings of $5 \mathrm{~mol} \%$ (entry 9). Here, decreased equivalents of $\mathrm{NaOPiv}$ resulted in diminished product yields (entries 10 and 11). Interestingly, the use of cobalt(III) acetate yielded isoquinolone 51aa in similar yields (entries 12 and 13). Shorter reaction times of $6 \mathrm{~h}$, along with an increased constant current of $8 \mathrm{~mA}$, resulted in a decreased isolated yield of product 51 aa (entry 14). Finally, control experiments verified the essential role of the electricity, the cobalt catalyst, and the base for the desired transformation (entries 15-17).

\subsubsection{Substrate Scope}

With the optimized reaction conditions in hand, the versatility and robustness of the cobaltaelectro-catalyzed $\mathrm{C}-\mathrm{H} / \mathrm{N}-\mathrm{H}$ functionalization of benzamides 34 was investigated. Thus, the resource-economical and water-tolerant cobalta-electrocatalysis efficiently converted differently substituted benzamides $\mathbf{3 4}$ to the corresponding isoquinolones $\mathbf{5 1}$ with overall high efficacy (Table 19). Among others, a wealth of functional groups, such as benzylic alkyl, ether, thioether, and halogen groups, were fully tolerated (entries 1-9).

Table 19. Versatility of the cobaltaelectro-catalyzed C-H activation in glycerol. ${ }^{[\mathrm{a}]}$

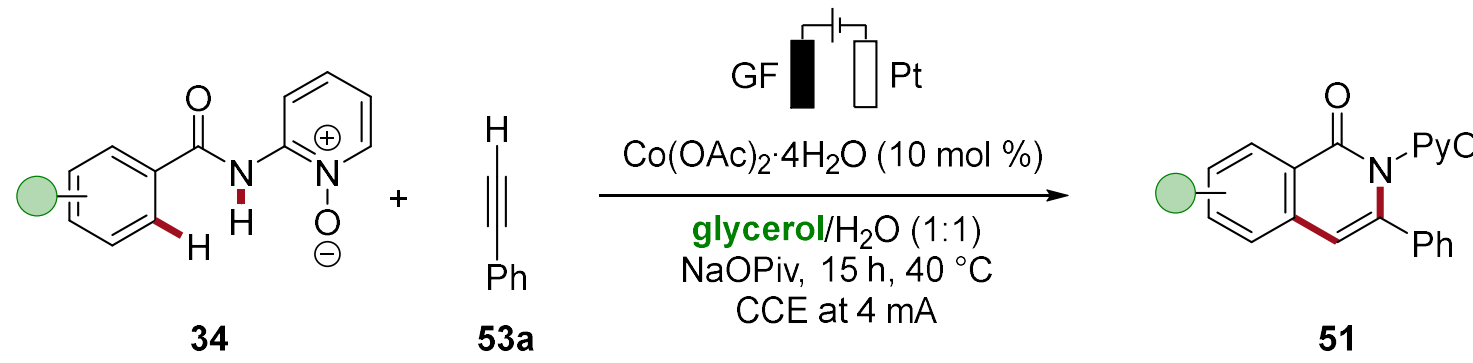

\begin{tabular}{llllll}
\hline Entry & Benzamide & $\mathbf{3 4}$ & Product & $\mathbf{5 1}$ & $\begin{array}{c}\text { Yield } \\
{[\%]}\end{array}$ \\
\hline 1 & $\mathbf{3 4 a}$ & $\mathbf{5 1 a a}$ & 92
\end{tabular}


2<smiles>Cc1ccc(C(=O)N[Pb])cc1</smiles>

3<smiles>COc1ccc(C(=O)NP=O)cc1</smiles><smiles>CSc1ccc(C(=O)NP=O)cc1</smiles>

5<smiles>O=C(N[Pb])c1ccc(I)cc1</smiles>

6<smiles>CC(C)(C)c1ccc(C(=O)NP=O)cc1</smiles>

7<smiles>O=C(NP)c1ccc(F)cc1</smiles>

8<smiles>Cc1cc(C)cc(C(=O)NPO)c1</smiles>

9<smiles>COc1cc(OC)cc(C(=O)NP(=O)(O)O)c1</smiles>

10 34b<smiles>Cc1ccc2c(=O)n(P(=O)(O)c3ccccc3)c(-c3ccccc3)cc2c1</smiles>

51ba $73^{[\mathrm{b}]}$

34d<smiles>COc1ccc2c(=O)n(P(=O)(O)c3ccccc3)c(-c3ccccc3)cc2c1</smiles>

51da 69

$34 \mathrm{e}$<smiles>CSc1ccc2c(=O)n(P(=O)(O)c3ccccc3)c(-c3ccccc3)cc2c1</smiles>

51ea 61

51ga 41 51ja 69

$34 \mathbf{j}$<smiles></smiles>

$34 k$<smiles></smiles>

$51 \mathrm{ka}$ 66

$34 u$<smiles>Cc1cc(C)c2cc(-c3ccccc3)n(PO)c(=O)c2c1</smiles>

51ua $63^{[\mathrm{c}]}$

34v<smiles>COc1cc(OC)c2cc(-c3ccccc3)n(P=O)c(=O)c2c1</smiles>

$51 v a$ 78

[a] Reaction conditions: Undivided cell, benzamide $34(0.50 \mathrm{mmol})$, alkyne 53a $(1.00 \mathrm{mmol})$, $\mathrm{Co}(\mathrm{OAc})_{2} \cdot 4 \mathrm{H}_{2} \mathrm{O}(10 \mathrm{~mol} \%)$, NaOPiv (2.00 equiv), glycerol $/ \mathrm{H}_{2} \mathrm{O}(1: 1,5.0 \mathrm{~mL}), 40^{\circ} \mathrm{C}, 4.0 \mathrm{~mA}, 15 \mathrm{~h}$, graphite felt anode, Pt-plate cathode. Isolated yields are given. [b] Under sonification, $\mathrm{Co}(\mathrm{OAc})_{3}(10 \mathrm{~mol} \%)$. Under standard conditions only $28 \%$ were isolated. [c] $\mathrm{Co}(\mathrm{OAc})_{3}(10 \mathrm{~mol} \%)$.

The positional selectivity of the reaction was mostly controlled by repulsive steric interactions, as was exemplified for the conversion of meta-methylated benzamide $\mathbf{3 4 \mathbf { w }}$ (entry 10). In addition, due to poor solubility of the alkyl-substituted benzamide $\mathbf{3 4 \mathbf { b }}$, a novel approach for sonoelectrochemical $\mathrm{C}-\mathrm{H}$ activation was developed (entry 2). In 
contrast to conventional electrochemical methods, sonoelectrochemistry is far less explored for organic synthesis. ${ }^{[295 \mathrm{c}, 302]}$ Thus, the ultrasound sonification resulted in a fine suspension of the substrate $\mathbf{3 4 b}$ and prooved to be beneficial for high catalytic efficiency of the cobalt catalyst in the glycerol $/ \mathrm{H}_{2} \mathrm{O}$ mixture, since only $28 \%$ of the product $\mathbf{5 1 b a}$ were isolated under the standard conditions.

Gratifyingly, the scope of the cobaltaelectro-catalyzed $\mathrm{C}-\mathrm{H}$ activation was not limited to benzamides 34. Indeed, the use of glycerol/ $\mathrm{H}_{2} \mathrm{O}$ as a solvent mixture proved also viable for the step-economical synthesis of 2-pyridones 189aa and 189ba via alkenylic $\mathrm{C}-\mathrm{H}$ activation (Table 20, entries 1 and 2). The robustness of the developed method even allowed for the conversion of furan 37e to deliver the fused heteroarene 189ea in 52\% yield, featuring high levels of regioselectivity (entry 3 ).

Table 20. Substrate scope of substituted alkenes and heteroarenes. ${ }^{[\mathrm{a}]}$<smiles>O=CC(O)C(=O)Nc1cccc[n+]1[O-]</smiles>

37

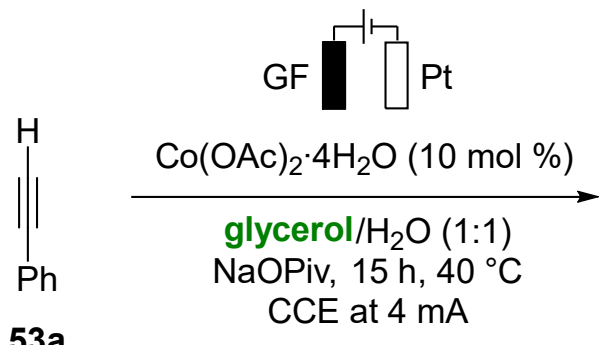

$53 a$

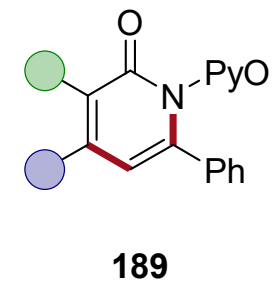

189

\begin{tabular}{|c|c|c|c|c|c|}
\hline Entry & Substrate & 37 & Product & 189 & $\begin{array}{c}\text { Yield } \\
{[\%]}\end{array}$ \\
\hline 1 & & $37 \mathbf{a}$ & & 189aa & 63 \\
\hline 2 & & $37 b$ & & $189 \mathrm{ba}$ & 55 \\
\hline 3 & & $37 e$ & & 189ea & $\begin{array}{c}52 \\
(10: 1)\end{array}$ \\
\hline
\end{tabular}

[a] Reaction conditions: Undivided cell, $37(0.50 \mathrm{mmol}), \mathbf{5 3 a}(1.00 \mathrm{mmol}), \mathrm{Co}(\mathrm{OAc})_{2} \cdot 4 \mathrm{H}_{2} \mathrm{O}(10 \mathrm{~mol} \%)$, NaOPiv (2.00 equiv), glycerol $/ \mathrm{H}_{2} \mathrm{O}(1: 1,5.0 \mathrm{~mL}), 40^{\circ} \mathrm{C}, 4.0 \mathrm{~mA}, 15 \mathrm{~h}$, graphite felt anode, Pt-plate cathode. Isolated yields are given.

The regioselectivity of the novel products 51ea and 189ea was unambiguously validated by X-ray crystal structure analysis (Figure 11). 

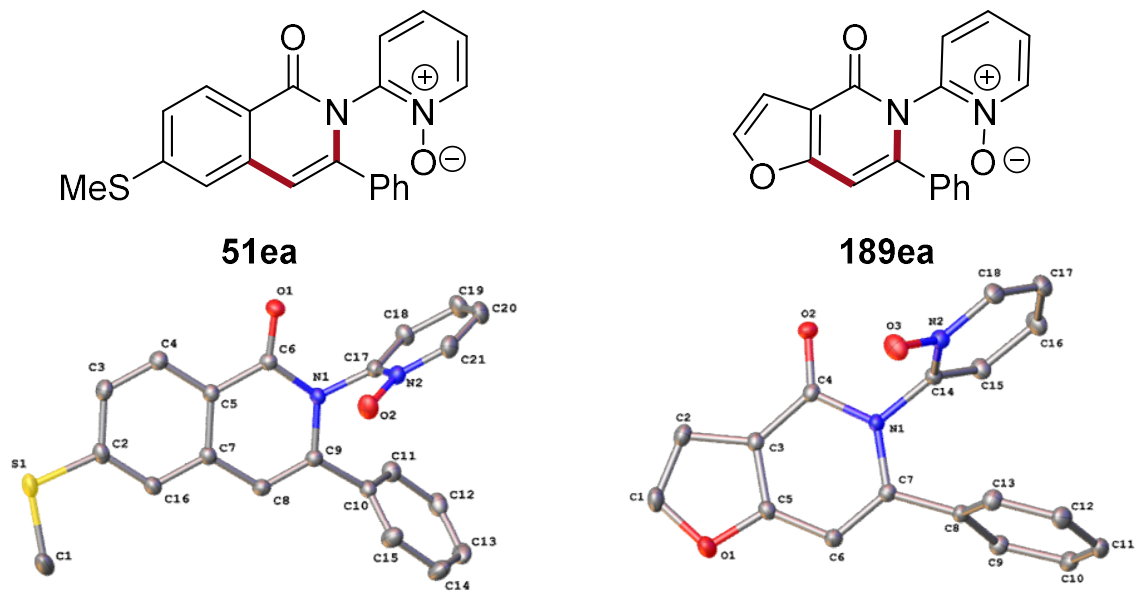

Figure 11. Molecular structures of 51ea and 189ea with thermal ellipsoids at $50 \%$ probability level. The hydrogen atoms are omitted for clarity.

Thereafter, a variety of alkynes $\mathbf{5 3}$ was tested toward the cobaltaelectro-catalyzed $\mathrm{C}-\mathrm{H} / \mathrm{N}-\mathrm{H}$ activation in biomass-derived glycerol (Table 21). Hence, the robustness of the electrocatalytic $\mathrm{C}-\mathrm{H}$ activation strategy was reflected by the annulation of diverse alkynes 53, fully tolerating valuable functional groups, including sensitive alkyl chloride (entry 4) and alkyl nitrile substituents (entry 5) or the strained cyclopropyl group (entry 6).

Table 21. Cobaltaelectro-catalyzed C-H annulation of alkynes $\mathbf{5 3}$ in glycerol. ${ }^{[\mathrm{a}]}$

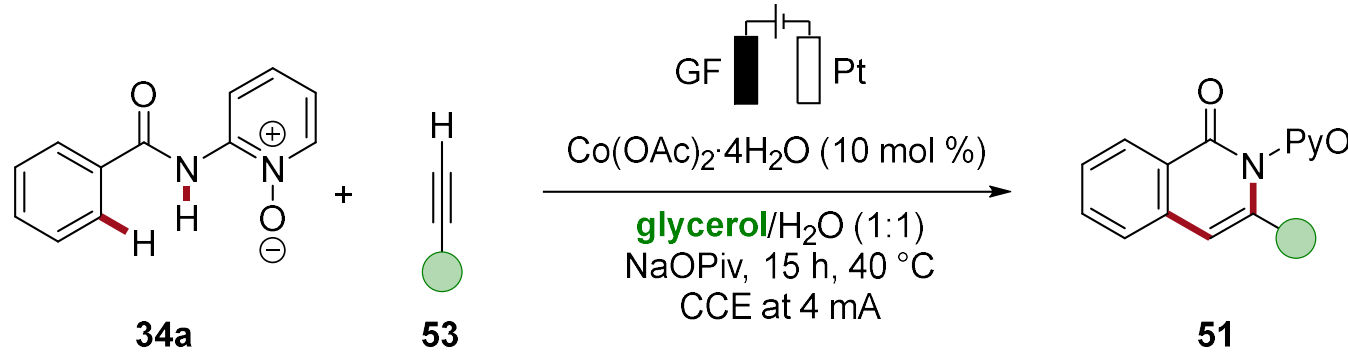

\begin{tabular}{|c|c|c|c|c|c|}
\hline Entry & Alkyne & 53 & Product & 51 & $\begin{array}{c}\text { Yield } \\
{[\%]}\end{array}$ \\
\hline 1 & $\mathrm{H}=$ & $53 a$ & & $51 a a$ & 92 \\
\hline 2 & $\mathrm{H} \equiv\langle$ & $53 b$ & & $51 \mathrm{ab}$ & 51 \\
\hline
\end{tabular}


3

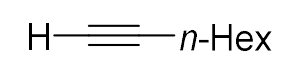

53c<smiles>CCCCCCc1cc2ccccc2c(=O)n1P(O)O</smiles>

51 ac 63

4

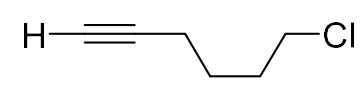

53d<smiles></smiles>

51 ad 65

5

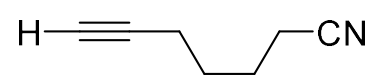

$53 e$<smiles>N#CCCCCc1cc2ccccc2c(=O)n1P(O)O</smiles>

51 ae 79

53f

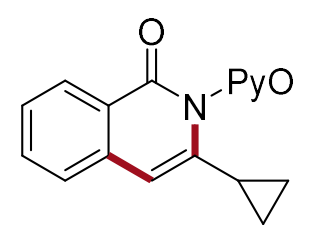

[a] Reaction conditions: Undivided cell, 34a $(0.50 \mathrm{mmol}), 53(1.00 \mathrm{mmol}), \mathrm{Co}(\mathrm{OAc})_{2} \cdot 4 \mathrm{H}_{2} \mathrm{O}(10 \mathrm{~mol} \%)$, NaOPiv (2.00 equiv), glycerol $/ \mathrm{H}_{2} \mathrm{O}(1: 1,5.0 \mathrm{~mL}), 40{ }^{\circ} \mathrm{C}, 4.0 \mathrm{~mA}, 15 \mathrm{~h}$, graphite felt anode, Pt-plate cathode. Isolated yields are given.

Notably, the intermolecular cobaltaelectro-catalyzed $\mathrm{C}-\mathrm{H} / \mathrm{N}-\mathrm{H}$ annulation proved further viable for challenging allene 75a (Scheme 63). Key to success was a slightly lower constant current of $2 \mathrm{~mA}$ for $15 \mathrm{~h}$ in the biomass-derived glycerol solvent system.<smiles>O=C(N[Pb](=O)[CH+]C=C=CP(=O)(c1ccccc1)c1ccccc1)c1ccccc1</smiles>

$34 a$

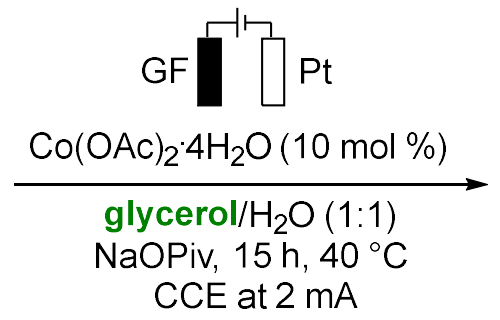

CCE at $2 \mathrm{~mA}$<smiles>O=c1c2ccccc2cc(CP(=O)(c2ccccc2)c2ccccc2)n1[O-]</smiles>

176aa: $63 \%$

Scheme 63. Cobaltaelectro-catalyzed C-H annulation with allene 75a in glycerol/ $\mathrm{H}_{2} \mathrm{O}$.

\subsubsection{Cobaltaelectro-Catalyzed C-H Activation Powered by Renewable Energies}

Finally, direct valorisation of renewable solar and wind energy was envisioned for the desired oxidative $\mathrm{C}-\mathrm{H}$ transformation. In general, within constant current electrolysis the potential at the working electrode will adjust until the substrate with the lowest oxidation potential is fully consumed. ${ }^{[170 \mathrm{~g}]}$ Evaluation of the redox properties of the reaction components by cyclic voltammetry revealed that the cobalt(II) salt has the lowest oxidation potential compared to solvent or reactants/product, thus preventing undesirable degradation or decomposition pathways ( $c f$. Figure 50 and 51). Hence, the working potential of the 
electrolysis would remain relatively constant during the course of the reaction. With this in mind, a proof-of-concept study was envisioned for the direct use of inexpensive and commercially available photovoltaic cells as the power supply for the depicted $\mathrm{C}-\mathrm{H}$ activation (Scheme 64). ${ }^{[300]}$ The applied current was fixed and regulated by the aid of a custom-made constant current regulator. With a commercially-available amorphous silicon photovoltaic cell in hand, the cobaltaelectro-catalyzed $\mathrm{C}-\mathrm{H} / \mathrm{N}-\mathrm{H}$ annulation - powered by sunlight - delivered isoquinolone 51aa in comparable yields to the conventional direct current galvanostat ( $c f$. Table 18, entry 14). The reaction was performed on the rooftop of the Institute of Organic and Biomolecular Chemistry in Göttingen, once on a sunny day during October (Scheme 64, left) and with similar reaction yields on the $20^{\text {th }}$ of December 2018, with less solar irradiance (Scheme 64, right).
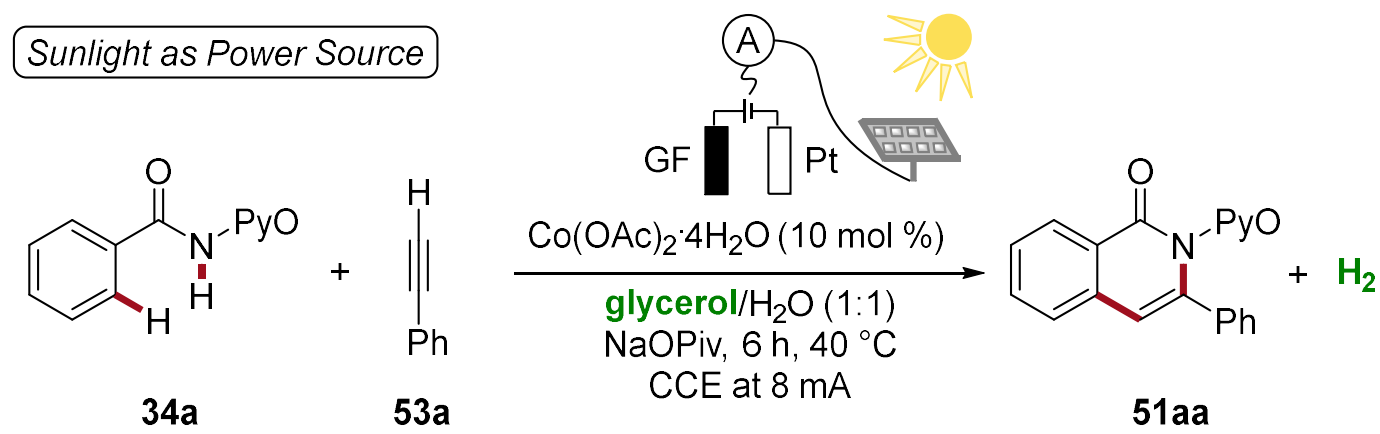

conventional galvanostat: $71 \%$ photovoltaic cell: $\mathbf{7 3} \%$

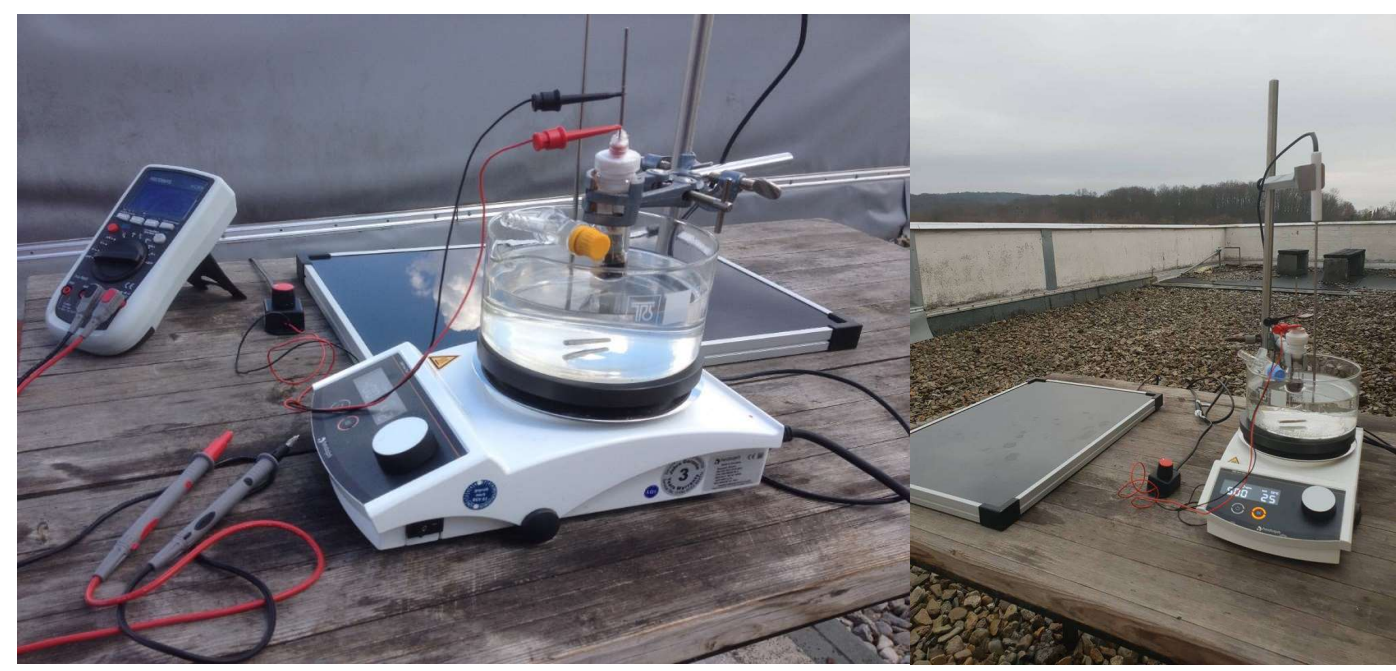

Scheme 64. Cobaltaelectro-catalyzed $\mathrm{C}-\mathrm{H}$ activation powered by sunlight.

Likewise, a similar reaction setup was used to perform the first organic electrocatalytic transformation powered by green wind energy (Scheme 65). Here, a commercially 
available wind turbine was connected via USB-cable with a custom-made constant current regulator. Under laboratory conditions, the wind turbine was propelled by a fan to enable a steady wind velocity. The electrolysis setup was connected and the wind-powered cobaltaelectro-catalyzed $\mathrm{C}-\mathrm{H} / \mathrm{N}-\mathrm{H}$ functionalization afforded isoquinolone 51aa in comparable yields with the conventional reaction setup ( $c f$. Table 18, entry 7 ). The slightly diminished performance can be rationalized by small current variations during the course of the reaction.
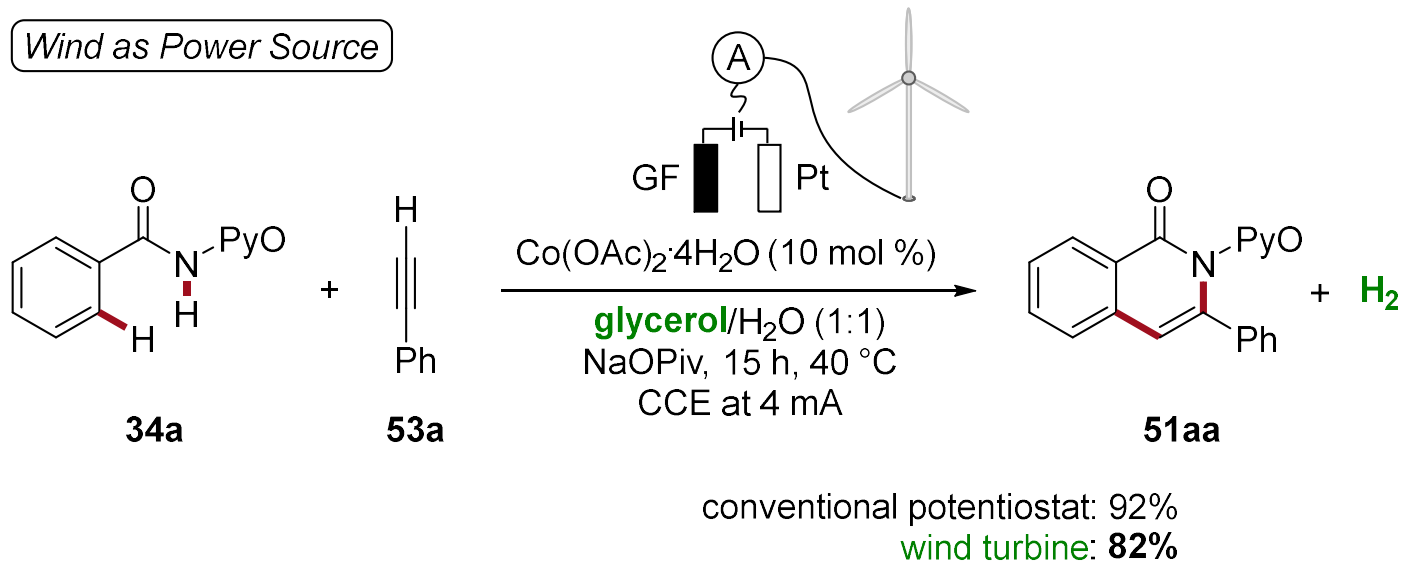

Scheme 65. Cobaltaelectro-catalyzed $\mathrm{C}-\mathrm{H}$ activation powered by wind energy.

In summary, the presented proof-of-concept study highlights the potential of metallaelectro-catalyzed $\mathrm{C}-\mathrm{H}$ activations toward fully resource economical and green organic synthesis. ${ }^{[9]}$ Herein, the unprecedented utilization of biomass-derived glycerol was disclosed as a suitable reaction medium for Earth-abundant cobaltaelectro-catalyzed $\mathrm{C}-\mathrm{H}$ activations. Additionally, renewable energy sources such as wind and solar energy were directly utilized for cobaltaelectro-catalyzed $\mathrm{C}-\mathrm{C} / \mathrm{N}-\mathrm{C}$ formations, with hydrogen gas as the sole byproduct. 


\subsection{Mechanistic Insights into Cobalta(III/IV/II)-Electrocatalysis}

In recent years, a plethora of oxidative cobalt-catalyzed $\mathrm{C}-\mathrm{H}$ transformations have successfully been developed - employing chemical redox reagents or electrochemical methods as terminal oxidant alike. Despite indisputable advances, the mechanistic understanding of their key-elementary steps continues to be underdeveloped and catalytic scenarios were thus far largely suggested to occur via a cobalt(II/III/I) manifold (vide supra). In contrast to Earth-abundant cobalt catalysis, $\left.{ }^{[55 a}, 60,154 \mathrm{~b}, 156 \mathrm{~h}, 303\right]$ in depth mechanistic studies have been conducted with $4 \mathrm{~d}$ and $5 \mathrm{~d}$ precious metals, such as palladium, ${ }^{[148 a, 304]}$ rhodium $^{[11 \mathrm{v}, 80-81]}$ and iridium. ${ }^{[305]}$ Here, particularly oxidation-induced reductive elimination has been identified as an important mechanistic feature to facilitate the often rate-determining reductive elimination within the $\mathrm{C}-\mathrm{H}$ activation catalytic cycle, as was described by Chang, ${ }^{[306]}$ Jones $^{[811]}$ and Tilset ${ }^{[307]}$ among others. ${ }^{[308]}$ Along the same lines, Ackermann ${ }^{[292]}$ and subsequently $X u^{[243 a]}$ have recently suggested that similar mechanistic scenarios are of equal importance for rhodaelectro-catalyzed $\mathrm{C}-\mathrm{H}$ activations and the formation of high-valent rhodium(IV) complexes was proposed to take place upon anodic oxidation. However, until the beginning of this study, limited mechanistic information on metallaelectro-catalyzed $\mathrm{C}-\mathrm{H}$ activation was available, ${ }^{[232]}$ especially for cobalta-electrocatalysis. Based on previously proposed mechanistic scenarios on cobaltaelectro-catalyzed $\mathrm{C}-\mathrm{H}$ activations, $[240 \mathrm{e}, 240 \mathrm{f}, 249-252,260]$ the isolation and full characterization of an ideally electrochemically generated, cyclometalated cobalt complex would thus be essential to support the suggested organometallic $\mathrm{C}-\mathrm{H}$ scission event, evaluate subsequent bond formations and allow for further mechanistic experimentation. ${ }^{[309]}$

\subsubsection{Isolation and Characterization of Cobalta(III)cycle 190}

The study was initiated by stoichiometric oxidation of amide 34a with the previously employed cobalt(II) acetate ${ }^{[249]}$ for the envisioned synthesis of a cyclometalated cobalt(III) complex 190 (Table 22). Acetonitrile was selected as an inert solvent to prevent follow-up reactions of the complex and to ensure a sufficient conductivity throughout the electrolysis. However, the desired complex 190 could not be detected when chemical oxidants such as silver or manganese salts were used (entries 1 and 2). ${ }^{\left[{ }^{95}, 158\right]}$ Likewise, oxygen failed to deliver the desired cobalt(III) complex 190 (entry 3). In contrast, when direct anodic oxidation under constant current conditions was applied, the desired cobalta(III)cycle 190 
was successfully isolated in $21 \%$ yield, along with various paramagnetic side products (entry 4). However, no cyclometalated complex was detected in the absence of sodium pivalate (entry 5), thus highlighting the importance of a carboxylate base to facilitate the

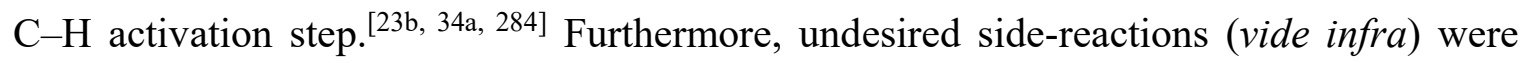
successfully suppressed by switching to more controlled constant potential electrolysis (CPE), at relatively low potentials of $1.3 \mathrm{~V}$ or $1.4 \mathrm{~V} v s . \mathrm{Ag} / \mathrm{Ag}^{+}$(entries 6-8). Here, the electrolysis was stopped after $1.0 \mathrm{~F}$ of electrons were consumed. The potential range for the chronoamperometric synthesis was chosen based on previously performed $\mathrm{CV}$-studies for cobaltaelectro-catalyzed C-H oxygenations ( $c f$. Figure 4). ${ }^{[249-250,258,260]}$

Table 22. Optimization of the reaction conditions. ${ }^{[a]}$<smiles></smiles>

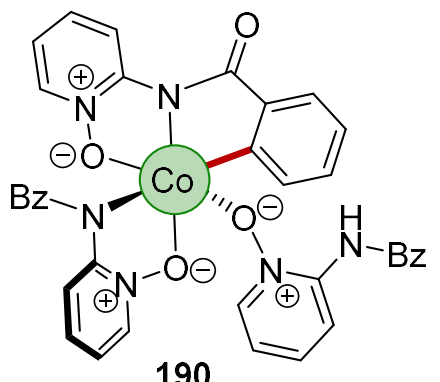

190

\begin{tabular}{cccc}
\hline Entry & {$[\mathrm{Co}]$} & Oxidant & Yield [\%] \\
\hline 1 & $\mathrm{Co}(\mathrm{OAc})_{2} \cdot 4 \mathrm{H}_{2} \mathrm{O}$ & $\mathrm{Ag}_{2} \mathrm{O}$ & --- \\
2 & $\mathrm{Co}(\mathrm{OAc})_{2} \cdot 4 \mathrm{H}_{2} \mathrm{O}$ & $\mathrm{Mn}(\mathrm{OAc})_{3}$ & traces \\
3 & $\mathrm{Co}(\mathrm{OAc})_{2} \cdot 4 \mathrm{H}_{2} \mathrm{O}$ & $\mathrm{O}_{2}($ air, $1 \mathrm{~atm})$ & $--{ }^{[\mathrm{b}]}$ \\
4 & $\mathrm{Co}(\mathrm{OAc})_{2}$ & $\mathrm{CCE}$ at $4 \mathrm{~mA}$ & 21 \\
5 & $\mathrm{Co}(\mathrm{OAc})_{2}$ & $\mathrm{CCE}$ at $4 \mathrm{~mA}$ & traces $^{[\mathrm{c}]}$ \\
6 & $\mathrm{Co}(\mathrm{OAc})_{2}$ & $\mathrm{CPE}$ at $1.3 \mathrm{~V}\left(v s . \mathrm{Ag} / \mathrm{Ag}^{+}\right)$ & $42^{[\mathrm{d}]}$ \\
7 & $\mathrm{Co}(\mathrm{OAc})_{2}$ & $\mathrm{CPE}$ at $1.4 \mathrm{~V}\left(v s . \mathrm{Ag} / \mathrm{Ag}^{+}\right)$ & $46^{[\mathrm{d}]}$ \\
8 & $\mathrm{Co}(\mathrm{OAc})_{2}$ & $\mathrm{CPE}$ at $4.0 \mathrm{~V}\left(v s . \mathrm{Ag} / \mathrm{Ag}^{+}\right)$ & $14^{[\mathrm{d}]}$ \\
9 & $\mathrm{Co}(\mathrm{OAc})_{3}{ }^{[\mathrm{e}]}$ & --- & 14 \\
\hline
\end{tabular}

[a] Reaction conditions: 34a (2.00 equiv), [Co] ( $0.33 \mathrm{mmol}), \mathrm{NaOPiv}$ (2.00 equiv), $\mathrm{MeCN}(5.0 \mathrm{~mL}), 7.5 \mathrm{~h}$, undivided cell, nitrogen atmosphere. Isolated yield is given. When electricity was applied, a graphite felt anode, Pt-plate cathode, and a silver-wire as the reference electrode were used. [b] The reaction was performed under air. [c] The reaction was performed with a constant current of $4 \mathrm{~mA}$ for $2.5 \mathrm{~h}$ in the absence of NaOPiv. [d] Electrolysis was stopped after 1.0 F was applied. Reaction conditions: 34a (1.00 equiv), [Co] $(0.50 \mathrm{mmol}), \mathrm{NaOPiv}(1.00$ equiv), $\mathrm{MeCN}(13 \mathrm{~mL})$. [e] Kochi and co-workers described the cobalt(III) acetate as a bi-metallic complex with the formula: $\mathrm{Co}_{2}(\mathrm{OAc})_{4}(\mathrm{OH})_{2}(\mathrm{HOAc})^{\left[{ }^{[30]}\right.}$

Interestingly, when cobalt(III) acetate was utilized as the cobalt source, only low conversion to the desired complex 190 was observed (entry 9). This can be rationalized by a slow ligand exchange rate at the cobalt(III) metal center or a competing 
disproportionation of the high-valent cobalt salt. ${ }^{[84 e, 98 a, 311]}$ However, the results are supportive of the $\mathrm{C}-\mathrm{H}$ activation occurring in the cobalt(III) oxidation state and oxidation of the cobalt(II) species being necessary prior the $\mathrm{C}-\mathrm{H}$ scission step. Likewise, it can be rationalized that deprotonation and coordination of substrate 34a to the cobalt(II) salt occurs prior to the oxidation step.

Molecular characterization of the cyclometalated cobalt(III) complex 190 was accomplished via NMR-spectroscopic analysis, high-resolution electrospray ionization mass spectrometry (ESI-MS), UV-vis and IR spectrophotometry. ${ }^{[309]}$ The solid state structure was unambiguously confirmed by means of X-ray diffraction analysis. Single crystals were obtained by slow evaporation of a saturated solution of $\mathbf{1 9 0}$ in $\mathrm{MeOH}$ (Figure 12).

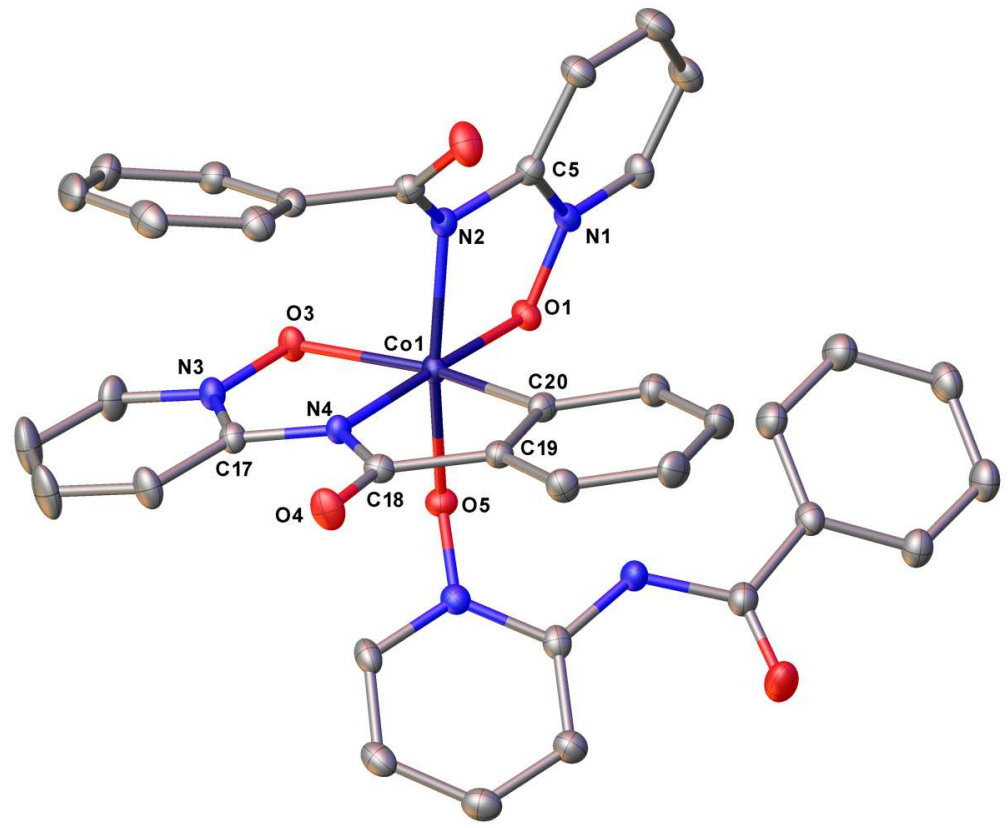

Figure 12. Molecular structure of $\mathbf{1 9 0}$ with thermal ellipsoids at 50\% probability level. The hydrogen atoms are omitted for clarity. Crystallization was performed by $D r . D$. Ghorai.

Analysis of the bond angles revealed a slightly distorted octahedral coordination geometry $\left(\mathrm{O}(1)-\mathrm{Co}(1)-\mathrm{O}(3)=96.41(8)^{\circ}, \mathrm{N}(2)-\mathrm{Co}(1)-\mathrm{O}(5)=166.23(10)^{\circ}\right)$. The bond length between the cobalt(III) center and the $\mathrm{C}-\mathrm{H}$ activated carbon $\mathrm{Co}-\mathrm{C} 20$ were found to be $1.916(3) \AA$ and similar to the previously reported cyclometalated cobalt(III) benzamide complexes (104 (Maiti): 1.916(3); ${ }^{[158]}$ Song: $1.923 ;^{[160]} 105$ (Ribas): 1.919(2) ${ }^{[159]}$ ). It was also detected that the axially bonded, terminal, oxygen-coordinated substrate 34a with a 
bond length of 1.9621(19) $\AA$ (Co-O5), readily dissociated in solution. The dissociation was confirmed by means of ESI-MS and NMR spectroscopic analysis.

In order to investigate the redox properties of the obtained cyclometalated complex 190, a series of cyclovoltammetric studies was performed (Figure 13). With a special focus on the mechanistic investigation of cobaltaelectro-catalyzed $\mathrm{C}-\mathrm{H}$ oxygenation, the analytical experiments were performed in methanol as the solvent and $n-\mathrm{Bu}_{4} \mathrm{NPF}_{6}(0.1 \mathrm{M})$ was used as the conducting salt. Complex 190 showed an irreversible redox event with an anodic peak potential of $E_{\mathrm{p}, \mathrm{a}}=0.95 \mathrm{~V}\left(v s . \mathrm{SCE}, 100 \mathrm{mV} \cdot \mathrm{s}^{-1}\right)$, while a half-peak potential of $E_{\mathrm{p}, 2}=$ $0.89 \mathrm{~V}\left(v s . \mathrm{SCE}, 100 \mathrm{mV} \cdot \mathrm{s}^{-1}\right)$ was determined for further comparison with computational studies (Table 23, entry 1). Even at higher scan rates of up to $v=1000 \mathrm{mV} \cdot \mathrm{s}^{-1}$, the oxidation event showed no reversible behavior, thus being indicative of a fast homogeneous, irreversible chemical follow-up reaction. Here, a possible EC-mechanism could consists of the electrochemical generation of a putative cobalt(IV) species, followed by ligand substitution or deprotonation. ${ }^{[154 b, 312]}$ The small shoulder with a slightly lower anodic peak potential of $E_{\mathrm{p}, \mathrm{a}}=0.76 \mathrm{~V}$ ( $v s$. SCE), was assigned to a different cobalt(III) complex 191.

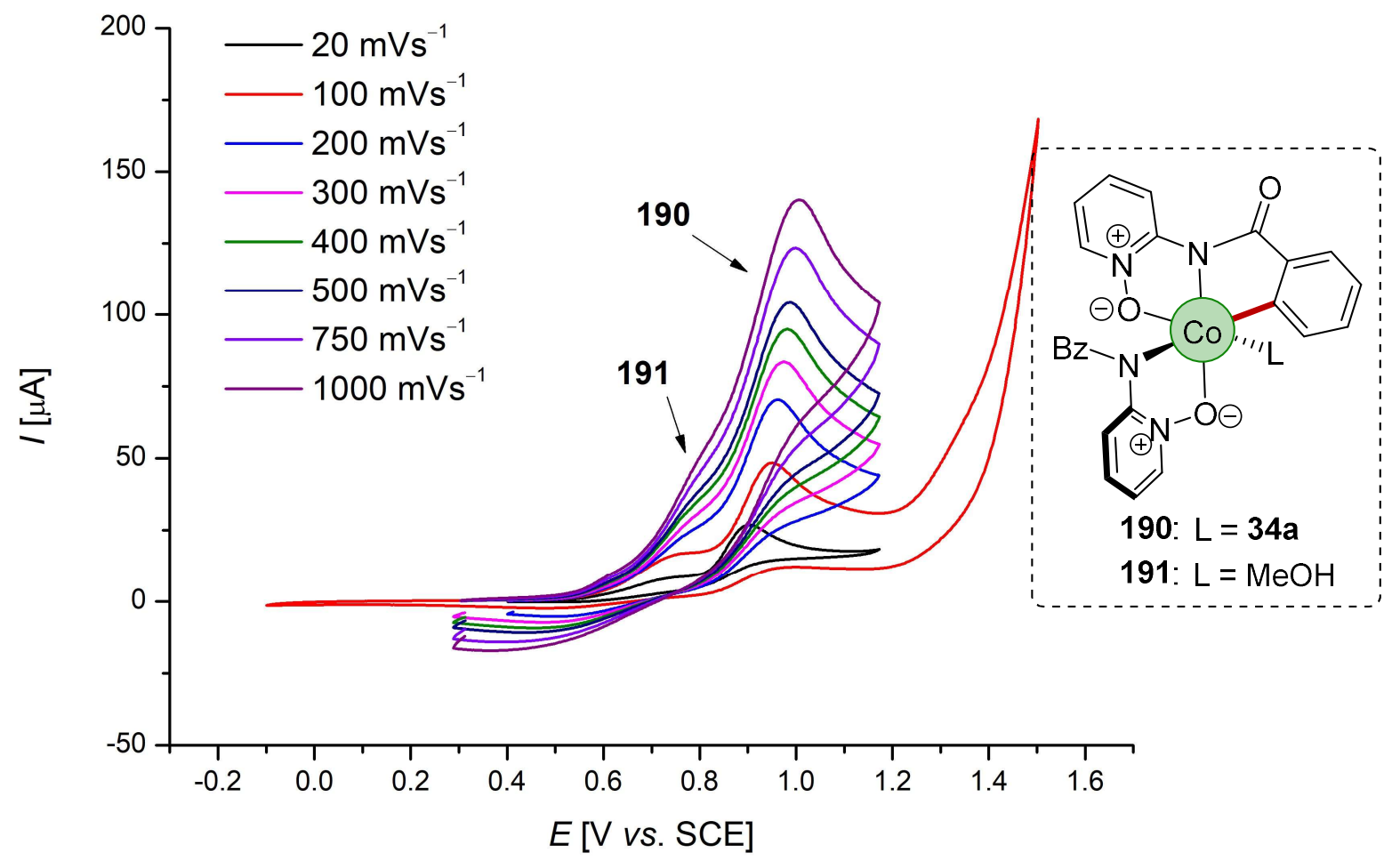

Figure 13. Cyclic voltammograms at $25^{\circ} \mathrm{C}$, with different scan rates using $\mathrm{MeOH}$ and $n-\mathrm{Bu}_{4} \mathrm{NPF}_{6}(0.1 \mathrm{M})$ as the electrolyte and a $\mathrm{GC}$ working electrode. 190 (3.5 mM).

With the aid of computational evaluated redox potentials, conducted by Dr. J. C.A. Oliveira (Table 23), it was found that substitution of the weakly mono $O$-coordinating 
substrate 34a with a methanol molecule had a stabilizing effect, as was reflected by the lower anodic oxidation potential of the cobalt(III) complex 191 (entry 2). ${ }^{[309]}$

Table 23. Calculated oxidation potentials. ${ }^{[a]}$

\begin{tabular}{ccc}
\hline Entry & Redox pair & Potential $(\mathrm{V}$ vs. SCE) \\
\hline 1 & $\mathbf{1 9 0 :}[\mathrm{Co}(\mathrm{III})] \rightarrow[\mathrm{Co}(\mathrm{IV})]+1 \mathrm{e}^{-}$ & 0.886 \\
2 & $\mathbf{1 9 1 :}[\mathrm{Co}(\mathrm{III})] \rightarrow[\mathrm{Co}(\mathrm{IV})]+1 \mathrm{e}^{-}$ & 0.818 \\
\hline
\end{tabular}

[a] DFT calculations were performed by Dr. J. C. A. Oliveira.

To decrease the reaction rate of chemical follow-up reactions, investigations of the oxidation events were performed at lower temperatures. ${ }^{[24 \mathrm{j}, 154-155,313]}$ When the CVexperiments were performed at a temperature of $0^{\circ} \mathrm{C}$ (Figure 14), a reversible $\mathrm{Co}(\mathrm{III}) / \mathrm{Co}(\mathrm{IV})$ redox-event was observed, albeit at a fast scan rate of more than $1.0 \mathrm{~V} \cdot \mathrm{s}^{-1}$. However, at lower scan rates, the oxidation remained irreversible. ${ }^{[309]}$

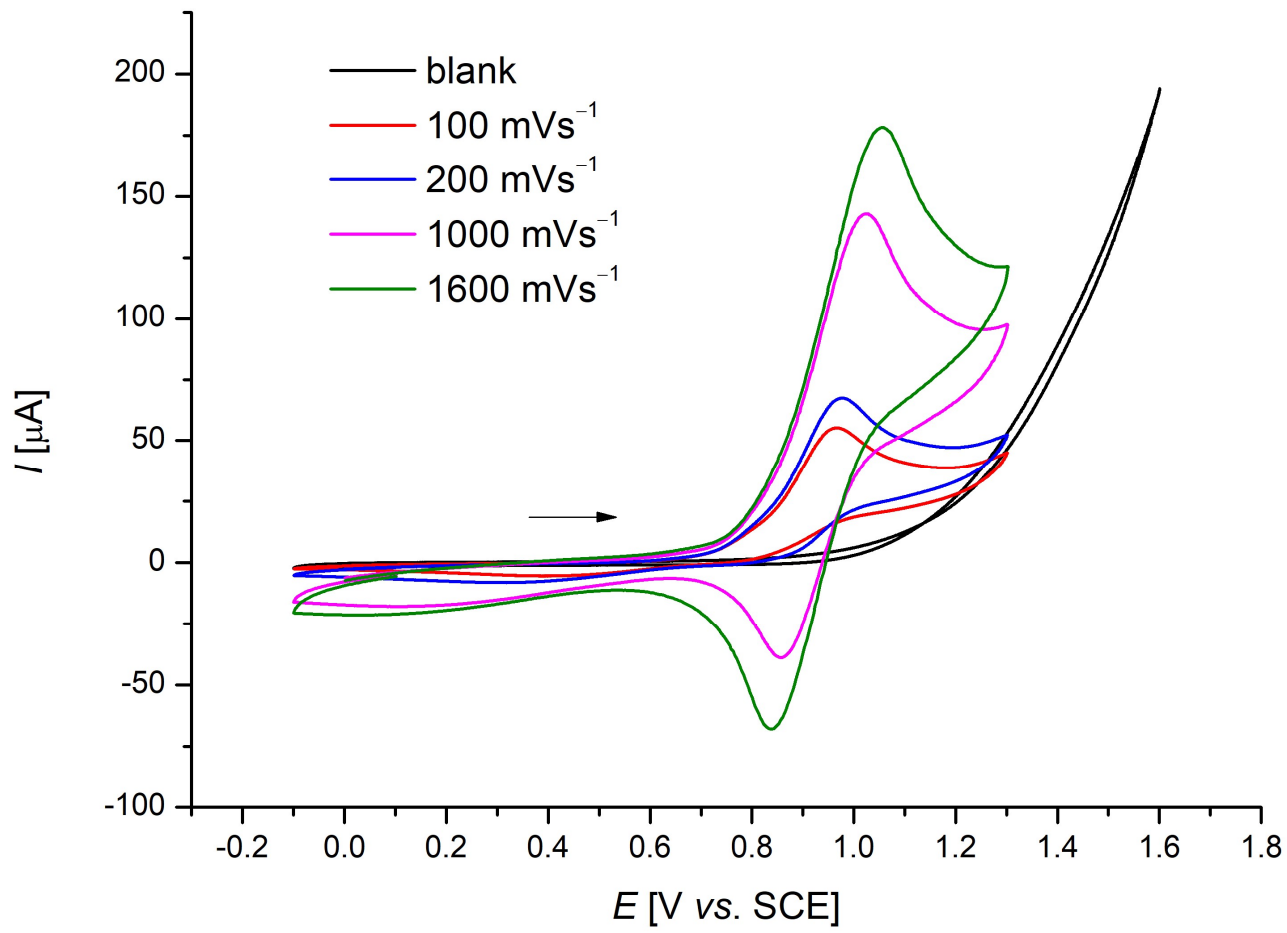

Figure 14. Cyclic voltammograms at $0{ }^{\circ} \mathrm{C}$ with different scan rates using $\mathrm{MeOH}$ and $n-\mathrm{Bu}_{4} \mathrm{NPF}_{6}(0.1 \mathrm{M})$ as the electrolyte and a $\mathrm{GC}$ working electrode. 190 (3.5 mM).

At decreased temperatures of $T=-78^{\circ} \mathrm{C}$, a reversible redox event was detected even at lower scan rates $\left(10 \mathrm{mV} \cdot \mathrm{s}^{-1}\right)$, showcasing the stability of a potentially formed high-valent cobalt(IV) complex after anodic oxidation of the cobalt(III) complex 190 (Figure 15). The recorded current response at $-78{ }^{\circ} \mathrm{C}$ was diminished, compared to the previous 
measurements at $T=0{ }^{\circ} \mathrm{C}$, due to the reduced electrolyte concentrations. Also, a silver-wire was used as the reference-electrode since the SCE was not applicable at these temperatures.

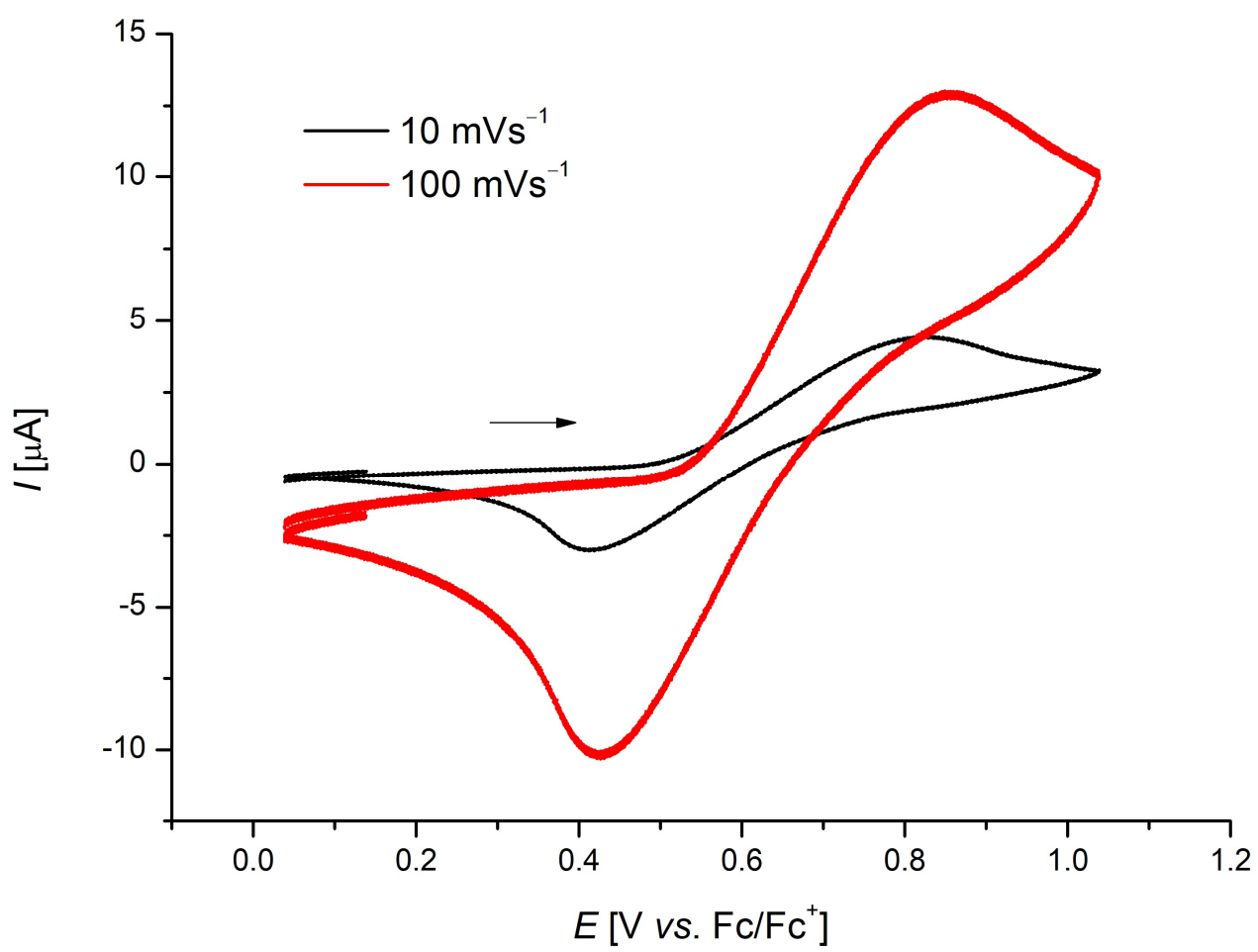

Figure 15. Cyclic voltammograms at $-78^{\circ} \mathrm{C}$ with different scan rates using $\mathrm{MeOH}$ and $n-\mathrm{Bu}_{4} \mathrm{NPF}_{6}(0.02 \mathrm{M})$ as the electrolyte and a $\mathrm{GC}$ working electrode. 190 (3.5 mM).

Cyclic voltammograms of different reaction components revealed observable changes in the redox events of cobalta(III)cycle 190 (Figure 16). Upon addition of NaOPiv, the irreversible oxidation wave at $E_{\mathrm{p}}=0.95 \mathrm{~V}(v s$. SCE) was shifted to lower potentials of $E_{\mathrm{p}, \mathrm{ox}}=0.84 \mathrm{~V}$ ( $\left.v s . \mathrm{SCE}\right)$. Likewise, the shift to lower oxidation potentials can be ascribed to the ligand exchange of the weakly $O$-coordinating substrate $\mathbf{3 4 a}$ by a pivalate anion (vide supra). The new oxidation wave at $E_{\mathrm{p}, \mathrm{ox}}=1.28 \mathrm{~V}$ (vs. SCE) can be attributed to the irreversible oxidation of the pivalate anion. Further addition of substrate 34a did not lead to significant changes in the voltammogram. 


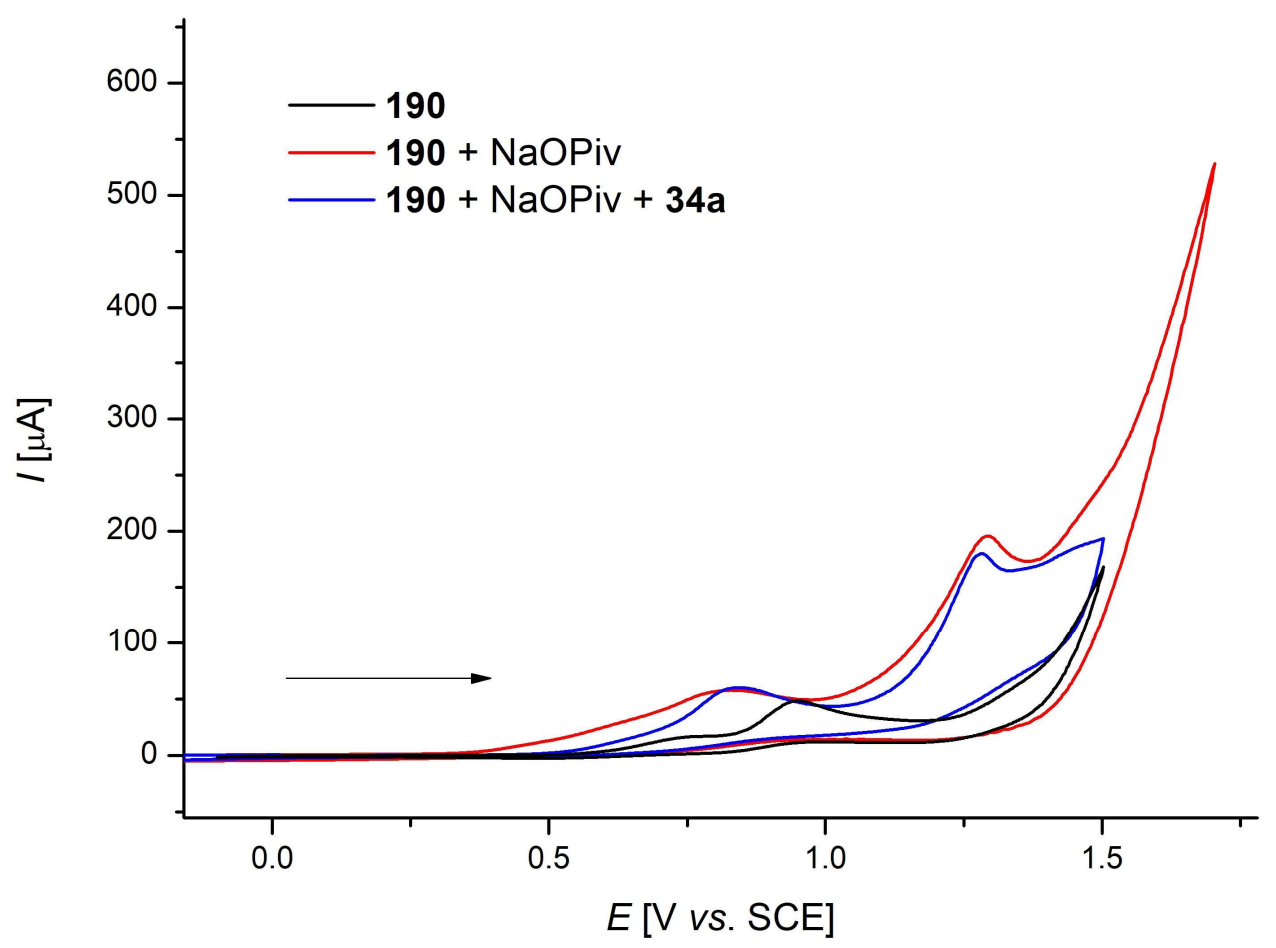

Figure 16. Cyclic voltammograms at $25^{\circ} \mathrm{C}$ and $100 \mathrm{mV} \cdot \mathrm{s}^{-1}$ using $\mathrm{MeOH}$ and $n-\mathrm{Bu}_{4} \mathrm{NPF}_{6}(0.1 \mathrm{M})$ as the electrolyte and a $\mathrm{GC}$ working electrode. (Black) 190 (3.5 mM); (red) 190 (3.5 mM) and NaOPiv (10 mM); (blue) 190 (3.5 mM), NaOPiv (10 mM) and 34a (10 mM).

Interestingly, with $\mathrm{MeCN}$ as the electrolyte, different redox properties of the cobalta(III)cycle 190 were observed (Figure 17 and 18). At scan rates of $100 \mathrm{mV} \cdot \mathrm{s}^{-1}$ and $200 \mathrm{mV} \cdot \mathrm{s}^{-1}$, respectively, one irreversible and one quasi-reversible oxidation event was observed. With an increased scan rate, it was found that the first oxidation event became reversible and a half-wave potential of $E_{1 / 2}=1.00 \mathrm{~V}$ ( $v s$. SCE) was detected. These observations strongly indicate that the chemical follow-up reaction is much slower in $\mathrm{MeCN}$ when comparing to the irreversible chemical reaction in $\mathrm{MeOH}$. It is noteworthy that unpublished computational investigations performed by $D r . J$. C. A. Oliveira revealed a metal-centered oxidation event. 


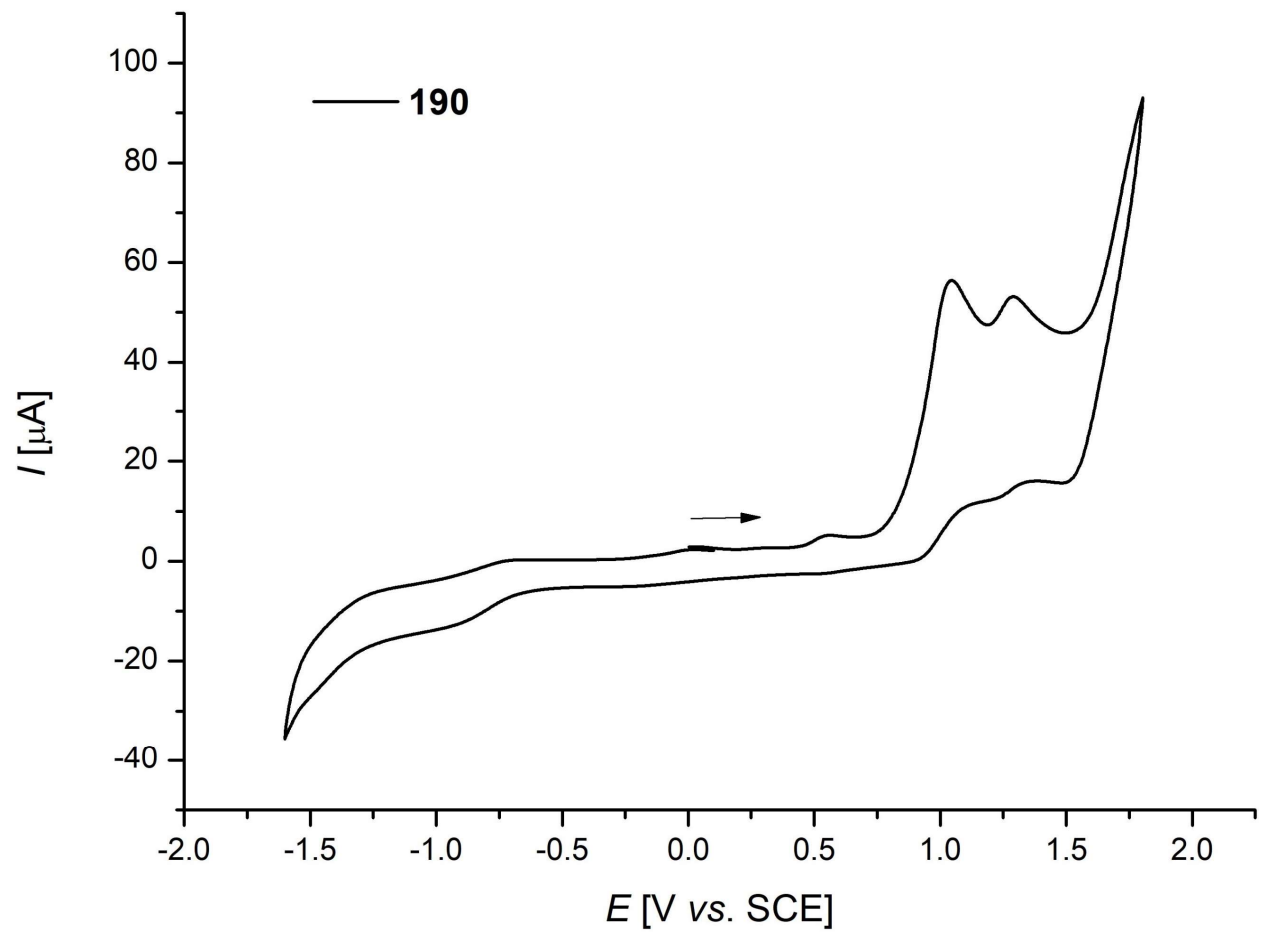

Figure 17. Cyclic voltammogram at $100 \mathrm{mV} \cdot \mathrm{s}^{-1}$ using $\mathrm{MeCN}$ and $n-\mathrm{Bu}_{4} \mathrm{NPF}_{6}(0.1 \mathrm{M})$ as the electrolyte and a GC working electrode. $190(3.5 \mathrm{mM})$.

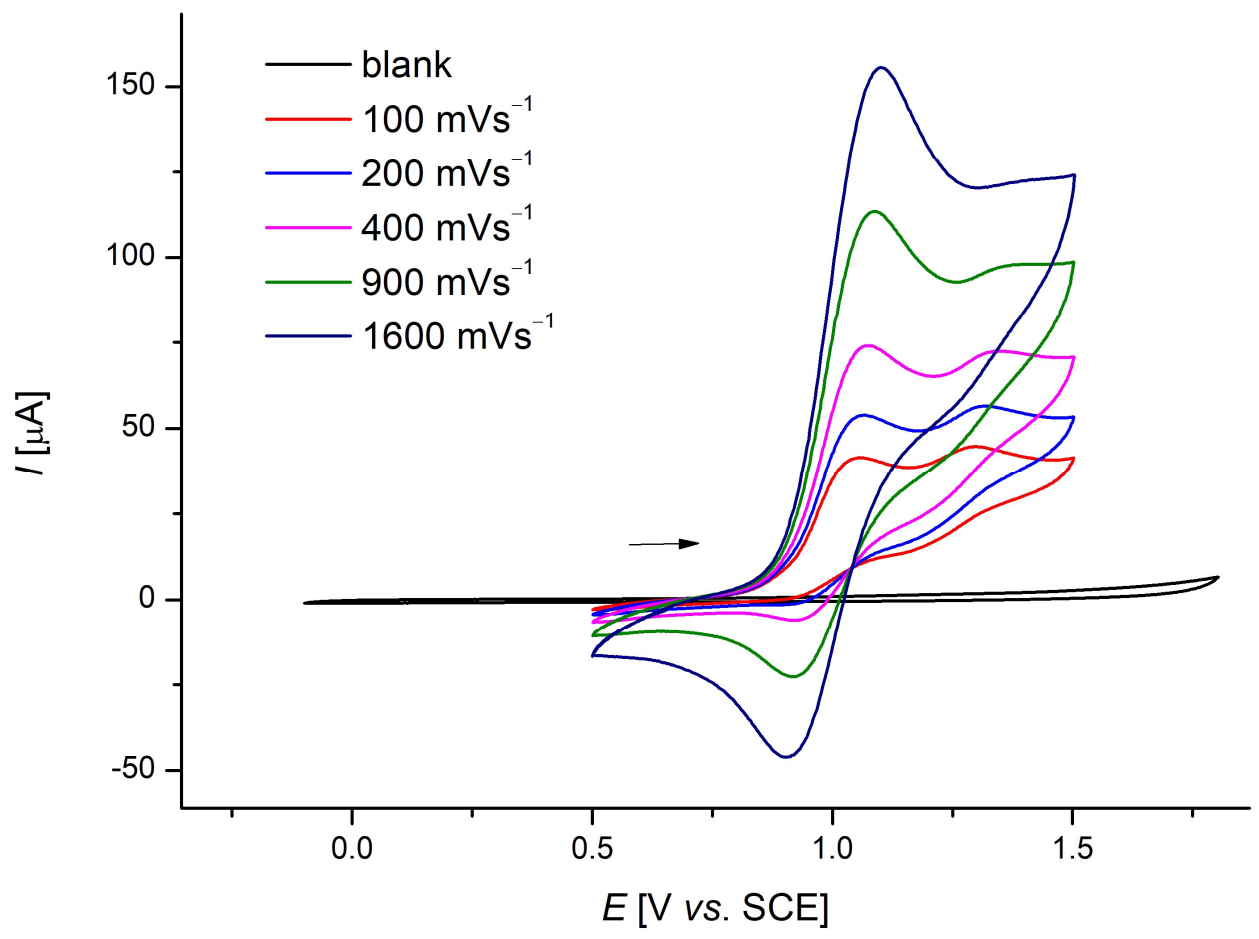

Figure 18. Cyclic voltammograms at $25^{\circ} \mathrm{C}$ with different scan rates using $\mathrm{MeCN}$ and $n-\mathrm{Bu}_{4} \mathrm{NPF}_{6}(0.1 \mathrm{M})$ as the electrolyte and a $\mathrm{GC}$ working electrode. 190 (3.5 mM). 
Additionally, spectrophotometric UV-vis studies of cobalt(II) acetate, cobalt(III) acetate and 190 were performed in $\mathrm{MeOH}$ at ambient temperature (Figure 19a). The simple cobalt salts revealed only low absorption in the ultraviolet region with absorption maxima at approximately $245 \mathrm{~nm}$. In contrast, the cyclometalated cobalt(III) complex 190 displayed a strong UV-vis absorption within the ultraviolet, violet and blue color region. Based on the intensive green color of the cobalt(III) complex (Figure 19b), an absorption at $380 \mathrm{~nm}$ should be observable. Indeed, a strong absorption maximum at $380 \mathrm{~nm}$ was detected, which is most likely caused by a ligand-to-metal charge-transfer.

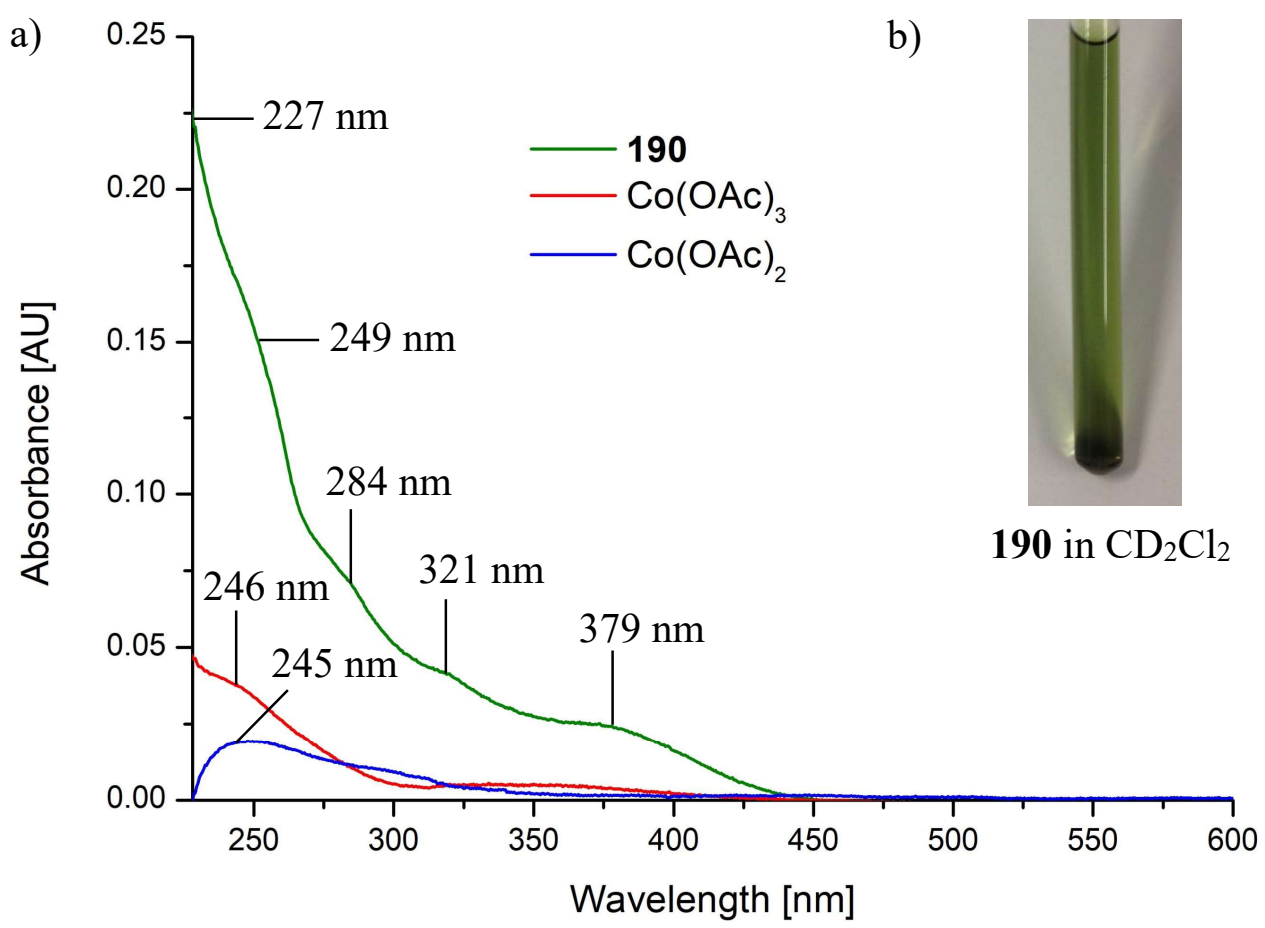

Figure 19. a) UV-vis studies in $\mathrm{MeOH}$. i) $\mathrm{Co}(\mathrm{OAc})_{2}\left(0.25 \mathrm{~mm}\right.$, blue). ii) $\mathrm{Co}(\mathrm{OAc})_{3}$ (0.25 mM, red). iii) 190 (0.08 mM, green). b) Characteristic dark green color of 190 solvated in $\mathrm{CD}_{2} \mathrm{Cl}_{2}$.

\subsubsection{Reaction Monitoring by Mass Spectrometry}

For the detection of potential reaction intermediates and to monitor the reaction profile upon electrochemical stimulus, in-operando analytical techniques were evaluated. Here, ${ }^{1} \mathrm{H}-\mathrm{NMR}$ spectroscopic analysis was not viable, due to the strong paramagnetic properties of the cobalt(II) species. Unfortunately, ${ }^{19} \mathrm{~F}-\mathrm{NMR}$ spectroscopy likewise resulted in unsatisfactory results. Previous results obtained by ESI-mass spectrometry in positive polarization mode exhibited high affinity of the pyridine- $N$-oxide group toward cations, especially sodium ions, thus rendering ESI-MS an ideal tool to probe the formation and the reaction profile of the cobaltacycle $190 .{ }^{[314]}$ However, it is noteworthy that the ion response 
in electrospray ionization is not linear, thus the signal intensity was not directly correlated with the concentration of the individual species in solutions. ${ }^{[315]}$ Initially, the formation of the cyclometalated complex under anodic oxidation in $\mathrm{MeCN}$ was monitored by ESI-mass spectrometry over a period of $120 \mathrm{~min}$ (Scheme 66). Notably, prior to anodic oxidation, almost complete complexation of substate 34a was detected. This indicates that the cobalt(II) acetate readily undergoes complexation with the deprotonated bidentate benzamide 34a to generate cobalt(II) bisamide 192. ${ }^{[309]}$

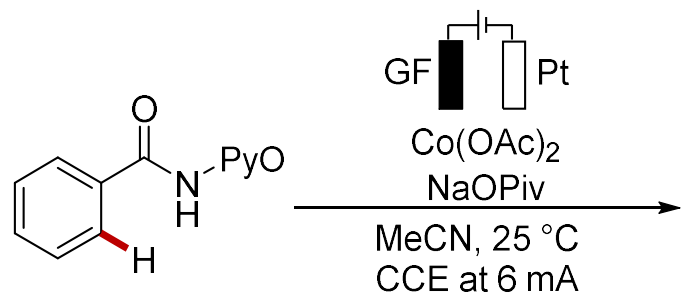

$34 a$

$m / z$

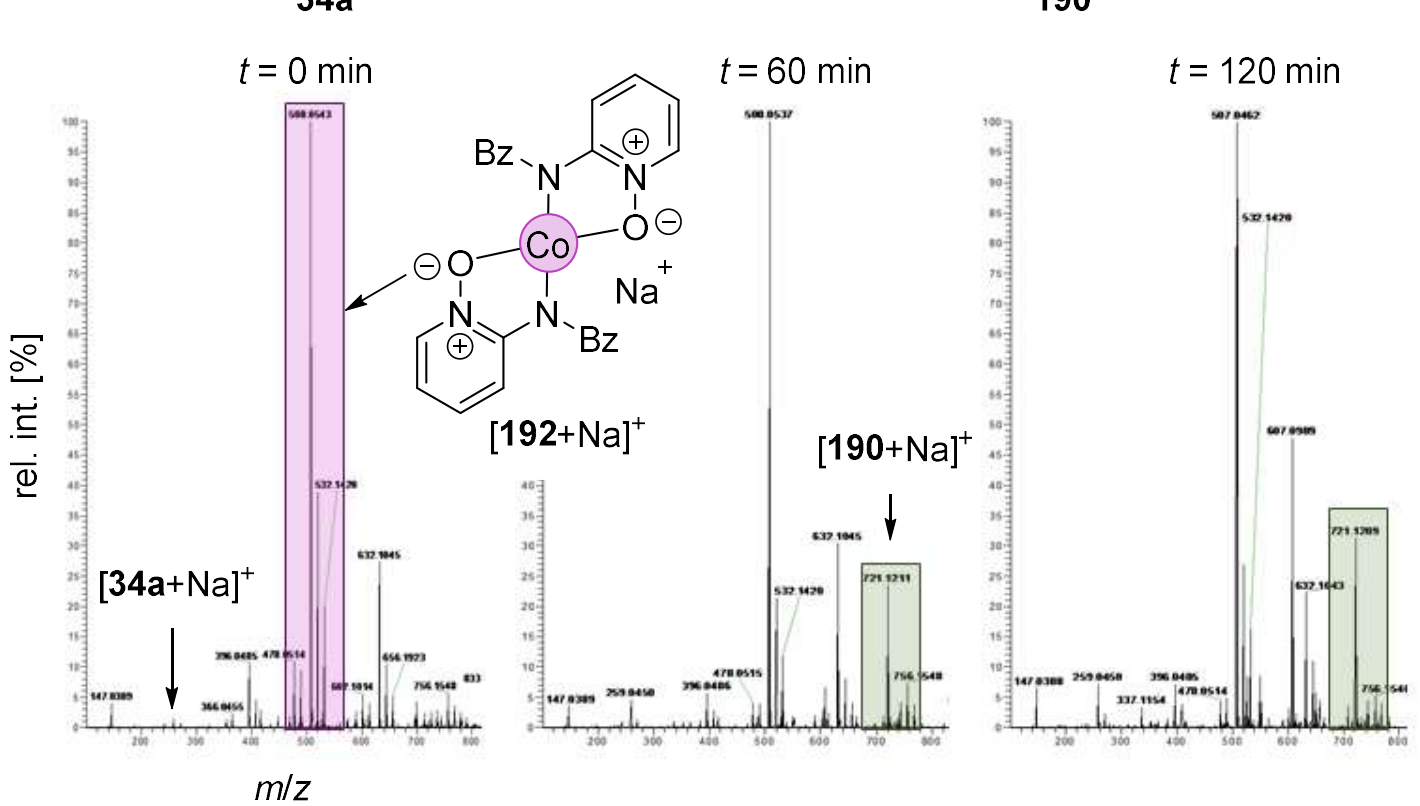

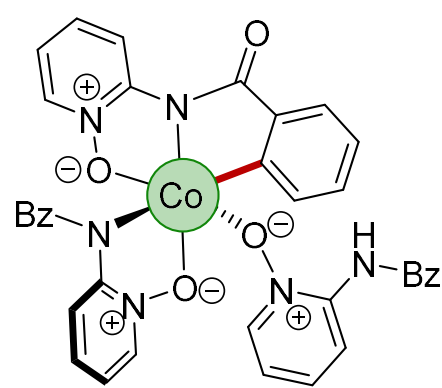

190

Scheme 66. ESI-MS monitoring of the formation of $\mathbf{1 9 0}$ upon anodic oxidation.

In contrast, when constant current electrolysis was applied to the reaction mixture, $\mathrm{C}-\mathrm{H}$ activation took place and the cyclometalated cobalt(III) complex 190 was detected. These results are in good agreement with the stoichiometric experiments and support the finding that ligation of substrate 34a occurred prior to anodic cobalt(II/III) oxidation (cf. Table 22). After successful reaction monitoring of the complex 190 formation, further experiments were performed to confirm the relevance of the anodically generated cobaltacycle $\mathbf{1 9 0}$ for the cobaltaelectro-catalyzed $\mathrm{C}-\mathrm{O}$ formation (Scheme 67). At $t=0 \mathrm{~min}$, small quantities of 
dissociated substrate 34a could be detected. However, no alkoxylated product 36ab was observed. With the start of electrolysis, a significant growth of product 36ab was observed, following a linear increase over time/applied current (Figure 20). Along with the decrease of cobalta(III)cycle $190[\mathbf{1 9 0}-\mathbf{3 4 a}+\mathrm{Na}]^{+}$, an increasing intensity of cobalt(III) complex 193 was detected.

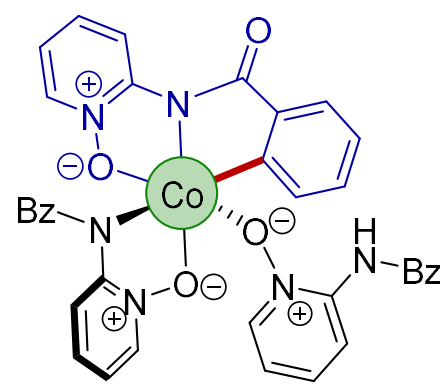

190
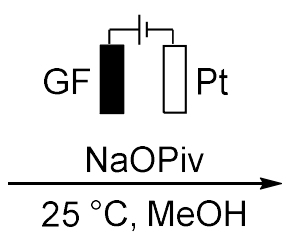
$\mathrm{CCE}$ at $6 \mathrm{~mA}$

$36 \mathrm{ab}$

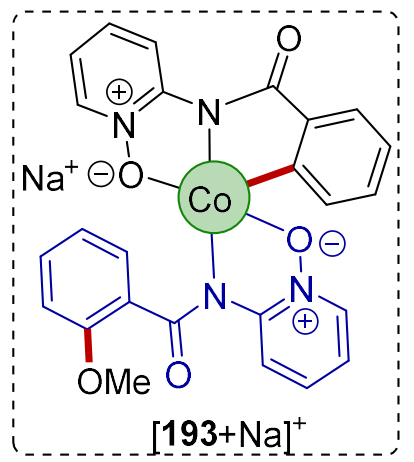

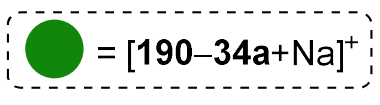

$t=0 \mathrm{~min}$
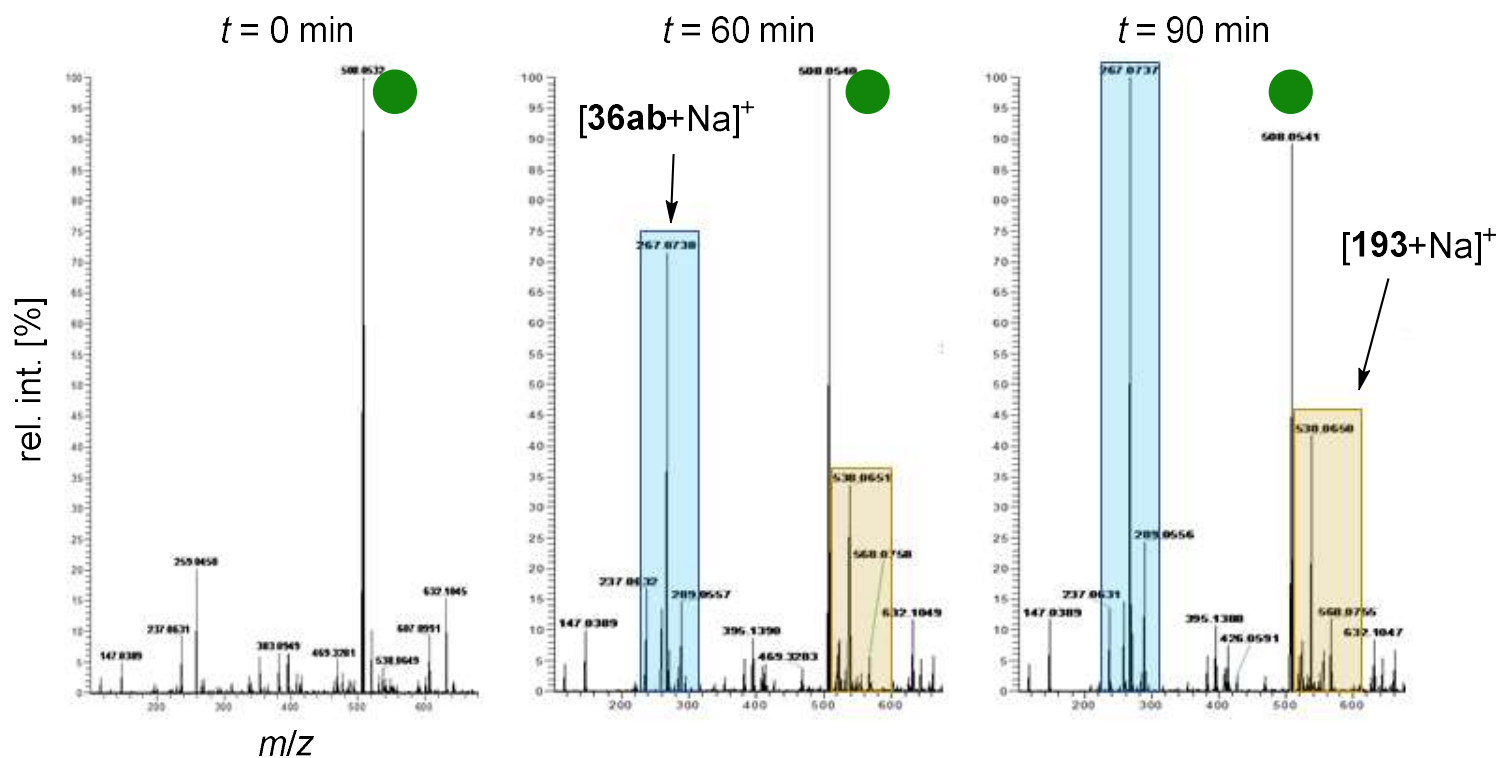

Scheme 67. ESI-MS monitoring of the formation of 36ab upon anodic oxidation. 


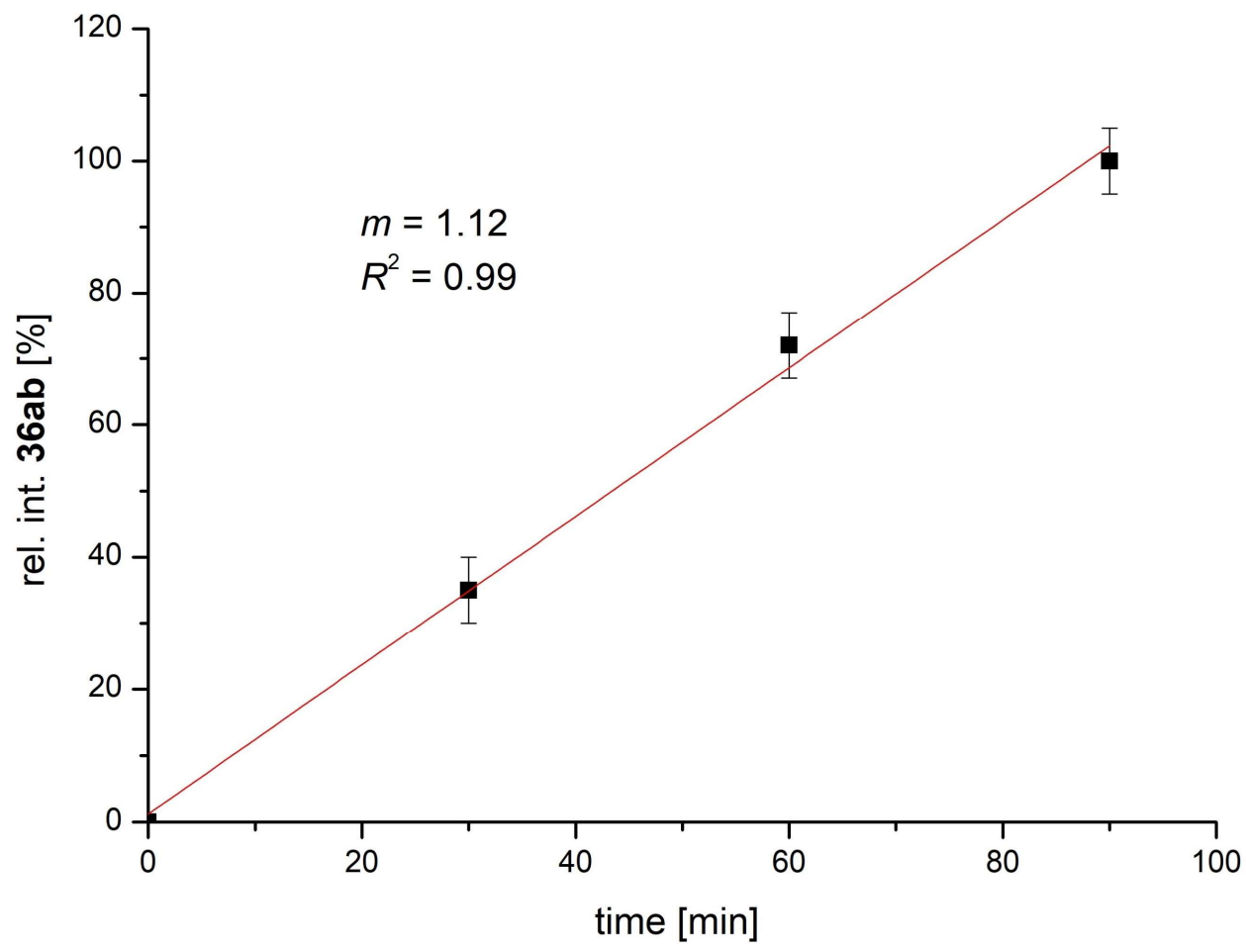

Figure 20. Relative intensity of 36ab detected with ESI-MS versus time.

\subsubsection{Reactivity of Cyclometalated Cobalt(III) Complex 190}

Stimulated by these findings, stoichiometric experiments were conducted to validate and quantify the initial hypothesis that oxidation of the cyclometalated cobalt(III) complexes 190 facilitates reductive elimination to release the $\mathrm{C}-\mathrm{O}$ coupled product. ${ }^{[309]}$ As was previously observed in the ESI-MS experiments, no alkoxylated product 36ab was formed in the absence of electricity (Scheme 68a). To assess if electricity is required to generate the potentially more nucleophilic alkoxide, a stoichiometric reaction with sodium methoxide was performed (Scheme 68b). Likewise, no $\mathrm{C}-\mathrm{H}$ oxygenated product 36ab was detected.

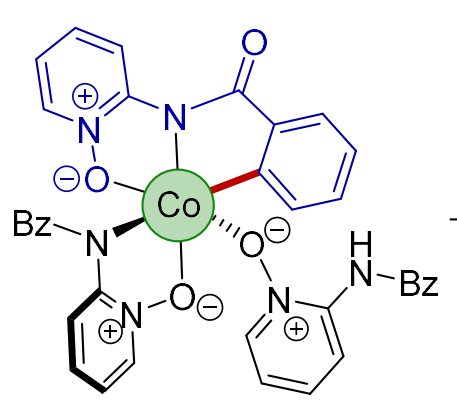

190

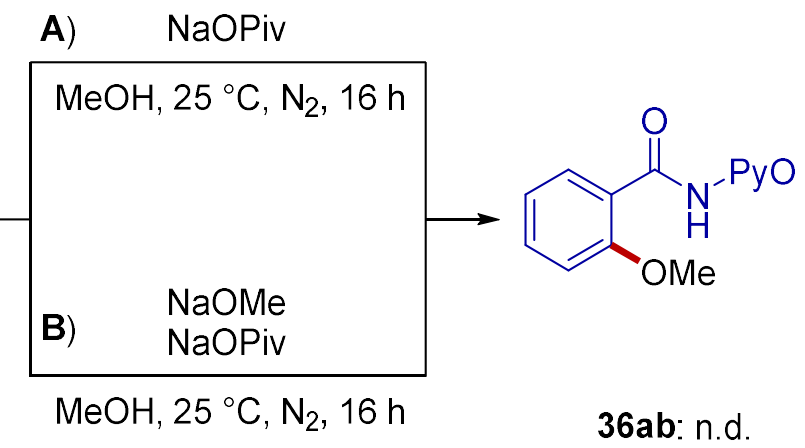

$\mathrm{MeOH}, 25^{\circ} \mathrm{C}, \mathrm{N}_{2}, 16 \mathrm{~h}$

36ab: n.d.

Scheme 68. Stoichiometric reactivity of $\mathbf{1 9 0}$ for $\mathrm{C}-\mathrm{O}$ formation without oxidants. 
Next, the oxidation-induced reductive elimination was probed by anodic oxidation of cobaltacycle 190 with constant potential electrolysis in $\mathrm{MeOH}$ (Scheme 69). In contrast to the redox-neutral reaction conditions, the desired product 36ab was isolated in $99 \%$ yield after chromatographic isolation, translating into quantitative conversion of the $\mathrm{C}-\mathrm{H}$ metalated substrate.

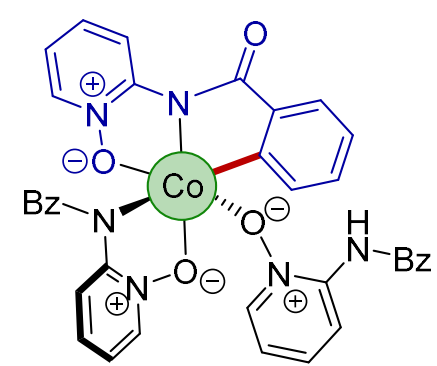

190

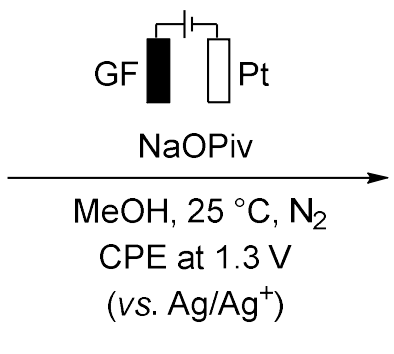

(vs. $\mathrm{Ag} / \mathrm{Ag}^{+}$)<smiles>COc1ccccc1C(=O)NPOC#N</smiles>

36ab: $99 \%$

Scheme 69. Oxidatively-induced reductive elimination for $\mathrm{C}-\mathrm{H}$ methoxylation.

In order to compare the results with the previously performed constant current electrolysis, an aliquot was removed before the reaction was stopped and analyzed by ESI time-of-flight mass spectrometry. Likewise, various cobalt complexes were detected, providing an overall insightful snapshot of the crude reaction mixture (Figure 21).

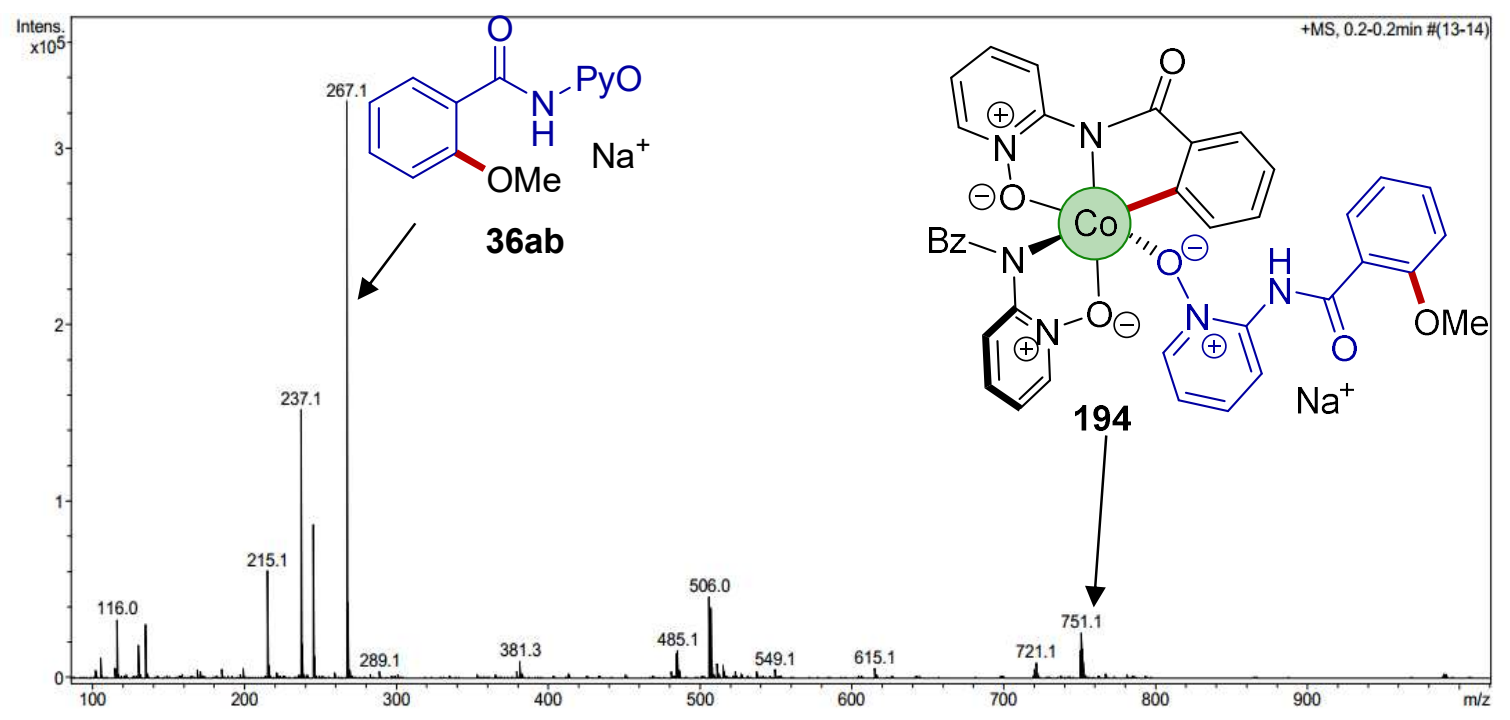

Figure 21. ESI-MS (positive ionization-mode) of the crude reaction mixture after $4 \mathrm{~h}$.

Two signals with a mass-to-charge ratio of 215 and 237 correspond to the free starting material 34a $\left([\mathrm{M}+\mathrm{H}]^{+}\right.$and $\left.[\mathrm{M}+\mathrm{Na}]^{+}\right)$. The detection of 34a in the crude mixture provides support for the weak coordination of the $O$-coordinating substrate in the cobalt(III) complex 
190. Furthermore, unreacted complex $[190+\mathrm{Na}]^{+}$was detected with small intensities, as well as the cobalt(III) complex 194, in which one substrate 34a was substituted by the product 36ab $(m / z=751)$.

To better understand and rationalize the nature of the $\mathrm{C}-\mathrm{O}$ bond forming reductive elimination step, density functional theory (DFT) calculations were performed by Dr. J. C. A. Oliveira. ${ }^{[309]}$ In accordance with the experimental results, DFT calculations were suggestive of an oxidatively-induced reductive elimination via a cobalt(III/IV/II) manifold. Here, facile reductive elimination from the cobalt(IV) precursor proved to be highly feasible with a calculated activation barrier of $9.9 \mathrm{kcal} \cdot \mathrm{mol}^{-1} \cdot{ }^{[309]}$

Encouraged by the results on cobaltaelectro-catalyzed $\mathrm{C}-\mathrm{H}$ oxygenations, analogous stoichiometric transformations of the cyclometalated cobalt(III) complex 190 with alkyne 53a and allene 75a were performed (Scheme 70). ${ }^{[250,260,300]}$

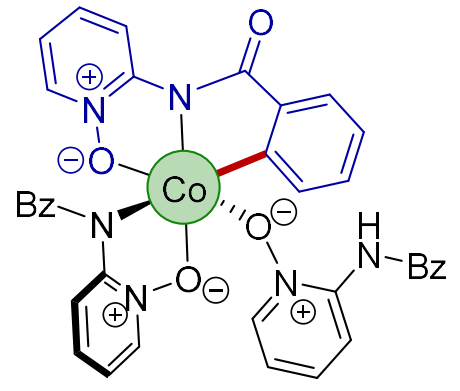

190

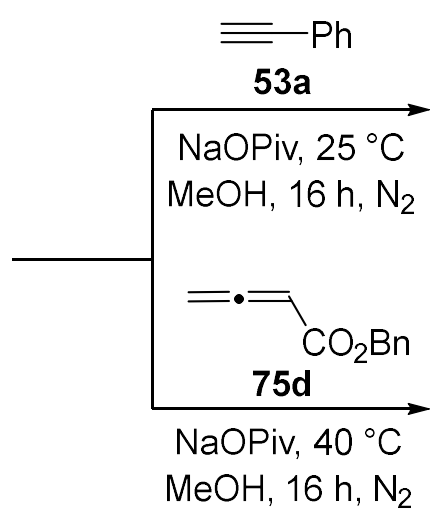

$\mathrm{MeOH}, 16 \mathrm{~h}, \mathrm{~N}_{2}$<smiles>O=c1c2ccccc2cc(-c2ccccc2)n1P(O)c1ccccc1</smiles>

51aa: $99 \%$<smiles>O=C(Cc1cc2ccccc2c(=O)n1PO)OCc1ccccc1</smiles>

176ad: $97 \%$

Scheme 70. Stoichiometric reactivity of $\mathbf{1 9 0}$ for $\mathrm{C}-\mathrm{C}$ formations without oxidants.

Interestingly, a facile product formation was observed for both desired transformations without the aid of additional oxidants. These results are in good agreement with previously performed computational studies for cobalt-catalyzed $\mathrm{C}-\mathrm{H} / \mathrm{N}-\mathrm{H}$ alkyne annulations with oxygen as the terminal oxidant and for cobaltaelectro-catalyzed $\mathrm{C}-\mathrm{H} / \mathrm{N}-\mathrm{H}$ allene annulations (vide supra), respectively. ${ }^{[112,260]}$ Based on these results, it can be assumed that the cobaltaelectro-catalyzed $\mathrm{C}-\mathrm{H}$ annulation reactions proceeds via a cobalt(II/III/I) catalytic manifold. It is further noteworthy that during ESI-MS studies of the alkyne annulation reaction, a mass signal was observed which could be assigned as the sevenmembered cobalta(III)cycle 195 (Figure 22). ${ }^{[112]}$ Unfortunately, the signal was of low intensity and further validation by MS/MS studies was not possible. ${ }^{[251,269]}$ 


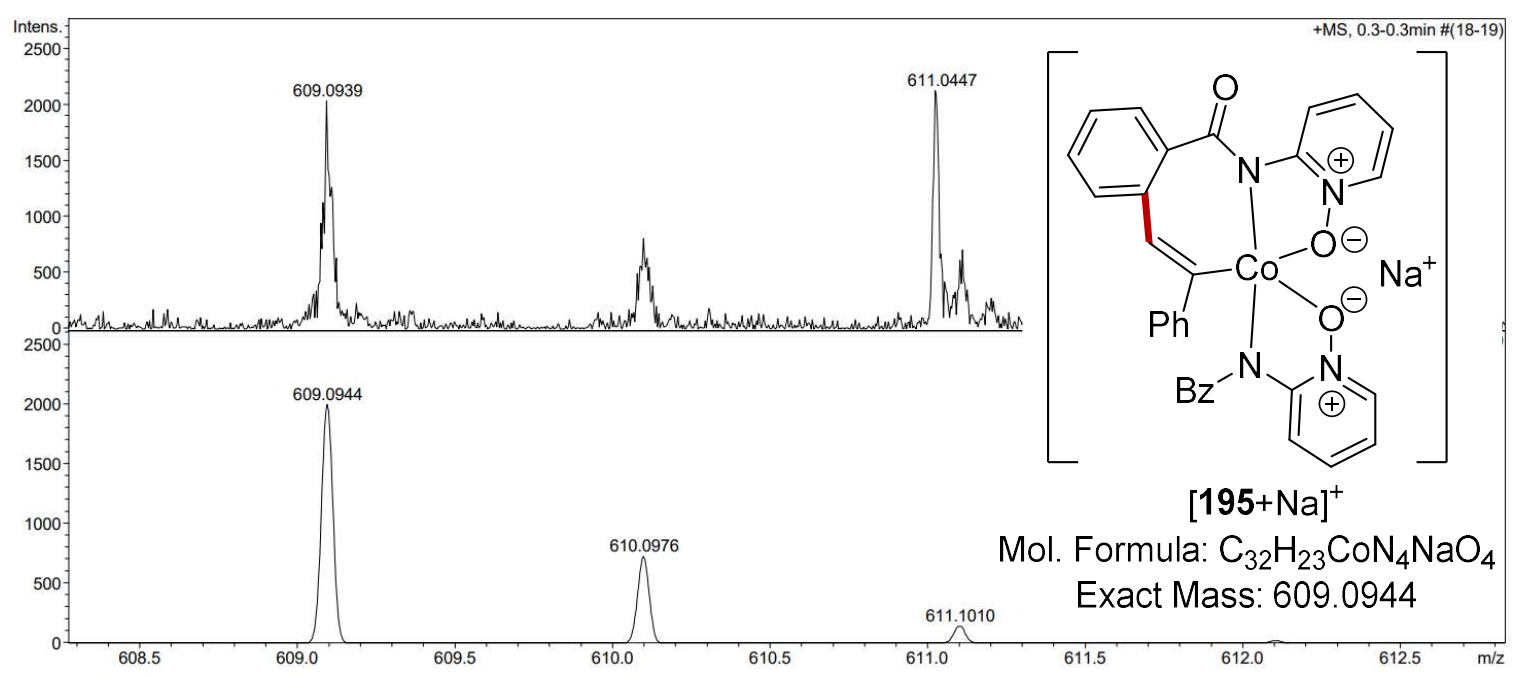

Figure 22. Attempted ESI-MS detection of seven-membered cobalt(III) complex 195.

\subsubsection{Hammett-Zuman Correlation}

A Hammett plot analysis is a commonly used empirical method to evaluate the rate dependence of a given reaction of interest with respect to electronic modifications of the reactant. ${ }^{[316]}$ In this study, a particularly interesting question was the relationship between the molecular structure of different cobalt(III) complexes and the electrochemical redox event:

$$
\begin{aligned}
{\left[\mathrm{Co}^{\mathrm{III}}\right] } & \rightarrow\left[\mathrm{Co}^{\mathrm{IV}}\right]+e^{-} \\
K_{0} & =\frac{\left[\mathrm{Co}^{\mathrm{IV}}\right]}{\left[\mathrm{Co}^{\mathrm{III}}\right]}
\end{aligned}
$$

with the equilibrium constant $K_{0}$ for the unsubstituted cobalt(III) complex 190. In this context, a Hammett-Zuman plot analysis was applied to investigate the influence of a substituent on the voltametric properties of the studied electron transfer process. ${ }^{\left[{ }^{[17]}\right.}$ Based on the established correlation (3.1), chemical reaction parameters such as the equilibrium constant $K(3.2)$ or reaction rates $k(3.3)$ can be directly related to different substituents on benzamide 34 .

$$
\begin{gathered}
\Delta E_{1 / 2}=\sigma_{\mathrm{p}} \times \rho \\
\log \left(\frac{K}{K_{0}}\right)=\sigma_{\mathrm{p}} \times \rho \\
\log \left(\frac{k}{k_{0}}\right)=\sigma_{\mathrm{p}} \times \rho
\end{gathered}
$$


In the Hammett-Zuman correlation, the Hammett-values $\sigma_{\mathrm{p}}$ represents the polar substituent constants, here in the para-position to the metalated carbon atom of the aromatic ring. The proportionality constant $\rho$, expresses the susceptibility of the electron transfer to the polar effects of the respective substituent and is, in contrast to the standard Hammett correlation (3.2 and 3.3), measured in volts. To study the influence of ligand substitutions on the oxidation-induced reductive elimination, differently substituted cobalt(III) complexes 190 and 196-201 were synthesized (Table 24). ${ }^{[309]}$

Table 24. Synthesis of differently substituted cyclometalated cobalt(III) complexes. ${ }^{[a]}$

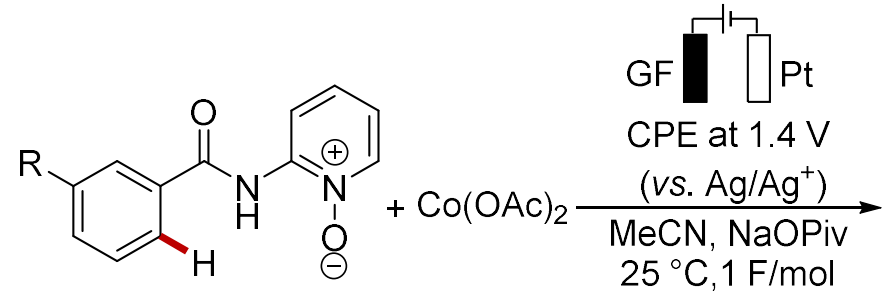

34

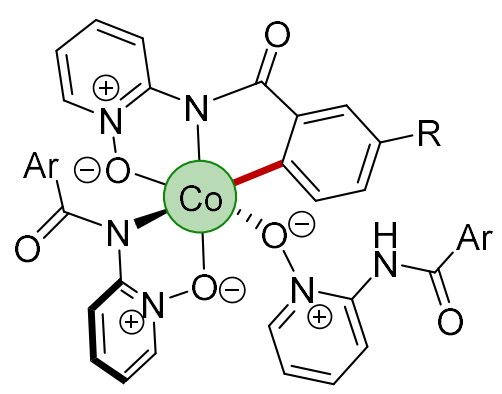

190, 196-201

\begin{tabular}{|c|c|c|c|c|c|}
\hline Entry & $\mathrm{R}=$ & 34 & Product, $\mathrm{Ar}=$ & & Yield [\%] \\
\hline 1 & $\mathrm{H}$ & $34 a$ & & 190 & 46 \\
\hline 2 & $\mathrm{Me}$ & 34b & & 196 & 51 \\
\hline 3 & $i-\operatorname{Pr}$ & $34 w$ & & 197 & 28 \\
\hline 4 & $\mathrm{OMe}$ & $34 p$ & & 198 & $13^{[\mathrm{b}]}$ \\
\hline 5 & $\mathrm{CN}$ & $34 x$ & & 199 & 19 \\
\hline 6 & $\mathrm{CF}_{3}$ & $34 y$ & & 200 & 13 \\
\hline 7 & $\mathrm{~F}$ & $34 r$ & & 201 & 51 \\
\hline
\end{tabular}

[a] Reaction conditions: Undivided cell, 34 (2.00 equiv), $\mathrm{Co}(\mathrm{OAc})_{2}(0.50 \mathrm{mmol}), \mathrm{NaOPiv}$ (2.00 equiv), $\mathrm{MeCN}(13.0 \mathrm{~mL}), 25^{\circ} \mathrm{C}$, constant potential electrolysis (CPE) at $1.4 \mathrm{~V} v s . \mathrm{Ag} / \mathrm{Ag}^{+}, 1 \mathrm{~F}$, graphite felt anode, Pt-plate cathode. Yields of isolated complexes are given. [b] C-H acyloxylation ${ }^{[254]}$ was detected with $3 \%$ conversion. 
Under the previously optimized reaction conditions for the electrosynthesis of $\mathbf{1 9 0}$, cyclometalated cobalt(III) complexes 196-198 decorated with electron-donating (entries 2-4), as well as electron-withdrawing groups 199-201 were synthesized (entries 5-7). The fluctuation in reaction yields can be attributed to a fast degradation of the complexes during workup or side reactions during the electrolysis. Especially with electron-donating substituents, larger amounts of purple, paramagnetic undesired products were observed (vide infra). In order to obtain detailed information about the influence of the substituent on the redox properties of the cyclometalated cobalt complexes, cyclic voltammograms were recorded in $\mathrm{MeOH}$ (Table 25). Notably, all cobalt(III) complexes displayed similar redox behaviors, yet electron-donating groups lowered the oxidation potential of the corresponding complexes, whereas electron-withdrawing substituents, para to the metalated carbon, led to increased oxidation potentials. ${ }^{[309]}$

Table 25. Oxidation potentials of cobalt(III) complexes 190, 196-201 and tabulated $\sigma_{\mathrm{p}}$-values. ${ }^{[\mathrm{a}]}$

\begin{tabular}{ccccc}
\hline Entry & Cobalt(III) complex & Substituent & $E_{\mathrm{p}, \text { ox }}(\mathrm{V} v s . \mathrm{SCE})$ & $\sigma_{\mathrm{p}}[316 \mathrm{a}]$ \\
\hline 1 & $\mathbf{1 9 0}$ & $\mathrm{H}$ & 1.01 & 0 \\
2 & $\mathbf{1 9 6}$ & $\mathrm{Me}$ & 0.96 & -0.17 \\
3 & $\mathbf{1 9 7}$ & $i-\mathrm{Pr}$ & 0.92 & -0.15 \\
4 & $\mathbf{1 9 8}$ & $\mathrm{OMe}$ & 0.91 & -0.27 \\
5 & $\mathbf{1 9 9}$ & $\mathrm{CN}$ & 1.16 & +0.66 \\
6 & $\mathbf{2 0 0}$ & $\mathrm{CF}_{3}$ & 1.08 & +0.54 \\
7 & $\mathbf{2 0 1}$ & $\mathrm{F}$ & 1.05 & +0.06 \\
\hline
\end{tabular}

[a] Cyclic voltammograms were recorded in $\mathrm{MeOH}$ with $n-\mathrm{Bu}_{4} \mathrm{NPF}_{6}(0.1 \mathrm{M})$ as the electrolyte. A GC working electrode and a saturated calomel electrode (SCE) reference electrode were used.

Next, the measured anodic peak potentials were plotted against the Hammett-values $\sigma_{\mathrm{p}}$ of the respective substituents (Figure 23). A linear correlation was uncovered with a reaction constant of $\rho=0.24 \mathrm{~V} \pm 0.04 \mathrm{~V}$. This finding illustrates that the electronic properties of the benzamide moiety have only little effect on the oxidation potential of the complex and thus minor inductive influence on the cobalt center. Nevertheless, the results indicate that electron-donating substituents stabilize the putative cobalt(III/IV) oxidation event. ${ }^{[1561]}$ 


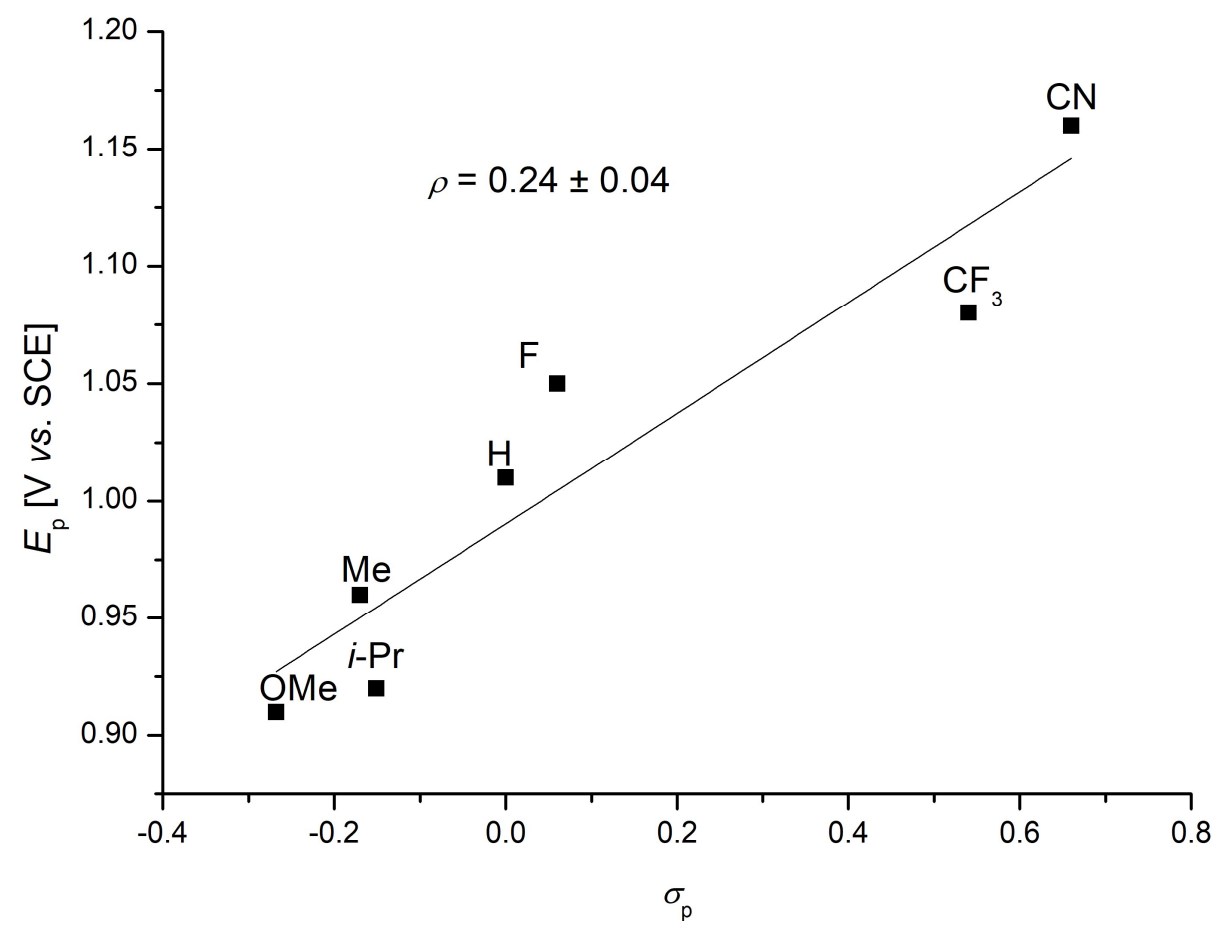

Figure 23. Hammett-Zuman plot of differently substituted cobalta(III)cycles 190, 196201.

Based on previous results for cobaltaelectro-catalyzed $\mathrm{C}-\mathrm{O}$ formations ( $c f$. Section 3.1), electron-donating substituents resulted in higher yields, whereas electron-withdrawing groups led to decreased product yields. ${ }^{[249-250,260]}$ Intuitively, it would be expected that electron-withdrawing substituents on the benzamide $\mathbf{3 4}$ would accelerate the reductive elimination step, since the high-valent cobalt complex would be destabilized. Based on the aforementioned findings, it is likely that the electron-donating substituents lower the activation energy of the oxidation event, prior to reductive elimination, thus being supportive of the oxidation ${ }^{[285]}$ as the bottleneck of the catalytic cycle. However, since the substrate $\mathbf{3 4}$ acts as an ancillary ligand, as well as the product forming carbon ligand, additional experiments with e.g. differently substituted pyridine- $N$-oxides or distinguishable ligand systems, ${ }^{[318]}$ collectively with computational studies, would be required to unambiguously evaluate the ligand effect on the cobaltaelectro-catalyzed $\mathrm{C}-\mathrm{H}$ activation.

\subsubsection{Cobaltaelectro-Catalyzed C-H Arylation via Twofold C-H Scission}

During the synthesis of the cyclometalated cobalt(III) complexes 190, 196-201, a considerable amount of unidentified paramagnetic byproducts were typically observed. Since the stoichiometric $\mathrm{C}-\mathrm{H}$ cobaltation reactions were performed in $\mathrm{MeCN}$ as an inert 
solvent, it was hypothesized that electrochemical oxidation of the cobalta(III) cycle to a cobalt(IV) species might result in an oxidation-induced dimerization of two of the coordinating substrates 34. ${ }^{[146]}$ Although, cyclovoltammetric studies in MeCN revealed a reversible oxidation event at scan rates higher than $900 \mathrm{mV} \cdot \mathrm{s}^{-1}$, at slower scan rates, the anodic event became irreversible, thus being indicative of chemical follow-up reactions ( $c f$. Figure 18). ${ }^{[309]}$ Unfortunately, attempts to recrystallize the crude paramagnetic mixture from $\mathrm{MeOH}$ failed to produce suitable crystals for X-ray diffraction analysis and only small amounts of greenish needles were isolated, thus revealed the octahedral cobalt(II) complex 202 (Figure 24).

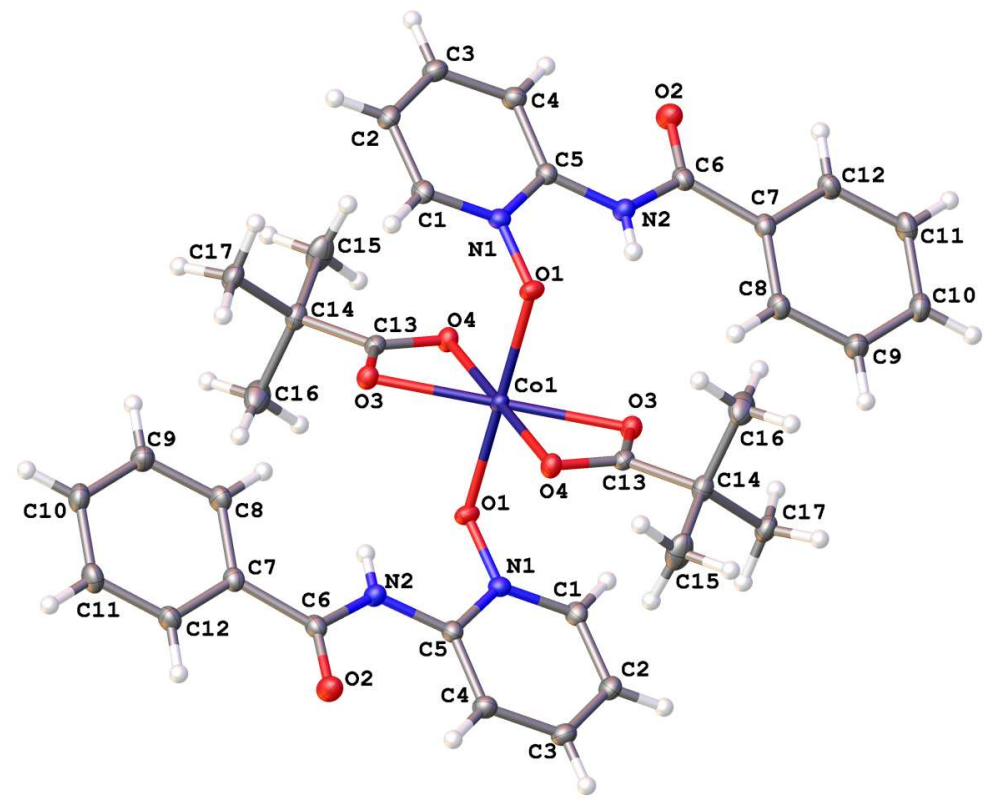

Figure 24. Molecular structure of $\mathbf{2 0 2}$ with thermal ellipsoids at 50\% probability level.

When the crude reaction mixture was purified by column chromatography, a purple fraction was separated. Based on ESI-MS data, a major signal at $m / z=719$ was indicative of the homocoupled product $[\mathbf{2 0 3}+\mathrm{Na}]^{+}$. To unambiguously characterize the isolated material, crystals suitable for X-ray diffraction analysis were grown via slow diffusion of $n$-hexane into a saturated solution of $\mathbf{2 0 3}$ in $\mathrm{CH}_{2} \mathrm{Cl}_{2}$ (Figure 25). The solid state structure revealed a pentacoordinated, distorted trigonal bipyramidal cobalt(II) complex 203 with a newly formed $\mathrm{C}-\mathrm{C}$ bond between two of the coordinated benzamides (C8-C9). Similar to the cyclometalated cobalt(III) complex 190, a third substrate 34a was found to coordinate via the pyridine- $N$-oxide group. Interestingly, when the crystallization was performed in $\mathrm{MeOH}$ as the solvent, the mono-O-coordinated substrate $\mathbf{3 4 a}$ was dissociated and a substrate-bridged polymeric cobalt(II) complex 204 was formed (Figure 26). 


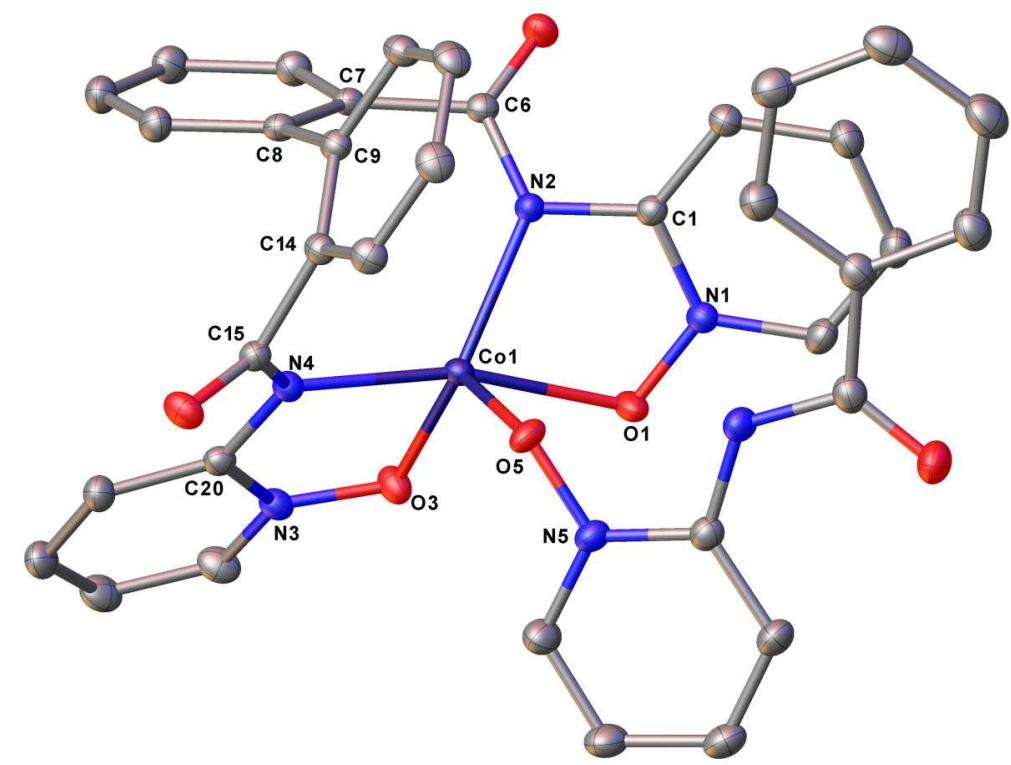

Figure 25. Molecular structure of $\mathbf{2 0 3}$ with thermal ellipsoids at 50\% probability level. The hydrogen atoms are omitted for clarity.

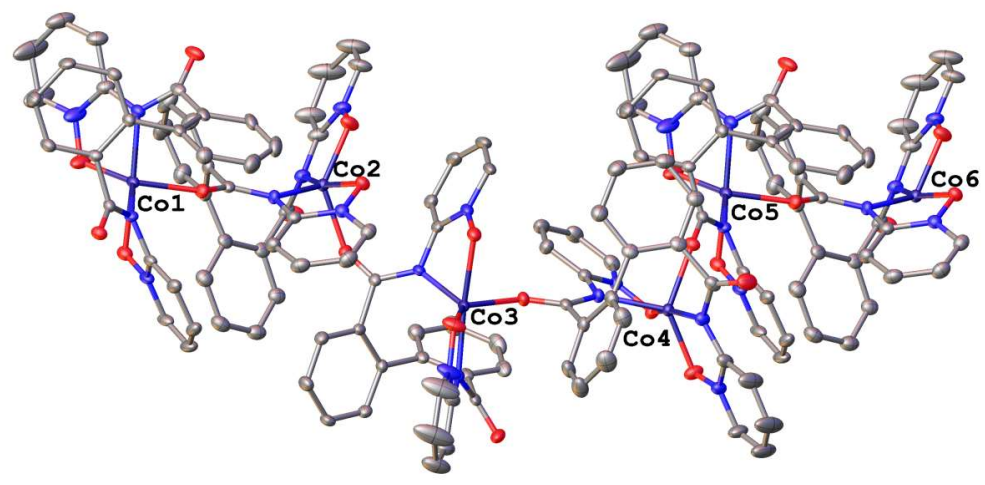

Figure 26. Molecular structure of 204 with thermal ellipsoids at 50\% probability level. The hydrogen atoms are omitted for clarity.

Inspired by these findings, attempts to optimize the cobaltaelectro-mediated two-fold $\mathrm{C}-\mathrm{H}$ arylation were conducted (Table 26). However, the yield of the dimerized product $\mathbf{2 0 3}$ was relatively low with either constant current or constant potential electrolysis (entries 1 and 2). After considerable experimentation, it was found that increased levels of homocoupling occurred at higher reaction temperatures and constant current electrolysis (entries 3 and 4). Also, solvents other than polar-aprotic $\mathrm{MeCN}$ proved to be unsuitable for the desired oxidation-induced reductive dimerization (entries 5-9). Noteworthily, with EtOH as the solvent, $70 \%$ of the $\mathrm{C}-\mathrm{H}$ ethoxylated product 36aa was isolated (entry 8 ), whereas no $\mathrm{C}-\mathrm{H}$ oxygenation was observed with a tertiary alcohol (entry 9). 
Table 26. Optimization of the twofold $\mathrm{C}-\mathrm{H}$ activation. ${ }^{[\mathrm{a}]}$

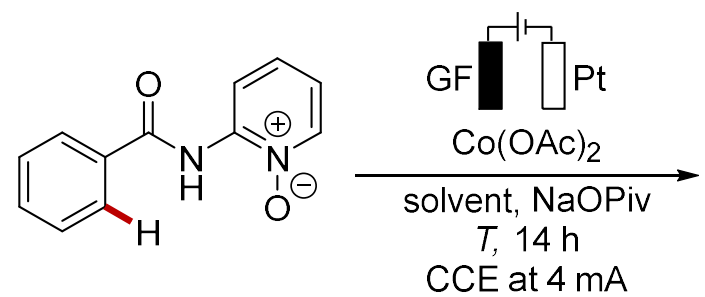

$34 a$

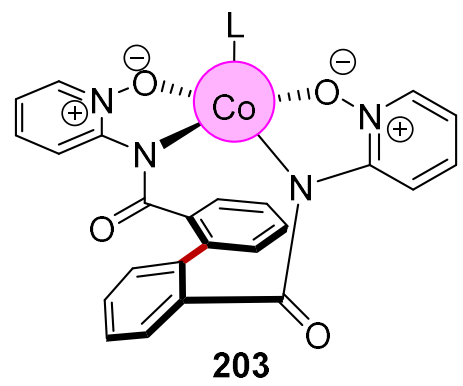

203

\begin{tabular}{cccc}
\hline Entry & Solvent & $T\left[{ }^{\circ} \mathrm{C}\right]$ & Yield [\%] \\
\hline 1 & $\mathrm{MeCN}$ & 25 & $25^{[\mathrm{b}]}$ \\
2 & $\mathrm{MeCN}$ & 25 & 20 \\
3 & $\mathrm{MeCN}$ & 40 & 32 \\
$\mathbf{4}$ & $\mathbf{M e C N}$ & $\mathbf{6 0}$ & $\mathbf{7 3}$ \\
5 & $\mathrm{CH}_{2} \mathrm{Cl}$ & 40 & $---{ }^{[\mathrm{c}]}$ \\
6 & $\mathrm{HFIP}$ & 40 & --- \\
7 & $\mathrm{GVL}$ & 60 & $\operatorname{traces}^{[\mathrm{c}]}$ \\
8 & $\mathrm{EtOH}$ & 40 & $\operatorname{traces}^{[\mathrm{d}]}$ \\
9 & $t-\mathrm{AmOH}$ & 40 & $\operatorname{traces}^{[\mathrm{c}]}$ \\
\hline
\end{tabular}

[a] Reaction conditions: 34a $(0.50 \mathrm{mmol}), \mathrm{Co}(\mathrm{OAc})_{2} \cdot 4 \mathrm{H}_{2} \mathrm{O}$ (0.50 equiv), NaOPiv (2.00 equiv), solvent $(5.0 \mathrm{~mL}), 14 \mathrm{~h}$, constant current electrolysis at $4 \mathrm{~mA}$, undivided cell, graphite felt anode, Pt-plate cathode. Isolated yields are given. [b] $\mathrm{CPE}$ at $1.4 \mathrm{~V}\left(v s . \mathrm{Ag} / \mathrm{Ag}^{+}\right)$. [c] $n-\mathrm{Bu}_{4} \mathrm{NPF}_{6}(1.00$ equiv) was added as a conducting salt. [d] 2-(2-Ethoxybenzamido)pyridine-1-oxide (36aa) was isolated in 70\% yield. ${ }^{[249]}$

The biphenyl backbone of the dimerized product $\mathbf{2 0 3}$ served as a strong linkage within the tetradentate ligand and thus formed a matching cavity for the cobalt(II) metal center. Attempts to demetalate and to release the homocoupling product $\mathbf{2 0 5}$ proved challenging and commonly used methods failed, such as acidic workup (aq. $\mathrm{HCl}(1 \mathrm{M})$ ) or washings with aqueous solutions of chelating sodium tartrate. Finally, it was found that the highlypolar demetalated product 205 could be isolated by stirring a solution of cobalt(II) complex 203 in $\mathrm{CH}_{2} \mathrm{Cl}_{2}$ for $12 \mathrm{~h}$ at $25^{\circ} \mathrm{C}$, with an aqueous EDTA solution (Scheme 71). 


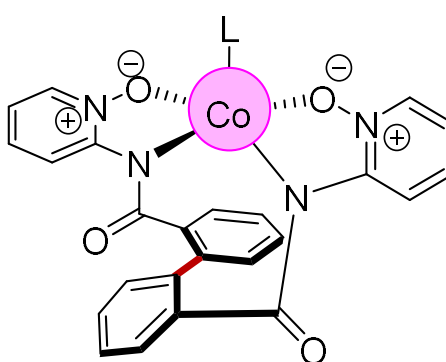

203<smiles>O=C(Nc1cccc[n+]1[O-])c1ccccc1-c1ccccc1NC(=O)c1cccc[n+]1[O-]</smiles>

205: $28 \%$

Scheme 71. Demetalation of the dimerized cobalt(II) complex 203.

\subsubsection{Catalyst Inhibition Studies}

The knowledge of possible catalyst deactivation pathways is of key importance to improve the efficacy of a catalyst and to decrease the required catalyst loadings. ${ }^{[319]}$ However, currently, there is only limited knowledge about factors controlling the catalyst lifetime in $\mathrm{Cp}^{*}$-free cobalt-catalyzed $\mathrm{C}-\mathrm{H}$ activations, including cobalta-electrocatalysis. Therefore, the isolated dimerized chelator 205 was investigated as an inhibitor in established cobaltaelectro-catalyzed $\mathrm{C}-\mathrm{H}$ activations (Scheme 72).

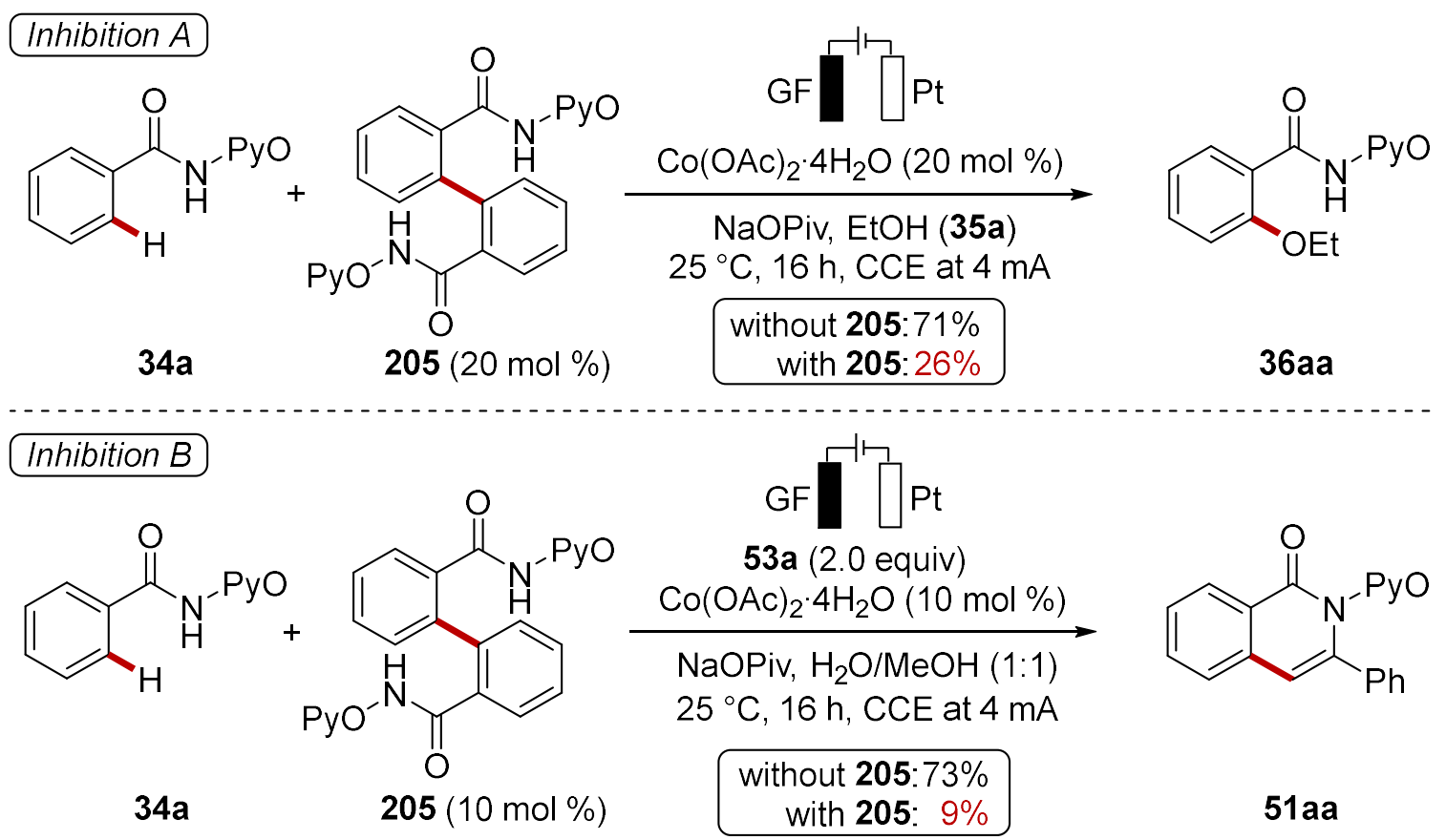

Scheme 72. Catalyst poisoning: A) Cobaltaelectro-catalyzed $\mathrm{C}-\mathrm{H}$ alkoxylation. B) Cobaltaelectro-catalyzed $\mathrm{C}-\mathrm{H}$ annulation with phenylacetylene (53a).

The catalyst poisoning studies were exemplified for the cobaltaelectro-catalyzed $\mathrm{C}-\mathrm{H}$ alkoxylation with ethanol (35a) (Scheme 72a) and for the cobaltaelectro-catalyzed $\mathrm{C}-\mathrm{H}$ annulation with phenylacetylene (51a) as the coupling product (Scheme 72b). Strikingly, 
both reactions showed a strong inhibition of the cobalta-electrocatalysis upon addition of the homocoupled product 205. Presumably, chelated cobalt(II) complex 203 was rapidly formed in-situ and thereby inhibited the regeneration of the active cobalt(III) species upon anodic oxidation. The presented ESI-MS studies (vide supra) also revealed that the dimerized cobalt(II) complex $\mathbf{2 0 3}$ was formed during the electrolysis, thus supporting the hypothesis that $\mathbf{2 0 3}$ is a possible off-cycle product.

\subsubsection{Proposed Catalytic Scenarios}

Based on the experimental studies summarized above ${ }^{[309]}$ and the mechanistic data obtained within previous projects, ${ }^{[112,249-252,260]}$ a plausible mechanistic rational for cobaltaelectro-catalyzed $\mathrm{C}-\mathrm{H}$ alkoxylations and $\mathrm{C}-\mathrm{H}$ annulations with alkynes $\mathbf{5 3}$ and allenes 75 can be proposed (Scheme 73). Initially, salt metathesis forms the cobalt(II) pivalate species, which upon deprotonation of substrate $\mathbf{3 4}$ produces the cobalt(II) complex 192 (A) ${ }^{[320]}$ Anodic oxidation (B), followed by concomitant $\mathrm{C}-\mathrm{H}$ activation via a baseassisted internal electrophilic-type substitution (BIES $)^{[35,41]}$ mechanism generates the key cyclometalated cobalt(III) complex 190 (C). At this stage of catalysis, the cobaltaelectrocatalyzed $\mathrm{C}-\mathrm{H}$ activation can divert into two different reaction pathways. In case of the $\mathrm{C}-$ $\mathrm{H} / \mathrm{N}-\mathrm{H}$ annulation reactions (Scheme 73, upper cycle), ligand substitution/coordination of the $\pi$-containing substrate $\mathbf{5 3}$ occurs (D), followed by migratory insertion into the Co-C bond to furnish the seven-membered cobaltacycle $195(\mathbf{E}) \cdot{ }^{[112,251]}$ Finally, reductive elimination releases the desired isoquinolones $\mathbf{5 1}$ and the reduced cobalt(I) complex 207 (F). Facile anodic oxidation and ligand coordination regenerates the cobalt(II) complex 192 (G). Thus, the cobaltaelectro-catalyzed alkyne $\mathbf{5 1}$ or allene $\mathbf{7 5}$ annulation reactions can be described to follow a cobalt(II/III/I) catalytic manifold. In the case of cobaltaelectrocatalyzed $\mathrm{C}-\mathrm{H}$ oxygenation reactions, a distinctively different catalytic scenario is proposed (Scheme 73, bottom cycle). Here, alcohol 35 coordination/ligand substitution at the key organometallic cobalta(III)cycle 190, followed by proton-coupled electron transfer $(\mathrm{PCET})^{[321]}$ via anodic oxidation is proposed to furnish the transient cobalt(IV) complex $208(\mathbf{H}) \cdot{ }^{[154 b]}$ Thereafter, facile reductive elimination (I) leads to intermediate 209. Finally, proto-demetalation $(\mathbf{J})$ delivers the desired alkoxylated product $\mathbf{3 6}$ and the reduced cobalt(II) complex 192. Conclusively, the cobaltaelectro-catalyzed C-O formation follows an oxidation-induced reductive elimination pathway via a cobalt(III/IV/II) manifold. For both catalytic scenarios, cathodic generation of hydrogen closes the electrical circuit and obviates the need for chemical oxidants. 


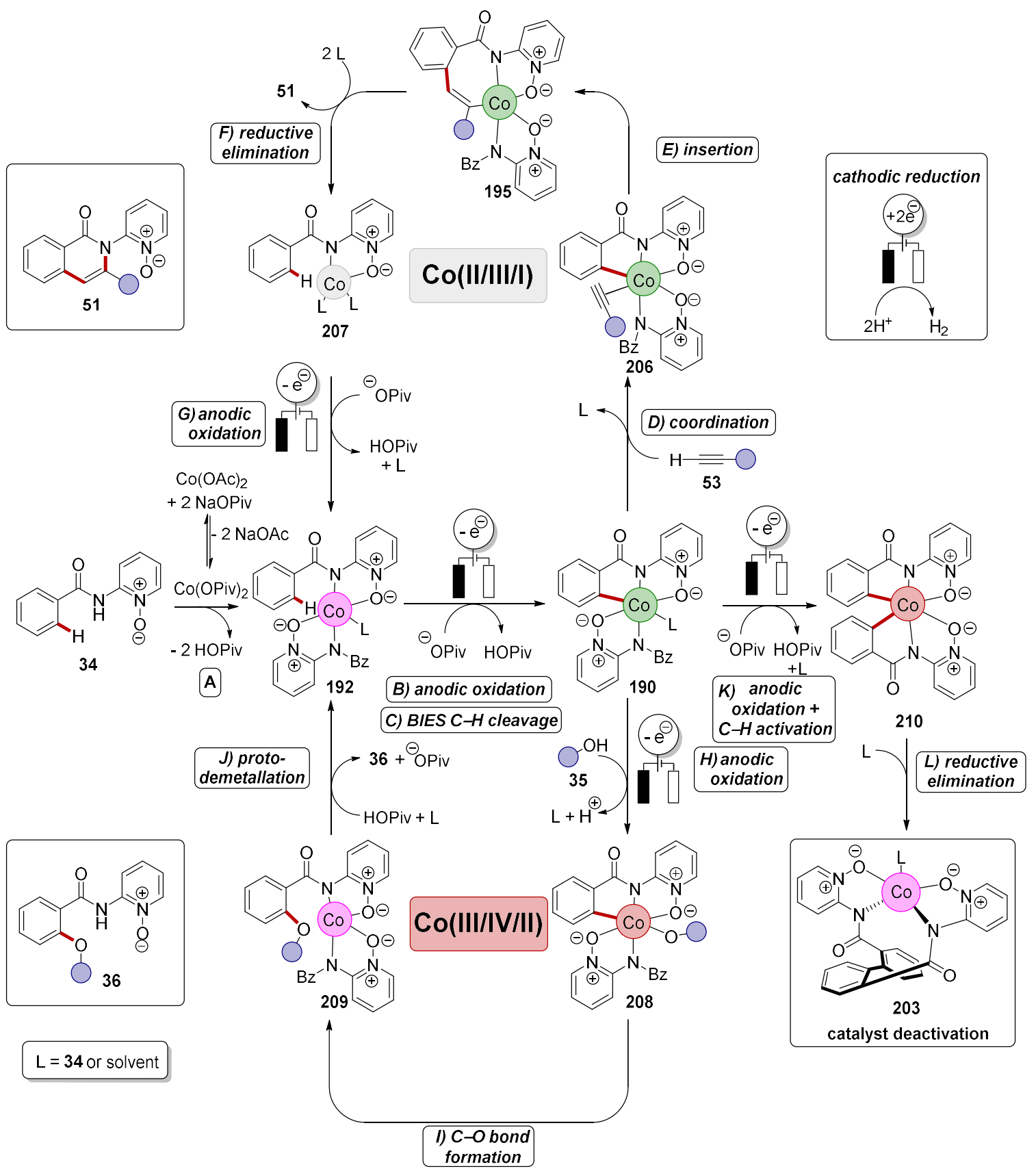

Scheme 73. Proposed general mechanism for cobaltaelectro-catalyzed C-H annulation reactions (top) and $\mathrm{C}-\mathrm{H}$ alkoxylation reactions (bottom). Color code: grey $\mathrm{Co}(\mathrm{I})$; purple $\mathrm{Co}(\mathrm{II})$; green $\mathrm{Co}(\mathrm{III})$; red $\mathrm{Co}(\mathrm{IV})$.

Notably, anodic oxidation of cobalt(III) complex 190 without any additional coupling partner furnishes the high-valent cobalt(IV) off-cycle intermediate 210, likely featuring two $\mathrm{Co}-\mathrm{C}$ bonds $(\mathbf{K})$. Likewise, facile reductive elimination generates the dimerized $\mathrm{C}-\mathrm{C}$ coupled cobalt(II) complex 203 (L). However, the $\mathrm{N}, \mathrm{O}$-tetradentate ligand operates as a strong chelator and thus stops the cobaltaelectro-catalyzed $\mathrm{C}-\mathrm{H}$ activation. Here, the mechanistic insights on the biaryl coupling ${ }^{[322]}$ would benefit from detailed DFT studies ${ }^{[100 \text {, }}$ 
$112,260,323]$ to support the proposed heterolytic $\mathrm{C}-\mathrm{C}$ formation via twofold $\mathrm{C}-\mathrm{H}$ activation. Alternative mechanistic scenarios, including homolytic $\mathrm{C}-\mathrm{H}$ cleavage via cobalt(IV) intermediates, have also been proposed based on DFT-calculations. ${ }^{[324]}$ Also, instead of a second $\mathrm{N}, \mathrm{O}$-bidentate substrate $\mathbf{3 4}$, additional pivalate coordination cannot be excluded.

After the presented work was published, a theoretical study on the mechanism of cobaltcatalyzed twofold $\mathrm{C}-\mathrm{H}$ activations with an 8-aminoquinoline directing group $\mathbf{2 6}$ was reported by Musaev and Macbeth. ${ }^{[325]}$ Interestingly, similar cobalt(II) and cobalt(III) complexes could be synthesized and analyzed. Experimental attempts for the synthesis of the dimerized products 94 were performed under harsh reaction conditions at $100{ }^{\circ} \mathrm{C}$, in DCE as the solvent, and with super-stoichiometric amounts of silver oxidants. The authors also supported the mobile ligand environment on the cobalt complexes and stated that fast ligand exchange between substrate $\mathbf{2 6}$ and pivalate was observed and theoretically feasible. Most notably, the catalytic scenario proposed by the authors was consistent of two consecutive CMD-type $\mathrm{C}-\mathrm{H}$ scission events for the oxidative cobalt-mediated dehydrogenative aryl-aryl coupling. Finally, DFT-calculations confirmed that the $\mathrm{C}-\mathrm{C}$ coupling was the rate-determining step of the overall reaction.

Also, Maseras and Pérez-Temprano recently reported that oxidation-induced reductive elimination likewise applies for $\mathrm{Cp} * \mathrm{Co}(\mathrm{III})$-catalyzed $\mathrm{C}-\mathrm{S}$ formations. Here, the generation of a key-cyclometalated cobalt(IV) species was supported by stoichiometric experiments, CV studies, and DFT calculations. ${ }^{[74 a]}$ 


\subsection{Mechanistic Studies of Metallaelectro-Catalyzed C-H} Activations

Electrochemistry offers a plethora of analytic tools to study the reaction mechanism of molecular transformations. ${ }^{[170 \mathrm{~g}, 209 \mathrm{c}, 263,278 \mathrm{a}, 326]}$ A profound knowledge of the respective redox potentials of the individual reaction components, as well as their interplay within the catalytic system is of key importance to delineate the catalyst's working mode, but also for the design of new catalysts and synthetic concepts. Here, a variety of electroanalytic studies were performed during the course of this thesis, with a major focus on cyclic voltametric studies, which are briefly summarized within the following chapter.

After the success of the proof-of-concept study on cobaltaelectro-catalyzed $\mathrm{C}-\mathrm{H}$ alkoxylation, ${ }^{[249]}$ Dr. C. Tian and L. Massignan have expanded the scope of the methodology on cobaltaelectro-catalyzed $\mathrm{C}-\mathrm{H}$ annulation reactions with alkynes $\mathbf{5 3}$ ( $c f$. Scheme 41). ${ }^{[250]}$ Within this study, I have performed cyclic voltametric studies to gain further insights into the catalyst modus operandi. The results of these experiments were related to the ones previously discussed in the chapters on cobaltaelectro-catalyzed $\mathrm{C}-\mathrm{H}$ allene and alkyne annulations in biomass-derived glycerol and are thus not further described (vide supra).

Further mechanistic studies have been performed in cooperation with M. Stangier and Dr. $Y$. Qiu on the first iridaelectro-catalyzed $\mathrm{C}-\mathrm{H}$ oxidation through weak $O$-coordination (Scheme 74). ${ }^{[327]}$ It is noteworthy that the reaction design, optimization, scope and other mechanistic experiments, including DFT-calculations by Dr. J. C. A. Oliveira, were investigated by the co-authors. In contrast to similarly reported rhodaelectro-catalyzed $\mathrm{C}-\mathrm{H}$ activations, ${ }^{[328]}$ the key for high levels of chemoselectivity control and excellent functional group tolerance within the iridaelectro-catalyzed manifold, was proposed to result from the employment of an additional redox catalyst 136. ${ }^{[220 \mathrm{q}, 224 \mathrm{~b}]}$ Within this project, a major focus was to unravel the mechanistic scenario of this novel mediated cooperation, between the benzoquinone redox mediator $\mathbf{1 3 6}$ and the active iridium(III) catalyst. ${ }^{[329]}$ First, it was found that the reduction and oxidation of the benzoquinone 136 occurred at less negative potentials, compared to the iridium(III/II) redox event (Scheme 74 , green $v s$. red). Second, the mixture of both catalysts resulted in irreversible redox behavior of the redox mediator 136, thus being indicative of sufficient electron transfer to the iridium complex (blue). However, the exact nature of the electron transfer step between 
the iridium complex 212 and the redox mediator 136 is still uncertain and might occur via pre-coordination of the benzoquinone $136 .{ }^{[330]}$

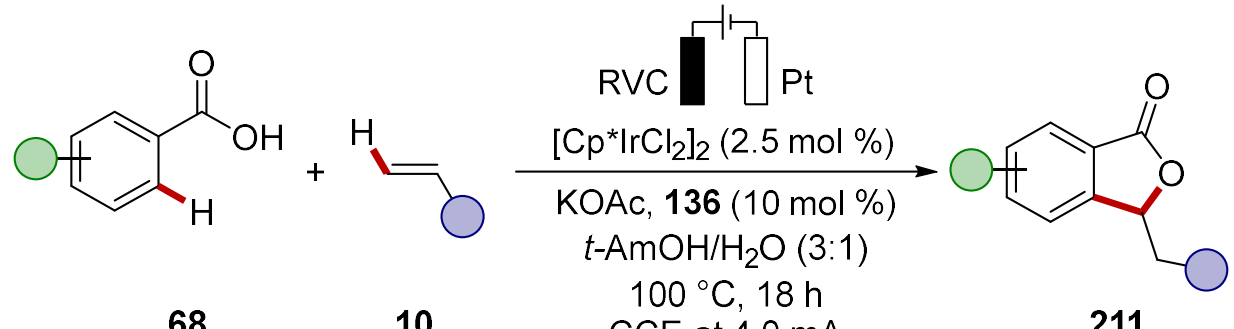

$68-10$

$\mathrm{CCE}$ at $4.0 \mathrm{~mA}$

211

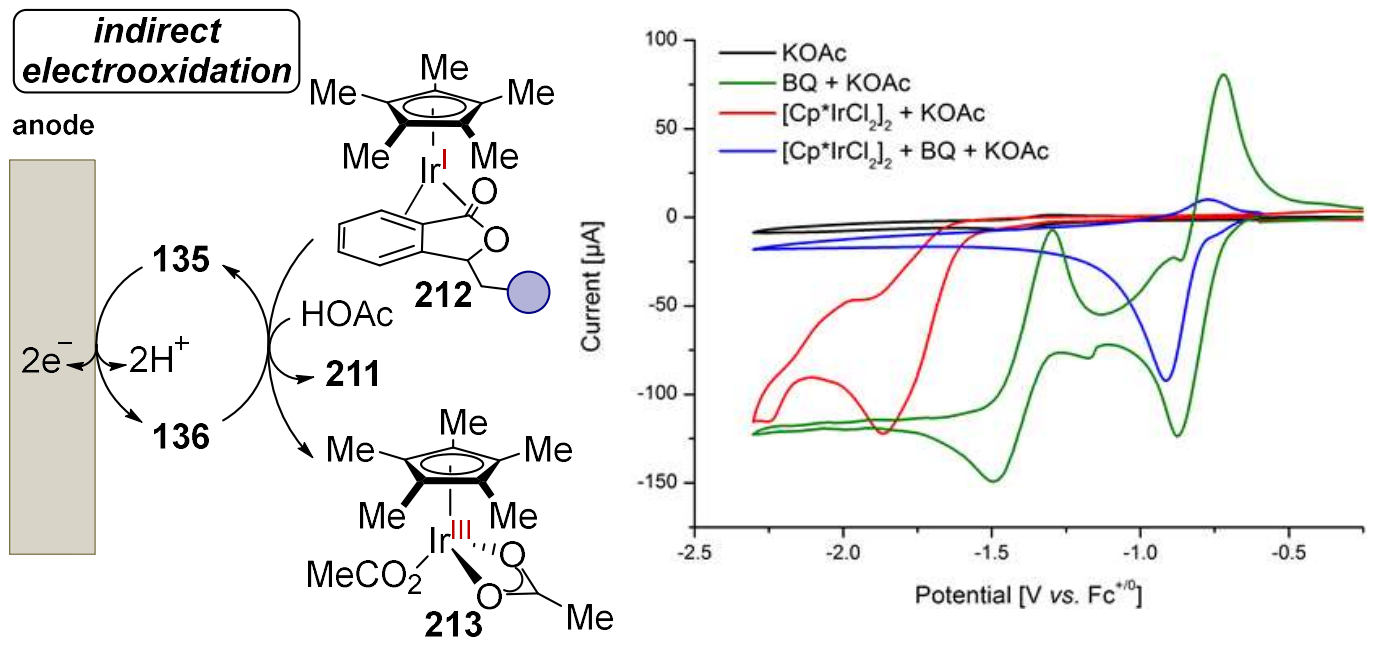

Scheme 74. Cooperated, indirect iridaelectro-catalyzed $\mathrm{C}-\mathrm{H}$ alkenylations of benzoic acids 68 with acrylates or acryl amidates 10. Cyclic voltammograms at $25^{\circ} \mathrm{C}$ and $100 \mathrm{mV} \cdot \mathrm{s}^{-1}$ using $\mathrm{MeCN}$ and $n-\mathrm{Bu}_{4} \mathrm{NPF}_{6}(0.1 \mathrm{M})$ as the electrolyte and a $\mathrm{GC}$ working electrode. a) Benzoquinone 136 (green), b) $\left[\mathrm{Cp}^{*} \mathrm{IrCl}_{2}\right]_{2}$ (red); and a mixture of a) and b) (blue).

In addition to metallaelectro-catalyzed transformations, also catalyst- and reagent-free electrochemical $\mathrm{C}-\mathrm{H}$ amination of azoles have been mechanistically examined. ${ }^{[331]}$ Within the study, mainly developed and performed by Dr. Y. Qiu, J. Struwe and Dr. J. C. A. Oliveira, I assisted the mechanistic assessment with detailed CV experiments. The experiments were performed together with $J$. Struwe during her master thesis and are thus not further discussed within this thesis.

Electroanalytical tools have been particularly effective when combined with other analytical techniques, such as in-operando IR or NMR spectroscopic analysis. Hence, the following cooperated project will be described in more detail to highlight the advantages of both analytical techniques for the mechanistic study of highly reactive reaction intermediates. 


\subsubsection{Mechanistic Studies for Ruthenaelectro-Catalyzed C-H Oxygenations}

The synthesis of phenol derivatives via direct $\mathrm{C}-\mathrm{H}$ oxygenation has become a valuable tool in modern organic synthesis and has particularly proven to be effective via ruthenium catalysis, with key contributions by Ackermann, ${ }^{[332]}$ among others. ${ }^{[319 a, 333]}$ The oxidative hydroxylation strategy, however, generally suffers from the employment of stoichiometric amounts of chemical oxidants and harsh reaction conditions, thus lowering the atomeconomy and ecological footprint of the overall process. ${ }^{[333 a, 334]}$ Previous studies demonstrated that the chemoselectivity of the ruthenium-catalyzed $\mathrm{C}-\mathrm{H}$ activation strongly depended on the terminal oxidant ${ }^{[335]}$ and that hypervalent-iodine(III) reagents ${ }^{[336]}$ solely resulted in intermolecular $\mathrm{C}\left(\mathrm{sp}^{2}\right)-\mathrm{H}$ hydroxylation. ${ }^{[332 \mathrm{~h}]}$ Based on these findings, and the recent achievements in electrochemical generation of hypervalent-iodine reagents from simple iodoarenes $\left.\mathbf{2 1 5},{ }^{[290 \mathrm{~d},} 299 \mathrm{~b}, 337\right]$ as well as the recent success in ruthenaelectrocatalysis, ${ }^{[243 b, 244 \mathrm{~d}-\mathrm{f}, 338]}$ it was reasoned to orchestrate both techniques within a userfriendly and resource-economic dual-catalytic manifold (Scheme 75). ${ }^{[339]}$ L. Massignan and Dr. X. Tan have developed, optimized and evaluated the ruthenaelectro-catalyzed $\mathrm{C}-\mathrm{H}$ hydroxylation in terms of substrate scope and mechanistic studies by experiment, other than the results presented below. Dr. R. Kuniyill performed DFT-calculations ${ }^{[340]}$ on the presented ruthena-electrocatalysis.

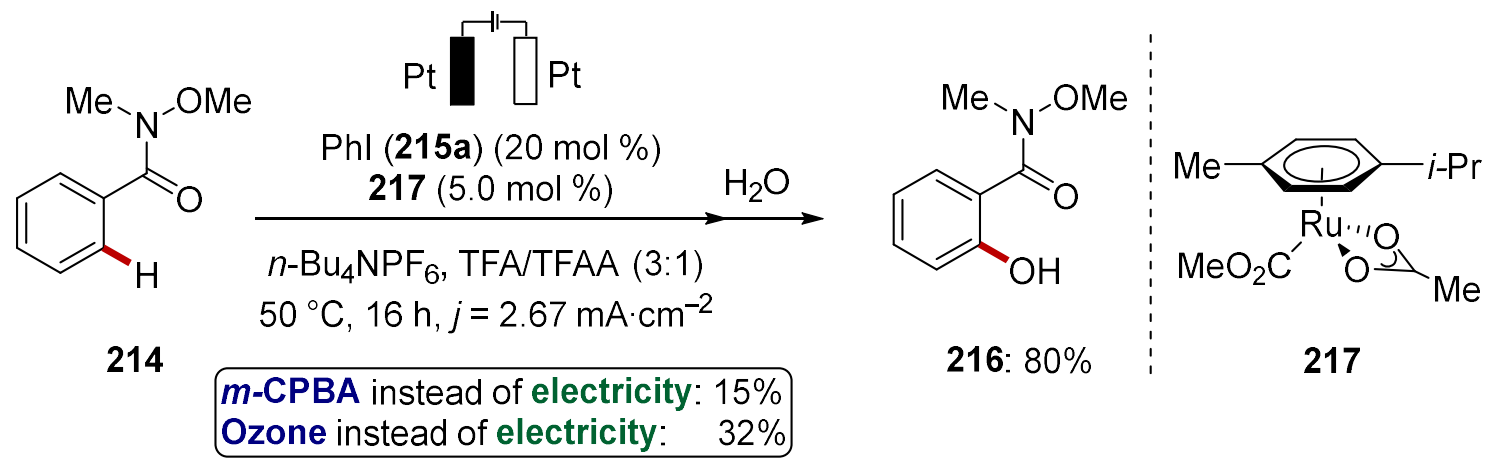

Scheme 75. Dual electrocatalysis in practice: iodine(III)/ruthenium(II) electrocatalytic $\mathrm{C}-\mathrm{H}$ hydroxylation.

It is noteworthy that instead of weakly-coordinating amides 214, also ketones and pyrazols were fully applicable as a directing group under the dual electrocatalytic conditions. Also, directing group-free remote $\mathrm{C}-\mathrm{H}$ oxygenation ${ }^{[332 \mathrm{e}]}$ was likewise accomplished. ${ }^{[339]}$ If, however, strong chemical oxidants, such as meta-chloroperoxybenzoic acid (m-CPBA) or 
ozone, commonly employed for the synthesis of hypervalent iodine reagents, were used instead of electricity, the desired product formation was drastically diminished.

Mechanistic studies, performed by L. Massignan and Dr. X. Tan revealed a reversible $\mathrm{C}-\mathrm{H}$ cleavage in the ortho-position of the substrate 214, along with a minor kinetic isotopic effect (KIE) of $k_{\mathrm{H}} / k_{\mathrm{D}} \approx 1.6$. Also, competition experiments were indicative of a BIES C-H activation manifold. ${ }^{[35,41]}$ In contrast, when chemical oxidants were used instead of electricity, the $\mathrm{C}-\mathrm{H}$ activation step was found to be the rate-determining step. ${ }^{[32 \mathrm{e}, 332 \mathrm{f}]}$ This remarkable finding suggested that different mechanistic scenarios were operative within the two catalytic manifolds. Given the efficiency of the unprecedented dual electrocatalytic $\mathrm{C}-\mathrm{H}$ oxygenation system, the authors became particularly interested in the cooperative effect of the in-situ formed iodine(III) reagent and the ruthenium(II) complex 217, since both catalyst proved to be essential to deliver the oxygenated product 216. ${ }^{[339]}$ However, the strong acidic solvent conditions and the transiently formed electrocatalytic species made the quest for potential reaction intermediates a major challenge. To tackle this issue, anodic oxidation in continuous flow has recently proven to be an efficient and safe tool for the synthesis and study of hypervalent iodine reagents. ${ }^{[290 \mathrm{~d}, 290 \mathrm{j}]}$ With this in mind, the reaction profile of the anodic formation of different iodine(III) reagents 218 and 219 was studied by in operando flow ${ }^{1} \mathrm{H}-\mathrm{NMR}$ spectroscopy (Scheme 76). 


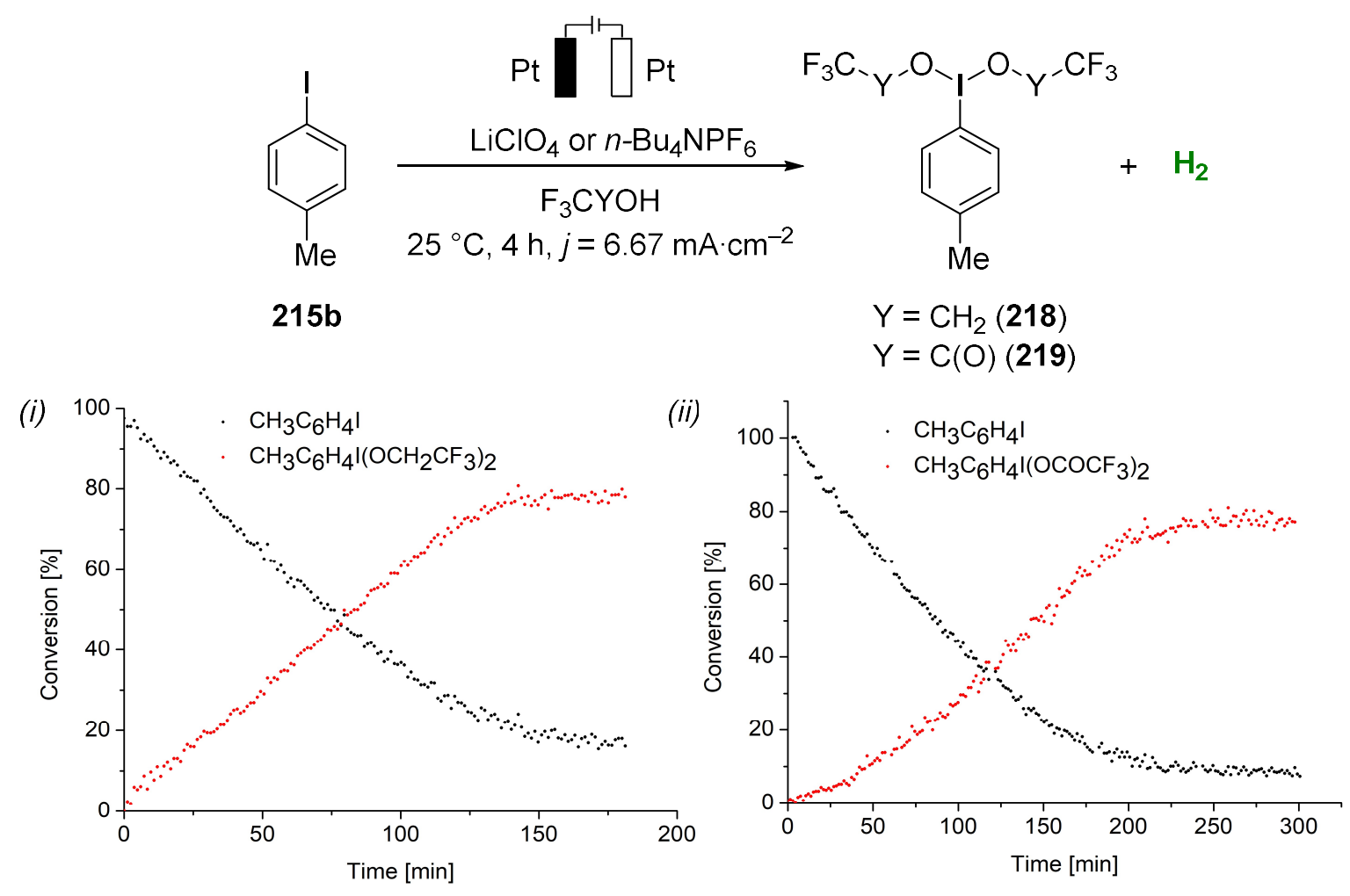

Scheme 76. In operando flow ${ }^{1} \mathrm{H}-\mathrm{NMR}$ studies for the formation of i) 218 and ii) 219 at $10 \mathrm{~mA}$ constant current electrolysis in trifluoroethanol (TFE) or trifluoroacetic acid (TFA), respectively. The conversion was determined by ${ }^{1} \mathrm{H}-\mathrm{NMR}$ analysis, using $\mathrm{CH}_{2} \mathrm{Br}_{2}$ as the internal standard.

Initially, the anodic conversion of 1-iodo-4-methylbenzene (215b) in trifluoroethanol (TFE) was monitored. Notably, almost full consumption of $\mathbf{2 1 5 b}$ was detected after $2.5 \mathrm{~h}$ at $10 \mathrm{~mA}$. Subsequently, the anodic generation of $\mathbf{2 1 9}$ from acidic TFA and $\mathbf{2 1 5} \mathbf{b}$ was found to occur with similar efficacy, with only slightly prolonged reaction times of $3 \mathrm{~h}$. The results were supportive of a facile anodic oxidation of the iodoarenes $\mathbf{2 1 5}$ and highlighted the stability of the formed products $\mathbf{2 1 8}$ and $\mathbf{2 1 9}$ under the reaction conditions. Thereafter, the redox properties of different haloarenes and substrate $\mathbf{2 1 4}$ were examined via cyclic voltammetry in TFA as the solvent (Figure 27). 

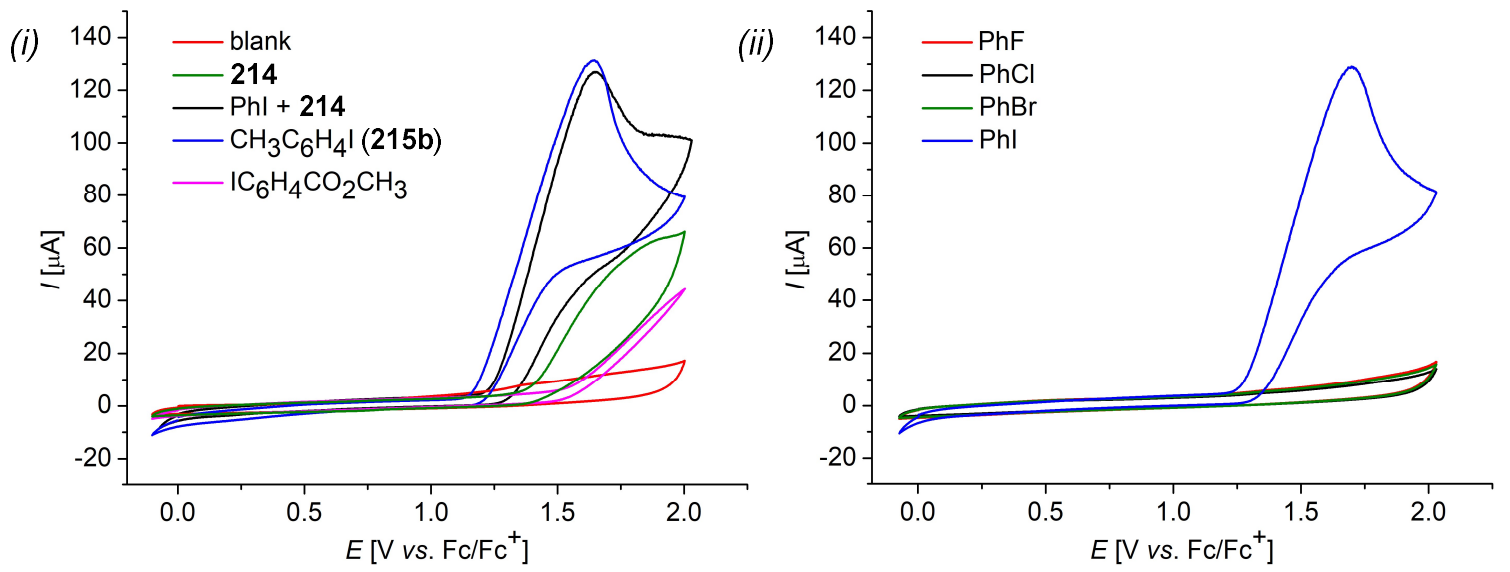

Figure 27. Cyclic voltammetry (TFA, $0.1 \mathrm{M} n-\mathrm{Bu}_{4} \mathrm{NPF}_{6}, 100 \mathrm{mV} \cdot \mathrm{s}^{-1}$ ) using $\mathrm{GC}$ as the working electrode. i) (red) blank; (green) 214; (black) iodobenzene (215a) + 214; (blue) 1-iodo-4-methylbenzene (215b); (magenta) methyl 4iodobenzoate. ii) (red) fluorobenzene; (black) chlorobenzene; (green) bromobenzene; (blue) iodobenzene (215a). The concentration of the individual analytes was $5.0 \mathrm{~mm}$.

The comparison of voltammograms of different reaction components highlighted the unique redox properties of electron-rich iodoarenes 215 in TFA with an onset potential of $E=1.25 \mathrm{~V} v s$. ferrocene for $\mathbf{2 1 5 b}$ (Figure 27i). Other haloarenes did not show any redox activity in the evaluated potential window (Figure 27ii). ${ }^{[341]}$ A mixture of iodobenzene 215a and 214 did not lead to significant changes in the voltammogram. This finding is in good agreement with the control experiments performed by L. Massignan, which showed no reaction in the absence of the ruthenium catalyst. However, the examination of the ruthenium complex 217 proved to be challenging under the corrosive solvent conditions and different solvent environments had to be investigated. Based on previous rutheniumcatalyzed C-H oxygenations with PIFA as a chemical oxidant, ${ }^{[332 \mathrm{~d}, 332 \mathrm{e}]}$ DCE was chosen as a less corrosive and more practical solvent for the evaluation of all reaction components and the well-defined ruthenium(II) carboxylate complex 217 (Figure 28). Cyclic voltammograms of the different reaction components revealed facile oxidation of the ruthenium complex 217 (Figure 28i). Notably, two irreversible oxidation reactions were detected $\left(E_{\mathrm{p}, \mathrm{ox}}=0.90 \mathrm{~V}\right.$ and $\left.E_{\mathrm{p}, \mathrm{ox}}=1.27 \mathrm{~V} v \mathrm{~s} . \mathrm{Fc} / \mathrm{Fc}^{+}, 100 \mathrm{mV} \cdot \mathrm{s}^{-1}\right)$, which were assigned to the formal $\mathrm{Ru}(\mathrm{II} / \mathrm{III})$ and $\mathrm{Ru}(\mathrm{III} / \mathrm{IV})$ oxidation, respectively. ${ }^{[342]}$ The irreversible character was further substantiated with voltammograms at different scan rates, being indicative of a fast chemical follow-up reaction (Figure 28ii). 
(i)

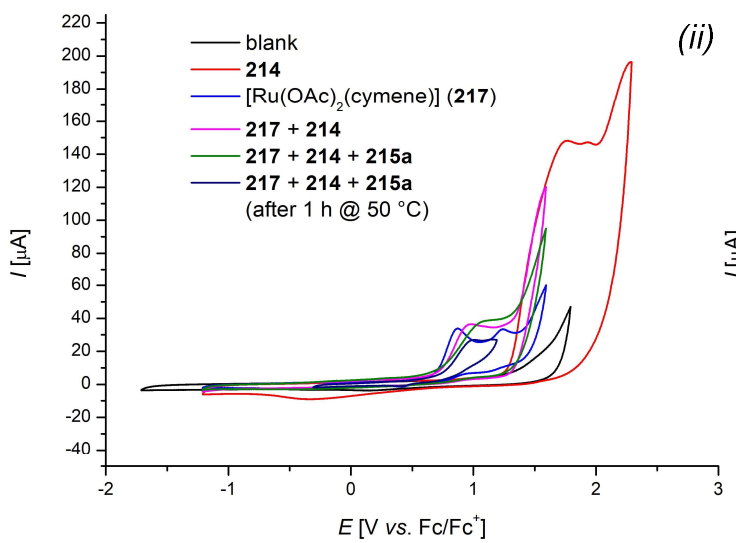

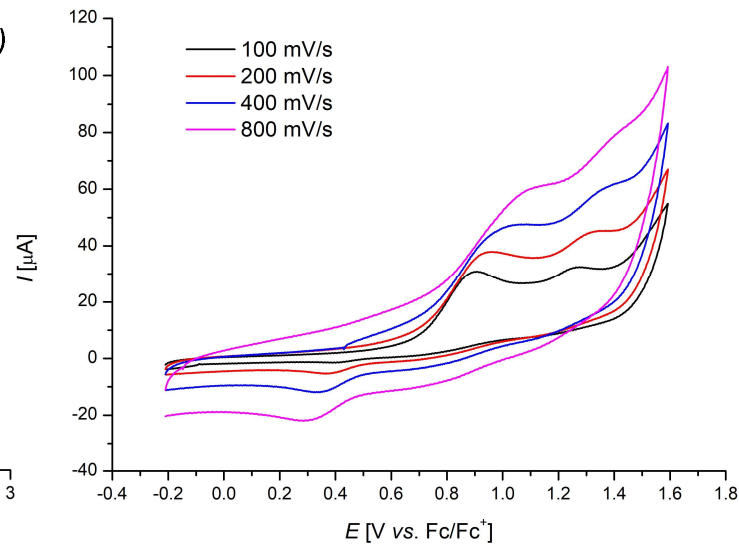

Figure 28. Cyclic voltammograms at (i) $100 \mathrm{mV} \cdot \mathrm{s}^{-1}$ and (ii) as depicted, using DCE and $n-\mathrm{Bu}_{4} \mathrm{NPF}_{6}(0.1 \mathrm{M})$ as the electrolyte and a $\mathrm{GC}$ working electrode; the concentration of the analytes was $5.0 \mathrm{mM}$; concentration of $\mathrm{Ru}(\mathrm{OAc})_{2}(p$ cymene) was 3.0 mM. i) (black) blank; (red) 214; (blue) [Ru]; (magenta) $[\mathrm{Ru}]+\mathbf{2 1 4}$; (green) $[\mathrm{Ru}]+\mathbf{2 1 4}+\mathbf{2 1 5 a}$; (navy blue) $[\mathrm{Ru}]+\mathbf{2 1 4}+\mathbf{2 1 5 a}$ was heated for $1 \mathrm{~h}$ at $50{ }^{\circ} \mathrm{C}$ and cooled to $25^{\circ} \mathrm{C}$ before the analysis was started. ii) $[\mathrm{Ru}]$ at different scan rates. $[\mathrm{Ru}]=\mathrm{Ru}(\mathrm{OAc})_{2}(p$-cymene) (217).

Given the key importance of a cyclometalated ruthenacycle $\mathrm{e}^{[25 \mathrm{a}, 343]}$ as a potential intermediate in the ruthenaelectro-catalyzed $\mathrm{C}-\mathrm{H}$ oxygenation, L. Massignan and Dr. A. Messinis have synthesized and fully characterized the cycloruthenated complex 221 (Scheme 77).

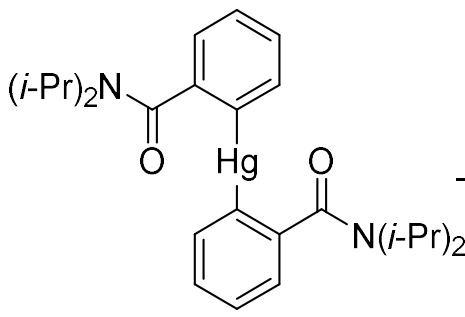

220

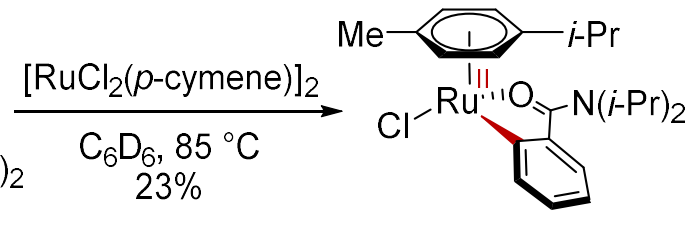

221

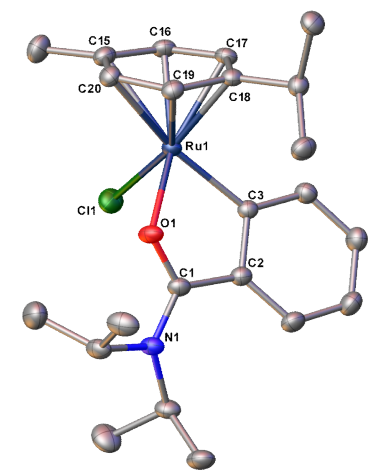

Scheme 77. Synthesis of cyclometalated ruthenium(II) complex 221. Reaction and characterization were performed by $\mathrm{Dr}$. A. Messinis.

With the well-defined ruthena(II)cycle 221 in hand, a detailed electroanalytical study was performed to unravel the nature of the oxidation reaction of the ruthenium complex. The study was initiated by cyclic voltammetry of complex 221 in DCE (Figure 29). 


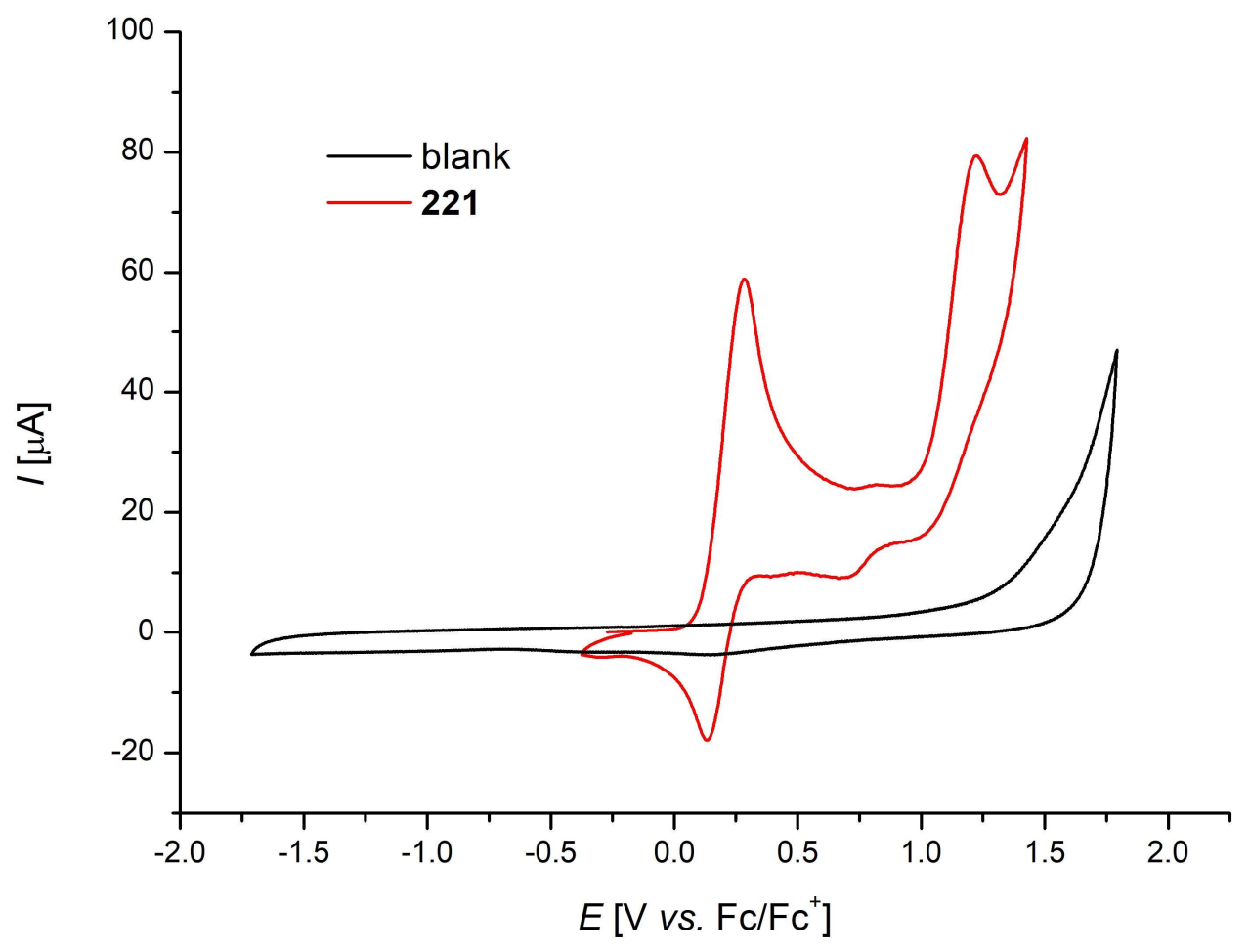

Figure 29. Cyclic voltammogram at $100 \mathrm{mV} \cdot \mathrm{s}^{-1}$ using DCE and $n-\mathrm{Bu}_{4} \mathrm{NPF}_{6}(0.1 \mathrm{M})$ as the electrolyte and a GC working electrode; concentration of complex 221 was $3.86 \mathrm{mM}$.

The voltammogram of complex 221 revealed a strong, reversible redox event at $E_{1 / 2}=$ $0.21 \mathrm{~V}\left(v s . \mathrm{Fc} / \mathrm{Fc}^{+} ; \Delta E_{\mathrm{p}}=170 \mathrm{mV}\right)$, a weak second redox event at $E_{1 / 2}=0.75 \mathrm{~V}\left(v s . \mathrm{Fc} / \mathrm{Fc}^{+}\right.$; $\left.\Delta E_{\mathrm{p}}=120 \mathrm{mV}\right)$ and an irreversible oxidation at $E_{\mathrm{p}}=1.22 \mathrm{~V}\left(v s . \mathrm{Fc} / \mathrm{Fc}^{+}\right)$. The latter, irreversible oxidation most likely corresponds to the coordinating chloride atom. To gain more information on the number of transferred electrons $n$ within the first redox event, ${ }^{[263]}$ a combined study by steady-state and non-steady state voltammetry was performed, following a known procedure. ${ }^{[344]}$ By comparison of the Randles-Ševčík equation (Eq. 3.4):

$$
i_{\mathrm{p}}=0.4463 \sqrt{\left(\frac{F^{3}}{R T}\right)} \operatorname{CAn}^{\frac{3}{2}} \sqrt{D} \sqrt{v}
$$

at $T=298 \mathrm{~K}$ :

$$
i_{\mathrm{p}}=\underbrace{\left(2.687 * 10^{5}\right) C A}_{X} n^{\frac{3}{2}} \sqrt{D} \sqrt{v}
$$

where $i_{\mathrm{p}}$ is the current maximum of the oxidation wave in amperes, $F$ is the Faraday constant in $\mathrm{C} \cdot \mathrm{mol}^{-1}, R$ is the gas constant in $\mathrm{J} \cdot \mathrm{K}^{-1} \cdot \mathrm{mol}^{-1}, C$ is the concentration of the ruthenium complex $221\left(3.9 \cdot 10^{-6} \mathrm{~mol} \cdot \mathrm{cm}^{-3}\right), A$ is the surface area of the working electrode 
(WE) $\left(0.071 \mathrm{~cm}^{2}\right), v$ is the scan rate in $\mathrm{V} \cdot \mathrm{s}^{-1}, D$ is the diffusion coefficient in $\mathrm{cm}^{2} \cdot \mathrm{s}^{-1}$ and $n$ is the electron transfer number, with the Levich equation (3.6):

$$
i_{\lim }=\underbrace{0.62 F A C v^{-\frac{1}{6}}}_{Y} n D^{\frac{2}{3}} \omega^{\frac{1}{2}}
$$

where $i_{\lim }$ is the limiting or Levich current in amperes, $\omega$ is the rotation rate of the GC-disc electrode in $\mathrm{rad} \cdot \mathrm{s}^{-1}\left(A=0.126 \mathrm{~cm}^{2}\right), v$ is the kinematic viscosity of DCE in $\mathrm{cm}^{2} \cdot \mathrm{s}^{-1}$ (defined by the ratio of the dynamic viscosity $\mu=0.7644 \mathrm{mPa} \cdot \mathrm{s}$ to the density $\rho=1.245 \mathrm{~kg} \cdot \mathrm{m}^{-3}$ at $T=298 \mathrm{~K}),{ }^{[345]}$ and $C$ is the concentration of the complex $221\left(1.0 \cdot 10^{-6} \mathrm{~mol} \cdot \mathrm{cm}^{-3}\right)$ it becomes visible that both equations are proportional with the transferred number of electrons $n$ during the redox event. Plotting the peak current of the ruthenium complex 221, measured via $\mathrm{CV}$, as a function of the square root of the scan rate (Figure 30i), alongside plotting the limiting current as a function of the square root of the rotation rate at the rotating ring disc electrode (RDE) (Figure 30ii), delivered two slopes $S_{1}$ and $S_{2}$, respectively, which directly depend on the function of $n$ (Eq. 3.7).

$$
n=\frac{\left(\frac{S_{1}}{X}\right)^{\frac{4}{3}}}{\left(\frac{S_{2}}{Y}\right)}=\frac{(0.0021)^{\frac{4}{3}}}{2.87 * 10^{-4}}=0.935 \pm 0.206
$$
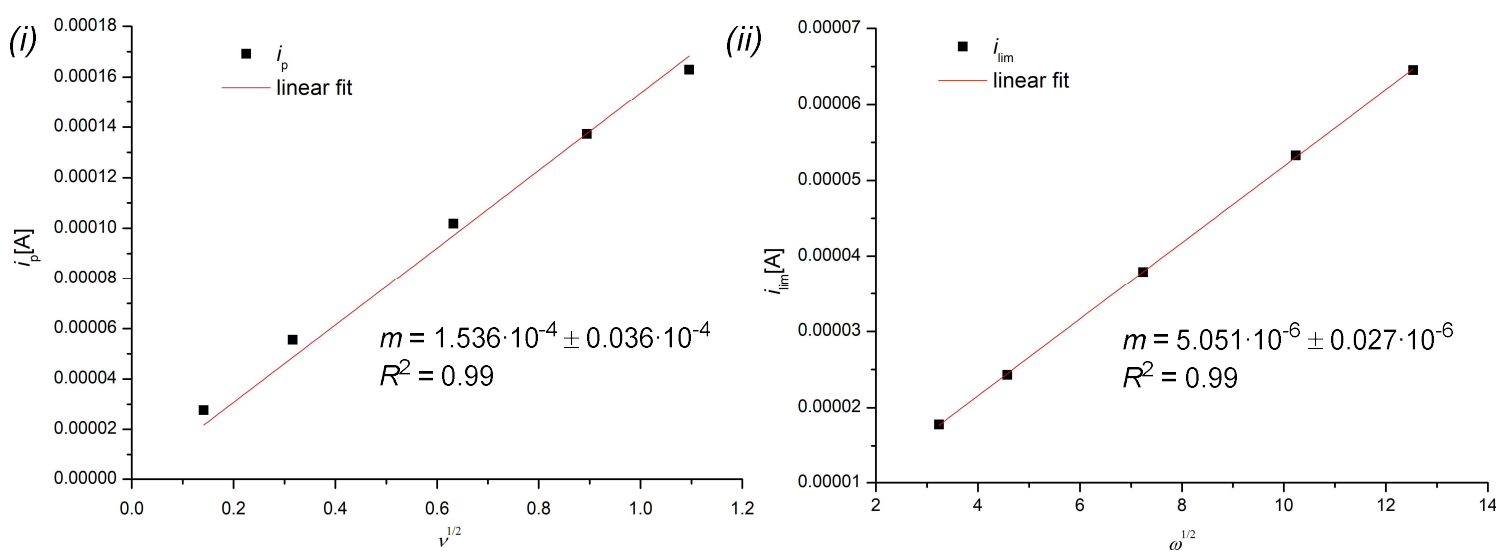

Figure 30. i) Linear fit for the Randles-Ševčik equation. ii) Linear fit for the Levich equation.

Thus, the results were supportive of a stepwise one-electron oxidation, rather than a concerted two-electron oxidation, facilitated by the electrochemically in-situ generated hypervalent iodine reagent. Recently reported detailed mechanistic studies on similar ruthenium-catalyzed $\mathrm{C}-\mathrm{H}$ oxygenation reactions supported the main mechanistic assumptions by experiment and computational DFT studies. ${ }^{[332 \mathrm{a}]}$ 
Based on the mechanistic studies by experiment and computation, a plausible catalytic cycle was proposed for the unprecedented dual electro-catalyzed $\mathrm{C}-\mathrm{H}$ oxygenation (Scheme 78). ${ }^{[339]}$ Initially, the pre-catalyst 217 undergoes fast ligand exchange in the TFA/TFAA solvent mixture, to form the catalytically potent cationic ruthenium complex 222. Carboxylate-assisted, reversible $\mathrm{C}-\mathrm{H}$ activation of amide $\mathbf{2 1 4}$ initiates the catalytic cycle to form the cyclometalated ruthenium(II) complex 223. ${ }^{[23 b, 34 a, 284,319 c]}$ Concurrently, the iodobenzene $\mathbf{2 1 5}$ is anodically oxidized to generate the hypervalent iodine(III) species 219. The transient redox catalyst 219 now mediates the oxidation of ruthenacycle 223 in a step-wise electron/carboxylate transfer process, to deliver the high-valent ruthenium(IV) complex 224. Oxidation-induced reductive elimination releases intermediate $\mathbf{2 2 5}$. The desired hydroxylated product $\mathbf{2 1 6}$ is formed after hydrolysis. Cathodic hydrogen formation closes the electrochemical cycle.

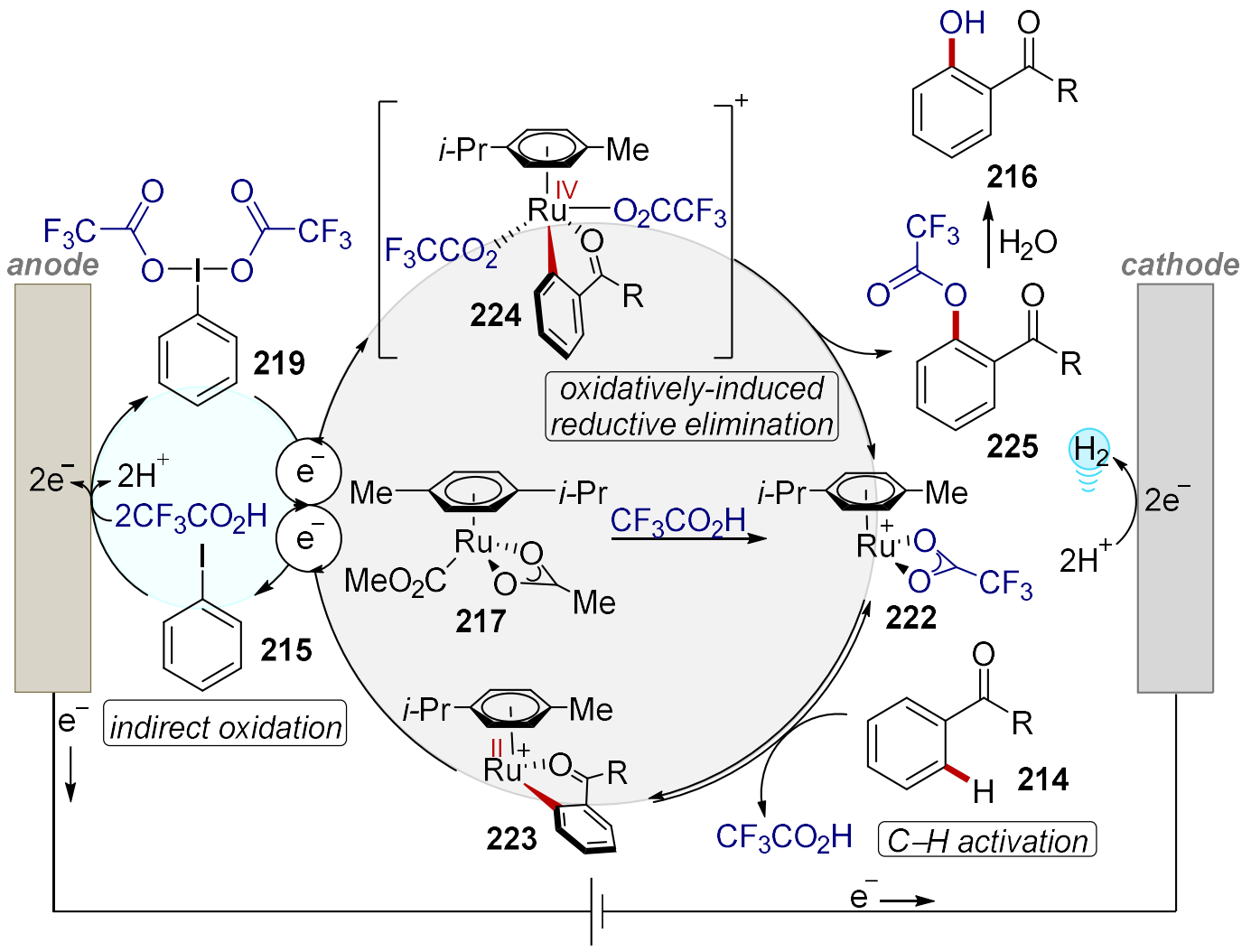

Scheme 78. Proposed catalytic scenario for the cooperative electrocatalytic $\mathrm{C}-\mathrm{H}$ oxygenation. 


\subsection{Manganaelectro-Catalyzed Undirected $\mathrm{C}\left(\mathrm{sp}^{3}\right)-\mathrm{H}$ Azidation}

The development of selective and resource-economic methods for the direct transformation of otherwise unreactive $\mathrm{C}\left(\mathrm{sp}^{3}\right)-\mathrm{H}$ bonds has remained as one of the most auspicious challenges in molecular syntheses. ${ }^{[23 \mathrm{~g}, 25 \mathrm{c}, 166]}$ While numerous biologically-

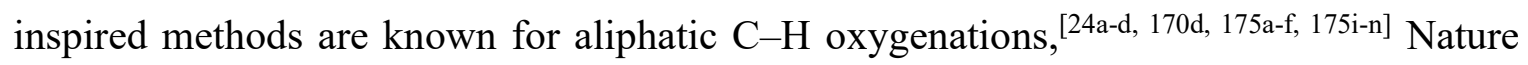
does not rely on $\mathrm{C}-\mathrm{H}$ amination reactions. ${ }^{[346]}$ Challenged by this topical quest, synthetic chemists have developed concepts for the direct amination of $\mathrm{C}\left(\mathrm{sp}^{3}\right)-\mathrm{H}$ bonds to overcome this lack of biosynthetic precedents. ${ }^{[347]}$ Among those methods, especially direct $\mathrm{C}-\mathrm{H}$ azidation reactions have been established as a step-economical tool to enable the desired C-N bond formation. ${ }^{[182 b, 182 d, 348]}$ Despite the significant progress in oxidative $\mathrm{C}-\mathrm{H}$ azidation reactions in recent years, previously developed strategies predominantly rely on stoichiometric amounts of strong, hazardous chemical energy carriers, such as persulfates, ${ }^{[189]} N$-fluorobenzenesulfonimide (NFSI) ${ }^{[203 a]}$ or hypervalent iodine reagents ${ }^{[185-}$ 187, 188b, 191, 201-202], among others. ${ }^{[169 b, 190,192]}$

In this context, a far more appealing, but thus far unprecedented strategy would involve the direct electrochemical $\mathrm{C}-\mathrm{H}$ azidation of aliphatic groups with valuable hydrogen gas as the sole byproduct. ${ }^{[349]}$

\subsubsection{Preliminary Studies toward Metal-Free Electrochemical C-H Azidation of Benzylic C-H Bonds}

Initially, different conditions were probed for the anodic generation of azidyl radicals from readily available inorganic azide sources. In accordance with previous reports, ${ }^{[350]}$ it was found that azide anions in water were successfully oxidized at a graphite-felt (GF) anode and resulted in vigorous nitrogen gas formation, while hydrogen gas was formed on the platinum cathode (Scheme 79).
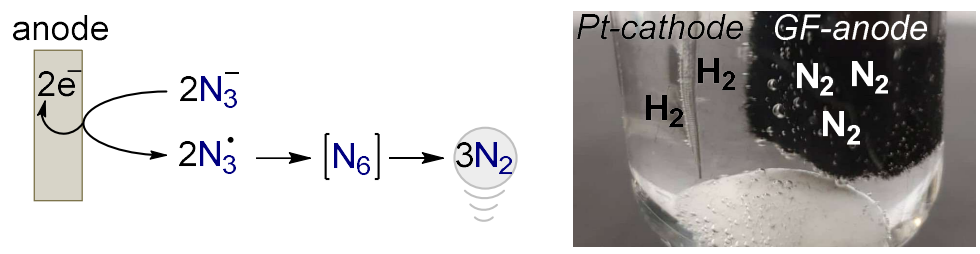

Scheme 79. Anodic oxidation of azide anions in water.

Based on these findings, the formation of azidyl-radicals via anodic oxidation was envisaged for metal-free $\mathrm{C}-\mathrm{H}$ azidations of activated benzylic $\mathrm{C}\left(\mathrm{sp}^{3}\right)-\mathrm{H}$ bonds $(\mathrm{BDE} \mathrm{C}-\mathrm{H}$ $\left.=82.9 \pm 1.2 \mathrm{kcal} \cdot \mathrm{mol}^{-1}\right)^{[15]}$ via hydrogen atom abstraction. ${ }^{[24 \mathrm{~d}, 169 \mathrm{~b}, 321 \mathrm{~d}, 351]}$ Preliminary 
attempts for the electrochemical $\mathrm{C}-\mathrm{H}$ azidation of 1,2,3,4-tetrahydronaphthalene (111a), by means of anodic oxidation under constant current electrolysis of an aqueous solution of sodium azide in a polar protic or aprotic co-solvent, resulted in a mixture of azidated product 113a and undesired oxygenated products 226a and 227, respectively (Table 27). Interestingly, when a biphasic mixture was used, only traces of oxidized products 226a and 227 were observed (entry 1). In contrast, when $\mathrm{MeCN}$ was employed as a polar-aprotic solvent, high conversion of the substrate 111a was obtained, albeit with only low formation of the desired organic azide 113a (entry 2). However, alcoholic solvents resulted in only low conversion of the benzylic substrate 111a (entries 3 and 4). Notably, high conversion of 111a was achieved, when acetic acid was tested as the solvent (entries 5-7), albeit with only poor chemoselecitvity toward the desired product $\mathbf{1 1 3 a}$.

Table 27. Initial results for metal-free electrochemical $\mathrm{C}-\mathrm{H}$ azidation. ${ }^{[\mathrm{a}]}$<smiles>c1ccc2c(c1)CCCC2</smiles>

$111 \mathrm{a}$

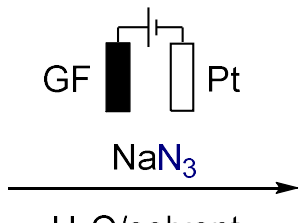

$\mathrm{H}_{2} \mathrm{O}$ /solvent

$4 \mathrm{~h}, 25^{\circ} \mathrm{C}$, air

CCE at $10 \mathrm{~mA}$<smiles>NC1CCCc2ccccc21</smiles>

$113 a$<smiles>O=C1CCCc2ccccc21</smiles>

$226 a$<smiles>CC(=O)OC1CCCc2ccccc21</smiles>

227

\begin{tabular}{cccc}
\hline Entry & Solvent & Yield [\%] $($ Ratio 113a/226a/227) & Conversion [\%] \\
\hline 1 & EtOAc & $2(1 / 1 / 0)$ & 8 \\
2 & $\mathrm{MeCN}$ & $38(6 / 32 / 0)$ & 67 \\
3 & $\mathrm{MeOH}$ & $4(0 / 4 / 0)$ & 31 \\
4 & $\mathrm{CF}_{3} \mathrm{CH}_{2} \mathrm{OH}$ & $9(5 / 4 / 0)$ & 38 \\
5 & $\mathrm{AcOH}$ & $88(15 / 48 / 25)$ & 89 \\
6 & $\mathrm{AcOH}$ & $85(15 / 38 / 32)$ & $95^{[\mathrm{b}]}$ \\
7 & $\mathrm{AcOH}$ & $51(20 / 28 / 3)$ & $70^{[\mathrm{c}]}$ \\
\hline
\end{tabular}

[a] Reaction conditions: 111a $(0.50 \mathrm{mmol})$, aq. $\mathrm{NaN}_{3}(2.0 \mathrm{~mL}, 2.0 \mathrm{M})$, additive (1.00 equiv), solvent $(2 \mathrm{~mL})$, $4 \mathrm{~h}, \mathrm{CCE}=10 \mathrm{~mA}, 25^{\circ} \mathrm{C}$. Undivided cell, graphite felt (GF) anode and platinum plate cathode. Conversion of 111a, yields and ratios were determined by gas chromatography (GC-FID) with $n$-dodecane as internal standard. Calibrations were conducted with authentic samples and compared with ${ }^{1} \mathrm{H}-\mathrm{NMR}$ spectroscopy, using 1,3,5-trimethoxybenzene as internal standard, and isolated yields, respectively. [b] Under nitrogen atmosphere. [c] Reticulated vitreous carbon (RVC) instead of GF.

In summary, the findings are suggestive of an efficient single-electron transfer (SET) oxidation of the benzylic substrate 111a with the azidyl radical as hydrogen atom abstractor (vide infra $).{ }^{[352]}$ Alternatively, $111 \mathrm{a}$ directly undergoes anodic oxidation $\left(E_{\mathrm{p}, \mathrm{a}}=2.1 \mathrm{~V} v s\right.$. $\mathrm{SCE}^{[353]}$ ) to form a stabilized ${ }^{[194]}$ benzylic radical cation. ${ }^{[354]}$ Subsequent deprotonation and 
a facile second single-electron-transfer oxidation would generate the benzylic cation, ${ }^{[355]}$ prone to nucleophilic trapping by among azide, water or acetate, to form the azidated product 113a, 1-tetralone (226a) ${ }^{[170 d, 356]}$ or the ester 227, respectively. It is noteworthy that a control experiment revealed facile overoxidation of the azidated product 113a to the corresponding ketone 226a, under constant current electrolysis in acetic acid/water mixtures (Scheme 80).
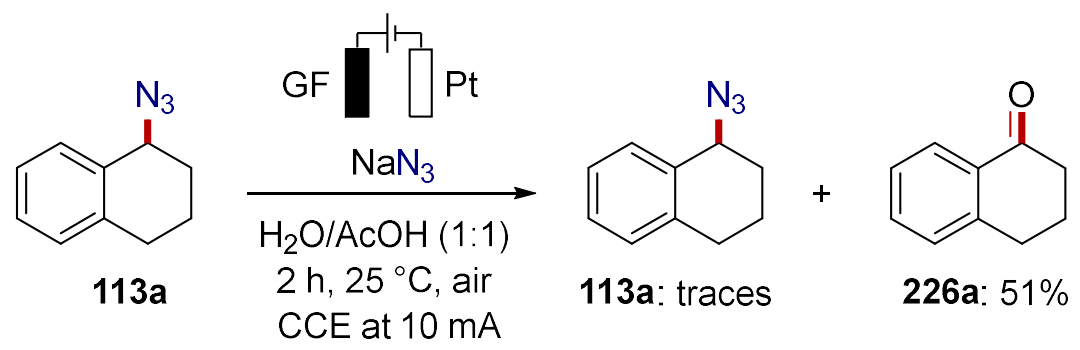

Scheme 80. Overoxidation of product 113a at constant current electrolysis.

Hence, it became apparent that a suitable catalyst was required to mediate the desired $\mathrm{C}\left(\mathrm{sp}^{3}\right)$-Het formation in a more selective fashion to avoid competitive overoxidation of the intermediates and products. In this context, synthetic manganese(III) porphyrin complexes represent a particularly versatile and attractive catalyst platform for bioinspired $\mathrm{C}-\mathrm{H}$ oxidation and subsequent heteroatom-transfer reactions to the otherwise inert aliphatic substrates. ${ }^{[175 f, 196 a, 197,347 b, 347 i, 357]}$ However, these methods commonly employ superstoichiometric amounts of iodosobenzene as the chemical oxidant and oxygen-surrogate to generate high-valent manganese(V)-oxo porphyrin complexes, ${ }^{[175 c, 358]}$ which represent the active catalyst for hydrogen atom abstraction of aliphatic $\mathrm{C}\left(\mathrm{sp}^{3}\right)-\mathrm{H}$ bonds to form the desired alkyl radical. ${ }^{[24 c, 24 i, 196 c, 359]}$ These hydroxo intermediates directly compete with the desired hetero-atom transfer reaction as was shown in a recent study by Groves on manganese-catalyzed $\mathrm{C}-\mathrm{H}$ azidations and therefore the reported azide to oxygen incorporation ratio was relatively low (2-4:1) (cf. Scheme 34). ${ }^{[198]}$ Hence, the undesired oxygenation reaction seemed unavoidable in $\mathrm{C}-\mathrm{H}$ azidations involving manganese-oxo species. Hence, it was reasoned that replacing the chemical charge carrier with reagent-free electrochemical potential would allow to generate high-valent manganese-catalyst in the absence of competing oxo-species.

Notably, manifolds have been developed to employ electrochemistry for manganesecatalyzed olefin functionalization ${ }^{[220 f, 360]}$ among others. ${ }^{[361]}$ However, the merger of manganese- and electrocatalysis for undirected $\mathrm{C}-\mathrm{H}$ functionalization reactions of saturated hydrocarbons has largely remained elusive. 


\subsubsection{Optimization Studies for Manganaelectro-Catalyzed C(sp $\left.{ }^{3}\right)-H$ Azidation}

The experimentation was initiated with cyclooctane (111b) as the model substrate for the desired manganaelectro-catalyzed $\mathrm{C}-\mathrm{H}$ azidation of unactivated (BDE $\mathrm{C}-\mathrm{H}$ : $\left.95.7 \mathrm{kcal} \cdot \mathrm{mol}^{-1}\right)^{[362]}$ alkanes (Table 28). Based on previous studies for the in-situ generation of hypervalent iodine species upon electrochemical stimulus (vide infra), ${ }^{[339]}$ it was envisioned that catalytic amounts of iodobenzene might replace the otherwise required super-stoichiometric amounts of potentially explosive iodosobenzene. Indeed, promising conversion of cyclooctane (111b) was observed in acetic acid/ $\mathrm{H}_{2} \mathrm{O}(\mathrm{v} / \mathrm{v} 1: 1)$ mixtures at $25{ }^{\circ} \mathrm{C}$ with simple sodium azide as the nitrogen source and catalytic amounts of iodobenzene (entry 1). It was further noticed that the manganese salen catalyst [Mn2] resulted in slightly higher conversion of the alkane $\mathbf{1 1 1 b}$, compared to manganese porphyrin complex [Mn1] (entry 2). Unfortunately, for both catalysts, the azide-to-oxygen

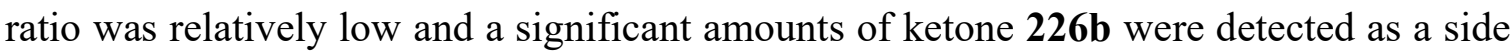
product. Surprisingly, and in contrast to the well-established protocols, ${ }^{[363]}$ it was noticed that similar outcome for the manganaelectrocatalytic manifold was achieved in the absence of any oxygen-transfer reagent (entry 3 ). Thus, the reactivity of the electrocatalysis was probed in $\mathrm{MeCN} / \mathrm{AcOH}$ mixtures. Interestingly, in the absence of water, the azide-tooxygen ratio was significantly improved with overall similar levels of efficacy of the manganaelectro-catalyzed $\mathrm{C}-\mathrm{H}$ functionalization (entry 4). Notably, if the reaction time was extended from five $\left(Q \cdot \mathrm{mol}^{-1}=2.24 \mathrm{~F}\right)$ to ten hours $\left(Q \cdot \mathrm{mol}^{-1}=4.48 \mathrm{~F}\right)$, higher overall conversion was detected, albeit with increased levels of overoxidation to the undesired oxygenated product $\mathbf{2 2 6 b}$ (entry 5).

After these initial successes for the manganaelectro-catalyzed $\mathrm{C}-\mathrm{H}$ azidation, $D r . R . C$. Samanta joined the project, and the minor further reaction optimization was conducted in a cooperative manner. The experiments performed by $D r . R$. C. Samanta are thus specifically highlighted in the following optimization tables. 
Table 28. Optimization of general reaction conditions for the $\mathrm{C}-\mathrm{H}$ azidation. ${ }^{[\mathrm{a}]}$

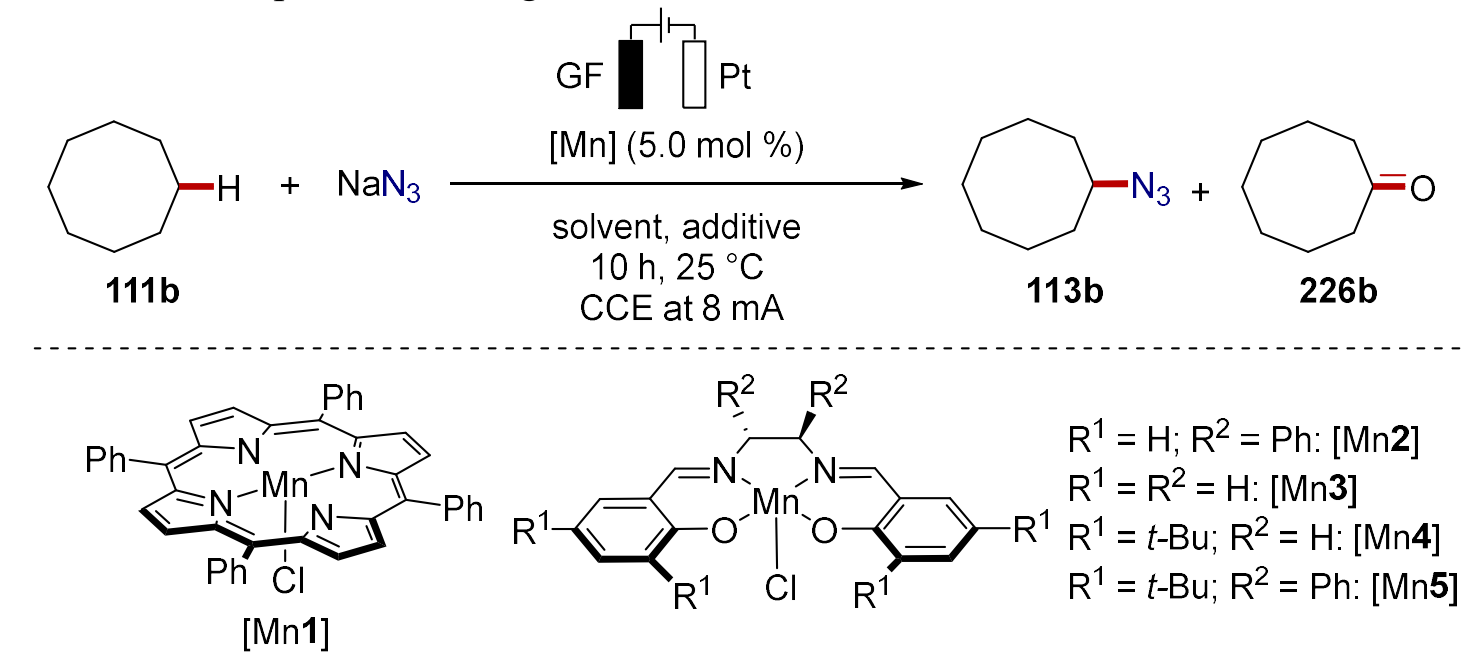

\begin{tabular}{cccccc}
\hline Entry & {$[\mathrm{Mn}]$} & $\mathrm{Solvent}$ & $\begin{array}{c}\text { Additive } \\
\text { (equiv) }\end{array}$ & $\begin{array}{c}\text { Yield [\%] } \\
\text { (Ratio 113b/226b) }\end{array}$ & $\begin{array}{c}\text { Conversion } \\
{[\%]}\end{array}$ \\
\hline 1 & {$[\mathrm{Mn} 1]$} & $\mathrm{AcOH} / \mathrm{H}_{2} \mathrm{O}$ & $\mathrm{PhI}(0.20)$ & $25(2.6: 1.0)$ & $37^{[\mathrm{b}]}$ \\
2 & {$[\mathrm{Mn2}]$} & $\mathrm{AcOH} / \mathrm{H}_{2} \mathrm{O}$ & $\mathrm{PhI}(0.20)$ & $33(2.0: 1.0)$ & $45^{[\mathrm{b}]}$ \\
3 & {$[\mathrm{Mn2}]$} & $\mathrm{AcOH} / \mathrm{H}_{2} \mathrm{O}$ & --- & $30(2.8: 1.0)$ & $41^{[\mathrm{b}]}$ \\
4 & {$[\mathrm{Mn2}]$} & $\mathrm{AcOH} / \mathrm{MeCN}$ & $\mathrm{LiClO}_{4}(1.00)$ & $31(5.2: 1.0)$ & $43^{[\mathrm{b}]}$ \\
5 & {$[\mathrm{Mn2}]$} & $\mathrm{AcOH} / \mathrm{MeCN}$ & $\mathrm{LiClO}_{4}(1.00)$ & $43(3.3: 1.0)$ & $72^{[\mathrm{c}]}$ \\
6 & {$[\mathrm{Mn2}]$} & $\mathrm{AcOH} / \mathrm{MeCN}$ & $\mathrm{LiClO}_{4}(1.00)$ & $40(12.3: 1.0)$ & $56^{*[\mathrm{c}, \mathrm{d}]}$ \\
7 & {$[\mathrm{Mn2}]$} & $\mathrm{AcOH} / \mathrm{MeCN}$ & $\mathbf{L i C l O}_{4}(\mathbf{1 . 0 0})$ & $\mathbf{4 5}(\mathbf{1 0 . 0} 1.0)$ & $70^{[\mathrm{d}]}$ \\
8 & {$[\mathrm{Mn2}]$} & $\mathrm{AcOH} / \mathrm{MeCN}$ & $\mathrm{LiClO}_{4}(1.00)$ & $30(1.0: 1.0)$ & $96^{*[\mathrm{~d}, \mathrm{e}]}$ \\
9 & {$[\mathrm{Mn2}]$} & $\mathrm{AcOH} / \mathrm{MeCN}$ & $\mathrm{LiClO}_{4}(1.00)$ & $42(9.5: 1.0)$ & $65^{[\mathrm{d}, \mathrm{f}]}$ \\
10 & {$[\mathrm{Mn2}]$} & $\mathrm{AcOH} / \mathrm{MeCN}$ & $\mathrm{LiClO}_{4}(1.00)$ & $42(10.0: 1.0)$ & $68^{[\mathrm{d}, \mathrm{g}]}$ \\
11 & {$[\mathrm{Mn2}]$} & $\mathrm{AcOH} / \mathrm{MeCN}$ & $\mathrm{LiClO}_{4}(1.00)$ & $46(5.6: 1.0)$ & $64^{*[\mathrm{~d}, \mathrm{~h}]}$ \\
12 & {$[\mathrm{Mn} 1]$} & $\mathrm{AcOH} / \mathrm{MeCN}$ & $\mathrm{LiClO}_{4}(1.00)$ & $44(1.6: 1.0)$ & $86^{[\mathrm{d}]}$ \\
13 & {$[\mathrm{Mn} 3]$} & $\mathrm{AcOH} / \mathrm{MeCN}$ & $\mathrm{LiClO}_{4}(1.00)$ & $10(4.0: 1.0)$ & $10^{[\mathrm{d}]}$ \\
14 & {$[\mathrm{Mn} 4]$} & $\mathrm{AcOH} / \mathrm{MeCN}$ & $\mathrm{LiClO}_{4}(1.00)$ & $39(12.0: 1.0)$ & $47^{[\mathrm{d}]}$ \\
15 & {$[\mathrm{Mn} 5]$} & $\mathrm{AcOH} / \mathrm{MeCN}$ & $\mathrm{LiClO}_{4}(1.00)$ & $47(8.4: 1.0)$ & $62^{[\mathrm{d}]}$ \\
16 & {$[\mathrm{Mn} 6]$} & $\mathrm{AcOH} / \mathrm{MeCN}$ & $\mathrm{LiClO}_{4}(1.00)$ & $32(9.7: 1.0)$ & $38^{[\mathrm{d}, \mathrm{i}]}$ \\
17 & --- & $\mathrm{AcOH} / \mathrm{MeCN}$ & $\mathrm{LiClO}_{4}(1.00)$ & $10(2.0: 1.0)$ & $49^{[\mathrm{d}]}$ \\
\hline
\end{tabular}

[a] Reaction conditions: $111 \mathrm{~b}(0.50 \mathrm{mmol}), \mathrm{NaN}_{3}(4.00 \mathrm{mmol}, 8.00$ equiv), [Mn] $(5.0 \mathrm{~mol} \%)$, additive (xx equiv), solvent $(1: 1,5.0 \mathrm{~mL}), 10 \mathrm{~h}, \mathrm{CCE}=8 \mathrm{~mA}, 25^{\circ} \mathrm{C}$. Undivided cell, graphite felt (GF) anode and platinum plate cathode. Conversion of $\mathbf{1 1 1} \mathbf{b}$, yields and ratios were determined by gas chromatography (GC-FID) with $n$-dodecane as internal standard. Calibrations were conducted with authentic samples and compared with ${ }^{1} \mathrm{H}$ NMR spectroscopy, using 1,3,5-trimethoxybenzene as internal standard, and isolated yields, respectively. [b] $5 \mathrm{~h}, 6 \mathrm{~mA}$. [c] $10 \mathrm{~h}, 6 \mathrm{~mA}$. [d] Under nitrogen atmosphere. [e] Light irradiation with blue LEDs. [f] $50{ }^{\circ} \mathrm{C}$. [g] Reaction was performed in dark. [h] [Mn2] (2.5 mol \%). [i] [Mn6] = (S,S)-Jacobsen's catalyst. *Performed by Dr. R. C. Samanta. 
Previous studies on electrochemical C-H oxygenations with manganese or iron ${ }^{[1751,364]}$ porphyrin and related complexes as the catalyst have indicated that oxygenation reactions can either occur via $\mathrm{O}_{2}$ from air or concurrent anodic water oxidation. ${ }^{[360 \mathrm{c}-\mathrm{f}, 360 \mathrm{~h}-\mathrm{j}, 365]}$ When the mangana-electrocatalysis was conducted with degassed solvents, under a nitrogen atmosphere, overall good conversion of cyclooctane (111b) to the desired azide 113b was observed, with only traces of cyclooctanon $(\mathbf{2 2 6} \mathbf{b})$ as the side-product and an overall satisfactory mass-balance (entry 6). When the current output was increased to $8 \mathrm{~mA}$ $\left(Q \cdot \mathrm{mol}^{-1}=5.97 \mathrm{~F}\right)$, a slightly increased yield of azide $113 \mathrm{~b}$ was obtained, with a satisfactory azide-to-ketone ratio of 10:1 (entry 7). Furthermore, light-irradiation or an increase in the reaction temperature did not have a beneficial effect on the reaction outcome (entries 8-10). A decrease in the catalyst loading to $2.5 \mathrm{~mol} \%$ did result in similar conversion, however with slightly diminished azide-to-ketone ratio of 5.6:1 (entry 11). Moreover, when other manganese(III) catalyst instead of [Mn2] were used, overall lower yields in azidation were detected (entries 12-16). Notably, complex [Mn3] with an unsubstituted salen ligand, resulted in a significantly reduced yield and chemoselectivity (entry 13). One possible catalyst deactivation pathway could be the oxidation of the Schiff base ligand backbone under the electrochemical conditions. ${ }^{[366]}$ In this context, the study of differently substituted salen-ligands could be interesting for future developments. ${ }^{[367]}$ Control experiments without catalyst revealed only minor background reactivity (entry 17). ${ }^{[368]}$ This was expected since the direct anodic electron transfer for unactivated alkanes can be excluded, simply due to their inherently high oxidation potential of $E_{\mathrm{ox}} \geq 2.5 \mathrm{~V} .^{[369]}$

Next, different solvent mixtures were evaluated for the manganaelectro-catalyzed $\mathrm{C}-\mathrm{H}$ azidation of unactivated alkane 111b (Table 29). Changing the solvent ratio for $\mathrm{MeCN} / \mathrm{AcOH}$ mixtures from 1:1 to 3:1 drastically enhanced the chemoselectivity toward the desired azidation reaction, with a concomitant drop in reactivity (entry 2). Interestingly, when aqueous solvent mixtures were degassed prior to use, $\mathrm{AcOH} / \mathrm{H}_{2} \mathrm{O}$ resulted in similar reaction outcome as of reactions under air (entry 3 vs. Table 28, entry 3 ), whereas when $\mathrm{MeCN} / \mathrm{H}_{2} \mathrm{O}$ was used, the mangana-electrocatalysis provided product $\mathbf{1 1 3 \mathbf { b }}$ with acceptable chemoselectivity, albeit in low yield (entry 4). Other solvent mixtures did not result in satisfactory conversion of the hydrocarbon under the electrocatalytic conditions (entries 5 10). Additional polar solvent mixtures were tested by Dr. R. C. Samanta, however all were inferior to $\mathrm{AcOH} / \mathrm{MeCN} .{ }^{[349]}$ 
Table 29. Optimization of solvents. ${ }^{[\mathrm{a}]}$

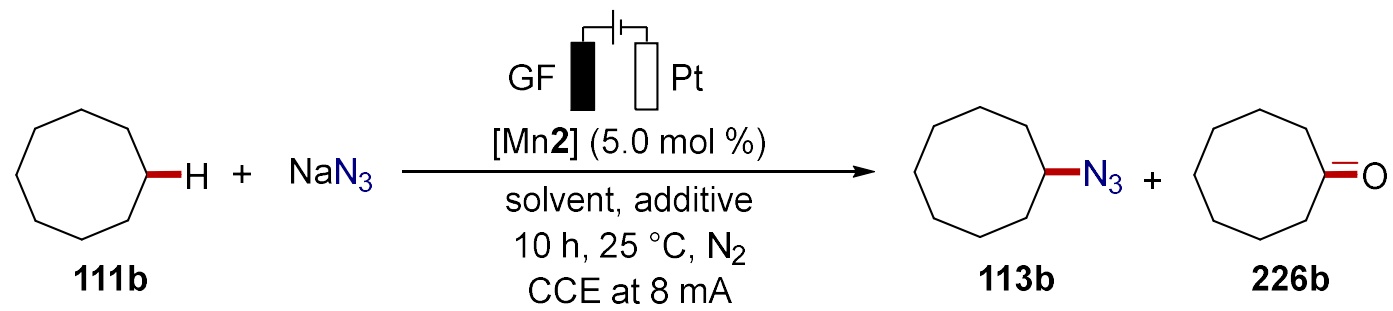

\begin{tabular}{|c|c|c|c|c|}
\hline Entry & Solvent & Additive (equiv) & $\begin{array}{c}\text { Yield }[\%] \\
\text { (Ratio } \mathbf{1 1 3 b} / \mathbf{2 2 6 b})\end{array}$ & Conversion [\%] \\
\hline 1 & $\mathrm{AcOH} / \mathrm{MeCN}$ & $\mathrm{LiClO}_{4}(\mathbf{1 . 0 0})$ & $45(10.0: 1.0)$ & 70 \\
\hline 2 & $\mathrm{AcOH} / \mathrm{MeCN}$ & $\mathrm{LiClO}_{4}(1.00)$ & $31(30.0: 1.0)$ & $40^{[\mathrm{b}]}$ \\
\hline 3 & $\mathrm{AcOH} / \mathrm{H}_{2} \mathrm{O}$ & --- & $25(2.1: 1.0)$ & 43 \\
\hline 4 & $\mathrm{MeCN} / \mathrm{H}_{2} \mathrm{O}$ & --- & $17(7.5: 1.0)$ & 28 \\
\hline 5 & $\mathrm{MeOH} / \mathrm{H}_{2} \mathrm{O}$ & --- & $4(1.0: 1.0)$ & 4 \\
\hline 6 & $\mathrm{HFIP} / \mathrm{H}_{2} \mathrm{O}$ & --- & $10(1.5: 1.0)$ & 67 \\
\hline 7 & $\mathrm{CH}_{2} \mathrm{Cl}_{2} / \mathrm{H}_{2} \mathrm{O}$ & --- & 0 & 1 \\
\hline 8 & $\mathrm{CF}_{3} \mathrm{COOH} / \mathrm{H}_{2} \mathrm{O}$ & --- & $3(0: 3.0)$ & 69 \\
\hline 9 & $\mathrm{AcOH} / \mathrm{EtOAc}$ & $\mathrm{LiClO}_{4}(1.00)$ & $4(3.0: 1.0)$ & $6^{[c]}$ \\
\hline 10 & $\mathrm{EtOAc} / \mathrm{H}_{2} \mathrm{O}$ & -- & $1(0: 1.0)$ & $1^{[\mathrm{d}]}$ \\
\hline
\end{tabular}

[a] Reaction conditions: $\mathbf{1 1 1 b}(0.50 \mathrm{mmol}), \mathrm{NaN}_{3}(4.00 \mathrm{mmol}, 8.00$ equiv), [Mn2] (5.0 mol \%), additive (xx equiv), solvent $(1: 1,5.0 \mathrm{~mL}), 10 \mathrm{~h}, \mathrm{CCE}=8 \mathrm{~mA}, 25^{\circ} \mathrm{C}$. Undivided cell, graphite felt (GF) anode and platinum plate cathode. Conversion of $\mathbf{1 1 1} \mathbf{b}$, yields and ratios were determined by gas chromatography (GC-FID) with $n$-dodecane as internal standard. Calibrations were conducted with authentic samples and compared with ${ }^{1} \mathrm{H}$ NMR spectroscopy, using 1,3,5-trimethoxybenzene as internal standard, and isolated yields, respectively. [b] $\mathrm{MeCN} / \mathrm{AcOH}$ (3:1). [c] $10 \mathrm{~h}, \mathrm{CPE}=2.0 \mathrm{~V}$. [d] [Mn1] instead of [Mn2].

Thereafter, various azide sources and different electrode materials were tested within the electrochemical manganese-catalyzed $\mathrm{C}\left(\mathrm{sp}^{3}\right)-\mathrm{H}$ azidation (Table 30$)$. When the amount of $\mathrm{NaN}_{3}$ was decreased, lower conversion was detected, together with increased levels of overoxidation to the ketone 226b (entry 2). Other nucleophilic azide sources delivered the desired product 113b in slightly lower yields (entries 3 and 4). In contrast, electrophilic azide sources fell short in the formation of the azidated product $\mathbf{1 1 3 b}$ within the

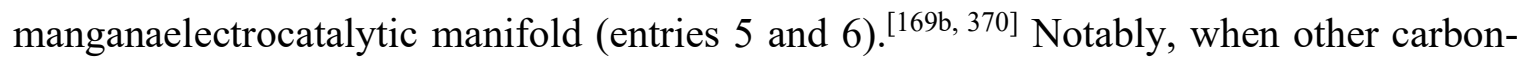
based electrodes were used as the anode material, almost no oxygenated side products $\mathbf{2 2 6} \mathbf{b}$ were observed, albeit overall lower conversion of cyclooctane (111b) was detected (entries 7-9). One reason for the low oxygenation could be the high overpotential of glassy carbon toward the oxygen evolution reaction. ${ }^{[170 \mathrm{~g}]}$ It also should be noted that full degassing of the 
graphite felt electrodes prior its insertion was challenging, due to its inherent high structural density and small pore size. When platinum was used as the anode material, no conversion of the alkane 111b was observed (entry 10).

Table 30. Optimization of electrode material and azide source. ${ }^{[\mathrm{a}]}$

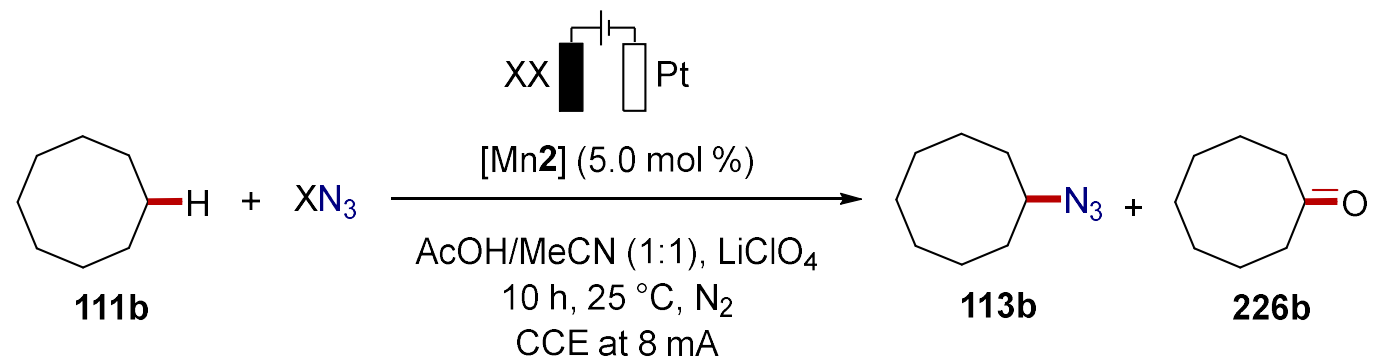

\begin{tabular}{ccccc}
\hline Entry & $\mathrm{XN}_{3}$ (equiv) & Anode $\mathrm{xx}$ & $\begin{array}{c}\text { Yield }[\%] \\
(\text { Ratio 113b/226b) }\end{array}$ & Conversion [\%] \\
\hline $\mathbf{1}$ & $\mathrm{NaN}_{\mathbf{3}}(\mathbf{8 . 0})$ & $\mathbf{G F}$ & $\mathbf{4 5}(\mathbf{1 0 . 0}: \mathbf{1 . 0})$ & $\mathbf{7 0}$ \\
2 & $\mathrm{NaN}_{3}(4.0)$ & $\mathrm{GF}$ & $35(4.8: 1.0)$ & 67 \\
3 & $\mathrm{KN}_{3}(8.0)$ & $\mathrm{GF}$ & $44(5.3: 1.0)$ & 69 \\
4 & $\mathrm{TMSN}_{3}(4.0)$ & $\mathrm{GF}$ & $30(8.0: 1.0)$ & $87^{*}$ \\
5 & $4-\mathrm{CF}_{3}-\mathrm{C}_{6} \mathrm{H}_{4} \mathrm{SO}_{2} \mathrm{~N}_{3}(2.0)$ & $\mathrm{GF}$ & 0 & 4 \\
6 & $\mathrm{TsN}_{3}(4.0)$ & $\mathrm{GF}$ & 0 & 25 \\
7 & $\mathrm{NaN}_{3}(8.0)$ & $\mathrm{GC}$ & $6(1.0: 0.0)$ & $11^{*}$ \\
8 & $\mathrm{NaN}_{3}(8.0)$ & $\mathrm{RVC}$ & $15(14.0: 1.0)$ & 23 \\
9 & $\mathrm{NaN}_{3}(8.0)$ & graphite & $19(1.0: 0.0)$ & $29 *$ \\
10 & $\mathrm{NaN}_{3}(8.0)$ & $\mathrm{Pt}$ & 0 & 1 \\
\hline
\end{tabular}

[a] Reaction conditions: 111b $(0.50 \mathrm{mmol}), \mathrm{XN}_{3}$ (xx equiv), [Mn2] $(5.0 \mathrm{~mol} \%), \mathrm{LiClO}_{4}$ (1.0 equiv), $\mathrm{AcOH} / \mathrm{MeCN}(1: 1,5.0 \mathrm{~mL}), 10 \mathrm{~h}, \mathrm{CCE}=8 \mathrm{~mA}, 25^{\circ} \mathrm{C}$. Undivided cell, anode $\mathrm{xx}$ and platinum plate cathode. Glassy carbon (GC). Reticulated vitreous carbon (RVC). Conversion of $\mathbf{1 1 1 b}$, yields and ratios were determined by gas chromatography (GC-FID) with $n$-dodecane as internal standard. Calibrations were conducted with authentic samples and compared with ${ }^{1} \mathrm{H}-\mathrm{NMR}$ spectroscopy, using 1,3,5-trimethoxybenzene as internal standard, and isolated yields, respectively. *Performed by Dr. R. C. Samanta.

Moreover, different current densities and reaction times were evaluated (Table 31). A variation in the current revealed that $8.0 \mathrm{~mA}\left(j=5.33 \mathrm{~mA} \cdot \mathrm{cm}^{-2}\right)$, applied over ten hours, was optimal in terms of conversion and chemoselectivity (entry 1). With lower applied current densities, the overall reaction yield and also the chemoselectivity was diminished. Likewise, higher current (entry 7) or longer reaction time with lower current than $8 \mathrm{~mA}$ led to overall diminished yields of the azidated product 113b (entries 2, 4 and 6). These findings are in line with the previously performed control experiment for metal-free $\mathrm{C}-\mathrm{H}$ 
azidations ( $c f$. Scheme 80 ), which supported that the organic azide easily undergoes overoxidation to the corresponding ketone during the course of the reaction. Importantly, in the absence of external charge, no azidation was observed (entries 8 and 9).

Table 31. Optimization of applied charge. ${ }^{[\mathrm{a}]}$

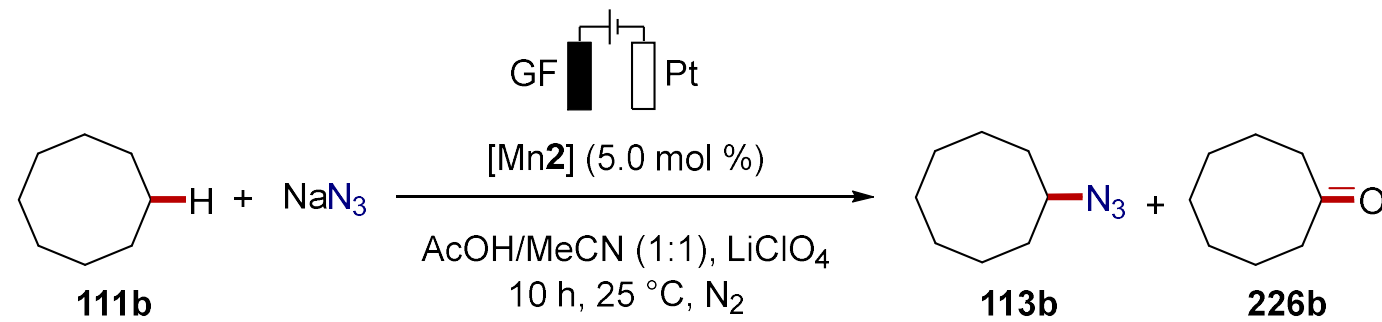

\begin{tabular}{cccc}
\hline Entry & Current & $\begin{array}{c}\text { Yield }[\%] \\
\text { (Ratio 113b/226b) }\end{array}$ & Conversion $[\%]$ \\
\hline $\mathbf{1}$ & CCE at $\mathbf{8 . 0} \mathbf{~ m A}$ & $\mathbf{4 5 ( 1 0 . 0 : 1 . 0 )}$ & $\mathbf{7 0}$ \\
2 & CCE at $8.0 \mathrm{~mA}$ & $33(2.0: 1.0)$ & $91^{[\mathrm{b}]}$ \\
3 & CCE at $4.0 \mathrm{~mA}$ & $28(8.3: 1.0)$ & 41 \\
4 & CCE at $4.0 \mathrm{~mA}$ & $44(6.3: 1.0)$ & $73^{[\mathrm{b}]}$ \\
5 & CCE at $6.0 \mathrm{~mA}$ & $43(3.3: 1.0)$ & 67 \\
6 & CCE at $6.0 \mathrm{~mA}$ & $32(3.3: 1.0)$ & $80^{[\mathrm{b}]}$ \\
7 & CCE at $10.0 \mathrm{~mA}$ & $26(2.6: 1.0)$ & 94 \\
8 & No current & 0 & 9 \\
9 & No current, under air & 0 & 10 \\
\hline
\end{tabular}

[a] Reaction conditions: 111b $(0.50 \mathrm{mmol}), \mathrm{NaN}_{3}$ (8.0 equiv), [Mn2] (5.0 mol \%), $\mathrm{LiClO}_{4}$ (1.0 equiv), $\mathrm{AcOH} / \mathrm{MeCN}(1: 1,5.0 \mathrm{~mL}), 10 \mathrm{~h}, 25^{\circ} \mathrm{C}$. Undivided cell, GF anode and Pt plate cathode. Conversion of 111b, yields and ratios were determined by gas chromatography (GC-FID) with $n$-dodecane as internal standard. Calibrations were conducted with authentic samples and compared with ${ }^{1} \mathrm{H}-\mathrm{NMR}$ spectroscopy, using 1,3,5trimethoxybenzene as internal standard, and isolated yields, respectively. [b] $15 \mathrm{~h}$.

\subsubsection{Substrate Scope of the Manganaelectro-Catalyzed C(sp $\left.{ }^{3}\right)-H$ Azidation}

With the optimized reaction conditions being identified, the performance of the manganaelectro-catalyzed manifold was explored for the intermolecular $\mathrm{C}-\mathrm{H}$ azidation of various activated and unactivated substrates 111 (Table 32). Secondary cyclic hydrocarbons $111 \mathrm{~b}$ and $111 \mathrm{~g}$ were converted to the corresponding organic azides $113 \mathrm{~b}$ and 113g in moderate yields (entries 1 and 2). In contrast to the metal-free $\mathrm{C}-\mathrm{H}$ functionalization (vide supra), manganaelectro-catalyzed $\mathrm{C}-\mathrm{H}$ azidation of tetraline (111a) was far superior and delivered the corresponding benzylic azide 113a in 56\% yield, with good levels of chemoselectivity (entry 3 ). 
Table 32. Reaction scope of manganaelectro-catalyzed $\mathrm{C}\left(\mathrm{sp}^{3}\right)-\mathrm{H}$ azidation. ${ }^{[\mathrm{a}]}$

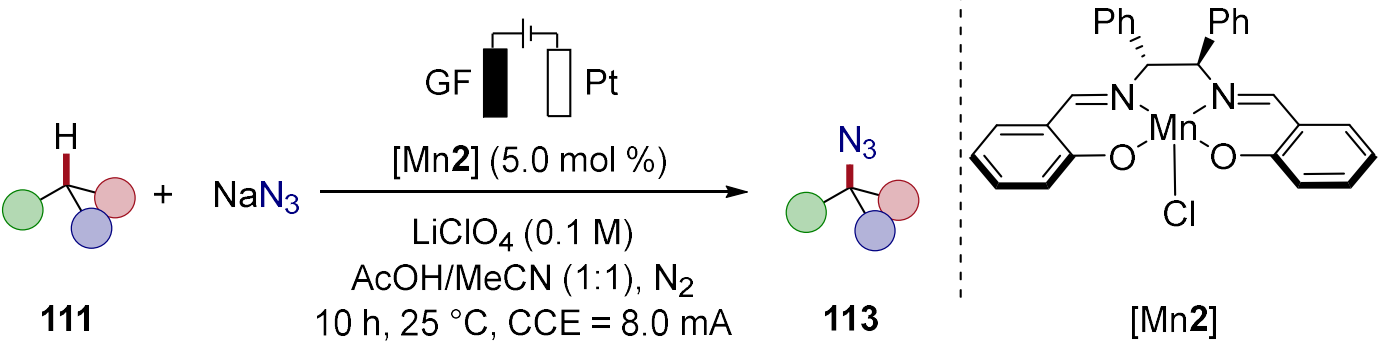

\begin{tabular}{|c|c|c|c|c|c|}
\hline Entry & Substrate & 111 & Product & 113 & $\begin{array}{c}\text { Yield } \\
{[\%]}\end{array}$ \\
\hline 1 & & 111b & & 113b & 40 \\
\hline 2 & & $111 \mathrm{~g}$ & & $113 g$ & 32 \\
\hline 3 & & 111a & & $113 a$ & $56^{[\mathrm{b}]}$ \\
\hline 4 & & 111h & & 113h & 62 \\
\hline 5 & $M$ & $111 i$ & & $113 i$ & $68(33: 1)$ \\
\hline 6 & & 111j & & 113j & $56(2.7: 1)$ \\
\hline 7 & & $111 \mathrm{k}$ & & 113k & 39 \\
\hline 8 & & 1111 & & 1131 & $48(3: 1)$ \\
\hline
\end{tabular}


9
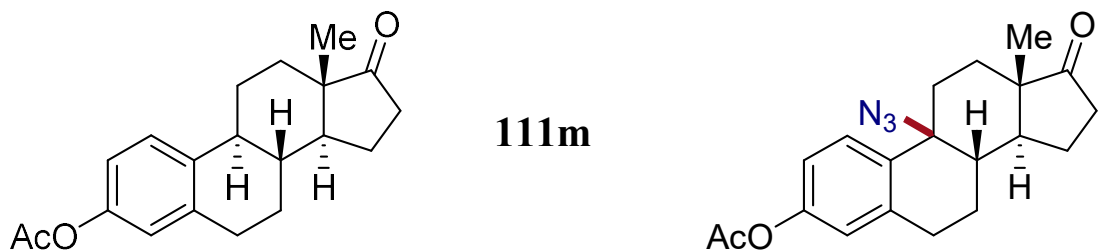

[a] Reaction conditions: substrate $111(0.50 \mathrm{mmol}), \mathrm{NaN}_{3}(4.00 \mathrm{mmol})$, [Mn2] $(5.0 \mathrm{~mol} \%), \mathrm{LiClO}_{4}$ $(0.50 \mathrm{mmol}), \mathrm{MeCN} / \mathrm{AcOH}(5 \mathrm{~mL}, 1: 1)$, nitrogen atmosphere, $10 \mathrm{~h}, 25^{\circ} \mathrm{C}$, constant current electrolysis at $8.0 \mathrm{~mA}$ in an undivided cell. All yields are isolated products; ratios for site-selectivity are determined by ${ }^{1} \mathrm{H}-$ NMR of the crude mixture. [b] 3,4-Dihydronaphthalen-1(2H)-one (226a) was detected in $10 \%$ from crude ${ }^{1} \mathrm{H}$-NMR with 1,3,5-trimethoxybenzene as internal standard. [c] $\mathrm{dr}=1: 1$.

Also, bibenzyl (111h) was selectively converted to the corresponding azide $113 \mathrm{~h}$ in $62 \%$ yield without detectable amounts of diazidation or overoxidation (entry 4 ). When $p$-cymene (111i) was used as the substrate with two distinct benzylic $\mathrm{C}-\mathrm{H}$ bonds, almost exclusive site-selective $\mathrm{C}-\mathrm{H}$ azidation was detected in the sterically more demanding tertiary position, with a tertiary-to-primary product ratio of 33:1 (entry 5). Likewise, site-selectivity studies with isobutylbenzene (111j) as the substrate revealed a preference for the benzylic $\mathrm{C}-\mathrm{H}$ cleavage over the tertiary $\mathrm{C}-\mathrm{H}$ bond in a ratio of $2.7: 1$ (entry 6 ).

Subsequently, the developed manganaelectro-catalyzed $\mathrm{C}-\mathrm{H}$ azidation protocol was employed for the late-stage functionalization of biologically relevant and pharmaceutically active molecules. ${ }^{[166 c, 202,371]}$ In this context, electrocatalytic $\mathrm{C}-\mathrm{H}$ azidation was successfully implemented for the conversion of biaryl $111 \mathbf{k}$, an analogue of a retinoic acid receptor agonist, ${ }^{[372]}$ to the corresponding azide derivative 113k (entry 7). In addition, acetoxylated (-)-menthol (111), with two electronically comparable tertiary $\mathrm{C}-\mathrm{H}$ bonds, reacted preferentially at the sterically more accessible isopropyl sidechain to provide azide 1131 in overall $48 \%$ yield (entry 8). Estrone acetate (111m) was transformed with high levels of efficacy under the standard conditions to provide the corresponding azide $113 \mathrm{~m}$ as a diastereomeric mixture in $75 \%$ yield (entry 9). The result is indicative of a radical pathway, with a persistent radical formed in the tertiary benzylic position. However, a second oxidation of the benzylic radical could not be excluded and would result in a stabilized carbocation, as was previously proposed for iron-catalyzed $\mathrm{C}-\mathrm{H}$ azidations. ${ }^{[202]}$ The results are supportive of an overall slow azide addition to yield the final product $\mathbf{1 1 3}$ and significantly improved site- and chemoselectivities were found when comparing with the previously reported findings on manganese-catalyzed $\mathrm{C}-\mathrm{H}$ azidation with chemical oxidants. $^{[198]}$

The versatility of the approach was further substantiated by Dr. A. Del Vecchio and Dr. R. C. Samanta with additional 22 scope examples for the manganaelectro-catalyzed $\mathrm{C}-\mathrm{H}$ 
azidation of secondary or tertiary $\mathrm{C}\left(\mathrm{sp}^{3}\right)-\mathrm{H}$ bonds in overall moderate to high yields (3295\%), including late-stage diversification of active pharmaceutical ingredients (API) such as ibuprofen or musk fragrance celestolide. Generally, when multiple reactive sites were present, $\mathrm{C}-\mathrm{H}$ azidation inherently took place at the tertiary $\mathrm{C}-\mathrm{H}$ bond over secondary or primary homolytic $\mathrm{C}-\mathrm{H}$ scission. Moreover, various functional groups including silyloxy, amides, ethers, esters, enolizable ketones and nitriles, were well tolerated. The scalability of the approach was further validated by $D r . R$. C. Samanta in a successful electrocatalytic gram-scale $\mathrm{C}\left(\mathrm{sp}^{3}\right)-\mathrm{H}$ azidation of TBS-protected 4-isopropylphenol at the isopropyl group, with similar levels of efficiency. ${ }^{[349]}$

\subsubsection{Mechanistic Studies}

\subsubsection{Radical Trap Experiment}

To support the involvement of radical intermediates, the effect of radical scavengers, such as (2,2,6,6-tetramethylpiperidin-1-yl)oxyl (TEMPO), was probed (Scheme 81). Here, complete inhibition of the reaction was observed, and no desired product 113n was detected. Nevertheless, reaction monitoring with ESI mass spectrometry or ${ }^{1} \mathrm{H}-\mathrm{NMR}$ spectroscopy failed to detect the putative radical adduct 228. Other radical scavengers such as 2,6-di-tert-butyl-4-methylphenol (BHT) provided similar results. ${ }^{[349]}$ In this context, it is noteworthy that recent studies by Lin reported the anodic generation of oxoammonium ions $\left(\mathrm{TEMPO}^{+}\right)$to mediate electrochemical $\mathrm{C}-\mathrm{H}$ azidation of olefins via a TEMPO-N ${ }_{3}$ charge-transfer complex. ${ }^{[373]}$ Thus, it can not be excluded that the easily oxidizable radical species, e.g. TEMPO or BHT, were anodically oxidized and resulted in a nonproductive redox bypass, with the $\mathrm{C}-\mathrm{H}$ bond remaining untouched.

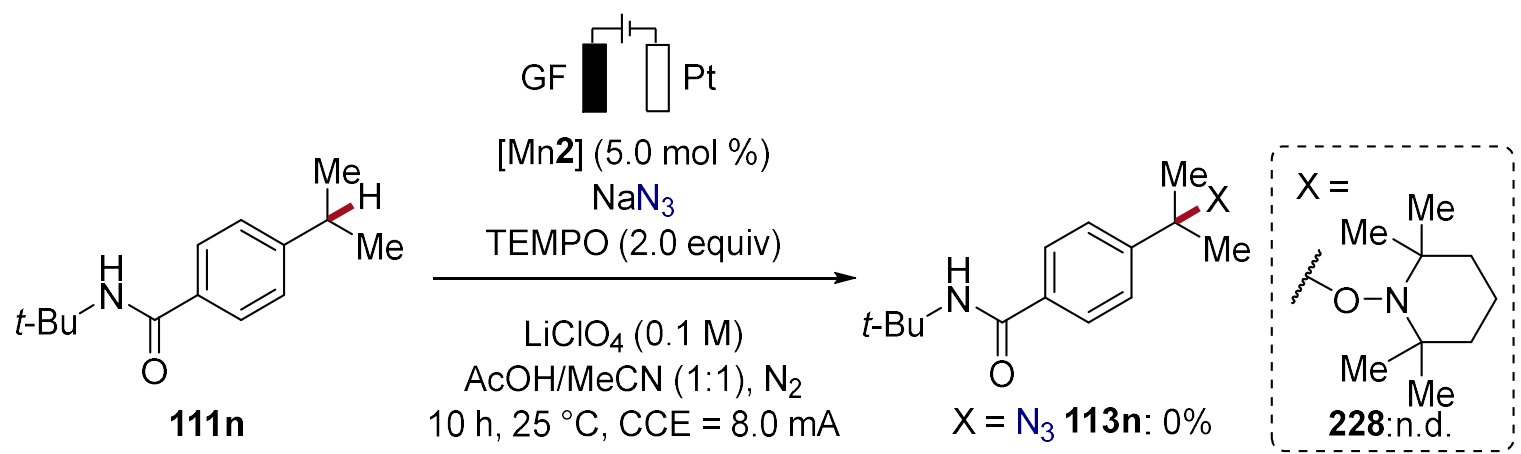

Scheme 81. Experiment with TEMPO as the radical scavenger. 


\subsubsection{Intermolecular Competition Experiment}

In addition, an intermolecular competition reaction with differently decorated cumene derivatives 1110 and 111p, with the substituent para to the isopropyl group, was performed to compare the effect of the electronic properties of the substrate on the reaction rates of the manganaelectro-catalyzed $\mathrm{C}-\mathrm{H}$ azidation. It was found that the electron-rich 1isopropyl-4-methoxybenzene (1110) reacted preferentially with a ratio 1130 vs. 113p of 6:1 (Scheme 82). The result highlighted the preferential $\mathrm{C}-\mathrm{H}$ functionalization of electronrich $\mathrm{C}-\mathrm{H}$ bonds.

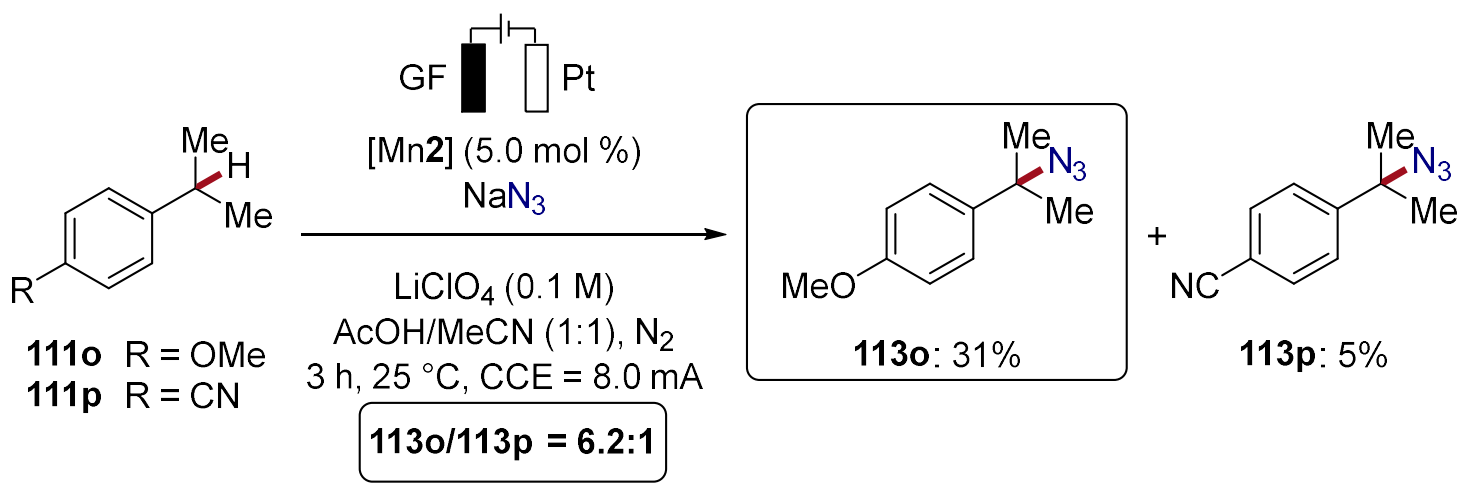

Scheme 82. Intermolecular competition reaction.

Furthermore, the kinetic isotope effect (KIE) for manganaelectro-catalyzed $\mathrm{C}-\mathrm{H}$ azidation of triphenylmethane and deuterated triphenylmethane- $d_{1}$ was evaluated by $\mathrm{Dr}$. A. Del Vecchio. Two parallel reactions were monitored via GC-FID and revealed a KIE of $k_{\mathrm{H}} / k_{\mathrm{D}}=3.0$, thus implying that the $\mathrm{C}-\mathrm{H}$ cleavage is the turnover-limiting step of the catalysis. $^{[349]}$

\subsubsection{Synthesis of Manganese Azide Complexes Mn5(III)- $\mathrm{N}_{3}$ and Mn5(IV)-(N3)2}

To identify the role of the manganese catalyst in the newly developed electrocatalytic $\mathrm{C}-\mathrm{H}$ transformation, studies were performed toward the preparation and full characterization of potentially active manganese azide complexes (Scheme 83). For the synthesis of the desired manganese salen complex Mn5(III)- $\mathrm{N}_{3}$ and $\mathrm{Mn5}(\mathrm{IV})-\left(\mathrm{N}_{3}\right)_{2}$, respectively, procedures by Fujii on related Jacobsen-azide-salen complexes were followed. ${ }^{[374]}$ Initially, Mn5(III)-N 3 was synthesized by treating the corresponding chloride complex [Mn5] with sodium azide in a $\mathrm{CH}_{2} \mathrm{Cl}_{2} / \mathrm{MeOH}$ mixture at $25^{\circ} \mathrm{C}$. The complex was isolated in $66 \%$ yield after recrystallization from hot $\mathrm{MeCN}$. The azide coordination was 
unambiguously confirmed by means of IR spectroscopy, ESI HR-MS and elemental analysis.

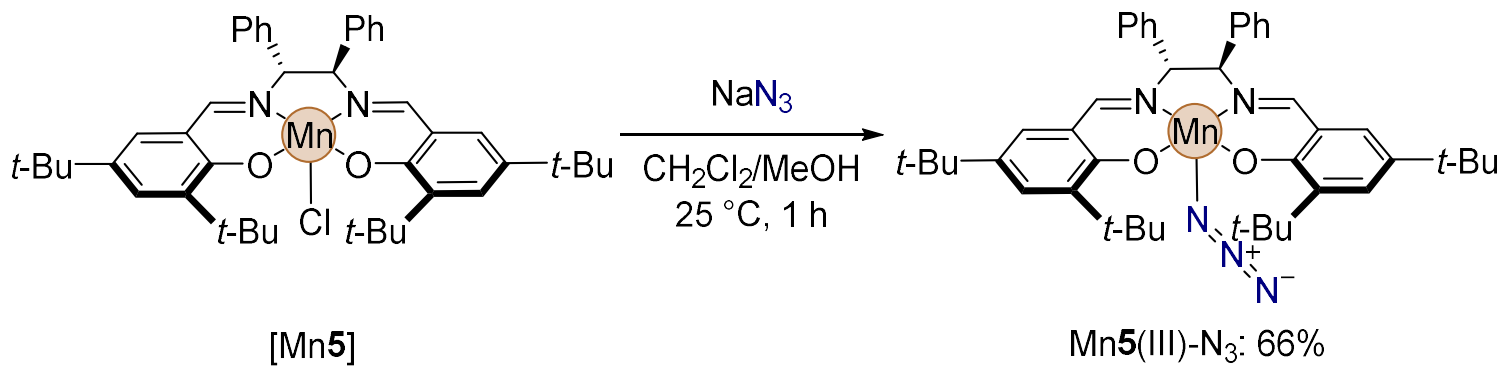

Scheme 83. Stoichiometric synthesis of the well-defined manganese(III) azide complex Mn5(III)-N 3 . Color code: brown Mn(III).

Likewise, the high-valent trans-diazidomanganese(IV) complex $\mathrm{Mn5}(\mathrm{IV})-\left(\mathrm{N}_{3}\right)_{2}$ was accessed by treating the manganese(III) complex Mn5(III)- $\mathrm{N}_{3}$ with a defined amount of $m$-CPBA as a chemical oxidant, in the presence of an excess of sodium azide (Scheme 84). Due to the potentially explosive nature of metal-azide complexes, the diazide complex was strictly handled at low temperatures and purification was performed by recrystallization from a $\mathrm{CH}_{2} \mathrm{Cl}_{2} / n$-pentane mixture at $-32{ }^{\circ} \mathrm{C}$. Elemental analysis and ESI mass spectrometry unambiguously confirmed the formation of the desired complex Mn5(IV)-( $\left.\mathrm{N}_{3}\right)_{2}$.

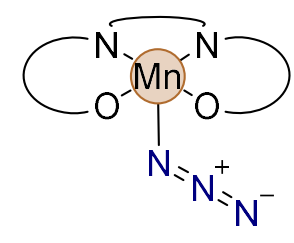

$\operatorname{Mn} 5$ (III)- $\mathrm{N}_{3}$

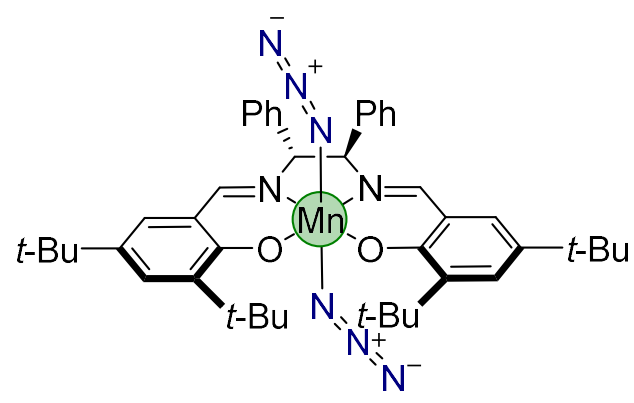

$\operatorname{Mn} 5(\mathrm{IV})-\left(\mathrm{N}_{3}\right)_{2}: 82 \%$

Scheme 84. Synthesis of the novel manganese(IV) diazide complex Mn5(IV)- $\left(\mathrm{N}_{3}\right)_{2}$. Color code: brown Mn(III); green Mn(IV).

To further compare the two novel manganese azide complexes, a detailed IR spectroscopic analysis was performed (Figure 31 ). 


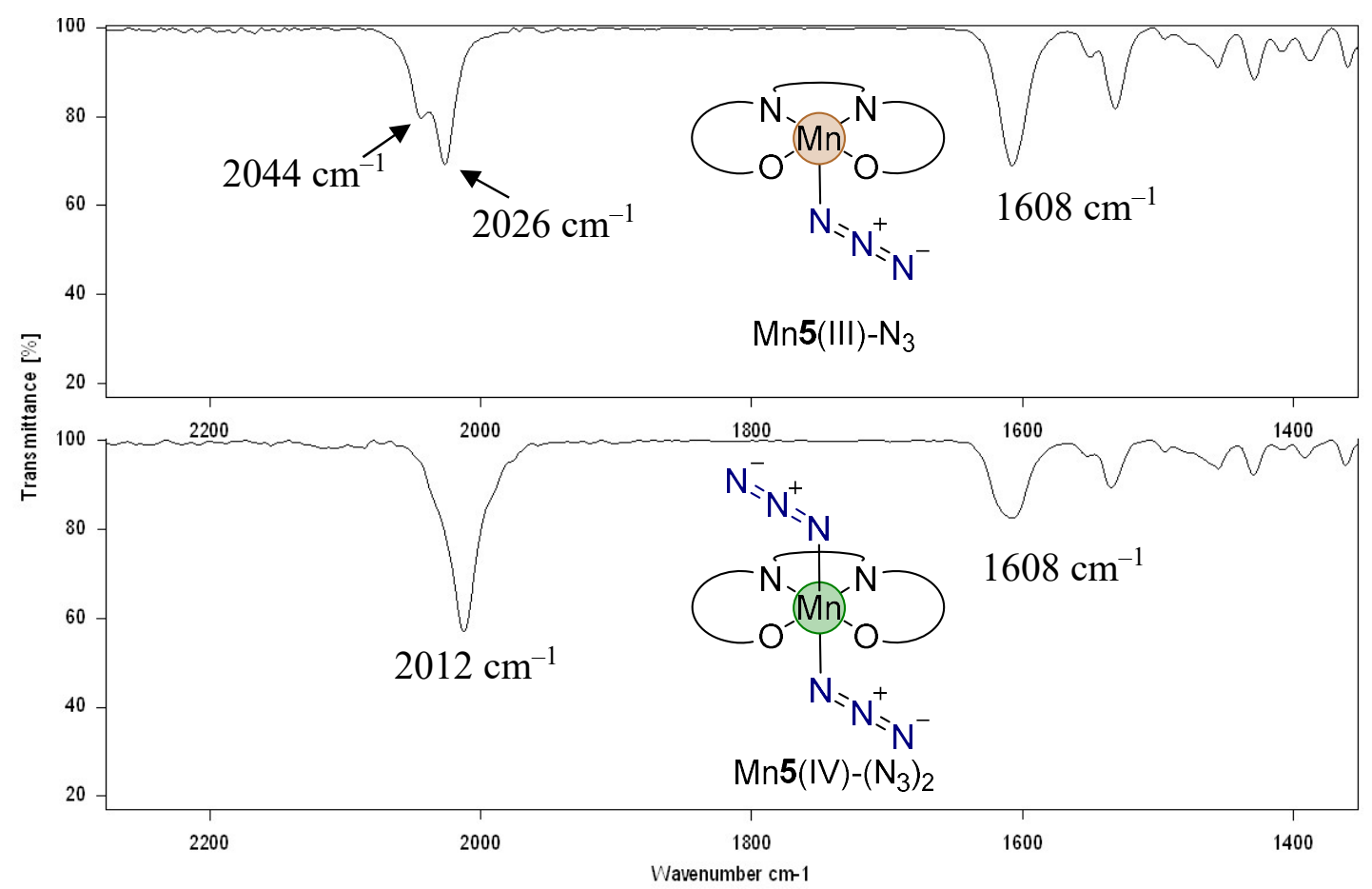

Figure 31. IR spectra comparison of the asymmetric stretching vibrations ( $\left.v_{\mathrm{as}}\right)$ of NNN at a) $\mathrm{Mn5}$ (III)- $\mathrm{N}_{3}$ and b) $\mathrm{Mn} 5(\mathrm{IV})-\left(\mathrm{N}_{3}\right)_{2}$, respectively.

The appearance of two distinct asymmetric stretching vibrations $\left(v_{\mathrm{as}}\right)^{[375]}$ at $2044 \mathrm{~cm}^{-1}$ and $2026 \mathrm{~cm}^{-1}$ for Mn5(III)-N 3 is in agreement with previous studies of Fujii ${ }^{[374 b]}$ and can be explained by the presence of differently azide-bridged, polymeric complexes in solution ${ }^{[376]}$ or by the presence of different complexes in asymmetric cells. ${ }^{[374 b]}$ For the high-valent $\operatorname{Mn5}(\mathrm{IV})-\left(\mathrm{N}_{3}\right)_{2}$, the asymmetric azide stretching vibration however was significantly shifted to lower wavelength of $v_{\mathrm{as}}=2012 \mathrm{~cm}^{-1}$. This finding is suggestive of a weakening of the coordinating azide bond upon oxidation of the complex to the corresponding highspin $\mathrm{d}^{3} \mathrm{Mn}(\mathrm{IV})$ species and increased ligand-to-metal charge transfer. ${ }^{[377]}$ The similar IR bands at $1608 \mathrm{~cm}^{-1}$ for $\mathrm{Mn5}$ (III)- $\mathrm{N}_{3}$ as well as for Mn5(IV)-(N $\left.{ }_{3}\right)_{2}$ can be assigned to the $\mathrm{C}=\mathrm{N}$ stretching mode of the salen ligand. Thus, revealing only minor or no changes in the salen ligand due to oxidation of the manganese central atom.

Finally, the molecular structure of the manganese(IV) diazide complex Mn5(IV)-(N $)_{2}$ was unambiguously confirmed by X-ray diffraction analysis (Figure 32). The solid state structure revealed an octahedral manganese(IV) complex with two azide groups in the axial positions. Interestingly, in a previous study by Fujii on related manganese(IV) azide complexes, a distorted stepped conformation was found with a Jacobsen's salen ligand. ${ }^{[374]}$ In contrast, the obtained molecular structure revealed a planar conformation of the salen ligand and only a minor twist of the two salicylidene rings was observed. 


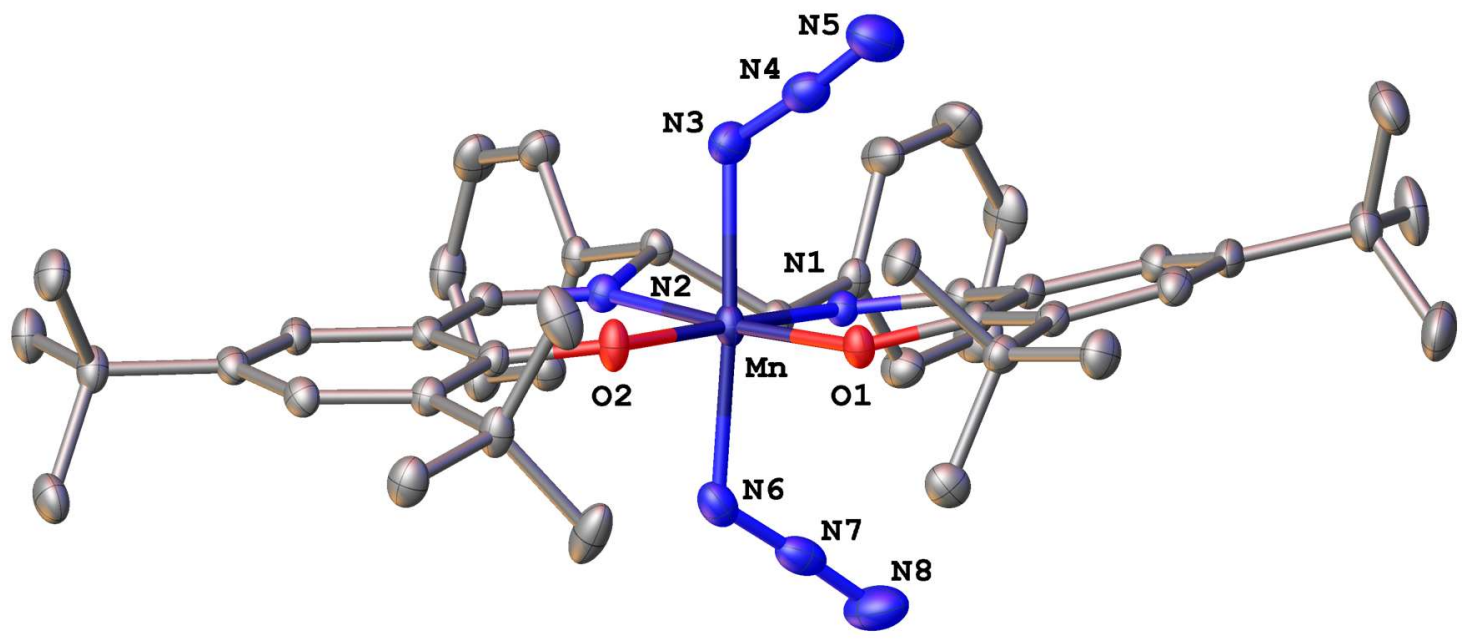

Figure 32. Molecular structure of $\operatorname{Mn5}(\mathrm{IV})-\left(\mathrm{N}_{3}\right)_{2}$ with thermal ellipsoids at $50 \%$ probability level. The hydrogen atoms are omitted for clarity.

\subsubsection{UV-Vis Absorption Spectroscopic Studies}

Next, UV-vis spectroscopy was used to evaluate the properties of the well-defined manganese azide complexes and different reaction components in solution. The study was initiated with the manganese(III) chloride complex [Mn5] in MeCN (Figure 33). Characteristic absorption maxima were detected at $244 \mathrm{~nm}, 328 \mathrm{~nm}$ and $440 \mathrm{~nm}$. Similar absorption maxima were observed when a solution of complex [Mn5] in $\mathrm{MeCN}$ was treated with $\mathrm{NaN}_{3}$, as well as for the well-defined manganese(III) azide complex Mn5(III)- $\mathrm{N}_{3}$. However, the oxidized manganese(IV) bis(azide) complex Mn5(IV)- $\left(\mathrm{N}_{3}\right)_{2}$ revealed significantly different absorption properties with maxima at $277 \mathrm{~nm}, 342 \mathrm{~nm}, 442 \mathrm{~nm}$, and $642 \mathrm{~nm}$. The strong increased absorption at $442 \mathrm{~nm}$ was assigned to the charge transfer bands from coordinating $\mathrm{N}_{3}$ to the manganese(IV) metal center. ${ }^{[374]}$ 


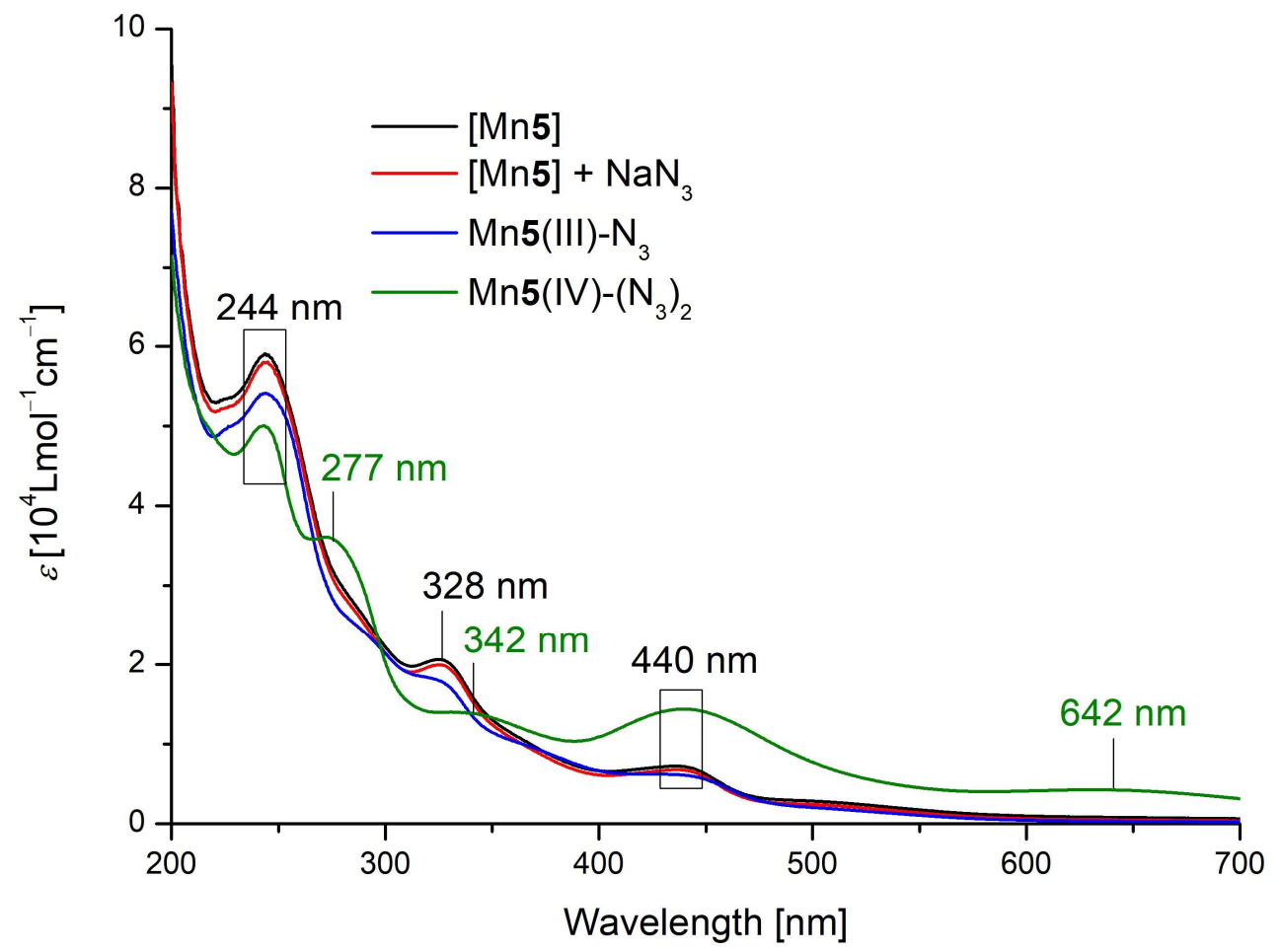

Figure 33. UV-vis studies in $\mathrm{MeCN}$ at $25^{\circ} \mathrm{C}$. a) [Mn5] (black). b) [Mn5] and $\mathrm{NaN}_{3}$ (red). c) $M n 5$ (III)- $\mathrm{N}_{3}$ (blue). d) $M n 5(I V)-\left(\mathrm{N}_{3}\right)_{2}$ (green).

With the characteristic absorption maxima identified, additional UV-vis experiments were performed to identify possible reaction intermediates and to evaluate the oxidation state of the manganese catalyst during the reaction. Therefore, three reaction mixtures were prepared and subsequently examined by UV-vis spectroscopy (Scheme 85 and Figure 34). To support the anodic oxidation of the manganese(III) complex, measurements were performed with reaction mixtures $\mathbf{A}$ and $\mathbf{B}$. Mixture A contained complex [Mn5] and $\mathrm{NaN}_{3}$ prior anodic oxidation and mixture $\mathbf{B}$ consisted of the same components, but was treated with constant current electrolysis for $30 \mathrm{~min}$, prior the spectroscopic analysis $\left(Q \cdot \mathrm{mol}^{-1}=\right.$ 5.96 F). Visually, the color of the reaction mixture changed drastically during the course of the electrolysis, from a clear brown solution into a dark green mixture, which was comparable to the dissolved well-defined manganese(IV)-bis(azide) complex Mn5(IV)$\left(\mathrm{N}_{3}\right)_{2}$ (Scheme 85). Thus, after anodic oxidation, mixture B showed similar absorption maxima to manganese(IV) complex Mn5(IV)-(N $\left.\mathrm{N}_{3}\right)_{2}$ (Figure 34, green line vs. red line). Interestingly, when the crude reaction mixture was analyzed, mixed absorption maxima of both species, manganese(III) (328 nm) and manganese(IV) (277, and $440 \mathrm{~nm})$, were detected, providing strong support for the catalytic formation and consumption of $\mathrm{Mn5}(\mathrm{IV})-\left(\mathrm{N}_{3}\right)_{2}$ during electrocatalytic $\mathrm{C}-\mathrm{H}$ azidations of hydrocarbons $\mathbf{1 1 1 b}$ (Figure 34, blue line). 


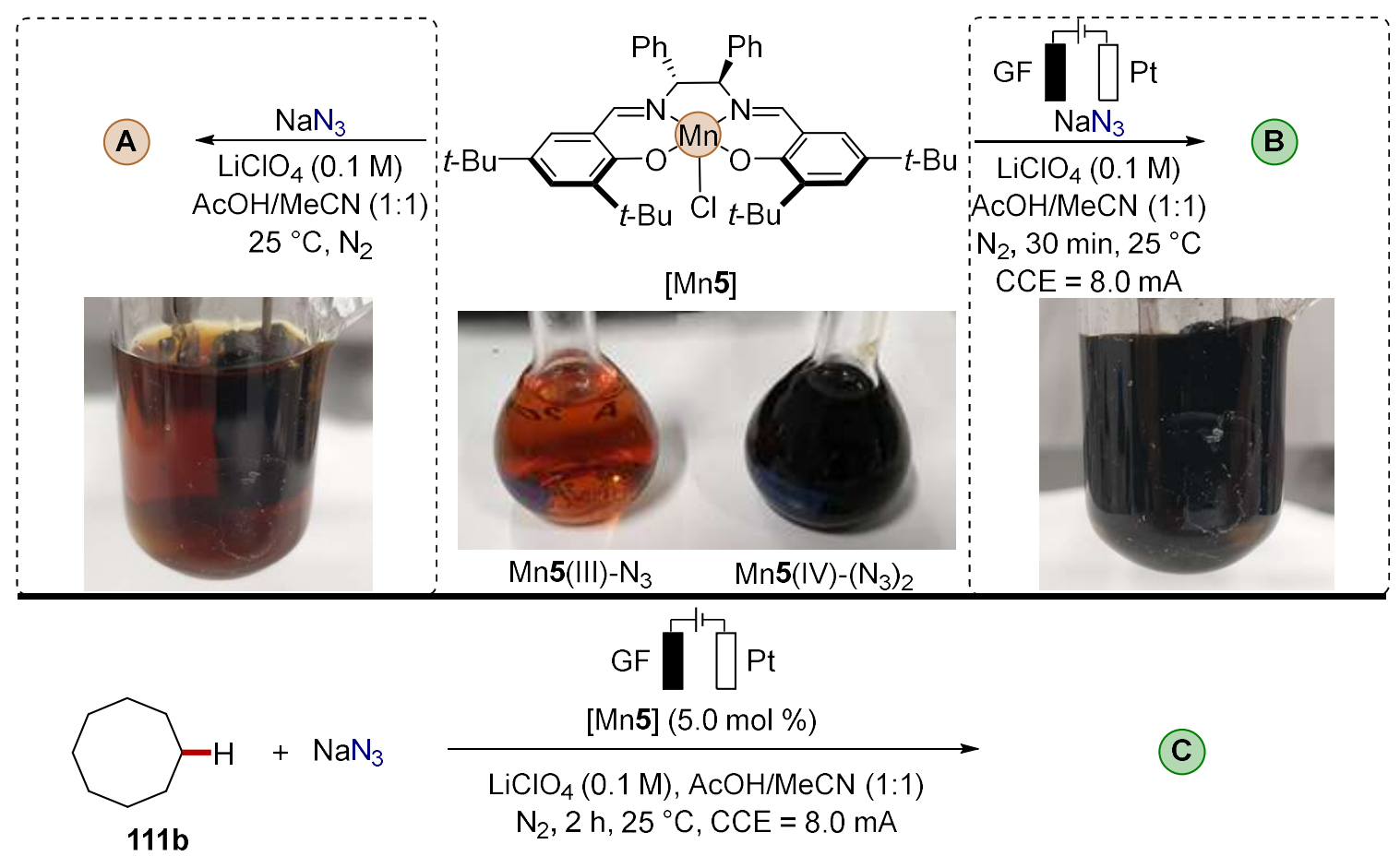

Scheme 85. Reaction mixture for UV-vis studies. A) [Mn5] $+\mathrm{NaN}_{3}$. B) $[\mathrm{Mn} 5]+\mathrm{NaN}_{3}$ and $\mathrm{CCE}$ at $8 \mathrm{~mA}$ for $30 \mathrm{~min} . \mathrm{C})[\mathrm{Mn} 5]+\mathrm{NaN}_{3}+\mathbf{1 1 1 b} \mathrm{CCE}$ at $8 \mathrm{~mA}, 2 \mathrm{~h}$.

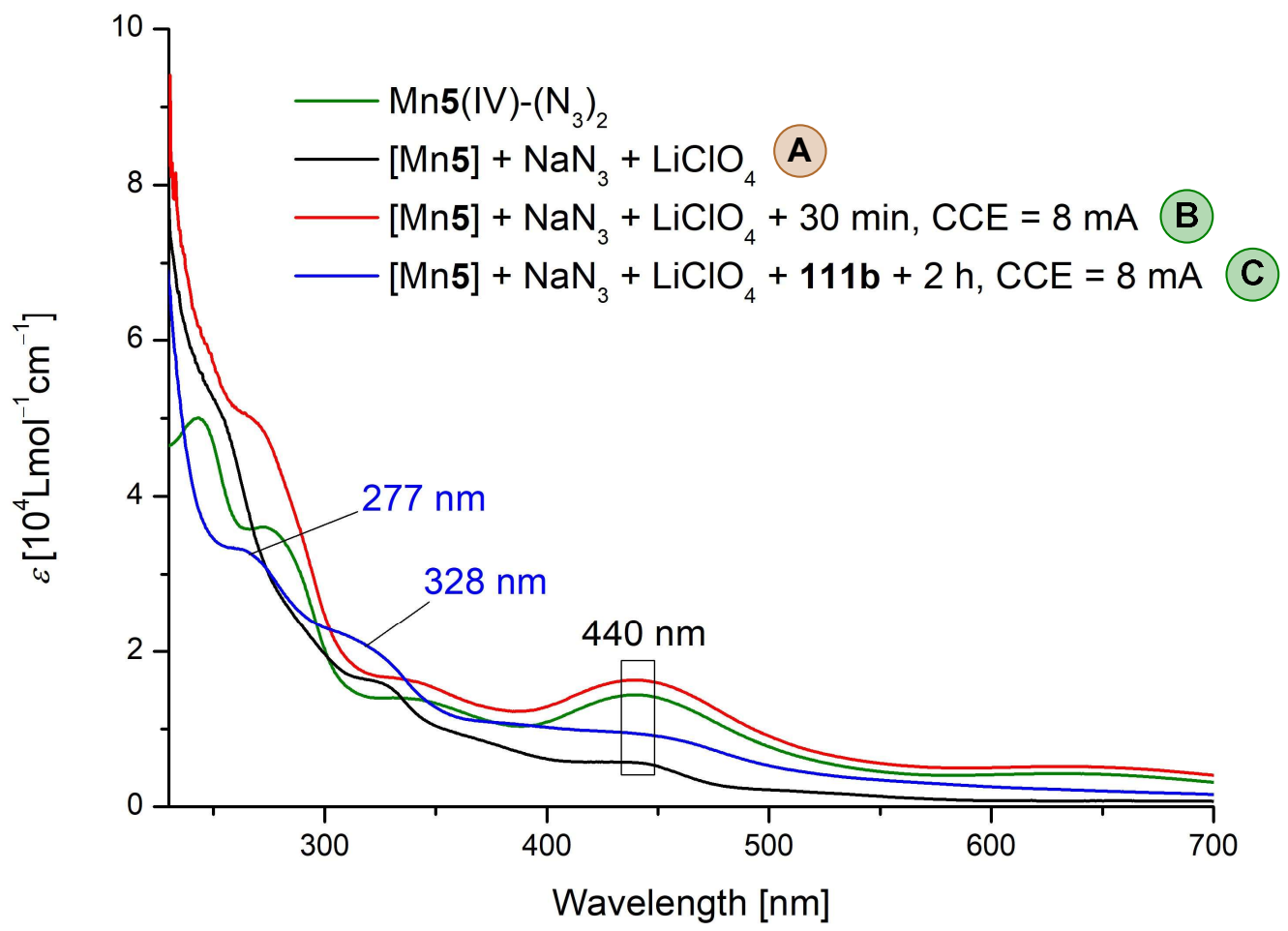

Figure 34. UV-vis studies in $\mathrm{MeCN} / \mathrm{AcOH}(1: 1)$ at $25^{\circ} \mathrm{C}$. a) $\mathrm{Mn5}(\mathrm{IV})-\left(\mathrm{N}_{3}\right)_{2}$ (green). b) Reaction mixture $\mathbf{A}$ : [Mn5], $\mathrm{NaN}_{3}$ and $\mathrm{LiClO}_{4}$ (black). c) Reaction mixture $\mathbf{A}$ after constant current electrolysis of $8 \mathrm{~mA}$ for $30 \mathrm{~min}$ (red). d) Reaction mixture B: [Mn5], $\mathrm{NaN}_{3}, \mathrm{LiClO}_{4}$ and cyclooctane (111b) after constant current electrolysis for $2 \mathrm{~h}$ (blue). 


\subsubsection{Electrochemical Analysis}

To further substantiate the obtained findings, detailed electrochemical analyses via cyclic voltammetry were performed. Initially, the anodic azide oxidation was probed in $\mathrm{MeCN}$ as the solvent, using different working electrodes (WE) (Figure 35). Due to poor solubility of $\mathrm{NaN}_{3}$, tetra- $n$-butylammonium azide was used as the azide source of choice. Under these conditions, the azide anion revealed an anodic onset potential of $+0.75 \mathrm{~V} v s$. $\mathrm{Ag} / \mathrm{AgCl}(3 \mathrm{M} \mathrm{KCl})$, with an irreversible peak potential of $E_{\mathrm{p}, \mathrm{a}}=0.88 \mathrm{~V}$ and $E_{\mathrm{p}, \mathrm{a}}=0.94 \mathrm{~V}$ for GC (black line) and Pt (red line) as the WE, respectively.

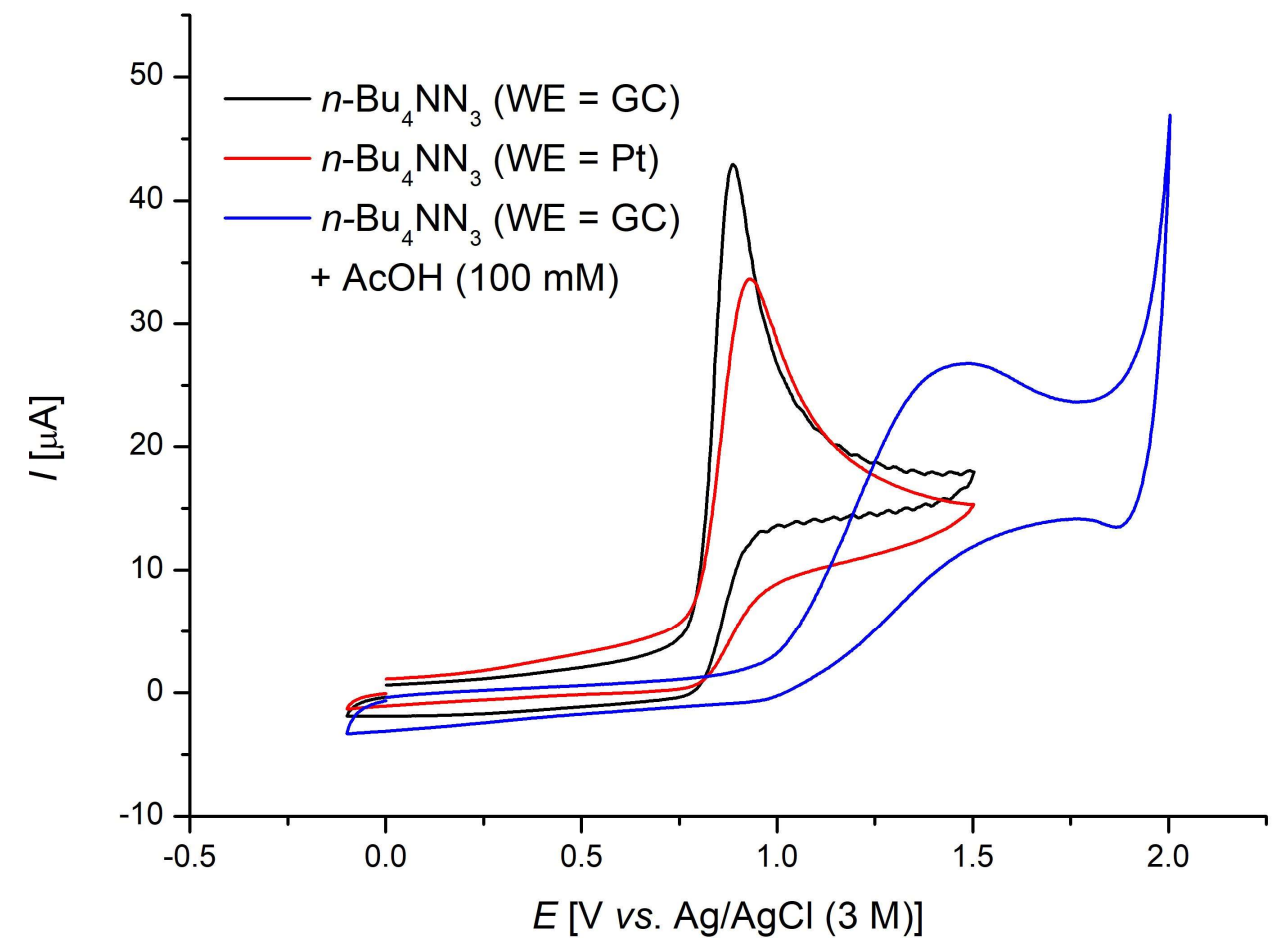

Figure 35. Cyclic voltammetry of tetra- $n$-butylammonium azide $(0.3 \mathrm{mM})$ at GC (black) or Pt (red, $3 \mathrm{~mm}$-diameter, disc-electrode) in the absence of or in the presence of an excess of $\mathrm{AcOH}$ (blue, $100 \mathrm{mM}$ ).

Based on these results, it is expected that different anodic working electrodes do not have a significant influence on the heterogeneous oxidation of azide anions. However, during the optimization studies, a platinum working electrode showed no product formation under the reaction conditions ( $c f$. Table 30, entry 10). This is a notable finding since previous studies on azidation of olefins under similar reaction conditions used platinum anodes. ${ }^{[378]}$ Hence, these outcomes were supportive of the important cooperation of manganese catalyst and carbon-based anode materials for electrocatalytic $\mathrm{C}-\mathrm{H}$ azidation reactions of unactivated hydrocarbons 111. Upon addition of glacial acetic acid (100 mM, blue line) the 
irreversible anodic oxidation event was shifted to an onset potential of $1.0 \mathrm{~V} v s . \mathrm{Ag} / \mathrm{AgCl}$ $(3 \mathrm{M} \mathrm{KCl})$ with a peak potential of $E_{\mathrm{p}, \mathrm{a}}=1.45 \mathrm{~V}$. The difference for the oxidation potential was illustrative of a $\mathrm{pH}$-dependent oxidation event, following the Nernst equation.

Likewise, the manganese(III) complex [Mn2] and other reaction components, such as 1,2,3,4-tetrahydronaphthalene (111a), were investigated by cyclic voltammetry (Figure 36). The manganese(III) chloride complex [Mn2] showed two distinct redox potentials in $\mathrm{MeCN}$ (black line). The first reversible redox event $\left(\Delta E_{\mathrm{p}}=91 \mathrm{mV}\right)$ at $E_{1 / 2}=-0.062 \mathrm{~V} v s$. $\mathrm{Ag} / \mathrm{AgCl}(3 \mathrm{M} \mathrm{KCl})$ was attributed to the $\mathrm{Mn}(\mathrm{II}) / \mathrm{Mn}(\mathrm{III})$ redox pair. The second, quasireversible redox-event $\left(\Delta E_{\mathrm{p}}=112 \mathrm{mV}\right)$ at $E_{1 / 2}=0.906 \mathrm{~V} v s . \mathrm{Ag} / \mathrm{AgCl}(3 \mathrm{M} \mathrm{KCl})$ was attributed to the $\mathrm{Mn}(\mathrm{III}) / \mathrm{Mn}(\mathrm{IV})$ redox couple. ${ }^{[379]}$ Reports by Fuji on similar complexes gave strong support for a ligand-centered electron transfer. ${ }^{[380]}$

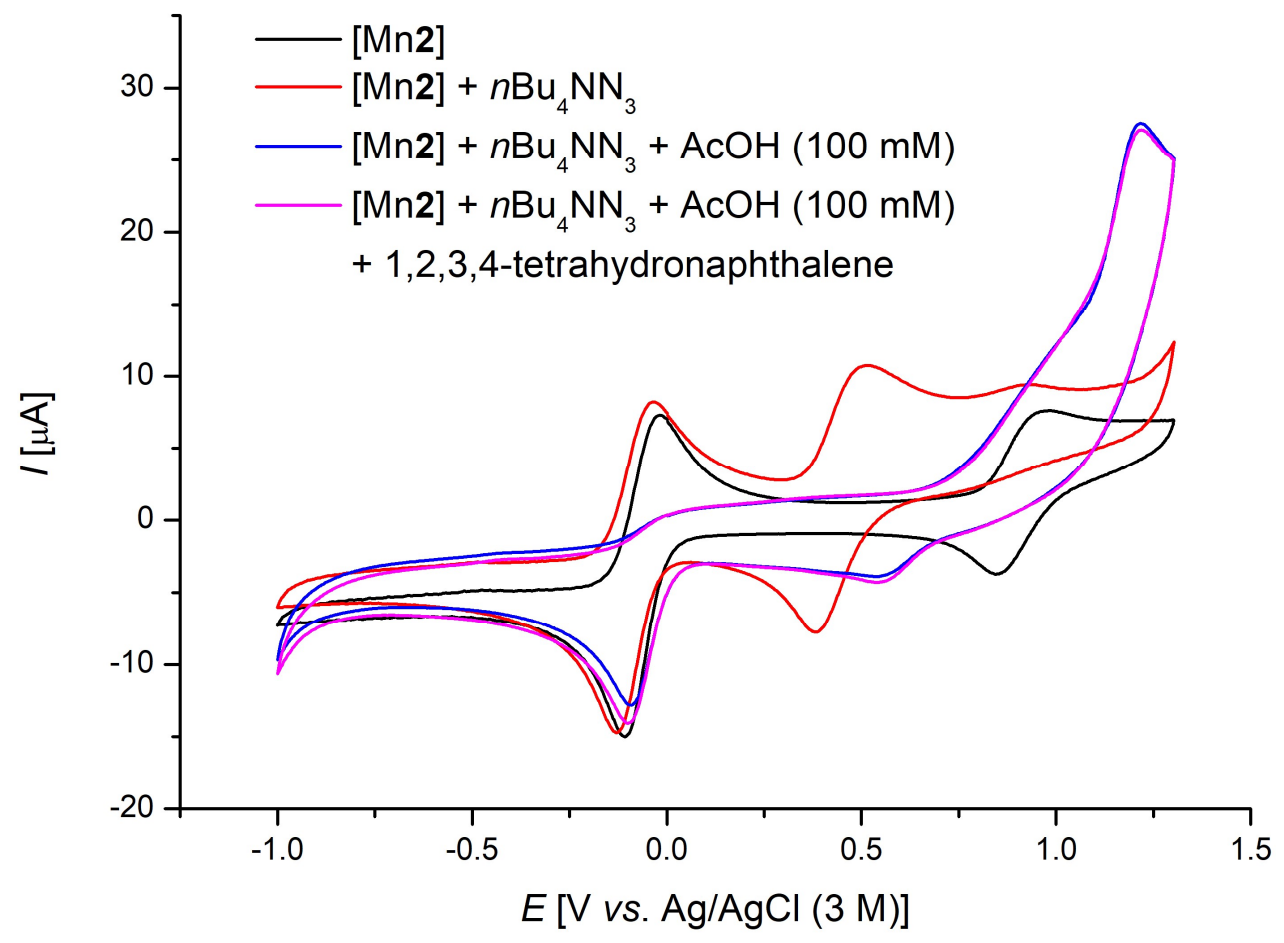

Figure 36. Cyclic voltammetry of a) [Mn2] (black); b) in the presence of tetra- $n$ butylammonium azide (red); c) as b) but with the addition of acetic acid $(100 \mathrm{mM}) ; \mathrm{d})$ as c) but in the presence of 111a.

Upon addition of the organic azide salt, the first reversible redox event $\left(\Delta E_{\mathrm{p}}=91 \mathrm{mV}\right)$ at $E_{1 / 2}=-0.086 \mathrm{~V} v s . \mathrm{Ag} / \mathrm{AgCl}(3 \mathrm{M} \mathrm{KCl})$ was only slightly shifted $\left(\Delta E_{1 / 2}=26 \mathrm{mV}\right)$ (red line). However, a significant change of the second quasi-reversible redox event $\left(\Delta E_{\mathrm{p}}=132 \mathrm{mV}\right)$ was detected $\left(E_{1 / 2}=0.448 \mathrm{~V} v s . \operatorname{Ag} / \operatorname{AgCl}(3 \mathrm{M} \mathrm{KCl})\right)$. The difference $\left(\Delta E_{1 / 2}=458 \mathrm{mV}\right) \mathrm{can}$ be explained by stabilization of the oxidized products by further addition of azide-anions, 
thus resulting in the corresponding $\mathrm{Mn} 2(\mathrm{IV})-\left(\mathrm{N}_{3}\right)_{2}$ complex (vide infra). Similar findings have been previously reported, albeit with chloride or cyanide anions. ${ }^{[379 a, 381]}$ The small irreversible anodic event at $E_{\mathrm{p}, \mathrm{a}}=0.93 \mathrm{~V}$ represents most-likely non-coordinating azide anions, dissociated chloride anions or free manganese(III) chloride complex [Mn2]. In the presence of an excess of acetic acid, the redox events are diminished and supportive for chemical follow-up reactions (blue line). Addition of substrate 111a did not result in significant changes of the voltammogram (magenta line).

In contrast to the manganese(III) chloride complex, the well-defined manganese(III) azide complex Mn5(III)- $\mathrm{N}_{3}$ showed a less-pronounced and broadened current response (Figure 37). The first, quasi-reversible redox event $\left(\Delta E_{\mathrm{p}}=372 \mathrm{mV}\right)$ at $E_{1 / 2}=-0.368 \mathrm{~V} v \mathrm{~s} . \mathrm{Ag} / \mathrm{AgCl}$ ( $3 \mathrm{M} \mathrm{KCl}$ ) was attributed to the $\mathrm{Mn}(\mathrm{II}) / \mathrm{Mn}(\mathrm{III})$ redox couple, thus revealing a more difficult reduction compared to the manganese(III) chloride complex [Mn2].

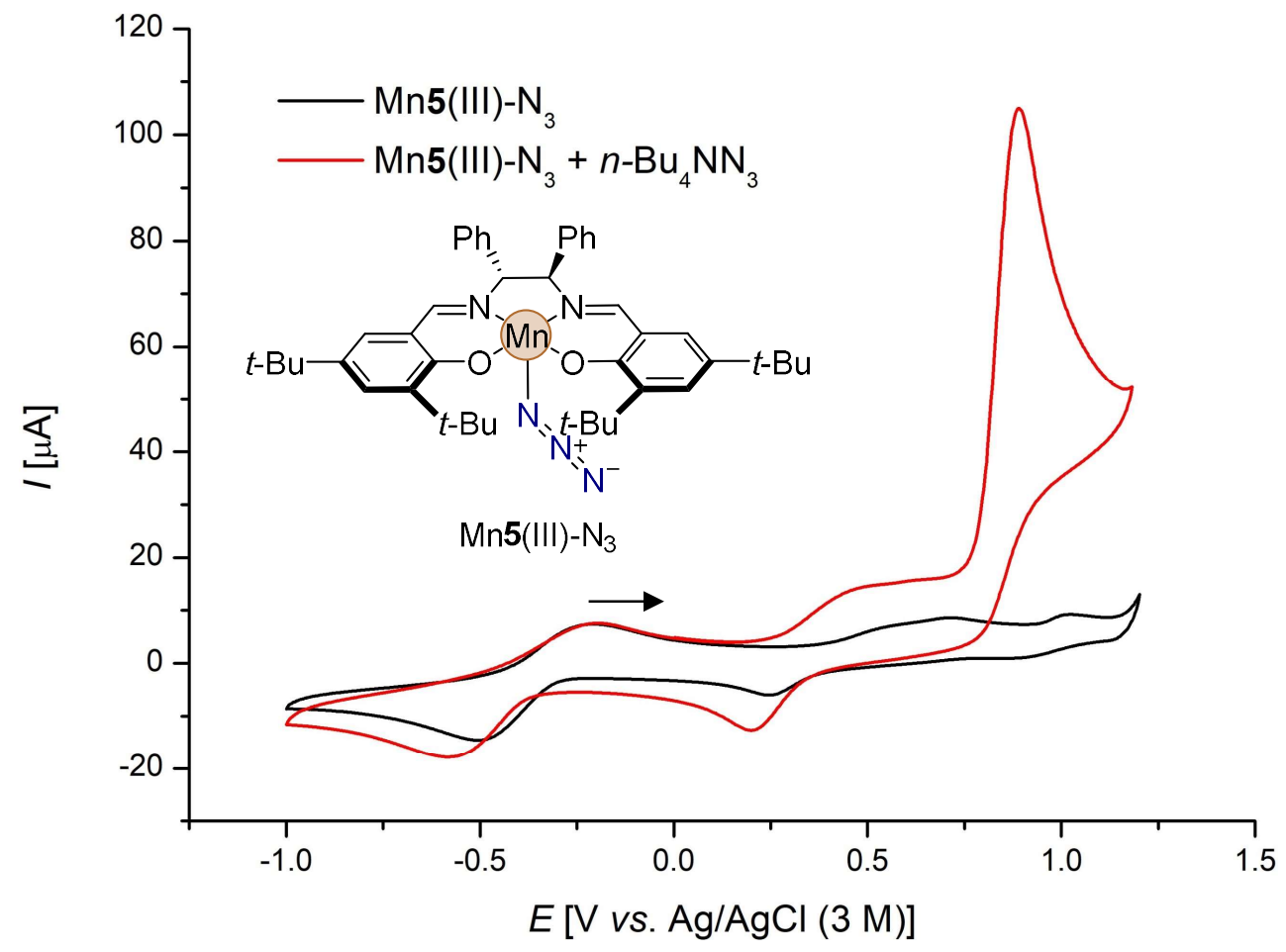

Figure 37. Cyclic voltammograms of $\mathrm{Mn5}$ (III)- $\mathrm{N}_{3}(0.3 \mathrm{mM})$ in the absence (black) and in the presence (red) of an excess of tetra- $n$-butylammonium azide.

The second, quasi-reversible redox-event $\left(\Delta E_{\mathrm{p}}=264 \mathrm{mV}\right)$ at $E_{1 / 2}=0.328 \mathrm{~V} v s . \mathrm{Ag} / \mathrm{AgCl}$ $(3 \mathrm{M} \mathrm{KCl})$ was assigned to the $\mathrm{Mn}(\mathrm{III}) / \mathrm{Mn}(\mathrm{IV})$ redox couple. Addition of tetra- $n$ butylammonium azide led to increased current response for the second redox event, which is supportive of the anodic formation of the $\operatorname{Mn5}(\mathrm{IV})-\left(\mathrm{N}_{3}\right)_{2}$ complex (red line). 
Finally, the well-defined manganese(IV) azide complex Mn5(IV)- $\left(\mathrm{N}_{3}\right)_{2}$ was investigated in electroanalytical studies. Similar to the previous complexes, a potential range of $+1.2 \mathrm{~V}$ to $-1.0 \mathrm{~V} v s . \mathrm{Ag} / \mathrm{AgCl}(3 \mathrm{M} \mathrm{KCl})$ was chosen. When the first voltammogram was recorded, with a starting potential of $E=0 \mathrm{~V}$ and a positive sweep rate of $100 \mathrm{mV} \cdot \mathrm{s}^{-1}$, a negative current response was recorded. The negative current gap is indicating that the manganese(IV) complex already undergoes reduction at a potential of below $E=0 \mathrm{~V}$ (Figure 38). However, previous reports by Fuji on similar complexes assigned the manganese(III/IV) redox couple to potentials at $E_{1 / 2}=-0.17 \mathrm{~V} v s . \mathrm{Fc} / \mathrm{Fc}^{+},{ }^{[374 b]}$ which was contradicted by our experiments.

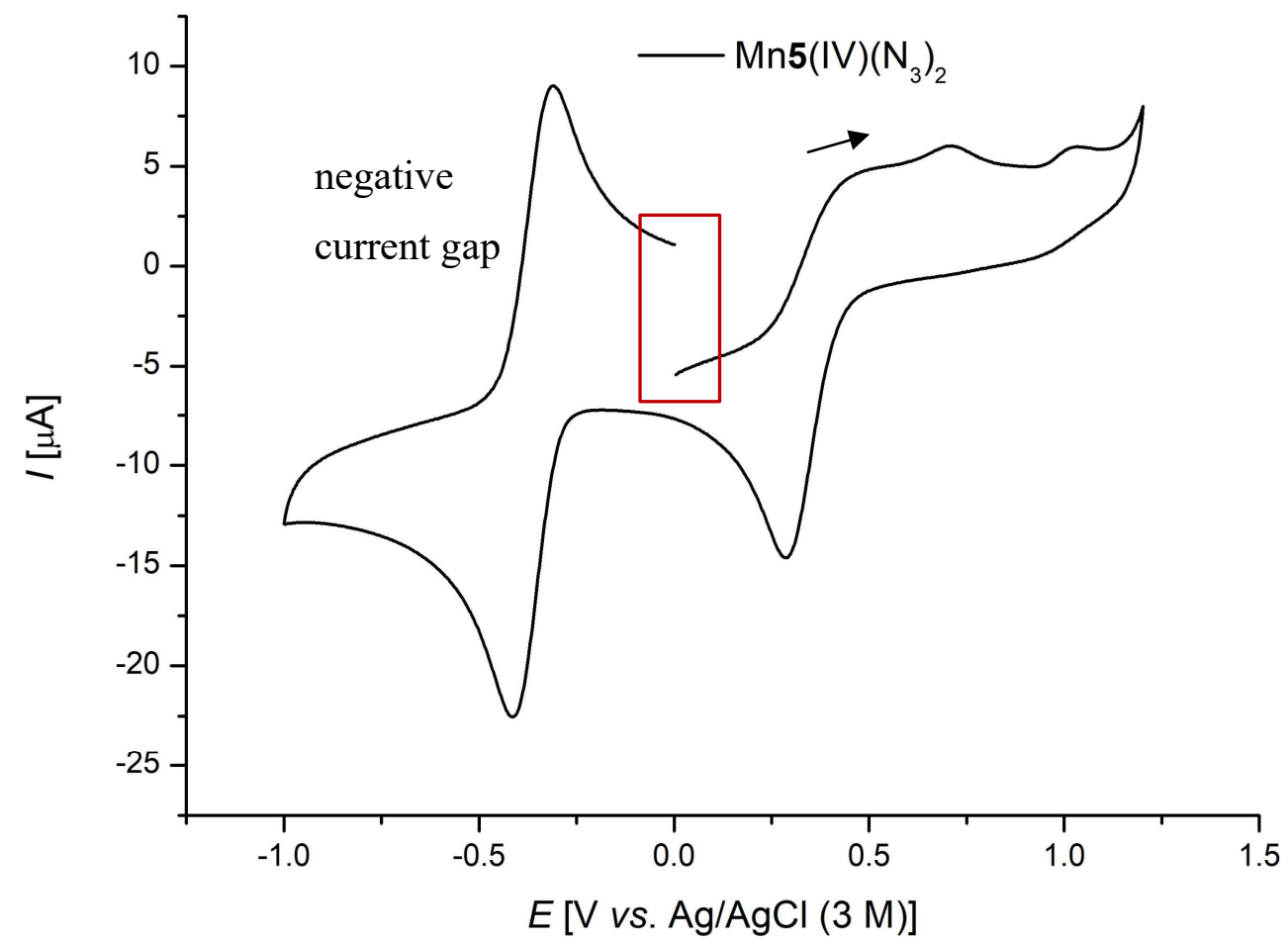

Figure 38. Cyclic voltammogram of Mn5(IV)-( $\left.\mathrm{N}_{3}\right)_{2}(0.3 \mathrm{~mm})$. Two voltammograms were recorded and the first voltammogram is presented. Starting potential was $E=0 \mathrm{~V}$, with a positive sweep rate.

To reveal the full redox properties of the complex, a second voltammogram was performed, with a starting potential of $1.2 \mathrm{~V}$ vs. $\mathrm{Ag} / \mathrm{AgCl}(3 \mathrm{M} \mathrm{KCl})$ and a negative sweep rate of $-100 \mathrm{mV} \cdot \mathrm{s}^{-1}$, to ensure that the complex is not reduced prior to the signal recording. Now, two redox events were measured (Figure 39). The first quasi-reversible redox event $\left(\Delta E_{\mathrm{p}}=\right.$ $0.136 \mathrm{~V})$ at $E_{1 / 2}=-0.370 \mathrm{~V} v s . \mathrm{Ag} / \mathrm{AgCl}(3 \mathrm{M} \mathrm{KCl})$ was assigned to the $\mathrm{Mn}(\mathrm{II}) / \mathrm{Mn}(\mathrm{III})$ redox couple with a concomitant loss of both azide ligands, which was reflected in a EC mechanism. The second, quasi-reversible redox-event $\left(\Delta E_{\mathrm{p}}=0.174 \mathrm{~V}\right)$ at $E_{1 / 2}=0.366 \mathrm{~V}$ 
vs. $\mathrm{Ag} / \mathrm{AgCl}(3 \mathrm{M} \mathrm{KCl})$ or $E_{1 / 2}=0.071 \mathrm{~V} v s . \mathrm{Fc} / \mathrm{Fc}^{+}$was attributed to the $\mathrm{Mn}(\mathrm{III}) / \mathrm{Mn}(\mathrm{IV})$ redox couple. ${ }^{[377]}$ Cyclic voltammograms at different scanning rates revealed a fast chemical follow-up reaction upon reduction of the manganese(IV) complex ( $c f$. Figures 64-67).

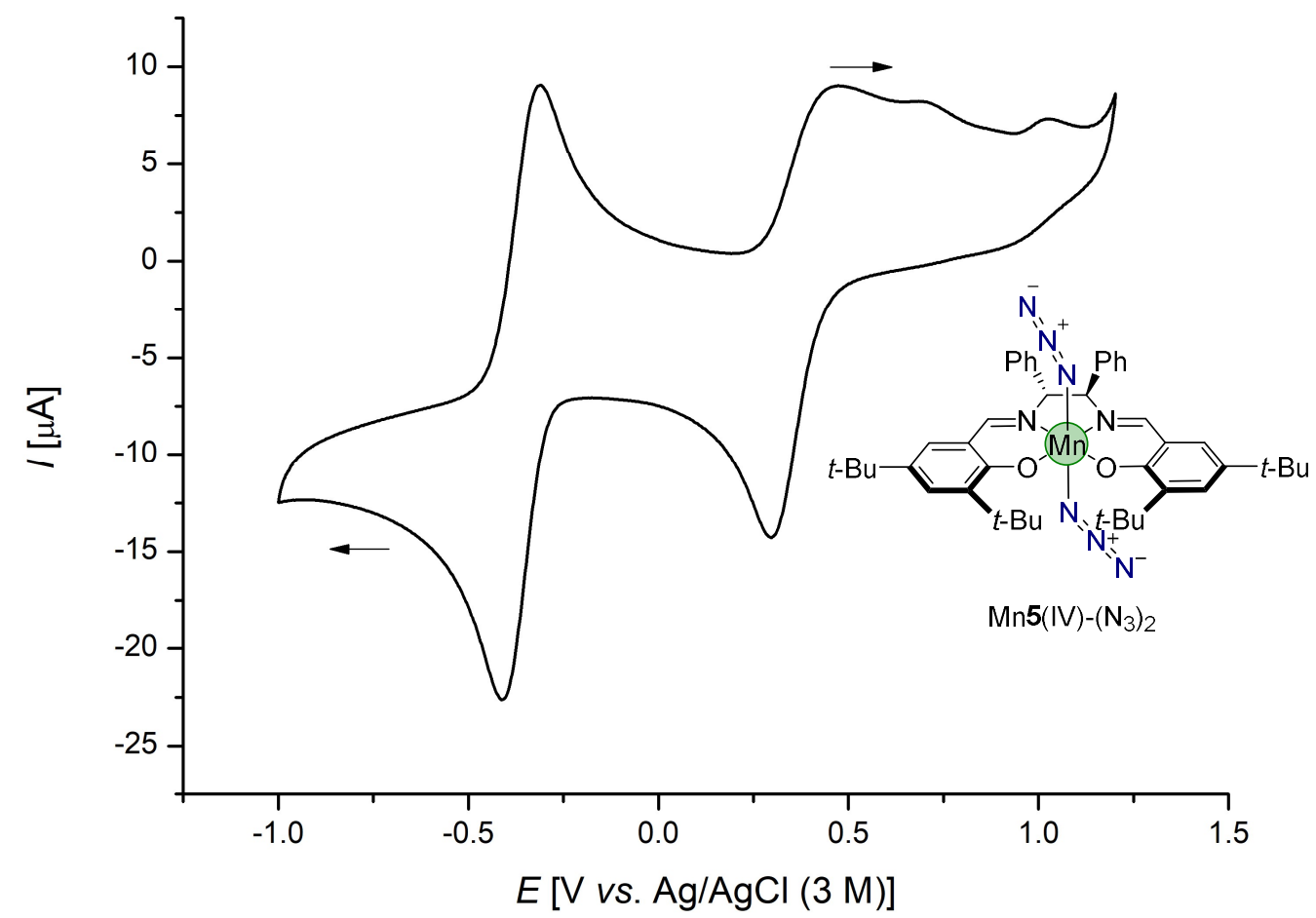

Figure 39. Cyclic voltammogram of Mn5(IV)-( $\left.\mathrm{N}_{3}\right)_{2}(0.3 \mathrm{mM})$. Two voltammograms were recorded and the second voltammogram is presented.

With the redox properties of all reaction components in hand, it became apparent that the anodic formation of manganese(IV) azide complexes is likely to occur under the reaction conditions and direct oxidation of the azide anion or the substrate $\mathbf{1 1 1}$ would require considerably higher working potentials. To put these findings into practice, a chronoamperometric experiment was performed to obtain electrocatalytic $\mathrm{C}-\mathrm{H}$ azidation reactions at exceedingly low oxidation potentials of $0.8 \mathrm{~V} v s . \mathrm{Ag} / \mathrm{Ag}^{+}$(Scheme 86). The detected current-time response revealed fast current consumption within the first two hours, followed by 8 hours of steady current response, indicative of a catalytic reaction profile. After overall $10 \mathrm{~h}$, the catalytic current response slowly decreased. Notably, substrate 111a was oxidized in $56 \%$ yield to the desired organic azide 113a, without any detection of overoxidation to the ketone 226a (vide supra). Based on the previous cyclovoltammetric analysis, none of the other reaction components were able to undergo anodic oxidation at 
these potentials, indicating the involvement of the manganese catalyst in both steps of the electrocatalytic $\mathrm{C}-\mathrm{H}$ azidation.

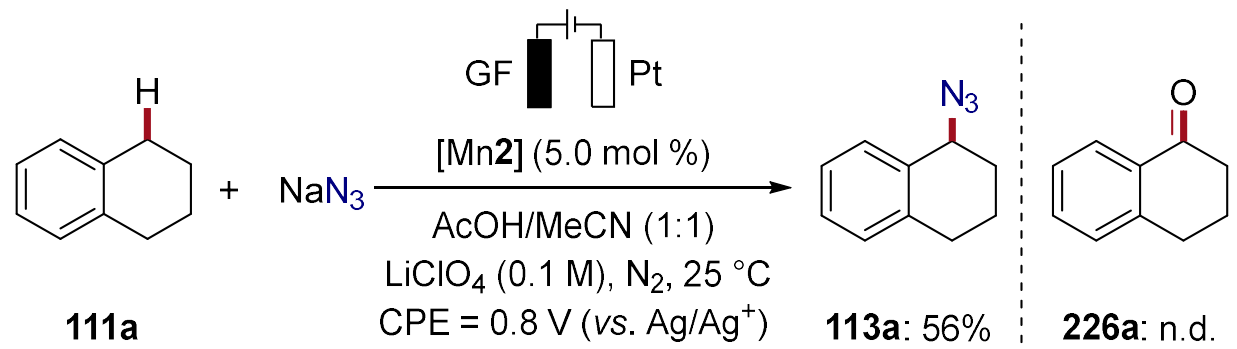

Scheme 86. Constant potential manganese-catalyzed $\mathrm{C}-\mathrm{H}$ azidation.

\subsubsection{Proposed Mechanism for Manganaelectro-Catalyzed C( $\left.\mathrm{sp}^{3}\right)-H$ Azidation}

In summary, it is proposed that the catalytic cycle is initiated by ligand-exchange of the manganese(III) chloride complex [Mn2] to form the active $\mathrm{Mn}(\mathrm{III})-\mathrm{N}_{3}$ complex 229, followed by facile anodic oxidation to generate the manganese(IV) diazide complex 230 (Scheme 87). Those catalytic steps were strongly supported by the electroanalytical and spectrophotometrical data. The high-valent $\mathrm{d}^{3}$ manganese(IV) complex 230 is prone to undergo hydrogen-atom-transfer $(\mathrm{HAT})^{[24 f, 321 \mathrm{~d}, 382]}$ or PCET $^{[321]}$ with the substrate 111, thus generating the aliphatic radical 121. These findings are supported by the radical quenching experiments ( $c f$. Scheme 81 ), the KIE studies and the results obtained within the substrate scope, inter alia for substrate $\mathbf{1 1 1} \mathrm{m}$ where a carbon-centered radical is generated that is sufficiently long-lived to epimerize before the formation of the $\mathrm{C}-\mathrm{N}$ bond ( $c f$. Table 32, entry 9). Subsequently, the key $\mathrm{C}-\mathrm{N}_{3}$ bond is formed via azide-radical rebound from manganese(IV) complex 230. ${ }^{[198,358 c, 363,383]}$ However, alternative azide transfer scenarios via radical polar cross-over cannot be ruled out. ${ }^{[171 c, 185 a, 203 a, 384]}$ It is further noteworthy that stoichiometric experiments with the well-defined manganese(III) azide complex $\mathrm{Mn5}$ (III)- $\mathrm{N}_{3}$ were unsuccessful in providing the desired $\mathrm{C}-\mathrm{H}$ azidated product $113 .{ }^{[349]}$ Finally, cathodic hydrogen formation closes the electrochemical cycle and obviates the employment of chemical oxidants. Future studies should include detailed computational studies on the manganese-catalyzed $\mathrm{C}-\mathrm{N}$ formation. 


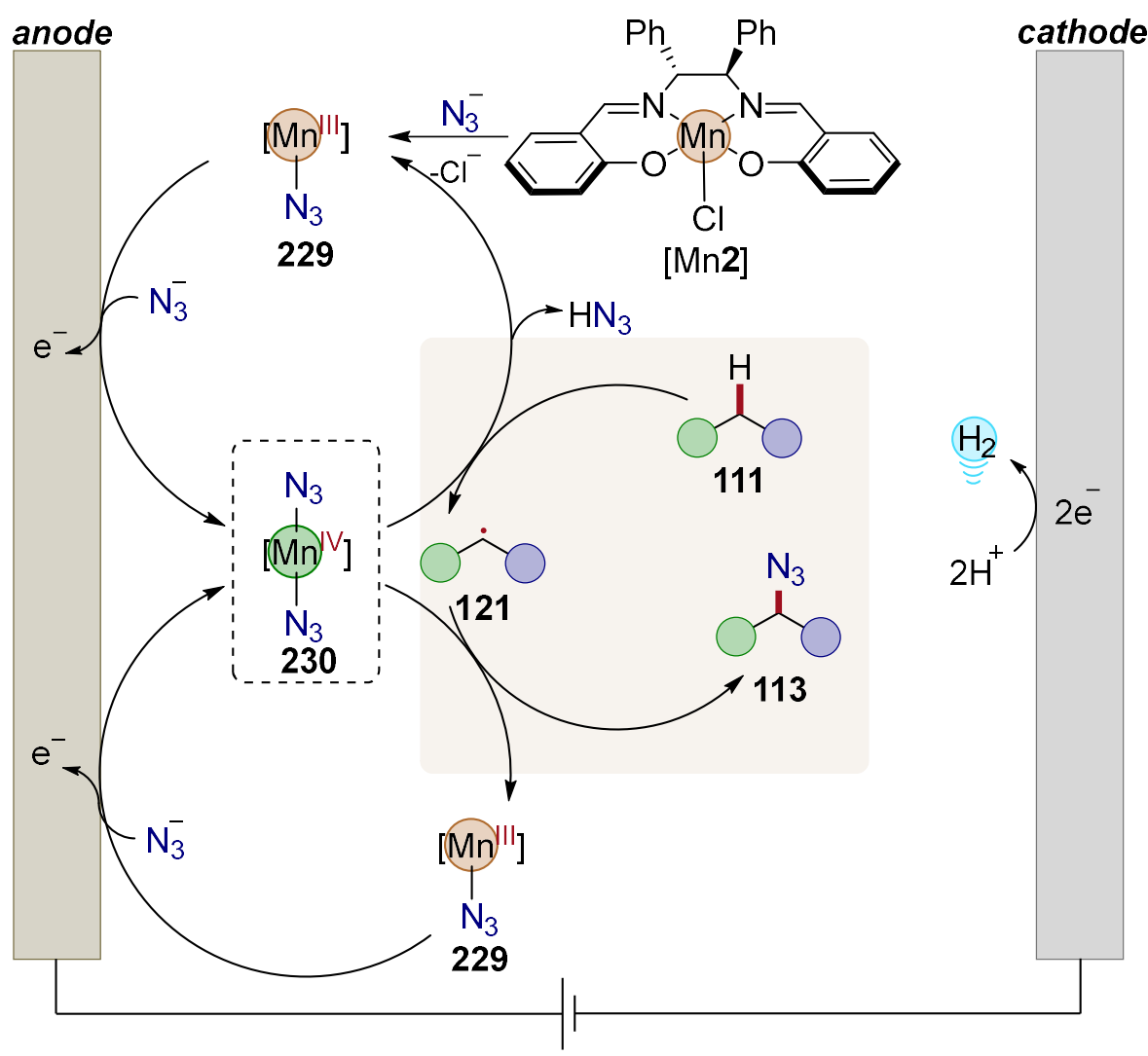

Scheme 87. Proposed catalytic cycle for the manganaelectro-catalyzed C-H azidation.

While our work represents the first manganaelectro-catalyzed $\mathrm{C}-\mathrm{H}$ azidation, it is noteworthy that during the preparation of our manuscript, the group of Lei reported a similar study on photoelectrochemical manganese-catalyzed $\mathrm{C}-\mathrm{H}$ azidation of saturated C-H bonds, with comparable levels of efficiency. ${ }^{[385]}$ Within their report, it was found that light-irradiation and catalytic amounts of a carbonyl photophor were essential to ensure high catalyst turnover. Furthermore, based on their mechanistic studies, it was proposed that the azide group transfer occurred within a manganese(II/III) manifold. Hydrogen atom transfer was proposed to take place via the photoexcited carbonyl-group or the azideradical. Possible side reactions of the photophore and the $\mathrm{C}\left(\mathrm{sp}^{3}\right)$-radical were not reported. ${ }^{[386]}$ 


\section{SUMMARY AND OUTLOOK}

The selective oxidative functionalization of otherwise inert $\mathrm{C}-\mathrm{H}$ bonds has emerged as an increasingly powerful tool in molecular syntheses. Despite remarkable advances in the field, most of the reported methods largely rely on precious metal catalysts, harsh reaction conditions and the necessity of stoichiometric amounts of chemical oxidants, compromising the overall sustainability of the strategy. In contrast, within this thesis, the merger of electrosynthesis and Earth-abundant $3 \mathrm{~d}$ metal-catalyzed $\mathrm{C}-\mathrm{H}$ activation has enabled a plethora of resource-economical strategies for the formation of $\mathrm{C}-\mathrm{C}$ and $\mathrm{C}-\mathrm{Het}$ bonds. The environmentally-sound approach enables molecular assembly under exceedingly mild reaction conditions and avoids the formation of undesired wasteproducts.

In the first project, the working hypothesis was put into practice for cobaltaelectrocatalyzed C-H oxygenations of benzamides 34 or alkenes 37 (Scheme 88). ${ }^{[249]}$ The optimized reaction conditions comprised of cost-effective cobalt(II) salts as the catalyst, mild reaction conditions at $23{ }^{\circ} \mathrm{C}$, user-friendly constant current electrolysis and $\mathrm{NaOPiv}$ as the sole additive.

(a) $\mathrm{C}-\mathrm{H}$ Oxygenation of benzamides 34

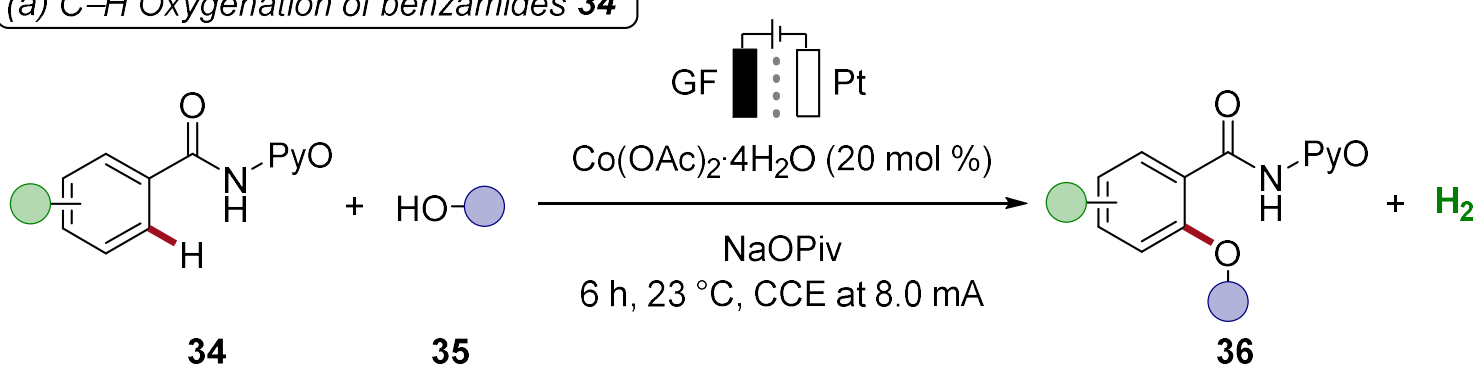

(b) $\mathrm{C}-\mathrm{H}$ Oxygenation of alkenes 37

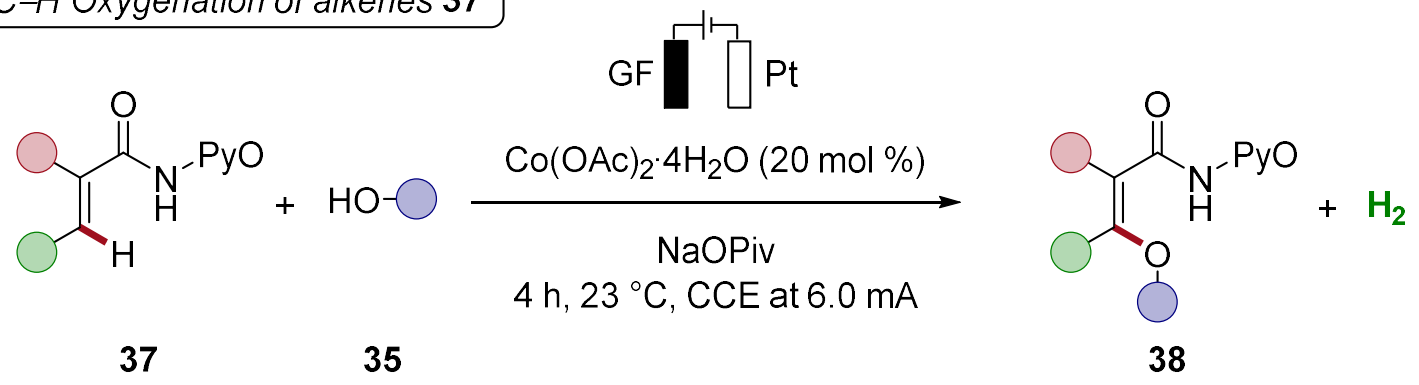

Scheme 88. Cobalt-catalyzed electrocatalytic C-H alkoxylation of (a) benzamides 34 and (b) alkenes 37. 
The robustness of the $\mathrm{C}-\mathrm{H}$ alkoxylation was characterized by ample substrate scope, including full tolerance of various oxidatively labile substituents on the amides $\mathbf{3 4}$ or $\mathbf{3 7}$, as well as on the alcohol coupling partner 35. Detailed mechanistic studies by cyclic voltammetry unraveled the key anodic formation of the active cobalt(III) catalyst and were suggestive of the formation of organometallic cyclometalated species.

The findings of this proof-of-concept study proved essential for various subsequent Earthabundant metallaelectro-catalyzed $\mathrm{C}-\mathrm{H}$ activations by inter alia nickel, ${ }^{[240 \mathrm{~b}]} \operatorname{copper}^{[387]}$ or iron. ${ }^{[388]}$

Based on the established strategy for sustainable $\mathrm{C}-\mathrm{H}$ oxygenations, an oxidative cobaltaelectro-catalyzed $\mathrm{C}-\mathrm{H} / \mathrm{N}-\mathrm{H}$ annulation reaction with allene $\mathbf{7 5}$ as a versatile coupling partner was devised (Scheme 89). ${ }^{[260]}$

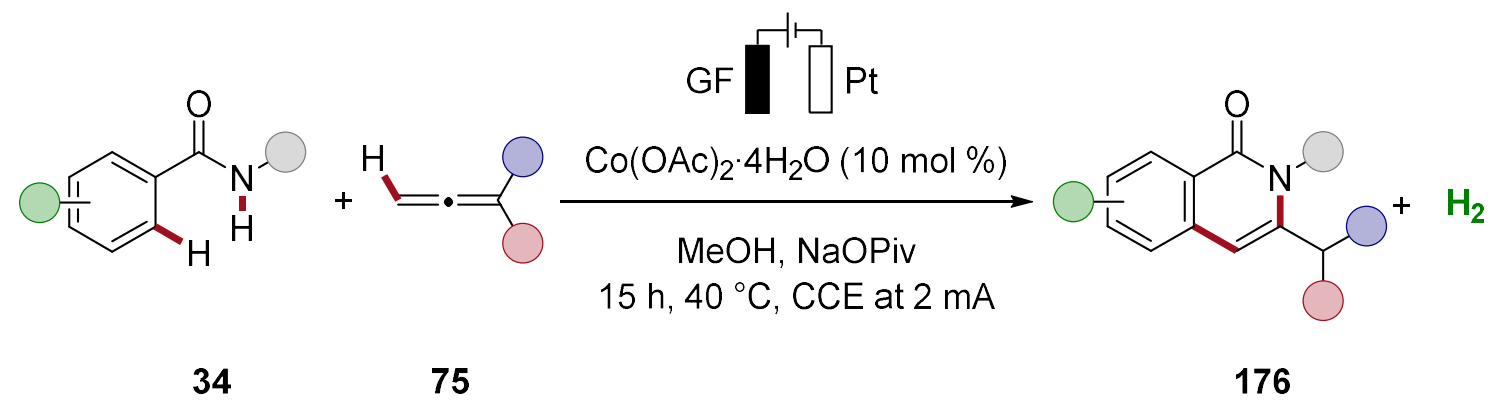

Scheme 89. Cobaltaeletro-catalyzed C-H/N-H activation with allenes $\mathbf{7 5}$.

Thus, electrocatalytic $\mathrm{C}-\mathrm{H} / \mathrm{N}-\mathrm{H}$ activation and subsequent allene 75 insertion was accomplished with excellent levels of chemo-, site- and regioselectivity under mild reaction conditions of $40^{\circ} \mathrm{C}$ with non-fluorinated solvents. The versatility of the approach was reflected by a broad substrate scope with differently decorated benzamides $\mathbf{3 4}$, being fully tolerant of oxidatively labile functional groups, such as thioethers or benzothiophene. The practical utility of the electrocatalytic allene annulation was furthermore highlighted by a multigram-scale isoquinolone 176 synthesis. Mechanistically guided constant potential electrolysis enabled the functionalization of oxidatively-labile allenes $\mathbf{7 5}$ with excellent levels of regio- and chemoselectivity. In addition, detailed kinetic studies by in-operando IR spectroscopy provided strong support for a facile $\mathrm{C}-\mathrm{H}$ cleavage and revealed the absence of an induction period for the electrocatalysis. Electroanalytical studies by cyclic voltammetry provided further insights into the electrochemical stability of the reaction components and unraveled the key anodic oxidation of organometallic cobalt intermediates. 
Furthermore, preliminary studies have been conducted for the first electrocatalytic $\mathrm{C}-\mathrm{H}$ activation with Earth-abundant metal catalyst under flow conditions.

The established cobaltaelectro-catalyzed allene annulation bears great potential for the development of the first asymmetric electrooxidative $\mathrm{C}-\mathrm{H}$ activation with $3 \mathrm{~d}$ a base metal catalyst, ${ }^{[11 e, 389]}$ for example when using chiral allenes ${ }^{[390]}$ as the coupling partner.

Inspired by the previously reported electrooxidative cobalt-catalyzed $\mathrm{C}-\mathrm{H}$ activation with alkynes 53 for the synthesis of biologically relevant isoquinolones 51 and pyridones 189, ${ }^{[250]}$ the first $\mathrm{C}-\mathrm{H}$ activation in biomass-derived glycerol was disclosed within the third project (Scheme 90). ${ }^{[300]}$ The strategy proved likewise viable for electrooxidative $\mathrm{C}-\mathrm{H} / \mathrm{N}-\mathrm{H}$ annulations with allene 75a.

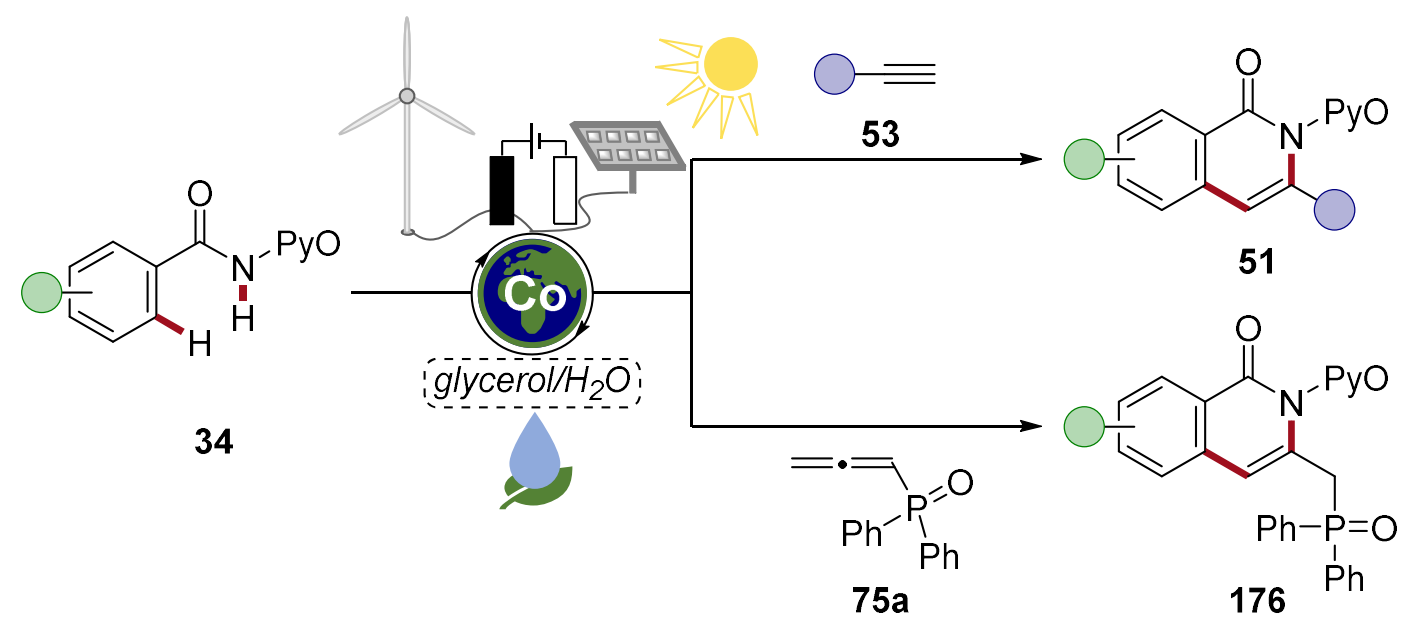

Scheme 90. Cobaltaelectro-catalyzed C-H activation in biomass-derived glycerol, powered by renewable energy sources.

To achieve full resource economy, the developed cobaltaelectro-catalyzed $\mathrm{C}-\mathrm{H}$ activation was powered by renewable energy sources, such as wind and solar energy, thus generating valuable hydrogen as the sole byproduct.

In a follow-up project, the electrochemical synthesis and full characterization of cobalt(II) complexes, such as 203, and key cyclometalated cobalt(III) complex 190, has led to manifold mechanistic insights for the previously established cobaltaelectro-catalyzed $\mathrm{C}-\mathrm{H}$ activation reactions (Scheme 91). ${ }^{[309]}$ Cyclic voltammetry and computational analysis gave strong support for the facile generation of high-valent cobalt(IV) complexes within cobaltaelectro-catalyzed $\mathrm{C}-\mathrm{O}$ formations. Detailed mass spectrometric analysis enabled insitu analysis of catalytically-relevant intermediates and proved viable for the tracking of 
cobaltaelectro-catalyzed $\mathrm{C}-\mathrm{H}$ oxygenations. Stoichiometric experiments likewise supported an oxidatively-induced reductive elimination via a cobalt(III/IV/II) modus operandi to be operative within the electrocatalytic $\mathrm{C}-\mathrm{O}$ formation. Hammett-Zuman correlations were applied to study the electronic influence on the benzamide $\mathbf{3 4}$ toward the catalytic efficacy of the respective cobalt complexes 190, 196-201. The mechanistically guided study furthermore enabled the development of unprecedented cobaltaelectrocatalyzed twofold $\mathrm{C}-\mathrm{H}$ arylations and provided insights for possible catalyst deactivation pathways. Here, future studies could include the development of novel ligand systems to avoid catalyst deactivation and to increase the catalyst lifetime. In addition, the report represents the first detailed study for the involvement of organometallic cobalt(IV) intermediates within synthetically-meaningful cobaltaelectro-catalyzed $\mathrm{C}-\mathrm{H}$ activation reactions. Noteworthy, oxidation-induced reductive elimination later also proved effective for nickela- ${ }^{[391]}$ ferra-,${ }^{[388]}$ or ruthenaelectro-catalyzed ${ }^{[392]} \mathrm{C}-\mathrm{H}$ activations. ${ }^{[225]}$

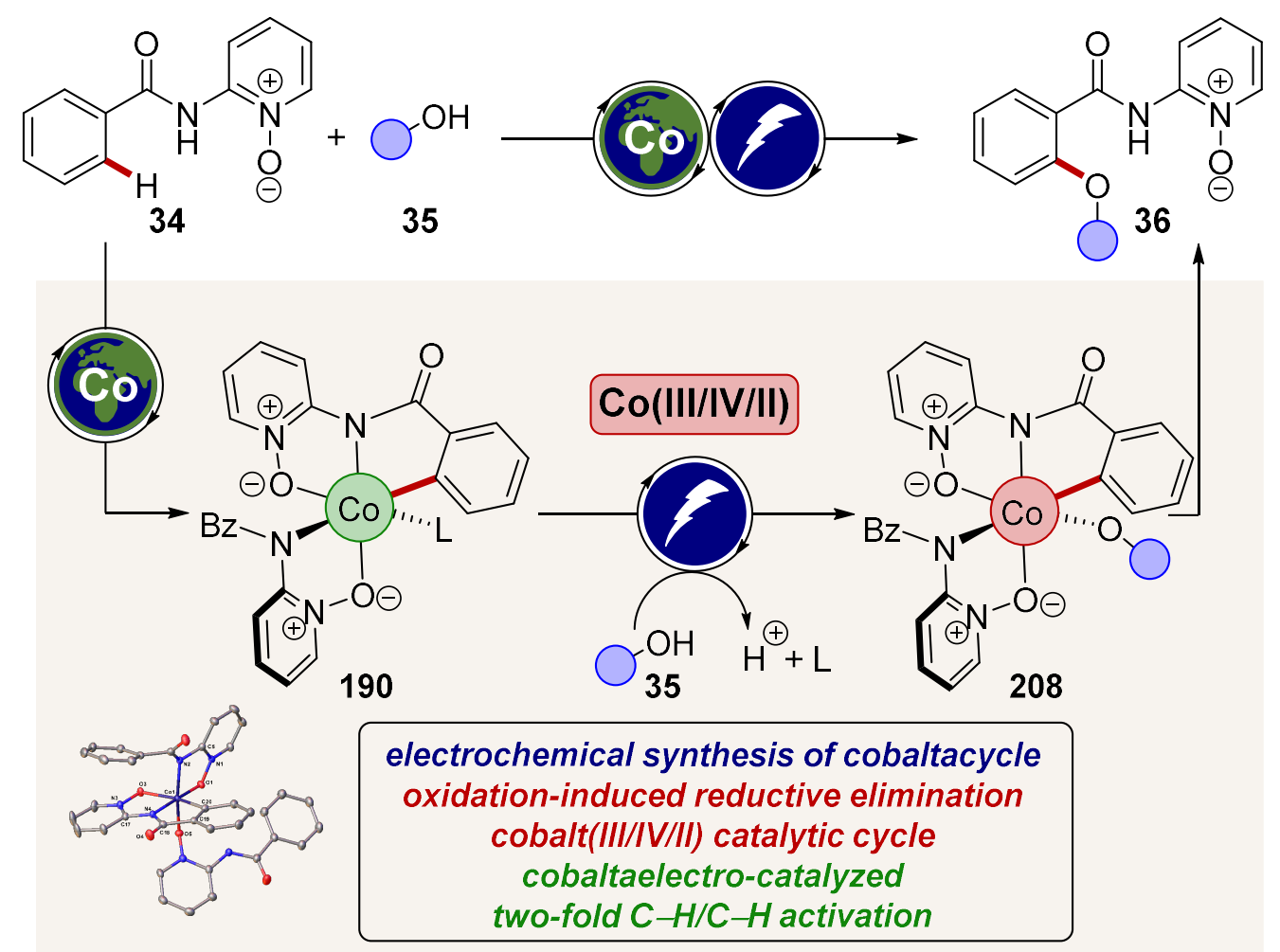

Scheme 91. Mechanistic insights into cobaltaelectro-catalyzed C-H activation.

Within different projects, detailed mechanistic studies via electroanalytical or in-operando spectroscopic techniques proved also viable to rationalize the working mode of other metallaelectro-catalyzed $\mathrm{C}-\mathrm{H}$ transformations. Notable examples include mediated 
iridaelectro-catalyzed $\mathrm{C}-\mathrm{H}$ alkenylations of benzoic acids $\mathbf{6 8}$ and novel dual electrocatalytic iodine(III)/ruthenium(II) $\mathrm{C}-\mathrm{H}$ oxygenation reactions.

In the last project, the merger of electrosynthesis and manganese catalysis enabled oxidative $\mathrm{C}-\mathrm{H}$ azidation of otherwise inert $\mathrm{C}\left(\mathrm{sp}^{3}\right)-\mathrm{H}$ bonds 111 with excellent levels of chemoselectivity (Scheme 92). ${ }^{[349]}$ Key features of the novel manganaelectro-catalyzed $\mathrm{C}-\mathrm{H}$ azidation include: no directing groups; the avoidance of chemical oxidants; hypervalent iodine transfer reagents or laborious photochemical irradiation; the utilization of most user-friendly sodium azide as nitrogen source and Earth-abundant, bioinspired manganese catalyst for full selectivity control. The exceedingly mild reaction conditions enabled the tolerance of various functional groups and undesired side reactions, such as overoxidation, were minimized. The robustness and utility of the resource-economic $\mathrm{C}-\mathrm{N}$ formation was further emphasized by the late-stage diversification of bioactive and pharmaceutically relevant compounds.

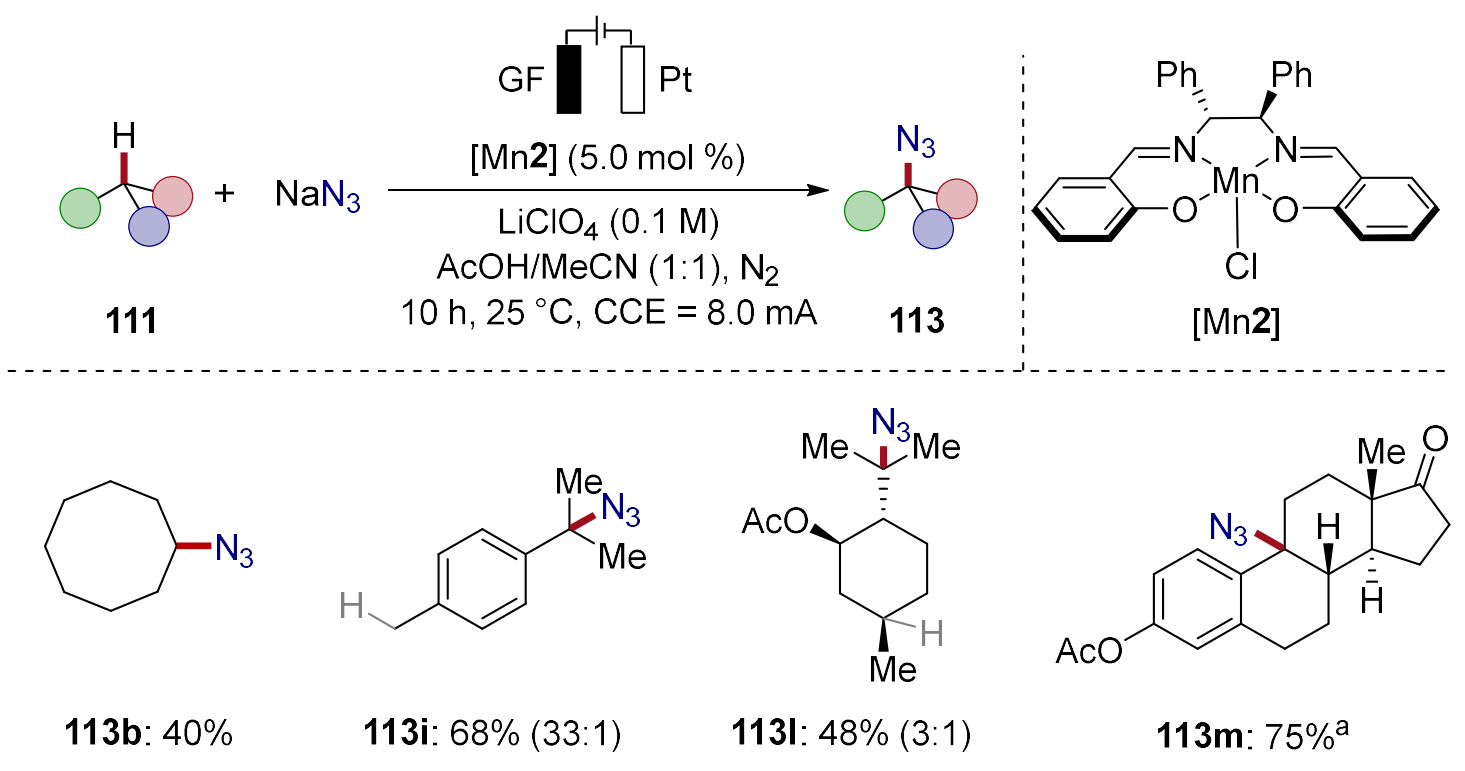

Scheme 92. Mangana(III/IV)electro-catalyzed $\mathrm{C}\left(\mathrm{sp}^{3}\right)-\mathrm{H}$ azidation. [a] $d r=1: 1$.

In addition, detailed mechanistic studies were performed to delineate the catalyst's mode of action. The investigations revealed a rate-determining $\mathrm{C}-\mathrm{H}$ cleavage event and provided support for the generation of radical intermediates. Furthermore, the synthesis and subsequent full characterization of high-valent manganese azide complexes by extensive spectrophotochemical and voltametric analysis thereof, gave strong support for a metalcatalyzed aliphatic radical formation, followed by an azidyl radical transfer within a manganese(III/IV) catalytic cycle. 
Given the topical interest in undirected $\mathrm{C}\left(\mathrm{sp}^{3}\right)-\mathrm{H}$ functionalization with sustainable electricity as the driving force, future prospects could include the functionalization of inert polymer scaffolds ${ }^{[393]}$ or light hydrocarbons. ${ }^{[394]}$ Moreover, a variety of other manganaelectro-catalyzed $\mathrm{C}-\mathrm{H}$ functionalizations are conceivable, such as selective $\mathrm{C}-\mathrm{H}$ isocyanations ${ }^{[395]}$ or valuable $\mathrm{C}-\mathrm{H}$ fluorinations. ${ }^{[196 b, 196 \mathrm{c}, 396]}$ The synthesized and fully characterized manganese azide complexes might also be of relevance for other research arenas such as the formation of manganese nitrido compounds ${ }^{[156 j, 397]}$ or nitrogen-based fuel cell technologies. ${ }^{[398]}$

A major element of this thesis also focused on the design and development of the electrochemical equipment, which were essential for the success of the presented projects. Parts of this efforts were published in a Nature Protocols article that also focused on a direct comparison of the custom-made setups and commercially available products. ${ }^{[274]}$ 


\section{EXPERimental PART}

\subsection{General Remarks}

Air and moisture sensitive reactions were carried out under inert gas atmosphere (HiQ nitrogen 5.0 with additional $\mathrm{CaH}_{2}$-filled drying funnel). Glassware was stored in an oven $\left(120^{\circ} \mathrm{C}\right)$ and dried under high-vacuum prior to use (three times, in between filled with nitrogen). Liquids and solutions were transferred via nitrogen-flushed syringes by BRAUN, with oven-dried stainless-steel cannulas $\left(120^{\circ} \mathrm{C}\right)$. Solids were added under counter flow of nitrogen (standard Schlenk technique). Solutions were concentrated under reduced pressure by rotary evaporation at $40{ }^{\circ} \mathrm{C}$ with either IKA $R V 10$ digital FLEX or a HEIDOLPH Hei-VAP Core. Non-volatile products were dried under high-vacuum for $14 \mathrm{~h}$. Air and moisture sensitive substances were stored in a MBRAUN glovebox. Yields refer to isolated compounds, estimated to be $>95 \%$ pure as was determined by ${ }^{1} \mathrm{H}-\mathrm{NMR}$ spectroscopy and GC analysis.

\subsubsection{Caution: Experimentation with Organic Azides ${ }^{[176 e, 180,399]}$}

Special safety consideration should be taken for any experimentation with organic azide compounds as they are considered as toxic and potentially explosive chemicals that can decompose under external impact such as heat, pressure, shock. For safety reasons, all reactions and processing steps involving azides were carried out behind a blast shield. Recommended storage of organic azides is below room temperature and away from light sources. As a recommended rule of thumb, azide compounds fulfilling equation (1) are generally stable.

$$
\frac{\left(N_{\text {Carbon }}+N_{\text {Oxygen }}\right)}{N_{\text {Nitrogen }}} \geq 3
$$

Azide compounds with a ratio of 1-3 can be isolated but should be stored in small amounts of less than 5 grams and in diluted concentrations. Special care should be taken for mixtures of azides with strong acids as highly toxic and explosive hydrazoic acid can be generated. Solutions of sodium azide can react with halogenated solvents and extremely explosive organic azides with a ratio $<1$ can be generated such as diazidomethane. ${ }^{[400]}$ The azide waste formed during the following procedures was separated, collected and diluted to aqueous solutions of less than $5 \%$ sodium azide and treated with freshly prepared nitrous acid. ${ }^{[401]}$ 


\subsubsection{Solvents and Reagents}

\section{Solvents}

All solvents for air- and moisture sensitive reactions were obtained from a solvent purification system (SPS-800 by MBRAUN) with the following exceptions:

Acetonitrile (MeCN)

1,2-Dichloroethane (DCE)

Methanol $(\mathrm{MeOH})$

tert-Amyl alcohol $(t-\mathrm{AmOH})$

Toluene was dried over $3 \AA$ molecular sieves for at least $24 \mathrm{~h}$ and degassed using multiple cycles of freeze-pump-thaw (FPT) method.

was stirred over $\mathrm{CaH}_{2}$ for $8 \mathrm{~h}$, degassed and distilled under reduced pressure.

was distilled over magnesium methanolate.

was stirred over $\mathrm{Na}$ chips for $5 \mathrm{~h}$ at $120{ }^{\circ} \mathrm{C}$, degassed and distilled under reduced pressure.

was pre-dried over $\mathrm{KH}$ and distilled under nitrogen over sodium and benzophenone.

For flash column chromatography technical grade solvents were used, which were distilled prior to use.

\section{Reagents}

Chemicals obtained from commercial sources with a purity higher than $95 \%$ were used without further purification. The following compounds were known from the literature and synthesized according to previously reported procedures:

Benzamides 34, ${ }^{[112]}$ allenes 75, ${ }^{[402]} \mathrm{Co}(\mathrm{OAc}) 3,{ }^{[310 \mathrm{~b}]}$ methyl 4'-hexyl-[1,1'-biphenyl]-4carboxylate $(\mathbf{1 1 1 k}),{ }^{[347 \mathrm{~d}]}$ menthol acetate $(\mathbf{1 1 1 1}),{ }^{[201]}$ estrone acetate $(\mathbf{1 1 1} \mathbf{m}),{ }^{[347 \mathrm{~d}]}$ salen $^{[403]}$ and porphyrin ligands, ${ }^{[404]}$ as well as the corresponding manganese complexes [Mn1]$[\mathrm{Mn6}]{ }^{[403 \mathrm{~b}, 404 \mathrm{a}]}$

The following chemicals were synthesized and generously provided by the people named below:

G. Chesnokov: 34e, 34q, and 34r

\section{Choi: $\mathbf{T s N}_{3}$}

S. Homölle: 341, 34m, and 34s, $62 \mathrm{a}$

I. Maksso: [Mn1] 


\section{Massginan: 214}

Dr. A. Messinis: 221

M. Oelschlegel: 26a, 75f, $75 \mathrm{~g}, \mathbf{7 5 h}$, and $\mathbf{7 5 m}$

Dr. R. C. Samanta: 111n, 1110, 111p, and [Mn5]

Dr. S. C. Sau: 75a, $\mathbf{7 5 j}$, 75k, and $\mathbf{7 5 l}$

Dr. N. Sauermann: 34h and 162a

\subsubsection{Analytical and Experimental Methods}

\section{Chromatography}

Analytical thin-layer chromatography (TLC) was used for reaction monitoring, analysis of column chromatography and determination of $R_{\mathrm{f}}$ values. TLC was performed on silica gel 60 coated aluminium-sheets, with fluorescence indicator F-254 (MACHEREY NAGEL, by MERCK). The plates were either visualized by UV light $(\lambda=254 \mathrm{~nm}$ or $366 \mathrm{~nm})$, if applicable, or by staining solution $\left(\mathrm{KMnO}_{4}\right.$ solution) followed by gentle heating by heatgun at $300{ }^{\circ} \mathrm{C}$.

Chromatographic purification of crude products was accomplished by flash column chromatography using MERCK silica gel, grade $60(40-63 \mu \mathrm{m}, 70-230$ mesh ASTM, Geduran SI 60). The crude products were loaded with the respective eluent. For acidsensitive compounds, such as azides, the silica gel was neutralized with $\mathrm{Et}_{3} \mathrm{~N}$ prior to use.

\section{Electrochemistry}

\section{Constant Potential Reactions under Amperometric Detection}

The constant potential electrolyses (CPE) were performed using a METROHM Autolab PGSTAT204 or a METROHM Dropsense 8000P workstation and Nova 2.1 or the Dropview 8400 software, respectively. A silver-wire $(d=1 \mathrm{~mm})$ was used as the pseudo-reference electrode in close proximity to the working electrode. To ensure comparable constant potential conditions with the results gained by cyclic voltammetry, calibration of the pseudo-reference electrode versus ferrocene was performed for each reaction system. If applicable, an aqueous $\mathrm{Ag} / \mathrm{AgCl}$ reference electrode was used instead of the silver-wire.

\section{Cyclic Voltammetry}

The cyclic voltammetry measurements were carried out using a METROHM Autolab PGSTAT204 workstation, and the following data analysis was performed with Nova 2.1. 
For the experiments, a glassy-carbon (GC) disc electrode $(d=3 \mathrm{~mm})$ or a platinum disc electrode $(d=3 \mathrm{~mm})$ was used as the working electrode (WE). Either a saturated calomel electrode ( $\mathrm{SCE})\left(\mathrm{Hg} / \mathrm{Hg}_{2} \mathrm{Cl}_{2}\right.$, ceramic frit, $\left.\mathrm{OD}=6 \mathrm{~mm}\right)$; an aqueous $\mathrm{Ag} / \mathrm{AgCl}\left(\mathrm{Ag} / \mathrm{Ag}^{+}\right.$, aq. $\mathrm{KCl}(3 \mathrm{M})$, ceramic frit, $\mathrm{OD}=6 \mathrm{~mm})$ or a non-aqueous $\mathrm{Ag} / \mathrm{AgNO}_{3}\left(\mathrm{Ag} / \mathrm{Ag}^{+}\right.$, solution of MeCN with $\mathrm{AgNO}_{3}(0.01 \mathrm{M})$ and $n-\mathrm{Bu}_{4} \mathrm{NClO}_{4}(0.1 \mathrm{M})$, vycor glass, $\left.\mathrm{OD}=6 \mathrm{~mm}\right)$ was used as the reference electrode (RE), if not stated otherwise. If a silver-wire $(d=1 \mathrm{~mm})$ was used as the pseudo-reference electrode, the voltammograms were referenced internally versus ferrocene. The counter electrode (CE) was a coiled platinum wire $(d=1 \mathrm{~mm})$. The electrodes were purchased from ALS JAPAN Co., Ltd. Measurements were recorded at a scan rate of $100 \mathrm{mV} \cdot \mathrm{s}^{-1}$, if not indicated otherwise. The working temperature was $298 \mathrm{~K}$, if not indicated otherwise. All solutions were degassed via freeze-pump-thaw method prior to use and nitrogen was bubbled through the solutions for at least $5 \mathrm{~min}$ before the experiment was performed. The voltametric studies were performed under a constant flow of dry nitrogen. The rotating-disc electrode (RDE) experiments were performed using a METROHM Autolab PGSTAT204 workstation and a RRDE-3A Rotating Ring Disk Electrode Apparatus Ver.2.0 purchased from ALS JAPAN Co., Ltd. For the RDE experiments a glassycarbon disc electrode ( $d=4 \mathrm{~mm}$, disc-electrode) was used as the working electrode, a coiled platinum wire $(d=1 \mathrm{~mm})$ was used as the counter electrode and a non-aqueous $\mathrm{Ag} / \mathrm{AgNO}_{3}$ $\left(\mathrm{Ag} / \mathrm{Ag}^{+}\right.$, solution of $\mathrm{MeCN}$ with $\mathrm{AgNO}_{3}(0.01 \mathrm{M})$ and $n-\mathrm{Bu}_{4} \mathrm{NClO}_{4}(0.1 \mathrm{M})$, vycor glass, $\mathrm{OD}=6 \mathrm{~mm}$ ) was used as the reference electrode. The operation temperature was $25^{\circ} \mathrm{C}$ and dry nitrogen was bubbled through the solution for at least $5 \mathrm{~min}$ before the analytical experiment was performed.

\section{Constant Current Electrolysis (CCE)}

The electrocatalytic reactions were performed in undivided electrochemical cells $(10 \mathrm{~mL})$ using pre-dried glassware, unless stated otherwise. Platinum electrodes $(10 \mathrm{~mm} \times 15 \mathrm{~mm}$ $\times 0.125 \mathrm{~mm}, 99.9 \%$, CHEMPUR Karlsruhe, Germany or 99.95\%; ESG-EDELMETALLHaNDEL GMBH \& Co. KG), graphite felt electrodes $(10 \mathrm{~mm} \times 15 \mathrm{~mm} \times 6 \mathrm{~mm}$, SIGRACELL®GFA 6 EA, SGL CARBON, Wiesbaden, Germany) and reticulated vitreous carbon (RVC) (40 mm x $5.0 \mathrm{~mm}$ x $6.0 \mathrm{~mm}$, Duocel ${ }^{\circledR}$ Reticulated Vitreous Carbon RVC100 (100 PPI) ERG AERosPace CoRPoration, Oakland, United States of America) were connected using stainless steel adapters, following the published protocol. ${ }^{[274]}$ Electrolysis was conducted using an AXIOMET $A X-3003 P$ galvanostat in constant current mode. Divided cells, separated by a P4 glass frit, were custom-made and obtained from GLASGERÄTEBAU 
Ochs LaborfachHAndel e. K. (Bovenden, Germany). A detailed description of the electrochemical equipment for the bulk electrolysis and further graphical information for the small electrolysis cells are given in the appendix (Figures 77-85). For reactions performed with the standardized electrochemistry kit, ElectraSyn 2.0 from IKA, the commercialized electrodes and $10 \mathrm{~mL}$ undivided cells were used, if not stated otherwise. If reactions were performed at temperatures other than room temperature, the vial was connected via the IKA ElectraSyn GOGO Module ${ }^{\circledR}$ and mounted in a silicon oil bath.

For reactions in flow, an ISMATEC REGLO Digital MS-2/12 (ISM 596) peristaltic pump and a KEYSIGHT E36104A galvanostat was employed. The flow-electrocatalysis was performed with either a commercially available IKA ElectraSyn flow system and the respective halfcell electrodes or custom-made flow reactor compartments for reactions with the graphitefelt electrode, designed together with Dr. L. Finger. ${ }^{\text {[292] }}$

For the electrocatalysis powered by sunlight, the commercially available amorphous silicon photovoltaic cell TPS-103 from CONRAD ELECTRONIC SE (6 W, $17.5 \mathrm{~V}$ max. voltage, $428 \mathrm{~mA}$ max. current, $467 \mathrm{~mm}$ x $161 \mathrm{~mm}$ x $19 \mathrm{~mm}$ ) was used. For the electrocatalysis powered by wind energy, the commercially available wind turbine Infinite Air obtained from TEXENERGY (7.5-10 W, 1.5-2 A at $5 \mathrm{~V}$ output voltage, blade diameter: $320 \mathrm{~mm}$ ) was employed. The wind turbine was powered with an external fan, to ensure a steady wind velocity. For both setups, the output current was controlled with a customized and normalized constant current regulator and regularly double checked with an ammeter. The reaction temperature was controlled with an oil bath temperature reservoir.

For experiments performed by sonoelectrochemistry, the reaction cell was mounted in a closed ultrasonic bath RK 100 SONOREX SUPER from BANDELIN in continuous operation mode. The ultrasonic bath was filled with water and covered with aluminum foil to avoid water evaporation. The sonication and electrolysis were started and stopped simultaneously.

\section{Elemental Analysis}

Elemental analyses were performed by the analytical laboratory in the Institute of Inorganic Chemistry of the University of Göttingen on a 4.1 vario EL 3 from ELEMENTAR. Analysis of the relative amounts of the elements: $\mathrm{C}, \mathrm{H}, \mathrm{N}, \mathrm{S}$ was reported as was received and are uncorrected. 


\section{Gas Chromatography}

Gas chromatographic analysis (GC) was performed on an AgILENT 7890 A GC System or AgILENT 7890B GC System equipped with an AgILENT HP-5MS column $(30 \mathrm{~m} \times 0.25 \mathrm{~mm}$ diameter, $0.25 \mu \mathrm{m}$ film thickness) and a flame-ionization detector (FID) using hydrogen as the carrier gas. Gas chromatography coupled with mass-spectrometry (GC-MS) was performed on the same instrument coupled with AGILENT 5875C Triple-Axis-Detector or AGILENT 5977B MSD. Mass spectra were obtained with electron ionization (EI) at $70 \mathrm{eV}$ in positive ion mode. Quantitative GC analyses were performed with the addition of $n$ dodecane as an internal standard.

\section{Infrared Spectroscopy (IR)}

Infrared (IR) spectra were recorded on a BRUKER Alpha-P FT-IR spectrometer with an ATR diamond probe for detection in the range of $\tilde{v}=4000-400 \mathrm{~cm}^{-1}$. Analysis of the spectra was performed with the software Opus 6.5 from BRUKER. For in situ-IR measurements, a ReactIR $^{\mathrm{TM}} 15$ from METTLER TOLLEDO was used. The analysis of the spectra was performed with the software $i C$ IR $4.3 \AA$ from METTLER TOLLEDO.

\section{Mass Spectrometry}

High resolution (HR) electrospray ionization (ESI) mass spectra were recorded on a micrOTOF or a maXis from BRUKER DALTONICS or a LTQ Orbitrab XL from THERMO SCIENTIFIC. EI-MS spectra were recorded on a JEOL AccuTOF (EI) instrument. Ionization of the samples was achieved using electrospray ionization (ESI) or electron ionization (EI). The ratio of mass to charge $(\mathrm{m} / \mathrm{z})$ is given, intensities $I$ relative to the base signal $(I=100)$ are written in parentheses.

\section{Nuclear Magnetic Resonance (NMR) Spectroscopy}

Nuclear magnetic resonance (NMR) spectra were recorded on BRUKER Avance 300, Avance III HD 400, Avance Neo 400, Avance III HD 500; VARIAN Mercury VX 300, Inova 500 or Inova 600 spectrometer at $300 \mathrm{MHz}, 400 \mathrm{MHz}, 500 \mathrm{MHz}, 600 \mathrm{MHz}\left({ }^{1} \mathrm{H}-\mathrm{NMR}\right), 75 \mathrm{MHz}$, $100 \mathrm{MHz}, 125 \mathrm{MHz}\left({ }^{13} \mathrm{C}-\mathrm{NMR}\right)$ and $282 \mathrm{MHz}\left({ }^{19} \mathrm{~F}-\mathrm{NMR}\right)$, respectively. Chemical shifts are reported as $\delta$-values in parts per million (ppm) relative to $\mathrm{SiMe}_{4}$ and are referenced to the residual proton peak or the carbon peak of the deuterated solvent: 


\begin{tabular}{c|cc} 
& & \\
& ${ }^{\mathbf{1}} \mathbf{H}-\mathbf{N M R}[\mathrm{ppm}]$ & $77.16 \mathrm{ppm}(\mathrm{t})$ \\
$\mathrm{CDCl}_{3}$ & $7.26(\mathrm{~s})$ & 53.84 (quint) \\
$\mathrm{CD}_{2} \mathrm{Cl}_{2}$ & $5.32(\mathrm{t})$ & $49.00($ sept$)$
\end{tabular}

For ${ }^{19} \mathrm{~F}-\mathrm{NMR}$ spectra, $\mathrm{CFCl}_{3}$ was used as an external standard. All measurements were performed at $298 \mathrm{~K}$, unless stated otherwise. The measured resonance multiplicities were reported by the following abbreviations: $\mathrm{s}$ (singlet), $\mathrm{d}$ (doublet), $\mathrm{t}$ (triplet), q (quartet), quint (quintet), sext (sextet), sept (septet), dd (doublet of doublets), dt (doublet of triplets), ddd (doublet of doublets of doublets), td (triplet of doublets), dddd (doublet of doublets of doublets of doublets), m (multiplet) and br (broad singlet). The coupling constants $J$ are reported in Hertz $(\mathrm{Hz})$. Analysis of all spectra until February 2019 was performed with MestReNova v10.0.2 and from February onwards with MestReNova v14.1.0 from Mestrelab RESEARCH S.L.

The ${ }^{1} \mathrm{H}-\mathrm{NMR}$ spectroscopic experiments in flow were performed on a Magritek Spinsolve $60^{\text {ULTRA }}$ from MAGRITEK GmbH, Germany. The spectra were batch-processed with the reaction monitoring wizard of the MestReNova v12.0.3 software. Arbitrary integral values were transformed to mmol and percentage values by referencing with dibromomethane as internal standard.

\section{Melting Points}

Melting points were measured using a Stuart ${ }^{\circledR}$ Melting Point Apparatus SMP 3 from BARLOWORLD SCIENTIFIC and are uncorrected.

\section{Vacuum}

The following average pressure was measured on the used rotary vane pump $R D 4$ from VACUUBRAND: $3 \cdot 10^{-2}$ mbar (uncorrected value).

\section{UV-Vis Spectroscopy}

Ultraviolet-visible spectroscopy was performed on a JASCO $V-770$ UV-vis/NIR spectrophotometer. UV-vis measurements were performed at $298 \mathrm{~K}$, unless stated otherwise. 


\subsection{General Procedures}

\subsubsection{General Procedure A: Cobaltaelectro-Catalyzed C-H Oxygenation in a Divided Cell Setup}

The electrocatalysis was carried out in an H-type divided cell (P4 sintered glass membrane), with a graphite felt anode $(10 \mathrm{~mm} \times 15 \mathrm{~mm} \times 6.0 \mathrm{~mm})$ and a platinum cathode $(10 \mathrm{~mm} \times 15 \mathrm{~mm} \times 0.125 \mathrm{~mm}) . \mathrm{NaOPiv}(122 \mathrm{mg}, 1.00 \mathrm{mmol}, 2.00$ equiv) was added in the cathodic chamber and dissolved in alcohol $35(7.0 \mathrm{~mL})$. The anodic chamber was charged with $\mathrm{Co}(\mathrm{OAc})_{2} \cdot 4 \mathrm{H}_{2} \mathrm{O}(25.7 \mathrm{mg}, 0.10 \mathrm{mmol}, 20 \mathrm{~mol} \%)$, NaOPiv (122 mg, $1.00 \mathrm{mmol}$, 2.00 equiv) and benzamide 34 ( $0.50 \mathrm{mmol}, 1.00$ equiv) and dissolved in alcohol 35 $(7.0 \mathrm{~mL})$. Electrolysis was started at ambient temperature with a constant current of $8 \mathrm{~mA}$ maintained for $6 \mathrm{~h}\left(Q \cdot \mathrm{mol}^{-1}=3.58 \mathrm{~F}\right)$. Then, the DC power supply was stopped and direct evaporation of the solvent and subsequent column chromatography on silica gel $\left(\mathrm{CH}_{2} \mathrm{Cl}_{2}\right.$ /acetone) yielded the desired product 36 .

\subsubsection{General Procedure B: Cobaltaelectro-Catalyzed C-H Oxygenation in an Undivided Cell Setup}

The electrocatalysis was carried out in an undivided cell, with a graphite felt anode $(10 \mathrm{~mm} \times 15 \mathrm{~mm} \times 6.0 \mathrm{~mm})$ and a platinum cathode $(10 \mathrm{~mm} \times 15 \mathrm{~mm} \times 0.125 \mathrm{~mm})$. A mixture of NaOPiv (122 mg, $1.00 \mathrm{mmol}, 2.00$ equiv), $\mathrm{Co}(\mathrm{OAc})_{2} \cdot 4 \mathrm{H}_{2} \mathrm{O} \quad(25.7 \mathrm{mg}$, $0.10 \mathrm{mmol}, 20 \mathrm{~mol} \%$ ), benzamide 34/acrylamide 37 (0.50 mmol, 1.00 equiv) and alcohol $35(7 \mathrm{~mL})$ was added to the electrochemical cell. Electrolysis was started at ambient temperature with a constant current of $8 \mathrm{~mA}$ maintained for $6 \mathrm{~h}\left(Q \cdot \mathrm{mol}^{-1}=3.58 \mathrm{~F}\right)$. Then, the DC power supply was stopped and direct evaporation of the solvent and subsequent column chromatography on silica gel $\left(\mathrm{CH}_{2} \mathrm{Cl}_{2} /\right.$ acetone $)$ yielded the desired product 36 or 38, respectively.

\subsubsection{General Procedure C: Cobaltaelectro-Catalyzed C-H Oxygenation in an Undivided Cell Setup}

The electrocatalysis was carried out in an undivided cell, with a graphite felt anode $(10 \mathrm{~mm} \times 15 \mathrm{~mm} \times 6.0 \mathrm{~mm})$ and a platinum cathode $(10 \mathrm{~mm} \times 15 \mathrm{~mm} \times 0.125 \mathrm{~mm})$. A mixture of $\mathrm{NaOPiv}\left(63.9 \mathrm{mg}, 0.50 \mathrm{mmol}, 2.00\right.$ equiv), $\mathrm{Co}(\mathrm{OAc})_{2} \cdot 4 \mathrm{H}_{2} \mathrm{O} \quad(12.7 \mathrm{mg}$, $0.05 \mathrm{mmol}, 20 \mathrm{~mol} \%$ ), acrylamide 37 ( $0.25 \mathrm{mmol}, 1.00$ equiv) and alcohol 35 (7 mL) was 
added to the electrochemical cell. Electrolysis was started at ambient temperature with a constant current of $6 \mathrm{~mA}$ maintained for $4 \mathrm{~h}\left(Q \cdot \mathrm{mol}^{-1}=3.58 \mathrm{~F}\right)$. Then, the DC power supply was stopped and direct evaporation of the solvent and subsequent column chromatography on silica gel $\left(\mathrm{CH}_{2} \mathrm{Cl}_{2} /\right.$ acetone $)$ yielded the desired product 38 .

\subsubsection{General Procedure D: Cobaltaelectro-Catalyzed C-H Activation for Allene} Annulations

The electrocatalysis was carried out in an undivided cell, with a graphite felt anode $(10 \mathrm{~mm} \times 15 \mathrm{~mm} \times 6.0 \mathrm{~mm})$ and a platinum cathode $(10 \mathrm{~mm} \times 15 \mathrm{~mm} \times 0.125 \mathrm{~mm})$. Benzamide 34 ( $0.50 \mathrm{mmol}, 1.00$ equiv), allene 75 (0.60 mmol, 1.20 equiv), NaOPiv (124 mg, $1.00 \mathrm{mmol}, 2.00$ equiv) and $\mathrm{Co}(\mathrm{OAc})_{2} \cdot 4 \mathrm{H}_{2} \mathrm{O}(12.7 \mathrm{mg}, 10 \mathrm{~mol} \%)$ were placed in a $10 \mathrm{~mL}$ cell and dissolved in $\mathrm{MeOH}(5.0 \mathrm{~mL})$. Electrolysis was performed at $40^{\circ} \mathrm{C}$ with a constant current of $2 \mathrm{~mA}$ maintained for $15 \mathrm{~h}\left(Q \cdot \mathrm{mol}^{-1}=2.24 \mathrm{~F}\right)$. At ambient temperature, the DC power supply was stopped, $\mathrm{H}_{2} \mathrm{O}(10 \mathrm{~mL})$ was added, and the graphite felt anode was washed with $\mathrm{CH}_{2} \mathrm{Cl}_{2}(10 \mathrm{~mL})$ in an ultrasonic bath. The combined phases were extracted with $\mathrm{CH}_{2} \mathrm{Cl}_{2}(3 \times 10 \mathrm{~mL})$, and then dried over $\mathrm{Na}_{2} \mathrm{SO}_{4}$. Evaporation of the solvents and column chromatography on silica gel, using $\mathrm{CH}_{2} \mathrm{Cl}_{2}$ /acetone to $\mathrm{CH}_{2} \mathrm{Cl}_{2} / \mathrm{MeOH}$ as the eluent, yielded the desired product $\mathbf{1 7 6 .}$

\subsubsection{General Procedure E: Cobaltaelectro-Catalyzed $\mathrm{C}-\mathrm{H}$ Activation in Aqueous Glycerol for Alkyne Annulations}

The electrocatalysis was carried out in an undivided cell, with a graphite felt anode $(25 \mathrm{~mm} \times 10 \mathrm{~mm} \times 6.0 \mathrm{~mm})$ and a platinum cathode $(25 \mathrm{~mm} \times 10 \mathrm{~mm} \times 0.125 \mathrm{~mm})$. Benzamide 34 ( $0.50 \mathrm{mmol}, 1.00$ equiv), alkyne 53 (1.00 mmol, 2.00 equiv), NaOPiv (124 mg, $1.00 \mathrm{mmol}, 2.00$ equiv), glycerol $/ \mathrm{H}_{2} \mathrm{O}(5.0 \mathrm{~mL}, 1: 1)$ and $\mathrm{Co}(\mathrm{OAc})_{2} \cdot 4 \mathrm{H}_{2} \mathrm{O}$ $(12.7 \mathrm{mg}, 10 \mathrm{~mol} \%)$ were placed in a $10 \mathrm{~mL}$ cell. Electrocatalysis was performed at $40{ }^{\circ} \mathrm{C}$ with a constant current of $4 \mathrm{~mA}$ maintained for $15 \mathrm{~h}\left(Q \cdot \mathrm{mol}^{-1}=4.48 \mathrm{~F}\right)$. Then, the DC power supply was stopped, and the reaction mixture was diluted with $\mathrm{CH}_{2} \mathrm{Cl}_{2}(2.0 \mathrm{~mL})$. The graphite felt anode was washed with $\mathrm{CH}_{2} \mathrm{Cl}_{2}(3 \times 5.0 \mathrm{~mL})$ in an ultrasonic bath $(3 \mathrm{x}$ $3 \mathrm{~min}$ ). The combined washings were added to the reaction mixture and the combined phases were washed with $\mathrm{H}_{2} \mathrm{O}(15 \mathrm{~mL})$. The aqueous phase was extracted with $\mathrm{CH}_{2} \mathrm{Cl}_{2}(4 \times$ $10 \mathrm{~mL}$ ). The crude extracts were then dried over $\mathrm{Na}_{2} \mathrm{SO}_{4}$. Evaporation of the solvent and subsequent column chromatography on silica gel afforded the corresponding product $\mathbf{5 1 .}$ 


\subsubsection{General Procedure F: Cobaltaelectro-Catalyzed $\mathrm{C}-\mathrm{H}$ Activation in Aqueous Glycerol for Allene Annulations}

The electrocatalysis was carried out in an undivided cell, with a graphite felt anode $(25 \mathrm{~mm} \times 10 \mathrm{~mm} \times 6.0 \mathrm{~mm})$ and a platinum cathode $(25 \mathrm{~mm} \times 10 \mathrm{~mm} \times 0.125 \mathrm{~mm})$. Benzamide 34 ( $0.50 \mathrm{mmol}, 1.00$ equiv), allene 75 (1.00 mmol, 2.00 equiv), NaOPiv (124 mg, $1.00 \mathrm{mmol}, 2.00$ equiv), glycerol/ $\mathrm{H}_{2} \mathrm{O}(5.0 \mathrm{~mL}, 1: 1)$ and $\mathrm{Co}(\mathrm{OAc})_{2} \cdot 4 \mathrm{H}_{2} \mathrm{O}$ $(12.7 \mathrm{mg}, 10 \mathrm{~mol} \%)$ were placed in a $10 \mathrm{~mL}$ cell. Electrocatalysis was performed at $40{ }^{\circ} \mathrm{C}$ with a constant current of $2 \mathrm{~mA}$ maintained for $15 \mathrm{~h}\left(Q \cdot \mathrm{mol}^{-1}=2.24 \mathrm{~F}\right)$. Then, the DCpower supply was stopped, and the reaction mixture was diluted with $\mathrm{CH}_{2} \mathrm{Cl}_{2}(2.0 \mathrm{~mL})$. The graphite felt anode was washed with $\mathrm{CH}_{2} \mathrm{Cl}_{2}(3 \times 5.0 \mathrm{~mL})$ in an ultrasonic bath $(3 \mathrm{x}$ $3 \mathrm{~min}$ ). The combined washings were added to the reaction mixture and the combined phases were washed with $\mathrm{H}_{2} \mathrm{O}(15 \mathrm{~mL})$. The aqueous phase was extracted with $\mathrm{CH}_{2} \mathrm{Cl}_{2}(4 \times$ $10 \mathrm{~mL}$ ). The crude extracts were then dried over $\mathrm{Na}_{2} \mathrm{SO}_{4}$. Evaporation of the solvent and subsequent column chromatography on silica gel afforded the corresponding product $\mathbf{1 7 6}$.

\subsubsection{General Procedure G: Electrosynthesis of Cobalta(III)cycle 190}

The electrosynthesis was carried out in an undivided cell, with a graphite felt (GF) anode $(25 \mathrm{~mm} \times 10 \mathrm{~mm} \times 6.0 \mathrm{~mm})$, a platinum cathode $(25 \mathrm{~mm} \times 10 \mathrm{~mm} \times 0.125 \mathrm{~mm})$ and a silver-wire $(100 \mathrm{~mm} \times 1.0 \mathrm{~mm})$ as the reference electrode (Figure 85$)$. Benzamide 34 (1.00 mmol, 2.00 equiv), NaOPiv (124 mg, $1.00 \mathrm{mmol}, 2.00$ equiv), MeCN (13 mL) and anhydrous $\mathrm{Co}(\mathrm{OAc})_{2}(88.5 \mathrm{mg}, 0.50 \mathrm{mmol}, 1.00$ equiv) were placed in a $20 \mathrm{~mL}$ cell under a nitrogen atmosphere. Electrolysis was performed at $25{ }^{\circ} \mathrm{C}$ with a constant potential of $1.4 \mathrm{~V}$ vs. $\mathrm{Ag} / \mathrm{Ag}^{+}$maintained until $1.0 \mathrm{~F}$ was passed through the cell. Then, the electrolysis was stopped, the suspension was filtered over Celite and the electrolysis cell was washed with $\mathrm{MeCN}(3 \times 5.0 \mathrm{~mL})$. The graphite felt anode was washed with $\mathrm{MeCN}(3 \times 5.0 \mathrm{~mL})$ in an ultrasonic bath ( $3 \times 3 \mathrm{~min})$. The combined washings were filtered over Celite and added to filtrate of the reaction mixture. Evaporation of the solvent and subsequent fast column chromatography on silica gel $\left(\mathrm{CH}_{2} \mathrm{Cl}_{2} / \mathrm{MeOH}\right)$ yielded the desired complex 190. The solvents were removed at low temperature, as decomposition was otherwise observed in $\mathrm{CH}_{2} \mathrm{Cl}_{2}$ within a few hours at room temperature. 


\subsubsection{General Procedure H: Metal-Free Electro C( ${\left(s^{3}\right)-H}^{3}$ Azidation}

The electrocatalysis was carried out in an undivided cell, with a graphite felt anode $(10 \mathrm{~mm} \times 15 \mathrm{~mm} \times 6 \mathrm{~mm})$ and a platinum cathode $(10 \mathrm{~mm} \times 15 \mathrm{~mm} \times 0.125 \mathrm{~mm})$. An aqueous solution of sodium azide $(2.0 \mathrm{~mL}, 2.0 \mathrm{M})$, AcOH $(2.0 \mathrm{~mL})$, and 1,2,3,4tetrahydronaphthalene (111a) $(66.1 \mathrm{mg}, 0.50 \mathrm{mmol})$ were placed in a $10 \mathrm{~mL}$ cell. The reaction mixture was stirred at $400 \mathrm{rpm}$. The electrodes were connected, and electrolysis was performed at $25^{\circ} \mathrm{C}$ with a constant current of $10 \mathrm{~mA}$ maintained for $4 \mathrm{~h}\left(Q \cdot \mathrm{mol}^{-1}=\right.$ $2.98 \mathrm{~F}$ ). At ambient temperature, the reaction mixture was quenched with sat. aq. $\mathrm{NaHCO}_{3}$ $(20 \mathrm{~mL})$ and EtOAc $(10 \mathrm{~mL})$ was added, and the graphite felt anode was washed with EtOAc $(3 \times 5 \mathrm{~mL})$ in an ultrasonic bath $(3 \times 3 \mathrm{~min})$. The separated aqueous layer was extracted with EtOAc $(3 \times 10 \mathrm{~mL})$. The combined organic layers were dried over $\mathrm{Na}_{2} \mathrm{SO}_{4}$, filtered and the solvents were removed in vacuo. The crude product mixture was purified by column chromatography.

\subsubsection{General Procedure I: Manganaelectro-Catalyzed C(sp $\left.{ }^{3}\right)-H$ Azidation}

The electrocatalysis was carried out in an undivided cell, with a graphite felt anode $(10 \mathrm{~mm} \times 15 \mathrm{~mm} \times 6.0 \mathrm{~mm})$ and a platinum cathode $(10 \mathrm{~mm} \times 15 \mathrm{~mm} \times 0.125 \mathrm{~mm})$. The manganese catalyst [Mn2] (12.7 mg, $25.0 \mu \mathrm{mol}, 5.0 \mathrm{~mol} \%$ ), sodium azide (260 mg, $4.00 \mathrm{mmol}, 8.00$ equiv), substrate 111 (0.50 mmol, 1.00 equiv) and $\mathrm{LiClO}_{4}$ (53.2 mg, $0.50 \mathrm{mmol}, 1.00$ equiv) were placed in a $10 \mathrm{~mL}$ cell under nitrogen atmosphere. The reaction components were dissolved in $\mathrm{MeCN}(2.5 \mathrm{~mL})$, followed by $\mathrm{AcOH}(2.5 \mathrm{~mL})$ and the reaction mixture was stirred at $400 \mathrm{rpm}$. The electrodes were connected under vigorous nitrogen-flow. Electrolysis was performed at $25^{\circ} \mathrm{C}$ with a constant current of $8 \mathrm{~mA}$ maintained for $10 \mathrm{~h}\left(Q \cdot \mathrm{mol}^{-1}=5.97 \mathrm{~F}\right)$. At ambient temperature, the reaction mixture was quenched with sat. aq. $\mathrm{NaHCO}_{3}(20 \mathrm{~mL})$ and EtOAc $(10 \mathrm{~mL})$ was added, and the graphite felt anode was washed with EtOAc $(3 \times 5 \mathrm{~mL})$ in an ultrasonic bath $(3 \times 3 \mathrm{~min})$. The combined organic mixture was washed with $\mathrm{H}_{2} \mathrm{O}(3 \times 10 \mathrm{~mL})$, and then dried over $\mathrm{Na}_{2} \mathrm{SO}_{4}$, filtered and the solvents were removed in vacuo. The crude product mixture was purified by column chromatography to afford the desired organic azides $\mathbf{1 1 3}$. 


\subsection{Cobaltaelectro-Catalyzed $\mathrm{C}-\mathrm{H}$ Oxygenation}

\subsubsection{Characterization Data}<smiles></smiles>

2-(2-Ethoxybenzamido)pyridine-1-oxide (36aa): The general procedure A was followed using 2-benzamidopyridine 1-oxide (34a) (107 $\mathrm{mg}, 0.50 \mathrm{mmol}$ ) and ethanol (35a) $(2 \times 7.0 \mathrm{~mL})$. Purification by column chromatography on silica gel $\left(\mathrm{CH}_{2} \mathrm{Cl}_{2}\right.$ /acetone $\left.3: 1\right)$ yielded 36aa (97.5 mg, $376 \mu \mathrm{mol}, 75 \%)$ as a white solid.

M. p.: $141-143{ }^{\circ} \mathrm{C}$.

${ }^{1}$ H-NMR $\left(500 \mathrm{MHz}, \mathrm{CDCl}_{3}\right): \delta=12.29(\mathrm{~s}, 1 \mathrm{H}), 8.72(\mathrm{dd}, J=8.6,1.9 \mathrm{~Hz}, 1 \mathrm{H}), 8.30-8.23$ (m, 2H), 7.50 (ddd, $J=8.4,2.1,0.7 \mathrm{~Hz} 1 \mathrm{H}), 7.32(\mathrm{dd}, J=8.6,1.9 \mathrm{~Hz}, 1 \mathrm{H}), 7.11-7.01$ (m, 2H), 6.97 (dd, $J=8.4,2.1 \mathrm{~Hz}, 1 \mathrm{H}), 4.29$ (q, $J=6.7 \mathrm{~Hz}, 2 \mathrm{H}), 1.69$ (t, $J=6.7 \mathrm{~Hz}, 3 \mathrm{H})$.

${ }^{13}$ C-NMR (125 MHz, $\left.\mathrm{CDCl}_{3}\right): \delta=163.8\left(\mathrm{C}_{\mathrm{q}}\right), 157.3\left(\mathrm{C}_{\mathrm{q}}\right), 145.3\left(\mathrm{C}_{\mathrm{q}}\right), 137.2(\mathrm{CH}), 134.2$ $(\mathrm{CH}), 132.5(\mathrm{CH}), 127.7(\mathrm{CH}), 121.0(\mathrm{CH}), 120.5\left(\mathrm{C}_{\mathrm{q}}\right), 118.3(\mathrm{CH}), 115.7(\mathrm{CH}), 112.3$ $(\mathrm{CH}), 65.3\left(\mathrm{CH}_{2}\right), 14.8\left(\mathrm{CH}_{3}\right)$.

IR (ATR): $\tilde{v}=3178,3060,1658,1507,1278,1241,1029,737 \mathrm{~cm}^{-1}$.

MS (EI) $m / z$ (relative intensity): 258 (10) [M] $]^{+}, 241$ (12), 197 (34), 149 (55), 121 (100), 93 (22).

HR-MS (EI) $m / z$ calcd. for $\mathrm{C}_{14} \mathrm{H}_{14} \mathrm{~N}_{2} \mathrm{O}_{3}[\mathrm{M}]^{+}: 258.1004$, found: 258.1009 .

The analytical data are in accordance with those previously reported in the literature. ${ }^{[99]}$<smiles>CCOc1ccccc1C(=O)Nc1cccc2cccnc12</smiles>

2-Ethoxy- $N$-(quinolin-8-yl)benzamide (161aa): The general procedure A was followed using $N$-(quinolin-8-yl)benzamide (26a) (124 mg, $0.50 \mathrm{mmol}$ ) and ethanol (35a) $(2 \times 7.0 \mathrm{~mL})$. Purification by column chromatography on silica gel $\left(\mathrm{CH}_{2} \mathrm{Cl}_{2}\right.$ /acetone $\left.6: 1\right)$ yielded $161 \mathrm{aa}(52.0 \mathrm{mg}, 178 \mu \mathrm{mol}, 36 \%)$ as a white solid.

M. p.: $101-103{ }^{\circ} \mathrm{C}$. 
${ }^{1}$ H-NMR (500 MHz, $\left.\mathrm{CDCl}_{3}\right): \delta=12.06(\mathrm{~s}, 1 \mathrm{H}), 9.08(\mathrm{dd}, J=7.6,2.2 \mathrm{~Hz}, 1 \mathrm{H}), 8.80(\mathrm{dd}$, $J=4.4,2.2 \mathrm{~Hz}, 1 \mathrm{H}), 8.34(\mathrm{dd}, J=7.7,2.3 \mathrm{~Hz}, 1 \mathrm{H}), 8.14(\mathrm{dd}, J=7.7,2.3 \mathrm{~Hz}, 1 \mathrm{H}), 7.57(\mathrm{dd}$, $J=8.0,7.7 \mathrm{~Hz}, 1 \mathrm{H}), 7.53-7.45(\mathrm{~m}, 2 \mathrm{H}), 7.43(\mathrm{dd}, J=4.4,4.2 \mathrm{~Hz} 1 \mathrm{H}), 7.10(\mathrm{dd}, J=8.0$, 7.7, 2.2 Hz, 1H), $7.05(\mathrm{dd}, J=7.7,2.3 \mathrm{~Hz}, 1 \mathrm{H}), 4.36$ (q, $J=7.5 \mathrm{~Hz}, 2 \mathrm{H}), 1.73$ (t, $J=7.5 \mathrm{~Hz}$, $3 \mathrm{H})$.

${ }^{13}$ C-NMR (125 MHz, $\left.\mathrm{CDCl}_{3}\right): \delta=164.0\left(\mathrm{C}_{\mathrm{q}}\right), 157.1\left(\mathrm{C}_{\mathrm{q}}\right), 147.7(\mathrm{CH}), 139.3\left(\mathrm{C}_{\mathrm{q}}\right), 136.2$ $(\mathrm{CH}), 135.9\left(\mathrm{C}_{\mathrm{q}}\right), 133.1(\mathrm{CH}), 132.6(\mathrm{CH}), 128.1\left(\mathrm{C}_{\mathrm{q}}\right), 127.5(\mathrm{CH}), 122.4\left(\mathrm{C}_{\mathrm{q}}\right), 121.5(\mathrm{CH})$, $121.4(\mathrm{CH}), 121.0(\mathrm{CH}), 117.7(\mathrm{CH}), 112.3(\mathrm{CH}), 66.3\left(\mathrm{CH}_{2}\right), 15.0\left(\mathrm{CH}_{3}\right)$.

IR (ATR): $\tilde{v}=3294,2985,1655,1524,1485,1324,1162,1039,752 \mathrm{~cm}^{-1}$.

MS (EI) $m / z$ (relative intensity): 292 (35) [M] ${ }^{+}, 259$ (25), 205 (12), 149 (65), 144 (69), 121 (100).

HR-MS (EI) $m / z$ calcd. for $\mathrm{C}_{14} \mathrm{H}_{14} \mathrm{~N}_{2} \mathrm{O}_{3}[\mathrm{M}]^{+}:$: 292.1212, found: 292.1221 .<smiles>CCOc1cc(C)ccc1C(=O)Nc1cccc[n+]1[O-]</smiles>

2-(2-Ethoxy-4-methylbenzamido)pyridine-1-oxide (36ba): The general procedure A was followed using benzamide 34b (114 mg, $0.50 \mathrm{mmol})$ and ethanol (35a) $(2 \times 7.0 \mathrm{~mL})$. Purification by column chromatography on silica gel $\left(\mathrm{CH}_{2} \mathrm{Cl}_{2} /\right.$ acetone $\left.3: 1\right)$ yielded $36 \mathbf{b a}$ (84.0 mg, $309 \mu \mathrm{mol}, 62 \%)$ as a white solid.

M. p.: $159-161{ }^{\circ} \mathrm{C}$.

${ }^{1}$ H-NMR (400 MHz, $\left.\mathrm{CDCl}_{3}\right): \delta=12.20(\mathrm{~s}, 1 \mathrm{H}), 8.72(\mathrm{dd}, J=8.6,1.8 \mathrm{~Hz}, 1 \mathrm{H}), 8.28(\mathrm{~d}, J$ $=6.5 \mathrm{~Hz}, 1 \mathrm{H}), 8.13(\mathrm{~d}, J=8.0 \mathrm{~Hz}, 1 \mathrm{H}), 7.33-7.24(\mathrm{~m}, 1 \mathrm{H}), 6.99-6.91(\mathrm{~m}, 1 \mathrm{H}), 6.87(\mathrm{~d}, J$ $=8.1 \mathrm{~Hz}, 1 \mathrm{H}), 6.81(\mathrm{~s}, 1 \mathrm{H}), 4.29(\mathrm{q}, J=7.0 \mathrm{~Hz}, 2 \mathrm{H}), 2.36(\mathrm{~s}, 3 \mathrm{H}), 1.67$ (t, $J=7.0 \mathrm{~Hz}, 3 \mathrm{H})$. ${ }^{13}$ C-NMR (125 MHz, $\left.\mathrm{CDCl}_{3}\right): \delta=164.0\left(\mathrm{C}_{\mathrm{q}}\right), 157.5\left(\mathrm{C}_{\mathrm{q}}\right), 145.7\left(\mathrm{C}_{\mathrm{q}}\right), 145.5\left(\mathrm{C}_{\mathrm{q}}\right), 137.5$ $(\mathrm{CH}), 132.6(\mathrm{CH}), 128.0(\mathrm{CH}), 122.0(\mathrm{CH}), 118.3(\mathrm{CH}), 117.8\left(\mathrm{C}_{\mathrm{q}}\right), 115.8(\mathrm{CH}), 113.1$ $(\mathrm{CH}), 65.3\left(\mathrm{CH}_{2}\right), 21.9\left(\mathrm{CH}_{3}\right), 14.8\left(\mathrm{CH}_{3}\right)$.

IR (ATR): $\tilde{v}=3195,3054,2988,1653,1607,1502,1395,1257,757,473 \mathrm{~cm}^{-1}$. MS (EI) $m / z$ (relative intensity): $272(10)\left[\mathrm{M}^{+}, 255\right.$ (10), 163 (100), 135 (95).

HR-MS (EI) $m / z$ calcd. for $\mathrm{C}_{15} \mathrm{H}_{16} \mathrm{~N}_{2} \mathrm{O}_{4}[\mathrm{M}]^{+}: 272.1161$, found: 272.1156 .

The analytical data are in accordance with those previously reported in the literature. ${ }^{[99]}$ 
<smiles>CCOc1cc(C(F)(F)F)ccc1C(=O)Nc1cccc[n+]1[O-]</smiles>

2-(2-Ethoxy-4-(trifluoromethyl)benzamido)pyridine-1-oxide (36ca): The general procedure A was followed using benzamide 34c (141 $\mathrm{mg}, 0.50 \mathrm{mmol})$ and ethanol (35a) $(2 \times 7.0 \mathrm{~mL})$. Purification by column chromatography on silica gel $\left(\mathrm{CH}_{2} \mathrm{Cl}_{2} /\right.$ acetone $\left.3: 1\right)$ yielded 36ca $(85.3 \mathrm{mg}, 262 \mu \mathrm{mol}, 52 \%)$ as a white solid.

M. p.: $186-189^{\circ} \mathrm{C}$.

${ }^{1}$ H-NMR $\left(400 \mathrm{MHz}, \mathrm{CDCl}_{3}\right): \delta=12.26(\mathrm{~s}, 1 \mathrm{H}), 8.68(\mathrm{dd}, J=8.4,1.7 \mathrm{~Hz}, 1 \mathrm{H}), 8.37(\mathrm{~d}$, $J=8.0 \mathrm{~Hz}, 1 \mathrm{H}), 8.28(\mathrm{dd}, J=6.7,1.4 \mathrm{~Hz}, 1 \mathrm{H}), 7.39-7.30(\mathrm{~m}, 2 \mathrm{H}), 7.28(\mathrm{~s}, 1 \mathrm{H}), 7.00(\mathrm{ddd}$, $J=8.4,6.4,1.7 \mathrm{~Hz}, 1 \mathrm{H}), 4.39$ (q, $J=7.0 \mathrm{~Hz}, 2 \mathrm{H}), 1.73(\mathrm{t}, J=7.0 \mathrm{~Hz}, 3 \mathrm{H})$.

${ }^{13}$ C-NMR (125 MHz, $\left.\mathrm{CDCl}_{3}\right): \delta=162.7\left(\mathrm{C}_{\mathrm{q}}\right), 157.4\left(\mathrm{C}_{\mathrm{q}}\right), 145.0\left(\mathrm{C}_{\mathrm{q}}\right), 137.3(\mathrm{CH}), 135.6$ $\left(\mathrm{q},{ }^{2} J_{\mathrm{C}-\mathrm{F}}=33.3 \mathrm{~Hz}, \mathrm{C}_{\mathrm{q}}\right), 133.5(\mathrm{CH}), 127.8(\mathrm{CH}), 123.5\left(\mathrm{C}_{\mathrm{q}}\right), 123.4\left(\mathrm{q},{ }^{1} J_{\mathrm{C}-\mathrm{F}}=280 \mathrm{~Hz}, \mathrm{C}_{\mathrm{q}}\right)$, $118.9(\mathrm{CH}), 117.6\left(\mathrm{q},{ }^{3} J_{\mathrm{C}-\mathrm{F}}=3.8 \mathrm{~Hz}, \mathrm{CH}\right), 116.0\left(\mathrm{q},{ }^{3} J_{\mathrm{C}-\mathrm{F}}=4.0 \mathrm{~Hz}, \mathrm{CH}\right), 109.5(\mathrm{CH}), 66.1$ $\left(\mathrm{CH}_{2}\right), 14.6\left(\mathrm{CH}_{3}\right)$.

${ }^{19}$ F-NMR $\left(282 \mathrm{MHz}, \mathrm{CDCl}_{3}\right): \delta=-63.19$.

IR (ATR): $\tilde{v}=3180,3061,1654,1501,1268,1071,760,744 \mathrm{~cm}^{-1}$.

MS (EI) $m / z$ (relative intensity): 326 (10) [M] $]^{+}, 209$ (19), 265 (31), 217 (40), 189 (100), 161 (28), $113(10)$.

HR-MS (EI) $m / z$ calcd. for $\mathrm{C}_{15} \mathrm{H}_{13} \mathrm{~F}_{3} \mathrm{~N}_{2} \mathrm{O}_{3}[\mathrm{M}]^{+}:$326.0878, found: 326.0876 .<smiles>CCOc1cc(OC)ccc1C(=O)Nc1cccc[n+]1[O-]</smiles>

2-(2-Ethoxy-4-methoxybenzamido)pyridine-1-oxide (36da): The general procedure $A$ was followed using benzamide 34d $(123 \mathrm{mg}, 0.50 \mathrm{mmol})$ and ethanol $(\mathbf{3 5 a})(2 \times 7.0 \mathrm{~mL})$. Purification by column chromatography on silica gel $\left(\mathrm{CH}_{2} \mathrm{Cl}_{2} /\right.$ acetone $\left.2: 1\right)$ yielded $\mathbf{3 6 d a}$ (106.3 mg, $369 \mu \mathrm{mol}, 74 \%$ ) as a white solid.

M. p.: $191-193^{\circ} \mathrm{C}$.

${ }^{1}$ H-NMR $\left(300 \mathrm{MHz}, \mathrm{CDCl}_{3}\right): \delta=12.13(\mathrm{~s}, 1 \mathrm{H}), 8.72(\mathrm{~d}, J=7.9 \mathrm{~Hz}, 1 \mathrm{H}), 8.28$ (d, $J=2.1 \mathrm{~Hz}, 1 \mathrm{H}), 8.22(\mathrm{~d}, J=9.3 \mathrm{~Hz}, 1 \mathrm{H}), 7.29(\mathrm{~d}, J=7.9,2.1 \mathrm{~Hz}, 1 \mathrm{H}), 6.96(\mathrm{dd}, J=7.4$, 
$7.1 \mathrm{~Hz}, 1 \mathrm{H}), 6.60(\mathrm{dd}, J=9.3,2.5 \mathrm{~Hz}, 1 \mathrm{H}), 6.53(\mathrm{~d}, J=2.5 \mathrm{~Hz}, 1 \mathrm{H}), 4.27(\mathrm{q}, J=7.0 \mathrm{~Hz}$, 2H), $3.84(\mathrm{~s}, 3 \mathrm{H}), 1.64(\mathrm{t}, J=7.0 \mathrm{~Hz}, 3 \mathrm{H})$.

${ }^{13}$ C-NMR (125 MHz, $\left.\mathrm{CDCl}_{3}\right): \delta=164.5\left(\mathrm{C}_{\mathrm{q}}\right), 163.6\left(\mathrm{C}_{\mathrm{q}}\right), 158.8\left(\mathrm{C}_{\mathrm{q}}\right), 145.7\left(\mathrm{C}_{\mathrm{q}}\right), 137.3$ $(\mathrm{CH}), 134.3(\mathrm{CH}), 127.8(\mathrm{CH}), 117.7(\mathrm{CH}), 115.6(\mathrm{CH}), 113.6\left(\mathrm{C}_{\mathrm{q}}\right), 105.7(\mathrm{CH}), 99.1(\mathrm{CH})$, $65.4\left(\mathrm{CH}_{2}\right), 55.6\left(\mathrm{CH}_{3}\right), 14.7\left(\mathrm{CH}_{3}\right)$.

IR (ATR): $\tilde{v}=3193,3057,2203,2120,1658,1504,1030,727,516 \mathrm{~cm}^{-1}$.

MS (EI) $m / z$ (relative intensity): 288 (12) [M] $]^{+}, 227$ (10), 179 (100), 151 (82), 95 (15).

HR-MS (EI) $m / z$ calcd. for $\mathrm{C}_{15} \mathrm{H}_{16} \mathrm{~N}_{2} \mathrm{O}_{4}[\mathrm{M}]^{+}: 288.1110$, found: 288.1120 .

The analytical data are in accordance with those previously reported in the literature. ${ }^{[99]}$<smiles></smiles>

2-[2-Ethoxy-4-(methylthio)benzamido]pyridine-1-oxide (36ea): The general procedure A was followed using benzamide $\mathbf{3 4 e}(130 \mathrm{mg}, 0.50 \mathrm{mmol})$ and ethanol $(\mathbf{3 5 a})(2 \times 7.0 \mathrm{~mL})$. Purification by column chromatography on silica gel $\left(\mathrm{CH}_{2} \mathrm{Cl}_{2} /\right.$ acetone $\left.5: 1\right)$ yielded 36ea (100 mg, $329 \mu \mathrm{mol}, 66 \%)$ as a white solid.

M. p.: $176-177^{\circ} \mathrm{C}$.

${ }^{1}$ H-NMR $\left(300 \mathrm{MHz}, \mathrm{CDCl}_{3}\right): \delta=12.15(\mathrm{~s}, 1 \mathrm{H}), 8.71(\mathrm{dd}, J=8.6,1.9 \mathrm{~Hz}, 1 \mathrm{H}), 8.30-8.24$ (m, 1H), 8.17 (d, $J=8.4 \mathrm{~Hz}, 1 \mathrm{H}), 7.31$ (ddd, $J=8.6,7.6,1.4 \mathrm{~Hz}, 1 \mathrm{H}), 6.96$ (ddd, $J=7.6$, $6.5,1.9 \mathrm{~Hz}, 1 \mathrm{H}), 6.90(\mathrm{dd}, J=8.4,1.7 \mathrm{~Hz}, 1 \mathrm{H}), 6.84(\mathrm{~d}, J=1.7 \mathrm{~Hz}, 1 \mathrm{H}), 4.30(\mathrm{q}, J=7.0 \mathrm{~Hz}$, $2 \mathrm{H}), 2.51(\mathrm{~s}, 3 \mathrm{H}), 1.70(\mathrm{t}, J=7.0 \mathrm{~Hz}, 3 \mathrm{H})$.

${ }^{13}$ C-NMR $\left(125 \mathrm{MHz}, \mathrm{CDCl}_{3}\right): \delta=163.6\left(\mathrm{C}_{\mathrm{q}}\right), 157.4\left(\mathrm{C}_{\mathrm{q}}\right), 147.1\left(\mathrm{C}_{\mathrm{q}}\right), 145.6\left(\mathrm{C}_{\mathrm{q}}\right), 137.4$ $(\mathrm{CH}), 132.9(\mathrm{CH}), 127.8(\mathrm{CH}), 118.3(\mathrm{CH}), 117.6(\mathrm{CH}), 117.1\left(\mathrm{C}_{\mathrm{q}}\right), 115.8(\mathrm{CH}), 109.2$ $(\mathrm{CH}), 65.6\left(\mathrm{CH}_{2}\right), 15.1\left(\mathrm{CH}_{3}\right), 14.9\left(\mathrm{CH}_{3}\right)$.

IR (ATR): $\tilde{v}=3225,3054,1651,1501,1269,896,734 \mathrm{~cm}^{-1}$.

MS (EI) $m / z$ (relative intensity): 304 (10) [M] $]^{+}, 243$ (14), 195 (100), 167 (70), 152 (10), $111(10), 43$ (24).

HR-MS (EI) $m / z$ calcd. for $\mathrm{C}_{14} \mathrm{H}_{14} \mathrm{~N}_{2} \mathrm{O}_{3}[\mathrm{M}]^{+}:$304.0882, found: 304.0877 . 
<smiles></smiles>

2-[3-Ethoxy-(1,1'-biphenyl)-4-carboxamido]pyridine-1-oxide (36fa): The general procedure A was followed using benzamide 34f (145 $\mathrm{mg}, 0.50 \mathrm{mmol}$ ) and ethanol (35a) $(2 \times 7.0 \mathrm{~mL})$. Purification by column chromatography on silica gel $\left(\mathrm{CH}_{2} \mathrm{Cl}_{2} /\right.$ acetone $\left.3: 1\right)$ yielded 36fa (101 mg, $302 \mu \mathrm{mol}, 61 \%$ ) as a white solid.

M. p.: $164-168^{\circ} \mathrm{C}$.

${ }^{1}$ H-NMR (400 MHz, $\mathrm{CDCl}_{3}$ ): $\delta=12.27$ (s, 1H), 8.72 (dd, $\left.J=8.6,1.8 \mathrm{~Hz}, 1 \mathrm{H}\right), 8.35-8.29$ (m, 2H), 7.62-7.57 (m, 2H), 7.47-7.41 (m, 2H), 7.40-7.34 (m, 1H), 7.34-7.27 (m, 2H), $7.22-7.20(\mathrm{~m}, 1 \mathrm{H}), 6.98-6.92(\mathrm{~m}, 1 \mathrm{H}), 4.39$ (q, $J=7.0 \mathrm{~Hz}, 2 \mathrm{H}), 1.73(\mathrm{t}, J=7.0 \mathrm{~Hz}, 3 \mathrm{H})$. ${ }^{13}$ C-NMR (125 MHz, $\left.\mathrm{CDCl}_{3}\right): \delta=163.8\left(\mathrm{C}_{\mathrm{q}}\right), 157.8\left(\mathrm{C}_{\mathrm{q}}\right), 147.4\left(\mathrm{C}_{\mathrm{q}}\right), 145.5\left(\mathrm{C}_{\mathrm{q}}\right), 140.0$ $\left(\mathrm{C}_{\mathrm{q}}\right), 137.4(\mathrm{CH}), 133.2(\mathrm{CH}), 129.0(\mathrm{CH}), 128.5(\mathrm{CH}), 127.9(\mathrm{CH}), 127.4(\mathrm{CH}), 120.0$ $(\mathrm{CH}), 119.4\left(\mathrm{C}_{\mathrm{q}}\right), 118.5(\mathrm{CH}), 115.9(\mathrm{CH}), 111.2(\mathrm{CH}), 65.7\left(\mathrm{CH}_{2}\right), 15.0\left(\mathrm{CH}_{3}\right)$.

IR (ATR): $\tilde{v}=3212,2980,1751,1667,1606,1500,1475,1210,753 \mathrm{~cm}^{-1}$.

MS (EI) $m / z$ (relative intensity): 334 (10) [M] $]^{+}, 317$ (10), 225 (100), 197 (59), 141 (20), 115 (14).

HR-MS (EI) $m / z$ calcd. for $\mathrm{C}_{20} \mathrm{H}_{18} \mathrm{~N}_{2} \mathrm{O}_{3}[\mathrm{M}]^{+}: 334.1317$, found: 334.1326 .

The analytical data are in accordance with those previously reported in the literature. ${ }^{\text {[99] }}$<smiles>CCOc1cc(I)ccc1C(=O)Nc1cccc[n+]1[O-]</smiles>

2-(2-Ethoxy-4-iodobenzamido)pyridine-1-oxide (36ga): The general procedure A was followed using benzamide $\mathbf{3 4 g}(170 \mathrm{mg}, 0.50 \mathrm{mmol})$ and ethanol (35a) $(2 \times 7.0 \mathrm{~mL})$. Purification by column chromatography on silica gel $\left(\mathrm{CH}_{2} \mathrm{Cl}_{2} /\right.$ acetone $\left.5: 1\right)$ yielded 36 ga (82.8 $\mathrm{mg}, 215 \mu \mathrm{mol}, 43 \%)$ as a white solid.

M. p.: $212-214{ }^{\circ} \mathrm{C}$.

${ }^{1}$ H-NMR $\left(300 \mathrm{MHz}, \mathrm{CDCl}_{3}\right): \delta=12.13(\mathrm{~s}, 1 \mathrm{H}), 8.69(\mathrm{~d}, J=8.5 \mathrm{~Hz}, 1 \mathrm{H}), 8.29(\mathrm{~d}, J=$ $6.5 \mathrm{~Hz}, 1 \mathrm{H}), 7.94(\mathrm{~d}, J=8.5 \mathrm{~Hz}, 1 \mathrm{H}), 7.45$ (dd, $J=8.3,1.5 \mathrm{~Hz}, 1 \mathrm{H}), 7.38$ (d, $J=1.5 \mathrm{~Hz}$, 1H), 7.35-7.28 (m, 1H), 7.05 5-6.96 (m, 1H), $4.30(\mathrm{q}, J=7.0 \mathrm{~Hz}, 2 \mathrm{H}), 1.68(\mathrm{t}, J=7.0 \mathrm{~Hz}$, $3 \mathrm{H})$. 
${ }^{13}$ C-NMR $\left(75 \mathrm{MHz}, \mathrm{CDCl}_{3}\right): \delta=163.4\left(\mathrm{C}_{\mathrm{q}}\right), 157.4\left(\mathrm{C}_{\mathrm{q}}\right), 145.5\left(\mathrm{C}_{\mathrm{q}}\right), 137.6(\mathrm{CH}), 133.8$ $(\mathrm{CH}), 130.7(\mathrm{CH}), 128.1(\mathrm{CH}), 122.0(\mathrm{CH}), 120.3\left(\mathrm{C}_{\mathrm{q}}\right), 118.7\left(\mathrm{C}_{\mathrm{q}}\right), 115.9(\mathrm{CH}), 101.2(\mathrm{CH})$, $66.1\left(\mathrm{CH}_{2}\right), 14.7\left(\mathrm{CH}_{3}\right)$.

IR (ATR): $\tilde{v}=3161,3053,1656,1511,1276,1242,799,763 \mathrm{~cm}^{-1}$.

MS (EI) $m / z$ (relative intensity): 384 (15) [M] $]^{+}, 367$ (30), 323 (25), 275 (100), 247 (80), 219 (25), $148(20), 120(40)$.

HR-MS (EI) $m / z$ calcd. for $\mathrm{C}_{14} \mathrm{H}_{13} \mathrm{IN}_{2} \mathrm{O}_{3}[\mathrm{M}]^{+}: 383.9971$ found: 383.9978 .

The analytical data are in accordance with those previously reported in the literature. ${ }^{[405]}$<smiles></smiles>

2-(5-Chloro-2-ethoxybenzamido)pyridine-1-oxide (36ha): The general procedure A was followed using benzamide 34h (124 mg, $0.50 \mathrm{mmol})$ and ethanol (35a) $(2 \times 7.0 \mathrm{~mL})$. Purification by column chromatography on silica gel $\left(\mathrm{CH}_{2} \mathrm{Cl}_{2}\right.$ /acetone $\left.5: 1\right)$ yielded $\mathbf{3 6 h a}$ (78.0 mg, $266 \mu \mathrm{mol}, 53 \%)$ as a white solid.

M. p.: $226-227^{\circ} \mathrm{C}$.

${ }^{1} \mathbf{H}-\mathbf{N M R}\left(300 \mathrm{MHz}, \mathrm{CDCl}_{3}\right) \delta=12.23(\mathrm{~s}, 1 \mathrm{H}), 8.70(\mathrm{dd}, J=8.4,1.9 \mathrm{~Hz}, 1 \mathrm{H}), 8.29(\mathrm{~d}, J=$ $6.5 \mathrm{~Hz}, 1 \mathrm{H}), 8.24(\mathrm{~d}, J=2.8 \mathrm{~Hz}, 1 \mathrm{H}), 7.46(\mathrm{dd}, J=8.9,2.8 \mathrm{~Hz}, 1 \mathrm{H}), 7.40-7.29(\mathrm{~m}, 1 \mathrm{H})$, 7.06-6.96 (m, 2H), 4.32 (q, $J=7.0 \mathrm{~Hz}, 2 \mathrm{H}), 1.70(\mathrm{t}, J=7.0 \mathrm{~Hz}, 3 \mathrm{H})$.

${ }^{13}$ C-NMR (125 MHz, $\left.\mathrm{CDCl}_{3}\right): \delta=162.6\left(\mathrm{C}_{\mathrm{q}}\right), 156.0\left(\mathrm{C}_{\mathrm{q}}\right), 145.3\left(\mathrm{C}_{\mathrm{q}}\right), 137.4(\mathrm{CH}), 133.9$ $(\mathrm{CH}), 132.3(\mathrm{CH}), 127.9(\mathrm{CH}), 126.6\left(\mathrm{C}_{\mathrm{q}}\right), 122.0\left(\mathrm{C}_{\mathrm{q}}\right), 118.8(\mathrm{CH}), 116.0(\mathrm{CH}), 110.1(\mathrm{CH})$, $66.1\left(\mathrm{CH}_{2}\right), 14.9\left(\mathrm{CH}_{3}\right)$.

IR (ATR): $\tilde{v}=3164,3061,1656,1563,1514,1431,1273,1031,754,507 \mathrm{~cm}^{-1}$.

MS (EI) $m / z$ (relative intensity): 294 (7) $\left[{ }^{37} \mathrm{Cl}-\mathrm{M}\right]^{+}, 292$ (20) $\left[{ }^{35} \mathrm{Cl}-\mathrm{M}\right]^{+}, 275$ (25), 231 (20), 183 (70), 155 (100), 43 (50).

HR-MS (EI) $m / z$ calcd. for $\mathrm{C}_{14} \mathrm{H}_{13} \mathrm{~N}_{2} \mathrm{O}_{3}{ }^{35} \mathrm{Cl}\left[{ }^{35} \mathrm{Cl}-\mathrm{M}\right]^{+}: 292.0615$, found: 292.0614 .

The analytical data are in accordance with those previously reported in the literature. ${ }^{[99]}$ 
<smiles>CCOc1ccc(I)cc1C(=O)Nc1cccc[n+]1[O-]</smiles>

2-(2-Ethoxy-5-iodobenzamido)pyridine-1-oxide (36ia): The general procedure $\mathbf{A}$ was followed using benzamide 34i $(170 \mathrm{mg}, 0.50 \mathrm{mmol})$ and ethanol $(\mathbf{3 5 a})(2 \times 7.0 \mathrm{~mL})$. Purification by column chromatography on silica gel $\left(\mathrm{CH}_{2} \mathrm{Cl}_{2} /\right.$ acetone 5:1) yielded 36ia (98.0 mg, $255 \mu \mathrm{mol}, 51 \%)$ as a white solid.

M. p.: $235-236^{\circ} \mathrm{C}$.

${ }^{1}$ H-NMR $\left(300 \mathrm{MHz}, \mathrm{CDCl}_{3}\right): \delta=12.20(\mathrm{~s}, 1 \mathrm{H}), 8.69(\mathrm{dd}, J=8.6,1.9 \mathrm{~Hz}, 1 \mathrm{H}), 8.55$ (d, $J=2.4 \mathrm{~Hz}, 1 \mathrm{H}), 8.32-8.26(\mathrm{~m}, 1 \mathrm{H}), 7.78(\mathrm{dd}, J=8.7,2.4 \mathrm{~Hz}, 1 \mathrm{H}), 7.36(\mathrm{ddd}, J=8.7,7.6$, $1.5 \mathrm{~Hz}, 1 \mathrm{H}), 7.01$ (ddd, $J=7.6,6.5,1.9 \mathrm{~Hz}, 1 \mathrm{H}), 6.83$ (d, $J=8.8 \mathrm{~Hz}, 1 \mathrm{H}), 4.32$ (q, $J=7.0 \mathrm{~Hz}, 2 \mathrm{H}), 1.70(\mathrm{t}, J=7.0 \mathrm{~Hz}, 3 \mathrm{H})$.

${ }^{13}$ C-NMR (126 MHz, $\left.\mathrm{CDCl}_{3}\right): \delta=162.4\left(\mathrm{C}_{\mathrm{q}}\right), 157.3\left(\mathrm{C}_{\mathrm{q}}\right), 145.2\left(\mathrm{C}_{\mathrm{q}}\right), 142.7(\mathrm{CH}), 141.2$ $(\mathrm{CH}), 137.4(\mathrm{CH}), 127.9(\mathrm{CH}), 122.7\left(\mathrm{C}_{\mathrm{q}}\right), 118.8(\mathrm{CH}), 116.0(\mathrm{CH}), 114.9(\mathrm{CH}), 83.3\left(\mathrm{C}_{\mathrm{q}}\right)$, $66.0\left(\mathrm{CH}_{2}\right), 14.9\left(\mathrm{CH}_{3}\right)$.

IR (ATR): $\tilde{v}=3166,3058,1656,1512,1278,1240,801,762 \mathrm{~cm}^{-1}$.

MS (EI) $m / z$ (relative intensity): 384 (20) [M] $]^{+}, 367$ (37), 323 (15), 275 (100), 247 (84), 219 (19), 148 (26), 120 (40).

HR-MS (EI) $m / z$ calcd. for $\mathrm{C}_{14} \mathrm{H}_{13} \mathrm{IN}_{2} \mathrm{O}_{3}[\mathrm{M}]^{+}: 383.9971$ found: 383.9980 .

The analytical data are in accordance with those previously reported in the literature. ${ }^{\text {[99] }}$<smiles></smiles>

2-(2-Methoxybenzamido)pyridine-1-oxide (36ab): The general procedure A was followed using benzamide 35a $(107 \mathrm{mg}, 0.50 \mathrm{mmol})$ and methanol $(\mathbf{3 5 b})(2 \times 7.0 \mathrm{~mL})$. Purification by column chromatography on silica gel $\left(\mathrm{CH}_{2} \mathrm{Cl}_{2}\right.$ /acetone $\left.2: 1\right)$ yielded $\mathbf{3 6 a b}$ (86.8 $\mathrm{mg}, 256 \mu \mathrm{mol}, 71 \%$ ) as a white solid.

M. p.: $121-124^{\circ} \mathrm{C}$.

${ }^{1}$ H-NMR (400 MHz, $\left.\mathrm{CDCl}_{3}\right): \delta=12.41(\mathrm{~s}, 1 \mathrm{H}), 8.67(\mathrm{dd}, J=8.3,1.7 \mathrm{~Hz}, 1 \mathrm{H}), 8.28-8.25$ (m, 2H), $7.53(\mathrm{ddd}, J=7.9,7.0,1.7 \mathrm{~Hz}, 1 \mathrm{H}), 7.35$ (ddd, $J=8.3,7.0,1.4 \mathrm{~Hz}, 1 \mathrm{H}), 7.10$ (ddd, 
$J=8.5,7.5,2.1 \mathrm{~Hz}, 1 \mathrm{H}), 7.05(\mathrm{dd}, J=8.5,1.0 \mathrm{~Hz}, 1 \mathrm{H}), 6.97(\mathrm{ddd}, J=8.0,7.5,1.0 \mathrm{~Hz}$, $1 \mathrm{H}), 4.13(\mathrm{~s}, 3 \mathrm{H})$.

${ }^{13}$ C-NMR (101 MHz, $\left.\mathrm{CDCl}_{3}\right): \delta=163.5\left(\mathrm{C}_{\mathrm{q}}\right), 157.9\left(\mathrm{C}_{\mathrm{q}}\right), 145.4\left(\mathrm{C}_{\mathrm{q}}\right), 137.3(\mathrm{CH}), 134.3$ $(\mathrm{CH}), 132.4(\mathrm{CH}), 128.3(\mathrm{CH}), 121.3(\mathrm{CH}), 120.4\left(\mathrm{C}_{\mathrm{q}}\right), 118.3(\mathrm{CH}), 115.5(\mathrm{CH}), 111.6$ $(\mathrm{CH}), 56.3\left(\mathrm{CH}_{3}\right)$.

IR (ATR): $\tilde{v}=3175,1671,1564,1479,1238,1043,744 \mathrm{~cm}^{-1}$.

MS (EI) $m / z$ (relative intensity): 244 (18) [M] ${ }^{+}, 227$ (11), 197 (10), 135 (100), 110 (15), 92 (22), $77(25)$.

HR-MS (ESI) $m / z$ calcd. for $\mathrm{C}_{14} \mathrm{H}_{13} \mathrm{~N}_{2} \mathrm{O}_{3}{ }^{79} \mathrm{Br}[\mathrm{M}]^{+}:$244.0848, found 244.0851.

The analytical data are in accordance with those previously reported in the literature. ${ }^{\text {[99] }}$<smiles></smiles>

2-(2-n-Propoxybenzamido)pyridine-1-oxide (36ac): The general procedure A was followed using benzamide $34 \mathrm{a}(107 \mathrm{mg}, 0.50 \mathrm{mmol})$, tetra- $n$-butylammonium acetate $(152 \mathrm{mg}, 0.50 \mathrm{mmol}, 1.00$ equiv in each half-cell) and $n$-propanol $(\mathbf{3 5 c})(2 \times 7.0 \mathrm{~mL})$. Purification by column chromatography on silica gel $\left(\mathrm{CH}_{2} \mathrm{Cl}_{2} /\right.$ acetone $\left.10: 1\right)$ yielded 36ac (88 $\mathrm{mg}, 324 \mu \mathrm{mol}, 65 \%$ ) as a white solid.

M. p.: $74-75^{\circ} \mathrm{C}$.

${ }^{1}$ H-NMR (300 MHz, $\left.\mathrm{CDCl}_{3}\right): \delta=12.23(\mathrm{~s}, 1 \mathrm{H}), 8.72(\mathrm{ddd}, J=8.6,1.9,0.5 \mathrm{~Hz}, 1 \mathrm{H}), 8.31-$ $8.24(\mathrm{~m}, 2 \mathrm{H}), 7.50(\mathrm{ddd}, J=8.3,7.3,1.9 \mathrm{~Hz}, 1 \mathrm{H}), 7.36-7.29(\mathrm{~m}, 1 \mathrm{H}), 7.12-7.02(\mathrm{~m}, 2 \mathrm{H})$, $6.97(\mathrm{ddd}, J=7.5,6.5,1.9 \mathrm{~Hz}, 1 \mathrm{H}), 4.21(\mathrm{t}, J=7.1 \mathrm{~Hz}, 2 \mathrm{H}), 2.22-2.06$ (m, 2H), 1.09 (t, $J=7.4 \mathrm{~Hz}, 3 \mathrm{H})$.

${ }^{13}$ C-NMR $\left(126 \mathrm{MHz}, \mathrm{CDCl}_{3}\right): \delta=164.0\left(\mathrm{C}_{\mathrm{q}}\right), 157.6\left(\mathrm{C}_{\mathrm{q}}\right), 145.4\left(\mathrm{C}_{\mathrm{q}}\right), 137.4(\mathrm{CH}), 134.3$ $(\mathrm{CH}), 132.7(\mathrm{CH}), 127.6(\mathrm{CH}), 121.1(\mathrm{CH}), 120.7\left(\mathrm{C}_{\mathrm{q}}\right), 118.5(\mathrm{CH}), 115.9(\mathrm{CH}), 112.6$ $(\mathrm{CH}), 71.6\left(\mathrm{CH}_{2}\right), 22.4\left(\mathrm{CH}_{2}\right), 10.7\left(\mathrm{CH}_{3}\right)$.

IR (ATR): $\tilde{v}=3212,2967,1668,1600,1504,1239,724 \mathrm{~cm}^{-1}$.

MS (EI) $m / z$ (relative intensity): 272 (10) [M] $]^{+}, 255$ (10), 230 (32), 163 (65), 135 (18), 121 (100), 93 (23), 65 (18).

HR-MS (EI) $m / z$ calcd. for $\mathrm{C}_{15} \mathrm{H}_{16} \mathrm{~N}_{2} \mathrm{O}_{3}[\mathrm{M}]^{+}: 272.1161$, found: 272.1171 . 
<smiles></smiles>

2-[2-(2,2,2-Trifluoroethoxy)benzamido]pyridine-1-oxide (36ad): The general procedure A was followed using benzamide 34a (107 $\mathrm{mg}, 0.50 \mathrm{mmol})$ and trifluoroethanol (35d) $(2 \times 7.0 \mathrm{~mL})$. Purification by column chromatography on silica gel $\left(\mathrm{CH}_{2} \mathrm{Cl}_{2} /\right.$ acetone 4:1) yielded 36ad (97.6 mg, $312 \mu \mathrm{mol}, 40 \%$ ) as a white solid.

M. p.: $152-154{ }^{\circ} \mathrm{C}$.

${ }^{1}$ H-NMR (300 MHz, $\left.\mathrm{CDCl}_{3}\right): \delta=11.93(\mathrm{~s}, 1 \mathrm{H}), 8.68(\mathrm{dd}, J=7.8,2.2 \mathrm{~Hz}, 1 \mathrm{H}), 8.33$ (dd, $J=6.6,1.2 \mathrm{~Hz}, 1 \mathrm{H}), 8.27(\mathrm{dd}, J=7.9,1.6 \mathrm{~Hz}, 1 \mathrm{H}), 7.59(\mathrm{ddd}, J=7.9,7.8,2.2 \mathrm{~Hz}, 1 \mathrm{H})$, $7.38(\mathrm{ddd}, J=8.1,6.6,1.7 \mathrm{~Hz}, 1 \mathrm{H}), 7.29(\mathrm{ddd}, J=8.1,6.6,1.2 \mathrm{~Hz}, 1 \mathrm{H}), 7.12(\mathrm{dd}, J=8.1$, $1.7 \mathrm{~Hz}, 1 \mathrm{H}), 7.03$ (ddd, $J=7.9,7.8,1.6 \mathrm{~Hz}, 1 \mathrm{H}), 4.76(\mathrm{t}, J=8.6 \mathrm{~Hz}, 2 \mathrm{H})$.

${ }^{13}$ C-NMR $\left(125 \mathrm{MHz}, \mathrm{CDCl}_{3}\right): \delta=163.0\left(\mathrm{C}_{\mathrm{q}}\right), 155.6\left(\mathrm{C}_{\mathrm{q}}\right), 144.9\left(\mathrm{C}_{\mathrm{q}}\right), 137.2(\mathrm{CH}), 134.1$ $(\mathrm{CH}), 132.8(\mathrm{CH}), 127.6(\mathrm{CH}), 123.3(\mathrm{CH}), 123.0\left(\mathrm{q},{ }^{1} J_{\mathrm{CF}}=258 \mathrm{~Hz}, \mathrm{C}_{\mathrm{q}}\right), 122.3\left(\mathrm{C}_{\mathrm{q}}\right), 118.7$ $(\mathrm{CH}), 115.5(\mathrm{CH}), 113.5(\mathrm{CH}), 66.5\left(\mathrm{q},{ }^{2} J_{\mathrm{CF}}=34.5 \mathrm{~Hz}, \mathrm{CH}_{2}\right)$.

${ }^{19}$ F-NMR $\left(282 \mathrm{MHz}, \mathrm{CDCl}_{3}\right): \delta=-72.81$.

IR (ATR): $\tilde{v}=3172,1669,1563,1504,1452,1092,670 \mathrm{~cm}^{-1}$.

MS (EI) m/z (relative intensity): 312 (16) [M] $]^{+}, 203$ (100), 197 (35), 183 (10) 155 (5), 120 (6), 92 (12).

HR-MS (ESI) $m / z$ calcd. for $\mathrm{C}_{14} \mathrm{H}_{12} \mathrm{~F}_{3} \mathrm{~N}_{2} \mathrm{O}_{3}[\mathrm{M}+\mathrm{H}]^{+}: 313.0795$, found: 313.0791 .<smiles>CCOC(C)=C(C)C(=O)Nc1cccc[n+]1[O-]</smiles>

(Z)-2-(3-Ethoxy-2-methylbut-2-enamido)pyridine-1-oxide (38aa): The general procedure $\mathbf{C}$ was followed using alkene 37a $(48.1 \mathrm{mg}, 0.25 \mathrm{mmol})$, and ethanol (35a) $(7.0 \mathrm{~mL})$. Purification by column chromatography on silica gel $\left(\mathrm{CH}_{2} \mathrm{Cl}_{2} /\right.$ acetone $\left.3: 1\right)$ yielded 38aa (41.1 mg, $174 \mu \mathrm{mol}, 69 \%$ ) as a white solid.

M. p.: $110-112^{\circ} \mathrm{C}$. 
${ }^{1}$ H-NMR $\left(300 \mathrm{MHz}, \mathrm{CDCl}_{3}\right): \delta=12.14(\mathrm{~s}, 1 \mathrm{H}), 8.66(\mathrm{dd}, J=8.5 \mathrm{~Hz}, 1 \mathrm{H}), 8.23(\mathrm{dd}, J=$ $6.4 \mathrm{~Hz}, 1 \mathrm{H}), 7.25$ (ddd, 1H), 6.92 (ddd, $J=6.6 \mathrm{~Hz}, 1 \mathrm{H}), 4.26$ (q, $J=7.6 \mathrm{~Hz}, 2 \mathrm{H}), 2.20$ (s, $3 \mathrm{H}), 1.96(\mathrm{~s}, 3 \mathrm{H}), 1.50$ (t, $J=7.6 \mathrm{~Hz}, 3 \mathrm{H})$.

${ }^{13}$ C-NMR $\left(101 \mathrm{MHz}, \mathrm{CDCl}_{3}\right): \delta=166.0\left(\mathrm{C}_{\mathrm{q}}\right), 160.0\left(\mathrm{C}_{\mathrm{q}}\right), 146.0\left(\mathrm{C}_{\mathrm{q}}\right), 137.4(\mathrm{CH}), 128.2$ $(\mathrm{CH}), 117.7(\mathrm{CH}), 115.4(\mathrm{CH}), 108.6\left(\mathrm{C}_{\mathrm{q}}\right), 65.4\left(\mathrm{CH}_{2}\right), 16.1\left(\mathrm{CH}_{3}\right), 15.5\left(\mathrm{CH}_{3}\right), 13.9\left(\mathrm{CH}_{3}\right)$. IR (ATR): $\tilde{v}=3201,2927,1701,1620,1503,1423,1161,1038,763 \mathrm{~cm}^{-1}$.

MS (EI) $m / z$ (relative intensity): 236 (5) [M] $]^{+}, 191$ (8), 165 (5), 149 (9), 127 (66), 99 (100), $84(23), 58(41)$.

HR-MS (EI) $m / z$ calcd. for $\mathrm{C}_{12} \mathrm{H}_{16} \mathrm{~N}_{2} \mathrm{O}_{3}[\mathrm{M}]^{+}:$236.1161, found: 236.1162 .

The analytical data are in accordance with those previously reported in the literature. ${ }^{\text {[99] }}$<smiles>COC(C)=C(C)C(=O)Nc1cccc[n+]1[O-]</smiles>

(Z)-2-(3-Methoxy-2-methylbut-2-enamido)pyridine-1-oxide (38ab): The general procedure $\mathbf{C}$ was followed using alkene $\mathbf{3 7 a}(96.1 \mathrm{mg}, 0.50 \mathrm{mmol})$ and methanol (35b) $(7.0 \mathrm{~mL})$. Purification by column chromatography on silica gel $\left(\mathrm{CH}_{2} \mathrm{Cl}_{2}\right.$ /acetone $\left.2: 1\right)$ yielded 38ab (69.0 mg, $310 \mu \mathrm{mol}, 62 \%)$ as a white solid.

M. p.: $116-118^{\circ} \mathrm{C}$.

${ }^{1}$ H-NMR $\left(300 \mathrm{MHz}, \mathrm{CDCl}_{3}\right): \delta=12.24(\mathrm{~s}, 1 \mathrm{H}), 8.59(\mathrm{dd}, J=8.5,2.1 \mathrm{~Hz}, 1 \mathrm{H}), 8.23(\mathrm{dd}$, $J=6.4,1.6 \mathrm{~Hz}, 1 \mathrm{H}), 7.35-7.23(\mathrm{~m}, 1 \mathrm{H}), 6.92(\mathrm{ddd}, J=8.5,6.4,2.1 \mathrm{~Hz}, 1 \mathrm{H}), 3.95(\mathrm{~s}, 3 \mathrm{H})$, $2.15(\mathrm{~d}, J=1.0 \mathrm{~Hz}, 3 \mathrm{H}), 1.91(\mathrm{~d}, J=1.0 \mathrm{~Hz}, 3 \mathrm{H})$.

${ }^{13}$ C-NMR $\left(125 \mathrm{MHz}, \mathrm{CDCl}_{3}\right): \delta=165.7\left(\mathrm{C}_{\mathrm{q}}\right), 160.3\left(\mathrm{C}_{\mathrm{q}}\right), 145.0\left(\mathrm{C}_{\mathrm{q}}\right), 137.5(\mathrm{CH}), 128.4$ $(\mathrm{CH}), 117.3(\mathrm{CH}), 114.8(\mathrm{CH}), 108.1(\mathrm{CH}), 56.2\left(\mathrm{CH}_{2}\right), 15.2\left(\mathrm{CH}_{3}\right), 13.7\left(\mathrm{CH}_{3}\right)$

IR (ATR): $\tilde{v}=3205,2947,1651,1501,1425,1163,1028,756 \mathrm{~cm}^{-1}$.

MS (EI) $m / z$ (relative intensity): 222 (5) [M] $]^{+}, 191$ (8), 175 (7), 148 (8), 113 (100), 105 (10), $78(8)$.

HR-MS (EI) $m / z$ calcd. for $\mathrm{C}_{11} \mathrm{H}_{14} \mathrm{~N}_{2} \mathrm{O}_{3}[\mathrm{M}]^{+}:$:22.1004, found: 222.1001 .

The analytical data are in accordance with those previously reported in the literature. ${ }^{[99]}$ 
<smiles></smiles>

2-(2-Methoxycyclohex-1-ene-1-carboxamido)pyridine-1-oxide (38bb): The general procedure $\mathbf{B}$ was followed using alkene $\mathbf{3 7 b}(54.6 \mathrm{mg}, 0.25 \mathrm{mmol})$, and methanol (35b) (7.0 mL). Purification by column chromatography on silica gel $\left(\mathrm{CH}_{2} \mathrm{Cl}_{2} /\right.$ acetone $\left.1: 1\right)$ yielded 38bb (44.9 mg, $181 \mu \mathrm{mol}, 72 \%$ ) as a white solid.

M. p.: $104-106^{\circ} \mathrm{C}$.

${ }^{1}$ H-NMR (400 MHz, $\left.\mathrm{CDCl}_{3}\right): \delta=12.17(\mathrm{~s}, 1 \mathrm{H}), 8.55(\mathrm{dd}, J=8.5,1.9 \mathrm{~Hz}, 1 \mathrm{H}), 8.19(\mathrm{~d}, J=$ $6.5 \mathrm{~Hz}, 1 \mathrm{H}), 7.28-7.21(\mathrm{~m}, 1 \mathrm{H}), 6.88$ (ddd, $J=8.0,6.5,1.9 \mathrm{~Hz}, 1 \mathrm{H}), 3.91$ (s, 3H), 2.44$2.34(\mathrm{~m}, 4 \mathrm{H}), 1.77-1.68(\mathrm{~m}, 2 \mathrm{H}), 1.64-1.55(\mathrm{~m}, 2 \mathrm{H})$.

${ }^{13}$ C-NMR (75 MHz, $\left.\mathrm{CDCl}_{3}\right): \delta=165.7\left(\mathrm{C}_{\mathrm{q}}\right), 162.4\left(\mathrm{C}_{\mathrm{q}}\right), 145.9\left(\mathrm{C}_{\mathrm{q}}\right), 137.3(\mathrm{CH}), 128.1$ $(\mathrm{CH}), 117.5(\mathrm{CH}), 115.0(\mathrm{CH}), 109.6\left(\mathrm{C}_{\mathrm{q}}\right), 55.2\left(\mathrm{CH}_{3}\right), 25.6\left(\mathrm{CH}_{2}\right), 24.3\left(\mathrm{CH}_{2}\right), 22.6\left(\mathrm{CH}_{2}\right)$, $22.0\left(\mathrm{CH}_{2}\right)$.

IR (ATR): $\tilde{v}=3210,2940,1654,1504,1427,1178,907,723 \mathrm{~cm}^{-1}$.

MS (EI) $m / z$ (relative intensity): 248 (6) [M] $]^{+}, 217$ (12), 201 (7), 139 (100), 128 (9), 79 (17).

HR-MS (EI) $m / z$ calcd. for $\mathrm{C}_{13} \mathrm{H}_{16} \mathrm{~N}_{2} \mathrm{O}_{3}$ [M] $]^{+}:$248.1161, found: 248.1164.

The analytical data are in accordance with those previously reported in the literature. ${ }^{[99]}$<smiles></smiles>

(Z)-2-(3-Ethoxy-2-methyl-3-phenylacrylamido)pyridine-1-oxide (38ca): The general procedure $\mathbf{C}$ was followed using 37c $(63.6 \mathrm{mg}, 0.25 \mathrm{mmol})$ and ethanol (35a) (7.0 $\mathrm{mL})$. Purification by column chromatography on silica gel $\left(\mathrm{CH}_{2} \mathrm{Cl}_{2} /\right.$ acetone $\left.3: 1\right)$ yielded 38ca (39.8 $\mathrm{mg}, 133 \mu \mathrm{mol}, 53 \%)$ as a white solid.

M. p.: $124-126^{\circ} \mathrm{C}$.

${ }^{1}$ H-NMR (400 MHz, $\left.\mathrm{CDCl}_{3}\right): \delta=12.38(\mathrm{~s}, 1 \mathrm{H}), 8.65$ (dd, $\left.J=7.9,1.8 \mathrm{~Hz}, 1 \mathrm{H}\right), 8.27$ (dd, $J=6.5,2.1 \mathrm{~Hz}, 1 \mathrm{H}), 7.51-7.43(\mathrm{~m}, 3 \mathrm{H}), 7.35-7.29(\mathrm{~m}, 3 \mathrm{H}), 6.96(\mathrm{ddd}, J=7.9,6.5,1.8 \mathrm{~Hz}$, $1 \mathrm{H}), 3.86(\mathrm{q}, J=7.3 \mathrm{~Hz}, 2 \mathrm{H}), 1.81(\mathrm{~s}, 3 \mathrm{H}), 1.40(\mathrm{t}, J=7.3 \mathrm{~Hz}, 3 \mathrm{H})$. 
${ }^{13}$ C-NMR $\left(125 \mathrm{MHz}, \mathrm{CDCl}_{3}\right): \delta=166.1\left(\mathrm{C}_{\mathrm{q}}\right), 160.8\left(\mathrm{C}_{\mathrm{q}}\right), 145.6\left(\mathrm{C}_{\mathrm{q}}\right), 137.3\left(\mathrm{C}_{\mathrm{q}}\right), 133.9$ $\left(\mathrm{C}_{\mathrm{q}}\right), 129.4(\mathrm{CH}), 128.9(\mathrm{CH}), 128.6(\mathrm{CH}), 127.8(\mathrm{CH}), 117.8(\mathrm{CH}), 115.3(\mathrm{CH}), 110.9$ $(\mathrm{CH}), 67.1\left(\mathrm{CH}_{2}\right), 15.1\left(\mathrm{CH}_{3}\right) 15.1\left(\mathrm{CH}_{3}\right)$.

IR (ATR): $\tilde{v}=3206,3058,2923,1649,1502,1389,1129,763,703 \mathrm{~cm}^{-1}$.

MS (ESI) $m / z$ (relative intensity): 619 (32) $[2 \mathrm{M}+\mathrm{Na}]^{+}, 321(100)[\mathrm{M}+\mathrm{Na}]^{+}, 299$ (52) $[\mathrm{M}+\mathrm{H}]^{+}, 189(67), 161(25), 137(9)$.

HR-MS (ESI) $m / z$ calcd. for $\mathrm{C}_{17} \mathrm{H}_{18} \mathrm{~N}_{2} \mathrm{O}_{3}[\mathrm{M}+\mathrm{H}]^{+}:$299.1394, found: 299.1390 .<smiles></smiles>

(Z)-2-(3-Methoxy-2-methyl-3-phenylacrylamido)pyridine-1-oxide (38cb): The general procedure $\mathbf{C}$ was followed using alkene $37 \mathbf{c}(63.6 \mathrm{mg}, 0.25 \mathrm{mmol})$ and methanol (35b) $(7.0 \mathrm{~mL})$. Purification by column chromatography on silica gel $\left(\mathrm{CH}_{2} \mathrm{Cl}_{2}\right.$ /acetone $\left.1: 1\right)$ yielded 38cb (50.7 mg, $178 \mu \mathrm{mol}, 71 \%)$ as a white solid.

M. p.: $131-133{ }^{\circ} \mathrm{C}$.

${ }^{1}$ H-NMR $\left(400 \mathrm{MHz}, \mathrm{CDCl}_{3}\right): \delta=12.48(\mathrm{~s}, 1 \mathrm{H}), 8.64(\mathrm{dd}, J=7.2,1.3 \mathrm{~Hz}, 1 \mathrm{H}), 8.30(\mathrm{dd}$, $J=7.7,2.1 \mathrm{~Hz}, 1 \mathrm{H}), 7.54-7.43(\mathrm{~m}, 3 \mathrm{H}), 7.42-7.34$ (m, 3H), 6.97 (ddd, $J=7.7,7.2,1.3 \mathrm{~Hz}$, $1 \mathrm{H}), 3.68(\mathrm{~s}, 3 \mathrm{H}), 1.81(\mathrm{~s}, 3 \mathrm{H})$.

${ }^{13}$ C-NMR $\left(125 \mathrm{MHz}, \mathrm{CDCl}_{3}\right): \delta=165.9\left(\mathrm{C}_{\mathrm{q}}\right), 161.6\left(\mathrm{C}_{\mathrm{q}}\right), 145.7\left(\mathrm{C}_{\mathrm{q}}\right), 137.2\left(\mathrm{C}_{\mathrm{q}}\right), 133.2$ $\left(\mathrm{C}_{\mathrm{q}}\right), 129.6(\mathrm{CH}), 129.0(\mathrm{CH}), 128.7(\mathrm{CH}), 128.3(\mathrm{CH}), 117.8(\mathrm{CH}), 115.1(\mathrm{CH}), 110.6$ $(\mathrm{CH}), 58.2\left(\mathrm{CH}_{3}\right), 15.0\left(\mathrm{CH}_{3}\right)$.

IR (ATR): $\tilde{v}=3203,2927,1656,1501,1425,1235,1103,751,701 \mathrm{~cm}^{-1}$.

MS (ESI) $m / z$ (relative intensity): $591(65)[2 \mathrm{M}+\mathrm{Na}]^{+}, 307(100)[\mathrm{M}+\mathrm{Na}]^{+}, 285(33)$

$[\mathrm{M}+\mathrm{H}]^{+}, 175(49)$.

HR-MS (ESI) $m / z$ calcd. for $\mathrm{C}_{16} \mathrm{H}_{16} \mathrm{~N}_{2} \mathrm{O}_{3}[\mathrm{M}+\mathrm{H}]^{+}:$: 285.1234, found: 285.1236 . 
<smiles>CO/C(=C(\C(=O)Nc1cccc[n+]1[O-])c1ccccc1)c1ccccc1</smiles>

(Z)-2-(3-Methoxy-2,3-diphenylacrylamido)pyridine-1-oxide (38db): The general procedure $\mathbf{C}$ was followed using $\mathbf{3 7 d}(79.1 \mathrm{mg}, 0.25 \mathrm{mmol})$ and methanol (35b) (7.0 mL). Purification by column chromatography on silica gel $\left(\mathrm{CH}_{2} \mathrm{Cl}_{2} /\right.$ acetone $\left.3: 1\right)$ yielded $\mathbf{3 8 d b}$ (54.0 mg, $156 \mu \mathrm{mol}, 62 \%)$ as a white solid.

M. p.: $205-207^{\circ} \mathrm{C}$.

${ }^{1}$ H-NMR $\left(400 \mathrm{MHz}, \mathrm{CDCl}_{3}\right): \delta=12.26(\mathrm{~s}, 1 \mathrm{H}), 8.60(\mathrm{dd}, J=8.5,1.8 \mathrm{~Hz}, 1 \mathrm{H}), 8.24(\mathrm{dd}$, $J=6.0,1.8 \mathrm{~Hz}, 1 \mathrm{H}), 7.37$ (ddd, $J=8.5,6.0,1.8 \mathrm{~Hz}, 1 \mathrm{H}), 7.23-7.19$ (m, 3H), 7.16-7.09 (m, 5H), 7.08-7.05 (m, 2H), 6.98 (ddd, $J=8.5,6.0,1.8 \mathrm{~Hz}, 1 \mathrm{H}), 3.75(\mathrm{~m}, 3 \mathrm{H})$.

${ }^{13}$ C-NMR $\left(125 \mathrm{MHz}, \mathrm{CDCl}_{3}\right): \delta=165.1\left(\mathrm{C}_{\mathrm{q}}\right), 163.6\left(\mathrm{C}_{\mathrm{q}}\right), 135.7\left(\mathrm{C}_{\mathrm{q}}\right), 132.6\left(\mathrm{C}_{\mathrm{q}}\right), 131.7$ $(\mathrm{CH}), 131.7(\mathrm{CH}), 129.7(\mathrm{CH}), 129.7(\mathrm{CH}), 129.3(\mathrm{CH}), 128.2(\mathrm{CH}), 128.2(\mathrm{CH}), 127.7$ $(\mathrm{CH}), 127.7(\mathrm{CH}), 126.8(\mathrm{CH}), 118.2\left(\mathrm{C}_{\mathrm{q}}\right), 114.9\left(\mathrm{C}_{\mathrm{q}}\right), 58.7\left(\mathrm{CH}_{3}\right)$.

IR (ATR): $\tilde{v}=3156,2947,1650,1557,1498,1426,1244,1012,760,703 \mathrm{~cm}^{-1}$.

MS (ESI) $m / z$ (relative intensity): 715 (83) $[2 \mathrm{M}+\mathrm{Na}]^{+}, 692(15)[2 \mathrm{M}+\mathrm{H}]^{+}, 369(100)$ $[\mathrm{M}+\mathrm{Na}]^{+}, 347(71)[\mathrm{M}+\mathrm{H}]^{+}, 237$ (96), $194(11)$.

HR-MS (ESI) $m / z$ calcd. for $\mathrm{C}_{21} \mathrm{H}_{18} \mathrm{~N}_{2} \mathrm{O}_{3}[\mathrm{M}+\mathrm{H}]^{+}: 347.1390$, found: 347.1395 . 


\subsection{Cobaltaelectro-Catalyzed C-H Activation for Allene Annulations}

\subsubsection{Characterization Data}<smiles></smiles>

2-\{3-[(Diphenylphosphoryl)methyl]-1-oxoisoquinolin-2(1H)-yl\}pyridine

1-oxide

(176aa): The general procedure $\mathbf{D}$ was followed using benzamide 34a (107 $\mathrm{mg}, 0.50 \mathrm{mmol})$ and allene 75a (144 mg, $0.60 \mathrm{mmol})$. Purification by column chromatography on silica gel $\left(\mathrm{CH}_{2} \mathrm{Cl}_{2} /\right.$ acetone 1:1 to $\mathrm{CH}_{2} \mathrm{Cl}_{2} / \mathrm{MeOH}$ 9:1) yielded 176aa (206 mg, $455 \mu \mathrm{mol}, 91 \%$ ) as a white solid.

M. p.: $128-130{ }^{\circ} \mathrm{C}$.

${ }^{1}$ H-NMR (600 MHz, $\mathrm{CDCl}_{3}$ ): $\delta=8.36-8.26$ (m, 2H), 7.76-7.70 (m, 2H), 7.60-7.54 (m, 2H), 7.51-7.44 (m, 6H), 7.43-7.35 (m, 3H), 7.33-7.30 (m, 1H), 7.29-7.25 (m, 2H), 6.36 $(\mathrm{d}, J=3.1 \mathrm{~Hz}, 1 \mathrm{H}), 3.74-3.61(\mathrm{~m}, 1 \mathrm{H}), 3.16-3.01(\mathrm{~m}, 1 \mathrm{H})$.

${ }^{13}$ C-NMR (126 MHz, $\left.\mathrm{CDCl}_{3}\right): \delta=162.6\left(\mathrm{C}_{\mathrm{q}}\right), 143.8\left(\mathrm{C}_{\mathrm{q}}\right), 140.0(\mathrm{CH}), 136.7\left(\mathrm{C}_{\mathrm{q}}\right), 133.3$ $(\mathrm{CH}), 132.9\left(\mathrm{~d},{ }^{2} J_{\mathrm{C}-\mathrm{P}}=7.9 \mathrm{~Hz}, \mathrm{C}_{\mathrm{q}}\right), 132.3\left(\mathrm{~d},{ }^{4} J_{\mathrm{C}-\mathrm{P}}=2.7 \mathrm{~Hz}, \mathrm{CH}\right), 132.2\left(\mathrm{~d},{ }^{4} J_{\mathrm{C}-\mathrm{P}}=2.7 \mathrm{~Hz}\right.$, $\mathrm{CH}), 132.1\left(\mathrm{~d},{ }^{1} J_{\mathrm{C}-\mathrm{P}}=100.1 \mathrm{~Hz}, \mathrm{C}_{\mathrm{q}}\right), 131.4\left(\mathrm{~d},{ }^{2} J_{\mathrm{C}-\mathrm{P}}=9.1 \mathrm{~Hz}, \mathrm{CH}\right), 131.1\left(\mathrm{~d},{ }^{1} J_{\mathrm{C}-\mathrm{P}}=\right.$ $\left.101.2 \mathrm{~Hz}, \mathrm{C}_{\mathrm{q}}\right), 130.6\left(\mathrm{~d},{ }^{2} J_{\mathrm{C}-\mathrm{P}}=9.4 \mathrm{~Hz}, \mathrm{CH}\right), 130.3(\mathrm{CH}), 128.9\left(\mathrm{~d},{ }^{3} J_{\mathrm{C}-\mathrm{P}}=4.2 \mathrm{~Hz}, \mathrm{CH}\right)$, $128.8\left(\mathrm{~d},{ }^{3} J_{\mathrm{C}-\mathrm{P}}=4.2 \mathrm{~Hz}, \mathrm{CH}\right), 128.2(\mathrm{CH}), 127.0(\mathrm{CH}), 126.1(\mathrm{CH}), 125.9(\mathrm{CH}), 125.8$ $(\mathrm{CH}), 124.9\left(\mathrm{C}_{\mathrm{q}}\right), 108.9\left(\mathrm{~d},{ }^{3} J_{\mathrm{C}-\mathrm{P}}=7.0 \mathrm{~Hz}, \mathrm{CH}\right), 34.5\left(\mathrm{~d},{ }^{1} J_{\mathrm{C}-\mathrm{P}}=65.6 \mathrm{~Hz}, \mathrm{CH}_{2}\right)$.

${ }^{31} \mathbf{P}\left\{{ }^{1} \mathbf{H}\right\}$-NMR $\left(162 \mathrm{MHz}, \mathrm{CDCl}_{3}\right): \delta=27.6$.

IR (ATR): $\tilde{v}=3057,1675,1628,1432,1271,1189,719,498 \mathrm{~cm}^{-1}$.

MS (EI) $m / z$ (relative intensity): 452 (25) [M] $]^{+}, 453$ (45), 319 (10), 251 (28), 234 (100), 201 (80), 77 (40).

HR-MS (EI) $m / z$ calcd. for $\mathrm{C}_{27} \mathrm{H}_{21} \mathrm{~N}_{2} \mathrm{O}_{3} \mathrm{P}[\mathrm{M}]^{+}:$452.1290, found: 452.1297 . 
<smiles>O=c1c2ccccc2cc(CP(=O)(c2ccccc2)c2ccccc2)n1-c1cccc2cccnc12</smiles>

3-[(Diphenylphosphoryl)methyl]-2-(quinolin-8-yl)isoquinolin-1(2H)-one (76aa): A modified general procedure $\mathbf{D}$ was followed using benzamide $\mathbf{2 6 a}$ (124 $\mathrm{mg}, 0.50 \mathrm{mmol})$, allene 75a (144 mg, $0.60 \mathrm{mmol})$ and TFE $(5.0 \mathrm{~mL})$ as the solvent. Purification by column chromatography on silica gel (EtOAc/n-hexane 9:1) yielded 76aa (102 mg, $209 \mu \mathrm{mol}$, $42 \%$ ) as a white solid.

M. p.: $233-234{ }^{\circ} \mathrm{C}$.

${ }^{1}$ H-NMR $\left(400 \mathrm{MHz}, \mathrm{CDCl}_{3}\right): \delta=8.86-8.80(\mathrm{~m}, 1 \mathrm{H}), 8.36-8.30(\mathrm{~m}, 1 \mathrm{H}), 8.22(\mathrm{dd}, J=8.4$, $1.8 \mathrm{~Hz}, 1 \mathrm{H}), 7.92(\mathrm{dd}, J=8.3,1.4 \mathrm{~Hz}, 1 \mathrm{H}), 7.65-7.49$ (m, 5H), 7.49-7.38 (m, 7H), 7.38$7.31(\mathrm{~m}, 3 \mathrm{H}), 7.21(\mathrm{dd}, J=7.3,1.4 \mathrm{~Hz}, 1 \mathrm{H}), 6.83(\mathrm{~d}, J=3.0 \mathrm{~Hz}, 1 \mathrm{H}), 3.52-3.33(\mathrm{~m}, 1 \mathrm{H})$, $3.03(\mathrm{dd}, J=16.0,12.7 \mathrm{~Hz}, 1 \mathrm{H})$.

${ }^{13}$ C-NMR $\left(101 \mathrm{MHz}, \mathrm{CDCl}_{3}\right): \delta=163.7\left(\mathrm{C}_{\mathrm{q}}\right), 151.6(\mathrm{CH}), 144.5\left(\mathrm{C}_{\mathrm{q}}\right), 136.9\left(\mathrm{~d},{ }^{4} J_{\mathrm{C}-\mathrm{P}}=\right.$ $\left.2.4 \mathrm{~Hz}, \mathrm{C}_{\mathrm{q}}\right), 136.5(\mathrm{CH}), 135.7\left(\mathrm{C}_{\mathrm{q}}\right), 134.3\left(\mathrm{~d},{ }^{2} J_{\mathrm{C}-\mathrm{P}}=6.2 \mathrm{~Hz}, \mathrm{C}_{\mathrm{q}}\right), 132.7(\mathrm{CH}), 132.6(\mathrm{~d}$, $\left.{ }^{1} J_{\mathrm{C}-\mathrm{P}}=100.8 \mathrm{~Hz}, \mathrm{C}_{\mathrm{q}}\right), 132.3\left(\mathrm{~d},{ }^{4} J_{\mathrm{C}-\mathrm{P}}=2.8 \mathrm{~Hz}, \mathrm{CH}\right), 132.2\left(\mathrm{~d},{ }^{4} J_{\mathrm{C}-\mathrm{P}}=2.8 \mathrm{~Hz}, \mathrm{CH}\right), 131.7$ $(\mathrm{CH}), 131.4\left(\mathrm{~d},{ }^{2} J_{\mathrm{C}-\mathrm{P}}=9.1 \mathrm{~Hz}, \mathrm{CH}\right), 131.2\left(\mathrm{~d},{ }^{1} J_{\mathrm{C}-\mathrm{P}}=100.3 \mathrm{~Hz}, \mathrm{C}_{\mathrm{q}}\right), 130.1\left(\mathrm{~d},{ }^{2} J_{\mathrm{C}-\mathrm{P}}=\right.$ $9.4 \mathrm{~Hz}, \mathrm{CH}), 129.4(\mathrm{CH}), 129.3\left(\mathrm{C}_{\mathrm{q}}\right), 128.9\left(\mathrm{~d},{ }^{3} J_{\mathrm{C}-\mathrm{P}}=4.8 \mathrm{~Hz}, \mathrm{CH}\right), 128.7\left(\mathrm{~d},{ }^{2} J_{\mathrm{C}-\mathrm{P}}=4.8 \mathrm{~Hz}\right.$, $\mathrm{CH}), 128.3(\mathrm{CH}), 126.7(\mathrm{CH}), 126.6(\mathrm{CH}), 126.2(\mathrm{CH}), 125.4\left(\mathrm{C}_{\mathrm{q}}\right), 122.0(\mathrm{CH}), 108.1(\mathrm{~d}$, $\left.{ }^{3} J_{\mathrm{C}-\mathrm{P}}=6.5 \mathrm{~Hz}, \mathrm{CH}\right), 35.0\left(\mathrm{~d},{ }^{1} J_{\mathrm{C}-\mathrm{P}}=66.9 \mathrm{~Hz}, \mathrm{CH}_{2}\right)$.

${ }^{31} \mathbf{P}\left\{{ }^{1} \mathbf{H}\right\}-\mathbf{N M R}\left(121 \mathrm{MHz}, \mathrm{CDCl}_{3}\right): \delta=27.7$.

IR (ATR): $\tilde{v}=1654,1623,1497,1247,880,830,764,538 \mathrm{~cm}^{-1}$.

MS (ESI) $m / z$ (relative intensity): $509(30)[\mathrm{M}+\mathrm{Na}]^{+}, 487(100)[\mathrm{M}+\mathrm{H}]^{+}$.

HR-MS (ESI) $m / z$ calcd. for $\mathrm{NaC}_{31} \mathrm{H}_{23} \mathrm{~N}_{2} \mathrm{O}_{2} \mathrm{P}[\mathrm{M}+\mathrm{Na}]^{+}:$509.1389, found: 509.1389.

The analytical data are in accordance with those previously reported in the literature. ${ }^{[133]}$ 
<smiles>CN(c1ccccn1)n1c(CP(=O)(c2ccccc2)c2ccccc2)cc2ccccc2c1=O</smiles>

3-[(Diphenylphosphoryl)methyl]-2-[methyl(pyridin-2-yl)amino]isoquinolin-1(2H)one (177aa): The general procedure D was followed using benzamide 62a (114 mg, $0.50 \mathrm{mmol}$ ) and allene 75a (144 $\mathrm{mg}, 0.60 \mathrm{mmol})$. Purification by column chromatography on silica gel (neutralized with $\mathrm{Et}_{3} \mathrm{~N}, \mathrm{CH}_{2} \mathrm{Cl}_{2} /$ acetone 5:1) yielded 177aa (200 mg, $430 \mu \mathrm{mol}, 86 \%$ ) as a white solid.

M. p.: $109-111^{\circ} \mathrm{C}$.

${ }^{1}$ H-NMR (500 MHz, $\left.\mathrm{CDCl}_{3}\right): \delta=8.27-8.24(\mathrm{~m}, 1 \mathrm{H}), 8.21$ (ddd, $\left.J=5.0,1.9,0.9 \mathrm{~Hz}, 1 \mathrm{H}\right)$, 7.78-7.68 (m, 4H), 7.60 (ddd, $J=8.3,7.1,1.4 \mathrm{~Hz}, 1 \mathrm{H}), 7.54-7.50$ (m, 2H), 7.48-7.37 (m, 7H), 6.79-6.74 (m, 2H), $6.27(\mathrm{~d}, J=8.5 \mathrm{~Hz}, 1 \mathrm{H}), 3.94(\mathrm{dd}, J=15.9,13.7 \mathrm{~Hz}, 1 \mathrm{H}), 3.71$ (dd, $J=16.0,12.6 \mathrm{~Hz}, 1 \mathrm{H}), 3.35(\mathrm{~s}, 3 \mathrm{H})$.

${ }^{13}$ C-NMR (126 MHz, $\left.\mathrm{CDCl}_{3}\right): \delta=161.5\left(\mathrm{C}_{\mathrm{q}}\right), 159.1\left(\mathrm{C}_{\mathrm{q}}\right), 148.2(\mathrm{CH}), 138.1(\mathrm{CH}), 136.4$ $\left(\mathrm{d},{ }^{2} J_{\mathrm{C}-\mathrm{P}}=5.3 \mathrm{~Hz}, \mathrm{C}_{\mathrm{q}}\right), 136.3\left(\mathrm{~d},{ }^{4} J_{\mathrm{C}-\mathrm{P}}=2.3 \mathrm{~Hz}, \mathrm{C}_{\mathrm{q}}\right), 133.0(\mathrm{CH}), 132.8\left(\mathrm{~d},{ }^{1} J_{\mathrm{C}-\mathrm{P}}=101.2 \mathrm{~Hz}\right.$, $\left.\mathrm{C}_{\mathrm{q}}\right), 132.3\left(\mathrm{~d},{ }^{4} J_{\mathrm{C}-\mathrm{P}}=3.0 \mathrm{~Hz}, \mathrm{CH}\right), 132.2\left(\mathrm{~d},{ }^{4} J_{\mathrm{C}-\mathrm{P}}=3.0 \mathrm{~Hz}, \mathrm{CH}\right), 131.3\left(\mathrm{~d},{ }^{2} J_{\mathrm{C}-\mathrm{P}}=9.3 \mathrm{~Hz}\right.$, $\mathrm{CH}), 131.2\left(\mathrm{~d},{ }^{1} J_{\mathrm{C}-\mathrm{P}}=100.1 \mathrm{~Hz}, \mathrm{C}_{\mathrm{q}}\right), 130.9\left(\mathrm{~d},{ }^{2} J_{\mathrm{C}-\mathrm{P}}=9.4 \mathrm{~Hz}, \mathrm{CH}\right), 128.9\left(\mathrm{~d},{ }^{3} J_{\mathrm{C}-\mathrm{P}}=4.7 \mathrm{~Hz}\right.$, $\mathrm{CH}), 128.9\left(\mathrm{~d},{ }^{3} J_{\mathrm{C}-\mathrm{P}}=4.7 \mathrm{~Hz}, \mathrm{CH}\right), 128.0(\mathrm{CH}), 126.7(\mathrm{CH}), 126.3(\mathrm{CH}), 126.2\left(\mathrm{C}_{\mathrm{q}}\right), 115.9$ $(\mathrm{CH}), 108.4\left(\mathrm{~d},{ }^{3} J_{\mathrm{C}-\mathrm{P}}=6.3 \mathrm{~Hz}, \mathrm{CH}\right), 107.0(\mathrm{CH}), 38.3\left(\mathrm{CH}_{3}\right), 32.3\left(\mathrm{~d},{ }^{1} J_{\mathrm{C}-\mathrm{P}}=67.7 \mathrm{~Hz}, \mathrm{CH}_{2}\right)$. ${ }^{31} \mathbf{P}\left\{{ }^{1} \mathbf{H}\right\}$-NMR $\left(162 \mathrm{MHz}, \mathrm{CDCl}_{3}\right): \delta=27.6$.

IR (ATR): $\tilde{v}=3057,1661,1622,1475,1192,1147,928,720 \mathrm{~cm}^{-1}$.

MS (EI) $m / z$ (relative intensity): 465 (5) [M] $]^{+}, 359$ (82), 282 (7), 235 (55), 201 (100), 107 (12), 77 (18).

HR-MS (EI) $m / z$ calcd. for $\mathrm{C}_{28} \mathrm{H}_{24} \mathrm{~N}_{3} \mathrm{O}_{2} \mathrm{P}[\mathrm{M}]^{+}: 465.1606$, found: 465.1611 .<smiles>Cc1ccc2c(=O)n(-c3cccc[n+]3[O-])c(CP(=O)(c3ccccc3)c3ccccc3)cc2c1</smiles>

2-\{3-[(Diphenylphosphoryl)methyl]-6-methyl-1-oxoisoquinolin-2(1H)-yl\}pyridine 1oxide (176ba): The general procedure D was followed using benzamide 34b (114 mg, 
$0.50 \mathrm{mmol})$ and allene $75 \mathrm{a}(144 \mathrm{mg}, 0.60 \mathrm{mmol})$. Purification by column chromatography on silica gel $\left(\mathrm{CH}_{2} \mathrm{Cl}_{2}\right.$ /acetone 1:1 to $\left.\mathrm{CH}_{2} \mathrm{Cl}_{2} / \mathrm{MeOH} 9: 1\right)$ yielded 176ba (224 mg, $481 \mu \mathrm{mol}$, $96 \%)$ as a white solid.

M. p.: $213-215^{\circ} \mathrm{C}$.

${ }^{1}$ H-NMR (400 MHz, $\left.\mathrm{CDCl}_{3}\right): \delta=8.35-8.25(\mathrm{~m}, 1 \mathrm{H}), 8.17$ (d, $\left.J=8.2 \mathrm{~Hz}, 1 \mathrm{H}\right), 7.74$ (ddd, $J=11.6,8.3,1.4 \mathrm{~Hz}, 2 \mathrm{H}), 7.61-7.55(\mathrm{~m}, 1 \mathrm{H}), 7.54-7.45(\mathrm{~m}, 5 \mathrm{H}), 7.44-7.35$ (m, 3H), 7.35$7.26(\mathrm{~m}, 2 \mathrm{H}), 7.26-7.20(\mathrm{~m}, 1 \mathrm{H}), 7.07(\mathrm{~s}, 1 \mathrm{H}), 6.33$ (d, $J=3.2 \mathrm{~Hz}, 1 \mathrm{H}), 3.67$ (dd, $J=15.9$, $11.9 \mathrm{~Hz}, 1 \mathrm{H}), 3.17-3.01(\mathrm{~m}, 1 \mathrm{H}), 2.42(\mathrm{~s}, 3 \mathrm{H})$.

${ }^{13}$ C-NMR (101 MHz, $\left.\mathrm{CDCl}_{3}\right): \delta=162.7\left(\mathrm{C}_{\mathrm{q}}\right), 144.1\left(\mathrm{C}_{\mathrm{q}}\right), 141.7\left(\mathrm{C}_{\mathrm{q}}\right), 140.2(\mathrm{CH}), 136.7$ $\left(\mathrm{d},{ }^{4} J_{\mathrm{C}-\mathrm{P}}=2.7 \mathrm{~Hz}, \mathrm{C}_{\mathrm{q}}\right), 133.0\left(\mathrm{~d},{ }^{2} J_{\mathrm{C}-\mathrm{P}}=7.8 \mathrm{~Hz}, \mathrm{C}_{\mathrm{q}}\right), 132.4\left(\mathrm{~d},{ }^{4} J_{\mathrm{C}-\mathrm{P}}=2.7 \mathrm{~Hz}, \mathrm{CH}\right), 132.3$ $\left(\mathrm{d},{ }^{4} J_{\mathrm{C}-\mathrm{P}}=2.7 \mathrm{~Hz}, \mathrm{CH}\right), 132.1\left(\mathrm{~d},{ }^{1} J_{\mathrm{C}-\mathrm{P}}=102.2 \mathrm{~Hz}, \mathrm{C}_{\mathrm{q}}\right), 131.5\left(\mathrm{~d},{ }^{2} J_{\mathrm{C}-\mathrm{P}}=9.2 \mathrm{~Hz}, \mathrm{CH}\right)$, $131.0\left(\mathrm{~d},{ }^{1} J_{\mathrm{C}-\mathrm{P}}=101.3 \mathrm{~Hz}, \mathrm{C}_{\mathrm{q}}\right), 130.7\left(\mathrm{~d},{ }^{2} J_{\mathrm{C}-\mathrm{P}}=9.5 \mathrm{~Hz}, \mathrm{CH}\right), 130.4(\mathrm{CH}), 129.0\left(\mathrm{~d},{ }^{3} J_{\mathrm{C}-\mathrm{P}}\right.$ $=5.3 \mathrm{~Hz}, \mathrm{CH}), 128.9\left(\mathrm{~d},{ }^{3} J_{\mathrm{C}-\mathrm{P}}=5.2 \mathrm{~Hz}, \mathrm{CH}\right), 128.7(\mathrm{CH}), 128.3(\mathrm{CH}), 126.1(\mathrm{CH}), 126.0$ $(\mathrm{CH}), 125.9(\mathrm{CH}), 122.8\left(\mathrm{C}_{\mathrm{q}}\right), 108.8\left(\mathrm{~d},{ }^{3} J_{\mathrm{C}-\mathrm{P}}=7.0 \mathrm{~Hz}, \mathrm{CH}\right), 34.4\left(\mathrm{~d},{ }^{1} J_{\mathrm{C}-\mathrm{P}}=66.3 \mathrm{~Hz}, \mathrm{CH}_{2}\right)$, $22.0\left(\mathrm{CH}_{3}\right)$.

${ }^{31} \mathbf{P}\left\{{ }^{1} \mathbf{H}\right\}$-NMR $\left(162 \mathrm{MHz}, \mathrm{CDCl}_{3}\right): \delta=27.6$.

IR (ATR): $\tilde{v}=3051,1656,1612,1488,1270,885,691,521 \mathrm{~cm}^{-1}$.

MS (EI) $m / z$ (relative intensity): 466 (12) [M] $]^{+}, 449$ (28), 319 (10), 265 (14), 248 (100), 201 (40), 77 (22).

HR-MS (EI) $m / z$ calcd. for $\mathrm{C}_{28} \mathrm{H}_{23} \mathrm{~N}_{2} \mathrm{O}_{3} \mathrm{P}[\mathrm{M}]^{+}:$466.1446, found: 466.1449 .<smiles>COc1ccc2c(=O)n(-c3cccc[n+]3[O-])c(CP(=O)(c3ccccc3)c3ccccc3)cc2c1</smiles>

\section{2-\{3-[(Diphenylphosphoryl)methyl]-6-methoxy-1-oxoisoquinolin-2(1H)-yl\}pyridine}

1-oxide (176da): The general procedure $\mathbf{D}$ was followed using benzamide 34d (122 mg, $0.50 \mathrm{mmol})$ and allene 75a (144 $\mathrm{mg}, 0.60 \mathrm{mmol})$. Purification by column chromatography on silica gel $\left(\mathrm{CH}_{2} \mathrm{Cl}_{2} /\right.$ acetone 1:1 to $\mathrm{CH}_{2} \mathrm{Cl}_{2} / \mathrm{MeOH}$ 9:1) yielded 176da (218 mg, $452 \mu \mathrm{mol}$, $90 \%$ ) as a white solid.

M. p.: $174-176^{\circ} \mathrm{C}$.

${ }^{1}$ H-NMR (400 MHz, $\left.\mathrm{CDCl}_{3}\right): \delta=8.30-8.16(\mathrm{~m}, 2 \mathrm{H}), 7.79-7.69(\mathrm{~m}, 2 \mathrm{H}), 7.59-7.52(\mathrm{~m}$, 1H), 7.52-7.43 (m, 5H), 7.43-7.34 (m, 3H), 7.35-7.29 (m, 1H), 7.26-7.16 (m, 2H), 6.97 
$(\mathrm{dd}, J=8.9,2.5 \mathrm{~Hz}, 1 \mathrm{H}), 6.67(\mathrm{~d}, J=2.5 \mathrm{~Hz}, 1 \mathrm{H}) 6.42-6.35(\mathrm{~m}, 1 \mathrm{H}), 3.85(\mathrm{~s}, 3 \mathrm{H}), 3.77-$ $3.58(\mathrm{~m}, 1 \mathrm{H}), 3.17-3.00(\mathrm{~m}, 1 \mathrm{H})$.

${ }^{13}$ C-NMR $\left(126 \mathrm{MHz}, \mathrm{CDCl}_{3}\right): \delta=163.5\left(\mathrm{C}_{\mathrm{q}}\right), 162.2\left(\mathrm{C}_{\mathrm{q}}\right), 140.4(\mathrm{CH}), 138.9\left(\mathrm{C}_{\mathrm{q}}\right), 136.7$ $\left(\mathrm{d},{ }^{4} J_{\mathrm{C}-\mathrm{P}}=2.7 \mathrm{~Hz}, \mathrm{C}_{\mathrm{q}}\right), 133.7\left(\mathrm{~d},{ }^{2} J_{\mathrm{C}-\mathrm{P}}=5.9 \mathrm{~Hz}, \mathrm{C}_{\mathrm{q}}\right), 132.3\left(\mathrm{~d},{ }^{4} J_{\mathrm{C}-\mathrm{P}}=2.0 \mathrm{~Hz}, \mathrm{CH}\right), 132.2$ $\left(\mathrm{d},{ }^{4} J_{\mathrm{C}-\mathrm{P}}=2.0 \mathrm{~Hz}, \mathrm{CH}\right), 132.1\left(\mathrm{~d},{ }^{1} J_{\mathrm{C}-\mathrm{P}}=103.9 \mathrm{~Hz}, \mathrm{C}_{\mathrm{q}}\right), 131.4\left(\mathrm{~d},{ }^{2} J_{\mathrm{C}-\mathrm{P}}=8.8 \mathrm{~Hz}, \mathrm{CH}\right)$, $131.0\left(\mathrm{~d},{ }^{1} J_{\mathrm{C}-\mathrm{P}}=100.9 \mathrm{~Hz}, \mathrm{C}_{\mathrm{q}}\right), 130.7\left(\mathrm{~d},{ }^{2} J_{\mathrm{C}-\mathrm{P}}=8.8 \mathrm{~Hz}, \mathrm{CH}\right), 130.3(\mathrm{CH}), 130.1(\mathrm{CH})$, $128.9\left(\mathrm{~d},{ }^{3} J_{\mathrm{C}-\mathrm{P}}=5.8 \mathrm{~Hz}, \mathrm{CH}\right), 128.8\left(\mathrm{~d},{ }^{3} J_{\mathrm{C}-\mathrm{P}}=5.8 \mathrm{~Hz}, \mathrm{CH}\right), 126.1(\mathrm{CH}), 125.7(\mathrm{CH}), 118.6$ $\left(\mathrm{C}_{\mathrm{q}}\right), 116.2(\mathrm{CH}), 108.8\left(\mathrm{~d},{ }^{3} J_{\mathrm{C}-\mathrm{P}}=6.3 \mathrm{~Hz}, \mathrm{CH}\right), 107.4(\mathrm{CH}), 55.7\left(\mathrm{CH}_{3}\right), 34.6\left(\mathrm{~d},{ }^{1} J_{\mathrm{C}-\mathrm{P}}=\right.$ $\left.67.7 \mathrm{~Hz}, \mathrm{CH}_{2}\right)$.

${ }^{31} \mathbf{P}\left\{{ }^{1} \mathbf{H}\right\}-N M R\left(162 \mathrm{MHz}, \mathrm{CDCl}_{3}\right): \delta=27.6$.

IR (ATR): $\tilde{v}=3057,1660,1599,1489,1250,1192,721,696,528 \mathrm{~cm}^{-1}$.

MS (ESI) $m / z$ (relative intensity): $987(80)[2 \mathrm{M}+\mathrm{Na}]^{+}, 965(20)[2 \mathrm{M}+\mathrm{H}]^{+}, 505(80)$ $[\mathrm{M}+\mathrm{Na}]^{+}, 483(100)[\mathrm{M}+\mathrm{H}]^{+}$.

HR-MS (ESI) $m / z$ calcd. for $\mathrm{C}_{28} \mathrm{H}_{24} \mathrm{~N}_{2} \mathrm{O}_{4} \mathrm{P}[\mathrm{M}+\mathrm{H}]^{+}$: 483.1468 , found: 483.1465 .<smiles>COc1ccc2c(=O)n(-c3cccc[n+]3[O-])c(CP(=O)(c3ccccc3)c3ccccc3)cc2c1</smiles>

\section{2-\{3-[(Diphenylphosphoryl)methyl]-6-(methylthio)-1-oxoisoquinolin-2(1H)-}

yl\}pyridine 1-oxide (176ea): The general procedure $D$ was followed using benzamide 34e $(130 \mathrm{mg}, 0.50 \mathrm{mmol})$ and allene $75 \mathbf{a}(144 \mathrm{mg}, 0.60 \mathrm{mmol})$. Purification by column chromatography on silica gel $\left(\mathrm{CH}_{2} \mathrm{Cl}_{2}\right.$ /acetone 1:1 to $\left.\mathrm{CH}_{2} \mathrm{Cl}_{2} / \mathrm{MeOH} 9: 1\right)$ yielded 176ea (226 mg, $454 \mu \mathrm{mol}, 91 \%$ ) as a white solid.

M. p.: $232-234{ }^{\circ} \mathrm{C}$.

${ }^{1}$ H-NMR $\left(400 \mathrm{MHz} \mathrm{CDCl}_{3}\right): \delta=8.33-8.21(\mathrm{~m}, 1 \mathrm{H}), 8.13(\mathrm{~d}, J=8.5 \mathrm{~Hz}, 1 \mathrm{H}), 7.78-7.67$ (m, 2H), 7.60-7.53 (m, 1H), 7.52-7.43 (m, 5H), 7.41-7.35 (m, 3H), 7.34-7.28 (m, 1H), 7.26-7.21 (m, 2H), $7.02(\mathrm{~d}, J=1.8 \mathrm{~Hz}, 1 \mathrm{H}), 6.37(\mathrm{~d}, J=2.9 \mathrm{~Hz}, 1 \mathrm{H}), 3.67(\mathrm{dd}, J=15.9$, $12.0 \mathrm{~Hz}, 1 \mathrm{H}), 3.13-3.01(\mathrm{~m}, 1 \mathrm{H}), 2.50(\mathrm{~s}, 3 \mathrm{H})$.

${ }^{13}$ C-NMR $\left(126 \mathrm{MHz}, \mathrm{CDCl}_{3}\right): \delta=162.3\left(\mathrm{C}_{\mathrm{q}}\right), 146.2\left(\mathrm{C}_{\mathrm{q}}\right), 140.2(\mathrm{CH}), 137.1\left(\mathrm{C}_{\mathrm{q}}\right), 133.9$ $\left(\mathrm{d},{ }^{2} J_{\mathrm{C}-\mathrm{P}}=7.2 \mathrm{~Hz}, \mathrm{C}_{\mathrm{q}}\right), 133.3\left(\mathrm{C}_{\mathrm{q}}\right), 132.7\left(\mathrm{~d},{ }^{1} J_{\mathrm{C}-\mathrm{P}}=99.8 \mathrm{~Hz}, \mathrm{C}_{\mathrm{q}}\right), 132.4\left(\mathrm{~d},{ }^{4} J_{\mathrm{C}-\mathrm{P}}=2.5 \mathrm{~Hz}\right.$, $\mathrm{CH}), 132.2\left(\mathrm{~d},{ }^{4} J_{\mathrm{C}-\mathrm{P}}=2.4 \mathrm{~Hz}, \mathrm{CH}\right), 131.4\left(\mathrm{~d},{ }^{2} J_{\mathrm{C}-\mathrm{P}}=9.1 \mathrm{~Hz}, \mathrm{CH}\right), 131.2\left(\mathrm{~d},{ }^{1} J_{\mathrm{C}-\mathrm{P}}=\right.$ 104.5 Hz, $\left.\mathrm{C}_{\mathrm{q}}\right), 130.6\left(\mathrm{~d},{ }^{2} J_{\mathrm{C}-\mathrm{P}}=9.3 \mathrm{~Hz}, \mathrm{CH}\right), 130.1(\mathrm{CH}), 128.9\left(\mathrm{~d},{ }^{3} J_{\mathrm{C}-\mathrm{P}}=5.4 \mathrm{~Hz}, \mathrm{CH}\right)$, 
$128.8\left(\mathrm{~d},{ }^{3} J_{\mathrm{C}-\mathrm{P}}=5.1 \mathrm{~Hz}, \mathrm{CH}\right), 128.3(\mathrm{CH}), 126.0(\mathrm{CH}), 124.6(\mathrm{CH}), 121.6\left(\mathrm{C}_{\mathrm{q}}\right), 120.9(\mathrm{CH})$, $108.3\left(\mathrm{~d},{ }^{3} J_{\mathrm{C}-\mathrm{P}}=6.7 \mathrm{~Hz}, \mathrm{CH}\right), 107.4(\mathrm{CH}), 34.5\left(\mathrm{~d},{ }^{1} J_{\mathrm{C}-\mathrm{P}}=65.5 \mathrm{~Hz}, \mathrm{CH}_{2}\right), 15.0\left(\mathrm{CH}_{3}\right)$.

${ }^{31} \mathbf{P}\left\{{ }^{1} \mathbf{H}\right\}-\mathbf{N M R}\left(162 \mathrm{MHz}, \mathrm{CDCl}_{3}\right): \delta=27.7$.

IR (ATR): $\tilde{v}=1660,1628,1434,1259,1196,723,683,521 \mathrm{~cm}^{-1}$.

MS (ESI) $m / z$ (relative intensity): 1019 (60) $[2 \mathrm{M}+\mathrm{Na}]^{+}, 537(5)[\mathrm{M}+\mathrm{K}]^{+}, 521$ (100) $[\mathrm{M}+\mathrm{Na}]^{+}, 499(90)[\mathrm{M}+\mathrm{H}]^{+}$.

HR-MS (ESI) $m / z$ calcd. for $\mathrm{C}_{28} \mathrm{H}_{24} \mathrm{~N}_{2} \mathrm{O}_{3} \mathrm{PS}[\mathrm{M}+\mathrm{H}]^{+}:$499.1240, found: 499.1238 .<smiles></smiles>

2-\{3-[(Diphenylphosphoryl)methyl]-1-oxo-6-phenylisoquinolin-2(1H)-yl\}pyridine 1oxide (176fa): The general procedure D was followed using benzamide 34f (145 mg, $0.50 \mathrm{mmol})$ and allene 75a (144 $\mathrm{mg}, 0.60 \mathrm{mmol})$. Purification by column chromatography on silica gel $\left(\mathrm{CH}_{2} \mathrm{Cl}_{2} /\right.$ acetone 1:1 to $\mathrm{CH}_{2} \mathrm{Cl}_{2} / \mathrm{MeOH}$ 9:1) yielded $\mathbf{1 7 6 f a}$ (185 mg, $350 \mu \mathrm{mol}$, $70 \%$ ) as a white solid.

M. p.: $146-148^{\circ} \mathrm{C}$.

${ }^{1}$ H-NMR (400 MHz, $\left.\mathrm{CDCl}_{3}\right): \delta=8.37-8.32(\mathrm{~m}, 2 \mathrm{H}), 7.80-7.72(\mathrm{~m}, 2 \mathrm{H}), 7.68-7.55(\mathrm{~m}$, 4H), 7.53-7.44 (m, 9H), 7.43-7.36 (m, 3H), 7.36-7.28 (m, 2H), $6.46(\mathrm{~d}, J=3.3 \mathrm{~Hz}, 1 \mathrm{H})$, $3.76-3.63(\mathrm{~m}, 1 \mathrm{H}), 3.12(\mathrm{dd}, J=16.0,13.3 \mathrm{~Hz}, 1 \mathrm{H})$.

${ }^{13}$ C-NMR (101 MHz, $\left.\mathrm{CDCl}_{3}\right): \delta=162.6\left(\mathrm{C}_{\mathrm{q}}\right), 146.2\left(\mathrm{C}_{\mathrm{q}}\right), 143.7\left(\mathrm{C}_{\mathrm{q}}\right), 140.1(\mathrm{CH}), 137.3$ $\left(\mathrm{d},{ }^{4} J_{\mathrm{C}-\mathrm{P}}=2.8 \mathrm{~Hz}, \mathrm{C}_{\mathrm{q}}\right), 133.5\left(\mathrm{~d},{ }^{2} J_{\mathrm{C}-\mathrm{P}}=7.9 \mathrm{~Hz}, \mathrm{C}_{\mathrm{q}}\right), 132.9\left(\mathrm{~d},{ }^{1} J_{\mathrm{C}-\mathrm{P}}=100.2 \mathrm{~Hz}, \mathrm{C}_{\mathrm{q}}\right), 132.5$ $\left(\mathrm{d},{ }^{4} J_{\mathrm{C}-\mathrm{P}}=2.8 \mathrm{~Hz}, \mathrm{CH}\right), 132.3\left(\mathrm{~d},{ }^{4} J_{\mathrm{C}-\mathrm{P}}=2.8 \mathrm{~Hz}, \mathrm{CH}\right), 131.7\left(\mathrm{C}_{\mathrm{q}}\right), 131.8\left(\mathrm{~d},{ }^{1} J_{\mathrm{C}-\mathrm{P}}=\right.$ 103.4 Hz, $\mathrm{C}_{\mathrm{q}}$ ), 131.5 (d, $\left.{ }^{3} J_{\mathrm{C}-\mathrm{P}}=9.0 \mathrm{~Hz}, \mathrm{CH}\right), 130.7$ (d, $\left.{ }^{2} J_{\mathrm{C}-\mathrm{P}}=9.2 \mathrm{~Hz}, \mathrm{CH}\right), 130.4(\mathrm{CH})$, $129.1(\mathrm{CH}), 129.1(\mathrm{CH}), 129.0\left(\mathrm{~d},{ }^{3} J_{\mathrm{C}-\mathrm{P}}=5.3 \mathrm{~Hz}, \mathrm{CH}\right), 128.9\left(\mathrm{~d},{ }^{3} J_{\mathrm{C}-\mathrm{P}}=5.3 \mathrm{~Hz}, \mathrm{CH}\right), 128.4$ $(\mathrm{CH}), 127.6(\mathrm{CH}), 126.3(\mathrm{CH}), 126.3(\mathrm{CH}), 125.8(\mathrm{CH}), 124.4(\mathrm{CH}), 123.9\left(\mathrm{C}_{\mathrm{q}}\right), 109.1(\mathrm{dd}$, $\left.{ }^{3} J_{\mathrm{C}-\mathrm{P}}=7.1 \mathrm{~Hz}, \mathrm{CH}\right), 34.5\left(\mathrm{~d},{ }^{1} J_{\mathrm{C}-\mathrm{P}}=65.9 \mathrm{~Hz}, \mathrm{CH}_{2}\right)$.

${ }^{31} \mathbf{P}\left\{{ }^{1} \mathbf{H}\right\}$-NMR $\left(126 \mathrm{MHz}, \mathrm{CDCl}_{3}\right): \delta=27.7$.

IR (ATR): $\tilde{v}=3054,1664,1598,1489,1273,1189,719,695,518 \mathrm{~cm}^{-1}$.

MS (EI) $m / z$ (relative intensity): 528 (5) [M] $]^{+}, 512$ (10), 327 (5), 301 (100), 281 (12), 201 (25), 77 (10).

HR-MS (EI) $m / z$ calcd. for $\mathrm{C}_{33} \mathrm{H}_{25} \mathrm{~N}_{2} \mathrm{O}_{3} \mathrm{P}[\mathrm{M}]^{+}:$528.1603, found: 528.1611. 
<smiles></smiles>

2-\{3-[(Diphenylphosphoryl)methyl]-6-iodo-1-oxoisoquinolin-2(1H)-yl\}pyridine 1oxide (176ga): The general procedure D was followed using benzamide 34g (170 mg, $0.50 \mathrm{mmol}$ ) and allene 75a (144 $\mathrm{mg}, 0.60 \mathrm{mmol})$. Purification by column chromatography on silica gel $\left(\mathrm{CH}_{2} \mathrm{Cl}_{2} /\right.$ acetone $1: 1$ to $\mathrm{CH}_{2} \mathrm{Cl}_{2} / \mathrm{MeOH}$ 9:1) yielded 176ga (160 mg, $278 \mu \mathrm{mol}$, $56 \%)$ as a white solid.

M. p.: $135-137^{\circ} \mathrm{C}$.

${ }^{1}$ H-NMR (400 MHz, $\left.\mathrm{CDCl}_{3}\right): \delta=8.38-8.26(\mathrm{~m}, 1 \mathrm{H}), 7.97$ (d, $\left.J=8.4 \mathrm{~Hz}, 1 \mathrm{H}\right), 7.78-7.69$ (m, 3H), 7.70-7.65 (m, 1H), 7.64-7.57 (m, 1H), 7.55-7.46 (m, 5H), 7.45-7.43 (m, 1H), 7.42-7.36 (m, 2H), 7.35-7.27 (m, 2H), $6.22(\mathrm{~d}, J=3.2 \mathrm{~Hz}, 1 \mathrm{H}), 3.65(\mathrm{dd}, J=15.8,11.4 \mathrm{~Hz}$, $1 \mathrm{H}), 3.13-3.03(\mathrm{~m}, 1 \mathrm{H})$.

${ }^{13}$ C-NMR (126 MHz, $\left.\mathrm{CDCl}_{3}\right): \delta=162.3\left(\mathrm{C}_{\mathrm{q}}\right), 143.4\left(\mathrm{C}_{\mathrm{q}}\right), 140.0(\mathrm{CH}), 138.1\left(\mathrm{C}_{\mathrm{q}}\right), 136.0$ $(\mathrm{CH}), 134.8(\mathrm{CH}), 134.6\left(\mathrm{~d},{ }^{2} J_{\mathrm{C}-\mathrm{P}}=8.1 \mathrm{~Hz}, \mathrm{C}_{\mathrm{q}}\right), 132.8\left(\mathrm{~d},{ }^{1} J_{\mathrm{C}-\mathrm{P}}=99.5 \mathrm{~Hz}, \mathrm{C}_{\mathrm{q}}\right), 132.5(\mathrm{~d}$, $\left.{ }^{4} J_{\mathrm{C}-\mathrm{P}}=2.7 \mathrm{~Hz}, \mathrm{CH}\right), 132.3\left(\mathrm{~d},{ }^{4} J_{\mathrm{C}-\mathrm{P}}=2.7 \mathrm{~Hz}, \mathrm{CH}\right), 131.4\left(\mathrm{~d},{ }^{2} J_{\mathrm{C}-\mathrm{P}}=9.2 \mathrm{~Hz}, \mathrm{CH}\right), 131.2(\mathrm{~d}$, $\left.{ }^{1} J_{\mathrm{C}-\mathrm{P}}=101.4 \mathrm{~Hz}, \mathrm{C}_{\mathrm{q}}\right), 130.6\left(\mathrm{~d},{ }^{2} J_{\mathrm{C}-\mathrm{P}}=9.4 \mathrm{~Hz}, \mathrm{CH}\right), 130.4(\mathrm{CH}), 129.7(\mathrm{CH}), 129.0\left(\mathrm{~d},{ }^{3} J_{\mathrm{C}-}\right.$ $\mathrm{P}=5.6 \mathrm{~Hz}, \mathrm{CH}), 128.9\left(\mathrm{~d},{ }^{3} J_{\mathrm{C}-\mathrm{P}}=5.6 \mathrm{~Hz}, \mathrm{CH}\right), 126.3(\mathrm{CH}), 125.8(\mathrm{CH}), 124.2\left(\mathrm{C}_{\mathrm{q}}\right), 107.3$ $\left(\mathrm{d},{ }^{3} J_{\mathrm{C}-\mathrm{P}}=6.9 \mathrm{~Hz}, \mathrm{CH}\right), 101.4\left(\mathrm{C}_{\mathrm{q}}\right), 34.7\left(\mathrm{~d},{ }^{1} J_{\mathrm{C}-\mathrm{P}}=65.3 \mathrm{~Hz}, \mathrm{CH}_{2}\right)$.

${ }^{31} \mathbf{P}\left\{{ }^{1} \mathbf{H}\right\}$-NMR $\left(162 \mathrm{MHz}, \mathrm{CDCl}_{3}\right): \delta=27.7$.

IR (ATR): $\tilde{v}=3057,1670,1490,1261,889,843,764,526 \mathrm{~cm}^{-1}$.

MS (EI) $m / z$ (relative intensity): 578 (12) [M] $]^{+}, 561$ (28), 377 (10), 360 (100), 234 (20), 201 (50), 78 (18).

HR-MS (EI) $m / z$ calcd. for $\mathrm{C}_{27} \mathrm{H}_{20} \mathrm{IN}_{2} \mathrm{O}_{3} \mathrm{P}[\mathrm{M}]^{+}:$578.0256, found: 578.0264.<smiles>CC(C)(C)c1ccc2c(=O)n(-c3cccc[n+]3[O-])c(CP(=O)(c3ccccc3)c3ccccc3)cc2c1</smiles>

2-\{6-(tert-Butyl)-3-[(diphenylphosphoryl)methyl]-1-oxoisoquinolin-2(1H)-yl\}pyridine 1-oxide (176ja): The general procedure $\mathbf{D}$ was followed using benzamide 34j (135 mg, $0.50 \mathrm{mmol}$ ) and allene 75a (144 $\mathrm{mg}, 0.60 \mathrm{mmol})$. Purification by column chromatography 
on silica gel $\left(\mathrm{CH}_{2} \mathrm{Cl}_{2} /\right.$ acetone 1:1 to $\mathrm{CH}_{2} \mathrm{Cl}_{2} / \mathrm{MeOH}$ 9:1) yielded 176ja (213 mg, $419 \mu \mathrm{mol}$, $84 \%$ ) as a white solid.

M. p.: $135-136^{\circ} \mathrm{C}$.

${ }^{1}$ H-NMR (400 MHz, $\left.\mathrm{CDCl}_{3}\right): \delta=8.35-8.17$ (m, 2H), 7.75 (ddd, $\left.J=11.6,8.1,1.4 \mathrm{~Hz}, 2 \mathrm{H}\right)$, 7.60-7.54 (m, 1H), 7.54-7.43 (m, 6H), 7.42-7.32 (m, 3H), 7.32-7.27 (m, 2H), 7.25-7.17 (m, 1H), $6.48(\mathrm{~d}, J=3.0 \mathrm{~Hz}, 1 \mathrm{H}), 3.78-3.59(\mathrm{~m}, 1 \mathrm{H}), 3.19-3.02(\mathrm{~m}, 1 \mathrm{H}), 1.34(\mathrm{~s}, 9 \mathrm{H})$.

${ }^{13}$ C-NMR (126 MHz, $\left.\mathrm{CDCl}_{3}\right): \delta=162.5\left(\mathrm{C}_{\mathrm{q}}\right), 157.0\left(\mathrm{C}_{\mathrm{q}}\right), 140.2(\mathrm{CH}), 136.7\left(\mathrm{C}_{\mathrm{q}}\right), 132.7$ $\left(\mathrm{d},{ }^{2} J_{\mathrm{C}-\mathrm{P}}=7.6 \mathrm{~Hz}, \mathrm{C}_{\mathrm{q}}\right), 132.3\left(\mathrm{~d},{ }^{4} J_{\mathrm{C}-\mathrm{P}}=1.9 \mathrm{~Hz}, \mathrm{CH}\right), 132.2\left(\mathrm{~d},{ }^{4} J_{\mathrm{C}-\mathrm{P}}=1.9 \mathrm{~Hz}, \mathrm{CH}\right), 132.0$ $\left(\mathrm{d},{ }^{1} J_{\mathrm{C}-\mathrm{P}}=101.9 \mathrm{~Hz}, \mathrm{C}_{\mathrm{q}}\right), 131.4\left(\mathrm{~d},{ }^{2} J_{\mathrm{C}-\mathrm{P}}=9.0 \mathrm{~Hz}, \mathrm{CH}\right), 131.2\left(\mathrm{~d},{ }^{1} J_{\mathrm{C}-\mathrm{P}}=101.2 \mathrm{~Hz}, \mathrm{C}_{\mathrm{q}}\right)$, $130.7\left(\mathrm{~d},{ }^{2} J_{\mathrm{C}-\mathrm{P}}=9.2 \mathrm{~Hz}, \mathrm{CH}\right), 130.1(\mathrm{CH}), 128.9\left(\mathrm{~d},{ }^{3} J_{\mathrm{C}-\mathrm{P}}=4.1 \mathrm{~Hz}, \mathrm{CH}\right), 128.8\left(\mathrm{~d},{ }^{3} J_{\mathrm{C}-\mathrm{P}}=\right.$ 4.1 Hz, CH), $128.0(\mathrm{CH}), 125.9(\mathrm{CH}), 125.8(\mathrm{CH}), 125.1(\mathrm{CH}), 122.7\left(\mathrm{C}_{\mathrm{q}}\right), 122.4(\mathrm{CH})$, $109.4\left(\mathrm{~d},{ }^{3} J_{\mathrm{C}-\mathrm{P}}=6.7 \mathrm{~Hz}, \mathrm{CH}\right), 35.4\left(\mathrm{C}_{\mathrm{q}}\right), 34.5\left(\mathrm{~d},{ }^{1} J_{\mathrm{C}-\mathrm{P}}=66.2 \mathrm{~Hz}, \mathrm{CH}_{2}\right), 31.2\left(\mathrm{CH}_{3}\right)$.

${ }^{31} \mathbf{P}\left\{{ }^{1} \mathbf{H}\right\}$-NMR $\left(162 \mathrm{MHz}, \mathrm{CDCl}_{3}\right): \delta=27.5$.

IR (ATR): $\tilde{v}=2967,1658,1596,1272,1193,725,689,541 \mathrm{~cm}^{-1}$.

MS (ESI) $m / z$ (relative intensity): $1039(40)[2 \mathrm{M}+\mathrm{Na}]^{+}, 1017(20)[2 \mathrm{M}+\mathrm{H}]^{+}, 531$ (40) $[\mathrm{M}+\mathrm{Na}]^{+}, 509(100)[\mathrm{M}+\mathrm{H}]^{+}$.

HR-MS (ESI) $m / z$ calcd. for $\mathrm{C}_{31} \mathrm{H}_{30} \mathrm{~N}_{2} \mathrm{O}_{3} \mathrm{P}[\mathrm{M}+\mathrm{H}]^{+}:$509.1989, found: 509.1984 .<smiles></smiles>

\section{2-\{3-[(Diphenylphosphoryl)methyl]-6-fluoro-1-oxoisoquinolin-2(1H)-yl\}pyridine 1-} oxide (176ka): The general procedure D was followed using benzamide 34k (116 mg, $0.50 \mathrm{mmol})$ and allene $75 \mathrm{a}(144 \mathrm{mg}, 0.60 \mathrm{mmol})$. Purification by column chromatography on silica gel $\left(\mathrm{CH}_{2} \mathrm{Cl}_{2} /\right.$ acetone 1:1 to $\mathrm{CH}_{2} \mathrm{Cl}_{2} / \mathrm{MeOH}$ 9:1) yielded 176ka (142 mg, $302 \mu \mathrm{mol}$, $60 \%)$ as a white solid.

M. p.: $198-200{ }^{\circ} \mathrm{C}$.

${ }^{1}$ H-NMR (400 MHz, $\left.\mathrm{CDCl}_{3}\right): \delta=8.35-8.26(\mathrm{~m}, 2 \mathrm{H}), 7.73$ (ddd, $\left.J=11.6,8.2,1.4 \mathrm{~Hz}, 2 \mathrm{H}\right)$, 7.62-7.55 (m, 1H), 7.55-7.46 (m, 5H), 7.46-7.42 (m, 1H), 7.42-7.36 (m, 2H), 7.36-7.26 (m, 2H), $7.10(\mathrm{td}, J=8.7,2.5 \mathrm{~Hz}, 1 \mathrm{H}), 6.90(\mathrm{dd}, J=9.2,2.5 \mathrm{~Hz}, 1 \mathrm{H}), 6.26(\mathrm{~d}, J=3.2 \mathrm{~Hz}$, 1H), 3.65 (dd, $J=15.9,11.5 \mathrm{~Hz}, 1 \mathrm{H}), 3.15-3.02(\mathrm{~m}, 1 \mathrm{H})$. 
${ }^{13}$ C-NMR $\left(126 \mathrm{MHz}, \mathrm{CDCl}_{3}\right): \delta=165.7\left(\mathrm{~d},{ }^{1} J_{\mathrm{C}-\mathrm{F}}=253.9 \mathrm{~Hz}, \mathrm{C}_{\mathrm{q}}\right), 161.8\left(\mathrm{C}_{\mathrm{q}}\right), 143.5\left(\mathrm{C}_{\mathrm{q}}\right)$, $140.0(\mathrm{CH}), 139.0\left(\mathrm{dd},{ }^{3} J_{\mathrm{C}-\mathrm{F}}=10.7 \mathrm{~Hz},{ }^{4} J_{\mathrm{C}-\mathrm{P}}=2.7 \mathrm{~Hz}, \mathrm{C}_{\mathrm{q}}\right), 134.6\left(\mathrm{~d},{ }^{2} J_{\mathrm{C}-\mathrm{P}}=8.0 \mathrm{~Hz}, \mathrm{C}_{\mathrm{q}}\right)$, $132.6\left(\mathrm{~d},{ }^{1} J_{\mathrm{C}-\mathrm{P}}=100.2 \mathrm{~Hz}, \mathrm{C}_{\mathrm{q}}\right), 132.4\left(\mathrm{~d},{ }^{4} J_{\mathrm{C}-\mathrm{P}}=2.6 \mathrm{~Hz}, \mathrm{CH}\right), 132.2\left(\mathrm{~d},{ }^{4} J_{\mathrm{C}-\mathrm{P}}=2.6 \mathrm{~Hz}\right.$, $\mathrm{CH}), 131.5\left(\mathrm{~d},{ }^{3} J_{\mathrm{C}-\mathrm{F}}=10.1 \mathrm{~Hz}, \mathrm{CH}\right), 131.3\left(\mathrm{~d},{ }^{2} J_{\mathrm{C}-\mathrm{P}}=9.2 \mathrm{~Hz}, \mathrm{CH}\right), 131.4\left(\mathrm{~d},{ }^{1} J_{\mathrm{C}-\mathrm{P}}=\right.$ $\left.102.2 \mathrm{~Hz}, \mathrm{C}_{\mathrm{q}}\right), 130.5\left(\mathrm{~d},{ }^{2} J_{\mathrm{C}-\mathrm{P}}=9.5 \mathrm{~Hz}, \mathrm{CH}\right), 130.2(\mathrm{CH}), 128.9\left(\mathrm{~d},{ }^{3} J_{\mathrm{C}-\mathrm{P}}=6.8 \mathrm{~Hz}, \mathrm{CH}\right)$, $128.8\left(\mathrm{~d},{ }^{3} J_{\mathrm{C}-\mathrm{P}}=6.6 \mathrm{~Hz}, \mathrm{CH}\right), 126.1(\mathrm{CH}), 125.9(\mathrm{CH}), 121.5\left(\mathrm{C}_{\mathrm{q}}\right), 115.5\left(\mathrm{~d},{ }^{2} J_{\mathrm{C}-\mathrm{F}}=\right.$ $23.5 \mathrm{~Hz}, \mathrm{CH}), 111.0\left(\mathrm{~d},{ }^{2} J_{\mathrm{C}-\mathrm{F}}=22.1 \mathrm{~Hz}, \mathrm{CH}\right), 108.0\left(\mathrm{dd},{ }^{3} J_{\mathrm{C}-\mathrm{P}}=7.0 \mathrm{~Hz},{ }^{4} J_{\mathrm{C}-\mathrm{F}}=3.1 \mathrm{~Hz}\right.$, $\mathrm{CH}), 34.4\left(\mathrm{~d},{ }^{1} J_{\mathrm{C}-\mathrm{P}}=65.3 \mathrm{~Hz}, \mathrm{CH}_{2}\right)$.

${ }^{19}$ F-NMR $\left(376 \mathrm{MHz}, \mathrm{CDCl}_{3}\right): \delta=-104.8(\mathrm{td}, J=8.8,5.9 \mathrm{~Hz})$.

${ }^{31} \mathbf{P}\left\{{ }^{1} \mathbf{H}\right\}$-NMR $\left(162 \mathrm{MHz}, \mathrm{CDCl}_{3}\right): \delta=27.7$.

IR (ATR): $\tilde{v}=3057,1666,1601,1430,1267,873,720,525 \mathrm{~cm}^{-1}$.

MS (EI) $m / z$ (relative intensity): 470 (15) [M] ${ }^{+}, 453$ (35), 319 (5), 251 (100), 224 (12), 201 (60), $78(20)$.

HR-MS (EI) $m / z$ calcd. for $\mathrm{C}_{27} \mathrm{H}_{20} \mathrm{FN}_{2} \mathrm{O}_{3} \mathrm{P}[\mathrm{M}]^{+}:$470.1196, found: 470.1183 .<smiles>CC(=O)c1ccc2c(=O)n(-c3cccc[n+]3[O-])c(CP(=O)(c3ccccc3)c3ccccc3)cc2c1</smiles>

2-\{6-Acetyl-3-[(diphenylphosphoryl)methyl]-1-oxoisoquinolin-2(1H)-yl\}pyridine 1oxide (176la): The general procedure D was followed using benzamide 341 (128 mg, $0.50 \mathrm{mmol}$ ) and allene 75a (144 $\mathrm{mg}, 0.60 \mathrm{mmol})$. Purification by column chromatography on silica gel $\left(\mathrm{CH}_{2} \mathrm{Cl}_{2} /\right.$ acetone 1:1 to $\mathrm{CH}_{2} \mathrm{Cl}_{2} / \mathrm{MeOH}$ 9:1) yielded 176la (161 mg, $326 \mu \mathrm{mol}$, $65 \%)$ as a white solid.

M. p.: $192-194{ }^{\circ} \mathrm{C}$.

${ }^{1}$ H-NMR $\left(400 \mathrm{MHz} \mathrm{CDCl}_{3}\right): \delta=8.41-8.29(\mathrm{~m}, 2 \mathrm{H}), 7.93(\mathrm{dd}, J=8.3,1.7 \mathrm{~Hz}, 1 \mathrm{H}), 7.85$ (s, 1H), 7.79-7.71 (m, 2H), 7.62-7.56 (m, 1H), 7.55-7.44 (m, 6H), 7.42-7.36 (m, 2H), 7.36-7.30 (m, 2H), $6.44(\mathrm{~d}, J=3.3 \mathrm{~Hz}, 1 \mathrm{H}), 3.68(\mathrm{dd}, J=15.9,11.3 \mathrm{~Hz}, 1 \mathrm{H}), 3.20-3.05$ (m, 1H), $2.65(\mathrm{~s}, 3 \mathrm{H})$.

${ }^{13}$ C-NMR $\left(126 \mathrm{MHz}, \mathrm{CDCl}_{3}\right): \delta=197.5\left(\mathrm{C}_{\mathrm{q}}\right), 162.0\left(\mathrm{C}_{\mathrm{q}}\right), 143.3\left(\mathrm{C}_{\mathrm{q}}\right), 140.5\left(\mathrm{C}_{\mathrm{q}}\right), 140.0$ $(\mathrm{CH}), 136.8\left(\mathrm{C}_{\mathrm{q}}\right), 134.4\left(\mathrm{~d},{ }^{2} J_{\mathrm{C}-\mathrm{P}}=7.8 \mathrm{~Hz}, \mathrm{C}_{\mathrm{q}}\right), 132.6\left(\mathrm{~d},{ }^{4} J_{\mathrm{C}-\mathrm{P}}=2.8 \mathrm{~Hz}, \mathrm{CH}\right), 132.3(\mathrm{~d}$, $\left.{ }^{4} J_{\mathrm{C}-\mathrm{P}}=2.8 \mathrm{~Hz}, \mathrm{CH}\right), 132.6\left(\mathrm{~d},{ }^{1} J_{\mathrm{C}-\mathrm{P}}=101.2 \mathrm{~Hz}, \mathrm{C}_{\mathrm{q}}\right), 131.3\left(\mathrm{~d},{ }^{2} J_{\mathrm{C}-\mathrm{P}}=9.2 \mathrm{~Hz}, \mathrm{CH}\right), 131.3$ $\left(\mathrm{d},{ }^{1} J_{\mathrm{C}-\mathrm{P}}=101.8 \mathrm{~Hz}, \mathrm{C}_{\mathrm{q}}\right), 130.6\left(\mathrm{~d},{ }^{2} J_{\mathrm{C}-\mathrm{P}}=9.5 \mathrm{~Hz}, \mathrm{CH}\right), 130.3(\mathrm{CH}), 129.0\left(\mathrm{~d},{ }^{3} J_{\mathrm{C}-\mathrm{P}}=\right.$ 
$12.8 \mathrm{~Hz}, \mathrm{CH}), 128.9\left(\mathrm{~d},{ }^{3} J_{\mathrm{C}-\mathrm{P}}=12.8 \mathrm{~Hz}, \mathrm{CH}\right), 128.7(\mathrm{CH}), 127.7\left(\mathrm{C}_{\mathrm{q}}\right), 126.5(\mathrm{CH}), 126.4$ $(\mathrm{CH}), 125.8(\mathrm{CH}), 125.7(\mathrm{CH}), 108.7\left(\mathrm{~d},{ }^{3} J_{\mathrm{C}-\mathrm{P}}=7.0 \mathrm{~Hz}, \mathrm{CH}\right), 34.6\left(\mathrm{~d},{ }^{1} J_{\mathrm{C}-\mathrm{P}}=65.5 \mathrm{~Hz}\right.$, $\left.\mathrm{CH}_{2}\right), 27.2\left(\mathrm{CH}_{3}\right)$.

${ }^{31} \mathbf{P}\left\{{ }^{1} \mathbf{H}\right\}$-NMR $\left(162 \mathrm{MHz}, \mathrm{CDCl}_{3}\right): \delta=27.7$.

IR (ATR): $\tilde{v}=3093,1672,1634,1433,1259,1201,717,537 \mathrm{~cm}^{-1}$.

MS (EI) $m / z$ (relative intensity): 494 (5) [M] $]^{+}, 478$ (12), 319 (5), 276 (100), 234 (10), 201 (35), 77 (12).

HR-MS (EI) $m / z$ calcd. for $\mathrm{C}_{29} \mathrm{H}_{23} \mathrm{~N}_{2} \mathrm{O}_{4} \mathrm{P}[\mathrm{M}]^{+}:$494.1395, found: 494.1402 .<smiles>CC(=O)c1ccc2c(=O)n(-c3cccc[n+]3[O-])c(CP(=O)(c3ccccc3)c3ccccc3)cc2c1</smiles>

\section{2-\{3-[(Diphenylphosphoryl)methyl]-6-(methoxycarbonyl)-1-oxoisoquinolin-2(1H)-}

yl\}pyridine 1-oxide (176ma): The general procedure D was followed using benzamide 34m (136 mg, $0.50 \mathrm{mmol})$ and allene 75a (144 mg, $1.00 \mathrm{mmol})$. Purification by column chromatography on silica gel $\left(\mathrm{CH}_{2} \mathrm{Cl}_{2}\right.$ /acetone $1: 1$ to $\left.\mathrm{CH}_{2} \mathrm{Cl}_{2} / \mathrm{MeOH} 9: 1\right)$ yielded $176 \mathbf{m a}$ (131 mg, $257 \mu \mathrm{mol}, 51 \%)$ as a white solid.

M. p.: $209-210^{\circ} \mathrm{C}$.

${ }^{1}$ H-NMR (400 MHz, $\left.\mathrm{CDCl}_{3}\right): \delta=8.40-8.24(\mathrm{~m}, 2 \mathrm{H}), 8.00(\mathrm{dd}, J=8.3,1.6 \mathrm{~Hz}, 1 \mathrm{H}), 7.98-$ $7.91(\mathrm{~m}, 1 \mathrm{H}), 7.79-7.67(\mathrm{~m}, 2 \mathrm{H}), 7.65-7.55(\mathrm{~m}, 2 \mathrm{H}), 7.55-7.44(\mathrm{~m}, 5 \mathrm{H}), 7.44-7.29(\mathrm{~m}$, 4H), 6.39-6.30 (m, 1H), 3.95 (s, 3H), 3.77-3.61 (m, 1H), 3.22-3.06 (m, 1H).

${ }^{13}$ C-NMR (126 MHz, $\left.\mathrm{CDCl}_{3}\right): \delta=166.2\left(\mathrm{C}_{\mathrm{q}}\right), 162.1\left(\mathrm{C}_{\mathrm{q}}\right), 143.8\left(\mathrm{C}_{\mathrm{q}}\right), 140.3(\mathrm{CH}), 136.5$ $\left(\mathrm{d},{ }^{2} J_{\mathrm{C}-\mathrm{P}}=8.1 \mathrm{~Hz}, \mathrm{C}_{\mathrm{q}}\right), 134.2\left(\mathrm{C}_{\mathrm{q}}\right), 133.9\left(\mathrm{~d},{ }^{1} J_{\mathrm{C}-\mathrm{P}}=104.4 \mathrm{~Hz}, \mathrm{C}_{\mathrm{q}}\right), 133.1\left(\mathrm{C}_{\mathrm{q}}\right), 132.5(\mathrm{CH})$, $132.4(\mathrm{CH}), 131.9\left(\mathrm{~d},{ }^{1} J_{\mathrm{C}-\mathrm{P}}=102.5 \mathrm{~Hz}, \mathrm{C}_{\mathrm{q}}\right), 131.4\left(\mathrm{~d},{ }^{2} J_{\mathrm{C}-\mathrm{P}}=9.1 \mathrm{~Hz}, \mathrm{CH}\right), 130.6\left(\mathrm{~d},{ }^{2} J_{\mathrm{C}-\mathrm{P}}\right.$ $=9.3 \mathrm{~Hz}, \mathrm{CH}), 130.3(\mathrm{CH}), 129.0\left(\mathrm{~d},{ }^{3} J_{\mathrm{C}-\mathrm{P}}=5.7 \mathrm{~Hz}, \mathrm{CH}\right), 128.9\left(\mathrm{~d},{ }^{3} J_{\mathrm{C}-\mathrm{P}}=5.7 \mathrm{~Hz}, \mathrm{CH}\right)$, $128.6(\mathrm{CH}), 127.9(\mathrm{CH}), 127.7\left(\mathrm{C}_{\mathrm{q}}\right), 127.0(\mathrm{CH}), 126.3(\mathrm{CH}), 126.1(\mathrm{CH}), 108.6\left(\mathrm{~d},{ }^{3} J_{\mathrm{C}-\mathrm{P}}\right.$ $=6.5 \mathrm{~Hz}, \mathrm{CH}), 52.7\left(\mathrm{CH}_{3}\right), 34.7\left(\mathrm{~d},{ }^{1} J_{\mathrm{C}-\mathrm{P}}=65.4 \mathrm{~Hz}, \mathrm{CH}_{2}\right)$.

${ }^{31} \mathbf{P}\left\{{ }^{1} \mathbf{H}\right\}$-NMR $\left(162 \mathrm{MHz}, \mathrm{CDCl}_{3}\right): \delta=28.0$.

IR (ATR): $\tilde{v}=3058,1723,1684,1434,1289,1260,1102,721 ; 518 \mathrm{~cm}^{-1}$.

MS (EI) $m / z$ (relative intensity): 510 (10) [M] $]^{+}, 493$ (18), 319 (5), 292 (100), 234 (10), 201 (45), 77 (15).

HR-MS (EI) $m / z$ calcd. for $\mathrm{C}_{29} \mathrm{H}_{23} \mathrm{~N}_{2} \mathrm{O}_{5} \mathrm{P}[\mathrm{M}]^{+}: 510.1345$, found: 510.1356. 
<smiles>Cc1cc2cc(CP(=O)(c3ccccc3)c3ccccc3)n(-c3cccc[n+]3[O-])c(=O)c2cc1C</smiles>

2-\{3-[(Diphenylphosphoryl)methyl]-6,7-dimethyl-1-oxoisoquinolin-2(1H)-yl\}pyridine

1-oxide (176na): The general procedure $\mathbf{D}$ was followed using benzamide 34n (121 mg, $0.50 \mathrm{mmol})$ and allene 75a (144 $\mathrm{mg}, 0.60 \mathrm{mmol})$. Purification by column chromatography on silica gel $\left(\mathrm{CH}_{2} \mathrm{Cl}_{2} /\right.$ acetone 1:1 to $\mathrm{CH}_{2} \mathrm{Cl}_{2} / \mathrm{MeOH}$ 9:1) yielded 176na (232 mg, $483 \mu \mathrm{mol}$, 97\%) as a white solid.

M. p.: $145-147^{\circ} \mathrm{C}$.

${ }^{1}$ H-NMR (400 MHz, $\left.\mathrm{CDCl}_{3}\right): \delta=8.35-8.25(\mathrm{~m}, 1 \mathrm{H}), 8.03(\mathrm{~s}, 1 \mathrm{H}), 7.77-7.69(\mathrm{~m}, 2 \mathrm{H})$, 7.60-7.54 (m, 1H), 7.52-7.46 (m, 4H), 7.46-7.43 (m, 1H), 7.41-7.34 (m, 3H), 7.34-7.23 (m, 2H), $7.05(\mathrm{~s}, 1 \mathrm{H}), 6.33$ (d, $J=3.2 \mathrm{~Hz}, 1 \mathrm{H}), 3.66$ (dd, $J=16.0,12.0 \mathrm{~Hz}, 1 \mathrm{H}), 3.13-3.01$ $(\mathrm{m}, 1 \mathrm{H}), 2.34(\mathrm{~s}, 3 \mathrm{H}), 2.33(\mathrm{~s}, 3 \mathrm{H})$.

${ }^{13}$ C-NMR (126 MHz, $\left.\mathrm{CDCl}_{3}\right): \delta=162.5\left(\mathrm{C}_{\mathrm{q}}\right), 144.2\left(\mathrm{C}_{\mathrm{q}}\right), 143.3\left(\mathrm{C}_{\mathrm{q}}\right), 140.1(\mathrm{CH}), 136.4$ $\left(\mathrm{C}_{\mathrm{q}}\right), 134.9\left(\mathrm{C}_{\mathrm{q}}\right), 132.3\left(\mathrm{~d},{ }^{1} J_{\mathrm{C}-\mathrm{P}}=99.2 \mathrm{~Hz}, \mathrm{C}_{\mathrm{q}}\right), 132.3\left(\mathrm{~d},{ }^{4} J_{\mathrm{C}-\mathrm{P}}=2.6 \mathrm{~Hz}, \mathrm{CH}\right), 132.1(\mathrm{~d}$, $\left.{ }^{4} J_{\mathrm{C}-\mathrm{P}}=2.6 \mathrm{~Hz}, \mathrm{CH}\right), 131.9\left(\mathrm{~d},{ }^{2} J_{\mathrm{C}-\mathrm{P}}=7.7 \mathrm{~Hz}, \mathrm{C}_{\mathrm{q}}\right), 131.4\left(\mathrm{~d},{ }^{2} J_{\mathrm{C}-\mathrm{P}}=9.0 \mathrm{~Hz}, \mathrm{CH}\right), 131.2(\mathrm{~d}$, $\left.{ }^{1} J_{\mathrm{C}-\mathrm{P}}=103.4 \mathrm{~Hz}, \mathrm{C}_{\mathrm{q}}\right), 130.6\left(\mathrm{~d},{ }^{2} J_{\mathrm{C}-\mathrm{P}}=9.3 \mathrm{~Hz}, \mathrm{CH}\right), 130.2(\mathrm{CH}), 128.8\left(\mathrm{~d},{ }^{3} J_{\mathrm{C}-\mathrm{P}}=5.7 \mathrm{~Hz}\right.$, $\mathrm{CH}), 128.8\left(\mathrm{~d},{ }^{3} J_{\mathrm{C}-\mathrm{P}}=5.6 \mathrm{~Hz}, \mathrm{CH}\right), 128.2(\mathrm{CH}), 126.5(\mathrm{CH}), 125.9(\mathrm{CH}), 125.7(\mathrm{CH}), 123.0$ $\left(\mathrm{C}_{\mathrm{q}}\right), 108.6\left(\mathrm{~d},{ }^{3} J_{\mathrm{C}-\mathrm{P}}=6.9 \mathrm{~Hz}, \mathrm{CH}\right), 34.4\left(\mathrm{~d},{ }^{1} J_{\mathrm{C}-\mathrm{P}}=66.2 \mathrm{~Hz}, \mathrm{CH}_{2}\right), 20.4\left(\mathrm{CH}_{3}\right), 20.0\left(\mathrm{CH}_{3}\right)$. ${ }^{31} \mathbf{P}\left\{{ }^{1} \mathbf{H}\right\}$-NMR $\left(162 \mathrm{MHz}, \mathrm{CDCl}_{3}\right): \delta=27.6$.

IR (ATR): $\tilde{v}=2996,1664,1596,1273,1201,840,733,511 \mathrm{~cm}^{-1}$.

MS (EI) $m / z$ (relative intensity): 480 (15) [M] $]^{+}, 463$ (20), 319 (15), 279 (15), 262 (100), 201 (39), 77 (15).

HR-MS (EI) $m / z$ calcd. for $\mathrm{C}_{29} \mathrm{H}_{25} \mathrm{~N}_{2} \mathrm{O}_{3} \mathrm{P}[\mathrm{M}]^{+}:$480.1603, found: 480.1608 .<smiles>O=c1c2c3c(ccc2cc(CP(=O)(c2ccccc2)c2ccccc2)n1-c1cccc[n+]1[O-])-c1ccccc1C3</smiles>

2-\{3-[(Diphenylphosphoryl)methyl]-1-oxo-1,11-dihydro-2H-indeno[1,2h]isoquinolin-2-yl\}pyridine 1-oxide (1760a): The general procedure D was followed 
using benzamide $34 \mathrm{o}(151 \mathrm{mg}, 0.50 \mathrm{mmol})$ and allene 75a (144 mg, $0.60 \mathrm{mmol})$. Purification by column chromatography on silica gel $\left(\mathrm{CH}_{2} \mathrm{Cl}_{2} /\right.$ acetone $1: 1$ to $\mathrm{CH}_{2} \mathrm{Cl}_{2} / \mathrm{MeOH} 9: 1$ ) yielded 176oa (240 mg, $444 \mu \mathrm{mol}, 89 \%$ ) as a white solid.

M. p.: $168-169^{\circ} \mathrm{C}$.

${ }^{1}$ H-NMR (400 MHz, $\left.\mathrm{CDCl}_{3}\right): \delta=8.41-8.33(\mathrm{~m}, 1 \mathrm{H}), 8.03(\mathrm{~d}, J=8.1 \mathrm{~Hz}, 1 \mathrm{H}), 7.84-7.71$ $(\mathrm{m}, 3 \mathrm{H}), 7.62-7.54(\mathrm{~m}, 3 \mathrm{H}), 7.54-7.46(\mathrm{~m}, 5 \mathrm{H}), 7.43-7.36(\mathrm{~m}, 3 \mathrm{H}), 7.36-7.29(\mathrm{~m}, 4 \mathrm{H})$, $6.43(\mathrm{~d}, J=3.3 \mathrm{~Hz}, 1 \mathrm{H}), 4.39$ (d, $J=3.2 \mathrm{~Hz}, 2 \mathrm{H}), 3.70(\mathrm{dd}, J=15.9,11.4 \mathrm{~Hz}, 1 \mathrm{H}), 3.20$ $3.09(\mathrm{~m}, 1 \mathrm{H})$.

${ }^{13}$ C-NMR (126 MHz, $\left.\mathrm{CDCl}_{3}\right): \delta=162.6\left(\mathrm{C}_{\mathrm{q}}\right), 145.2\left(\mathrm{C}_{\mathrm{q}}\right), 144.5\left(\mathrm{C}_{\mathrm{q}}\right), 143.8\left(\mathrm{C}_{\mathrm{q}}\right), 141.2$ $\left(\mathrm{C}_{\mathrm{q}}\right), 140.3\left(\mathrm{C}_{\mathrm{q}}\right), 140.0(\mathrm{CH}), 136.2\left(\mathrm{~d},{ }^{4} J_{\mathrm{C}-\mathrm{P}}=2.8 \mathrm{~Hz}, \mathrm{C}_{\mathrm{q}}\right), 132.9\left(\mathrm{~d},{ }^{1} J_{\mathrm{C}-\mathrm{P}}=99.5 \mathrm{~Hz}, \mathrm{C}_{\mathrm{q}}\right)$, $132.4\left(\mathrm{~d},{ }^{4} J_{\mathrm{C}-\mathrm{P}}=2.7 \mathrm{~Hz}, \mathrm{CH}\right), 132.2\left(\mathrm{~d},{ }^{4} J_{\mathrm{C}-\mathrm{P}}=2.7 \mathrm{~Hz}, \mathrm{CH}\right), 132.0\left(\mathrm{~d},{ }^{2} J_{\mathrm{C}-\mathrm{P}}=8.3 \mathrm{~Hz}, \mathrm{C}_{\mathrm{q}}\right)$, $131.4\left(\mathrm{~d},{ }^{2} J_{\mathrm{C}-\mathrm{P}}=9.2 \mathrm{~Hz}, \mathrm{CH}\right), 131.2\left(\mathrm{~d},{ }^{1} J_{\mathrm{C}-\mathrm{P}}=101.9 \mathrm{~Hz}, \mathrm{C}_{\mathrm{q}}\right), 130.6\left(\mathrm{~d},{ }^{2} J_{\mathrm{C}-\mathrm{P}}=9.5 \mathrm{~Hz}\right.$, $\mathrm{CH}), 130.5(\mathrm{CH}), 128.9\left(\mathrm{~d},{ }^{3} J_{\mathrm{C}-\mathrm{P}}=4.6 \mathrm{~Hz}, \mathrm{CH}\right), 128.8\left(\mathrm{~d},{ }^{3} J_{\mathrm{C}-\mathrm{P}}=4.4 \mathrm{~Hz}, \mathrm{CH}\right), 127.0(\mathrm{CH})$, $126.6(\mathrm{CH}), 126.1(\mathrm{CH}), 125.8(\mathrm{CH}), 125.5(\mathrm{CH}), 125.1(\mathrm{CH}), 124.9(\mathrm{CH}), 121.9\left(\mathrm{C}_{\mathrm{q}}\right)$, $119.6(\mathrm{CH}), 109.6\left(\mathrm{~d},{ }^{3} J_{\mathrm{C}-\mathrm{P}}=7.1 \mathrm{~Hz}, \mathrm{CH}\right), 39.5\left(\mathrm{CH}_{2}\right), 34.6\left(\mathrm{~d},{ }^{1} J_{\mathrm{C}-\mathrm{P}}=65.8 \mathrm{~Hz}, \mathrm{CH}_{2}\right)$.

${ }^{31} \mathbf{P}\left\{{ }^{1} \mathbf{H}\right\}$-NMR $\left(162 \mathrm{MHz}, \mathrm{CDCl}_{3}\right): \delta=27.7$.

IR (ATR): $\tilde{v}=3054,1665,1601,1431,1265,1186,846,692,522 \mathrm{~cm}^{-1}$.

MS (EI) $m / z$ (relative intensity): 540 (15) [M] $]^{+}, 524$ (45), 339 (15), 322 (100), 293 (15), 201 (49), 77 (15).

HR-MS (EI) $m / z$ calcd. for $\mathrm{C}_{34} \mathrm{H}_{25} \mathrm{~N}_{2} \mathrm{O}_{3} \mathrm{P}[\mathrm{M}]^{+}:$540.1603, found: 540.1593 .<smiles>COc1ccc2cc(CP(=O)(c3ccccc3)c3ccccc3)n(-c3cccc[n+]3[O-])c(=O)c2c1</smiles><smiles>COc1cccc2c1C(=O)N(c1cccc[n+]1CCP(=O)(c1ccccc1)c1ccccc1)C2=O</smiles>

\section{2-\{3-[(Diphenylphosphoryl)methyl]-7-methoxy-1-oxoisoquinolin-2(1H)-yl\}pyridine} 1-oxide (176pa); 2-\{3-[(diphenylphosphoryl)methyl]-5-methoxy-1-oxoisoquinolin2(1H)-yl\}pyridine 1-oxide (176pa') : The general procedure D was followed using benzamide 34p (122 mg, $0.50 \mathrm{mmol}$ ) and allene 75a (144 mg, $0.60 \mathrm{mmol})$. Purification by column chromatography on silica gel $\left(\mathrm{CH}_{2} \mathrm{Cl}_{2}\right.$ /acetone $1: 1$ to $\left.\mathrm{CH}_{2} \mathrm{Cl}_{2} / \mathrm{MeOH} 9: 1\right)$ yielded 176pa/176pa' (231 mg, $479 \mu \mathrm{mol}, 96 \%$ ) as a white solid. The ratio of 176pa/176pa' (75:25) was determined by ${ }^{1} \mathrm{H}-\mathrm{NMR}$ spectroscopy. Resonances are reported for 176pa. 
M. p.: $175-177^{\circ} \mathrm{C}$.

${ }^{1}$ H-NMR $\left(600 \mathrm{MHz}, \mathrm{CDCl}_{3}\right): \delta=8.34-8.28(\mathrm{~m}, 1 \mathrm{H}), 7.75-7.68(\mathrm{~m}, 3 \mathrm{H}), 7.58-7.52(\mathrm{~m}$, 1H), 7.50-7.45 (m, 6H), 7.40-7.27 (m, 4H), 7.23-7.18 (m, 2H), 6.34 (d, $J=3.4 \mathrm{~Hz}, 1 \mathrm{H})$, $3.65(\mathrm{dd}, J=16.0,11.5 \mathrm{~Hz}, 1 \mathrm{H}), 3.08(\mathrm{dd}, J=15.8,13.4 \mathrm{~Hz}, 1 \mathrm{H})$.

${ }^{13}$ C-NMR (126 MHz, $\left.\mathrm{CDCl}_{3}\right): \delta=162.3\left(\mathrm{C}_{\mathrm{q}}\right), 158.8\left(\mathrm{C}_{\mathrm{q}}\right), 143.8\left(\mathrm{C}_{\mathrm{q}}\right), 140.0(\mathrm{CH}), 132.9$ $\left(\mathrm{d},{ }^{1} J_{\mathrm{C}-\mathrm{P}}=100.0 \mathrm{~Hz}, \mathrm{C}_{\mathrm{q}}\right), 132.3\left(\mathrm{~d},{ }^{4} J_{\mathrm{C}-\mathrm{P}}=2.8 \mathrm{~Hz}, \mathrm{CH}\right), 132.1\left(\mathrm{~d},{ }^{4} J_{\mathrm{C}-\mathrm{P}}=2.0 \mathrm{~Hz}, \mathrm{CH}\right)$, $131.6\left(\mathrm{~d},{ }^{1} J_{\mathrm{C}-\mathrm{P}}=101.4 \mathrm{~Hz}, \mathrm{C}_{\mathrm{q}}\right), 131.4\left(\mathrm{~d},{ }^{2} J_{\mathrm{C}-\mathrm{P}}=9.1 \mathrm{~Hz}, \mathrm{CH}\right), 130.8\left(\mathrm{~d},{ }^{4} J_{\mathrm{C}-\mathrm{P}}=2.9 \mathrm{~Hz}, \mathrm{C}_{\mathrm{q}}\right)$, $130.6\left(\mathrm{~d},{ }^{2} J_{\mathrm{C}-\mathrm{P}}=9.4 \mathrm{~Hz}, \mathrm{CH}\right), 130.2(\mathrm{CH}), 128.9\left(\mathrm{~d},{ }^{3} J_{\mathrm{C}-\mathrm{P}}=4.1 \mathrm{~Hz}, \mathrm{CH}\right), 128.8\left(\mathrm{~d},{ }^{3} J_{\mathrm{C}-\mathrm{P}}=\right.$ 4.1 Hz, CH), $127.7(\mathrm{CH}), 126.0\left(\mathrm{C}_{\mathrm{q}}\right), 125.6(\mathrm{CH}), 123.6(\mathrm{CH}), 119.8(\mathrm{CH}), 112.8(\mathrm{CH})$, $108.7\left(\mathrm{~d},{ }^{3} J_{\mathrm{C}-\mathrm{P}}=7.1 \mathrm{~Hz}, \mathrm{CH}\right), 108.4(\mathrm{CH}), 55.7\left(\mathrm{CH}_{3}\right), 34.3\left(\mathrm{~d},{ }^{1} J_{\mathrm{C}-\mathrm{P}}=66.3 \mathrm{~Hz}, \mathrm{CH}_{2}\right)$.

${ }^{31} \mathbf{P}\left\{{ }^{1} \mathbf{H}\right\}$-NMR $\left(162 \mathrm{MHz}, \mathrm{CDCl}_{3}\right): \delta=27.7$.

IR (ATR): $\tilde{v}=1665,1598,1489,1432,1256,1189,1256,719,505 \mathrm{~cm}^{-1}$.

MS (EI) $m / z$ (relative intensity): 482 (5) [M] ${ }^{+}, 466$ (20), 319 (10), 264 (100), 249 (50), 201 (48), 77 (25).

HR-MS (EI) $m / z$ calcd. for $\mathrm{C}_{28} \mathrm{H}_{23} \mathrm{~N}_{2} \mathrm{O}_{4} \mathrm{P}[\mathrm{M}]^{+}:$482.1395, found: 482.1389 .<smiles></smiles>

$176 q a$<smiles>O=c1c2cc3c(cc2cc(CP(=O)(c2ccccc2)c2ccccc2)n1-c1cccc[n+]1[O-])OCO3</smiles>

$176 q a^{\prime}$

2-\{8-[(Diphenylphosphoryl)methyl]-6-oxo-[1,3]dioxolo[4,5-f]isoquinolin-7(6H)yl\}pyridine 1-oxide (176qa); 2-\{7-[(diphenylphosphoryl)methyl]-5-oxo[1,3]dioxolo[4,5-g]isoquinolin-6(5H)-yl\}pyridine 1-oxide (176qa'): The general procedure D was followed using benzamide $\mathbf{3 4 q}(129 \mathrm{mg}, 0.50 \mathrm{mmol})$ and allene $\mathbf{7 5 a}$ (144 mg, $0.60 \mathrm{mmol}$ ). Purification by column chromatography on silica gel $\left(\mathrm{CH}_{2} \mathrm{Cl}_{2}\right.$ /acetone 1:1 to $\mathrm{CH}_{2} \mathrm{Cl}_{2} / \mathrm{MeOH}$ 9:1) yielded 176qa/176qa' (216 mg, $435 \mu \mathrm{mol}$, $87 \%$ ) as a white solid. The ratio of $\mathbf{1 7 6 q a}$ /176qa' (81:19) was determined by ${ }^{1} \mathrm{H}-\mathrm{NMR}$ spectroscopy. Resonances are reported for 176qa.

M. p.: $202-204^{\circ} \mathrm{C}$.

${ }^{1}$ H-NMR (600 MHz, $\left.\mathrm{CDCl}_{3}\right): \delta=8.32-8.28(\mathrm{~m}, 1 \mathrm{H}), 7.91(\mathrm{~d}, J=8.4 \mathrm{~Hz}, 1 \mathrm{H}), 7.76-7.70$ (m, 2H), 7.59-7.55 (m, 1H), 7.52-7.46 (m, 6H), 7.40-7.36 (m, 2H), 7.31-7.26 (m, 2H), 
$6.94(\mathrm{~d}, J=8.4 \mathrm{~Hz}, 1 \mathrm{H}), 6.32(\mathrm{~d}, J=3.4 \mathrm{~Hz}, 1 \mathrm{H}), 6.06(\mathrm{dd}, J=4.4,1.5 \mathrm{~Hz}, 2 \mathrm{H}), 3.67(\mathrm{dd}$, $J=16.0,11.6 \mathrm{~Hz}, 1 \mathrm{H}), 3.10(\mathrm{dd}, J=15.3,13.5 \mathrm{~Hz}, 1 \mathrm{H})$.

${ }^{13}$ C-NMR (126 MHz, $\left.\mathrm{CDCl}_{3}\right): \delta=161.9\left(\mathrm{C}_{\mathrm{q}}\right), 150.7\left(\mathrm{C}_{\mathrm{q}}\right), 143.7\left(\mathrm{C}_{\mathrm{q}}\right), 141.4\left(\mathrm{C}_{\mathrm{q}}\right), 139.9$ $(\mathrm{CH}), 133.6\left(\mathrm{~d},{ }^{2} J_{\mathrm{C}-\mathrm{P}}=8.7 \mathrm{~Hz}, \mathrm{C}_{\mathrm{q}}\right), 132.9\left(\mathrm{~d},{ }^{1} J_{\mathrm{C}-\mathrm{P}}=99.5 \mathrm{~Hz}, \mathrm{C}_{\mathrm{q}}\right), 132.4\left(\mathrm{~d},{ }^{4} J_{\mathrm{C}-\mathrm{P}}=2.8 \mathrm{~Hz}\right.$, $\mathrm{CH}), 132.2\left(\mathrm{~d},{ }^{4} J_{\mathrm{C}-\mathrm{P}}=2.8 \mathrm{~Hz}, \mathrm{CH}\right), 131.4\left(\mathrm{~d},{ }^{2} J_{\mathrm{C}-\mathrm{P}}=9.1 \mathrm{~Hz}, \mathrm{CH}\right), 131.1\left(\mathrm{~d},{ }^{1} J_{\mathrm{C}-\mathrm{P}}=\right.$ $\left.102.1 \mathrm{~Hz}, \mathrm{C}_{\mathrm{q}}\right), 130.6\left(\mathrm{~d},{ }^{2} J_{\mathrm{C}-\mathrm{P}}=9.4 \mathrm{~Hz}, \mathrm{CH}\right), 130.3(\mathrm{CH}), 128.9\left(\mathrm{~d},{ }^{3} J_{\mathrm{C}-\mathrm{P}}=5.7 \mathrm{~Hz}, \mathrm{CH}\right)$, $128.8\left(\mathrm{~d},{ }^{3} J_{\mathrm{C}-\mathrm{P}}=5.4 \mathrm{~Hz}, \mathrm{CH}\right), 126.0(\mathrm{CH}), 125.6(\mathrm{CH}), 124.0(\mathrm{CH}), 120.7\left(\mathrm{C}_{\mathrm{q}}\right), 119.8\left(\mathrm{C}_{\mathrm{q}}\right)$, $108.7(\mathrm{CH}), 102.4\left(\mathrm{CH}_{2}\right), 101.6\left(\mathrm{~d},{ }^{3} J_{\mathrm{C}-\mathrm{P}}=7.3 \mathrm{~Hz}, \mathrm{CH}\right), 35.0\left(\mathrm{~d},{ }^{1} J_{\mathrm{C}-\mathrm{P}}=65.4 \mathrm{~Hz}, \mathrm{CH}_{2}\right)$. ${ }^{31} \mathbf{P}\left\{{ }^{1} \mathbf{H}\right\}$-NMR $\left(162 \mathrm{MHz}, \mathrm{CDCl}_{3}\right): \delta=27.3$.

IR (ATR): $\tilde{v}=1666,1625,1464,1430,1291,1064,715,515 \mathrm{~cm}^{-1}$.

MS (EI) $m / z$ (relative intensity): 496 (10) [M] $]^{+}, 480$ (15), 319 (10), 278 (100), 249 (10), 201 (35), 77 (15).

HR-MS (EI) $m / z$ calcd. for $\mathrm{C}_{28} \mathrm{H}_{21} \mathrm{~N}_{2} \mathrm{O}_{5} \mathrm{P}[\mathrm{M}]^{+}:$496.1188, found: 496.1186 .<smiles></smiles>

176ra<smiles></smiles>

176ra'

2-\{3-[(Diphenylphosphoryl)methyl]-7-fluoro-1-oxoisoquinolin-2(1H)-yl\}pyridine 1oxide (176ra); 2-\{3-[(diphenylphosphoryl)methyl]-7-fluoro-1-oxoisoquinolin-2(1 H)yl\}pyridine 1-oxide (176ra'): The general procedure $\mathbf{D}$ was followed using benzamide 34r $(116 \mathrm{mg}, 0.50 \mathrm{mmol})$ and allene 75a $(144 \mathrm{mg}, 0.60 \mathrm{mmol})$. Purification by column chromatography on silica gel $\left(\mathrm{CH}_{2} \mathrm{Cl}_{2}\right.$ /acetone 1:1 to $\mathrm{CH}_{2} \mathrm{Cl}_{2} / \mathrm{MeOH}$ 9:1) yielded 176ra/176ra' (191 mg, $406 \mu \mathrm{mol}, 81 \%$ ) as a white solid. The ratio of 176ra/176ra' (50:50) was determined by ${ }^{1} \mathrm{H}-\mathrm{NMR}$ spectroscopy. Resonances are reported for both isomers.

M. p.: $188-190{ }^{\circ} \mathrm{C}$.

${ }^{1}$ H-NMR (600 MHz, $\left.\mathrm{CDCl}_{3}\right): \delta=8.34-8.28(\mathrm{~m}, 2 \mathrm{H}), 8.06(\mathrm{~d}, J=7.0 \mathrm{~Hz}, 1 \mathrm{H}), 7.91$ (dd, $J=9.1,2.7 \mathrm{~Hz}, 1 \mathrm{H}), 7.74-7.68(\mathrm{~m}, 4 \mathrm{H}), 7.63$ (t, $J=4.9 \mathrm{~Hz}, 1 \mathrm{H}), 7.58-7.54(\mathrm{~m}, 2 \mathrm{H}), 7.51-$ $7.43(\mathrm{~m}, 12 \mathrm{H}), 7.39-7.35(\mathrm{~m}, 4 \mathrm{H}), 7.33-7.25(\mathrm{~m}, 7 \mathrm{H}), 6.37$ (d, $J=3.6 \mathrm{~Hz}, 1 \mathrm{H}), 6.34$ (d, $J=3.3 \mathrm{~Hz}, 1 \mathrm{H}), 3.71-3.59(\mathrm{~m}, 2 \mathrm{H}), 3.16-3.04(\mathrm{~m}, 2 \mathrm{H})$.

${ }^{13}$ C-NMR $\left(126 \mathrm{MHz}, \mathrm{CDCl}_{3}\right): \delta=161.3\left(\mathrm{~d},{ }^{1} J_{\mathrm{C}-\mathrm{F}}=247.8 \mathrm{~Hz}, \mathrm{C}_{\mathrm{q}}\right), 161.6\left(\mathrm{~d},{ }^{4} J_{\mathrm{C}-\mathrm{F}}=3.3 \mathrm{~Hz}\right.$, $\left.\mathrm{C}_{\mathrm{q}}\right), 161.5\left(\mathrm{C}_{\mathrm{q}}\right), 157.1\left(\mathrm{~d},{ }^{1} J_{\mathrm{C}-\mathrm{F}}=252.1 \mathrm{~Hz}, \mathrm{C}_{\mathrm{q}}\right), 143.3\left(\mathrm{C}_{\mathrm{q}}\right), 139.8(\mathrm{CH}), 139.7(\mathrm{CH}), 134.0$ 
$\left(\mathrm{d},{ }^{2} J_{\mathrm{C}-\mathrm{P}}=9.1 \mathrm{~Hz}, \mathrm{C}_{\mathrm{q}}\right), 133.1\left(\mathrm{dd},{ }^{3} J_{\mathrm{C}-\mathrm{F}}=10.5 \mathrm{~Hz},{ }^{2} J_{\mathrm{C}-\mathrm{P}}=9.0 \mathrm{~Hz}, \mathrm{C}_{\mathrm{q}}\right), 132.3\left(\mathrm{~d},{ }^{4} J_{\mathrm{C}-\mathrm{P}}=\right.$ $2.9 \mathrm{~Hz}, \mathrm{CH}), 132.3\left(\mathrm{~d},{ }^{4} J_{\mathrm{C}-\mathrm{P}}=2.8 \mathrm{~Hz}, \mathrm{CH}\right), 132.1\left(\mathrm{~d},{ }^{1} J_{\mathrm{C}-\mathrm{P}}=102.9 \mathrm{~Hz}, \mathrm{C}_{\mathrm{q}}\right), 132.1(\mathrm{CH})$, $131.2\left(\mathrm{~d},{ }^{2} J_{\mathrm{C}-\mathrm{P}}=9.4 \mathrm{~Hz}, \mathrm{CH}\right), 130.5\left(\mathrm{~d},{ }^{3} J_{\mathrm{C}-\mathrm{F}}=12.3 \mathrm{~Hz}, \mathrm{CH}\right), 130.4\left(\mathrm{~d},{ }^{2} J_{\mathrm{C}-\mathrm{P}}=9.9 \mathrm{~Hz}, \mathrm{CH}\right)$, $130.3\left(\mathrm{C}_{\mathrm{q}}\right), 130.3\left(\mathrm{~d},{ }^{3} J_{\mathrm{C}-\mathrm{F}}=12.3 \mathrm{~Hz}, \mathrm{CH}\right), 130.1(\mathrm{CH}), 128.8\left(\mathrm{~d},{ }^{3} J_{\mathrm{C}-\mathrm{P}}=5.2 \mathrm{~Hz}, \mathrm{CH}\right), 128.7$ $\left(\mathrm{d},{ }^{3} J_{\mathrm{C}-\mathrm{P}}=4.9 \mathrm{~Hz}, \mathrm{CH}\right), 128.3(\mathrm{CH}), 128.2(\mathrm{CH}), 127.0(\mathrm{CH}), 127.0(\mathrm{CH}), 126.5\left(\mathrm{C}_{\mathrm{q}}\right), 126.4$ $\left(\mathrm{d},{ }^{3} J_{\mathrm{C}-\mathrm{F}}=6.8 \mathrm{~Hz}, \mathrm{C}_{\mathrm{q}}\right), 126.1(\mathrm{CH}), 125.9\left(\mathrm{~d},{ }^{2} J_{\mathrm{C}-\mathrm{F}}=20.5 \mathrm{~Hz}, \mathrm{C}_{\mathrm{q}}\right), 125.6(\mathrm{CH}), 123.8(\mathrm{CH})$, $121.9\left(\mathrm{~d},{ }^{2} J_{\mathrm{C}-\mathrm{F}}=23.5 \mathrm{~Hz}, \mathrm{CH}\right), 113.3\left(\mathrm{~d},{ }^{2} J_{\mathrm{C}-\mathrm{F}}=23.0 \mathrm{~Hz}, \mathrm{CH}\right), 108.0\left(\mathrm{~d},{ }^{3} J_{\mathrm{C}-\mathrm{P}}=7.2 \mathrm{~Hz}\right.$, $\mathrm{CH}), 100.8\left(\mathrm{~d},{ }^{3} J_{\mathrm{C}-\mathrm{P}}=6.6 \mathrm{~Hz}, \mathrm{CH}\right), 35.1\left(\mathrm{~d},{ }^{1} J_{\mathrm{C}-\mathrm{P}}=64.8 \mathrm{~Hz}, \mathrm{CH}_{2}\right), 34.3\left(\mathrm{~d},{ }^{1} J_{\mathrm{C}-\mathrm{P}}=65.6 \mathrm{~Hz}\right.$, $\left.\mathrm{CH}_{2}\right)$.

${ }^{19} \mathbf{F}-\mathbf{N M R}\left(376 \mathrm{MHz}, \mathrm{CDCl}_{3}\right): \delta=(-112.6)-(-112.5)\left(\mathrm{m}, \mathrm{F}_{\mathrm{A}}\right),-122.5(\mathrm{dd}, J=9.7,5.3 \mathrm{~Hz}$, $\mathrm{F}_{\mathrm{B}) \text {. }}$

${ }^{31} \mathbf{P}\left\{{ }^{1} \mathbf{H}\right\}$-NMR $\left(162 \mathrm{MHz}, \mathrm{CDCl}_{3}\right): \delta=27.6$.

IR (ATR): $\tilde{v}=1672,1602,1490,1430,1260,1186,854,726,526 \mathrm{~cm}^{-1}$.

MS (EI) $m / z$ (relative intensity): 470 (30) [M] $]^{+}, 453$ (40), 319 (10), 252 (100), 223 (15), 201 (75), 77 (25).

HR-MS (EI) $m / z$ calcd. for $\mathrm{C}_{27} \mathrm{H}_{20} \mathrm{FN}_{2} \mathrm{O}_{3} \mathrm{P}[\mathrm{M}]^{+}: 470.1196$, found: 470.1201 .<smiles>Cc1cc(C)c2c(=O)n(-c3cccc[n+]3[O-])c(CP(=O)(c3ccccc3)c3ccccc3)cc2c1</smiles>

\section{2-\{3-[(Diphenylphosphoryl)methyl]-6,8-dimethyl-1-oxoisoquinolin-2(1H)-yl\}pyridine}

1-oxide (176sa): The general procedure $\mathbf{D}$ was followed using benzamide 34s $(121 \mathrm{mg}$, $0.50 \mathrm{mmol}$ ) and allene 75a (144 $\mathrm{mg}, 0.60 \mathrm{mmol})$. Purification by column chromatography on silica gel $\left(\mathrm{CH}_{2} \mathrm{Cl}_{2} /\right.$ acetone $1: 1$ to $\mathrm{CH}_{2} \mathrm{Cl}_{2} / \mathrm{MeOH}$ 9:1) yielded 176sa (175 mg, $364 \mu \mathrm{mol}$, $73 \%)$ as a white solid.

M. p.: $148-149{ }^{\circ} \mathrm{C}$.

${ }^{1}$ H-NMR (500 MHz, $\left.\mathrm{CDCl}_{3}\right): \delta=8.36-8.31(\mathrm{~m}, 1 \mathrm{H}), 7.74-7.70(\mathrm{~m}, 2 \mathrm{H}), 7.60-7.55$ (m, 1H), 7.52-7.45 (m, 5H), 7.45-7.42 (m, 1H), 7.41-7.36 (m, 2H), 7.33-7.27 (m, 2H), 6.99 (d, $J=1.7 \mathrm{~Hz}, 1 \mathrm{H}), 6.87(\mathrm{~d}, J=1.7 \mathrm{~Hz}, 1 \mathrm{H}), 6.24$ (d, $J=3.2 \mathrm{~Hz}, 1 \mathrm{H}), 3.61$ (dd, $J=16.0$, $11.5 \mathrm{~Hz}, 1 \mathrm{H}), 3.04$ (dd, $J=16.0,13.6 \mathrm{~Hz}, 1 \mathrm{H}), 2.74$ (s, 3H), 2.34 (s, 3H).

${ }^{13}$ C-NMR $\left(126 \mathrm{MHz}, \mathrm{CDCl}_{3}\right): \delta=163.3\left(\mathrm{C}_{\mathrm{q}}\right), 144.3\left(\mathrm{C}_{\mathrm{q}}\right), 143.2\left(\mathrm{C}_{\mathrm{q}}\right), 142.4\left(\mathrm{C}_{\mathrm{q}}\right), 140.1$ $(\mathrm{CH}), 138.7\left(\mathrm{C}_{\mathrm{q}}\right), 133.7\left(\mathrm{C}_{\mathrm{q}}\right), 133.5\left(\mathrm{~d},{ }^{1} J_{\mathrm{C}-\mathrm{P}}=100.2 \mathrm{~Hz}, \mathrm{C}_{\mathrm{q}}\right), 132.5\left(\mathrm{~d},{ }^{2} J_{\mathrm{C}-\mathrm{P}}=7.9 \mathrm{~Hz}, \mathrm{C}_{\mathrm{q}}\right)$, 
$132.4\left(\mathrm{~d},{ }^{4} J_{\mathrm{C}-\mathrm{P}}=2.8 \mathrm{~Hz}, \mathrm{CH}\right), 132.3\left(\mathrm{~d},{ }^{4} J_{\mathrm{C}-\mathrm{P}}=2.8 \mathrm{~Hz}, \mathrm{CH}\right), 131.7(\mathrm{CH}), 131.5\left(\mathrm{~d},{ }^{2} J_{\mathrm{C}-\mathrm{P}}=\right.$ $9.2 \mathrm{~Hz}, \mathrm{CH}), 131.2\left(\mathrm{~d},{ }^{1} J_{\mathrm{C}-\mathrm{P}}=103.3 \mathrm{~Hz}, \mathrm{C}_{\mathrm{q}}\right), 130.5(\mathrm{CH}), 129.0\left(\mathrm{~d},{ }^{3} J_{\mathrm{C}-\mathrm{P}}=3.4 \mathrm{~Hz}, \mathrm{CH}\right)$, $128.8\left(\mathrm{~d},{ }^{3} J_{\mathrm{C}-\mathrm{P}}=3.4 \mathrm{~Hz}, \mathrm{CH}\right), 126.0(\mathrm{CH}), 125.9(\mathrm{CH}), 124.5(\mathrm{CH}), 121.1\left(\mathrm{C}_{\mathrm{q}}\right), 109.4(\mathrm{~d}$, $\left.{ }^{3} J_{\mathrm{C}-\mathrm{P}}=7.0 \mathrm{~Hz}, \mathrm{CH}\right), 34.2\left(\mathrm{~d},{ }^{1} J_{\mathrm{C}-\mathrm{P}}=66.3 \mathrm{~Hz}, \mathrm{CH}_{2}\right), 23.5\left(\mathrm{CH}_{3}\right), 21.6\left(\mathrm{CH}_{3}\right)$.

${ }^{31} \mathbf{P}\left\{{ }^{1} \mathbf{H}\right\}$-NMR $\left(121 \mathrm{MHz}, \mathrm{CDCl}_{3}\right): \delta=27.6$.

IR (ATR): $\tilde{v}=2920,1666,1561,1263,1195,860,729,516 \mathrm{~cm}^{-1}$.

MS (ESI) $m / z$ (relative intensity): $503(60)[\mathrm{M}+\mathrm{Na}]^{+}, 481(100)[\mathrm{M}+\mathrm{H}]^{+}$.

HR-MS (ESI) $m / z$ calcd. for $\mathrm{C}_{29} \mathrm{H}_{25} \mathrm{~N}_{2} \mathrm{O}_{3} \mathrm{P}[\mathrm{M}+\mathrm{H}]^{+}: 481.1676$, found: 481.1673 .

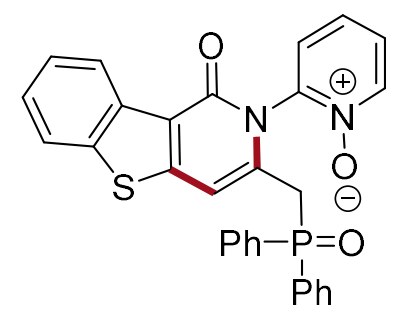

2-\{3-[(Diphenylphosphoryl)methyl]-1-oxobenzo[4,5] thieno[3,2-c]pyridin-2(1H)-

yl\}pyridine 1-oxide (176ta): The general procedure $D$ was followed using benzamide 34t (135 mg, $0.50 \mathrm{mmol})$ and allene $75 \mathrm{a}(144 \mathrm{mg}, 0.60 \mathrm{mmol})$. Purification by column chromatography on silica gel $\left(\mathrm{CH}_{2} \mathrm{Cl}_{2} /\right.$ acetone $1: 1$ to $\mathrm{CH}_{2} \mathrm{Cl}_{2} / \mathrm{MeOH}$ 9:1) yielded 176ta (135 mg, $266 \mu \mathrm{mol}, 53 \%)$ as a white solid.

M. p.: $206-208^{\circ} \mathrm{C}$.

${ }^{1}$ H-NMR $\left(400 \mathrm{MHz}, \mathrm{CDCl}_{3}\right): \delta=8.35(\mathrm{~d}, J=6.4 \mathrm{~Hz}, 1 \mathrm{H}), 7.91(\mathrm{~d}, J=8.1 \mathrm{~Hz}, 1 \mathrm{H}), 7.81-$ $7.68(\mathrm{~m}, 3 \mathrm{H}), 7.63-7.57(\mathrm{~m}, 1 \mathrm{H}), 7.57-7.48(\mathrm{~m}, 6 \mathrm{H}), 7.48-7.38(\mathrm{~m}, 4 \mathrm{H}), 7.38-7.28(\mathrm{~m}$, $2 \mathrm{H}), 6.86-6.75(\mathrm{~m}, 1 \mathrm{H}), 3.86-3.74(\mathrm{~m}, 1 \mathrm{H}), 3.27-3.10(\mathrm{~m}, 1 \mathrm{H})$.

${ }^{13}$ C-NMR (126 MHz, $\left.\mathrm{CDCl}_{3}\right): \delta=158.7\left(\mathrm{C}_{\mathrm{q}}\right), 143.2\left(\mathrm{C}_{\mathrm{q}}\right), 142.9\left(\mathrm{C}_{\mathrm{q}}\right), 141.6\left(\mathrm{C}_{\mathrm{q}}\right), 140.0$ $(\mathrm{CH}), 135.6\left(\mathrm{~d},{ }^{2} J_{\mathrm{C}-\mathrm{P}}=7.8 \mathrm{~Hz}, \mathrm{C}_{\mathrm{q}}\right), 134.8\left(\mathrm{C}_{\mathrm{q}}\right), 132.5\left(\mathrm{~d},{ }^{4} J_{\mathrm{C}-\mathrm{P}}=2.3 \mathrm{~Hz}, \mathrm{CH}\right), 132.4\left(\mathrm{~d},{ }^{4} J_{\mathrm{C}-}\right.$ $\mathrm{P}=2.1 \mathrm{~Hz}, \mathrm{CH}) 132.3\left(\mathrm{~d},{ }^{1} J_{\mathrm{C}-\mathrm{P}}=100.8 \mathrm{~Hz}, \mathrm{C}_{\mathrm{q}}\right), 131.5\left(\mathrm{~d},{ }^{2} J_{\mathrm{C}-\mathrm{P}}=7.8 \mathrm{~Hz}, \mathrm{CH}\right), 131.2(\mathrm{~d}$, $\left.{ }^{1} J_{\mathrm{C}-\mathrm{P}}=101.5 \mathrm{~Hz}, \mathrm{C}_{\mathrm{q}}\right), 130.7\left(\mathrm{~d},{ }^{2} J_{\mathrm{C}-\mathrm{P}}=8.8 \mathrm{~Hz}, \mathrm{CH}\right), 130.3(\mathrm{CH}), 129.0\left(\mathrm{~d},{ }^{3} J_{\mathrm{C}-\mathrm{P}}=4.1 \mathrm{~Hz}\right.$, $\mathrm{CH}), 128.9\left(\mathrm{~d},{ }^{3} J_{\mathrm{C}-\mathrm{P}}=4.1 \mathrm{~Hz}, \mathrm{CH}\right), 128.5\left(\mathrm{C}_{\mathrm{q}}\right), 128.4(\mathrm{CH}), 126.4(\mathrm{CH}), 125.7(\mathrm{CH}), 124.9$ $(\mathrm{CH}), 123.5(\mathrm{CH}), 123.3(\mathrm{CH}), 103.6\left(\mathrm{~d},{ }^{3} J_{\mathrm{C}-\mathrm{P}}=5.9 \mathrm{~Hz}, \mathrm{CH}\right), 35.1\left(\mathrm{~d},{ }^{1} J_{\mathrm{C}-\mathrm{P}}=64.2 \mathrm{~Hz}\right.$, $\mathrm{CH}_{2}$ ).

${ }^{31} \mathbf{P}\left\{{ }^{1} \mathbf{H}\right\}$-NMR $\left(162 \mathrm{MHz}, \mathrm{CDCl}_{3}\right): \delta=27.5$.

IR (ATR): 1661, 1586, 1489, 1268, 1199, 852, 710, $525 \mathrm{~cm}^{-1}$.

MS (EI) $m / z$ (relative intensity): 508 (10) [M] $]^{+}, 492$ (20), 319 (10), 290 (100), 261 (12), 201 (35), 77 (12). 
HR-MS (EI) $m / z$ calcd. for $\mathrm{C}_{29} \mathrm{H}_{21} \mathrm{~N}_{2} \mathrm{O}_{3} \mathrm{PS}[\mathrm{M}]^{+}:$508.1010, found: 508.1020.<smiles></smiles>

\section{6-[(Diphenylphosphoryl)methyl]-3,4-dimethyl-2-oxo-2 $H$-[1,2'-bipyridine] $\quad$ 1'-oxide}

(182aa): The general procedure $\mathbf{D}$ was followed using alkane $37 \mathbf{a}(96 \mathrm{mg}, 0.50 \mathrm{mmol})$ and allene 75a (144 mg, $0.60 \mathrm{mmol})$. Purification by column chromatography on silica gel $\left(\mathrm{CH}_{2} \mathrm{Cl}_{2}\right.$ /acetone 1:1 to $\left.\mathrm{CH}_{2} \mathrm{Cl}_{2} / \mathrm{MeOH} 9: 1\right)$ yielded 182aa (140 mg, $\left.326 \mu \mathrm{mol}, 65 \%\right)$ as a white solid.

M. p.: $131-132{ }^{\circ} \mathrm{C}$.

${ }^{1}$ H-NMR $\left(400 \mathrm{MHz}, \mathrm{CDCl}_{3}\right): \delta=8.37-8.19(\mathrm{~m}, 1 \mathrm{H}), 7.75-7.64(\mathrm{~m}, 2 \mathrm{H}), 7.62-7.54(\mathrm{~m}$, 1H), 7.53-7.41 (m, 5H), 7.41-7.34 (m, 3H), 7.33-7.26 (m, 1H), 7.24-7.16 (m, 1H), 5.85 (d, $J=3.1 \mathrm{~Hz}, 1 \mathrm{H}), 3.63-3.49$ (m, 1H), 3.10-2.95 (m, 1H), $2.00(\mathrm{~s}, 6 \mathrm{H})$.

${ }^{13}$ C-NMR (126 MHz, $\left.\mathrm{CDCl}_{3}\right): \delta=162.7\left(\mathrm{C}_{\mathrm{q}}\right), 147.0\left(\mathrm{~d},{ }^{4} J_{\mathrm{C}-\mathrm{P}}=2.4 \mathrm{~Hz}, \mathrm{C}_{\mathrm{q}}\right), 144.6\left(\mathrm{C}_{\mathrm{q}}\right)$, $140.0(\mathrm{CH}), 134.8\left(\mathrm{~d},{ }^{2} J_{\mathrm{C}-\mathrm{P}}=7.9 \mathrm{~Hz}, \mathrm{C}_{\mathrm{q}}\right), 132.4\left(\mathrm{~d},{ }^{4} J_{\mathrm{C}-\mathrm{P}}=2.8 \mathrm{~Hz}, \mathrm{CH}\right), 132.2\left(\mathrm{~d},{ }^{4} J_{\mathrm{C}-\mathrm{P}}=\right.$ $2.8 \mathrm{~Hz}, \mathrm{CH}), 132.4\left(\mathrm{~d},{ }^{1} J_{\mathrm{C}-\mathrm{P}}=100.3 \mathrm{~Hz}, \mathrm{C}_{\mathrm{q}}\right), 131.3\left(\mathrm{~d},{ }^{2} J_{\mathrm{C}-\mathrm{P}}=9.2 \mathrm{~Hz}, \mathrm{CH}\right), 131.1\left(\mathrm{~d},{ }^{1} J_{\mathrm{C}-\mathrm{P}}\right.$ $\left.=102.4 \mathrm{~Hz}, \mathrm{C}_{\mathrm{q}}\right), 130.6\left(\mathrm{~d},{ }^{2} J_{\mathrm{C}-\mathrm{P}}=9.4 \mathrm{~Hz}, \mathrm{CH}\right), 129.9(\mathrm{CH}), 128.9\left(\mathrm{~d},{ }^{3} J_{\mathrm{C}-\mathrm{P}}=3.9 \mathrm{~Hz}, \mathrm{CH}\right)$, $128.8\left(\mathrm{~d},{ }^{3} J_{\mathrm{C}-\mathrm{P}}=3.9 \mathrm{~Hz}, \mathrm{CH}\right), 125.9(\mathrm{CH}), 125.8(\mathrm{CH}), 125.4\left(\mathrm{~d},{ }^{4} J_{\mathrm{C}-\mathrm{P}}=2.9 \mathrm{~Hz}, \mathrm{C}_{\mathrm{q}}\right), 111.9$ $\left(\mathrm{d},{ }^{3} J_{\mathrm{C}-\mathrm{P}}=6.4 \mathrm{~Hz} \mathrm{CH}\right), 34.5\left(\mathrm{~d},{ }^{1} J_{\mathrm{C}-\mathrm{P}}=65.1 \mathrm{~Hz}, \mathrm{CH}_{2}\right), 20.1\left(\mathrm{CH}_{3}\right), 12.7\left(\mathrm{CH}_{3}\right)$.

${ }^{31} \mathbf{P}\left\{{ }^{1} \mathbf{H}\right\}$-NMR $\left(162 \mathrm{MHz}, \mathrm{CDCl}_{3}\right): \delta=27.4$.

IR (ATR): $\tilde{v}=1656,1550,1432,1268,1195,757,729,697 ; 489 \mathrm{~cm}^{-1}$.

MS (ESI) $m / z$ (relative intensity): $883(60)[2 \mathrm{M}+\mathrm{Na}]^{+}, 453(100)[\mathrm{M}+\mathrm{Na}]^{+}, 431$ (70) $[\mathrm{M}+\mathrm{H}]^{+}$.

HR-MS (ESI) $m / z$ calcd. for $\mathrm{C}_{25} \mathrm{H}_{24} \mathrm{~N}_{2} \mathrm{O}_{3} \mathrm{P}[\mathrm{M}+\mathrm{H}]^{+}: 431.1519$, found: 431.1515 .<smiles></smiles>

2-[3-(2-Ethoxy-2-oxoethyl)-1-oxoisoquinolin-2(1H)-yl]pyridine 1-oxide (176ac): The general procedure $\mathbf{D}$ was followed using benzamide 34a $(107 \mathrm{mg}, 0.50 \mathrm{mmol})$ and allene 
75c $(67.2 \mathrm{mg}, 0.60 \mathrm{mmol})$. Purification by column chromatography on silica gel $\left(\mathrm{CH}_{2} \mathrm{Cl}_{2}\right.$ /acetone 5:1) yielded $\mathbf{1 7 6 a c}(92.3 \mathrm{mg}, 285 \mu \mathrm{mol}, 57 \%)$ as a white solid.

M. p.: $172-174{ }^{\circ} \mathrm{C}$.

${ }^{1}$ H-NMR (500 MHz, $\left.\mathrm{CDCl}_{3}\right): \delta=8.39-8.31$ (m, 2H), 7.67 (ddd, $\left.J=7.9,7.2,1.3 \mathrm{~Hz}, 1 \mathrm{H}\right)$, 7.53-7.44 (m, 3H), 7.41-7.37 (m, 1H), 7.37-7.30 (m, 1H), $6.57(\mathrm{~s}, 1 \mathrm{H}), 4.06-3.92(\mathrm{~m}, 2 \mathrm{H})$, $3.53(\mathrm{~d}, J=17.3 \mathrm{~Hz}, 1 \mathrm{H}), 3.34(\mathrm{~d}, J=17.3 \mathrm{~Hz}, 1 \mathrm{H}), 1.13(\mathrm{t}, J=7.1 \mathrm{~Hz}, 3 \mathrm{H})$.

${ }^{13}$ C-NMR (126 MHz, $\left.\mathrm{CDCl}_{3}\right): \delta=169.4\left(\mathrm{C}_{\mathrm{q}}\right), 162.6\left(\mathrm{C}_{\mathrm{q}}\right), 143.9\left(\mathrm{C}_{\mathrm{q}}\right), 140.7(\mathrm{CH}), 137.1$ $\left(\mathrm{C}_{\mathrm{q}}\right), 135.2\left(\mathrm{C}_{\mathrm{q}}\right), 133.5(\mathrm{CH}), 129.4(\mathrm{CH}), 128.4(\mathrm{CH}), 127.2(\mathrm{CH}), 126.3(\mathrm{CH}), 126.2(\mathrm{CH})$, 125.5 (CH), $125.2\left(\mathrm{C}_{\mathrm{q}}\right), 108.9(\mathrm{CH}), 61.5\left(\mathrm{CH}_{2}\right), 38.8\left(\mathrm{CH}_{2}\right), 14.2\left(\mathrm{CH}_{3}\right)$.

IR (ATR): $\tilde{v}=1736,1668,1628,1491,1433,1258,1149,759,691 \mathrm{~cm}^{-1}$.

MS (EI) $m / z$ (relative intensity): 324 (50) [M] $]^{+}, 279$ (25), 251 (25), 235 (100), 205 (45), 145 (35), 78 (90).

HR-MS (EI) $m / z$ calcd. for $\mathrm{C}_{18} \mathrm{H}_{16} \mathrm{~N}_{2} \mathrm{O}_{4}[\mathrm{M}]^{+}: 324.1110$, found: 324.1115 .<smiles>O=C(Cc1cc2ccccc2c(=O)n1-c1cccc[n+]1[O-])OCc1ccccc1</smiles>

2-\{3-[2-(Benzyloxy)-2-oxoethyl]-1-oxoisoquinolin-2(1H)-yl\}pyridine 1-oxide (176ad): The general procedure $\mathbf{D}$ was followed using benzamide 34a $(107 \mathrm{mg}, 0.50 \mathrm{mmol})$ and allene 75d (104 mg, $0.60 \mathrm{mmol})$. Purification by column chromatography on silica gel $\left(\mathrm{CH}_{2} \mathrm{Cl}_{2}\right.$ /acetone 5:1) yielded 176ad (137 mg, $\left.355 \mu \mathrm{mol}, 71 \%\right)$ as a yellow solid.

M. p.: $109-110^{\circ} \mathrm{C}$.

${ }^{1}$ H-NMR (400 MHz, $\left.\mathrm{CDCl}_{3}\right): \delta=8.38-8.27$ (m, 2H), 7.67 (ddd, $\left.J=8.2,7.2,1.4 \mathrm{~Hz}, 1 \mathrm{H}\right)$, 7.52-7.44 (m, 2H), 7.37-7.31 (m, 3H), 7.30-7.20 (m, 4H), 7.07 (td, $J=7.8,1.4 \mathrm{~Hz}, 1 \mathrm{H})$, 6.57 (s, 1H), 4.99 (s, 2H), 3.58 (d, $J=17.4 \mathrm{~Hz}, 1 \mathrm{H}), 3.38$ (d, $J=17.4 \mathrm{~Hz}, 1 \mathrm{H})$.

${ }^{13}$ C-NMR $\left(101 \mathrm{MHz}, \mathrm{CDCl}_{3}\right): \delta=169.1\left(\mathrm{C}_{\mathrm{q}}\right), 162.6\left(\mathrm{C}_{\mathrm{q}}\right), 143.6\left(\mathrm{C}_{\mathrm{q}}\right), 140.5(\mathrm{CH}), 137.0$ $\left(\mathrm{C}_{\mathrm{q}}\right), 135.3\left(\mathrm{C}_{\mathrm{q}}\right), 135.0\left(\mathrm{C}_{\mathrm{q}}\right), 133.5(\mathrm{CH}), 129.3(\mathrm{CH}), 128.8(\mathrm{CH}), 128.7(\mathrm{CH}), 128.7(\mathrm{CH})$, 128.4 (CH), $127.3(\mathrm{CH}), 126.3(\mathrm{CH}), 126.3(\mathrm{CH}), 125.5(\mathrm{CH}), 125.3\left(\mathrm{C}_{\mathrm{q}}\right), 109.0(\mathrm{CH}), 67.2$ $\left(\mathrm{CH}_{2}\right), 38.9\left(\mathrm{CH}_{2}\right)$.

IR (ATR): $\tilde{v}=1732,1668,1633,1483,1255,1151,756,691 \mathrm{~cm}^{-1}$.

MS (EI) $m / z$ (relative intensity): 386 (25) [M] $]^{+}, 279$ (20), 251 (15), 235 (50), 205 (15), 91 (100), 78 (30).

HR-MS (EI) $m / z$ calcd. for $\mathrm{C}_{23} \mathrm{H}_{18} \mathrm{~N}_{2} \mathrm{O}_{4}[\mathrm{M}]^{+}: 386.1267$, found: 386.1265 . 
<smiles></smiles>

2-[3-Benzyl-1-oxoisoquinolin-2(1H)-yl]pyridine 1-oxide (176ab): The electrocatalysis was carried out in an undivided cell, with a graphite felt anode $(10 \mathrm{~mm} \times 15 \mathrm{~mm} \times 6.0 \mathrm{~mm})$, a platinum cathode $(10 \mathrm{~mm} \times 15 \mathrm{~mm} \times 0.125 \mathrm{~mm})$ and a $\mathrm{Ag}$-wire $(100 \mathrm{~mm} \times 1.0 \mathrm{~mm})$ as the reference electrode. Benzamide 34a (53.6 mg, $0.25 \mathrm{mmol}, 1.00$ equiv), allene $75 \mathbf{b}$ (116 mg, $1.00 \mathrm{mmol}, 4.00$ equiv), NaOPiv (62.1 mg, $1.00 \mathrm{mmol}, 2.00$ equiv) and $\mathrm{Co}(\mathrm{OAc})_{2} \cdot 4 \mathrm{H}_{2} \mathrm{O}(12.7 \mathrm{mg}, 20 \mathrm{~mol} \%)$ were placed in a $10 \mathrm{~mL}$ cell and dissolved in $\mathrm{MeOH}$ $(7.0 \mathrm{~mL})$. Electrolysis was performed at $40{ }^{\circ} \mathrm{C}$ with a constant potential of $1.25 \mathrm{~V} v s$. $\mathrm{Ag} / \mathrm{Ag}^{+}$maintained for $3 \mathrm{~h}\left(Q \cdot \mathrm{mol}^{-1}=3.88 \mathrm{~F}\right)$. At ambient temperature, the electrolysis was stopped, $\mathrm{H}_{2} \mathrm{O}(10 \mathrm{~mL})$ was added, and the graphite felt anode was washed with $\mathrm{CH}_{2} \mathrm{Cl}_{2}$ $(10 \mathrm{~mL})$ in an ultrasonic bath. The combined phases were extracted with $\mathrm{CH}_{2} \mathrm{Cl}_{2}(3 \times$ $10 \mathrm{~mL}$ ), and then dried over $\mathrm{Na}_{2} \mathrm{SO}_{4}$. Evaporation of the solvents and subsequent column chromatography on silica gel using, a mixture of $\mathrm{CH}_{2} \mathrm{Cl}_{2}$ /acetone $(5: 1)$ as the eluent yielded 176ab (42.3 mg, $128 \mu \mathrm{mol}, 51 \%)$ as a white solid.

M. p.: $208-209^{\circ} \mathrm{C}$.

${ }^{1}$ H-NMR (400 MHz, $\left.\mathrm{CDCl}_{3}\right): \delta=8.40-8.31(\mathrm{~m}, 2 \mathrm{H}), 7.66(\mathrm{ddd}, J=8.2,7.2,1.4 \mathrm{~Hz}, 1 \mathrm{H})$, 7.50-7.42 (m, 2H), 7.29 (ddd, $J=8.5,6.6,2.0 \mathrm{~Hz}, 1 \mathrm{H}), 7.22-7.17$ (m, 3H), 7.10-7.03 (m, 1H), 6.98-6.90 (m, 3H), 6.42 (s, 1H), 3.85 (d, $J=16.3 \mathrm{~Hz}, 1 \mathrm{H}), 3.60$ (d, $J=16.3 \mathrm{~Hz}, 1 \mathrm{H})$. ${ }^{13}$ C-NMR (101 MHz, $\left.\mathrm{CDCl}_{3}\right): \delta=162.9\left(\mathrm{C}_{\mathrm{q}}\right), 144.3\left(\mathrm{C}_{\mathrm{q}}\right), 141.3\left(\mathrm{C}_{\mathrm{q}}\right), 140.5(\mathrm{CH}), 137.3$ $\left(\mathrm{C}_{\mathrm{q}}\right), 136.7\left(\mathrm{C}_{\mathrm{q}}\right), 133.4(\mathrm{CH}), 128.8(\mathrm{CH}), 128.7(\mathrm{CH}), 128.5(\mathrm{CH}), 128.4(\mathrm{CH}), 127.2(\mathrm{CH})$, $126.8(\mathrm{CH}), 126.0(\mathrm{CH}), 125.9(\mathrm{CH}), 125.2(\mathrm{CH}), 124.9\left(\mathrm{C}_{\mathrm{q}}\right), 107.8(\mathrm{CH}), 39.1\left(\mathrm{CH}_{2}\right)$.

IR (ATR): $\tilde{v}=1660,1627,1431,1273,904,860,759,693 \mathrm{~cm}^{-1}$.

MS (EI) $m / z$ (relative intensity): 328 (10) [M] $]^{+}, 311$ (70), 249 (60), 235 (100), 209 (45), 168 (25), 78 (90).

HR-MS (ESI) $m / z$ calcd. for $\mathrm{C}_{21} \mathrm{H}_{17} \mathrm{~N}_{2} \mathrm{O}_{2}[\mathrm{M}+\mathrm{H}]^{+}: 329.1285$, found: 329.1283 . 


\subsubsection{Multigram-Scale Synthesis of 176na}

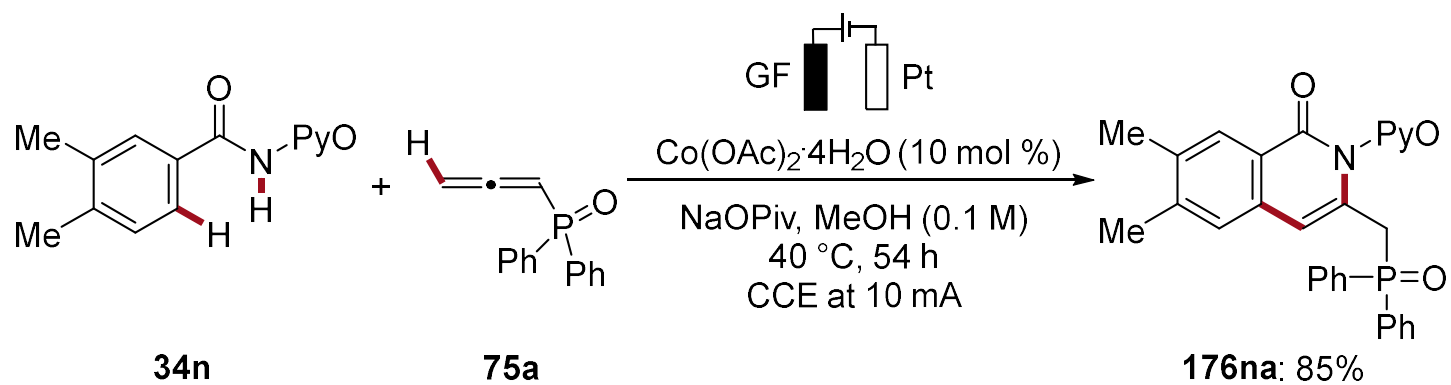

Benzamide 34n (2.18 g, 9.00 mmol, 1.00 equiv), allene 75a (2.59 g, 1.20 equiv), NaOPiv $\left(2.23 \mathrm{~g}, 2.00\right.$ equiv) and $\mathrm{Co}(\mathrm{OAc})_{2} \cdot 4 \mathrm{H}_{2} \mathrm{O}(234 \mathrm{mg}, 10 \mathrm{~mol} \%)$ were placed in an undivided cell $(100 \mathrm{~mL})$ together with a graphite felt anode $(25 \mathrm{~mm} \times 50 \mathrm{~mm} \times 6.0 \mathrm{~mm})$ and a platinum cathode $(25 \mathrm{~mm} \times 50 \mathrm{~mm} \times 0.125 \mathrm{~mm})$. The components were dissolved in $\mathrm{MeOH}(90 \mathrm{~mL})$. Electrocatalysis was performed at $40{ }^{\circ} \mathrm{C}$ with a constant current of $10 \mathrm{~mA}$ maintained for $54 \mathrm{~h}\left(Q \cdot \mathrm{mol}^{-1}=2.24 \mathrm{~F}\right)$. At ambient temperature, $\mathrm{H}_{2} \mathrm{O}(200 \mathrm{~mL})$ was added, and the graphite felt anode was washed with $\mathrm{CH}_{2} \mathrm{Cl}_{2}(2 \times 50 \mathrm{~mL})$ in an ultrasonic bath. The combined phases were extracted with $\mathrm{CH}_{2} \mathrm{Cl}_{2}(3 \times 150 \mathrm{~mL})$, and then dried over $\mathrm{Na}_{2} \mathrm{SO}_{4}$. Evaporation of the solvents and subsequent column chromatography $\left(\mathrm{CH}_{2} \mathrm{Cl}_{2} /\right.$ acetone $1: 1$ to $\mathrm{CH}_{2} \mathrm{Cl}_{2} / \mathrm{MeOH} 9: 1$ ) yielded 176na (3.66 g, $7.62 \mathrm{mmol}, 85 \%$ ) as a white solid.

\subsubsection{H/D Exchange Experiments}<smiles>O=C(NP)c1ccccc1</smiles><smiles>O=P(C=CC=CCO)(c1ccccc1)c1ccccc1</smiles>

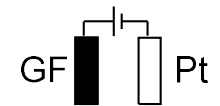<smiles>O=C(O)c1ccccc1C(=O)N[Pb]</smiles>

$[D]_{n}-34 a: 13 \%$

$34 a$ $176 a$ $\frac{\mathrm{Co}(\mathrm{OAc})_{2} \cdot 4 \mathrm{H}_{2} \mathrm{O}(10 \mathrm{~mol} \%)}{\mathrm{NaOPiv}, \mathrm{CD}_{3} \mathrm{OD}}$

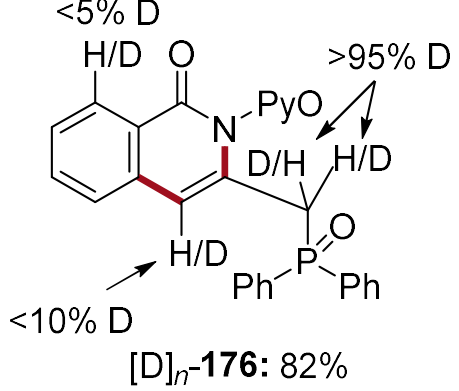

Benzamide 34a (107 mg, $0.50 \mathrm{mmol}, 1.00$ equiv), allene 75a (144 mg, 1.20 equiv), NaOPiv (124 mg, $1.00 \mathrm{mmol}, 2.00$ equiv) and $\mathrm{Co}(\mathrm{OAc})_{2} \cdot 4 \mathrm{H}_{2} \mathrm{O}(12.7 \mathrm{mg}, 10 \mathrm{~mol} \%)$ were placed in 
a $10 \mathrm{~mL}$ undivided cell together with a graphite felt anode and a platinum cathode. The components were dissolved in $\mathrm{CD}_{3} \mathrm{OD}(5.0 \mathrm{~mL})$. Electrocatalysis was performed at $40{ }^{\circ} \mathrm{C}$ with a constant current of $2 \mathrm{~mA}$ maintained for $6 \mathrm{~h}$. At ambient temperature, $\mathrm{H}_{2} \mathrm{O}(10 \mathrm{~mL})$ was added, and the graphite felt anode was washed with $\mathrm{CH}_{2} \mathrm{Cl}_{2}(10 \mathrm{~mL})$ in an ultrasonic bath. The combined phases were extracted with $\mathrm{CH}_{2} \mathrm{Cl}_{2}(3 \times 10 \mathrm{~mL})$, and then dried over $\mathrm{Na}_{2} \mathrm{SO}_{4}$. Evaporation of the solvents and subsequent column chromatography $\left(\mathrm{CH}_{2} \mathrm{Cl}_{2}\right.$ /acetone 1:1 to $\left.\mathrm{CH}_{2} \mathrm{Cl}_{2} / \mathrm{MeOH} 9: 1\right)$ yielded $[\mathrm{D}]_{n}-34 \mathrm{a}(14.1 \mathrm{mg}, 13 \%)$ as a white solid and $[\mathrm{D}]_{n}$-176aa $(81.9 \mathrm{mg}, 82 \%)$ as a white solid. The D-incorporation was estimated by ${ }^{1} \mathrm{H}-\mathrm{NMR}$ spectroscopy.

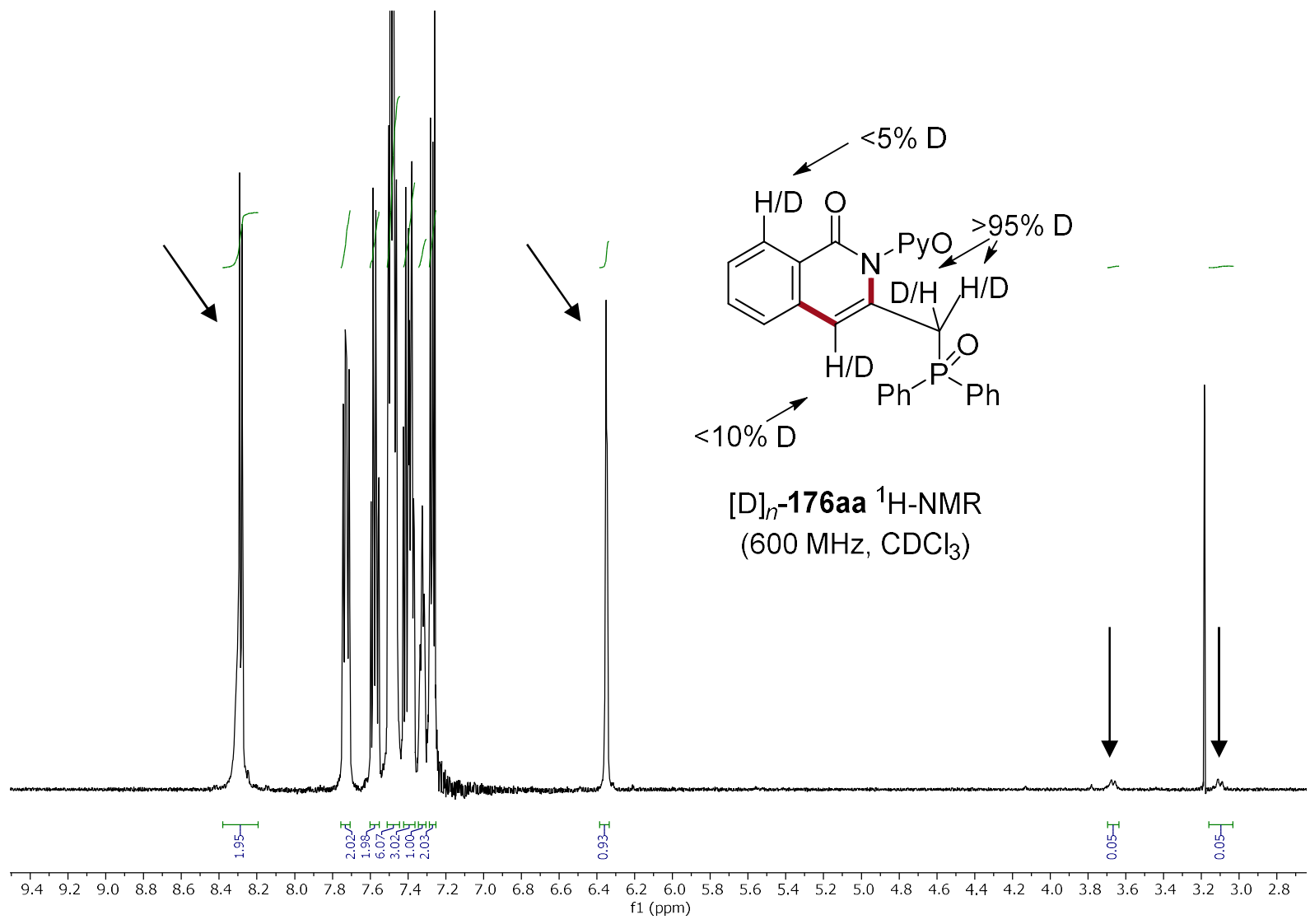

Figure 40. $\quad{ }^{1} \mathrm{H}-\mathrm{NMR}$ of $[\mathrm{D}]_{n}$-176aa from the deuteration study. 


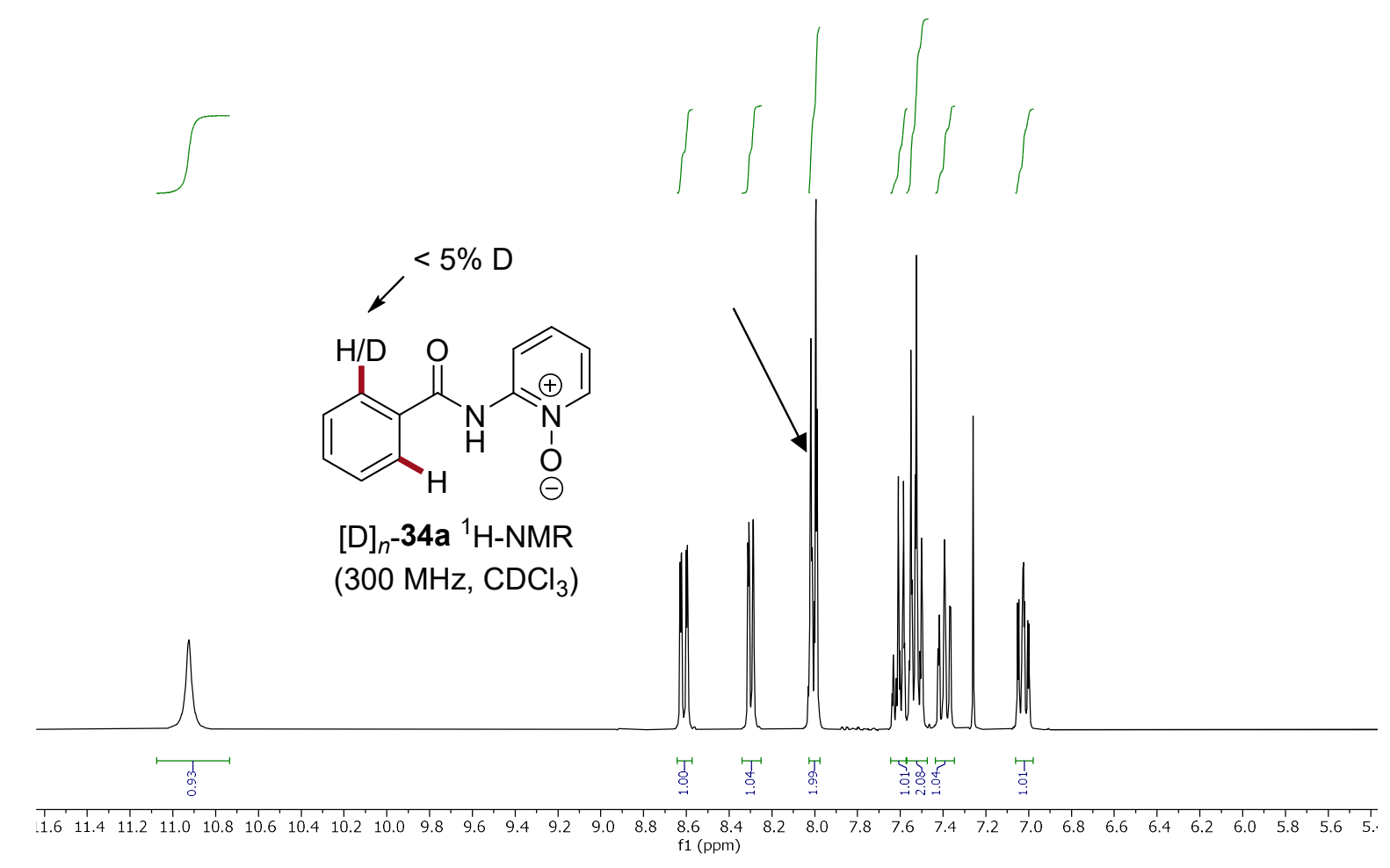

Figure 41. $\quad{ }^{1} \mathrm{H}-\mathrm{NMR}$ of reisolated $[\mathrm{D}]_{n}-\mathbf{3 4 a}$ from the deuteration study.

\subsubsection{Competition Experiments}

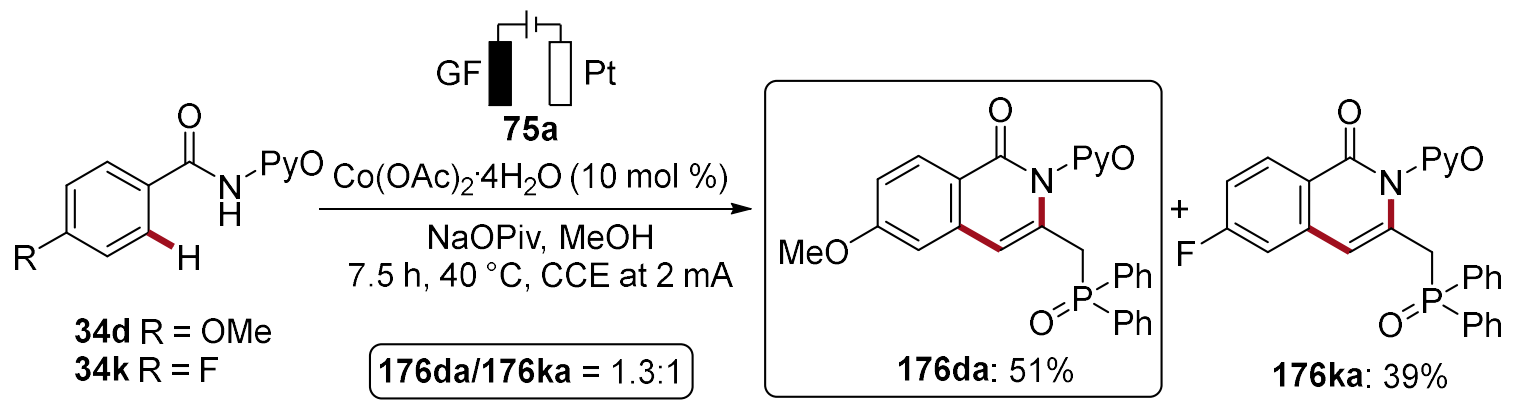

The electrocatalysis was carried out in an undivided cell, with a graphite felt anode and a platinum cathode. Benzamide 34d (61 mg, $0.25 \mathrm{mmol}$ ), benzamide 34k (58 mg, $0.25 \mathrm{mmol}$ ), allene 75a ( $0.60 \mathrm{mmol}, 1.20$ equiv), NaOPiv (124 mg, $1.00 \mathrm{mmol}, 2.00$ equiv) and $\mathrm{Co}(\mathrm{OAc})_{2} \cdot 4 \mathrm{H}_{2} \mathrm{O} \quad(12.7 \mathrm{mg}, 10 \mathrm{~mol} \%)$ were dissolved in $\mathrm{MeOH}(5.0 \mathrm{~mL})$. Electrocatalysis was performed at $40{ }^{\circ} \mathrm{C}$ with a constant current of $2 \mathrm{~mA}$ maintained for $7.5 \mathrm{~h}$. At ambient temperature, $\mathrm{H}_{2} \mathrm{O}(10 \mathrm{~mL})$ was added, and the graphite felt anode was washed with $\mathrm{CH}_{2} \mathrm{Cl}_{2}(10 \mathrm{~mL})$ in an ultrasonic bath. The combined phases were extracted with $\mathrm{CH}_{2} \mathrm{Cl}_{2}(3 \times 10 \mathrm{~mL})$, then dried over $\mathrm{Na}_{2} \mathrm{SO}_{4}$. Evaporation of the solvents and analysis of the crude mixture by ${ }^{1} \mathrm{H}-\mathrm{NMR}$ using 1,3,5-trimethoxybenzene (TMB) (14.0 mg, 
$83.3 \mu \mathrm{mol}, 0.33$ equiv) as an internal standard, showed a product distribution of 1.3:1 in favor of 176da.
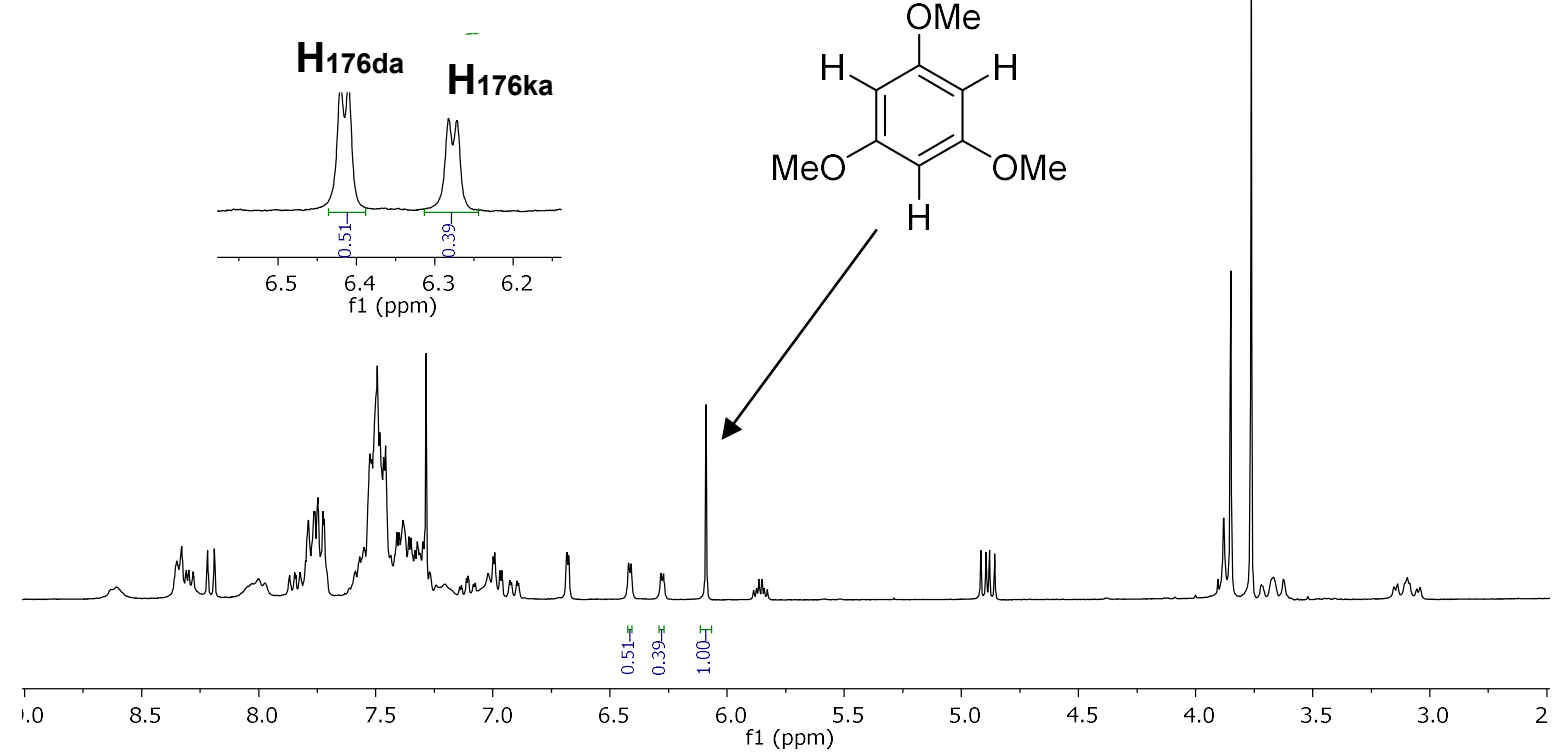

Figure 42. $\quad{ }^{1} \mathrm{H}-\mathrm{NMR}$ spectra of the crude reaction mixture of 176da and 176ka.

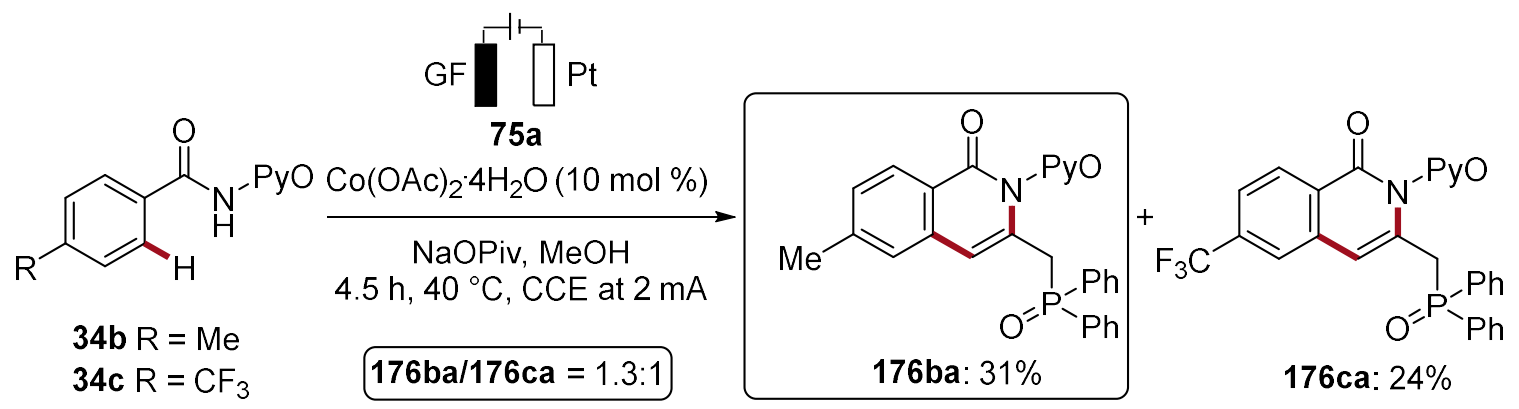

The electrocatalysis was carried out in an undivided cell, with a graphite felt anode and a platinum cathode. Benzamide 34b (34 mg, $0.15 \mathrm{mmol}$ ), benzamide 34c (42 mg, $0.15 \mathrm{mmol}$ ), allene 75a ( $86 \mathrm{mg}, 0.36 \mathrm{mmol}, 1.20$ equiv), NaOPiv (74 mg, $0.60 \mathrm{mmol}$, 2.00 equiv) and $\mathrm{Co}(\mathrm{OAc})_{2} \cdot 4 \mathrm{H}_{2} \mathrm{O}(12.7 \mathrm{mg}, 10 \mathrm{~mol} \%)$ were dissolved in $\mathrm{MeOH}(5.0 \mathrm{~mL})$. Electrocatalysis was performed at $40{ }^{\circ} \mathrm{C}$ with a constant current of $2 \mathrm{~mA}$ maintained for $4.5 \mathrm{~h}$. At ambient temperature, $\mathrm{H}_{2} \mathrm{O}(10 \mathrm{~mL})$ was added, and the graphite felt anode was washed with $\mathrm{CH}_{2} \mathrm{Cl}_{2}(10 \mathrm{~mL})$ in an ultrasonic bath. The combined phases were extracted with $\mathrm{CH}_{2} \mathrm{Cl}_{2}(3 \times 10 \mathrm{~mL})$, then dried over $\mathrm{Na}_{2} \mathrm{SO}_{4}$. Evaporation of the solvents and analysis 
of the crude mixture by ${ }^{1} \mathrm{H}-\mathrm{NMR}$ using 1,3,5-trimethoxybenzene (TMB) (8.4 mg, $50.0 \mu \mathrm{mol}, 0.33$ equiv) as an internal standard, showed a product distribution of 1.3:1 in favor of 176ba.
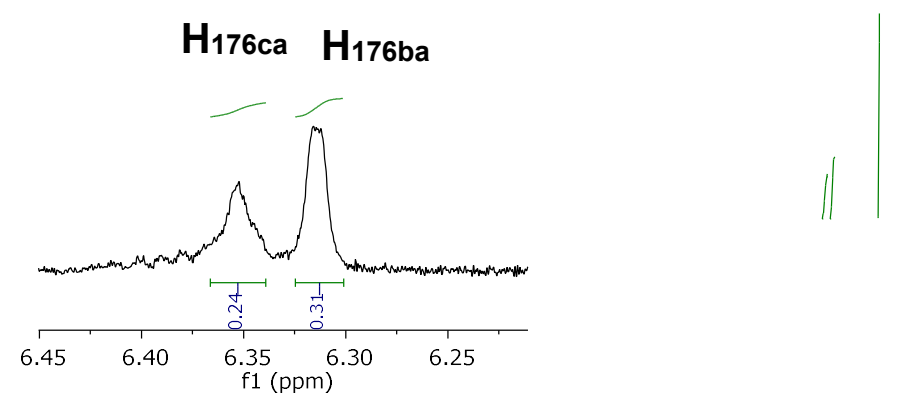<smiles>COc1ccc(OC)cc1</smiles>

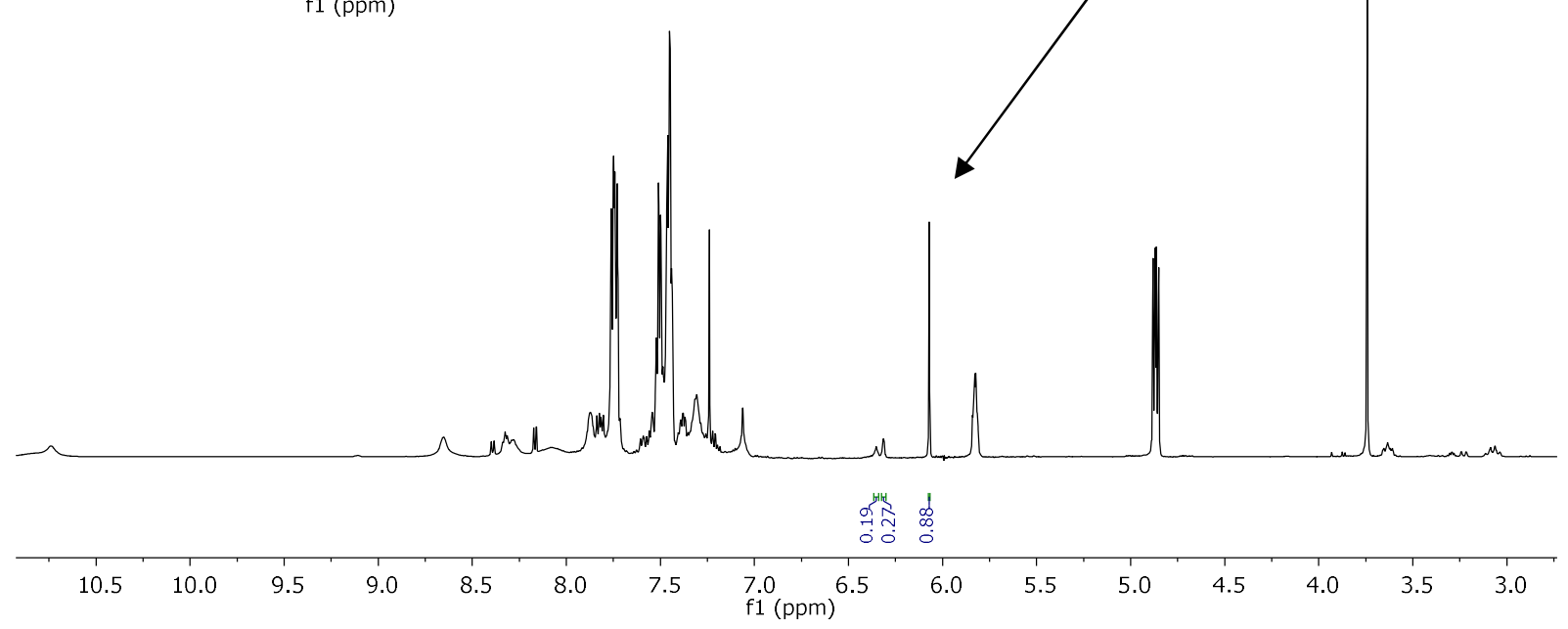

Figure 43. $\quad{ }^{1} \mathrm{H}-\mathrm{NMR}$ spectra of the crude reaction mixture of $\mathbf{1 7 6 b a}$ and 176 ca.

\subsubsection{Reaction Profile}

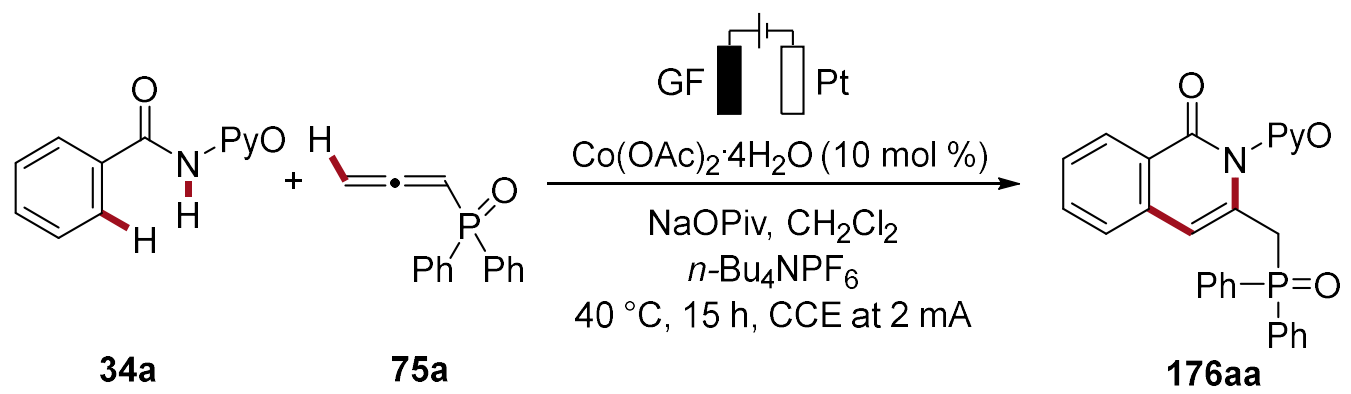

The electrocatalysis was carried out in an undivided cell, with a graphite felt anode and a platinum cathode. Benzamide 34a (107 mg, $0.50 \mathrm{mmol}, 1.00$ equiv), allene 75a (144 mg, 1.20 equiv), $\mathrm{NaOPiv}$ (124 mg, $1.00 \mathrm{mmol}, 2.00$ equiv), $n-\mathrm{Bu}_{4} \mathrm{NPF}_{6}$ (387 mg, 2.00 equiv) and $\mathrm{Co}(\mathrm{OAc})_{2} \cdot 4 \mathrm{H}_{2} \mathrm{O}(12.7 \mathrm{mg}, 10 \mathrm{~mol} \%)$ were placed in a $10 \mathrm{~mL}$ cell and dissolved in $\mathrm{CH}_{2} \mathrm{Cl}_{2}(10 \mathrm{~mL})$. A MetTler TOLEDO ReactIR was used. The solution was heated for 
20 min to $40{ }^{\circ} \mathrm{C}$, until a stable temperature was achieved. Then, electrocatalysis was performed at a constant current of $2 \mathrm{~mA}$ maintained for $16 \mathrm{~h}$. Every minute (first $8 \mathrm{~h}$ ) and every two minutes (further $8 \mathrm{~h}$ ) an IR spectrum was recorded. After the first $2 \mathrm{~h}$, the product conversion was determined by ${ }^{1} \mathrm{H}-\mathrm{NMR}$ spectroscopy using 1,3,5-trimethoxybenzene (TMB) as an internal standard to correlate the signal intensity for the initial rate studies. Signals at $1510 \mathrm{~cm}^{-1}$ and $1675 \mathrm{~cm}^{-1}$ were identified to originate from the starting material and the product, respectively (Figures 44 and 45).

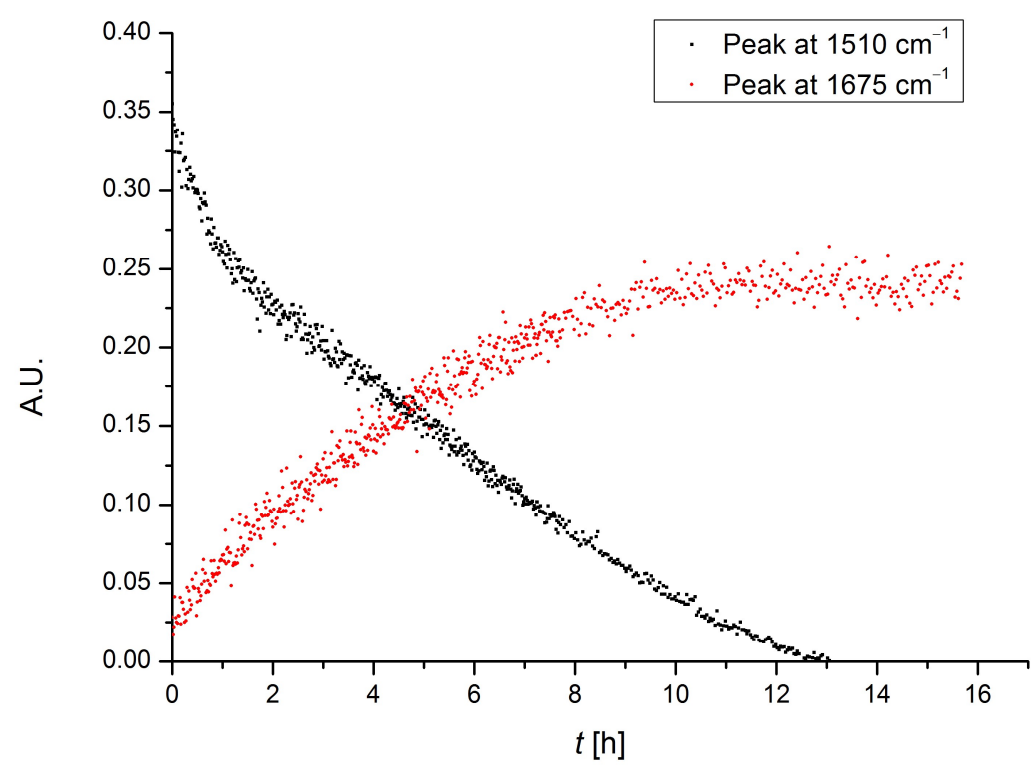

Figure 44. Plot of the observed vibrations of interest over time.

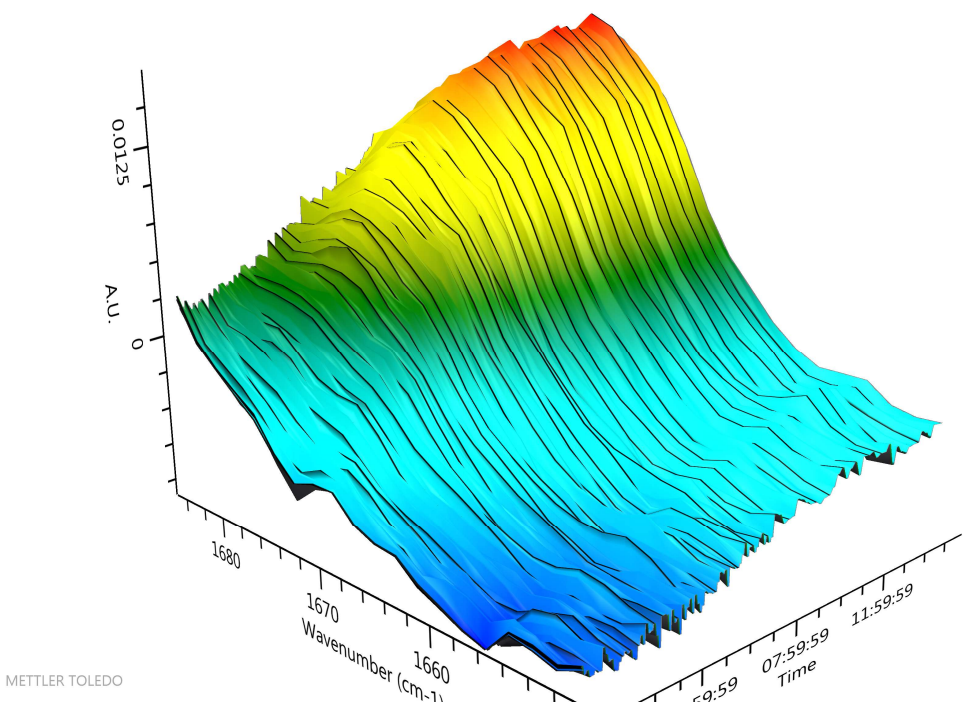

Figure 45. 3D-Surface plot of one of the observed vibrations at $1675 \mathrm{~cm}^{-1}$. 


\subsubsection{Kinetic Isotope Effect}

\subsubsection{KIE Studies by ${ }^{1} \mathrm{H}-\mathrm{NMR}$ Analysis}

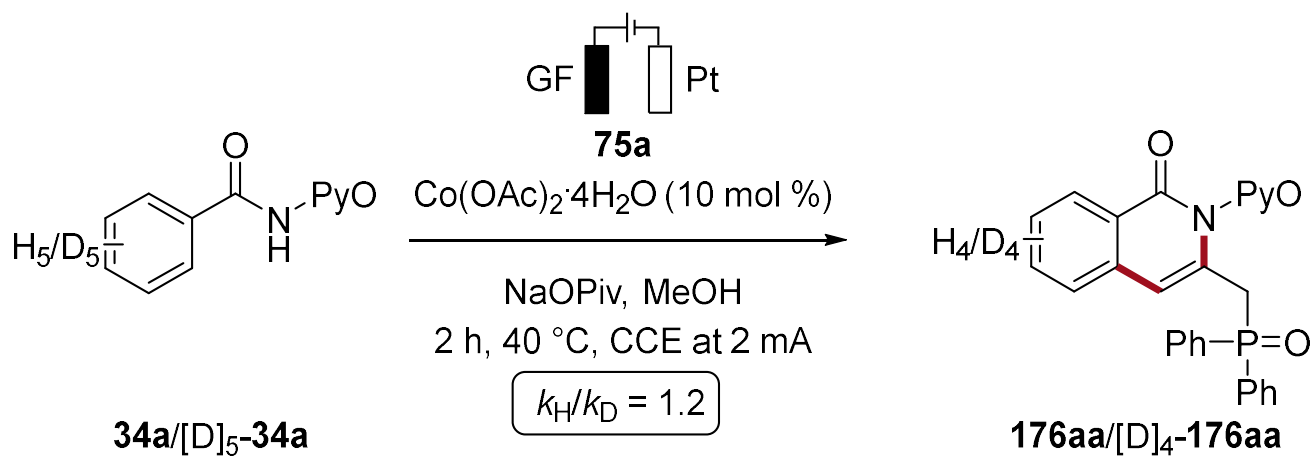

Two parallel reactions using substrates 34a and $[D]_{5}-34 \mathbf{a}(0.50 \mathrm{mmol}$ each) were carried out to determine the kinetic isotope effect (KIE) by comparison of the initial rates. After 5 minutes, aliquots of $0.2 \mathrm{~mL}$ were removed from the cell. The mixture was extracted with $\mathrm{CH}_{2} \mathrm{Cl}_{2}(3 \times 2 \mathrm{~mL})$. After evaporation of the solvents, the crude mixture was analyzed by ${ }^{1} \mathrm{H}-\mathrm{NMR}$ spectroscopy using 1,3,5-trimethoxybenzene as an internal standard. The measured yields for 176aa and $[D]_{4}-176 a a$ were plotted and a linear fit revealed a KIE of $k_{\mathrm{H}} / k_{\mathrm{D}} \approx 1.2$ (Figure 8 ).

\begin{tabular}{cccccccc}
\hline Time $[\mathrm{min}]$ & 5 & 10 & 20 & 30 & 40 & 50 & 60 \\
\hline 176aa $[\%]$ & 2.8 & 5.1 & 8.8 & 10.6 & 12.5 & 13.9 & 14.6 \\
{$[\mathrm{D}]_{4-176 a a}[\%]$} & 2.5 & 4.6 & 7.3 & 8.3 & 9.7 & 11.1 & 13.5 \\
\hline
\end{tabular}

\subsubsection{KIE Studies by In-Operando IR Analysis}

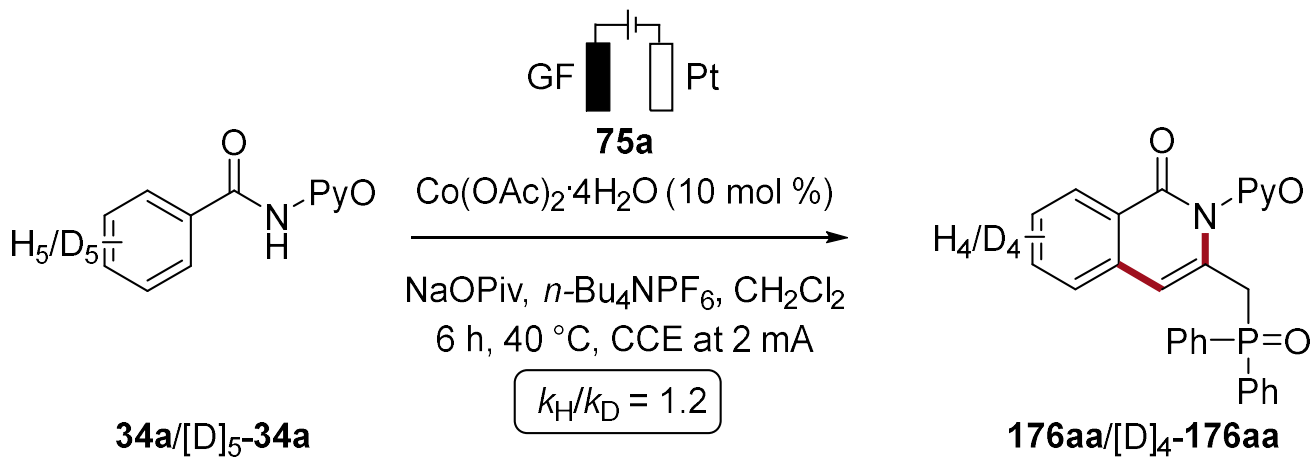

Two parallel reactions using substrates 34a and [D]5-34a $(0.50 \mathrm{mmol}$ each) were carried out, following the procedure for the ReactIR studies (vide supra), to determine the KIE. Every minute an IR spectrum was recorded. The KIE was determined by measuring the initial rates from the increase of the signal at $1675 \mathrm{~cm}^{-1}$. The measured relative conversion 
for 176aa and $[\mathrm{D}]_{4}$-176aa were plotted and a linear fit revealed a KIE of $k_{\mathrm{H}} / k_{\mathrm{D}} \approx 1.2$ (Figures 46 and 47).

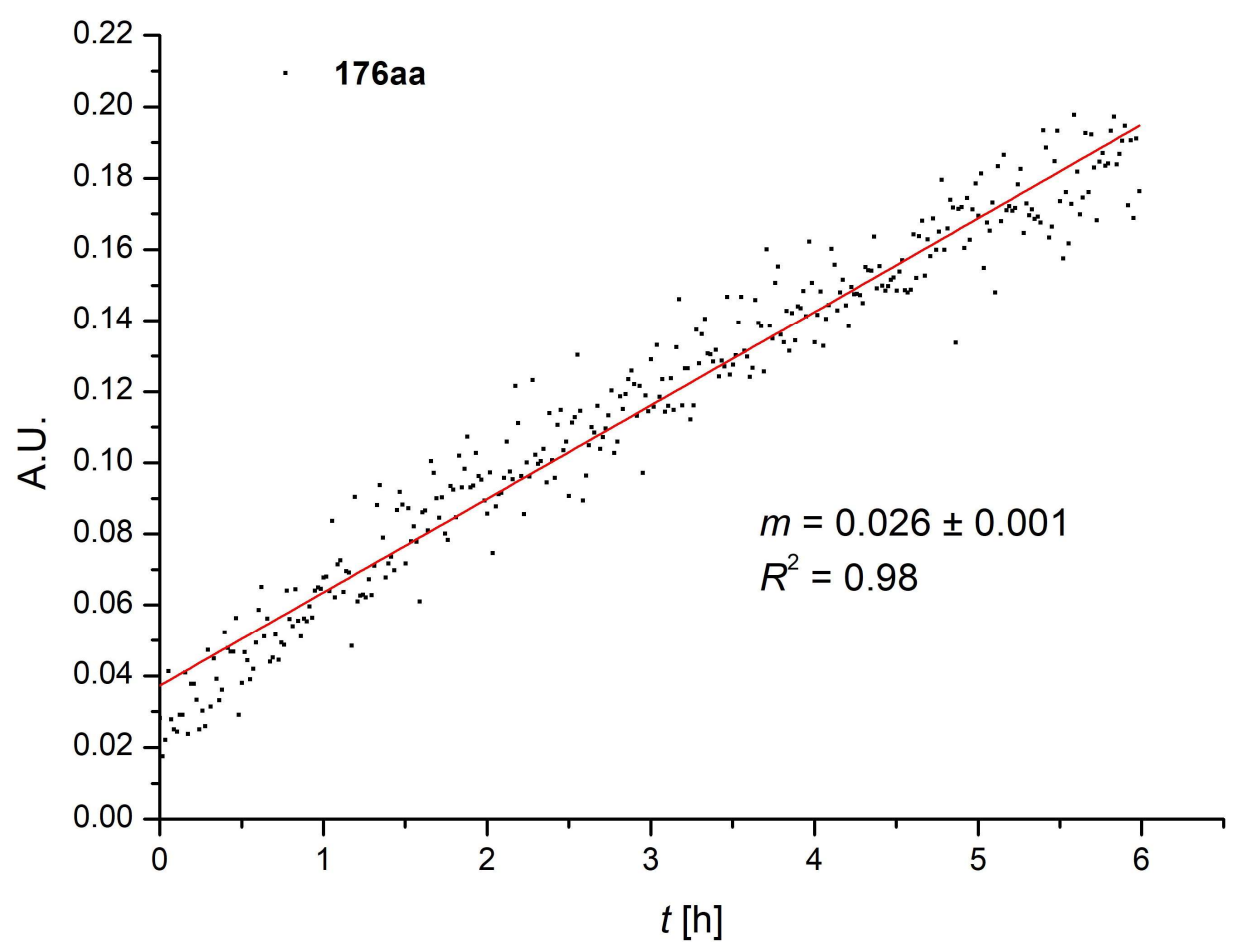

Figure 46. Initial rate analysis of 176aa.

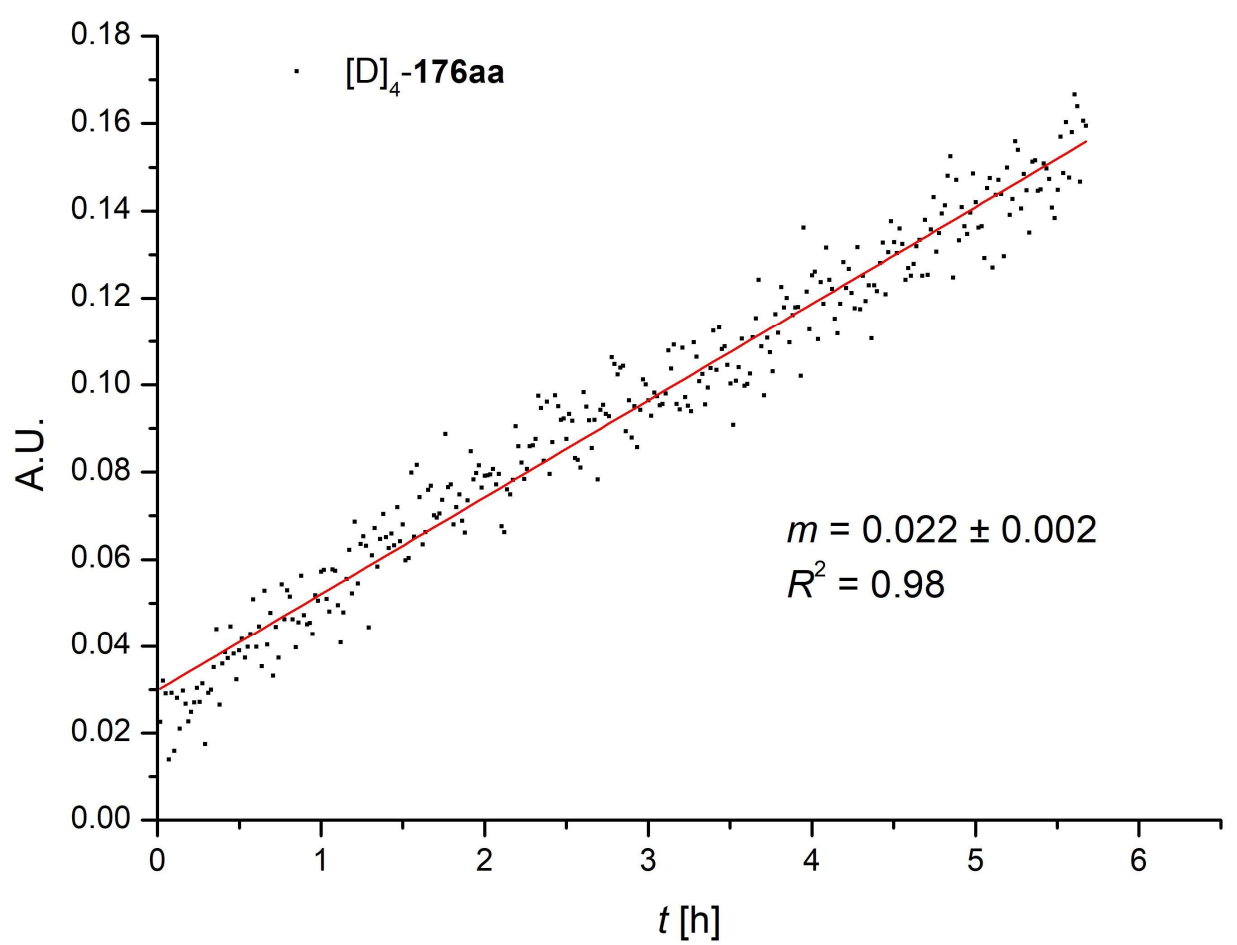

Figure 47. Initial rate analysis of $[D]_{4}-176 a a$. 


\subsection{Cobaltaelectro-Catalyzed $\mathrm{C}-\mathrm{H}$ Activation in Biomass-Derived Glycerol Powered by Renewable Energy Sources}

\subsubsection{Characterization Data}<smiles></smiles>

2-[1-Oxo-3-phenylisoquinolin-2(1H)-yl]-pyridine-1-oxide $\quad(51 \mathrm{aa}): \quad$ The general procedure E was followed using benzamide 34a (107 $\mathrm{mg}, 0.50 \mathrm{mmol})$ and alkyne 53a $(102 \mathrm{mg}, \quad 1.00 \mathrm{mmol})$. Purification by column chromatography on silica gel $\left(\mathrm{CH}_{2} \mathrm{Cl}_{2} /\right.$ acetone $\left.3: 1\right)$ yielded $51 \mathrm{aa}(145 \mathrm{mg}, 92 \%)$ as a white solid.

M. p.: $223-225^{\circ} \mathrm{C}$.

${ }^{1}$ H-NMR $\left(300 \mathrm{MHz}, \mathrm{CDCl}_{3}\right): \delta=8.46-8.37(\mathrm{~m}, 1 \mathrm{H}), 8.23-8.14(\mathrm{~m}, 1 \mathrm{H}), 7.70$ (ddd, $J=$ 8.0, 7.1, $1.4 \mathrm{~Hz}, 1 \mathrm{H}), 7.53(\mathrm{dd}, J=7.2,0.7 \mathrm{~Hz}, 1 \mathrm{H}), 7.50$ (ddd, $J=8.2,7.1,1.3 \mathrm{~Hz}, 1 \mathrm{H})$, 7.44-7.35 (m, 2H), 7.28-7.16 (m, 3H), 7.16-7.08 (m, 2H), $7.06(\mathrm{ddd}, J=8.5,6.9,1.1 \mathrm{~Hz}$, $1 \mathrm{H}), 6.61(\mathrm{~s}, 1 \mathrm{H})$.

${ }^{13}$ C-NMR (126 MHz, $\left.\mathrm{CDCl}_{3}\right): \delta=162.0\left(\mathrm{C}_{\mathrm{q}}\right), 145.3\left(\mathrm{C}_{\mathrm{q}}\right), 142.7\left(\mathrm{C}_{\mathrm{q}}\right), 139.8(\mathrm{CH}), 136.9$ $\left(\mathrm{C}_{\mathrm{q}}\right), 134.7\left(\mathrm{C}_{\mathrm{q}}\right), 133.2(\mathrm{CH}), 128.9(\mathrm{CH}), 128.2(\mathrm{CH}), 128.0(\mathrm{CH}), 127.8(\mathrm{CH}), 127.5(\mathrm{CH})$, $126.9(\mathrm{CH}), 126.2(\mathrm{CH}), 125.3(\mathrm{CH}), 124.9(\mathrm{CH}), 124.8\left(\mathrm{C}_{\mathrm{q}}\right), 107.9(\mathrm{CH})$.

IR (ATR): $\tilde{v}=3074,1658,1482,1425,1382,1257,1143,890,518 \mathrm{~cm}^{-1}$. MS (EI) $m / z$ (relative intensity): 314 (20) [M] $]^{+}, 298$ (50), 269 (60), 194 (30), 181 (100), 165 (30), 78.0 (93).

HR-MS (EI) $m / z$ calcd. for $\mathrm{C}_{20} \mathrm{H}_{14} \mathrm{~N}_{2} \mathrm{O}_{2}[\mathrm{M}]^{+}: 314.1055$, found: 314.1052 .

The analytical data are in accordance with those previously reported in the literature. ${ }^{[250]}$<smiles>Cc1ccc2c(=O)n(-c3cccc[n+]3[O-])c(-c3ccccc3)cc2c1</smiles>

2-[6-Methyl-1-oxo-3-phenylisoquinolin-2(1H)-yl]-pyridine-1-oxide (51ba): A modified procedure $\mathbf{E}$ was followed using benzamide 34b (114 mg, $0.50 \mathrm{mmol}$ ), alkyne 53a (102 mg, 
$1.00 \mathrm{mmol}), \mathrm{Co}(\mathrm{OAc})_{3}(11.3 \mathrm{mg}, 10 \mathrm{~mol} \%)$. The reaction cell was mounted in a closed ultrasonic bath. The sonication and electrolysis were started and stopped simultaneously. Purification by column chromatography on silica gel $\left(\mathrm{CH}_{2} \mathrm{Cl}_{2} /\right.$ acetone $\left.3: 1\right)$ yielded $\mathbf{5 1 b a}$ (120 $\mathrm{mg}, 73 \%)$ as a white solid.

M. p. $=222-223^{\circ} \mathrm{C}$.

${ }^{1}$ H-NMR $\left(400 \mathrm{MHz}, \mathrm{CDCl}_{3}\right): \delta=8.31(\mathrm{~d}, J=8.1 \mathrm{~Hz}, 1 \mathrm{H}), 8.24-8.18(\mathrm{~m}, 1 \mathrm{H}), 7.43-7.36$ (m, 2H), 7.35-7.29 (m, 2H), 7.26-7.18 (m, 3H), 7.16-7.10 (m, 2H), 7.09-7.02 (m, 1H), $6.53(\mathrm{~s}, 1 \mathrm{H}), 2.48(\mathrm{~s}, 3 \mathrm{H})$.

${ }^{13}$ C-NMR (101 MHz, $\left.\mathrm{CDCl}_{3}\right): \delta=162.0\left(\mathrm{C}_{\mathrm{q}}\right), 145.8\left(\mathrm{C}_{\mathrm{q}}\right), 144.2\left(\mathrm{C}_{\mathrm{q}}\right), 142.8\left(\mathrm{C}_{\mathrm{q}}\right), 140.2$ $(\mathrm{CH}), 137.3\left(\mathrm{C}_{\mathrm{q}}\right), 135.1\left(\mathrm{C}_{\mathrm{q}}\right), 129.1(\mathrm{CH}), 128.8(\mathrm{CH}), 128.5(\mathrm{CH}), 128.4(\mathrm{CH}), 128.1(\mathrm{CH})$, $127.9(\mathrm{CH}), 126.3(\mathrm{CH}), 125.5(\mathrm{CH}), 125.2(\mathrm{CH}), 122.8\left(\mathrm{C}_{\mathrm{q}}\right), 108.1(\mathrm{CH}), 22.0\left(\mathrm{CH}_{3}\right)$.

IR (ATR): $\tilde{v}=3064,1665,1620,1479,1261,902,763,725 \mathrm{~cm}^{-1}$.

MS (EI) $m / z$ (relative intensity): 328 (20) [M] $]^{+}, 312.1$ (15), 283 (15), 208 (25), 181 (100), 165 (15), 78 (65).

HR-MS (EI) $m / z$ calcd. for $\mathrm{C}_{21} \mathrm{H}_{16} \mathrm{~N}_{2} \mathrm{O}_{2}[\mathrm{M}]^{+}:$328.1212, found: 328.1206 .

The analytical data are in accordance with those previously reported in the literature. ${ }^{[250]}$<smiles>COc1ccc2c(=O)n(-c3cccc[n+]3[O-])c(-c3ccccc3)cc2c1</smiles>

2-[6-Methoxy-1-oxo-3-phenylisoquinolin-2(1H)-yl]-pyridine-1-oxide (51da): The general procedure $\mathbf{E}$ was followed using benzamide 34d (122 $\mathrm{mg}, 0.50 \mathrm{mmol})$ and alkyne 53a (102 mg, $1.00 \mathrm{mmol})$. Purification by column chromatography on silica gel $\left(\mathrm{CH}_{2} \mathrm{Cl}_{2}\right.$ /acetone $\left.3: 1\right)$ yielded $\mathbf{5 1 d a}(119 \mathrm{mg}, 69 \%)$ as a white solid.

M. p.: $201-202{ }^{\circ} \mathrm{C}$.

${ }^{1}$ H-NMR $\left(400 \mathrm{MHz}, \mathrm{CDCl}_{3}\right): \delta=8.38-8.29(\mathrm{~m}, 1 \mathrm{H}), 8.26-8.11(\mathrm{~m}, 1 \mathrm{H}), 7.44-7.34(\mathrm{~m}$, 2H), 7.26-7.17 (m, 3H), 7.17-7.09 (m, 2H), 7.08-7.02 (m, 2H), 6.91 (d, $J=2.4 \mathrm{~Hz}, 1 \mathrm{H})$, $6.52(\mathrm{~s}, 1 \mathrm{H}), 3.92(\mathrm{~s}, 3 \mathrm{H})$.

${ }^{13}$ C-NMR (125 MHz, $\left.\mathrm{CDCl}_{3}\right): \delta=163.8\left(\mathrm{C}_{\mathrm{q}}\right), 161.6\left(\mathrm{C}_{\mathrm{q}}\right), 145.8\left(\mathrm{C}_{\mathrm{q}}\right), 143.4\left(\mathrm{C}_{\mathrm{q}}\right), 140.2$ $(\mathrm{CH}), 139.4\left(\mathrm{C}_{\mathrm{q}}\right), 135.1\left(\mathrm{C}_{\mathrm{q}}\right), 130.7(\mathrm{CH}), 129.2(\mathrm{CH}), 128.3(\mathrm{CH}), 128.0(\mathrm{CH}), 127.9(\mathrm{CH})$, $125.5(\mathrm{CH}), 125.1(\mathrm{CH}), 118.8\left(\mathrm{C}_{\mathrm{q}}\right), 116.4(\mathrm{CH}), 108.0(\mathrm{CH}), 107.7(\mathrm{CH}), 55.7\left(\mathrm{CH}_{3}\right)$.

IR (ATR): $\tilde{v}=1656,1604,1486,1378,1247,1145,767,692 \mathrm{~cm}^{-1}$. 
MS (EI) $m / z$ (relative intensity): 344 (20) $\left[\mathrm{M}^{+}\right], 299$ (10), 255 (15), 224 (20), 181 (100), 135 (10), 78 (45).

HR-MS (EI) $m / z$ calcd. for $\mathrm{C}_{21} \mathrm{H}_{16} \mathrm{~N}_{2} \mathrm{O}_{3}\left[\mathrm{M}^{+}\right] 344.1161$, found 344.1149.

The analytical data are in accordance with those previously reported in the literature. ${ }^{[117]}$<smiles>CSc1ccc2c(=O)n(-c3cccc[n+]3[O-])c(-c3ccccc3)cc2c1</smiles>

2-[6-(Methylthio)-1-oxo-3-phenylisoquinolin-2(1H)-yl]-pyridine-1-oxide (51ea): The general procedure $\mathbf{E}$ was followed using benzamide $34 \mathrm{e}(130 \mathrm{mg}, 0.50 \mathrm{mmol})$ and alkyne 53a $(102 \mathrm{mg}, 1.00 \mathrm{mmol})$. Purification by column chromatography on silica gel $\left(\mathrm{CH}_{2} \mathrm{Cl}_{2} /\right.$ acetone $\left.3: 1\right)$ yielded 51ea $(110 \mathrm{mg}, 61 \%)$ as a white solid.

M. p.: $218-220^{\circ} \mathrm{C}$.

${ }^{1} \mathbf{H}-\mathbf{N M R}\left(500 \mathrm{MHz}, \mathrm{CDCl}_{3}\right): \delta=8.28(\mathrm{dd}, J=8.4,1.8 \mathrm{~Hz}, 1 \mathrm{H}), 8.25-8.14$ (m, 1H), 7.427.36 (m, 2H), 7.32 (dd, $J=8.4,1.8 \mathrm{~Hz}, 1 \mathrm{H}), 7.28$ (d, $J=1.9 \mathrm{~Hz}, 1 \mathrm{H}), 7.26-7.19$ (m, 3H), 7.18-7.11 (m, 2H), 7.11-7.02 (m, 1H), $6.51(\mathrm{~s}, 1 \mathrm{H}), 2.57(\mathrm{~s}, 3 \mathrm{H})$.

${ }^{13}$ C-NMR $\left(126 \mathrm{MHz}, \mathrm{CDCl}_{3}\right): \delta=161.8\left(\mathrm{C}_{\mathrm{q}}\right), 146.4\left(\mathrm{C}_{\mathrm{q}}\right), 145.6\left(\mathrm{C}_{\mathrm{q}}\right), 143.6\left(\mathrm{C}_{\mathrm{q}}\right), 140.2$ $(\mathrm{CH}), 137.6\left(\mathrm{C}_{\mathrm{q}}\right), 134.9\left(\mathrm{C}_{\mathrm{q}}\right), 129.3(\mathrm{CH}), 128.7(\mathrm{CH}), 128.4(\mathrm{CH}), 128.0(\mathrm{CH}), 127.9(\mathrm{CH})$, 125.6 (CH), $125.4(\mathrm{CH}), 124.8(\mathrm{CH}), 121.7\left(\mathrm{C}_{\mathrm{q}}\right), 121.2(\mathrm{CH}), 107.6(\mathrm{CH}), 15.0\left(\mathrm{CH}_{3}\right)$.

IR (ATR): $\tilde{v}=1663,1587,1431,1372,1269,916,730,703 \mathrm{~cm}^{-1}$.

MS (EI) $m / z$ (relative intensity): 360 (20) [M] ${ }^{+}, 315$ (10), 240 (20), 193 (10), 181 (100), 78 (45).

HR-MS (EI) $m / z$ calcd. for $\mathrm{C}_{21} \mathrm{H}_{16} \mathrm{~N}_{2} \mathrm{O}_{2} \mathrm{~S}[\mathrm{M}]^{+}: 360.0932$, found: 360.0925 .

Crystals suitable for X-ray crystallography were grown by slow evaporation from a saturated solution of 51ea in EtOAc. 
<smiles></smiles>

2-[6-Iodo-1-oxo-3-phenylisoquinolin-2(1H)-yl]-pyridine-1-oxide (51ga): The general procedure E was followed using benzamide $\mathbf{3 4 g}(170 \mathrm{mg}, 0.50 \mathrm{mmol})$ and alkyne 53a $(102 \mathrm{mg}, 1.00 \mathrm{mmol})$. Purification by column chromatography on silica gel $\left(\mathrm{CH}_{2} \mathrm{Cl}_{2}\right.$ /acetone 6:1) yielded $\mathbf{5 1 g a}(91.4 \mathrm{mg}, 41 \%)$ as a white solid.

M. p.: $251-252^{\circ} \mathrm{C}$.

${ }^{1}$ H-NMR $\left(400 \mathrm{MHz}, \mathrm{CDCl}_{3}\right): \delta=8.22(\mathrm{ddd}, J=6.5,1.5,0.7 \mathrm{~Hz}, 1 \mathrm{H}), 8.11(\mathrm{dd}, J=8.5$, $0.6 \mathrm{~Hz}, 1 \mathrm{H}), 7.96(\mathrm{~d}, J=1.6 \mathrm{~Hz}, 1 \mathrm{H}), 7.80(\mathrm{dd}, J=8.5,1.7 \mathrm{~Hz}, 1 \mathrm{H}), 7.41-7.35(\mathrm{~m}, 2 \mathrm{H})$, 7.30-7.26 (m, 1H), 7.26-7.21 (m, 2H), 7.18-7.11 (m, 2H), 7.10-7.05 (m, 1H), 6.49 (s, 1H). ${ }^{13}$ C-NMR (101 MHz, $\left.\mathrm{CDCl}_{3}\right): \delta=161.9\left(\mathrm{C}_{\mathrm{q}}\right), 145.4\left(\mathrm{C}_{\mathrm{q}}\right), 144.1\left(\mathrm{C}_{\mathrm{q}}\right), 140.2(\mathrm{CH}), 138.6$ $\left(\mathrm{C}_{\mathrm{q}}\right), 136.3(\mathrm{CH}), 135.3(\mathrm{CH}), 134.7\left(\mathrm{C}_{\mathrm{q}}\right), 130.1(\mathrm{CH}), 129.5(\mathrm{CH}), 128.5(\mathrm{CH}), 128.0(\mathrm{CH})$, $127.8(\mathrm{CH}), 125.7(\mathrm{CH}), 125.2(\mathrm{CH}), 124.3\left(\mathrm{C}_{\mathrm{q}}\right), 106.8(\mathrm{CH}), 101.6\left(\mathrm{C}_{\mathrm{q}}\right)$.

IR (ATR): $\tilde{v}=1666,1618,1597,1578,1271,898,761,726 \mathrm{~cm}^{-1}$.

MS (EI) $m / z$ (relative intensity): 440 (20) [M] $]^{+}, 395$ (15), 320 (20), 268 (10), 181 (100), 78 (50).

HR-MS (EI) $m / z$ calcd. for $\mathrm{C}_{20} \mathrm{H}_{13} \mathrm{IN}_{2} \mathrm{O}_{2}[\mathrm{M}]^{+}$: 440.0022, found: 440.0024 .

The analytical data are in accordance with those previously reported in the literature. ${ }^{[250]}$<smiles></smiles>

2-[6-(tert-Butyl)-1-oxo-3-phenylisoquinolin-2(1H)-yl]-pyridine-1-oxide (51ja): The general procedure $\mathbf{E}$ was followed using benzamide $\mathbf{3 4} \mathbf{j}(135 \mathrm{mg}, 0.50 \mathrm{mmol}$ ) and alkyne 53a $(102 \mathrm{mg}, 1.00 \mathrm{mmol})$. Purification by column chromatography on silica gel $\left(\mathrm{CH}_{2} \mathrm{Cl}_{2} /\right.$ acetone $\left.3: 1\right)$ yielded $\left.\mathbf{5 1 j a ~ ( 1 2 8 ~} \mathrm{mg}, \mathbf{6 9 \%}\right)$ as a white solid.

M. p.: $248-250{ }^{\circ} \mathrm{C}$. 
${ }^{1}$ H-NMR (300 MHz, $\left.\mathrm{CDCl}_{3}\right): \delta=8.39-8.33(\mathrm{~m}, 1 \mathrm{H}), 8.24-8.19(\mathrm{~m}, 1 \mathrm{H}), 7.57(\mathrm{dd}, J=8.5$, $1.9 \mathrm{~Hz}, 1 \mathrm{H}), 7.54-7.52(\mathrm{~m}, 1 \mathrm{H}), 7.43-7.37$ (m, 2H), 7.26-7.18 (m, 3H), 7.17-7.10 (m, 2H), 7.09-7.03 (m, 1H), $6.60(\mathrm{~s}, 1 \mathrm{H}), 1.40(\mathrm{~s}, 9 \mathrm{H})$.

${ }^{13}$ C-NMR $\left(126 \mathrm{MHz}, \mathrm{CDCl}_{3}\right): \delta=161.9\left(\mathrm{C}_{\mathrm{q}}\right), 157.1\left(\mathrm{C}_{\mathrm{q}}\right), 145.7\left(\mathrm{C}_{\mathrm{q}}\right), 142.6\left(\mathrm{C}_{\mathrm{q}}\right), 140.1$ $(\mathrm{CH}), 137.1\left(\mathrm{C}_{\mathrm{q}}\right), 135.1\left(\mathrm{C}_{\mathrm{q}}\right), 129.1(\mathrm{CH}), 128.3(\mathrm{CH}), 128.3(\mathrm{CH}), 128.1(\mathrm{CH}), 127.8(\mathrm{CH})$, $125.4(\mathrm{CH}), 125.3(\mathrm{CH}), 125.0(\mathrm{CH}), 122.8\left(\mathrm{C}_{\mathrm{q}}\right), 122.6(\mathrm{CH}), 108.6(\mathrm{CH}), 35.5\left(\mathrm{C}_{\mathrm{q}}\right), 31.3$ $\left(\mathrm{CH}_{3}\right)$.

IR (ATR): $\tilde{v}=1681,1617,1606,1488,1270,939,764,698 \mathrm{~cm}^{-1}$.

MS (EI) $m / z$ (relative intensity): 370 (15) [M] ${ }^{+}, 325$ (10), 250 (12), 235 (15), 181 (100), 78 (40).

HR-MS (EI) $m / z$ calcd. for $\mathrm{C}_{24} \mathrm{H}_{22} \mathrm{~N}_{2} \mathrm{O}_{2}[\mathrm{M}]^{+}: 370.1681$, found: 370.1683 .

The analytical data are in accordance with those previously reported in the literature. ${ }^{[250]}$<smiles></smiles>

2-[6-Fluoro-1-oxo-3-phenylisoquinolin-2(1H)-yl]-pyridine-1-oxide (51 ka): The general procedure $\mathbf{E}$ was followed using benzamide $\mathbf{3 4 k}(116 \mathrm{mg}, 0.50 \mathrm{mmol})$ and alkyne $\mathbf{5 3 a}$ $(102 \mathrm{mg}, 1.00 \mathrm{mmol})$. Purification by column chromatography on silica gel $\left(\mathrm{CH}_{2} \mathrm{Cl}_{2} /\right.$ acetone $\left.6: 1\right)$ yielded $51 \mathrm{ka}(109 \mathrm{mg}, 66 \%)$ as a white solid.

M. p.: $191-192^{\circ} \mathrm{C}$.

${ }^{1} \mathbf{H}-\mathrm{NMR}\left(300 \mathrm{MHz}, \mathrm{CDCl}_{3}\right): \delta=8.44(\mathrm{dd}, J=9.6,5.7 \mathrm{~Hz}, 1 \mathrm{H}), 8.21(\mathrm{dd}, J=6.4,1.6 \mathrm{~Hz}$, 1H), 7.40 (dd, $J=7.8,1.8 \mathrm{~Hz}, 2 \mathrm{H}), 7.31-7.26(\mathrm{~m}, 1 \mathrm{H}), 7.25-7.14(\mathrm{~m}, 5 \mathrm{H}), 7.14-7.11(\mathrm{~m}$, 1H), 7.10-7.03 (m, 1H), $6.54(\mathrm{~s}, 1 \mathrm{H})$.

${ }^{13} \mathrm{C}-\mathrm{NMR}\left(125 \mathrm{MHz}, \mathrm{CDCl}_{3}\right): \delta=166.1\left(\mathrm{~d},{ }^{1} J_{\mathrm{C}-\mathrm{F}}=254.1 \mathrm{~Hz}, \mathrm{C}_{\mathrm{q}}\right), 161.4\left(\mathrm{C}_{\mathrm{q}}\right), 145.4\left(\mathrm{C}_{\mathrm{q}}\right)$, $144.3\left(\mathrm{C}_{\mathrm{q}}\right), 140.2(\mathrm{CH}), 139.6\left(\mathrm{~d},{ }^{3} J_{\mathrm{C}-\mathrm{F}}=10.6 \mathrm{~Hz}, \mathrm{C}_{\mathrm{q}}\right), 134.7\left(\mathrm{C}_{\mathrm{q}}\right), 131.9\left(\mathrm{~d},{ }^{3} J_{\mathrm{C}-\mathrm{F}}=10.2 \mathrm{~Hz}\right.$, $\mathrm{CH}), 129.5(\mathrm{CH}), 128.5(\mathrm{CH}), 128.0(\mathrm{CH}), 127.8(\mathrm{CH}), 125.7(\mathrm{CH}), 125.3(\mathrm{CH}), 121.7(\mathrm{~d}$, $\left.{ }^{4} J_{\mathrm{C}-\mathrm{F}}=1.8 \mathrm{~Hz}, \mathrm{C}_{\mathrm{q}}\right), 115.8\left(\mathrm{~d},{ }^{2} J_{\mathrm{C}-\mathrm{F}}=23.4 \mathrm{~Hz}, \mathrm{CH}\right), 111.5\left(\mathrm{~d},{ }^{2} J_{\mathrm{C}-\mathrm{F}}=22.1 \mathrm{~Hz}, \mathrm{CH}\right), 107.5$ $\left(\mathrm{d},{ }^{4} J_{\mathrm{C}-\mathrm{F}}=3.1 \mathrm{~Hz}, \mathrm{CH}\right)$.

${ }^{19}$ F-NMR (376 MHz, $\left.\mathrm{CDCl}_{3}\right): \delta=-104.7(\mathrm{td}, J=8.9,5.7 \mathrm{~Hz})$.

IR (ATR): $\tilde{v}=1666,1610,1506,1479,1421,1270,866,758 \mathrm{~cm}^{-1}$. 
MS (EI) $m / z$ (relative intensity): 332 (55) [M] $]^{+}, 315$ (10), 287 (15), 212 (25), 181 (100), 78 (65).

HR-MS (EI) $m / z$ calcd. for $\mathrm{C}_{20} \mathrm{H}_{13} \mathrm{FN}_{2} \mathrm{O}_{2}[\mathrm{M}+\mathrm{H}]^{+}: 332.0961$, found: 332.0966 .

The analytical data are in accordance with those previously reported in the literature. ${ }^{[250]}$<smiles>Cc1cc(C)c2cc(-c3ccccc3)n(-c3cccc[n+]3[O-])c(=O)c2c1</smiles>

2-[5,7-Dimethyl-1-oxo-3-phenylisoquinolin-2(1H)-yl]-pyridine-1-oxide (51ua): A modified general procedure $\mathbf{E}$ was followed using benzamide $\mathbf{3 4} \mathbf{u}$ (121 $\mathrm{mg}, 0.50 \mathrm{mmol}$ ), alkyne 53a $(102 \mathrm{mg}, 1.00 \mathrm{mmol})$ and $\mathrm{Co}(\mathrm{OAc})_{3}(11.3 \mathrm{mg}, 10 \mathrm{~mol} \%)$. Purification by column chromatography on silica gel $\left(\mathrm{CH}_{2} \mathrm{Cl}_{2} /\right.$ acetone $\left.3: 1\right)$ yielded 51 ua $(108 \mathrm{mg}, 63 \%)$ as a white solid.

M. p.: $238-239{ }^{\circ} \mathrm{C}$.

${ }^{1}$ H-NMR $\left(400 \mathrm{MHz}, \mathrm{CDCl}_{3}\right): \delta=8.20(\mathrm{dd}, J=6.9,1.5 \mathrm{~Hz}, 1 \mathrm{H}), 8.11(\mathrm{~s}, 1 \mathrm{H}), 7.45-7.39$ (m, 2H), $7.37(\mathrm{~s}, 1 \mathrm{H}), 7.26-7.20(\mathrm{~m}, 3 \mathrm{H}), 7.16-7.11(\mathrm{~m}, 2 \mathrm{H}), 7.06(\mathrm{ddd}, J=8.2,7.1,1.4$ $\mathrm{Hz}, 1 \mathrm{H}), 6.68(\mathrm{~d}, J=0.8 \mathrm{~Hz}, 1 \mathrm{H}), 2.51(\mathrm{~s}, 3 \mathrm{H}), 2.45$ (s, 3H).

${ }^{13}$ C-NMR $\left(125 \mathrm{MHz}, \mathrm{CDCl}_{3}\right): \delta=162.3\left(\mathrm{C}_{\mathrm{q}}\right), 145.9\left(\mathrm{C}_{\mathrm{q}}\right), 141.3\left(\mathrm{C}_{\mathrm{q}}\right), 140.2(\mathrm{CH}), 137.0$ $\left(\mathrm{C}_{\mathrm{q}}\right), 135.9(\mathrm{CH}), 135.5\left(\mathrm{C}_{\mathrm{q}}\right), 133.7\left(\mathrm{C}_{\mathrm{q}}\right), 133.6\left(\mathrm{C}_{\mathrm{q}}\right), 129.1(\mathrm{CH}), 128.3(\mathrm{CH}), 128.2(\mathrm{CH})$, $127.8(\mathrm{CH}), 126.1(\mathrm{CH}), 125.5(\mathrm{CH}), 125.3\left(\mathrm{C}_{\mathrm{q}}\right), 125.0(\mathrm{CH}), 105.1(\mathrm{CH}), 21.6\left(\mathrm{CH}_{3}\right), 19.0$ $\left(\mathrm{CH}_{3}\right)$.

IR (ATR): $\tilde{v}=1709,1668,1489,1251,851,756,700,578 \mathrm{~cm}^{-1}$.

MS (EI) $m / z$ (relative intensity): 342 (10) [M] $]^{+}, 326$ (10), 297 (10), 222 (25), 207 (15), 181 (100), $78(60)$.

HR-MS (ESI) $m / z$ calcd. for $\mathrm{C}_{22} \mathrm{H}_{19} \mathrm{~N}_{2} \mathrm{O}_{2}[\mathrm{M}+\mathrm{H}]^{+}: 343.1441$, found: 343.1444 .

The analytical data are in accordance with those previously reported in the literature. ${ }^{[250]}$ 
<smiles>COc1cc(OC)c2cc(-c3ccccc3)n(-c3cccc[n+]3[O-])c(=O)c2c1</smiles>

2-[5,7-Dimethoxy-1-oxo-3-phenylisoquinolin-2(1H)-yl]-pyridine-1-oxide (51va): The general procedure $\mathbf{E}$ was followed using benzamide $\mathbf{3 4 v}(137 \mathrm{mg}, 0.50 \mathrm{mmol})$ and alkyne 53a $(102 \mathrm{mg}, 1.00 \mathrm{mmol})$. Purification by column chromatography on silica gel $\left(\mathrm{CH}_{2} \mathrm{Cl}_{2} /\right.$ acetone $\left.3: 1\right)$ yielded $\mathbf{5 1 v a}(146 \mathrm{mg}, 78 \%)$ as a white solid.

M. p.: $254-255^{\circ} \mathrm{C}$.

${ }^{1}$ H-NMR $\left(400 \mathrm{MHz}, \mathrm{CDCl}_{3}\right): \delta=8.23(\mathrm{dd}, J=6.6,1.3 \mathrm{~Hz}, 1 \mathrm{H}), 7.44(\mathrm{dd}, J=2.4,0.6 \mathrm{~Hz}$, 1H), 7.41-7.37 (m, 2H), 7.24-7.17 (m, 3H), 7.17-7.11 (m, 1H), 7.11-7.02 (m, 2H), 6.94 $(\mathrm{d}, J=0.7 \mathrm{~Hz}, 1 \mathrm{H}), 6.74(\mathrm{~d}, J=2.4 \mathrm{~Hz}, 1 \mathrm{H}), 3.91(\mathrm{~s}, 3 \mathrm{H}), 3.90(\mathrm{~s}, 3 \mathrm{H})$.

${ }^{13}$ C-NMR $\left(101 \mathrm{MHz}, \mathrm{CDCl}_{3}\right): \delta=161.6\left(\mathrm{C}_{\mathrm{q}}\right), 159.8\left(\mathrm{C}_{\mathrm{q}}\right), 156.2\left(\mathrm{C}_{\mathrm{q}}\right), 146.0\left(\mathrm{C}_{\mathrm{q}}\right), 140.2$ $(\mathrm{CH}), 139.7\left(\mathrm{C}_{\mathrm{q}}\right), 135.5\left(\mathrm{C}_{\mathrm{q}}\right), 128.9(\mathrm{CH}), 128.3(\mathrm{CH}), 128.2(\mathrm{CH}), 127.6(\mathrm{CH}), 126.7\left(\mathrm{C}_{\mathrm{q}}\right)$, $125.5(\mathrm{CH}), 125.1(\mathrm{CH}), 122.9\left(\mathrm{C}_{\mathrm{q}}\right), 104.0(\mathrm{CH}), 102.7(\mathrm{CH}), 99.8(\mathrm{CH}), 56.0\left(\mathrm{CH}_{3}\right), 55.9$ $\left(\mathrm{CH}_{3}\right)$.

IR (ATR): $\tilde{v}=1665,1606,1489,1429,1351,1041,788,750 \mathrm{~cm}^{-1}$.

MS (EI) $m / z$ (relative intensity): 374 (10) [M] $]^{+}, 358$ (10), 269 (10), 254 (10), 181 (100), 78 (45).

HR-MS (EI) $m / z$ calcd. for $\mathrm{C}_{22} \mathrm{H}_{18} \mathrm{~N}_{2} \mathrm{O}_{4}[\mathrm{M}]^{+}: 374.1267$, found: 374.1259 .

The analytical data are in accordance with those previously reported in the literature. ${ }^{[250]}$<smiles></smiles>

51wa<smiles>Cc1cccc2c(=O)n(-c3cccc[n+]3[O-])c(-c3ccccc3)cc12</smiles>

51wa'

2-[7-Methyl-1-oxo-3-phenylisoquinolin-2(1H)-yl]-pyridine-1-oxide (51wa); 2-[5Methyl-1-oxo-3-phenylisoquinolin-2(1H)-yl]-pyridine-1-oxide (51wa'): The general procedure $\mathbf{E}$ was followed using benzamide $\mathbf{3 4 w}$ (114 $\mathrm{mg}, 0.50 \mathrm{mmol}$ ) and alkyne 53a (102 mg, $1.00 \mathrm{mmol})$. Purification by column chromatography on silica gel $\left(\mathrm{CH}_{2} \mathrm{Cl}_{2} /\right.$ acetone $\left.3: 1\right)$ yielded $\mathbf{5 1 w a} / \mathbf{5 1} \mathbf{w a},(130 \mathrm{mg}, 83 \%)$ as a white solid. The ratio of 
51wa/51wa' (4:1) was determined by ${ }^{1} \mathrm{H}-\mathrm{NMR}$ spectroscopy. Resonances are reported for 51 wa.

M. p.: $218-220^{\circ} \mathrm{C}$.

${ }^{1}$ H-NMR (400 MHz, $\mathrm{CDCl}_{3}$ ): $\delta=8.24-8.29(\mathrm{~m}, 2 \mathrm{H}), 7.55-7.50(\mathrm{~m}, 1 \mathrm{H}), 7.47-7.44$ (m, 1H), 7.41-7.37 (m, 2H), 7.25-7.20 (m, 3H), 7.14-7.10 (m, 2H), 7.09-7.03 (m, 1H), 6.57 $(\mathrm{s}, 1 \mathrm{H}), 2.49(\mathrm{~s}, 3 \mathrm{H})$.

${ }^{13}$ C-NMR (101 MHz, $\left.\mathrm{CDCl}_{3}\right): \delta=162.0\left(\mathrm{C}_{\mathrm{q}}\right), 145.8\left(\mathrm{C}_{\mathrm{q}}\right), 141.8\left(\mathrm{C}_{\mathrm{q}}\right), 140.1(\mathrm{CH}), 137.4$ $\left(\mathrm{C}_{\mathrm{q}}\right), 135.1\left(\mathrm{C}_{\mathrm{q}}\right), 134.8(\mathrm{CH}), 134.8\left(\mathrm{C}_{\mathrm{q}}\right), 129.1(\mathrm{CH}), 128.3(\mathrm{CH}), 128.1(\mathrm{CH}), 128.1(\mathrm{CH})$, $127.8(\mathrm{CH}), 126.4(\mathrm{CH}), 125.5(\mathrm{CH}), 125.1(\mathrm{CH}), 125.0\left(\mathrm{C}_{\mathrm{q}}\right), 108.1(\mathrm{CH}), 21.6\left(\mathrm{CH}_{3}\right)$.

IR (ATR): $\tilde{v}=1664,1492,1430,1385,1277,847,758,704 \mathrm{~cm}^{-1}$.

MS (EI) $m / z$ (relative intensity): 328 (20) [M] $]^{+}, 283$ (10), 208 (35), 193 (10), 181 (100), 78 (65).

HR-MS (EI) $m / z$ calcd. for $\mathrm{C}_{21} \mathrm{H}_{16} \mathrm{~N}_{2} \mathrm{O}_{2}[\mathrm{M}]^{+}: 328.1212$, found: 328.1221 .<smiles>Cc1cc(-c2ccccc2)n(-c2cccc[n+]2[O-])c(=O)c1C</smiles>

3,4-Dimethyl-2-oxo-6-phenyl-2H-[1,2'-bipyridine]-1'-oxide (189aa): The general procedure $\mathbf{E}$ was followed using alkene $\mathbf{3 7 a}(96.0 \mathrm{mg}, 0.50 \mathrm{mmol})$ and alkyne 53a (102 $\mathrm{mg}$, $1.00 \mathrm{mmol})$. Purification by column chromatography on silica gel $\left(\mathrm{CH}_{2} \mathrm{Cl}_{2}\right.$ /acetone $\left.1: 1\right)$ yielded 189aa (92.1 mg, 63\%) as a white solid.

M. p.: $203-204{ }^{\circ} \mathrm{C}$.

${ }^{1}$ H-NMR $\left(300 \mathrm{MHz}, \mathrm{CDCl}_{3}\right): \delta=8.21(\mathrm{ddd}, J=6.4,1.0,0.4 \mathrm{~Hz}, 1 \mathrm{H}), 7.36-7.30(\mathrm{~m}, 2 \mathrm{H})$, 7.25-7.21 (m, 2H), 7.20-7.16 (m, 1H), 7.15-7.10 (m, 1H), 7.08-7.03 (m, 2H), $6.15(\mathrm{~s}, 1 \mathrm{H})$, $2.25(\mathrm{~s}, 3 \mathrm{H}), 2.15(\mathrm{~s}, 3 \mathrm{H})$.

${ }^{13}$ C-NMR $\left(125 \mathrm{MHz}, \mathrm{CDCl}_{3}\right): \delta=162.1\left(\mathrm{C}_{\mathrm{q}}\right), 147.3\left(\mathrm{C}_{\mathrm{q}}\right), 145.8\left(\mathrm{C}_{\mathrm{q}}\right), 144.3\left(\mathrm{C}_{\mathrm{q}}\right), 140.1$ $(\mathrm{CH}), 134.5\left(\mathrm{C}_{\mathrm{q}}\right), 129.2(\mathrm{CH}), 128.3(\mathrm{CH}), 127.8(\mathrm{CH}), 127.4(\mathrm{CH}), 125.6\left(\mathrm{C}_{\mathrm{q}}\right), 125.4(\mathrm{CH})$, $125.1(\mathrm{CH}), 111.3(\mathrm{CH}), 20.3\left(\mathrm{CH}_{3}\right), 12.7\left(\mathrm{CH}_{3}\right)$.

IR (ATR): $\tilde{v}=1659,1558,1488,1434,1258,851,767,701 \mathrm{~cm}^{-1}$. MS (EI) $m / z$ (relative intensity): 292 (35) [M] ${ }^{+}, 276$ (20), 198 (100), 181 (42), 170 (40), 78 (70).

HR-MS (EI) $m / z$ calcd. for $\mathrm{C}_{18} \mathrm{H}_{16} \mathrm{~N}_{2} \mathrm{O}_{2}[\mathrm{M}]^{+}:$292.1212, found: 292.1200 . 
The analytical data are in accordance with those previously reported in the literature. ${ }^{[250]}$<smiles></smiles>

\section{2-[1-Oxo-3-phenyl-5,6,7,8-tetrahydroisoquinolin-2(1H)-yl]-pyridine-1-oxide (189ba):}

The general procedure $\mathbf{E}$ was followed using alkene $\mathbf{3 7 b}$ (109 $\mathrm{mg}, 0.50 \mathrm{mmol})$ and alkyne 53a $(102 \mathrm{mg}, 1.00 \mathrm{mmol})$. Purification by column chromatography on silica gel $\left(\mathrm{CH}_{2} \mathrm{Cl}_{2}\right.$ /acetone $\left.2: 1\right)$ yielded $\mathbf{1 8 9 b a}(87.0 \mathrm{mg}, 55 \%)$ as a colorless oil.

${ }^{1}$ H-NMR (400 MHz, $\left.\mathrm{CDCl}_{3}\right): \delta=8.19(\mathrm{dd}, J=6.3,0.8 \mathrm{~Hz}, 1 \mathrm{H}), 7.35-7.30$ (m, 2H), 7.24$7.16(\mathrm{~m}, 3 \mathrm{H}), 7.15-7.10(\mathrm{~m}, 1 \mathrm{H}), 7.09-7.02(\mathrm{~m}, 2 \mathrm{H}), 6.05(\mathrm{~s}, 1 \mathrm{H}), 2.66-2.50(\mathrm{~m}, 4 \mathrm{H}), 1.83-$ $1.73(\mathrm{~m}, 4 \mathrm{H})$.

${ }^{13}$ C-NMR (101 MHz, $\left.\mathrm{CDCl}_{3}\right): \delta=161.9\left(\mathrm{C}_{\mathrm{q}}\right), 148.5\left(\mathrm{C}_{\mathrm{q}}\right), 145.7\left(\mathrm{C}_{\mathrm{q}}\right), 144.3\left(\mathrm{C}_{\mathrm{q}}\right), 140.1$ $(\mathrm{CH}), 134.7\left(\mathrm{C}_{\mathrm{q}}\right), 129.2(\mathrm{CH}), 128.4(\mathrm{CH}), 127.8(\mathrm{CH}), 127.5(\mathrm{CH}), 126.7\left(\mathrm{C}_{\mathrm{q}}\right), 125.5(\mathrm{CH})$, $125.2(\mathrm{CH}), 110.2(\mathrm{CH}), 29.7\left(\mathrm{CH}_{2}\right), 23.5\left(\mathrm{CH}_{2}\right), 22.1\left(\mathrm{CH}_{2}\right), 22.0\left(\mathrm{CH}_{2}\right)$.

IR (ATR): $\tilde{v}=3021,1655,1481,1431,1249,905,840,728 \mathrm{~cm}^{-1}$.

MS (EI) $m / z$ (relative intensity): 318 (30) [M] ${ }^{+}, 301$ (10), 224 (100), 195 (20), 181 (60), 78 (60).

HR-MS (EI) $m / z$ calcd. for $\mathrm{C}_{20} \mathrm{H}_{18} \mathrm{~N}_{2} \mathrm{O}_{2}[\mathrm{M}]^{+}: 318.1368$, found: 318.1363 .

The analytical data are in accordance with those previously reported in the literature. ${ }^{[250]}$<smiles></smiles>

189ea<smiles></smiles>

189ea'

2-[4-Oxo-6-phenylfuro[3,2-c]pyridin-5(4H)-yl]-pyridine-1-oxide (189ea); 2-[4-Oxo-6phenylfuro[3,4-c]pyridin-5(4H)-yl]-pyridine 1-oxide (189ea'): The general procedure $\mathbf{E}$ was followed using 2-(furan-3-carboxamido)pyridine 1-oxide 37e (102 mg, $0.50 \mathrm{mmol}$ ) and alkyne 53a (102 mg, $1.00 \mathrm{mmol}$ ). Purification by column chromatography on silica gel $\left(\mathrm{CH}_{2} \mathrm{Cl}_{2} /\right.$ acetone 5:1) yielded 189ea/189ea' $(79.0 \mathrm{mg}, 52 \%)$ as a white solid. The ratio of 
189ea/189ea' (10:1) was determined by ${ }^{1} \mathrm{H}-\mathrm{NMR}$ spectroscopy. Resonances are reported for 189ea.

M. p.: $199-200{ }^{\circ} \mathrm{C}$.

${ }^{1}$ H-NMR (600 MHz, $\left.\mathrm{CDCl}_{3}\right): \delta=8.23-8.13(\mathrm{~m}, 1 \mathrm{H}), 7.55-7.52(\mathrm{~m}, 1 \mathrm{H}), 7.39-7.36(\mathrm{~m}$, 2H), 7.26-7.25 (m, 1H), 7.23-7.20 (m, 2H), 7.16-7.11 (m, 2H), 7.09-7.04 (m, 1H), 7.02$7.00(\mathrm{~m}, 1 \mathrm{H}), 6.78(\mathrm{~s}, 1 \mathrm{H})$.

${ }^{13}$ C-NMR $\left(126 \mathrm{MHz}, \mathrm{CDCl}_{3}\right): \delta=159.8\left(\mathrm{C}_{\mathrm{q}}\right), 158.4\left(\mathrm{C}_{\mathrm{q}}\right), 145.6\left(\mathrm{C}_{\mathrm{q}}\right), 145.1\left(\mathrm{C}_{\mathrm{q}}\right), 143.6$ $(\mathrm{CH}), 139.9(\mathrm{CH}), 134.4\left(\mathrm{C}_{\mathrm{q}}\right), 129.3(\mathrm{CH}), 128.2(\mathrm{CH}), 127.1(\mathrm{CH}), 127.8(\mathrm{CH}), 125.5$ $(\mathrm{CH}), 125.0(\mathrm{CH}), 114.9\left(\mathrm{C}_{\mathrm{q}}\right), 107.8(\mathrm{CH}), 98.2(\mathrm{CH})$.

IR (ATR): $\tilde{v}=3112,1675,1574,1488,1266,730,590,517 \mathrm{~cm}^{-1}$.

MS (EI) $m / z$ (relative intensity): 304 (35) [M] ${ }^{+}, 288$ (70), 259 (45), 231 (35), 181 (100), 78 (60).

HR-MS (ESI) $m / z$ calcd. for $\mathrm{C}_{18} \mathrm{H}_{13} \mathrm{~N}_{2} \mathrm{O}_{3}[\mathrm{M}+\mathrm{H}]^{+}: 305.0921$, found: 305.0922 .

Crystals suitable for X-ray crystallography were grown by slow evaporation from a saturated solution of 189ea in EtOAc.<smiles></smiles>

2-\{3-[4-(tert-Butyl)phenyl]-1-oxoisoquinolin-2(1H)-yl\}-pyridine-1-oxide (51ab): The general procedure $\mathbf{E}$ was followed using benzamide 34a (107 $\mathrm{mg}, 0.50 \mathrm{mmol}$ ) and alkyne 53b $(158 \mathrm{mg}, 1.00 \mathrm{mmol})$. Purification by column chromatography on silica gel $\left(\mathrm{CH}_{2} \mathrm{Cl}_{2}\right.$ /acetone $\left.4: 1\right)$ yielded 51ab (119 $\left.\mathrm{mg}, 51 \%\right)$ as a white solid.

M. p.: $162-163{ }^{\circ} \mathrm{C}$.

${ }^{1}$ H-NMR $\left(400 \mathrm{MHz}, \mathrm{CDCl}_{3}\right): \delta=8.41(\mathrm{dd}, J=8.0,0.7 \mathrm{~Hz}, 1 \mathrm{H}), 8.28-8.17(\mathrm{~m}, 1 \mathrm{H}), 7.69$ (ddd, $J=8.2,7.1,1.2 \mathrm{~Hz}, 1 \mathrm{H}), 7.54(\mathrm{dd}, J=8.0,1.1 \mathrm{~Hz}, 1 \mathrm{H}), 7.49$ (ddd, $J=8.1,7.1,1.1$ $\mathrm{Hz}, 1 \mathrm{H}), 7.35-7.28(\mathrm{~m}, 2 \mathrm{H}), 7.25-7.20(\mathrm{~m}, 2 \mathrm{H}), 7.18-7.12(\mathrm{~m}, 2 \mathrm{H}), 7.12-7.04(\mathrm{~m}, 1 \mathrm{H})$, $6.60(\mathrm{~s}, 1 \mathrm{H}), 1.24(\mathrm{~s}, 9 \mathrm{H})$.

${ }^{13}$ C-NMR $\left(126 \mathrm{MHz}, \mathrm{CDCl}_{3}\right): \delta=162.1\left(\mathrm{C}_{\mathrm{q}}\right), 152.3\left(\mathrm{C}_{\mathrm{q}}\right), 145.7\left(\mathrm{C}_{\mathrm{q}}\right), 142.8\left(\mathrm{C}_{\mathrm{q}}\right), 140.1$ $(\mathrm{CH}), 137.3\left(\mathrm{C}_{\mathrm{q}}\right), 133.4(\mathrm{CH}), 132.0\left(\mathrm{C}_{\mathrm{q}}\right), 128.5(\mathrm{CH}), 127.9(\mathrm{CH}), 127.7(\mathrm{CH}), 127.0(\mathrm{CH})$, $126.3(\mathrm{CH}), 125.4(\mathrm{CH}), 125.2(\mathrm{CH}), 125.1(\mathrm{CH}), 125.0\left(\mathrm{C}_{\mathrm{q}}\right), 108.1(\mathrm{CH}), 34.8\left(\mathrm{C}_{\mathrm{q}}\right), 31.3$ $\left(\mathrm{CH}_{3}\right)$. 
IR (ATR): $\tilde{v}=3056,1678,1621,1478,1432,1272,876,758 \mathrm{~cm}^{-1}$.

MS (EI) $m / z$ (relative intensity): 370 (25) [M] $]^{+}, 269$ (10), 237 (100), 194 (25), 78 (40), 57 (10).

HR-MS (EI) $m / z$ calcd. for $\mathrm{C}_{24} \mathrm{H}_{22} \mathrm{~N}_{2} \mathrm{O}_{2}[\mathrm{M}]^{+}: 370.1681$, found: 370.1680 .

The analytical data are in accordance with those previously reported in the literature. ${ }^{[250]}$<smiles>CCCCCc1cc2ccccc2c(=O)n1-c1cccc[n+]1[O-]</smiles>

2-[3-Hexyl-1-oxoisoquinolin-2(1H)-yl]-pyridine-1-oxide (51ac): The general procedure E was followed using benzamide 34a $(107 \mathrm{mg}, 0.50 \mathrm{mmol})$ and alkyne 53c (110 $\mathrm{mg}$, $1.00 \mathrm{mmol})$. Purification by column chromatography on silica gel $\left(\mathrm{CH}_{2} \mathrm{Cl}_{2} /\right.$ acetone $\left.3: 1\right)$ yielded 51ac (102 mg, 63\%) as a white solid.

M. p.: $138-140{ }^{\circ} \mathrm{C}$.

${ }^{1}$ H-NMR (400 MHz, $\left.\mathrm{CDCl}_{3}\right): \delta=8.40(\mathrm{dd}, J=5.0,2.9 \mathrm{~Hz}, 1 \mathrm{H}), 8.35-8.31(\mathrm{~m}, 1 \mathrm{H}), 7.64$ (ddd, $J=8.3,7.1,1.4 \mathrm{~Hz}, 1 \mathrm{H}), 7.50-7.44(\mathrm{~m}, 2 \mathrm{H}), 7.44-7.36(\mathrm{~m}, 3 \mathrm{H}), 6.44(\mathrm{~s}, 1 \mathrm{H}), 2.43-$ $2.09(\mathrm{~m}, 2 \mathrm{H}), 1.62-1.45(\mathrm{~m}, 2 \mathrm{H}), 1.31-1.11(\mathrm{~m}, 6 \mathrm{H}), 0.84(\mathrm{t}, J=6.9 \mathrm{~Hz}, 3 \mathrm{H})$.

${ }^{13}$ C-NMR (101 MHz, $\left.\mathrm{CDCl}_{3}\right): \delta=162.8\left(\mathrm{C}_{\mathrm{q}}\right), 144.6\left(\mathrm{C}_{\mathrm{q}}\right), 142.7\left(\mathrm{C}_{\mathrm{q}}\right), 140.8(\mathrm{CH}), 137.6$ $\left(\mathrm{C}_{\mathrm{q}}\right), 133.3(\mathrm{CH}), 128.3(\mathrm{CH}), 128.2(\mathrm{CH}), 126.5(\mathrm{CH}), 126.1(\mathrm{CH}), 125.9(\mathrm{CH}), 125.6$ $(\mathrm{CH}), 124.6\left(\mathrm{C}_{\mathrm{q}}\right), 105.1(\mathrm{CH}), 32.1\left(\mathrm{CH}_{2}\right), 31.5\left(\mathrm{CH}_{2}\right), 28.9\left(\mathrm{CH}_{2}\right), 27.7\left(\mathrm{CH}_{2}\right), 22.5\left(\mathrm{CH}_{2}\right)$, $14.1\left(\mathrm{CH}_{3}\right)$.

IR (ATR): $\tilde{v}=2927,1630,1426,1396,1265,876,570,493 \mathrm{~cm}^{-1}$.

MS (EI) $m / z$ (relative intensity): 328 (30) [M] $]^{+}, 306$ (48), 251 (45), 234 (100), 78.0 (61).

HR-MS (EI) $m / z$ calcd. for $\mathrm{C}_{20} \mathrm{H}_{22} \mathrm{~N}_{2} \mathrm{O}_{2}[\mathrm{M}]^{+}: 322.1681$, found: 322.1684 .<smiles>O=c1c2ccccc2cc(CCCCCl)n1-c1cccc[n+]1[O-]</smiles>

2-[3-(4-Chlorobutyl)-1-oxoisoquinolin-2(1H)-yl]-pyridine-1-oxide (51ad): The general procedure $\mathbf{E}$ was followed using benzamide 34a $(107 \mathrm{mg}, 0.50 \mathrm{mmol})$ and alkyne 53d $(117 \mathrm{mg}, \quad 1.00 \mathrm{mmol})$. Purification by column chromatography on silica gel $\left(\mathrm{CH}_{2} \mathrm{Cl}_{2}\right.$ /acetone 3:1) yielded $\mathbf{5 1 a d}(107 \mathrm{mg}, 65 \%)$ as a white solid. 
M. p.: $169-170{ }^{\circ} \mathrm{C}$.

${ }^{1}$ H-NMR (300 MHz, $\left.\mathrm{CDCl}_{3}\right): \delta=8.39(\mathrm{dd}, J=4.9,3.0 \mathrm{~Hz}, 1 \mathrm{H}), 8.36-8.28(\mathrm{~m}, 1 \mathrm{H}), 7.65$ (ddd, $J=8.3,7.1,1.4 \mathrm{~Hz}, 1 \mathrm{H}), 7.52-7.45(\mathrm{~m}, 2 \mathrm{H}), 7.45-7.41(\mathrm{~m}, 1 \mathrm{H}), 7.41-7.36(\mathrm{~m}, 2 \mathrm{H})$, $6.45(\mathrm{~s}, 1 \mathrm{H}), 3.55-3.38(\mathrm{~m}, 2 \mathrm{H}), 2.44-2.15(\mathrm{~m}, 2 \mathrm{H}), 1.82-1.59(\mathrm{~m}, 4 \mathrm{H})$.

${ }^{13}$ C-NMR (125 MHz, $\left.\mathrm{CDCl}_{3}\right): \delta=162.6\left(\mathrm{C}_{\mathrm{q}}\right), 144.3\left(\mathrm{C}_{\mathrm{q}}\right), 141.7\left(\mathrm{C}_{\mathrm{q}}\right), 140.7(\mathrm{CH}), 137.3$ $\left(\mathrm{C}_{\mathrm{q}}\right), 133.3(\mathrm{CH}), 128.2(\mathrm{CH}), 128.1(\mathrm{CH}), 126.6(\mathrm{CH}), 126.2(\mathrm{CH}), 125.8(\mathrm{CH}), 125.7$ $(\mathrm{CH}), 124.6\left(\mathrm{C}_{\mathrm{q}}\right), 105.3(\mathrm{CH}), 44.6\left(\mathrm{CH}_{2}\right), 31.9\left(\mathrm{CH}_{2}\right), 31.4\left(\mathrm{CH}_{2}\right), 24.9\left(\mathrm{CH}_{2}\right)$.

IR (ATR): $\tilde{v}=3092,1631,1561,1490,1424,1259,823,768 \mathrm{~cm}^{-1}$.

MS (EI) $m / z$ (relative intensity): 328 (25) [M] $]^{+}, 311$ (40), 251 (50), 234 (100), 171 (30), $78.0(72)$.

HR-MS (EI) $m / z$ calcd. for $\mathrm{C}_{18} \mathrm{H}_{17} \mathrm{ClN}_{2} \mathrm{O}_{2}[\mathrm{M}]^{+}: 328.0979$, found: 328.0971 .

The analytical data are in accordance with those previously reported in the literature. ${ }^{[250]}$<smiles>N#CCCCc1cc2ccccc2c(=O)n1-c1cccc[n+]1[O-]</smiles>

2-[3-(3-Cyanobutyl)-1-oxoisoquinolin-2(1H)-yl]-pyridine-1-oxide (51ae): The general procedure $\mathbf{E}$ was followed using benzamide 34a $(107 \mathrm{mg}, 0.50 \mathrm{mmol})$ and alkyne 53e (117 mg, $1.00 \mathrm{mmol})$. Purification by column chromatography on silica gel $\left(\mathrm{CH}_{2} \mathrm{Cl}_{2} /\right.$ acetone $\left.3: 1\right)$ yielded 51 ae $(120 \mathrm{mg}, 79 \%)$ as a white solid.

M. p.: $176-177^{\circ} \mathrm{C}$.

${ }^{1}$ H-NMR (300 MHz, $\left.\mathrm{CDCl}_{3}\right): \delta=8.42-8.36(\mathrm{~m}, 1 \mathrm{H}), 8.33(\mathrm{dd}, J=8.1,1.3 \mathrm{~Hz}, 1 \mathrm{H}), 7.67$ (ddd, $J=8.3,7.1,1.4 \mathrm{~Hz}, 1 \mathrm{H}), 7.53-7.47$ (m, 2H), 7.46-7.44 (m, 1H), 7.44-7.37 (m, 2H), $6.47(\mathrm{~s}, 1 \mathrm{H}), 2.56-2.43(\mathrm{~m}, 2 \mathrm{H}), 2.42-2.26(\mathrm{~m}, 2 \mathrm{H}), 1.99-1.74(\mathrm{~m}, 2 \mathrm{H})$.

${ }^{13}$ C-NMR (125 MHz, $\left.\mathrm{CDCl}_{3}\right): \delta=162.5\left(\mathrm{C}_{\mathrm{q}}\right), 144.1\left(\mathrm{C}_{\mathrm{q}}\right), 140.8(\mathrm{CH}), 139.7\left(\mathrm{C}_{\mathrm{q}}\right), 136.9$ $\left(\mathrm{C}_{\mathrm{q}}\right), 133.5(\mathrm{CH}), 128.3(\mathrm{CH}), 128.0(\mathrm{CH}), 127.0(\mathrm{CH}), 126.5(\mathrm{CH}), 125.9(\mathrm{CH}), 125.9$ $(\mathrm{CH}), 124.8\left(\mathrm{C}_{\mathrm{q}}\right), 118.9\left(\mathrm{C}_{\mathrm{q}}\right), 106.1(\mathrm{CH}), 31.1\left(\mathrm{CH}_{2}\right), 23.6\left(\mathrm{CH}_{2}\right), 16.7\left(\mathrm{CH}_{2}\right)$.

IR (ATR): $\tilde{v}=2921,1630,1596,1428,1247,890,766,751 \mathrm{~cm}^{-1}$.

MS (EI) $m / z$ (relative intensity): 305 (26) [M] $]^{+}, 265$ (37), 249 (100), 234 (68), 171 (63), $78.0(66)$.

HR-MS (EI) $m / z$ calcd. for $\mathrm{C}_{18} \mathrm{H}_{15} \mathrm{~N}_{3} \mathrm{O}_{2}[\mathrm{M}]^{+}:$305.1164, found: 305.1156 . 
<smiles></smiles>

2-[3-Cyclopropyl-1-oxoisoquinolin-2(1H)-yl]-pyridine-1-oxide (51af): The general procedure E was followed using benzamide 34a (107 $\mathrm{mg}, 0.50 \mathrm{mmol})$ and alkyne 53f $(66.1 \mathrm{mg}, \quad 1.00 \mathrm{mmol})$. Purification by column chromatography on silica gel $\left(\mathrm{CH}_{2} \mathrm{Cl}_{2}\right.$ /acetone $\left.3: 1\right)$ yielded 51 af $(80.2 \mathrm{mg}, 57 \%)$ as a white solid.

M. p.: $224-226^{\circ} \mathrm{C}$.

${ }^{1} \mathbf{H}-\mathbf{N M R}\left(300 \mathrm{MHz}, \mathrm{CDCl}_{3}\right): \delta=8.40(\mathrm{dd}, J=5.0,2.9 \mathrm{~Hz}, 1 \mathrm{H}), 8.34(\mathrm{dd}, J=8.0,0.6 \mathrm{~Hz}$, $1 \mathrm{H}), 7.63$ (ddd, $J=8.2,7.1,1.4 \mathrm{~Hz}, 1 \mathrm{H}), 7.51-7.44$ (m, 2H), 7.44-7.40 (m, 1H), 7.40-7.35 (m, 2H), 6.38 (s, 1H), 1.59-1.44 (m, 1H), 1.00-0.89 (m, 1H), 0.70-0.46 (m, 3H).

${ }^{13}$ C-NMR (126 MHz, $\left.\mathrm{CDCl}_{3}\right): \delta=162.5\left(\mathrm{C}_{\mathrm{q}}\right), 145.1\left(\mathrm{C}_{\mathrm{q}}\right), 143.4\left(\mathrm{C}_{\mathrm{q}}\right), 140.5(\mathrm{CH}), 137.4$ $\left(\mathrm{C}_{\mathrm{q}}\right), 133.2(\mathrm{CH}), 128.3(\mathrm{CH}), 128.0(\mathrm{CH}), 126.6(\mathrm{CH}), 125.9(\mathrm{CH}), 125.9(\mathrm{CH}), 125.5$ $(\mathrm{CH}), 124.8\left(\mathrm{C}_{\mathrm{q}}\right), 104.9(\mathrm{CH}), 13.3(\mathrm{CH}), 7.4\left(\mathrm{CH}_{2}\right), 5.4\left(\mathrm{CH}_{2}\right)$.

IR (ATR): $\tilde{v}=1666,1632,1592,1428,1392,1266,758,728 \mathrm{~cm}^{-1}$.

MS (EI) $m / z$ (relative intensity): 278 (40) [M] $]^{+}, 261$ (85), 234 (80), 193 (50), 145 (55), 78 (100).

HR-MS (EI) $m / z$ calcd. for $\mathrm{C}_{17} \mathrm{H}_{14} \mathrm{~N}_{2} \mathrm{O}_{2}[\mathrm{M}]^{+}: 278.1055$, found: 278.1057 .

The analytical data are in accordance with those previously reported in the literature. ${ }^{[250]}$<smiles></smiles>

2-\{3-[(Diphenylphosphoryl)methyl]-1-oxoisoquinolin-2(1H)-yl\}pyridine

1-oxide

(176aa): The general procedure $\mathbf{F}$ was followed using benzamide 34a (107 mg, $0.50 \mathrm{mmol}$ ) and allene 75a (144 mg, $0.60 \mathrm{mmol})$. Purification by column chromatography on silica gel $\left(\mathrm{CH}_{2} \mathrm{Cl}_{2} /\right.$ acetone 1:1 to $\mathrm{CH}_{2} \mathrm{Cl}_{2} / \mathrm{MeOH}$ 9:1) yielded 176aa (206 mg, $455 \mu \mathrm{mol}, 63 \%$ ) as a white solid. The analytical data are in accordance with those previously reported ( $c f$. Chapter 5.4.1). 


\subsubsection{Renewable Energy Power Setup}

\subsubsection{Cobaltaelectro-Catalyzed C-H Activation Powered by Sunlight}

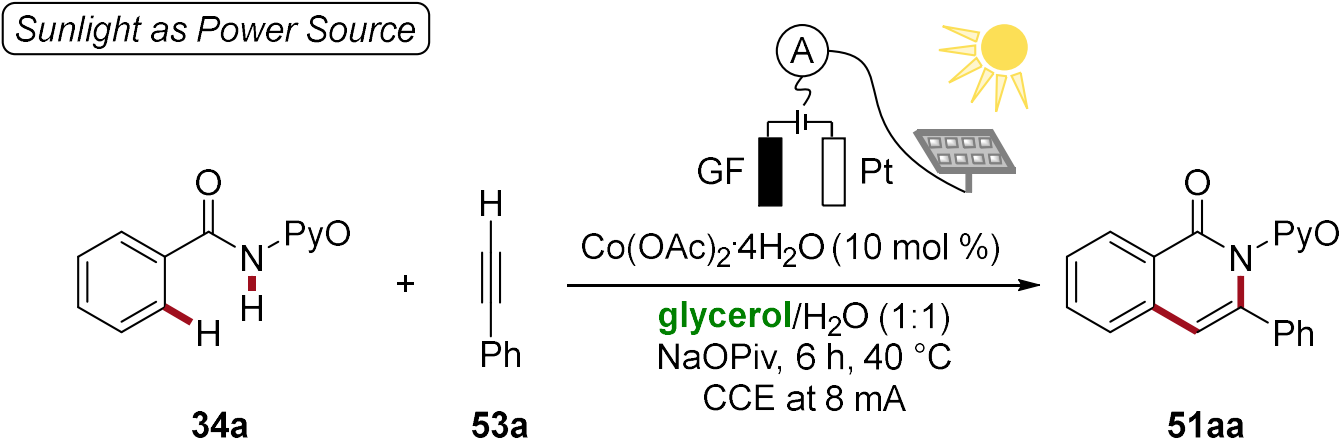

conventional galvanostat: $71 \%$ photovoltaic cell: $\mathbf{7 3} \%$

The electrocatalysis was carried out in an undivided cell, with a graphite felt (GF) anode $(25 \mathrm{~mm} \times 10 \mathrm{~mm} \times 6.0 \mathrm{~mm})$ and a platinum cathode $(25 \mathrm{~mm} \times 10 \mathrm{~mm} \times 0.125 \mathrm{~mm})$. Benzamide 34a ( $0.50 \mathrm{mmol}, 1.00$ equiv), alkyne 53a (1.00 mmol, 2.00 equiv), NaOPiv (124 mg, 1.00 mmol, 2.00 equiv), glycerol $/ \mathrm{H}_{2} \mathrm{O}(5.0 \mathrm{~mL}, 1: 1)$ and $\mathrm{Co}(\mathrm{OAc})_{2} \cdot 4 \mathrm{H}_{2} \mathrm{O}$ $(12.7 \mathrm{mg}, 10 \mathrm{~mol} \%)$ were placed in a $10 \mathrm{~mL}$ cell. Electrocatalysis was performed at $40{ }^{\circ} \mathrm{C}$ with a constant current of $8 \mathrm{~mA}$ maintained for $6 \mathrm{~h}$. The solar panel was disconnected, and the reaction mixture was diluted with $\mathrm{CH}_{2} \mathrm{Cl}_{2}(2.0 \mathrm{~mL})$. The graphite felt anode was washed with $\mathrm{CH}_{2} \mathrm{Cl}_{2}(3 \times 5.0 \mathrm{~mL})$ in an ultrasonic bath $(3 \times 3 \mathrm{~min})$. The combined washings were added to the reaction mixture and the combined phases were washed with $\mathrm{H}_{2} \mathrm{O}(15 \mathrm{~mL})$. The aqueous phase was extracted with $\mathrm{CH}_{2} \mathrm{Cl}_{2}(4 \times 10 \mathrm{~mL})$. The crude extracts were then dried over $\mathrm{Na}_{2} \mathrm{SO}_{4}$. Evaporation of the solvent and subsequent column chromatography on silica gel afforded the corresponding product 51 aa $(114 \mathrm{mg}, 73 \%)$ as a white solid.

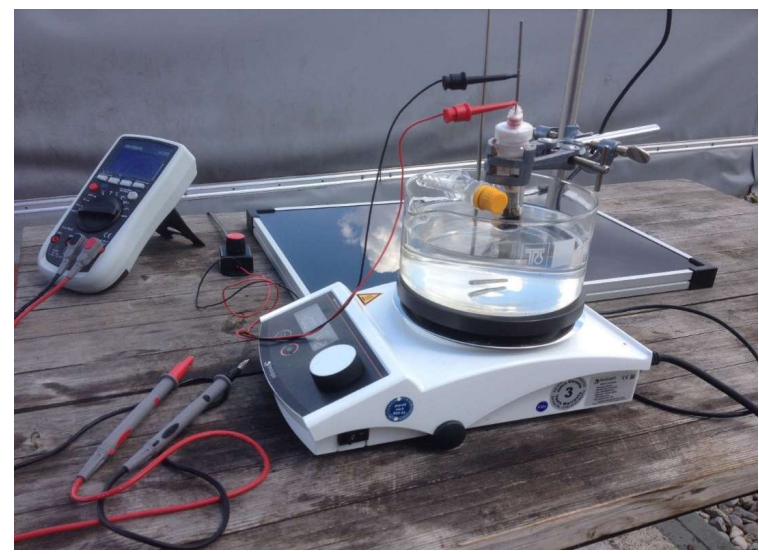

Figure 48. Cobalta-electrocatalysis powered by a photovoltaic cell. 


\subsubsection{Cobaltaelectro-Catalyzed C-H Activation Powered by Wind Energy}

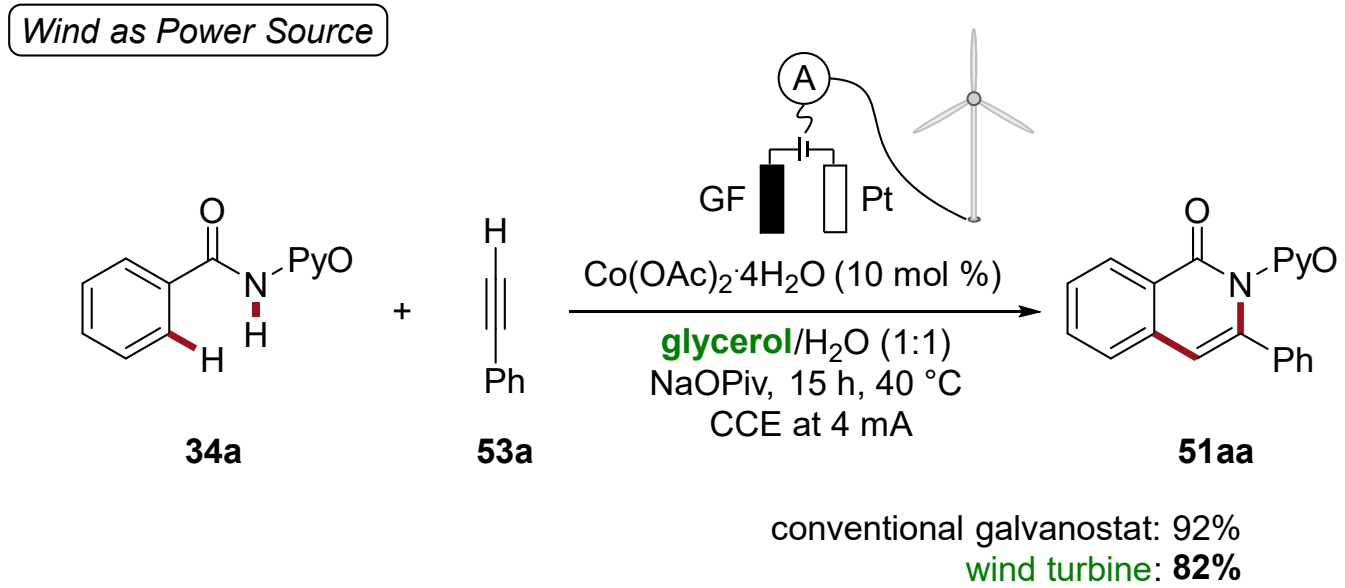

The electrocatalysis was carried out in an undivided cell, with a graphite felt (GF) anode $(25 \mathrm{~mm} \times 10 \mathrm{~mm} \times 6.0 \mathrm{~mm})$ and a platinum cathode $(25 \mathrm{~mm} \times 10 \mathrm{~mm} \times 0.125 \mathrm{~mm})$. Benzamide 34a ( $0.50 \mathrm{mmol}, 1.00$ equiv), alkyne 53a (1.00 mmol, 2.00 equiv), NaOPiv (124 mg, $1.00 \mathrm{mmol}, 2.00$ equiv), glycerol/ $\mathrm{H}_{2} \mathrm{O}(5.0 \mathrm{~mL}, 1: 1)$ and $\mathrm{Co}(\mathrm{OAc})_{2} \cdot 4 \mathrm{H}_{2} \mathrm{O}$ $(12.7 \mathrm{mg}, 10 \mathrm{~mol} \%)$ were placed in a $10 \mathrm{~mL}$ cell. Electrocatalysis was performed at $40{ }^{\circ} \mathrm{C}$ with a constant current of $4 \mathrm{~mA}$ maintained for $15 \mathrm{~h}$. The wind turbine was stopped, and the reaction mixture was diluted with $\mathrm{CH}_{2} \mathrm{Cl}_{2}(2.0 \mathrm{~mL})$. The graphite felt anode was washed with $\mathrm{CH}_{2} \mathrm{Cl}_{2}(3 \times 5.0 \mathrm{~mL})$ in an ultrasonic bath $(3 \times 3 \mathrm{~min})$. The combined washings were added to the reaction mixture and the combined phases were washed with $\mathrm{H}_{2} \mathrm{O}(15 \mathrm{~mL})$. The aqueous phase was extracted with $\mathrm{CH}_{2} \mathrm{Cl}_{2}(4 \times 10 \mathrm{~mL})$. The crude extracts were then dried over $\mathrm{Na}_{2} \mathrm{SO}_{4}$. Evaporation of the solvent and subsequent column chromatography on silica gel afforded the corresponding product 51 aa $(128 \mathrm{mg}, 82 \%)$ as a white solid.

A)

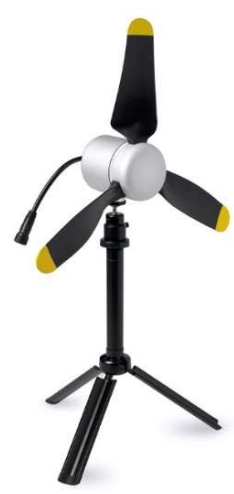

B)

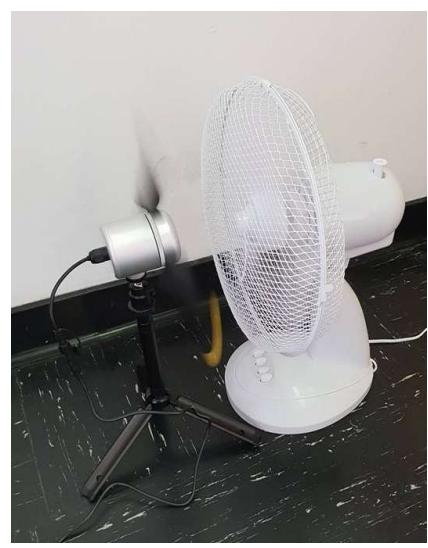

Figure 49. A) TEXENERGY Limited, Infinite Air wind turbine. B) Wind turbine powered by a fan. 


\subsubsection{Cyclic Voltammetry}

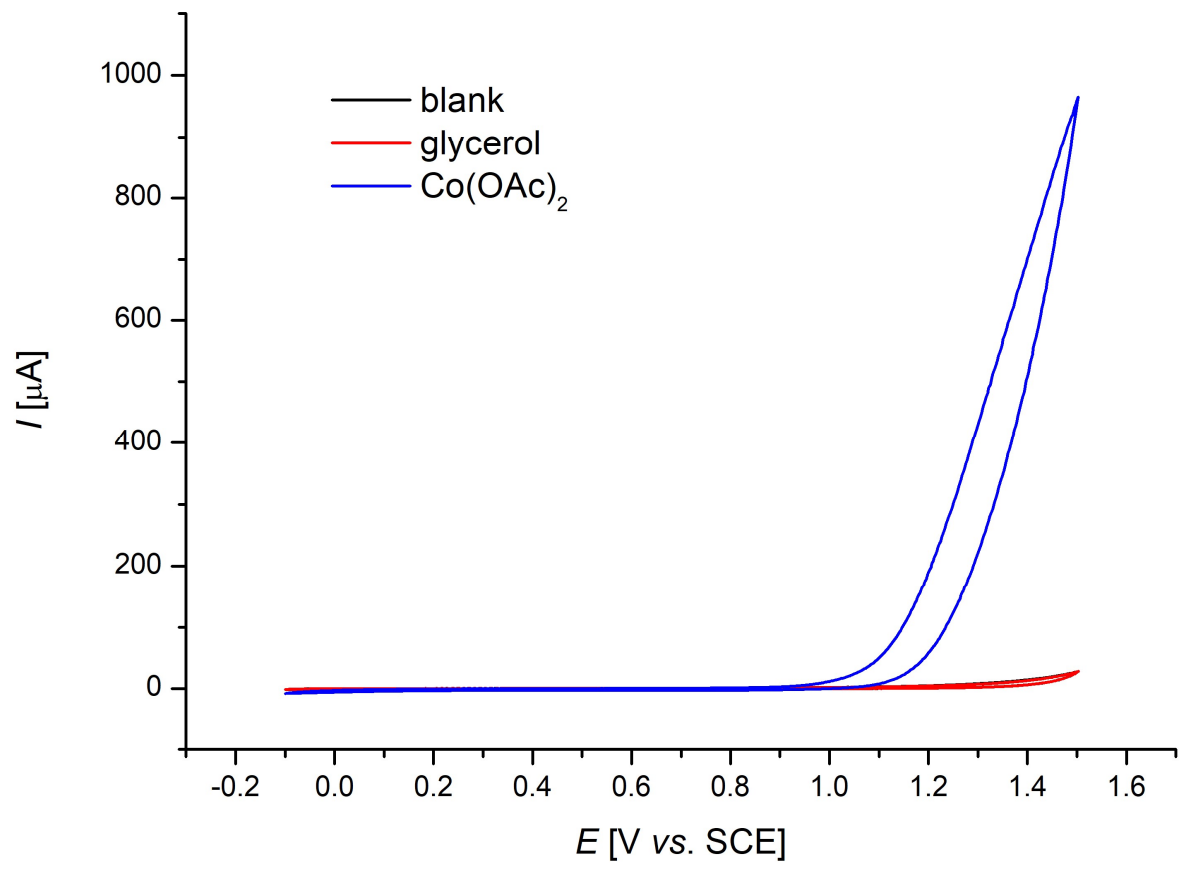

Figure 50. Cyclic voltammograms at $100 \mathrm{mV} \cdot \mathrm{s}^{-1}$ using $\mathrm{H}_{2} \mathrm{O}$ and $\mathrm{NaOAc}(0.2 \mathrm{M})$ as the electrolyte and a GC working electrode. (black) Blank; (red) glycerol (50 mM); (blue) $\mathrm{Co}(\mathrm{OAc})_{2}(10 \mathrm{mM})$.

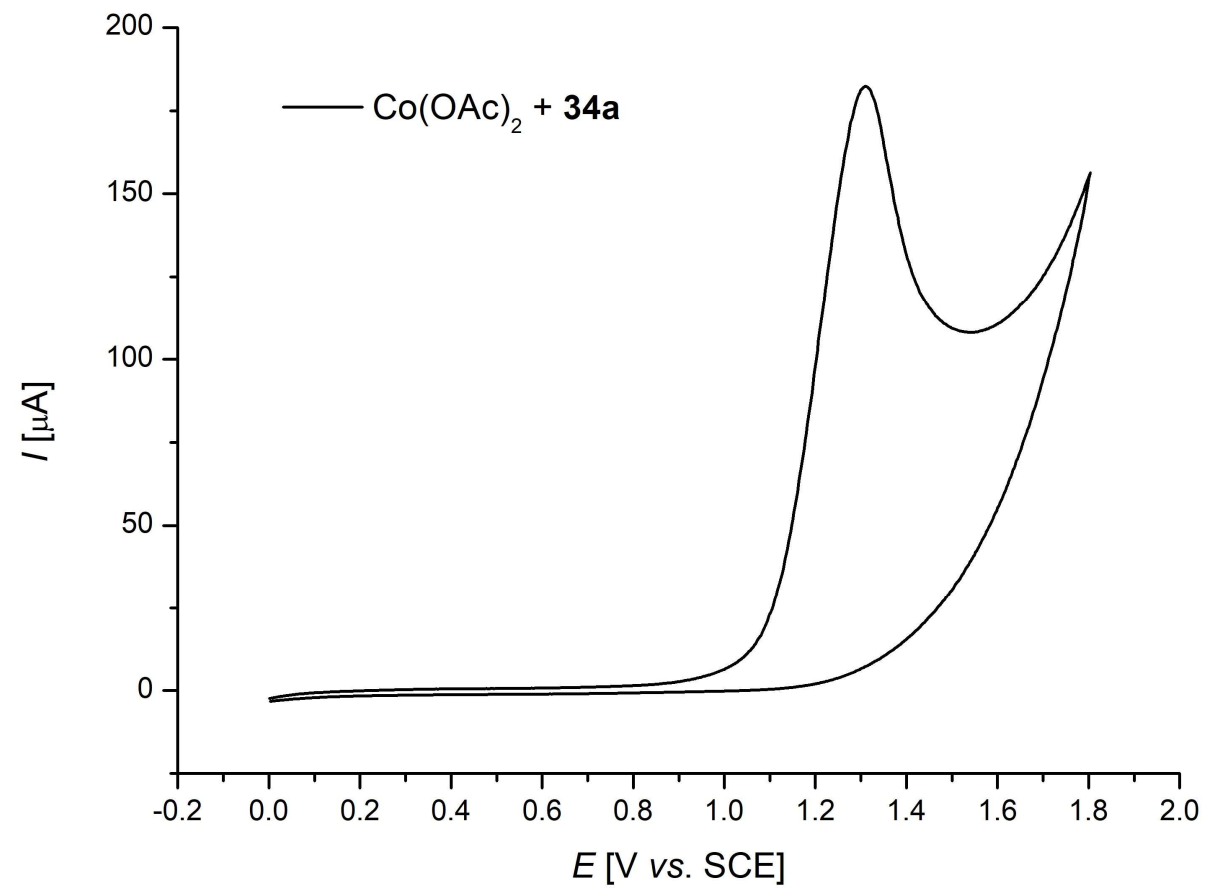

Figure 51. Cyclic voltammogram at $100 \mathrm{mV} \cdot \mathrm{s}^{-1}$ using $\mathrm{H}_{2} \mathrm{O}$ and $\mathrm{NaOAc}(0.2 \mathrm{M})$ as the electrolyte and a $\mathrm{GC}$ working electrode. $\mathrm{Co}(\mathrm{OAc})_{2}(10 \mathrm{mM})$ and benzamide 34a (10 mM). 


\subsection{Mechanistic Insights into Cobalta(III/IV/II)-Electrocatalysis}

\subsubsection{Characterization Data}

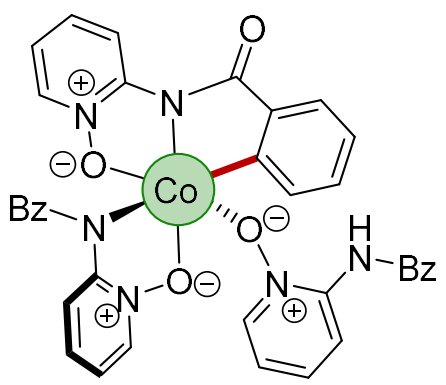

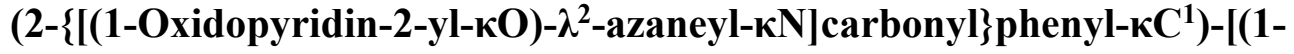

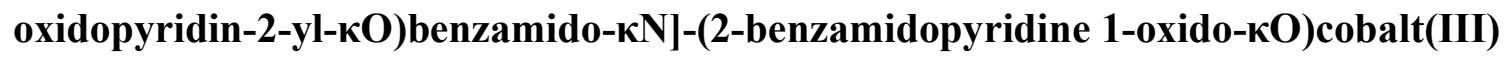
(190): The general procedure $\mathbf{G}$ was followed using benzamide 34a $(214 \mathrm{mg}, 1.00 \mathrm{mmol}$, 2.00 equiv), NaOPiv (124 mg, $1.00 \mathrm{mmol}, 2.00$ equiv), $\mathrm{MeCN}(13 \mathrm{~mL})$ and $\mathrm{Co}(\mathrm{OAc})_{2}$ (88.5 mg, $0.50 \mathrm{mmol})$. Purification by column chromatography on silica gel $\left(\mathrm{CH}_{2} \mathrm{Cl}_{2} / \mathrm{MeOH}\right.$ 99:1 $\rightarrow$ 30:1) yielded $190(161 \mathrm{mg}, 46 \%)$ as a dark green solid. NMR spectroscopy and ESI-MS studies revealed the dissociation of the weakly mono-Ocoordinated substrate 34a in solution. With increased ionization energy for the ESI-MS, a significant decrease of the corresponding cobalta(III)cycle 190 signal with three coordinating substrates $\left([\mathrm{M}+\mathrm{Na}]^{+}, m / z=721.1\right)$ was observed.

${ }^{1}$ H-NMR $\left(400 \mathrm{MHz}, \mathrm{CD}_{2} \mathrm{Cl}_{2}\right): \delta=8.66(\mathrm{dd}, J=6.5,0.8 \mathrm{~Hz}, 1 \mathrm{H}), 8.05(\mathrm{dd}, J=7.7,0.6 \mathrm{~Hz}$, $1 \mathrm{H}), 7.96(\mathrm{dd}, J=8.8,1.6 \mathrm{~Hz}, 1 \mathrm{H}), 7.51-7.44(\mathrm{~m}, 1 \mathrm{H}), 7.37-7.29(\mathrm{~m}, 2 \mathrm{H}), 7.18-7.06(\mathrm{~m}$, $3 \mathrm{H}), 6.87-6.74(\mathrm{~m}, 4 \mathrm{H}), 6.60-6.49(\mathrm{~m}, 3 \mathrm{H}), 6.18-6.10(\mathrm{~m}, 1 \mathrm{H})$.

${ }^{13}$ C-NMR $\left(101 \mathrm{MHz}, \mathrm{CD}_{2} \mathrm{Cl}_{2}\right): \delta=182.1\left(\mathrm{C}_{\mathrm{q}}\right), 174.3\left(\mathrm{C}_{\mathrm{q}}\right), 157.0\left(\mathrm{C}_{\mathrm{q}}\right), 156.6\left(\mathrm{C}_{\mathrm{q}}\right), 148.7$ $\left(\mathrm{C}_{\mathrm{q}}\right), 139.3(\mathrm{CH}), 138.3(\mathrm{CH}), 137.6\left(\mathrm{C}_{\mathrm{q}}\right), 137.2\left(\mathrm{C}_{\mathrm{q}}\right), 136.2(\mathrm{CH}), 136.1(\mathrm{CH}), 131.8(\mathrm{CH})$, $131.4(\mathrm{CH}), 131.0(\mathrm{CH}), 128.4(\mathrm{CH}), 128.3(\mathrm{CH}), 128.1(\mathrm{CH}), 126.0(\mathrm{CH}), 119.3(\mathrm{CH})$, $118.8(\mathrm{CH}), 114.1(\mathrm{CH}), 114.1(\mathrm{CH})$.

IR (ATR): $\tilde{v}=3059,1640,1606,1557,1486,1361,1261,751 \mathrm{~cm}^{-1}$.

MS (ESI) $m / z$ (relative intensity): 991.1 (25) $[2 \mathrm{xM}-2 \times 34 a+N a]^{+}, 721.1(20)[\mathrm{M}+\mathrm{Na}]^{+}$, $699.1(5)[\mathrm{M}+\mathrm{H}]^{+}, 507.0(100)[\mathrm{M}-\mathbf{3 4 a}+\mathrm{Na}]^{+}, 485.1(25)[\mathrm{M}-\mathbf{3 4 a}+\mathrm{H}]^{+}$.

HR-MS (ESI) $m / z$ calcd. for $\mathrm{C}_{36} \mathrm{H}_{27} \mathrm{CoN}_{6} \mathrm{NaO}_{6}[\mathrm{M}+\mathrm{Na}]^{+}: 721.1216$, found 721.1221.

Crystals suitable for an X-ray diffraction study were obtained from slow solvent evaporation of a saturated solution of $\mathbf{1 9 0}$ in $\mathrm{MeOH}$. Crystallization studies were performed together with Dr. D. Ghorai. 


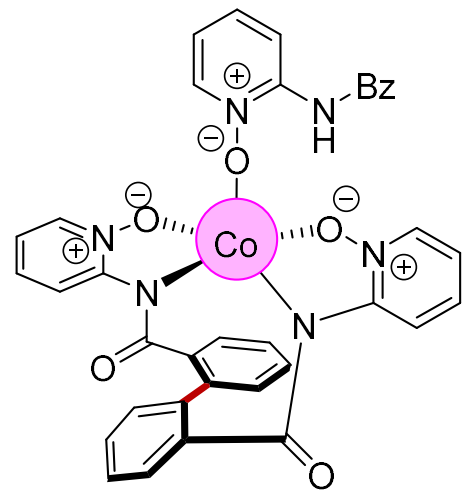

(2-Benzamidopyridine

1-oxide)

$\left(2,2^{\prime}-\left\{\left[\left(1,1^{\prime}-\right.\right.\right.\right.$ biphenyl $)-2,2^{\prime}-$

dicarbonyl]bis(azanediyl)\}bis(pyridine 1-oxido)cobalt(II) (203): Benzamide 34a (107 mg, $0.50 \mathrm{mmol}, 1.00$ equiv), NaOPiv (124 mg, $1.00 \mathrm{mmol}, 2.00$ equiv), MeCN $(5 \mathrm{~mL})$ and $\mathrm{Co}(\mathrm{OAc})_{2} \cdot 4 \mathrm{H}_{2} \mathrm{O}(62.3 \mathrm{mg}, 0.25 \mathrm{mmol}, 0.50$ equiv $)$ were placed in a $10 \mathrm{~mL}$ cell. Electrosynthesis was performed at $60^{\circ} \mathrm{C}$ with a constant current of $4 \mathrm{~mA}$ for $14 \mathrm{~h}$. Then, the DC-power supply was stopped and the reaction mixture was diluted with $\mathrm{CH}_{2} \mathrm{Cl}_{2}$ $(2.0 \mathrm{~mL})$. The graphite felt anode was washed with $\mathrm{CH}_{2} \mathrm{Cl}_{2}(3 \times 5.0 \mathrm{~mL})$ in an ultrasonic bath $(3 \times 3 \mathrm{~min})$. The combined washings were added to the reaction mixture and the combined phases were stirred with aq. sodium tartrate $(15 \mathrm{~mL}, 1.0 \mathrm{M})$. The aqueous phase was extracted with $\mathrm{CH}_{2} \mathrm{Cl}_{2}(4 \times 10 \mathrm{~mL})$. The crude extracts were dried over $\mathrm{Na}_{2} \mathrm{SO}_{4}$. Evaporation of the solvents and subsequent column chromatography on silica gel $\left(\mathrm{CH}_{2} \mathrm{Cl}_{2} / \mathrm{MeOH}\right.$ 99:1 $\left.\rightarrow 10: 1\right)$, followed by recrystallisation in $\mathrm{CH}_{2} \mathrm{Cl}_{2}$ yielded 203 (92.6 mg, $132 \mu \mathrm{mol}, 53 \%)$ as pink crystals and $205(21.0 \mathrm{mg}, 49.2 \mu \mathrm{mol}, 20 \%)$ as white crystals. Paramagnetic ${ }^{1} \mathrm{H}-\mathrm{NMR}$ spectroscopy and ESI-MS analysis showed dissociation of the mono-O-coordinated substrate $\mathbf{3 4 a}$ in solution, which could be confirmed by X-ray diffraction analysis.

${ }^{1}$ H-NMR (300 MHz, paramagnetic, $\mathrm{CD}_{2} \mathrm{Cl}_{2}$ ): $\delta=132.26$ (br, 1H), 128.80 (br, 1H), 103.86 (br, 1H), 78.86 (br, 1H), 10.20-9.20 (m, 1H), 9.34-9.08 (m, 1H), 8.41-7.19 (m, 7H), -3.96 (br, 1H), $-17.52(\mathrm{br}, 1 \mathrm{H}),-22.60(\mathrm{br}, 1 \mathrm{H})$.

IR (ATR): $\tilde{v}=3062,2942,1687,1617,1556,1479,1430,1339,1135,750,704 \mathrm{~cm}^{-1}$. MS (ESI) $m / z$ (relative intensity): 1472.1 (25) $[3 \times \mathrm{xM}-3 \times 34 a+N a]^{+}, 1202.1$ (20) $[2 \times \mathrm{x}-$ $\mathbf{3 4 a}+\mathrm{Na}]^{+}, 989.0(50)[2 \times \mathrm{M}-2 \times 34 \mathbf{a}+\mathrm{Na}]^{+}, 719.1(60)[\mathrm{M}+\mathrm{Na}]^{+}, 506.0(100)[\mathrm{M}-\mathbf{3 4 a}+\mathrm{Na}]^{+}$, $484.1(25)[\mathrm{M}-\mathbf{3 4 a}+\mathrm{H}]^{+}$.

HR-MS (ESI) $m / z$ calcd. for $\mathrm{C}_{36} \mathrm{H}_{25} \mathrm{CoN}_{6} \mathrm{NaO}_{6}[\mathrm{M}+\mathrm{Na}]^{+}: 719.1060$, found 719.1048. 
Crystals suitable for X-ray diffraction studies were obtained from slow diffusion of $n$-hexane into a saturated solution of $\mathbf{2 0 3}$ in $\mathrm{CH}_{2} \mathrm{Cl}_{2}$ or concentrated solution of $\mathbf{2 0 3}$ in $\mathrm{MeOH}$.<smiles>O=C(Nc1cccc[n+]1[O-])c1ccccc1-c1ccccc1NC(=O)c1cccc[n+]1[O-]</smiles>

2,2'-\{[(1,1'-Biphenyl)-2,2'-dicarbonyl]bis(azanediyl)\}bis(pyridine 1-oxide) (205): The representative synthesis of $\mathbf{2 0 3}$ was followed, using benzamide $\mathbf{3 4 a}$ (107 mg, $0.50 \mathrm{mmol}$, 1.00 equiv), NaOPiv (124 mg, $1.00 \mathrm{mmol}, 2.00$ equiv), $\mathrm{MeCN} \quad(5 \mathrm{~mL})$ and $\mathrm{Co}(\mathrm{OAc})_{2} \cdot 4 \mathrm{H}_{2} \mathrm{O}(62.3 \mathrm{mg}, 0.25 \mathrm{mmol}, 0.50$ equiv $)$. The obtained purple crystals were dissolved in $\mathrm{CH}_{2} \mathrm{Cl}_{2}(5.0 \mathrm{~mL})$ and vigorously stirred with aq. EDTA solution $(10 \mathrm{~mL}$, $0.1 \mathrm{M})$ for $12 \mathrm{~h}$ at $25^{\circ} \mathrm{C}$. The phases were separated, and the aqueous phase was extracted with $\mathrm{CH}_{2} \mathrm{Cl}_{2}(4 \times 5.0 \mathrm{~mL})$. The crude extracts were dried over $\mathrm{Na}_{2} \mathrm{SO}_{4}$. Evaporation of the solvents and subsequent column chromatography on silica gel $\left(\mathrm{CH}_{2} \mathrm{Cl}_{2} / \mathrm{MeOH} 15: 1 \rightarrow 8: 1\right)$ yielded 205 (30.2 mg, $70.8 \mu \mathrm{mol}, 28 \%$ ) as white crystals.

M. p.: $209-210^{\circ} \mathrm{C}$.

${ }^{1}$ H-NMR $\left(600 \mathrm{MHz} \mathrm{CDCl}_{3}\right): \delta=10.60(\mathrm{~s}, 2 \mathrm{H}), 8.42(\mathrm{ddd}, J=8.5,1.9,0.6 \mathrm{~Hz}, 2 \mathrm{H}), 8.11$ (ddd, $J=6.5,0.9,0.6 \mathrm{~Hz}, 2 \mathrm{H}), 7.89-7.81(\mathrm{~m}, 2 \mathrm{H}), 7.53-7.43(\mathrm{~m}, 4 \mathrm{H}), 7.33-7.28(\mathrm{~m}, 2 \mathrm{H})$, $7.23(\mathrm{ddd}, J=8.5,7.6,1.5 \mathrm{~Hz}, 2 \mathrm{H}), 6.90(\mathrm{ddd}, J=7.6,6.5,1.9 \mathrm{~Hz}, 2 \mathrm{H})$. Even at $-35{ }^{\circ} \mathrm{C}$, no hindered rotation was detected.

${ }^{13}$ C-NMR (151 MHz, $\left.\mathrm{CDCl}_{3}\right): \delta=167.4\left(\mathrm{C}_{\mathrm{q}}\right), 144.4\left(\mathrm{C}_{\mathrm{q}}\right), 139.7\left(\mathrm{C}_{\mathrm{q}}\right), 137.1(\mathrm{CH}), 134.7$ $\left(\mathrm{C}_{\mathrm{q}}\right), 131.4(\mathrm{CH}), 130.6(\mathrm{CH}), 128.5(\mathrm{CH}), 128.4(\mathrm{CH}), 127.8(\mathrm{CH}), 118.8(\mathrm{CH}), 115.2$ $(\mathrm{CH})$.

IR (ATR): $\tilde{v}=3257,1678,1566,1501,1425,1282,1208,756,725 \mathrm{~cm}^{-1}$.

MS (ESI) $m / z$ (relative intensity): $875.2(30)[2 \mathrm{xM}+\mathrm{Na}]^{+}, 449.1(100)[\mathrm{M}+\mathrm{Na}]^{+}, 427.1$ (10) $[\mathrm{M}+\mathrm{H}]^{+}$.

HR-MS (ESI) $m / z$ calcd. for $\mathrm{C}_{24} \mathrm{H}_{18} \mathrm{~N}_{4} \mathrm{NaO}_{4}[\mathrm{M}+\mathrm{Na}]^{+}:$449.1220, found 449.1216. 


\subsubsection{Reaction Monitoring by Mass Spectrometry}

\subsubsection{ESI-MS Monitoring of the Formation of 190 upon Anodic Oxidation}

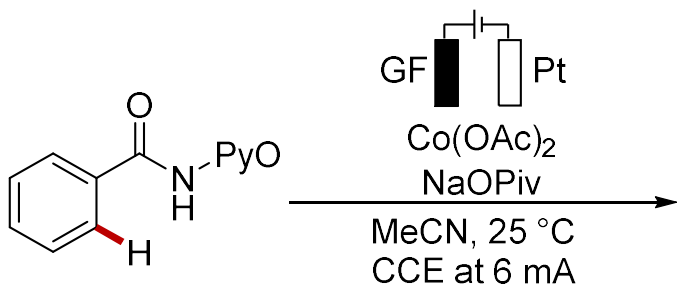

$34 a$

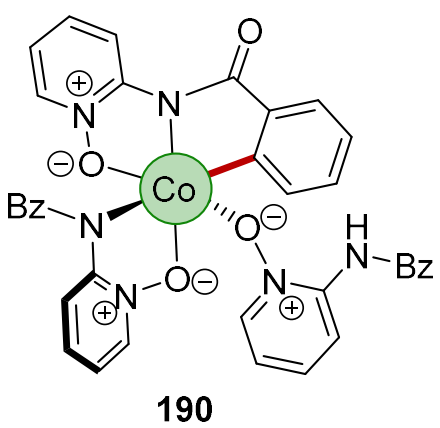

190

Benzamide 34a (107 mg, $0.50 \mathrm{mmol}, 1.00$ equiv), $\mathrm{Co}(\mathrm{OAc})_{2} \cdot 4 \mathrm{H}_{2} \mathrm{O}(125 \mathrm{mg}, 0.50 \mathrm{mmol}$, 1.00 equiv), NaOPiv (124 mg, $1.00 \mathrm{mmol}, 2.00$ equiv), and $\mathrm{MeCN}(9.0 \mathrm{~mL})$ were placed in a $20 \mathrm{~mL}$ undivided cell. Electrosynthesis was performed at $25^{\circ} \mathrm{C}$ with a constant current of $6 \mathrm{~mA}$ maintained for $2 \mathrm{~h}$. Aliquots $(100 \mu \mathrm{L})$ were collected after $0,60,120 \mathrm{~min}$ and were diluted with $\mathrm{MeCN}$ to give a $0.01 \mathrm{~mm}$ solution.

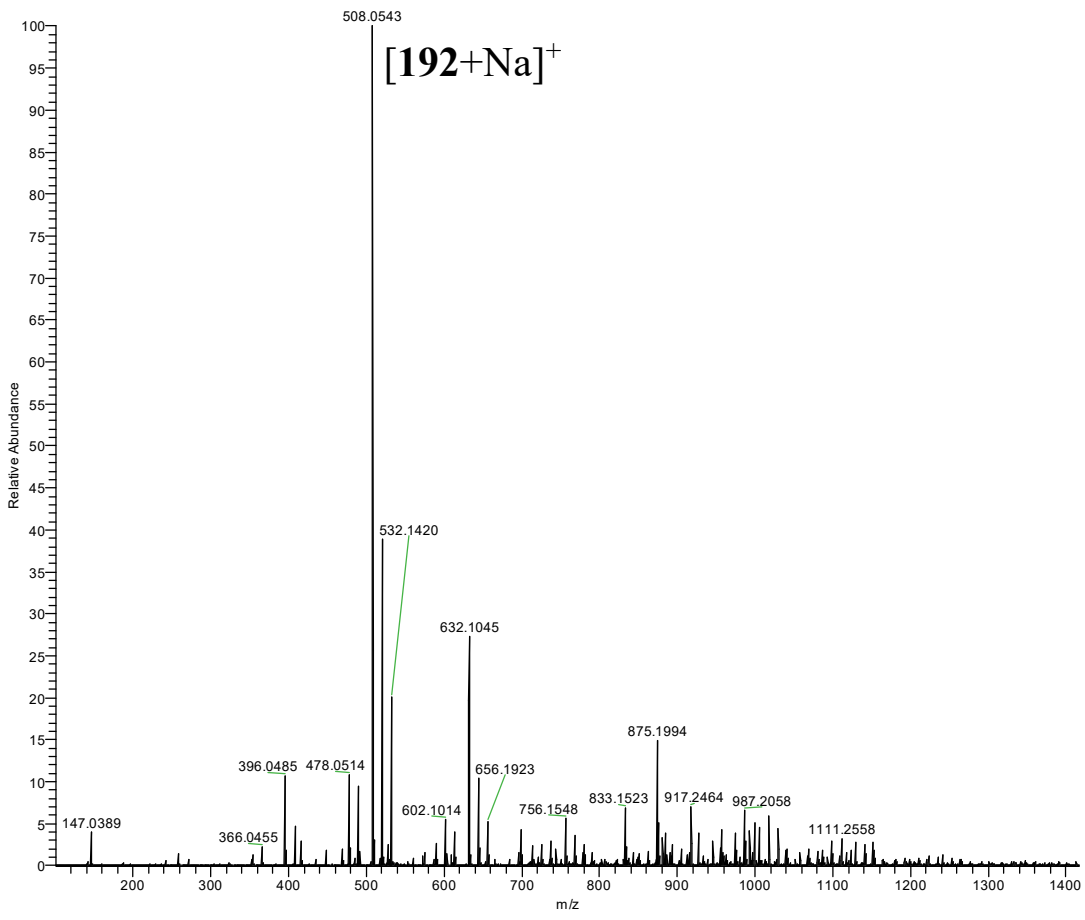

Figure 52. ESI-MS (positive ionization-mode) after 0 min. 


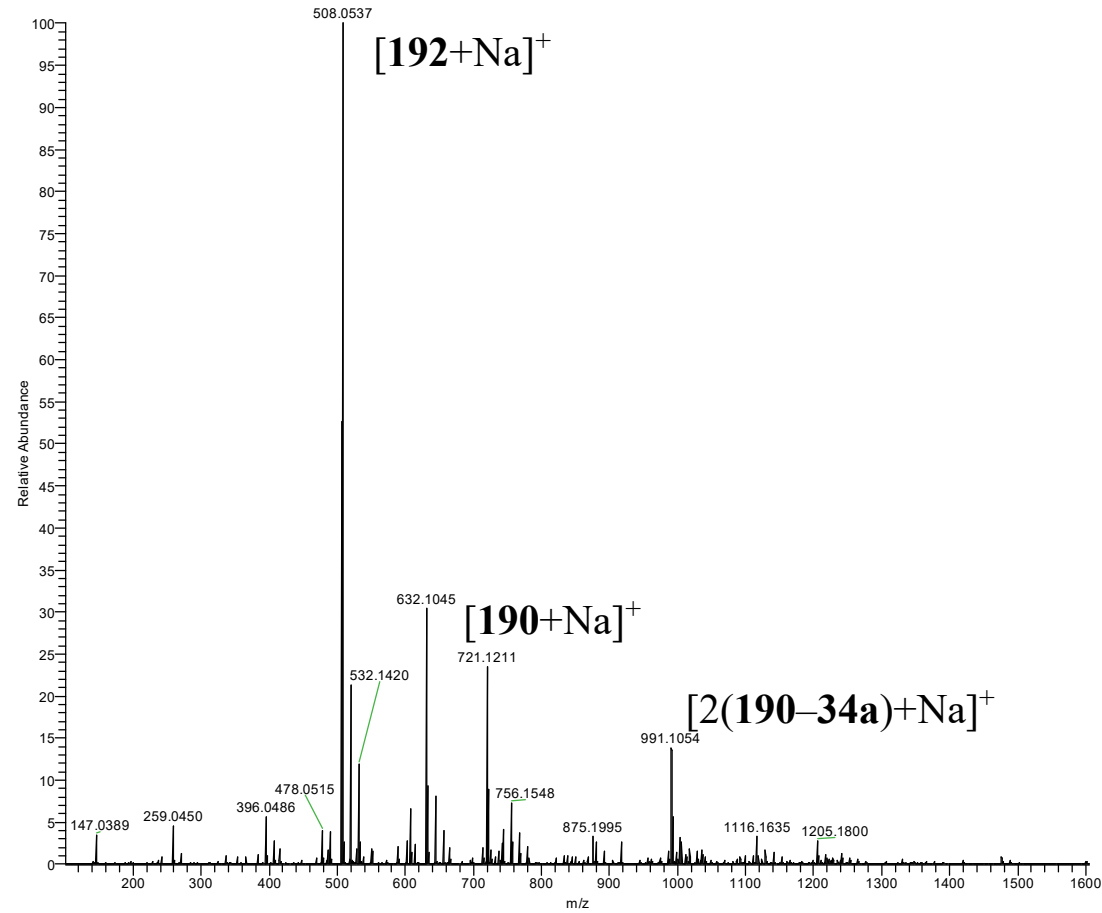

Figure 53. ESI-MS (positive ionization-mode) after $60 \mathrm{~min}$.

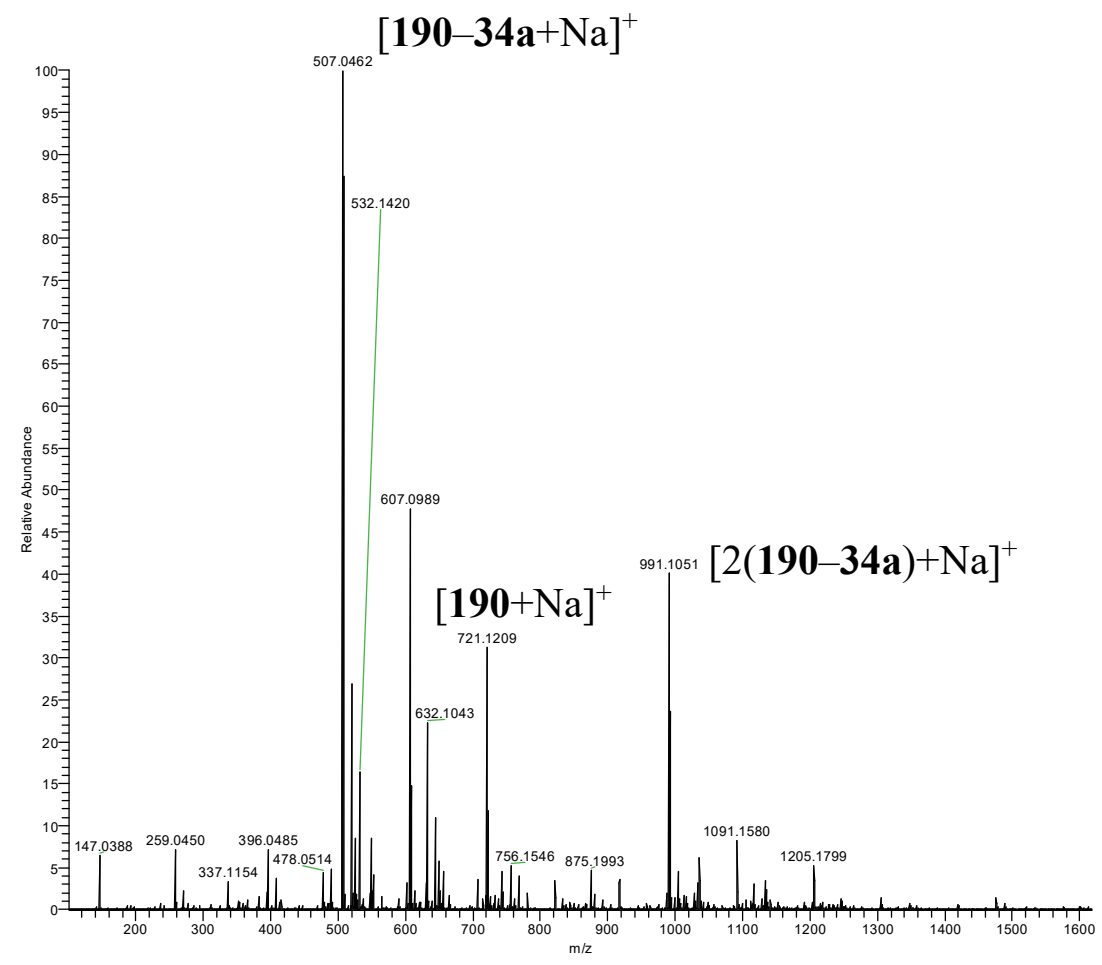

Figure 54. ESI-MS (positive ionization-mode) after $120 \mathrm{~min}$. 
5.6.2.2 ESI-MS Monitoring of the Formation of 36ab upon Anodic Oxidation of 190

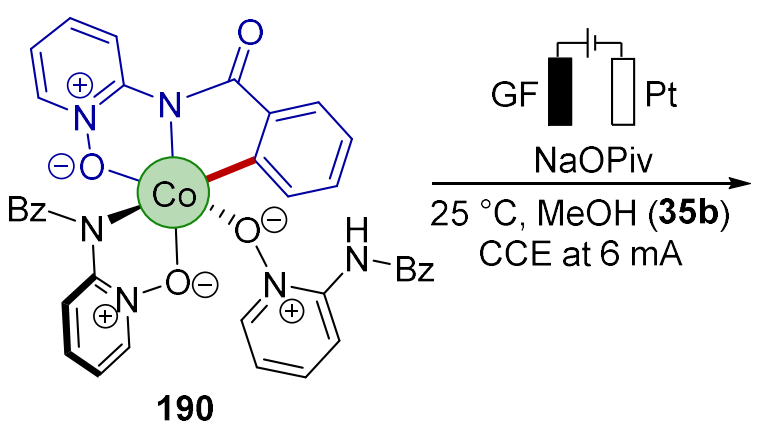<smiles>COc1ccccc1C(=O)NPC=O</smiles>

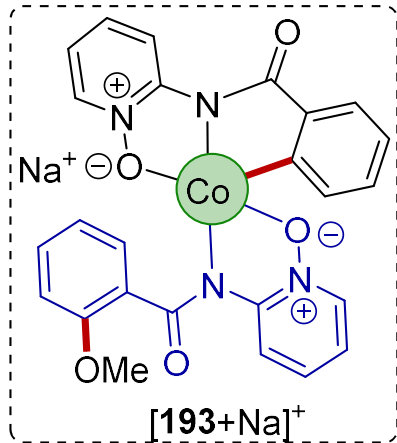

34 (140 mg, $200 \mu \mathrm{mol}, 1.00$ equiv), NaOPiv (49.6 mg, $400 \mu \mathrm{mol}, 2.00$ equiv), and $\mathrm{MeOH}$ (35b) $(9.0 \mathrm{~mL})$ were placed in a $20 \mathrm{~mL}$ cell. Electrosynthesis was performed at $25^{\circ} \mathrm{C}$ with a constant current of $6 \mathrm{~mA}$ maintained for $90 \mathrm{~min}$. Aliquots $(100 \mu \mathrm{L})$ were collected after 0, 30, 60, $90 \mathrm{~min}$ and were diluted with $\mathrm{MeOH}$ to give a $0.01 \mathrm{~mm}$ solution.

Table 33. Plot of relative intensity of $\mathbf{3 6} \mathbf{a b}$, detected with ESI-MS, versus time.

\begin{tabular}{ccc}
\hline Entry & Time $[\mathrm{min}]$ & Rel. Int. 36ab [\%] \\
\hline 1 & 0 & 0 \\
2 & 30 & 35 \\
3 & 60 & 72 \\
4 & 90 & 100 \\
\hline
\end{tabular}

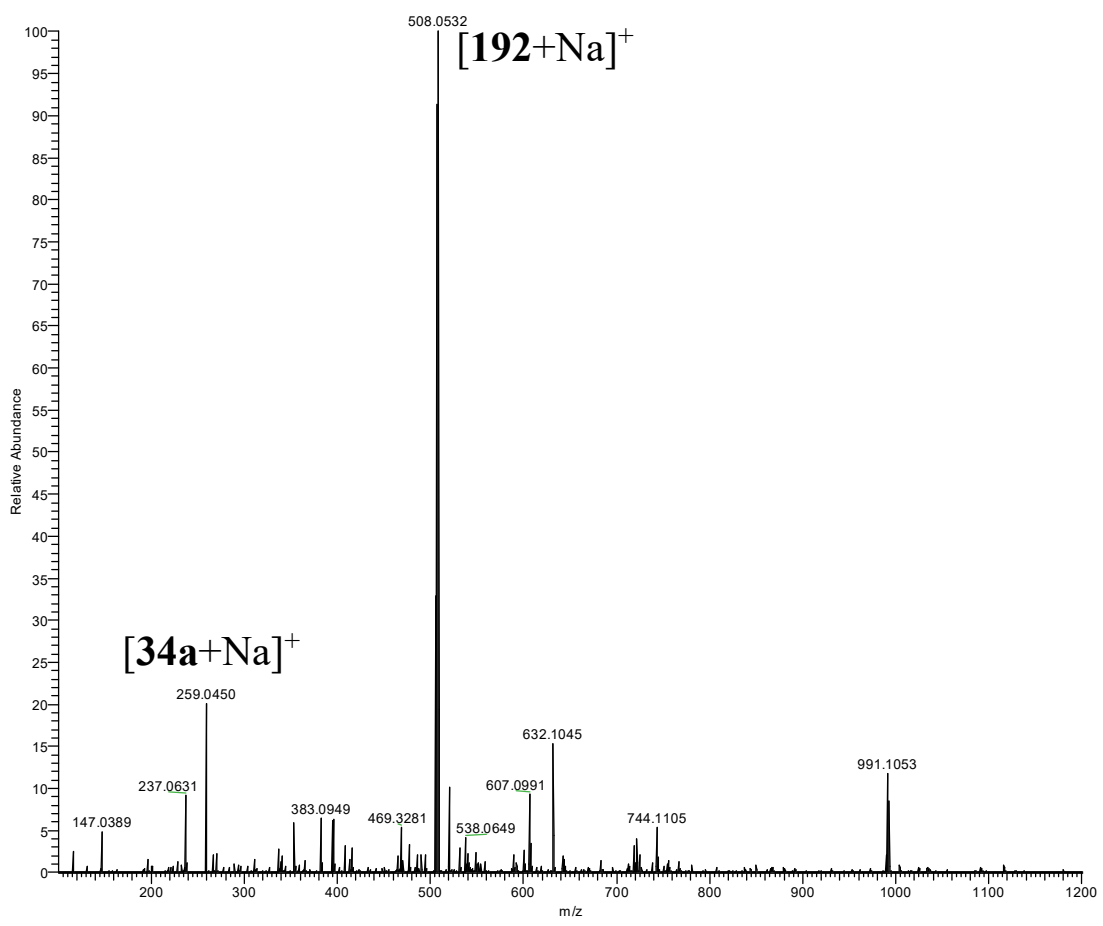

Figure 55. ESI-MS (positive ionization-mode) after $0 \mathrm{~min}$. 


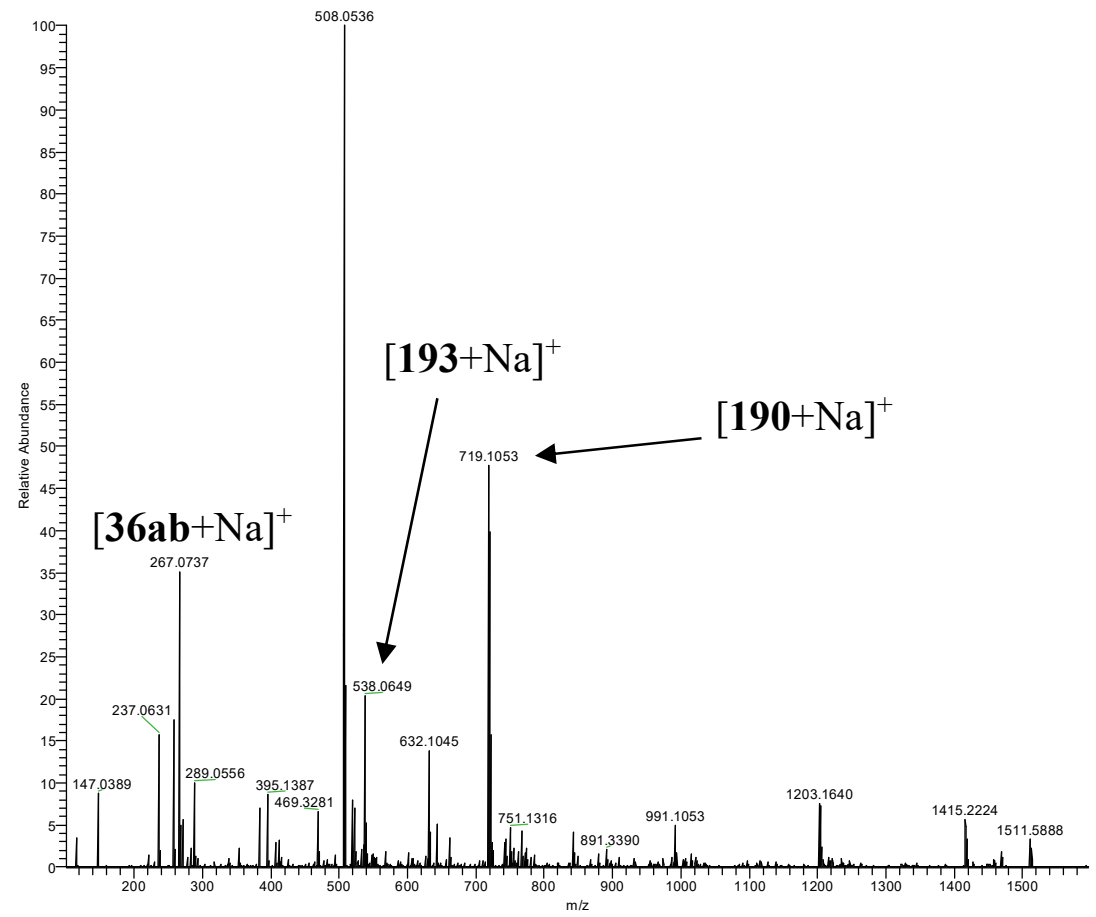

Figure 56. ESI-MS (positive ionization-mode) after $30 \mathrm{~min}$.

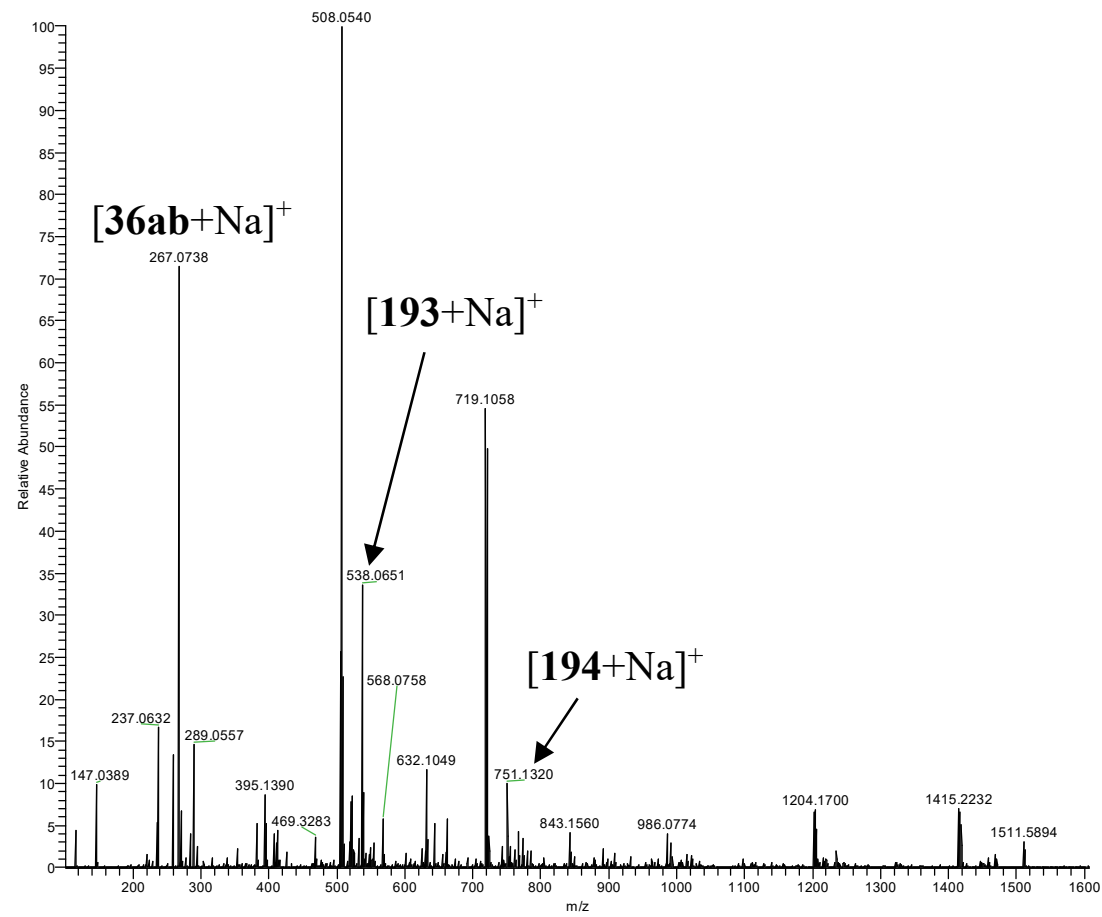

Figure 57. ESI-MS (positive ionization-mode) after $60 \mathrm{~min}$. 


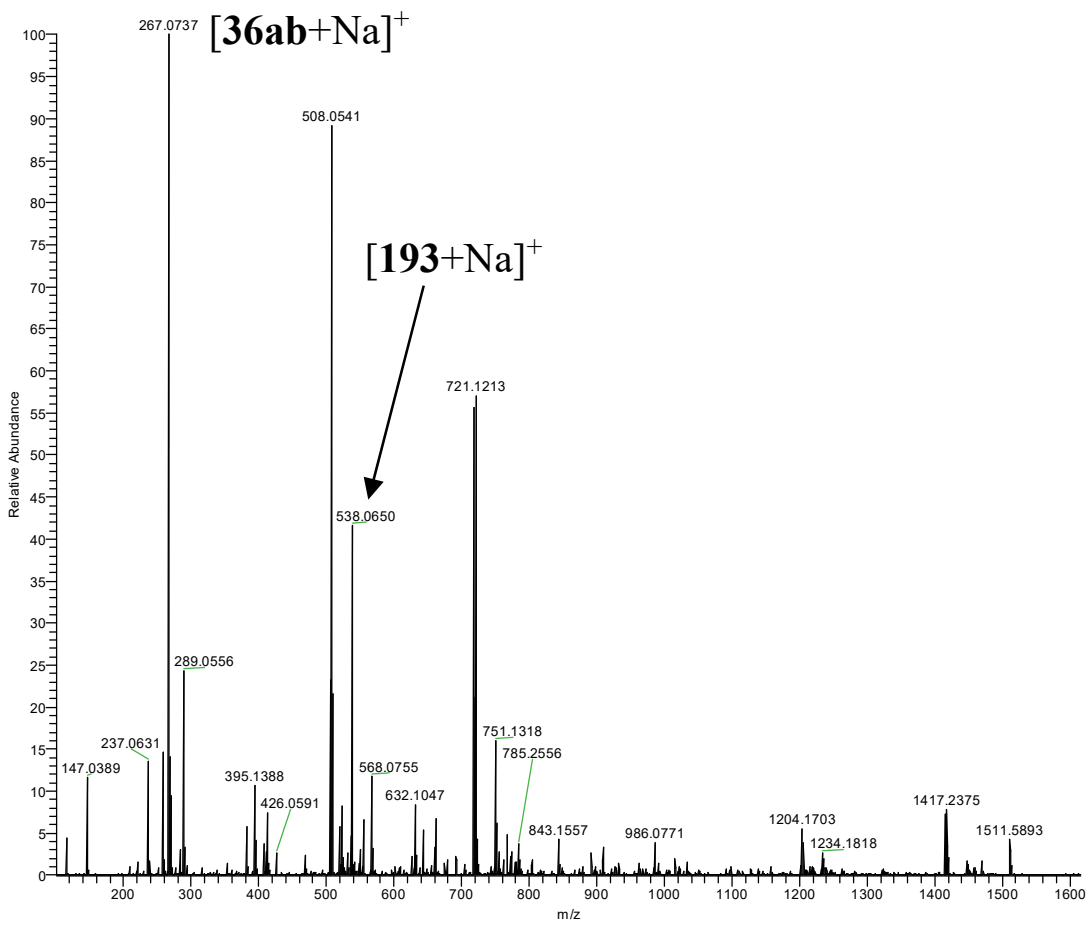

Figure 58. ESI-MS (positive ionization-mode) after $90 \mathrm{~min}$.

\subsubsection{Reactivity of Cyclometalated Cobalt(III) Complex 190}

\subsubsection{Reactivity of Cobalt(III)-Complex 190 toward C-H Alkoxylation in the}

\section{Absence of Oxidants}

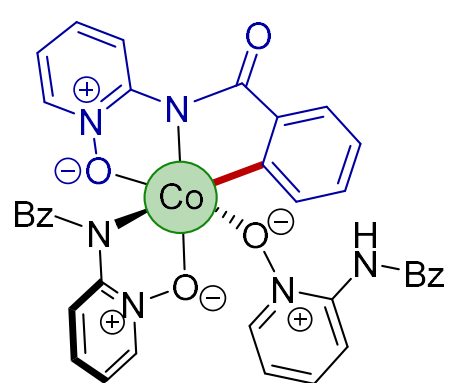

190

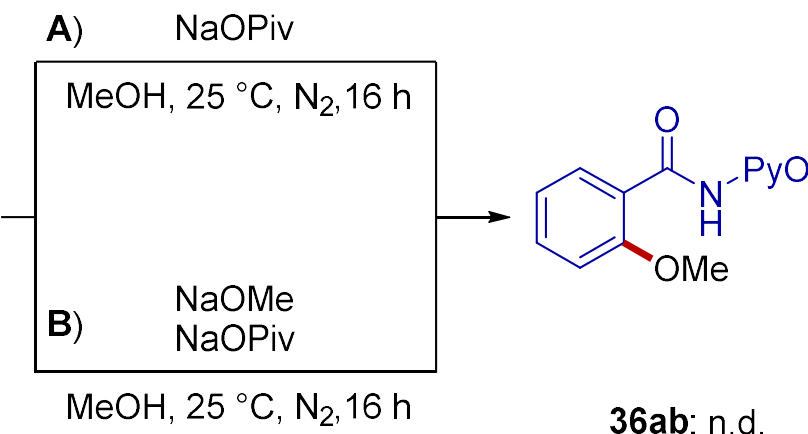

36ab: n.d.

Reaction A) Cobalt(III) complex 190 (45.1 mg, $64.4 \mu$ mol, 1.00 equiv), NaOPiv (16.0 mg, $128 \mu \mathrm{mol}, 2.00$ equiv), and $\mathrm{MeOH}(\mathbf{3 5 b})(3.0 \mathrm{~mL})$ were placed in a $10 \mathrm{~mL}$ Schlenk tube. The mixture was degassed and purged with nitrogen for 3 times and stirred at $25{ }^{\circ} \mathrm{C}$ for $16 \mathrm{~h}$. Then, 1,3,5-trimethoxybenzene (10.8 mg, 1.00 equiv) was added as an internal standard. The crude mixture was filtered over Celite and the Schlenk tube was washed with $\mathrm{MeOH}(3 \times 5.0 \mathrm{~mL})$. Evaporation of the solvent in vacuo and subsequent ${ }^{1} \mathrm{H}-\mathrm{NMR}-$ spectroscopic analysis, as well as ESI-MS analysis did not show any product formation. 
Reaction B) Cobalt(III) complex 190 (45.1 mg, $64.4 \mu \mathrm{mol}, 1.00$ equiv), $\mathrm{NaOMe}$ (13.8 mg, $256 \mu \mathrm{mol}, 4.00$ equiv), NaOPiv (16.0 mg, $128 \mu \mathrm{mol}, 2.00$ equiv), and $\mathrm{MeOH}$ (35b) $(3.0 \mathrm{~mL})$ were placed in a $10 \mathrm{~mL}$ Schlenk tube. The mixture was degassed and purged with nitrogen for 3 times and stirred at $25^{\circ} \mathrm{C}$ for $16 \mathrm{~h}$. Then, 1,3,5-trimethoxybenzene $(10.8 \mathrm{mg}$, 1.00 equiv) was added as an internal standard. The crude mixture was filtered over Celite and the Schlenk tube was washed with $\mathrm{MeOH}(3 \times 5.0 \mathrm{~mL})$. Evaporation of the solvent in vacuo and subsequent ${ }^{1} \mathrm{H}-\mathrm{NMR}$-spectroscopic analysis, as well as ESI-MS analysis did not show any product formation.

\subsubsection{Reactivity of Cobalt(III)-Complex 190 toward Electrochemical C-H} Alkoxylation
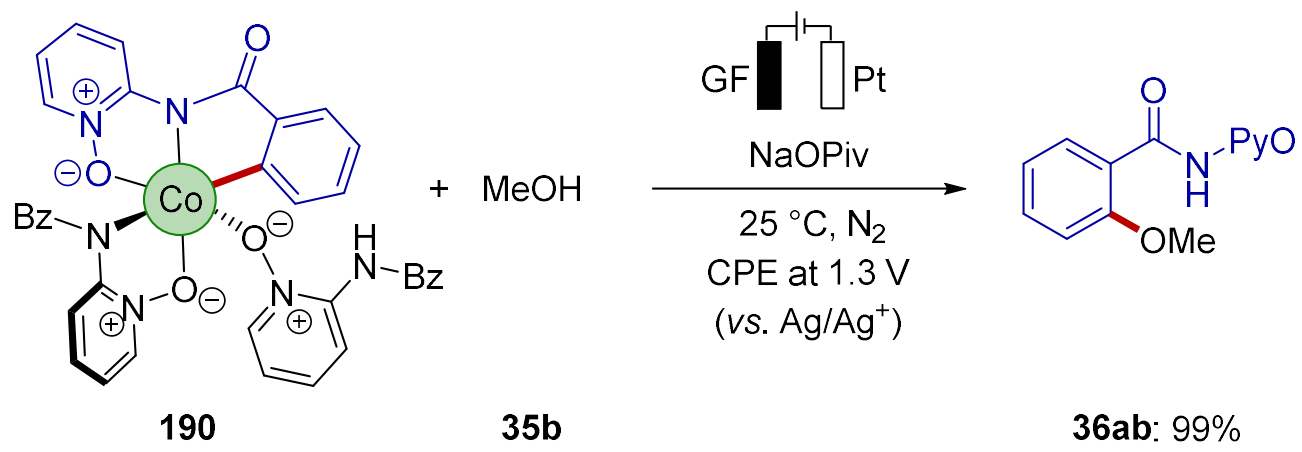

36ab: $99 \%$

190 (100 mg, $143 \mu \mathrm{mol}, 1.00$ equiv), NaOPiv (36.0 mg, $0.60 \mathrm{mmol}, 4.20$ equiv), and $\mathrm{MeOH}(\mathbf{3 5 b})(4.0 \mathrm{~mL})$ were placed in a $10 \mathrm{~mL}$ cell. Electrosynthesis was performed at $25{ }^{\circ} \mathrm{C}$ with a constant potential of $1.3 \mathrm{~V}$ versus $\mathrm{Ag} / \mathrm{Ag}^{+}$maintained until $1.5 \mathrm{~F}$ was passed through the cell $(4 \mathrm{~h})$. Then, the electrolysis was stopped, the suspension was filtered over Celite and the electrolysis cell was washed with $\mathrm{CH}_{2} \mathrm{Cl}_{2}(3 \times 5.0 \mathrm{~mL})$. The graphite felt anode was washed with $\mathrm{CH}_{2} \mathrm{Cl}_{2}(3 \times 5.0 \mathrm{~mL})$ in an ultrasonic bath $(3 \times 3 \mathrm{~min})$. The combined washings were added to the reaction mixture and the combined phases were washed with $\mathrm{H}_{2} \mathrm{O}(15 \mathrm{~mL})$. The aqueous phase was extracted with $\mathrm{CH}_{2} \mathrm{Cl}_{2}(4 \times 10 \mathrm{~mL})$. The crude extracts were then dried over $\mathrm{Na}_{2} \mathrm{SO}_{4}$. Evaporation of the solvent in vacuo and subsequent column chromatography on silica gel $\left(\mathrm{CH}_{2} \mathrm{Cl}_{2} /\right.$ acetone 3:1) afforded 2-(2methoxybenzamido)pyridine-1-oxide (36ab) (35 mg, 99\%) as a white solid. The yield of $99 \%$ is based on the conversion of one of the coordinating substrates $34 \mathbf{a}$. 


\subsubsection{Reactivity of Cobalt(III)-Complex 190 toward C-H Annulation with} Alkyne 53a
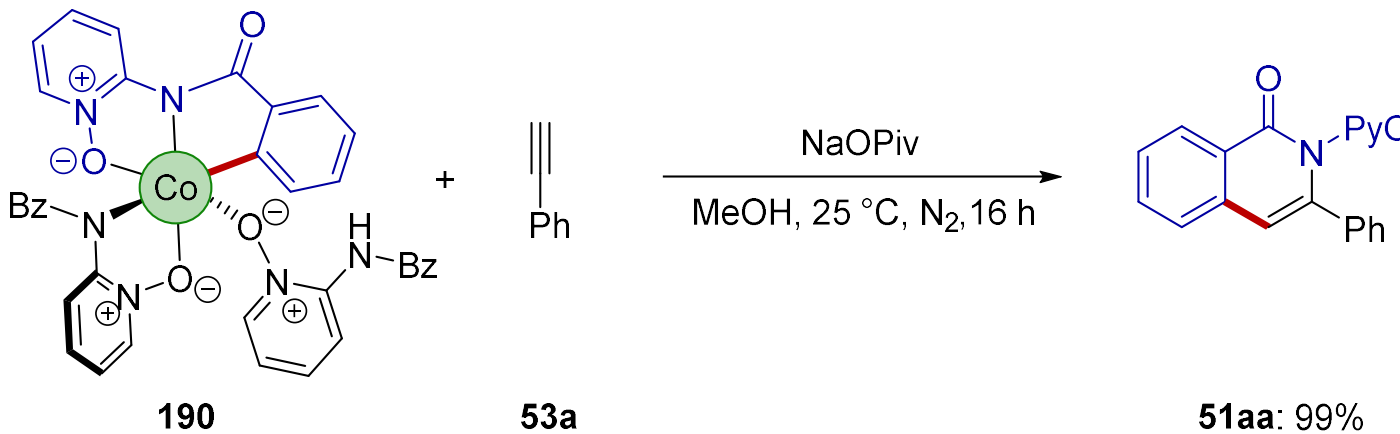

Cobalt(III) complex 190 (45.1 mg, $64.4 \mu \mathrm{mol}, 1.00$ equiv), phenylacetylene (53a) (26.3 mg, $258 \mu \mathrm{mol}, 4.00$ equiv), NaOPiv (16.0 mg, $128 \mu \mathrm{mol}, 2.00$ equiv), and $\mathrm{MeOH}$ $(2.0 \mathrm{~mL})$ were placed in a $10 \mathrm{~mL}$ Schlenk tube. The mixture was degassed and purged with nitrogen for 3 times and stirred at $25^{\circ} \mathrm{C}$ for $16 \mathrm{~h}$. Then, 1,3,5-trimethoxybenzene $(10.8 \mathrm{mg}$, 1.00 equiv) was added as an internal standard. The crude mixture was filtered over Celite and the Schlenk tube was washed with $\mathrm{MeOH}(3 \times 5.0 \mathrm{~mL})$. Evaporation of the solvent in vacuo and subsequent ${ }^{1} \mathrm{H}-\mathrm{NMR}$-spectroscopic analysis revealed $99 \%{ }^{1} \mathrm{H}-\mathrm{NMR}$-conversion. Recombination of the reaction mixture with the NMR sample and subsequent column chromatography on silica gel $\left(\mathrm{CH}_{2} \mathrm{Cl}_{2} /\right.$ acetone $\left.3: 1\right)$ afforded the corresponding product 2[1-oxo-3-phenylisoquinolin-2(1H)-yl]-pyridine-1-oxide (51aa) $(21 \mathrm{mg}, 99 \%)$ as a white solid. The yield of $99 \%$ is based on the conversion of one of the coordinating substrates. At the end of the reaction and before the work-up, an aliquot $(10 \mu \mathrm{L})$ was removed and was diluted with dry $\mathrm{MeOH}$ to give a $0.01 \mathrm{mM}$ solution. The solution was sealed under a flow of nitrogen and the sample was analyzed via ESI-MS to detect a non-converted, sevenmembered cobalta(III)cycle 195. However, only traces were detected, being supportive for fast reductive elimination to release the desired product 51aa. 
5.6.3.4 Reactivity of Cobalt(III)-Complex 190 toward C-H Annulation with Allene 75d

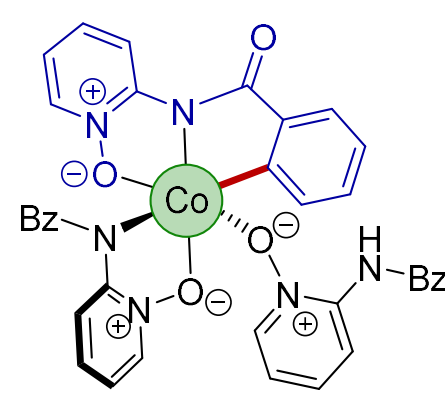

190

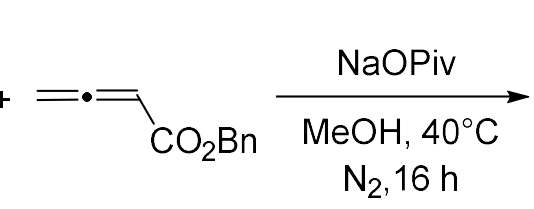

$75 d$<smiles>O=C(Cc1cc2ccccc2c(=O)n1P(O)O)Oc1ccccc1</smiles>

176ad: $97 \%$

Cobalt(III) complex 190 (45.1 mg, $64.4 \mu \mathrm{mol}, 1.00$ equiv), benzyl buta-2,3-dienoate (75d) (44.5 mg, $256 \mu \mathrm{mol}, 4.00$ equiv), NaOPiv (16.0 mg, $128 \mu \mathrm{mol}, 2.00$ equiv), and $\mathrm{MeOH}$ $(2.0 \mathrm{~mL})$ were placed in a $10 \mathrm{~mL}$ Schlenk tube. The mixture was degassed and purged with nitrogen for 3 times and stirred at $25^{\circ} \mathrm{C}$ for $16 \mathrm{~h}$. Then, 1,3,5-trimethoxybenzene $(10.8 \mathrm{mg}$, 1.00 equiv) was added as an internal standard. The crude mixture was filtered over Celite and the Schlenk tube was washed with $\mathrm{MeOH}(3 \times 5.0 \mathrm{~mL})$. Evaporation of the solvent in vacuo and subsequent ${ }^{1} \mathrm{H}-\mathrm{NMR}$-spectroscopic analysis revealed 99\% NMR-conversion. Recombination of the reaction mixture with the NMR sample and subsequent column chromatography on silica gel $\left(\mathrm{CH}_{2} \mathrm{Cl}_{2} /\right.$ acetone $\left.3: 1\right)$ afforded the corresponding 2-\{3-[2(benzyloxy)-2-oxoethyl]-1-oxoisoquinolin-2(1H)-yl\}pyridine 1-oxide (176ad) (24 mg, $97 \%$ ) as a yellow solid. The yield of $97 \%$ is based on the conversion of only one of the coordinating substrates. 


\subsubsection{Hammett-Zuman Plot}

\section{Characterization Data}

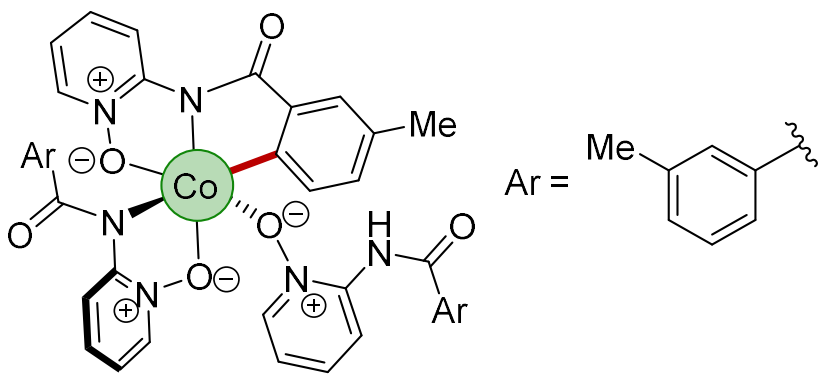

Tris[2-(3-methylbenzamido)pyridine 1-oxido]cobalt(III) (196): The general procedure G was followed using benzamide 34b (228 mg, $1.00 \mathrm{mmol}, 2.00$ equiv) and $\mathrm{MeCN}$ (13.0 mL). Purification by column chromatography on silica gel $\left(\mathrm{CH}_{2} \mathrm{Cl}_{2} / \mathrm{MeOH}\right.$ 99:1 $\rightarrow 30: 1)$ yielded $196(190 \mathrm{mg}, 257 \mu \mathrm{mol}, 51 \%)$ as a dark green solid.

${ }^{1}$ H-NMR (400 MHz, CD $\left.{ }_{3} \mathrm{OD}\right): \delta=8.60-8.46(\mathrm{~m}, 1 \mathrm{H}), 8.45-8.25(\mathrm{~m}, 1 \mathrm{H}), 7.57-7.38(\mathrm{~m}$, 2H), 7.36-7.20 (m, 4H), 7.14-7.08 (m, 1H), 7.06-6.94 (m, 2H), 6.90-6.83 (m, 1H), 6.83$6.75(\mathrm{~m}, 1 \mathrm{H}), 6.72-6.61(\mathrm{~m}, 1 \mathrm{H}), 2.51(\mathrm{~s}, 3 \mathrm{H}), 2.14(\mathrm{~s}, 3 \mathrm{H})$.

${ }^{13}$ C-NMR (101 MHz, CD $\left.{ }_{3} \mathrm{OD}\right): \delta=185.7\left(\mathrm{C}_{\mathrm{q}}\right), 156.3\left(\mathrm{C}_{\mathrm{q}}\right), 150.8\left(\mathrm{C}_{\mathrm{q}}\right), 148.6\left(\mathrm{C}_{\mathrm{q}}\right), 138.5$ $\left(\mathrm{C}_{\mathrm{q}}\right), 137.2\left(\mathrm{C}_{\mathrm{q}}\right), 137.1\left(\mathrm{C}_{\mathrm{q}}\right), 135.9(\mathrm{CH}), 135.7(\mathrm{CH}), 135.3(\mathrm{CH}), 134.1(\mathrm{CH}), 131.9\left(\mathrm{C}_{\mathrm{q}}\right)$, $131.5(\mathrm{CH}), 131.2(\mathrm{CH}), 131.1(\mathrm{CH}), 127.7(\mathrm{CH}), 127.1(\mathrm{CH}), 126.8(\mathrm{CH}), 124.2(\mathrm{CH})$, $120.3\left(\mathrm{C}_{\mathrm{q}}\right), 119.2(\mathrm{CH}), 118.8(\mathrm{CH}), 116.6(\mathrm{CH}), 114.9(\mathrm{CH}), 20.1\left(\mathrm{CH}_{3}\right), 19.3\left(\mathrm{CH}_{3}\right)$. IR (ATR): $\tilde{v}=2920,1627,1598,1557,1483,1361,1191,755 \mathrm{~cm}^{-1}$.

MS (ESI) $m / z$ (relative intensity): 1047.2 (20) $[2 \mathrm{xM}-2 \times 34 b+N a]^{+}, 763.2(10)[\mathrm{M}+\mathrm{Na}]^{+}$, $535.1(100)[\mathrm{M}-\mathbf{3 4 b}+\mathrm{Na}]^{+}, 513.1(20)[\mathrm{M}-\mathbf{3 4 b}+\mathrm{H}]^{+}$.

HR-MS (ESI) $m / z$ calcd. for $\mathrm{C}_{39} \mathrm{H}_{33} \mathrm{CoN}_{6} \mathrm{NaO}_{6}[\mathrm{M}+\mathrm{Na}]^{+}:$763.1686, found 763.1686.<smiles>CC(C)c1cccc(C(C)C)c1</smiles>

Tris[2-(3-isopropylbenzamido)pyridine 1-oxido]cobalt(III) (197): The general procedure $\mathbf{G}$ was followed using benzamide $\mathbf{3 4 w}(256 \mathrm{mg}, 1.00 \mathrm{mmol}, 2.00$ equiv) and 
MeCN (13.0 mL). Purification by column chromatography on silica gel $\left(\mathrm{CH}_{2} \mathrm{Cl}_{2} / \mathrm{MeOH}\right.$ 99:1 $\rightarrow 30: 1)$ yielded $197(116 \mathrm{mg}, 141 \mu \mathrm{mol}, 28 \%)$ as a dark green solid.

${ }^{1}$ H-NMR (400 MHz, CD $\left.{ }_{3} \mathrm{OD}\right): \delta=8.74-8.53(\mathrm{~m}, 1 \mathrm{H}), 8.54-8.36(\mathrm{~m}, 1 \mathrm{H}), 7.77-7.65$ (m, 1H), 7.64-7.48 (m, 2H), 7.48-7.38 (m, 1H), 7.37-7.19 (m, 2H), 7.19-7.00 (m, 4H), 7.00$6.88(\mathrm{~m}, 1 \mathrm{H}), 6.88-6.75(\mathrm{~m}, 1 \mathrm{H}), 6.75-6.54(\mathrm{~m}, 1 \mathrm{H}), 3.05-2.96(\mathrm{~m}, 1 \mathrm{H}), 2.82-2.64(\mathrm{~m}$, $1 \mathrm{H}), 1.36-1.29(\mathrm{~m}, 6 \mathrm{H}), 1.16(\mathrm{~d}, J=6.5 \mathrm{~Hz}, 6 \mathrm{H})$.

${ }^{13}$ C-NMR (101 MHz, CD $\left.{ }_{3} \mathrm{OD}\right): \delta=186.2\left(\mathrm{C}_{\mathrm{q}}\right), 157.6\left(\mathrm{C}_{\mathrm{q}}\right), 157.0\left(\mathrm{C}_{\mathrm{q}}\right), 149.8\left(\mathrm{C}_{\mathrm{q}}\right), 149.6$ $\left(\mathrm{C}_{\mathrm{q}}\right), 146.9(\mathrm{CH}), 146.9(\mathrm{CH}), 139.6\left(\mathrm{C}_{\mathrm{q}}\right), 138.5\left(\mathrm{C}_{\mathrm{q}}\right), 137.4\left(\mathrm{C}_{\mathrm{q}}\right), 137.0(\mathrm{CH}), 136.9(\mathrm{CH})$, $133.2\left(\mathrm{C}_{\mathrm{q}}\right), 132.7(\mathrm{CH}), 132.6(\mathrm{CH}), 130.1(\mathrm{CH}), 129.9(\mathrm{CH}), 128.5(\mathrm{CH}), 128.3(\mathrm{CH})$, $127.0(\mathrm{CH}), 126.3(\mathrm{CH}), 125.5(\mathrm{CH}), 120.3(\mathrm{CH}), 120.2(\mathrm{CH}), 117.8(\mathrm{CH}), 116.0(\mathrm{CH})$, $34.9(\mathrm{CH}), 34.7(\mathrm{CH}), 24.9\left(2 \mathrm{xCH}_{3}\right), 24.2\left(2 \mathrm{xCH}_{3}\right)$.

IR (ATR): $\tilde{v}=2960,1625,1599,1556,1485,1363,1200,756 \mathrm{~cm}^{-1}$.

MS (ESI) $m / z$ (relative intensity): 1159.3 (10) $[2 \times \mathrm{M}-2 \times 34 w+N a]^{+}, 847.3(20)[\mathrm{M}+\mathrm{Na}]^{+}$, $591.1(100)[\mathrm{M}-34 \mathbf{w}+\mathrm{Na}]^{+}, 569.2(20)[\mathrm{M}-34 \mathbf{w}+\mathrm{H}]^{+}$.

HR-MS (ESI) $m / z$ calcd. for $\mathrm{C}_{45} \mathrm{H}_{45} \mathrm{CoN}_{6} \mathrm{NaO}_{6}[\mathrm{M}+\mathrm{Na}]^{+}$: 847.2625, found 847.2612.

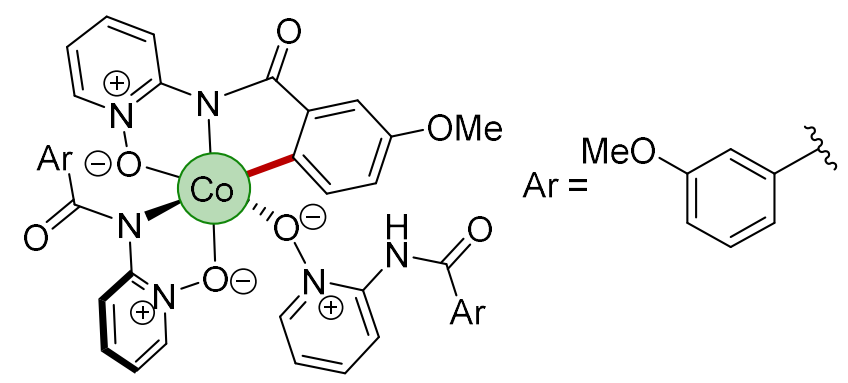

Tris[2-(3-methoxybenzamido)pyridine 1-oxido]cobalt(III) (198): The general procedure $\mathbf{G}$ was followed using benzamide $34 \mathbf{p}$ (244 $\mathrm{mg}, 1.00 \mathrm{mmol}, 2.00$ equiv) and $\mathrm{MeCN}(13.0 \mathrm{~mL})$. Purification by column chromatography on silica gel $\left(\mathrm{CH}_{2} \mathrm{Cl}_{2} / \mathrm{MeOH}\right.$ 99:1 $\rightarrow 20: 1)$ yielded $198(52.1 \mathrm{mg}, 66.1 \mu \mathrm{mol}, 13 \%)$ as a dark green solid.

${ }^{1}$ H-NMR (400 MHz, CD $\left.{ }_{3} \mathrm{OD}\right): \delta=8.54-8.25$ (m, 2H), 7.42-7.34 (m, 1H), 7.28-7.18 (m, 3H), 7.16-7.10 (m, 1H), 7.08-7.01 (m, 1H), 6.95-6.88 (m, 1H), 6.88-6.75 (m, 2H), 6.75$6.63(\mathrm{~m}, 3 \mathrm{H}), 6.63-6.51(\mathrm{~m}, 1 \mathrm{H}), 3.85(\mathrm{~s}, 3 \mathrm{H}), 3.50$ (s, 3H).

${ }^{13}$ C-NMR (101 MHz, $\left.\mathrm{CD}_{3} \mathrm{OD}\right): \delta=160.5,160.3,158.2,157.7,145.9,141.0,138.6,137.2$, 137.1, 136.9, 135.6, 135.3, 132.9, 129.6, 121.4, 120.6, 120.2, 118.9, 118.7, 118.0, 117.7, 116.2, 115.8, 112.7, 112.4, $55.9\left(\mathrm{CH}_{3}\right), 55.5\left(\mathrm{CH}_{3}\right) .{ }^{13} \mathrm{C}-\mathrm{NMR}$ signals could not be clearly assigned, due to decomposition of the complex during the measurement.

IR (ATR): $\tilde{v}=2963,1626,1581,1558,1485,1363,1268,756 \mathrm{~cm}^{-1}$. 
MS (ESI) $m / z$ (relative intensity): 1111.1 (5) $[2 \mathrm{xM}-2 \times 34 p+N a]^{+}, 811.2(20)[\mathrm{M}+\mathrm{Na}]^{+}$, $789.1(3)[\mathrm{M}+\mathrm{H}]^{+}, 667.1(20)[\mathrm{M}-\mathbf{3 4 p}+\mathrm{OPiv}+\mathrm{Na}]^{+}, 567.1(100)[\mathrm{M}-\mathbf{3 4 p}+\mathrm{Na}]^{+}, 545.1(25)$ $[\mathrm{M}-34 \mathbf{p}+\mathrm{H}]^{+}$.

HR-MS (ESI) $m / z$ calcd. for $\mathrm{C}_{39} \mathrm{H}_{33} \mathrm{CoN}_{6} \mathrm{NaO}_{9}[\mathrm{M}+\mathrm{Na}]^{+}:$811.1533, found 811.1517.

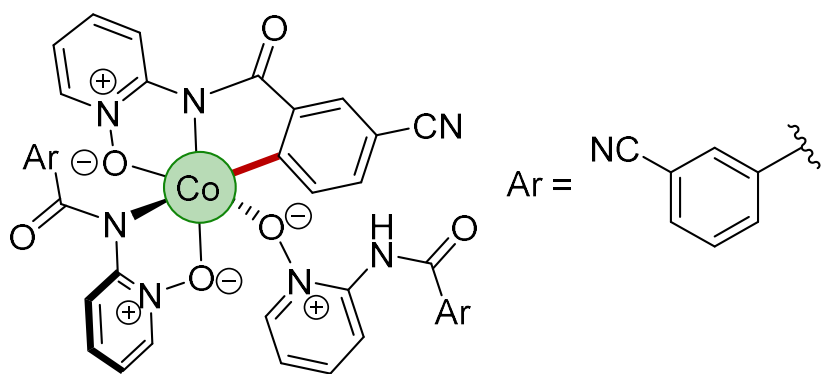

Tris[2-(3-cyanobenzamido)pyridine 1-oxido]cobalt(III) (199): The general procedure G was followed using benzamide $34 \mathbf{x}(239 \mathrm{mg}, 1.00 \mathrm{mmol}, 2.00$ equiv) and $\mathrm{MeCN}$ $(13.0 \mathrm{~mL})$. Purification by column chromatography on silica gel $\left(\mathrm{CH}_{2} \mathrm{Cl}_{2} / \mathrm{MeOH}\right.$ 99:1 $\rightarrow 10: 1)$ yielded $199(74.9 \mathrm{mg}, 96.9 \mu \mathrm{mol}, 19 \%)$ as a brown solid.

${ }^{1}$ H-NMR (400 MHz, CD $\left.{ }_{3} \mathrm{OD}\right): \delta=8.72-8.63(\mathrm{~m}, 1 \mathrm{H}), 7.83-7.69(\mathrm{~m}, 3 \mathrm{H}), 7.64-7.48(\mathrm{~m}$, 3H), 7.44-7.30 (m, 4H), 7.27-7.19 (m, 1H), 7.13-7.09 (m, 1H), 7.01-6.86 (m, 2H).

${ }^{13}$ C-NMR (101 MHz, $\left.\mathrm{CD}_{3} \mathrm{OD}\right): \delta=182.2,157.3,151.7,142.8,141.2,139.3,138.4,137.4$, 135.1, 133.6, 133.1, 132.2, 131.2, 130.9, 130.9, 129.9, 129.1, 120.0, 119.4, 118.7, 112.6, 109.9. ${ }^{13} \mathrm{C}-\mathrm{NMR}$ resonances could not be clearly assigned, due to partial decomposition during the measurement.

IR (ATR): $\tilde{v}=3081,2229,1625,1598,1489,1370,1337,1178,759 \mathrm{~cm}^{-1}$.

MS (ESI) $m / z$ (relative intensity): $1091.1(10)[2 \times M-2 \times 34 x+N a]^{+}, 796.1(10)[\mathrm{M}+\mathrm{Na}]^{+}$, $774.1(3)[\mathrm{M}+\mathrm{H}]^{+}, 557.0(100)[\mathrm{M}-\mathbf{3 4 x}+\mathrm{Na}]^{+}, 535.1(10)[\mathrm{M}-\mathbf{3 4 x}+\mathrm{H}]^{+}$.

HR-MS (ESI) $m / z$ calcd. for $\mathrm{C}_{39} \mathrm{H}_{24} \mathrm{CoN}_{9} \mathrm{NaO}_{6}[\mathrm{M}+\mathrm{Na}]^{+}:$796.1074, found 796.1068.

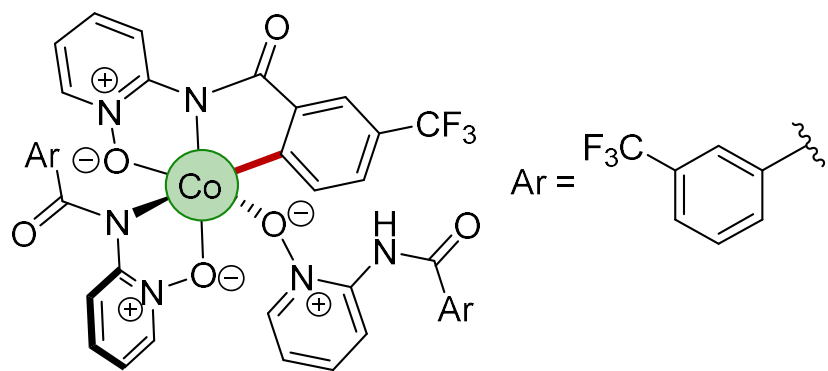

Tris \{2-[3-(trifluoromethyl)benzamido]pyridine 1-oxido\}cobalt(III) (200): The general procedure $\mathbf{G}$ was followed using benzamide $\mathbf{3 4 y}$ (282 $\mathrm{mg}, 1.00 \mathrm{mmol}, 2.00$ equiv) and 
MeCN $(13.0 \mathrm{~mL})$. Purification by column chromatography on silica gel $\left(\mathrm{CH}_{2} \mathrm{Cl}_{2} / \mathrm{MeOH}\right.$ 99:1 $\rightarrow 10: 1)$ yielded 200 (58.9 $\mathrm{mg}, 65.3 \mu \mathrm{mol}, 13 \%)$ as a dark green solid.

${ }^{1}$ H-NMR (400 MHz, CD $\left.{ }_{3} \mathrm{OD}\right): \delta=9.78-9.53(\mathrm{~m}, 1 \mathrm{H}), 9.02-8.80(\mathrm{~m}, 1 \mathrm{H}), 7.95-7.79(\mathrm{~m}$, 2H), 7.75-7.52 (m, 4H), 7.46-7.31 (m, 2H), 7.30-7.21 (m, 2H), 7.10-6.98 (m, 1H), 6.90$6.65(\mathrm{~m}, 1 \mathrm{H}), 6.90-6.65(\mathrm{~m}, 1 \mathrm{H})$.

${ }^{13}$ C-NMR (101 MHz, $\left.\mathrm{CD}_{3} \mathrm{OD}\right): \delta=165.2,156.8,153.1,150.2,144.2,140.7,139.4,138.0$, $137.3,136.7,135.5,134.6,132.7,132.3,131.0,130.2,129.2,128.7,127.8,126.7,125.5$, 125.1, 123.2, 121.8, 120.9, 119.9, 118.6, 116.9, 116.8. ${ }^{13} \mathrm{C}-\mathrm{NMR}$ resonances could not be clearly assigned, due to partial decomposition during the measurement.

${ }^{19} \mathbf{F}\left\{{ }^{1} \mathbf{H}\right\}$-NMR $\left(377 \mathrm{MHz}, \mathrm{CD}_{3} \mathrm{OD}\right): \delta=-63.8,-64.4$.

IR (ATR): $\tilde{v}=3083,1697,1614,1577,1511,1491,1431,1329,1250,1128,761,696 \mathrm{~cm}^{-1}$.

MS (ESI) $m / z$ (relative intensity): $925.2(30)[\mathrm{M}+\mathrm{Na}]^{+}, 903.2(5)[\mathrm{M}+\mathrm{H}]^{+}, 643.1(100)[\mathrm{M}-$ $\mathbf{3 4 y}+\mathrm{Na}]^{+}, 621.1(10)[\mathrm{M}-\mathbf{3 4 y}+\mathrm{H}]^{+}, 305.1(50)[\mathbf{3 4 y}+\mathrm{Na}]^{+}$.

HR-MS (ESI) $m / z$ calcd. for $\mathrm{C}_{39} \mathrm{H}_{24} \mathrm{CoF}_{9} \mathrm{~N}_{6} \mathrm{NaO}_{6}[\mathrm{M}+\mathrm{Na}]^{+}$: 925.0838, found 925.0846.

Based on NMR spectroscopy and ESI-MS studies, the cobalt(III) complex 200 still contained unreacted substrate $\mathbf{3 4 y}$, which could not be fully separated by either column chromatography or recrystallisation. However, $\mathrm{CV}$-experiments were not influenced by the contamination since $\mathbf{3 4 y}$ showed a significantly higher oxidation potential.

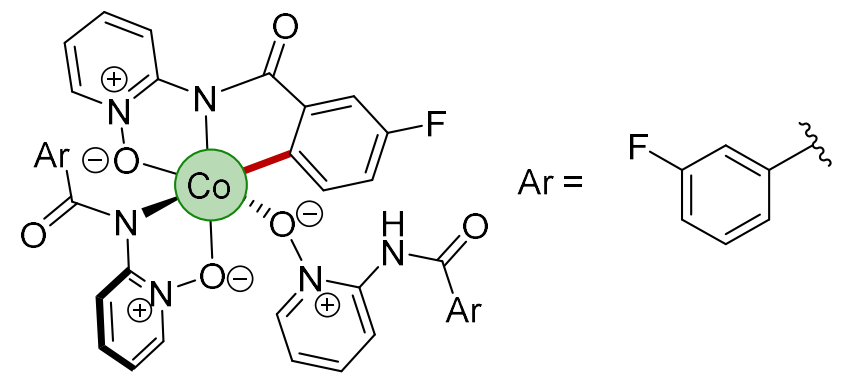

Tris[2-(3-fluorobenzamido)pyridine 1-oxido]cobalt(III) (201): The general procedure $\mathbf{G}$ was followed using benzamide 34r (232 mg, $1.00 \mathrm{mmol}, 2.00$ equiv) and $\mathrm{MeCN}$ $(13.0 \mathrm{~mL})$. Purification by column chromatography on silica gel $\left(\mathrm{CH}_{2} \mathrm{Cl}_{2} / \mathrm{MeOH}\right.$ 99:1 $\rightarrow 30: 1)$ yielded $201(187 \mathrm{mg}, 255 \mu \mathrm{mol}, 51 \%)$ as a green solid.

${ }^{1}$ H-NMR (400 MHz, CD $\left.{ }_{3} \mathrm{OD}\right): \delta=8.46-8.24(\mathrm{~m}, 1 \mathrm{H}), 7.83-7.64(\mathrm{~m}, 1 \mathrm{H}), 7.63-7.38(\mathrm{~m}$, 4H), 7.40-7.17 (m, 3H), 7.14-7.02 (m, 1H), 6.98-6.81 (m, 3H), 6.79-6.59 (m, 2H).

${ }^{13}$ C-NMR (101 MHz, $\left.\mathrm{CD}_{3} \mathrm{OD}\right): \delta=165.1,163.2,157.4,152.9,147.1,138.9,138.5,136.8$, $133.6,133.2,132.8,132.1,130.6,127.2$, 126.6, 125.0, 124.3, 124.2, 121.2, 121.1, 120.4, 
120.2, 119.8, 119.5, 118.6, 118.4, 118.2, 116.5, 92.8. ${ }^{13} \mathrm{C}-\mathrm{NMR}$ resonances could not be clearly assigned, due to partial decomposition during the measurement.

${ }^{19} \mathbf{F}\left\{{ }^{1} \mathbf{H}\right\}$-NMR $\left(376 \mathrm{MHz}, \mathrm{CD}_{2} \mathrm{Cl}_{2}\right): \delta=-112.5,-113.7$.

IR (ATR): $\tilde{v}=3079,1696,1632,1587,1486,1437,1365,757 \mathrm{~cm}^{-1}$.

MS (ESI) $m / z$ (relative intensity): $775.1(35)[\mathrm{M}+\mathrm{Na}]^{+}, 753.1(5)[\mathrm{M}+\mathrm{H}]^{+}, 543.0(100)[\mathrm{M}-$ 34r $+\mathrm{Na}]^{+}, 521.0(20)[\mathrm{M}-\mathbf{3 4} \mathbf{r}+\mathrm{H}]^{+}, 255.1(50)[34 \mathbf{r}+\mathrm{Na}]^{+}$.

HR-MS (ESI) $m / z$ calcd. for $\mathrm{C}_{36} \mathrm{H}_{24} \mathrm{CoFN}_{6} \mathrm{O}_{6}[\mathrm{M}+\mathrm{Na}]^{+}:$755.0934, found 755.0932.

Based on NMR spectroscopy and ESI-MS studies, the cobalt(III) complex 201 contained unreacted substrate $\mathbf{3 4 r}$, which could not be fully separated by either column chromatography or recrystallisation. However, $\mathrm{CV}$-experiments were not influenced by the contamination since 34r showed a significantly higher oxidation potential.

\subsubsection{Cyclic Voltammetry}

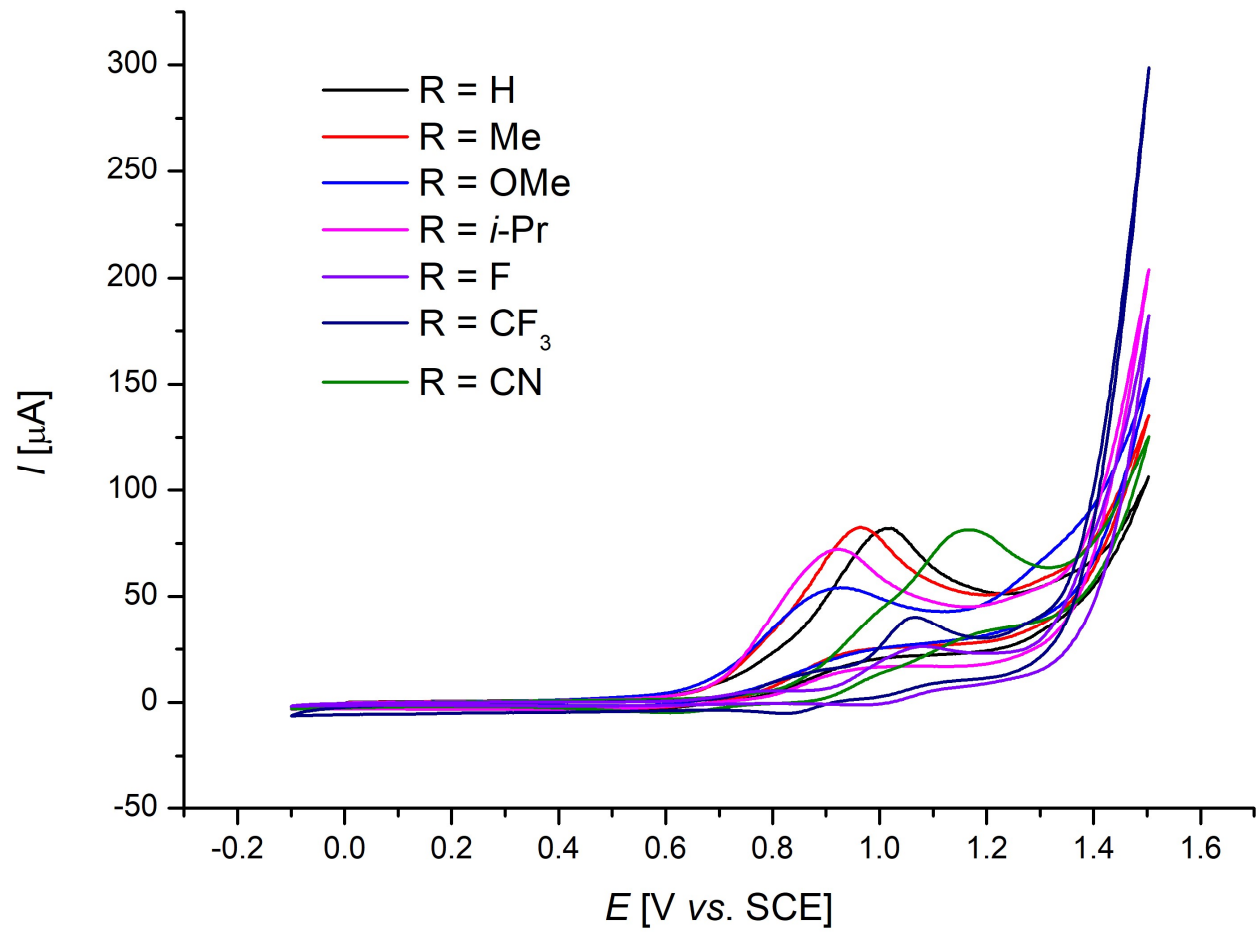

Figure 59. Cyclic voltammograms at $100 \mathrm{mV} \cdot \mathrm{s}^{-1}$ using $\mathrm{MeOH}$ and $n-\mathrm{Bu}_{4} \mathrm{NPF}_{6}(0.1 \mathrm{M})$ as the electrolyte and a GC working electrode. Substrate concentration was $3.5 \mathrm{mM}$. 


\subsubsection{Inhibition Experiments}

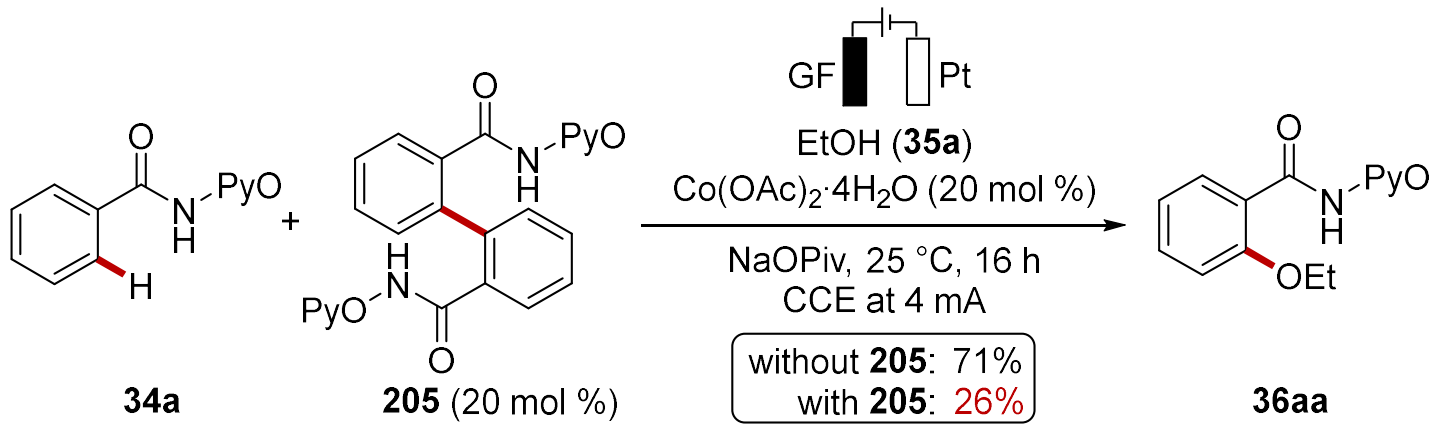

Benzamide 34a (107 mg, $0.50 \mathrm{mmol}, 1.00$ equiv), NaOPiv (124 mg, $1.00 \mathrm{mmol}$, 2.00 equiv), $\mathrm{Co}(\mathrm{OAc})_{2} \cdot 4 \mathrm{H}_{2} \mathrm{O}$ (25.4 mg, $20 \mathrm{~mol} \%$ ), 205 (42.6 mg, $0.10 \mathrm{mmol}, 20 \mathrm{~mol} \%$ ), and $\mathrm{EtOH}(\mathbf{3 5 a})(5.0 \mathrm{~mL})$ were placed in a $10 \mathrm{~mL}$ cell. Prior to electrolysis, the reaction mixture was stirred for $10 \mathrm{~min}$ at $25{ }^{\circ} \mathrm{C}$. Electrosynthesis was performed at $25{ }^{\circ} \mathrm{C}$ with a constant current of $4 \mathrm{~mA}$, maintained for $16 \mathrm{~h}$. Then, 1,3,5-trimethoxybenzene $(28.0 \mathrm{mg}$, 0.33 equiv) was added as an internal standard and the reaction mixture was diluted with $\mathrm{CH}_{2} \mathrm{Cl}_{2}(2.0 \mathrm{~mL})$. The graphite felt anode was washed with $\mathrm{CH}_{2} \mathrm{Cl}_{2}(3 \times 5.0 \mathrm{~mL})$ in an ultrasonic bath $(3 \times 3 \mathrm{~min})$. The combined washings were added to the reaction mixture and the combined phases were washed with $\mathrm{H}_{2} \mathrm{O}(15 \mathrm{~mL})$. The aqueous phase was extracted with $\mathrm{CH}_{2} \mathrm{Cl}_{2}(4 \times 10 \mathrm{~mL})$. The crude extracts were then dried over $\mathrm{Na}_{2} \mathrm{SO}_{4}$. Evaporation of the solvent in vacuo and subsequent ${ }^{1} \mathrm{H}$-NMR spectroscopic analysis revealed $75 \%{ }^{1} \mathrm{H}$ NMR-conversion of substrate 34a due to overoxidation and only low formation of the desired product 36aa (26\%). The yield of the dimerized product 205 was determined to be $4 \%$. In parallel, a control reaction without the dimerized product delivered product 36aa in $71 \%{ }^{1} \mathrm{H}-\mathrm{NMR}$ conversion.

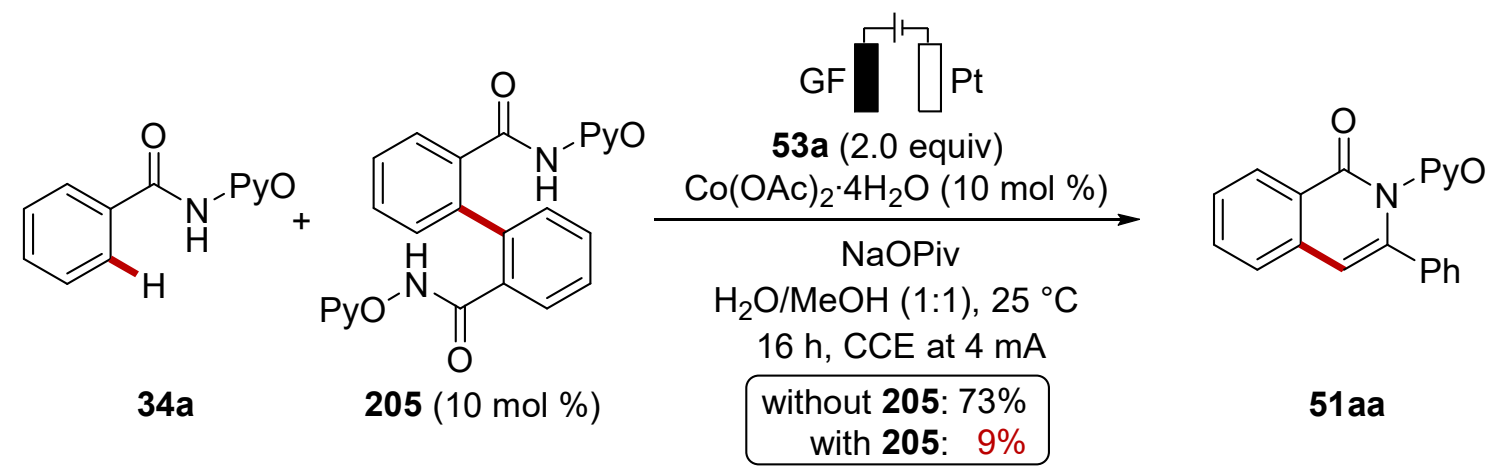

Benzamide 34a (107 mg, $0.50 \mathrm{mmol}, 1.00$ equiv), phenylacetylene (102 mg, $1.00 \mathrm{mmol}$, 2.00 equiv), NaOPiv (124 mg, $1.00 \mathrm{mmol}, 2.00$ equiv), $\mathrm{Co}(\mathrm{OAc})_{2} \cdot 4 \mathrm{H}_{2} \mathrm{O} \quad(12.7 \mathrm{mg}$, $10 \mathrm{~mol} \%$ ), 205 (42.6 mg, $50.0 \mu \mathrm{mol}, 10 \mathrm{~mol} \%$ ), and $\mathrm{H}_{2} \mathrm{O} / \mathrm{MeOH}(\mathrm{v} / \mathrm{v}=1: 1,5.0 \mathrm{~mL}$ ) were 
placed in a $10 \mathrm{~mL}$ cell. Prior to electrolysis, the reaction mixture was stirred for $10 \mathrm{~min}$ at $25^{\circ} \mathrm{C}$. Electrosynthesis was performed at $25^{\circ} \mathrm{C}$ with a constant current of $4 \mathrm{~mA}$, maintained for $16 \mathrm{~h}$. Then, 1,3,5-trimethoxybenzene ( $28.0 \mathrm{mg}, 0.33$ equiv) was added as an internal standard and the reaction mixture was diluted with $\mathrm{CH}_{2} \mathrm{Cl}_{2}(2.0 \mathrm{~mL})$. The graphite felt anode was washed with $\mathrm{CH}_{2} \mathrm{Cl}_{2}(3 \times 5.0 \mathrm{~mL})$ in an ultrasonic bath $(3 \times 3 \mathrm{~min})$. The combined washings were added to the reaction mixture and the combined phases were washed with $\mathrm{H}_{2} \mathrm{O}(15 \mathrm{~mL})$. The aqueous phase was extracted with $\mathrm{CH}_{2} \mathrm{Cl}_{2}(4 \times 10 \mathrm{~mL})$. The crude extracts were then dried over $\mathrm{Na}_{2} \mathrm{SO}_{4}$. Evaporation of the solvent in vacuo and subsequent ${ }^{1} \mathrm{H}-\mathrm{NMR}$ spectroscopic analysis revealed 97\% NMR conversion of substrate 34a, due to overoxidation and only traces of the desired product 51 aa $(9 \%)$. The dimerized product $\mathbf{2 0 5}$ could only be detected in traces. In parallel, a control reaction without the dimerized product delivered product $\mathbf{5 1}$ aa in 73\% ${ }^{1} \mathrm{H}-\mathrm{NMR}$ conversion. 


\subsection{Mechanistic Studies for Ruthenaelectro-Catalyzed C-H Oxygenation}

\subsubsection{Reaction Profile for the Formation of Hypervalent Iodine Reagents}<smiles>Cc1ccc(I)cc1</smiles>
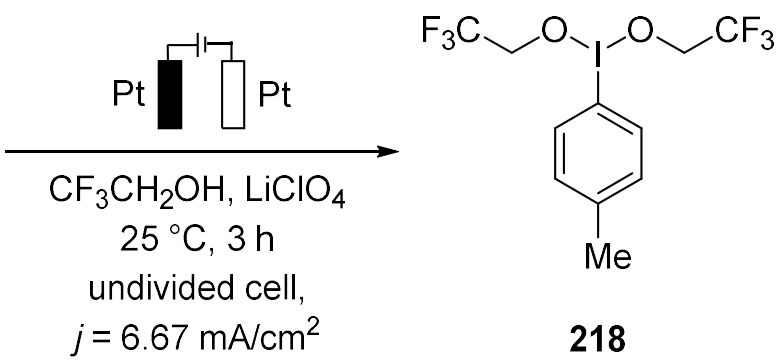

The flow NMR study was carried out in an undivided cell with two platinum electrodes $(10 \mathrm{~mm} \times 15 \mathrm{~mm} \times 0.125 \mathrm{~mm})$. The cell was charged with 4-iodotoluene (215b) (109 $\mathrm{mg}$, $0.50 \mathrm{mmol}, 1.00$ equiv) and $\mathrm{LiClO}_{4}(63.8 \mathrm{mg}, 0.60 \mathrm{mmol}, 1.20$ equiv) in TFE (10 mL). After recording initial spectra, the electrolysis was initiated by applying a constant current of $10 \mathrm{~mA}$ at $25^{\circ} \mathrm{C}$. The in-line electrolysis and NMR monitoring were performed for $3 \mathrm{~h}$. The reaction solution was circulated between the benchtop NMR spectrometer and the electrochemical cell with a flow speed of $0.8 \mathrm{~mL} \cdot \mathrm{min}^{-1}$. The reaction profile was monitored with ${ }^{1} \mathrm{H}-\mathrm{NMR}$ spectra recorded after every 90 seconds. After the electrolysis was stopped, the spectra were batch processed with the reaction monitoring wizard of the MestreNova 12.0.3 software. Arbitrary integral values were transformed to mmol and percentage values by referencing with dibromomethane as internal standard. Accordingly, the product $p$ tolylbis(2,2,2-trifluoroethoxy)- $\lambda^{3}$-iodane (218) was formed in $78 \%$ NMR conversion. ${ }^{1} \mathrm{H}$ NMR signals at a chemical shift (referenced against the trifluoroethanol solvent signal at $4.81 \mathrm{ppm}$ ) of $1.96 \mathrm{ppm}$ and $2.19 \mathrm{ppm}$ were identified to originate from the starting material 215b and 218, respectively.<smiles>Cc1ccc(I)cc1</smiles>

215b

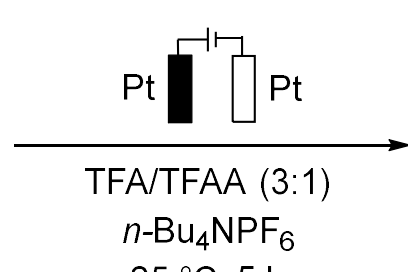

$25^{\circ} \mathrm{C}, 5 \mathrm{~h}$ undivided cell, $j=6.67 \mathrm{~mA} / \mathrm{cm}^{2}$<smiles>Cc1ccc(OC(=O)C(F)(F)F)c(C(F)(F)F)c1</smiles>

219

The flow NMR study was carried out in an undivided cell with two platinum electrodes $(10 \mathrm{~mm} \times 15 \mathrm{~mm} \times 0.125 \mathrm{~mm})$. The cell was charged with 4-iodotoluene (215b) (109 $\mathrm{mg}$, 
$0.50 \mathrm{mmol}, 1.00$ equiv) and $n-\mathrm{Bu}_{4} \mathrm{NPF}_{6}(194 \mathrm{mg}, 0.50 \mathrm{mmol}, 1.00$ equiv) in TFA/TFAA $(10 \mathrm{~mL}, 3: 1 \mathrm{v} / \mathrm{v})$. After recording initial spectra, the electrolysis was initiated by applying a constant current of $10 \mathrm{~mA}$ at $25^{\circ} \mathrm{C}$. The in-line electrolysis and NMR monitoring were performed for $5 \mathrm{~h}$. The reaction solution was circulated between the benchtop NMR and the electrochemical cell with a flow speed of $0.8 \mathrm{~mL} \cdot \mathrm{min}^{-1}$. The reaction profile was monitored with ${ }^{1} \mathrm{H}-\mathrm{NMR}$ spectra recorded after every 90 seconds. After the electrolysis was stopped, the spectra were batch processed with the reaction monitoring wizard of the MestreNova 12.0.3 software. Arbitrary integral values were transformed to mmol and percentage values by referencing with dibromomethane as internal standard. Accordingly, the product $p$-tolyl- $\lambda^{3}$-iodanediyl bis(2,2,2-trifluoroacetate) (219) was formed in 76\% NMR conversion. ${ }^{1} \mathrm{H}-\mathrm{NMR}$ resonances at a chemical shift (referenced against the trifluoroacetic acid residual solvent peak of $11.50 \mathrm{ppm}$ ) of $8.08 \mathrm{ppm}$ and $6.83 \mathrm{ppm}$ were identified to originate from the starting material $\mathbf{2 1 5 b}$ and $\mathbf{2 1 9}$, respectively.

\subsubsection{Evaluation of the Electron Transfer Number by CV and RDE}

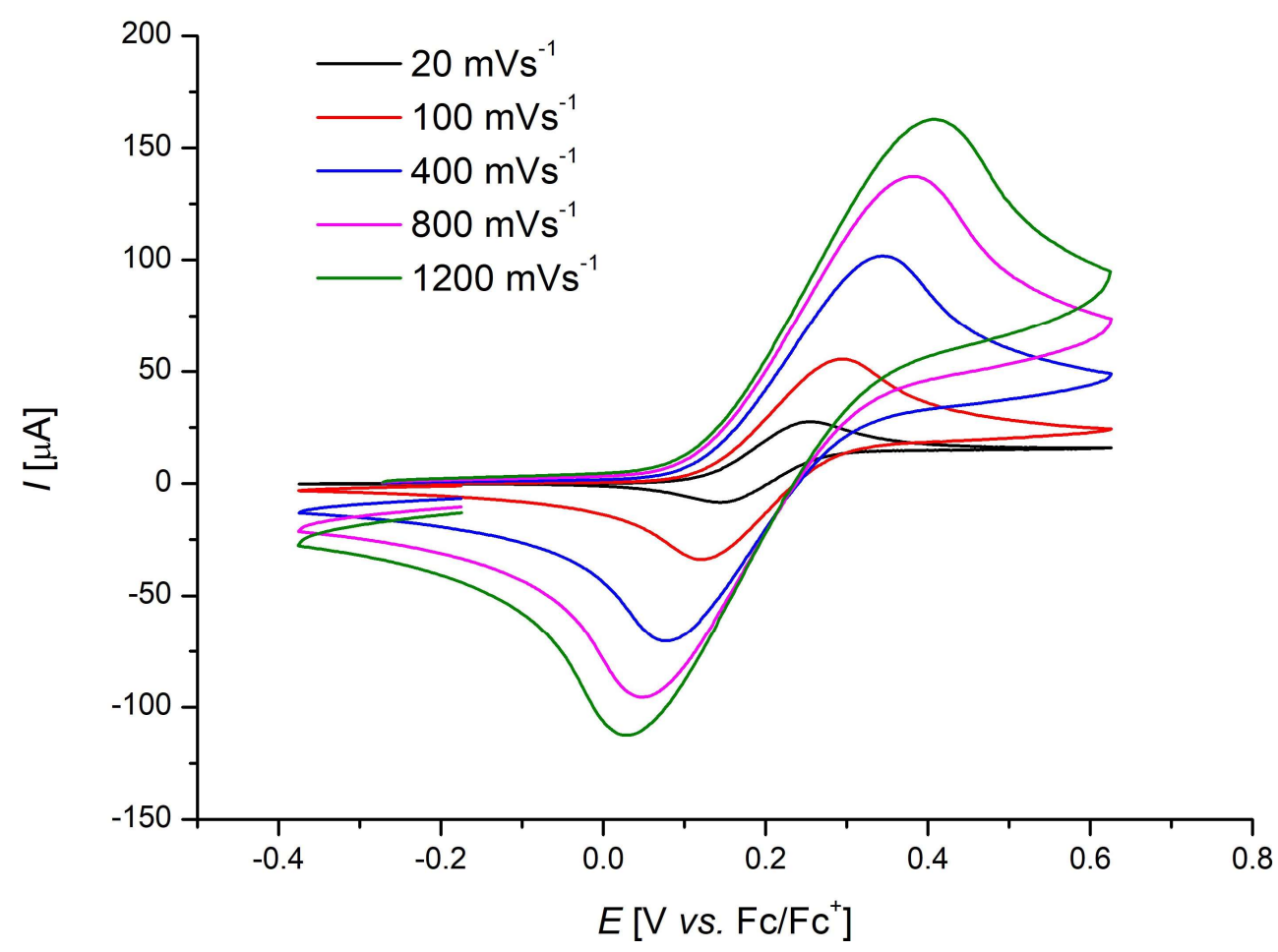

Figure 60. Cyclic voltammograms at different scan rates using DCE and $n$-Bu $\mathrm{Bu}_{4} \mathrm{NF}_{6}$ $(0.1 \mathrm{M})$ as the electrolyte and a GC working electrode; concentration of complex 221 was $3.86 \mathrm{~mm}$. A non-aqueous $\mathrm{Ag} / \mathrm{AgNO} \mathrm{H}_{3}$ in $\mathrm{MeCN}$ was used as the reference electrode. 
Table 34. Plot data of oxidative peak current $i_{\mathrm{p}}$ in amperes versus square root of scan rate $v$.

\begin{tabular}{ccc}
\hline Entry & $i_{\mathrm{p}}[\mathrm{A}]$ & $v^{1 / 2}\left[\mathrm{~V}^{1 / 2} \cdot \mathrm{s}^{-1 / 2}\right]$ \\
\hline 1 & $2.76 \cdot 10^{-5}$ & 0.141 \\
2 & $5.55 \cdot 10^{-5}$ & 0.316 \\
3 & $1.02 \cdot 10^{-4}$ & 0.632 \\
4 & $1.37 \cdot 10^{-4}$ & 0.894 \\
5 & $1.63 \cdot 10^{-4}$ & 1.095 \\
\hline
\end{tabular}

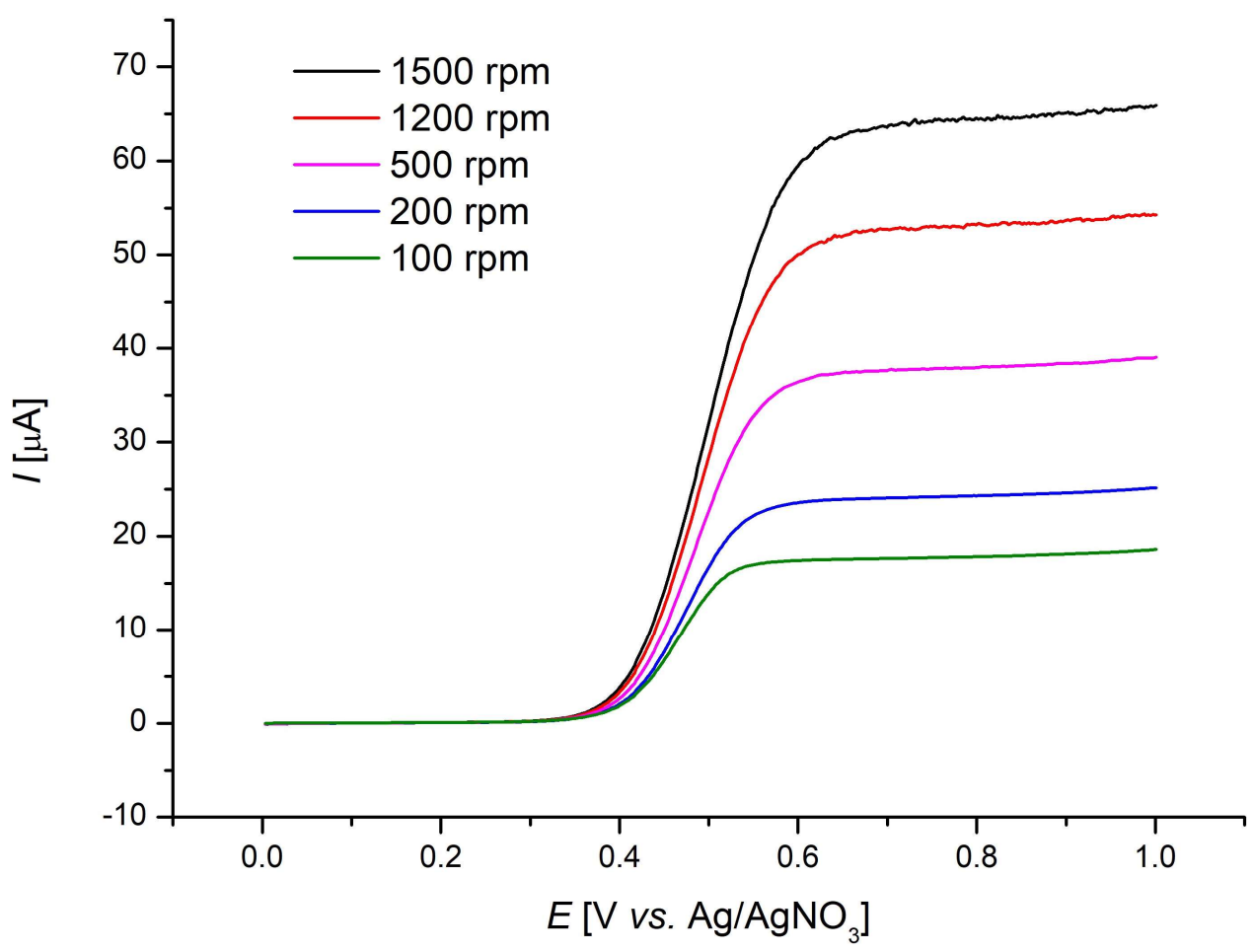

Figure 61. Linear sweep voltammetry of complex 221 at different rotation speeds, using a GC rotating-disc electrode. DCE and $n-\mathrm{Bu}_{4} \mathrm{NPF}_{6}(0.1 \mathrm{M})$ was used as the electrolyte; a scan rate of $20 \mathrm{mV} \cdot \mathrm{s}^{-1}$ and a concentration of complex 221 of $3.859 \mathrm{~mm}$, was used. A non-aqueous $\mathrm{Ag} / \mathrm{AgNO}_{3}$ in acetonitrile was used as the reference electrode. 
Table 35. Plot data of Levich current $i_{\text {lim }}$ in amperes versus square root of rotation rate $\omega$.

\begin{tabular}{ccc}
\hline Entry & $i_{\lim }\left[10^{-5} \mathrm{~A}\right]$ & $\omega^{1 / 2}\left[\mathrm{rad}^{1 / 2} \cdot \mathrm{s}^{-1 / 2}\right]$ \\
\hline 1 & 1.78 & 3.236 \\
2 & 2.43 & 4.576 \\
3 & 3.79 & 7.236 \\
4 & 5.33 & 10.23 \\
5 & 6.45 & 12.53 \\
\hline
\end{tabular}

The error number of the electron transfer number $n$ was calculated via propagation of uncertainty, using the following values: $\sigma A_{\mathrm{RDE}}= \pm 0.0001 \mathrm{~cm}^{2} ; \sigma A_{\mathrm{CV}}= \pm 0.01 \mathrm{~cm}^{2} ; \sigma S_{1}=$ $\pm 0.036 \cdot 10^{-4} ; \sigma S_{2}= \pm 0.036 \cdot 10^{-6} ; C_{\mathrm{RDE}}= \pm 0.1 \cdot 10^{-6} \mathrm{~mol} \cdot \mathrm{cm}^{-3} ; C_{\mathrm{CV}}= \pm 0.1 \cdot 10^{-6} \mathrm{~mol} \cdot \mathrm{cm}^{-3}$. 


\subsection{Manganaelectro-Catalyzed Undirected C( $\left(\mathrm{sp}^{3}\right)-\mathrm{H}$ Azidation}

\subsubsection{Characterization Data}<smiles>NC1CCCc2ccccc21</smiles>

1-Azido-1,2,3,4-tetrahydronaphthalene (113a): The general procedure I was followed using 1,2,3,4-tetrahydronaphthalene (111a) $(66.1 \mathrm{mg}, 0.50 \mathrm{mmol})$ and sodium azide (260 mg, $4.00 \mathrm{mmol})$. Purification by column chromatography on silica gel ( $n$ pentane/EtOAc 99:1) yielded 113a (48.6 mg, $281 \mu \mathrm{mol}, 56 \%$ ) as a colorless liquid.

${ }^{1}$ H-NMR (400 MHz, $\left.\mathrm{CDCl}_{3}\right): \delta=7.33-7.27(\mathrm{~m}, 1 \mathrm{H}), 7.26-7.19(\mathrm{~m}, 2 \mathrm{H}), 7.17-7.10(\mathrm{~m}$, $1 \mathrm{H}), 4.57(\mathrm{t}, J=4.9 \mathrm{~Hz}, 1 \mathrm{H}), 2.90-2.81(\mathrm{~m}, 1 \mathrm{H}), 2.81-2.68(\mathrm{~m}, 1 \mathrm{H}), 2.08-1.91(\mathrm{~m}, 3 \mathrm{H})$, $1.89-1.72(\mathrm{~m}, 1 \mathrm{H})$.

${ }^{13}$ C-NMR (101 MHz, $\left.\mathrm{CDCl}_{3}\right): \delta=137.5\left(\mathrm{C}_{\mathrm{q}}\right), 133.9\left(\mathrm{C}_{\mathrm{q}}\right), 129.6(\mathrm{CH}), 129.3(\mathrm{CH}), 127.8$ $(\mathrm{CH}), 126.3(\mathrm{CH}), 59.6(\mathrm{CH}), 29.3\left(\mathrm{CH}_{2}\right), 28.9\left(\mathrm{CH}_{2}\right), 19.1\left(\mathrm{CH}_{2}\right)$.

IR (ATR): $\tilde{v}=2937,2870,2093,1454,1233,943,766,741 \mathrm{~cm}^{-1}$.

MS (EI) $m / z$ (relative intensity): 155 (10), 145 (20) [M- $\left.{ }_{2}\right]^{+}, 131$ (100), 115 (45), 102 (5), 91 (20), 77 (5).

HR-MS (ESI) $m / z$ calcd. for $\mathrm{C}_{10} \mathrm{H}_{13} \mathrm{~N}_{3} \mathrm{Na}[\mathrm{M}+\mathrm{Na}]^{+}: 196.0845$, found: 196.0845 .

The analytical data corresponds with those reported in the literature. ${ }^{[06]}$<smiles>NC1CCCCCCC1</smiles>

Azidocyclooctane (113b): The general procedure I was followed using cyclooctane (111b) (56.1 $\mathrm{mg}, 0.50 \mathrm{mmol})$ and sodium azide $(260 \mathrm{mg}, 4.00 \mathrm{mmol})$. Purification by column chromatography on silica gel ( $n$-pentane/EtOAc 99:1) yielded 113b (30.8 mg, $201 \mu \mathrm{mol}$, $40 \%$ ) as a colorless liquid.

${ }^{1}$ H-NMR (400 MHz, $\left.\mathrm{CDCl}_{3}\right): \delta=3.64-3.51(\mathrm{~m}, 1 \mathrm{H}), 1.94-1.84(\mathrm{~m}, 2 \mathrm{H}), 1.79-1.67$ (m, $4 \mathrm{H}), 1.61-1.48(\mathrm{~m}, 8 \mathrm{H})$.

${ }^{13}$ C-NMR $\left(101 \mathrm{MHz}, \mathrm{CDCl}_{3}\right): \delta=62.4(\mathrm{CH}), 31.0\left(\mathrm{CH}_{2}\right), 27.4\left(\mathrm{CH}_{2}\right), 25.3\left(\mathrm{CH}_{2}\right), 23.3$ $\left(\mathrm{CH}_{2}\right)$.

IR (ATR): $\tilde{v}=2927,2854,2091,1474,1259,1095,940,668 \mathrm{~cm}^{-1}$. 
MS (EI) $m / z$ (relative intensity): 125 (5) [M- $\left.\mathrm{N}_{2}\right]^{+}, 110$ (10), 96 (50), 82 (100), 67 (70), 55 (15).

HR-MS (EI) $m / z$ calcd. for $\mathrm{C}_{8} \mathrm{H}_{15} \mathrm{~N}_{1}\left[\mathrm{M}-\mathrm{N}_{2}\right]^{+}:$125.1204, found: 125.1200 .

The analytical data corresponds with those reported in the literature. ${ }^{[407]}$<smiles>[N]C1CCCCCC1</smiles>

Azidocycloheptane (113g): The general procedure I was followed using cycloheptane (111g) (49.1 mg, $0.50 \mathrm{mmol})$ and sodium azide (260 mg, $4.00 \mathrm{mmol}$ ). Purification by column chromatography on silica gel (n-pentane/EtOAc 99:1) yielded 113g (23.1 mg, $165 \mu \mathrm{mol}, 33 \%)$ as a colorless liquid.

${ }^{1}$ H-NMR (400 MHz, $\left.\mathrm{CDCl}_{3}\right): \delta=3.57-3.48(\mathrm{~m}, 1 \mathrm{H}), 1.99-1.88(\mathrm{~m}, 2 \mathrm{H}), 1.74-1.51$ (m, $8 \mathrm{H}), 1.49-1.36(\mathrm{~m}, 2 \mathrm{H})$.

${ }^{13}$ C-NMR $\left(101 \mathrm{MHz}, \mathrm{CDCl}_{3}\right): \delta=62.8(\mathrm{CH}), 33.9\left(\mathrm{CH}_{2}\right), 28.0\left(\mathrm{CH}_{2}\right), 23.6\left(\mathrm{CH}_{2}\right)$.

IR (ATR): $\tilde{v}=2927,2858,2083,1459,1249,958,830,560 \mathrm{~cm}^{-1}$.

MS (EI) $m / z$ (relative intensity): 110 (15) $\left[\mathrm{M}-\mathrm{N}_{2}\right]^{+}, 96$ (25), 91 (5), 82 (100), 68 (40), 55 (55).

HR-MS (EI) $m / z$ calcd. for $\mathrm{C}_{7} \mathrm{H}_{13} \mathrm{~N}_{1}\left[\mathrm{M}-\mathrm{N}_{2}\right]^{+}: 111.1048$, found: 111.1044 .

The analytical data corresponds with those reported in the literature. ${ }^{[408]}$<smiles>NC(Cc1ccccc1)c1ccccc1</smiles>

(1-Azidoethane-1,2-diyl)dibenzene (113h): The general procedure I was followed using 1,2-diphenylethane (111h) (91.2 mg, $0.50 \mathrm{mmol})$ and sodium azide (260 mg, $4.00 \mathrm{mmol})$. Purification by column chromatography on silica gel (n-pentane/EtOAc 99:1) yielded 113h (72.1 mg, $309 \mu \mathrm{mol}, 62 \%)$ as a colorless liquid.

${ }^{1}$ H-NMR (400 MHz, $\left.\mathrm{CDCl}_{3}\right): \delta=7.40-7.32(\mathrm{~m}, 3 \mathrm{H}), 7.31-7.23(\mathrm{~m}, 5 \mathrm{H}), 7.17-7.13(\mathrm{~m}$, $2 \mathrm{H}), 4.68(\mathrm{td}, J=8.4,6.2 \mathrm{~Hz}, 1 \mathrm{H}), 3.13-3.00(\mathrm{~m}, 2 \mathrm{H})$.

${ }^{13}$ C-NMR (101 MHz, $\left.\mathrm{CDCl}_{3}\right): \delta=139.5\left(\mathrm{C}_{\mathrm{q}}\right), 137.6\left(\mathrm{C}_{\mathrm{q}}\right), 129.5(\mathrm{CH}), 128.9(\mathrm{CH}), 128.6$ $(\mathrm{CH}), 128.5(\mathrm{CH}), 127.1(\mathrm{CH}), 126.9(\mathrm{CH}), 67.8(\mathrm{CH}), 43.2\left(\mathrm{CH}_{2}\right)$.

IR (ATR): $\tilde{v}=3030,2921,2094,1495,1453,1247,757,696 \mathrm{~cm}^{-1}$.

MS (ESI) $m / z$ (relative intensity): $246(2)[\mathrm{M}+\mathrm{Na}]^{+}, 181(100)\left[\mathrm{M}-\mathrm{N}_{3}\right]^{+}, 117$ (60).

HR-MS (ESI) $m / z$ calcd. for $\mathrm{C}_{14} \mathrm{H}_{13} \mathrm{~N}_{3} \mathrm{Na}[\mathrm{M}+\mathrm{Na}]^{+}:$246.1002, found: 246.1002 . 
The analytical data corresponds with those reported in the literature. ${ }^{[406]}$<smiles>Cc1ccc(C(C)(C)C)cc1</smiles>

$113 i$<smiles>CC(C)c1ccc(CN)cc1</smiles>

$113 \mathrm{i}^{\prime}$

1-(2-Azidopropan-2-yl)-4-methylbenzene (113i): The general procedure I was followed using $p$-cymene (111i) $(67.1 \mathrm{mg}, 0.50 \mathrm{mmol})$ and sodium azide $(260 \mathrm{mg}, 4.00 \mathrm{mmol})$. The ratio of 113i/113i' (33:1) was determined by ${ }^{1} \mathrm{H}-\mathrm{NMR}$ spectroscopy of the crude reaction mixture. Purification by column chromatography on silica gel (n-pentane/EtOAc 99:1) yielded 113i/113i' (59.4 mg, $339 \mu \mathrm{mol}, 66 \%)$ as a colorless liquid. Analytical data is reported for $\mathbf{1 1 3 i}$.

${ }^{1}$ H-NMR (400 MHz, $\left.\mathrm{CDCl}_{3}\right): \delta=7.36(\mathrm{~d}, J=8.3 \mathrm{~Hz}, 2 \mathrm{H}), 7.20(\mathrm{~d}, J=8.3 \mathrm{~Hz}, 2 \mathrm{H}), 2.37$ (s, 3H), $1.65(\mathrm{~s}, 6 \mathrm{H})$.

${ }^{13}$ C-NMR (101 MHz, $\left.\mathrm{CDCl}_{3}\right): \delta=141.8\left(\mathrm{C}_{\mathrm{q}}\right), 137.2\left(\mathrm{C}_{\mathrm{q}}\right), 129.3(\mathrm{CH}), 125.2(\mathrm{CH}), 63.8$ $\left(\mathrm{C}_{\mathrm{q}}\right), 28.6\left(\mathrm{CH}_{3}\right), 21.1\left(\mathrm{CH}_{3}\right)$.

IR (ATR): $\tilde{v}=2978,2925,2103,1515,1460,1256,1099,816 \mathrm{~cm}^{-1}$.

MS (EI) $m / z$ (relative intensity): 133 (20) [M-N $]^{+}, 132$ (98), 117 (100), 105 (10), 91 (40), 77 (5).

HR-MS (EI) $m / z$ calcd. for $\mathrm{C}_{10} \mathrm{H}_{13}\left[\mathrm{M}-\mathrm{N}_{3}\right]^{+}: 133.1012$, found: 133.1012 .

The analytical data corresponds with those reported in the literature. ${ }^{[191]}$<smiles>CC(C)C(N)c1ccccc1</smiles>

113j<smiles>CC(C)(C)Cc1ccccc1</smiles>

(1-Azido-2-methylpropyl)benzene (113j): The general procedure I was followed using isobutylbenzene (111j) (67.1 $\mathrm{mg}, 0.50 \mathrm{mmol})$ and sodium azide $(260 \mathrm{mg}, 4.00 \mathrm{mmol})$. The ratio of $\mathbf{1 1 3 j}$ /113j' $(2.7: 1)$ was determined by ${ }^{1} \mathrm{H}-\mathrm{NMR}$ spectroscopy of the crude reaction mixture. ${ }^{[409]}$ Purification by column chromatography on silica gel ( $n$-pentane/EtOAc 99:1) yielded $\mathbf{1 1 3 j} / \mathbf{1 1 3 j}$ ' $(49.2 \mathrm{mg}, 281 \mu \mathrm{mol}, 56 \%)$ as a colorless liquid. Analytical data is reported for $\mathbf{1 1 3 j}$.

${ }^{1} \mathbf{H}-\mathbf{N M R}\left(400 \mathrm{MHz}, \mathrm{CDCl}_{3}\right): \delta=7.41-7.34(\mathrm{~m}, 2 \mathrm{H}), 7.34-7.29(\mathrm{~m}, 1 \mathrm{H}), 7.28-7.24(\mathrm{~m}$, 2H), $4.13(\mathrm{~d}, J=8.0 \mathrm{~Hz}, 1 \mathrm{H}), 2.00(\mathrm{dh}, J=8.0,6.7 \mathrm{~Hz}, 1 \mathrm{H}), 1.02(\mathrm{~d}, J=6.7 \mathrm{~Hz}, 3 \mathrm{H}), 0.80$ $(\mathrm{d}, J=6.7 \mathrm{~Hz}, 3 \mathrm{H})$. 
${ }^{13}$ C-NMR (101 MHz, $\left.\mathrm{CDCl}_{3}\right): \delta=139.1\left(\mathrm{C}_{\mathrm{q}}\right), 128.7(\mathrm{CH}), 128.2(\mathrm{CH}), 127.6(\mathrm{CH}), 73.4$ $(\mathrm{CH}), 34.2(\mathrm{CH}), 19.7\left(\mathrm{CH}_{3}\right), 19.4\left(\mathrm{CH}_{3}\right)$.

IR (ATR): $\tilde{v}=2963,2928,2096,1468,1249,1153,747,701 \mathrm{~cm}^{-1}$.

MS (EI) $m / z$ (relative intensity): 146 (20) [M-N $]^{+}, 132$ (30), 117 (60), 104 (100), 91 (55), $77(55)$.

HR-MS (EI) $m / z$ calcd. for $\mathrm{C}_{10} \mathrm{H}_{12} \mathrm{~N}_{1}\left[\mathrm{M}-\mathrm{N}_{2}\right]^{+}:$146.0964, found: 146.0966 .

The analytical data corresponds with those reported in the literature. ${ }^{[203 a]}$<smiles>CCCCCC(N)c1ccc(-c2ccc(C(=O)OC)cc2)cc1</smiles>

Methyl 4'-(1-azidohexyl)-[1,1'-biphenyl]-4-carboxylate (113k): The general procedure I was followed using methyl 4'-hexyl-[1,1'-biphenyl]-4-carboxylate (111k) (148 mg, $0.50 \mathrm{mmol})$ and sodium azide $(260 \mathrm{mg}, 4.00 \mathrm{mmol})$. Purification by column chromatography on silica gel ( $n$-hexane/EtOAc 10:1) yielded 113k (65.7 mg, $195 \mu \mathrm{mol}$, $39 \%)$ as a colorless liquid.

${ }^{1}$ H-NMR (600 MHz, $\left.\mathrm{CDCl}_{3}\right): \delta=8.13-8.10(\mathrm{~m}, 2 \mathrm{H}), 7.68-7.65(\mathrm{~m}, 2 \mathrm{H}), 7.64(\mathrm{~d}, J=8.4$ $\mathrm{Hz}, 2 \mathrm{H}), 7.41-7.38$ (m, 2H), 4.46 (dd, $J=7.8,6.6 \mathrm{~Hz}, 1 \mathrm{H}), 3.95$ (s, 3H), 1.92-1.82 (m, 1H), 1.81-1.73 (m, 1H), 1.47-1.36 (m, 1H), 1.36-1.25 (m, 5H), 0.91-0.86 (m, 3H).

${ }^{13}$ C-NMR $\left(101 \mathrm{MHz}, \mathrm{CDCl}_{3}\right): \delta=167.1\left(\mathrm{C}_{\mathrm{q}}\right), 145.1\left(\mathrm{C}_{\mathrm{q}}\right), 140.1\left(\mathrm{C}_{\mathrm{q}}\right), 139.9\left(\mathrm{C}_{\mathrm{q}}\right), 130.3$ $(\mathrm{CH}), 129.2\left(\mathrm{C}_{\mathrm{q}}\right), 127.8(\mathrm{CH}), 127.6(\mathrm{CH}), 127.1(\mathrm{CH}), 66.2(\mathrm{CH}), 52.3\left(\mathrm{CH}_{3}\right), 36.3\left(\mathrm{CH}_{2}\right)$, 31.6 $\left(\mathrm{CH}_{2}\right), 26.1\left(\mathrm{CH}_{2}\right), 22.6\left(\mathrm{CH}_{2}\right), 14.1\left(\mathrm{CH}_{3}\right)$.

IR (ATR): $\tilde{v}=2932,2859,2093,1719,1608,1435,1275,1110,773 \mathrm{~cm}^{-1}$.

MS (ESI) $m / z$ (relative intensity): $697(100)[2 \mathrm{M}+\mathrm{Na}]^{+}, 360(70)[\mathrm{M}+\mathrm{Na}]^{+}, 295(10)[\mathrm{M}-$ $\left.\mathrm{N}_{3}\right]^{+}$.

HR-MS (ESI) $m / z$ calcd. for $\mathrm{C}_{20} \mathrm{H}_{23} \mathrm{~N}_{3} \mathrm{O}_{2} \mathrm{Na}[\mathrm{M}+\mathrm{Na}]^{+}: 360.1682$, found: 360.1681 . 
<smiles>CC(=O)O[C@H]1C[C@H](C)CC[C@@H]1C(C)(C)N</smiles>

1131<smiles>CC(=O)O[C@H]1CC(C)(N)CC[C@@H]1C(C)C</smiles>

(1R,2S,5R)-2-(2-Azidopropan-2-yl)-5-methylcyclohexyl acetate (1131): The general procedure I was followed using (-)-menthol acetate (1111) $(99.2 \mathrm{mg}, 0.50 \mathrm{mmol})$ and sodium azide ( $260 \mathrm{mg}, 4.00 \mathrm{mmol}$ ). The ratio of 1131/1131' (3:1) was determined by ${ }^{1} \mathrm{H}-$ NMR of the crude reaction mixture. Purification by column chromatography on silica gel (n-pentane/EtOAc 95:5) yielded 1131/113l' (57.6 mg, $241 \mu \mathrm{mol}, 48 \%$ ) as a colorless liquid. Analytical data is reported for $\mathbf{1 1 3 1}$.

${ }^{1}$ H-NMR $\left(600 \mathrm{MHz}, \mathrm{CDCl}_{3}\right): \delta=4.81(\mathrm{td}, J=10.7,4.5 \mathrm{~Hz}, 1 \mathrm{H}), 2.05(\mathrm{~s}, 3 \mathrm{H}), 1.99-1.94$ (m, 1H), $1.92(\mathrm{dq}, J=13.4,3.5 \mathrm{~Hz}, 1 \mathrm{H}), 1.71$ (ddq, $J=11.9,3.6,2.3 \mathrm{~Hz}, 1 \mathrm{H}), 1.68-1.63$ $(\mathrm{m}, 1 \mathrm{H}), 1.53-1.45(\mathrm{~m}, 1 \mathrm{H}), 1.26(\mathrm{~s}, 3 \mathrm{H}), 1.24(\mathrm{~s}, 3 \mathrm{H}), 1.11$ (tdd, $J=13.3,12.3,3.6 \mathrm{~Hz}$, $1 \mathrm{H}), 1.01(\mathrm{td}, J=12.3,10.9 \mathrm{~Hz}, 1 \mathrm{H}), 0.93-0.90(\mathrm{~m}, 1 \mathrm{H}), 0.90(\mathrm{~d}, J=6.5 \mathrm{~Hz}, 3 \mathrm{H})$.

${ }^{13}$ C-NMR (101 MHz, $\left.\mathrm{CDCl}_{3}\right): \delta=170.5\left(\mathrm{C}_{\mathrm{q}}\right), 73.4(\mathrm{CH}), 63.6\left(\mathrm{C}_{\mathrm{q}}\right), 49.1(\mathrm{CH}), 41.4\left(\mathrm{CH}_{2}\right)$, $34.2\left(\mathrm{CH}_{2}\right), 31.3(\mathrm{CH}), 26.6\left(\mathrm{CH}_{2}\right), 25.1\left(\mathrm{CH}_{3}\right), 24.7\left(\mathrm{CH}_{3}\right), 21.8\left(\mathrm{CH}_{3}\right), 21.8\left(\mathrm{CH}_{3}\right)$.

IR (ATR): $\tilde{v}=2955,2872,2099,1734,1457,1371,1239,1027 \mathrm{~cm}^{-1}$.

MS (ESI) $m / z$ (relative intensity): 379 (10), $262(100)[\mathrm{M}+\mathrm{Na}]^{+}, 197(10)\left[\mathrm{M}-\mathrm{N}_{3}\right]^{+}, 152$ (10).

HR-MS (ESI) $m / z$ calcd. for $\mathrm{C}_{12} \mathrm{H}_{21} \mathrm{~N}_{3} \mathrm{O}_{2} \mathrm{Na}[\mathrm{M}+\mathrm{Na}]^{+}:$: 262.1526, found: 262.1528 .

The analytical data corresponds with those reported in the literature. ${ }^{[201]}$<smiles>[M]C12CCC3c4ccc(OC(C)=O)cc4CCC3C1CCC2=O</smiles>

$113 m$<smiles>CC(=O)Oc1ccc2c(c1)CCC1C2CCC2(C)C(=O)CCC12</smiles>

$113 m^{\prime}$

$(8 S, 9 R, 13 S, 14 S)-9$-Azido-13-methyl-17-oxo-7,8,9,11,12,13,14,15,16,17-decahydro-6Hcyclopenta[a]phenanthren-3-yl acetate $(\mathbf{1 1 3 m})$ : The general procedure I was followed using estrone acetate $111 \mathrm{~m}$ (156 mg, $0.50 \mathrm{mmol}$ ) and sodium azide (260 mg, $4.00 \mathrm{mmol})$. Purification by column chromatography on silica gel ( $n$-hexane/EtOAc 99:1 $\rightarrow$ 80:20) yielded 113m (66.3 mg, $188 \mu \mathrm{mol}, 38 \%$ ) and 113m' (65.3 mg, $185 \mu \mathrm{mol}, 37 \%$ ) as a mixture of diastereomers as colorless liquids. 
${ }^{1}$ H-NMR $\left(600 \mathrm{MHz}, \mathrm{CDCl}_{3}\right): \delta=7.43(\mathrm{~d}, J=8.5 \mathrm{~Hz}, 1 \mathrm{H}), 6.96(\mathrm{dd}, J=8.3,2.5 \mathrm{~Hz}, 1 \mathrm{H})$, $6.93(\mathrm{~d}, J=2.5 \mathrm{~Hz}, 1 \mathrm{H}), 3.00-2.88(\mathrm{~m}, 3 \mathrm{H}), 2.66-2.59$ (m, 1H), 2.49 (ddd, $J=19.2,8.9$, $1.1 \mathrm{~Hz}, 1 \mathrm{H}), 2.30(\mathrm{~s}, 3 \mathrm{H}), 2.17(\mathrm{dt}, J=19.2,8.9 \mathrm{~Hz}, 1 \mathrm{H}), 2.05-1.95(\mathrm{~m}, 2 \mathrm{H}), 1.89(\mathrm{td}, J=$ 11.4, 10.7, $2.9 \mathrm{~Hz}, 1 \mathrm{H}), 1.84-1.74(\mathrm{~m}, 4 \mathrm{H}), 1.61-1.56$ (m, 1H), 0.89 (s, 3H).

${ }^{13}$ C-NMR $\left(101 \mathrm{MHz}, \mathrm{CDCl}_{3}\right): \delta=219.7\left(\mathrm{C}_{\mathrm{q}}\right), 169.6\left(\mathrm{C}_{\mathrm{q}}\right), 150.5\left(\mathrm{C}_{\mathrm{q}}\right), 138.5\left(\mathrm{C}_{\mathrm{q}}\right), 134.3$ $\left(\mathrm{C}_{\mathrm{q}}\right), 126.2(\mathrm{CH}), 122.9(\mathrm{CH}), 119.2(\mathrm{CH}), 65.3\left(\mathrm{C}_{\mathrm{q}}\right), 47.6\left(\mathrm{C}_{\mathrm{q}}\right), 43.6(\mathrm{CH}), 40.8(\mathrm{CH}), 35.9$ $\left(\mathrm{CH}_{2}\right), 29.5\left(\mathrm{CH}_{2}\right), 28.9\left(\mathrm{CH}_{2}\right), 28.0\left(\mathrm{CH}_{2}\right), 21.4\left(\mathrm{CH}_{2}\right), 21.3\left(\mathrm{CH}_{3}\right), 20.5\left(\mathrm{CH}_{2}\right), 13.4\left(\mathrm{CH}_{3}\right)$. IR (ATR): $\tilde{v}=2937,2856,2093,1763,1738,1494,1262,1200,756 \mathrm{~cm}^{-1}$.

MS (ESI) $m / z$ (relative intensity): $729(10)[2 \mathrm{M}+\mathrm{Na}]^{+}, 376(100)[\mathrm{M}+\mathrm{Na}]^{+}, 311(50)[\mathrm{M}-$ $\left.\mathrm{N}_{3}\right]^{+}$.

HR-MS (ESI) $m / z$ calcd. for $\mathrm{C}_{20} \mathrm{H}_{23} \mathrm{~N}_{3} \mathrm{O}_{3} \mathrm{Na}[\mathrm{M}+\mathrm{Na}]^{+}: 376.1632$, found: 376.1629 .

The analytical data corresponds with those reported in the literature. ${ }^{[406]}$

$(8 S, 9 S, 13 S, 14 S)-9-A z i d o-13-m e t h y l-17-0 x 0-7,8,9,11,12,13,14,15,16,17-d e c a h y d r o-6 H-$ cyclopenta $[a]$ phenanthren-3-yl acetate $\left(113 m^{\prime}\right)$

${ }^{1}$ H-NMR (600 MHz, $\left.\mathrm{CDCl}_{3}\right): \delta=7.44(\mathrm{~d}, J=8.6 \mathrm{~Hz}, 1 \mathrm{H}), 6.99(\mathrm{dd}, J=8.6,2.6 \mathrm{~Hz}, 1 \mathrm{H})$, $6.95(\mathrm{~d}, J=2.5 \mathrm{~Hz}, 1 \mathrm{H}), 2.84-2.79(\mathrm{~m}, 3 \mathrm{H}), 2.71(\mathrm{dt}, J=14.4,3.3 \mathrm{~Hz}, 1 \mathrm{H}), 2.47$ (ddd, $J=$ 19.4, 8.9, 1.1 Hz, 2H), 2.29 (s, 4H), 2.11-2.02 (m, 4H), 1.96-1.91 (m, 1H), 1.81-1.75 (m, 2H), $1.71(\mathrm{dt}, J=13.6,3.4 \mathrm{~Hz}, 2 \mathrm{H}), 1.66(\mathrm{ddd}, J=12.5,9.0,3.5 \mathrm{~Hz}, 1 \mathrm{H}), 1.61-1.53$ (m, $3 \mathrm{H}), 1.14(\mathrm{td}, J=13.8,3.5 \mathrm{~Hz}, 2 \mathrm{H}), 1.02(\mathrm{~s}, 4 \mathrm{H})$.

${ }^{13}$ C-NMR $\left(101 \mathrm{MHz}, \mathrm{CDCl}_{3}\right): \delta=218.8\left(\mathrm{C}_{\mathrm{q}}\right), 169.4\left(\mathrm{C}_{\mathrm{q}}\right), 150.4\left(\mathrm{C}_{\mathrm{q}}\right), 138.9\left(\mathrm{C}_{\mathrm{q}}\right), 130.5$ $\left(\mathrm{C}_{\mathrm{q}}\right), 127.1(\mathrm{CH}), 123.0(\mathrm{CH}), 119.8(\mathrm{CH}), 65.9\left(\mathrm{C}_{\mathrm{q}}\right), 47.5\left(\mathrm{C}_{\mathrm{q}}\right), 43.2(\mathrm{CH}), 39.4(\mathrm{CH}), 35.8$ $\left(\mathrm{CH}_{2}\right), 30.6\left(\mathrm{CH}_{2}\right), 28.4\left(\mathrm{CH}_{2}\right), 25.2\left(\mathrm{CH}_{2}\right), 21.9\left(\mathrm{CH}_{2}\right), 21.3\left(\mathrm{CH}_{3}\right), 19.6\left(\mathrm{CH}_{2}\right), 13.6\left(\mathrm{CH}_{3}\right)$. IR (ATR): $\tilde{v}=2948,2864,2093,1763,1740,1495,1204,1013,755 \mathrm{~cm}^{-1}$.

MS (ESI) $m / z$ (relative intensity): $729.4(10)[2 \mathrm{M}+\mathrm{Na}]^{+}, 376.2(100)[\mathrm{M}+\mathrm{Na}]^{+}, 311.2(30)$ $\left[\mathrm{M}-\mathrm{N}_{3}\right]^{+}$.

HR-MS (ESI) $m / z$ calcd. for $\mathrm{C}_{20} \mathrm{H}_{23} \mathrm{~N}_{3} \mathrm{O}_{3} \mathrm{Na}[\mathrm{M}+\mathrm{Na}]^{+}: 376.1632$, found: 376.1634 . 


\subsubsection{Mechanistic Studies}

\subsubsection{Radical Trap Experiments}

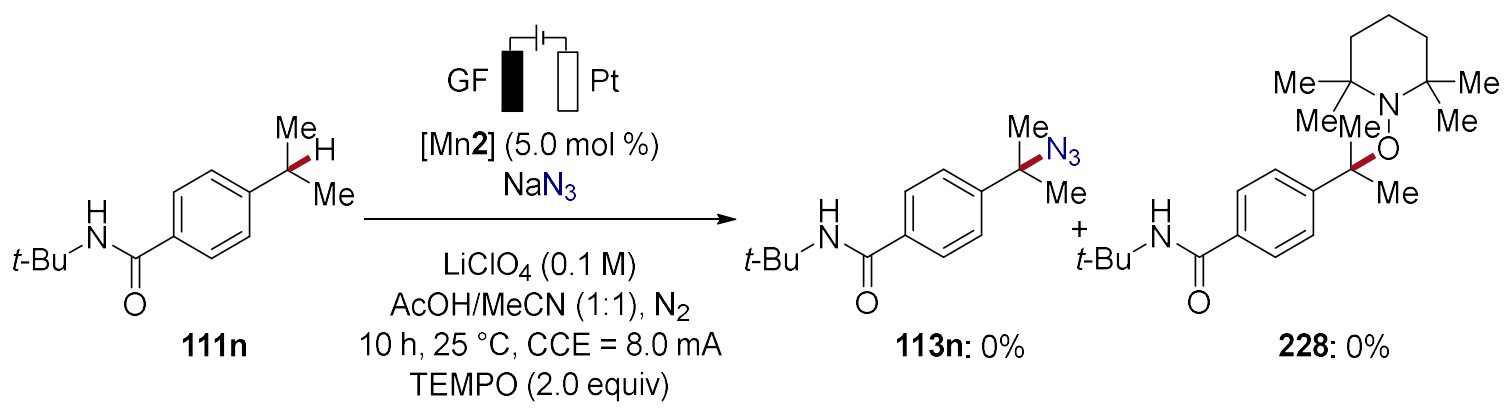

[Mn2] (12.7 mg, $25.0 \mu \mathrm{mol}, 5.0 \mathrm{~mol} \%$ ), sodium azide (260 mg, $4.00 \mathrm{mmol}, 8.00$ equiv), $N$-(tert-butyl)-4-isopropylbenzamide (111n) $\left(110 \mathrm{mg}, 0.50 \mathrm{mmol}, 1.00\right.$ equiv), $\mathrm{LiClO}_{4}$ (53.2 mg, $0.50 \mathrm{mmol}, 1.00$ equiv) and (2,2,6,6-tetramethylpiperidin-1-yl)oxyl (TEMPO) (156 mg, $1.00 \mathrm{mmol}, 2.0$ equiv) were placed in an undivided cell under nitrogen atmosphere. The reaction components were dissolved in $\mathrm{MeCN}(2.5 \mathrm{~mL})$, followed by $\mathrm{AcOH}(2.5 \mathrm{~mL})$ and the reaction mixture was stirred at $400 \mathrm{rpm}$. The electrodes were connected under vigorous nitrogen flow. Electrolysis was performed at $25^{\circ} \mathrm{C}$ with a constant current of $8 \mathrm{~mA}$ maintained for $10 \mathrm{~h}\left(Q \cdot \mathrm{mol}^{-1}=5.97 \mathrm{~F}\right)$. The reaction was monitored with ESI mass spectrometry to detect putative reaction intermediates. At ambient temperature, the reaction mixture was quenched with $\mathrm{H}_{2} \mathrm{O}(20 \mathrm{~mL})$ and EtOAc $(10 \mathrm{~mL})$ was added, and the graphite felt anode was washed with EtOAc $(3 \times 5 \mathrm{~mL})$ in an ultrasonic bath. The combined mixture was washed with $\mathrm{H}_{2} \mathrm{O}(3 \times 10 \mathrm{~mL})$, and then dried over $\mathrm{Na}_{2} \mathrm{SO}_{4}$, filtered and the solvents were removed in vacuo. The crude mixture was analyzed by ${ }^{1} \mathrm{H}-\mathrm{NMR}$ spectroscopy, but no desired product was detected. Column chromatography of the crude reaction mixture afforded substrate $111 \mathrm{n}(98.1 \mathrm{mg}, 446 \mu \mathrm{mol}$, $89 \%$ ) without any of the desired products $113 \mathrm{n}$ or $\mathbf{2 2 8}$.

\subsubsection{Intermolecular Competition Experiment}

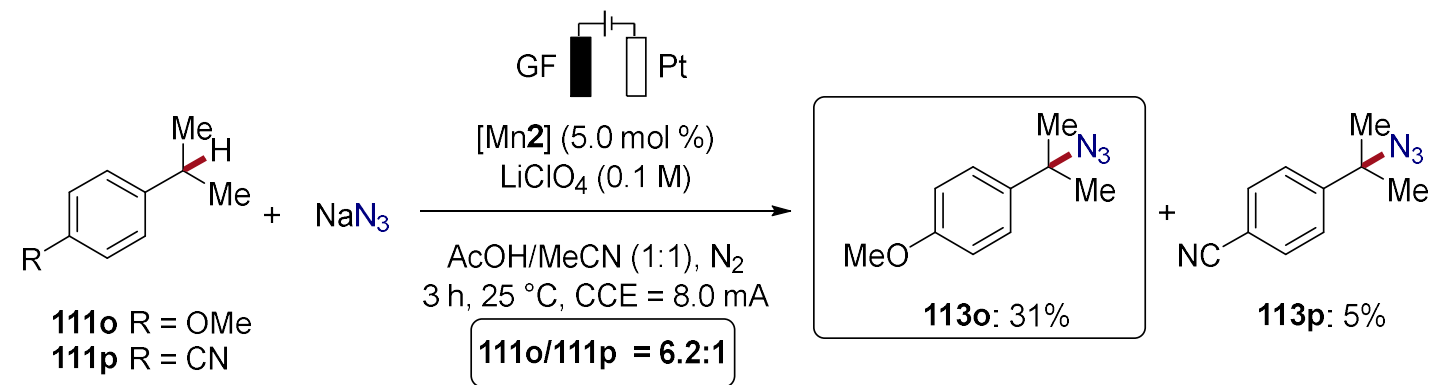


[Mn2] (12.7 mg, $25.0 \mu \mathrm{mol}, 5.0 \mathrm{~mol} \%$ ), sodium azide (260 mg, $4.00 \mathrm{mmol}, 8.00$ equiv), 1-isopropyl-4-methoxybenzene $\quad(\mathbf{1 1 1 0}) \quad(75.1 \mathrm{mg}, \quad 0.50 \mathrm{mmol}, \quad 1.00$ equiv), 4isopropylbenzonitrile (111p) $\left(72.6 \mathrm{mg}, 0.50 \mathrm{mmol}, 1.00\right.$ equiv) and $\mathrm{LiClO}_{4}(53.2 \mathrm{mg}$, $0.50 \mathrm{mmol}, 1.00$ equiv) were placed in an undivided cell under nitrogen atmosphere. The reaction components were dissolved in $\mathrm{MeCN}(2.5 \mathrm{~mL})$, followed by $\mathrm{AcOH}(2.5 \mathrm{~mL})$ and the reaction mixture was stirred at $400 \mathrm{rpm}$. The electrodes were connected under vigorous nitrogen-flow. Electrolysis was performed at $25{ }^{\circ} \mathrm{C}$ with a constant current of $8 \mathrm{~mA}$ maintained for $3 \mathrm{~h}$. At ambient temperature, the reaction mixture was quenched with $\mathrm{H}_{2} \mathrm{O}$ $(20 \mathrm{~mL})$ and EtOAc $(10 \mathrm{~mL})$ was added, and the graphite felt anode was washed with EtOAc $(3 \times 5 \mathrm{~mL})$ in an ultrasonic bath. ${ }^{1} \mathrm{H}-\mathrm{NMR}$ spectroscopic analysis did not lead to clean signal separation of both products but revealed a clear favor of $\mathbf{1 1 1 0}$. The combined mixture was washed with $\mathrm{H}_{2} \mathrm{O}(3 \times 10 \mathrm{~mL})$, and then dried over $\mathrm{Na}_{2} \mathrm{SO}_{4}$, filtered and the solvents were removed in vacuo. Column chromatography (n-pentane/EtOAc 99:1 $\rightarrow$ 10:1) of the crude reaction mixture afforded product $1130(29.6 \mathrm{mg}, 155 \mu \mathrm{mol}, 31 \%)$ and product $113 p$ ( $4.2 \mathrm{mg}, 24.9 \mu \mathrm{mol}, 5 \%)$ in a distribution of $6.2: 1$ in favor of $\mathbf{1 1 3 0}$.

\subsubsection{Synthesis of Manganese Salen Azide Complexes Mn5(III)- $\mathrm{N}_{3}$ and

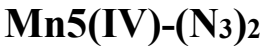

\section{Caution}

Special care should be taken for the preparation of the following metal-azide complexes (vide supra). The azide complexes are potentially toxic and explosive and should be handled only in small amounts. Due to high solubility of the complexes in chlorinated solvents, synthesis and purification was performed in $\mathrm{CH}_{2} \mathrm{Cl}_{2}$. To prevent unwanted generation of diazidomethane, ${ }^{[400]}$ solutions should always be kept at low temperatures and residues should be neutralized immediately after the procedure was completed.

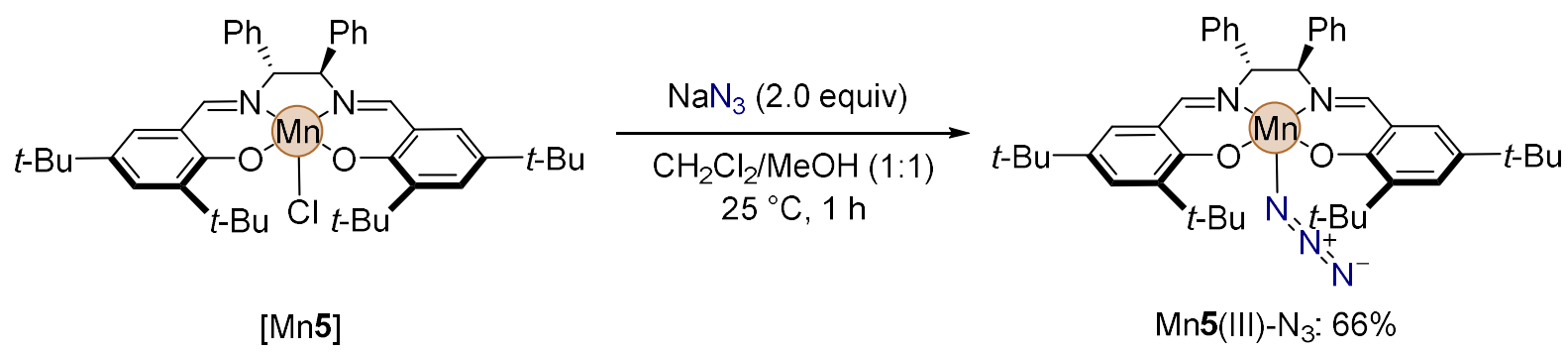

A Schlenk tube was charged with [Mn5] (366 mg, $0.50 \mathrm{mmol}, 1.00$ equiv), $\mathrm{CH}_{2} \mathrm{Cl}_{2}$ $(5.0 \mathrm{~mL})$ and $\mathrm{MeOH}(5.0 \mathrm{~mL}) . \mathrm{NaN}_{3}(65.0 \mathrm{mg}, 1.00 \mathrm{mmol}, 2.00$ equiv) was dissolved in 
distilled water $(1.0 \mathrm{~mL})$ and added to the mixture. The reaction mixture was stirred at $25^{\circ} \mathrm{C}$ for $1 \mathrm{~h}$. Subsequently, the solvent was removed under reduced pressure, at ambient temperature. To the crude solids, $\mathrm{CH}_{2} \mathrm{Cl}_{2}(5.0 \mathrm{~mL})$ was added and the dark brown solution was filtered over a pad of celite, followed by a second filtration through a membrane syringe filter. Then, the $\mathrm{CH}_{2} \mathrm{Cl}_{2}$ was removed in vacuo at ambient temperature and the remaining solid was recrystallized from hot $\mathrm{MeCN}$ to yield Mn5(III)- $\mathrm{N}_{3}$ (256 mg, $332 \mu \mathrm{mol}, 66 \%$ ) as dark brown crystalline needles. The azide complex was subsequently stored in a freezer at $-32{ }^{\circ} \mathrm{C}$.

IR (ATR): $\tilde{v}=2955,2905,2869,2044,2026,1608,1532,1429,1313,1251,699 \mathrm{~cm}^{-1}$.

MS (ESI) $m / z$ (relative intensity): 697 (100) $\left[\mathrm{M}-\mathrm{N}_{3}\right]^{+}$.

HR-MS (ESI) $m / z$ calcd. for $\mathrm{C}_{44} \mathrm{H}_{54} \mathrm{MnN}_{2} \mathrm{O}_{2}\left[\mathrm{M}-\mathrm{N}_{3}\right]^{+}: 697.3560$, found: 697.3560 .

Anal. calcd. for $\mathrm{C}_{44} \mathrm{H}_{54} \mathrm{MnN}_{5} \mathrm{O}_{2}$ : C, 71.43; H, 7.36; N, 9.47. Found: C, 69.19; H, 7.28; N, 9.11 .

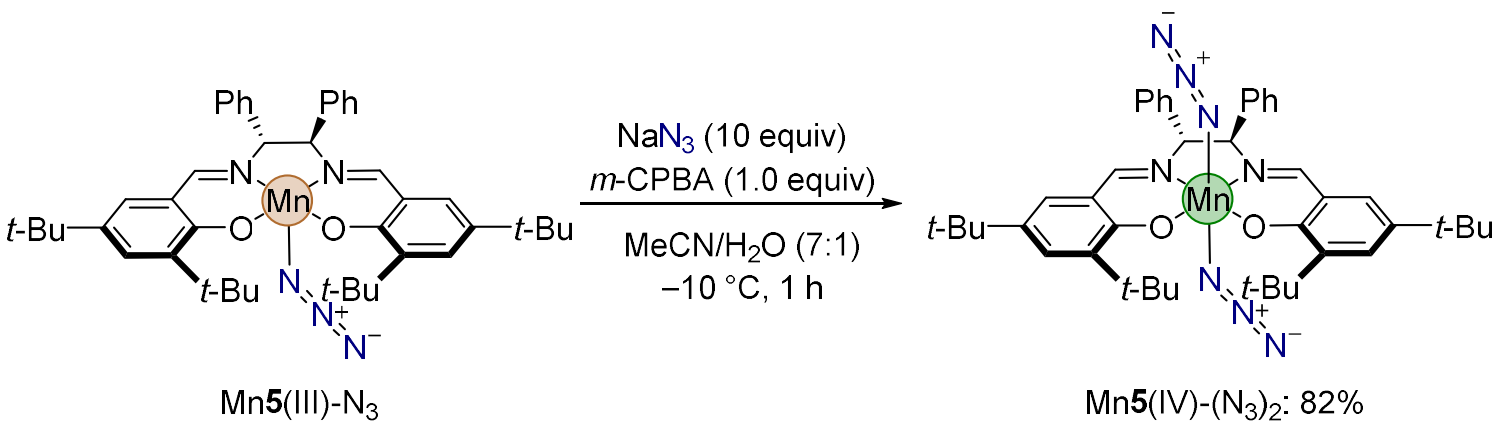

A Schlenk tube was charged with $\mathrm{Mn5}$ (III)-N $\mathrm{N}_{3}$ (74.0 mg, $0.10 \mathrm{mmol}, 1.00$ equiv) and $\mathrm{MeCN}(5.0 \mathrm{~mL}) . \mathrm{NaN}_{3}(65.0 \mathrm{mg}, 1.00 \mathrm{mmol}, 10.0$ equiv) and meta-chloroperoxybenzoic acid (m-CPBA) $(24.7 \mathrm{mg}, 0.1 \mathrm{mmol}, 1.00$ equiv) were dissolved in distilled water $(1.0 \mathrm{~mL})$ and slowly added to the mixture at $-10^{\circ} \mathrm{C}$. The reaction mixture was stirred at $-10^{\circ} \mathrm{C}$ for $1 \mathrm{~h}$. Subsequently, the solvent was removed under reduced pressure, at $-10{ }^{\circ} \mathrm{C}$. To the crude solids, $\mathrm{CH}_{2} \mathrm{Cl}_{2}(5.0 \mathrm{~mL})$ was added and the dark green solution was filtered over a pad of celite, followed by a second filtration through a membrane syringe filter. Recrystallisation from $\mathrm{CH}_{2} \mathrm{Cl}_{2}$ and pentane at $-32{ }^{\circ} \mathrm{C}$ afforded $\mathrm{Mn5}$ (IV)-( $\left.\mathrm{N}_{3}\right)_{2}(64.1 \mathrm{mg}, 82.0 \mu \mathrm{mol}, 82 \%)$ as dark green crystallin solid. The azide complex was subsequently stored in a freezer at $-32{ }^{\circ} \mathrm{C}$.

IR (ATR): $\tilde{v}=2956,2906,2869,2012,1608,1534,1430,1309,1249,699 \mathrm{~cm}^{-1}$.

MS (ESI) $m / z$ (relative intensity): 1438 (15) $\left[2 \mathrm{M}-3 \mathrm{~N}_{3}\right]^{+}, 781$ (5) $[\mathrm{M}]^{+}, 762$ (5) $[\mathrm{M}-$ $\left.\mathrm{N}_{3}+\mathrm{Na}\right]^{+}, 739(5)\left[\mathrm{M}-\mathrm{N}_{3}\right]^{+}, 697(100)\left[\mathrm{M}-2 \mathrm{~N}_{3}\right]^{+}$. 
HR-MS (ESI) $m / z$ calcd. for $\mathrm{C}_{44} \mathrm{H}_{54} \mathrm{MnN}_{5} \mathrm{O}_{2} \mathrm{Na}\left[\mathrm{M}-\mathrm{N}_{3}+\mathrm{Na}\right]^{+}:$762.3550, found: 762.3535 . Anal. calcd. for $\mathrm{C}_{44} \mathrm{H}_{54} \mathrm{MnN}_{8} \mathrm{O}_{2}$ : C, 67.59; H, 6.96; N, 14.33. Found: C, 67.01; H, 6.91; N, 13.94.

Crystals suitable for X-ray diffraction studies were obtained from a saturated solution of Mn5(IV)- $\left(\mathrm{N}_{3}\right)_{2}$ in toluene at $278 \mathrm{~K}$. Crystallization studies were performed together with Dr. A. Messinis.

\subsubsection{UV-Vis Spectroscopy}

The UV-vis absorption spectroscopy was carried out using a JASCO $V-770$ UV-vis/NIR spectrophotometer. The working temperature was $25^{\circ} \mathrm{C}$ and a quartz cell $(l=1 \mathrm{~cm})$ was used. The experiments were performed under a nitrogen atmosphere if not stated otherwise. The concentration of the analyte was $4 \cdot 10^{-5} \mathrm{~mol} \cdot \mathrm{L}^{-1}$ and $5 \cdot 10^{-5} \mathrm{~mol} \cdot \mathrm{L}^{-1}$ for the reaction mixtures, respectively. Acetonitrile (HPLC-grade) was used as the solvent for dilution.

\section{Preparation of Reaction mixtures A and B}

The constant current electrolysis was carried out using standard conditions (vide supra). The manganese catalyst [Mn5] (18.3 mg, $25.0 \mu \mathrm{mol}, 5.0 \mathrm{~mol} \%$ ), sodium azide (260 mg, $4.00 \mathrm{mmol}, 8.00$ equiv) and $\mathrm{LiClO}_{4}(53.2 \mathrm{mg}, 0.50 \mathrm{mmol}, 1.00$ equiv) were placed in a $10 \mathrm{~mL}$ cell under nitrogen atmosphere. The reaction components were dissolved in $\mathrm{MeCN}$ $(2.5 \mathrm{~mL})$, followed by $\mathrm{AcOH}(2.5 \mathrm{~mL})$ and the reaction mixture was stirred at $400 \mathrm{rpm}$. An aliquot was removed, diluted, and was transferred into the UV-vis cuvette (reaction mixture A). Now, the electrodes were connected under vigorous nitrogen-flow. Electrolysis was performed at $25{ }^{\circ} \mathrm{C}$ with a constant potential of $8 \mathrm{~mA}$ maintained for $30 \mathrm{~min}$. Within few seconds, the dark brown solution turned dark green/black ( $c f$. Scheme 85). At ambient temperature, $1.0 \mathrm{~mL}$ of the crude mixture was removed and diluted to $5 \cdot 10^{-5} \mathrm{~mol} \cdot \mathrm{L}^{-1}$. The diluted mixture $\mathbf{B}$ was transferred into the cuvette and a UV-vis spectrum was recorded immediately.

\section{Preparation of Reaction mixture C}

The constant current electrolysis was carried out using standard conditions (vide supra). The manganese catalyst [Mn5] (18.3 mg, $25.0 \mu \mathrm{mol}, 5.0 \mathrm{~mol} \%$ ), sodium azide (260 mg, $4.00 \mathrm{mmol}, 8.00$ equiv), $\mathrm{LiClO}_{4}(53.2 \mathrm{mg}, 0.50 \mathrm{mmol}, 1.00$ equiv) and cyclooctane (111b) (56.1 mg, $0.50 \mathrm{mmol}$ ) were placed in a $10 \mathrm{~mL}$ cell under nitrogen atmosphere. The reaction components were dissolved in $\mathrm{MeCN}(2.5 \mathrm{~mL})$, followed by $\mathrm{AcOH}(2.5 \mathrm{~mL})$ and the 
reaction mixture was stirred at $400 \mathrm{rpm}$. The electrodes were connected under vigorous nitrogen-flow. Electrolysis was performed at $25^{\circ} \mathrm{C}$ with a constant potential of $8 \mathrm{~mA}$ maintained for $120 \mathrm{~min}$. Similar to reaction mixture A, the dark brown solution turned dark green/black within few seconds ( $c f$. Scheme 85 ). At ambient temperature, $1.0 \mathrm{~mL}$ of the crude mixture was removed and diluted to $5 \cdot 10^{-5} \mathrm{~mol} \cdot \mathrm{L}^{-1}$. The diluted mixture was transferred into the cuvette and a UV-vis spectrum was recorded immediately.

\subsubsection{Cyclic Voltammetry}

Cyclic voltammetry was performed according to the general procedure (vide supra). Acetonitrile $(\mathrm{MeCN})$ with $0.1 \mathrm{~mol} \cdot \mathrm{L}^{-1} \mathrm{LiClO}_{4}$ as a conducting salt served as electrolyte for the measurements. The concentration of the analyte was $0.3 \mathrm{mmol} \cdot \mathrm{L}^{-1}$ if not stated otherwise.

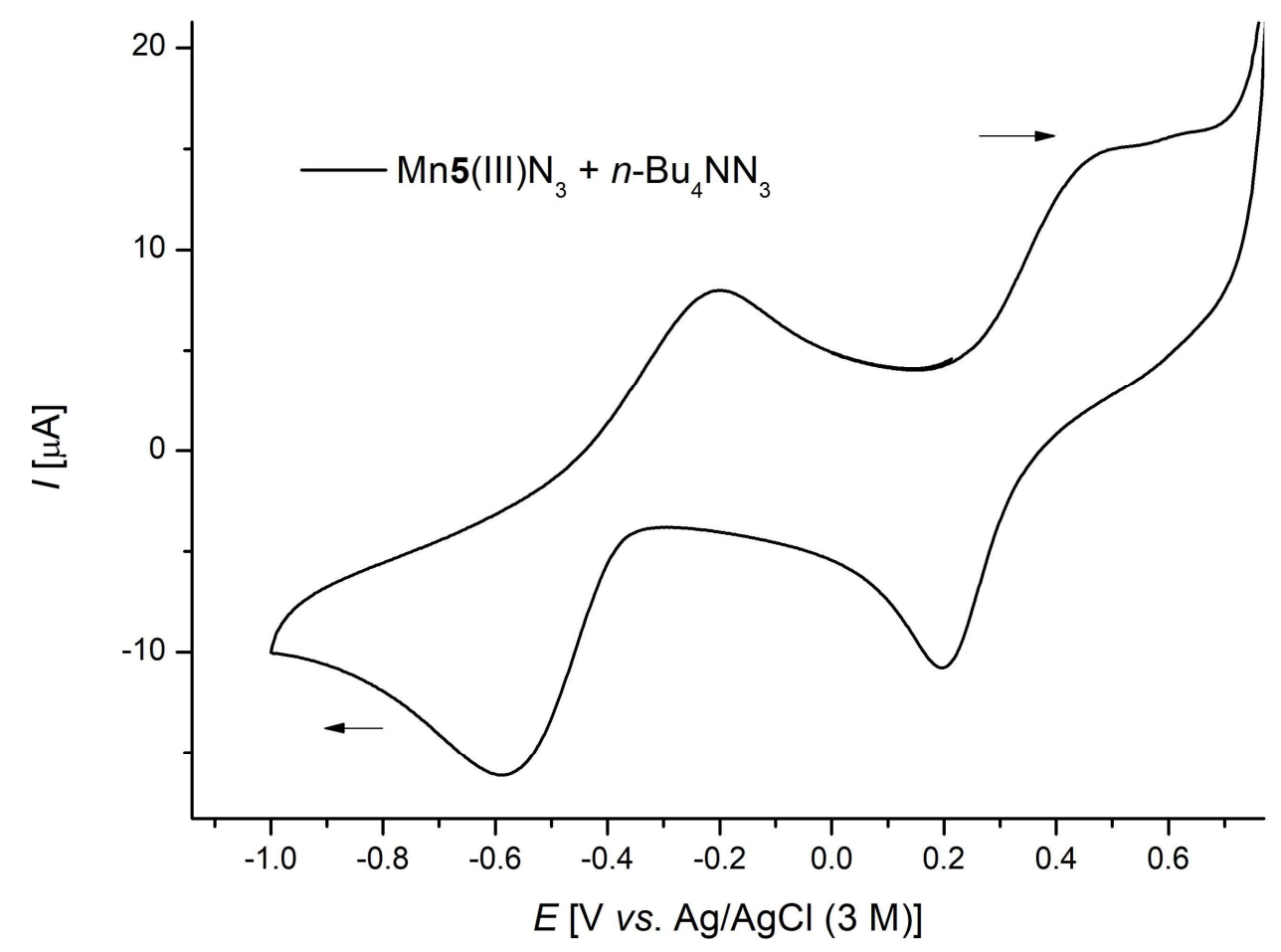

Figure 62. Cyclic voltammogram of $\mathrm{Mn5}(\mathrm{III})-\mathrm{N}_{3}(0.3 \mathrm{mM})$ in the presence of tetra- $n$ butylammonium azide. 


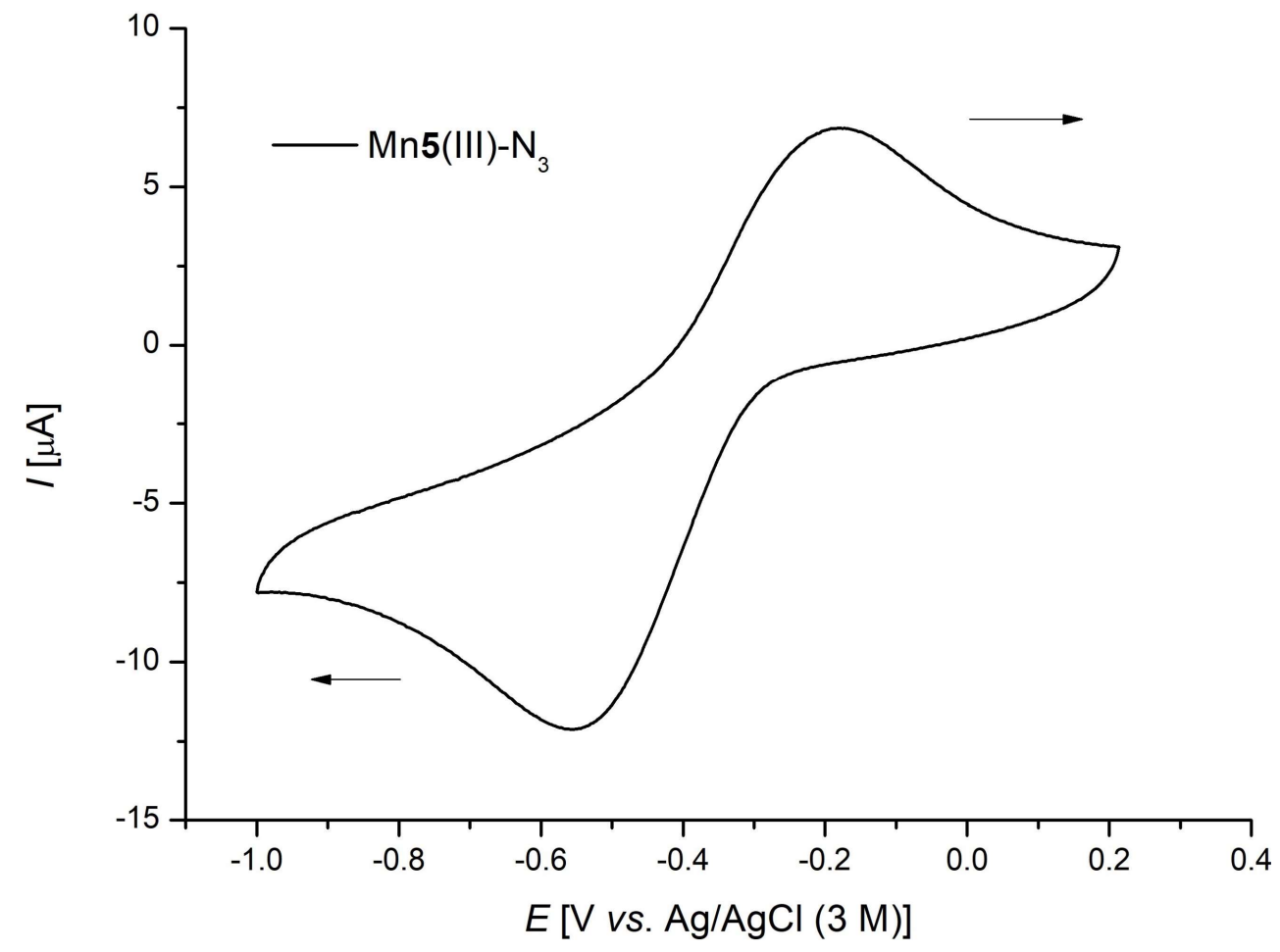

Figure 63. Cyclic voltammogram of $\mathrm{Mn5}(\mathrm{III})-\mathrm{N}_{3} \quad(0.3 \mathrm{mM})$. Two cyclic voltammograms were recorded, and the second voltammogram is presented.

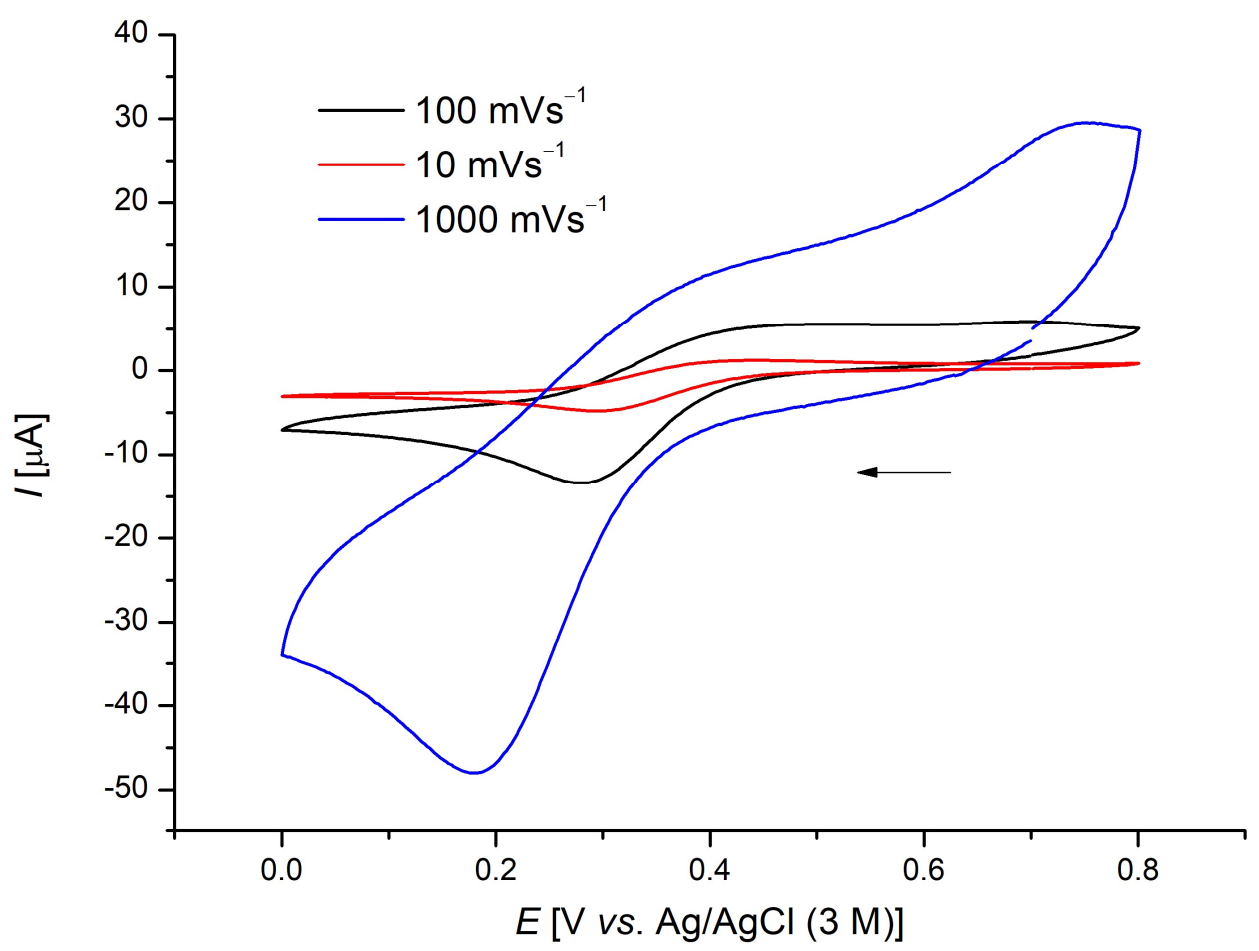

Figure 64. CVs of complex Mn5(IV)- $\left(\mathrm{N}_{3}\right)_{2}(0.3 \mathrm{mM})$ at different scan rates. Sweep direction was negative, with a starting potential of $0.8 \mathrm{~V}$ on the WE. 


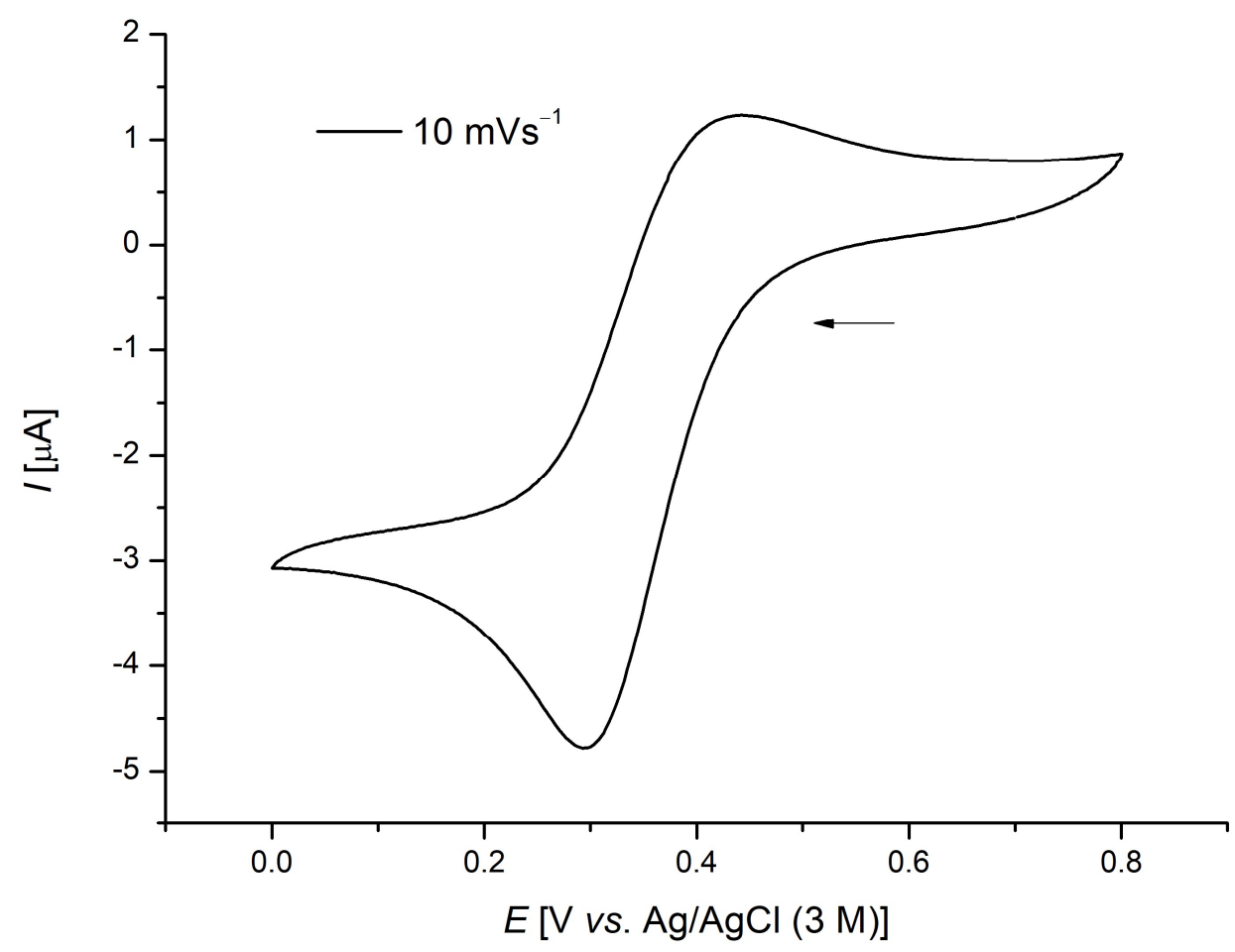

Figure 65. $\mathrm{CV}$ of complex Mn5(IV)-(N $)_{2}(0.3 \mathrm{mM})$ at $10 \mathrm{mV} \cdot \mathrm{s}^{-1}$. Sweep direction was negative, with a starting potential of $0.8 \mathrm{~V}$ on the WE.

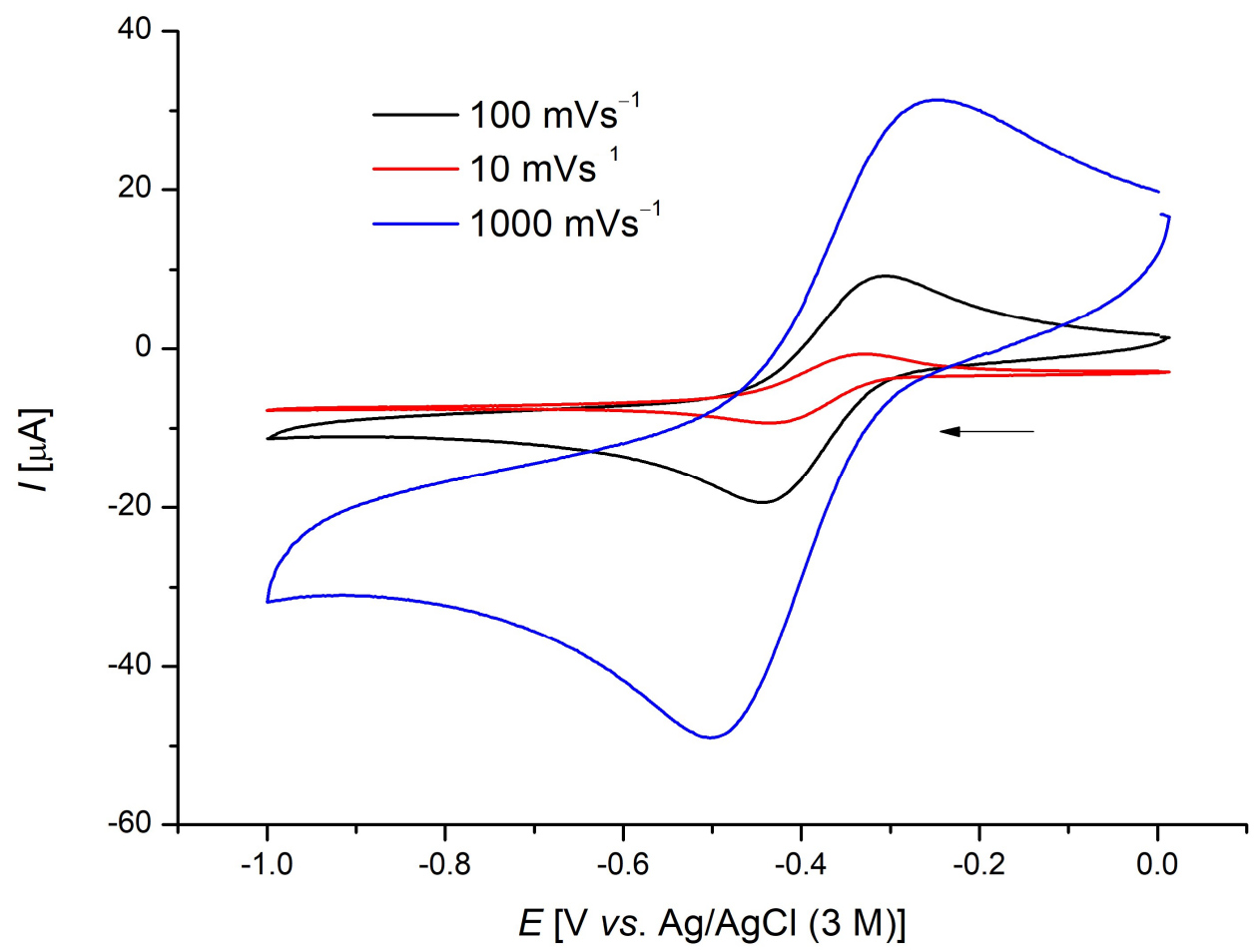

Figure 66. CVs of complex $\mathrm{Mn5}(\mathrm{IV})-\left(\mathrm{N}_{3}\right)_{2}(0.3 \mathrm{~mm})$ at different scan rates. Sweep direction was negative, with a starting potential of $0.0 \mathrm{~V}$ on the $\mathrm{WE}$. 


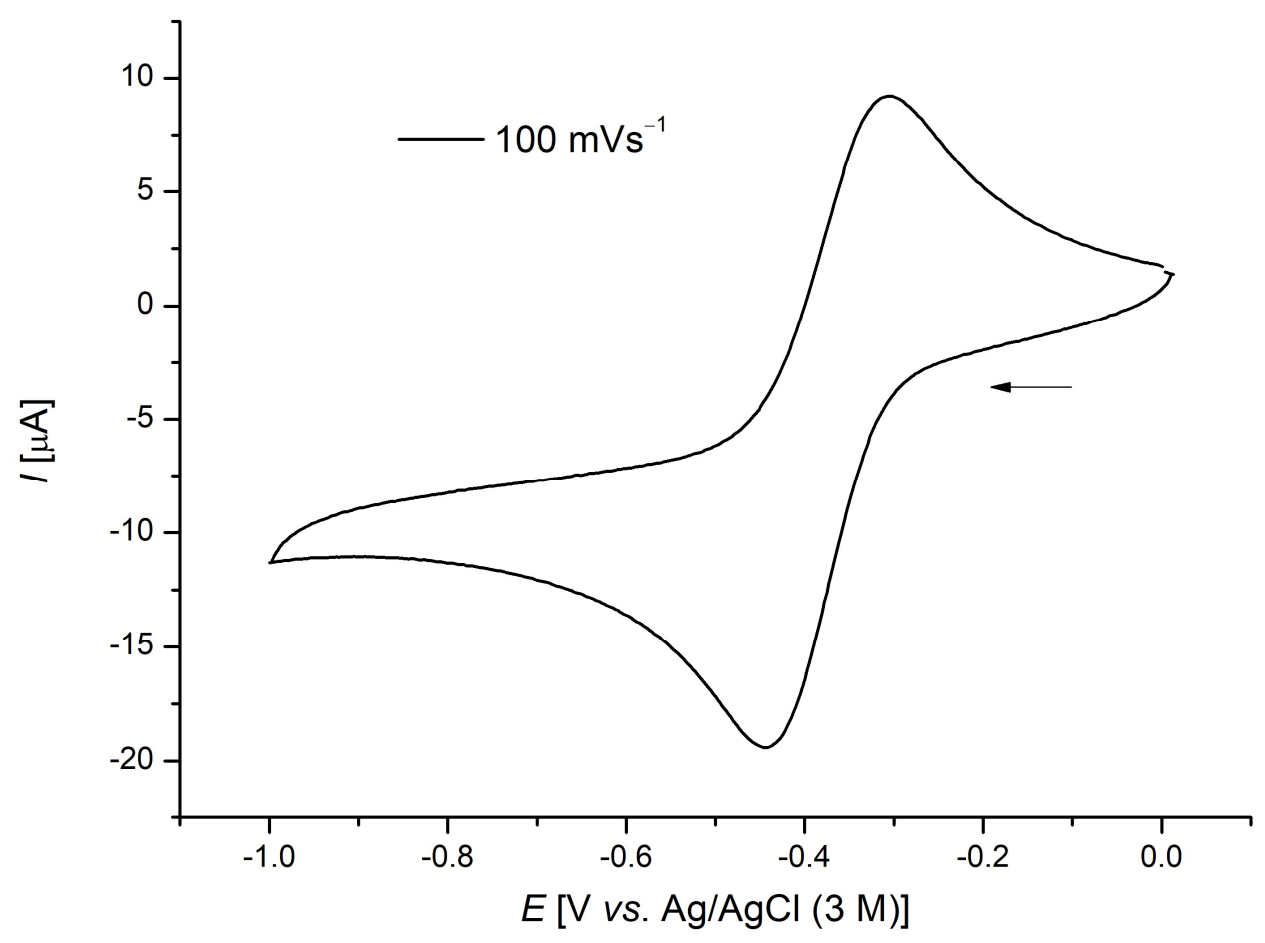

Figure 67. $\mathrm{CV}$ of complex $\mathrm{Mn5}(\mathrm{IV})-\left(\mathrm{N}_{3}\right)_{2}(0.3 \mathrm{mM})$ at $100 \mathrm{mV} \cdot \mathrm{s}^{-1}$. Sweep direction was negative, with a starting potential of $0.0 \mathrm{~V}$ on the WE.

\subsubsection{Constant Potential Electrolysis}

For chronoamperometric experiments, a silver-wire $(d=1 \mathrm{~mm})$ was used as the pseudoreference electrode in close proximity to the graphite felt working electrode. To ensure comparable constant potential conditions with the results gained by cyclic voltammetry, calibration of the silver-wire reference electrode versus ferrocene was performed.

$$
\begin{aligned}
& E_{\frac{1}{2}}\left(\mathrm{Fc} \mid \mathrm{Fc}^{+}\right)=0.36 \mathrm{~V}(\text { vs. } \mathrm{Ag} \mid \mathrm{AgCl}) \\
& E_{\frac{1}{2}}\left(\mathrm{Fc} \mid \mathrm{Fc}^{+}\right)=0.29 \mathrm{~V}\left(\text { vs. } \mathrm{Ag} \mid \mathrm{Ag}^{+}\right)
\end{aligned}
$$

Based on previous CV studies, no direct azide oxidation should occur in $\mathrm{MeCN} / \mathrm{AcOH}$ solvent mixtures at potentials below $1.0 \mathrm{~V} v s . \mathrm{Ag} / \mathrm{Ag}^{+}$.

The constant potential electrolysis was carried out in an undivided cell, with a graphite felt anode $(10 \mathrm{~mm} \times 15 \mathrm{~mm} \times 6 \mathrm{~mm})$, a platinum cathode $(10 \mathrm{~mm} \times 15 \mathrm{~mm} \times 0.125 \mathrm{~mm})$ and a silver-wire as the reference electrode. The manganese catalyst [Mn2] (12.7 mg, $25.0 \mu \mathrm{mol}, \quad 5.0 \mathrm{~mol} \%$ ), sodium azide (260 mg, $4.00 \mathrm{mmol}, 8.00$ equiv), 1,2,3,4tetrahydronaphthalene (111a) $\left(66.1 \mathrm{mg}, 0.50 \mathrm{mmol}, 1.00\right.$ equiv) and $\mathrm{LiClO}_{4}(53.2 \mathrm{mg}$, 
$0.50 \mathrm{mmol}, 1.00$ equiv) were placed in a $10 \mathrm{~mL}$ cell under nitrogen atmosphere. The reaction components were dissolved in $\mathrm{MeCN}(2.5 \mathrm{~mL})$, followed by $\mathrm{AcOH}(2.5 \mathrm{~mL})$ and the reaction mixture was stirred at $400 \mathrm{rpm}$. The electrodes were connected under vigorous nitrogen-flow. Electrolysis was performed at $25^{\circ} \mathrm{C}$ with a constant potential of $0.8 \mathrm{~V}$ ( $v s$. $\mathrm{Ag} / \mathrm{Ag}^{+}$) maintained for $20 \mathrm{~h}$. At ambient temperature, the reaction mixture was quenched with sat. aq. $\mathrm{NaHCO}_{3}(20 \mathrm{~mL})$ and EtOAc $(10 \mathrm{~mL})$ was added, and the graphite felt anode was washed with EtOAc $(3 \times 5 \mathrm{~mL})$ in an ultrasonic bath. The combined mixture was washed with $\mathrm{H}_{2} \mathrm{O}(3 \times 10 \mathrm{~mL})$, and then dried over $\mathrm{Na}_{2} \mathrm{SO}_{4}$, filtered and the solvents were removed in vacuo. Purification by column chromatography on silica gel ( $n$-pentane/EtOAc 99:1) yielded 113a (48.8 mg, $282 \mu \mathrm{mol}, 56 \%)$ as a colorless liquid.

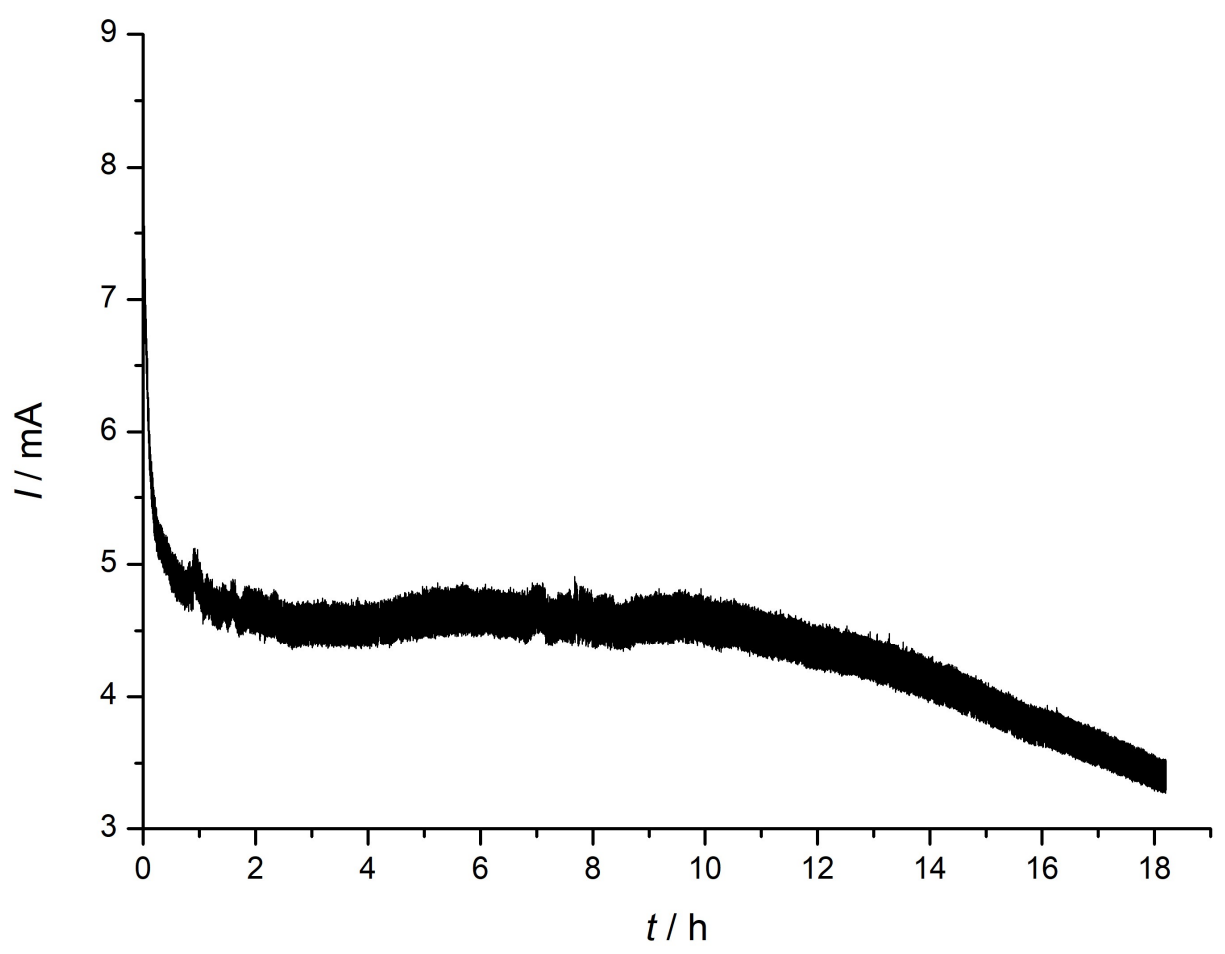

Figure 68. Chronoamperometry at $0.8 \mathrm{~V} v s . \mathrm{Ag} / \mathrm{Ag}^{+}$; resulting current-time response.

The strong interferences in current response during the recoding are most likely caused by the stirring plate below the electrolysis cell. 


\section{Crystallographic Data}

\section{X-Ray Crystallography}

The following crystal structures were measured and solved by $\mathrm{Dr}$. C. Golz. A suitable single crystal was selected via a SMZ1270 stereomicroscope from NiKON METROLOGY. The crystal was mounted on a MicroMounts or MicroLoops from MITEGEN holder, in NVH oil, on a BRUKER D 8 Venture four-circle-diffractometer with a photon II detector. A microfocus I $\mu S \mathrm{Cu} / \mathrm{Mo}$ from INCOATEC with mirror optics HELIOS and single-hole collimator from BRUKER were used as the X-ray sources. The crystal was cooled to given temperature with Cryostream 800 from OXFORD CRYOSYSTEMS. The data was collected with APEX3 Suite from BRUKER and were integrated with SAINTV8.38A and SADABS 2016/2 was used for the absorption correction. The structure was solved with the $S H E L X T^{[410]}$ structure solution program using Intrinsic Phasing and refined with the SHELXL ${ }^{[411]}$ refinement package using Least Squares minimization. Final data was processed and visualized with Olex2 (v1.3.0) from OLEXSYS. ${ }^{[412]}$

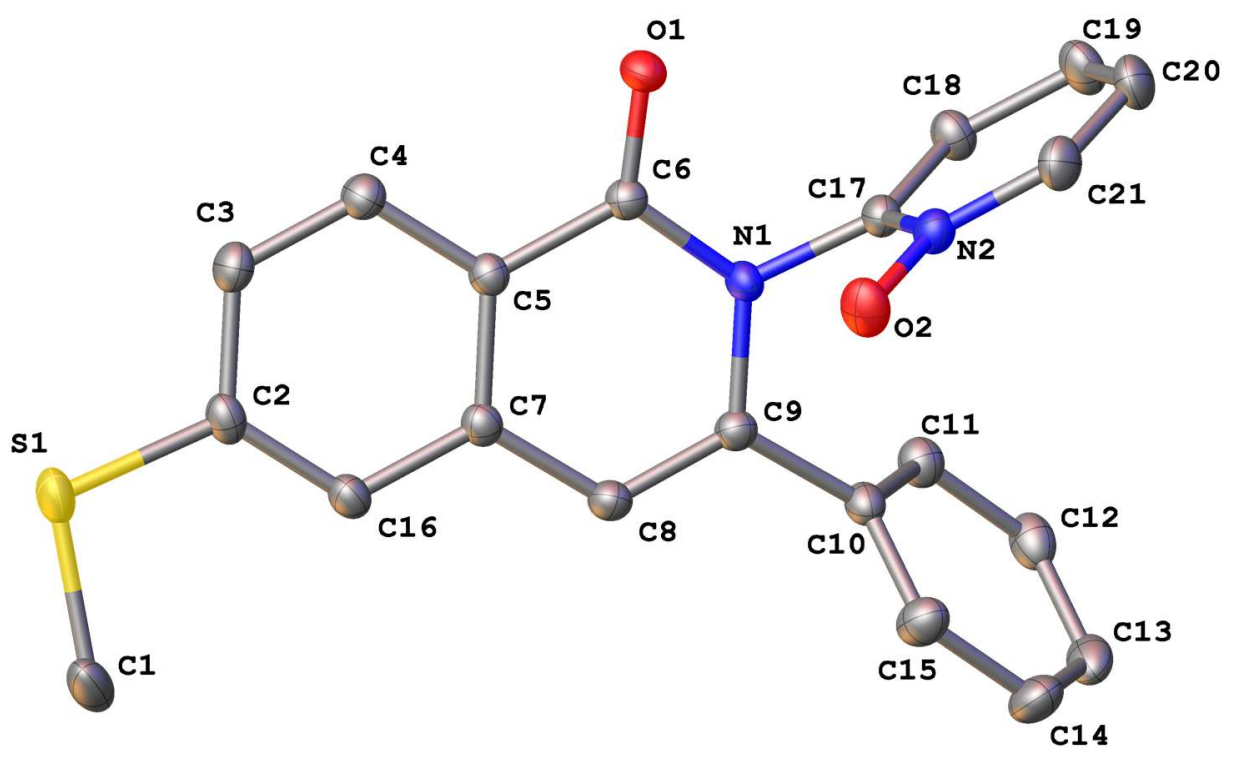

Figure 69. Molecular structure of 51 ea with thermal ellipsoids at 50\% probability level. The hydrogen atoms are omitted for clarity.

Table 36. Crystal data and structure refinement for 51ea.

Compound

CCDC number

Empirical formula

\section{1ea}

2044869

$\mathrm{C}_{21} \mathrm{H}_{16} \mathrm{~N}_{2} \mathrm{O}_{2} \mathrm{~S}$ 
Formula weight

Temperature

Wavelength

Crystal system

Space group

Unit cell dimensions

Volume

Z

Density (calculated)

Absorption coefficient

$\mathrm{F}(000)$

Crystal size

$2 \Theta$ range for data collection

Index ranges

Reflections collected

Independent reflections

Data / restraints / parameters

Goodness-of-fit on $\mathrm{F}^{2}$

Final $R$ indices [I $>2 \sigma(\mathrm{I})]$

$R$ indices (all data)

Largest diff. peak and hole
360.42

$100.0 \mathrm{~K}$

$0.71073 \AA$

monoclinic

$P 21 / \mathrm{c}$

$$
\begin{array}{ll}
a=20.2946(17) \AA & \alpha=90^{\circ} \\
b=8.5484(8) \AA & \beta=115.149(2)^{\circ} \\
c=22.0044(15) \AA & \gamma=90^{\circ}
\end{array}
$$

3455.6(5) $\AA^{3}$

8

$1.386 \mathrm{~g} / \mathrm{m}^{3}$

$0.206 \mathrm{~mm}^{-1}$

1504.0

$(0.26 \times 0.25 \times 0.12) \mathrm{mm}^{3}$

$4.434^{\circ}$ to $57.51^{\circ}$

$-27 \leq \mathrm{h} \leq 27,-11 \leq \mathrm{k} \leq 11,-29 \leq 1 \leq 29$

86523

$8954\left[R_{\text {int }}=0.0456, R_{\text {sigma }}=0.0245\right]$

$8954 / 0 / 471$

1.036

$R_{1}=0.0406, \mathrm{w} R_{2}=0.1044$

$R_{1}=0.0460, \mathrm{w} R_{2}=0.1082$

$0.65 \mathrm{e} \cdot \AA^{-3}$ and $-0.40 \mathrm{e} \cdot \AA^{-3}$

Table 37. Bond lengths in $[\AA]$.

\begin{tabular}{cccc}
\hline Atoms & Bond Length $[\AA]$ & Atoms & Bond Length $[\AA]$ \\
\hline $\mathrm{S}(1)-\mathrm{C}(1)$ & $1.7963(18)$ & $\mathrm{C}(7)-\mathrm{C}(8)$ & $1.4357(18)$ \\
$\mathrm{S}(1)-\mathrm{C}(2)$ & $1.7548(14)$ & $\mathrm{C}(7)-\mathrm{C}(16)$ & $1.4072(17)$ \\
$\mathrm{O}(1)-\mathrm{C}(6)$ & $1.2343(16)$ & $\mathrm{C}(8)-\mathrm{C}(9)$ & $1.3482(18)$ \\
$\mathrm{O}(2)-\mathrm{N}(2)$ & $1.2970(15)$ & $\mathrm{C}(9)-\mathrm{C}(10)$ & $1.4834(18)$ \\
$\mathrm{N}(1)-\mathrm{C}(6)$ & $1.3994(16)$ & $\mathrm{C}(10)-\mathrm{C}(11)$ & $1.4019(19)$ \\
$\mathrm{N}(1)-\mathrm{C}(9)$ & $1.4077(16)$ & $\mathrm{C}(10)-\mathrm{C}(15)$ & $1.3887(19)$ \\
$\mathrm{N}(1)-\mathrm{C}(17)$ & $1.4256(16)$ & $\mathrm{C}(11)-\mathrm{C}(12)$ & $1.390(2)$
\end{tabular}




$\begin{array}{cccc}\mathrm{N}(2)-\mathrm{C}(17) & 1.3722(17) & \mathrm{C}(12)-\mathrm{C}(13) & 1.390(2) \\ \mathrm{N}(2)-\mathrm{C}(21) & 1.3679(18) & \mathrm{C}(13)-\mathrm{C}(14) & 1.383(2) \\ \mathrm{C}(2)-\mathrm{C}(3) & 1.414(2) & \mathrm{C}(14)-\mathrm{C}(15) & 1.394(2) \\ \mathrm{C}(2)-\mathrm{C}(16) & 1.3866(19) & \mathrm{C}(17)-\mathrm{C}(18) & 1.3774(18) \\ \mathrm{C}(3)-\mathrm{C}(4) & 1.3754(19) & \mathrm{C}(18)-\mathrm{C}(19) & 1.3925(19) \\ \mathrm{C}(4)-\mathrm{C}(5) & 1.4050(18) & \mathrm{C}(19)-\mathrm{C}(20) & 1.389(2) \\ \mathrm{C}(5)-\mathrm{C}(6) & 1.4581(17) & \mathrm{C}(20)-\mathrm{C}(21) & 1.372(2) \\ \mathrm{C}(5)-\mathrm{C}(7) & 1.4061(18) & & \end{array}$

Table 38. Bond angles in $\left[{ }^{\circ}\right]$.

\begin{tabular}{cccc}
\hline Atoms & Angle [ $\left.{ }^{\circ}\right]$ & Atoms & Angle [ $\left.{ }^{\circ}\right]$ \\
\hline $\mathrm{C}(2)-\mathrm{S}(1)-\mathrm{C}(1)$ & $102.79(7)$ & $\mathrm{C}(9)-\mathrm{C}(8)-\mathrm{C}(7)$ & $120.95(12)$ \\
$\mathrm{C}(6)-\mathrm{N}(1)-\mathrm{C}(9)$ & $123.07(11)$ & $\mathrm{N}(1)-\mathrm{C}(9)-\mathrm{C}(10)$ & $118.18(11)$ \\
$\mathrm{C}(6)-\mathrm{N}(1)-\mathrm{C}(17)$ & $114.84(11)$ & $\mathrm{C}(8)-\mathrm{C}(9)-\mathrm{N}(1)$ & $120.03(12)$ \\
$\mathrm{C}(9)-\mathrm{N}(1)-\mathrm{C}(17)$ & $119.70(11)$ & $\mathrm{C}(8)-\mathrm{C}(9)-\mathrm{C}(10)$ & $121.60(12)$ \\
$\mathrm{O}(2)-\mathrm{N}(2)-\mathrm{C}(17)$ & $120.70(11)$ & $\mathrm{C}(11)-\mathrm{C}(10)-\mathrm{C}(9)$ & $120.24(12)$ \\
$\mathrm{O}(2)-\mathrm{N}(2)-\mathrm{C}(21)$ & $120.38(12)$ & $\mathrm{C}(15)-\mathrm{C}(10)-\mathrm{C}(9)$ & $119.82(12)$ \\
$\mathrm{C}(22)-\mathrm{N}(2)-\mathrm{C}(17)$ & $118.91(12)$ & $\mathrm{C}(15)-\mathrm{C}(10)-\mathrm{C}(12)$ & $119.69(13)$ \\
$\mathrm{C}(3)-\mathrm{C}(2)-\mathrm{S}(1)$ & $115.94(10)$ & $\mathrm{C}(12)-\mathrm{C}(11)-\mathrm{C}(10)$ & $119.87(13)$ \\
$\mathrm{C}(16)-\mathrm{C}(2)-\mathrm{S}(1)$ & $124.23(11)$ & $\mathrm{C}(11)-\mathrm{C}(12)-\mathrm{C}(13)$ & $120.12(14)$ \\
$\mathrm{C}(16)-\mathrm{C}(2)-\mathrm{C}(3)$ & $119.82(12)$ & $\mathrm{C}(14)-\mathrm{C}(13)-\mathrm{C}(12)$ & $120.06(14)$ \\
$\mathrm{C}(4)-\mathrm{C}(3)-\mathrm{C}(2)$ & $120.59(13)$ & $\mathrm{C}(13)-\mathrm{C}(14)-\mathrm{C}(15)$ & $120.23(14)$ \\
$\mathrm{C}(3)-\mathrm{C}(4)-\mathrm{C}(5)$ & $119.84(13)$ & $\mathrm{C}(10)-\mathrm{C}(15)-\mathrm{C}(14)$ & $120.02(14)$ \\
$\mathrm{C}(4)-\mathrm{C}(5)-\mathrm{C}(6)$ & $119.35(12)$ & $\mathrm{C}(2)-\mathrm{C}(16)-\mathrm{C}(7)$ & $120.03(12)$ \\
$\mathrm{C}(4)-\mathrm{C}(5)-\mathrm{C}(7)$ & $120.07(12)$ & $\mathrm{N}(2)-\mathrm{C}(17)-\mathrm{N}(1)$ & $114.50(11)$ \\
$\mathrm{C}(7)-\mathrm{C}(5)-\mathrm{C}(6)$ & $120.56(12)$ & $\mathrm{N}(2)-\mathrm{C}(17)-\mathrm{C}(18)$ & $121.15(12)$ \\
$\mathrm{O}(1)-\mathrm{C}(6)-\mathrm{N}(1)$ & $119.85(12)$ & $\mathrm{C}(18)-\mathrm{C}(17)-\mathrm{N}(1)$ & $124.22(12)$ \\
$\mathrm{O}(1)-\mathrm{C}(6)-\mathrm{C}(5)$ & $124.11(12)$ & $\mathrm{C}(17)-\mathrm{C}(18)-\mathrm{C}(19)$ & $119.94(13)$ \\
$\mathrm{N}(1)-\mathrm{C}(6)-\mathrm{C}(5)$ & $116.03(11)$ & $\mathrm{C}(20)-\mathrm{C}(19)-\mathrm{C}(18)$ & $118.48(13)$ \\
$\mathrm{C}(5)-\mathrm{C}(7)-\mathrm{C}(8)$ & $119.01(12)$ & $\mathrm{C}(21)-\mathrm{C}(20)-\mathrm{C}(19)$ & $120.30(13)$ \\
$\mathrm{C}(5)-\mathrm{C}(7)-\mathrm{C}(16)$ & $119.54(12)$ & $\mathrm{N}(2)-\mathrm{C}(21)-\mathrm{C}(20)$ & $121.22(13)$ \\
$\mathrm{C}(16)-\mathrm{C}(7)-\mathrm{C}(8)$ & $121.44(12)$ & & \\
\hline
\end{tabular}




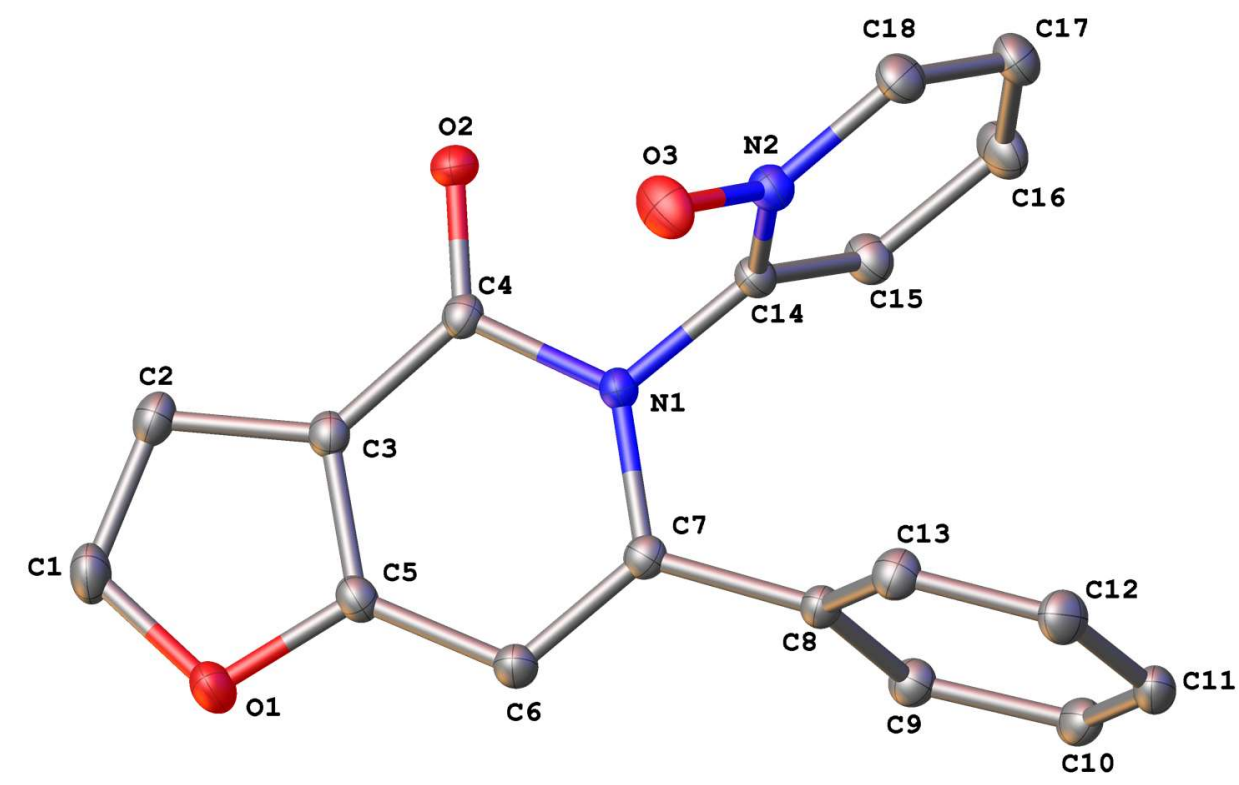

Figure 70. Molecular structure of 189ea with thermal ellipsoids at 50\% probability level. The hydrogen atoms are omitted for clarity.

Table 39. Crystal data and structure refinement for 189ea.

Compound

CCDC number

Empirical formula

Formula weight

Temperature

Crystal system

Space group

Unit cell dimensions

Volume

Z

Density (calculated)

Absorption coefficient

$\mathrm{F}(000)$

Crystal size

Radiation

$2 \Theta$ range for data collection

\section{9ea}

2044870

$\mathrm{C}_{18} \mathrm{H}_{12} \mathrm{~N}_{2} \mathrm{O}_{3}$

304.30

$100.0 \mathrm{~K}$

triclinic

$P-1$

$a=8.8017(9) \AA$

$\alpha=62.870(4)^{\circ}$

$b=9.5871(13) \AA$

$\beta=75.576(5)^{\circ}$

$c=10.2305(14$

(14) $\AA$

$\gamma=68.099(4)^{\circ}$

709.58(16) $\AA^{3}$

2

$1.424 \mathrm{~g} / \mathrm{m}^{3}$

$0.099 \mathrm{~mm}^{-1}$

316.0

$(0.245 \times 0.229 \times 0.227) \mathrm{mm}^{3}$

$\operatorname{MoK} \alpha(\lambda=0.71073)$

$5.006^{\circ}$ to $59.176^{\circ}$ 
Index ranges

Reflections collected

Independent reflections

Data / restraints / parameters

Goodness-of-fit on $\mathrm{F}^{2}$

Final $R$ indices $[\mathrm{I}>2 \sigma(\mathrm{I})]$

$R$ indices (all data)

Largest diff. peak and hole
$-11 \leq \mathrm{h} \leq 12,-13 \leq \mathrm{k} \leq 13,-14 \leq 1 \leq 14$

35405

$3953\left[R_{\text {int }}=0.0249, R_{\text {sigma }}=0.0132\right]$

$3953 / 0 / 208$

1.056

$R_{1}=0.0395, \mathrm{w} R_{2}=0.1040$

$R_{1}=0.0430, \mathrm{w} R_{2}=0.1072$

$0.41 \mathrm{e} \cdot \AA^{-3}$ and $-0.32 \mathrm{e} \cdot \AA^{-3}$

Table 40. Bond lengths in $[\AA]$.

\begin{tabular}{cccc}
\hline Atoms & Bond Length $[\AA]$ & Atoms & Bond Length $[\AA]$ \\
\hline $\mathrm{O}(1)-\mathrm{C}(1)$ & $1.3876(13)$ & $\mathrm{C}(5)-\mathrm{C}(6)$ & $1.4120(13)$ \\
$\mathrm{O}(1)-\mathrm{C}(5)$ & $1.3588(11)$ & $\mathrm{C}(6)-\mathrm{C}(7)$ & $1.3577(13)$ \\
$\mathrm{O}(2)-\mathrm{C}(4)$ & $1.2302(12)$ & $\mathrm{C}(7)-\mathrm{C}(8)$ & $1.4864(13)$ \\
$\mathrm{O}(3)-\mathrm{N}(2)$ & $1.2924(11)$ & $\mathrm{C}(8)-\mathrm{C}(9)$ & $1.3943(13)$ \\
$\mathrm{N}(1)-\mathrm{C}(4)$ & $1.4143(12)$ & $\mathrm{C}(8)-\mathrm{C}(13)$ & $1.3950(13)$ \\
$\mathrm{N}(1)-\mathrm{C}(7)$ & $1.4005(12)$ & $\mathrm{C}(9)-\mathrm{C}(10)$ & $1.3916(14)$ \\
$\mathrm{N}(1)-\mathrm{C}(14)$ & $1.4200(12)$ & $\mathrm{C}(10)-\mathrm{C}(11)$ & $1.3910(15)$ \\
$\mathrm{N}(2)-\mathrm{C}(14)$ & $1.3681(12)$ & $\mathrm{C}(11)-\mathrm{C}(12)$ & $1.3895(15)$ \\
$\mathrm{N}(2)-\mathrm{C}(18)$ & $1.3647(13)$ & $\mathrm{C}(12)-\mathrm{C}(13)$ & $1.3919(14)$ \\
$\mathrm{C}(1)-\mathrm{C}(2)$ & $1.3529(15)$ & $\mathrm{C}(14)-\mathrm{C}(15)$ & $1.3774(14)$ \\
$\mathrm{C}(2)-\mathrm{C}(3)$ & $1.4314(13)$ & $\mathrm{C}(15)-\mathrm{C}(16)$ & $1.3866(14)$ \\
$\mathrm{C}(3)-\mathrm{C}(4)$ & $1.4324(13)$ & $\mathrm{C}(16)-\mathrm{C}(17)$ & $1.3896(15)$ \\
$\mathrm{C}(3)-\mathrm{C}(5)$ & $1.3752(13)$ & $\mathrm{C}(17)-\mathrm{C}(18)$ & $1.3746(15)$ \\
\hline
\end{tabular}

Table 41. Bond angles in $\left[^{\circ}\right]$.

\begin{tabular}{cccc}
\hline Atoms & ${\text { Angle }\left[{ }^{\circ}\right]}^{\circ}$ & Atoms & Angle [ $\left.{ }^{\circ}\right]$ \\
\hline $\mathrm{C}(5)-\mathrm{O}(1)-\mathrm{C}(1)$ & $105.65(8)$ & $\mathrm{N}(1)-\mathrm{C}(7)-\mathrm{C}(8)$ & $116.23(8)$ \\
$\mathrm{C}(4)-\mathrm{N}(1)-\mathrm{C}(14)$ & $115.42(8)$ & $\mathrm{C}(6)-\mathrm{C}(7)-\mathrm{N}(1)$ & $120.35(8)$ \\
$\mathrm{C}(7)-\mathrm{N}(1)-\mathrm{C}(4)$ & $125.54(8)$ & $\mathrm{C}(6)-\mathrm{C}(7)-\mathrm{C}(8)$ & $123.37(8)$ \\
$\mathrm{C}(7)-\mathrm{N}(1)-\mathrm{C}(14)$ & $118.96(8)$ & $\mathrm{C}(9)-\mathrm{C}(8)-\mathrm{C}(7)$ & $118.99(8)$ \\
$\mathrm{O}(3)-\mathrm{N}(2)-\mathrm{C}(14)$ & $120.69(8)$ & $\mathrm{C}(9)-\mathrm{C}(8)-\mathrm{C}(13)$ & $119.85(9)$
\end{tabular}




$\begin{array}{cccc}\mathrm{O}(3)-\mathrm{N}(2)-\mathrm{C}(18) & 120.68(8) & \mathrm{C}(13)-\mathrm{C}(8)-\mathrm{C}(7) & 121.13(8) \\ \mathrm{C}(18)-\mathrm{N}(2)-\mathrm{C}(14) & 118.63(8) & \mathrm{C}(10)-\mathrm{C}(9)-\mathrm{C}(8) & 120.18(9) \\ \mathrm{C}(2)-\mathrm{C}(1)-\mathrm{O}(1) & 111.66(9) & \mathrm{C}(11)-\mathrm{C}(20)-\mathrm{C}(9) & 119.78(9) \\ \mathrm{C}(1)-\mathrm{C}(2)-\mathrm{C}(3) & 105.36(9) & \mathrm{C}(12)-\mathrm{C}(11)-\mathrm{C}(10) & 120.17(9) \\ \mathrm{C}(2)-\mathrm{C}(3)-\mathrm{C}(4) & 132.32(9) & \mathrm{C}(11)-\mathrm{C}(12)-\mathrm{C}(13) & 120.20(9) \\ \mathrm{C}(5)-\mathrm{C}(3)-\mathrm{C}(2) & 106.86(8) & \mathrm{C}(12)-\mathrm{C}(13)-\mathrm{C}(8) & 119.79(9) \\ \mathrm{C}(5)-\mathrm{C}(3)-\mathrm{C}(4) & 120.83(8) & \mathrm{N}(2)-\mathrm{C}(14)-\mathrm{N}(1) & 115.80(8) \\ \mathrm{O}(2)-\mathrm{C}(4)-\mathrm{N}(1) & 119.91(9) & \mathrm{N}(2)-\mathrm{C}(14)-\mathrm{C}(15) & 121.29(9) \\ \mathrm{O}(2)-\mathrm{C}(4)-\mathrm{C}(3) & 127.33(9) & \mathrm{C}(15)-\mathrm{C}(14)-\mathrm{N}(1) & 122.90(9) \\ \mathrm{N}(1)-\mathrm{C}(4)-\mathrm{C}(3) & 112.73(8) & \mathrm{C}(14)-\mathrm{C}(15)-\mathrm{C}(16) & 120.23(9) \\ \mathrm{O}(1)-\mathrm{C}(5)-\mathrm{C}(3) & 110.47(8) & \mathrm{C}(15)-\mathrm{C}(16)-\mathrm{C}(17) & 118.18(10) \\ \mathrm{O}(1)-\mathrm{C}(5)-\mathrm{C}(6) & 125.07(9) & \mathrm{C}(18)-\mathrm{C}(17)-\mathrm{C}(16) & 120.27(9) \\ \mathrm{C}(3)-\mathrm{C}(5)-\mathrm{C}(6) & 124.45(9) & \mathrm{N}(2)-\mathrm{C}(18)-\mathrm{C}(17) & 121.39(9) \\ \mathrm{C}(7)-\mathrm{C}(6)-\mathrm{C}(5) & 116.07(9) & & \end{array}$

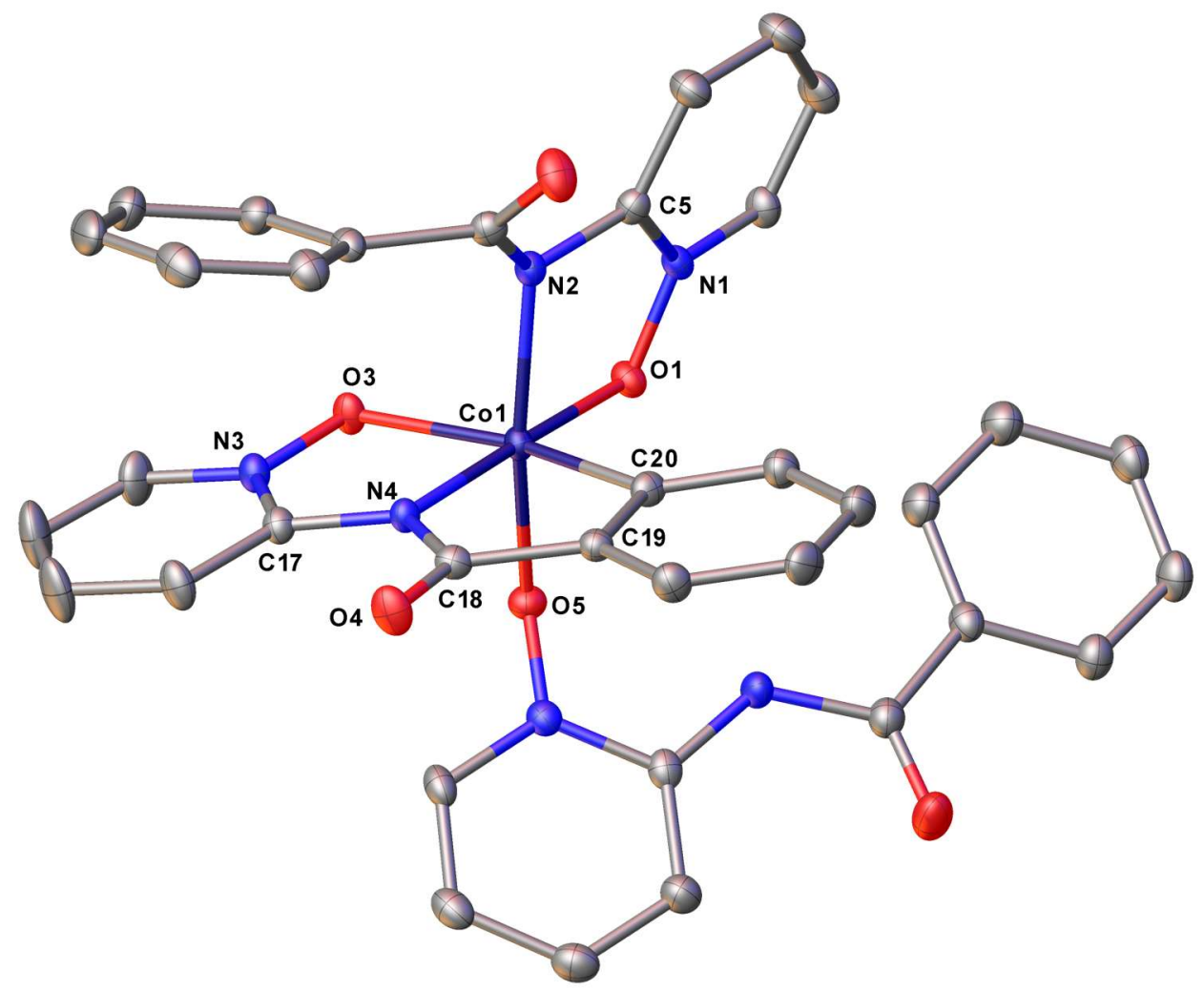

Figure 71. Molecular structure of $\mathbf{1 9 0}$ with thermal ellipsoids at 50\% probability level. The hydrogen atoms are omitted for clarity. 
Table 42. Crystal data and structure refinement for $\mathbf{1 9 0}$.

Compound

CCDC number

Empirical formula

Formula weight

Temperature

Crystal system

Space group

Unit cell dimensions

Volume

Z

Density (calculated)

Absorption coefficient

$\mathrm{F}(000)$

Crystal size

Radiation

$2 \Theta$ range for data collection

Index ranges

Reflections collected

Independent reflections

Data / restraints / parameters

Goodness-of-fit on $\mathrm{F}^{2}$

Final $R$ indices $[\mathrm{I}>2 \sigma(\mathrm{I})]$

$R$ indices (all data)

Largest diff. peak and hole

Flack parameter
190

1983755

$\mathrm{C}_{36} \mathrm{H}_{27} \mathrm{CoN}_{6} \mathrm{O}_{6}$

698.56

$99.98 \mathrm{~K}$

orthorhombic

$P 2{ }_{1}{ }_{1} 2_{1}$

$a=9.2770(12) \AA$

$\alpha=90^{\circ}$

$b=20.177(3) \AA$

$\beta=90^{\circ}$

$c=32.452(7) \AA$

$\gamma=90^{\circ}$

6074.6(17) $\AA^{3}$

8

$1.528 \mathrm{~g} / \mathrm{m}^{3}$

$0.626 \mathrm{~mm}^{-1}$

0.626

$(0.288 \times 0.241 \times 0.062) \mathrm{mm}^{3}$

$\operatorname{MoK} \alpha(\lambda=0.71073)$

$4.272^{\circ}$ to $59.138^{\circ}$

$-12 \leq \mathrm{h} \leq 10,-28 \leq \mathrm{k} \leq 20,-45 \leq 1 \leq 44$

45233

$16976\left[R_{\text {int }}=0.0422, R_{\text {sigma }}=0.0552\right]$

$16976 / 0 / 891$

1.014

$R_{1}=0.0382, \mathrm{w} R_{2}=0.0801$

$R_{1}=0.0454, \mathrm{w} R_{2}=0.0834$

$0.67 \mathrm{e} \cdot \AA^{-3}$ and $-0.58 \mathrm{e} \cdot \AA^{-3}$

$0.019(6)$

Table 43. Bond lengths in $[\AA]$.

\begin{tabular}{cccc}
\hline Atoms & Bond Length $[\AA]$ & Atoms & Bond Length $[\AA]$ \\
\hline $\mathrm{Co}-\mathrm{O}(1)$ & $1.896(2)$ & $\mathrm{C}(7)-\mathrm{C}(8)$ & $1.396(4)$ \\
$\mathrm{Co}-\mathrm{O}(3)$ & $2.0522(19)$ & $\mathrm{C}(7)-\mathrm{C}(12)$ & $1.389(4)$
\end{tabular}




\begin{tabular}{cccc}
$\mathrm{Co}-\mathrm{O}(5)$ & $1.9621(19)$ & $\mathrm{C}(8)-\mathrm{C}(9)$ & $1.393(4)$ \\
$\mathrm{Co}-\mathrm{N}(2)$ & $1.947(2)$ & $\mathrm{C}(9)-\mathrm{C}(10)$ & $1.381(5)$ \\
$\mathrm{Co}-\mathrm{N}(4)$ & $1.881(2)$ & $\mathrm{C}(10)-\mathrm{C}(11)$ & $1.391(5)$ \\
$\mathrm{Co}-\mathrm{C}(20)$ & $1.916(3)$ & $\mathrm{C}(11)-\mathrm{C}(12)$ & $1.391(4)$ \\
$\mathrm{O}(1)-\mathrm{N}(1)$ & $1.360(3)$ & $\mathrm{C}(13)-\mathrm{C}(14)$ & $1.371(5)$ \\
$\mathrm{O}(2)-\mathrm{C}(6)$ & $1.222(4)$ & $\mathrm{C}(14)-\mathrm{C}(15)$ & $1.384(5)$ \\
$\mathrm{O}(3)-\mathrm{N}(3)$ & $1.342(3)$ & $\mathrm{C}(15)-\mathrm{C}(16)$ & $1.379(4)$ \\
$\mathrm{O}(4)-\mathrm{C}(18)$ & $1.231(3)$ & $\mathrm{C}(16)-\mathrm{C}(17)$ & $1.396(4)$ \\
$\mathrm{O}(5)-\mathrm{N}(5)$ & $1.340(3)$ & $\mathrm{C}(18)-\mathrm{C}(19)$ & $1.491(4)$ \\
$\mathrm{O}(6)-\mathrm{C}(30)$ & $1.216(4)$ & $\mathrm{C}(19)-\mathrm{C}(20)$ & $1.397(4)$ \\
$\mathrm{N}(1)-\mathrm{C}(1)$ & $1.348(4)$ & $\mathrm{C}(19)-\mathrm{C}(24)$ & $1.395(4)$ \\
$\mathrm{N}(1)-\mathrm{C}(5)$ & $1.360(4)$ & $\mathrm{C}(20)-\mathrm{C}(21)$ & $1.401(4)$ \\
$\mathrm{N}(2)-\mathrm{C}(5)$ & $1.365(4)$ & $\mathrm{C}(21)-\mathrm{C}(22)$ & $1.396(4)$ \\
$\mathrm{N}(2)-\mathrm{C}(6)$ & $1.386(4)$ & $\mathrm{C}(22)-\mathrm{C}(23)$ & $1.388(5)$ \\
$\mathrm{N}(3)-\mathrm{C}(13)$ & $1.351(4)$ & $\mathrm{C}(23)-\mathrm{C}(24)$ & $1.390(4)$ \\
$\mathrm{N}(3)-\mathrm{C}(17)$ & $1.377(4)$ & $\mathrm{C}(25)-\mathrm{C}(26)$ & $1.366(4)$ \\
$\mathrm{N}(4)-\mathrm{C}(17)$ & $1.362(3)$ & $\mathrm{C}(26)-\mathrm{C}(27)$ & $1.392(4)$ \\
$\mathrm{N}(4)-\mathrm{C}(18)$ & $1.376(3)$ & $\mathrm{C}(27)-\mathrm{C}(28)$ & $1.376(4)$ \\
$\mathrm{N}(5)-\mathrm{C}(25)$ & $1.358(4)$ & $\mathrm{C}(28)-\mathrm{C}(29)$ & $1.397(4)$ \\
$\mathrm{N}(5)-\mathrm{C}(29)$ & $1.366(4)$ & $\mathrm{C}(30)-\mathrm{C}(31)$ & $1.495(4)$ \\
$\mathrm{N}(6)-\mathrm{C}(29)$ & $1.374(4)$ & $\mathrm{C}(31)-\mathrm{C}(32)$ & $1.403(4)$ \\
$\mathrm{N}(6)-\mathrm{C}(30)$ & $\mathrm{C}(31)-\mathrm{C}(36)$ & $1.391(4)$ \\
$\mathrm{C}(1)-\mathrm{C}(2)$ & $\mathrm{C}(32)-\mathrm{C}(33)$ & $1.383(5)$ \\
$\mathrm{C}(2)-\mathrm{C}(3)$ & $\mathrm{C}(33)-\mathrm{C}(34)$ & $1.389(5)$ \\
$\mathrm{C}(3)-\mathrm{C}(4)$ & $\mathrm{C}(34)-\mathrm{C}(35)$ & $1.396(4)$ \\
$\mathrm{C}(4)-\mathrm{C}(5)$ & $1.382(35)-\mathrm{C}(36)$ & \\
$\mathrm{C}(6)-\mathrm{C}(7)$ & $1.371(4)$ & & \\
& $1.396(5)$ & & \\
\hline
\end{tabular}

Table 44. Bond angles in $\left[^{\circ}\right]$.

\begin{tabular}{cccc}
\hline Atoms & Angle $\left[{ }^{\circ}\right]$ & Atoms & Angle $\left[{ }^{\circ}\right]$ \\
\hline $\mathrm{O}(1)-\mathrm{Co}(1)-\mathrm{O}(3)$ & $96.41(8)$ & $\mathrm{C}(12)-\mathrm{C}(7)-\mathrm{C}(6)$ & $119.5(3)$ \\
$\mathrm{O}(1)-\mathrm{Co}(1)-\mathrm{O}(5)$ & $83.35(9)$ & $\mathrm{C}(12)-\mathrm{C}(7)-\mathrm{C}(8)$ & $119.4(3)$
\end{tabular}




\begin{tabular}{|c|c|c|c|}
\hline $\mathrm{O}(1)-\mathrm{Co}(1)-\mathrm{N}(2)$ & $83.08(10)$ & $\mathrm{C}(9)-\mathrm{C}(8)-\mathrm{C}(7)$ & $120.0(3)$ \\
\hline $\mathrm{O}(1)-\mathrm{Co}(1)-\mathrm{C}(20)$ & $98.11(11)$ & $C(10)-C(9)-C(8)$ & $120.1(3)$ \\
\hline $\mathrm{O}(5)-\mathrm{Co}(1)-\mathrm{O}(3)$ & $84.45(8)$ & $\mathrm{C}(9)-\mathrm{C}(10)-\mathrm{C}(11)$ & $120.5(3)$ \\
\hline $\mathrm{N}(2)-\mathrm{Co}(1)-\mathrm{O}(3)$ & $94.76(9)$ & $\mathrm{C}(12)-\mathrm{C}(11)-\mathrm{C}(10)$ & $119.4(3)$ \\
\hline $\mathrm{N}(2)-\mathrm{Co}(1)-\mathrm{O}(5)$ & $166.23(10)$ & $\mathrm{C}(7)-\mathrm{C}(12)-\mathrm{C}(11)$ & $120.7(3)$ \\
\hline $\mathrm{N}(4)-\mathrm{Co}(1)-\mathrm{O}(1)$ & $176.08(9)$ & $\mathrm{N}(3)-\mathrm{C}(13)-\mathrm{C}(14)$ & $120.8(3)$ \\
\hline $\mathrm{N}(4)-\mathrm{Co}(1)-\mathrm{O}(3)$ & $81.43(9)$ & $C(13)-C(14)-C(15)$ & $119.2(3)$ \\
\hline $\mathrm{N}(4)-\mathrm{Co}(1)-\mathrm{O}(5)$ & $93.16(9)$ & $\mathrm{C}(16)-\mathrm{C}(15)-\mathrm{C}(14)$ & $120.3(3)$ \\
\hline $\mathrm{N}(4)-\mathrm{Co}(1)-\mathrm{N}(2)$ & $100.33(10)$ & $C(15)-C(16)-C(17)$ & $119.9(3)$ \\
\hline $\mathrm{N}(4)-\mathrm{Co}(1)-\mathrm{C}(20)$ & $84.05(12)$ & $\mathrm{N}(3)-\mathrm{C}(17)-\mathrm{C}(16)$ & $118.3(3)$ \\
\hline $\mathrm{C}(20)-\mathrm{Co}(1)-\mathrm{O}(3)$ & $165.48(11)$ & $\mathrm{N}(4)-\mathrm{C}(17)-\mathrm{N}(3)$ & $112.7(3)$ \\
\hline $\mathrm{C}(20)-\mathrm{Co}(1)-\mathrm{O}(5)$ & $96.86(10)$ & $\mathrm{N}(4)-\mathrm{C}(17)-\mathrm{C}(16)$ & $128.9(3)$ \\
\hline $\mathrm{C}(20)-\mathrm{Co}(1)-\mathrm{N}(2)$ & $87.35(11)$ & $\mathrm{O}(4)-\mathrm{C}(18)-\mathrm{N}(4)$ & $126.8(3)$ \\
\hline $\mathrm{N}(1)-\mathrm{O}(1)-\mathrm{Co}(1)$ & $111.33(16)$ & $\mathrm{O}(4)-\mathrm{C}(18)-\mathrm{C}(19)$ & $124.8(3)$ \\
\hline $\mathrm{N}(3)-\mathrm{O}(3)-\mathrm{Co}(1)$ & $108.38(16)$ & $\mathrm{N}(4)-\mathrm{C}(18)-\mathrm{C}(19)$ & $108.4(2)$ \\
\hline $\mathrm{N}(5)-\mathrm{O}(5)-\mathrm{Co}(1)$ & $122.51(16)$ & $\mathrm{C}(20)-\mathrm{C}(19)-\mathrm{C}(18)$ & $116.2(3)$ \\
\hline $\mathrm{O}(1)-\mathrm{N}(1)-\mathrm{C} 5)$ & $117.6(2)$ & $\mathrm{C}(24)-\mathrm{C}(19)-\mathrm{C}(18)$ & $121.4(3)$ \\
\hline $\mathrm{C}(1)-\mathrm{N}(1)-\mathrm{O}(1)$ & $118.2(2)$ & $\mathrm{C}(24)-\mathrm{C}(19)-\mathrm{C}(20)$ & $122.4(3)$ \\
\hline $\mathrm{C}(1)-\mathrm{N}(1)-\mathrm{C}(5)$ & $124.2(3)$ & $\mathrm{C}(19)-\mathrm{C}(20)-\mathrm{Co}(1)$ & $112.3(2)$ \\
\hline $\mathrm{C}(5)-\mathrm{N}(2)-\mathrm{Co}(1)$ & $110.57(18)$ & $\mathrm{C}(19)-\mathrm{C}(20)-\mathrm{C}(21)$ & $117.8(3)$ \\
\hline $\mathrm{C}(5)-\mathrm{N}(2)-\mathrm{C}(6)$ & $118.0(2)$ & $\mathrm{C}(21)-\mathrm{C}(20)-\mathrm{Co}(1)$ & $129.8(2)$ \\
\hline $\mathrm{C}(6)-\mathrm{N}(2)-\mathrm{Co}(1)$ & $125.0(2)$ & $\mathrm{C}(22)-\mathrm{C}(21)-\mathrm{C}(20)$ & $120.2(3)$ \\
\hline $\mathrm{O}(3)-\mathrm{N}(3)-\mathrm{C}(13)$ & $118.9(3)$ & $\mathrm{C}(23)-\mathrm{C}(22)-\mathrm{C}(21)$ & $120.9(3)$ \\
\hline $\mathrm{O}(3)-\mathrm{N}(3)-\mathrm{C}(17)$ & $119.6(2)$ & $\mathrm{C}(22)-\mathrm{C}(23)-\mathrm{C}(24)$ & $119.9(3)$ \\
\hline $\mathrm{C}(13)-\mathrm{N}(3)-\mathrm{C}(17)$ & $121.5(3)$ & $\mathrm{C}(23)-\mathrm{C}(24)-\mathrm{C}(19)$ & $118.9(3)$ \\
\hline $\mathrm{C}(17)-\mathrm{N}(4)-\mathrm{Co}(1)$ & $117.14(19)$ & $\mathrm{N}(5)-\mathrm{C}(25)-\mathrm{C}(26)$ & $121.0(3)$ \\
\hline$C(17)-N(4)-C(18)$ & $123.7(3)$ & $\mathrm{C}(25)-\mathrm{C}(26)-\mathrm{C}(27)$ & $118.4(3)$ \\
\hline $\mathrm{C}(18)-\mathrm{N}(4)-\mathrm{Co}(1)$ & $118.99(18)$ & $\mathrm{C}(28)-\mathrm{C}(27)-\mathrm{C}(26)$ & $120.8(3)$ \\
\hline $\mathrm{O}(5)-\mathrm{N}(5)-\mathrm{C}(25)$ & $119.5(2)$ & $\mathrm{C}(27)-\mathrm{C}(28)-\mathrm{C}(29)$ & $119.6(3)$ \\
\hline $\mathrm{O}(5)-\mathrm{N}(5)-\mathrm{C}(29)$ & $118.5(2)$ & $\mathrm{N}(5)-\mathrm{C}(29)-\mathrm{N}(6)$ & $113.5(3)$ \\
\hline $\mathrm{C}(25)-\mathrm{N}(5)-\mathrm{C}(29)$ & $121.7(3)$ & $\mathrm{N}(5)-\mathrm{C}(29)-\mathrm{C}(28)$ & $118.5(3)$ \\
\hline$C(29)-N(6)-C(30)$ & $125.6(3)$ & $\mathrm{N}(6)-\mathrm{C}(29)-\mathrm{C}(28)$ & $128.0(3)$ \\
\hline $\mathrm{N}(1)-\mathrm{C}(1)-\mathrm{C}(2)$ & $120.2(3)$ & $\mathrm{O}(6)-\mathrm{C}(30)-\mathrm{N}(6)$ & $121.9(3)$ \\
\hline
\end{tabular}




$\begin{array}{llll}\mathrm{C}(1)-\mathrm{C}(2)-\mathrm{C}(3) & 118.1(3) & \mathrm{O}(6)-\mathrm{C}(30)-\mathrm{C}(31) & 122.0(3) \\ \mathrm{C}(4)-\mathrm{C}(3)-\mathrm{C}(2) & 120.7(3) & \mathrm{N}(6)-\mathrm{C}(30)-\mathrm{C}(31) & 116.1(3) \\ \mathrm{C}(3)-\mathrm{C}(4)-\mathrm{C}(5) & 120.5(3) & \mathrm{C}(32)-\mathrm{C}(31)-\mathrm{C}(30) & 116.3(3) \\ \mathrm{N}(1)-\mathrm{C}(5)-\mathrm{N}(2) & 113.8(2) & \mathrm{C}(36)-\mathrm{C}(31)-\mathrm{C}(30) & 124.4(3) \\ \mathrm{N}(1)-\mathrm{C}(5)-\mathrm{C}(4) & 116.1(3) & \mathrm{C}(36)-\mathrm{C}(31)-\mathrm{C}(32) & 119.2(3) \\ \mathrm{N}(2)-\mathrm{C}(5)-\mathrm{C}(4) & 129.8(3) & \mathrm{C}(33)-\mathrm{C}(32)-\mathrm{C}(31) & 120.5(3) \\ \mathrm{O}(2)-\mathrm{C}(6)-\mathrm{N}(2) & 124.5(3) & \mathrm{C}(32)-\mathrm{C}(33)-\mathrm{C}(34) & 120.5(3) \\ \mathrm{O}(2)-\mathrm{C}(6)-\mathrm{C}(7) & 118.9(3) & \mathrm{C}(33)-\mathrm{C}(34)-\mathrm{C}(35) & 119.5(3) \\ \mathrm{N}(2)-\mathrm{C}(6)-\mathrm{C}(7) & 116.4(2) & \mathrm{C}(34)-\mathrm{C}(35)-\mathrm{C}(36) & 120.3(3)\end{array}$

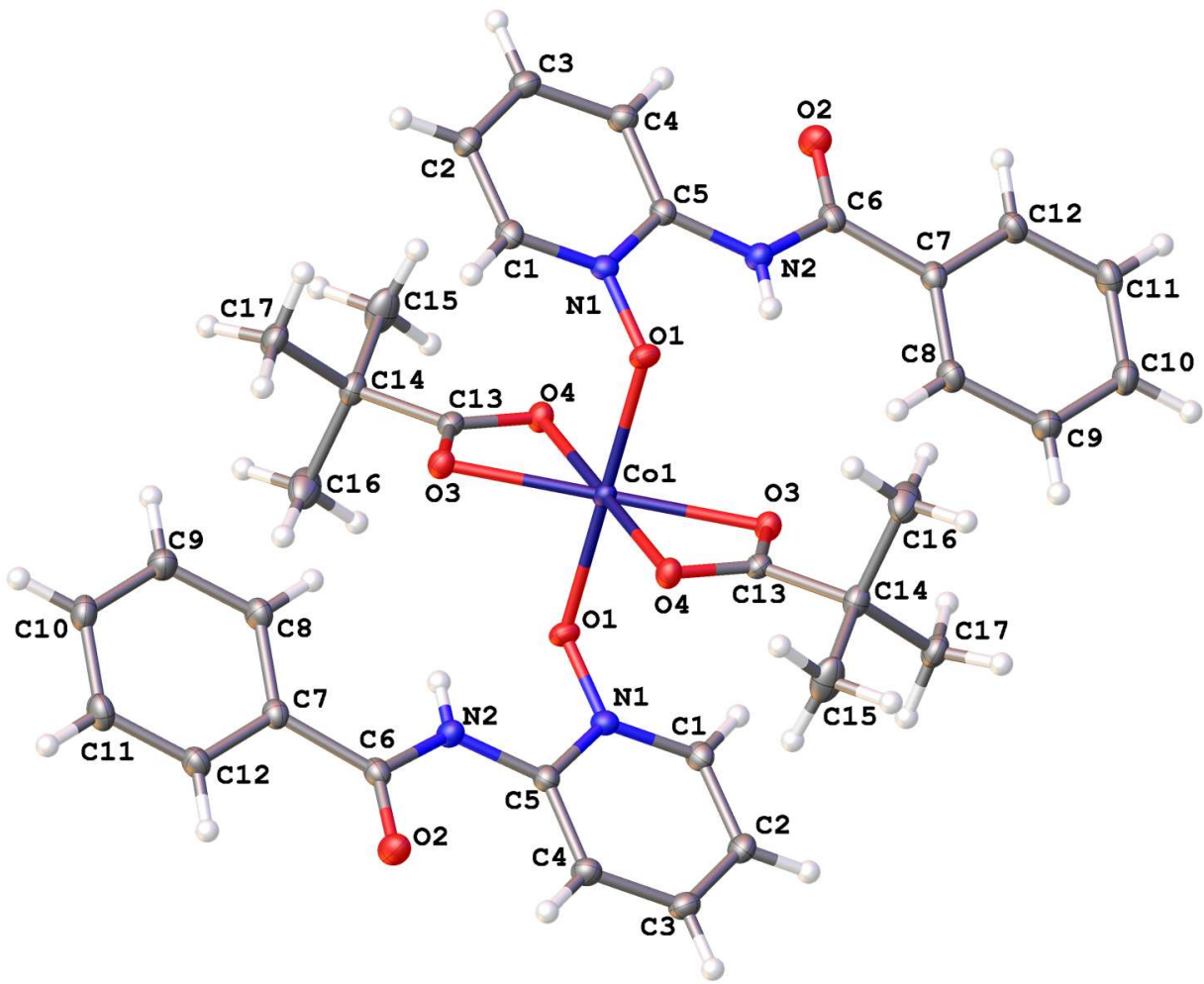

Figure 72. Molecular structure of $\mathbf{2 0 2}$ with thermal ellipsoids at 50\% probability level.

Table 45. Crystal data and structure refinement for 202 .

Compound

CCDC number

Empirical formula

Formula weight

Temperature

Crystal system
202

2045464

$\mathrm{C}_{34} \mathrm{H}_{38} \mathrm{CoN}_{4} \mathrm{O}_{8}$

689.61

$100.0 \mathrm{~K}$

triclinic 
Space group

Unit cell dimensions

Volume

Z

Density (calculated)

Absorption coefficient

$\mathrm{F}(000)$

Crystal size

Radiation

$2 \Theta$ range for data collection

Index ranges

Reflections collected

Independent reflections

Data / restraints / parameters

Goodness-of-fit on $\mathrm{F}^{2}$

Final $R$ indices $[\mathrm{I}>2 \sigma(\mathrm{I})]$

$R$ indices (all data)

Largest diff. peak and hole
$P-1$

$a=6.0548(11) \AA$

$\alpha=75.515(6)^{\circ}$

$b=11.117(2) \AA$

$\beta=82.271(5)^{\circ}$

$c=12.341(3) \AA$

$\gamma=87.820(5)^{\circ}$

$797.0(3) \AA^{3}$

1

$1.437 \mathrm{~g} / \mathrm{m}^{3}$

$0.598 \mathrm{~mm}^{-1}$

361.0

$(0.368 \times 0.233 \times 0.032) \mathrm{mm}^{3}$

$\operatorname{MoK} \alpha(\lambda=0.71073)$

$5.708^{\circ}$ to $59.146^{\circ}$

$-8 \leq \mathrm{h} \leq 8,-15 \leq \mathrm{k} \leq 15,-17 \leq 1 \leq 17$

8815

$8815\left[R_{\text {int }}=?, R_{\text {sigma }}=0.0158\right]$

$8815 / 0 / 221$

1.080

$R_{1}=0.0260, \mathrm{w} R_{2}=0.0689$

$R_{1}=0.0272, \mathrm{w} R_{2}=0.0700$

$0.45 \mathrm{e} \cdot \AA^{-3}$ and $-0.49 \mathrm{e} \cdot \AA^{-3}$

Table 46. Bond lengths in $[\AA]$.

\begin{tabular}{cccc}
\hline Atoms & Bond Length $[\AA]$ & Atoms & Bond Length $[\AA]$ \\
\hline $\mathrm{Co}(1)-\mathrm{O}\left(1^{1}\right)$ & $2.0527(10)$ & $\mathrm{C}(1)-\mathrm{C}(2)$ & $1.3804(18)$ \\
$\mathrm{Co}(1)-\mathrm{O}(1)$ & $2.0527(10)$ & $\mathrm{C}(2)-\mathrm{C}(3)$ & $1.3919(19)$ \\
$\mathrm{Co}(1)-\mathrm{O}(3)$ & $2.1192(10)$ & $\mathrm{C}(3)-\mathrm{C}(4)$ & $1.3859(19)$ \\
$\mathrm{Co}(1)-\mathrm{O}\left(3^{1}\right)$ & $2.1193(10)$ & $\mathrm{C}(4)-\mathrm{C}(5)$ & $1.3958(18)$ \\
$\mathrm{Co}(1)-\mathrm{O}\left(4^{1}\right)$ & $2.1372(10)$ & $\mathrm{C}(6)-\mathrm{C}(7)$ & $1.4975(18)$ \\
$\mathrm{Co}(1)-\mathrm{O}(4)$ & $2.1372(10)$ & $\mathrm{C}(7)-\mathrm{C}(8)$ & $1.3986(19)$ \\
$\mathrm{Co}(1)-\mathrm{C}(13)$ & $2.4588(13)$ & $\mathrm{C}(7)-\mathrm{C}(12)$ & $1.3981(17)$ \\
$\mathrm{Co}(1)-\mathrm{C}\left(13^{1}\right)$ & $2.4588(13)$ & $\mathrm{C}(8)-\mathrm{C}(9)$ & $1.3950(19)$ \\
$\mathrm{O}(1)-\mathrm{N}(1)$ & $1.3400(14)$ & $\mathrm{C}(9)-\mathrm{C}(10)$ & $1.3908(19)$ \\
$\mathrm{O}(2)-\mathrm{C}(6)$ & $1.2216(17)$ & $\mathrm{C}(10)-\mathrm{C}(11)$ & $1.392(2)$
\end{tabular}




$\begin{array}{lllc}\mathrm{O}(3)-\mathrm{C}(13) & 1.2729(15) & \mathrm{C}(11)-\mathrm{C}(12) & 1.3917(19) \\ \mathrm{O}(4)-\mathrm{C}(13) & 1.2673(15) & \mathrm{C}(13)-\mathrm{C}(14) & 1.5272(18) \\ \mathrm{N}(1)-\mathrm{C}(1) & 1.3506(17) & \mathrm{C}(14)-\mathrm{C}(15) & 1.530(2) \\ \mathrm{N}(1)-\mathrm{C}(5) & 1.3680(16) & \mathrm{C}(14)-\mathrm{C}(16) & 1.540(2) \\ \mathrm{N}(2)-\mathrm{C}(5) & 1.3792(16) & \mathrm{C}(14)-\mathrm{C}(17) & 1.5324(19) \\ \mathrm{N}(2)-\mathrm{C}(6) & 1.3844(15) & & \end{array}$

Table 47. Bond angles in $\left[^{\circ}\right]$.

\begin{tabular}{|c|c|c|c|}
\hline Atoms & Angle $\left[{ }^{\circ}\right]$ & Atoms & Angle $\left[{ }^{\circ}\right]$ \\
\hline $\mathrm{O}\left(1^{1}\right)-\mathrm{Co}(1)-\mathrm{O}(1)$ & 180.0 & $\mathrm{O}(1)-\mathrm{N}(1)-\mathrm{C}(1)$ & $119.79(11)$ \\
\hline $\mathrm{O}(1)-\mathrm{Co}(1)-\mathrm{O}\left(3^{1}\right)$ & $94.83(4)$ & $\mathrm{O}(1)-\mathrm{N}(1)-\mathrm{C}(5)$ & $118.15(10)$ \\
\hline $\mathrm{O}\left(1^{1}\right)-\mathrm{Co}(1)-\mathrm{O}\left(3^{1}\right)$ & $85.17(4)$ & $\mathrm{C}(1)-\mathrm{N}(1)-\mathrm{C}(5)$ & $122.04(11)$ \\
\hline $\mathrm{O}(1)-\mathrm{Co}(1)-\mathrm{O}(3)$ & $85.17(4)$ & $\mathrm{C}(5)-\mathrm{N}(2)-\mathrm{C}(6)$ & $125.69(12)$ \\
\hline $\mathrm{O}\left(1^{1}\right)-\mathrm{Co}(1)-\mathrm{O}(3)$ & $94.83(4)$ & $\mathrm{N}(1)-\mathrm{C}(1)-\mathrm{C}(2)$ & $120.52(12)$ \\
\hline $\mathrm{O}(1)-\mathrm{Co}(1)-\mathrm{O}(4)$ & $87.63(4)$ & $C(1)-C(2)-C(3)$ & $118.70(12)$ \\
\hline $\mathrm{O}\left(1^{1}\right)-\mathrm{Co}(1)-\mathrm{O}(4)$ & $92.37(4)$ & $C(4)-C(3)-C(2)$ & $120.53(12)$ \\
\hline $\mathrm{O}(1)-\mathrm{Co}(1)-\mathrm{O}\left(4^{1}\right)$ & $92.37(4)$ & $C(3)-C(4)-C(5)$ & $119.36(12)$ \\
\hline $\mathrm{O}\left(1^{1}\right)-\mathrm{Co}(1)-\mathrm{O}\left(4^{1}\right)$ & $87.63(4)$ & $\mathrm{N}(1)-\mathrm{C}(5)-\mathrm{N}(2)$ & $113.85(11)$ \\
\hline $\mathrm{O}(1)-\mathrm{Co}(1)-\mathrm{C}(13)$ & $84.90(4)$ & $\mathrm{N}(1)-\mathrm{C}(5)-\mathrm{C}(4)$ & $118.84(12)$ \\
\hline $\mathrm{O}\left(1^{1}\right)-\mathrm{Co}(1)-\mathrm{C}\left(13^{1}\right)$ & $84.90(4)$ & $\mathrm{N}(2)-\mathrm{C}(5)-\mathrm{C}(4)$ & $127.30(12)$ \\
\hline $\mathrm{O}(1)-\mathrm{Co}(1)-\mathrm{C}\left(13^{1}\right)$ & $95.10(4)$ & $\mathrm{O}(2)-\mathrm{C}(6)-\mathrm{N}(2)$ & $122.08(12)$ \\
\hline $\mathrm{O}\left(1^{1}\right)-\mathrm{Co}(1)-\mathrm{C}(13)$ & $95.10(4)$ & $\mathrm{O}(2)-\mathrm{C}(6)-\mathrm{C}(7)$ & $121.39(11)$ \\
\hline $\mathrm{O}(3)-\mathrm{Co}(1)-\mathrm{O}\left(3^{1}\right)$ & $180.00(4)$ & $\mathrm{N}(2)-\mathrm{C}(6)-\mathrm{C}(7)$ & $116.53(12)$ \\
\hline $\mathrm{O}\left(3^{1}\right)-\mathrm{Co}(1)-\mathrm{O}(4)$ & $117.83(3)$ & $C(8)-C(7)-C(6)$ & $124.32(12)$ \\
\hline $\mathrm{O}(3)-\mathrm{Co}(1)-\mathrm{O}(4)$ & $62.17(3)$ & $C(12)-C(7)-C(6)$ & $115.98(12)$ \\
\hline $\mathrm{O}\left(3^{1}\right)-\mathrm{Co}(1)-\mathrm{O}\left(4^{1}\right)$ & $62.17(3)$ & $\mathrm{C}(12)-\mathrm{C}(7)-\mathrm{C}(8)$ & $119.63(12)$ \\
\hline $\mathrm{O}(3)-\mathrm{Co}(1)-\mathrm{O}\left(4^{1}\right)$ & $117.83(3)$ & $\mathrm{C}(9)-\mathrm{C}(8)-\mathrm{C}(7)$ & $119.51(12)$ \\
\hline $\mathrm{O}(3)-\mathrm{Co}(1)-\mathrm{C}\left(13^{1}\right)$ & $148.83(4)$ & $\mathrm{C}(10)-\mathrm{C}(9)-\mathrm{C}(8)$ & $120.68(13)$ \\
\hline $\mathrm{O}\left(3^{1}\right)-\mathrm{Co}(1)-\mathrm{C}\left(13^{1}\right)$ & $31.17(4)$ & $\mathrm{C}(9)-\mathrm{C}(10)-\mathrm{C}(11)$ & $119.85(13)$ \\
\hline $\mathrm{O}(3)-\mathrm{Co}(1)-\mathrm{C}(13)$ & $31.17(4)$ & $C(12)-C(11)-C(10)$ & $119.86(13)$ \\
\hline $\mathrm{O}\left(3^{1}\right)-\mathrm{Co}(1)-\mathrm{C}(13)$ & $148.83(4)$ & $C(11)-C(12)-C(7)$ & $120.47(13)$ \\
\hline $\mathrm{O}(41)-\mathrm{Co}(1)-\mathrm{O}(4)$ & 180.0 & $\mathrm{O}(3)-\mathrm{C}(13)-\mathrm{C}(14)$ & $119.59(11)$ \\
\hline
\end{tabular}




$\begin{array}{cccc}\mathrm{O}(4)-\mathrm{Co}(1)-\mathrm{C}(13) & 31.02(4) & \mathrm{O}(4)-\mathrm{C}(13)-\mathrm{O}(3) & 119.79(12) \\ \mathrm{O}\left(4^{1}\right)-\mathrm{Co}(1)-\mathrm{C}\left(13^{1}\right) & 31.02(4) & \mathrm{O}(4)-\mathrm{C}(13)-\mathrm{C}(14) & 120.58(11) \\ \mathrm{O}\left(4^{1}\right)-\mathrm{Co}(1)-\mathrm{C}(13) & 148.98(4) & \mathrm{C}(13)-\mathrm{C}(14)-\mathrm{C}(15) & 110.39(11) \\ \mathrm{O}(4)-\mathrm{Co}(1)-\mathrm{C}\left(13^{1}\right) & 148.98(4) & \mathrm{C}(13)-\mathrm{C}(14)-\mathrm{C}(16) & 106.56(11) \\ \mathrm{C}(13)-\mathrm{Co}(1)-\mathrm{C}\left(13^{1}\right) & 180.0 & \mathrm{C}(13)-\mathrm{C}(14)-\mathrm{C}(17) & 109.88(11) \\ \mathrm{N}(1)-\mathrm{O}(1)-\mathrm{Co}(1) & 118.31(7) & \mathrm{C}(15)-\mathrm{C}(14)-\mathrm{C}(16) & 110.11(13) \\ \mathrm{C}(13)-\mathrm{O}(3)-\mathrm{Co}(1) & 89.30(7) & \mathrm{C}(15)-\mathrm{C}(14)-\mathrm{C}(17) & 110.14(12) \\ \mathrm{C}(13)-\mathrm{O}(4)-\mathrm{Co}(1) & 88.65(8) & \mathrm{C}(17)-\mathrm{C}(14)-\mathrm{C}(16) & 109.70(11)\end{array}$

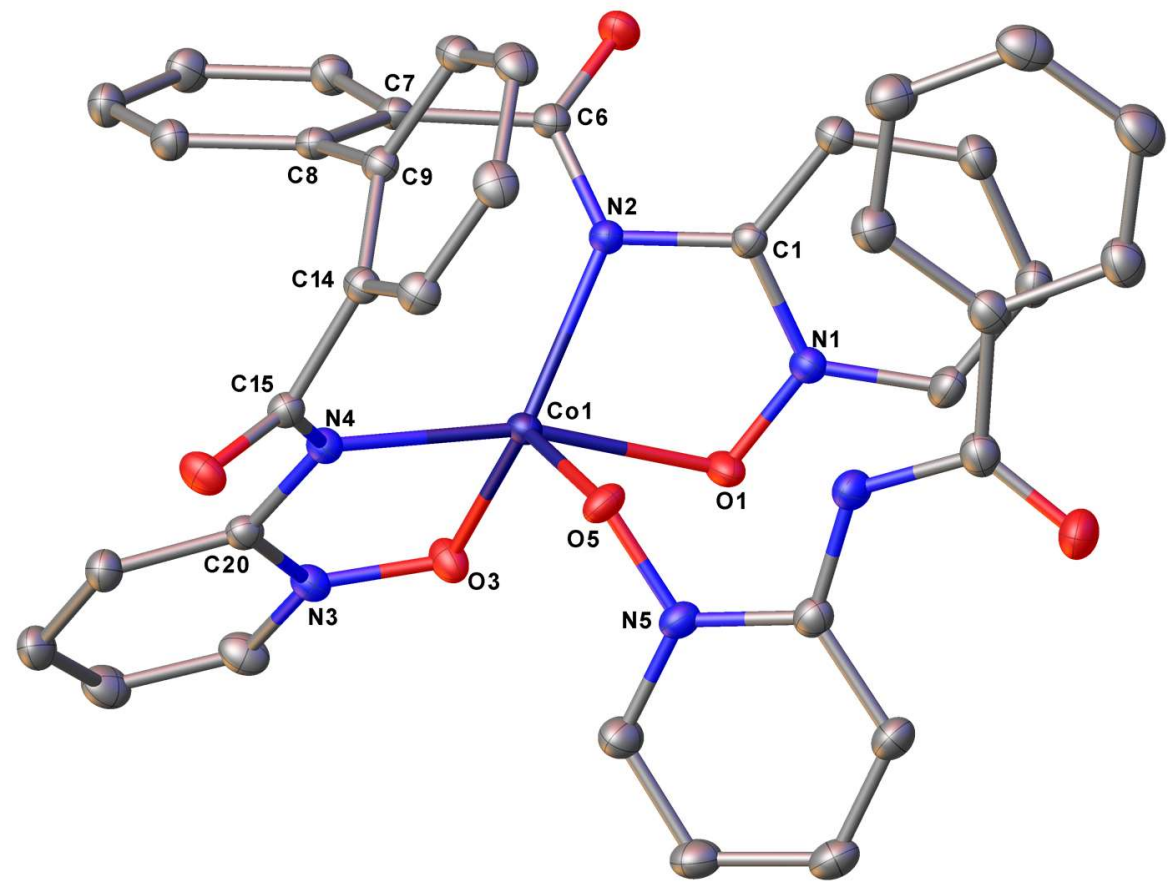

Figure 73. Molecular structure of $\mathbf{2 0 3}$ with thermal ellipsoids at $50 \%$ probability level. The hydrogen atoms and one molecule of $\mathrm{CH}_{2} \mathrm{Cl}_{2}$ are omitted for clarity.

Table 48. Crystal data and structure refinement for 203 .

Compound

CCDC number

Empirical formula

Formula weight

Temperature

Crystal system

Space group

Unit cell dimensions
203

1983756

$\mathrm{C}_{37} \mathrm{H}_{28} \mathrm{Cl}_{2} \mathrm{CoN}_{6} \mathrm{O}_{6}$

782.48

$99.97 \mathrm{~K}$

triclinic

$P-1$

$a=8.1484(3) \AA \quad \alpha=77.154(2)^{\circ}$ 
Volume

Z

Density (calculated)

Absorption coefficient

$\mathrm{F}(000)$

Crystal size

Radiation

$2 \Theta$ range for data collection

Index ranges

Reflections collected

Independent reflections

Data / restraints / parameters

Goodness-of-fit on $\mathrm{F}^{2}$

Final $R$ indices [I $>2 \sigma(\mathrm{I})]$

$R$ indices (all data)

Largest diff. peak and hole

$$
\begin{array}{ll}
b=11.8103(5) \AA & \beta=84.964(2)^{\circ} \\
c=18.0756(8) \AA & \gamma=87.7910(10)^{\circ}
\end{array}
$$

\section{2}

$1.539 \mathrm{~g} / \mathrm{m}^{3}$

$0.725 \mathrm{~mm}^{-1}$

802.0

$(0.625 \times 0.586 \times 0.484) \mathrm{mm}^{3}$

$\operatorname{MoK} \alpha(\lambda=0.71073)$

$4.638^{\circ}$ to $61.064^{\circ}$

$-11 \leq \mathrm{h} \leq 11,-16 \leq \mathrm{k} \leq 16,-25 \leq 1 \leq 25$

59902

$10220\left[R_{\text {int }}=0.0231, R_{\text {sigma }}=0.0150\right]$

$10220 / 0 / 483$

1.034

$R_{1}=0.0279, \mathrm{w} R_{2}=0.0714$

$R_{1}=0.0293, \mathrm{w} R_{2}=0.0733$

$0.45 \mathrm{e} \cdot \AA^{-3}$ and $-0.44 \mathrm{e} \cdot \AA^{-3}$

Table 49. Bond lengths in $[\AA]$.

\begin{tabular}{cccc}
\hline Atoms & Bond Length $[\AA]$ & Atoms & Bond Length $[\AA]$ \\
\hline $\mathrm{Co}(1)-\mathrm{O}(1)$ & $2.1228(8)$ & $\mathrm{C}(8)-\mathrm{C}(9)$ & $1.4900(14)$ \\
$\mathrm{Co}(1)-\mathrm{O}(3)$ & $1.9785(8)$ & $\mathrm{C}(8)-\mathrm{C}(16)$ & $1.3992(14)$ \\
$\mathrm{Co}(1)-\mathrm{O}(5)$ & $2.0825(8)$ & $\mathrm{C}(9)-\mathrm{C}(10)$ & $1.4010(14)$ \\
$\mathrm{Co}(1)-\mathrm{N}(2)$ & $2.0123(9)$ & $\mathrm{C}(9)-\mathrm{C}(14)$ & $1.4030(14)$ \\
$\mathrm{Co}(1)-\mathrm{N}(4)$ & $2.0428(9)$ & $\mathrm{C}(10)-\mathrm{C}(11)$ & $1.3863(16)$ \\
$\mathrm{O}(1)-\mathrm{N}(1)$ & $1.3388(11)$ & $\mathrm{C}(11)-\mathrm{C}(12)$ & $1.3946(16)$ \\
$\mathrm{O}(2)-\mathrm{C}(6)$ & $1.2334(12)$ & $\mathrm{C}(12)-\mathrm{C}(13)$ & $1.3873(15)$ \\
$\mathrm{O}(3)-\mathrm{N}(3)$ & $1.3537(12)$ & $\mathrm{C}(13)-\mathrm{C}(14)$ & $1.3967(14)$ \\
$\mathrm{O}(4)-\mathrm{C}(15)$ & $1.2328(13)$ & $\mathrm{C}(14)-\mathrm{C}(15)$ & $1.5000(14)$ \\
$\mathrm{O}(5)-\mathrm{N}(5)$ & $1.3436(11)$ & $\mathrm{C}(16)-\mathrm{C}(17)$ & $1.3899(16)$ \\
$\mathrm{O}(6)-\mathrm{C}(30)$ & $1.2187(14)$ & $\mathrm{C}(17)-\mathrm{C}(18)$ & $1.3905(16)$ \\
$\mathrm{N}(1)-\mathrm{C}(1)$ & $1.3665(13)$ & $\mathrm{C}(18)-\mathrm{C}(19)$ & $1.3875(15)$
\end{tabular}




$\begin{array}{cccc}\mathrm{N}(1)-\mathrm{C}(5) & 1.3540(14) & \mathrm{C}(20)-\mathrm{C}(21) & 1.4087(14) \\ \mathrm{N}(2)-\mathrm{C}(1) & 1.3740(13) & \mathrm{C}(21)-\mathrm{C}(22) & 1.3807(16) \\ \mathrm{N}(2)-\mathrm{C}(6) & 1.3687(13) & \mathrm{C}(22)-\mathrm{C}(23) & 1.3946(18) \\ \mathrm{N}(3)-\mathrm{C}(20) & 1.3708(14) & \mathrm{C}(23)-\mathrm{C}(24) & 1.3695(17) \\ \mathrm{N}(3)-\mathrm{C}(24) & 1.3550(14) & \mathrm{C}(25)-\mathrm{C}(26) & 1.3983(15) \\ \mathrm{N}(4)-\mathrm{C}(15) & 1.3718(13) & \mathrm{C}(26)-\mathrm{C}(27) & 1.3812(17) \\ \mathrm{N}(4)-\mathrm{C}(20) & 1.3609(13) & \mathrm{C}(27)-\mathrm{C}(28) & 1.3922(18) \\ \mathrm{N}(5)-\mathrm{C}(25) & 1.3593(14) & \mathrm{C}(28)-\mathrm{C}(29) & 1.3746(16) \\ \mathrm{N}(5)-\mathrm{C}(29) & 1.3500(14) & \mathrm{C}(30)-\mathrm{C}(31) & 1.4902(16) \\ \mathrm{N}(6)-\mathrm{C}(25) & 1.3750(14) & \mathrm{C}(31)-\mathrm{C}(32) & 1.3980(16) \\ \mathrm{N}(6)-\mathrm{C}(30) & 1.3819(14) & \mathrm{C}(31)-\mathrm{C}(36) & 1.3978(17) \\ \mathrm{C}(1)-\mathrm{C}(2) & 1.4002(14) & \mathrm{C}(32)-\mathrm{C}(33) & 1.3893(18) \\ \mathrm{C}(2)-\mathrm{C}(3) & 1.3816(15) & \mathrm{C}(33)-\mathrm{C}(34) & 1.388(2) \\ \mathrm{C}(3)-\mathrm{C}(4) & 1.3973(16) & \mathrm{C}(34)-\mathrm{C}(35) & 1.3905(18) \\ \mathrm{C}(4)-\mathrm{C}(5) & 1.3735(16) & \mathrm{C}(35)-\mathrm{C}(36) & 1.3894(17) \\ \mathrm{C}(6)-\mathrm{C}(7) & 1.5003(14) & \mathrm{C}(11 \mathrm{~B})-\mathrm{C}(37) & 1.719(7) \\ \mathrm{C}(7)-\mathrm{C}(8) & 1.4061(14) & \mathrm{C}(12)-\mathrm{C}(37) & 1.7778(15) \\ \mathrm{C}(7)-\mathrm{C}(19) & 1.3978(14) & \mathrm{C}(37)-\mathrm{C}(11 \mathrm{~A}) & 1.776(8)\end{array}$

Table 50. Bond angles in $\left[{ }^{\circ}\right]$.

\begin{tabular}{cccc}
\hline Atoms & Angle $\left[{ }^{\circ}\right]$ & Atoms & Angle [ $\left.{ }^{\circ}\right]$ \\
\hline $\mathrm{O}(3)-\mathrm{Co}(1)-\mathrm{O}(1)$ & $88.00(3)$ & $\mathrm{C}(10)-\mathrm{C}(9)-\mathrm{C}(8)$ & $117.94(9)$ \\
$\mathrm{O}(3)-\mathrm{Co}(1)-\mathrm{O}(5)$ & $119.75(3)$ & $\mathrm{C}(10)-\mathrm{C}(9)-\mathrm{C}(14)$ & $119.01(10)$ \\
$\mathrm{O}(3)-\mathrm{Co}(1)-\mathrm{N}(2)$ & $132.24(4)$ & $\mathrm{C}(14)-\mathrm{C}(9)-\mathrm{C}(8)$ & $122.96(9)$ \\
$\mathrm{O}(3)-\mathrm{Co}(1)-\mathrm{N}(4)$ & $79.99(3)$ & $\mathrm{C}(11)-\mathrm{C}(10)-\mathrm{C}(9)$ & $120.76(10)$ \\
$\mathrm{O}(5)-\mathrm{Co}(1)-\mathrm{O}(1)$ & $85.77(3)$ & $\mathrm{C}(10)-\mathrm{C}(11)-\mathrm{C}(12)$ & $120.14(10)$ \\
$\mathrm{N}(2)-\mathrm{Co}(1)-\mathrm{O}(1)$ & $77.13(3)$ & $\mathrm{C}(13)-\mathrm{C}(12)-\mathrm{C}(11)$ & $119.53(10)$ \\
$\mathrm{N}(2)-\mathrm{Co}(1)-\mathrm{O}(5)$ & $104.28(3)$ & $\mathrm{C}(12)-\mathrm{C}(13)-\mathrm{C}(14)$ & $120.84(10)$ \\
$\mathrm{N}(2)-\mathrm{Co}(1)-\mathrm{N}(4)$ & $118.12(4)$ & $\mathrm{C}(9)-\mathrm{C}(14)-\mathrm{C}(15)$ & $122.33(9)$ \\
$\mathrm{N}(4)-\mathrm{Co}(1)-\mathrm{O}(1)$ & $164.62(3)$ & $\mathrm{C}(13)-\mathrm{C}(14)-\mathrm{C}(9)$ & $119.70(9)$ \\
$\mathrm{N}(4)-\mathrm{Co}(1)-\mathrm{O}(5)$ & $91.84(3)$ & $\mathrm{C}(13)-\mathrm{C}(14)-\mathrm{C}(15)$ & $117.94(9)$ \\
$\mathrm{N}(1)-\mathrm{O}(1)-\mathrm{Co}(1)$ & $107.10(6)$ & $\mathrm{O}(4)-\mathrm{C}(15)-\mathrm{N}(4)$ & $126.02(10)$
\end{tabular}




\begin{tabular}{|c|c|c|c|}
\hline $\mathrm{N}(3)-\mathrm{O}(3)-\mathrm{Co}(1)$ & $112.91(6)$ & $\mathrm{O}(4)-\mathrm{C}(15)-\mathrm{C}(14)$ & $120.60(9)$ \\
\hline $\mathrm{N}(5)-\mathrm{O}(5)-\mathrm{Co}(1)$ & $115.12(6)$ & $\mathrm{N}(4)-\mathrm{C}(15)-\mathrm{C}(14)$ & $113.32(9)$ \\
\hline $\mathrm{O}(1)-\mathrm{N}(1)-\mathrm{C}(1)$ & $118.51(9)$ & $C(17)-C(16)-C(8)$ & $120.88(10)$ \\
\hline $\mathrm{O}(1)-\mathrm{N}(1)-\mathrm{C}(5)$ & $119.28(9)$ & $\mathrm{C}(16)-\mathrm{C}(17)-\mathrm{C}(18)$ & $120.19(10)$ \\
\hline $\mathrm{C}(5)-\mathrm{N}(1)-\mathrm{C}(1)$ & $122.22(9)$ & $\mathrm{C}(19)-\mathrm{C}(18)-\mathrm{C}(17)$ & $119.75(10)$ \\
\hline $\mathrm{C}(1)-\mathrm{N}(2)-\mathrm{Co}(1)$ & $111.24(6)$ & $C(18)-C(19)-C(7)$ & $120.36(10)$ \\
\hline $\mathrm{C}(6)-\mathrm{N}(2)-\mathrm{Co}(1)$ & $129.30(7)$ & $N(3)-C(20)-C(21)$ & $116.77(9)$ \\
\hline $\mathrm{C}(6)-\mathrm{N}(2)-\mathrm{C}(1)$ & $116.11(9)$ & $\mathrm{N}(4)-\mathrm{C}(20)-\mathrm{N}(3)$ & $113.65(9)$ \\
\hline $\mathrm{O}(3)-\mathrm{N}(3)-\mathrm{C}(20)$ & $119.17(9)$ & $N(4)-C(20)-C(21)$ & $129.47(10)$ \\
\hline $\mathrm{O}(3)-\mathrm{N}(3)-\mathrm{C}(24)$ & $117.77(9)$ & $\mathrm{C}(22)-\mathrm{C}(21)-\mathrm{C}(20)$ & $120.32(11)$ \\
\hline $\mathrm{C}(24)-\mathrm{N}(3)-\mathrm{C}(20)$ & $123.06(10)$ & $\mathrm{C}(21)-\mathrm{C}(22)-\mathrm{C}(23)$ & $120.44(11)$ \\
\hline $\mathrm{C}(15)-\mathrm{N}(4)-\mathrm{Co}(1)$ & $122.74(7)$ & $\mathrm{C}(24)-\mathrm{C}(23)-\mathrm{C}(22)$ & $118.61(11)$ \\
\hline $\mathrm{C}(20)-\mathrm{N}(4)-\mathrm{Co}(1)$ & $113.55(7)$ & $\mathrm{N}(3)-\mathrm{C}(24)-\mathrm{C}(23)$ & $120.44(11)$ \\
\hline $\mathrm{C}(20)-\mathrm{N}(4)-\mathrm{C}(15)$ & $120.82(9)$ & $\mathrm{N}(5)-\mathrm{C}(25)-\mathrm{N}(6)$ & $113.01(9)$ \\
\hline $\mathrm{O}(5)-\mathrm{N}(5)-\mathrm{C}(25)$ & $118.21(9)$ & $\mathrm{N}(5)-\mathrm{C}(25)-\mathrm{C}(26)$ & $118.98(10)$ \\
\hline $\mathrm{O}(5)-\mathrm{N}(5)-\mathrm{C}(29)$ & $119.92(9)$ & $\mathrm{N}(6)-\mathrm{C}(25)-\mathrm{C}(26)$ & $127.99(10)$ \\
\hline $\mathrm{C}(29)-\mathrm{N}(5)-\mathrm{C}(25)$ & $121.82(10)$ & $\mathrm{C}(27)-\mathrm{C}(26)-\mathrm{C}(25)$ & $119.64(11)$ \\
\hline $\mathrm{C}(25)-\mathrm{N}(6)-\mathrm{C}(30)$ & $126.72(10)$ & $\mathrm{C}(26)-\mathrm{C}(27)-\mathrm{C}(28)$ & $119.81(11)$ \\
\hline $\mathrm{N}(1)-\mathrm{C}(1)-\mathrm{N}(2)$ & $114.63(9)$ & $\mathrm{C}(29)-\mathrm{C}(28)-\mathrm{C}(27)$ & $119.22(11)$ \\
\hline $\mathrm{N}(1)-\mathrm{C}(1)-\mathrm{C}(2)$ & $118.18(9)$ & $\mathrm{N}(5)-\mathrm{C}(29)-\mathrm{C}(28)$ & $120.51(11)$ \\
\hline $\mathrm{N}(2)-\mathrm{C}(1)-\mathrm{C}(2)$ & 127.01(9) & $\mathrm{O}(6)-\mathrm{C}(30)-\mathrm{N}(6)$ & $122.01(11)$ \\
\hline$C(3)-C(2)-C(1)$ & $120.18(10)$ & $\mathrm{O}(6)-\mathrm{C}(30)-\mathrm{C}(31)$ & $122.73(11)$ \\
\hline$C(2)-C(3)-C(4)$ & $119.75(10)$ & $\mathrm{N}(6)-\mathrm{C}(30)-\mathrm{C}(31)$ & $115.26(10)$ \\
\hline$C(5)-C(4)-C(3)$ & 119.14(10) & $\mathrm{C}(32)-\mathrm{C}(31)-\mathrm{C}(30)$ & $116.43(11)$ \\
\hline $\mathrm{N}(1)-\mathrm{C}(5)-\mathrm{C}(4)$ & $120.44(10)$ & $\mathrm{C}(36)-\mathrm{C}(31)-\mathrm{C}(30)$ & $123.77(10)$ \\
\hline $\mathrm{O}(2)-\mathrm{C}(6)-\mathrm{N}(2)$ & $124.96(10)$ & $\mathrm{C}(36)-\mathrm{C}(31)-\mathrm{C}(32)$ & $119.80(11)$ \\
\hline $\mathrm{O}(2)-\mathrm{C}(6)-\mathrm{C}(7)$ & $119.72(9)$ & $\mathrm{C}(33)-\mathrm{C}(32)-\mathrm{C}(31)$ & $119.69(12)$ \\
\hline $\mathrm{N}(2)-\mathrm{C}(6)-\mathrm{C}(7)$ & $115.14(9)$ & $\mathrm{C}(34)-\mathrm{C}(33)-\mathrm{C}(32)$ & $120.33(12)$ \\
\hline$C(8)-C(7)-C(6)$ & $122.93(9)$ & $\mathrm{C}(33)-\mathrm{C}(34)-\mathrm{C}(35)$ & $120.21(12)$ \\
\hline$C(19)-C(7)-C(6)$ & $116.84(9)$ & $\mathrm{C}(36)-\mathrm{C}(35)-\mathrm{C}(34)$ & $119.86(12)$ \\
\hline$C(19)-C(7)-C(8)$ & $120.23(10)$ & $\mathrm{C}(35)-\mathrm{C}(36)-\mathrm{C}(31)$ & $120.11(11)$ \\
\hline$C(7)-C(8)-C(9)$ & $121.28(9)$ & $\mathrm{C}(11 \mathrm{~B})-\mathrm{C}(37)-\mathrm{C}(12)$ & $110.6(2)$ \\
\hline $\mathrm{C}(16)-\mathrm{C}(8)-\mathrm{C}(7)$ & 118.51(10) & & \\
\hline
\end{tabular}




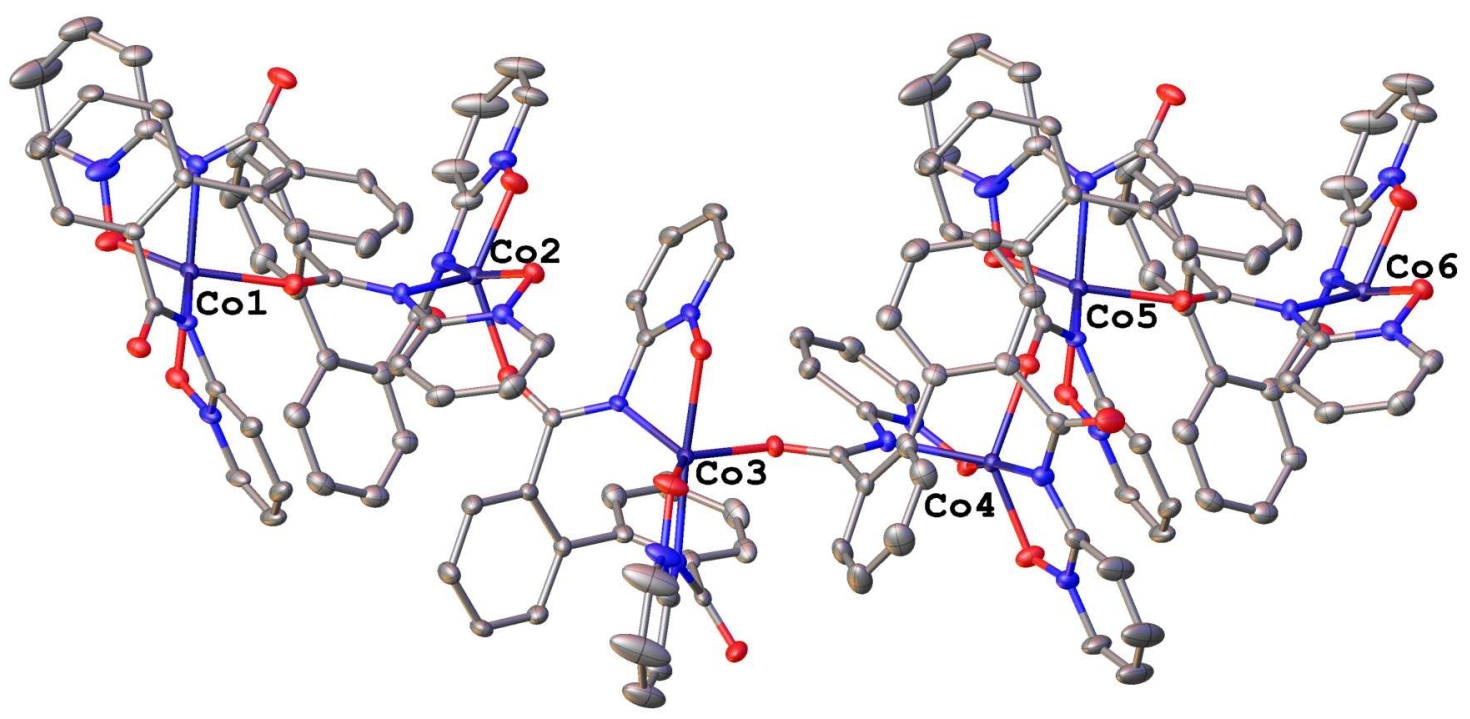

Figure 74. Molecular structure of 204 as a polymer $\left(\mathrm{C}_{48} \mathrm{H}_{32} \mathrm{Co}_{2} \mathrm{~N}_{8} \mathrm{O}_{8}\right)_{n}$, with thermal ellipsoids at $50 \%$ probability level. The hydrogen atoms are omitted for clarity.

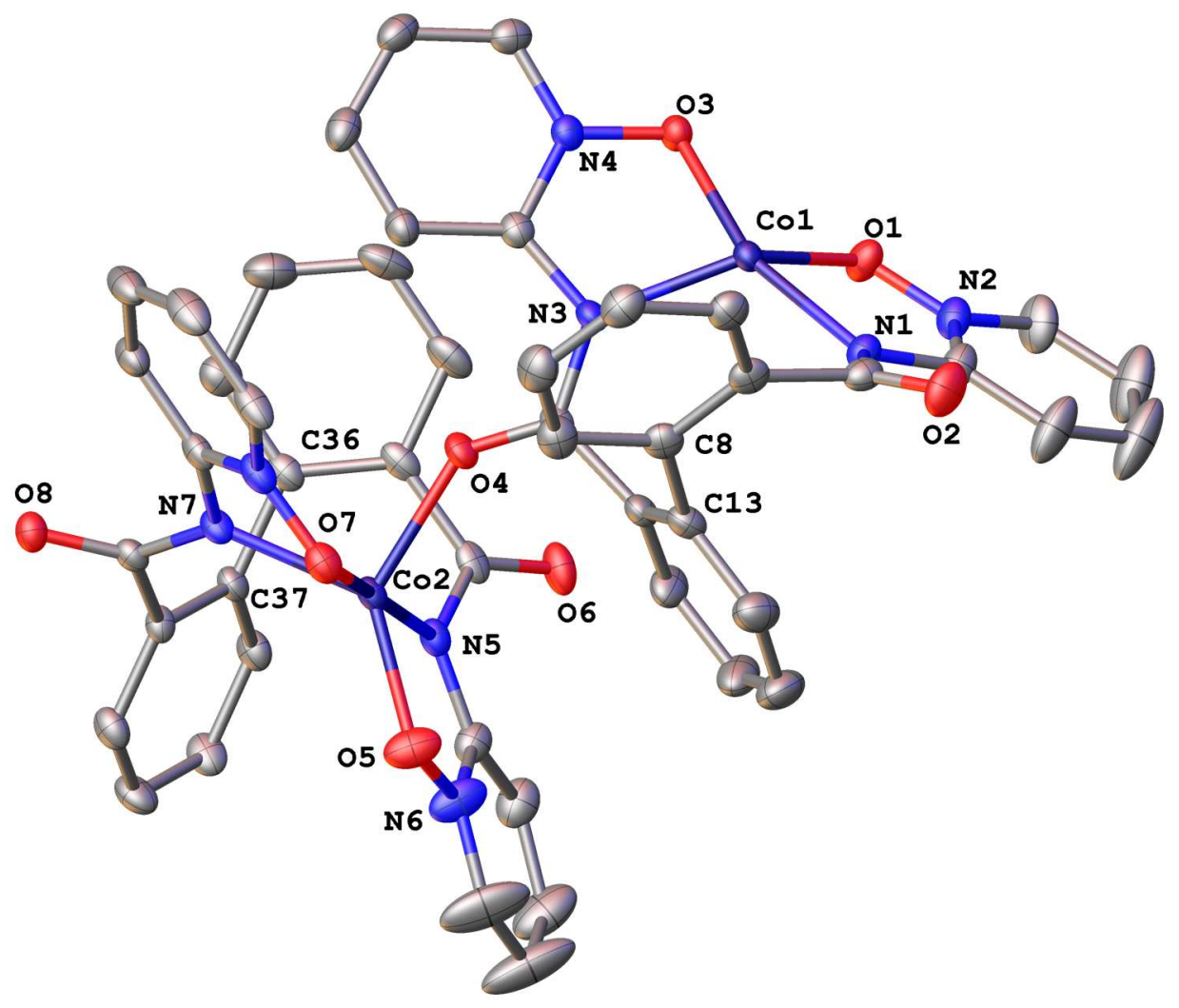

Figure 75. Molecular structure of 204 with thermal ellipsoids at 50\% probability level. The hydrogen atoms and one molecule of $\mathrm{MeOH}$ are omitted for clarity.

Table 51. Crystal data and structure refinement for 204. 
CCDC number

Empirical formula

Formula weight

Temperature

Crystal system

Space group

Unit cell dimensions

Volume

Z

Density (calculated)

Absorption coefficient

$\mathrm{F}(000)$

Crystal size

Radiation

$2 \Theta$ range for data collection

Index ranges

Reflections collected

Independent reflections

Data / restraints / parameters

Goodness-of-fit on $\mathrm{F}^{2}$

Final $R$ indices [I $>2 \sigma(\mathrm{I})]$

$R$ indices (all data)

Largest diff. peak and hole
1983757

$\mathrm{C}_{50} \mathrm{Co}_{2} \mathrm{H}_{40} \mathrm{~N}_{8} \mathrm{O}_{10}$

1030.76

$100.0 \mathrm{~K}$

monoclinic

$P 2{ }_{1} / \mathrm{c}$

$a=10.2832(5) \AA$

$\alpha=90^{\circ}$

$b=18.3959(9) \AA$

$\beta=90.550(2)^{\circ}$

$c=23.9133(13) \AA$

$\gamma=90^{\circ}$

4

$1.514 \mathrm{~g} / \mathrm{m}^{3}$

$0.805 \mathrm{~mm}^{-1}$

2120.0

$(0.251 \times 0.154 \times 0.058) \mathrm{mm}^{3}$

$\operatorname{MoK} \alpha(\lambda=0.71073)$

$3.962^{\circ}$ to $55.794^{\circ}$

$-13 \leq \mathrm{h} \leq 13,-24 \leq \mathrm{k} \leq 24,-31 \leq 1 \leq 31$

276458

$10795\left[R_{\text {int }}=0.0360, R_{\text {sigma }}=0.0107\right]$

$10795 / 0 / 595$

1.157

$R_{1}=0.0588, \mathrm{w} R_{2}=0.1343$

$R_{1}=0.0602, \mathrm{w} R_{2}=0.1350$

$1.57 \mathrm{e} \cdot \AA^{-3}$ and $-1.13 \mathrm{e} \cdot \AA^{-3}$

Table 52. Bond lengths in $[\AA]$.

\begin{tabular}{cccc}
\hline Atoms & Bond Length $[\AA]$ & Atoms & Bond Length $[\AA]$ \\
\hline $\mathrm{Co}(1)-\mathrm{O}(1)$ & $1.956(2)$ & $\mathrm{C}(7)-\mathrm{C}(12)$ & $1.394(5)$ \\
$\mathrm{Co}(1)-\mathrm{O}(3)$ & $2.086(2)$ & $\mathrm{C}(8)-\mathrm{C}(9)$ & $1.402(5)$ \\
$\mathrm{Co}(1)-\mathrm{O}\left(8^{1}\right)$ & $2.028(2)$ & $\mathrm{C}(8)-\mathrm{C}(13)$ & $1.498(5)$ \\
$\mathrm{Co}(1)-\mathrm{N}(1)$ & $2.056(3)$ & $\mathrm{C}(9)-\mathrm{C}(10)$ & $1.393(5)$ \\
$\mathrm{Co}(1)-\mathrm{N}(3)$ & $2.038(3)$ & $\mathrm{C}(10)-\mathrm{C}(11)$ & $1.379(5)$
\end{tabular}




\begin{tabular}{|c|c|c|c|}
\hline $\mathrm{Co}(2)-\mathrm{O}(4)$ & $2.040(2)$ & $\mathrm{C}(11)-\mathrm{C}(12)$ & $1.385(5)$ \\
\hline $\mathrm{Co}(2)-\mathrm{O}(5)$ & $1.954(3)$ & $C(13)-C(14)$ & $1.394(5)$ \\
\hline $\mathrm{Co}(2)-\mathrm{O}(7)$ & $2.129(2)$ & $\mathrm{C}(13)-\mathrm{C}(19)$ & $1.399(5)$ \\
\hline $\mathrm{Co}(2)-\mathrm{N}(5)$ & $2.054(3)$ & $C(14)-C(15)$ & $1.515(4)$ \\
\hline $\mathrm{Co}(2)-\mathrm{N}(7)$ & $2.043(3)$ & $C(14)-C(16)$ & $1.395(5)$ \\
\hline $\mathrm{O}(1)-\mathrm{N}(2)$ & $1.331(4)$ & $C(16)-C(17)$ & $1.389(5)$ \\
\hline $\mathrm{O}(2)-\mathrm{C}(6)$ & $1.242(4)$ & $\mathrm{C}(17)-\mathrm{C}(18)$ & $1.390(6)$ \\
\hline $\mathrm{O}(3)-\mathrm{N}(4)$ & $1.330(3)$ & $\mathrm{C}(18)-\mathrm{C}(19)$ & $1.384(6)$ \\
\hline $\mathrm{O}(4)-\mathrm{C}(15)$ & $1.245(4)$ & $\mathrm{C}(20)-\mathrm{C}(21)$ & $1.396(4)$ \\
\hline $\mathrm{O}(5)-\mathrm{N}(6)$ & $1.355(4)$ & $\mathrm{C}(21)-\mathrm{C}(22)$ & $1.386(5)$ \\
\hline $\mathrm{O}(6)-\mathrm{C}(30)$ & $1.232(4)$ & $\mathrm{C}(22)-\mathrm{C}(23)$ & $1.384(5)$ \\
\hline $\mathrm{O}(7)-\mathrm{N}(8)$ & $1.334(4)$ & $C(23)-C(24)$ & $1.372(5)$ \\
\hline $\mathrm{O}(8)-\mathrm{C}(39)$ & $1.252(4)$ & $C(25)-C(26)$ & $1.414(5)$ \\
\hline $\mathrm{N}(1)-\mathrm{C}(1)$ & $1.368(4)$ & $C(26)-C(27)$ & $1.360(6)$ \\
\hline $\mathrm{N}(1)-\mathrm{C}(6)$ & $1.365(4)$ & $\mathrm{C}(27)-\mathrm{C}(28)$ & $1.386(7)$ \\
\hline $\mathrm{N}(2)-\mathrm{C}(1)$ & $1.363(4)$ & $\mathrm{C}(28)-\mathrm{C}(29)$ & $1.377(7)$ \\
\hline $\mathrm{N}(2)-\mathrm{C}(5)$ & $1.362(4)$ & $C(30)-C(31)$ & $1.497(5)$ \\
\hline $\mathrm{N}(3)-\mathrm{C}(15)$ & $1.342(4)$ & $\mathrm{C}(31)-\mathrm{C}(32)$ & $1.392(5)$ \\
\hline $\mathrm{N}(3)-\mathrm{C}(20)$ & $1.388(4)$ & $C(31)-C(36)$ & $1.399(5)$ \\
\hline $\mathrm{N}(4)-\mathrm{C}(20)$ & $1.383(4)$ & $C(32)-C(33)$ & $1.376(6)$ \\
\hline $\mathrm{N}(4)-\mathrm{C}(24)$ & $1.356(4)$ & $\mathrm{C}(33)-\mathrm{C}(34)$ & $1.388(6)$ \\
\hline $\mathrm{N}(5)-\mathrm{C}(25)$ & $1.350(4)$ & $\mathrm{C}(34)-\mathrm{C}(35)$ & $1.395(5)$ \\
\hline $\mathrm{N}(5)-\mathrm{C}(30)$ & $1.365(4)$ & $C(35)-C(36)$ & $1.400(5)$ \\
\hline $\mathrm{N}(6)-\mathrm{C}(25)$ & $1.366(5)$ & $C(36)-C(37)$ & $1.490(4)$ \\
\hline $\mathrm{N}(6)-\mathrm{C}(29)$ & $1.334(5)$ & $\mathrm{C}(37)-\mathrm{C}(38)$ & $1.400(4)$ \\
\hline $\mathrm{N}(7)-\mathrm{C}(39)$ & $1.336(4)$ & $\mathrm{C}(37)-\mathrm{C}(43)$ & $1.395(4)$ \\
\hline $\mathrm{N}(7)-\mathrm{C}(44)$ & $1.391(4)$ & $\mathrm{C}(38)-\mathrm{C}(39)$ & $1.499(4)$ \\
\hline $\mathrm{N}(8)-\mathrm{C}(44)$ & $1.358(4)$ & $\mathrm{C}(38)-\mathrm{C}(40)$ & $1.392(5)$ \\
\hline $\mathrm{N}(8)-\mathrm{C}(48)$ & $1.359(4)$ & $\mathrm{C}(40)-\mathrm{C}(41)$ & $1.390(5)$ \\
\hline$C(1)-C(2)$ & $1.410(5)$ & $\mathrm{C}(41)-\mathrm{C}(42)$ & $1.392(5)$ \\
\hline $\mathrm{C}(2)-\mathrm{C}(3)$ & $1.371(6)$ & $\mathrm{C}(42)-\mathrm{C}(43)$ & $1.383(5)$ \\
\hline$C(3)-C(4)$ & $1.388(7)$ & $C(44)-C(45)$ & $1.388(5)$ \\
\hline$C(4)-C(5)$ & $1.370(6)$ & $C(45)-C(46)$ & $1.390(5)$ \\
\hline
\end{tabular}




$\begin{array}{llll}\mathrm{C}(6)-\mathrm{C}(7) & 1.503(5) & \mathrm{C}(46)-\mathrm{C}(47) & 1.392(6) \\ \mathrm{C}(7)-\mathrm{C}(8) & 1.404(5) & \mathrm{C}(47)-\mathrm{C}(48) & 1.371(6)\end{array}$

Table 53. Bond angles in $\left[^{\circ}\right]$.

\begin{tabular}{|c|c|c|c|}
\hline Atoms & Angle $\left[^{\circ}\right]$ & Atoms & Angle $\left[^{\circ}\right]$ \\
\hline $\mathrm{O}(1)-\mathrm{Co}(1)-\mathrm{O}(3)$ & $85.26(9)$ & $C(9)-C(8)-C(13)$ & $119.9(3)$ \\
\hline $\mathrm{O}(1)-\mathrm{Co}(1)-\mathrm{O}\left(8^{1}\right)$ & $134.65(10)$ & $\mathrm{C}(10)-\mathrm{C}(9)-\mathrm{C}(8)$ & $120.1(3)$ \\
\hline $\mathrm{O}(1)-\mathrm{Co}(1)-\mathrm{N}(1)$ & $80.21(10)$ & $\mathrm{C}(11)-\mathrm{C}(10)-\mathrm{C}(9)$ & $120.3(3)$ \\
\hline $\mathrm{O}(1)-\mathrm{Co}(1)-\mathrm{N}(3)$ & $123.24(11)$ & $\mathrm{C}(10)-\mathrm{C}(11)-\mathrm{C}(12)$ & $120.2(3)$ \\
\hline $\mathrm{O}\left(8^{1}\right)-\mathrm{Co}(1)-\mathrm{O}(3)$ & $86.95(9)$ & $C(11)-C(12)-C(7)$ & $120.6(3)$ \\
\hline $\mathrm{O}\left(8^{1}\right)-\mathrm{Co}(1)-\mathrm{N}(1)$ & $95.43(10)$ & $C(14)-C(13)-C(8)$ & $122.4(3)$ \\
\hline $\mathrm{O}\left(8^{1}\right)-\mathrm{Co}(1)-\mathrm{N}(3)$ & $98.24(10)$ & $C(14)-C(13)-C(19)$ & $119.2(3)$ \\
\hline $\mathrm{N}(1)-\mathrm{Co}(1)-\mathrm{O}(3)$ & $161.77(10)$ & $C(19)-C(13)-C(8)$ & $118.4(3)$ \\
\hline $\mathrm{N}(3)-\mathrm{Co}(1)-\mathrm{O}(3)$ & $77.48(10)$ & $C(13)-C(14)-C(15)$ & $122.3(3)$ \\
\hline $\mathrm{N}(3)-\mathrm{Co}(1)-\mathrm{N}(1)$ & $119.84(11)$ & $C(13)-C(14)-C(16)$ & $119.9(3)$ \\
\hline $\mathrm{O}(4)-\mathrm{Co}(2)-\mathrm{O}(7)$ & $85.86(9)$ & $C(16)-C(14)-C(15)$ & $117.7(3)$ \\
\hline $\mathrm{O}(4)-\mathrm{Co}(2)-\mathrm{N}(5)$ & $94.89(10)$ & $\mathrm{O}(4)-\mathrm{C}(15)-\mathrm{N}(3)$ & $124.1(3)$ \\
\hline $\mathrm{O}(4)-\mathrm{Co}(2)-\mathrm{N}(7)$ & $98.59(10)$ & $\mathrm{O}(4)-\mathrm{C}(15)-\mathrm{C}(14)$ & $118.5(3)$ \\
\hline $\mathrm{O}(5)-\mathrm{Co}(2)-\mathrm{O}(4)$ & $131.18(11)$ & $\mathrm{N}(3)-\mathrm{C}(15)-\mathrm{C}(14)$ & $117.4(3)$ \\
\hline $\mathrm{O}(5)-\mathrm{Co}(2)-\mathrm{O}(7)$ & $93.07(10)$ & $C(17)-C(16)-C(14)$ & $120.2(3)$ \\
\hline $\mathrm{O}(5)-\mathrm{Co}(2)-\mathrm{N}(5)$ & $80.66(11)$ & $C(16)-C(17)-C(18)$ & $120.2(4)$ \\
\hline $\mathrm{O}(5)-\mathrm{Co}(2)-\mathrm{N}(7)$ & $128.60(12)$ & $\mathrm{C}(19)-\mathrm{C}(18)-\mathrm{C}(17)$ & $119.6(4)$ \\
\hline $\mathrm{N}(5)-\mathrm{Co}(2)-\mathrm{O}(7)$ & $172.30(10)$ & $\mathrm{C}(18)-\mathrm{C}(19)-\mathrm{C}(13)$ & $120.9(4)$ \\
\hline $\mathrm{N}(7)-\mathrm{Co}(2)-\mathrm{O}(7)$ & $76.38(10)$ & $\mathrm{N}(3)-\mathrm{C}(20)-\mathrm{C}(21)$ & $129.4(3)$ \\
\hline $\mathrm{N}(7)-\mathrm{Co}(2)-\mathrm{N}(5)$ & $111.02(10)$ & $\mathrm{N}(4)-\mathrm{C}(20)-\mathrm{N}(3)$ & $113.4(3)$ \\
\hline $\mathrm{N}(2)-\mathrm{O}(1)-\mathrm{Co}(1)$ & 113.59(19) & $\mathrm{N}(4)-\mathrm{C}(20)-\mathrm{C}(21)$ & $117.1(3)$ \\
\hline $\mathrm{N}(4)-\mathrm{O}(3)-\mathrm{Co}(1)$ & 107.97(17) & $C(22)-C(21)-C(20)$ & $120.4(3)$ \\
\hline $\mathrm{C}(15)-\mathrm{O}(4)-\mathrm{Co}(2)$ & $138.0(2)$ & $\mathrm{C}(23)-\mathrm{C}(22)-\mathrm{C}(21)$ & $120.5(3)$ \\
\hline $\mathrm{N}(6)-\mathrm{O}(5)-\mathrm{Co}(2)$ & $112.9(2)$ & $C(24)-C(23)-C(22)$ & $118.9(3)$ \\
\hline $\mathrm{N}(8)-\mathrm{O}(7)-\mathrm{Co}(2)$ & $105.79(17)$ & $\mathrm{N}(4)-\mathrm{C}(24)-\mathrm{C}(23)$ & $120.6(3)$ \\
\hline $\mathrm{C}(39)-\mathrm{O}(8)-\mathrm{Co}\left(1^{2}\right)$ & $141.2(2)$ & $\mathrm{N}(5)-\mathrm{C}(25)-\mathrm{N}(6)$ & $114.6(3)$ \\
\hline $\mathrm{C}(1)-\mathrm{N}(1)-\mathrm{Co}(1)$ & $112.2(2)$ & $\mathrm{N}(5)-\mathrm{C}(25)-\mathrm{C}(26)$ & $128.3(3)$ \\
\hline $\mathrm{C}(6)-\mathrm{N}(1)-\mathrm{Co}(1)$ & $124.6(2)$ & $\mathrm{N}(6)-\mathrm{C}(25)-\mathrm{C}(26)$ & $116.9(3)$ \\
\hline
\end{tabular}




\begin{tabular}{|c|c|c|c|}
\hline $\mathrm{C}(6)-\mathrm{N}(1)-\mathrm{C}(1)$ & $120.9(3)$ & $\mathrm{C}(27)-\mathrm{C}(26)-\mathrm{C}(25)$ & $120.5(4)$ \\
\hline $\mathrm{O}(1)-\mathrm{N}(2)-\mathrm{C}(1)$ & $120.3(3)$ & $\mathrm{C}(26)-\mathrm{C}(27)-\mathrm{C}(28)$ & $120.4(4)$ \\
\hline $\mathrm{O}(1)-\mathrm{N}(2)-\mathrm{C}(5)$ & $116.8(3)$ & $\mathrm{C}(29)-\mathrm{C}(28)-\mathrm{C}(27)$ & $118.6(4)$ \\
\hline $\mathrm{C}(5)-\mathrm{N}(2)-\mathrm{C}(1)$ & $122.9(3)$ & $\mathrm{N}(6)-\mathrm{C}(29)-\mathrm{C}(28)$ & $120.7(4)$ \\
\hline $\mathrm{C}(15)-\mathrm{N}(3)-\mathrm{Co}(1)$ & $128.8(2)$ & $\mathrm{O}(6)-\mathrm{C}(30)-\mathrm{N}(5)$ & $126.1(3)$ \\
\hline $\mathrm{C}(15)-\mathrm{N}(3)-\mathrm{C}(20)$ & $120.1(3)$ & $\mathrm{O}(6)-\mathrm{C}(30)-\mathrm{C}(31)$ & $119.7(3)$ \\
\hline $\mathrm{C}(20)-\mathrm{N}(3)-\mathrm{Co}(1)$ & $111.1(2)$ & $\mathrm{N}(5)-\mathrm{C}(30)-\mathrm{C}(31)$ & $114.1(3)$ \\
\hline $\mathrm{O}(3)-\mathrm{N}(4)-\mathrm{C}(20)$ & $118.9(3)$ & $\mathrm{C}(32)-\mathrm{C}(31)-\mathrm{C}(30)$ & $118.9(3)$ \\
\hline $\mathrm{O}(3)-\mathrm{N}(4)-\mathrm{C}(24)$ & $118.7(3)$ & $C(32)-C(31)-C(36)$ & $120.4(3)$ \\
\hline $\mathrm{C}(24)-\mathrm{N}(4)-\mathrm{C}(20)$ & $122.4(3)$ & $C(36)-C(31)-C(30)$ & $120.6(3)$ \\
\hline $\mathrm{C}(25)-\mathrm{N}(5)-\mathrm{Co}(2)$ & $112.3(2)$ & $\mathrm{C}(33)-\mathrm{C}(32)-\mathrm{C}(31)$ & $120.7(3)$ \\
\hline $\mathrm{C}(25)-\mathrm{N}(5)-\mathrm{C}(30)$ & $120.2(3)$ & $\mathrm{C}(32)-\mathrm{C}(33)-\mathrm{C}(34)$ & $119.8(3)$ \\
\hline $\mathrm{C}(30)-\mathrm{N}(5)-\mathrm{Co}(2)$ & $123.4(2)$ & $\mathrm{C}(33)-\mathrm{C}(34)-\mathrm{C}(35)$ & $120.0(4)$ \\
\hline $\mathrm{O}(5)-\mathrm{N}(6)-\mathrm{C}(25)$ & $119.4(3)$ & $C(34)-C(35)-C(36)$ & $120.6(3)$ \\
\hline $\mathrm{C}(29)-\mathrm{N}(6)-\mathrm{O}(5)$ & $117.6(3)$ & $\mathrm{C}(31)-\mathrm{C}(36)-\mathrm{C}(35)$ & $118.4(3)$ \\
\hline $\mathrm{C}(29)-\mathrm{N}(6)-\mathrm{C}(25)$ & $122.9(3)$ & $\mathrm{C}(31)-\mathrm{C}(36)-\mathrm{C}(37)$ & $120.6(3)$ \\
\hline $\mathrm{C}(39)-\mathrm{N}(7)-\mathrm{Co}(2)$ & $130.4(2)$ & $\mathrm{C}(35)-\mathrm{C}(36)-\mathrm{C}(37)$ & $120.9(3)$ \\
\hline $\mathrm{C}(39)-\mathrm{N}(7)-\mathrm{C}(44)$ & $118.6(3)$ & $\mathrm{C}(38)-\mathrm{C}(37)-\mathrm{C}(36)$ & $121.2(3)$ \\
\hline $\mathrm{C}(44)-\mathrm{N}(7)-\mathrm{Co}(2)$ & $110.0(2)$ & $\mathrm{C}(43)-\mathrm{C}(37)-\mathrm{C}(36)$ & $120.3(3)$ \\
\hline $\mathrm{O}(7)-\mathrm{N}(8)-\mathrm{C}(44)$ & $119.8(3)$ & $\mathrm{C}(43)-\mathrm{C}(37)-\mathrm{C}(38)$ & $118.5(3)$ \\
\hline $\mathrm{O}(7)-\mathrm{N}(8)-\mathrm{C}(48)$ & 119.1(3) & $\mathrm{C}(37)-\mathrm{C}(38)-\mathrm{C}(39)$ & $120.8(3)$ \\
\hline $\mathrm{C}(44)-\mathrm{N}(8)-\mathrm{C}(48)$ & 121.1(3) & $\mathrm{C}(40)-\mathrm{C}(38)-\mathrm{C}(37)$ & $120.5(3)$ \\
\hline $\mathrm{N}(1)-\mathrm{C}(1)-\mathrm{C}(2)$ & $129.6(3)$ & $\mathrm{C}(40)-\mathrm{C}(38)-\mathrm{C}(39)$ & $118.6(3)$ \\
\hline $\mathrm{N}(2)-\mathrm{C}(1)-\mathrm{N}(1)$ & $113.7(3)$ & $\mathrm{O}(8)-\mathrm{C}(39)-\mathrm{N}(7)$ & $127.0(3)$ \\
\hline $\mathrm{N}(2)-\mathrm{C}(1)-\mathrm{C}(2)$ & 116.6(3) & $\mathrm{O}(8)-\mathrm{C}(39)-\mathrm{C}(38)$ & $117.0(3)$ \\
\hline$C(3)-C(2)-C(1)$ & $120.8(4)$ & $\mathrm{N}(7)-\mathrm{C}(39)-\mathrm{C}(38)$ & $116.0(3)$ \\
\hline$C(2)-C(3)-C(4)$ & $120.7(4)$ & $\mathrm{C}(41)-\mathrm{C}(40)-\mathrm{C}(38)$ & $120.1(3)$ \\
\hline$C(5)-C(4)-C(3)$ & $118.3(4)$ & $\mathrm{C}(40)-\mathrm{C}(41)-\mathrm{C}(42)$ & $119.7(3)$ \\
\hline $\mathrm{N}(2)-\mathrm{C}(5)-\mathrm{C}(4)$ & $120.6(4)$ & $C(43)-C(42)-C(41)$ & $120.1(3)$ \\
\hline $\mathrm{O}(2)-\mathrm{C}(6)-\mathrm{N}(1)$ & $126.5(3)$ & $\mathrm{C}(42)-\mathrm{C}(43)-\mathrm{C}(37)$ & $121.1(3)$ \\
\hline $\mathrm{O}(2)-\mathrm{C}(6)-\mathrm{C}(7)$ & $119.4(3)$ & $\mathrm{N}(8)-\mathrm{C}(44)-\mathrm{N}(7)$ & $113.5(3)$ \\
\hline $\mathrm{N}(1)-\mathrm{C}(6)-\mathrm{C}(7)$ & 114.1(3) & $\mathrm{N}(8)-\mathrm{C}(44)-\mathrm{C}(45)$ & $119.3(3)$ \\
\hline$C(8)-C(7)-C(6)$ & $121.5(3)$ & $\mathrm{C}(45)-\mathrm{C}(44)-\mathrm{N}(7)$ & $127.0(3)$ \\
\hline
\end{tabular}




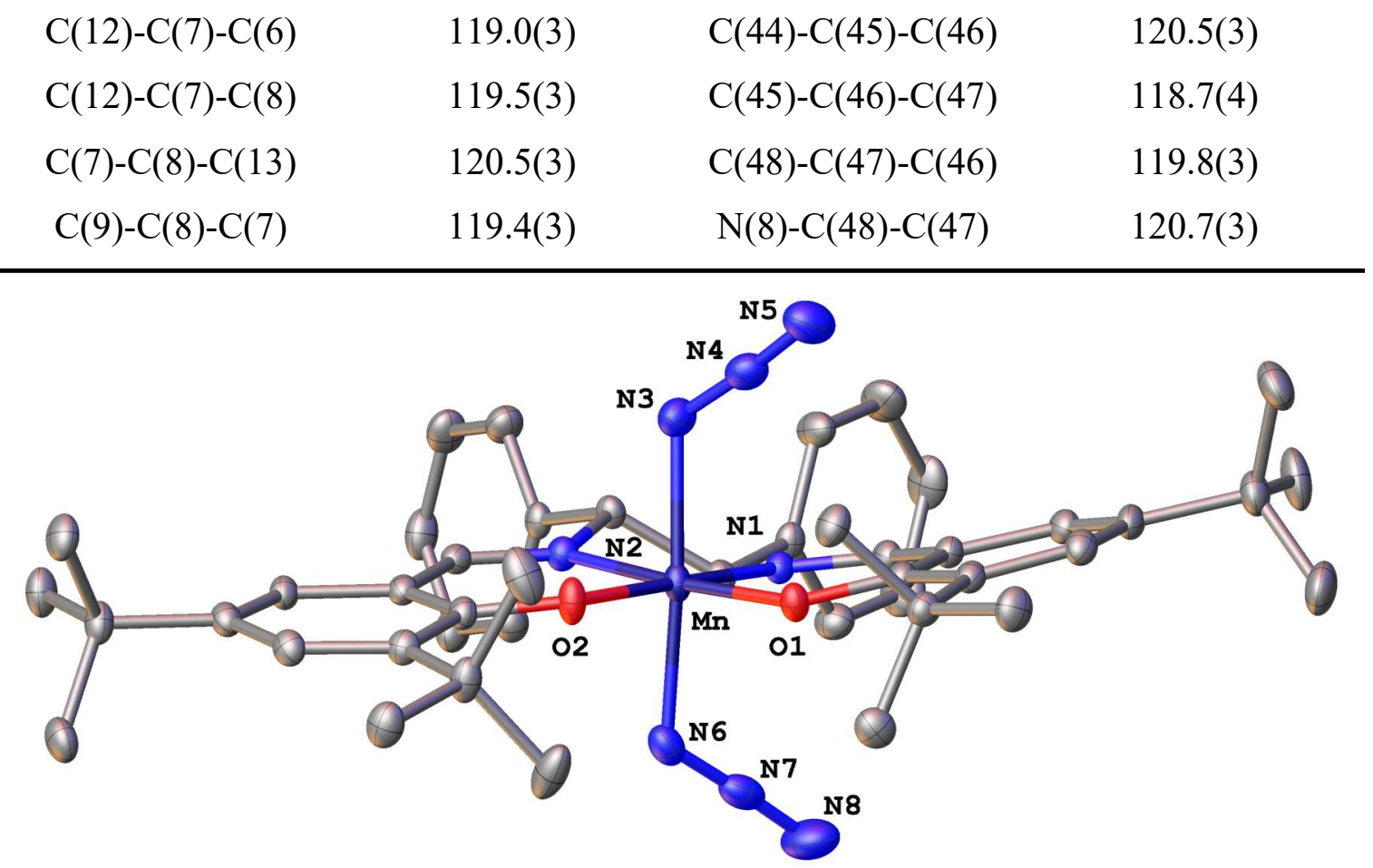

Figure 76. Molecular structure of $\operatorname{Mn} 5(\mathrm{IV})-\left(\mathrm{N}_{3}\right)_{2}$ with thermal ellipsoids at $50 \%$ probability level. The hydrogen atoms are omitted for clarity.

Table 54. Crystal data and structure refinement for $\operatorname{Mn} 5(\mathrm{IV})-\left(\mathrm{N}_{3}\right)_{2}$.

Compound

Empirical formula

Formula weight

Temperature

Crystal system

Space group

Unit cell dimensions

Volume

Z

Density (calculated)

Absorption coefficient

$\mathrm{F}(000)$

Crystal size

Radiation

$$
\begin{aligned}
& \mathrm{Mn5}(\mathrm{IV})-\left(\mathrm{N}_{3}\right)_{2} \\
& \mathrm{C}_{44} \mathrm{H}_{54} \mathrm{MnN}_{8} \mathrm{O}_{2}
\end{aligned}
$$

781.89

$100.0 \mathrm{~K}$

monoclinic

$P 2_{1}$

$$
\begin{array}{ll}
a=18.0677(6) \AA & \alpha=90^{\circ} \\
b=12.8060(4) & \beta=108.4020(10)^{\circ} \\
c=18.9198(5) & \gamma=90^{\circ}
\end{array}
$$

4

$1.250 \mathrm{~g} / \mathrm{m}^{3}$

$0.364 \mathrm{~mm}^{-1}$

1660.0

$(0.225 \times 0.185 \times 0.046) \mathrm{mm}^{3}$

$\operatorname{MoK} \alpha(\lambda=0.71073)$ 
$2 \Theta$ range for data collection

Index ranges

Reflections collected

Independent reflections

Data / restraints / parameters

Goodness-of-fit on $\mathrm{F}^{2}$

Final $R$ indices $[\mathrm{I}>2 \sigma(\mathrm{I})]$

$R$ indices (all data)

Largest diff. peak and hole

Flack parameter $3.768^{\circ}$ to $55.868^{\circ}$

$-23 \leq \mathrm{h} \leq 23,-16 \leq \mathrm{k} \leq 16,-24 \leq 1 \leq 24$

93554

$19819\left[R_{\text {int }}=0.0391, R_{\text {sigma }}=0.0343\right]$

$19819 / 100 / 1070$

1.029

$R_{1}=0.0340, \mathrm{w} R_{2}=0.0831$

$R_{1}=0.0399, \mathrm{w} R_{2}=0.0866$

$0.28 \mathrm{e} \cdot \AA^{-3}$ and $-0.47 \mathrm{e} \cdot \AA^{-3}$

$0.019(6)$

Table 55. Bond lengths in $[\AA]$.

\begin{tabular}{cccc}
\hline Atoms & Bond Length $[\AA]$ & Atoms & Bond Length $[\AA]$ \\
\hline $\mathrm{Mn}(12)-\mathrm{O}(12)$ & $1.8486(19)$ & $\mathrm{C}(162)-\mathrm{C}(172)$ & $1.427(4)$ \\
$\mathrm{Mn}(12)-\mathrm{O}(22)$ & $1.8440(19)$ & $\mathrm{C}(162)-\mathrm{C}(462)$ & $1.414(4)$ \\
$\mathrm{Mn}(12)-\mathrm{N}(12)$ & $2.000(2)$ & $\mathrm{C}(172)-\mathrm{C}(182)$ & $1.379(4)$ \\
$\mathrm{Mn}(12)-\mathrm{N}(42)$ & $1.999(2)$ & $\mathrm{C}(172)-\mathrm{C}(272)$ & $1.542(4)$ \\
$\mathrm{Mn}(12)-\mathrm{N}(72)$ & $1.963(2)$ & $\mathrm{C}(182)-\mathrm{C}(192)$ & $1.413(4)$ \\
$\mathrm{Mn}(12)-\mathrm{N}(82)$ & $1.978(2)$ & $\mathrm{C}(192)-\mathrm{C}(202)$ & $1.372(4)$ \\
$\mathrm{O}(12)-\mathrm{C}(12)$ & $1.330(3)$ & $\mathrm{C}(192)-\mathrm{C}(232)$ & $1.531(4)$ \\
$\mathrm{O}(22)-\mathrm{C}(162)$ & $1.320(3)$ & $\mathrm{C}(202)-\mathrm{C}(462)$ & $1.427(3)$ \\
$\mathrm{N}(12)-\mathrm{N}(22)$ & $1.212(4)$ & $\mathrm{C}(222)-\mathrm{C}(462)$ & $1.439(4)$ \\
$\mathrm{N}(22)-\mathrm{N}(32)$ & $1.147(4)$ & $\mathrm{C}(232)-\mathrm{C}(242)$ & $1.540(4)$ \\
$\mathrm{N}(42)-\mathrm{N}(52)$ & $1.208(3)$ & $\mathrm{C}(232)-\mathrm{C}(252)$ & $1.535(4)$ \\
$\mathrm{N}(52)-\mathrm{N}(62)$ & $1.140(4)$ & $\mathrm{C}(232)-\mathrm{C}(262)$ & $1.532(4)$ \\
$\mathrm{N}(72)-\mathrm{C}(72)$ & $1.293(3)$ & $\mathrm{C}(272)-\mathrm{C}(282)$ & $1.537(4)$ \\
$\mathrm{N}(72)-\mathrm{C}(312)$ & $1.489(3)$ & $\mathrm{C}(272)-\mathrm{C}(292)$ & $1.534(4)$ \\
$\mathrm{N}(82)-\mathrm{C}(222)$ & $1.293(3)$ & $\mathrm{C}(272)-\mathrm{C}(302)$ & $1.535(4)$ \\
$\mathrm{N}(82)-\mathrm{C}(322)$ & $1.483(3)$ & $\mathrm{C}(312)-\mathrm{C}(322)$ & $1.542(3)$ \\
$\mathrm{C}(12)-\mathrm{C}(22)$ & $1.426(4)$ & $\mathrm{C}(312)-\mathrm{C}(392)$ & $1.508(4)$ \\
$\mathrm{C}(12)-\mathrm{C}(62)$ & $1.406(4)$ & $\mathrm{C}(322)-\mathrm{C}(332)$ & $1.514(3)$ \\
$\mathrm{C}(22)-\mathrm{C}(32)$ & $1.386(4)$ & $\mathrm{C}(332)-\mathrm{C}(342)$ & $1.392(4)$ \\
$\mathrm{C}(22)-\mathrm{C}(122)$ & $1.529(4)$ & $\mathrm{C}(332)-\mathrm{C}(382)$ & $1.393(4)$ \\
& & &
\end{tabular}




$\begin{array}{rlll}\mathrm{C}(32)-\mathrm{C}(42) & 1.403(4) & \mathrm{C}(342)-\mathrm{C}(352) & 1.391(4) \\ \mathrm{C}(42)-\mathrm{C}(52) & 1.378(4) & \mathrm{C}(352)-\mathrm{C}(362) & 1.379(4) \\ \mathrm{C}(42)-\mathrm{C}(82) & 1.530(4) & \mathrm{C}(362)-\mathrm{C}(372) & 1.379(4) \\ \mathrm{C}(52)-\mathrm{C}(62) & 1.417(4) & \mathrm{C}(372)-\mathrm{C}(382) & 1.393(4) \\ \mathrm{C}(62)-\mathrm{C}(72) & 1.441(4) & \mathrm{C}(392)-\mathrm{C}(402) & 1.386(4) \\ \mathrm{C}(82)-\mathrm{C}(92) & 1.549(5) & \mathrm{C}(392)-\mathrm{C}(442) & 1.392(4) \\ \mathrm{C}(82)-\mathrm{C}(102) & 1.523(5) & \mathrm{C}(402)-\mathrm{C}(472) & 1.389(4) \\ \mathrm{C}(82)-\mathrm{C}(452) & 1.532(4) & \mathrm{C}(422)-\mathrm{C}(432) & 1.383(4) \\ \mathrm{C}(122)-\mathrm{C}(132) & 1.539(4) & \mathrm{C}(422)-\mathrm{C}(472) & 1.370(5) \\ \mathrm{C}(122)-\mathrm{C}(142) & 1.526(4) & \mathrm{C}(432)-\mathrm{C}(442) & 1.379(4) \\ \mathrm{C}(122)-\mathrm{C}(152) & 1.543(4) & & \end{array}$

Table 56. Bond angles in $\left[^{\circ}\right]$.

\begin{tabular}{cccc}
\hline Atoms & Angle [ $\left.{ }^{\circ}\right]$ & Atoms & Angle [ ${ }^{\circ}$ \\
\hline $\mathrm{O}(12)-\mathrm{Mn}(12)-\mathrm{N}(12)$ & $93.25(9)$ & $\mathrm{C}(142)-\mathrm{C}(122)-\mathrm{C}(132)$ & $108.0(2)$ \\
$\mathrm{O}(12)-\mathrm{Mn}(12)-\mathrm{N}(42)$ & $93.30(9)$ & $\mathrm{C}(142)-\mathrm{C}(122)-\mathrm{C}(152)$ & $106.9(2)$ \\
$\mathrm{O}(12)-\mathrm{Mn}(12)-\mathrm{N}(72)$ & $91.48(9)$ & $\mathrm{O}(22)-\mathrm{C}(162)-\mathrm{C}(172)$ & $118.8(2)$ \\
$\mathrm{O}(12)-\mathrm{Mn}(12)-\mathrm{N}(82)$ & $174.26(9)$ & $\mathrm{O}(22)-\mathrm{C}(162)-\mathrm{C}(462)$ & $122.2(2)$ \\
$\mathrm{O}(22)-\mathrm{Mn}(12)-\mathrm{O}(12)$ & $92.99(8)$ & $\mathrm{C}(462)-\mathrm{C}(162)-\mathrm{C}(172)$ & $119.0(2)$ \\
$\mathrm{O}(22)-\mathrm{Mn}(12)-\mathrm{N}(12)$ & $89.27(10)$ & $\mathrm{C}(162)-\mathrm{C}(172)-\mathrm{C}(272)$ & $121.1(2)$ \\
$\mathrm{O}(22)-\mathrm{Mn}(12)-\mathrm{N}(42)$ & $92.18(10)$ & $\mathrm{C}(182)-\mathrm{C}(172)-\mathrm{C}(162)$ & $117.8(3)$ \\
$\mathrm{O}(22)-\mathrm{Mn}(12)-\mathrm{N}(72)$ & $175.05(9)$ & $\mathrm{C}(182)-\mathrm{C}(172)-\mathrm{C}(272)$ & $121.1(2)$ \\
$\mathrm{O}(22)-\mathrm{Mn}(12)-\mathrm{N}(82)$ & $92.29(9)$ & $\mathrm{C}(172)-\mathrm{C}(182)-\mathrm{C}(192)$ & $124.4(3)$ \\
$\mathrm{N}(42)-\mathrm{Mn}(12)-\mathrm{N}(12)$ & $173.21(11)$ & $\mathrm{C}(182)-\mathrm{C}(192)-\mathrm{C}(232)$ & $117.6(2)$ \\
$\mathrm{N}(72)-\mathrm{Mn}(12)-\mathrm{N}(12)$ & $88.35(10)$ & $\mathrm{C}(202)-\mathrm{C}(192)-\mathrm{C}(182)$ & $117.3(2)$ \\
$\mathrm{N}(72)-\mathrm{Mn}(12)-\mathrm{N}(42)$ & $89.70(10)$ & $\mathrm{C}(202)-\mathrm{C}(192)-\mathrm{C}(232)$ & $125.0(3)$ \\
$\mathrm{N}(72)-\mathrm{Mn}(12)-\mathrm{N}(82)$ & $83.33(9)$ & $\mathrm{C}(192)-\mathrm{C}(202)-\mathrm{C}(462)$ & $121.1(3)$ \\
$\mathrm{N}(82)-\mathrm{Mn}(12)-\mathrm{N}(12)$ & $89.06(10)$ & $\mathrm{N}(82)-\mathrm{C}(222)-\mathrm{C}(462)$ & $126.1(3)$ \\
$\mathrm{N}(82)-\mathrm{Mn}(12)-\mathrm{N}(42)$ & $84.26(10)$ & $\mathrm{C}(192)-\mathrm{C}(232)-\mathrm{C}(242)$ & $108.6(2)$ \\
$\mathrm{C}(12)-\mathrm{O}(12)-\mathrm{Mn}(12)$ & $130.10(18)$ & $\mathrm{C}(192)-\mathrm{C}(232)-\mathrm{C}(252)$ & $109.8(2)$ \\
$\mathrm{C}(162)-\mathrm{O}(22)-\mathrm{Mn}(12)$ & $130.85(18)$ & $\mathrm{C}(192)-\mathrm{C}(232)-\mathrm{C}(262)$ & $112.3(2)$ \\
$\mathrm{N}(22)-\mathrm{N}(12)-\mathrm{Mn}(12)$ & $120.2(2)$ & $\mathrm{C}(252)-\mathrm{C}(232)-\mathrm{C}(242)$ & $110.1(2)$ \\
$\mathrm{N}(32)-\mathrm{N}(22)-\mathrm{N}(12)$ & $177.4(3)$ & $\mathrm{C}(262)-\mathrm{C}(232)-\mathrm{C}(242)$ & $107.6(2)$
\end{tabular}




\begin{tabular}{|c|c|c|c|}
\hline $\mathrm{N}(52)-\mathrm{N}(42)-\mathrm{Mn}(12)$ & $120.9(2)$ & $C(262)-C(232)-C(252)$ & $108.4(2)$ \\
\hline $\mathrm{N}(62)-\mathrm{N}(52)-\mathrm{N}(42)$ & $175.8(3)$ & $\mathrm{C}(282)-\mathrm{C}(272)-\mathrm{C}(172)$ & $108.8(2)$ \\
\hline $\mathrm{C}(72)-\mathrm{N}(72)-\mathrm{Mn}(12)$ & $125.59(19)$ & $C(292)-C(272)-C(172)$ & $110.1(2)$ \\
\hline $\mathrm{C}(72)-\mathrm{N}(72)-\mathrm{C}(312)$ & $122.2(2)$ & $\mathrm{C}(292)-\mathrm{C}(272)-\mathrm{C}(282)$ & $110.9(3)$ \\
\hline $\mathrm{C}(312)-\mathrm{N}(72)-\mathrm{Mn}(12)$ & $112.11(15)$ & $\mathrm{C}(292)-\mathrm{C}(272)-\mathrm{C}(302)$ & $107.5(3)$ \\
\hline $\mathrm{C}(222)-\mathrm{N}(82)-\mathrm{Mn}(12)$ & $124.6(2)$ & $C(302)-C(272)-C(172)$ & $111.5(2)$ \\
\hline $\mathrm{C}(222)-\mathrm{N}(82)-\mathrm{C}(322)$ & $122.7(2)$ & $\mathrm{C}(302)-\mathrm{C}(272)-\mathrm{C}(282)$ & $108.1(2)$ \\
\hline $\mathrm{C}(322)-\mathrm{N}(82)-\mathrm{Mn}(12)$ & $112.27(15)$ & $\mathrm{N}(72)-\mathrm{C}(312)-\mathrm{C}(322)$ & $105.45(18)$ \\
\hline $\mathrm{O}(12)-\mathrm{C}(12)-\mathrm{C}(22)$ & $118.6(2)$ & $\mathrm{N}(72)-\mathrm{C}(312)-\mathrm{C}(392)$ & $114.1(2)$ \\
\hline $\mathrm{O}(12)-\mathrm{C}(12)-\mathrm{C}(62)$ & $122.0(2)$ & $\mathrm{C}(392)-\mathrm{C}(312)-\mathrm{C}(322)$ & $115.2(2)$ \\
\hline$C(62)-C(12)-C(22)$ & $119.3(2)$ & $\mathrm{N}(82)-\mathrm{C}(322)-\mathrm{C}(312)$ & $105.71(19)$ \\
\hline$C(12)-C(22)-C(122)$ & $121.1(2)$ & $\mathrm{N}(82)-\mathrm{C}(322)-\mathrm{C}(332)$ & $116.6(2)$ \\
\hline$C(32)-C(22)-C(12)$ & $117.1(3)$ & $\mathrm{C}(332)-\mathrm{C}(322)-\mathrm{C}(312)$ & $111.31(19)$ \\
\hline $\mathrm{C}(32)-\mathrm{C}(22)-\mathrm{C}(122)$ & $121.8(2)$ & $\mathrm{C}(342)-\mathrm{C}(332)-\mathrm{C}(322)$ & $120.0(2)$ \\
\hline$C(22)-C(32)-C(42)$ & $124.9(3)$ & $\mathrm{C}(342)-\mathrm{C}(332)-\mathrm{C}(382)$ & $118.8(3)$ \\
\hline $\mathrm{C}(32)-\mathrm{C}(42)-\mathrm{C}(82)$ & 119.6(3) & $\mathrm{C}(382)-\mathrm{C}(332)-\mathrm{C}(322)$ & $121.0(2)$ \\
\hline$C(52)-C(42)-C(32)$ & $117.3(2)$ & $\mathrm{C}(352)-\mathrm{C}(342)-\mathrm{C}(332)$ & $120.4(3)$ \\
\hline $\mathrm{C}(52)-\mathrm{C}(42)-\mathrm{C}(82)$ & $123.1(3)$ & $\mathrm{C}(362)-\mathrm{C}(352)-\mathrm{C}(342)$ & $120.2(3)$ \\
\hline $\mathrm{C}(42)-\mathrm{C}(52)-\mathrm{C}(62)$ & $120.8(3)$ & $\mathrm{C}(372)-\mathrm{C}(362)-\mathrm{C}(352)$ & $120.0(3)$ \\
\hline$C(12)-C(62)-C(52)$ & $120.6(2)$ & $\mathrm{C}(362)-\mathrm{C}(372)-\mathrm{C}(382)$ & $120.1(3)$ \\
\hline$C(12)-C(62)-C(72)$ & $122.5(2)$ & $\mathrm{C}(372)-\mathrm{C}(382)-\mathrm{C}(332)$ & $120.5(3)$ \\
\hline$C(52)-C(62)-C(72)$ & $116.8(2)$ & $\mathrm{C}(402)-\mathrm{C}(392)-\mathrm{C}(312)$ & $119.0(2)$ \\
\hline $\mathrm{N}(72)-\mathrm{C}(72)-\mathrm{C}(62)$ & $125.3(2)$ & $\mathrm{C}(402)-\mathrm{C}(392)-\mathrm{C}(442)$ & $118.7(3)$ \\
\hline $\mathrm{C}(42)-\mathrm{C}(82)-\mathrm{C}(92)$ & $108.6(3)$ & $\mathrm{C}(442)-\mathrm{C}(392)-\mathrm{C}(312)$ & $122.2(2)$ \\
\hline $\mathrm{C}(42)-\mathrm{C}(82)-\mathrm{C}(452)$ & $109.4(2)$ & $C(392)-C(402)-C(472)$ & $120.6(3)$ \\
\hline $\mathrm{C}(102)-\mathrm{C}(82)-\mathrm{C}(42)$ & $112.0(3)$ & $\mathrm{C}(472)-\mathrm{C}(422)-\mathrm{C}(432)$ & $120.0(3)$ \\
\hline$C(102)-C(82)-C(92)$ & $109.4(3)$ & $\mathrm{C}(442)-\mathrm{C}(432)-\mathrm{C}(422)$ & $120.2(3)$ \\
\hline $\mathrm{C}(102)-\mathrm{C}(82)-\mathrm{C}(452)$ & 109.2(3) & $\mathrm{C}(432)-\mathrm{C}(442)-\mathrm{C}(392)$ & $120.5(3)$ \\
\hline $\mathrm{C}(452)-\mathrm{C}(82)-\mathrm{C}(92)$ & $108.2(3)$ & $C(162)-C(462)-C(202)$ & $120.1(2)$ \\
\hline $\mathrm{C}(22)-\mathrm{C}(122)-\mathrm{C}(132)$ & $109.8(2)$ & $\mathrm{C}(162)-\mathrm{C}(462)-\mathrm{C}(222)$ & $122.6(2)$ \\
\hline $\mathrm{C}(22)-\mathrm{C}(122)-\mathrm{C} 152)$ & $110.1(2)$ & $C(202)-C(462)-C(222)$ & $117.2(2)$ \\
\hline$C(132)-C(122)-C 152)$ & $110.3(2)$ & $\mathrm{C}(422)-\mathrm{C}(472)-\mathrm{C}(402)$ & $120.0(3)$ \\
\hline $\mathrm{C}(142)-\mathrm{C}(122)-\mathrm{C} 22)$ & $111.8(2)$ & & \\
\hline
\end{tabular}




\section{REFERENCES}

[1] H. Remane, A. Hantschmann, M. Weißenfels, Z. Chem. 1984, 24, 393-403.

[2] R. Keen, The life and work of Friedrich Wöhler (1800-1882), Traugott Bautz $\mathrm{GmbH}$, Nordhausen, 2005.

[3] a) C. Jimenez-Gonzalez, C. S. Ponder, Q. B. Broxterman, J. B. Manley, Org. Process Res. Dev. 2011, 15, 912-917; b) R. A. Sheldon, Green Chem. 2007, 9, $1273-$ 1283; c) D. J. C. Constable, A. D. Curzons, V. L. Cunningham, Green Chem. 2002, 4, 521-527.

[4] a) J. B. Zimmerman, P. T. Anastas, H. C. Erythropel, W. Leitner, Science 2020, 367, 397-400; b) T. Keijer, V. Bakker, J. C. Slootweg, Nat. Chem. 2019, 11, 190195; c) G. T. Whiteker, Org. Process Res. Dev. 2019, 23, 2109-2121; d) H. C. Erythropel, J. B. Zimmerman, T. M. de Winter, L. Petitjean, F. Melnikov, C. H. Lam, A. W. Lounsbury, K. E. Mellor, N. Z. Janković, Q. Tu, L. N. Pincus, M. M. Falinski, W. Shi, P. Coish, D. L. Plata, P. T. Anastas, Green Chem. 2018, 20, 19291961; e) D. Cespi, E. S. Beach, T. E. Swarr, F. Passarini, I. Vassura, P. J. Dunn, P. T. Anastas, Green Chem. 2015, 17, 3390-3400; f) D. Kralisch, D. Ott, D. Gericke, Green Chem. 2015, 17, 123-145; g) P. T. Anastas, J. C. Warner, Green chemistry : theory and practice, Oxford University Press, New York, 2014; h) S. Y. Tang, R. A. Bourne, R. L. Smith, M. Poliakoff, Green Chem. 2008, 10, 268-269; i) P. T. Anastas, R. L. Lankey, Green Chem. 2000, 2, 289-295.

[5] a) B. Cornils, W. A. Herrmann, M. Beller, R. Paciello, Applied homogeneous catalysis with organometallic compounds a comprehensive handbook in four volumes, Wiley-VCH, Weinheim, 2018; b) A. De Meijere, S. Bräse, M. Oestreich, Metal Catalyzed Cross-Coupling Reactions and More, Wiley-VCH, Weinheim, 2014; c) C. A. Busacca, D. R. Fandrick, J. J. Song, C. H. Senanayake, Adv. Synth. Catal. 2011, 353, 1825-1864; d) L. Ackermann, Modern arylation methods, WileyVCH, Weinheim, 2009; e) F. Diederich, Metal catalyzed cross coupling reactions, Wiley-VCH, Weinheim, 2001.

[6] Nobel Prize Home Page: https://www.nobelprize.org/prizes/chemistry/2010/ summary/ (accessed on 10.Apr.2021)

[7] T. Laird, Org. Process Res. Dev. 2012, 16, 1-2.

[8] C. C. C. Johansson Seechurn, M. O. Kitching, T. J. Colacot, V. Snieckus, Angew. Chem. Int. Ed. 2012, 51, 5062-5085.

[9] T. H. Meyer, L. H. Finger, P. Gandeepan, L. Ackermann, Trends Chem. 2019, 1, 63-76.

[10] a) B. M. Trost, Angew. Chem. Int. Ed. Engl. 1995, 34, 259-281; b) B. Trost, Science 1991, 254, 1471-1477.

[11] a) T. Dalton, T. Faber, F. Glorius, ACS Cent Sci 2021, 7, 245-261; b) S. Rej, Y. Ano, N. Chatani, Chem. Rev. 2020, 120, 1788-1887; c) O. Baudoin, Angew. Chem. Int. Ed. 2020, 59, 17798-17809; d) Ł. Woźniak, N. Cramer, Trends Chem. 2019, 1, 471-484; e) J. Loup, U. Dhawa, F. Pesciaioli, J. Wencel-Delord, L. Ackermann, Angew. Chem. Int. Ed. 2019, 58, 12803-12818; f) W. Wang, M. M. Lorion, J. Shah, A. R. Kapdi, L. Ackermann, Angew. Chem. Int. Ed. 2018, 57, 14700-14717; g) J. He, M. Wasa, K. S. L. Chan, Q. Shao, J.-Q. Yu, Chem. Rev. 2017, 117, 8754-8786; h) Y. Park, Y. Kim, S. Chang, Chem. Rev. 2017, 117, 9247-9301; i) M. M. Lorion, K. Maindan, A. R. Kapdi, L. Ackermann, Chem. Soc. Rev. 2017, 46, 7399-7420; j) J. A. Leitch, C. G. Frost, Chem. Soc. Rev. 2017, 46, 7145-7153; k) C. G. Newton, 
S.-G. Wang, C. C. Oliveira, N. Cramer, Chem. Rev. 2017, 117, 8908-8976; 1) Y. Wei, P. Hu, M. Zhang, W. Su, Chem. Rev. 2017, 117, 8864-8907; m) J. Li, S. De Sarkar, L. Ackermann, Top. Organomet. Chem. 2016, 217-257; n) Q.-Z. Zheng, N. Jiao, Chem. Soc. Rev. 2016, 45, 4590-4627; o) L. Ackermann, Acc. Chem. Res. 2014, 47, 281-295; p) J. Wencel-Delord, F. Glorius, Nat Chem 2013, 5, 369-375; q) J. Yamaguchi, A. D. Yamaguchi, K. Itami, Angew. Chem. Int. Ed. 2012, 51, 8960-9009; r) P. B. Arockiam, C. Bruneau, P. H. Dixneuf, Chem. Rev. 2012, 112, 5879-5918; s) L. McMurray, F. O'Hara, M. J. Gaunt, Chem. Soc. Rev. 2011, 40, 1885-1898; t) T. Satoh, M. Miura, Chem. Eur. J. 2010, 16, 11212-11222; u) T. W. Lyons, M. S. Sanford, Chem. Rev. 2010, 110, 1147-1169; v) D. A. Colby, R. G. Bergman, J. A. Ellman, Chem. Rev. 2010, 110, 624-655; w) X. Chen, K. M. Engle, D.-H. Wang, J.-Q. Yu, Angew. Chem. Int. Ed. 2009, 48, 5094-5115; x) R. G. Bergman, Nature 2007, 446, 391-393; y) L.-C. Campeau, D. R. Stuart, K. Fagnou, Aldrichim. Acta 2007, 40, 35-41; z) F. Kakiuchi, Top. Organomet. Chem. 2007, 24, $1-33$.

[12] a) P. A. Wender, B. L. Miller, Nature 2009, 460, 197-201; b) P. A. Wender, V. A. Verma, T. J. Paxton, T. H. Pillow, Acc. Chem. Res. 2008, 41, 40-49; c) P. A. Wender, M. P. Croatt, B. Witulski, Tetrahedron 2006, 62, 7505-7511.

[13] a) R. C. Samanta, T. H. Meyer, I. Siewert, L. Ackermann, Chem. Sci. 2020, 11, 8657-8670; b) O. Daugulis, Top. Curr. Chem. 2010, 292, 57-84.

[14] a) C.-Y. Huang, H. Kang, J. Li, C.-J. Li, J. Org. Chem. 2019, 84, 12705-12721; b) Y. Yang, J. Lan, J. You, Chem. Rev. 2017, 117, 8787-8863; c) C. S. Yeung, V. M. Dong, Chem. Rev. 2011, 111, 1215-1292; d) S. H. Cho, J. Y. Kim, J. Kwak, S. Chang, Chem. Soc. Rev. 2011, 40, 5068-5083; e) J. A. Ashenhurst, Chem. Soc. Rev. 2010, 39, 540-548; f) C.-J. Li, Acc. Chem. Res. 2009, 42, 335-344.

[15] X.-S. Xue, P. Ji, B. Zhou, J.-P. Cheng, Chem. Rev. 2017, 117, 8622-8648.

[16] a) Z. Huang, G. Dong, Acc. Chem. Res. 2017, 50, 465-471; b) L. Ackermann, Top. Organomet. Chem. 2007, 24, 35-60.

[17] F. G. Bordwell, Acc. Chem. Res. 1988, 21, 456-463.

[18] E. M. Beck, M. J. Gaunt, Top. Curr. Chem. 2010, 292, 85-121.

[19] T. P. Pabst, P. J. Chirik, Organometallics 2021.

[20] S. De Sarkar, W. Liu, S. I. Kozhushkov, L. Ackermann, Adv. Synth. Catal. 2014, 356, 1461-1479.

[21] W. Ma, P. Gandeepan, J. Li, L. Ackermann, Org. Chem. Front. 2017, 4, 1435-1467.

[22] P. Gandeepan, L. Ackermann, Chem 2018, 4, 199-222.

[23] a) P. Gandeepan, T. Müller, D. Zell, G. Cera, S. Warratz, L. Ackermann, Chem. Rev. 2019, 119, 2192-2452; b) L. Ackermann, Chem. Rev. 2011, 111, 1315-1345; c) R. Giri, B.-F. Shi, K. M. Engle, N. Maugel, J.-Q. Yu, Chem. Soc. Rev. 2009, 38, 3242-3272; d) R. G. Bergman, Nature 2007, 446, 391-393; e) G. Dyker, Handbook of $\mathrm{C}-\mathrm{H}$ transformations: applications in organic synthesis, Wiley-VCH, Weinheim, 2005; f) R. H. Crabtree, J. Organomet. Chem. 2004, 689, 4083-4091; g) J. A. Labinger, J. E. Bercaw, Nature 2002, 417, 507-514.

[24] a) M. Costas, in Remote $C-H$ Bond Functionalizations, Eds.: D. Maiti, S. Guin, Wiley-VCH, Weinheim 2021, pp. 383-421; b) L. Vicens, G. Olivo, M. Costas, $A C S$ Catal. 2020, 10, 8611-8631; c) X. Huang, J. T. Groves, Chem. Rev. 2018, 118, 2491-2553; d) M. Milan, M. Salamone, M. Costas, M. Bietti, Acc. Chem. Res. 2018, 51, 1984-1995; e) H. Yi, G. Zhang, H. Wang, Z. Huang, J. Wang, A. K. Singh, A. Lei, Chem. Rev. 2017, 117, 9016-9085; f) X. Huang, J. T. Groves, JBIC J. Biolog. Inorg. Chem. 2017, 22, 185-207; g) R. A. Baglia, J. P. T. Zaragoza, D. P. Goldberg, Chem. Rev. 2017, 117, 13320-13352; h) A. S. Borovik, Chem. Soc. Rev. 2011, 40, 
1870-1874; i) M. Costas, Coord. Chem. Rev. 2011, 255, 2912-2932; j) A. Gunay, K. H. Theopold, Chem. Rev. 2010, 110, 1060-1081.

[25] a) M. Albrecht, Chem. Rev. 2010, 110, 576-623; b) D. Balcells, E. Clot, O. Eisenstein, Chem. Rev. 2010, 110, 749-823; c) B. A. Arndtsen, R. G. Bergman, T. A. Mobley, T. H. Peterson, Acc. Chem. Res. 1995, 28, 154-162.

[26] T. G. P. Harper, P. J. Desrosiers, T. C. Flood, Organometallics 1990, 9, 2523-2528.

[27] G. Erker, J. Organomet. Chem. 1977, 134, 189-202.

[28] M. Lersch, M. Tilset, Chem. Rev. 2005, 105, 2471-2526.

[29] J. Kua, X. Xu, R. A. Periana, W. A. Goddard, Organometallics 2002, 21, 511-525.

[30] J. Oxgaard, W. J. Tenn, R. J. Nielsen, R. A. Periana, W. A. Goddard, Organometallics 2007, 26, 1565-1567.

[31] a) J. L. Bennett, P. T. Wolczanski, J. Am. Chem. Soc. 1997, 119, 10696-10719; b) C. C. Cummins, S. M. Baxter, P. T. Wolczanski, J. Am. Chem. Soc. 1988, 110, 8731-8733; c) P. J. Walsh, F. J. Hollander, R. G. Bergman, J. Am. Chem. Soc. 1988, 110, 8729-8731.

[32] I. Özdemir, S. Demir, B. Çetinkaya, C. Gourlaouen, F. Maseras, C. Bruneau, P. H. Dixneuf, J. Am. Chem. Soc. 2008, 130, 1156-1157.

[33] a) L. Ackermann, Synthesis 2006, 1557-1571; b) L. Ackermann, Org. Lett. 2005, 7, 3123-3125.

[34] a) D. L. Davies, S. A. Macgregor, C. L. McMullin, Chem. Rev. 2017, 117, 86498709; b) B. Biswas, M. Sugimoto, S. Sakaki, Organometallics 2000, 19, 3895-3908.

[35] T. Rogge, J. C. A. Oliveira, R. Kuniyil, L. Hu, L. Ackermann, ACS Catal. 2020, 10, 10551-10558.

[36] S. I. Gorelsky, D. Lapointe, K. Fagnou, J. Am. Chem. Soc. 2008, 130, 10848-10849.

[37] L. David, F. Keith, Chem. Lett. 2010, 39, 1118-1126.

[38] D. García-Cuadrado, A. A. C. Braga, F. Maseras, A. M. Echavarren, J. Am. Chem. Soc. 2006, 128, 1066-1067.

[39] a) L.-C. Campeau, D. R. Stuart, J.-P. Leclerc, M. Bertrand-Laperle, E. Villemure, H.-Y. Sun, S. Lasserre, N. Guimond, M. Lecavallier, K. Fagnou, J. Am. Chem. Soc. 2009, 131, 3291-3306; b) L.-C. Campeau, M. Bertrand-Laperle, J.-P. Leclerc, E. Villemure, S. Gorelsky, K. Fagnou, J. Am. Chem. Soc. 2008, 130, 3276-3277; c) J.P. Leclerc, K. Fagnou, Angew. Chem. Int. Ed. 2006, 45, 7781-7786; d) L.-C. Campeau, S. Rousseaux, K. Fagnou, J. Am. Chem. Soc. 2005, 127, 18020-18021.

[40] a) Y. Boutadla, D. L. Davies, S. A. Macgregor, A. I. Poblador-Bahamonde, Dalton Trans. 2009, 5820-5831; b) Y. Boutadla, D. L. Davies, S. A. Macgregor, A. I. Poblador-Bahamonde, Dalton Trans. 2009, 5887-5893; c) D. L. Davies, S. M. A. Donald, S. A. Macgregor, J. Am. Chem. Soc. 2005, 127, 13754-13755.

[41] a) L. Wang, B. P. Carrow, ACS Catal. 2019, 9, 6821-6836; b) K. Naksomboon, J. Poater, F. M. Bickelhaupt, M. Á. Fernández-Ibáñez, J. Am. Chem. Soc. 2019, 141, 6719-6725; c) E. Tan, O. Quinonero, M. Elena de Orbe, A. M. Echavarren, $A C S$ Catal. 2018, 8, 2166-2172; d) D. Zell, M. Bursch, V. Müller, S. Grimme, L. Ackermann, Angew. Chem. Int. Ed. 2017, 56, 10378-10382; e) W. Liu, S. C. Richter, Y. Zhang, L. Ackermann, Angew. Chem. Int. Ed. 2016, 55, 7747-7750; f) W. Ma, R. Mei, G. Tenti, L. Ackermann, Chem. Eur. J. 2014, 20, 15248-15251.

[42] P. Nuss, M. J. Eckelman, PLoS One 2014, 9, e101298.

[43] P. Gandeepan, L. H. Finger, T. H. Meyer, L. Ackermann, Chem. Soc. Rev. 2020, 49, 4254-4272.

[44] a) S. M. Khake, N. Chatani, Trends Chem. 2019, 1, 524-539; b) R. Shang, L. Ilies, E. Nakamura, Chem. Rev. 2017, 117, 9086-9139; c) M. Moselage, J. Li, L. Ackermann, ACS Catal. 2016, 6, 498-525; d) W. Liu, L. Ackermann, ACS Catal. 
2016, 6, 3743-3752; e) G. Cera, L. Ackermann, Top. Curr. Chem. 2016, 374, 191224; f) N. Yoshikai, ChemCatChem 2015, 7, 732-734; g) X.-X. Guo, D.-W. Gu, Z. Wu, W. Zhang, Chem. Rev. 2015, 115, 1622-1651; h) J. Yamaguchi, K. Muto, K. Itami, Eur. J. Org. Chem. 2013, 19-30; i) A. A. Kulkarni, O. Daugulis, Synthesis 2009, 4087-4109.

[45] a) L. Vaccaro, Eur. J. Org. Chem. 2020, 4273-4283; b) P. Gandeepan, N. Kaplaneris, S. Santoro, L. Vaccaro, L. Ackermann, ACS Sustain. Chem. Eng. 2019, 7, 8023-8040; c) S. Santoro, F. Ferlin, L. Luciani, L. Ackermann, L. Vaccaro, Green Chem. 2017, 19, 1601-1612; d) F. Jérôme, R. Luque, Bio-based solvents, WileyVCH, Hoboken, 2017.

[46] www.Infomine.com/investment/ (accessed on 10.Apr.2021)

[47] a) F. Hebrard, P. Kalck, Chem. Rev. 2009, 109, 4272-4282; b) A. Y. Khodakov, W. Chu, P. Fongarland, Chem. Rev. 2007, 107, 1692-1744.

[48] D. Leckel, Energy Fuels 2009, 23, 2342-2358.

[49] M. S. Kharasch, E. K. Fields, J. Am. Chem. Soc. 1941, 63, 2316-2320.

[50] M. Giedyk, K. Goliszewska, D. Gryko, Chem. Soc. Rev. 2015, 44, 3391-3404.

[51] a) P. Gandeepan, C.-H. Cheng, Acc. Chem. Res. 2015, 48, 1194-1206; b) B. Heller, M. Hapke, Chem. Soc. Rev. 2007, 36, 1085-1094.

[52] a) B. J. Teobald, Tetrahedron 2002, 58, 4133-4170; b) K. M. Nicholas, Acc. Chem. Res. 1987, 20, 207-214.

[53] a) T. Shibata, Adv. Synth. Catal. 2006, 348, 2328-2336; b) I. U. Khand, G. R. Knox, P. L. Pauson, W. E. Watts, J. Chem. Soc., Perkin Trans. 1 1973, 975-977.

[54] a) J. R. Ludwig, E. M. Simmons, S. R. Wisniewski, P. J. Chirik, Org. Lett. 2021, 23, 625-630; b) O. Planas, C. J. Whiteoak, X. Ribas, in Non-Noble Metal Catalysis, Eds.: R. J. M. Klein Gebbink, M.-E. Moret, Wiley-VCH, Weinheim 2019, pp. 297328; c) S. Asghar, S. B. Tailor, D. Elorriaga, R. B. Bedford, Angew. Chem. Int. Ed. 2017, 56, 16367-16370; d) G. Cahiez, A. Moyeux, Chem. Rev. 2010, 110, 14351462.

[55] a) R. Mei, U. Dhawa, R. C. Samanta, W. Ma, J. Wencel-Delord, L. Ackermann, ChemSusChem 2020, 13, 3306-3356; b) L. Ackermann, J. Org. Chem. 2014, 79, 8948-8954.

[56] Y. Kommagalla, N. Chatani, Coord. Chem. Rev. 2017, 350, 117-135.

[57] S. Prakash, R. Kuppusamy, C.-H. Cheng, ChemCatChem 2018, 10, 683-705.

[58] a) T. Yoshino, S. Matsunaga, Synlett 2019, 30, 1384-1400; b) T. Yoshino, S. Matsunaga, Adv. Synth. Catal. 2017, 359, 1245-1262; c) T. Yoshino, S. Matsunaga, Adv. Organomet. Chem. 2017, 68, 197-247; d) T. Yoshino, H. Ikemoto, S. Matsunaga, M. Kanai, Angew. Chem. Int. Ed. 2013, 52, 2207-2211.

[59] D. Wei, X. Zhu, J.-L. Niu, M.-P. Song, ChemCatChem 2016, 8, 1242-1263.

[60] O. Planas, P. G. Chirila, C. J. Whiteoak, X. Ribas, Adv. Organomet. Chem. 2018, 69, 209-282.

[61] a) N. Yoshikai, in Cobalt Catalysis in Organic Synthesis, Eds.: M. Hapke, G. Hilt, Wiley-VCH, Weinheim 2020, pp. 89-161; b) K. Gao, N. Yoshikai, Acc. Chem. Res. 2014, 47, 1208-1219; c) N. Yoshikai, Bull. Chem. Soc. Jpn. 2014, 87, 843-857.

[62] a) L. Lukasevics, L. Grigorjeva, Org. Biomol. Chem. 2020, 18, 7460-7466; b) A. Baccalini, S. Vergura, P. Dolui, G. Zanoni, D. Maiti, Org. Biomol. Chem. 2019, 17, 10119-10141; c) S. Wang, S.-Y. Chen, X.-Q. Yu, Chem. Commun. 2017, 53, $3165-$ 3180; d) P. G. Chirila, C. J. Whiteoak, Dalton Trans. 2017, 46, 9721-9739.

[63] S. Murahashi, J. Am. Chem. Soc. 1955, 77, 6403-6404.

[64] S. Murahashi, S. Horiie, J. Am. Chem. Soc. 1956, 78, 4816-4817.

[65] J. P. Kleiman, M. Dubeck, J. Am. Chem. Soc. 1963, 85, 1544-1545. 
[66] M. I. Bruce, M. Z. Iqbal, F. G. A. Stone, J. Chem. Soc. A 1970, 3204-3209.

[67] H.-F. Klein, M. Helwig, U. Koch, U. Flörke, H.-J. Haupt, Z. Naturforsch. B 1993, $48 b, 778-784$.

[68] S. Camadanli, R. Beck, U. Flörke, H.-F. Klein, Dalton Trans. 2008, 5701-5704.

[69] a) H.-F. Klein, R. Beck, U. Flörke, H.-J. Haupt, Eur. J. Inorg. Chem. 2003, 13801387; b) H.-F. Klein, S. Schneider, M. He, U. Floerke, H.-J. Haupt, Eur. J. Inorg. Chem. 2000, 2295-2301.

[70] H.-F. Klein, S. Camadanli, R. Beck, D. Leukel, U. Flörke, Angew. Chem. Int. Ed. 2005, 44, 975-977.

[71] G. Halbritter, F. Knoch, A. Wolski, H. Kisch, Angew. Chem. Int. Ed. Engl. 1994, 33, 1603-1605.

[72] H. Gstach, H. Kisch, Z. Naturforsch. B 1983, 38, 251-252.

[73] C. P. Lenges, M. Brookhart, J. Am. Chem. Soc. 1997, 119, 3165-3166.

[74] a) S. López-Resano, S. M. de Salinas, F. A. Garcés-Pineda, A. Moneo-Corcuera, J. R. Galán-Mascarós, F. Maseras, M. H. Perez-Temprano, Angew. Chem. Int. Ed. 2021, 60, 11217-11221; b) S. Martínez de Salinas, J. Sanjosé-Orduna, C. Odena, S. Barranco, J. Benet-Buchholz, M. H. Pérez-Temprano, Angew. Chem. Int. Ed. 2020, 59, 6239-6243; c) J. Sanjosé-Orduna, D. Gallego, A. Garcia-Roca, E. Martin, J. Benet-Buchholz, M. H. Pérez-Temprano, Angew. Chem. Int. Ed. 2017, 56, $12137-$ 12141.

[75] K. Gao, P.-S. Lee, T. Fujita, N. Yoshikai, J. Am. Chem. Soc. 2010, 132, 1224912251.

[76] P.-S. Lee, T. Fujita, N. Yoshikai, J. Am. Chem. Soc. 2011, 133, 17283-17295.

[77] a) T. Yamakawa, N. Yoshikai, Tetrahedron 2013, 69, 4459-4465; b) K. Gao, N. Yoshikai, Angew. Chem. Int. Ed. 2011, 50, 6888-6892.

[78] Z. Ding, N. Yoshikai, Angew. Chem. Int. Ed. 2012, 51, 4698-4701.

[79] a) B. J. Fallon, E. Derat, M. Amatore, C. Aubert, F. Chemla, F. Ferreira, A. PerezLuna, M. Petit, Org. Lett. 2016, 18, 2292-2295; b) B. J. Fallon, J.-B. Garsi, E. Derat, M. Amatore, C. Aubert, M. Petit, ACS Catal. 2015, 5, 7493-7497; c) B. J. Fallon, E. Derat, M. Amatore, C. Aubert, F. Chemla, F. Ferreira, A. Perez-Luna, M. Petit, J. Am. Chem. Soc. 2015, 137, 2448-2451.

[80] a) K. Ueura, T. Satoh, M. Miura, Org. Lett. 2007, 9, 1407-1409; b) K. Ueura, T. Satoh, M. Miura, J. Org. Chem. 2007, 72, 5362-5367.

[81] Selected examples: a) S. Rakshit, C. Grohmann, T. Besset, F. Glorius, J. Am. Chem. Soc. 2011, 133, 2350-2353; b) T. Satoh, M. Miura, Chem. Eur. J. 2010, 16, 1121211222; c) G. Song, D. Chen, C.-L. Pan, R. H. Crabtree, X. Li, J. Org. Chem. 2010, 75, 7487-7490; d) N. Guimond, K. Fagnou, J. Am. Chem. Soc. 2009, 131, 1205012051; e) L. Li, W. W. Brennessel, W. D. Jones, J. Am. Chem. Soc. 2008, 130, 12414-12419; f) D. R. Stuart, M. Bertrand-Laperle, K. M. N. Burgess, K. Fagnou, J. Am. Chem. Soc. 2008, 130, 16474-16475. Selected reviews: g) T. Piou, T. Rovis, Acc. Chem. Res. 2018, 51, 170-180; h) J. Wencel-Delord, F. W. Patureau, F. Glorius, Top. Organomet. Chem. 2015, 55, 1-27; i) G. Song, X. Li, Acc. Chem. Res. 2015, 48, 1007-1020; j) B. Ye, N. Cramer, Acc. Chem. Res. 2015, 48, 1308-1318; k) G. Song, F. Wang, X. Li, Chem. Soc. Rev. 2012, 41, 3651-3678; 1) J. Y. Kim, S. H. Park, J. Ryu, S. H. Cho, S. H. Kim, S. Chang, J. Am. Chem. Soc. 2012, 134, 9110-9113; m) D. A. Colby, A. S. Tsai, R. G. Bergman, J. A. Ellman, Acc. Chem. Res. 2012, 45, 814-825.

[82] a) W. E. Broderick, K. Kanamori, R. D. Willett, J. I. Legg, Inorg. Chem. 1991, 30, 3875-3881; b) K. Kanamori, W. E. Broderick, R. F. Jordan, R. D. Willett, J. I. Legg, J. Am. Chem. Soc. 1986, 108, 7122-7124. 
[83] R. H. Crabtree, Angew. Chem. Int. Ed. Engl. 1993, 32, 789-805.

[84] a) R. H. Crabtree, Chem. Rev. 1985, 85, 245-269; b) S. R. Jones, J. M. Mellor, J. Chem. Soc., Perkin Trans. 2 1977, 511-517; c) A. Onopchenko, J. G. D. Schulz, J. Org. Chem. 1973, 38, 909-912; d) J. Hanotier, P. Camerman, M. Hanotier-Bridoux, P. de Radzitzky, J. Chem. Soc., Perkin Trans. 2 1972, 2247-2252; e) S. S. Lande, J. K. Kochi, J. Am. Chem. Soc. 1968, 90, 5196-5207.

[85] T. Avilés, A. Dinis, M. J. Calhorda, P. C. Pinto, V. Félix, M. G. B. Drew, J. Organomet. Chem. 2001, 625, 186-194.

[86] M. Brookhart, A. F. Volpe, D. M. Lincoln, I. T. Horvath, J. M. Millar, J. Am. Chem. Soc. 1990, 112, 5634-5636.

[87] M. Brookhart, B. E. Grant, C. P. Lenges, M. H. Prosenc, P. S. White, Angew. Chem. Int. Ed. 2000, 39, 1676-1679.

[88] a) U. Koelle, B. Fuss, M. V. Rajasekharan, B. L. Ramakrishna, J. H. Ammeter, M. C. Boehm, J. Am. Chem. Soc. 1984, 106, 4152-4160; b) T. Aviles, M. L. H. Green, J. Chem. Soc., Dalton Trans. 1979, 1116-1120.

[89] a) M. E. Tauchert, C. D. Incarvito, A. L. Rheingold, R. G. Bergman, J. A. Ellman, J. Am. Chem. Soc. 2012, 134, 1482-1485; b) Y. Li, X.-S. Zhang, H. Li, W.-H. Wang, K. Chen, B.-J. Li, Z.-J. Shi, Chem. Sci. 2012, 3, 1634-1639.

[90] a) J. C. A. Oliveira, U. Dhawa, L. Ackermann, ACS Catal. 2021, 11, 1505-1515; b) J. Guihaumé, S. Halbert, O. Eisenstein, R. N. Perutz, Organometallics 2012, 31, $1300-1314$.

[91] A. L. Allred, J. Inorg. Nucl. Chem. 1961, 17, 215-221.

[92] D. Zell, Q. Bu, M. Feldt, L. Ackermann, Angew. Chem. Int. Ed. 2016, 55, 74087412.

[93] L. N. Lewis, J. F. Smith, J. Am. Chem. Soc. 1986, 108, 2728-2735.

[94] K. Fumitoshi, Y. Yoshimi, C. Naoto, M. Shinji, Chem. Lett. 1995, 24, 681-682.

[95] L. Grigorjeva, O. Daugulis, Angew. Chem. Int. Ed. 2014, 53, 10209-10212.

[96] a) O. Daugulis, J. Roane, L. D. Tran, Acc. Chem. Res. 2015, 48, 1053-1064; b) D. Shabashov, O. Daugulis, J. Am. Chem. Soc. 2010, 132, 3965-3972; c) V. G. Zaitsev, D. Shabashov, O. Daugulis, J. Am. Chem. Soc. 2005, 127, 13154-13155.

[97] C. Sambiagio, D. Schönbauer, R. Blieck, T. Dao-Huy, G. Pototschnig, P. Schaaf, T. Wiesinger, M. F. Zia, J. Wencel-Delord, T. Besset, B. U. W. Maes, M. Schnürch, Chem. Soc. Rev. 2018, 47, 6603-6743.

[98] a) J. K. Kochi, R. T. Tang, T. Bernath, J. Am. Chem. Soc. 1973, 95, 7114-7123; b) R. Tang, J. K. Kochi, J. Inorg. Nucl. Chem. 1973, 35, 3845-3856.

[99] L.-B. Zhang, X.-Q. Hao, S.-K. Zhang, Z.-J. Liu, X.-X. Zheng, J.-F. Gong, J.-L. Niu, M.-P. Song, Angew. Chem. Int. Ed. 2015, 54, 272-275.

[100] X.-K. Guo, L.-B. Zhang, D. Wei, J.-L. Niu, Chem. Sci. 2015, 6, 7059-7071.

[101] S.-K. Liu, X.-F. Cui, X.-X. Liu, H.-J. Ma, D.-D. Wei, X.-L. Luo, G.-S. Huang, ChemistrySelect 2018, 3, 3989-3992.

[102] D. Nageswar Rao, S. Rasheed, G. Raina, Q. N. Ahmed, C. K. Jaladanki, P. V. Bharatam, P. Das, J. Org. Chem. 2017, 82, 7234-7244.

[103] J. Lan, H. Xie, X. Lu, Y. Deng, H. Jiang, W. Zeng, Org. Lett. 2017, 19, 4279-4282.

[104] R. Ueno, S. Natsui, N. Chatani, Org. Lett. 2018, 20, 1062-1065.

[105] C. Lin, Z. Chen, Z. Liu, Y. Zhang, Adv. Synth. Catal. 2018, 360, 519-532.

[106] a) Z.-1. Li, P.-Y. Wu, C. Cai, Org. Chem. Front. 2019, 6, 2043-2047; b) W. Sarkar, A. Bhowmik, A. Mishra, T. K. Vats, I. Deb, Adv. Synth. Catal. 2018, 360, 32283232.

[107] K. Raghuvanshi, K. Rauch, L. Ackermann, Chem. Eur. J. 2015, 21, 1790-1794. 
[108] Q. Gou, X. Tan, M. Zhang, M. Ran, T. Yuan, S. He, L. Zhou, T. Cao, F. Luo, Org. Lett. 2020, 22, 1966-1971.

[109] L. Grigorjeva, O. Daugulis, Org. Lett. 2014, 16, 4684-4687.

[110] X.-L. Han, P.-P. Lin, Q. Li, Chin. Chem. Lett. 2019, 30, 1495-1502.

[111] R. Gujjarappa, N. Vodnala, C. C. Malakar, Adv. Synth. Catal. 2020, 362, 48964990.

[112] R. Mei, H. Wang, S. Warratz, S. A. Macgregor, L. Ackermann, Chem. Eur. J. 2016, 22, 6759-6763.

[113] a) S. Warratz, C. Kornhaaß, A. Cajaraville, B. Niepötter, D. Stalke, L. Ackermann, Angew. Chem. Int. Ed. 2015, 54, 5513-5517; b) P. M. Osterberg, J. K. Niemeier, C. J. Welch, J. M. Hawkins, J. R. Martinelli, T. E. Johnson, T. W. Root, S. S. Stahl, Org. Process Res. Dev. 2015, 19, 1537-1543; c) A. N. Campbell, S. S. Stahl, Acc. Chem. Res. 2012, 45, 851-863; d) A. E. King, T. C. Brunold, S. S. Stahl, J. Am. Chem. Soc. 2009, 131, 5044-5045; e) S. S. Stahl, Angew. Chem. Int. Ed. 2004, 43, 3400-3420.

[114] J. Wencel-Delord, F. Colobert, Org. Chem. Front. 2016, 3, 394-400.

[115] S. G. Bratsch, J. Phys. Chem. Ref. Data 1989, 18, 1-21.

[116] K. Cheng, N. J. Rahier, B. M. Eisenhauer, R. Gao, S. J. Thomas, S. M. Hecht, J. Am. Chem. Soc. 2005, 127, 838-839.

[117] X.-Q. Hao, C. Du, X. Zhu, P.-X. Li, J.-H. Zhang, J.-L. Niu, M.-P. Song, Org. Lett. 2016, 18, 3610-3613.

[118] L.-B. Zhang, X.-Q. Hao, Z.-J. Liu, X.-X. Zheng, S.-K. Zhang, J.-L. Niu, M.-P. Song, Angew. Chem. Int. Ed. 2015, 54, 10012-10015.

[119] V. G. Landge, G. Jaiswal, E. Balaraman, Org. Lett. 2016, 18, 812-815.

[120] S. Zhai, S. Qiu, X. Chen, J. Wu, H. Zhao, C. Tao, Y. Li, B. Cheng, H. Wang, H. Zhai, Chem. Commun. 2018, 54, 98-101.

[121] H. Zhao, X. Shao, T. Wang, S. Zhai, S. Qiu, C. Tao, H. Wang, H. Zhai, Chem. Commun. 2018, 54, 4927-4930.

[122] C. Kuai, L. Wang, B. Li, Z. Yang, X. Cui, Org. Lett. 2017, 19, 2102-2105.

[123] A. M. Martinez, N. Rodriguez, R. Gomez-Arrayas, J. C. Carretero, Chem. Eur. J. 2017, 23, 11669-11676.

[124] F. Ling, Z. Xie, J. Chen, C. Ai, H. Shen, Z. Wang, X. Yi, W. Zhong, Adv. Synth. Catal. 2019, 361, 3094-3101.

[125] a) Y. Ran, Y. Yang, L. Zhang, Tetrahedron Lett. 2016, 57, 3322-3325; b) O. Planas, C. J. Whiteoak, A. Company, X. Ribas, Adv. Synth. Catal. 2015, 357, 4003-4012;

c) D. Kalsi, B. Sundararaju, Org. Lett. 2015, 17, 6118-6121.

[126] T. T. Nguyen, L. Grigorjeva, O. Daugulis, ACS Catal. 2016, 6, 551-554.

[127] T. T. Nguyen, L. Grigorjeva, O. Daugulis, Angew. Chem. Int. Ed. 2018, 57, 16881691.

[128] S. Kathiravan, I. A. Nicholls, Org. Lett. 2017, 19, 4758-4761.

[129] D. Kalsi, S. Dutta, N. Barsu, M. Rueping, B. Sundararaju, ACS Catal. 2018, 8, 8115-8120.

[130] D. Kalsi, N. Barsu, S. Chakrabarti, P. Dahiya, M. Rueping, B. Sundararaju, Chem. Commun. 2019, 55, 11626-11629.

[131] J. Zhang, H. Chen, C. Lin, Z. Liu, C. Wang, Y. Zhang, J. Am. Chem. Soc. 2015, 137, 12990-12996.

[132] a) B. Yang, Y. Qiu, J.-E. Bäckvall, Acc. Chem. Res. 2018, 51, 1520-1531; b) J. Ye, S. Ma, Acc. Chem. Res. 2014, 47, 989-1000; c) S. Yu, S. Ma, Angew. Chem. Int. Ed. 2012, 51, 3074-3112; d) S. Yu, S. Ma, Angew. Chem. Int. Ed. 2012, 51, 30743112; e) C. Aubert, L. Fensterbank, P. Garcia, M. Malacria, A. Simonneau, Chem. 
Rev. 2011, 111, 1954-1993; f) S. Ma, Chem. Rev. 2005, 105, 2829-2872; g) A. Hoffmann-Röder, N. Krause, Angew. Chem. Int. Ed. 2004, 43, 1196-1216; h) N. Krause, A. S. K. Hashmi, Modern Allene Chemistry, Wiley-VCH, Weinheim, 2004; i) A. S. K. Hashmi, Angew. Chem. Int. Ed. 2000, 39, 3590-3593.

[133] N. Thrimurtulu, A. Dey, D. Maiti, C. M. Volla, Angew. Chem. Int. Ed. 2016, 55, 12361-12365.

[134] a) X. F. Xia, Y. Q. Wang, L. L. Zhang, X. R. Song, X. Y. Liu, Y. M. Liang, Chem. Eur. J. 2014, 20, 5087-5091; b) A. Rodriguez, J. Albert, X. Ariza, J. Garcia, J. Granell, J. Farras, A. La Mela, E. Nicolas, J. Org. Chem. 2014, 79, 9578-9585; c) H. Wang, F. Glorius, Angew. Chem. Int. Ed. 2012, 51, 7318-7322.

[135] T. Li, C. Zhang, Y. Tan, W. Pan, Y. Rao, Org. Chem. Front. 2017, 4, 204-209.

[136] R. Boobalan, R. Kuppusamy, R. Santhoshkumar, P. Gandeepan, C. H. Cheng, Chem CatChem 2017, 9, 273-277.

[137] N. Thrimurtulu, R. Nallagonda, C. M. Volla, Chem. Commun. 2017, 53, 1872-1875.

[138] T. Lan, L. Wang, Y. Rao, Org. Lett. 2017, 19, 972-975.

[139] X. Yao, L. Jin, Y. Rao, Asian J. Org. Chem. 2017, 6, 825-830.

[140] S. Zhai, S. Qiu, X. Chen, C. Tao, Y. Li, B. Cheng, H. Wang, H. Zhai, ACS Catal. 2018, 8, 6645-6649.

[141] a) F.-X. Felpin, S. Sengupta, Chem. Soc. Rev. 2019, 48, 1150-1193; b) G. Bringmann, T. Gulder, T. A. M. Gulder, M. Breuning, Chem. Rev. 2011, 111, 563639; c) R. Martin, S. L. Buchwald, Acc. Chem. Res. 2008, 41, 1461-1473; d) D. A. Horton, G. T. Bourne, M. L. Smythe, Chem. Rev. 2003, 103, 893-930; e) J. Hassan, M. Sévignon, C. Gozzi, E. Schulz, M. Lemaire, Chem. Rev. 2002, 102, 1359-1470.

[142] a) L. Ackermann, Org. Process Res. Dev. 2015, 19, 260-269; b) L. Ackermann, R. Vicente, A. R. Kapdi, Angew. Chem. Int. Ed. 2009, 48, 9792-9826.

[143] J. D. Hayler, D. K. Leahy, E. M. Simmons, Organometallics 2019, 38, 36-46.

[144] a) Q. Bu, E. Gońka, K. Kuciński, L. Ackermann, Chem. Eur. J. 2019, 25, 22132216; b) P. B. De, S. Pradhan, S. Banerjee, T. Punniyamurthy, Chem. Commun. 2018, 54, 2494-2497; c) X. Zhu, J.-H. Su, C. Du, Z.-L. Wang, C.-J. Ren, J.-L. Niu, M.-P. Song, Org. Lett. 2017, 19, 596-599; d) L. Hu, Q. Gui, X. Chen, Z. Tan, G. Zhu, Org. Biomol. Chem. 2016, 14, 11070-11075; e) B. Li, Z.-H. Wu, Y.-F. Gu, C.L. Sun, B.-Q. Wang, Z.-J. Shi, Angew. Chem. Int. Ed. 2011, 50, 1109-1113.

[145] a) S. Lei, H. Cao, L. Chen, J. Liu, H. Cai, J. Tan, Adv. Synth. Catal. 2015, 357, 3109-3114; b) M. Nishino, K. Hirano, T. Satoh, M. Miura, Angew. Chem. Int. Ed. 2013, 52, 4457-4461; c) M. Kitahara, N. Umeda, K. Hirano, T. Satoh, M. Miura, $J$. Am. Chem. Soc. 2011, 133, 2160-2162; d) M. Zhu, K.-i. Fujita, R. Yamaguchi, Chem. Commun. 2011, 47, 12876-12878; e) D. Monguchi, A. Yamamura, T. Fujiwara, T. Somete, A. Mori, Tetrahedron Lett. 2010, 51, 850-852; f) Y. Li, J. Jin, W. Qian, W. Bao, Org. Biomol. Chem. 2010, 8, 326-330; g) X. Chen, G. Dobereiner, X.-S. Hao, R. Giri, N. Maugel, J.-Q. Yu, Tetrahedron 2009, 65, 30853089; h) H.-Q. Do, O. Daugulis, J. Am. Chem. Soc. 2009, 131, 17052-17053; i) X. Chen, X.-S. Hao, C. E. Goodhue, J.-Q. Yu, J. Am. Chem. Soc. 2006, 128, 67906791.

[146] L. Grigorjeva, O. Daugulis, Org. Lett. 2015, 17, 1204-1207.

[147] C. Du, P.-X. Li, X. Zhu, J.-F. Suo, J.-L. Niu, M.-P. Song, Angew. Chem. Int. Ed. 2016, 55, 13571-13575.

[148] a) L.-M. Xu, B.-J. Li, Z. Yang, Z.-J. Shi, Chem. Soc. Rev. 2010, 39, 712-733; b) S. R. Whitfield, M. S. Sanford, J. Am. Chem. Soc. 2007, 129, 15142-15143; c) K. L. Hull, E. L. Lanni, M. S. Sanford, J. Am. Chem. Soc. 2006, 128, 14047-14049.

[149] W. Yang, Y. Li, J. Zhu, W. Liu, J. Ke, C. He, Chem. Sci. 2020, 11, 10149-10158. 
[150] a) T. Rogge, L. Ackermann, Angew. Chem. Int. Ed. 2019, 58, 15640-15645; b) L. Ackermann, P. Novák, R. Vicente, V. Pirovano, H. K. Potukuchi, Synthesis 2010, 2245-2253; c) X. Guo, G. Deng, C.-J. Li, Adv. Synth. Catal. 2009, 351, 2071-2074.

[151] N. Lv, Z. Chen, Y. Liu, Z. Liu, Y. Zhang, Org. Lett. 2018, 20, 5845-5848.

[152] G. Tan, S. He, X. Huang, X. Liao, Y. Cheng, J. You, Angew. Chem. Int. Ed. 2016, 55, 10414-10418.

[153] X. Wang, Y. Chen, H. Song, Y. Liu, Q. Wang, Org. Lett. 2020, 22, 9331-9336.

[154] a) J. Cheng, L. Wang, P. Wang, L. Deng, Chem. Rev. 2018, 118, 9930-9987; b) M. E. Vol'pin, I. Y. Levitin, A. L. Sigan, A. T. Nikitaev, J. Organomet. Chem. 1985, 279, 263-280.

[155] a) E. K. Byrne, K. H. Theopold, J. Am. Chem. Soc. 1989, 111, 3887-3896; b) B. K. Bower, H. G. Tennent, J. Am. Chem. Soc. 1972, 94, 2512-2514.

[156] a) Y. M. Kwon, Y. Lee, G. E. Evenson, T. A. Jackson, D. Wang, J. Am. Chem. Soc. 2020, 142, 13435-13441; b) Y. Li, S. Handunneththige, J. Xiong, Y. Guo, M. R. Talipov, D. Wang, J. Am. Chem. Soc. 2020, 142, 21670-21678; c) Y. Li, S. Handunneththige, E. R. Farquhar, Y. Guo, M. R. Talipov, F. Li, D. Wang, J. Am. Chem. Soc. 2019, 141, 20127-20136; d) J. A. Bellow, S. A. Stoian, J. van Tol, A. Ozarowski, R. L. Lord, S. Groysman, J. Am. Chem. Soc. 2016, 138, 5531-5534; e) E. M. Zolnhofer, M. Käß, M. M. Khusniyarov, F. W. Heinemann, L. Maron, M. van Gastel, E. Bill, K. Meyer, J. Am. Chem. Soc. 2014, 136, 15072-15078; f) S. Hong, F. F. Pfaff, E. Kwon, Y. Wang, M.-S. Seo, E. Bill, K. Ray, W. Nam, Angew. Chem. Int. Ed. 2014, 53, 10403-10407; g) R. A. Lewis, S. P. George, A. Chapovetsky, G. Wu, J. S. Figueroa, T. W. Hayton, Chem. Commun. 2013, 49, 2888-2890; h) E. R. King, G. T. Sazama, T. A. Betley, J. Am. Chem. Soc. 2012, 134, 17858-17861; i) F. F. Pfaff, S. Kundu, M. Risch, S. Pandian, F. Heims, I. Pryjomska-Ray, P. Haack, R. Metzinger, E. Bill, H. Dau, P. Comba, K. Ray, Angew. Chem. Int. Ed. 2011, 50, 1711-1715; j) C. C. Hojilla Atienza, A. C. Bowman, E. Lobkovsky, P. J. Chirik, J. Am. Chem. Soc. 2010, 132, 16343-16345; k) T. J. Collins, R. D. Powell, C. Slebodnick, E. S. Uffelman, J. Am. Chem. Soc. 1991, 113, 8419-8425; 1) M. E. Vol'pin, I. Y. Levitin, A. L. Sigan, J. Halpern, G. M. Tom, Inorg. Chim. Acta 1980, 41, 271-277; m) I. Levitin, A. L. Sigan, M. E. Vol'pin, J. Chem. Soc., Chem. Commun. 1975, 469-470.

[157] a) J. Sanjosé-Orduna, Á. L. Mudarra, S. Martínez de Salinas, M. H. PérezTemprano, ChemSusChem 2019, 12, 2882-2897; b) J. Sanjosé-Orduna, J. BenetBuchholz, M. H. Pérez-Temprano, Inorg. Chem. 2019, 58, 10569-10577; c) J. Sanjosé-Orduna, J. M. Sarria Toro, M. H. Pérez-Temprano, Angew. Chem. Int. Ed. 2018, 57, 11369-11373.

[158] S. Maity, R. Kancherla, U. Dhawa, E. Hoque, S. Pimparkar, D. Maiti, ACS Catal. 2016, 6, 5493-5499.

[159] O. Planas, C. J. Whiteoak, V. Martin-Diaconescu, I. Gamba, J. M. Luis, T. Parella, A. Company, X. Ribas, J. Am. Chem. Soc. 2016, 138, 14388-14397.

[160] C. Du, P.-X. Li, X. Zhu, J.-N. Han, J.-L. Niu, M.-P. Song, ACS Catal. 2017, 7, 2810-2814.

[161] Y. Kommagalla, N. Chatani, Org. Lett. 2019, 21, 5971-5976.

[162] L. Lukasevics, A. Cizikovs, L. Grigorjeva, Org. Lett. 2021, 23, 2748-2753.

[163] a) X. Ribas, M. Devillard, Chem. Eur. J. 2018, 24, 1222-1230; b) X. Zhou, A. I. Day, A. J. Edwards, A. C. Willis, W. G. Jackson, Inorg. Chem. 2005, 44, 452-460.

[164] O. Planas, S. Roldan-Gomez, V. Martin-Diaconescu, T. Parella, J. M. Luis, A. Company, X. Ribas, J. Am. Chem. Soc. 2017, 139, 14649-14655. 
[165] O. Planas, S. Roldán-Gómez, V. Martin-Diaconescu, J. M. Luis, A. Company, X. Ribas, Chem. Sci. 2018, 9, 5736-5746.

[166] a) J. C. K. Chu, T. Rovis, Angew. Chem. Int. Ed. 2018, 57, 62-101; b) J. F. Hartwig, M. A. Larsen, ACS Cent. Sci. 2016, 2, 281-292; c) T. Cernak, K. D. Dykstra, S. Tyagarajan, P. Vachal, S. W. Krska, Chem. Soc. Rev. 2016, 45, 546-576; d) M. C. White, Science 2012, 335, 807-809; e) T. Brück1, R. D. Baxter, Y. Ishihara, P. S. Baran, Acc. Chem. Res. 2012, 45, 826-839.

[167] a) M. P. Doyle, R. Duffy, M. Ratnikov, L. Zhou, Chem. Rev. 2010, 110, 704-724; b) H. M. L. Davies, J. R. Manning, Nature 2008, 451, 417-424; c) H. M. L. Davies, R. E. J. Beckwith, Chem. Rev. 2003, 103, 2861-2904; d) R. H. Crabtree, J. Chem. Soc., Dalton Trans. 2001, 2437-2450; e) A. E. Shilov, G. B. Shul'pin, Chem. Rev. 1997, 97, 2879-2932.

[168] H. Wang, X. Gao, Z. Lv, T. Abdelilah, A. Lei, Chem. Rev. 2019, 119, 6769-6787.

[169] a) R. S. J. Proctor, R. J. Phipps, Angew. Chem. Int. Ed. 2019, 58, 13666-13699; b) K. A. Margrey, W. L. Czaplyski, D. A. Nicewicz, E. J. Alexanian, J. Am. Chem. Soc. 2018, 140, 4213-4217; c) C. R. J. Stephenson, T. P. Yoon, D. W. C. MacMillan, Visible light photocatalysis in organic chemistry, Wiley-VCH, Weinheim, 2018; d) M. K. Nielsen, B. J. Shields, J. Liu, M. J. Williams, M. J. Zacuto, A. G. Doyle, Angew. Chem. Int. Ed. 2017, 56, 7191-7194; e) W. L. Czaplyski, C. G. Na, E. J. Alexanian, J. Am. Chem. Soc. 2016, 138, 13854-13857; f) D. R. Heitz, J. C. Tellis, G. A. Molander, J. Am. Chem. Soc. 2016, 138, 1271512718; g) F. Minisci, R. Bernardi, F. Bertini, R. Galli, M. Perchinummo, Tetrahedron 1971, 27, 3575-3579.

[170] a) Z.-W. Hou, D.-J. Liu, P. Xiong, X.-L. Lai, J. Song, H.-C. Xu, Angew. Chem. Int. Ed. 2021, 60, 2943-2947; b) F. Wang, S. S. Stahl, Acc. Chem. Res. 2020, 53, 561574; c) J.-I. Yoshida, R. Hayashi, A. Shimizu, in Green Oxidation in Organic Synthesis, Eds.: N. Jiao, S. S. Stahl, Wiley-VCH, Weinheim 2019, pp. 409-437; d) A. Das, J. E. Nutting, S. S. Stahl, Chem. Sci. 2019, 10, 7542-7548; e) S. Herold, D. Bafaluy, K. Muñiz, Green Chem. 2018, 20, 3191-3196; f) M. Rafiee, F. Wang, D. P. Hruszkewycz, S. S. Stahl, J. Am. Chem. Soc. 2018, 140, 22-25; g) O. Hammerich, B. Speiser, Organic electrochemistry, 5th edn, CRC Press, Boca Raton, 2016; h) E. J. Horn, B. R. Rosen, P. S. Baran, ACS Cent. Sci. 2016, 2, 302-308; i) M. Yan, Y. Kawamata, P. S. Baran, Chem. Rev. 2017, 117, 13230-13319; j) E. J. Horn, B. R. Rosen, Y. Chen, J. Tang, K. Chen, M. D. Eastgate, P. S. Baran, Nature 2016, 533, 77-81; k) H. J. Schäfer, Anodic Reactions of Alkanes, Alkenes, and Aromatic Compounds, in Encyclopedia of Electrochemistry, Ed.: A. J. Bard, Wiley-VCH, Boston, 2007.

[171] a) K. P. Bryliakov, Chem. Rev. 2017, 117, 11406-11459; b) R. Lin, A. P. Amrute, J. Pérez-Ramírez, Chem. Rev. 2017, 117, 4182-4247; c) A. P. Antonchick, L. Burgmann, Angew. Chem. Int. Ed. 2013, 52, 3267-3271; d) D. H. R. Barton, D. Doller, Acc. Chem. Res. 1992, 25, 504-512; e) D. P. Curran, Synthesis 1988, 489513; f) G. Balavoine, D. H. R. Barton, J. Boivin, A. Gref, N. Ozbalik, H. Rivière, Tetrahedron Lett. 1986, 27, 2849-2852; g) J. M. Tedder, Angew. Chem. Int. Ed. Engl. 1982, 21, 401-410; h) C. E. Frank, Chem. Rev. 1950, 46, 155-169; i) H. Hock, S. Lang, Ber. Dtsch. Chem. Ges. B 1944, 77, 257-264; j) H. J. H. Fenton, J. Chem. Soc., Trans. 1894, 65, 899-910.

[172] a) P. A. Williams, J. Cosme, D. M. Vinković, A. Ward, H. C. Angove, P. J. Day, C. Vonrhein, I. J. Tickle, H. Jhoti, Science 2004, 305, 683-686; b) M. Merkx, D. A. Kopp, M. H. Sazinsky, J. L. Blazyk, J. Müller, S. J. Lippard, Angew. Chem. Int. Ed. 2001, 40, 2782-2807; c) A. C. Rosenzweig, S. J. Lippard, Acc. Chem. Res. 1994, 
27, 229-236; d) J. T. Groves, G. A. McClusky, R. E. White, M. J. Coon, Biochem. Biophys. Res. Commun. 1978, 81, 154-160.

[173] a) J. C. Lewis, P. S. Coelho, F. H. Arnold, Chem. Soc. Rev. 2011, 40, 2003-2021; b) A. Butler, M. Sandy, Nature 2009, 460, 848-854.

[174] a) M. T. Reetz, J. Am. Chem. Soc. 2013, 135, 12480-12496; b) K. Drauz, H. Gröger, O. May, Enzyme catalysis in organic synthesis, Wiley-VCH, Weinheim, 2012; c) F. H. Arnold, Acc. Chem. Res. 1998, 31, 125-131.

[175] a) J. Chen, Z. Jiang, S. Fukuzumi, W. Nam, B. Wang, Coord. Chem. Rev. 2020, 421, 213443; b) J. Serrano-Plana, C. Rumo, J. G. Rebelein, R. L. Peterson, M. Barnet, T. R. Ward, J. Am. Chem. Soc. 2020, 142, 10617-10623; c) M. Guo, T. Corona, K. Ray, W. Nam, ACS Cent. Sci. 2019, 5, 13-28; d) F. Burg, M. Gicquel, S. Breitenlechner, A. Pöthig, T. Bach, Angew. Chem. Int. Ed. 2018, 57, 2953-2957; e) F. Schwizer, Y. Okamoto, T. Heinisch, Y. Gu, M. M. Pellizzoni, V. Lebrun, R. Reuter, V. Köhler, J. C. Lewis, T. R. Ward, Chem. Rev. 2018, 118, 142-231; f) X. Engelmann, I. Monte-Pérez, K. Ray, Angew. Chem. Int. Ed. 2016, 55, 7632-7649; g) H. Renata, Z. J. Wang, F. H. Arnold, Angew. Chem. Int. Ed. 2015, 54, 33513367; h) M. L. Matthews, W.-C. Chang, A. P. Layne, L. A. Miles, C. Krebs, J. M. Bollinger, Nat. Chem. Biol. 2014, 10, 209-215; i) R. Fasan, ACS Catal. 2012, 2, 647-666; j) L. M. Podust, D. H. Sherman, Nat. Prod. Rep. 2012, 29, 1251-1266; k) S.-I. Murahashi, D. Zhang, Chem. Soc. Rev. 2008, 37, 1490-1501; 1) I. G. Denisov, T. M. Makris, S. G. Sligar, I. Schlichting, Chem. Rev. 2005, 105, 2253-2278; m) M. Sono, M. P. Roach, E. D. Coulter, J. H. Dawson, Chem. Rev. 1996, 96, 2841-2888; n) J. T. Groves, T. E. Nemo, R. S. Myers, J. Am. Chem. Soc. 1979, 101, 1032-1033.

[176] a) M. Goswami, B. de Bruin, Eur. J. Org. Chem. 2017, 1152-1176; b) W. Song, S. I. Kozhushkov, L. Ackermann, Angew. Chem. Int. Ed. 2013, 52, 6576-6578; c) S. Chiba, Synlett 2012, 2012, 21-44; d) N. Jung, S. Bräse, Angew. Chem. Int. Ed. 2012, 51, 12169-12171; e) S. Bräse, C. Gil, K. Knepper, V. Zimmermann, Angew. Chem. Int. Ed. 2005, 44, 5188-5240; f) M. Köhn, R. Breinbauer, Angew. Chem. Int. Ed. 2004, 43, 3106-3116.

[177] a) P. P. Geurink, W. A. van der Linden, A. C. Mirabella, N. Gallastegui, G. de Bruin, A. E. M. Blom, M. J. Voges, E. D. Mock, B. I. Florea, G. A. van der Marel, C. Driessen, M. van der Stelt, M. Groll, H. S. Overkleeft, A. F. Kisselev, J. Med. Chem. 2013, 56, 1262-1275; b) D. B. Smith, G. Kalayanov, C. Sund, A. Winqvist, T. Maltseva, V. J. P. Leveque, S. Rajyaguru, S. L. Pogam, I. Najera, K. Benkestock, X.-X. Zhou, A. C. Kaiser, H. Maag, N. Cammack, J. A. Martin, S. Swallow, N. G. Johansson, K. Klumpp, M. Smith, J. Med. Chem. 2009, 52, 2971-2978; c) G. C. Tron, T. Pirali, R. A. Billington, P. L. Canonico, G. Sorba, A. A. Genazzani, Med. Res. Rev. 2008, 28, 278-308; d) A. G. Habeeb, P. N. Praveen Rao, E. E. Knaus, J. Med. Chem. 2001, 44, 3039-3042.

[178] a) W. Xi, T. F. Scott, C. J. Kloxin, C. N. Bowman, Adv. Funct. Mater. 2014, 24, 2572-2590; b) R. Kluger, J. Am. Chem. Soc. 2010, 132, 6611-6612.

[179] a) P. Thirumurugan, D. Matosiuk, K. Jozwiak, Chem. Rev. 2013, 113, 4905-4979; b) L. A. Canalle, D. W. P. M. Löwik, J. C. M. van Hest, Chem. Soc. Rev. 2010, 39, 329-353; c) M. D. Best, Biochemistry 2009, 48, 6571-6584; d) D. M. Huryn, M. Okabe, Chem. Rev. 1992, 92, 1745-1768.

[180] H. C. Kolb, M. G. Finn, K. B. Sharpless, Angew. Chem. Int. Ed. 2001, 40, 20042021.

[181] S. Bräse, K. Banert, Organic Azides: Syntheses and Applications, Wiley-VCH, Chicester, 2011. 
[182] a) P. Sivaguru, Y. Ning, X. Bi, Chem. Rev. 2021; b) L. Ge, M.-F. Chiou, Y. Li, H. Bao, Green Synthesis and Catalysis 2020, 1, 86-120; c) R. Sala, C. Loro, F. Foschi, G. Broggini, Catalysts 2020, 10, 1173; d) X. Huang, J. T. Groves, ACS Catal. 2016, 6, 751-759.

[183] P. Magnus, J. Lacour, J. Am. Chem. Soc. 1992, 114, 767-769.

[184] P. Magnus, C. Hulme, W. Weber, J. Am. Chem. Soc. 1994, 116, 4501-4502.

[185] a) P. Magnus, J. Lacour, P. A. Evans, M. B. Roe, C. Hulme, J. Am. Chem. Soc. 1996, 118, 3406-3418; b) P. Magnus, J. Lacour, W. Weber, J. Am. Chem. Soc. 1993, $115,9347-9348$

[186] Y. Kita, H. Tohma, T. Takada, S. Mitoh, S. Fujita, M. Gyoten, Synlett 1994, 427428.

[187] a) V. V. Zhdankin, A. P. Krasutsky, C. J. Kuehl, A. J. Simonsen, J. K. Woodward, B. Mismash, J. T. Bolz, J. Am. Chem. Soc. 1996, 118, 5192-5197; b) A. P. Krasutsky, C. J. Kuehl, V. V. Zhdankin, Synlett 1995, 1081-1082; c) V. V. Zhdankin, C. J. Kuehl, A. P. Krasutsky, M. S. Formaneck, J. T. Bolz, Tetrahedron Lett. 1994, 35, 9677-9680.

[188] a) C. M. Pedersen, L. G. Marinescu, M. Bols, Org. Biomol. Chem. 2005, 3, 816822; b) C. Viuf, M. Bols, Angew. Chem. Int. Ed. 2001, 40, 623-625.

[189] X. Zhang, H. Yang, P. Tang, Org. Lett. 2015, 17, 5828-5831.

[190] Y. Wang, G.-X. Li, G. Yang, G. He, G. Chen, Chem. Sci. 2016, 7, 2679-2683.

[191] P. T. G. Rabet, G. Fumagalli, S. Boyd, M. F. Greaney, Org. Lett. 2016, 18, $1646-$ 1649.

[192] S. Kamijo, M. Watanabe, K. Kamijo, K. Tao, T. Murafuji, Synthesis 2016, 48, 115 121.

[193] X. Wang, A. Studer, Acc. Chem. Res. 2017, 50, 1712-1724.

[194] D. Leifert, A. Studer, Angew. Chem. Int. Ed. 2020, 59, 74-108.

[195] C. L. Hill, J. A. Smegal, T. J. Henly, J. Org. Chem. 1983, 48, 3277-3281.

[196] a) W. Liu, J. T. Groves, Acc. Chem. Res. 2015, 48, 1727-1735; b) W. Liu, J. T. Groves, Angew. Chem. Int. Ed. 2013, 52, 6024-6027; c) W. Liu, X. Huang, M.-J. Cheng, R. J. Nielsen, W. A. Goddard, J. T. Groves, Science 2012, 337, 1322-1325; d) W. Liu, J. T. Groves, J. Am. Chem. Soc. 2010, 132, 12847-12849.

[197] P. R. Ortiz de Montellano, Chem. Rev. 2010, 110, 932-948.

[198] X. Huang, T. M. Bergsten, J. T. Groves, J. Am. Chem. Soc. 2015, 137, 5300-5303.

[199] B. Hong, T. Luo, X. Lei, ACS Cent. Sci. 2020, 6, 622-635.

[200] M. Ochiai, Chem. Rec. 2007, 7, 12-23.

[201] A. Sharma, J. F. Hartwig, Nature 2015, 517, 600-604.

[202] R. R. Karimov, A. Sharma, J. F. Hartwig, ACS Cent. Sci. 2016, 2, 715-724.

[203] a) S.-E. Suh, S.-J. Chen, M. Mandal, I. A. Guzei, C. J. Cramer, S. S. Stahl, J. Am. Chem. Soc. 2020, 142, 11388-11393; b) X. Bao, Q. Wang, J. Zhu, Nat. Commun. 2019, 10, 769 .

[204] H. Chen, W. Yang, W. Wu, H. Jiang, Org. Biomol. Chem. 2014, 12, 3340-3343.

[205] a) Q.-H. Deng, T. Bleith, H. Wadepohl, L. H. Gade, J. Am. Chem. Soc. 2013, 135, 5356-5359; b) M. V. Vita, J. Waser, Org. Lett. 2013, 15, 3246-3249.

[206] N. G. Connelly, W. E. Geiger, Chem. Rev. 1996, 96, 877-910.

[207] S. Caron, R. W. Dugger, S. G. Ruggeri, J. A. Ragan, D. H. B. Ripin, Chem. Rev. 2006, 106, 2943-2989.

[208] T. H. Meyer, L. Ackermann, Aldrichim. Acta 2021, 54, in press.

[209] a) K. Scott, Sustainable and green electrochemical science and technology, John Wiley \& Sons, Inc., Hoboken, 2017; b) T. Fuchigami, M. Atobe, S. Inagi, Fundamentals and Applications of Organic Electrochemistry, Wiley-VCH, 
Weinheim, 2014; c) A. J. Bard, L. R. Faulkner, Electrochemical methods: fundamentals and applications, John Wiley \& Sons, Inc., New York, 2001.

[210] M. Faraday, Pogg. Ann. Phys. Chem 1834, 33, 433-451.

[211] H. Kolbe, Liebigs Ann. Chem. 1849, 69, 257-294.

[212] H.-J. Schäfer, Top. Curr. Chem. 1990, 152, 91-151.

[213] a) T. H. Meyer, I. Choi, C. Tian, L. Ackermann, Chem 2020, 6, 2484-2496; b) H. J. Schäfer, C. R. Chim. 2011, 14, 745-765; c) H. Lund, J. Electrochem. Soc. 2002, 149, S21; d) M. M. Baizer, ACS Symp. Ser. Am. Chem. Soc. 1989, 390, 172-175.

[214] M. M. Baizer, J. Electrochem. Soc. 1964, 111, 215.

[215] T. Shono, Tetrahedron 1984, 40, 811-850.

[216] J. H. Simons, J. Electrochem. Soc. 1949, 95, 47.

[217] a) T. Shono, Top. Curr. Chem. 1988, 148, 131-151; b) E. J. Corey, R. R. Sauers, J. Am. Chem. Soc. 1959, 81, 1739-1743.

[218] a) R. D. Little, J. Org. Chem. 2020; b) Y. Kawamata, P. S. Baran, Joule 2020, 4, 701-704; c) B. A. Frontana-Uribe, R. D. Little, J. G. Ibanez, A. Palma, R. VasquezMedrano, Green Chem. 2010, 12, 2099-2119.

[219] A. Jutand, in Organic Electrochemistry, 5th edn, Eds.: O. Hammerich, B. Speiser, CRC Press, Boca Raton 2015, pp. 1393-1432.

[220] a) N. Chen, H.-C. Xu, Chem. Rec. 2021, 21, DOI: 10.1002/tcr.202100048; b) S.-H. Shi, Y. Liang, N. Jiao, Chem. Rev. 2021, 121, 485-505; c) C. Kingston, M. D. Palkowitz, Y. Takahira, J. C. Vantourout, B. K. Peters, Y. Kawamata, P. S. Baran, Acc. Chem. Res. 2020, 53, 72-83; d) G. Hilt, ChemElectroChem 2020, 7, 395-405; e) M. C. Leech, K. Lam, Acc. Chem. Res. 2020, 53, 121-134; f) J. C. Siu, N. Fu, S. Lin, Acc. Chem. Res. 2020, 53, 547-560; g) H. Chen, O. Simoska, K. Lim, M. Grattieri, M. Yuan, F. Dong, Y. S. Lee, K. Beaver, S. Weliwatte, E. M. Gaffney, S. D. Minteer, Chem. Rev. 2020, 120, 12903-12993; h) K. Yamamoto, M. Kuriyama, O. Onomura, Acc. Chem. Res. 2020, 53, 105-120; i) P. Xiong, H.-C. Xu, Acc. Chem. Res. 2019, 52, 3339-3350; j) Y. Okada, K. Chiba, Chem. Rev. 2018, 118, 45924630; k) S. Tang, Y. Liu, A. Lei, Chem 2018, 4, 27-45; 1) J.-I. Yoshida, A. Shimizu, R. Hayashi, Chem. Rev. 2018, 118, 4702-4730; m) A. Wiebe, T. Gieshoff, S. Möhle, E. Rodrigo, M. Zirbes, S. R. Waldvogel, Angew. Chem. Int. Ed. 2018, 57, 55945619; n) Y. Jiang, K. Xu, C. Zeng, Chem. Rev. 2018, 118, 4485-4540; o) D. S. P. Cardoso, B. Sljukic, D. M. F. Santos, C. A. C. Sequeira, Org. Process Res. Dev. 2017, 21, 1213-1226; p) R. Feng, J. A. Smith, K. D. Moeller, Acc. Chem. Res. 2017, 50, 2346-2352; q) R. Francke, R. D. Little, Chem. Soc. Rev. 2014, 43, 2492-2521; r) J.-I. Yoshida, K. Kataoka, R. Horcajada, A. Nagaki, Chem. Rev. 2008, 108, 22652299.

[221] M. S. Freund, J. A. Labinger, N. S. Lewis, J. E. Bercaw, J. Mol. Catal. 1994, 87, L11-L15.

[222] C. Amatore, C. Cammoun, A. Jutand, Adv. Synth. Catal. 2007, 349, 292-296.

[223] a) C. Jia, T. Kitamura, Y. Fujiwara, Acc. Chem. Res. 2001, 34, 633-639; b) Y. Fujiwara, I. Moritani, S. Danno, R. Asano, S. Teranishi, J. Am. Chem. Soc. 1969, 91, 7166-7169; c) I. Moritanl, Y. Fujiwara, Tetrahedron Lett. 1967, 8, 1119-1122.

[224] a) A. Dey, T. B. Gunnoe, V. R. Stamenkovic, ACS Catal. 2020, 10, 13156-13158; b) R. Francke, R. D. Little, ChemElectroChem 2019, 6, 4373-4382.

[225] C. Zhu, N. W. J. Ang, T. H. Meyer, Y. Qiu, L. Ackermann, ACS Cent. Sci. 2021.

[226] F. Kakiuchi, T. Kochi, H. Mutsutani, N. Kobayashi, S. Urano, M. Sato, S. Nishiyama, T. Tanabe, J. Am. Chem. Soc. 2009, 131, 11310-11311.

[227] D. Kalyani, A. R. Dick, W. Q. Anani, M. S. Sanford, Org. Lett. 2006, 8, 2523-2526. 
[228] H. Aiso, T. Kochi, H. Mutsutani, T. Tanabe, S. Nishiyama, F. Kakiuchi, J. Org. Chem. 2012, 77, 7718-7724.

[229] F. Saito, H. Aiso, T. Kochi, F. Kakiuchi, Organometallics 2014, 33, 6704-6707.

[230] a) K. Sano, N. Kimura, T. Kochi, F. Kakiuchi, Asian J. Org. Chem. 2018, 7, 13111314; b) M. Konishi, K. Tsuchida, K. Sano, T. Kochi, F. Kakiuchi, J. Org. Chem. 2017, 82, 8716-8724.

[231] Q.-L. Yang, X.-Y. Wang, T.-L. Wang, X. Yang, D. Liu, X. Tong, X.-Y. Wu, T.-S. Mei, Org. Lett. 2019, 21, 2645-2649.

[232] Q.-L. Yang, Y.-Q. Li, C. Ma, P. Fang, X.-J. Zhang, T.-S. Mei, J. Am. Chem. Soc. 2017, 139, 3293-3298.

[233] Y.-Q. Li, Q.-L. Yang, P. Fang, T.-S. Mei, D. Zhang, Org. Lett. 2017, 19, 29052908.

[234] A. Shrestha, M. Lee, A. L. Dunn, M. S. Sanford, Org. Lett. 2018, 20, 204-207.

[235] H. Wu, Q. An, C. He, X. Fan, W. Guo, M. Zuo, C. Xu, R. Guo, W. Chu, Z. Sun, Adv. Synth. Catal. 2020, 362, 2459-2465.

[236] a) J. E. Erchinger, M. van Gemmeren, Asian J. Org. Chem. 2021, 10, 50-60; b) F. Kakiuchi, T. Kochi, Chem. Lett. 2020, 49, 1256-1269; c) F. Kakiuchi, T. Kochi, Isr. J. Chem. 2017, 57, 953-963; d) K.-J. Jiao, C.-Q. Zhao, P. Fang, T.-S. Mei, Tetrahedron Lett. 2017, 58, 797-802; e) T. V. Grayaznova, Y. B. Dudkina, D. R. Islamov, O. N. Kataeva, O. G. Sinyashin, D. A. Vicic, Y. H. Budnikova, J. Organomet. Chem. 2015, 785, 68-71.

[237] Q.-L. Yang, C.-Z. Li, L.-W. Zhang, Y.-Y. Li, X. Tong, X.-Y. Wu, T.-S. Mei, Organometallics 2019, 38, 1208-1212.

[238] C. Ma, C.-Q. Zhao, Y.-Q. Li, L.-P. Zhang, X.-T. Xu, K. Zhang, T.-S. Mei, Chem. Commun. 2017, 53, 12189-12192.

[239] U. Dhawa, C. Tian, T. Wdowik, J. C. A. Oliveira, J. Hao, L. Ackermann, Angew. Chem. Int. Ed. 2020.

[240] a) Y. Qiu, C. Zhu, M. Stangier, J. Struwe, L. Ackermann, CCS Chem. 2021, 3, 15291552; b) S.-K. Zhang, R. C. Samanta, A. Del Vecchio, L. Ackermann, Chem. Eur. J. 2020, 26, 10936-10947; c) L. Ackermann, Acc. Chem. Res. 2020, 53, 84-104; d) Y. Qiu, J. Struwe, L. Ackermann, Synlett 2019, 30, 1164-1173; e) N. Sauermann, T. H. Meyer, L. Ackermann, Chem. Eur. J. 2018, 24, 16209-16217; f) N. Sauermann, T. H. Meyer, Y. Qiu, L. Ackermann, ACS Catal. 2018, 8, 7086-7103.

[241] a) K.-J. Jiao, Y.-K. Xing, Q.-L. Yang, H. Qiu, T.-S. Mei, Acc. Chem. Res. 2020, 53, 300-310; b) C. Ma, P. Fang, T.-S. Mei, ACS Catal. 2018, 8, 7179-7189; c) Q.-L. Yang, P. Fang, T.-S. Mei, Chin. J. Chem. 2018, 36, 338-352.

[242] a) P. Wang, X. Gao, P. Huang, A. Lei, ChemCatChem 2020, 12, 27-40; b) Y. Yuan, A. Lei, Acc. Chem. Res. 2019, 52, 3309-3324; c) S. Tang, L. Zeng, A. Lei, J. Am. Chem. Soc. 2018, 140, 13128-13135.

[243] a) Z. J. Wu, F. Su, W. Lin, J. Song, T. B. Wen, H. J. Zhang, H. C. Xu, Angew. Chem. Int. Ed. 2019, 58, 16770-16774; b) F. Xu, Y.-J. Li, C. Huang, H.-C. Xu, $A C S$ Catal. 2018, 8, 3820-3824.

[244] a) Y. H. Budnikova, Chem. Rec. 2021, 21, DOI: 10.1002/tcr.202100009; b) X. Ye, C. Wang, S. Zhang, J. Wei, C. Shan, L. Wojtas, Y. Xie, X. Shi, ACS Catal. 2020, 10, 11693-11699; c) S. Kathiravan, S. Suriyanarayanan, I. A. Nicholls, Org. Lett. 2019, 21, 1968-1972; d) Z.-Q. Wang, C. Hou, Y.-F. Zhong, Y.-X. Lu, Z.-Y. Mo, Y.-M. Pan, H.-T. Tang, Org. Lett. 2019, 21, 9841-9845; e) M.-J. Luo, M. Hu, R.-J. Song, D.-L. He, J.-H. Li, Chem. Commun. 2019, 55, 1124-1127; f) M.-J. Luo, T.T. Zhang, F.-J. Cai, J.-H. Li, D.-L. He, Chem. Commun. 2019, 55, 7251-7254. 
[245] a) P. Gomes, C. Gosmini, J. Périchon, J. Org. Chem. 2003, 68, 1142-1145; b) P. Gomes, C. Gosmini, J. Périchon, Tetrahedron 2003, 59, 2999-3002; c) P. Gomes, H. Fillon, C. Gosmini, E. Labbé, J. Périchon, Tetrahedron 2002, 58, 8417-8424; d) P. Gomes, C. Gosmini, J.-Y. Nédélec, J. Périchon, Tetrahedron Lett. 2002, 43, 5901-5903; e) J. Chaussard, J.-C. Folest, J.-Y. Nedelec, J. Perichon, S. Sibille, M. Troupel, Synthesis 1990, 369-381.

[246] a) B.-L. Chen, H.-W. Zhu, Y. Xiao, Q.-L. Sun, H. Wang, J.-X. Lu, Electrochem. Commun. 2014, 42, 55-59; b) N. W. J. Ang, J. C. A. Oliveira, L. Ackermann, Angew. Chem. Int. Ed. 2020, 59, 12842-12847; c) P. L. Fabre, O. Reynes, Electrochem. Commun. 2010, 12, 1360-1362; d) J. Damodar, S. Krishna Mohan, S. K. Khaja Lateef, S. Jayarama Reddy, Synth. Commun. 2005, 35, 1143-1150; e) W.H. Chung, P. Guo, K.-Y. Wong, C.-P. Lau, J. Electroanal. Chem. 2000, 486, 3239; f) A. A. Isse, A. Gennaro, E. Vianello, J. Chem. Soc., Dalton Trans. 1996, 16131618; g) O. Sock, M. Troupel, J. Perichon, Tetrahedron Lett. 1985, 26, 1509-1512; h) J.-C. Folest, J.-M. Duprilot, J. Perichon, Y. Robin, J. Devynck, Tetrahedron Lett. 1985, 26, 2633-2636.

[247] a) H. Shimakoshi, Y. Hisaeda, Curr. Opin. Electrochem. 2018, 8, 24-30; b) A. M. Khenkin, M. Somekh, R. Carmieli, R. Neumann, Angew. Chem. Int. Ed. 2018, 57, 5403-5407; c) I. A. Dereven'kov, D. S. Salnikov, R. Silaghi-Dumitrescu, S. V. Makarov, O. I. Koifman, Coord. Chem. Rev. 2016, 309, 68-83; d) M. Giedyk, H. Shimakoshi, K. Goliszewska, D. Gryko, Y. Hisaeda, Dalton Trans. 2016, 45, 83408346; e) L. Eberson, J. Am. Chem. Soc. 1983, 105, 3192-3199.

[248] J. Zhong, Y. Yu, D. Zhang, K. Ye, Chin. Chem. Lett. 2020, 32, 963-972.

[249] N. Sauermann, T. H. Meyer, C. Tian, L. Ackermann, J. Am. Chem. Soc. 2017, 139, $18452-18455$.

[250] C. Tian, L. Massignan, T. H. Meyer, L. Ackermann, Angew. Chem. Int. Ed. 2018, 57, 2383-2387.

[251] R. Mei, N. Sauermann, J. C. A. Oliveira, L. Ackermann, J. Am. Chem. Soc. 2018, 140, 7913-7921.

[252] N. Sauermann, R. Mei, L. Ackermann, Angew. Chem. Int. Ed. 2018, 57, 5090-5094.

[253] X. Gao, P. Wang, L. Zeng, S. Tang, A. Lei, J. Am. Chem. Soc. 2018, 140, 41954199.

[254] C. Tian, U. Dhawa, J. Struwe, L. Ackermann, Chin. J. Chem. 2019, 37, 552-556.

[255] S. C. Sau, R. Mei, J. Struwe, L. Ackermann, ChemSusChem 2019, 12, 3023-3027.

[256] L. Zeng, H. Li, S. Tang, X. Gao, Y. Deng, G. Zhang, C.-W. Pao, J.-L. Chen, J.-F. Lee, A. Lei, ACS Catal. 2018, 8, 5448-5453.

[257] Y. Cao, Y. Yuan, Y. Lin, X. Jiang, Y. Weng, T. Wang, F. Bu, L. Zeng, A. Lei, Green Chem. 2020, 22, 1548-1552.

[258] U. Dhawa, C. Tian, W. Li, L. Ackermann, ACS Catal. 2020, 10, 6457-6462.

[259] R. Mei, W. Ma, Y. Zhang, X. Guo, L. Ackermann, Org. Lett. 2019, 21, 6534-6538.

[260] T. H. Meyer, J. C. A. Oliveira, S. C. Sau, N. W. J. Ang, L. Ackermann, ACS Catal. 2018, 8, 9140-9147.

[261] a) P. De Luna, C. Hahn, D. Higgins, S. A. Jaffer, T. F. Jaramillo, E. H. Sargent, Science 2019, 364, eaav3506; b) R. F. Service, Science 2019, 365, 1236-1239; c) Z. J. Schiffer, K. Manthiram, Joule 2017, 1, 10-14; d) T. R. Cook, D. K. Dogutan, S. Y. Reece, Y. Surendranath, T. S. Teets, D. G. Nocera, Chem. Rev. 2010, 110, 6474-6502.

[262] L. Orha, G. Akien, I. T. Horváth, in Handbook of Green Chemistry, Ed.: P. Anastas, Wiley-VCH, Weinheim 2010, pp. 93-120. 
[263] C. Sandford, M. A. Edwards, K. J. Klunder, D. P. Hickey, M. Li, K. Barman, M. S. Sigman, H. S. White, S. D. Minteer, Chem. Sci. 2019, 10, 6404-6422.

[264] V. A. Larson, B. Battistella, K. Ray, N. Lehnert, W. Nam, Nat. Rev. Chem. 2020, 4, 404-419.

[265] a) J. J. Li, D. S. Johnson, Innovative drug synthesis, Wiley-Blackwell, New York, 2016; b) J. J. Li, Contemporary drug synthesis, John Wiley \& Sons, Inc., New York, 2010; c) H.-G. Elias, An introduction to polymer science, Wiley-VCH, Weinheim, 2005.

[266] a) S. Enthaler, A. Company, Chem. Soc. Rev. 2011, 40, 4912-4924; b) J. F. Hartwig, Nature 2008, 455, 314-322; c) A. R. Muci, S. L. Buchwald, Top. Curr. Chem. 2002, 219, 131-209.

[267] a) F. Monnier, M. Taillefer, Angew. Chem. Int. Ed. 2009, 48, 6954-6971; b) G. Evano, N. Blanchard, M. Toumi, Chem. Rev. 2008, 108, 3054-3131; c) D. Ma, Q. Cai, Acc. Chem. Res. 2008, 41, 1450-1460; d) S. V. Ley, A. W. Thomas, Angew. Chem. Int. Ed. 2003, 42, 5400-5449.

[268] a) B. Liu, B.-F. Shi, Tetrahedron Lett. 2015, 56, 15-22; b) X.-Q. Hao, L.-J. Chen, B. Ren, L.-Y. Li, X.-Y. Yang, J.-F. Gong, J.-L. Niu, M.-P. Song, Org. Lett. 2014, 16, 1104-1107; c) A. M. Suess, M. Z. Ertem, C. J. Cramer, S. S. Stahl, J. Am. Chem. Soc. 2013, 135, 9797-9804; d) J. Roane, O. Daugulis, Org. Lett. 2013, 15, 58425845; e) D.-H. Wang, M. Wasa, R. Giri, J.-Q. Yu, J. Am. Chem. Soc. 2008, 130, 7190-7191; f) L. V. Desai, H. A. Malik, M. S. Sanford, Org. Lett. 2006, 8, 11411144; g) A. R. Dick, K. L. Hull, M. S. Sanford, J. Am. Chem. Soc. 2004, 126, 23002301.

[269] N. Sauermann, PhD thesis 2018, Georg-August-Universität Göttingen.

[270] T. Gieshoff, A. Kehl, D. Schollmeyer, K. D. Moeller, S. R. Waldvogel, J. Am. Chem. Soc. 2017, 139, 12317-12324.

[271] B. Elsler, A. Wiebe, D. Schollmeyer, K. M. Dyballa, R. Franke, S. R. Waldvogel, Chem. Eur. J. 2015, 21, 12321-12325.

[272] D. M. Heard, A. J. J. Lennox, Angew. Chem. Int. Ed. 2020, 59, 18866-18884.

[273] a) N. Dubouis, A. Grimaud, Chem. Sci. 2019, 10, 9165-9181; b) M. Zeng, Y. Li, J. Mater. Chem. A 2015, 3, 14942-14962.

[274] C. Tian, T. H. Meyer, M. Stangier, U. Dhawa, K. Rauch, L. H. Finger, L. Ackermann, Nat. Protoc. 2020, 15, 1760-1774.

[275] T. Morofuji, A. Shimizu, J.-I. Yoshida, Chem. Eur. J. 2015, 21, 3211-3214.

[276] J. L. Röck1, D. Pollok, R. Franke, S. R. Waldvogel, Acc. Chem. Res. 2020, 53, 4561.

[277] J. K. Beattie, C. U. Beck, P. A. Lay, A. F. Masters, Inorg. Chem. 2003, 42, 83668370.

[278] a) K. J. Lee, B. D. McCarthy, J. L. Dempsey, Chem. Soc. Rev. 2019, 48, 2927-2945; b) J. Malyszko, S. Michalkiewicz, D. Goral, M. Scendo, J. Appl. Electrochem. 1998, 28, 107-113.

[279] a) A. F. M. Noisier, M. A. Brimble, Chem. Rev. 2014, 114, 8775-8806; b) W. Wang, M. M. Lorion, J. Shah, A. R. Kapdi, L. Ackermann, Angew. Chem. Int. Ed. 2018, 57, 14700-14717.

[280] a) T. Li, J. Li, Z. Zhu, Y. Chen, X. Li, Q. Yang, J. Xia, W. Zhang, C. Zhang, W. Pan, S. Wu, Org. Chem. Front. 2021, 8, 928-935; b) A. M. Messinis, L. H. Finger, L. Hu, L. Ackermann, J. Am. Chem. Soc. 2020, 142, 13102-13111; c) R. K. Shukla, A. M. Nair, S. Khan, C. M. R. Volla, Angew. Chem. Int. Ed. 2020, 59, 17042-17048; d) H. Wang, N. Kaplaneris, L. Ackermann, Cell Rep. Phys. Sci. 2020, 1, 100178; e) W. Ding, Y. K. T. Ho, Y. Okuda, C. K. Wijaya, Z. H. Tan, N. Yoshikai, Org. Lett. 
2019, 21, 6173-6178; f) R. Kuppusamy, R. Santhoshkumar, R. Boobalan, H.-R. Wu, C.-H. Cheng, ACS Catal. 2018, 8, 1880-1883; g) J. Mo, T. Müller, J. C. A. Oliveira, L. Ackermann, Angew. Chem. Int. Ed. 2018, 57, 7719-7723; h) S. Nakanowatari, T. Muller, J. C. A. Oliveira, L. Ackermann, Angew. Chem. Int. Ed. 2017, 56, 15891-15895; i) S. Nakanowatari, R. Mei, M. Feldt, L. Ackermann, ACS Catal. 2017, 7, 2511-2515; j) S.-Y. Chen, X.-L. Han, J.-Q. Wu, Q. Li, Y. Chen, H. Wang, Angew. Chem. Int. Ed. 2017, 56, 9939-9943; k) S.-Y. Chen, Q. Li, X.-G. Liu, J.-Q. Wu, S.-S. Zhang, H. Wang, ChemSusChem 2017, 10, 2360-2364; 1) S. Y. Chen, Q. Li, H. Wang, J. Org. Chem. 2017, 82, 11173-11181.

[281] a) D. S. Kong, Y. F. Wang, Y. S. Zhao, Q. H. Li, Y. X. Chen, P. Tian, G. Q. Lin, Org. Lett. 2018, 20, 1154-1157; b) Z.-J. Jia, C. Merten, R. Gontla, C. G. Daniliuc, A. P. Antonchick, H. Waldmann, Angew. Chem. Int. Ed. 2017, 56, 2429-2434; c) S. Nakanowatari, L. Ackermann, Chem. Eur. J. 2015, 21, 16246-16251; d) B. Ye, N. Cramer, J. Am. Chem. Soc. 2013, 135, 636-639; e) H. Wang, B. Beiring, D. G. Yu, K. D. Collins, F. Glorius, Angew. Chem. Int. Ed. 2013, 52, 12430-12434; f) R. Zeng, C. Fu, S. Ma, J. Am. Chem. Soc. 2012, 134, 9597-9600; g) D. N. Tran, N. Cramer, Angew. Chem. Int. Ed. 2010, 49, 8181-8184; h) Y. J. Zhang, E. Skucas, M. J. Krische, Org. Lett. 2009, 11, 4248-4250.

[282] a) S. Torii, H. Okumoto, M. A. Rashid, M. Mohri, Synlett 1992, 721-722; b) G. Pattenden, G. M. Robertson, Tetrahedron Lett. 1985, 41, 4001-4011; c) G. Schlegel, H. J. Schäfer, Chem. Ber. 1983, 116, 960-969.

[283] R. Santhoshkumar, C.-H. Cheng, Asian J. Org. Chem. 2018, 7, 1151-1163.

[284] D. R. Stuart, K. Fagnou, in Inventing Reactions, Ed.: L. J. Gooßen, Springer, Berlin Heidelberg, 2013, pp. 91-119.

[285] S. Tang, D. Wang, Y. Liu, L. Zeng, A. Lei, Nat. Commun. 2018, 9, 798.

[286] R. Mei, X. Fang, L. He, J. Sun, L. Zou, W. Ma, L. Ackermann, Chem. Commun. 2020, 56, 1393-1396.

[287] D. J. C. Constable, C. Jimenez-Gonzalez, R. K. Henderson, Org. Process Res. Dev. 2007, 11, 133-137.

[288] a) D. L. Hughes, Org. Process Res. Dev. 2020, 24, 1850-1860; b) M. Baumann, T. S. Moody, M. Smyth, S. Wharry, Org. Process Res. Dev. 2020, 24, 1802-1813; c) M. B. Plutschack, B. Pieber, K. Gilmore, P. H. Seeberger, Chem. Rev. 2017, 117, 11796-11893; d) H. P. L. Gemoets, Y. Su, M. Shang, V. Hessel, R. Luque, T. Noël, Chem. Soc. Rev. 2016, 45, 83-117; e) M. Movsisyan, E. I. P. Delbeke, J. K. E. T. Berton, C. Battilocchio, S. V. Ley, C. V. Stevens, Chem. Soc. Rev. 2016, 45, 48924928; f) B. Gutmann, D. Cantillo, C. O. Kappe, Angew. Chem. Int. Ed. 2015, 54, 6688-6728.

[289] a) T. Wirth, Curr. Opin. Electrochem. 2021, 28, 100701; b) T. P. Nicholls, C. Schotten, C. E. Willans, Curr. Opin. Green Sust. Chem. 2020, 26, 100355; c) N. Tanbouza, T. Ollevier, K. Lam, iScience 2020, 23, 101720; d) M. Elsherbini, T. Wirth, Acc. Chem. Res. 2019, 52, 3287-3296; e) T. Noël, Y. Cao, G. Laudadio, Acc. Chem. Res. 2019, 52, 2858-2869; f) M. Atobe, H. Tateno, Y. Matsumura, Chem. Rev. 2018, 118, 4541-4572; g) K. Mitsudo, Y. Kurimoto, K. Yoshioka, S. Suga, Chem. Rev. 2018, 118, 5985-5999; h) D. Pletcher, R. A. Green, R. C. D. Brown, Chem. Rev. 2018, 118, 4573-4591; i) C. Gütz, A. Stenglein, S. R. Waldvogel, Org. Process Res. Dev. 2017, 21, 771-778.

[290] a) M. Lehmann, C. C. Scarborough, E. Godineau, C. Battilocchio, Ind. Eng. Chem. Res. 2020, 59, 7321-7326; b) L. Chen, L. M. Barton, J. C. Vantourout, Y. Xu, C. Chu, E. C. Johnson, J. J. Sabatini, P. S. Baran, Org. Process Res. Dev. 2020; c) S. Maljuric, W. Jud, C. O. Kappe, D. Cantillo, J. Flow Chem. 2020, 10, 181-190; d) 
M. Elsherbini, B. Winterson, H. Alharbi, A. A. Folgueiras-Amador, C. Génot, T. Wirth, Angew. Chem. Int. Ed. 2019, 58, 9811-9815; e) G. Laudadio, E. Barmpoutsis, C. Schotten, L. Struik, S. Govaerts, D. L. Browne, T. Noël, J. Am. Chem. Soc. 2019, 141, 5664-5668; f) B. K. Peters, K. X. Rodriguez, S. H. Reisberg, S. B. Beil, D. P. Hickey, Y. Kawamata, M. Collins, J. Starr, L. Chen, S. Udyavara, K. Klunder, T. J. Gorey, S. L. Anderson, M. Neurock, S. D. Minteer, P. S. Baran, Science 2019, 363, 838-845; g) M. Santi, J. Seitz, R. Cicala, T. Hardwick, N. Ahmed, T. Wirth, Chem. Eur. J. 2019, 25, 16230-16235; h) P. Nikolaienko, M. Jentsch, A. P. Kale, Y. Cai, M. Rueping, Chem. Eur. J. 2019, 25, 7177-7184; i) R. A. Green, R. C. D. Brown, D. Pletcher, B. Harji, Org. Process Res. Dev. 2015, 19, 1424-1427; j) K. Watts, W. Gattrell, T. Wirth, Beilstein J. Org. Chem. 2011, 7, 1108-1114; k) D. Horii, T. Fuchigami, M. Atobe, J. Am. Chem. Soc. 2007, 129, 11692-11693; 1) C. A. Paddon, M. Atobe, T. Fuchigami, P. He, P. Watts, S. J. Haswell, G. J. Pritchard, S. D. Bull, F. Marken, J. Appl. Electrochem. 2006, 36, 617; m) S. Suga, M. Okajima, K. Fujiwara, J.-I. Yoshida, J. Am. Chem. Soc. 2001, 123, 7941-7942.

[291] S. Santoro, F. Ferlin, L. Ackermann, L. Vaccaro, Chem. Soc. Rev. 2019, 48, 27672782.

[292] W.-J. Kong, L. H. Finger, A. M. Messinis, R. Kuniyil, J. C. A. Oliveira, L. Ackermann, J. Am. Chem. Soc. 2019, 141, 17198-17206.

[293] a) G. S. Kumar, A. Peshkov, A. Brzozowska, P. Nikolaienko, C. Zhu, M. Rueping, Angew. Chem. Int. Ed. 2020, 59, 6513-6519; b) N. S. Lewis, Science 2016, 351, aad1920; c) B. H. Nguyen, R. J. Perkins, J. A. Smith, K. D. Moeller, Beilstein J. Org. Chem. 2015, 11, 280-287; d) B. H. Nguyen, A. Redden, K. D. Moeller, Green Chem. 2014, 16, 69-72; e) S. Chu, A. Majumdar, Nature 2012, 488, 294; f) R. E. Blankenship, D. M. Tiede, J. Barber, G. W. Brudvig, G. Fleming, M. Ghirardi, M. R. Gunner, W. Junge, D. M. Kramer, A. Melis, T. A. Moore, C. C. Moser, D. G. Nocera, A. J. Nozik, D. R. Ort, W. W. Parson, R. C. Prince, R. T. Sayre, Science 2011, 332, 805-809; g) L. A. Anderson, A. Redden, K. D. Moeller, Green Chem. 2011, 13, 1652-1654; h) H. B. Gray, Nat. Chem. 2009, $1,7$.

[294] a) R. Chen, C. Yang, Z. Zhou, F. Haeffner, A. Dersjant, N. Dulock, Q. Dong, D. He, J. Jin, Y. Zhao, J. Niu, D. Wang, Angew. Chem. Int. Ed. 2021, 60, 7534-7539; b) O. Simoska, Z. Rhodes, S. Weliwatte, J. R. Cabrera-Pardo, E. M. Gaffney, K. Lim, S. D. Minteer, ChemSusChem 2021, 14, 1674-1686; c) C. Xu, E. Paone, D. Rodríguez-Padrón, R. Luque, F. Mauriello, Chem. Soc. Rev. 2020, 49, 4273-4306; d) K. Li, Y. Sun, Chem. Eur. J. 2018, 24, 18258-18270.

[295] a) Q. He, J. McNutt, J. Yang, Renew. Sustain. Energy Rev. 2017, 71, 63-76; b) L. J. Diorazio, D. R. J. Hose, N. K. Adlington, Org. Process Res. Dev. 2016, 20, 760773; c) P. Cintas, S. Tagliapietra, E. Calcio Gaudino, G. Palmisano, G. Cravotto, Green Chem. 2014, 16, 1056-1065; d) M. Simões, S. Baranton, C. Coutanceau, ChemSusChem 2012, 5, 2106-2124; e) M. Pagliaro, R. Ciriminna, H. Kimura, M. Rossi, C. Della Pina, Angew. Chem. Int. Ed. 2007, 46, 4434-4440.

[296] C. Tian, PhD Thesis 2020, Georg-August-Universität Göttingen.

[297] a) A. Bechtoldt, M. E. Baumert, L. Vaccaro, L. Ackermann, Green Chem. 2018, 20, 398-402; b) F. Ferlin, L. Luciani, S. Santoro, A. Marrocchi, D. Lanari, A. Bechtoldt, L. Ackermann, L. Vaccaro, Green Chem. 2018, 20, 2888-2893.

[298] J. Luo, Y. He, M. Zhong, Z. Jin, Appl. Phys. Lett. 2006, 89, 013104.

[299] a) C. Stang, F. Harnisch, ChemSusChem 2016, 9, 50-60; b) T. Broese, R. Francke, Org. Lett. 2016, 18, 5896-5899; c) S. J. Yoo, L.-J. Li, C.-C. Zeng, R. D. Little, Angew. Chem. Int. Ed. 2015, 54, 3744-3747. 
[300] T. H. Meyer, G. A. Chesnokov, L. Ackermann, ChemSusChem 2020, 13, 668-671.

[301] a) L. Liu, D. Wang, in Handbook of Green Chemistry, Ed.: P. T. Anastas, WileyVCH, Weinheim 2010, pp. 207-228; b) R. Breslow, in Handbook of Green Chemistry, Ed.: P. T. Anastas, Wiley-VCH, Weinheim 2010, pp. 1-29.

[302] a) J. González-García, M. D. Esclapez, P. Bonete, Y. V. Hernández, L. G. Garretón, V. Sáez, Ultrasonics 2010, 50, 318-322; b) R. G. Compton, J. C. Eklund, F. Marken, Electroanalysis 1997, 9, 509-522; c) J. Reisse, H. Francois, J. Vandercammen, O. Fabre, A. Kirsch-de Mesmaeker, C. Maerschalk, J. L. Delplancke, Electrochim. Acta 1994, 39, 37-39; d) T. J. Mason, J. P. Lorimer, D. J. Walton, Ultrasonics 1990, 28, 333-337.

[303] a) R. Arevalo, P. J. Chirik, J. Am. Chem. Soc. 2019, 141, 9106-9123; b) H. Xu, W. H. Bernskoetter, J. Am. Chem. Soc. 2011, 133, 14956-14959; c) J. Halpern, Angew. Chem. Int. Ed. Engl. 1985, 24, 274-282.

[304] a) D. C. Powers, T. Ritter, in Comprehensive Organic Synthesis II, Ed.: P. Knochel, Elsevier, Amsterdam 2014, pp. 719-743; b) D. C. Powers, T. Ritter, Acc. Chem. Res. 2012, 45, 840-850; c) A. J. Hickman, M. S. Sanford, Nature 2012, 484, 177185; d) P. Sehnal, R. J. K. Taylor, I. J. S. Fairlamb, Chem. Rev. 2010, 110, 824-889.

[305] a) S. Y. Hong, Y. Park, Y. Hwang, Y. B. Kim, M.-H. Baik, S. Chang, Science 2018, 359, 1016-1021; b) P. Gao, W. Guo, J. Xue, Y. Zhao, Y. Yuan, Y. Xia, Z. Shi, J. Am. Chem. Soc. 2015, 137, 12231-12240; c) L. Huang, D. Hackenberger, L. J. Gooßen, Angew. Chem. Int. Ed. 2015, 54, 12607-12611; d) J. Kim, S.-W. Park, M.H. Baik, S. Chang, J. Am. Chem. Soc. 2015, 137, 13448-13451; e) C. Suzuki, K. Hirano, T. Satoh, M. Miura, Org. Lett. 2015, 17, 1597-1600; f) H. Wang, F. Xie, Z. Qi, X. Li, Org. Lett. 2015, 17, 920-923; g) F. Xie, Z. Qi, S. Yu, X. Li, J. Am. Chem. Soc. 2014, 136, 4780-4787; h) H. Kim, K. Shin, S. Chang, J. Am. Chem. Soc. 2014, 136, 5904-5907; i) J. Kim, S. Chang, Angew. Chem. Int. Ed. 2014, 53, 2203-2207; j) L. Li, W. W. Brennessel, W. D. Jones, Organometallics 2009, 28, 3492-3500; k) D. L. Davies, S. M. A. Donald, O. Al-Duaij, S. A. Macgregor, M. Pölleth, J. Am. Chem. Soc. 2006, 128, 4210-4211; 1) H. Tamura, H. Yamazaki, H. Sato, S. Sakaki, J. Am. Chem. Soc. 2003, 125, 16114-16126; m) S. R. Klei, J. T. Golden, T. D. Tilley, R. G. Bergman, J. Am. Chem. Soc. 2002, 124, 2092-2093; n) J.-Y. Cho, M. K. Tse, D. Holmes, R. E. Maleczka, M. R. Smith, Science 2002, 295, 305-308; o) B. A. Arndtsen, R. G. Bergman, Science 1995, 270, 1970-1973.

[306] a) J. Kim, S. Jin, D. Kim, S. Chang, Bull. Korean Chem. Soc. 2021, 42, 529-532; b) J. Kim, K. Shin, S. Jin, D. Kim, S. Chang, J. Am. Chem. Soc. 2019, 141, 4137-4146; c) K. Shin, Y. Park, M.-H. Baik, S. Chang, Nat. Chem. 2017, 10, 218-224; d) S. H. Park, J. Kwak, K. Shin, J. Ryu, Y. Park, S. Chang, J. Am. Chem. Soc. 2014, 136, 2492-2502.

[307] a) E. Fooladi, M. Tilset, Inorg. Chem. 1997, 36, 6021-6027; b) A. Pedersen, M. Tilset, Organometallics 1993, 12, 56-64.

[308] a) R. J. Harris, J. Park, T. A. F. Nelson, N. Iqbal, D. C. Salgueiro, J. Bacsa, C. E. MacBeth, M.-H. Baik, S. B. Blakey, J. Am. Chem. Soc. 2020, 142, 5842-5851; b) S. Vásquez-Céspedes, X. Wang, F. Glorius, ACS Catal. 2018, 8, 242-257; c) A. J. Canty, Dalton Trans. 2009, 10409-10417; d) D. M. Crumpton-Bregel, K. I. Goldberg, J. Am. Chem. Soc. 2003, 125, 9442-9456; e) B. S. Williams, A. W. Holland, K. I. Goldberg, J. Am. Chem. Soc. 1999, 121, 252-253.

[309] T. H. Meyer, J. C. A. Oliveira, D. Ghorai, L. Ackermann, Angew. Chem. Int. Ed. 2020, 59, 10955-10960. 
[310] a) J. K. Beattie, T. W. Hambley, J. A. Klepetko, A. F. Masters, P. Turner, Polyhedron 1997, 16, 2109-2112; b) S. S. Lande, C. D. Falk, J. K. Kochi, J. Inorg. Nucl. Chem. 1971, 33, 4101-4109.

[311] A. G. Blackman, Cobalt: Inorganic \& Coordination Chemistry, in Encyclopedia of Inorganic Chemistry, Eds.: R. B. King, R. H. Crabtree, C. M. Lukehart, D. A. Atwood, R. A. Scott, Wiley-VCH, Weinheim, 2005.

[312] a) Y. Surendranath, M. W. Kanan, D. G. Nocera, J. Am. Chem. Soc. 2010, 132, 16501-16509; b) J. G. McAlpin, Y. Surendranath, M. Dincă, T. A. Stich, S. A. Stoian, W. H. Casey, D. G. Nocera, R. D. Britt, J. Am. Chem. Soc. 2010, 132, 68826883.

[313] a) F. C. Anson, T. J. Collins, R. J. Coots, S. L. Gipson, T. G. Richmond, J. Am. Chem. Soc. 1984, 106, 5037-5038; b) J. Topich, J. Halpern, Inorg. Chem. 1979, 18, 1339-1343; c) J. Halpern, M. S. Chan, J. Hanson, T. S. Roche, J. A. Topich, J. Am. Chem. Soc. 1975, 97, 1606-1608.

[314] J. Mehara, J. Roithová, Chem. Sci. 2020, 11, 11960-11972.

[315] I. Omari, P. Randhawa, J. Randhawa, J. Yu, J. S. McIndoe, J. Am. Soc. Mass Spectrom. 2019, 30, 1750-1757.

[316] a) C. Hansch, A. Leo, R. W. Taft, Chem. Rev. 1991, 91, 165-195; b) C. Hansch, A. Leo, S. H. Unger, K. H. Kim, D. Nikaitani, E. J. Lien, J. Med. Chem. 1973, 16, 1207-1216; c) H. H. Jaffé, Chem. Rev. 1953, 53, 191-261; d) L. P. Hammett, J. Am. Chem. Soc. 1937, 59, 96-103; e) L. P. Hammett, Chem. Rev. 1935, 17, 125-136.

[317] a) F. J. González, C. Frontana, M. Gómez, I. González, General Aspects of Redox Chemistry, in Encyclopedia of Physical Organic Chemistry, Eds.: Z. Wang, U. Wille, E. Juaristi, Wiley-VCH, Weinheim, 2017, pp. 1-19; b) W. Shan, N. Desbois, V. Blondeau-Patissier, M. L. Naitana, V. Quesneau, Y. Rousselin, C. P. Gros, Z. Ou, K. M. Kadish, Chem. Eur. J. 2017, 23, 12833-12844; c) M. Ostermeier, M.-A. Berlin, R. M. Meudtner, S. Demeshko, F. Meyer, C. Limberg, S. Hecht, Chem. Eur. J. 2010, 16, 10202-10213; d) M. Sjödin, J. Gätjens, L. C. Tabares, P. Thuéry, V. L. Pecoraro, S. Un, Inorg. Chem. 2008, 47, 2897-2908; e) V. Chandrasekhar, R. Thirumoorthi, R. Azhakar, Organometallics 2007, 26, 26-29; f) J. Chambers, B. Eaves, D. Parker, R. Claxton, P. S. Ray, S. J. Slattery, Inorg. Chim. Acta 2006, 359, 2400-2406; g) P. Zuman, General Equation for the Relation Between the Polarographic Half Wave Potential and Substituent Effects, in Substituent Effects in Organic Polarography, Springer, New York 1967, pp. 23-41.

[318] T. Furuya, D. Benitez, E. Tkatchouk, A. E. Strom, P. Tang, W. A. Goddard, T. Ritter, J. Am. Chem. Soc. 2010, 132, 3793-3807.

[319] a) J. B. C. Mack, K. L. Walker, S. G. Robinson, R. N. Zare, M. S. Sigman, R. M. Waymouth, J. Du Bois, J. Am. Chem. Soc. 2019, 141, 972-980; b) T. Gensch, M. J. James, T. Dalton, F. Glorius, Angew. Chem. Int. Ed. 2018, 57, 2296-2306; c) R. D. John C. Chadwick, Zoraida Freixa, Piet W. N. M. van Leeuwen, Homogeneous Catalysts: Activity - Stability - Deactivation, Wiley-VCH, Weinheim, 2011; d) C. H. Bartholomew, Appl. Catal. A Gen. 2001, 212, 17-60; e) P. W. N. M. van Leeuwen, Appl. Cataly. A Gen. 2001, 212, 61-81.

[320] M. Lafrance, K. Fagnou, J. Am. Chem. Soc. 2006, 128, 16496-16497.

[321] a) J. R. Mayfield, E. N. Grotemeyer, T. A. Jackson, Chem. Commun. 2020, 56, 9238-9255; b) C. Costentin, J.-M. Savéant, Curr. Opin. Electrochem. 2017, 1, 104109; c) N. Hoffmann, Eur. J. Org. Chem. 2017, 1982-1992; d) J. M. Mayer, Acc. Chem. Res. 2011, 44, 36-46; e) J. J. Warren, T. A. Tronic, J. M. Mayer, Chem. Rev. 2010, 110, 6961-7001; f) C. Costentin, Chem. Rev. 2008, 108, 2145-2179; g) M. H. V. Huynh, T. J. Meyer, Chem. Rev. 2007, 107, 5004-5064. 
[322] E. Reisenhofer, G. Costa, Inorg. Chim. Acta 1981, 49, 121-124.

[323] K. Yamazaki, Y. Kommagalla, Y. Ano, N. Chatani, Org. Chem. Front. 2019, 6, 537-543.

[324] X.-R. Chen, S.-Q. Zhang, T. H. Meyer, C.-H. Yang, Q.-H. Zhang, J.-R. Liu, H.-J. Xu, F.-H. Cao, L. Ackermann, X. Hong, Chem. Sci. 2020, 11, 5790-5796.

[325] L.-P. Xu, E. E. L. N. Liu, J. Bacsa, C. E. MacBeth, D. G. Musaev, Chem. Sci. 2020, 11, 6085-6096.

[326] a) S. G. Robinson, M. S. Sigman, Acc. Chem. Res. 2020, 53, 289-299; b) E. Labbé, O. Buriez, ChemElectroChem 2019, 6, 4118-4125; c) C. Costentin, J.-M. Savéant, ChemElectroChem 2014, 1, 1226-1236; d) E. S. Rountree, B. D. McCarthy, T. T. Eisenhart, J. L. Dempsey, Inorg. Chem. 2014, 53, 9983-10002; e) J.-M. Savéant, Chem. Rev. 2008, 108, 2348-2378; f) W. E. Geiger, Organometallics 2007, 26, 5738-5765; g) C. Amatore, A. Jutand, Acc. Chem. Res. 2000, 33, 314-321; h) P. T. Kissinger, W. R. Heineman, Laboratory techniques in electroanalytical chemistry, CRC Press, Boca Raton, 1996.

[327] Y. Qiu, M. Stangier, T. H. Meyer, J. C. A. Oliveira, L. Ackermann, Angew. Chem. Int. Ed. 2018, 57, 14179-14183.

[328] Y. Qiu, W.-J. Kong, J. Struwe, N. Sauermann, T. Rogge, A. Scheremetjew, L. Ackermann, Angew. Chem. Int. Ed. 2018, 57, 5828-5832.

[329] D. Lionetti, V. W. Day, J. D. Blakemore, Organometallics 2017, 36, 1897-1905.

[330] J. Moussa, C. Guyard-Duhayon, P. Herson, H. Amouri, M. N. Rager, A. Jutand, Organometallics 2004, 23, 6231-6238.

[331] Y. Qiu, J. Struwe, T. H. Meyer, J. C. A. Oliveira, L. Ackermann, Chem. Eur. J. 2018, 24, 12784-12789.

[332] a) Q. Bu, R. Kuniyil, Z. Shen, E. Gońka, L. Ackermann, Chem. Eur. J. 2020, 26, 16450-16454; b) F. Yang, H. Zhang, B. Wang, L. Ackermann, Chin. J. Org. Chem. 2019, 39, 59-73; c) G. G. Dias, T. Rogge, R. Kuniyil, C. Jacob, R. F. S. MennaBarreto, E. N. da Silva Júnior, L. Ackermann, Chem. Commun. 2018, 54, 1284012843; d) F. Yang, K. Rauch, K. Kettelhoit, L. Ackermann, Angew. Chem. Int. Ed. 2014, 53, 11285-11288; e) W. Liu, L. Ackermann, Org. Lett. 2013, 15, 3484-3486; f) F. Yang, L. Ackermann, Org. Lett. 2013, 15, 718-720; g) V. S. Thirunavukkarasu, L. Ackermann, Org. Lett. 2012, 14, 6206-6209; h) V. S. Thirunavukkarasu, J. Hubrich, L. Ackermann, Org. Lett. 2012, 14, 4210-4213.

[333] a) Z. Iqbal, A. Joshi, S. Ranjan De, Adv. Synth. Catal. 2020, 362, 5301-5351; b) S. G. Robinson, J. B. C. Mack, S. N. Alektiar, J. Du Bois, M. S. Sigman, Org. Lett. 2020, 22, 7060-7063; c) Y.-C. Yuan, C. Bruneau, V. Dorcet, T. Roisnel, R. Gramage-Doria, J. Org. Chem. 2019, 84, 1898-1907; d) Y.-C. Yuan, C. Bruneau, T. Roisnel, R. Gramage-Doria, Org. Biomol. Chem. 2019, 17, 7517-7525; e) J. B. C. Mack, J. D. Gipson, J. Du Bois, M. S. Sigman, J. Am. Chem. Soc. 2017, 139, 9503-9506; f) Y.-H. Sun, T.-Y. Sun, Y.-D. Wu, X. Zhang, Y. Rao, Chem. Sci. 2016, 7, 2229-2238; g) X. Yang, Y. Sun, Z. Chen, Y. Rao, Adv. Synth. Catal. 2014, 356, 1625-1630; h) E. McNeill, J. Du Bois, J. Am. Chem. Soc. 2010, 132, 10202-10204; i) J. C. Dobson, T. J. Meyer, Inorg. Chem. 1988, 27, 3283-3291.

[334] a) C. Dai, Y. Han, L. Liu, Z.-B. Huang, D.-Q. Shi, Y. Zhao, Org. Chem. Front. 2020, 7, 1703-1708; b) Y.-F. Liang, X. Wang, Y. Yuan, Y. Liang, X. Li, N. Jiao, ACS Catal. 2015, 5, 6148-6152; c) V. S. Thirunavukkarasu, S. I. Kozhushkov, L. Ackermann, Chem. Commun. 2014, 50, 29-39; d) P. Y. Choy, F. Y. Kwong, Org. Lett. 2013, 15, 270-273; e) H.-Y. Zhang, H.-M. Yi, G.-W. Wang, B. Yang, S.-D. Yang, Org. Lett. 2013, 15, 6186-6189; f) G. Shan, X. Yang, L. Ma, Y. Rao, Angew. Chem. Int. Ed. 2012, 51, 13070-13074; g) F. Mo, L. J. Trzepkowski, G. Dong, 
Angew. Chem. Int. Ed. 2012, 51, 13075-13079; h) J. M. Racowski, N. D. Ball, M. S. Sanford, J. Am. Chem. Soc. 2011, 133, 18022-18025; i) D. A. Alonso, C. Nájera, I. M. Pastor, M. Yus, Chem. Eur. J. 2010, 16, 5274-5284; j) T. Yoneyama, R. H. Crabtree, J. Mol. Catal. A: Chem. 1996, 108, 35-40.

[335] a) L. Ackermann, J. Pospech, K. Graczyk, K. Rauch, Org. Lett. 2012, 14, 930-933;

b) L. Ackermann, J. Pospech, Org. Lett. 2011, 13, 4153-4155.

[336] a) D. P. Hari, P. Caramenti, J. Waser, Acc. Chem. Res. 2018, 51, 3212-3225; b) X. Li, P. Chen, G. Liu, Beilstein J. Org. Chem. 2018, 14, 1813-1825; c) Y. Li, D. P. Hari, M. V. Vita, J. Waser, Angew. Chem. Int. Ed. 2016, 55, 4436-4454; d) A. Yoshimura, V. V. Zhdankin, Chem. Rev. 2016, 116, 3328-3435; e) F. V. Singh, T. Wirth, Chem. Asian J. 2014, 9, 950-971; f) T. Dohi, M. Ito, N. Yamaoka, K. Morimoto, H. Fujioka, Y. Kita, Tetrahedron 2009, 65, 10797-10815; g) V. V. Zhdankin, P. J. Stang, Chem. Rev. 2002, 102, 2523-2584.

[337] a) A. Maity, B. L. Frey, N. D. Hoskinson, D. C. Powers, J. Am. Chem. Soc. 2020, 142, 4990-4995; b) W.-C. Gao, Z.-Y. Xiong, S. Pirhaghani, T. Wirth, Synthesis 2019, 51, 276-284; c) R. Francke, Curr. Opin. Electrochem. 2019, 15, 83-88; d) J. D. Haupt, M. Berger, S. R. Waldvogel, Org. Lett. 2019, 21, 242-245; e) M. Elsherbini, T. Wirth, Chem. Eur. J. 2018, 24, 13399-13407; f) R. Möckel, E. Babaoglu, G. Hilt, Chem. Eur. J. 2018, 24, 15781-15785; g) K. Inoue, Y. Ishikawa, S. Nishiyama, Org. Lett. 2010, 12, 436-439; h) D. Kajiyama, K. Inoue, Y. Ishikawa, S. Nishiyama, Tetrahedron 2010, 66, 9779-9784; i) S. Nishiyama, Y. Amano, Heterocycles 2008, 75, 1997; j) R. D. Richardson, T. Wirth, Angew. Chem. Int. Ed. 2006, 45, 4402-4404; k) T. Fuchigami, T. Fujita, J. Org. Chem. 1994, 59, 71907192.

[338] a) Y. Qiu, C. Tian, L. Massignan, T. Rogge, L. Ackermann, Angew. Chem. Int. Ed. 2018, 57, 5818-5822; b) R. Mei, J. Koeller, L. Ackermann, Chem. Commun. 2018, $54,12879-12882$.

[339] L. Massignan, X. Tan, T. H. Meyer, R. Kuniyil, A. M. Messinis, L. Ackermann, Angew. Chem. Int. Ed. 2020, 59, 3184-3189.

[340] C. Shan, L. Zhu, L.-B. Qu, R. Bai, Y. Lan, Chem. Soc. Rev. 2018, 47, 7552-7576.

[341] H. G. Roth, N. A. Romero, D. A. Nicewicz, Synlett 2016, 27, 714-723.

[342] P. Gandeepan, J. Koeller, K. Korvorapun, J. Mohr, L. Ackermann, Angew. Chem. Int. Ed. 2019, 58, 9820-9825.

[343] a) L. Zhang, L. Zhu, Y. Zhang, Y. Yang, Y. Wu, W. Ma, Y. Lan, J. You, ACS Catal. 2018, 8, 8324-8335; b) J. Li, K. Korvorapun, S. De Sarkar, T. Rogge, D. J. Burns, S. Warratz, L. Ackermann, Nat. Commun. 2017, 8, 15430; c) J. Li, S. Warratz, D. Zell, S. De Sarkar, E. E. Ishikawa, L. Ackermann, J. Am. Chem. Soc. 2015, 137, 13894-13901; d) B. Li, T. Roisnel, C. Darcel, P. H. Dixneuf, Dalton Trans. 2012, 41, 10934-10937; e) J.-P. Djukic, J.-B. Sortais, L. Barloy, M. Pfeffer, Eur. J. Inorg. Chem. 2009, 817-853; f) H. C. L. Abbenhuis, M. Pfeffer, J. P. Sutter, A. de Cian, J. Fischer, H. L. Ji, J. H. Nelson, Organometallics 1993, 12, 4464-4472.

[344] a) C. Amatore, M. Azzabi, P. Calas, A. Jutand, C. Lefrou, Y. Rollin, J. Electroanal. Chem. 1990, 288, 45-63; b) S. Treimer, A. Tang, D. C. Johnson, Electroanalysis 2002, 14, 165-171.

[345] L. Mussari, M. Postigo, C. Lafuente, F. M. Royo, J. S. Urieta, J. Chem. Eng. Data 2000, 45, 86-91.

[346] a) A. Das, C. Hessin, Y. Ren, M. Desage-El Murr, Chem. Soc. Rev. 2020, 49, 88408867; b) R. Hili, A. K. Yudin, Nat. Chem. Biol. 2006, 2, 284-287.

[347] a) Z.-J. Jia, S. Gao, F. H. Arnold, J. Am. Chem. Soc. 2020, 142, 10279-10283; b) R. Singh, A. Mukherjee, ACS Catal. 2019, 9, 3604-3617; c) A. E. Bosnidou, K. Muñiz, 
Angew. Chem. Int. Ed. 2019, 58, 7485-7489; d) J. R. Clark, K. Feng, A. Sookezian, M. C. White, Nat. Chem. 2018, 10, 583-591; e) S. M. Paradine, J. R. Griffin, J. Zhao, A. L. Petronico, S. M. Miller, M. Christina White, Nat. Chem. 2015, 7, 987994; f) A. McNally, B. Haffemayer, B. S. L. Collins, M. J. Gaunt, Nature 2014, 510, 129-133; g) J. L. Jeffrey, R. Sarpong, Chem. Sci. 2013, 4, 4092-4106; h) J. L. Roizen, M. E. Harvey, J. Du Bois, Acc. Chem. Res. 2012, 45, 911-922; i) H. Lu, X. P. Zhang, Chem. Soc. Rev. 2011, 40, 1899-1909; j) F. Collet, R. H. Dodd, P. Dauban, Chem. Commun. 2009, 5061-5074; k) H. M. L. Davies, M. S. Long, Angew. Chem. Int. Ed. 2005, 44, 3518-3520; 1) X.-Q. Yu, J.-S. Huang, X.-G. Zhou, C.-M. Che, Org. Lett. 2000, 2, 2233-2236.

[348] a) Y. Yang, F. H. Arnold, Acc. Chem. Res. 2021, 54, 1209-1225; b) P.-G. Ding, X.S. Hu, F. Zhou, J. Zhou, Org. Chem. Front. 2018, 5, 1542-1559.

[349] T. H. Meyer, R. C. Samanta, A. Del Vecchio, L. Ackermann, Chem. Sci. 2021, 12 , 2890-2897.

[350] a) V. Plzak, H. Wendt, Ber. Bunsenges. Phys. Chem. 1979, 83, 481-486; b) G. A. Ward, C. M. Wright, J. Electroanal. Chem. 1964, 8, 302-309.

[351] a) W. Li, C. Zhu, Radically Initiated Distal C(sp $\left.{ }^{3}\right)-H$ Functionalization, in Remote C-H Bond Functionalizations: Methods and Strategies in Organic Synthesis, Eds.: D. Maiti, S. Guin, Wiley-VCH, Weinheim 2021, pp. 315-341; b) L. Capaldo, D. Ravelli, Eur. J. Org. Chem. 2017, 2056-2071.

[352] a) A. S. H. Ryder, W. B. Cunningham, G. Ballantyne, T. Mules, A. G. Kinsella, J. Turner-Dore, C. M. Alder, L. J. Edwards, B. S. J. McKay, M. N. Grayson, A. J. Cresswell, Angew. Chem. Int. Ed. 2020, 59, 14986-14991; b) A. Studer, D. P. Curran, Angew. Chem. Int. Ed. 2016, 55, 58-102; c) A. Studer, D. P. Curran, Nat. Chem. 2014, 6, 765-773.

[353] M. Zhang, Z.-F. Lu, Y. Liu, G. Grampp, H.-W. Hu, J.-H. Xu, Tetrahedron 2006, 62, 5663-5674.

[354] J. A. Marko, A. Durgham, S. L. Bretz, W. Liu, Chem. Commun. 2019, 55, 937-940.

[355] a) M. Schmittel, A. Burghart, Angew. Chem. Int. Ed. Engl. 1997, 36, 2550-2589; b) D. D. M. Wayner, D. J. McPhee, D. Griller, J. Am. Chem. Soc. 1988, 110, 132-137; c) H. Wendt, Angew. Chem. Int. Ed. Engl. 1982, 21, 256-270.

[356] a) B. Chandra, H. K. M, S. Pattanayak, S. S. Gupta, Chem. Sci. 2020, 11, $11877-$ 11885; b) Y. Sun, X. Li, M. Yang, W. Xu, J. Xie, M. Ding, Green Chem. 2020, 22 , 7543-7551; c) J. E. Nutting, M. Rafiee, S. S. Stahl, Chem. Rev. 2018, 118, 48344885; d) D. P. Hruszkewycz, K. C. Miles, O. R. Thiel, S. S. Stahl, Chem. Sci. 2017, 8, 1282-1287; e) Y. Zhu, Z. Chen, J. Zhang, Q. Wu, C. Ma, R. D. Little, Electrochim. Acta 2016, 207, 308-312; f) Y. Zhu, Y. Zhu, H. Zeng, Z. Chen, R. D. Little, C. Ma, J. Electroanal. Chem. 2015, 751, 105-110; g) G. Falgayrac, A. Savall, Catal. Today 1995, 24, 189-194; h) C. Ueda, M. Noyama, H. Ohmori, M. Masui, Chem. Pharm. Bull. 1987, 35, 1372-1377; i) M. Masui, S. Hara, T. Ueshima, T. Kawaguchi, S. Ozaki, Chem. Pharm. Bull. 1983, 31, 4209-4211.

[357] a) C.-M. Che, V. K.-Y. Lo, C.-Y. Zhou, J.-S. Huang, Chem. Soc. Rev. 2011, 40, 1950-1975; b) C. L. Hill, B. C. Schardt, J. Am. Chem. Soc. 1980, 102, 6374-6375.

[358] a) M. Guo, M. S. Seo, Y.-M. Lee, S. Fukuzumi, W. Nam, J. Am. Chem. Soc. 2019, 141, 12187-12191; b) M. Guo, Y.-M. Lee, M. S. Seo, Y.-J. Kwon, X.-X. Li, T. Ohta, W.-S. Kim, R. Sarangi, S. Fukuzumi, W. Nam, Inorg. Chem. 2018, 57, 1023210240; c) W. Liu, M.-J. Cheng, R. J. Nielsen, W. A. Goddard, J. T. Groves, ACS Catal. 2017, 7, 4182-4188; d) M. Oszajca, A. Franke, M. Brindell, G. Stochel, R. van Eldik, Coord. Chem. Rev. 2016, 306, 483-509; e) M. Y. Hyun, Y. D. Jo, J. H. Lee, H. G. Lee, H. M. Park, I. H. Hwang, K. B. Kim, S. J. Lee, C. Kim, Chem. Eur. 
J. 2013, 19, 1810-1818; f) W. J. Song, M. S. Seo, S. DeBeer George, T. Ohta, R. Song, M.-J. Kang, T. Tosha, T. Kitagawa, E. I. Solomon, W. Nam, J. Am. Chem. Soc. 2007, 129, 1268-1277; g) J. A. Gustafsson, L. Rondahl, J. Bergman, Biochemistry 1979, 18, 865-870.

[359] a) V. Lyaskovskyy, A. I. O. Suarez, H. Lu, H. Jiang, X. P. Zhang, B. de Bruin, J. Am. Chem. Soc. 2011, 133, 12264-12273; b) J. E. Lyons, P. E. Ellis, S. N. Shaikh, Inorg. Chim. Acta 1998, 270, 162-168; c) P. Battioni, J. P. Renaud, J. F. Bartoli, M. Reina-Artiles, M. Fort, D. Mansuy, J. Am. Chem. Soc. 1988, 110, 8462-8470; d) J. T. Groves, W. J. Kruper, R. C. Haushalter, J. Am. Chem. Soc. 1980, 102, 63756377.

[360] a) K. Jin, J. H. Maalouf, N. Lazouski, N. Corbin, D. Yang, K. Manthiram, J. Am. Chem. Soc. 2019, 141, 6413-6418; b) N. Fu, G. S. Sauer, A. Saha, A. Loo, S. Lin, Science 2017, 357, 575-579; c) K.-P. Ho, K.-Y. Wong, T. H. Chan, Tetrahedron 2006, 62, 6650-6658; d) H. Tanaka, M. Kuroboshi, H. Takeda, H. Kanda, S. Torii, J. Electroanal. Chem. 2001, 507, 75-81; e) P. Guo, K.-Y. Wong, Electrochem. Commun. 1999, 1, 559-563; f) J.-C. Moutet, A. Ourari, Electrochim. Acta 1997, 42, 2525-2531; g) F. Bedioui, J. Devynck, C. Bied-Charreton, J. Mol. Catal. A: Chem. 1996, 113, 3-11; h) C. P. Horwitz, P. J. Winslow, J. T. Warden, C. A. Lisek, Inorg. Chem. 1993, 32, 82-88; i) H. Nishihara, K. Pressprich, R. W. Murray, J. P. Collman, Inorg. Chem. 1990, 29, 1000-1006; j) C. P. Horwitz, S. E. Creager, R. W. Murray, Inorg. Chem. 1990, 29, 1006-1011.

[361] a) J. H. Zagal, S. Griveau, J. F. Silva, T. Nyokong, F. Bedioui, Coord. Chem. Rev. 2010, 254, 2755-2791; b) F. Bedioui, J. Devynck, C. Bied-Charreton, Acc. Chem. Res. 1995, 28, 30-36.

[362] A. Mukherjee, M. Martinho, E. L. Bominaar, E. Münck, L. Que Jr., Angew. Chem. Int. Ed. 2009, 48, 1780-1783.

[363] L. Zhang, Y.-M. Lee, M. Guo, S. Fukuzumi, W. Nam, J. Am. Chem. Soc. 2020, 142, 19879-19884.

[364] a) M. Mukherjee, A. Dey, ACS Cent. Sci. 2019, 5, 671-682; b) N. Kostopoulos, C. Achaibou, J.-M. Noël, F. Kanoufi, M. Robert, C. Fave, E. Anxolabéhère-Mallart, Inorg. Chem. 2020, 59, 11577-11583; c) M. Bugnola, R. Carmieli, R. Neumann, ACS Catal. 2018, 8, 3232-3236; d) W. Nam, Acc. Chem. Res. 2007, 40, 522-531.

[365] a) N. Kostopoulos, F. Banse, C. Fave, E. Anxolabéhère-Mallart, Chem. Commun. 2021, 57, 1198-1201; b) E. Anxolabéhère-Mallart, F. Banse, Curr. Opin. Electrochem. 2019, 15, 118-124; c) X. Han, K. Wang, G. Zhang, W. Gao, J. Chen, Adv. Synth. Catal. 2019, 361, 2804-2824; d) S. Sahu, D. P. Goldberg, J. Am. Chem. Soc. 2016, 138, 11410-11428; e) S. E. Creager, R. W. Murray, Inorg. Chem. 1987, 26, 2612-2618; f) S. E. Creager, S. A. Raybuck, R. W. Murray, J. Am. Chem. Soc. 1986, 108, 4225-4227.

[366] E. M. McGarrigle, D. G. Gilheany, Chem. Rev. 2005, 105, 1563-1602.

[367] a) H. Sterckx, B. Morel, B. U. W. Maes, Angew. Chem. Int. Ed. 2019, 58, 79467970; b) B. Meunier, Chem. Rev. 1992, 92, 1411-1456.

[368] A. A. Fokin, P. R. Schreiner, Chem. Rev. 2002, 102, 1551-1594.

[369] H. J. Schäfer, in Alkanes and Cycloalkanes, Eds.: S. Patai, Z. Rappoport, John Wiley \& Sons Inc., Hoboken 1992, pp. 781-808.

[370] H. Lu, V. Subbarayan, J. Tao, X. P. Zhang, Organometallics 2010, 29, 389-393.

[371] J. Börgel, T. Ritter, Chem 2020, 6, 1877-1887.

[372] B. W. Lund, F. Piu, N. K. Gauthier, A. Eeg, E. Currier, V. Sherbukhin, M. R. Brann, U. Hacksell, R. Olsson, J. Med. Chem. 2005, 48, 7517-7519. 
[373] a) H. M. Nelson, J. C. Siu, A. Saha, D. Cascio, S. N. MacMillan, S.-B. Wu, C. Lu, J. A. Rodríguez, K. N. Houk, S. Lin, Org. Lett. 2021, 23, 454-458; b) J. C. Siu, G. S. Sauer, A. Saha, R. L. Macey, N. Fu, T. Chauviré, K. M. Lancaster, S. Lin, J. Am. Chem. Soc. 2018, 140, 12511-12520.

[374] a) T. Kurahashi, M. Hada, H. Fujii, J. Am. Chem. Soc. 2009, 131, 12394-12405; b) T. Kurahashi, H. Fujii, Inorg. Chem. 2008, 47, 7556-7567.

[375] R. S. Czernuszewicz, W.-D. Wagner, G. B. Ray, K. Nakamoto, J. Mol. Struct. 1991, 242, 99-117.

[376] M. Yuan, F. Zhao, W. Zhang, Z.-M. Wang, S. Gao, Inorg. Chem. 2007, 46, 1123511242.

[377] B. C. Dave, R. S. Czernuszewicz, J. Coord. Chem. 1994, 33, 257-269.

[378] H. J. Schäfer, Angew. Chem. Int. Ed. Engl. 1970, 9, 158-159.

[379] a) M. Kasuno, M. Hayano, M. Fujiwara, T. Matsushita, Polyhedron 2009, 28, $425-$ 430; b) A. Panja, N. Shaikh, M. Ali, P. Vojtíšek, P. Banerjee, Polyhedron 2003, 22, 1191-1198.

[380] T. Kurahashi, H. Fujii, J. Am. Chem. Soc. 2011, 133, 8307-8316.

[381] T. Kurahashi, Inorg. Chem. 2018, 57, 1066-1078.

[382] a) R. Ren, H. Zhao, L. Huan, C. Zhu, Angew. Chem. Int. Ed. 2015, 54, 12692-12696; b) K. Wieghardt, U. Bossek, B. Nuber, J. Weiss, J. Bonvoisin, M. Corbella, S. E. Vitols, J. J. Girerd, J. Am. Chem. Soc. 1988, 110, 7398-7411.

[383] K.-B. Cho, H. Hirao, S. Shaik, W. Nam, Chem. Soc. Rev. 2016, 45, 1197-1210.

[384] a) R. J. Wiles, G. A. Molander, Isr. J. Chem. 2020, 60, 281-293; b) H. Hu, S.-J. Chen, M. Mandal, S. M. Pratik, J. A. Buss, S. W. Krska, C. J. Cramer, S. S. Stahl, Nat. Catal. 2020, 3, 358-367; c) L. Pitzer, J. L. Schwarz, F. Glorius, Chem. Sci. 2019, 10, 8285-8291; d) Y. Wang, X. Hu, C. A. Morales-Rivera, G.-X. Li, X. Huang, G. He, P. Liu, G. Chen, J. Am. Chem. Soc. 2018, 140, 9678-9684.

[385] L. Niu, C. Jiang, Y. Liang, D. Liu, F. Bu, R. Shi, H. Chen, A. D. Chowdhury, A. Lei, J. Am. Chem. Soc. 2020, 142, 17693-17702.

[386] G. Dormán, H. Nakamura, A. Pulsipher, G. D. Prestwich, Chem. Rev. 2016, 116, 15284-15398.

[387] C. Tian, U. Dhawa, A. Scheremetjew, L. Ackermann, ACS Catal. 2019, 9, 76907696.

[388] C. Zhu, M. Stangier, J. C. A. Oliveira, L. Massignan, L. Ackermann, Chem. Eur. J. 2019, 25, 16382-16389.

[389] a) T. K. Achar, S. Maiti, S. Jana, D. Maiti, ACS Catal. 2020, 10, 13748-13793; b) F. Pesciaioli, U. Dhawa, J. C. A. Oliveira, R. Yin, M. John, L. Ackermann, Angew. Chem. Int. Ed. 2018, 57, 15425-15429.

[390] a) M. M. Yamano, A. V. Kelleghan, Q. Shao, M. Giroud, B. J. Simmons, B. Li, S. Chen, K. N. Houk, N. K. Garg, Nature 2020, 586, 242-247; b) B. M. Trost, D. Zell, C. Hohn, G. Mata, A. Maruniak, Angew. Chem. Int. Ed. 2018, 57, 12916-12920; c) M. Wang, Z.-L. Liu, X. Zhang, P.-P. Tian, Y.-H. Xu, T.-P. Loh, J. Am. Chem. Soc. 2015, 137, 14830-14833; d) T. Hashimoto, K. Sakata, F. Tamakuni, M. J. Dutton, K. Maruoka, Nat. Chem. 2013, 5, 240-244; e) S. Yu, S. Ma, Angew. Chem. Int. Ed. 2012, 51, 3074-3112; f) S. Yu, S. Ma, Chem. Commun. 2011, 47, 5384-5418.

[391] a) S.-K. Zhang, J. Struwe, L. Hu, L. Ackermann, Angew. Chem. Int. Ed. 2020, 59, 3178-3183; b) S.-K. Zhang, R. C. Samanta, N. Sauermann, L. Ackermann, Chem. Eur. J. 2018, 24, 19166-19170.

[392] a) X. Tan, X. Hou, T. Rogge, L. Ackermann, Angew. Chem. Int. Ed. 2021, 60, 46194624; b) L. Yang, R. Steinbock, A. Scheremetjew, R. Kuniyil, L. H. Finger, A. M. Messinis, L. Ackermann, Angew. Chem. Int. Ed. 2020, 59, 11130-11135. 
[393] a) L. Chen, K. G. Malollari, A. Uliana, D. Sanchez, P. B. Messersmith, J. F. Hartwig, Chem 2021, 7, 137-145; b) A. Bunescu, S. Lee, Q. Li, J. F. Hartwig, ACS Cent. Sci. 2017, 3, 895-903.

[394] G. Laudadio, Y. Deng, K. van der Wal, D. Ravelli, M. Nuño, M. Fagnoni, D. Guthrie, Y. Sun, T. Noël, Science 2020, 369, 92-96.

[395] a) N. Liu, X. Chen, L. Jin, Y.-F. Yang, Y.-B. She, Org. Chem. Front. 2021, 8, 1858 1866; b) X. Huang, T. Zhuang, P. A. Kates, H. Gao, X. Chen, J. T. Groves, J. Am. Chem. Soc. 2017, 139, 15407-15413.

[396] a) T. Fuchigami, S. Inagi, Acc. Chem. Res. 2020, 53, 322-334; b) Y. Takahira, M. Chen, Y. Kawamata, P. Mykhailiuk, H. Nakamura, B. K. Peters, S. H. Reisberg, C. Li, L. Chen, T. Hoshikawa, T. Shibuguchi, P. S. Baran, Synlett 2019, 30, 11781182; c) K. Miyamoto, H. Nishiyama, I. Tomita, S. Inagi, ChemElectroChem 2019, 6, 97-100; d) G. Li, A. K. Dilger, P. T. Cheng, W. R. Ewing, J. T. Groves, Angew. Chem. Int. Ed. 2018, 57, 1251-1255; e) W. Liu, X. Huang, M. S. Placzek, S. W. Krska, P. McQuade, J. M. Hooker, J. T. Groves, Chem. Sci. 2018, 9, 1168-1172; f) M. Balandeh, A. Rios, N. Allison, D. Shirazi, A. Gomez, L. Rambaran, T. Holloway, S. Sadeghi, ChemElectroChem 2018, 5, 3353-3356; g) T. Sawamura, K. Takahashi, S. Inagi, T. Fuchigami, Angew. Chem. Int. Ed. 2012, 51, 4413-4416.

[397] a) Y. Liu, T.-C. Lau, J. Am. Chem. Soc. 2019, 141, 3755-3766; b) C.-M. Ho, T.-C. Lau, H.-L. Kwong, W.-T. Wong, J. Chem. Soc., Dalton Trans. 1999, 2411-2414; c) J. T. Groves, T. Takahashi, J. Am. Chem. Soc. 1983, 105, 2073-2074; d) C. L. Hill, F. J. Hollander, J. Am. Chem. Soc. 1982, 104, 7318-7319.

[398] a) C. Rebreyend, Y. Gloaguen, M. Lutz, J. I. van der Vlugt, I. Siewert, S. Schneider, B. de Bruin, Chem. Eur. J. 2017, 23, 17438-17443; b) M. G. Scheibel, B. Askevold, F. W. Heinemann, E. J. Reijerse, B. de Bruin, S. Schneider, Nat. Chem. 2012, 4, 552-558.

[399] H. C. Kolb, K. B. Sharpless, Drug Discov. Today 2003, 8, 1128-1137.

[400] R. E. Conrow, W. D. Dean, Org. Process Res. Dev. 2008, 12, 1285-1286.

[401] https://drs.illinois.edu/Page/SafetyLibrary/SodiumAzide (accessed: 01.Oct.2020).

[402] a) C. Hurtado-Rodrigo, S. Hoehne, M. P. Muñoz, Chem. Commun. 2014, 50, $1494-$ 1496; b) L. Rout, A. M. Harned, Chem. Eur. J. 2009, 15, 12926-12928; c) H. Guo, R. Qian, Y. Guo, S. Ma, J. Org. Chem. 2008, 73, 7934-7938.

[403] a) R. K. Al-Shewiki, M. Korb, A. Hildebrandt, S. Zahn, S. Naumov, R. Buschbeck, T. Rüffer, H. Lang, Dalton Trans. 2019, 48, 1578-1585; b) W. Zhang, E. N. Jacobsen, J. Org. Chem. 1991, 56, 2296-2298; c) E. N. Jacobsen, W. Zhang, M. L. Guler, J. Am. Chem. Soc. 1991, 113, 6703-6704; d) T. Matsushita, T. Shono, Polyhedron 1983, 2, 613-617.

[404] a) L. Kaustov, M. E. Tal, A. I. Shames, Z. Gross, Inorg. Chem. 1997, 36, 35033511; b) A. D. Adler, F. R. Longo, F. Kampas, J. Kim, J. Inorg. Nucl. Chem. 1970, $32,2443-2445$.

[405] L.-B. Zhang, X.-Q. Hao, S.-K. Zhang, K. Liu, B. Ren, J.-F. Gong, J.-L. Niu, M.-P. Song, J. Org. Chem. 2014, 79, 10399-10409.

[406] X. Huang, T. M. Bergsten, J. T. Groves, J. Am. Chem. Soc. 2015, 137, 5300-5303.

[407] J. Waser, B. Gaspar, H. Nambu, E. M. Carreira, J. Am. Chem. Soc. 2006, 128, 11693-11712.

[408] K.-P. Shing, Y. Liu, B. Cao, X.-Y. Chang, T. You, C.-M. Che, Angew. Chem. Int. Ed. 2018, 57, 11947-11951.

[409] M. Dryzhakov, M. Hellal, E. Wolf, F. C. Falk, J. Moran, J. Am. Chem. Soc. 2015, 137, 9555-9558.

[410] G. Sheldrick, Acta Cryst. C 2015, 71, 3-8. 
[411] G. Sheldrick, Acta Cryst. A 2008, 64, 112-122.

[412] O. V. Dolomanov, L. J. Bourhis, R. J. Gildea, J. A. K. Howard, H. Puschmann, J. Appl. Crystallogr. 2009, 42, 339-341. 



\section{DANKSAGUNG}

Zuallererst möchte ich mich herzlich bei meinem Doktorvater Herrn Prof. Dr. Lutz Ackermann für die Aufnahme in seinen Arbeitskreis, die herausragende Betreuung meiner Promotion, die uneingeschränkte Unterstützung in den vergangenen Jahren und das Vertrauen in mich und meine Arbeit bedanken. Seine wissenschaftliche Kreativität sowie seine effiziente und produktive Arbeitsmoral waren und sind stets ein Vorbild für mich.

Furthermore, I would like to thank Prof. Dr. Shoubhik Das for accepting to be my second $\mathrm{PhD}$ supervisor and for the valuable feedback and suggestions during our meetings and progress reports. Ebenfalls möchte ich mich herzlich bei Herrn Prof. Dr. Konrad Koszinowski, Herrn Jun.-Prof. Dr. Johannes C. L. Walker, Herrn Dr. Holm Frauendorf und Herrn Dr. Daniel Janßen-Müller für ihr bereitwilliges Mitwirken als Teil der Prüfungskommission bedanken.

I thank Robert, Maximilian, João, Torben, Leonardo, Ramesh, Alexej and Uttam from our group for thoroughly proofreading this thesis and improving its quality with their comments and suggestions.

Furthermore, I sincerely thank each and every of my co-workers for the inspiring collaboration within the projects - it has been a pleasure and honor to work with you.

Außerdem möchte ich mich herzlich bei den Werkstätten der Georg-August-Universität Göttingen für die Unterstützung und rasche Bearbeitung der Aufträge bedanken. Besonderer Dank gilt an dieser Stelle Heiko Renziehausen, Van Thao Nguyen und Herrn Heymel für die Konstruktion der Elektrolysezellen und die sorgfältige Bearbeitung der mechanischen Projekte; Herrn Ehrhardt und der gesamten Elektronikwerkstatt für die Konstruktion, Reparatur und ausführliche Beratung der elektrotechnischen Komponenten sowie Frau von Roden-Wendhausen und den Mitarbeitern der Glastechnik für ihre hervorragende Arbeit. Besonders möchte ich mich zudem bei den analytischen Abteilungen des IOBCs and IACs für die rasche und gewissenhafte Bearbeitung der zahlreichen analytischen Proben bedanken - vor allem bei Herrn Dr. Holm Frauendorf für die hervorragende Zusammenarbeit bei den massenspektrometrischen Projekten. Vielen Dank an dieser Stelle auch an Herrn Dr. Christopher Golz für die gewissenhaften Kristallstrukturanalysen. 
Ein großer Dank gilt außerdem Gabi Keil-Knepel und Bianca Spitalieri für die sorgfältige Lösung aller organisatorischen und administrativen Fragen; Stefan Beußhausen für die unentbehrliche Unterstützung im Bereich EDV und vielem mehr sowie bei Karsten Rauch für die großartige Hilfe aller praktischer Belange im Labor.

I particularly thank the whole Ackermann Group for the unique, fun and constructive working environment during the years. I could list each and every one, and a special thanks goes to Maximilian, Leonardo, Ramesh, Svenja \& Lars, Antonis, Cong, Kun, Julia, Lina, Nikos, Alexej and the former group members Nicolas, Marc, Daniel, Alex, Thomas, Julian, Joachim, and Alan, for all the fun during BBQ, football matches, volleyball, kayaking, Christmas markets, Cuxhaven trips etc...

Außerdem danke ich meinem Bachelorstudent Simon Homölle und allen weiteren Studierenden für die Zuarbeit bei den Projekten und das angenehme Miteinander im Labor.

Bedanken möchte ich mich an dieser Stelle auch bei meinen ehemaligen Kommilitonen Niels, Freed, Lorenz, Peter, Lukas und Thore für die großartige gemeinsame Zeit während des Studiums und danach!

Das abschließende und größte Dankeschön geht an meine Familie für Eure uneingeschränkte Unterstützung, Geduld und den starken Rückhalt in allen Lebenslagen. Meinem Bruder Jann und meinen Eltern möchte ich dafür danken, dass ihr immer an meiner Seite steht und ich stets auf euch zählen kann.

Sarah danke ich für so viel mehr als ich hier schreiben kann, Deine Liebe und Unterstützung und so viel Wichtigeres, als alle Ergebnisse dieser Arbeit. 


\section{Curriculum Vitae}

\section{PERSONAL INFORMATION}

Name:

Tjark Hannes Meyer

Date of Birth:

$07^{\text {th }}$ of January 1991 in Leer (Germany)

Place of Birth:

Nationality:

Leer, Ostfriesland (Germany)

German

\section{Professional EXPerience}

01/2017 - present Ph.D. Student, Institute of Organic and Biomolecular Chemistry Georg-August-University Göttingen, Germany

$\mathrm{PhD}$ thesis "Merging Electrosynthesis with 3d Transition MetalCatalyzed C-H Transformations“, with Prof. Dr. Lutz Ackermann

01/2016 - 03/2016 Industrial Placement, Bayer Pharma AG, Wuppertal, Germany Research \& Development, laboratory of Dr. Johannes Platzek

2014-2016 Teaching Assistant, Institute of Organic and Biomolecular Chemistry, Göttingen, Germany

06/2010 - 09/2011 Paramedic, Rettungsdienst-Friesland gGmbH, Germany

\section{EDUCATION}

10/2014 - 09/2016 Master's degree in chemistry (M.Sc., Final Grade: 1.4 with distinction)

Georg-August-University Göttingen, Germany

Master Thesis: „Manganese-Catalyzed C-H/C-C Activation“, with Prof. Dr. Lutz Ackermann (grade: 1.0).

09/2015 - 12/2015 Research Stay in the United Kingdom

University of Edinburgh, United Kingdom

Development of novel manganese-catalyzed hydrofunctionalization of alkenes, with Dr. Stephen Thomas.

10/2011 - 08/2014 Bachelor's degree in chemistry (B.Sc., Final Grade: 1.9)

Georg-August-University Göttingen, Germany

Bachelor Thesis: "Secondary Phosphine Oxides as Preligands for Ruthenium-Catalyzed Direct Arylations", with Prof. Dr. Lutz Ackermann (grade: 1.0).

$06 / 2010$

Abitur/A-Levels (Final Grade: 1.9)

Kooperative Gesamtschule Wiesmoor, Germany 
Teaching award for the "Heterocyclic Chemistry" tutorial

2018

Best poster award, $21^{\text {st }}$ International Symposium on Homogeneous Catalysis

$2015 \quad$ Erasmus-fellowship

\section{SCIENTIFIC ACHIEVEMENTS}

2021

Selected to attend the DSM Technical Business Course, MATCH.

2020

Selected to attend the Bayer Postdoc Workshop, Chemical R\&D.

2020

Presented my research on invitation at the American NSF CCHF Symposium (Link).

2019

Selected to attend the Boehringer Ingelheim MedChem PhD Course.

2019 Invited oral presentation at the Göttingen GDCh-Christmas Colloquium.

\section{TEACHING EXPERIENCE}

WiSe 19/20

Senior lecture assistant of the course "Synthetic Methods of Organic Chemistry"

SoSe 18-20 Thrice: Senior lecture assistant of the course "Modern Organic and Biomolecular Chemistry - Electrocatalysis"

SoSe 17-19 Thrice: Senior lecture assistant of the course "Organic Chemistry I" WiSe 18/19 Senior lecture assistant of the course "Heterocyclic Chemistry"

WiSe 17/18 Assistant of the practical course "Methods of Modern Organic Chemistry"

WiSe 17/18 Assistant within the FoLL Program (Research-Oriented Teaching and Learning)

\section{SCIENTIFIC CONTRIBUTIONS}

\section{Publications}

1) T. H. Meyer, L. Ackermann, "Chemistry with Potential-Present Challenges and Emerging Trends in Organic Electrocatalysis", Aldrichimica Acta 2021, 54, in press.

2) T. H. Meyer, $^{+}$R. C. Samanta, ${ }^{+}$A. Del Vecchio, L. Ackermann, "Mangana(III/IV)electro-Catalyzed $C\left(s p^{3}\right)-H$ Azidation" Chem. Sci. 2021, 11, 2890-2897.

3) C. Zhu, ${ }^{+}$N. W. J. Ang, ${ }^{+}$T. H. Meyer, Y. Qiu, L. Ackermann, "Organic Electrochemistry: Molecular Syntheses with Potential" ACS Cent. Sci. 2021, 7, 415431.

4) T. H. Meyer, I. Choi, C. Tian, L. Ackermann, "Power the Future - How Can Electrochemistry Make a Difference in Organic Synthesis?" Chem 2020, 6, 24842496. 
5) R. C. Samanta, T. H. Meyer, I. Siewert, L. Ackermann, "Renewable Resources for Sustainable Metallaelectro-Catalysed C-H Activation" Chem. Sci. 2020, 11, 86578670 .

6) P. Gandeepan, L. H. Finger, T. H. Meyer, L. Ackermann, "3d Metallaelectrocatalysis for Resource Economical Syntheses" Chem. Soc. Rev. 2020, 49, 4254-4272.

7) X.-R. Chen, S.-Q. Zhang, T. H. Meyer, C.-H. Yang, Q.-H. Zhang, J.-R. Liu, H.-J. $\mathrm{Xu}, \mathrm{F} . \mathrm{-H}$. Cao, L. Ackermann, X Hong, "Carboxylate Breaks Arene $\mathrm{C}-\mathrm{H}$ Bond via a Hydrogen-Atom-Transfer Mechanism in Electrochemical Cobalt Catalysis" Chem. Sci. 2020, 11, 5790-5796.

8) T. H. Meyer, J. C. A. Oliveira, D. Ghorai, L. Ackermann, "Insights into Cobalta(III/IV/II)-Electrocatalysis: Oxidation-Induced Reductive Elimination for Twofold C-H Activation" Angew. Chem. Int. Ed. 2020, 59, 10955-10960.

9) T. H. Meyer, G. A. Chesnokov, L. Ackermann, "Cobaltaelectro-Catalyzed $C-H$ Activation in Biomass-Derived Glycerol: Powered by Renewable Wind and Solar Energy" ChemSusChem 2020, 13, 668-671.

10) C. Tian, ${ }^{+}$T. H. Meyer, ${ }^{+}$M. Stangier, U. Dhawa, K. Rauch, L. H. Finger, L. Ackermann, "Cobaltaelectro-Catalyzed C-H Activation for Resource-Economical Molecular Syntheses" Nature Protoc 2020, 15, 1760-1774.

11) L. Massignan, ${ }^{+}$X. Tan, ${ }^{+}$T. H. Meyer, R. Kuniyil, A. M. Messinis, L. Ackermann, "C-H Oxygenation Reactions Enabled by Dual Catalysis with Electrogenerated Hypervalent Iodine Species and Ruthenium Complexes" Angew. Chem. Int. Ed. 2020, 59, 3184-3189.

12) L. Capdevila, T. H. Meyer, S. Roldán-Gómez, J. M. Luis, L. Ackermann, X. Ribas, "Chemo-Divergent Nickel(0)-Catalyzed Arene $C-F$ Activation with Alkynes: Unprecedented C-F/C-H Double-Insertion" ACS Catal. 2019, 9, 11074-11081.

13) T. H. Meyer, L. H. Finger, P. Gandeepan, L. Ackermann, "Resource Economy by Metallaelectrocatalysis: Merging Electrochemistry and $\mathrm{C}-\mathrm{H}$ Activation" Trends Chem. 2019, 1, 63-76.

14) Y. Qiu, ${ }^{+}$M. Stangier, ${ }^{+}$T. H. Meyer, J. C. A. Oliveira, L. Ackermann, "IridiumCatalyzed Electrooxidative $C-H$ Activation by Chemo-Selective Redox-Catalyst Cooperation" Angew. Chem. Int. Ed. 2018, 57, 14179-14183.

15) T. H. Meyer, J. C. A. Oliveira, S. Chandra Sau, N. W. J. Ang, L. Ackermann, "Electrooxidative Allene Annulations by Mild Cobalt-Catalyzed C-H Activation" ACS Catal. 2018, 8, 9140-9147.

16) N. Sauermann, T. H. Meyer, Y. Qiu, L. Ackermann, "Electrocatalytic $C-H$ Activation" ACS Catal. 2018, 8, 7086-7103.

17) N. Sauermann, T. H. Meyer, L. Ackermann, "Electrochemical Cobalt-Catalyzed CH Activation" Chem. Eur. J. 2018, 24, 16209-16217.

18) Y. Qiu, ${ }^{+}$J. Struwe, ${ }^{+}$T. H. Meyer, J. C. A. Oliveira, L. Ackermann, "Catalyst- and Reagent-free Electrochemical Azole C-H Amination" Chem. Eur. J. 2018, 24, 12784-12789.

19) C. Tian, L. Massignan, T. H. Meyer, L. Ackermann, "Electrochemical $C-H / N-H$ Activation by Water-Tolerant Cobalt-Catalysis at Room Temperature" Angew. Chem. Int. Ed. 2018, 57, 2383-2387. 
20) N. Sauermann, ${ }^{+}$T. H. Meyer, ${ }^{+}$C. Tian, L. Ackermann, "Electrochemical CobaltCatalyzed C-H Oxygenation at Room Temperature" J. Am. Chem. Soc. 2017, 139, 18452-18455.

21) T. H. Meyer, ${ }^{+}$W. Liu, ${ }^{+}$M. Feldt, A. Wuttke, R. A. Mata, L. Ackermann, "Manganese(I)-Catalyzed Dispersion-Enabled C-H/C-C Activation" Chem. Eur. J. 2017, 23, 5443-5447.

${ }^{+}$These authors contributed equally.

\section{Presentations}

1) T. H. Meyer, "Merging Electrochemistry and Metal-Catalyzed $\mathrm{C}-\mathrm{H}$ Transformations", Bayer Postdoc Workshop in Chemical R\&D, virtual meeting, November 2020 (oral presentation).

2) T. H. Meyer, J. C. A. Oliveira, D. Ghorai, L. Ackermann, "Mechanistic Insights into Cobalta(III/IV/II)-Electrocatalysis", Beilstein Organic Chemistry Symposium - 3d Metal Catalysis, virtual meeting, September 2020 (poster presentation).

3) T. H. Meyer, "Merging Electrochemistry and Metal-Catalyzed C-H Activation", NSF CCHF Beyond Telluride Symposium, virtual meeting, April 2020 (oral presentation).

4) $\quad$ T. H. Meyer, "Merging Electrochemistry and Metal-Catalyzed C-H Activation", GDCh-Weihnachtskolloquium, Göttingen, Dezember 2019 (oral presentation).

5) $\quad$ T. H. Meyer, "Merging Electrochemistry and Metal-Catalyzed C-H Activation", Boehringer-Ingelheim MedChem PhD Workshop, Biberach an der Riss, September 2019 (oral presentation).

6) T. H. Meyer, N. Sauermann, C. Tian, L. Ackermann, "Resource Economy by Cobaltaelectrocatalysis: Merging Electrochemistry and C-H Activation", $21^{\text {st }}$ European Symposium on Organic Chemistry, Wien, Austria, July 2019 (poster presentation).

7) T. H. Meyer, N. Sauermann, C. Tian, L. Ackermann, "Electrochemical CobaltCatalyzed C-H-Oxygenation at Room Temperature", Beilstein Organic Chemistry Symposium - Electrifying Organic Synthesis, Mainz, April 2019 (poster presentation).

8) T. H. Meyer, N. Sauermann, C. Tian, L. Ackermann, "Electrochemical CobaltCatalyzed C-H-Oxygenation at Room Temperature", $21^{\text {st }}$ International Symposium on Homogeneous Catalysis, Amsterdam, Netherlands, July 2018 (poster presentation + Best Poster Award).

9) T. H. Meyer, W. Liu, M. Feldt, A. Wuttke, R. A. Mata, L. Ackermann, "Manganese(I)-Catalyzed C-H/C-C Bond Activation", Summer School on Organic Catalysis for Energy Conversion, Göttingen, September 2017 (poster presentation).

10) T. H. Meyer, W. Liu, M. Feldt, A. Wuttke, R. A. Mata, L. Ackermann, "Manganese(I)-Catalyzed C-H/C-C Bond Activation", $26^{\text {th }}$ International Society of Heterocyclic Chemistry Congress, Regensburg, September 2017 (poster presentation). 


\section{ERKLÄRUNG}

Hiermit erkläre ich, dass die vorliegende Dissertation in dem Zeitraum von Januar 2017 bis Mai 2021 am Institut für Organische und Biomolekulare Chemie der Georg-AugustUniversität Göttingen unter Anleitung von

\section{Herrn Prof. Dr. Lutz Ackermann}

selbständig durchgeführt wurde und keine anderen als die angegebenen Hilfsmittel und Quellen verwendet wurden. Zudem erkläre ich, dass ich mich an keiner anderen Universität um einen Doktortitel beworben habe.

Göttingen, den 31.05.2021 



\section{APPENDIX}

NMR Spectra

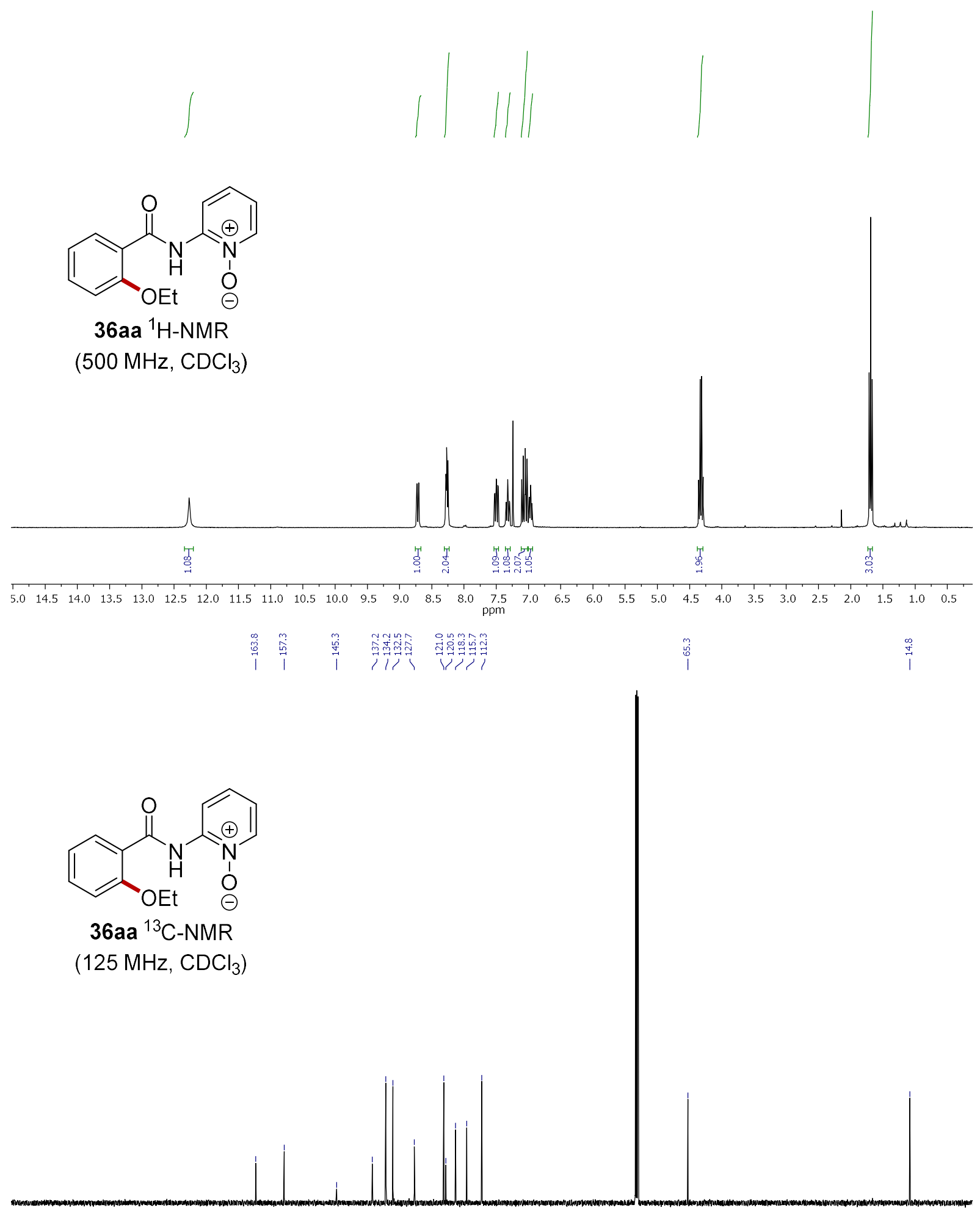

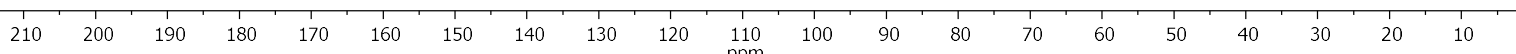



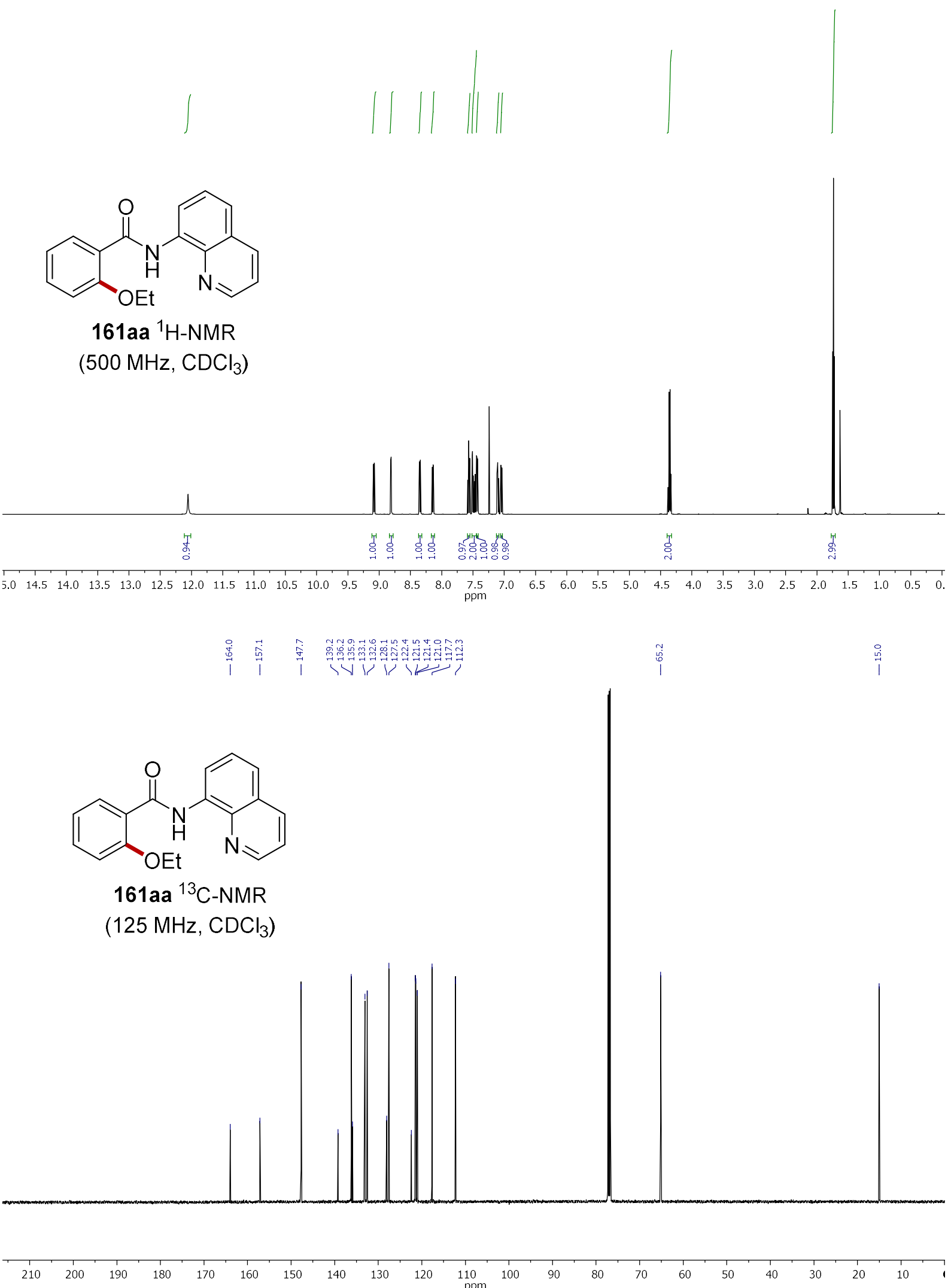

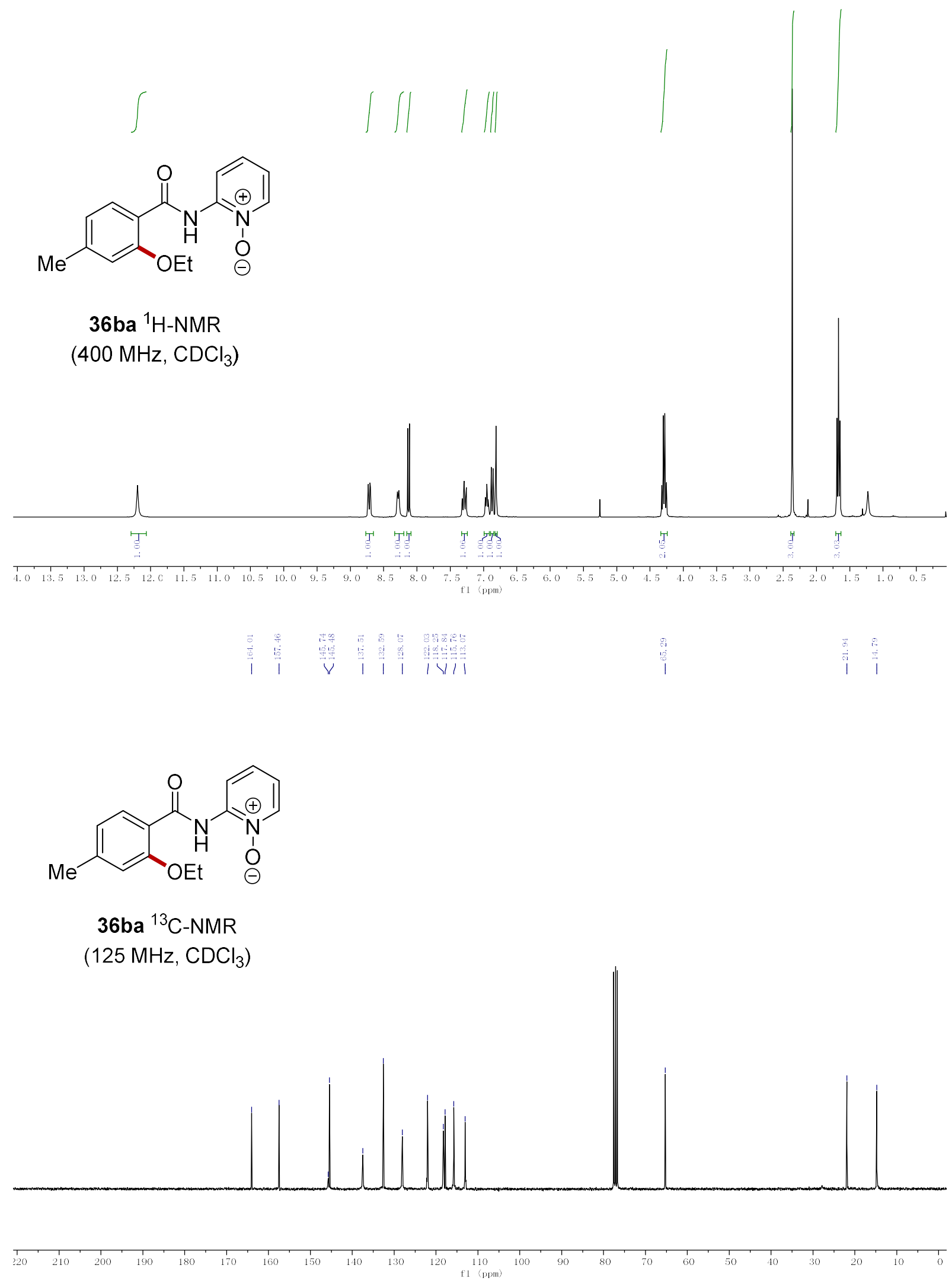
<smiles>[R]NC(C)Oc1cc(C(F)(F)F)ccc1C(=O)Nc1cccc[n+]1[O-]</smiles>

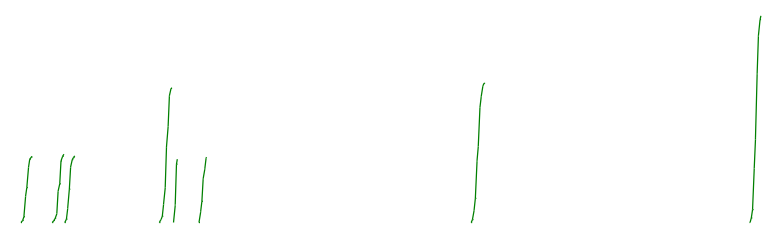

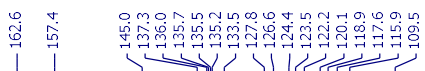

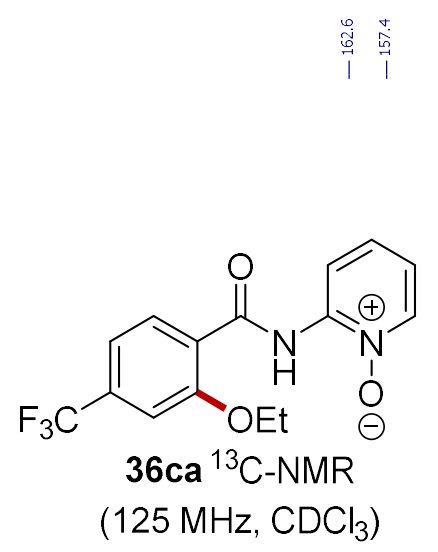

(125 MHz, $\mathrm{CDCl}_{3}$ )
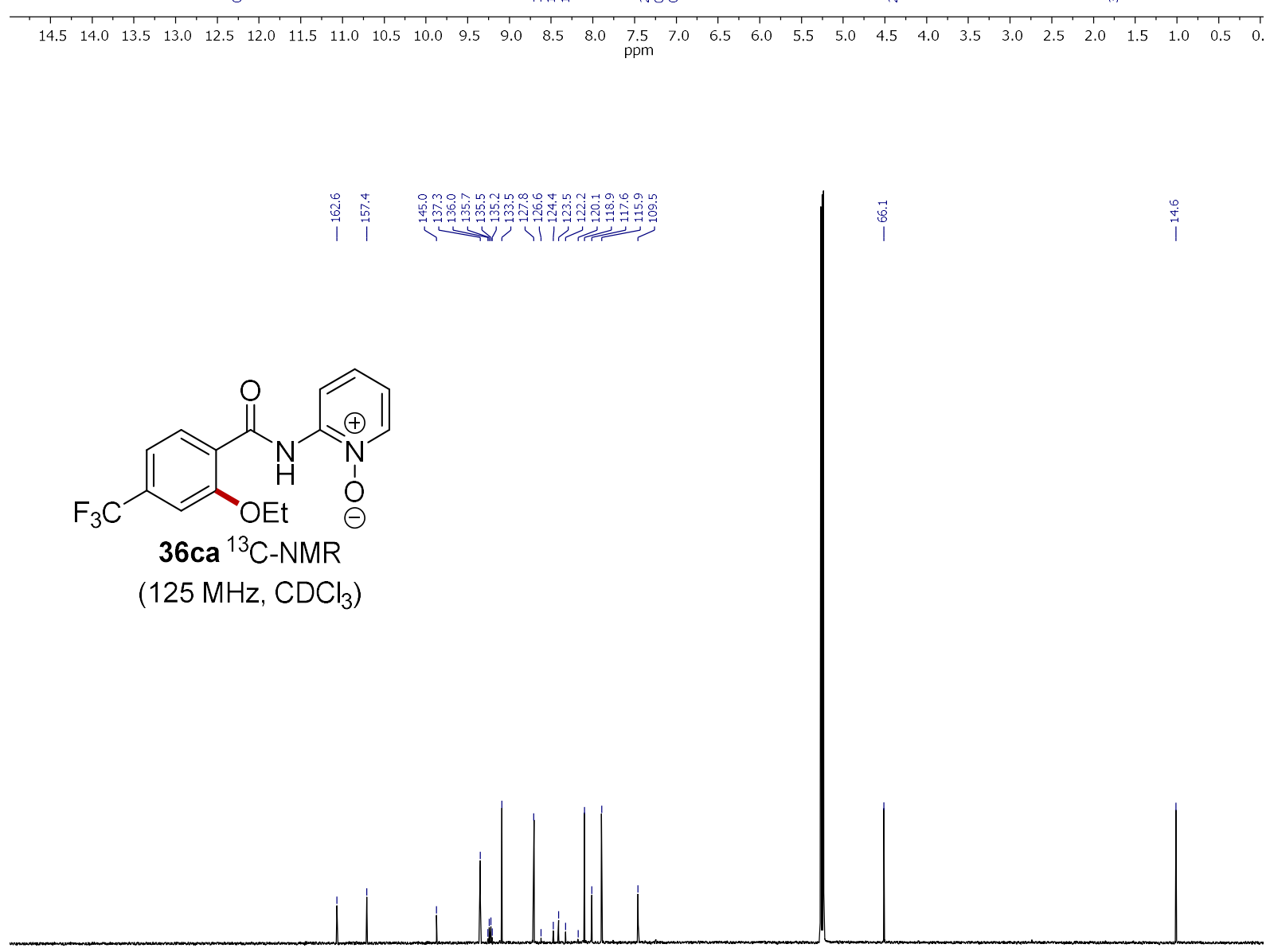

\begin{tabular}{llllllllllllllllllllllll}
\hline & 20 & 210 & 200 & 190 & 180 & 170 & 160 & 150 & 140 & 130 & 120 & 110 & 100 & 90 & 80 & 70 & 60 & 50 & 40 & 30 & 20 & 10 & 1
\end{tabular} 


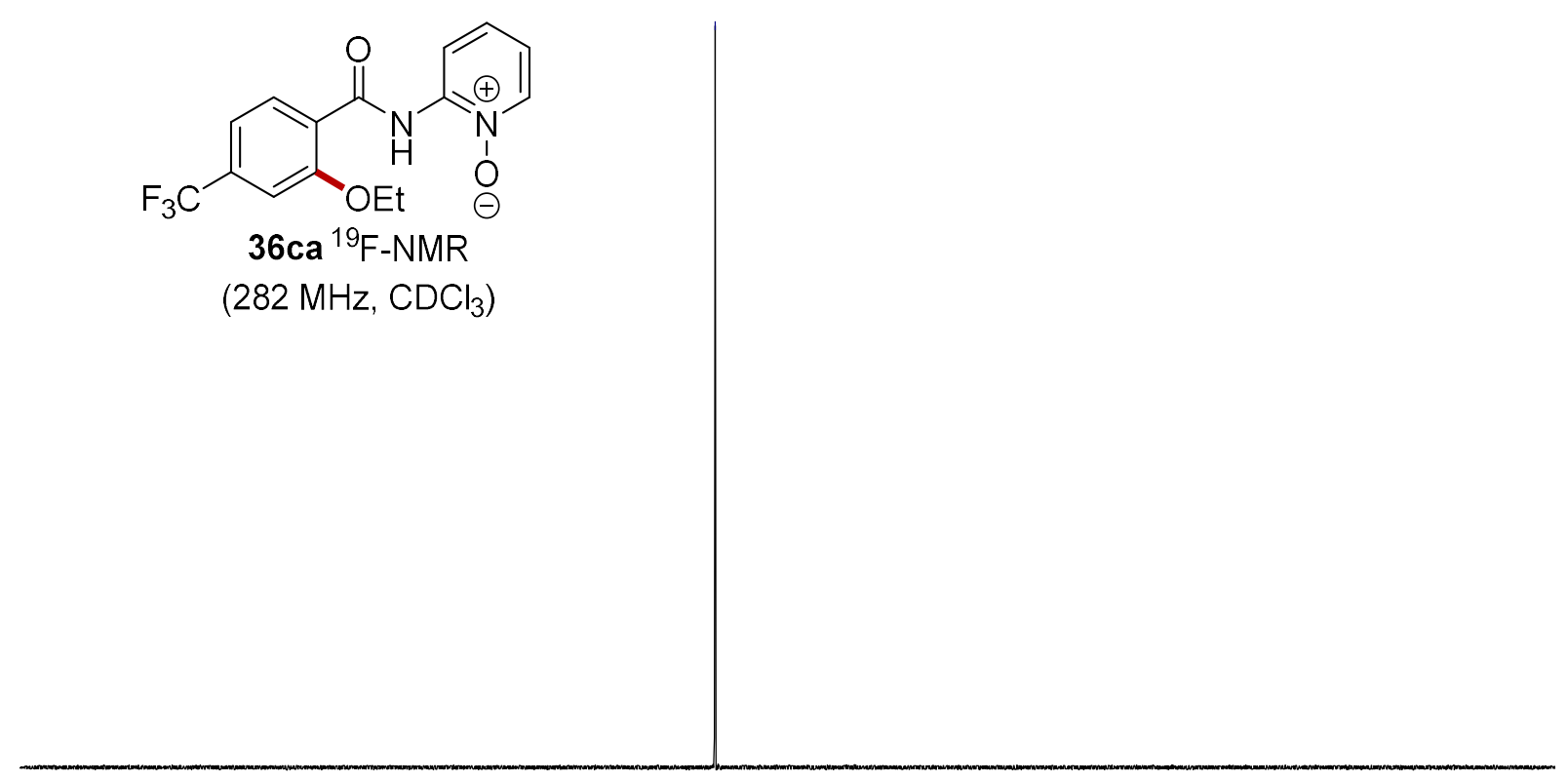<smiles>C=CC</smiles><smiles>c1ccccc1</smiles>

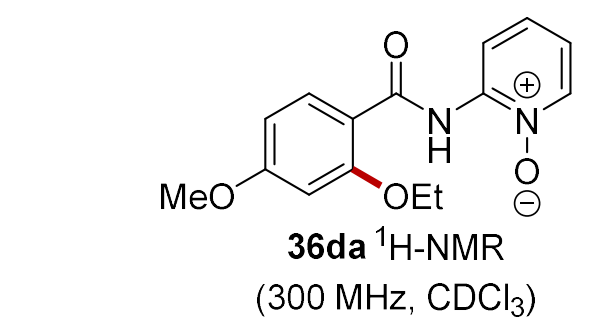

$\left(300 \mathrm{MHz}, \mathrm{CDCl}_{3}\right.$ )
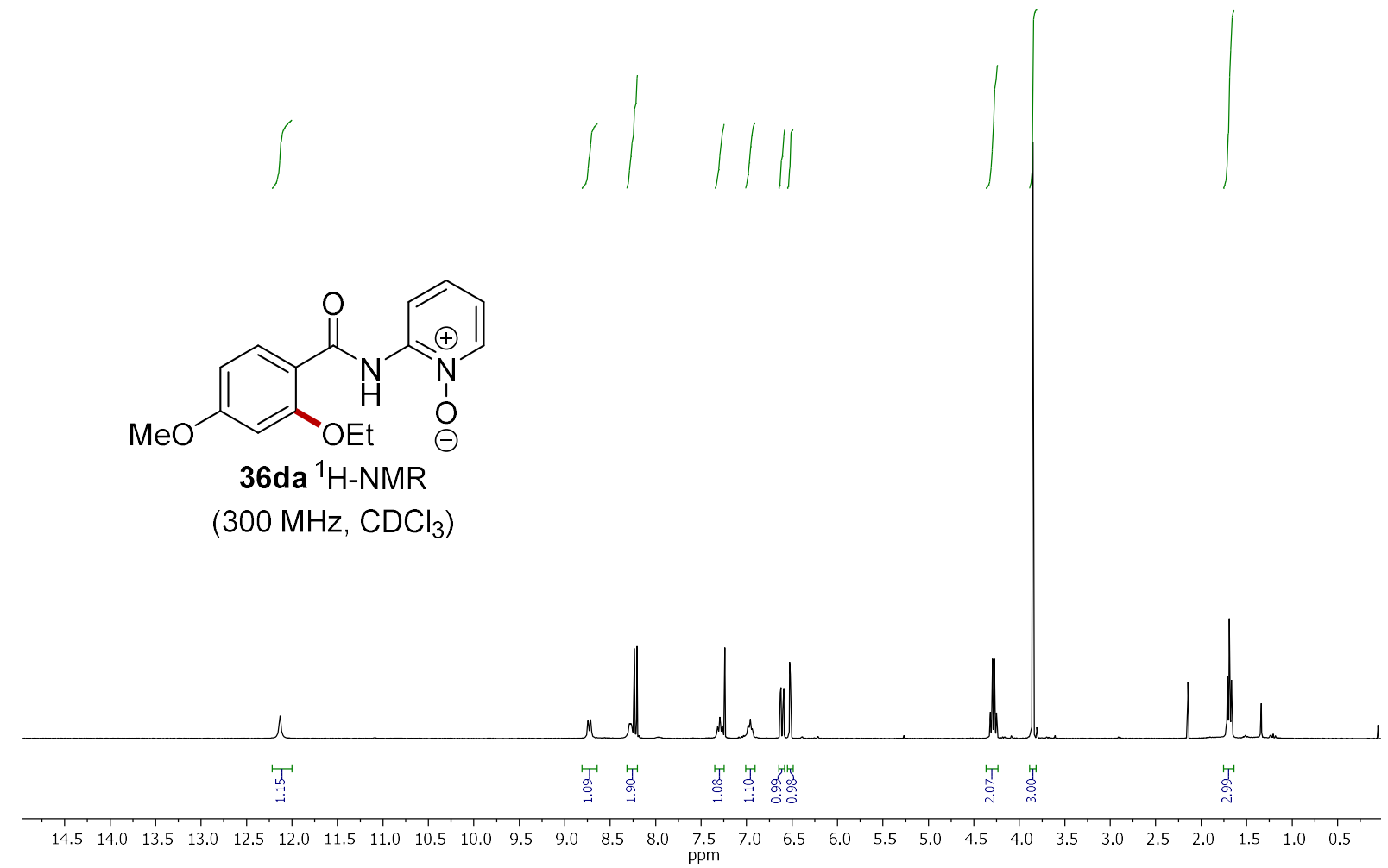


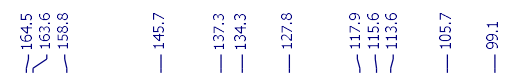<smiles>CCCCCOc1cc(OC)ccc1C(=O)Nc1cccc[n+]1[O-]</smiles>

(125 MHz, $\mathrm{CDCl}_{3}$ )
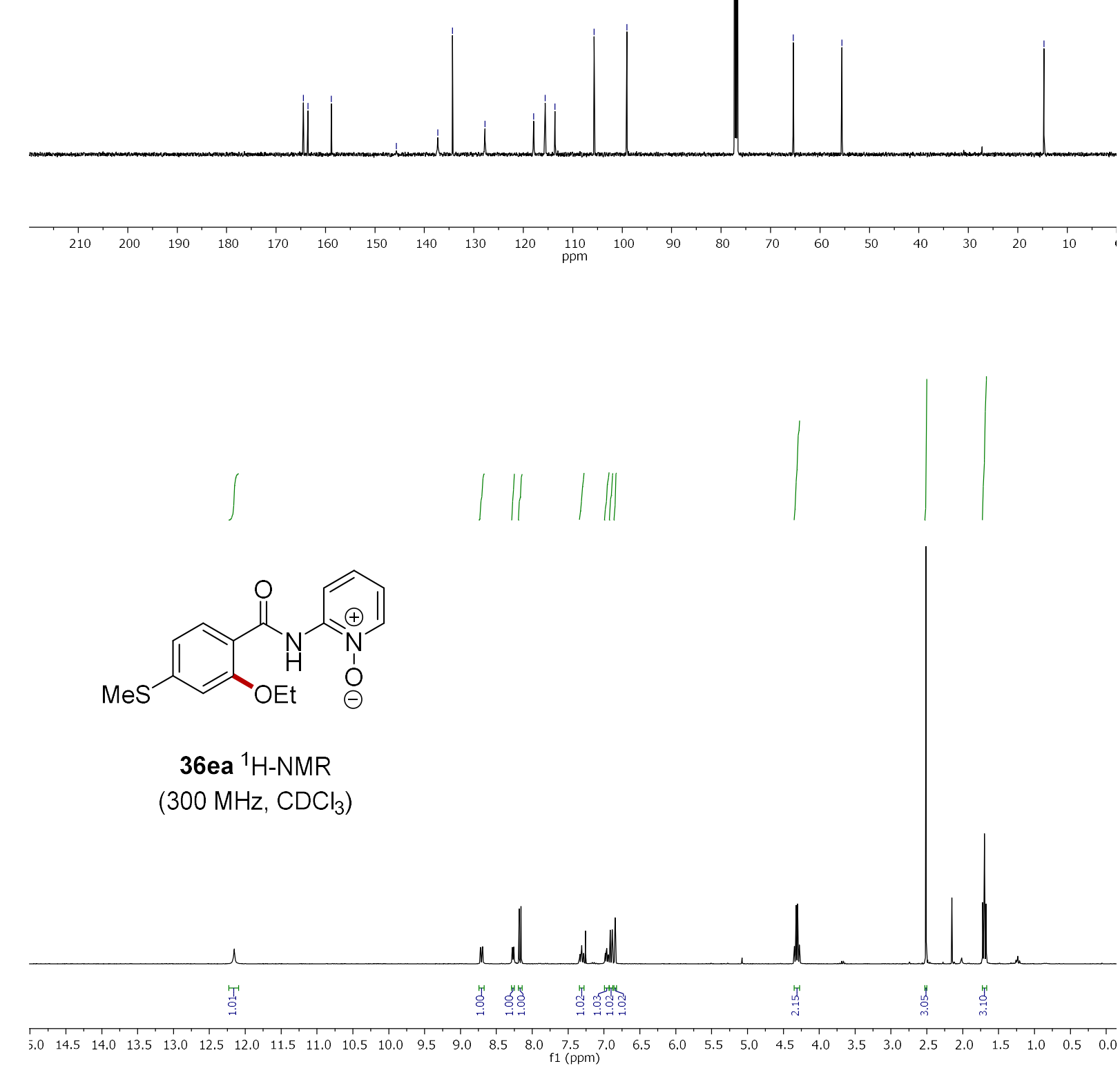

36ea ${ }^{1} \mathrm{H}-\mathrm{NMR}$

(300 MHz, CDCl $)_{3}$ )

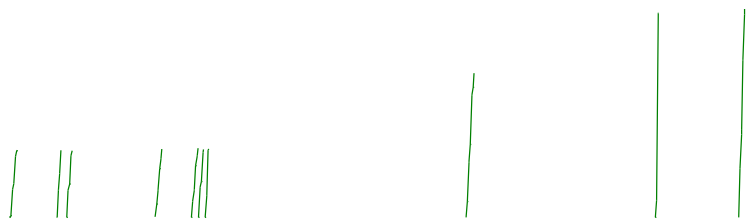




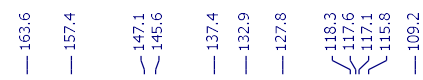<smiles>CCOc1cc(S(C)(=O)=O)ccc1C(=O)Nc1cccc[n+]1[O-]</smiles>

36ea ${ }^{13} \mathrm{C}-\mathrm{NMR}$

(126 MHz, $\mathrm{CDCl}_{3}$ )

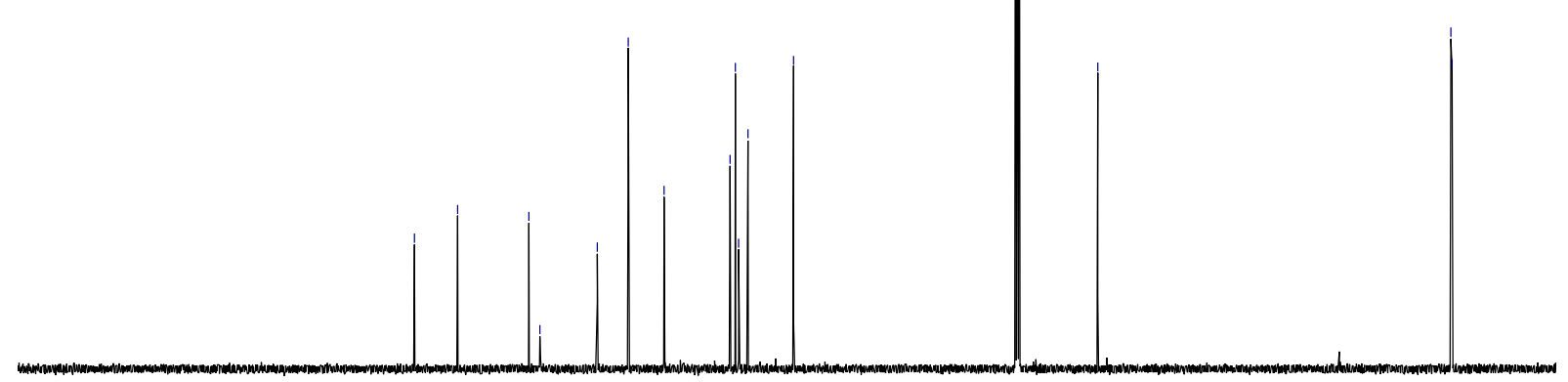

\begin{tabular}{lllllllllllllllllllllll}
\hline & 210 & 200 & 190 & 180 & 170 & 160 & 150 & 140 & 130 & 120 & 110 & 100 & 90 & 80 & 70 & 60 & 50 & 40 & 30 & 20 & 10 & 1
\end{tabular}<smiles></smiles>

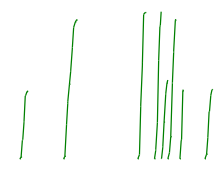

36fa ${ }^{1} \mathrm{H}-\mathrm{NMR}$ (400 MHz, $\mathrm{CDCl}_{3}$ )

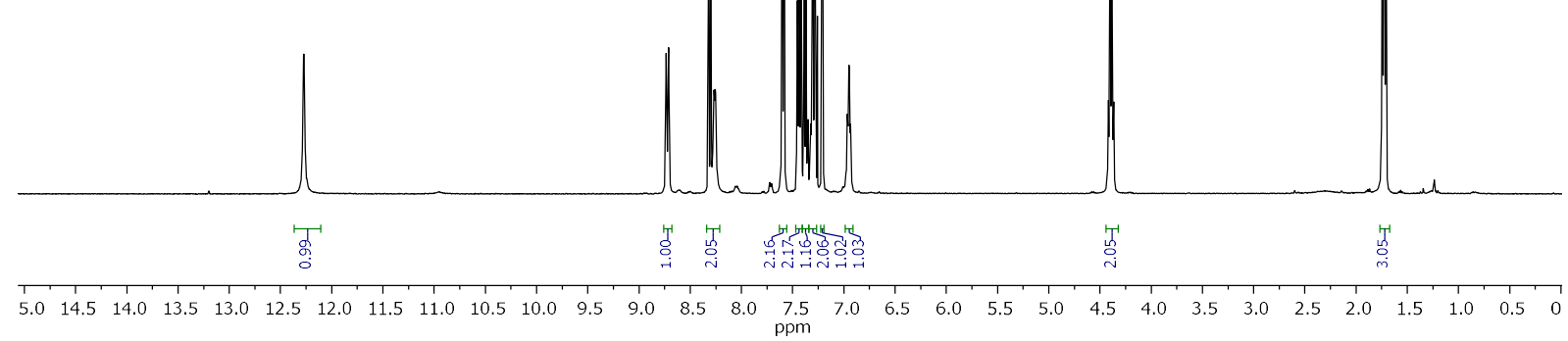




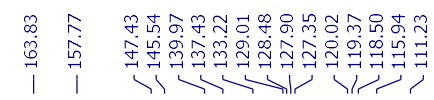

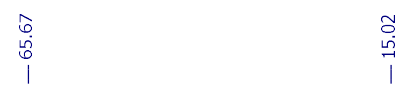<smiles></smiles>

36fa ${ }^{13} \mathrm{C}-\mathrm{NMR}$

$\left(125 \mathrm{MHz}, \mathrm{CDCl}_{3}\right.$ )

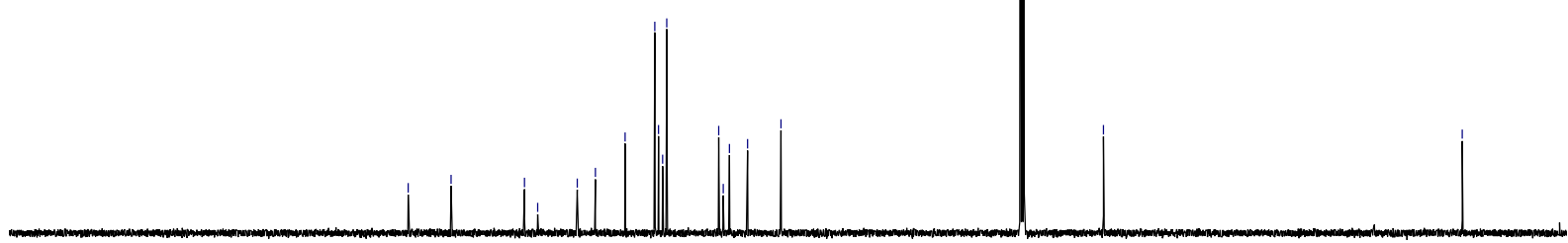

$\begin{array}{lllllllllllllllllllllllllll}20 & 210 & 200 & 190 & 180 & 170 & 160 & 150 & 140 & 130 & 120 & 110 & 100 & 90 & 80 & 70 & 60 & 50 & 40 & 30 & 20 & 10\end{array}$<smiles>C1CCCCC1</smiles>
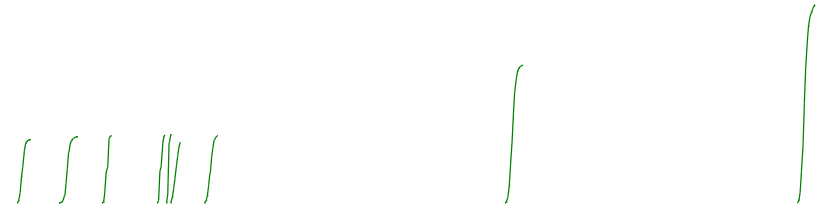<smiles>CCOc1cc(I)ccc1C(=O)Nc1cccc[n+]1[O-]</smiles>

36ga ${ }^{1} \mathrm{H}-\mathrm{NMR}$ $\left(300 \mathrm{MHz}, \mathrm{CDCl}_{3}\right.$ )

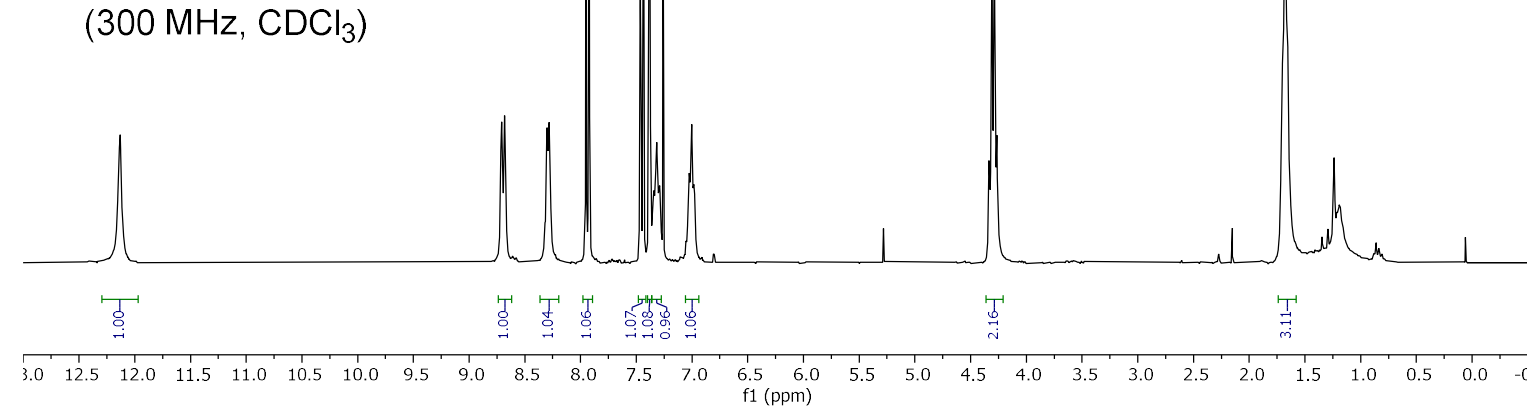




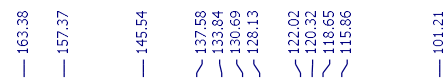<smiles>CCOc1cc(I)ccc1C(=O)Nc1cccc[n+]1[O-]</smiles>

36ga ${ }^{13} \mathrm{C}-\mathrm{NMR}$

$\left(75 \mathrm{MHz}, \mathrm{CDCl}_{3}\right.$ )

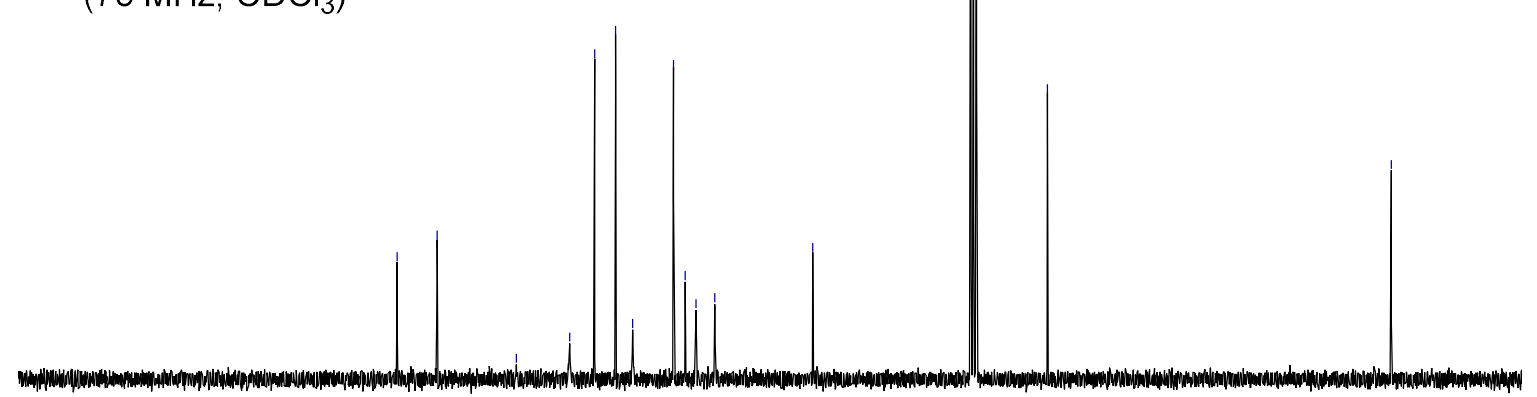

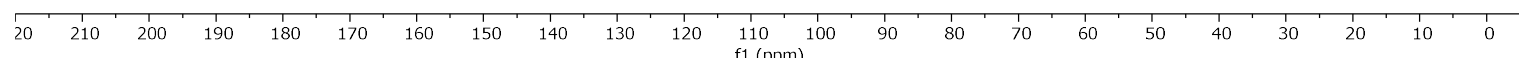<smiles>CC=CC</smiles>

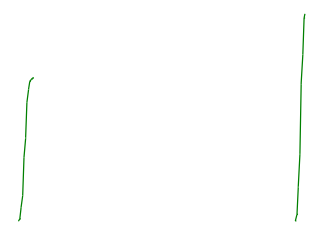<smiles>CCOc1ccc(Cl)cc1C(=O)Nc1cccc[n+]1[O-]</smiles>

36ha ${ }^{1} \mathrm{H}-\mathrm{NMR}$

(300 MHz, $\mathrm{CDCl}_{3}$ )

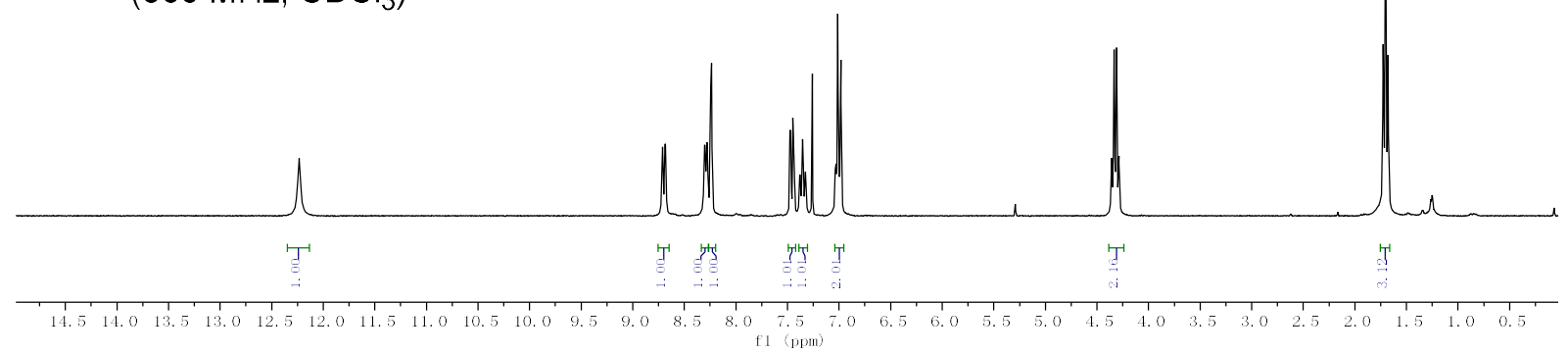


<smiles>CCOc1ccc(Cl)cc1C(=O)Nc1cccc[n+]1[O-]</smiles>

36ha ${ }^{13} \mathrm{C}-\mathrm{NMR}$

(125 MHz, $\mathrm{CDCl}_{3}$ )
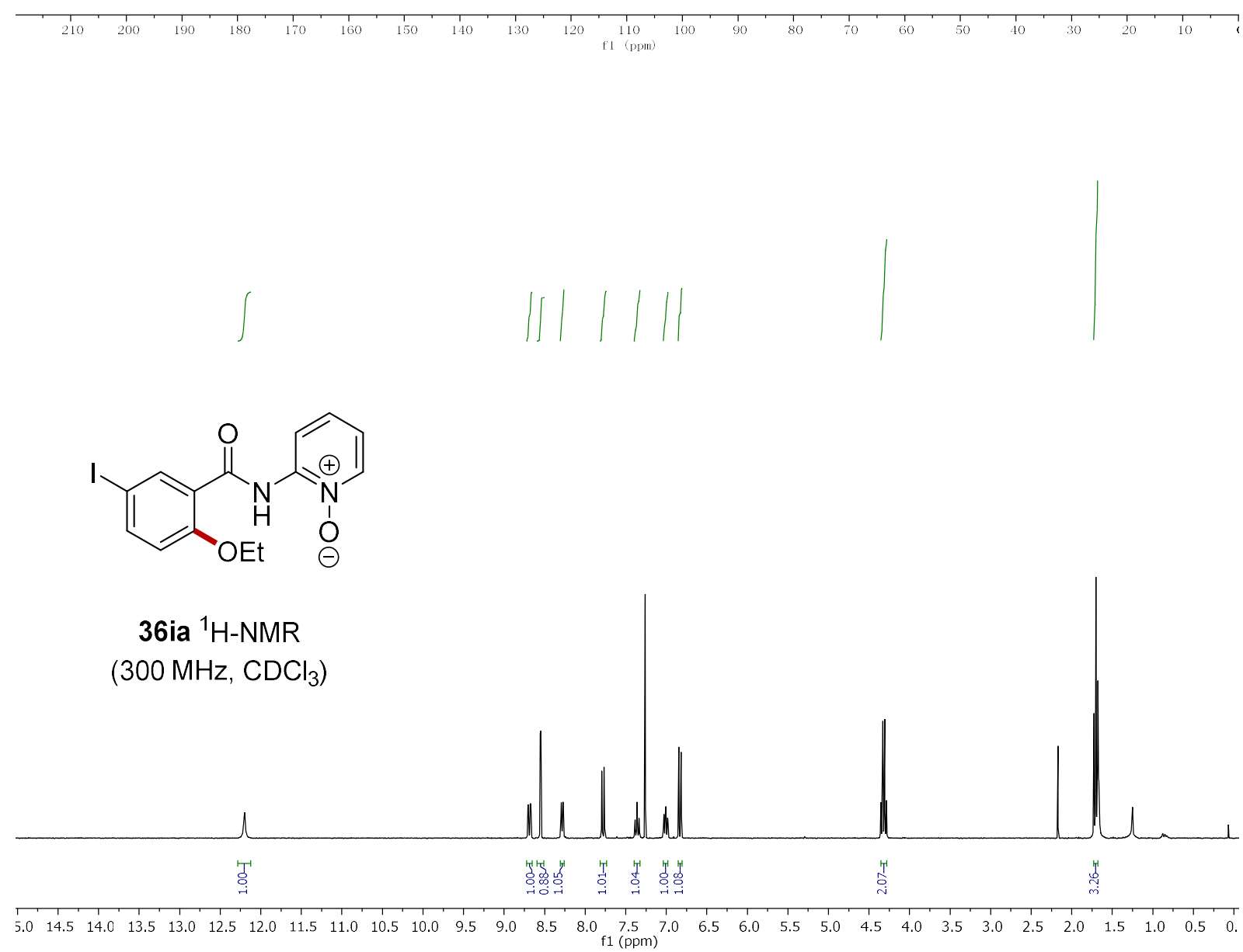
<smiles>CCOc1ccc(I)cc1C(=O)Nc1cccc[n+]1[O-]</smiles>

36ia ${ }^{13} \mathrm{C}-\mathrm{NMR}$

(125 MHz, $\mathrm{CDCl}_{3}$ )
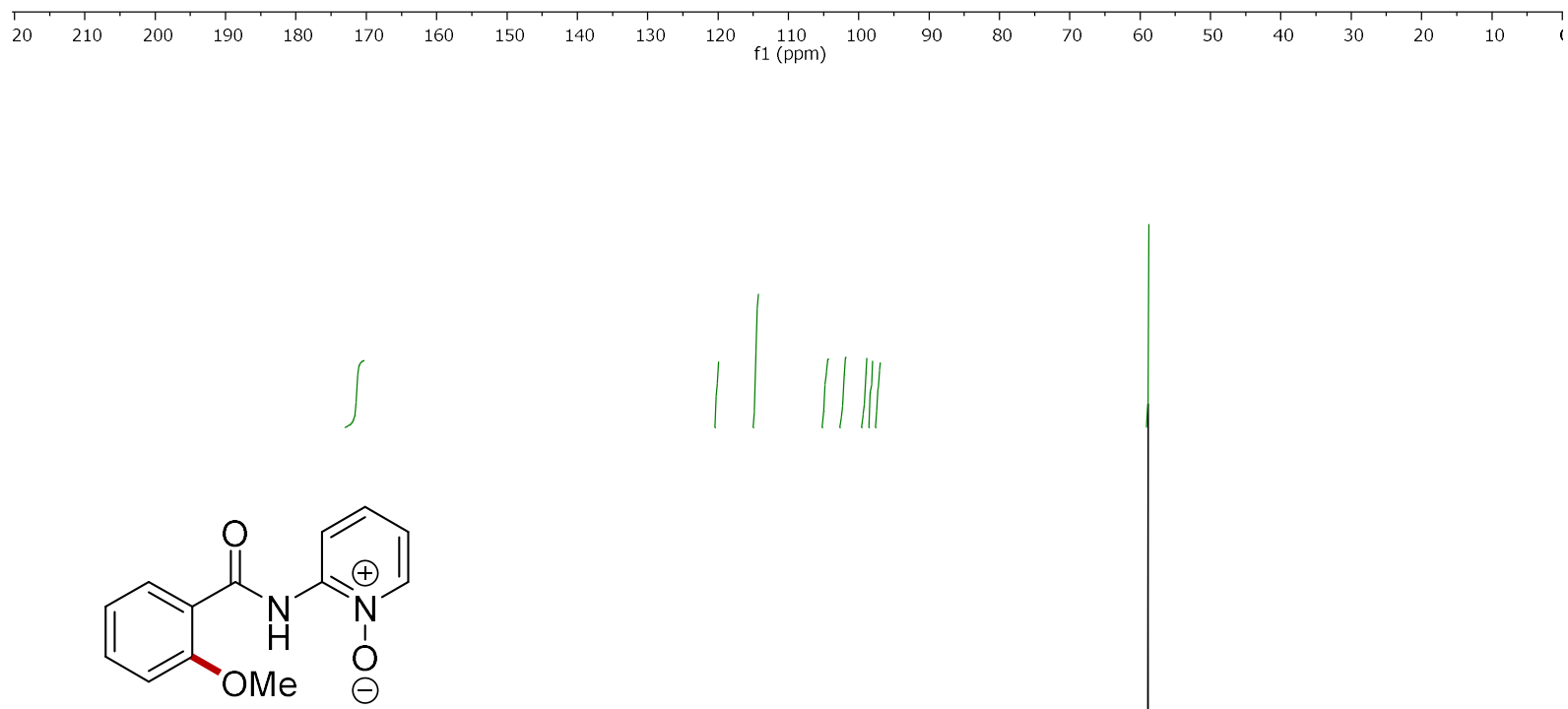

36ab ${ }^{1} \mathrm{H}-\mathrm{NMR}$

$\left(400 \mathrm{MHz}, \mathrm{CDCl}_{3}\right.$ )

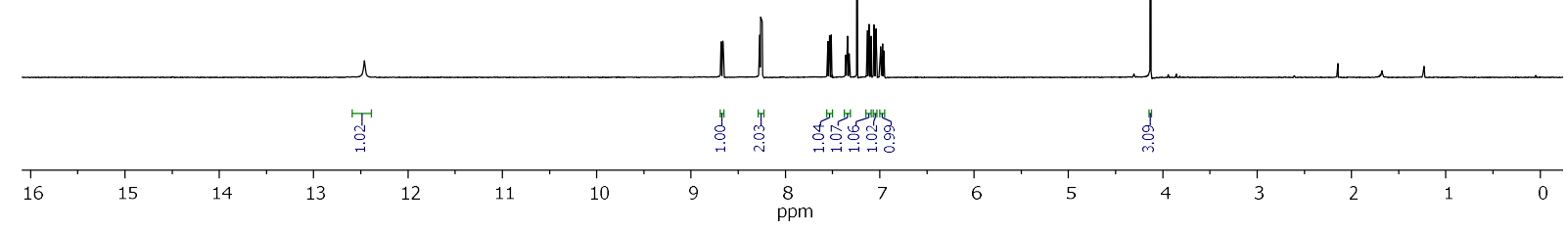




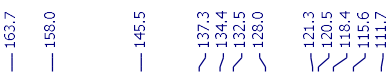<smiles>COc1ccccc1C(=O)Nc1cccc[n+]1[O-]</smiles>

36ab ${ }^{13} \mathrm{C}-\mathrm{NMR}$

(101 MHz, $\mathrm{CDCl}_{3}$ )
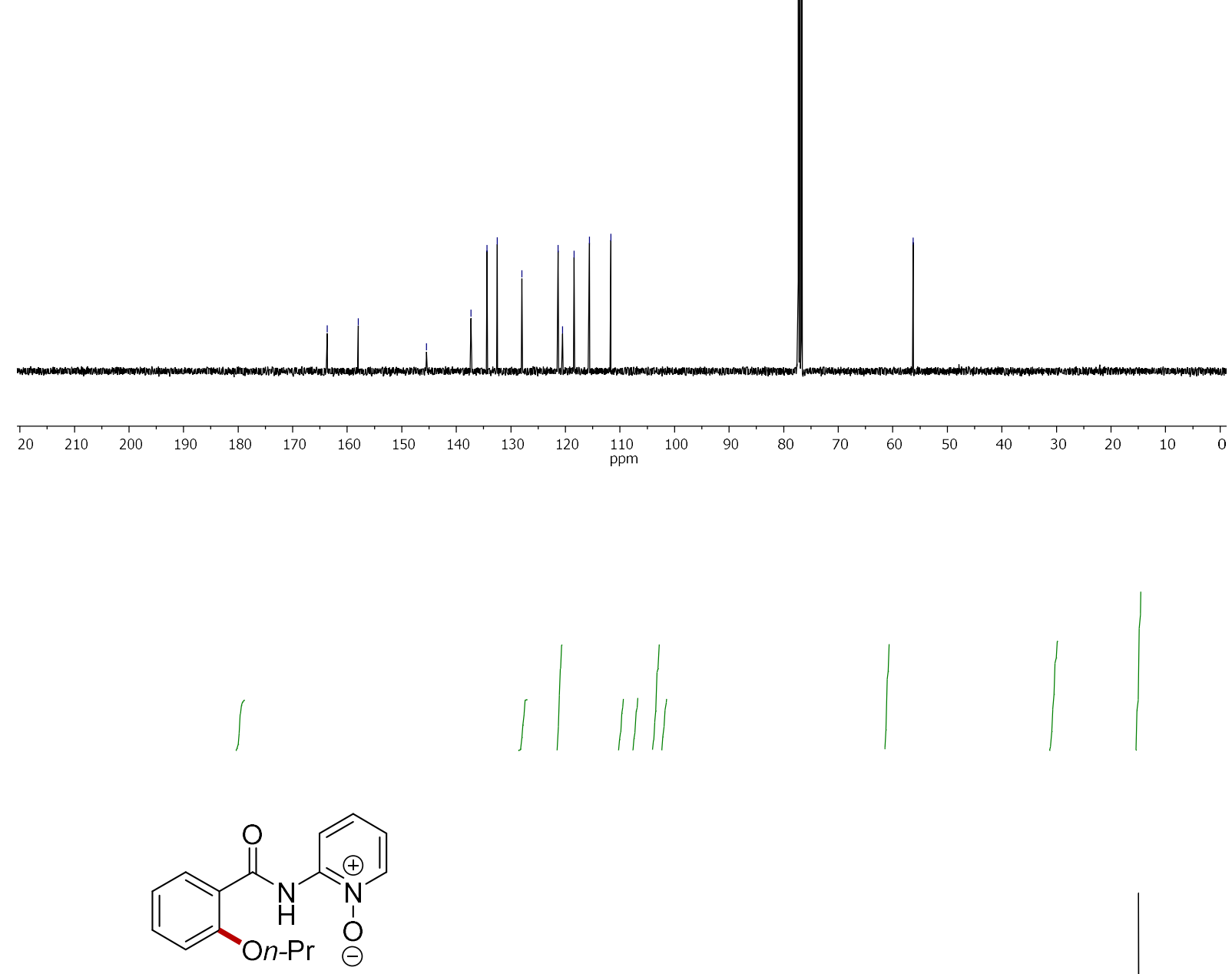

36ac ${ }^{1} \mathrm{H}-\mathrm{NMR}$

$\left(300 \mathrm{MHz}, \mathrm{CDCl}_{3}\right.$ )

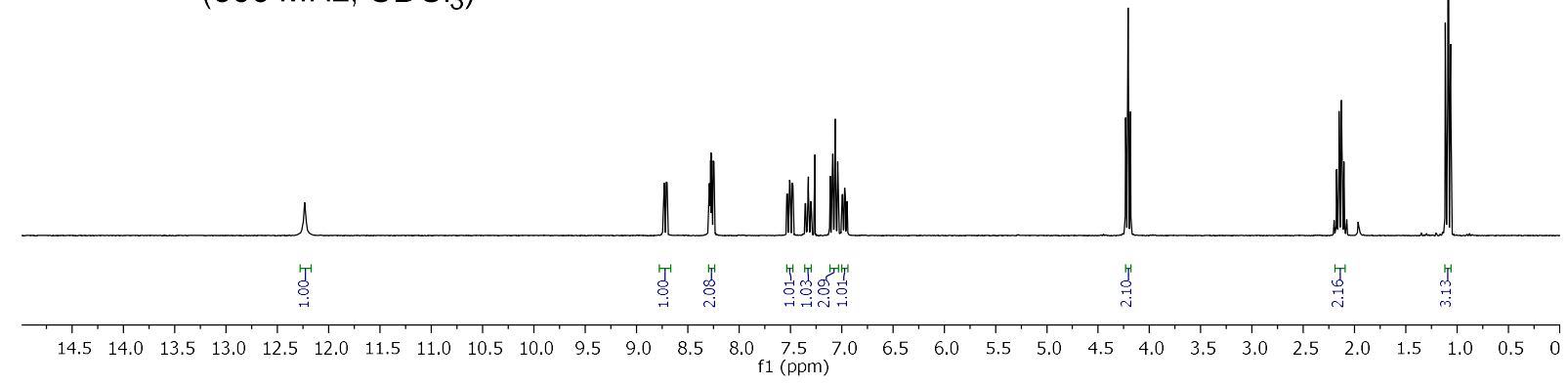




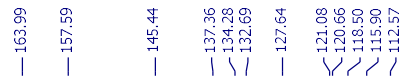<smiles>CCCOc1ccccc1C(=O)Nc1cccc[n+]1[O-]</smiles>

$36 a c{ }^{13} \mathrm{C}-\mathrm{NMR}$

(126 MHz, $\mathrm{CDCl}_{3}$ )
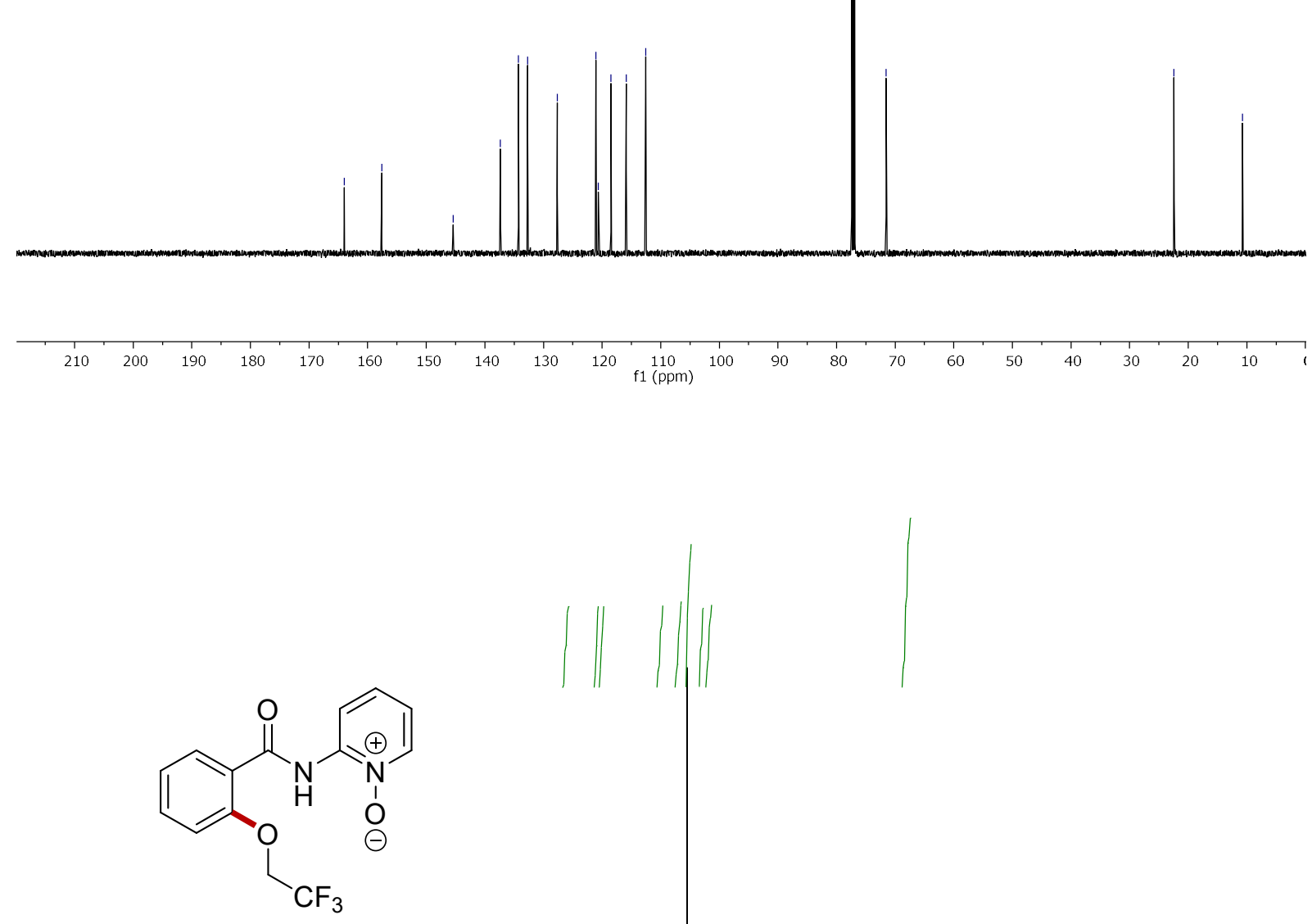

36ad ${ }^{1} \mathrm{H}-\mathrm{NMR}$

$\left(300 \mathrm{MHz}, \mathrm{CDCl}_{3}\right.$ )

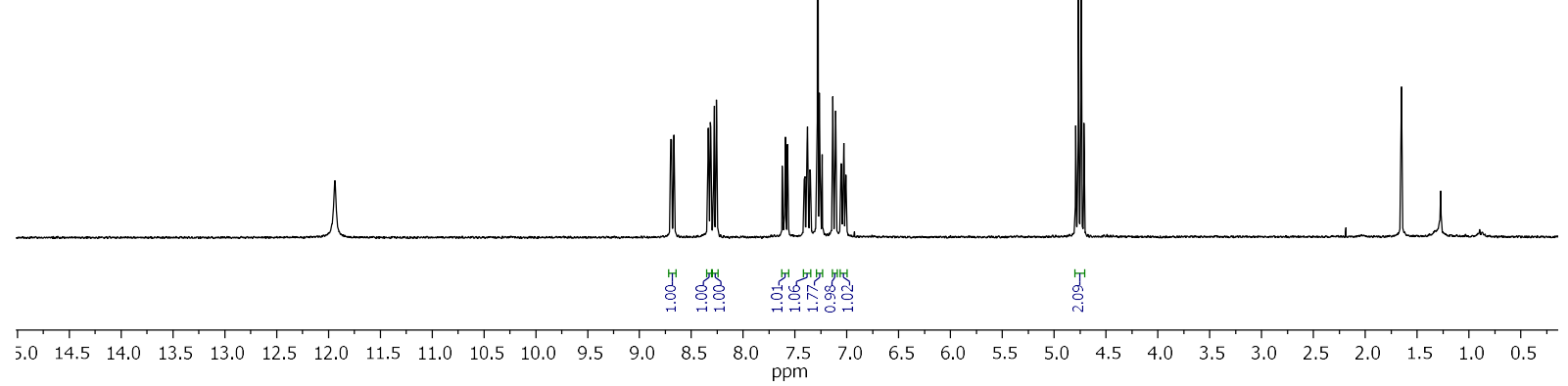



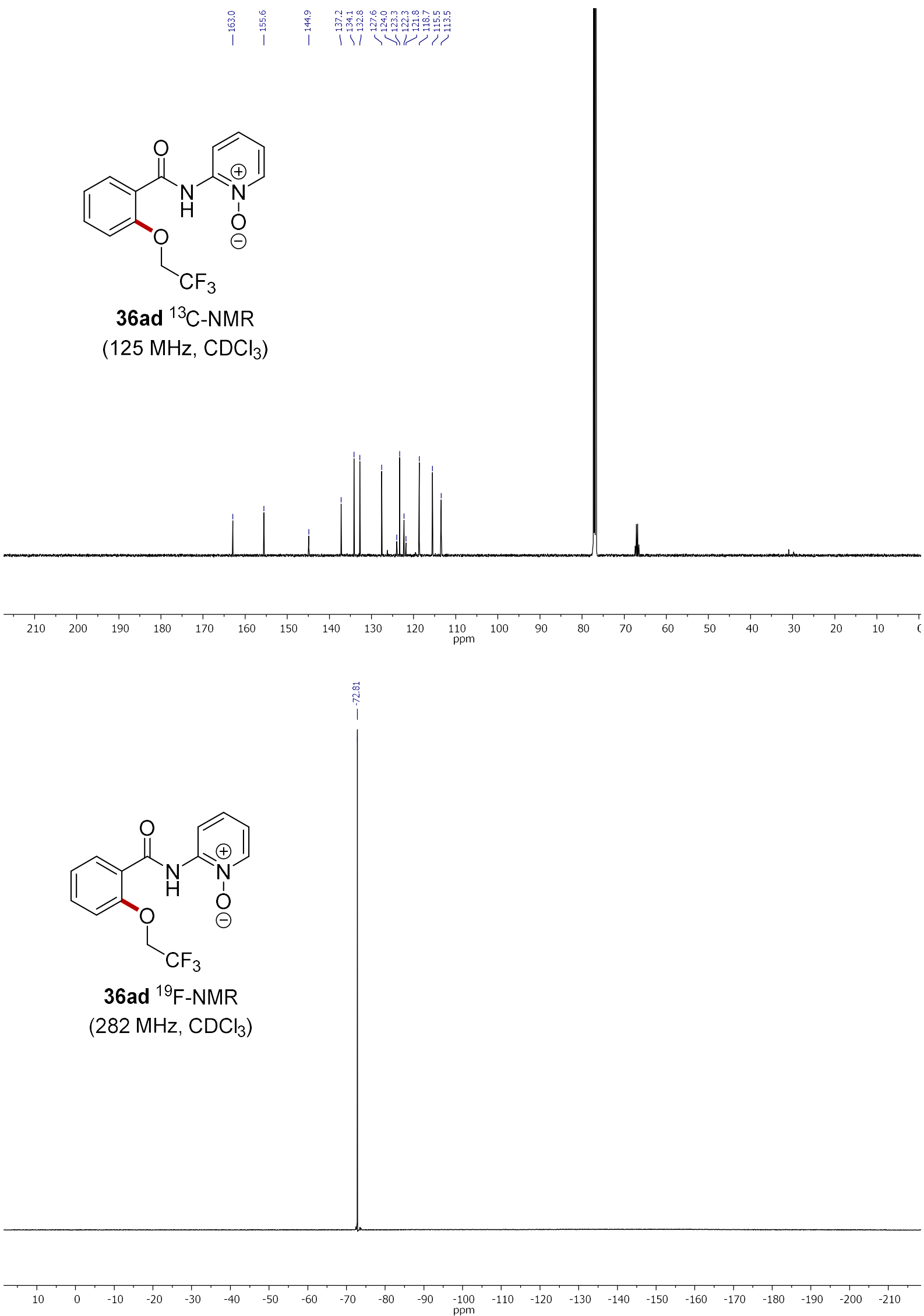


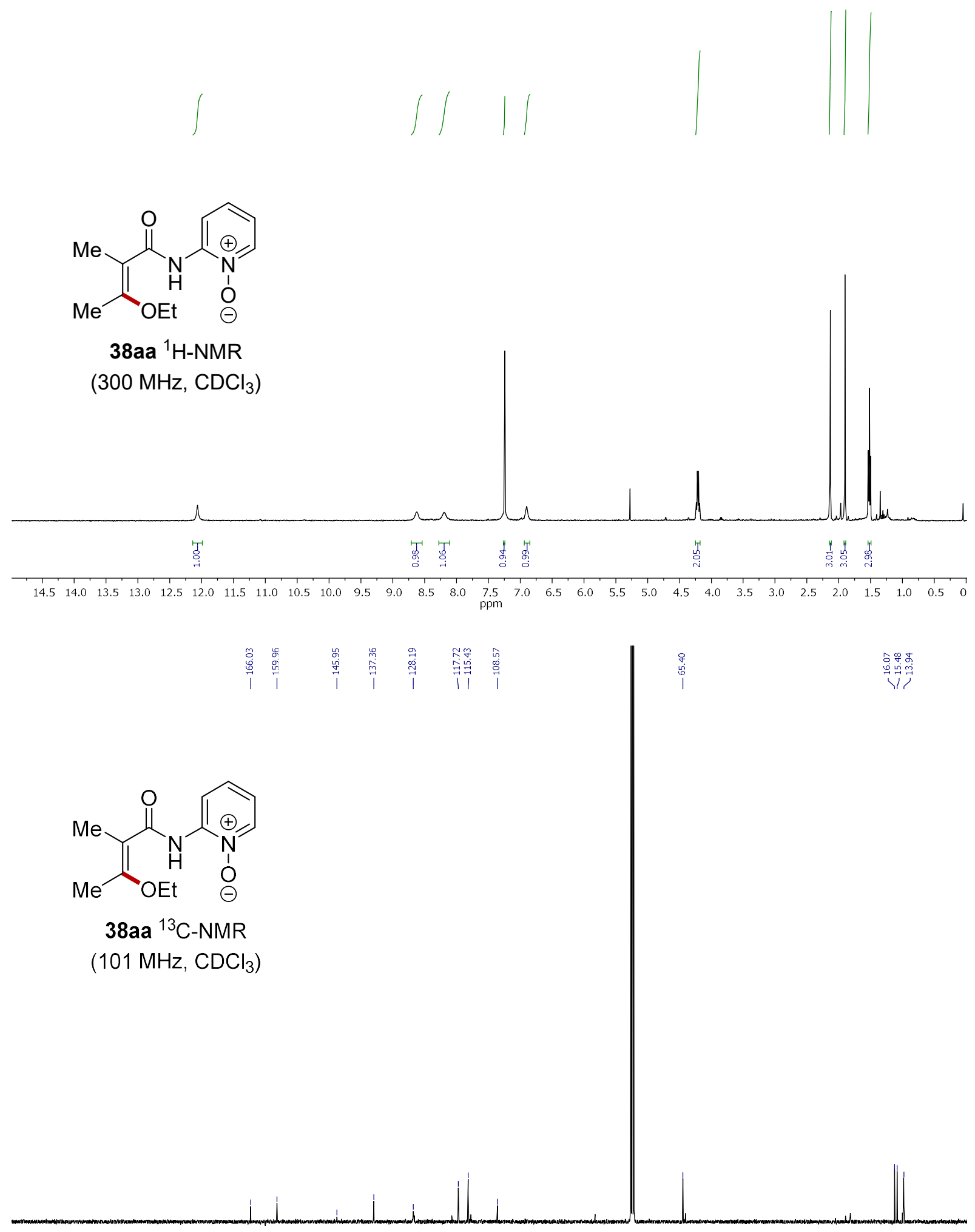

$\begin{array}{lllllllllllllllllllllll}1 & 1 \\ 220 & 210 & 200 & 190 & 180 & 170 & 160 & 150 & 140 & 130 & 120 & 110 & 100 & 90 & 80 & 70 & 60 & 50 & 40 & 30 & 20 & 10 & 0\end{array}$ 

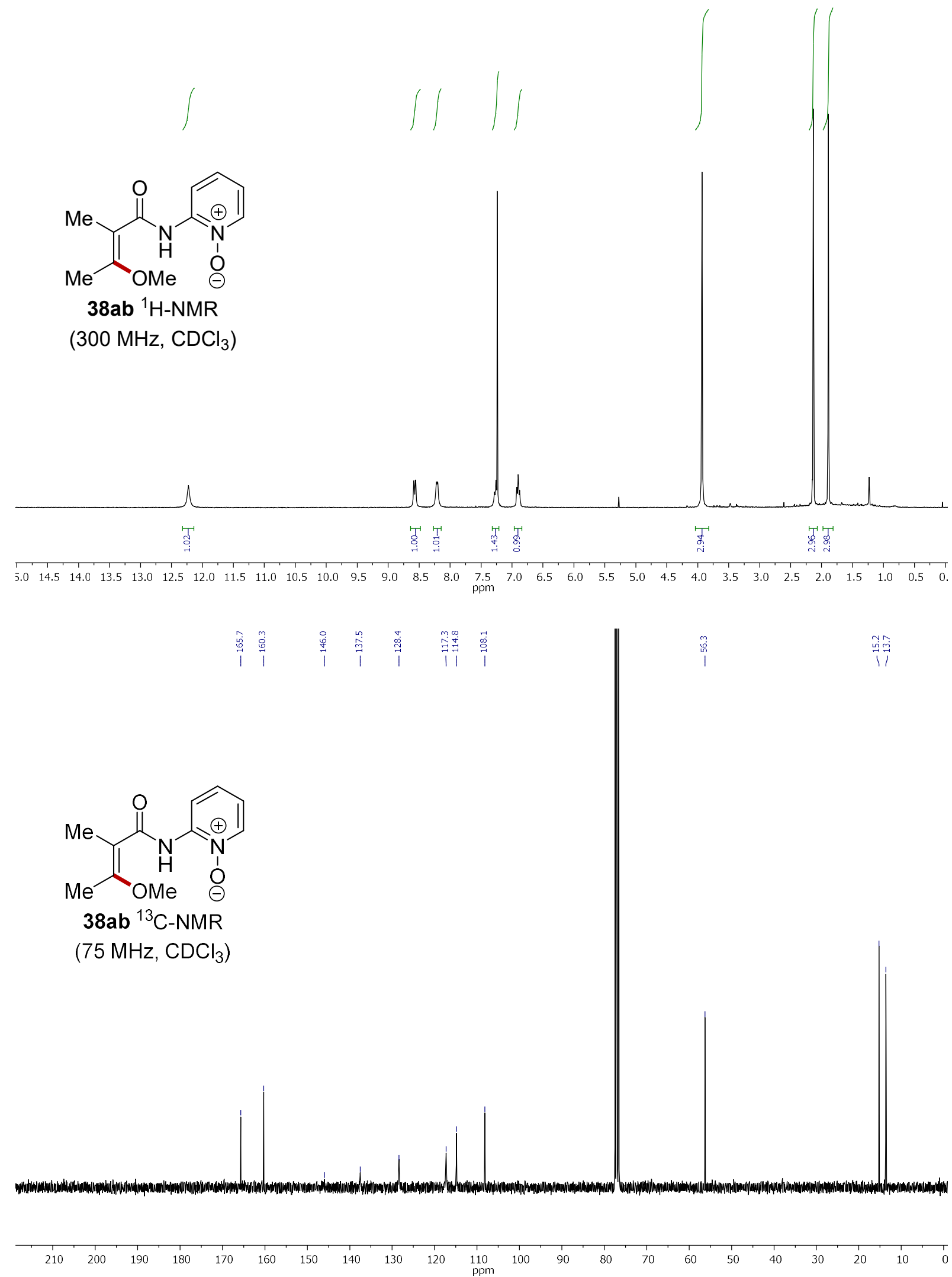


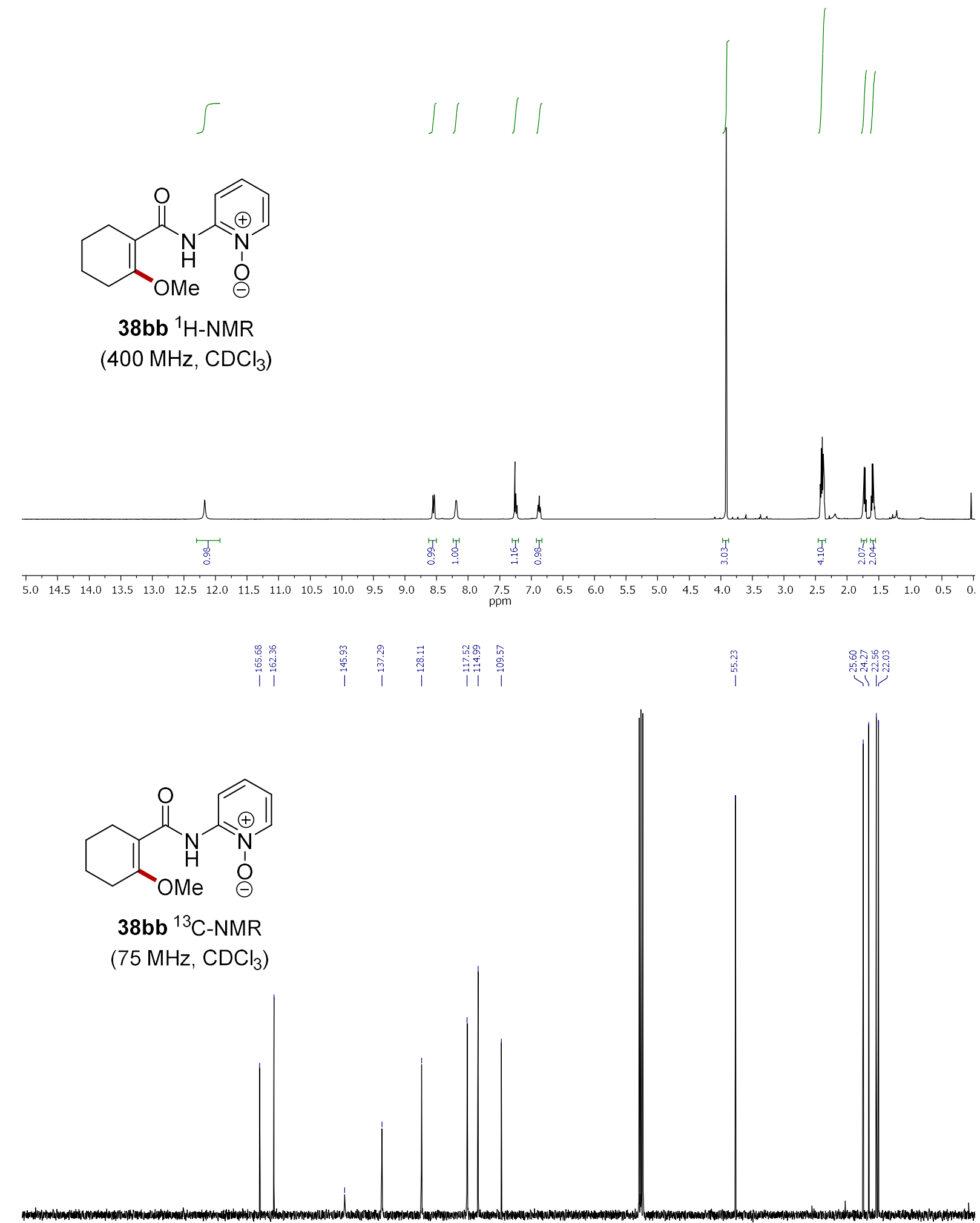

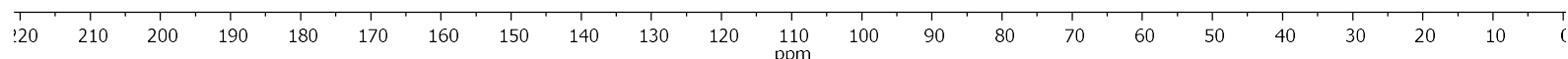



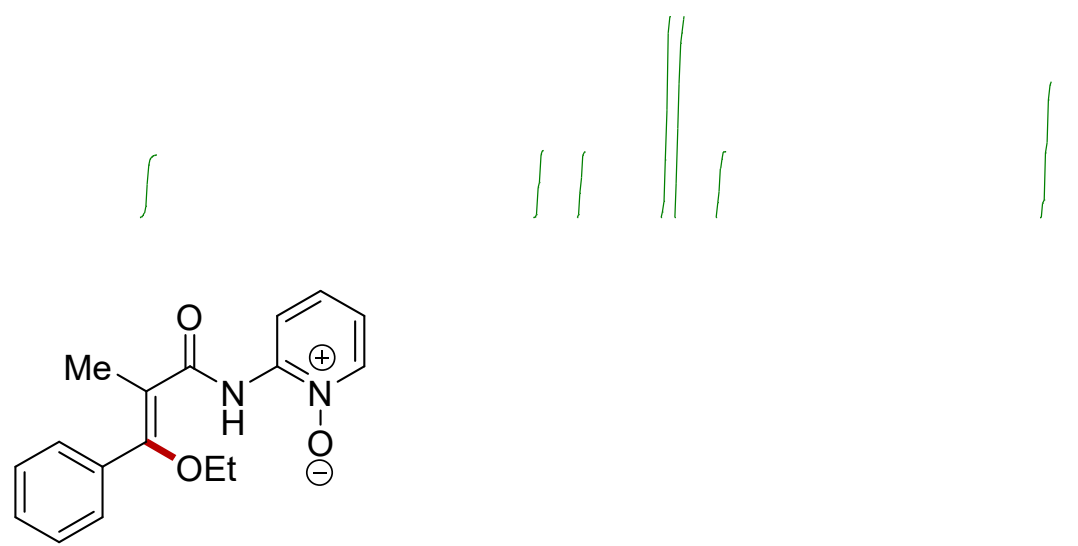

38ca ${ }^{1} \mathrm{H}-\mathrm{NMR}$

$\left(300 \mathrm{MHz}, \mathrm{CDCl}_{3}\right)$

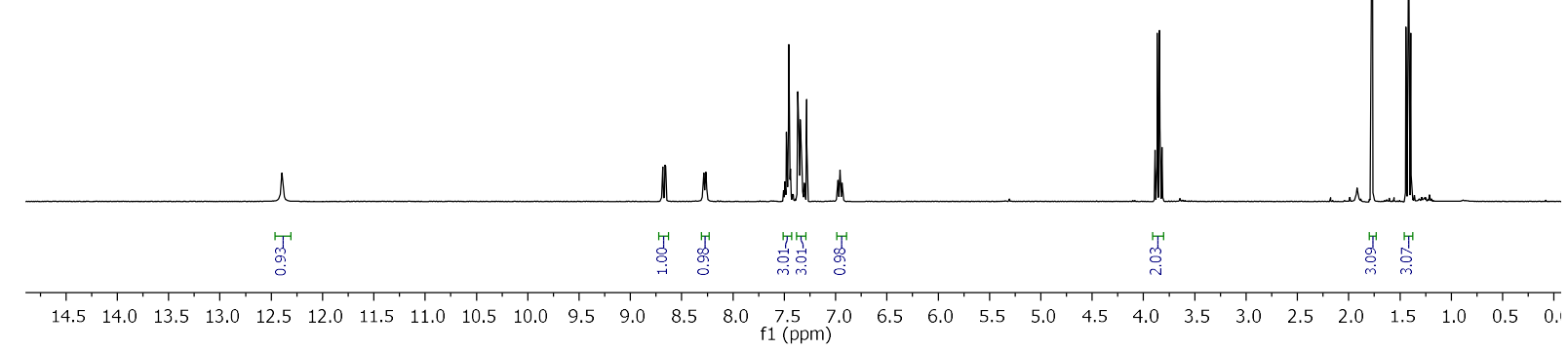

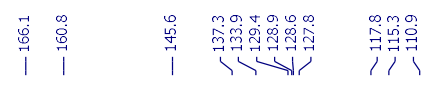<smiles>CCO/C(=C(/C)C(=O)Nc1cccc[n+]1[O-])c1ccccc1</smiles>

38ca ${ }^{13} \mathrm{C}-\mathrm{NMR}$

$\left(75 \mathrm{MHz}, \mathrm{CDCl}_{3}\right.$ )
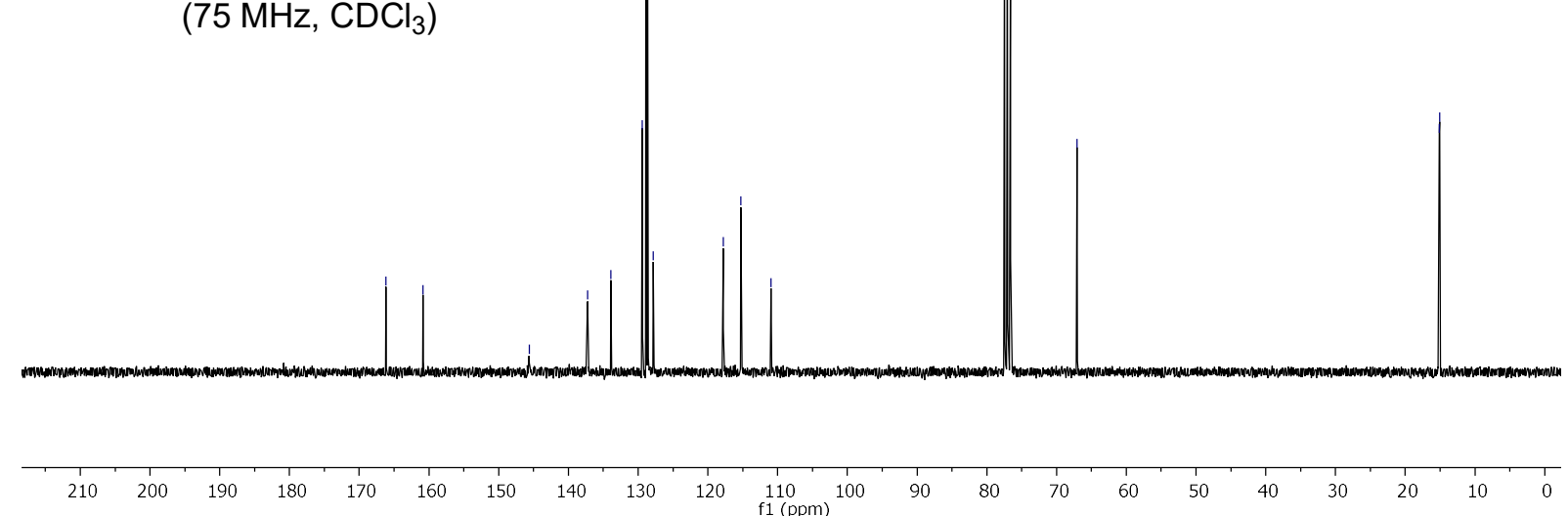


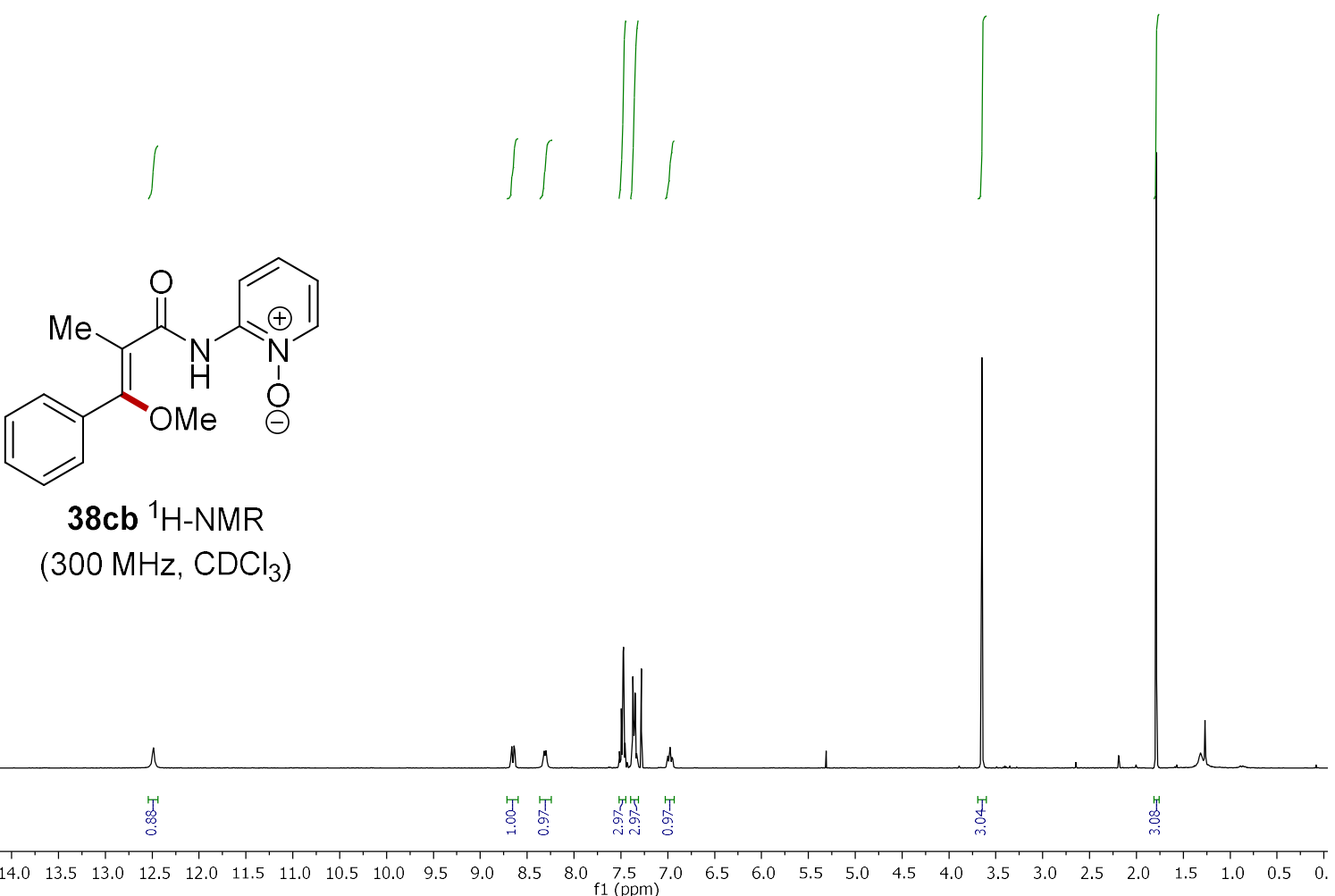

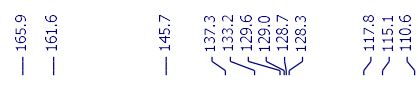<smiles>CO/C(=C(/C)C(=O)Nc1cccc[n+]1[O-])c1ccccc1</smiles>

$38 \mathrm{cb}^{13} \mathrm{C}-\mathrm{NMR}$

$\left(75 \mathrm{MHz}, \mathrm{CDCl}_{3}\right.$ )

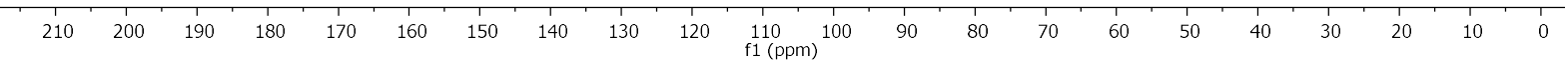



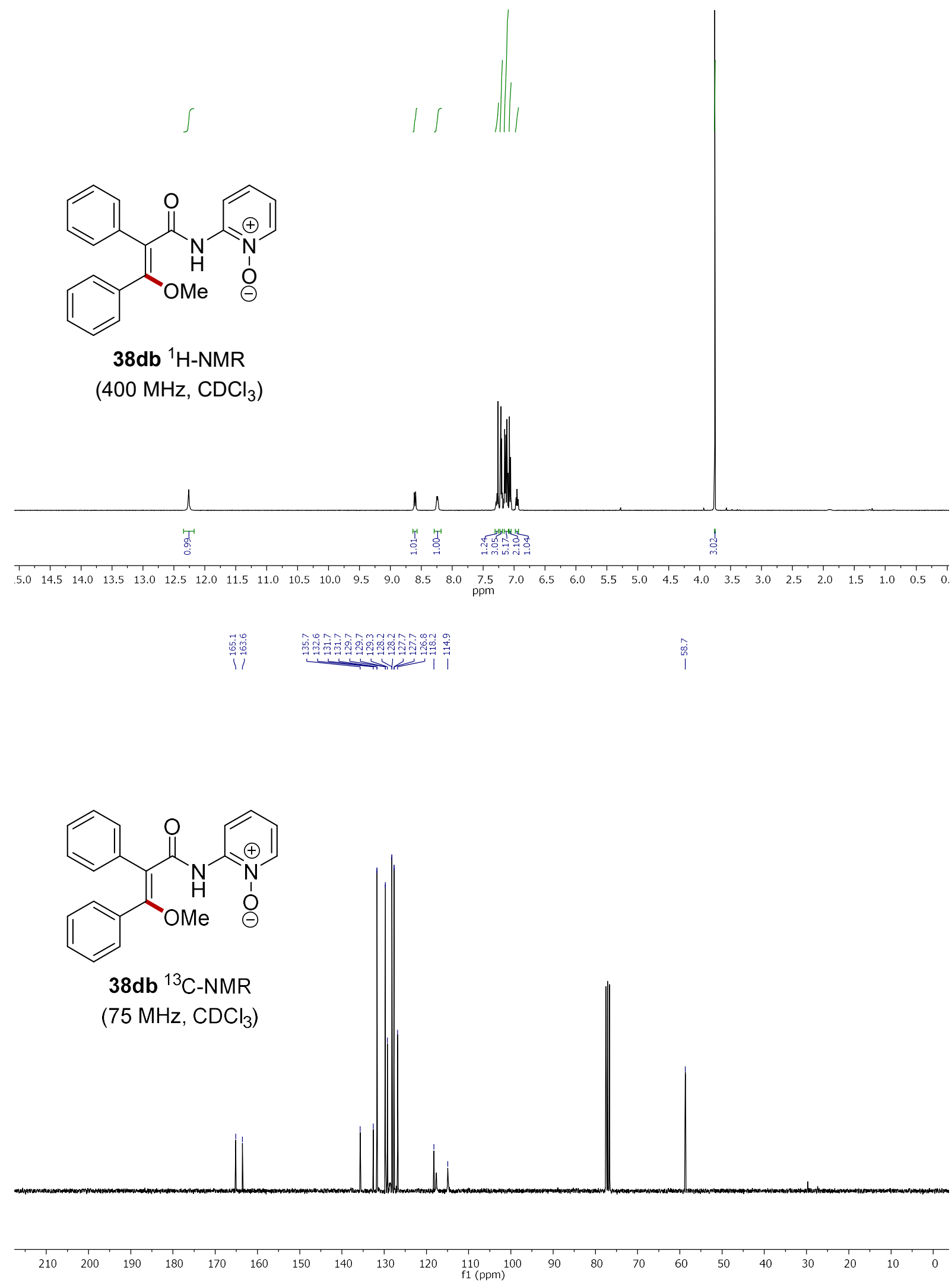
<smiles>O=c1c2ccccc2cc(CP(=O)(c2ccccc2)c2ccccc2)n1-c1cccc[n+]1[O-]</smiles>

176aa ${ }^{1} \mathrm{H}-\mathrm{NMR}$

(600 MHz, $\mathrm{CDCl}_{3}$ )
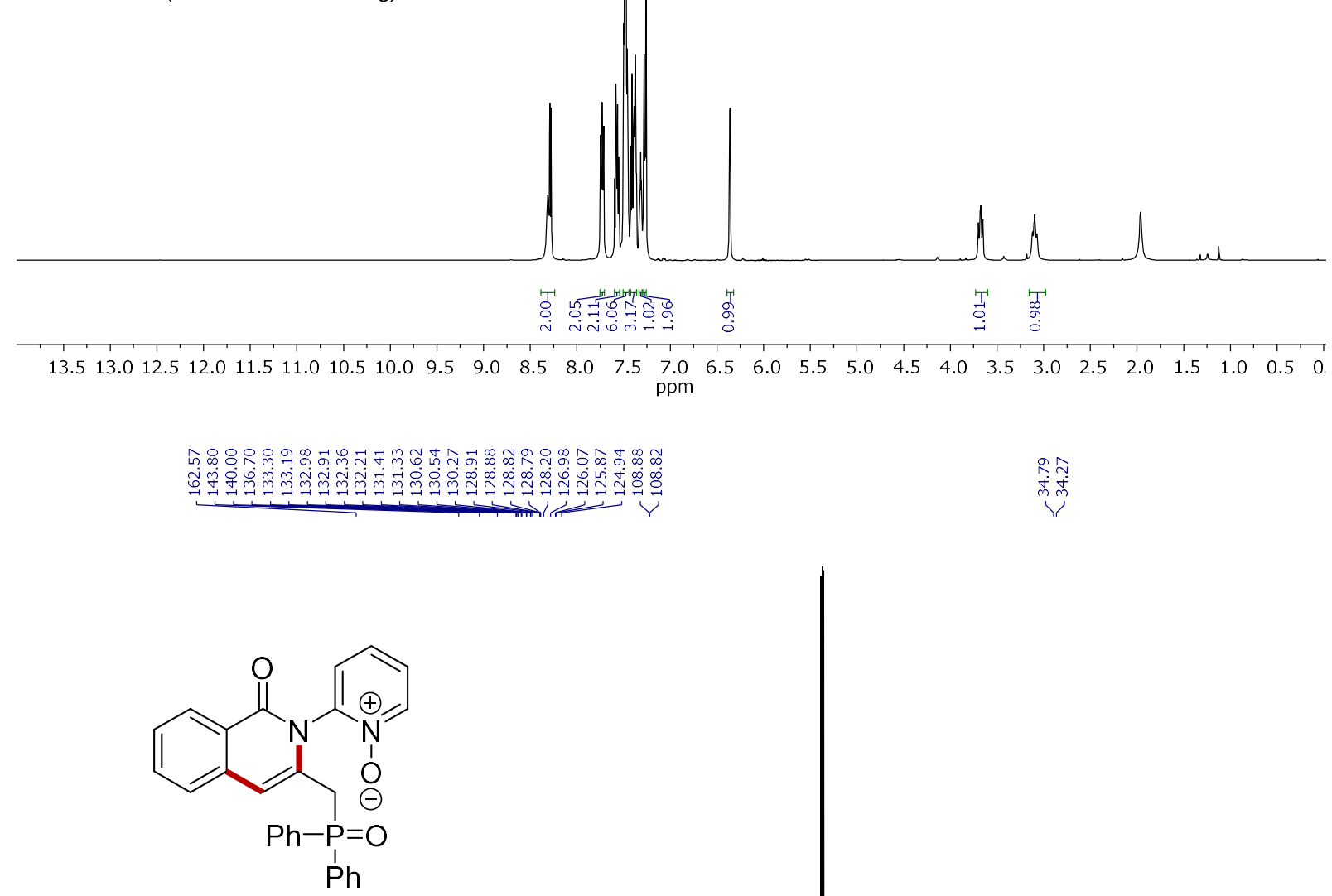

176aa ${ }^{13} \mathrm{C}-\mathrm{NMR}$

(126 MHz, $\mathrm{CDCl}_{3}$ )

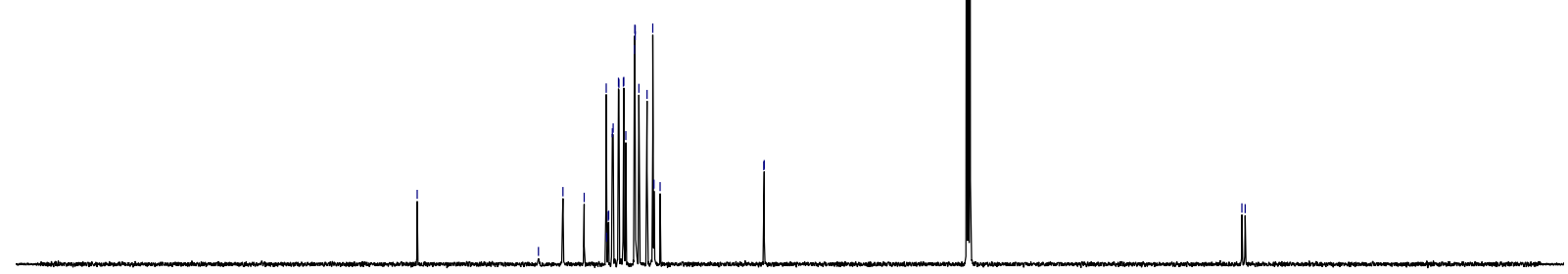

$\begin{array}{lllllllllllllllllllllllll}220 & 210 & 200 & 190 & 180 & 170 & 160 & 150 & 140 & 130 & 120 & 110 & 100 & 90 & 80 & 70 & 60 & 50 & 40 & 30 & 20 & 10 & 0 & -10\end{array}$ 


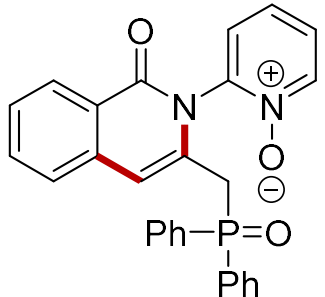

176aa ${ }^{31} \mathrm{P}-\mathrm{NMR}$

(162 MHz, $\mathrm{CDCl}_{3}$ )

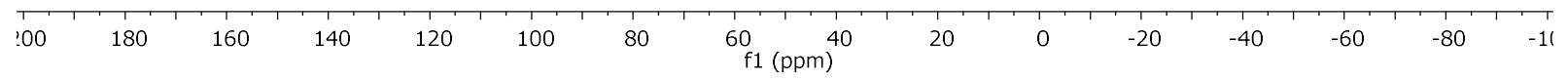<smiles>O=c1c2ccccc2cc(CP(=O)(c2ccccc2)c2ccccc2)n1-c1cccc2cccnc12</smiles>

76aa ${ }^{1} \mathrm{H}-\mathrm{NMR}$ $\left(400 \mathrm{MHz}, \mathrm{CDCl}_{3}\right.$ )

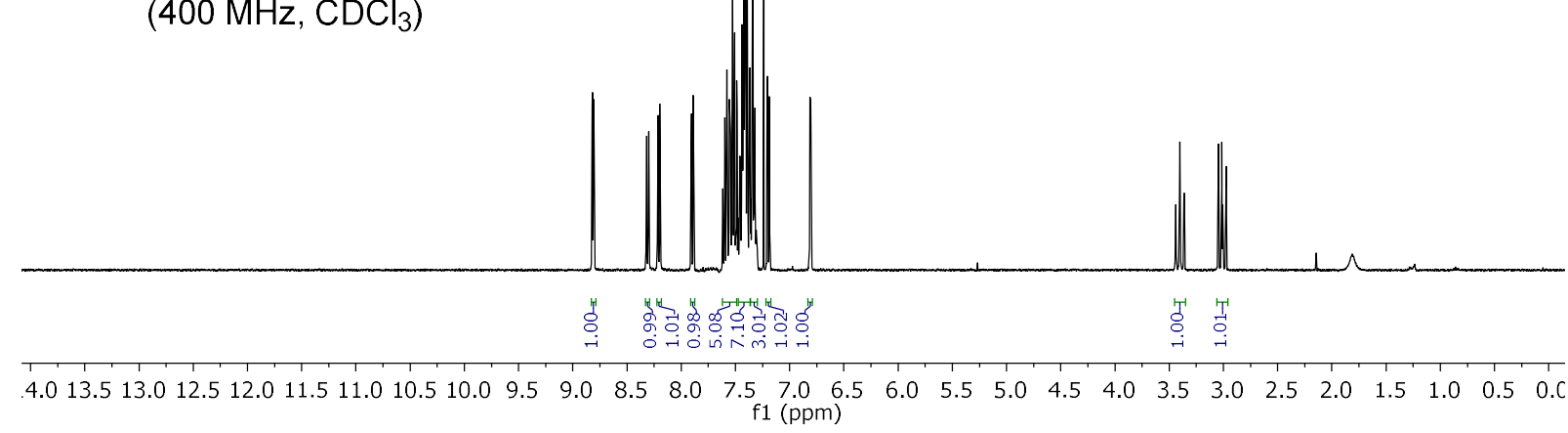




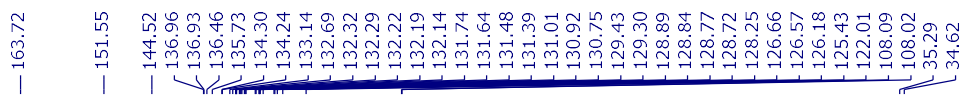<smiles>O=c1c2ccccc2cc(CP(=O)(c2ccccc2)c2ccccc2)n1-c1cccc2ccccc12</smiles>

76aa ${ }^{13} \mathrm{C}-\mathrm{NMR}$

(101 MHz, $\mathrm{CDCl}_{3}$ )

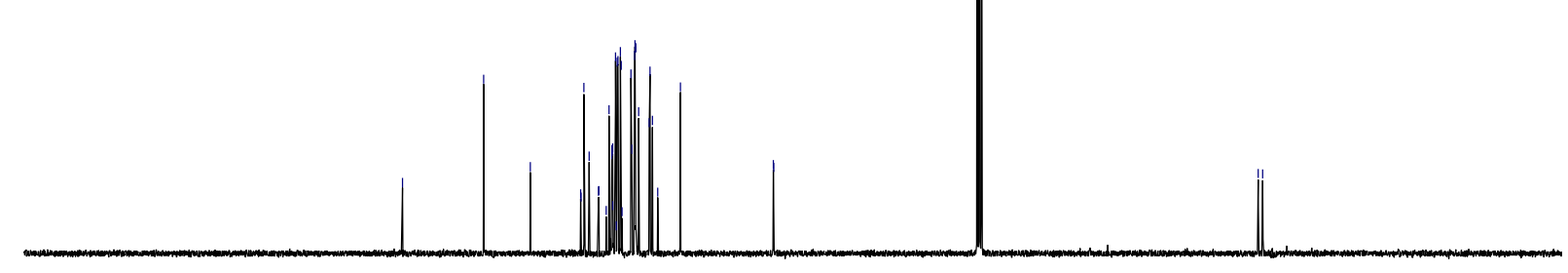

$\begin{array}{lllllllllllllllllllllllll}20 & 210 & 200 & 190 & 180 & 170 & 160 & 150 & 140 & 130 & 120 & 110 & 100 & 90 & 80 & 70 & 60 & 50 & 40 & 30 & 20 & 10 & 0 & -\end{array}$ $\underset{8}{\stackrel{8}{N}}$<smiles>O=c1c2ccccc2cc(CP(=O)(c2ccccc2)c2ccccc2)n1-c1cccc2ccccc12</smiles>

76aa ${ }^{31}$ P-NMR

(121 MHz, $\mathrm{CDCl}_{3}$ )

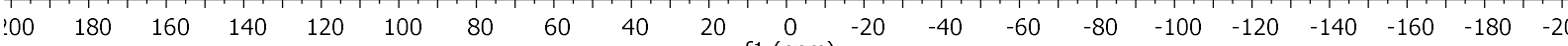


<smiles>CN(c1ccccn1)n1c(CP(=O)(c2ccccc2)c2ccccc2)cc2ccccc2c1=O</smiles>

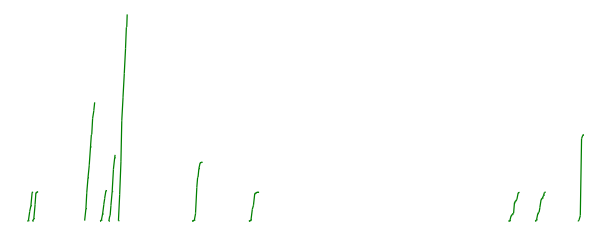

177aa ${ }^{1} \mathrm{H}-\mathrm{NMR}$

(500 MHz, CDCl 3 )

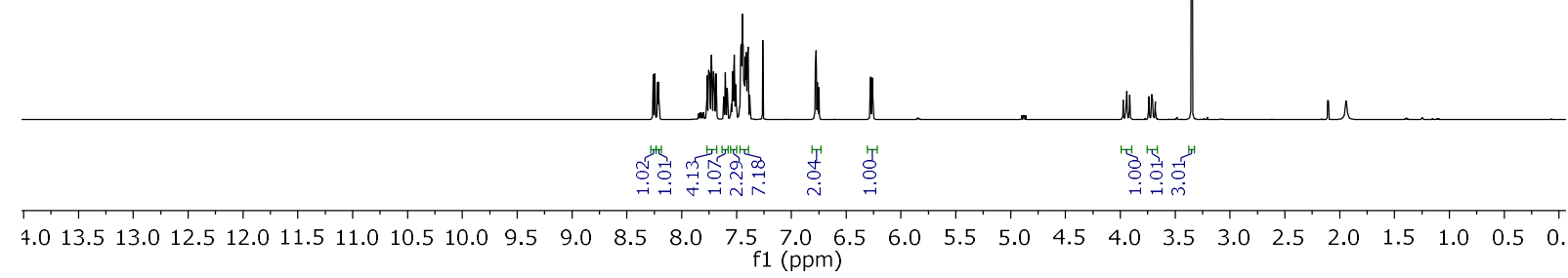<smiles>CN(c1ccccn1)n1c(CP(=O)(c2ccccc2)c2ccccc2)cc2ccccc2c1=O</smiles>

177aa ${ }^{13} \mathrm{C}-\mathrm{NMR}$

$\left(126 \mathrm{MHz}, \mathrm{CDCl}_{3}\right.$ )

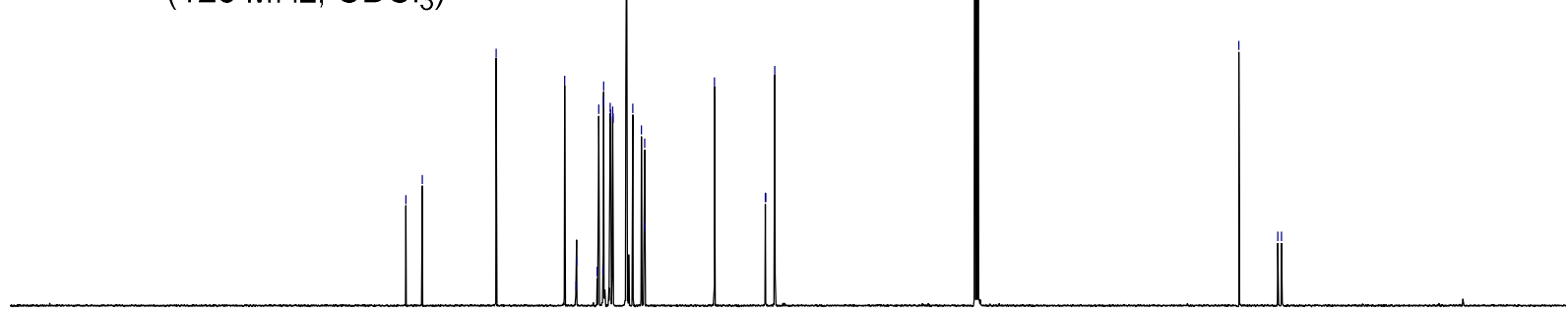

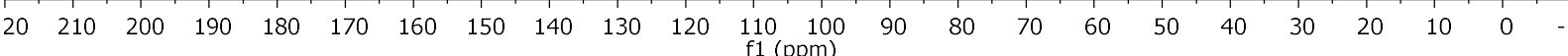


<smiles>CN(c1ccccn1)n1c(CP(=O)(c2ccccc2)c2ccccc2)cc2ccccc2c1=O</smiles>

177aa ${ }^{31}$ P-NMR

(162 MHz, $\mathrm{CDCl}_{3}$ )

$\begin{array}{lllllllllllllllllllllllllllllllllllll}45 & 44 & 43 & 42 & 41 & 40 & 39 & 38 & 37 & 36 & 35 & 34 & 33 & 32 & 31 & 30 & 29 & 28 & 27 & 26 & 25 & 24 & 23 & 22 & 21 & 20 & 19 & 18 & 17 & 16 & 15 & 14 & 13 & 12 & 11 & 1\end{array}$<smiles>C#CC#CC=CC1=CC=C(n2c(CP(=O)(c3ccccc3)c3ccccc3)cc3cc(C)ccc3c2=O)N([O-])C=C1</smiles>

176ba ${ }^{1} \mathrm{H}-\mathrm{NMR}$

$\left(400 \mathrm{MHz}, \mathrm{CDCl}_{3}\right.$ )

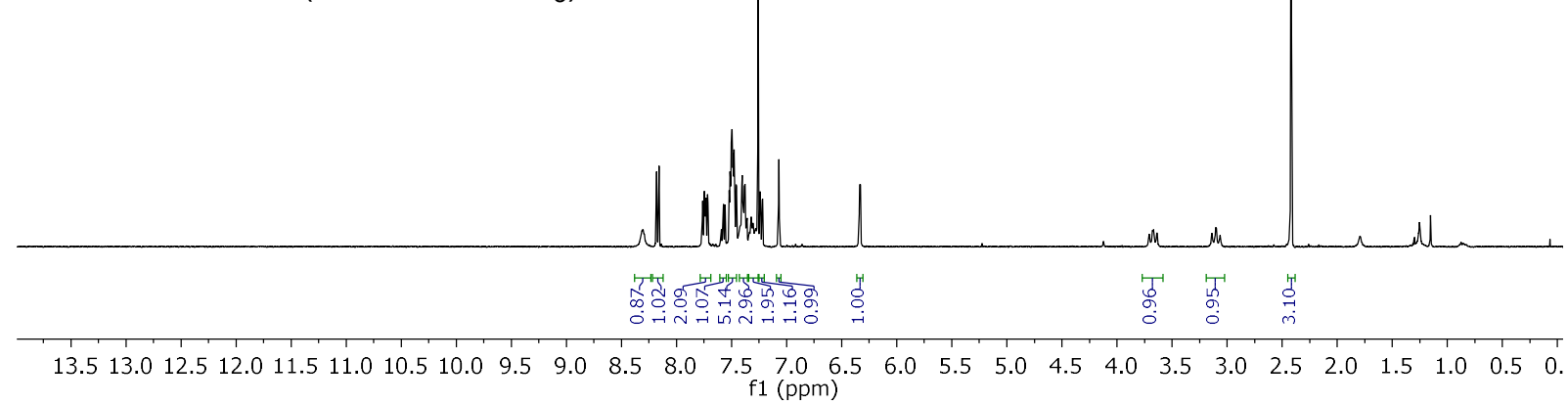


<smiles>Cc1ccc2c(=O)n(-c3cccc[n+]3CCP(=O)(c3ccccc3)c3ccccc3)cc(Cl)c2c1</smiles>

176ba ${ }^{13} \mathrm{C}-\mathrm{NMR}$

$\left(101 \mathrm{MHz}, \mathrm{CDCl}_{3}\right)$

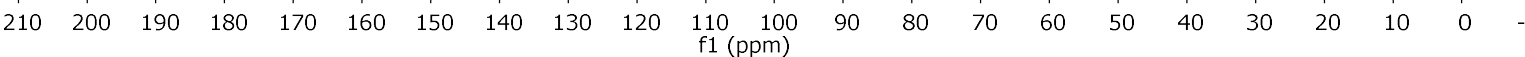<smiles></smiles>

176ba ${ }^{31} \mathrm{P}-\mathrm{NMR}$

$\left(162 \mathrm{MHz}, \mathrm{CDCl}_{3}\right)$

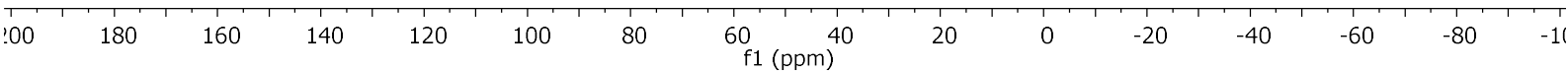


<smiles>COc1ccc2c(=O)n(-c3cccc[n+]3[O-])c(CP(=O)(c3ccccc3)c3ccccc3)cc2c1</smiles>

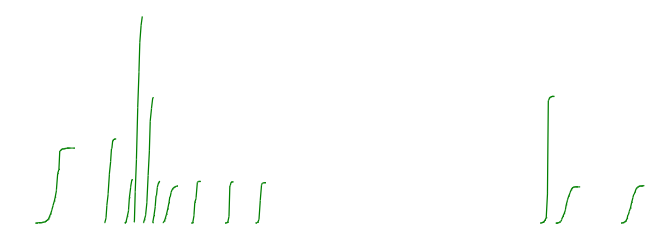

176da ${ }^{1} \mathrm{H}-\mathrm{NMR}$

(400 MHz, $\mathrm{CDCl}_{3}$ )<smiles>COc1ccc2c(=O)n(-c3cccc[n+]3[O-])c(CP(=O)(c3ccccc3)c3ccccc3)cc2c1</smiles>

176da ${ }^{13} \mathrm{C}-\mathrm{NMR}$

(126 MHz, $\mathrm{CDCl}_{3}$ )

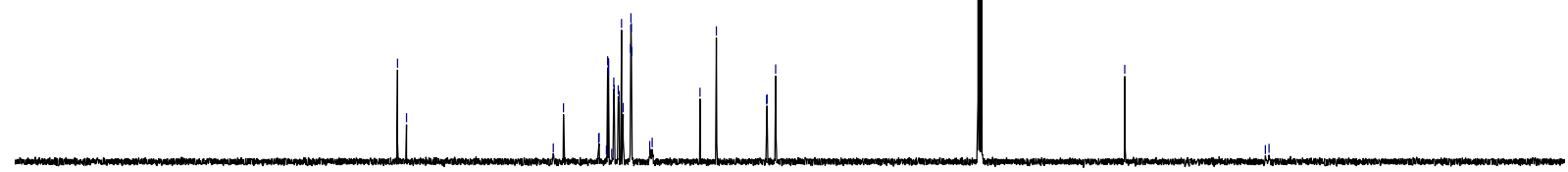

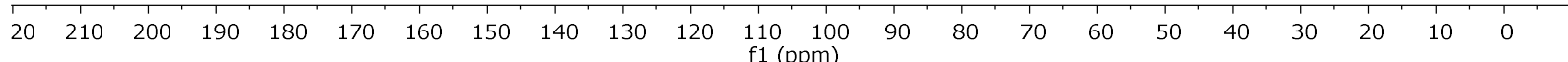


<smiles>COc1ccc2c(=O)n(-c3cccc[n+]3[O-])c(CP(=O)(c3ccccc3)c3ccccc3)cc2c1</smiles>

176da ${ }^{31} \mathrm{P}-\mathrm{NMR}$

(162 MHz, $\mathrm{CDCl}_{3}$ )

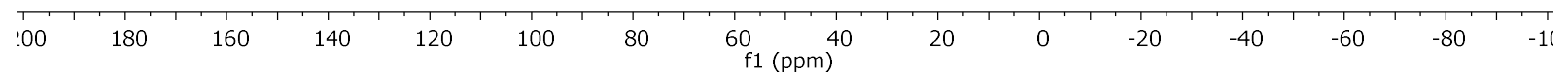<smiles>CSc1ccc2c(=O)n(-c3cccc[n+]3[O-])c(CP(=O)(c3ccccc3)c3ccccc3)cc2c1</smiles>

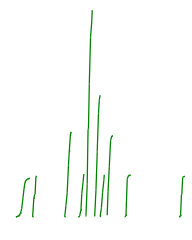

176ea ${ }^{1} \mathrm{H}-\mathrm{NMR}$

(400 MHz, $\mathrm{CDCl}_{3}$ )

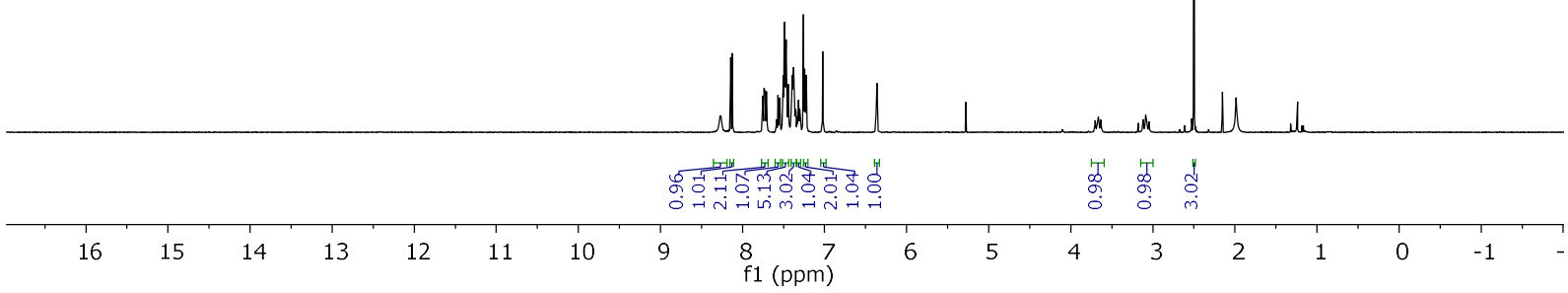


<smiles>COc1ccc2c(=O)n(-c3cccc[n+]3[O-])c(CP(=O)(c3ccccc3)c3ccccc3)cc2c1</smiles>

176ea ${ }^{13} \mathrm{C}-\mathrm{NMR}$

$\left(126 \mathrm{MHz}, \mathrm{CDCl}_{3}\right.$ )

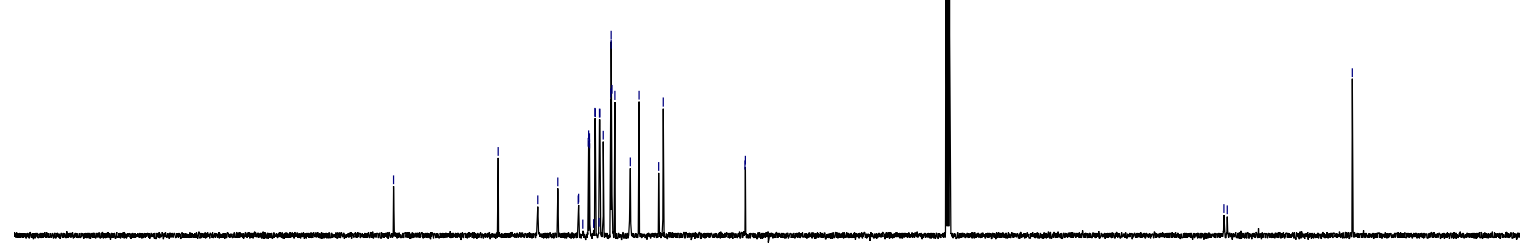

$\begin{array}{llllllllllllllllllllllllll}20 & 210 & 200 & 190 & 180 & 170 & 160 & 150 & 140 & 130 & 120 & \begin{array}{l}110 \\ \mathrm{f} 1(\mathrm{ppm})\end{array} & 90 & 80 & 70 & 60 & 50 & 40 & 30 & 20 & 10 & 0 & -1\end{array}$<smiles>COc1ccc2c(=O)n(-c3cccc[n+]3[O-])c(CP(=O)(c3ccccc3)c3ccccc3)cc2c1</smiles>

176ea ${ }^{31} \mathrm{P}-\mathrm{NMR}$

(162 MHz, $\mathrm{CDCl}_{3}$ )

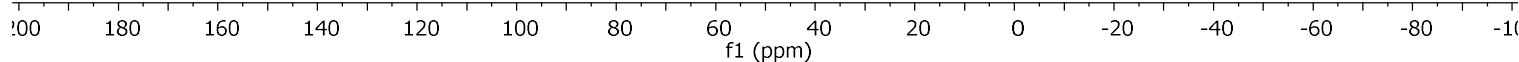




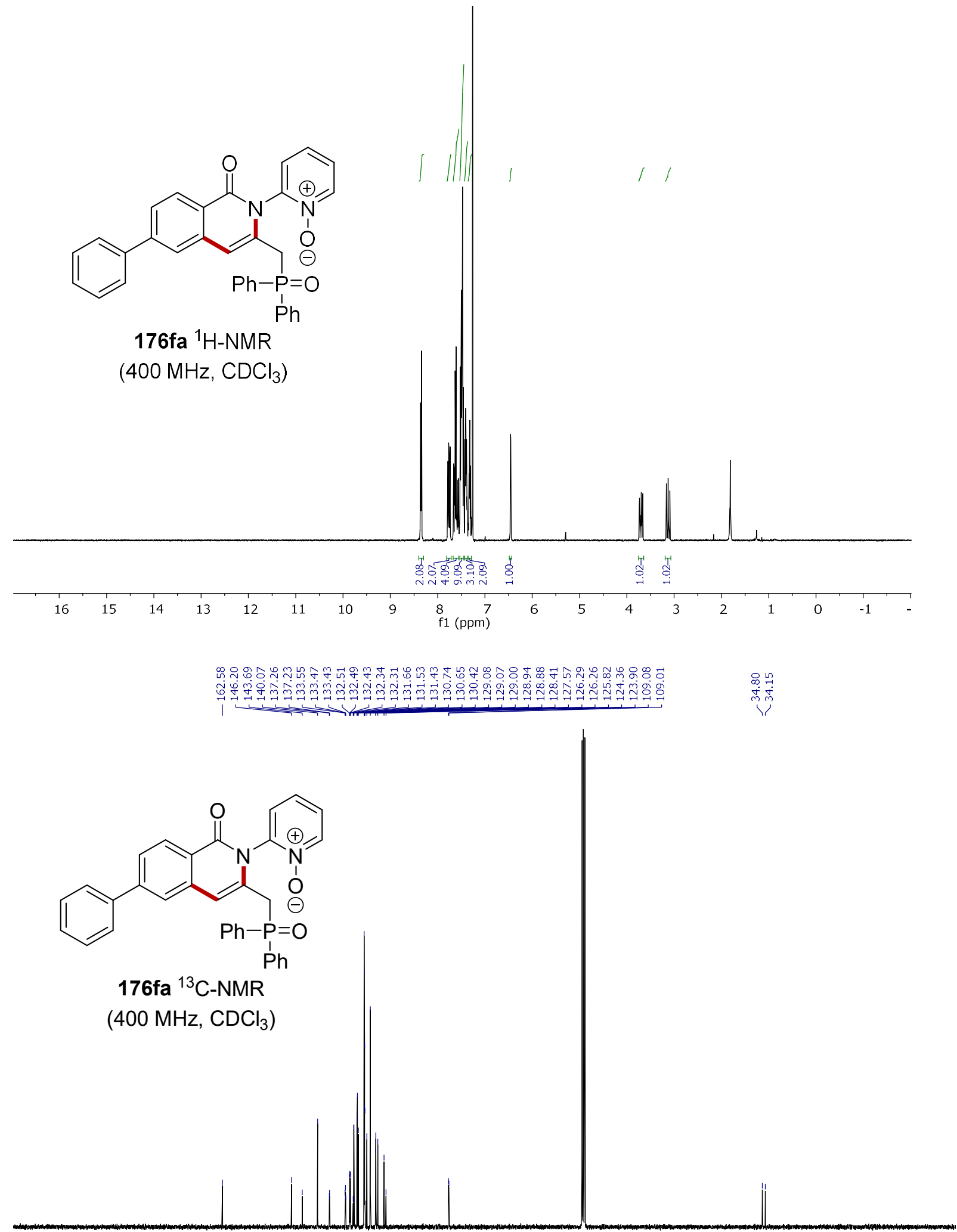

$\begin{array}{lllllllllllllllllllllll}210 & 200 & 190 & 180 & 170 & 160 & 150 & 140 & 130 & 120 & 110 & 100 & 90 & 80 & 70 & 60 & 50 & 40 & 30 & 20 & 10 & 0 & -1\end{array}$ 
<smiles>O=c1c2ccc(-c3ccccc3)cc2cc(CP(=O)(c2ccccc2)c2ccccc2)n1-c1cccc[n+]1[O-]</smiles>

176fa ${ }^{31} \mathrm{P}-\mathrm{NMR}$

$\left(126 \mathrm{MHz}, \mathrm{CDCl}_{3}\right.$ )

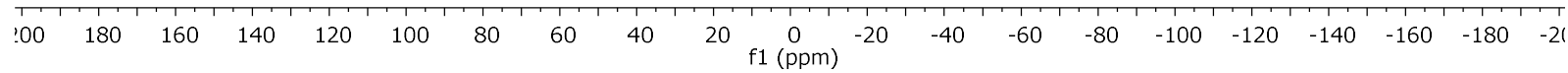<smiles>O=c1c2ccc(I)cc2cc(CP(=O)(c2ccccc2)c2ccccc2)n1-c1cccc[n+]1[O-]</smiles><smiles>CC#CC</smiles>

176ga ${ }^{1} \mathrm{H}-\mathrm{NMR}$ (400 MHz, $\mathrm{CDCl}_{3}$ )

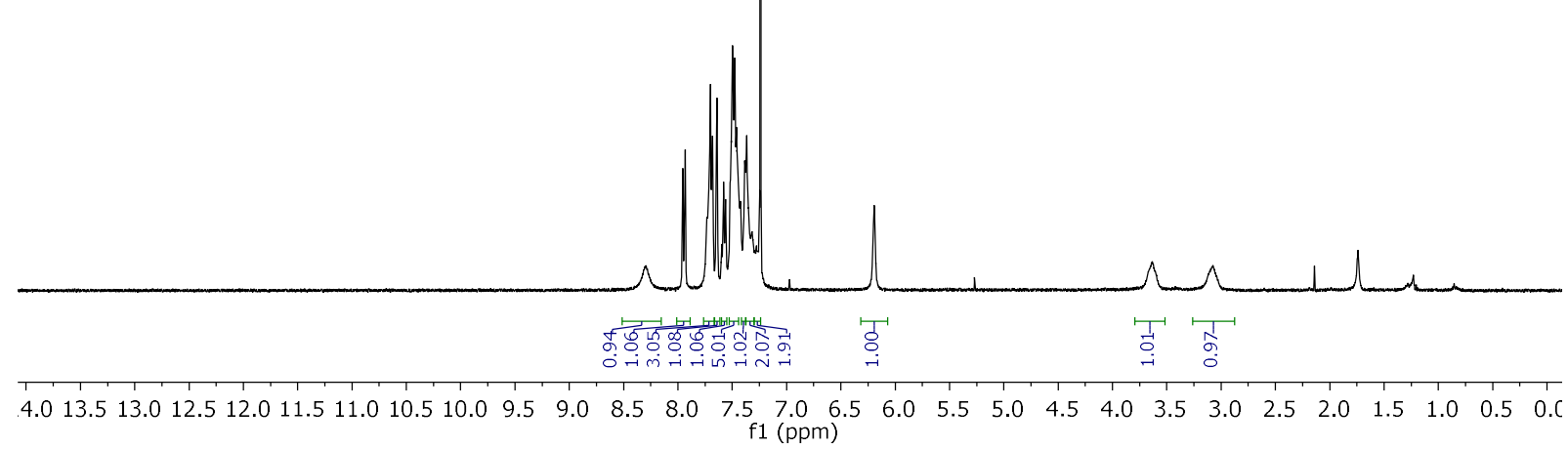




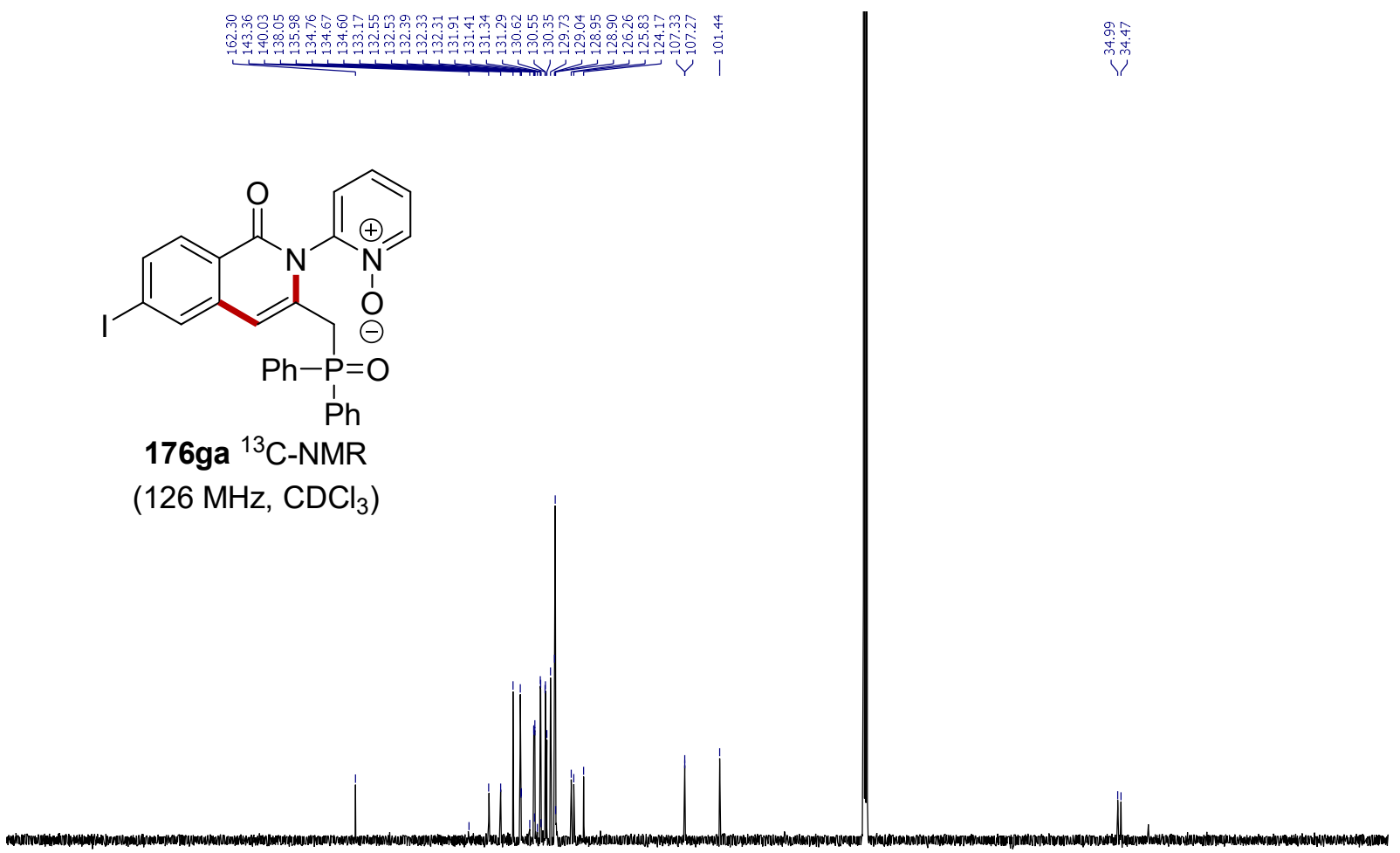

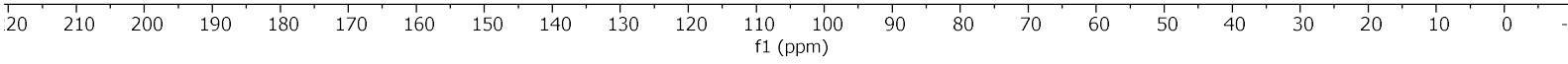<smiles></smiles>

176ga ${ }^{31} \mathrm{P}-\mathrm{NMR}$

$\left(162 \mathrm{MHz}, \mathrm{CDCl}_{3}\right)$ 
<smiles>CC(C)(C)c1ccc2c(=O)n(-c3cccc[n+]3[O-])c(CP(=O)(c3ccccc3)c3ccccc3)cc2c1</smiles>

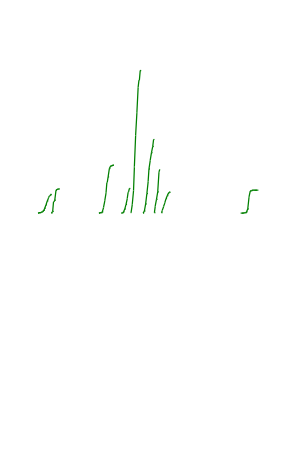

176ja ${ }^{1} \mathrm{H}-\mathrm{NMR}$

(400 MHz, $\mathrm{CDCl}_{3}$ )
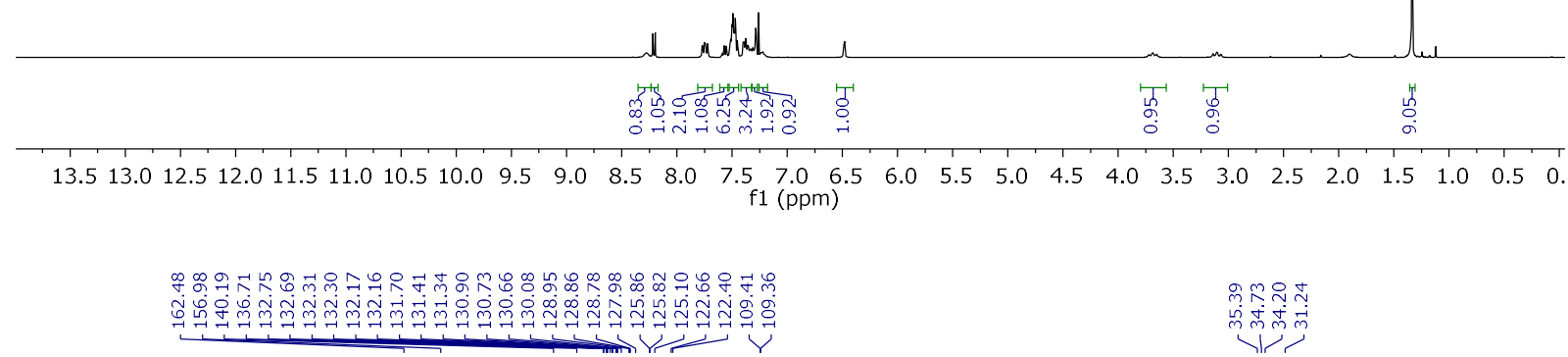<smiles>CC(C)(C)c1ccc2c(=O)n(-c3cccc[n+]3[O-])c(CP(=O)(c3ccccc3)c3ccccc3)cc2c1</smiles>

176 ja ${ }^{13} \mathrm{C}-\mathrm{NMR}$ (126 MHz, $\mathrm{CDCl}_{3}$ )

$\begin{array}{lllllllllllll}20 & 210 & 200 & 190 & 180 & 170 & 160 & 150 & 140 & 130 & 120 & 110 & 100\end{array}$ $1(\mathrm{ppm})$ 
<smiles>CC(C)(C)c1ccc2c(=O)n(-c3cccc[n+]3[O-])c(CP(=O)(c3ccccc3)c3ccccc3)cc2c1</smiles>

176ja ${ }^{31} \mathrm{P}-\mathrm{NMR}$

(162 $\mathrm{MHz}, \mathrm{CDCl}_{3}$ )

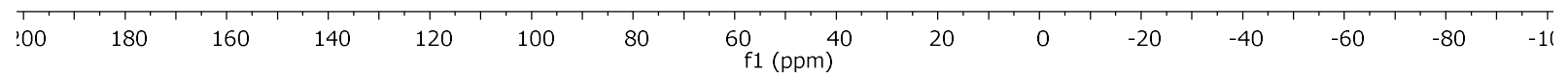

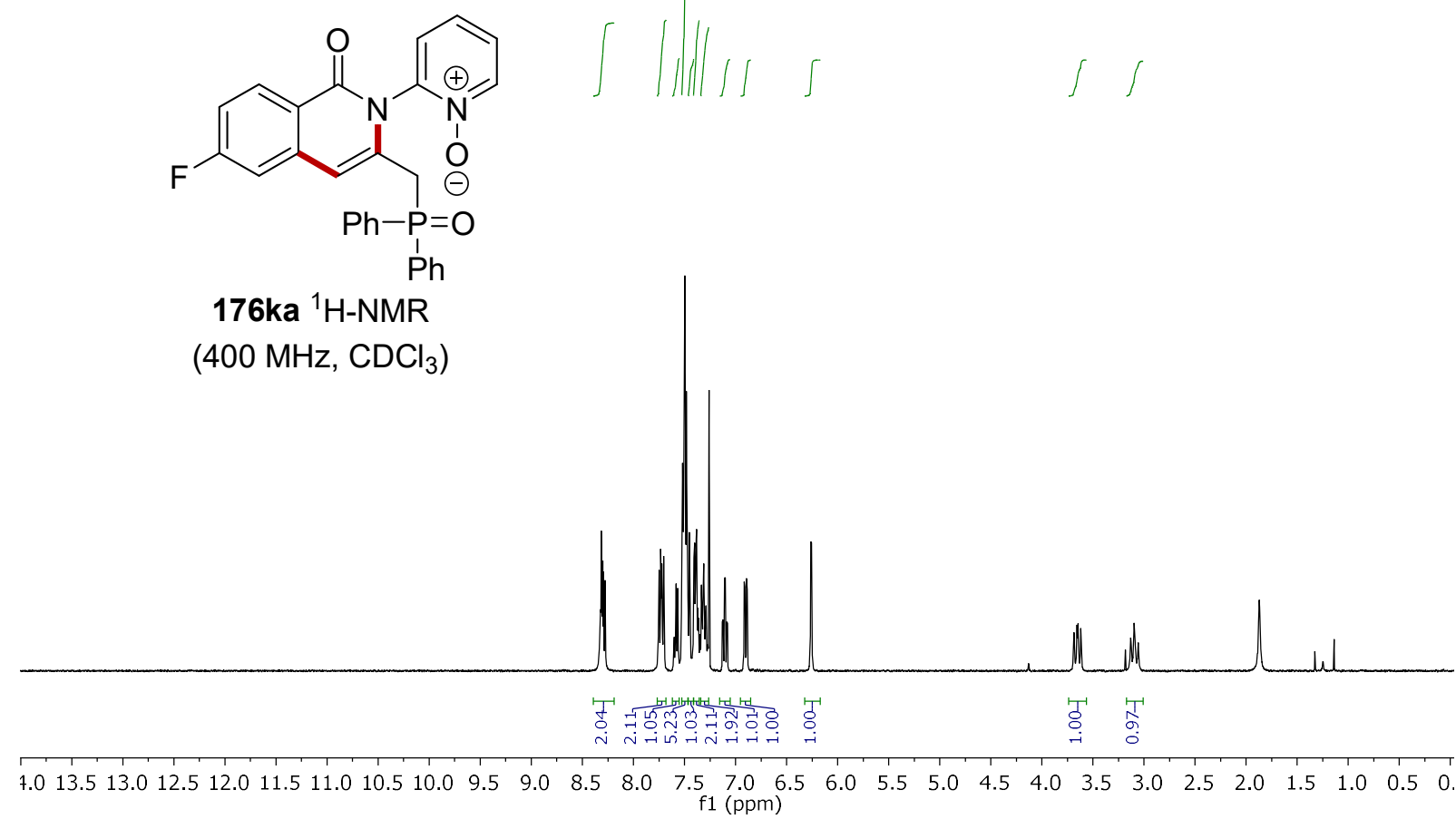




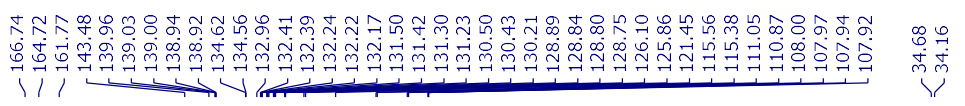<smiles></smiles>

176ka ${ }^{13} \mathrm{C}-\mathrm{NMR}$

$\left(126 \mathrm{MHz}, \mathrm{CDCl}_{3}\right.$ )

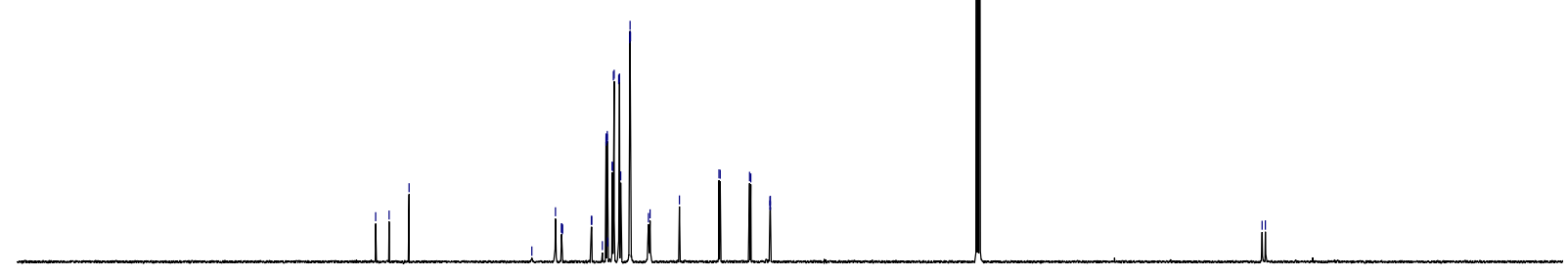

$\begin{array}{lllllllllllllllllllllll}20 & 210 & 200 & 190 & 180 & 170 & 160 & 150 & 140 & 130 & 120 & \begin{array}{c}110 \\ \mathrm{f} 1(\mathrm{ppm})\end{array} & 100 & 80 & 70 & 60 & 50 & 40 & 30 & 20 & 10 & 0 & -\end{array}$

每

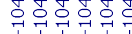

iv<smiles>O=c1c2ccc(F)cc2cc(CP(=O)(c2ccccc2)c2ccccc2)n1-c1cccc[n+]1[O-]</smiles>

$\mathrm{Ph}$

176ka ${ }^{19} \mathrm{~F}-\mathrm{NMR}$

(376 MHz, $\mathrm{CDCl}_{3}$ ) 
<smiles>O=c1c2ccc(F)cc2cc(CP(=O)(c2ccccc2)c2ccccc2)n1-c1cccc[n+]1[O-]</smiles>

176ka ${ }^{31} \mathrm{P}-\mathrm{NMR}$

(162 MHz, $\mathrm{CDCl}_{3}$ )

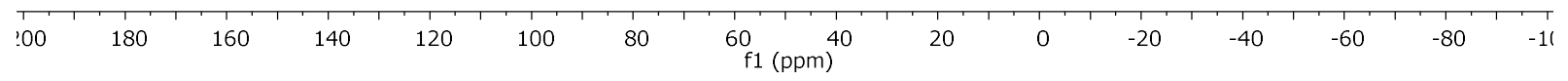<smiles>CC(=O)c1ccc2c(=O)n(-c3cccc[n+]3[O-])c(CP(=O)(c3ccccc3)c3ccccc3)cc2c1</smiles>

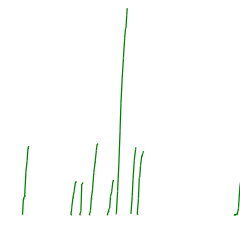

176la ${ }^{1} \mathrm{H}-\mathrm{NMR}$

(400 MHz, $\mathrm{CDCl}_{3}$ )

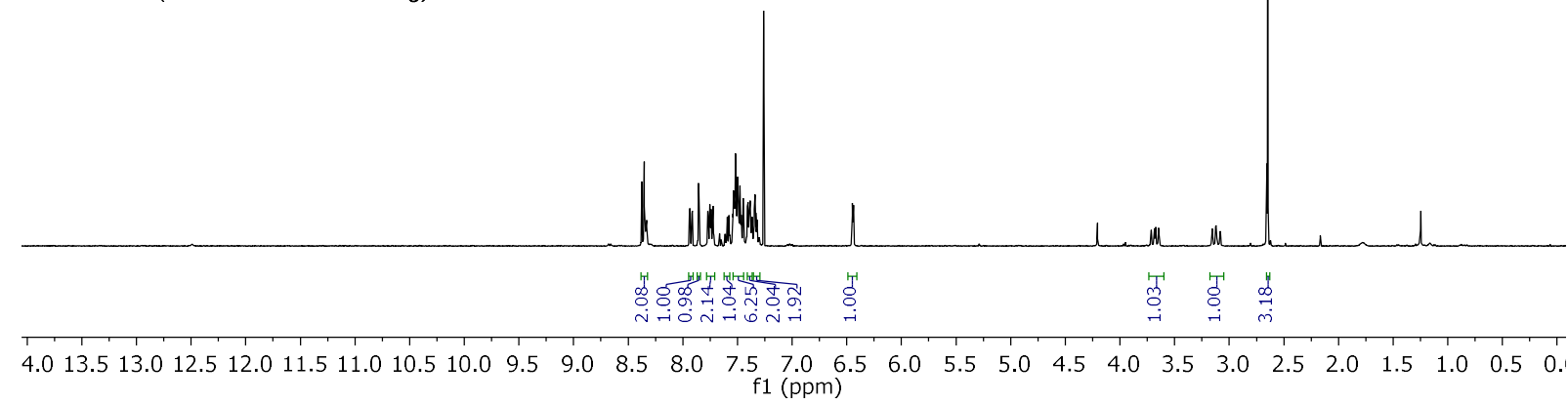


<smiles>CC(=O)c1ccc2c(=O)n(-c3cccc[n+]3[O-])c(CP(=O)(c3ccccc3)c3ccccc3)cc2c1</smiles>

176la ${ }^{13} \mathrm{C}-\mathrm{NMR}$

(126 MHz, $\mathrm{CDCl}_{3}$ )

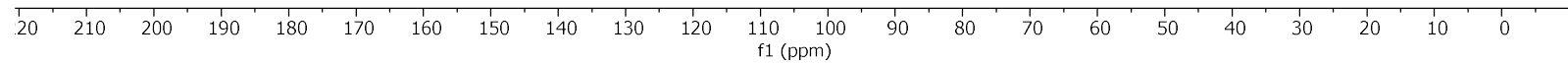<smiles>CC(=O)c1ccc2c(=O)n(-c3cccc[n+]3[O-])c(CP(=O)(c3ccccc3)c3ccccc3)cc2c1</smiles>

176la ${ }^{31} \mathrm{P}-\mathrm{NMR}$

$\left(162 \mathrm{MHz}, \mathrm{CDCl}_{3}\right.$ )

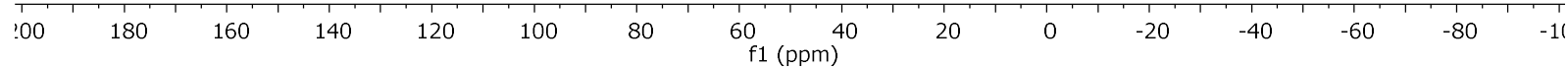


<smiles>CC(=O)c1ccc2c(=O)n(-c3cccc[n+]3[O-])c(CP(=O)(c3ccccc3)c3ccccc3)cc2c1</smiles>

$\mathrm{Ph}$

$176 \mathrm{ma}^{1} \mathrm{H}-\mathrm{NMR}$

(400 MHz, $\mathrm{CDCl}_{3}$ )

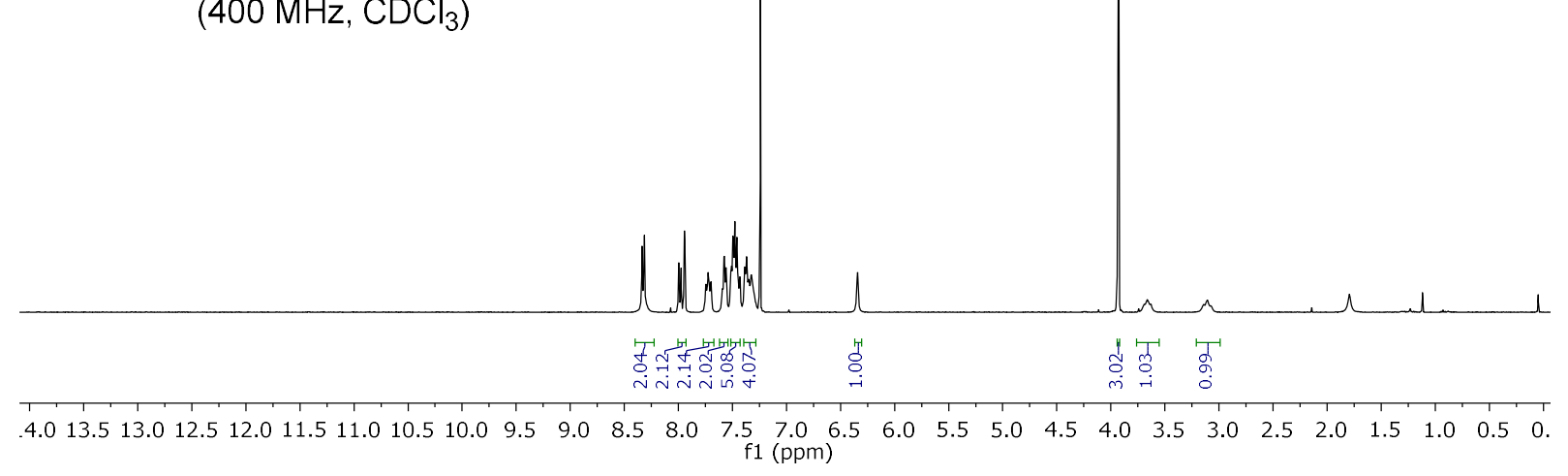<smiles>CC(=O)c1ccc2c(=O)n(-c3cccc[n+]3[O-])c(CP(=O)(c3ccccc3)c3ccccc3)cc2c1</smiles>

176ma ${ }^{13} \mathrm{C}-\mathrm{NMR}$

(126 MHz, $\mathrm{CDCl}_{3}$ )

\begin{tabular}{lllllllllllll}
120 & 210 & 200 & 190 & 180 & 170 & 160 & 150 & 140 & 130 & 120 & $\begin{array}{c}110 \\
f 1(\mathrm{ppm})\end{array}$ \\
\hline
\end{tabular} 


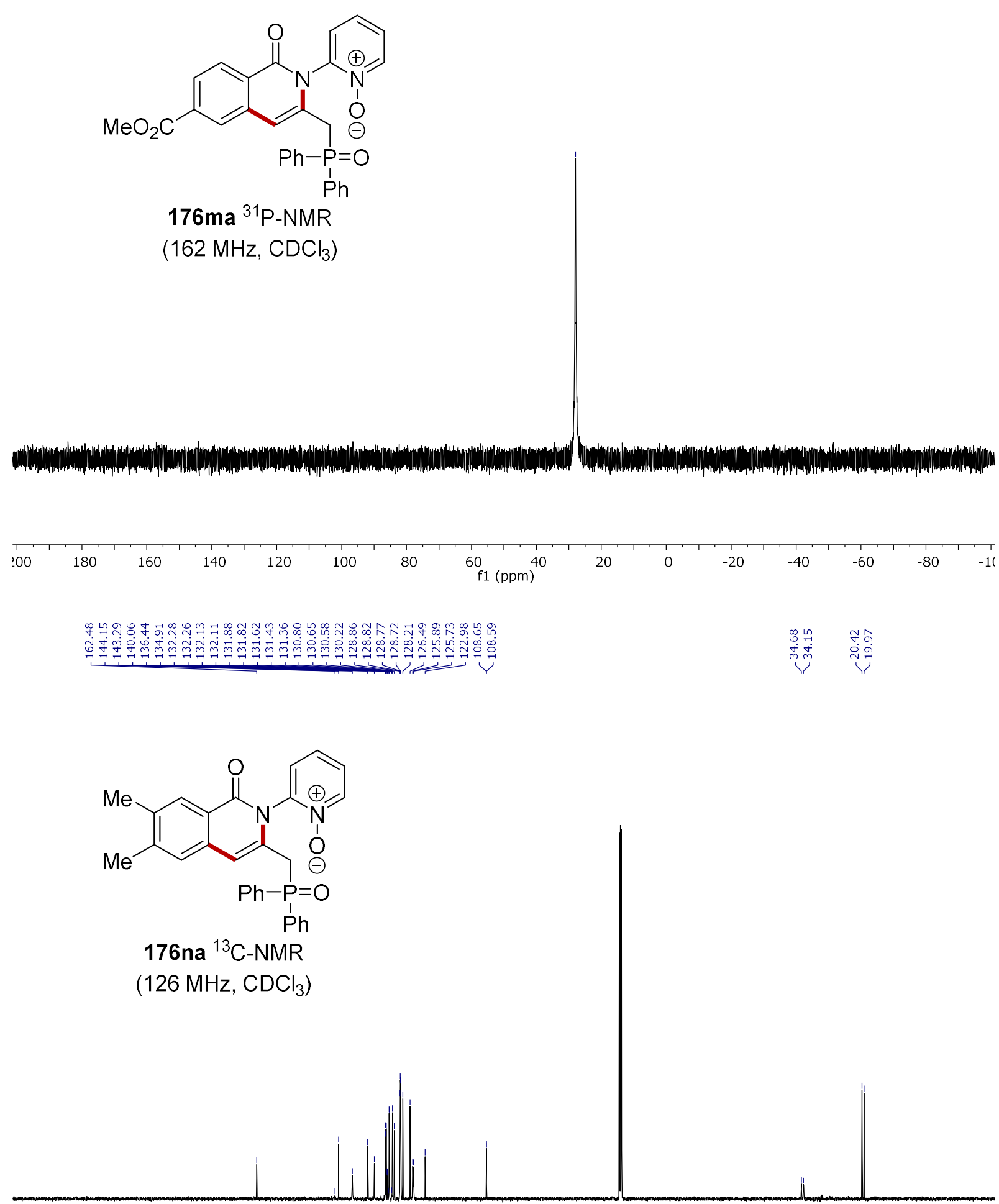

176ma ${ }^{31} \mathrm{P}-\mathrm{NMR}$

(162 $\mathrm{MHz}, \mathrm{CDCl}_{3}$ )

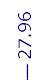

$\begin{array}{lllllllllllllllllllllll}110 & 200 & 190 & 180 & 170 & 160 & 150 & 140 & 130 & 120 & \begin{array}{c}110 \\ \mathrm{f} 1(\mathrm{ppm})\end{array} & 90 & 80 & 70 & 60 & 50 & 40 & 30 & 20 & 10 & 0 & -1\end{array}$ 

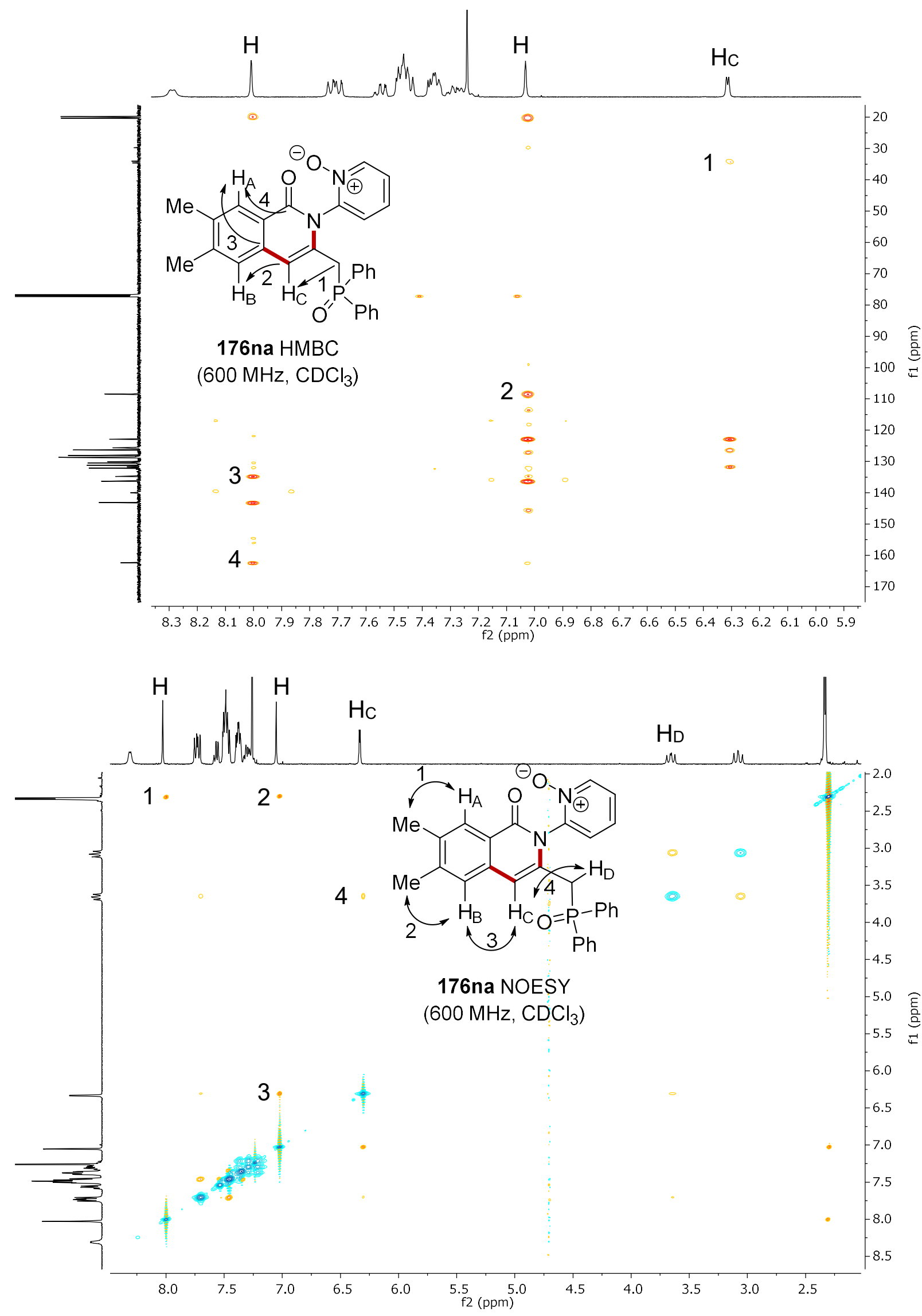
<smiles>Cc1cc2cc(CP(=O)(c3ccccc3)c3ccccc3)n(-c3cccc[n+]3[O-])c(=O)c2cc1C</smiles>

176na ${ }^{31} \mathrm{P}-\mathrm{NMR}$

(162 MHz, $\mathrm{CDCl}_{3}$ )
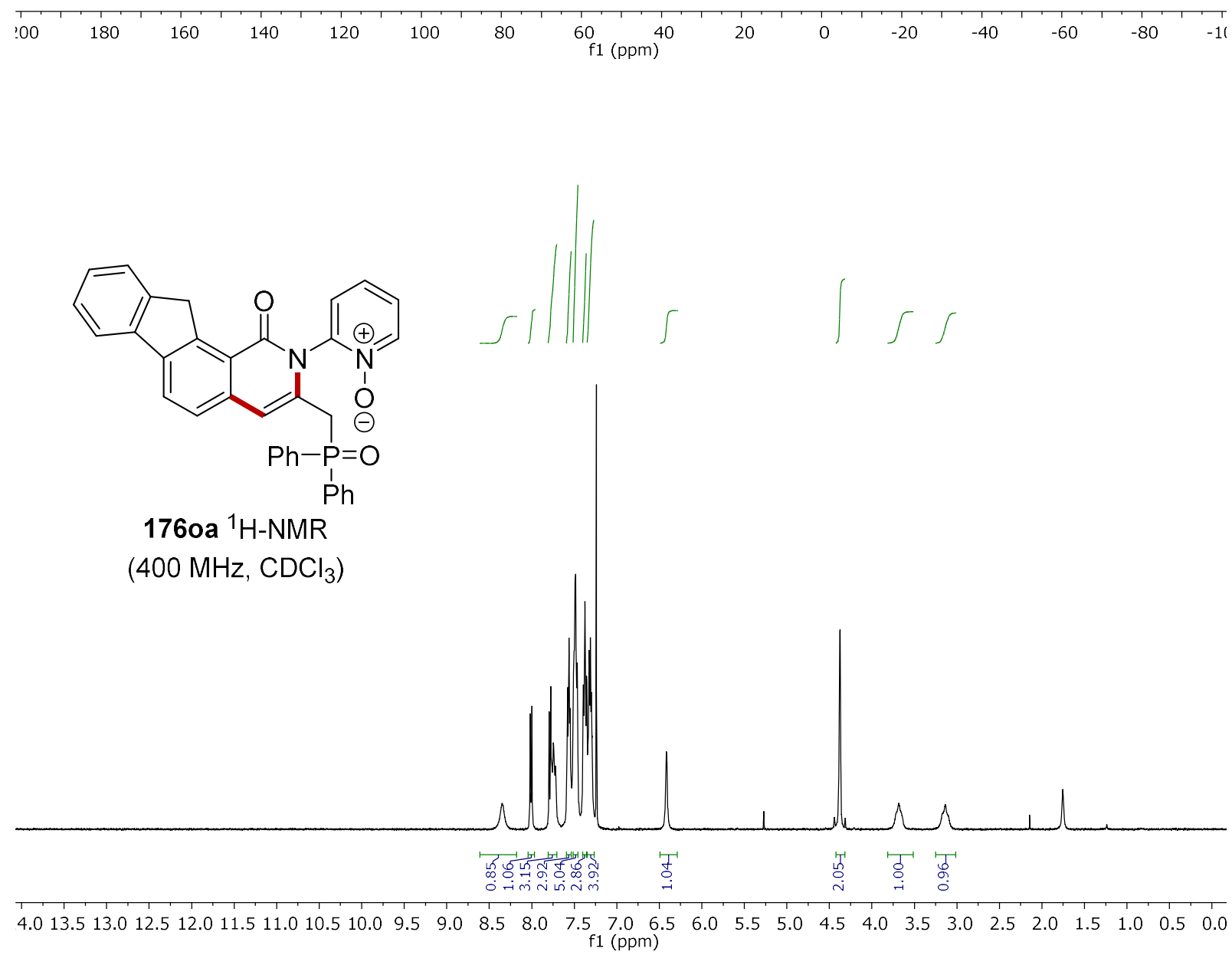


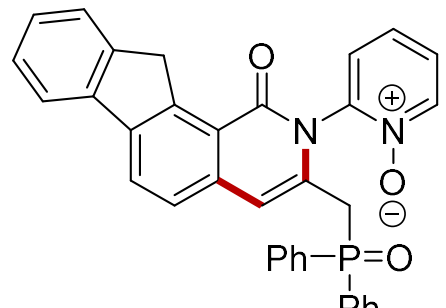

$1760 a^{13} \mathrm{C}-\mathrm{NMR}$

$\left(126 \mathrm{MHz}, \mathrm{CDCl}_{3}\right)$
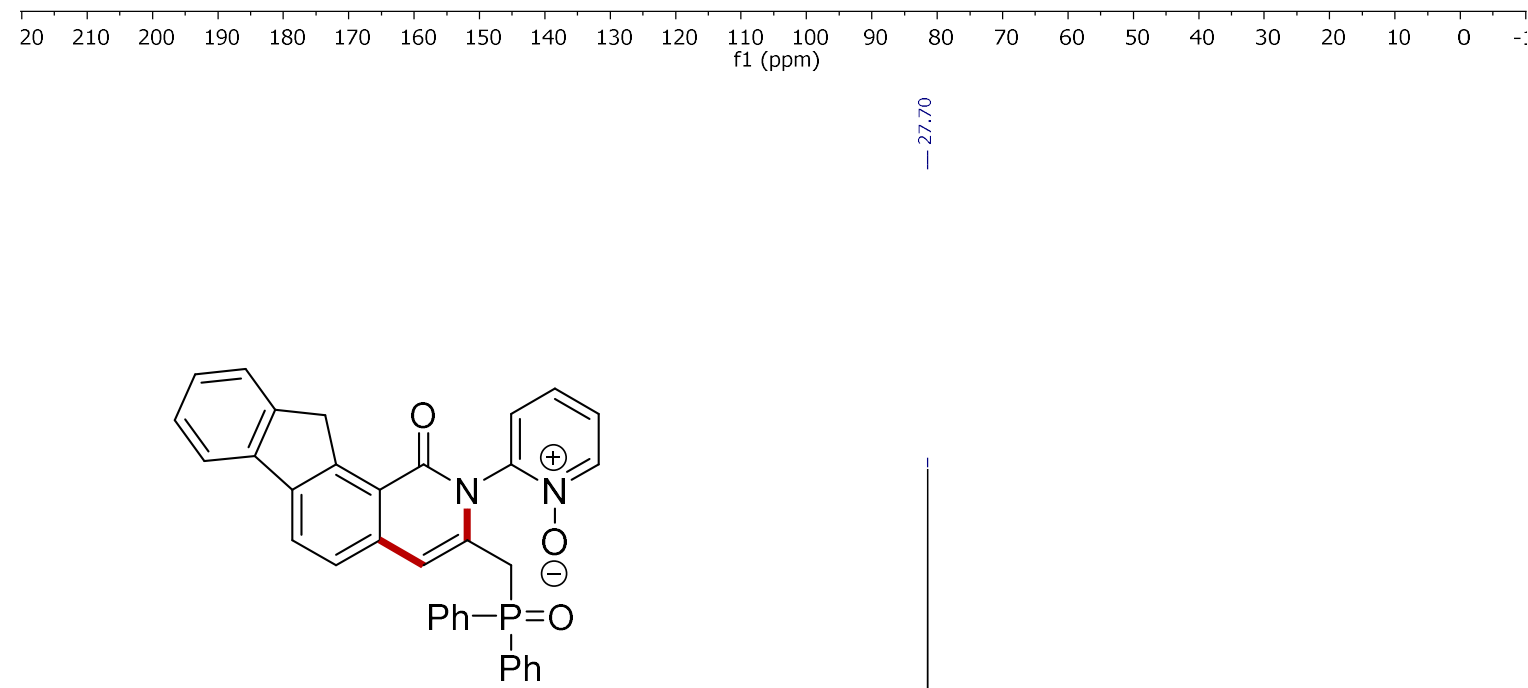

176oa ${ }^{31} \mathrm{P}-\mathrm{NMR}$

$\left(162 \mathrm{MHz}, \mathrm{CDCl}_{3}\right)$

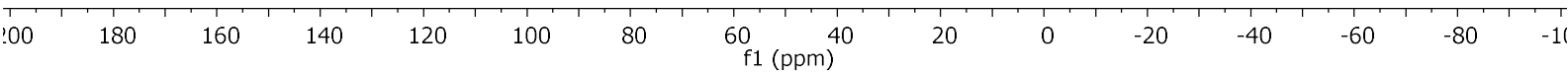




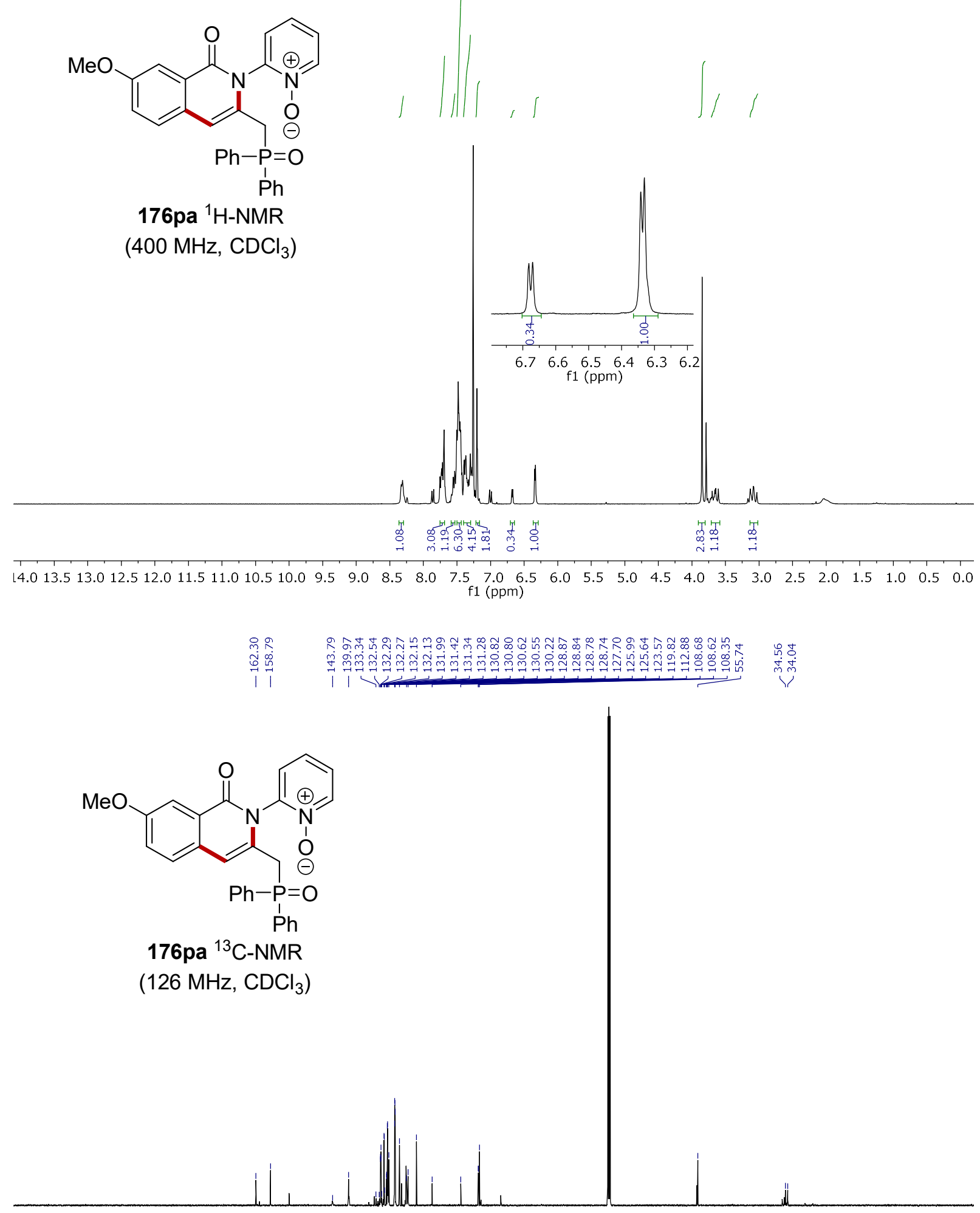

$\begin{array}{lllllllllllllllllllllllllll}20 & 210 & 200 & 190 & 180 & 170 & 160 & 150 & 140 & 130 & 120 & 110 & 100 & 90 & 80 & 70 & 60 & 50 & 40 & 30 & 20 & 10 & 0 & -1\end{array}$ 


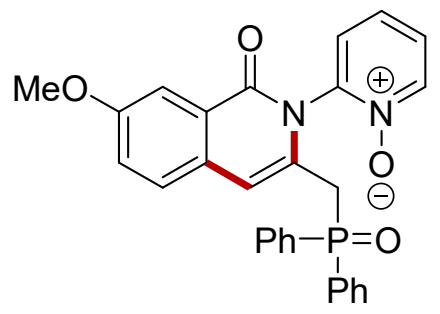

176pa ${ }^{31} \mathrm{P}-\mathrm{NMR}$

(162 MHz, $\mathrm{CDCl}_{3}$ )

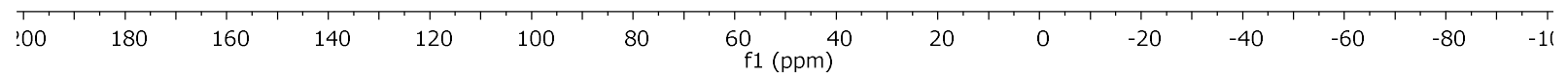<smiles>O=c1c2ccc3c(c2cc(CP(=O)(c2ccccc2)c2ccccc2)n1-c1cccc[n+]1[O-])OCO3</smiles>

176qa ${ }^{1} \mathrm{H}-\mathrm{NMR}$

$\left(600 \mathrm{MHz}, \mathrm{CDCl}_{3}\right.$ )

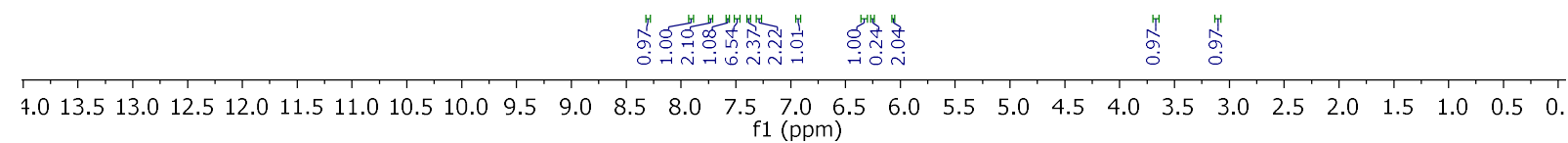




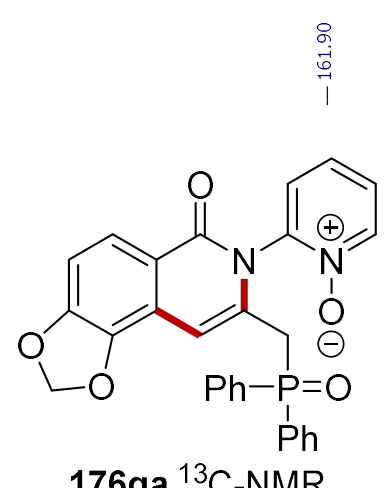

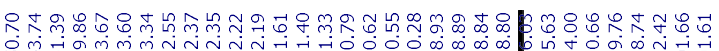

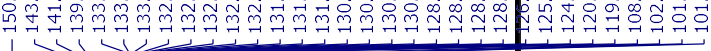

$\left(126 \mathrm{MHz} \mathrm{CDCl}_{3}\right)$

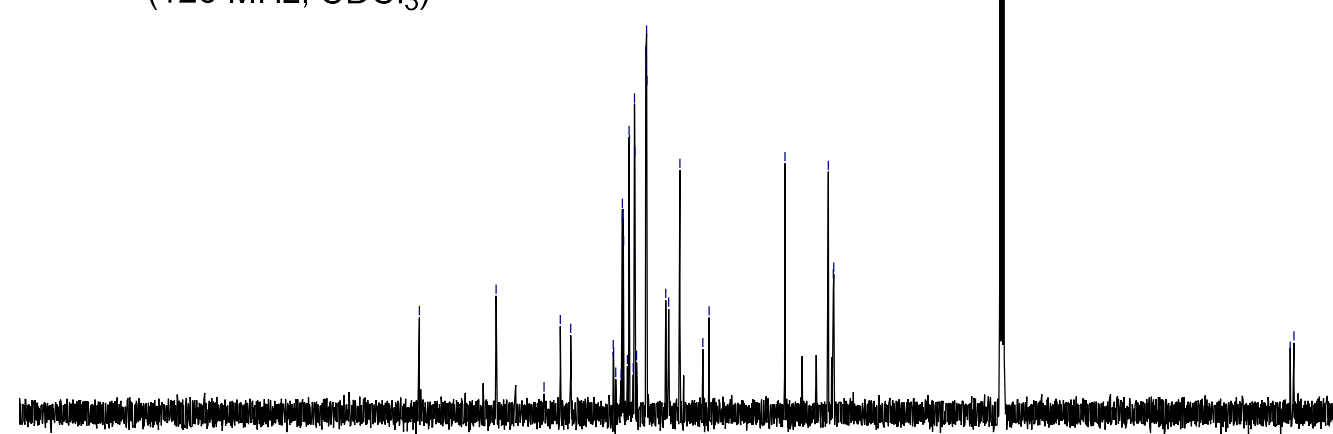

$\begin{array}{llllllllllllllllllllllll}20 & 210 & 200 & 190 & 180 & 170 & 160 & 150 & 140 & 130 & 120 & 110 & 100 & 90 & 80 & 70 & 60 & 50 & 40 & 30 & 20 & 10 & 0\end{array}$ $\stackrel{\substack{i \\ i}}{1}$<smiles>O=c1c2ccc3c(c2cc(CP(=O)(c2ccccc2)c2ccccc2)n1-c1cccc[n+]1[O-])OCO3</smiles>

176qa ${ }^{31}$ P-NMR

(162 MHz, $\mathrm{CDCl}_{3}$ )

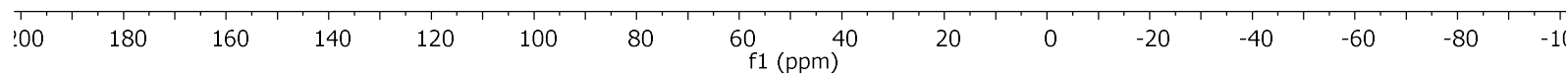


<smiles></smiles>

176ra<smiles></smiles>

${ }^{1} \mathrm{H}-\mathrm{NMR}$

(600 MHz, $\mathrm{CDCl}_{3}$ )
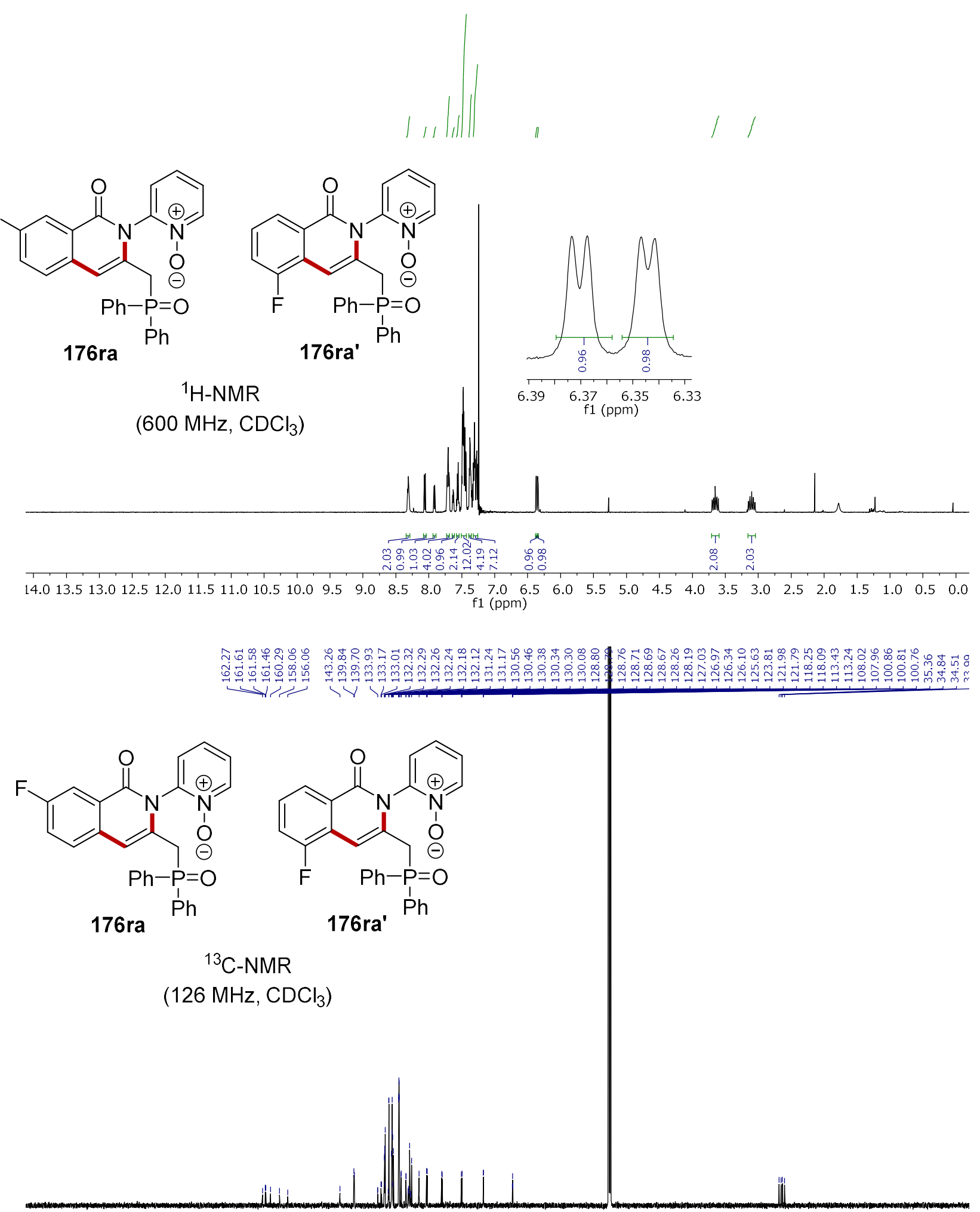

$\begin{array}{lllllllllllllllllllllllllll}20 & 210 & 200 & 190 & 180 & 170 & 160 & 150 & 140 & 130 & 120 & 110 & 100 & 90 & 80 & 70 & 60 & 50 & 40 & 30 & 20 & 10 & 0 & -1\end{array}$ 
<smiles></smiles>

176ra<smiles>O=c1c2cccc(F)c2cc(CP(=O)(c2ccccc2)c2ccccc2)n1-c1cccc[n+]1[O-]</smiles>

176ra'

${ }^{19} \mathrm{~F}-\mathrm{NMR}$

(376 MHz, $\mathrm{CDCl}_{3}$ )

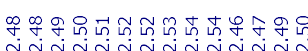

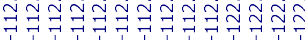

年

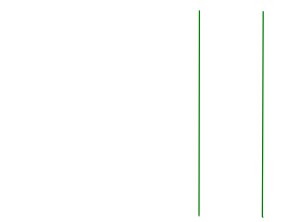

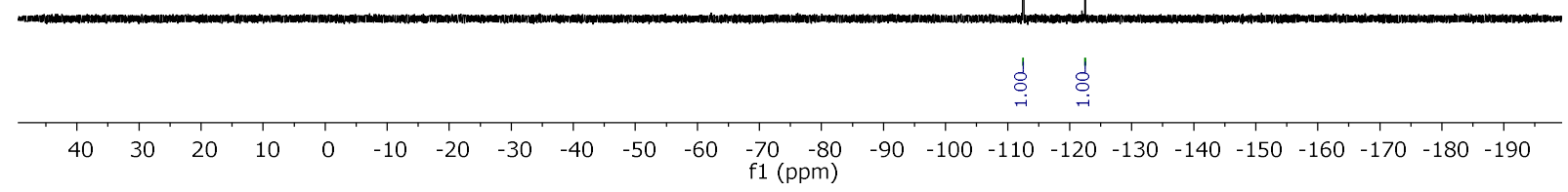<smiles></smiles>

176ra<smiles>CP(=O)(Cc1cc2c(F)cccc2c(=O)n1-c1cccc[n+]1[O-])c1ccccc1</smiles>

176ra'

${ }^{31} \mathrm{P}-\mathrm{NMR}$

(162 MHz, $\mathrm{CDCl}_{3}$ )

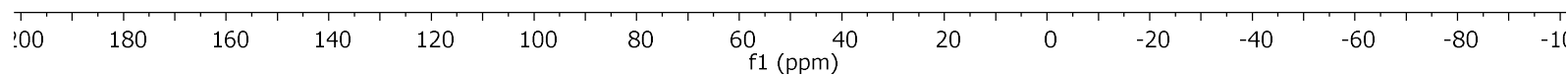


<smiles>Cc1cc(C)c2c(=O)n(-c3cccc[n+]3[O-])c(CP(=O)(c3ccccc3)c3ccccc3)cc2c1</smiles>

$\mathrm{Ph}$

176sa ${ }^{1} \mathrm{H}-\mathrm{NMR}$

$\left(500 \mathrm{MHz}, \mathrm{CDCl}_{3}\right.$ )

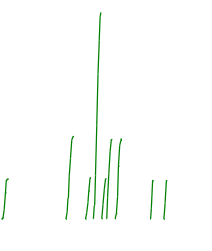


<smiles>Cc1cc(C)c2c(=O)n(-c3cccc[n+]3[O-])c(CP(=O)(c3ccccc3)c3ccccc3)cc2c1</smiles>

176sa ${ }^{31}$ P-NMR

(121 MHz, $\mathrm{CDCl}_{3}$ )
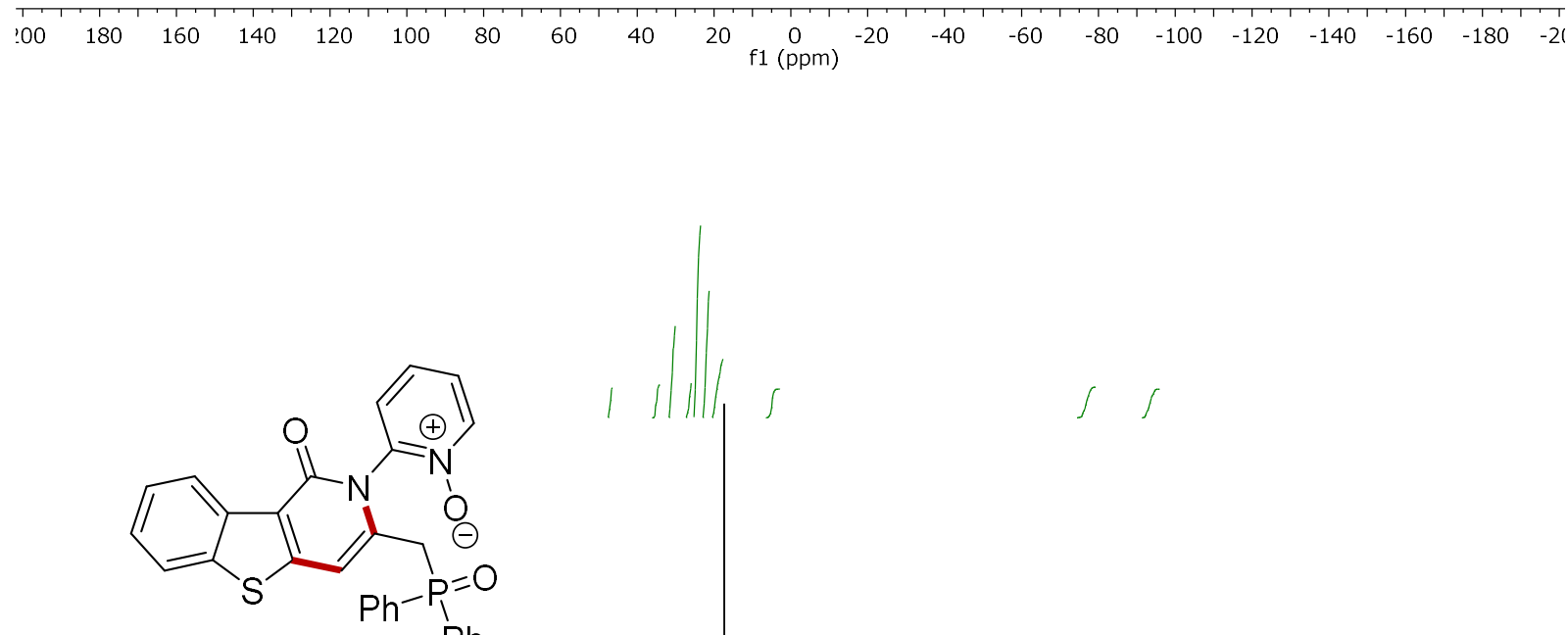

176ta ${ }^{1} \mathrm{H}-\mathrm{NMR}$

$\left(400 \mathrm{MHz}, \mathrm{CDCl}_{3}\right)$

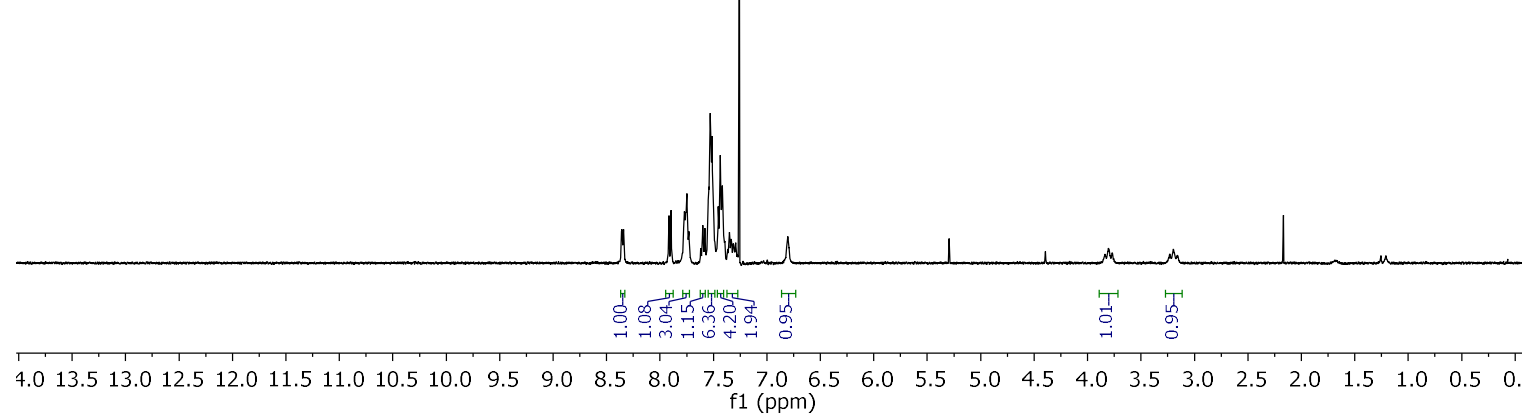


<smiles></smiles>

176ta ${ }^{13} \mathrm{C}-\mathrm{NMR}$

$\left(126 \mathrm{MHz}, \mathrm{CDCl}_{3}\right)$

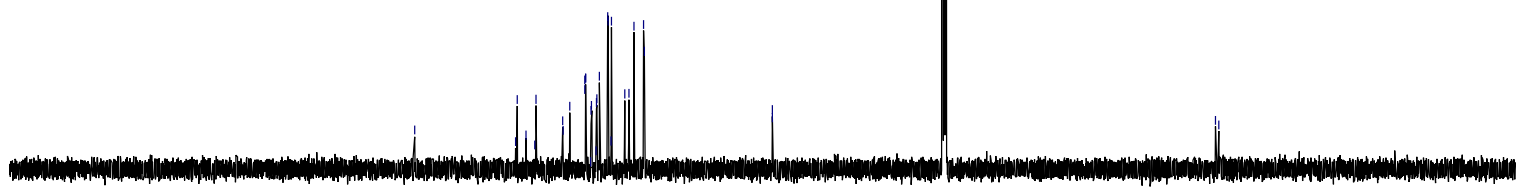

$\begin{array}{llllllllllllllllllllllllllll}20 & 210 & 200 & 190 & 180 & 170 & 160 & 150 & 140 & 130 & 120 & \begin{array}{l}110 \\ \mathrm{f} 1(\mathrm{ppm})\end{array} & 100 & 90 & 80 & 70 & 60 & 50 & 40 & 30 & 20 & 10 & 0 & -1\end{array}$<smiles></smiles>

176 ta ${ }^{31} \mathrm{P}-\mathrm{NMR}$

$\left(162 \mathrm{MHz}, \mathrm{CDCl}_{3}\right.$ )

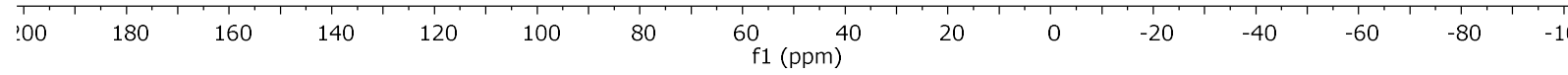


<smiles>Cc1cc(CP(=O)(c2ccccc2)c2ccccc2)n(-c2cccc[n+]2[O-])c(=O)c1C</smiles>

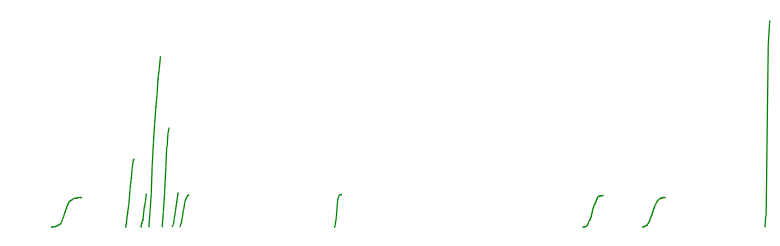

182aa ${ }^{1} \mathrm{H}-\mathrm{NMR}$

$\left(400 \mathrm{MHz}, \mathrm{CDCl}_{3}\right.$ )

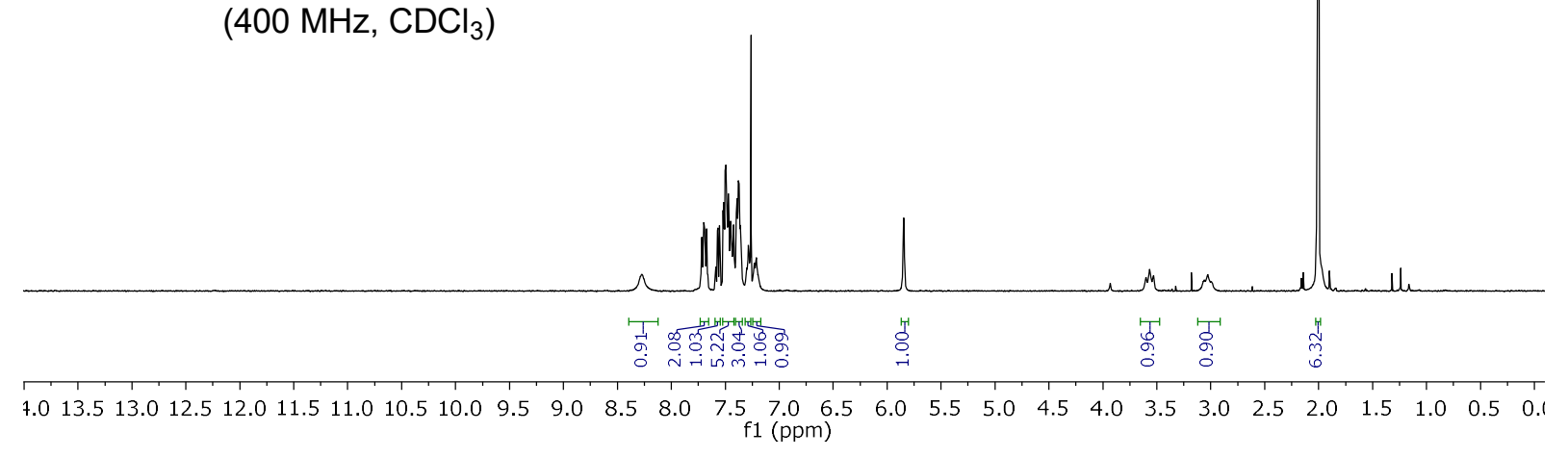<smiles>Cc1cc(CP(=O)(c2ccccc2)c2ccccc2)n(-c2cccc[n+]2[O-])c(=O)c1C</smiles>

182aa ${ }^{13} \mathrm{C}-\mathrm{NMR}$

$\left(126 \mathrm{MHz}, \mathrm{CDCl}_{3}\right)$

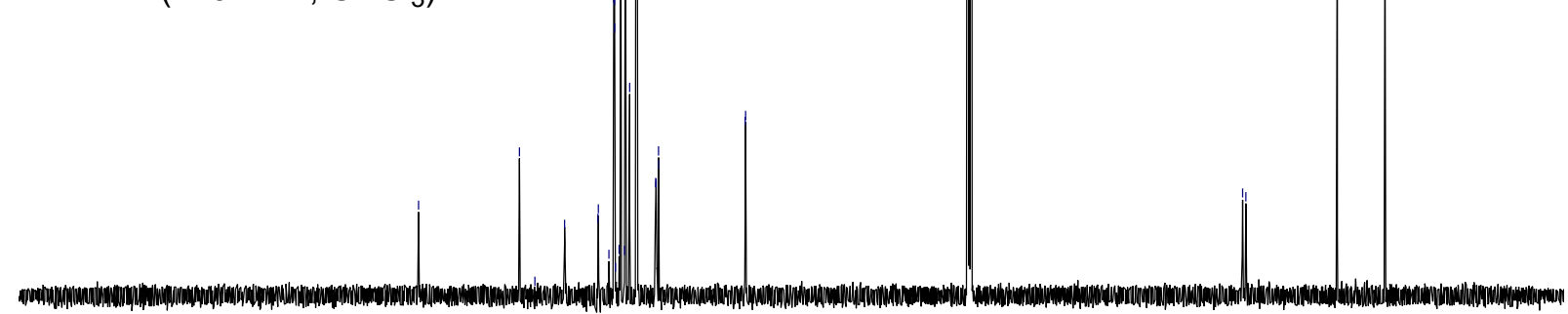




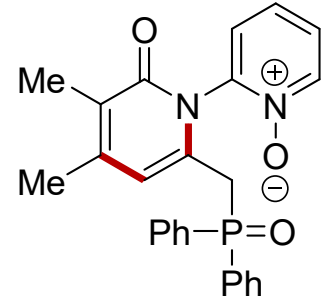

182aa ${ }^{31}$ P-NMR

(162 MHz, $\mathrm{CDCl}_{3}$ )

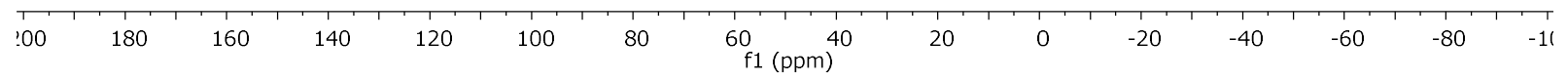<smiles>CCOCc1cc2ccccc2c(=O)n1-c1cccc[n+]1[O-]</smiles>

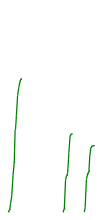

176ac ${ }^{1} \mathrm{H}-\mathrm{NMR}$

$\left(500 \mathrm{MHz}, \mathrm{CDCl}_{3}\right)$

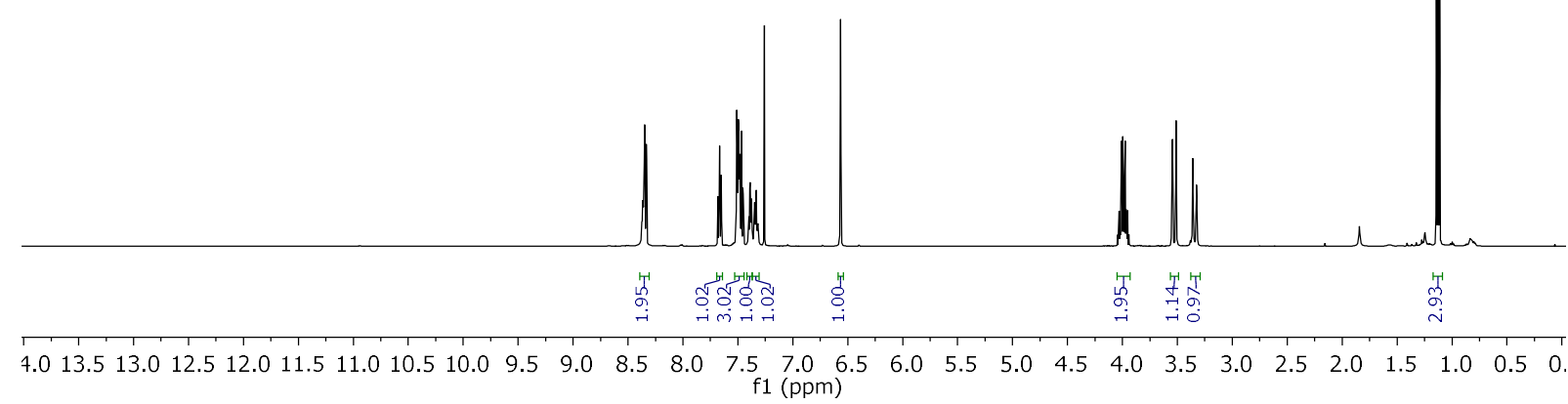


<smiles>CCOCCc1cc2ccccc2c(=O)n1-c1cccc[n+]1[O-]</smiles>

176ac ${ }^{13} \mathrm{C}-\mathrm{NMR}$

$\left(126 \mathrm{MHz}, \mathrm{CDCl}_{3}\right)$

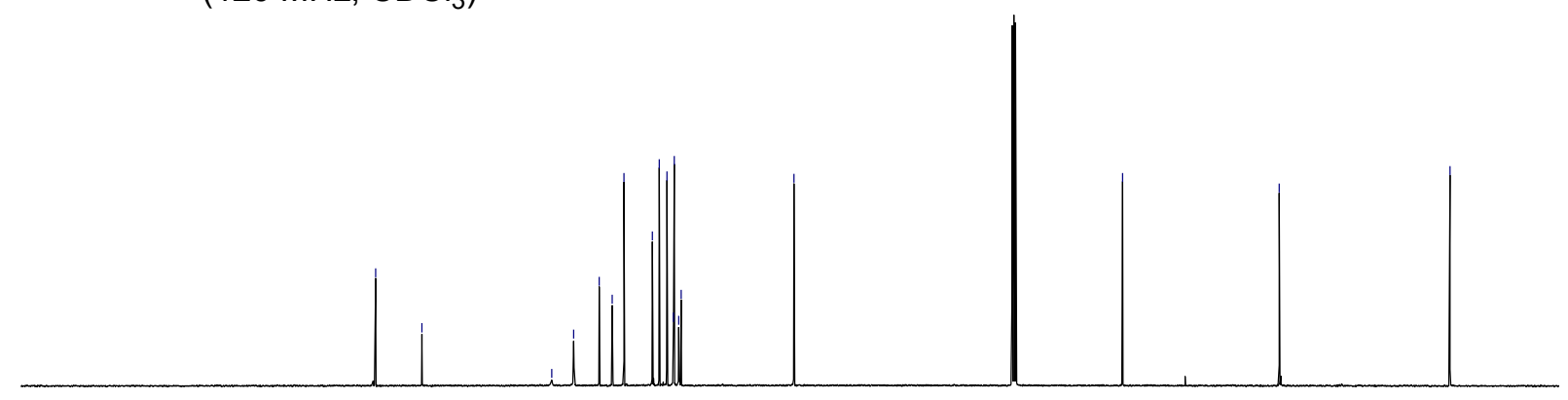

$\begin{array}{lllllllllllllllllllllllllll}1 & 210 & 200 & 190 & 180 & 170 & 160 & 150 & 140 & 130 & 120 & \begin{array}{l}110 \\ \mathrm{f} 1(\mathrm{ppm})\end{array} & 100 & 90 & 80 & 70 & 60 & 50 & 40 & 30 & 20 & 10 & 0\end{array}$

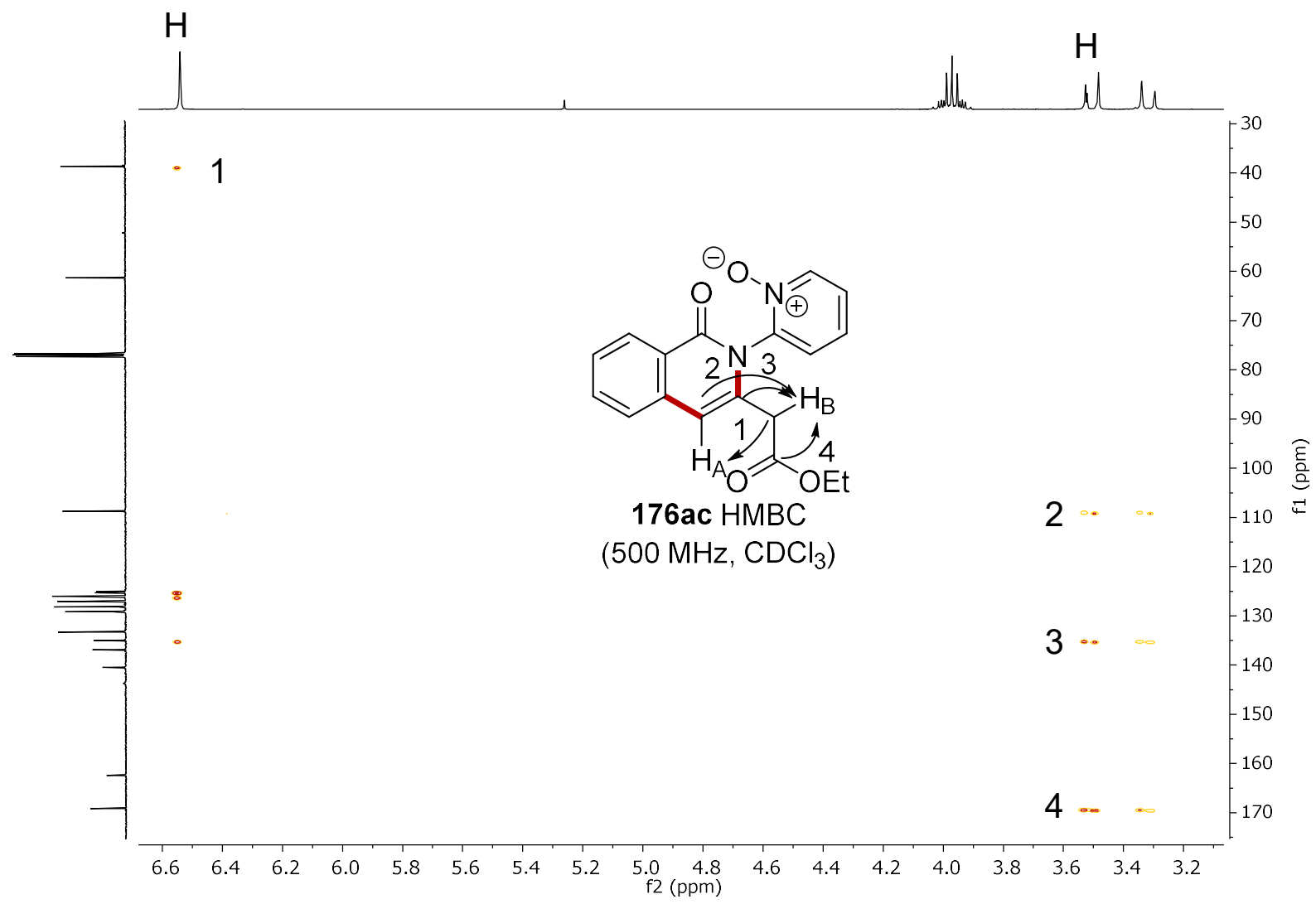




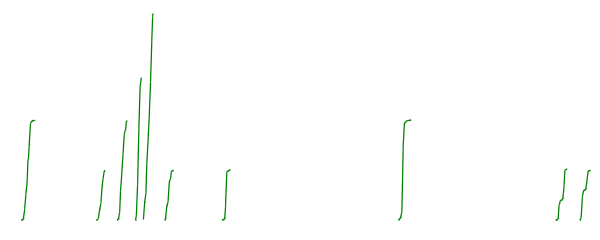<smiles>O=C(Cc1cc2ccccc2c(=O)n1-c1cccc[n+]1[O-])OCc1ccccc1</smiles>

176ad ${ }^{1} \mathrm{H}-\mathrm{NMR}$ $\left(400 \mathrm{MHz}, \mathrm{CDCl}_{3}\right)$

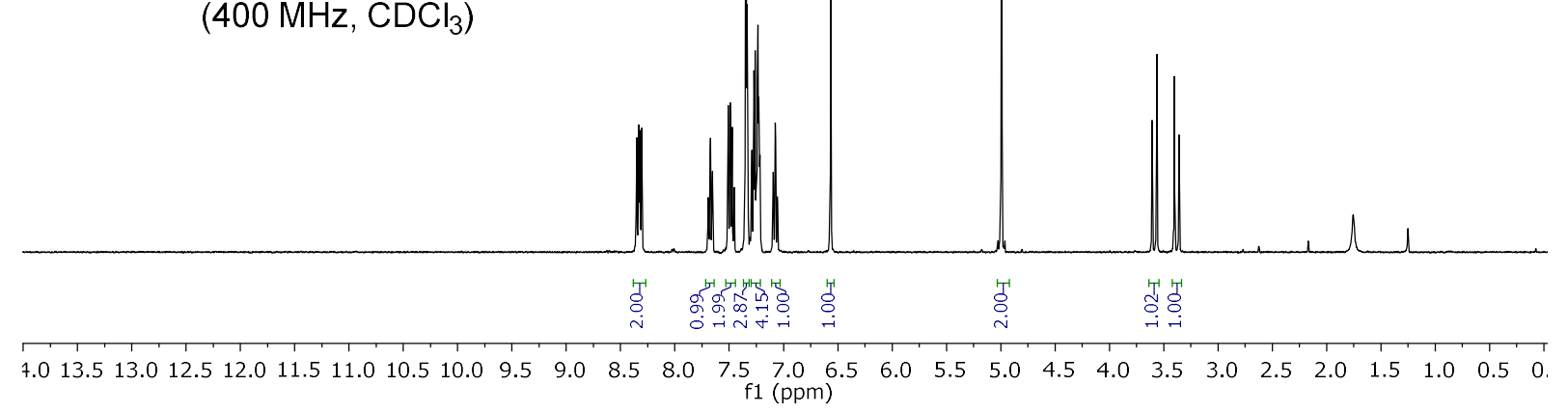

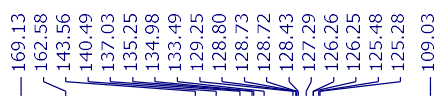<smiles>O=C(Cc1cc2ccccc2c(=O)n1-c1cccc[n+]1[O-])OCc1ccccc1</smiles>

176ad ${ }^{13} \mathrm{C}-\mathrm{NMR}$ $\left(101 \mathrm{MHz}, \mathrm{CDCl}_{3}\right.$ )

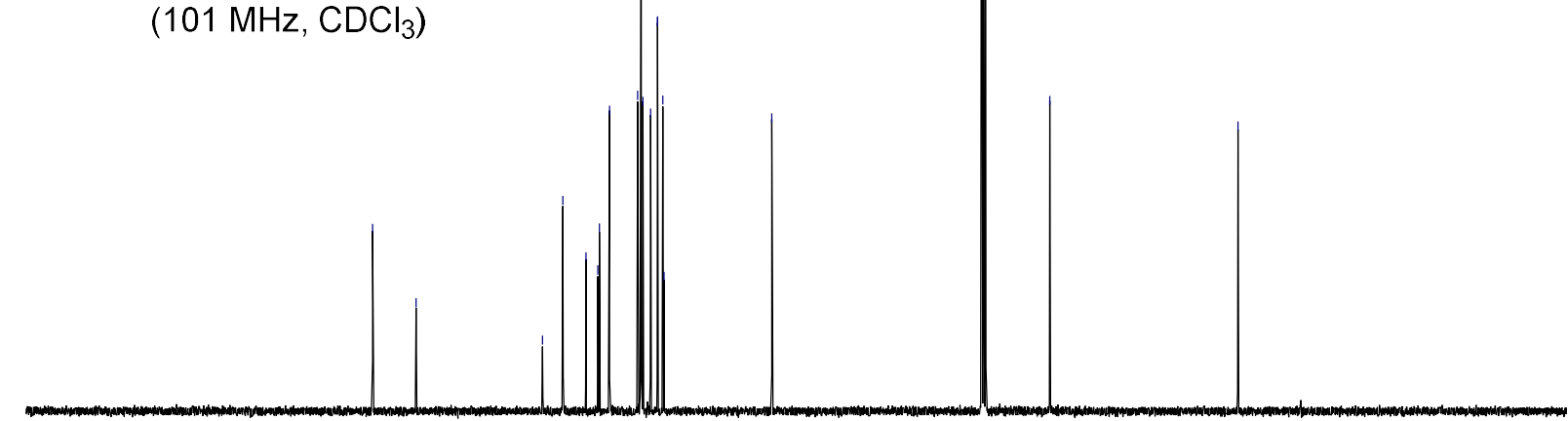

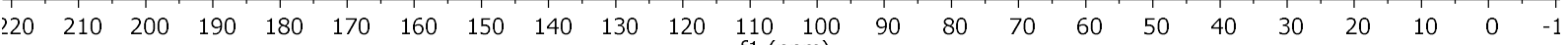
f1 (ppm) 


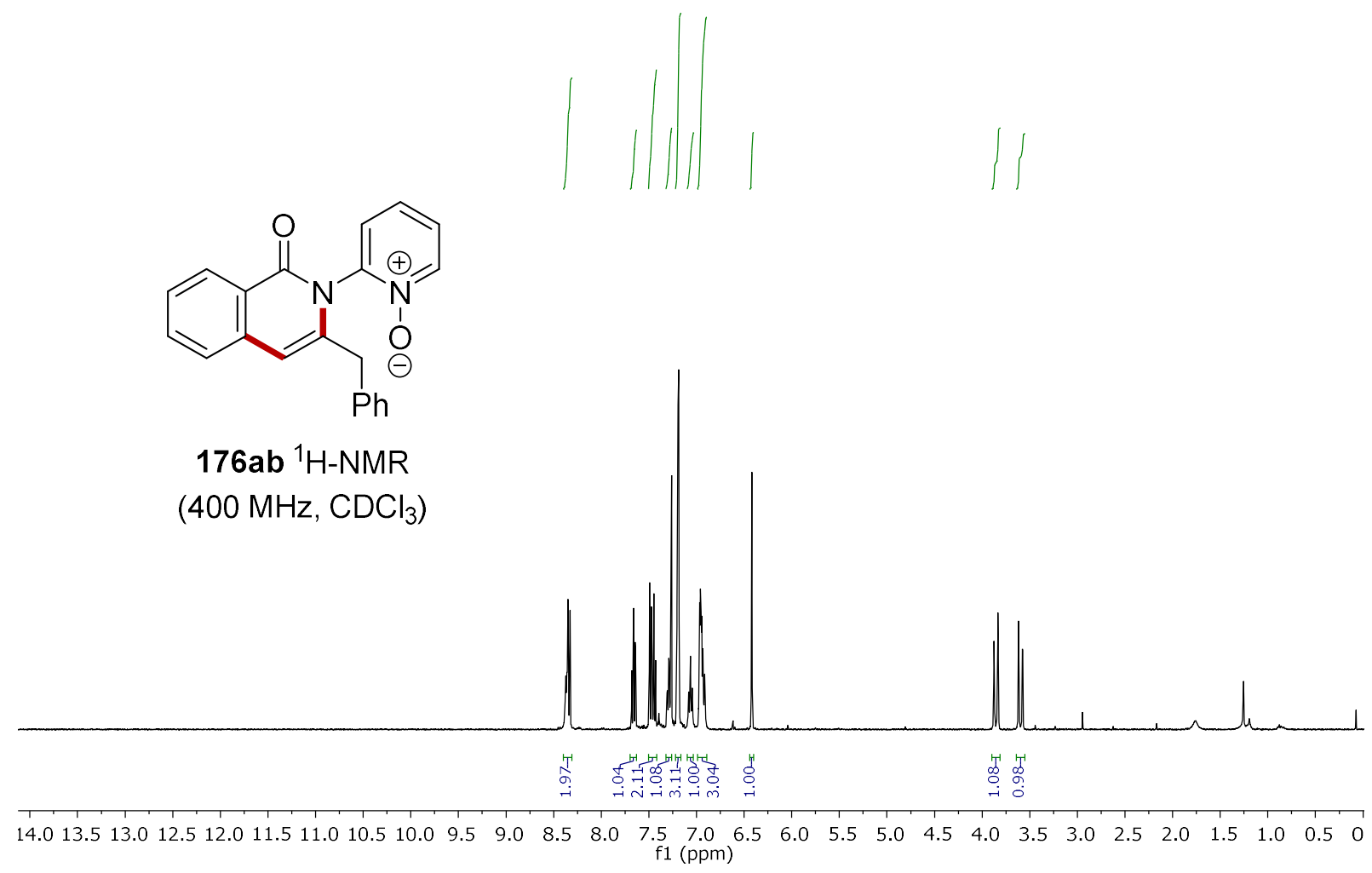

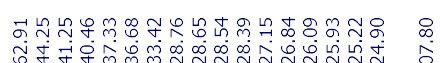

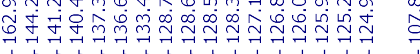

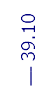<smiles>O=c1c2ccccc2cc(Cc2ccccc2)n1-c1cccc[n+]1[O-]</smiles>

176ab ${ }^{13} \mathrm{C}-\mathrm{NMR}$

$\left(101 \mathrm{MHz}, \mathrm{CDCl}_{3}\right.$ )

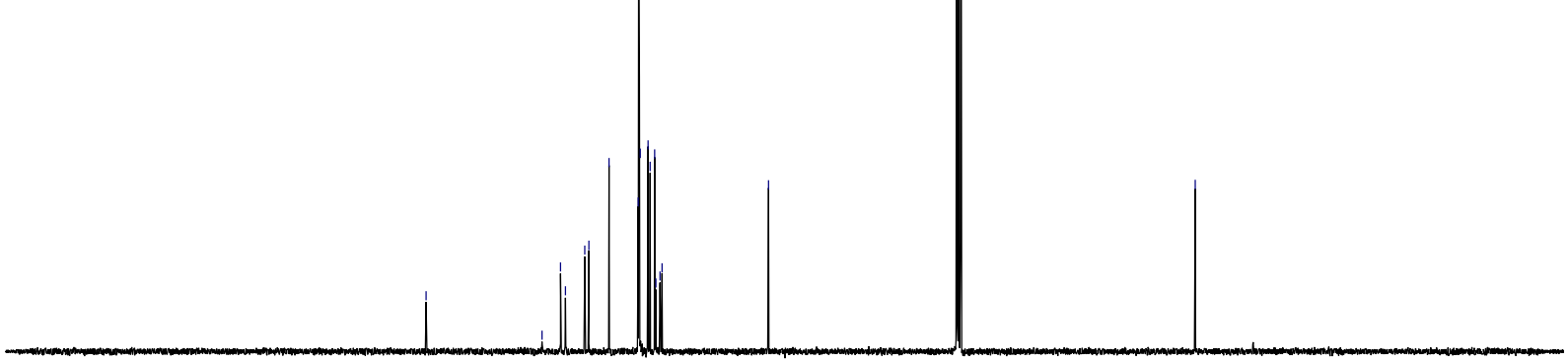

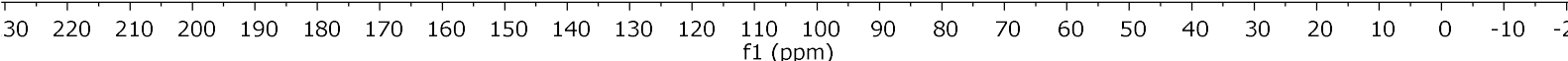




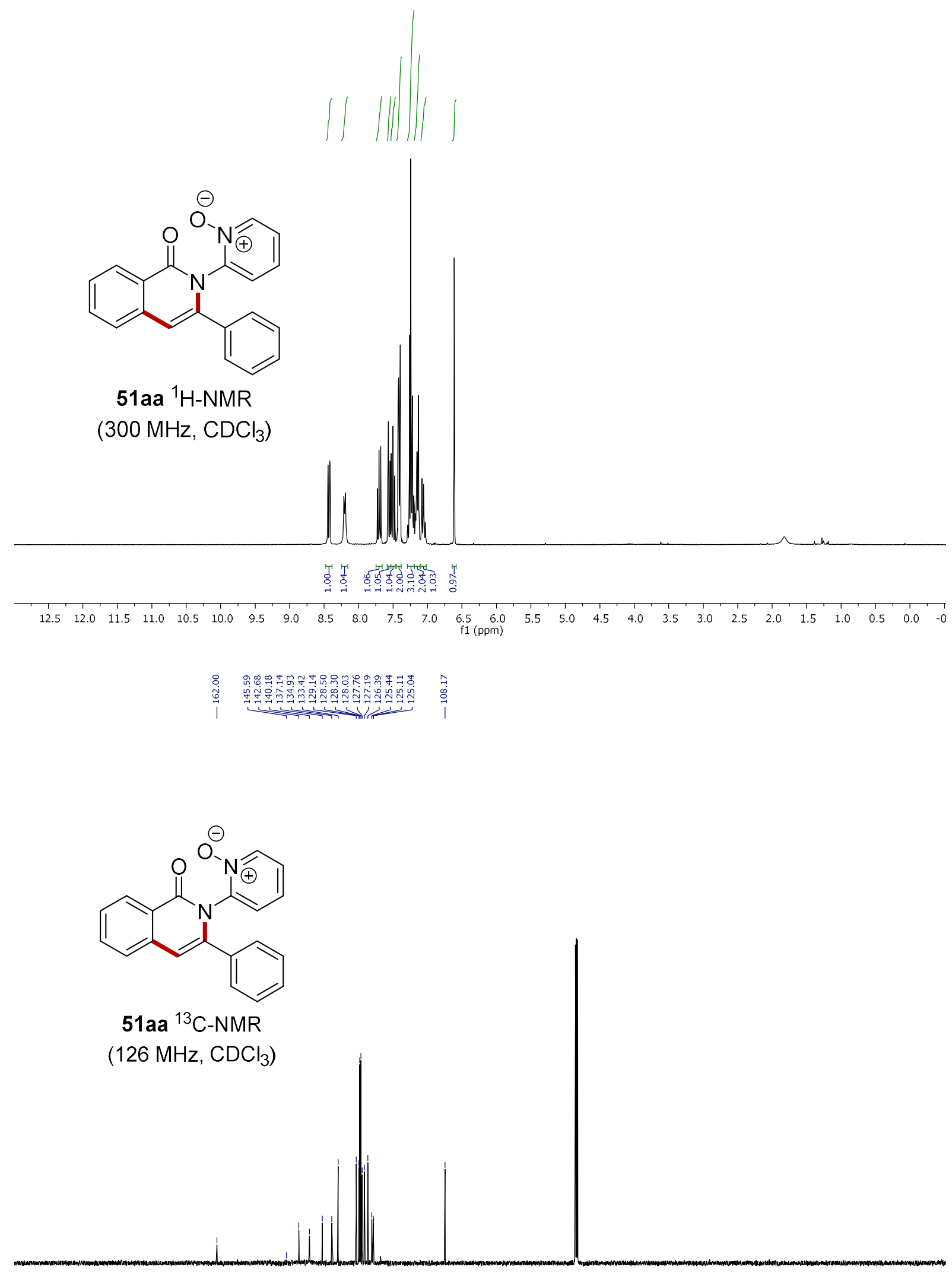

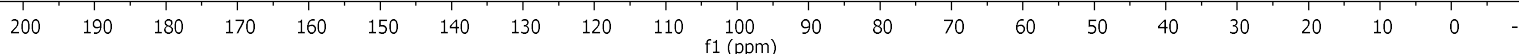




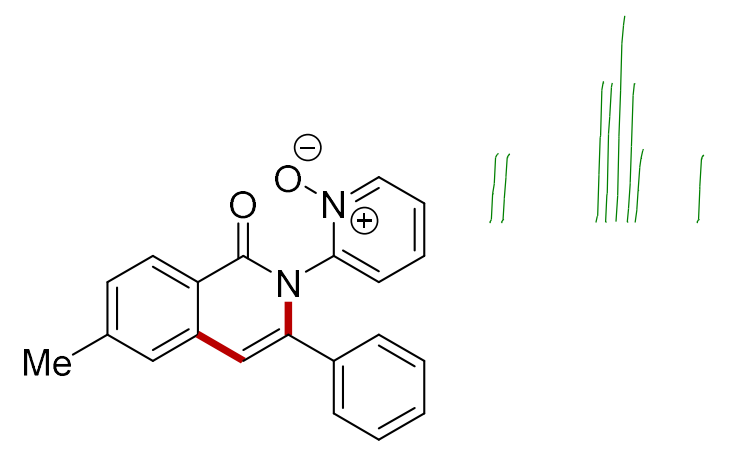

51 ba ${ }^{1} \mathrm{H}-\mathrm{NMR}$

$\left(400 \mathrm{MHz}, \mathrm{CDCl}_{3}\right.$ )
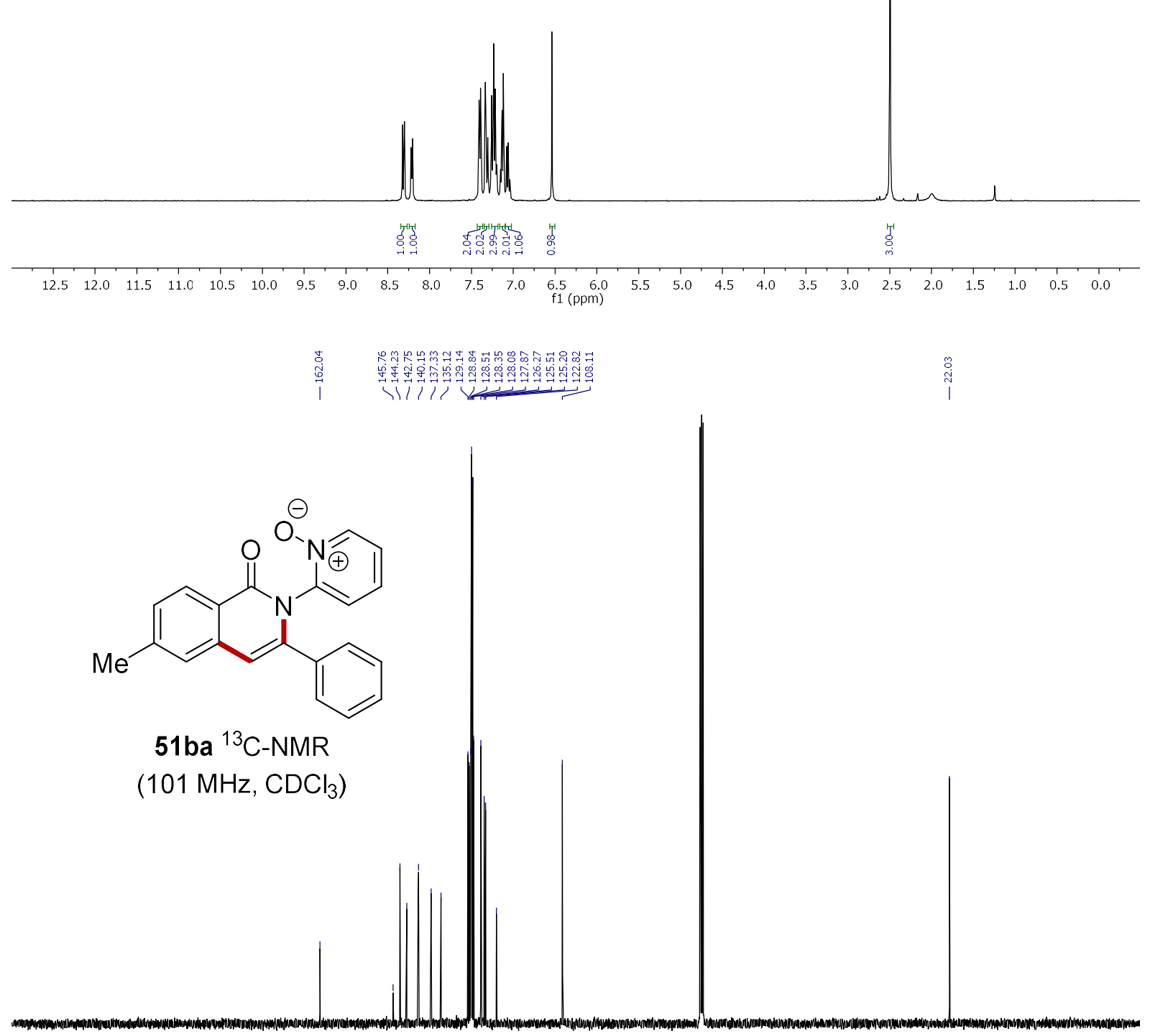

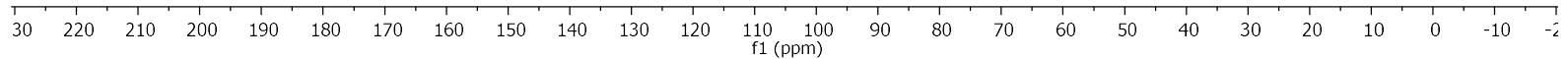




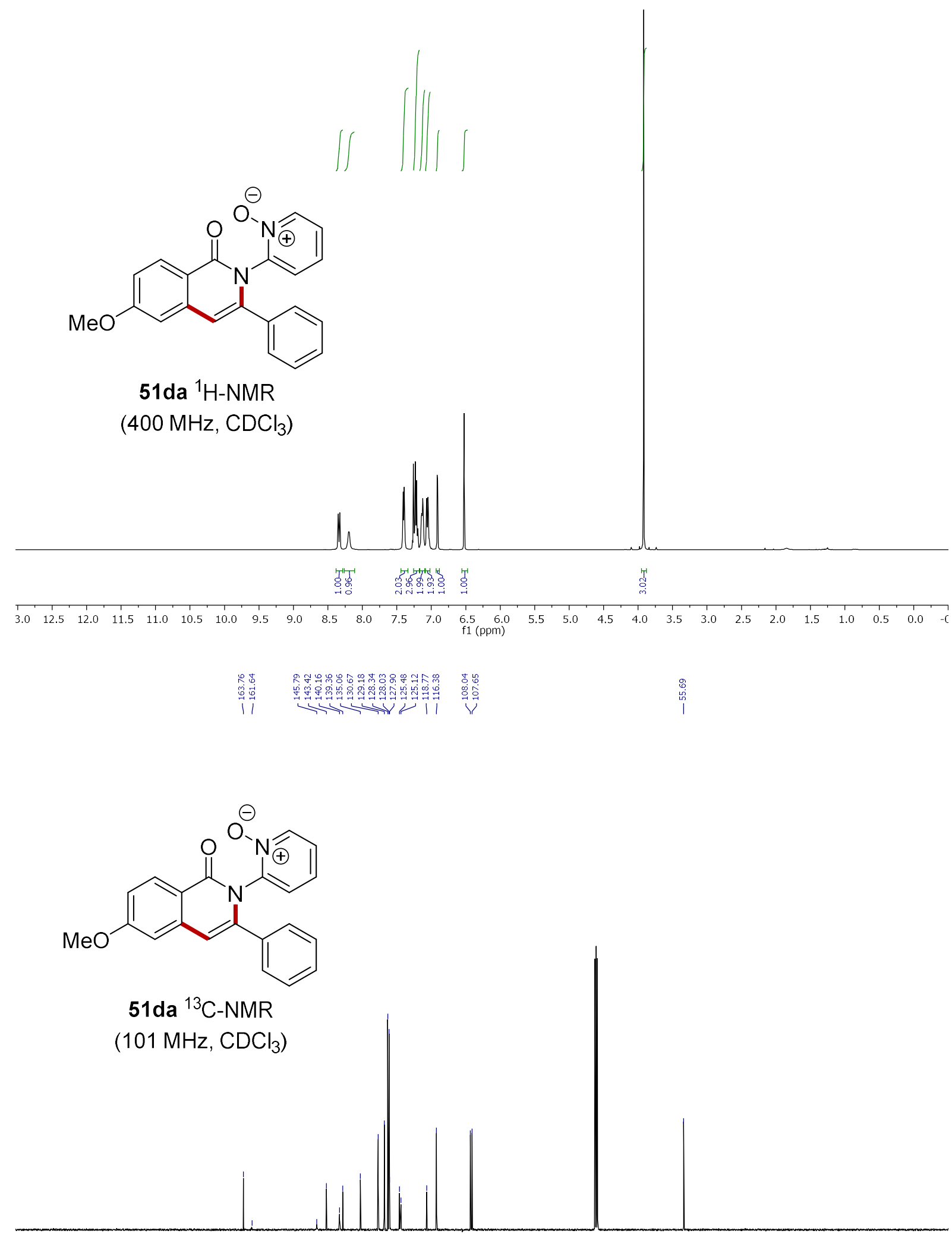

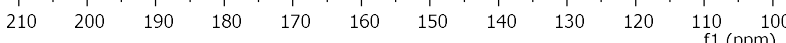



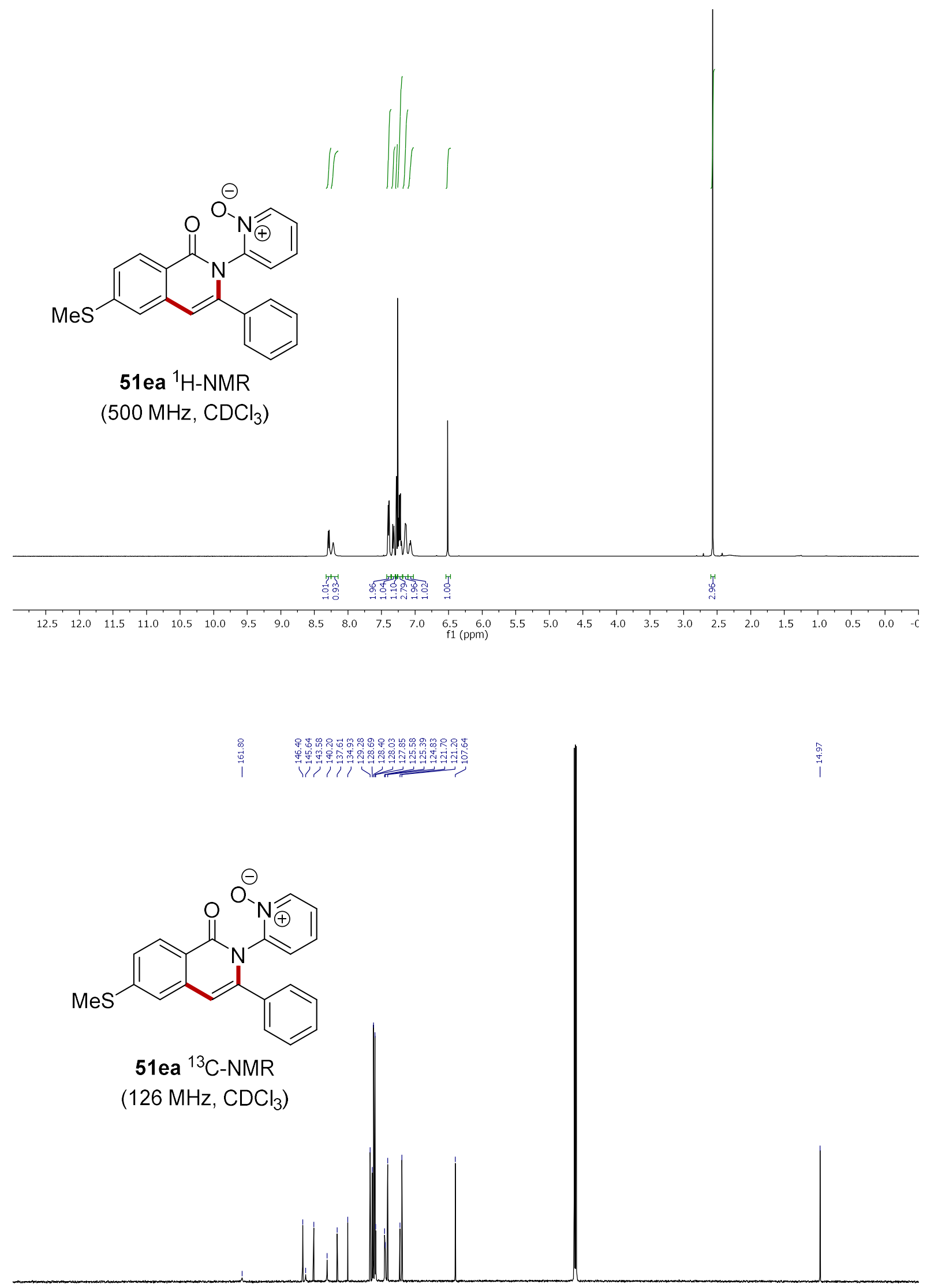

$\begin{array}{lllllllllllllllllllllllllllllll} & 210 & 200 & 190 & 180 & 170 & 160 & 150 & 140 & 130 & 120 & 110 & 100 & 90 & 80 & 70 & 60 & 50 & 40 & 30 & 20 & 10 & 0 & -\end{array}$ 


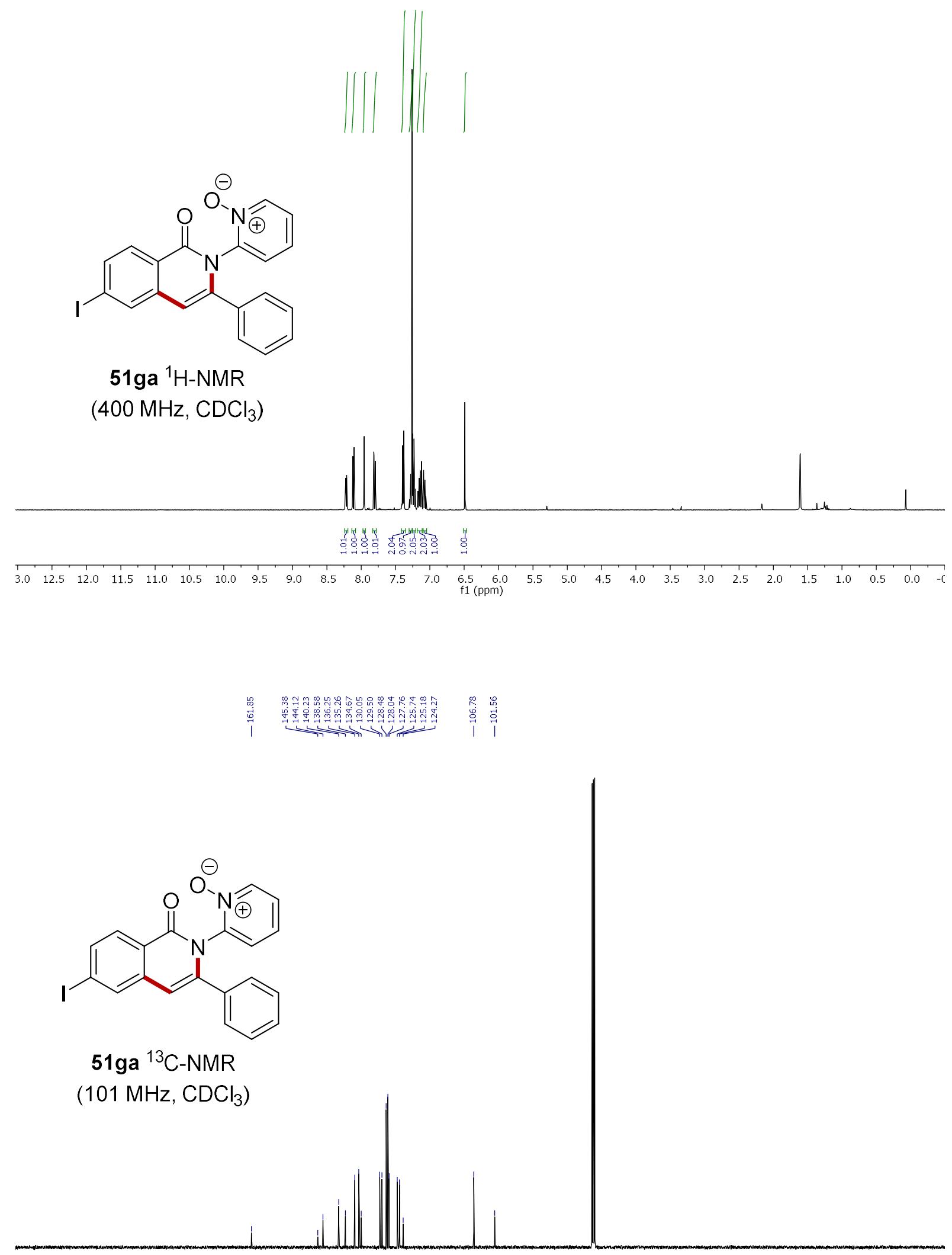

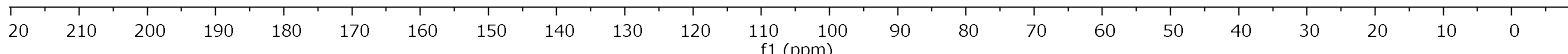


<smiles>CC(C)(C)c1ccc2c(=O)n(-c3cccc[n+]3[O-])c(-c3ccccc3)cc2c1</smiles>

51ja ${ }^{1} \mathrm{H}-\mathrm{NMR}$

(300 $\mathrm{MHz} \mathrm{CDCl}_{3}$ )

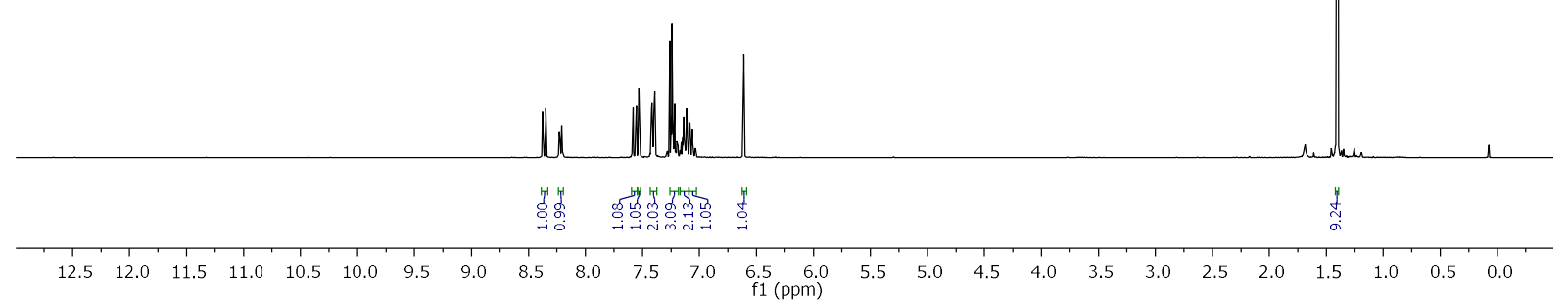

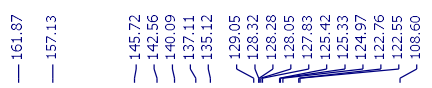

Fis<smiles>CC(C)(C)c1ccc2c(=O)n(-c3cccc[n+]3[O-])c(-c3ccccc3)cc2c1</smiles>

51ja ${ }^{13} \mathrm{C}-\mathrm{NMR}$

$\left(126 \mathrm{MHz}, \mathrm{CDCl}_{3}\right.$ )

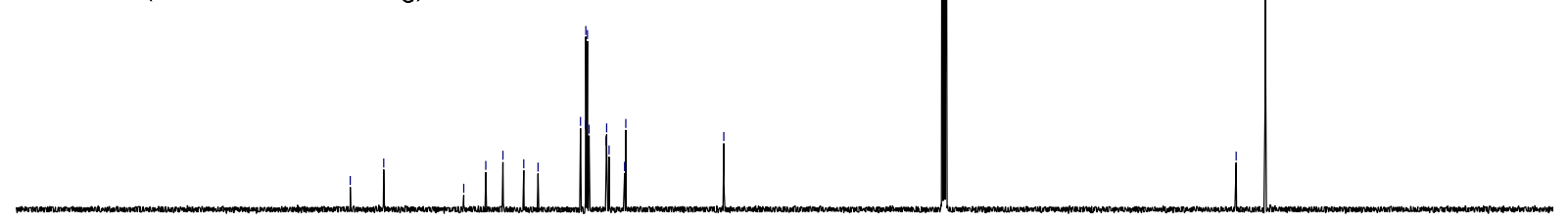

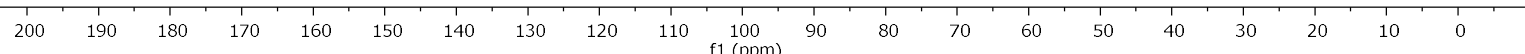




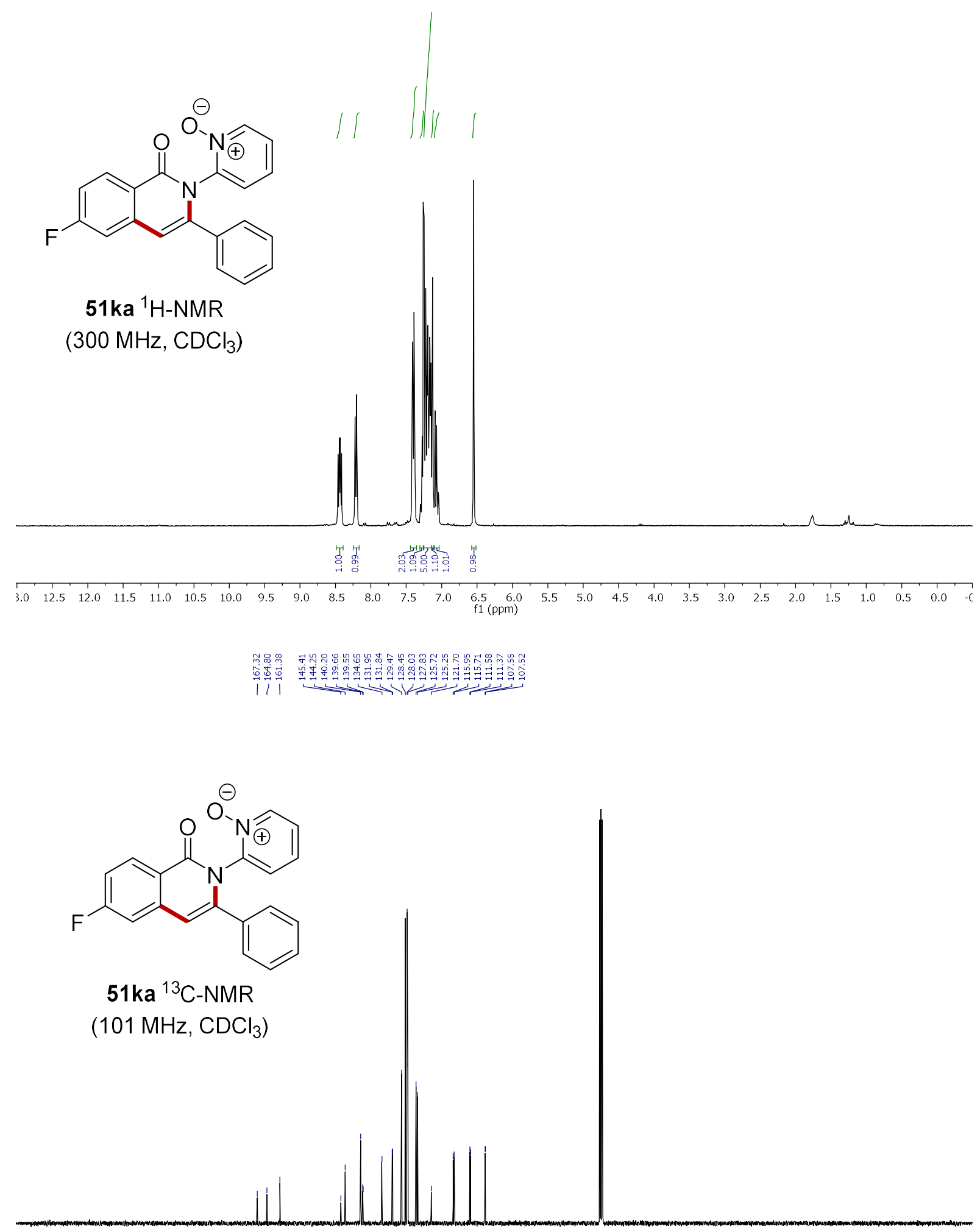

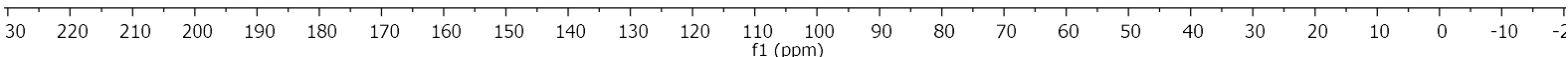



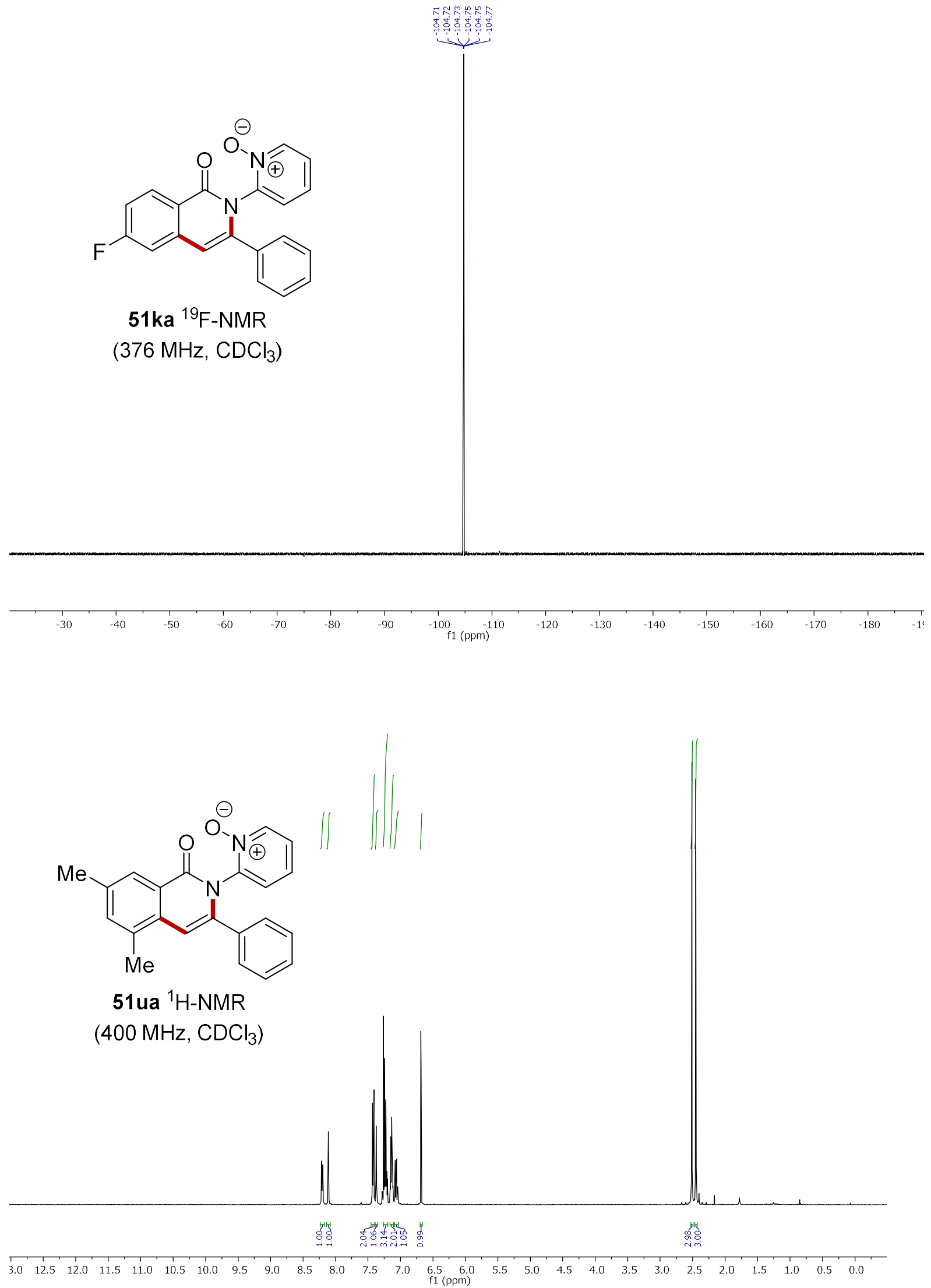

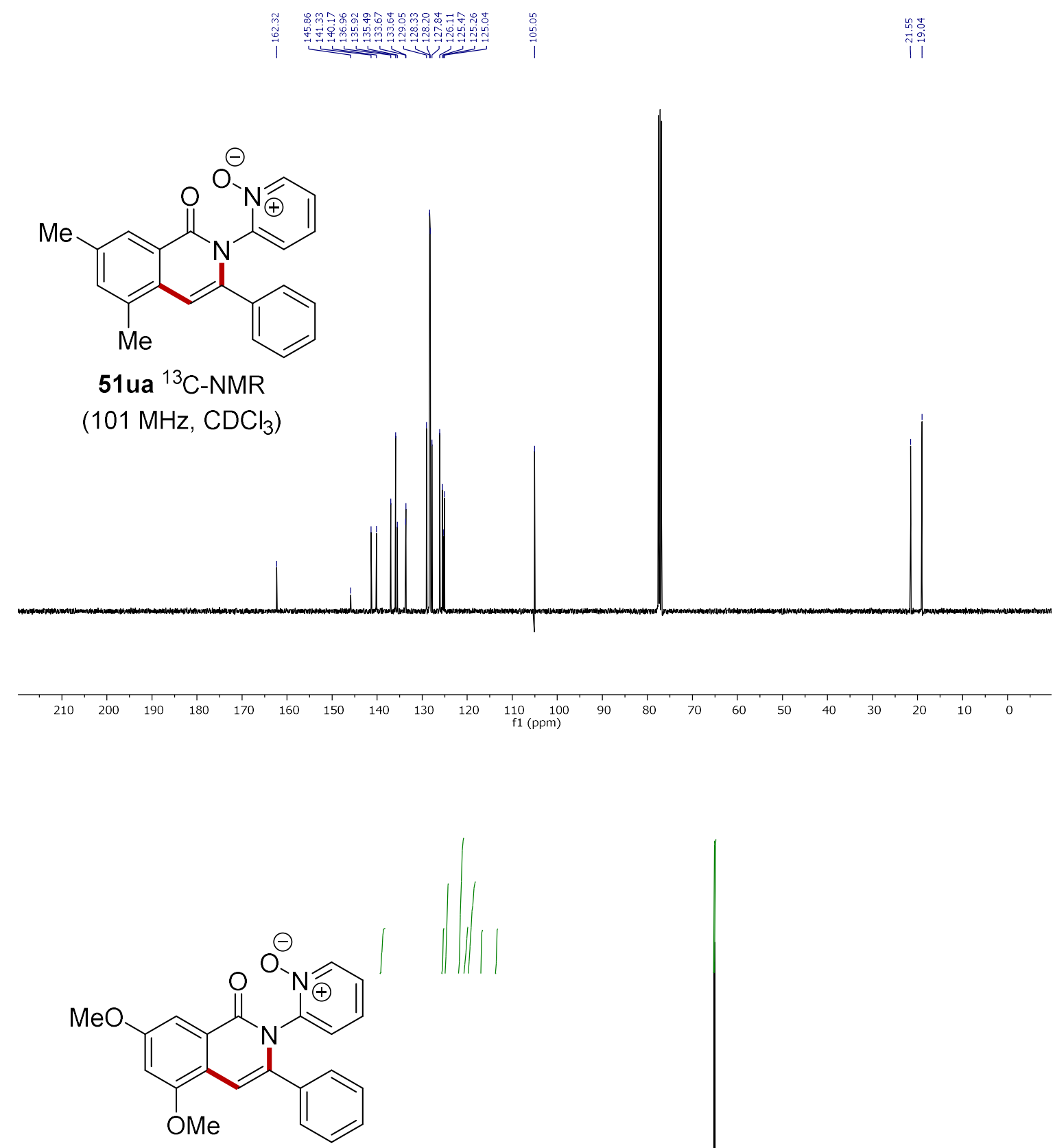

51va ${ }^{1} \mathrm{H}-\mathrm{NMR}$

$\left(400 \mathrm{MHz}, \mathrm{CDCl}_{3}\right)$

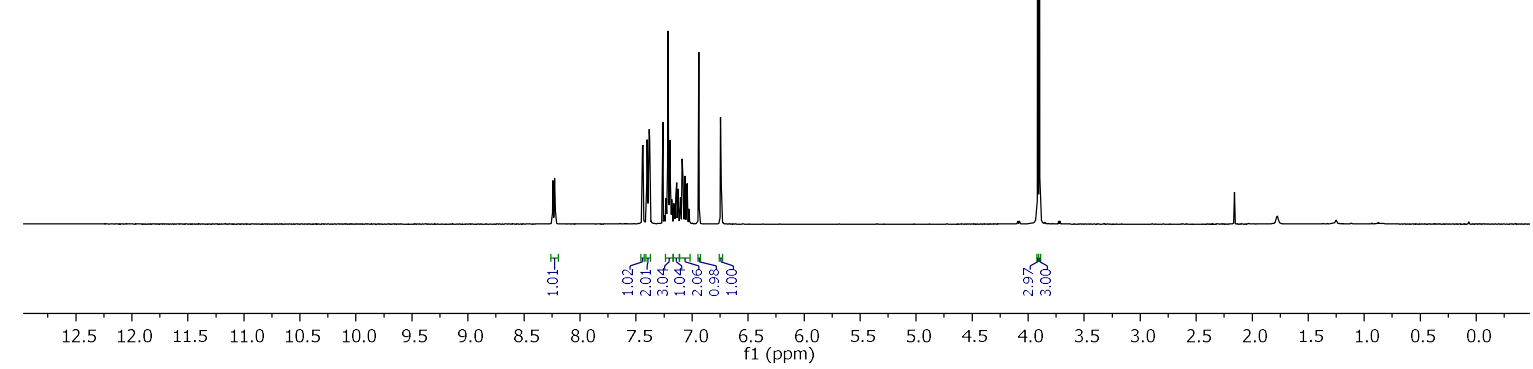




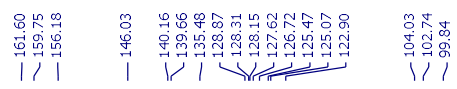<smiles>COc1cc(OC)c2cc(-c3ccccc3)n(-c3cccc[n+]3[O-])c(=O)c2c1</smiles>

51 va ${ }^{13} \mathrm{C}-\mathrm{NMR}$

(101 MHz, $\mathrm{CDCl}_{3}$ )

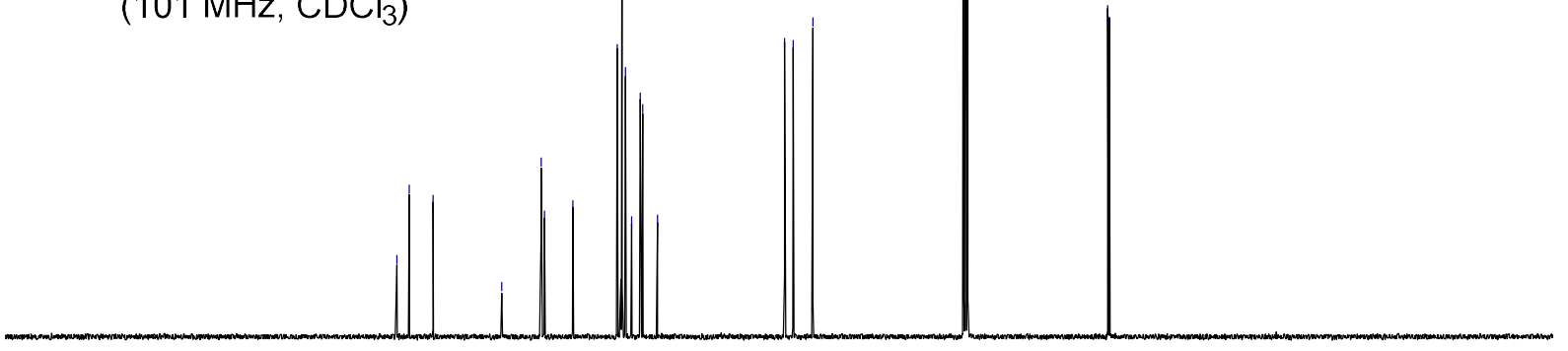

$\begin{array}{llllllllllllllllllllllllll}210 & 200 & 190 & 180 & 170 & 160 & 150 & 140 & 130 & 120 & 110 & 100 & 90 & 80 & 70 & 60 & 50 & 40 & 30 & 20 & 10 & 0 & -10\end{array}$

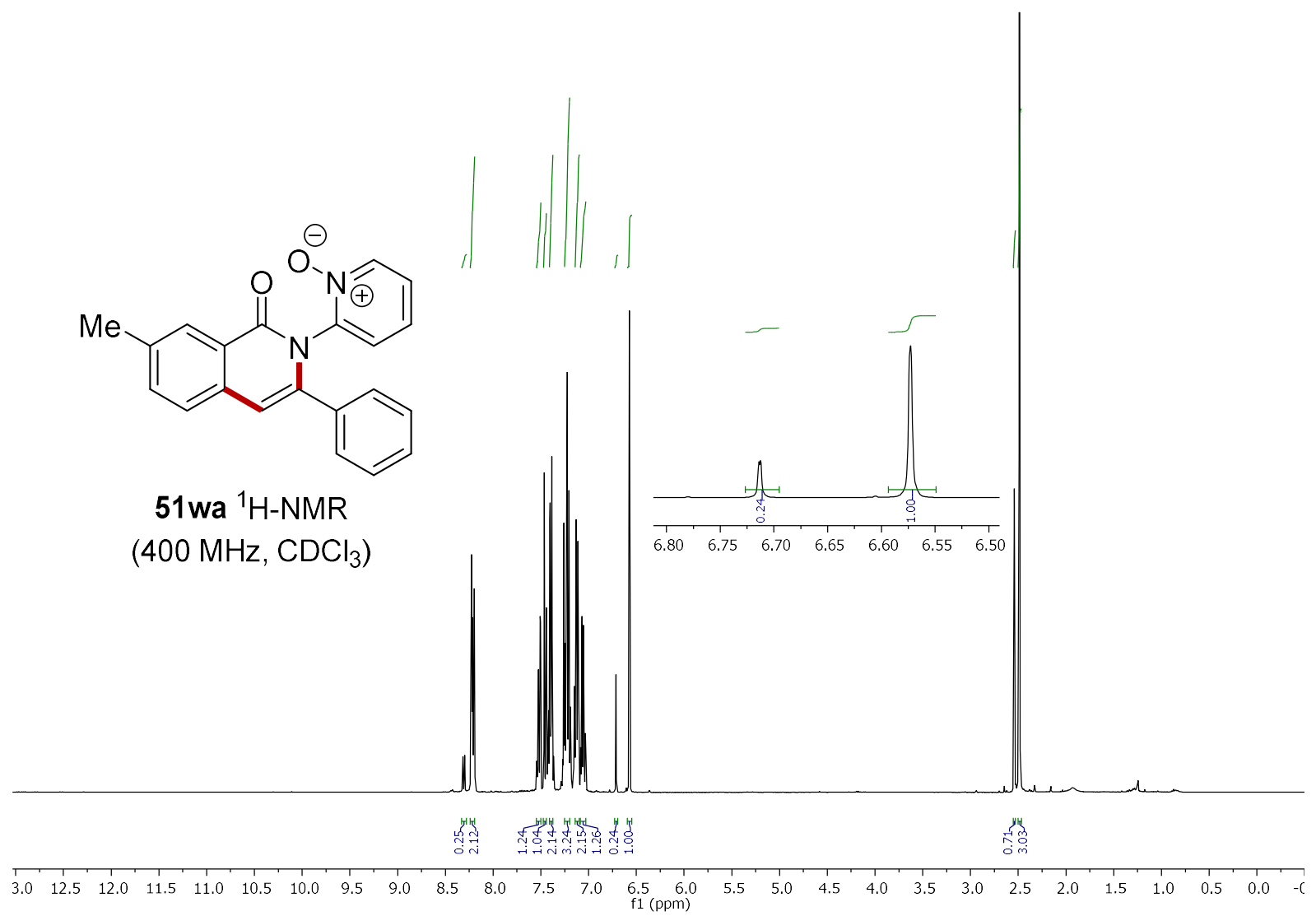




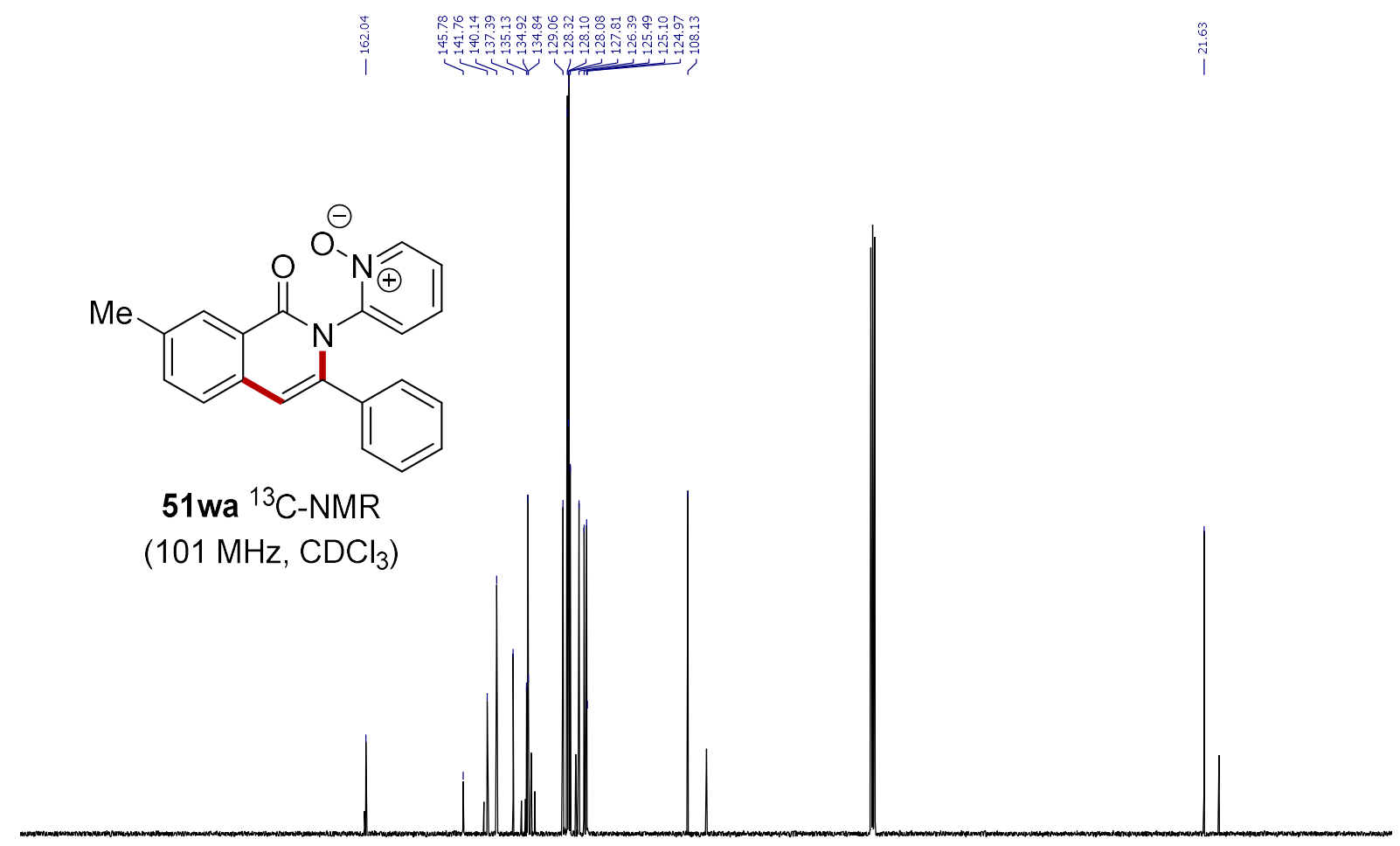

$\begin{array}{lllllllllll}210 & 200 & 190 & 180 & 170 & 160 & 150 & 140 & 130 & 120 & \begin{array}{c}110 \\ \mathrm{f} 1(\mathrm{ppm})\end{array}\end{array}$<smiles>Cc1cc(-c2ccccc2)n(-c2cccc[n+]2[O-])c(=O)c1C</smiles>

189aa ${ }^{1} \mathrm{H}-\mathrm{NMR}$

$\left(300 \mathrm{MHz}, \mathrm{CDCl}_{3}\right.$ )

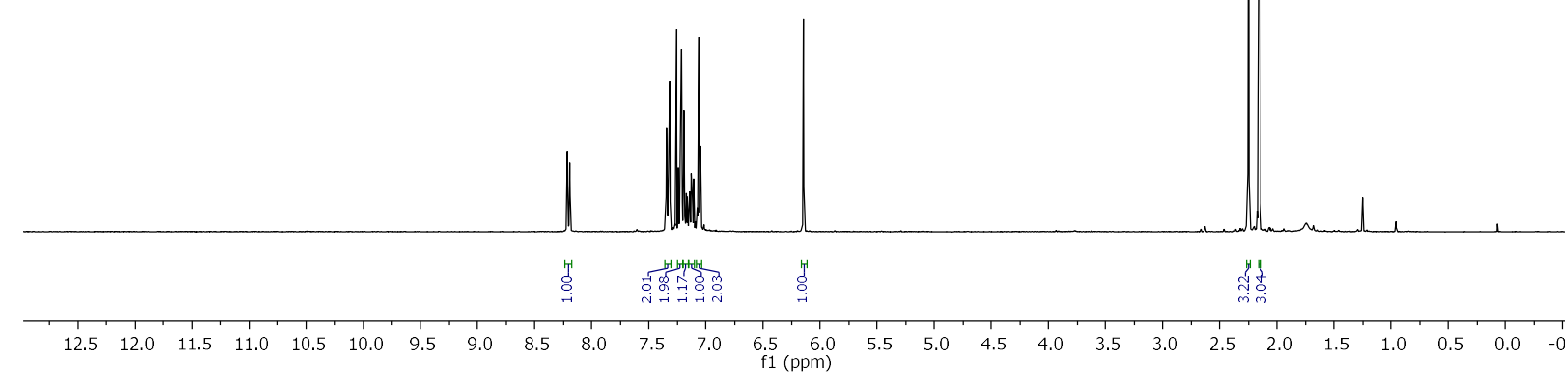


<smiles>Cc1cc(-c2ccccc2)n(-c2cccc[n+]2[O-])c(=O)c1C</smiles>

189aa ${ }^{13} \mathrm{C}-\mathrm{NMR}$

(125 MHz, $\mathrm{CDCl}_{3}$ )
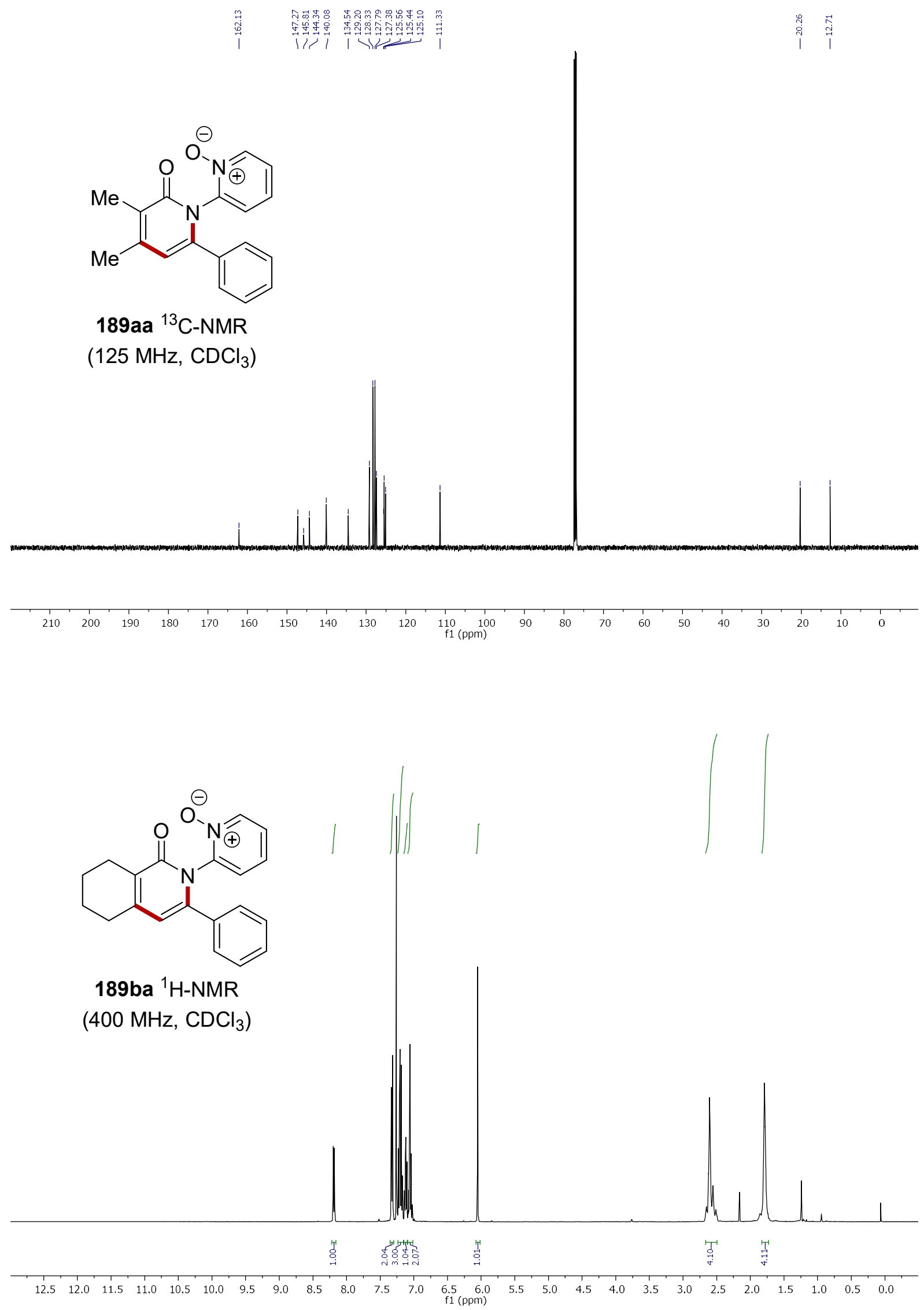
<smiles>O=c1c2c(cc(-c3ccccc3)n1-c1cccc[n+]1[O-])CCCC2</smiles>

189ba ${ }^{13} \mathrm{C}-\mathrm{NMR}$ $\left(101 \mathrm{MHz}, \mathrm{CDCl}_{3}\right.$ )
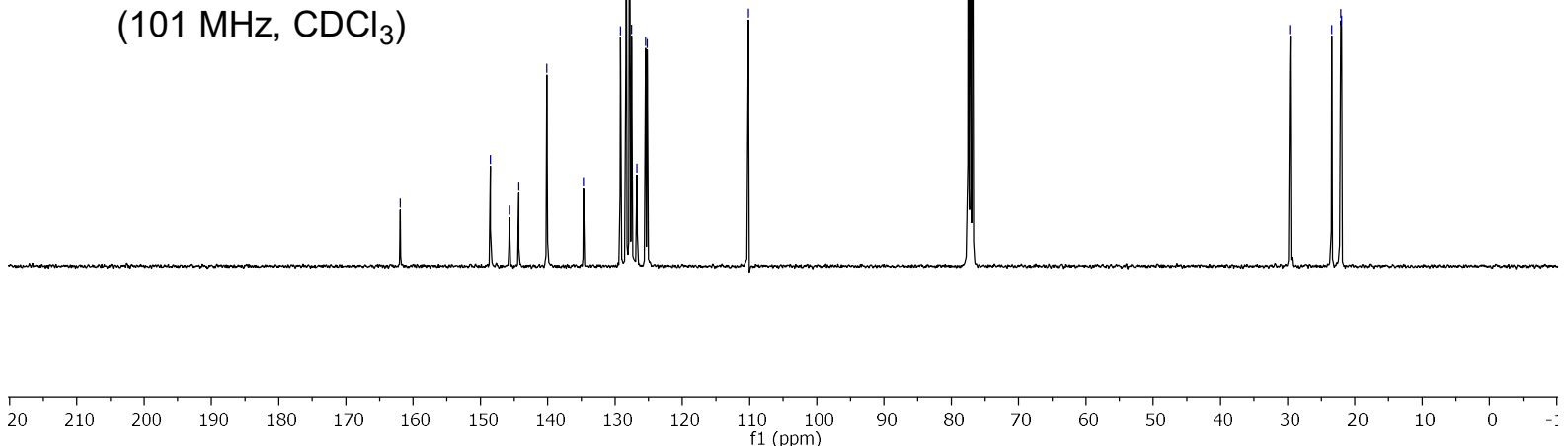

V<smiles></smiles>

189ea ${ }^{1} \mathrm{H}-\mathrm{NMR}$

$\left(600 \mathrm{MHz}, \mathrm{CDCl}_{3}\right.$ )
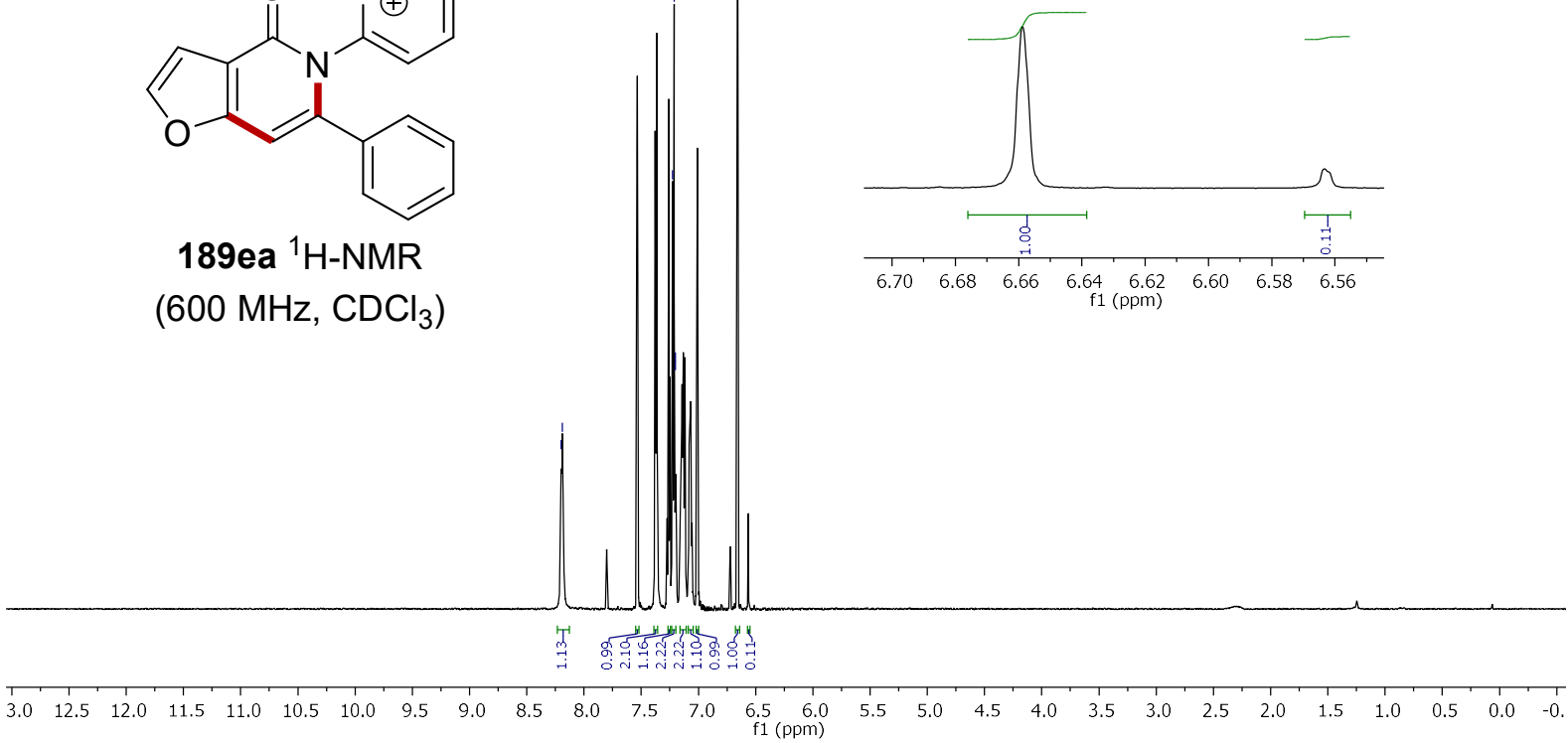

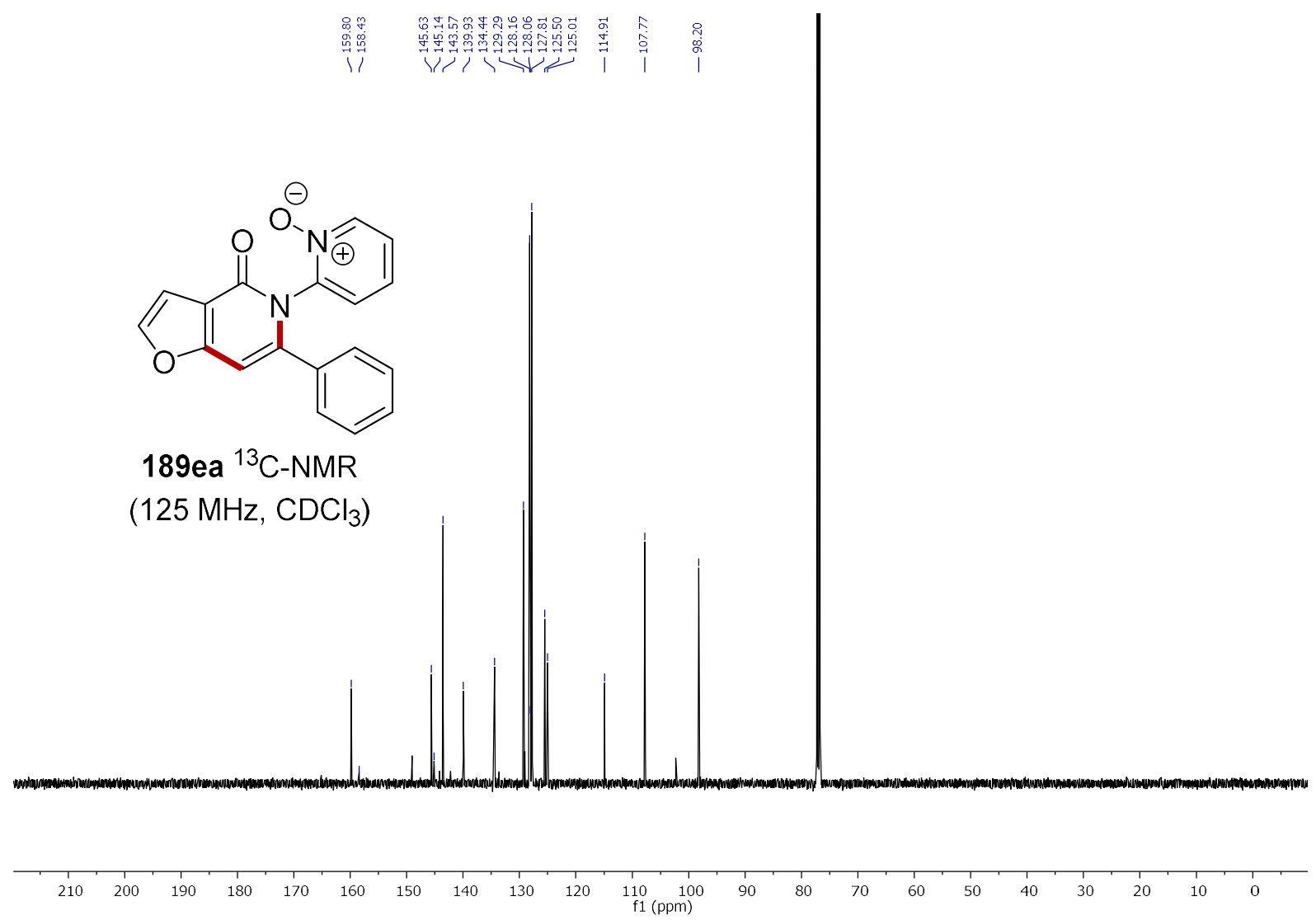

\section{is I I Illli i}<smiles>CC(C)(C)c1ccc(-c2cc3ccccc3c(=O)n2-c2cccc[n+]2[O-])cc1</smiles>

51ab ${ }^{1} \mathrm{H}-\mathrm{NMR}$

$\left(400 \mathrm{MHz}, \mathrm{CDCl}_{3}\right.$ )

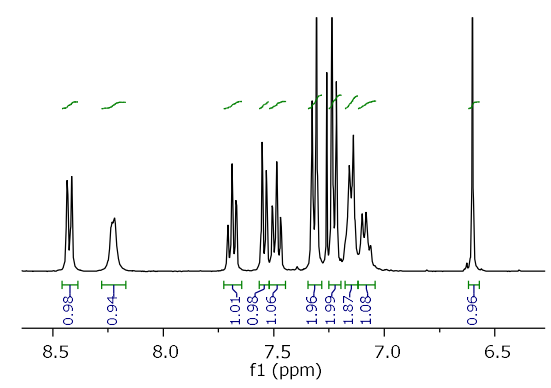

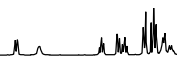

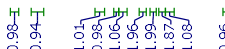

$\begin{array}{llllllllllllllllll}3.0 & 12.5 & 12.0 & 11.5 & 11.0 & 10.5 & 10.0 & 9.5 & 9.0 & 8.5 & 8.0 & 7.5 & 7.0 & 6.5 & 6.0 \\ f 1(\mathrm{ppm})\end{array}$ 

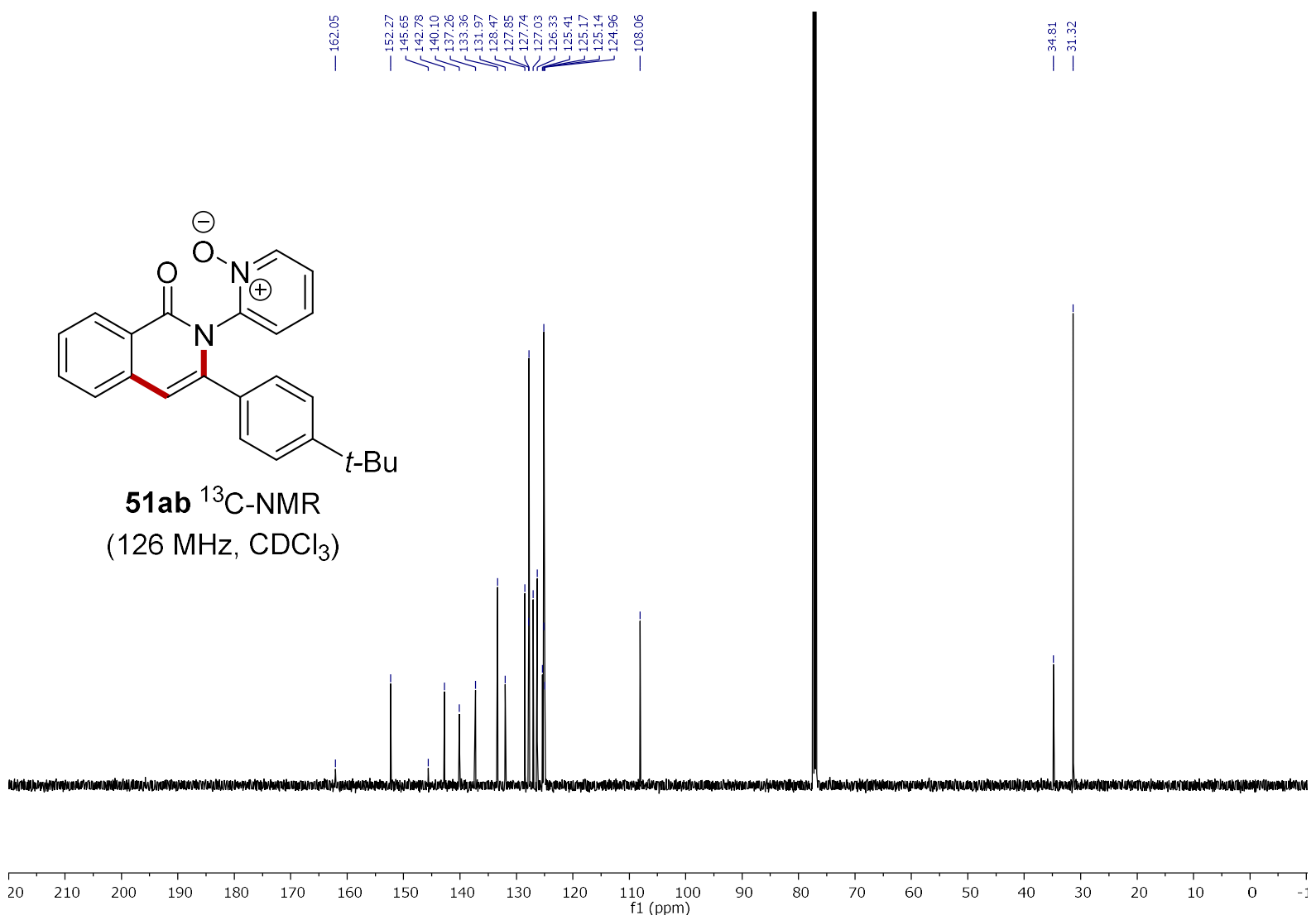<smiles>CCCCCCc1cc2ccccc2c(=O)n1-c1cccc[n+]1[O-]</smiles>

$51 \mathrm{ac}{ }^{1} \mathrm{H}-\mathrm{NMR}$ (400 MHz, $\mathrm{CDCl}_{3}$ )

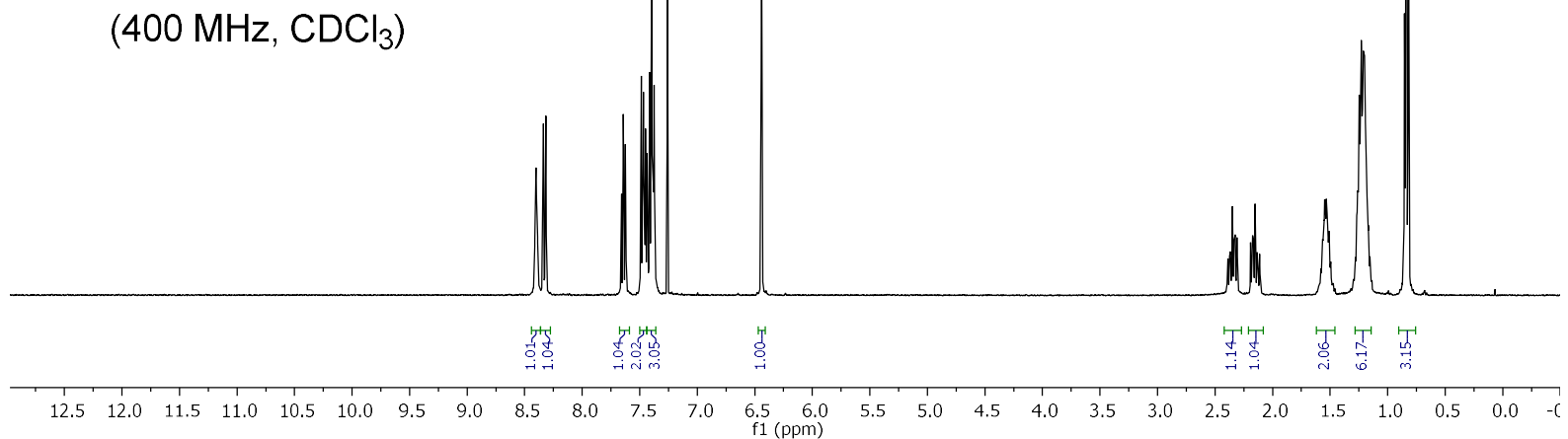



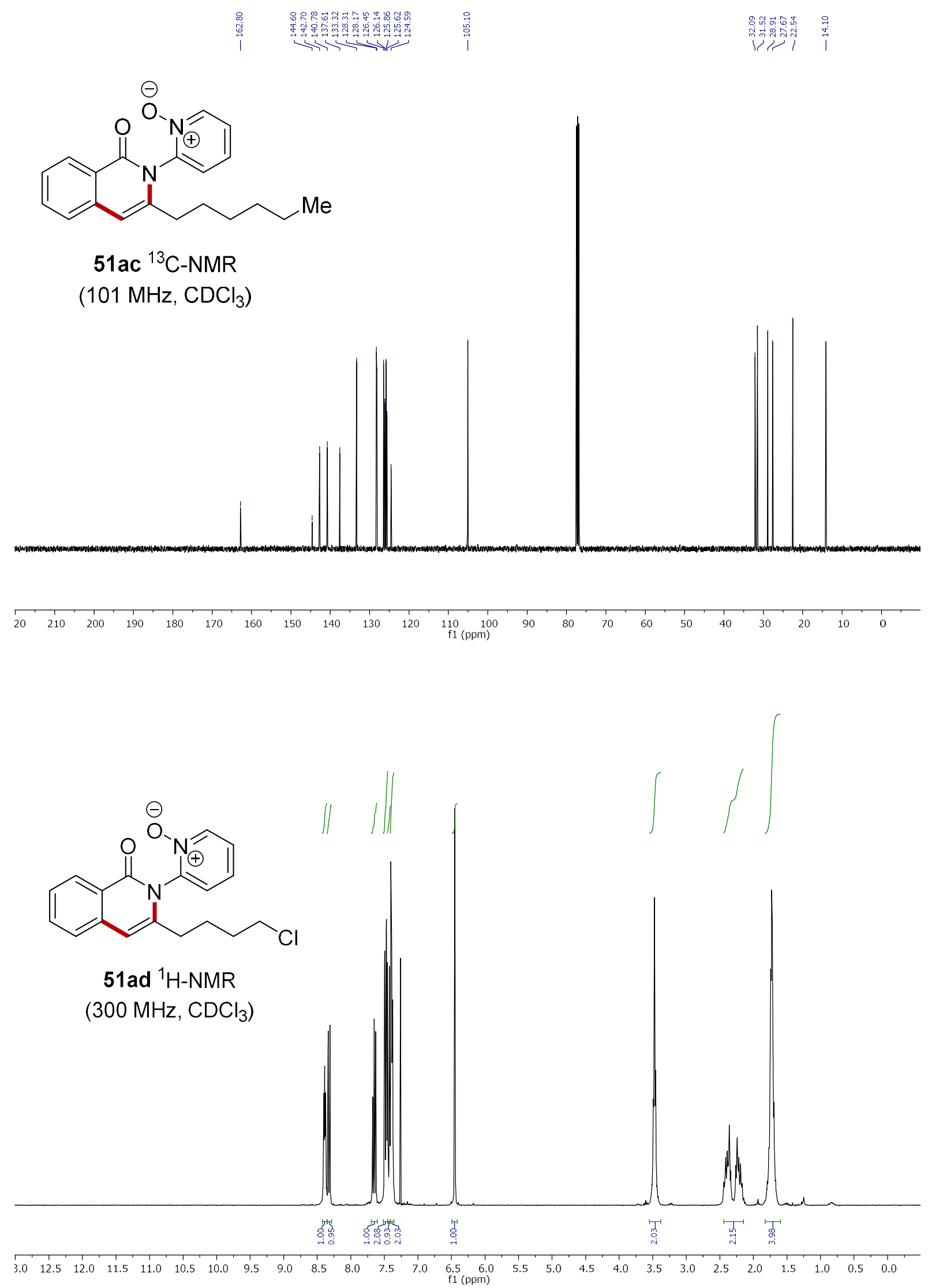

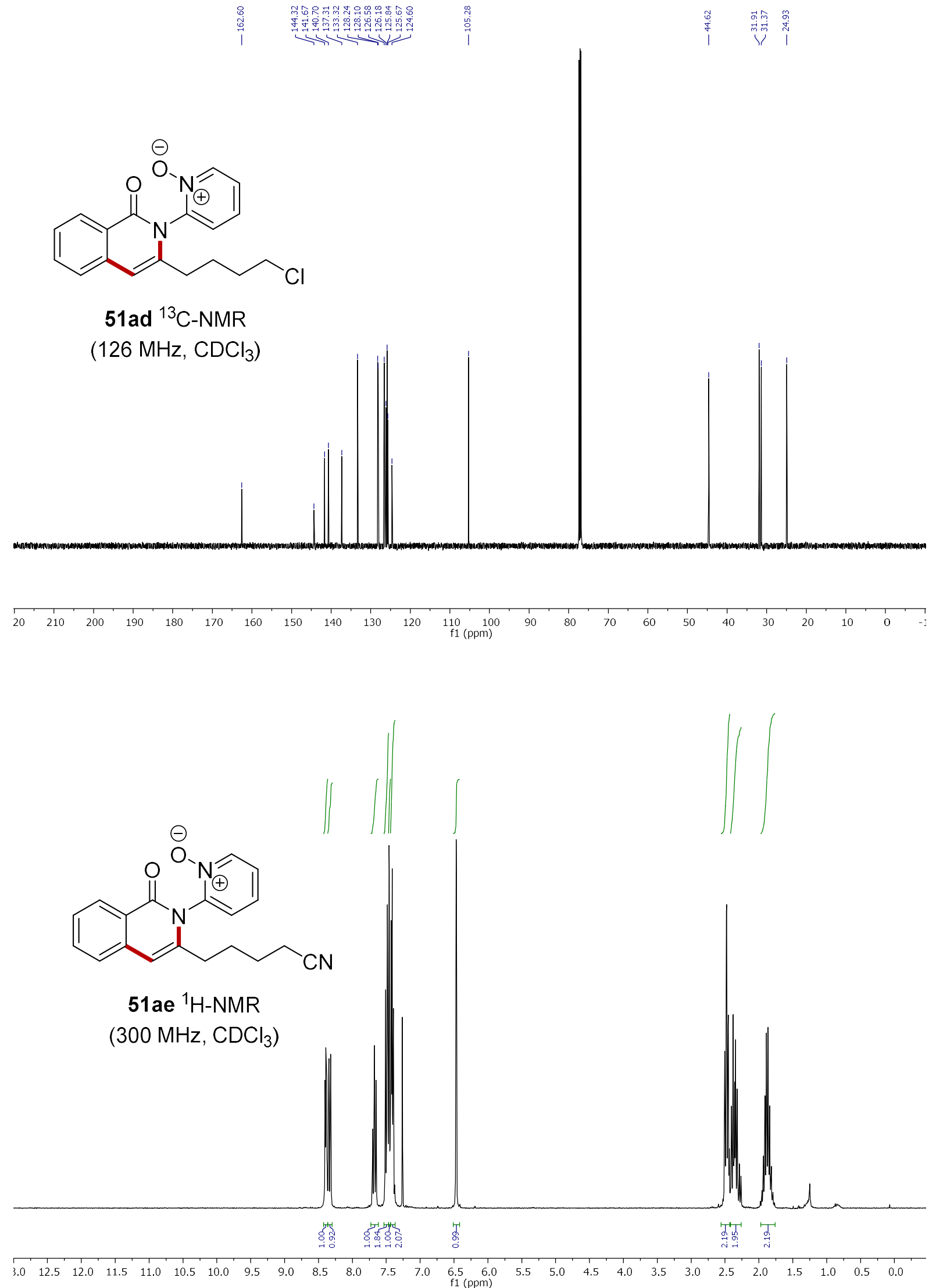

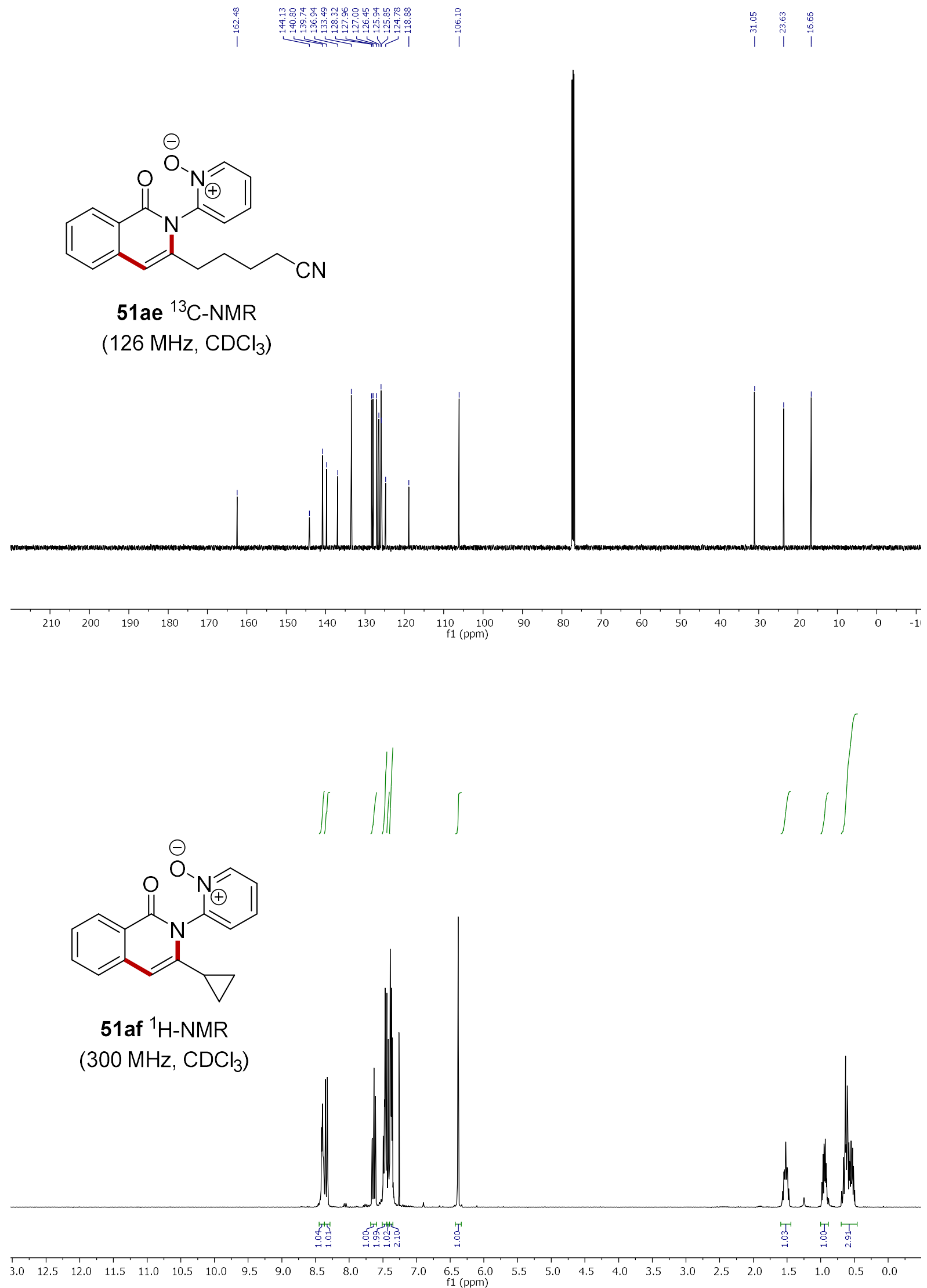


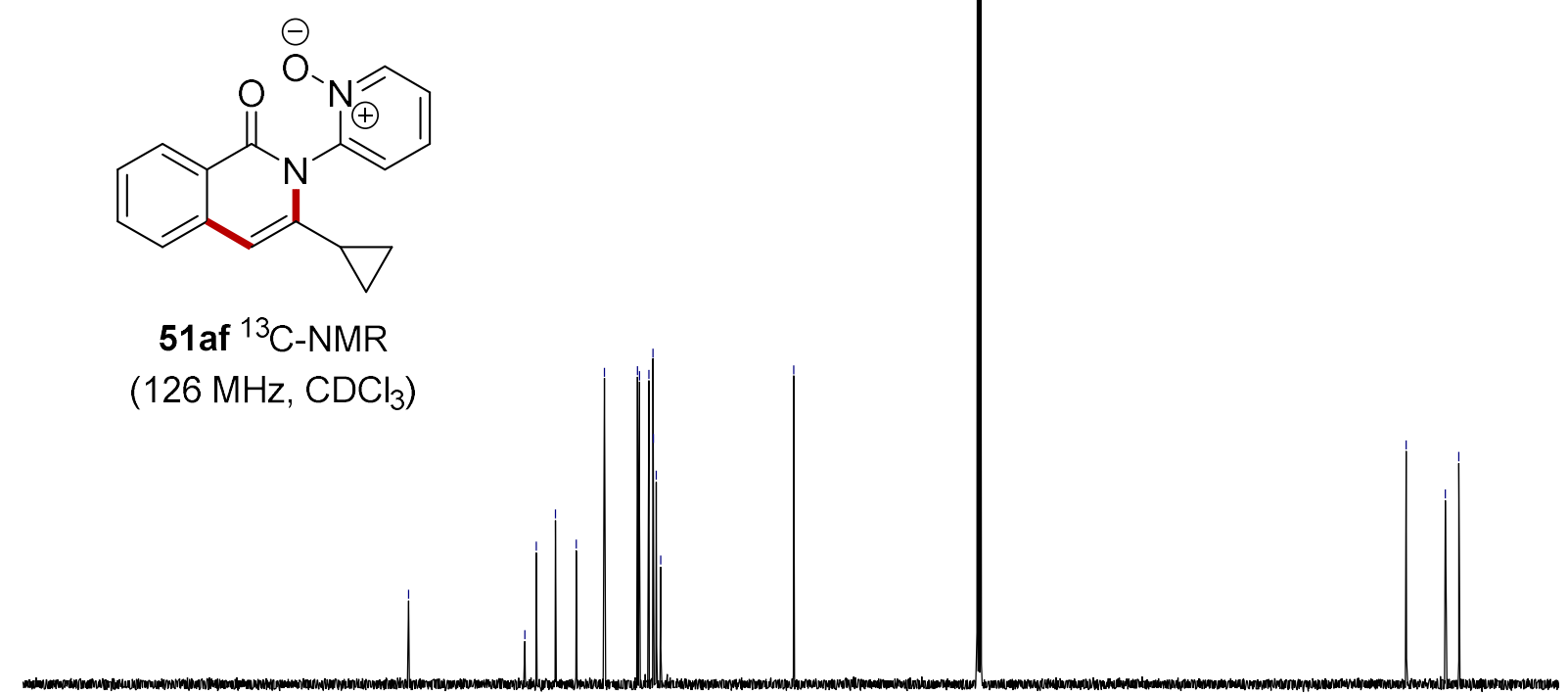

51af ${ }^{13} \mathrm{C}-\mathrm{NMR}$ (126 MHz, $\mathrm{CDCl}_{3}$ )
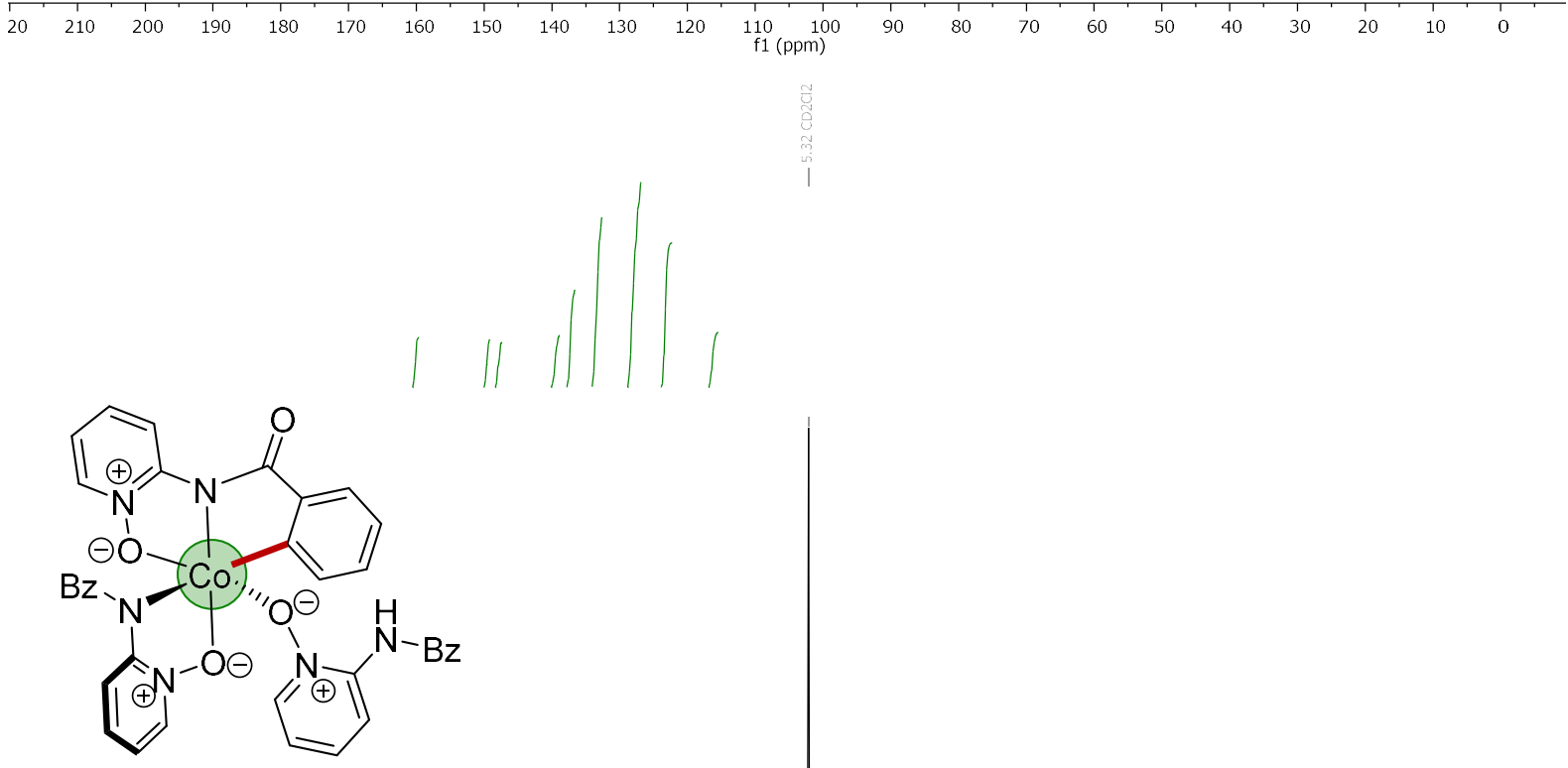

190, ${ }^{1} \mathrm{H}-\mathrm{NMR}$ (400 MHz, $\mathrm{CD}_{2} \mathrm{Cl}_{2}$ )

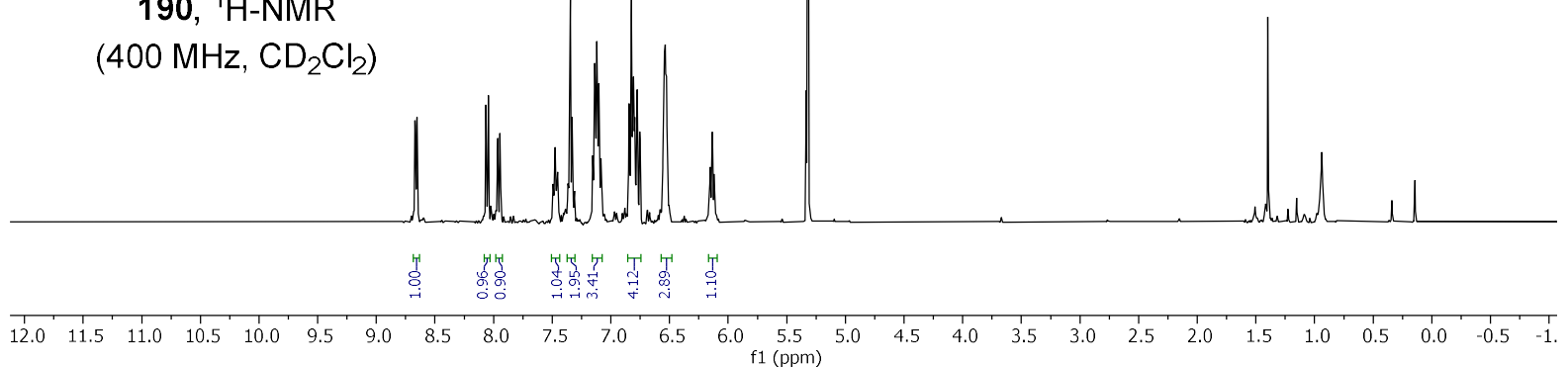




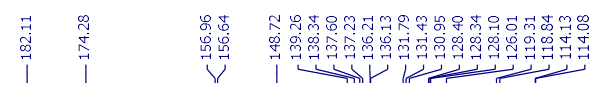

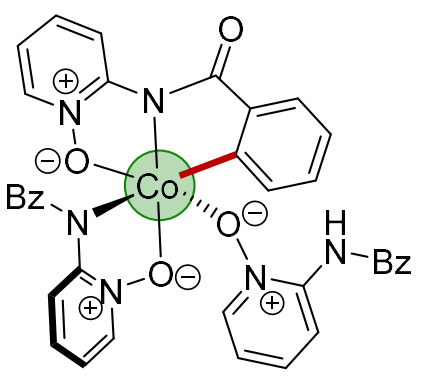

190, ${ }^{13} \mathrm{C}-\mathrm{NMR}$

(101 MHz, $\mathrm{CD}_{2} \mathrm{Cl}_{2}$ )
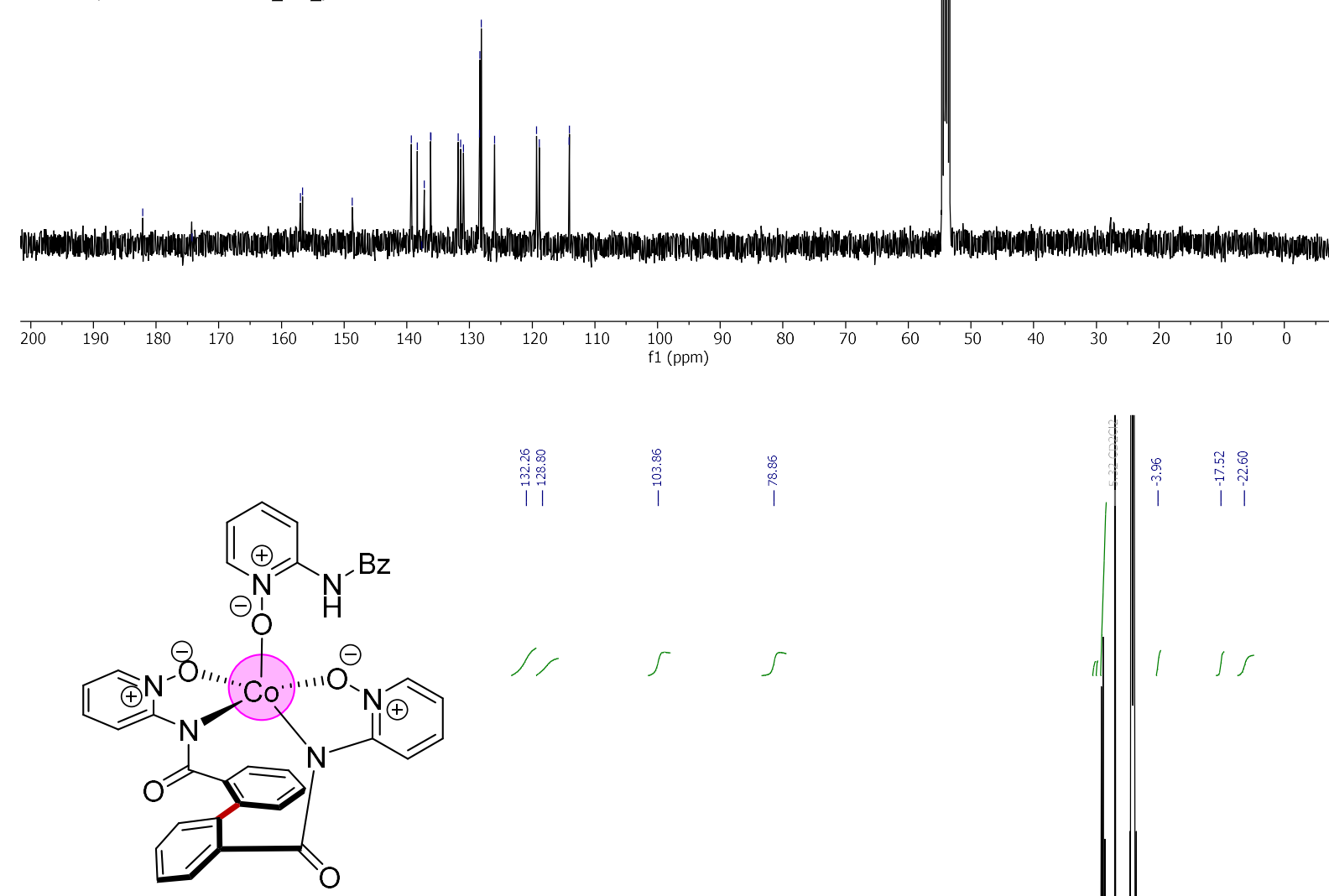

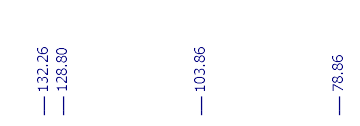

$203{ }^{1} \mathrm{H}-\mathrm{NMR}$

(300 $\mathrm{MHz}, \mathrm{CD}_{2} \mathrm{Cl}_{2}$ )

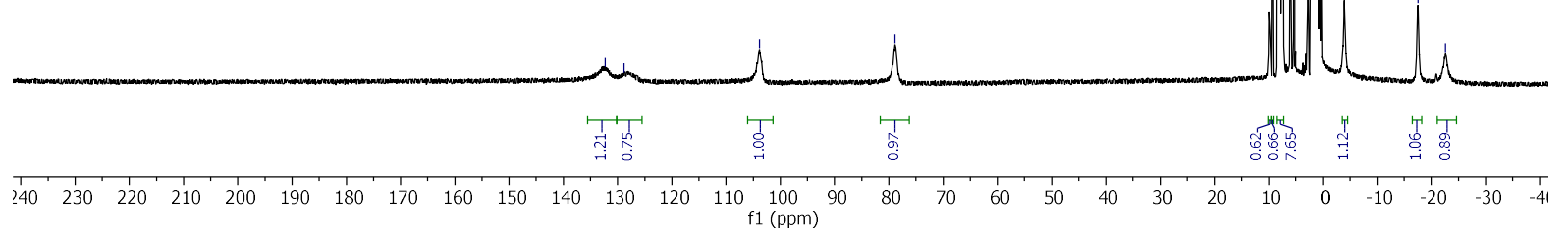



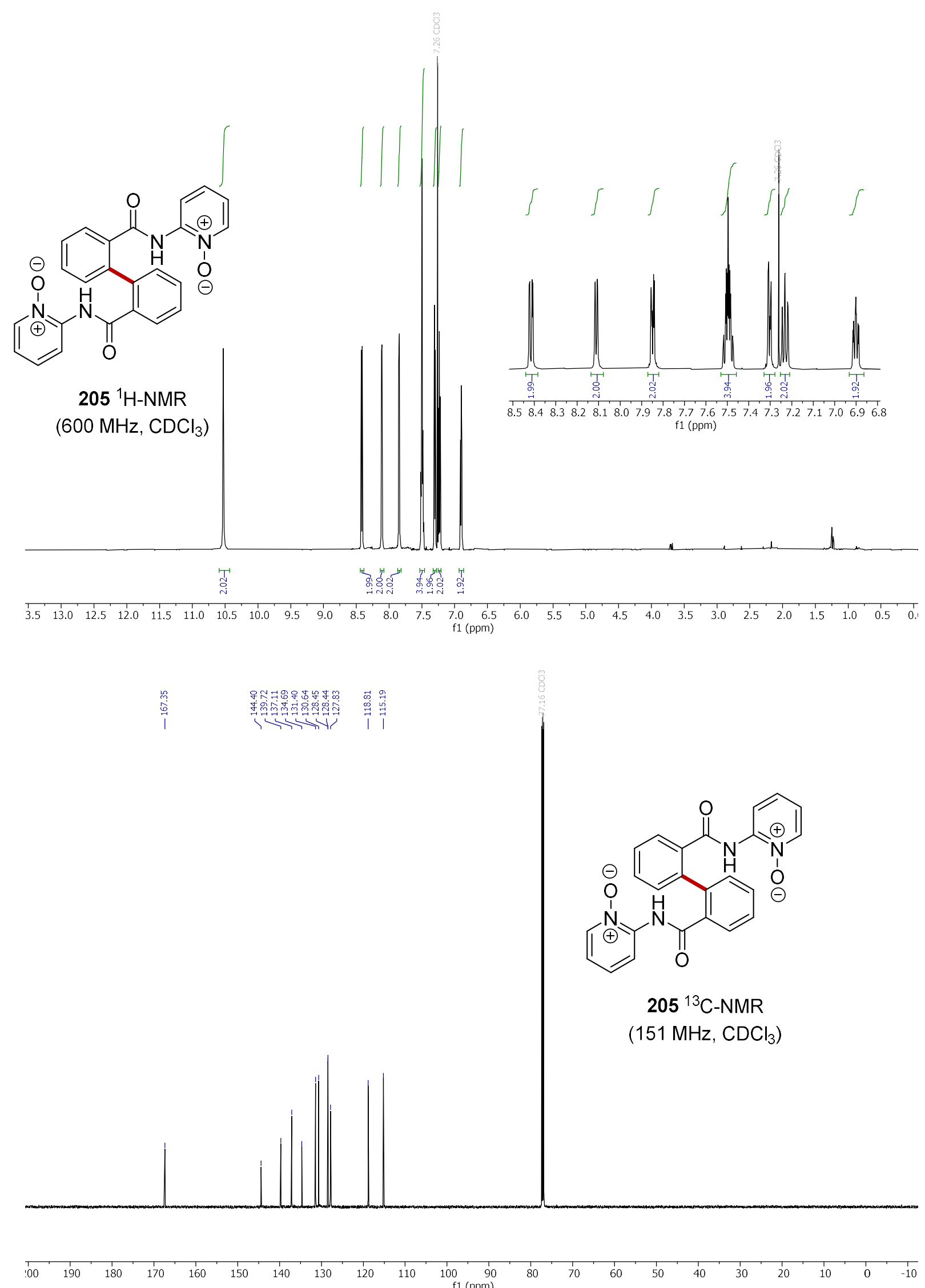

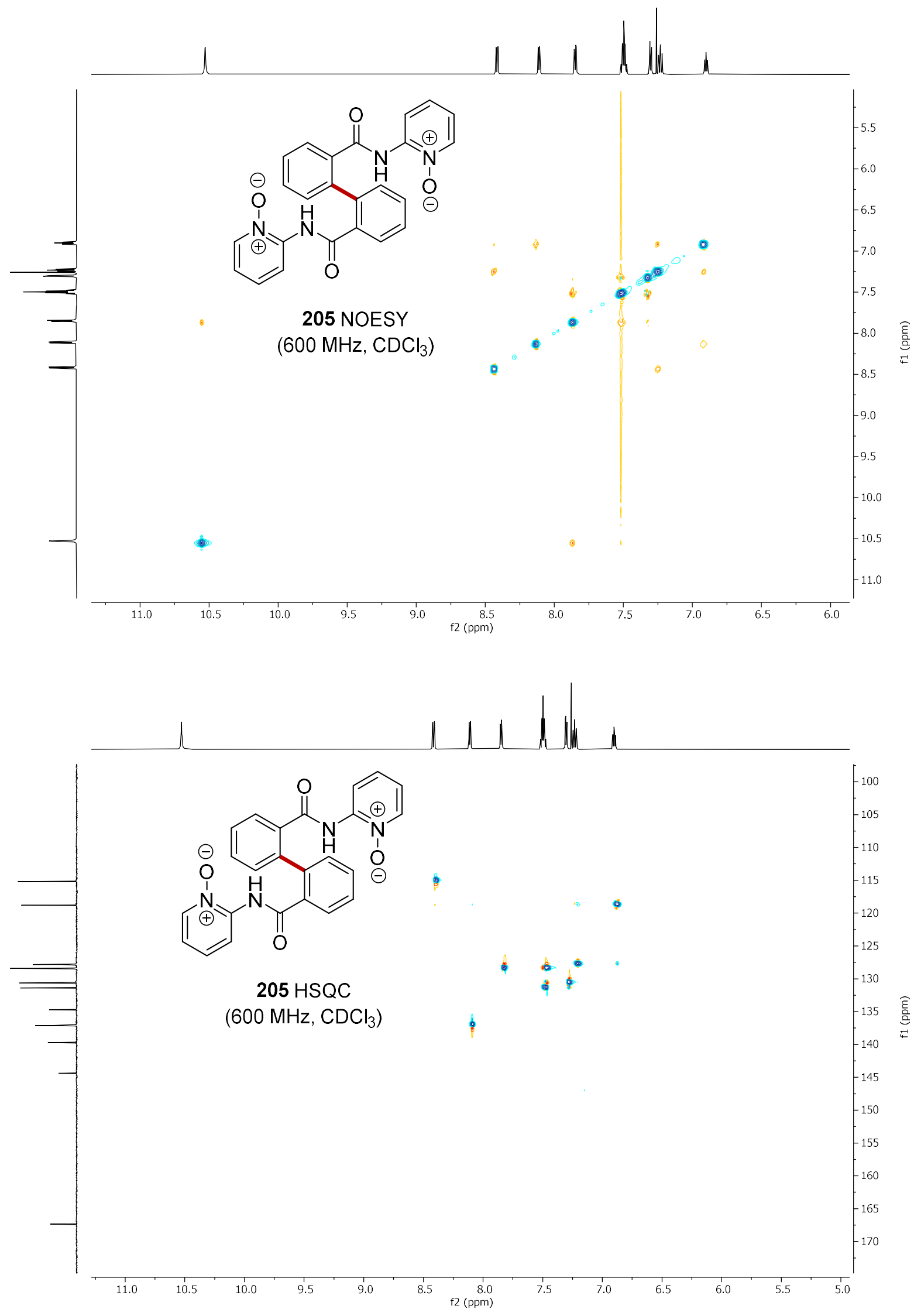
406

Appendix
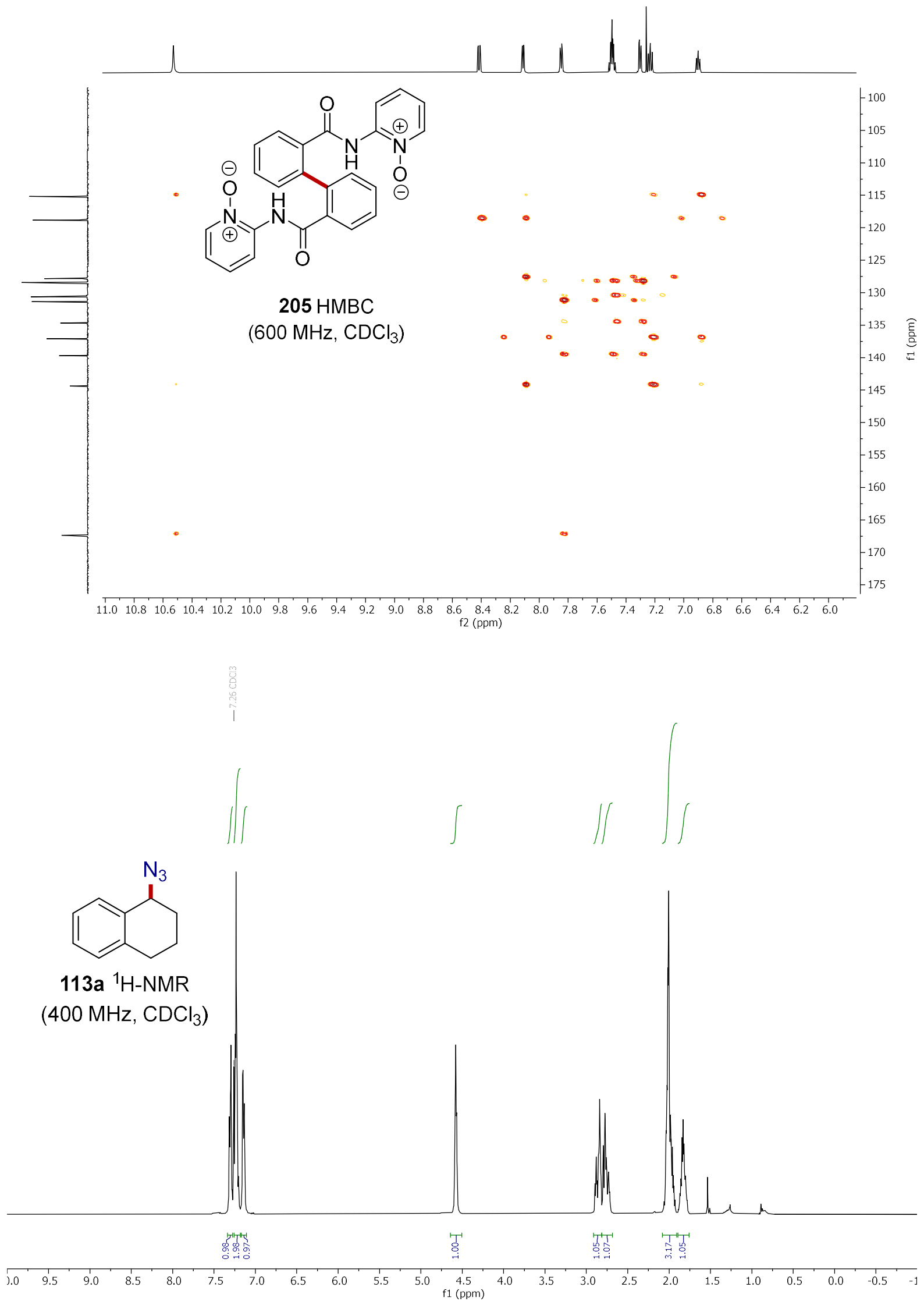

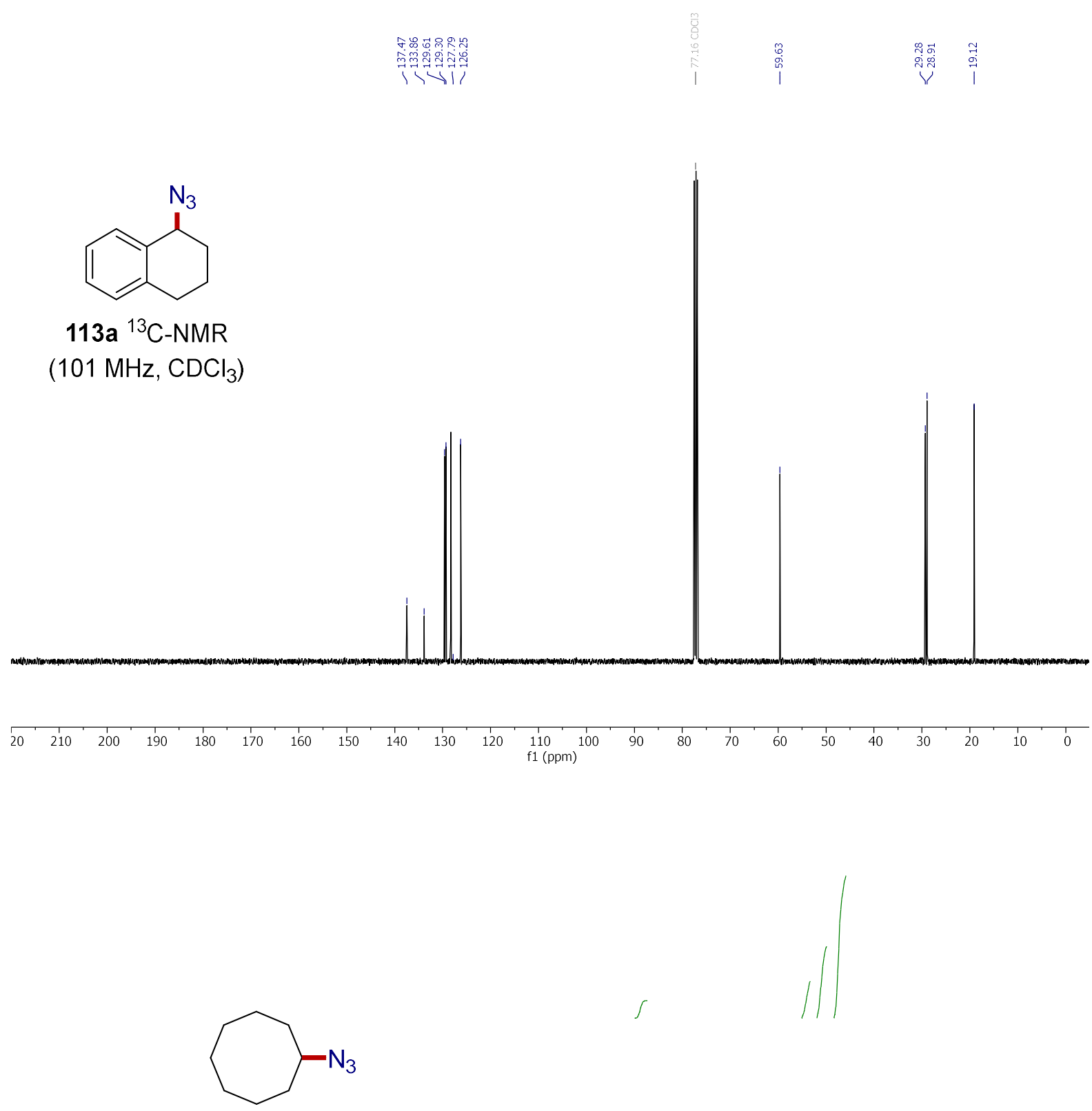

113b ${ }^{1} \mathrm{H}-\mathrm{NMR}$

(400 MHz, $\mathrm{CDCl}_{3}$ )

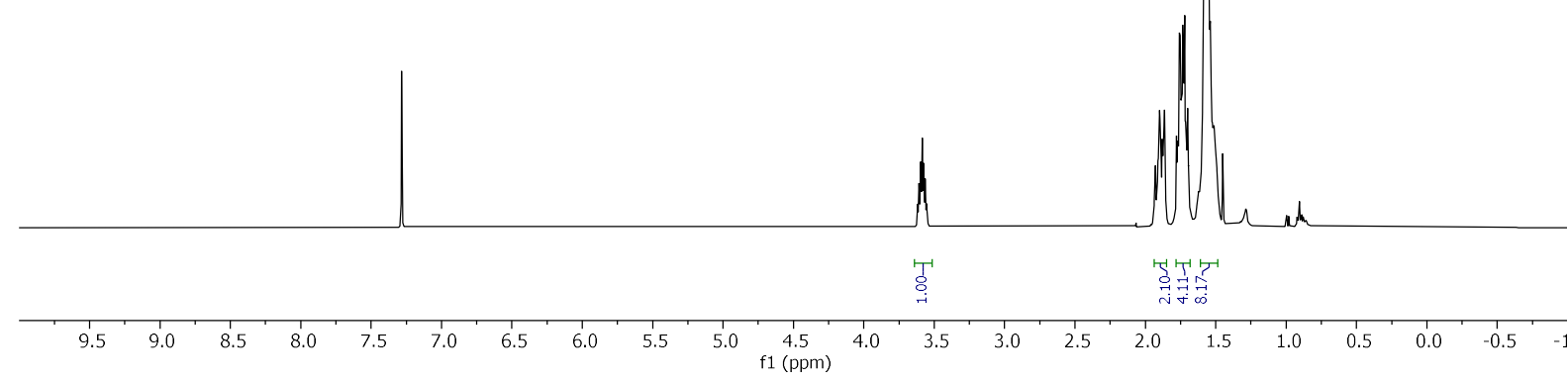



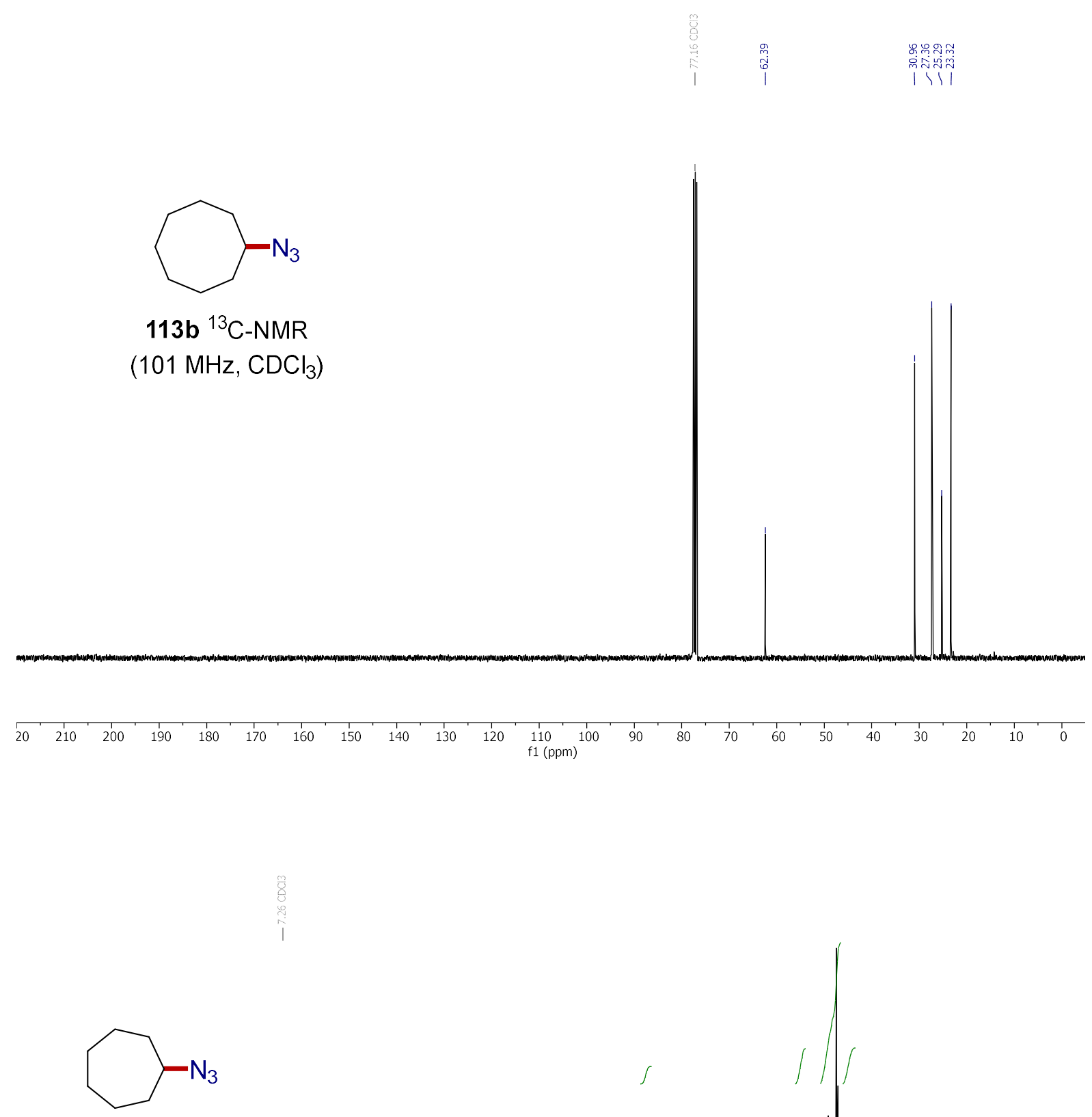

$113 \mathrm{~g}{ }^{1} \mathrm{H}-\mathrm{NMR}$

$\left(400 \mathrm{MHz}, \mathrm{CDCl}_{3}\right)$
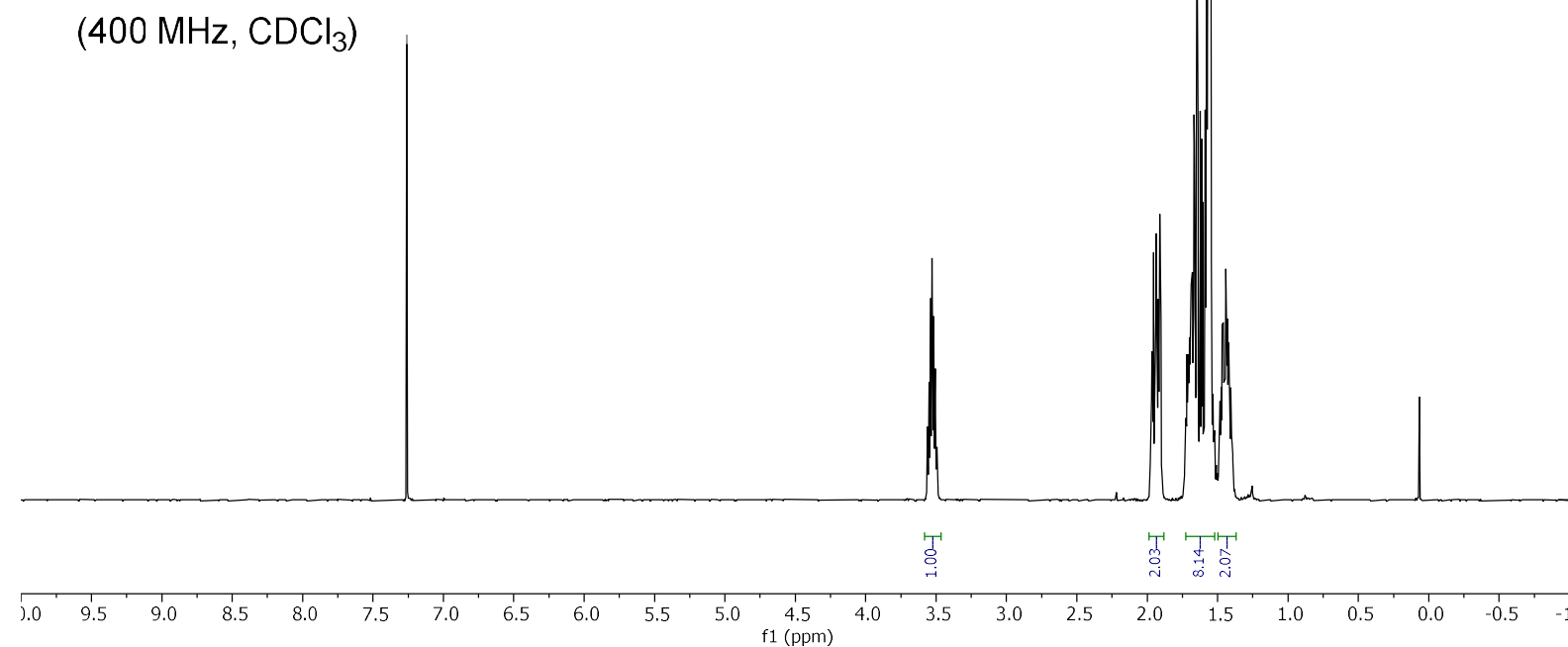

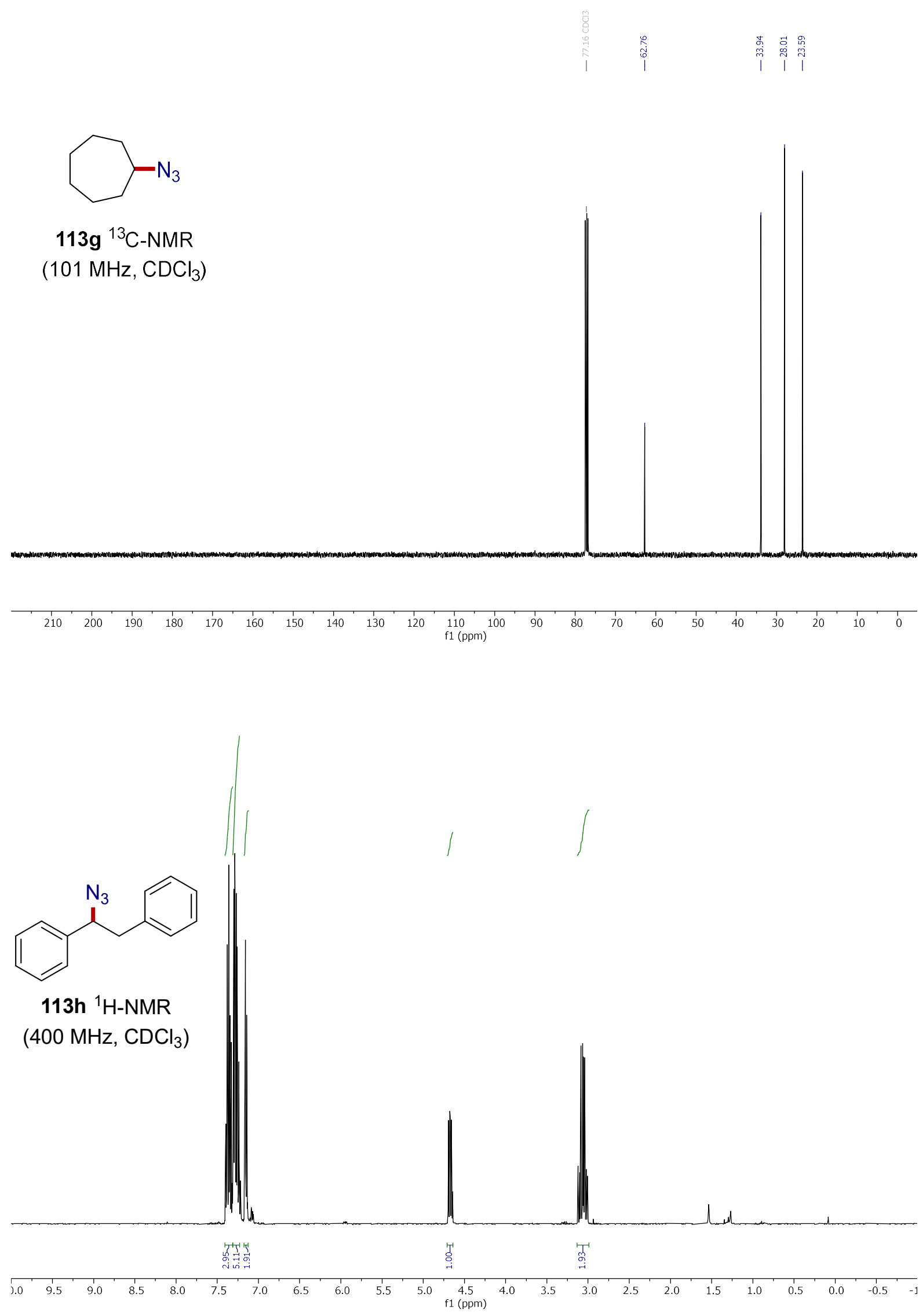

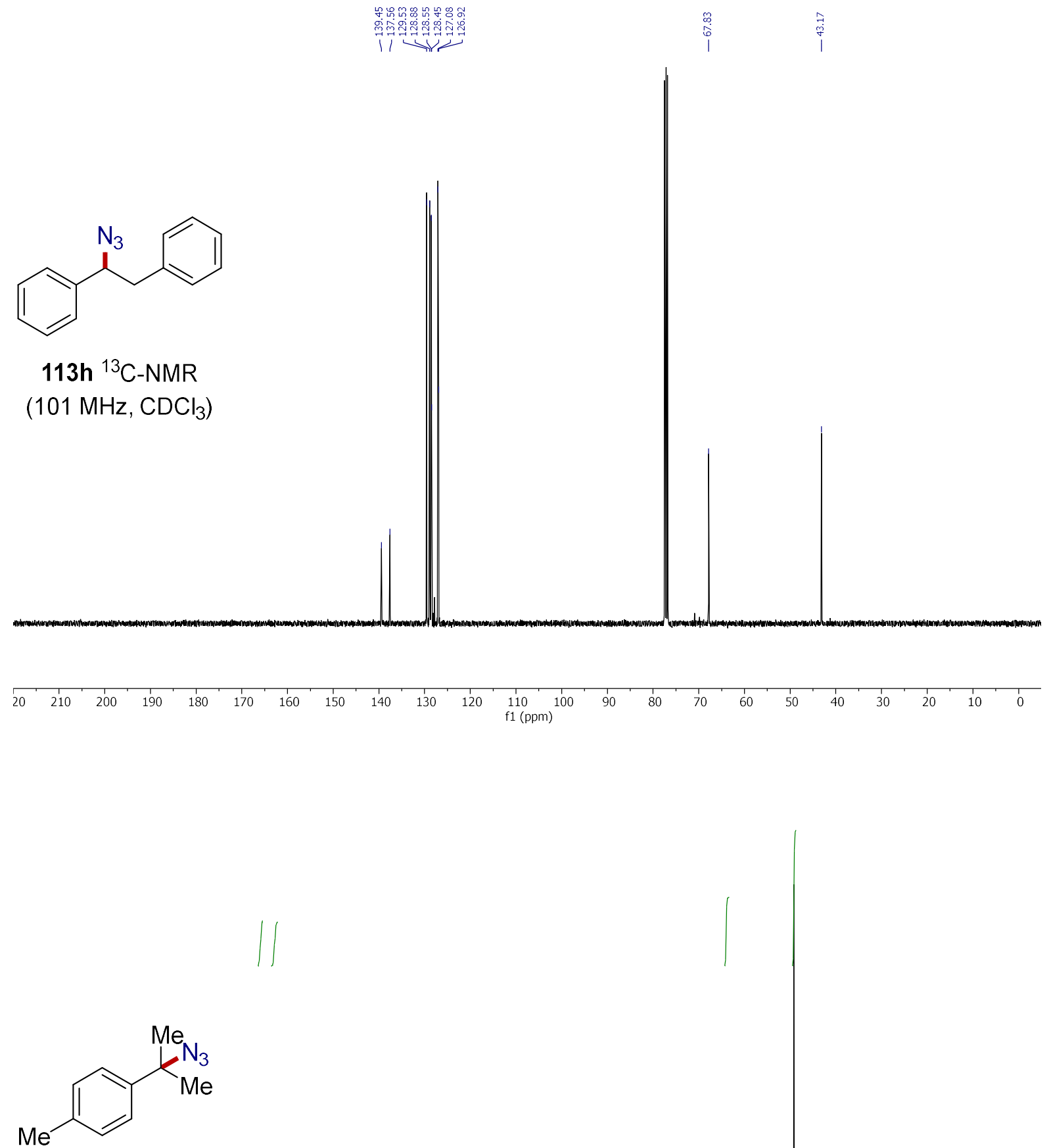

$113 \mathrm{i}^{1} \mathrm{H}-\mathrm{NMR}$

$\left(400 \mathrm{MHz}, \mathrm{CDCl}_{3}\right)$

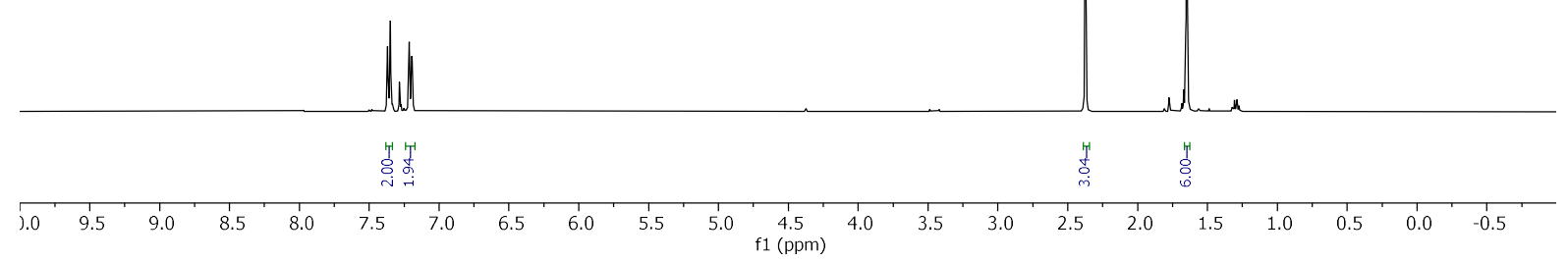



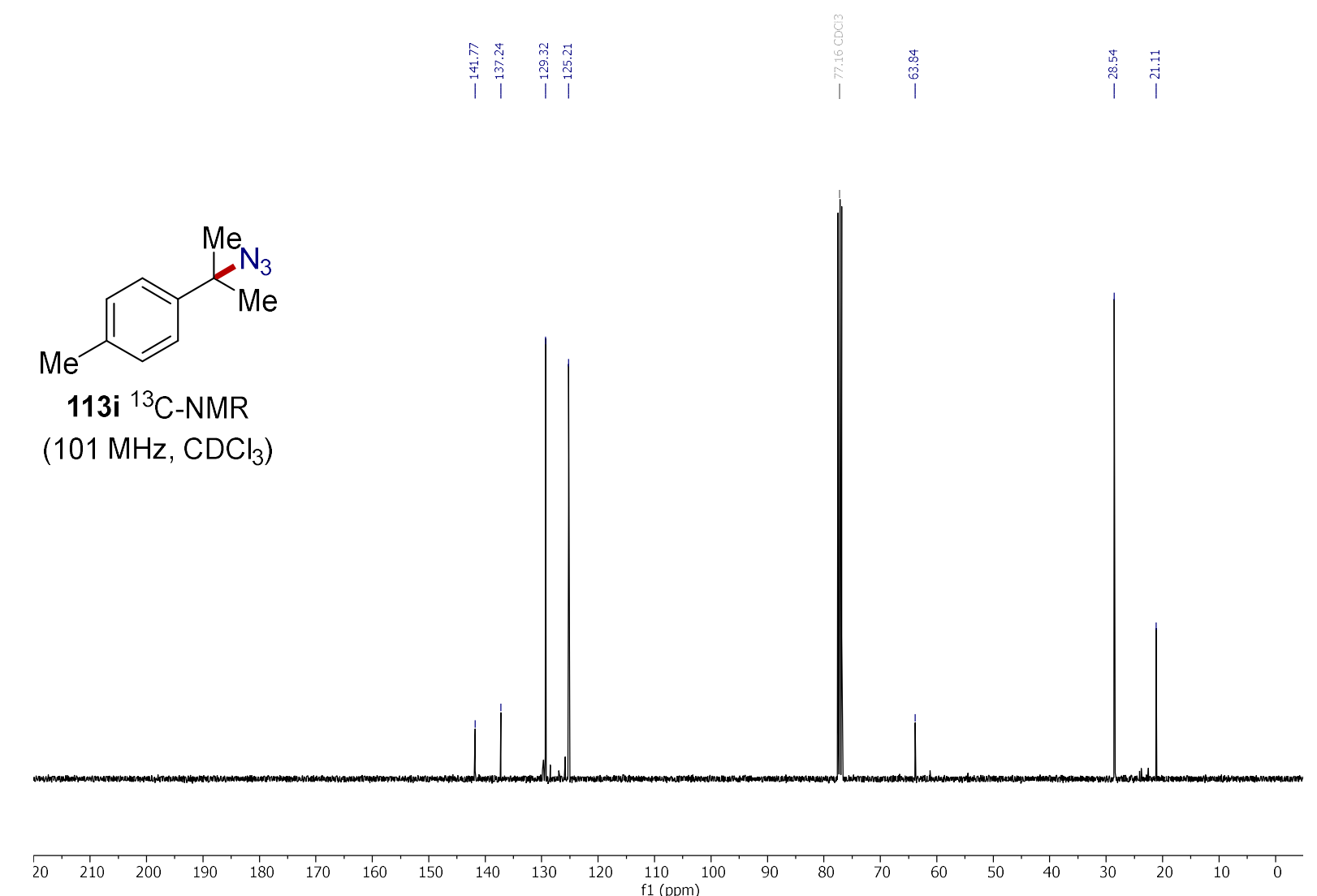<smiles>CC(C)C(N)c1ccccc1</smiles>

113j ${ }^{1} \mathrm{H}-\mathrm{NMR}$ (400 MHz, $\mathrm{CDCl}_{3}$ )

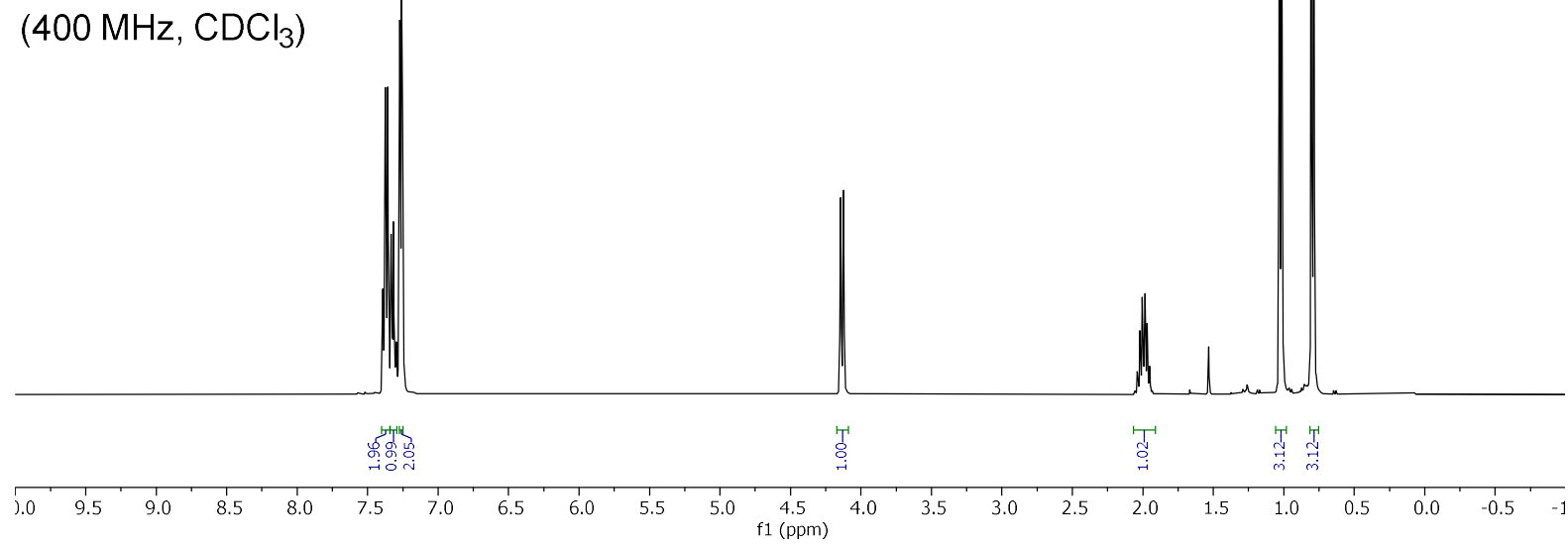




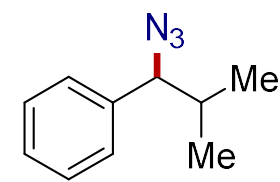

113j ${ }^{13} \mathrm{C}-\mathrm{NMR}$

$\left(101 \mathrm{MHz}, \mathrm{CDCl}_{3}\right.$ )
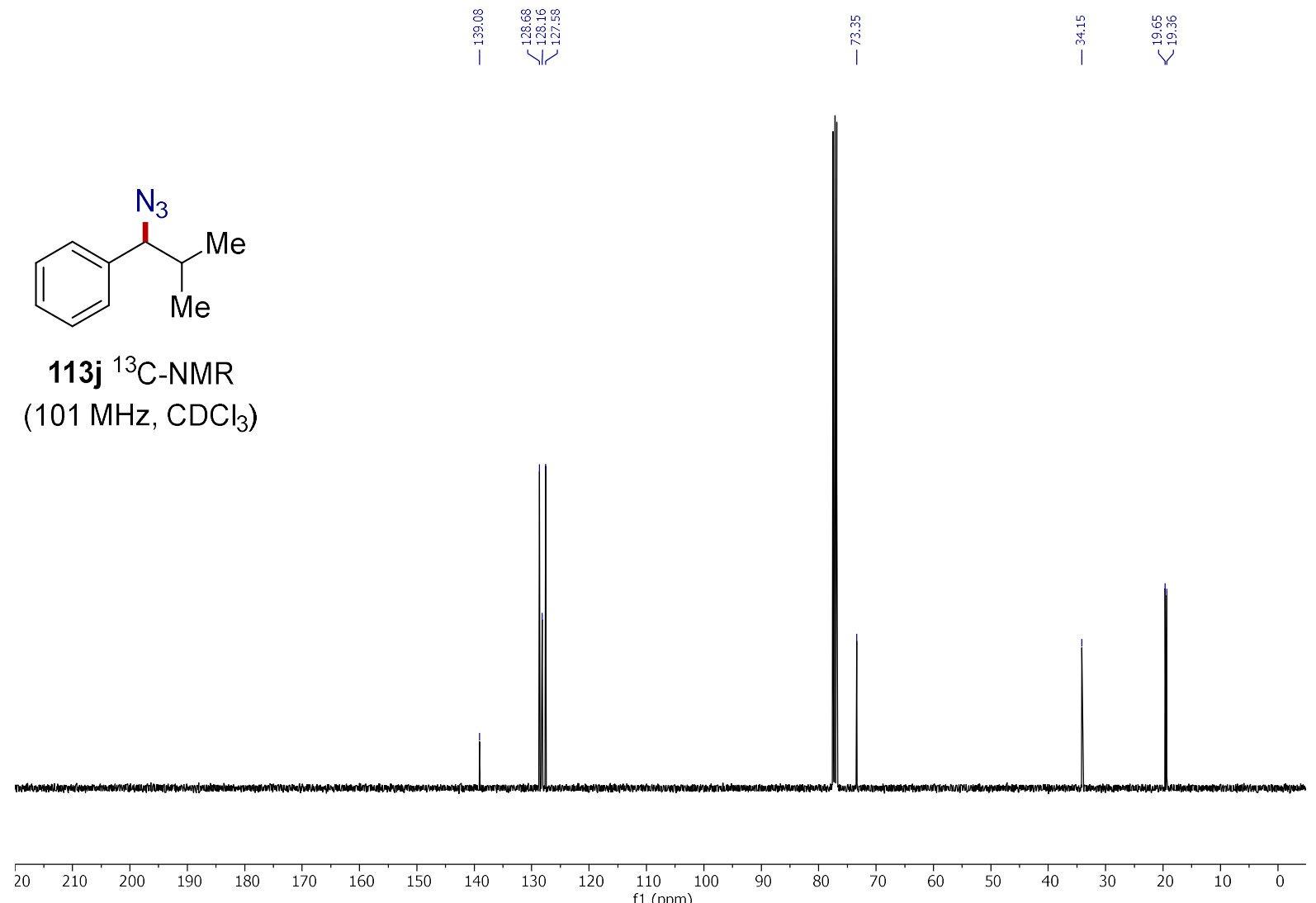<smiles>CCCCCC(N)c1ccc(-c2ccc(C(=O)OC)cc2)cc1</smiles>

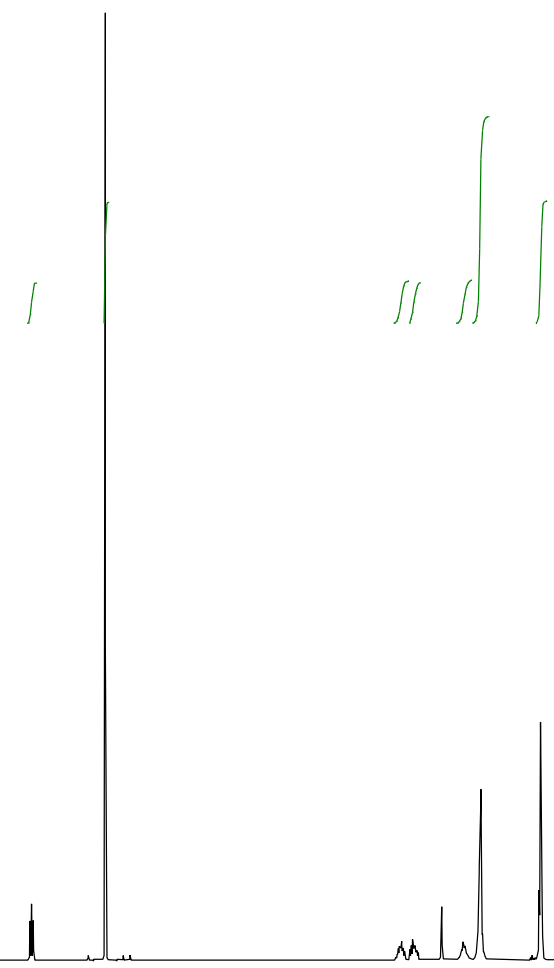

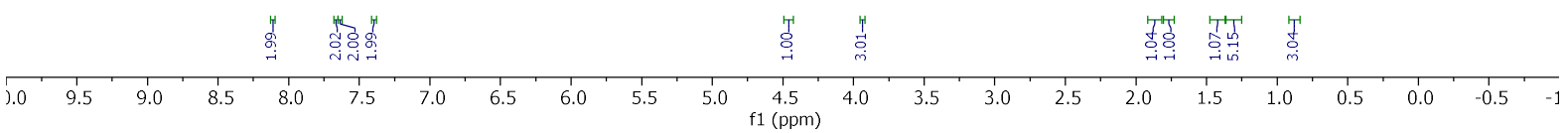



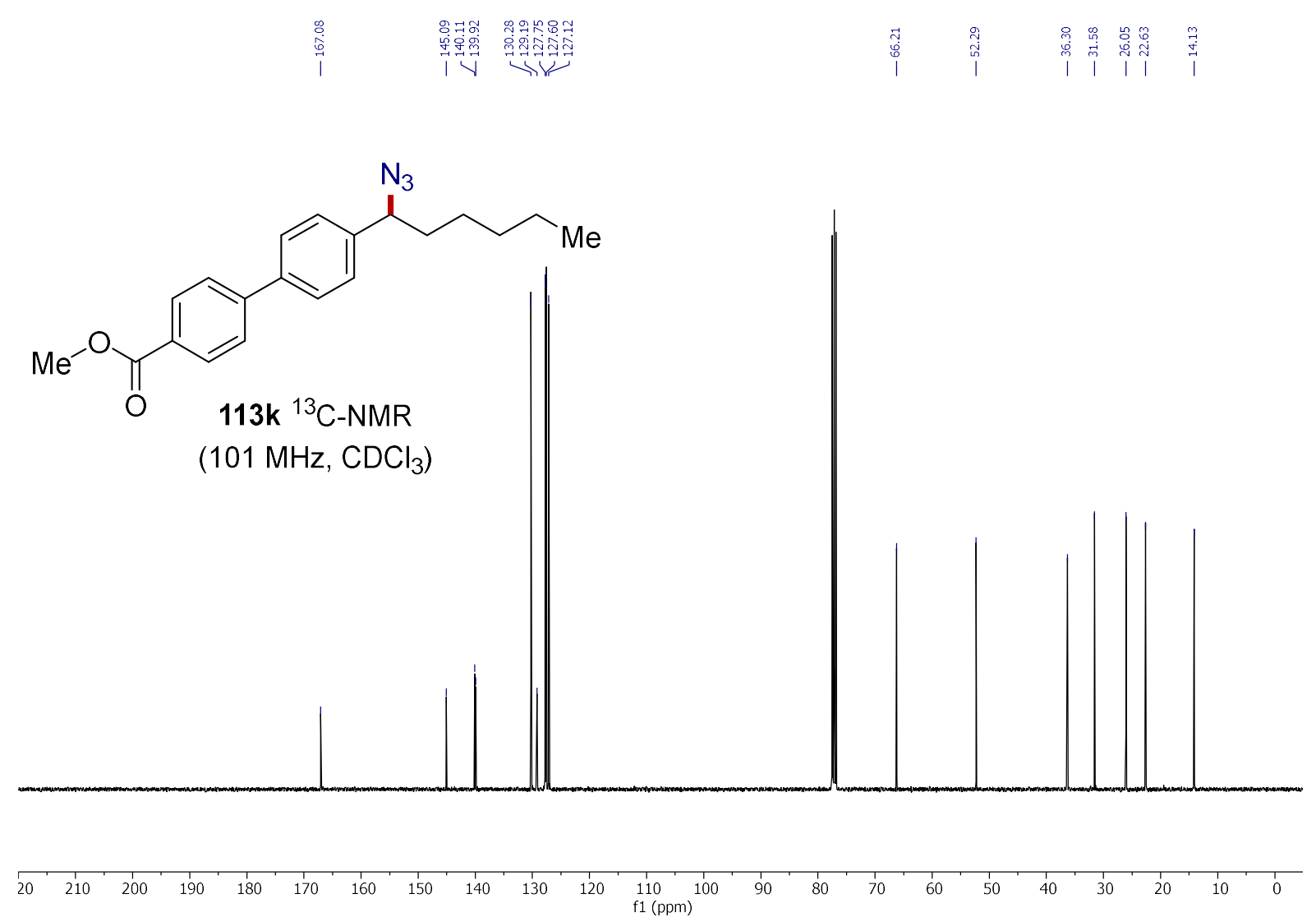<smiles>CC(=O)O[C@H]1CC(C)CC[C@@H]1C(C)(C)N</smiles>

113I ${ }^{1} \mathrm{H}-\mathrm{NMR}$

(600 MHz, $\mathrm{CDCl}_{3}$ )

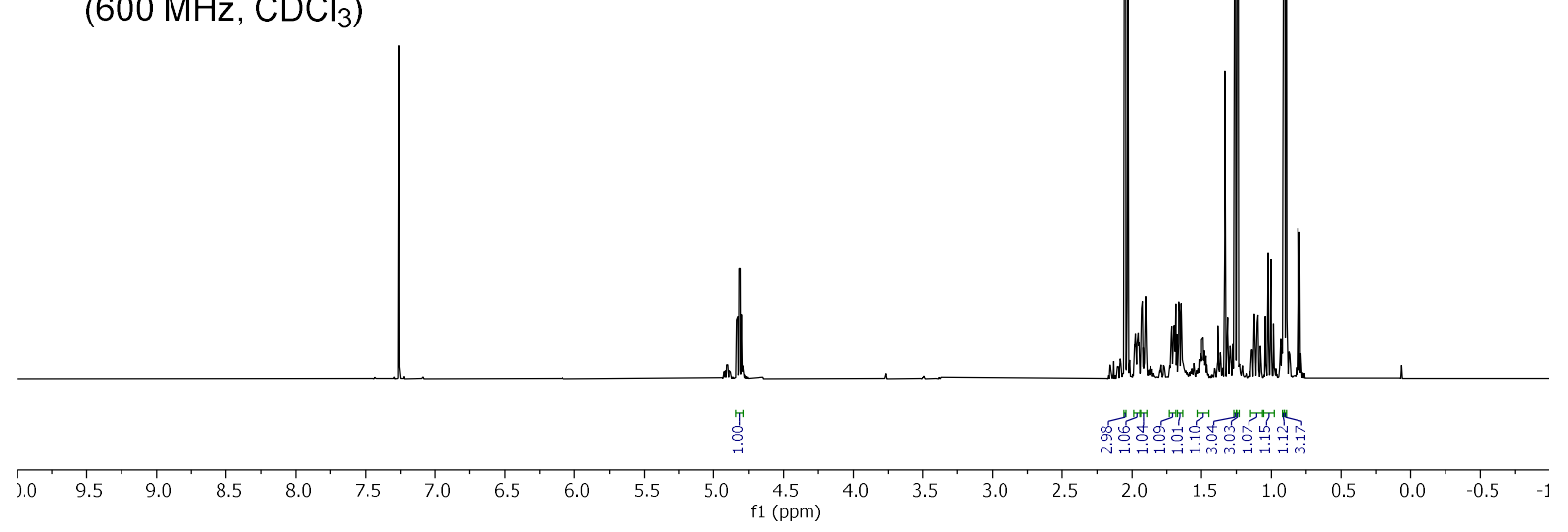



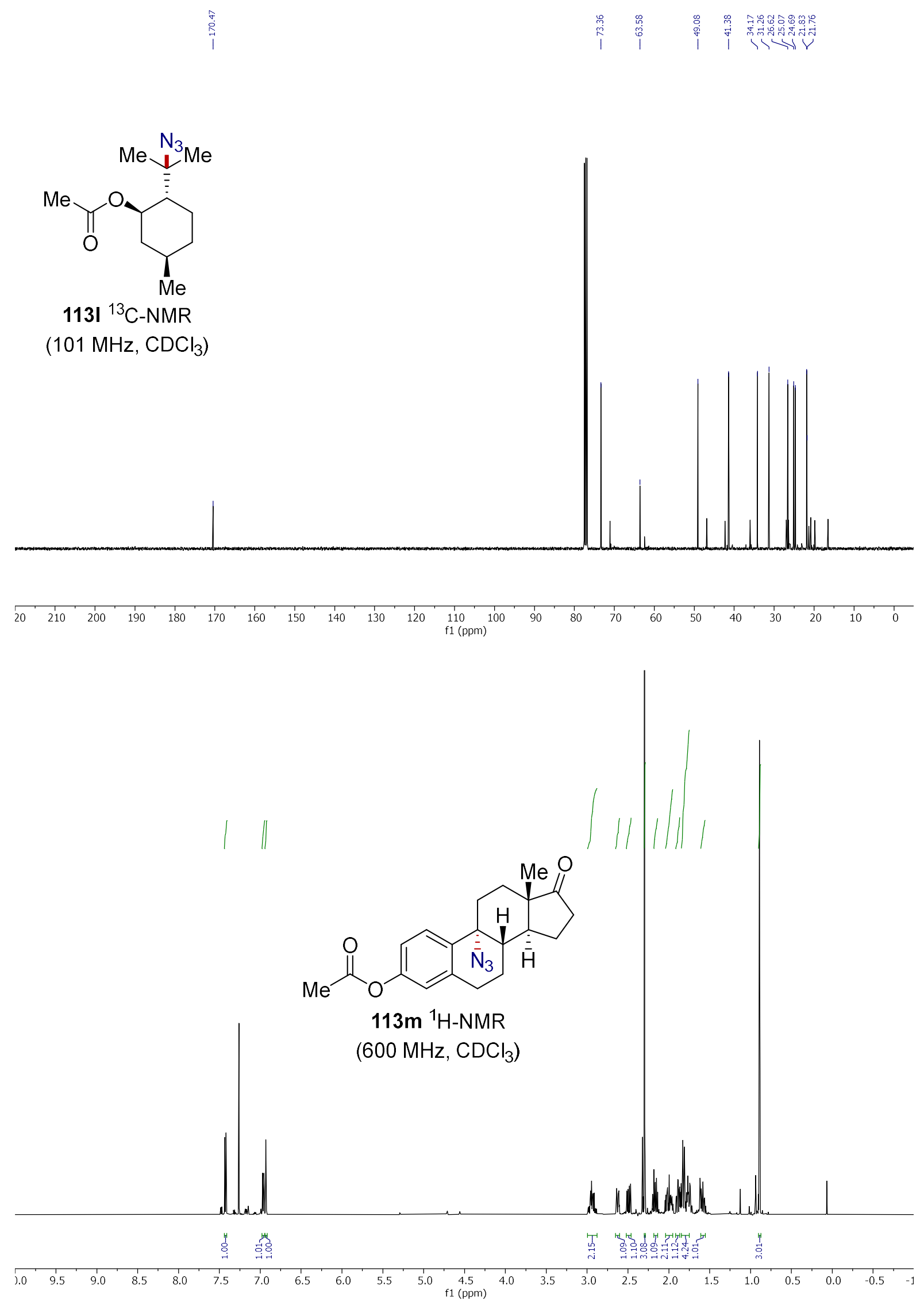


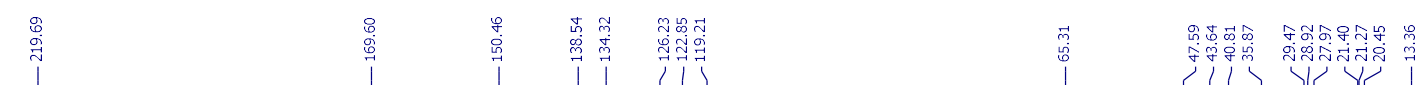<smiles>CC(=O)Oc1ccc2c(c1)CC[C@@H]1C2CC[C@]2(C)C(=O)CC[C@H]12</smiles>

$113 \mathrm{~m}{ }^{13} \mathrm{C}-\mathrm{NMR}$

$\left(101 \mathrm{MHz}, \mathrm{CDCl}_{3}\right)$

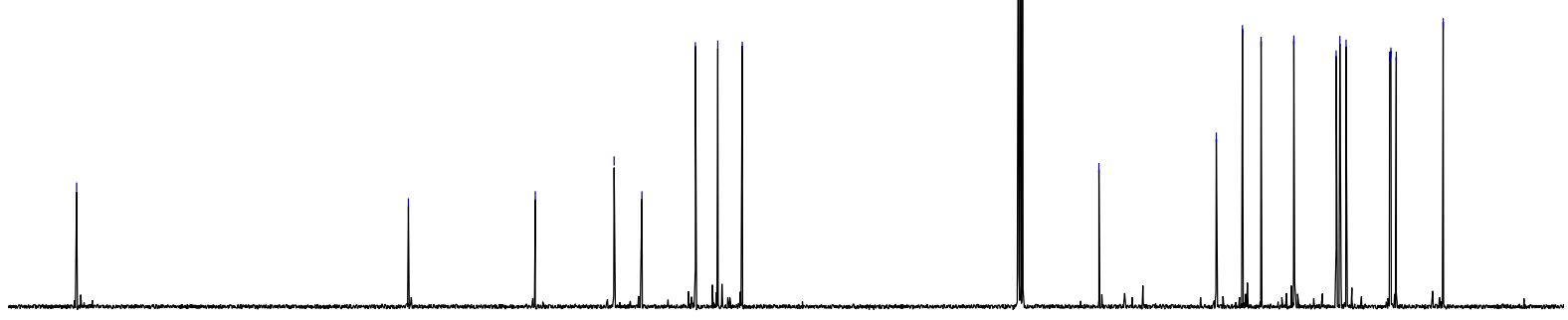

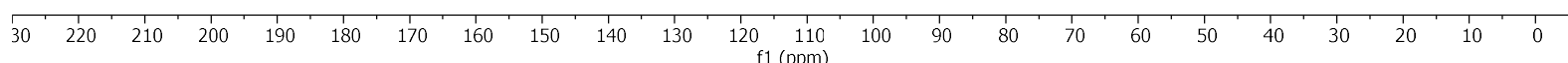

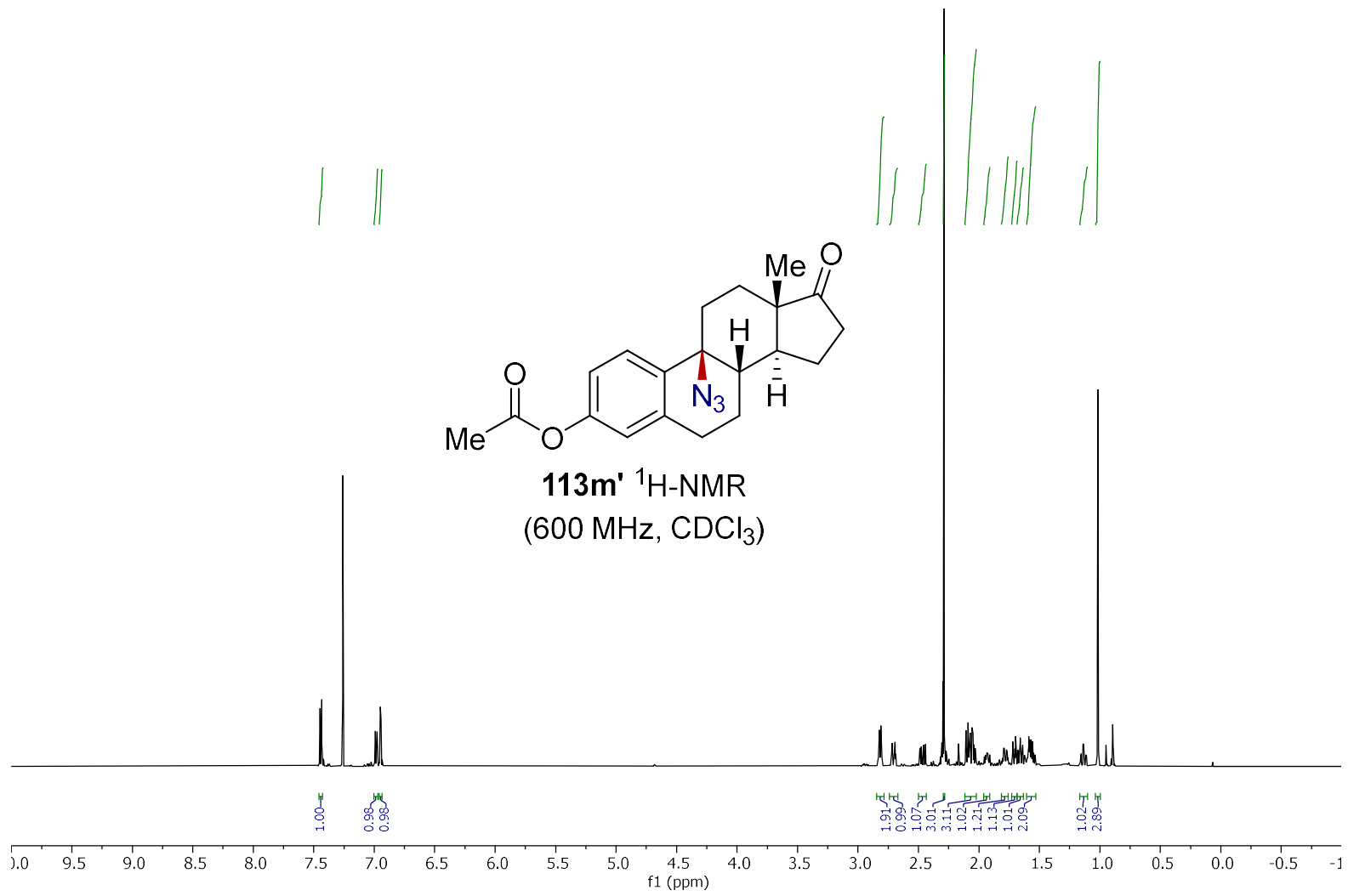




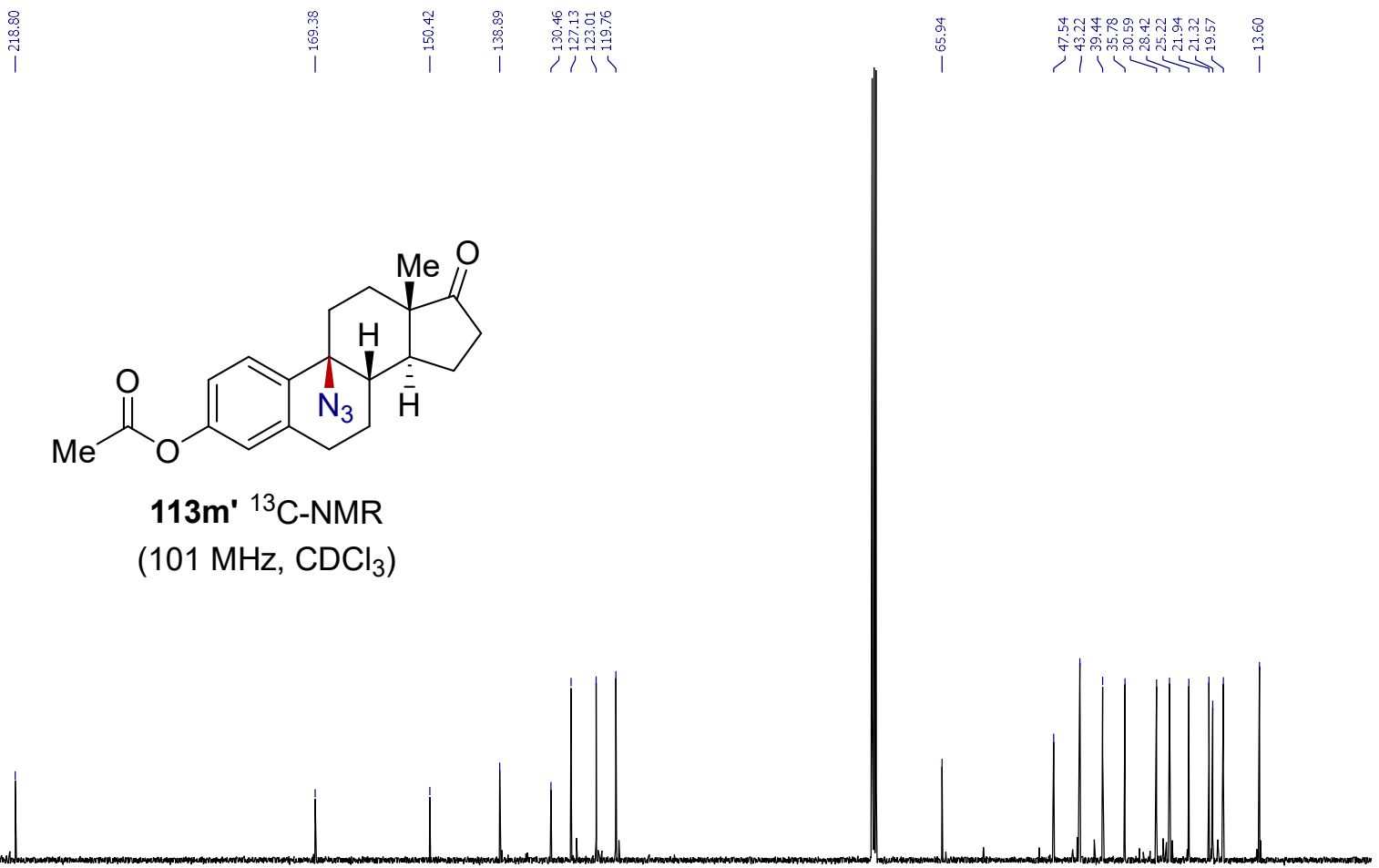

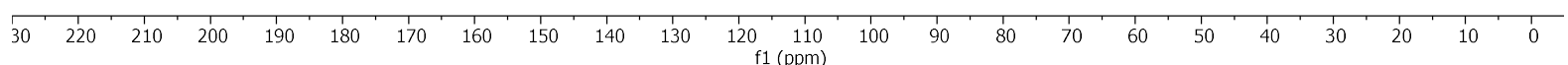




\section{Apparatus}

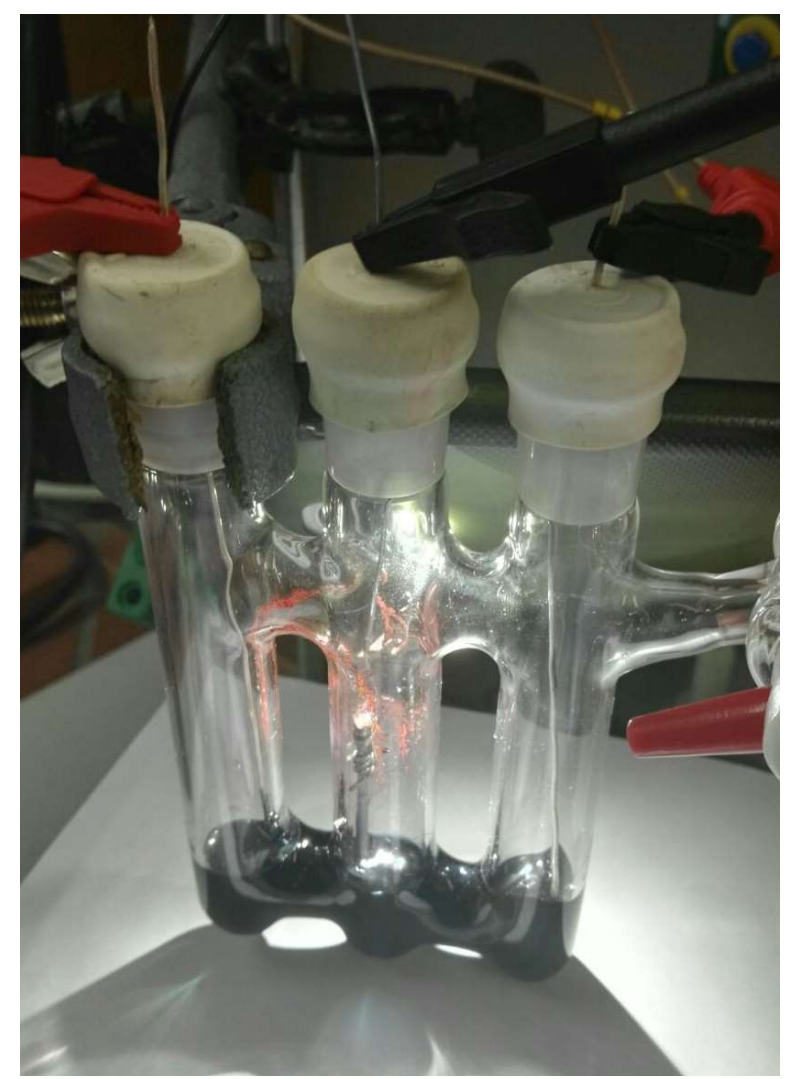

Figure 77. Initial undivided cell with a three-electrode setup.

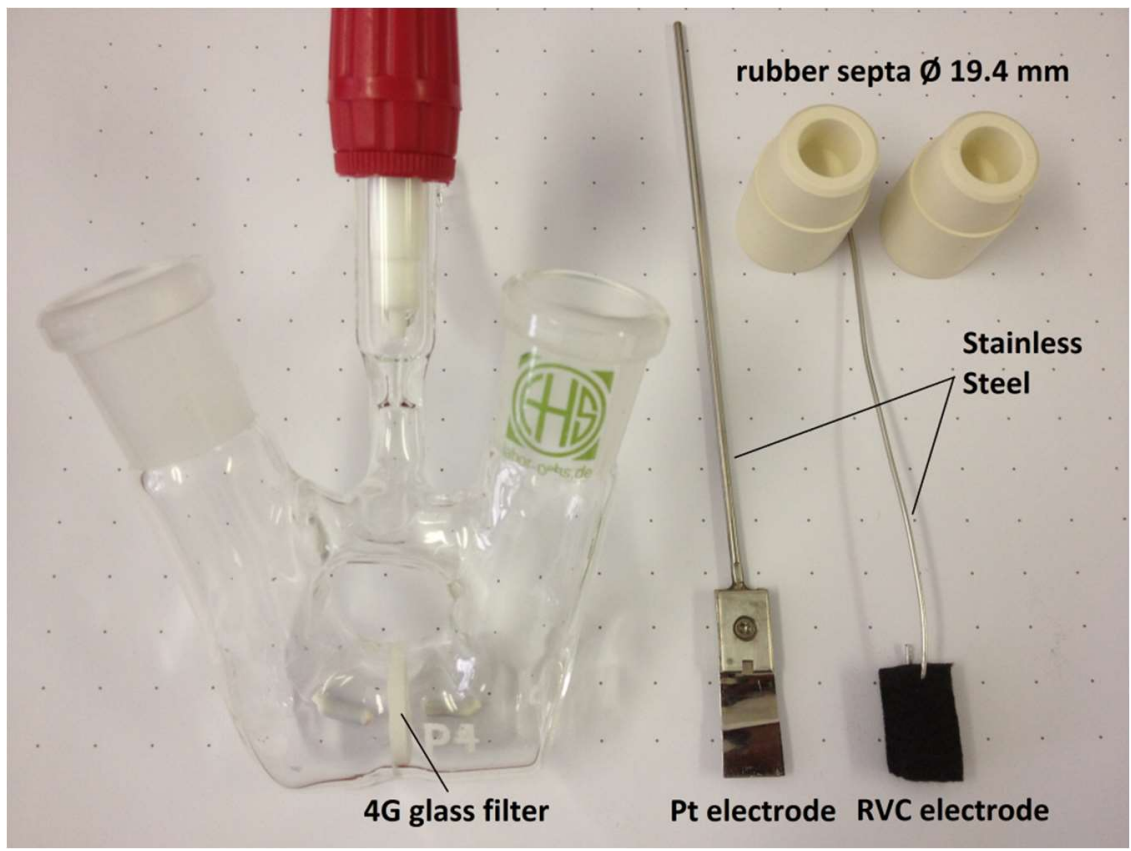

Figure 78. Divided cell for constant current electrolysis. ${ }^{[249]}$ 


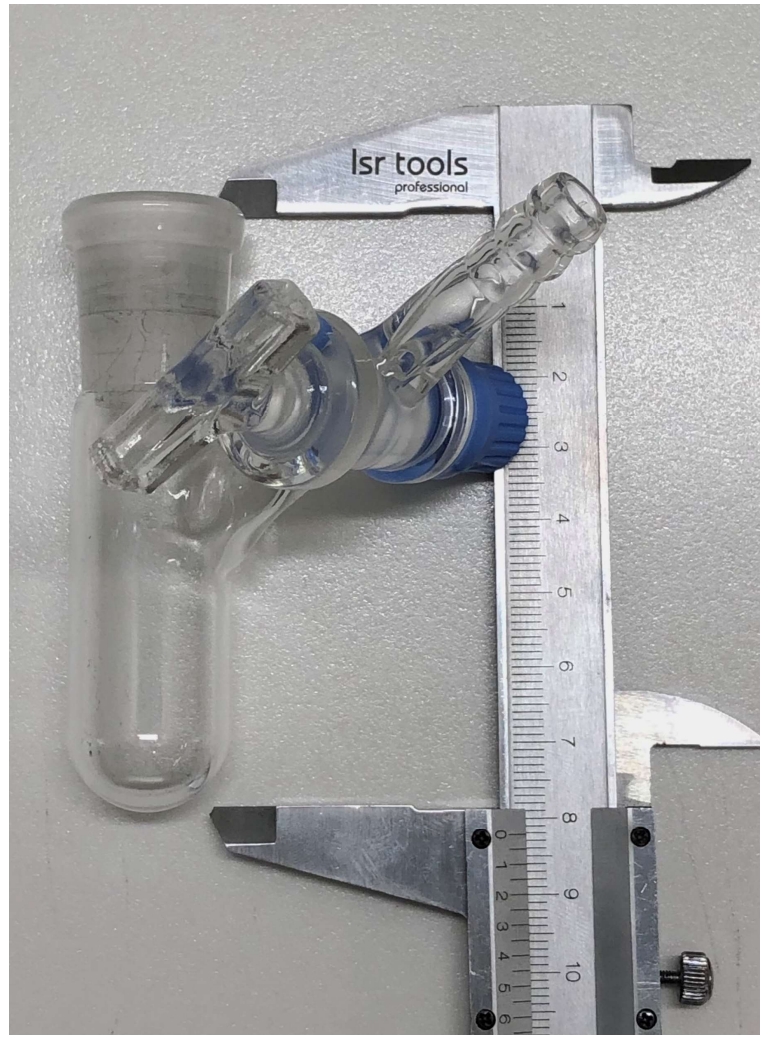

Figure 79. Improved undivided cell for constant current electrolysis. ${ }^{[274]}$
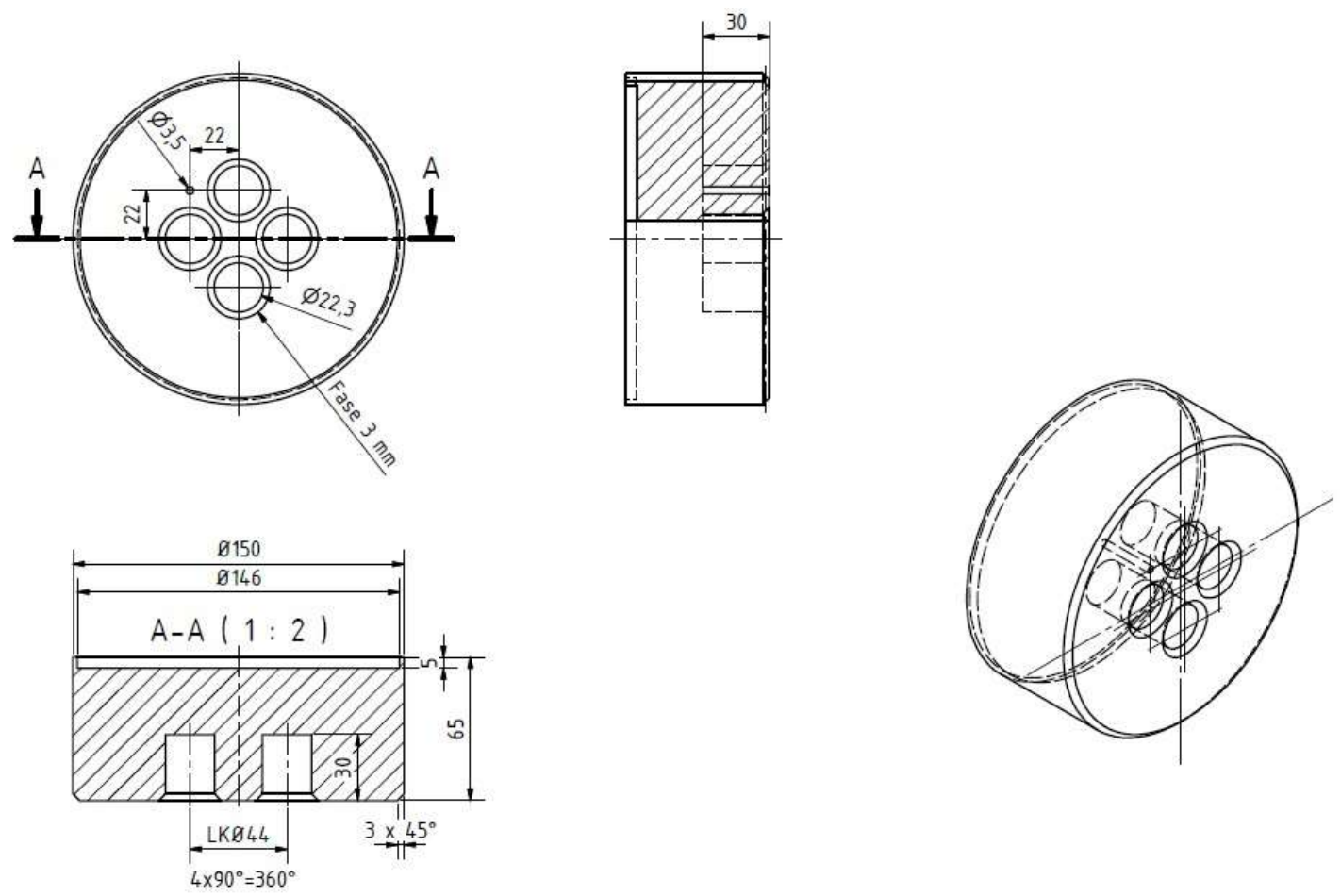

Figure 80. Technical drawing of the thermal reservoir. Values are given in millimeter. Technical drawings were performed by H.-J. Heymel. ${ }^{[274]}$ 

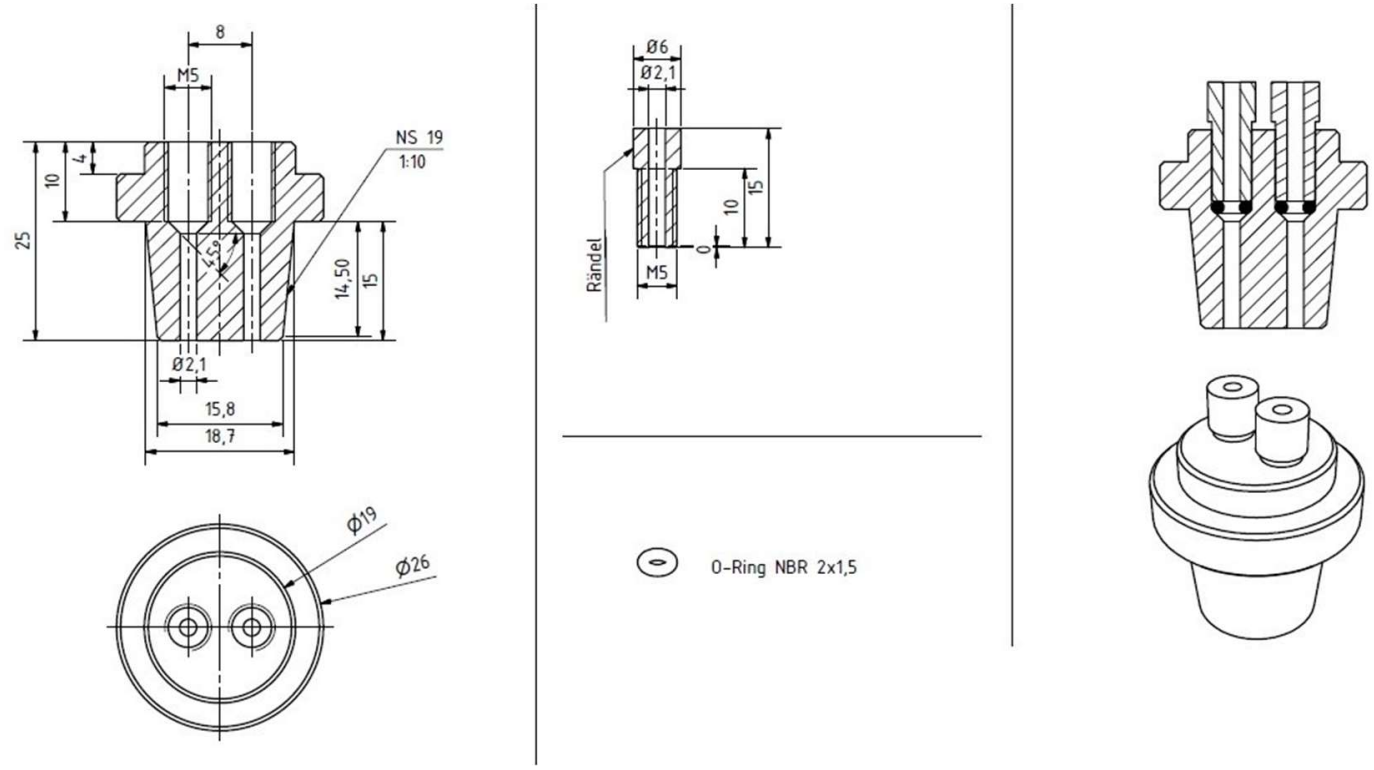

Figure 81. Technical drawing of the reaction vessel caps. Values are given in millimeter. Technical drawings were performed by H.-J. Heymel. ${ }^{[274]}$
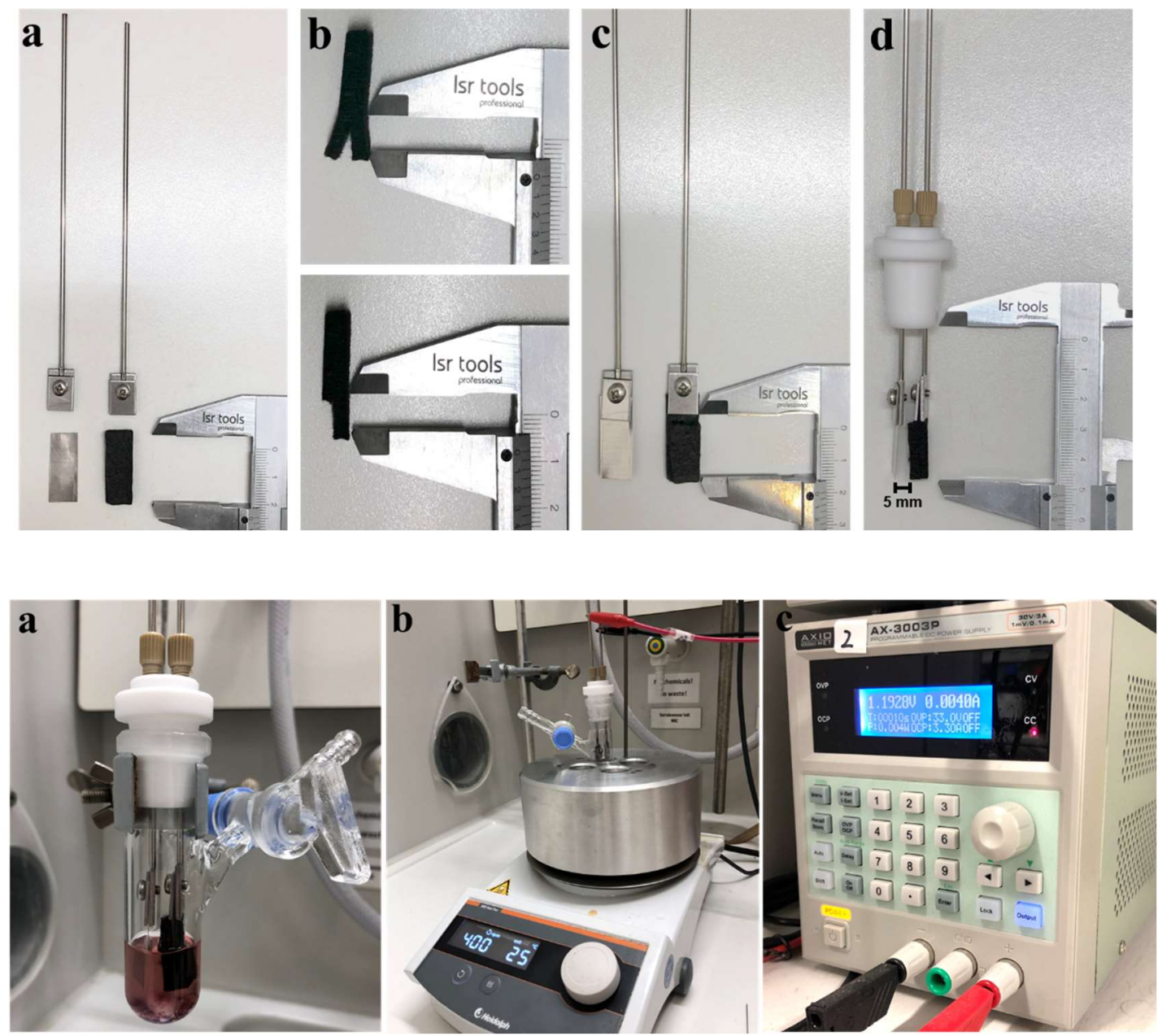

Figure 82. General reaction preparation for the cobaltaelectro-catalyzed C-H activation in an undivided cell. Photo credit: $M$. Stangier. ${ }^{[274]}$ 


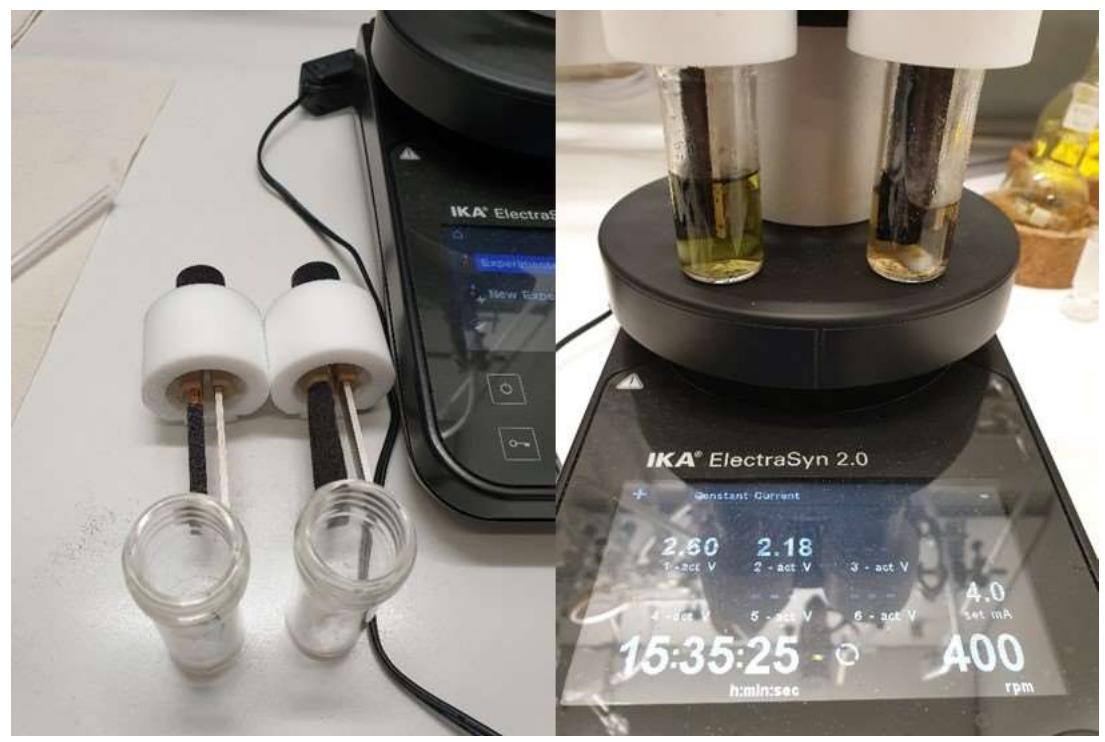

Figure 83. Equipment for the reactions performed with the IKA Electrasyn 2.0. ${ }^{[274]}$

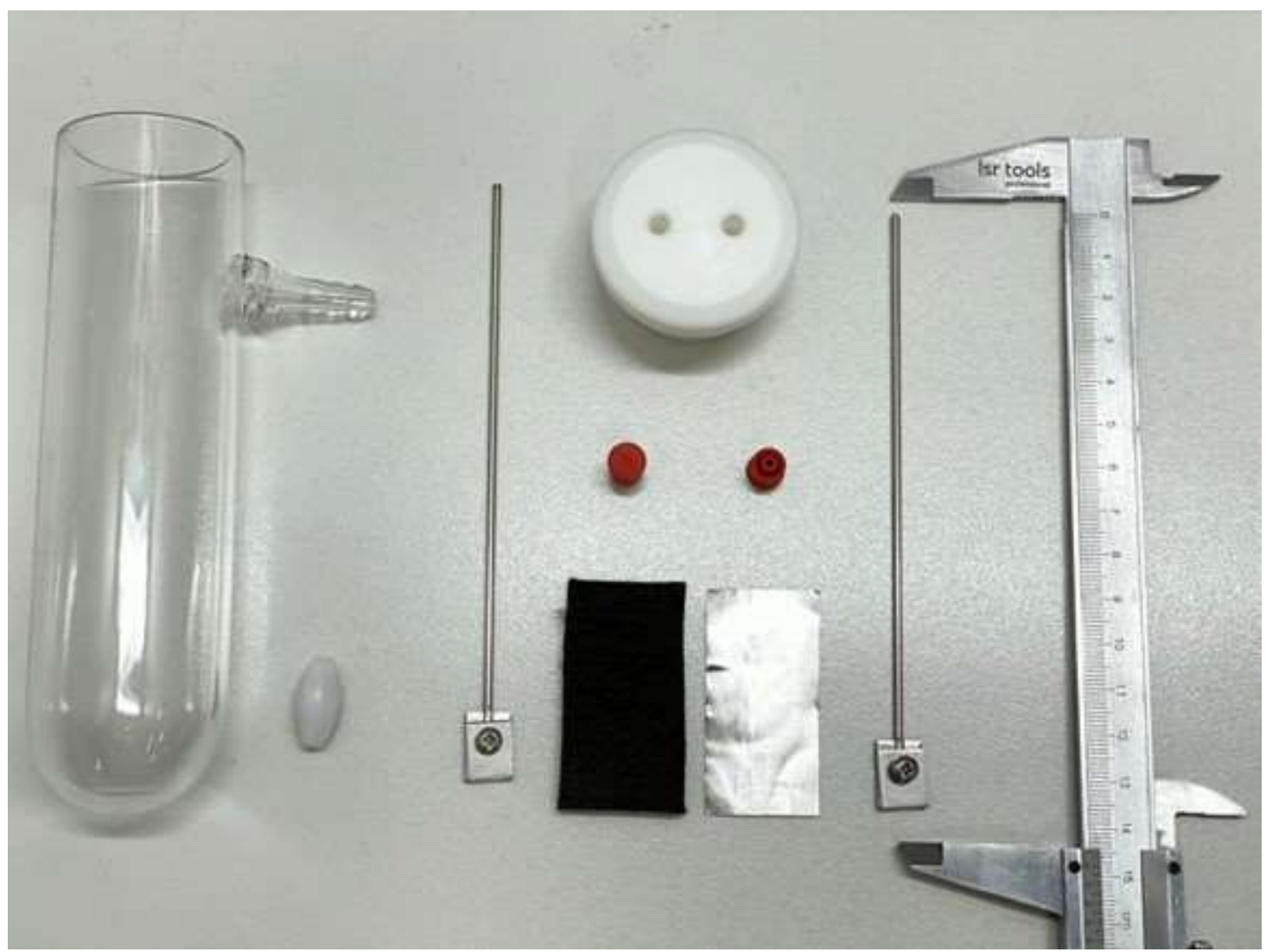

Figure 84. General equipment for the large-scale electrolysis. ${ }^{[274]}$ 


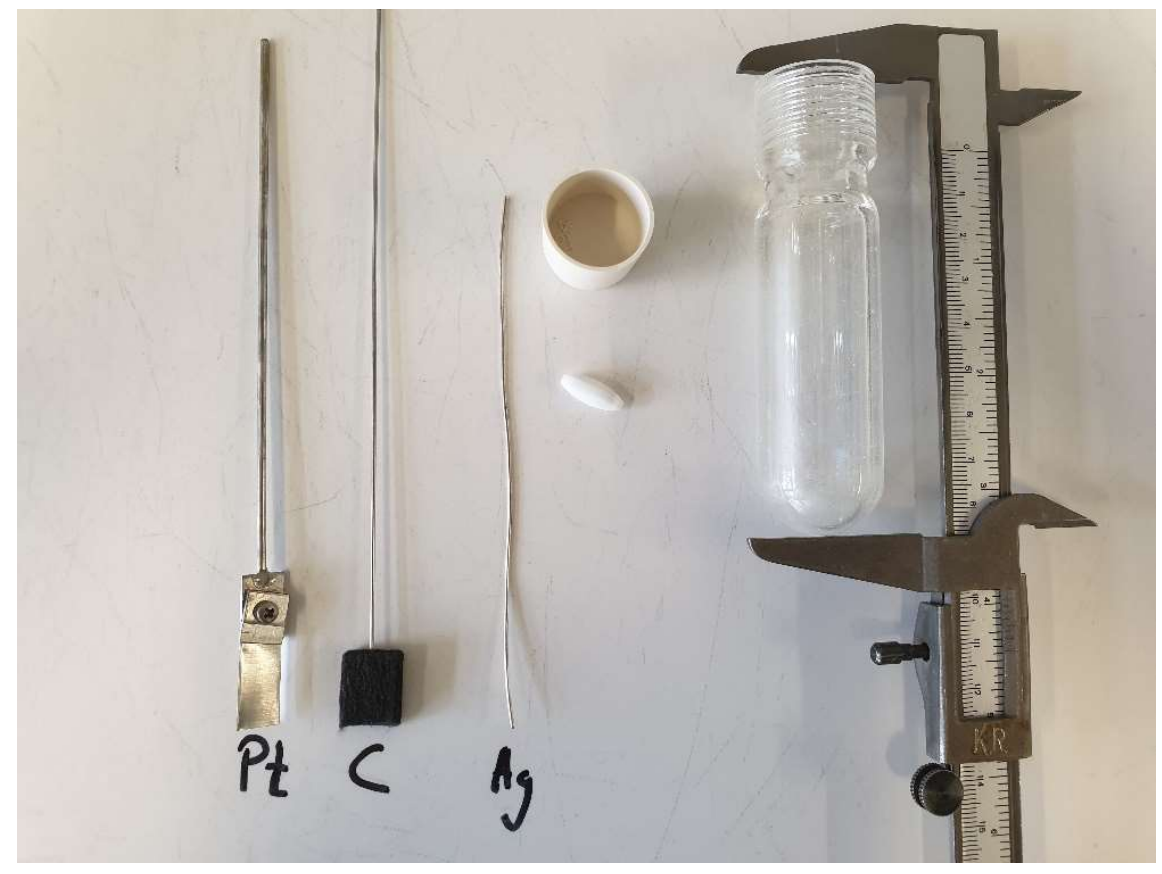

Figure 85. General equipment for the constant potential electrolysis. ${ }^{[309]}$ 